\title{
DATA SUPPLEMENT TO THE U.S. GEOLOGICAL SURVEY 1:2,000,000-SCALE MAP OF SHORELINE EROSION AND ACCRETION OF THE MID-ATLANTIC COAST
}

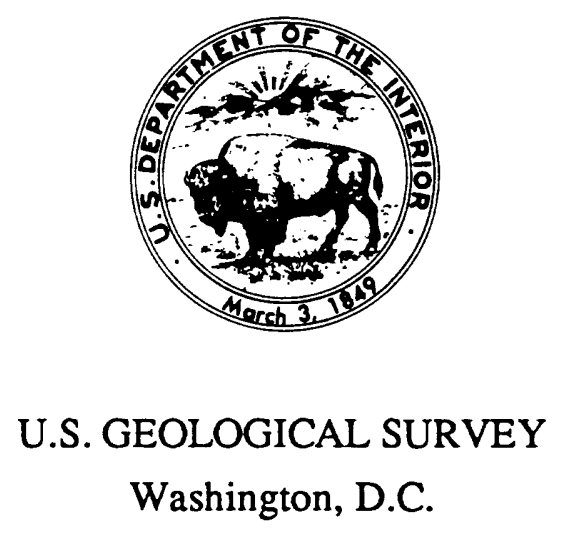

OPEN-FILE REPORT 92-377

1 December 1992

Robert Dolan and Judith Peatross

Department of Environmental Sciences

University of Virginia

Charlottesville, Virginia 22903 


\title{
DATA SUPPLEMENT TO THE U.S. GEOLOGICAL SURVEY 1:2,000,000-SCALE MAP OF SHORELINE EROSION AND ACCRETION OF THE MIDDLE ATLANTIC COAST
}

\author{
Robert Dolan and Judith Peatross \\ Department of Environmental Sciences \\ University of Virginia \\ Charlottesville, Virginia 22903
}

\section{INTRODUCTION}

In 1982, the U.S. Geological Survey published a 1:7,500,000-scale National Atlas map of the shoreline rates of erosion and accretion for the United States. A national data storage and retrieval system, termed the Coastal Erosion Information System (CEIS), was developed as part of that map compilation project (May and others, 1982). This report includes a tabulation and statistical summaries of the data in the CEIS used to compile the middle Atlantic coast map.

\section{THE COASTAL EROSION INFORMATION SYSTEM (CEIS)}

The CEIS was designed to provide a framework to store, manage, and analyze large volumes of geographically referenced data on shoreline rates of change. Shoreline data are stored in records identified by longitude and latitude. Each record contains the rate of shoreline change as well as supplemental information on the location, such as the county, the township, or key place names, and the name of the U.S. Geological Survey (USGS) 7.5 minute topographic quadrangle map covering the area.

No standardized methodology has been adopted by coastal engineers and scientists for recording and analyzing changes in shorelines. For this reason, the data in the CEIS were generated in many ways. Each data record includes reference to the methods used to delineate the shoreline changes and the methods that were used to calculate rates of change. There is no attempt to judge the accuracy or reliability of the data.

The shoreline change data used for the middle Atlantic coast map were obtained from several sources (table 1) and include records that span periods ranging from 20 to 165 years. Most of this information, which is listed in the bibliography that follows, was obtained in the form of published tabulated data. 


\section{Table 1}

\section{Sources of Data for the Middle Atlantic Coast Map, USGS, 1992}

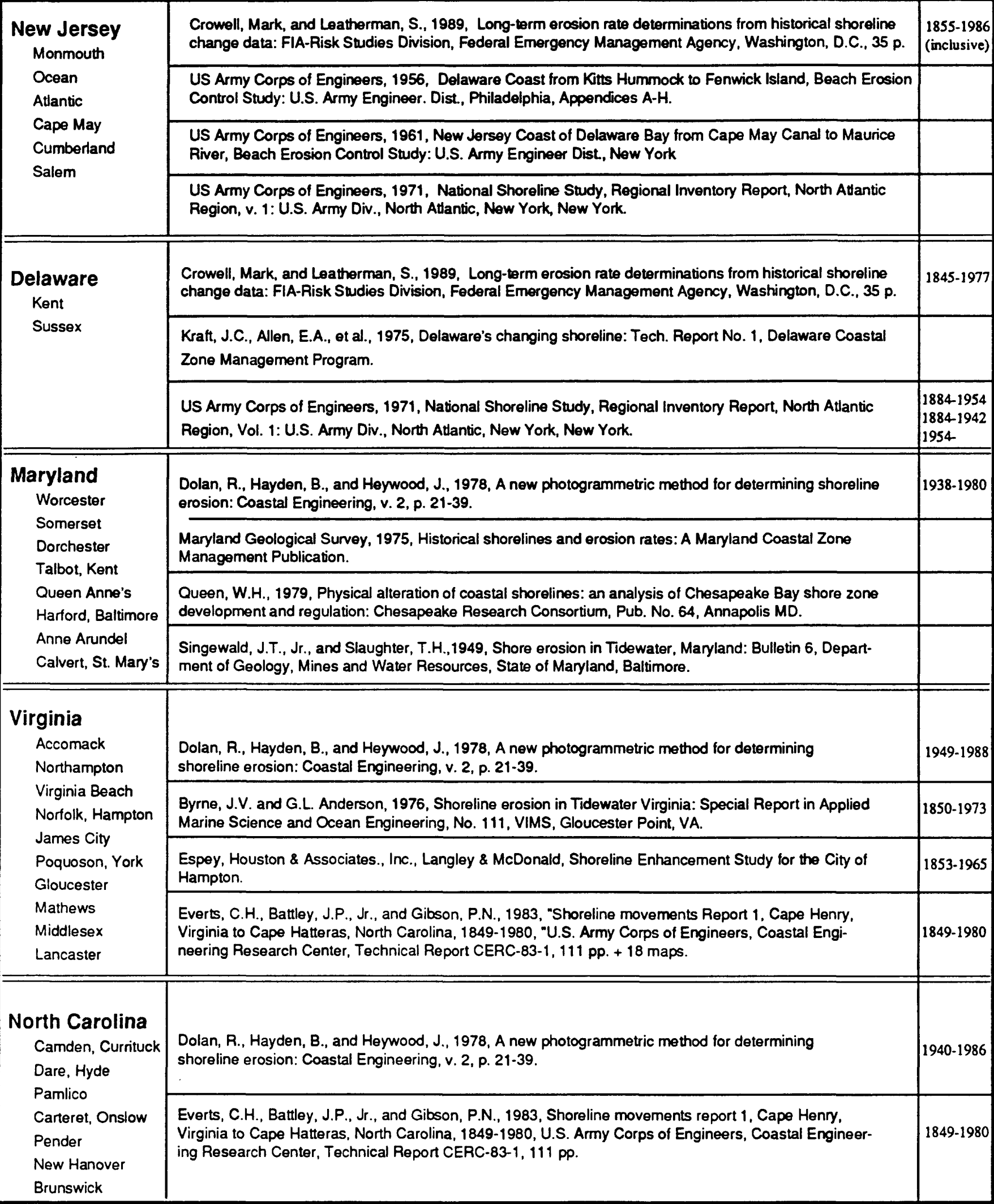




\section{REPRESENTING SHORELINE MOVEMENT WITH STATISTICS}

The shoreline rate-of-change statistic is expected to reflect a cumulative summary of the processes that have impacted the coast through time. The precision with which values reflect this summary is dependent on: (1) the accuracy of the original digitized or mapped shorelines, (2) the temporal variability of the shoreline, (3) the number of data points (measured shoreline positions) used in calculating the rate, (4) the proximity of each observation to the time of an actual change in the trend of shoreline movement, (5) the period of time between the shoreline measurements, (6) the total time span of shoreline data, and (7) the method used to calculate the rate.

The movement of the shoreline over time usually consists of a predictable component of variation that can be regarded as the trend and short-term variation, or noise. Long-term phenomena, such as a rise in sea level or a systematic change in the natural sediment supply, occur over periods of decades to centuries and produce the more predictable trends. Short-term variation, introduced over periods ranging from days to seasons (Anders and Byrnes, 1991; National Research Council, 1990), contribute to substantial fluctuations in the position of the shoreline. This suggests that both the long- and short-term trends are discernible, although it may be difficult to determine if the processes responsible for these changes function independently or dependently. Some coastal groups rely on long-term trends in shoreline change for policy decisions (Federal Emergency Management Agency, 1988; North Carolina Division of Coastal Management, 1986) and others use current or short-term trends (Savage and Foster, 1989).

Little quantitative information is available for estimating the reliability of long-term versus short-term rates of shoreline change for prediction (Morton, 1991; Dolan and others, 1991, 1992). Furthermore, the definition of long-term depends on the purpose of the investigation, the availability of data, and the temporal variability of the shoreline under study. Naturally, if a shoreline is undergoing change at a constant and uniform rate, the time interval is not an important consideration and the precision of the estimate of change would be high. In reality, few shorelines behave in this manner.

\section{SHORELINE MAPPING METHODS}

When developing statistics for predicting shoreline change from highly variable shoreline data, the reliability of the data used is a fundamental issue (Dolan and others, 1991, 1992). The first step in calculating rates of shoreline change is to measure the position of the shoreline as accurately as possible. The methods used to accomplish this procedure are numerous (Crowell and others, 1989; Dolan and others, 1978; Leatherman, 1983; Smith and Zarillo, 1990). The most common sources for shoreline data include aerial photographs, maps (quadrangles) produced by the USGS, nautical charts or topographic sheets (Tsheets) produced by the National Ocean Service, and beach profiles. Shoreline 
datums used include the high water line (Dolan and others, 1980), the approximate mean high water line (Foster and Savage, 1989), and the sedimentwater interface (White and Morton, 1987).

Shoreline datums for maps and charts vary (Crowell and others, 1991). The NOS uses the mean high water line on T-sheets. For aerial photographs, the actual shoreline position at any given time is dependent on the prevailing oceanographic conditions during the flight. Map and chart shorelines (derived from aerial photographs) are typically an average based on post-survey correction factors (National Research Council, 1990).

The measurement error associated with shoreline delineation methods is well-documented (Anders and Byrnes, 1991; Crowell and others, 1991; Dolan and others, 1980; Leatherman, 1983). Crowell and others (1991) point out that the implications of the photographic and mapping errors is more significant in areas where changes in shoreline positions are small.

Shoreline measurement errors are documented as $\pm 8.4 \mathrm{~m}$ (about $25 \mathrm{ft}$ ) for T-sheets mapped prior to the use of aerial photographs, $\pm 5.0 \mathrm{~m}$ (about $16 \mathrm{ft}$ ) for modern T-sheets, and $\pm 12.2 \mathrm{~m}(35 \mathrm{ft})$ for 1:24,000-scale USGS quadrangles (Crowell and others, 1991). For aerial photographs, measurement error is a function of scale and the quality of the photography. Crowell and others (1991) calculated, using a root-mean-square approach, that the error range for nontidal coordinated aerial photography $(1: 10,000)$ is approximately $\pm 7.0 \mathrm{~m},(23 \mathrm{ft})$, not including the potential error associated with interpretations of the high water line.

\section{DATA COLLECTION AND STORAGE}

For data used to compile this map, preference was given to regional or statewide data sets spanning a relatively long period of time. All of the data used for the open-ocean coastline of the middle Atlantic coast map was obtained from two high-spatial-resolution data sets (table 1): the "COASTS" data base, generated from aerial photographic analysis at the University of Virginia (Dolan and others, 1978), and the METRIC MAPPING data, compiled by the coastal research group at the University of Maryland (Leatherman, 1983; Crowell and Leatherman, 1989). COASTS data were used for the shorelines of North Carolina, Virginia, and Maryland; the METRIC MAPPING data were used for Delaware and New Jersey, and parts of Virginia and Maryland. Both of these data bases provide shoreline erosion rates at $150-\mathrm{ft}(50-\mathrm{m})$ intervals along the coast. Because the minimum mapping unit (along the coast) for a map of 1:2,000,000-scale is $3 \mathrm{mi}(5 \mathrm{~km})$, considerable smoothing and generalization were required to reduce the more than 20,000 individual values to obtain the patterns shown on the map. Several smaller data sets were used for the summaries of erosion and accretion along the shorelines of Chesapeake Bay and Delaware Bay. 


\section{PATTERNS OF EROSION AND ACCRETION: MIDDLE ATLANTIC STATES}

Table 2 and figure 1 summarize the patterns of shoreline erosion and accretion rates for the middle Atlantic States weighted by length of coastline. Overall, 46 percent of the 662 miles of open ocean-facing coastline from New Jersey to North Carolina is eroding, 17 percent is accreting, and 37 percent of the data is in the stable to minor rate-of-change category. Figures 2 a-d include color strip maps of the data and pie diagrams showing the State by State patterns of change. Twenty-two percent of New Jersey's shoreline, 93 percent of Delaware's, 74.5 percent of Maryland's, 49.5 percent of Virginia's, and 42 percent of North Carolina's shorelines are eroding at rates greater than $0.5 \mathrm{~m} / \mathrm{yr}(1.5 \mathrm{ft} / \mathrm{yr})$. The average rate of bay shoreline erosion is $1.6 \mathrm{~m} / \mathrm{yr}(5.2 \mathrm{ft} / \mathrm{yr})$ for the Chesapeake Bay and $1.1 \mathrm{~m} / \mathrm{yr}(3.6 \mathrm{ft} / \mathrm{yr})$ for Delaware Bay.

There are also numerous significant differences in shoreline erosion and accretion rates within states and between geomorphologic regions along the middle Atlantic coast. One example is given in figures $3 a$ and $b$. The erosion rate for the reach of shoreline south of Chesapeake Bay, between Cape Hatteras and Cape Henry, averages $1.7 \mathrm{~m} / \mathrm{yr}(5.1 \mathrm{ft} / \mathrm{yr})$; whereas, for the reach immediately north of the bay, between Cape Charles and Chincoteague (the Virginia barrier islands), the average is $3.7 \mathrm{~m} / \mathrm{yr}(11.2 \mathrm{ft} / \mathrm{yr})$. Explanations for these differences have challenged geologists and oceanographers for many years.

\section{THE DATA PROCESSING AND TABULATIONS}

As indicated earlier, two large data sets and several smaller ones were selected from the national CEIS data base to produce the color patterns of shoreline change shown on the middle Atlantic coast map (table 1). The patterns are based on average rates of erosion and accretion calculated at more than 20,000 transects (sites) measured from maps, charts, and aerial photographs spanning periods ranging from 20 to 150 years.

Although the CEIS was originally designed to store and analyze national shoreline trends in 3-minute cells $(3.5 \mathrm{mi})$ around the coastline, a hierarchical structure for data storage and retrieval was added that makes it possible to include data at varying spatial densities (fig. 4). For the middle Atlantic coast map, most of the data were selected from sources that are spaced at $50-\mathrm{m}(150-\mathrm{ft})$ intervals. Only shoreline change data from Delaware Bay and Chesapeake Bay are spaced at 5-km (3-mi) intervals.

To display the CEIS information graphically, the middle Atlantic data sets for New Jersey, Delaware, Maryland, Virginia, and North Carolina were transferred from the CEIS PC-based system to a Macintosh-based system. The following fields from each CEIS record were selected for transfer: (1) transect number, (2) average rate of shoreline change, (3) latitude and longitude, (4) county and state, (5) source of information, (6) period of record, and (7) the spacing level along the coast (spatial resolution). These records were grouped by State for the first phase of the smoothing. Atlas Mapmaker 4.52 and Excel 4.0 were used to transfer and cluster the individual records into the patterns shown. 


\begin{tabular}{|l|llll|}
\hline \multicolumn{5}{|c|}{$\begin{array}{c}\text { Table 2 } \\
\text { Summary of Shoreline Erosion and Accretion by States }\end{array}$} \\
\hline $\begin{array}{c}\text { STATES } \\
\text { (excluding Bays) }\end{array}$ & Shoreline (mi) & Erosion (\%) & Accretion (\%) & Minor/Stable (\%) \\
\hline \hline New Jersey & 130 & 22.6 & 27.4 & 50.2 \\
Delaware & 25 & 92.5 & 7.5 & 7.4 \\
Maryland & 31 & 74.5 & 0.6 & 25.0 \\
Virginia & 159 & 49.5 & 19.4 & 31.1 \\
North Carolina & 317 & 48.2 & 14.6 & 37.3 \\
\hline \hline BAYS & Shoreline (mi) & Erosion (\%) & Accretion (\%) & Minor/Stable (\%) \\
\hline \hline
\end{tabular}




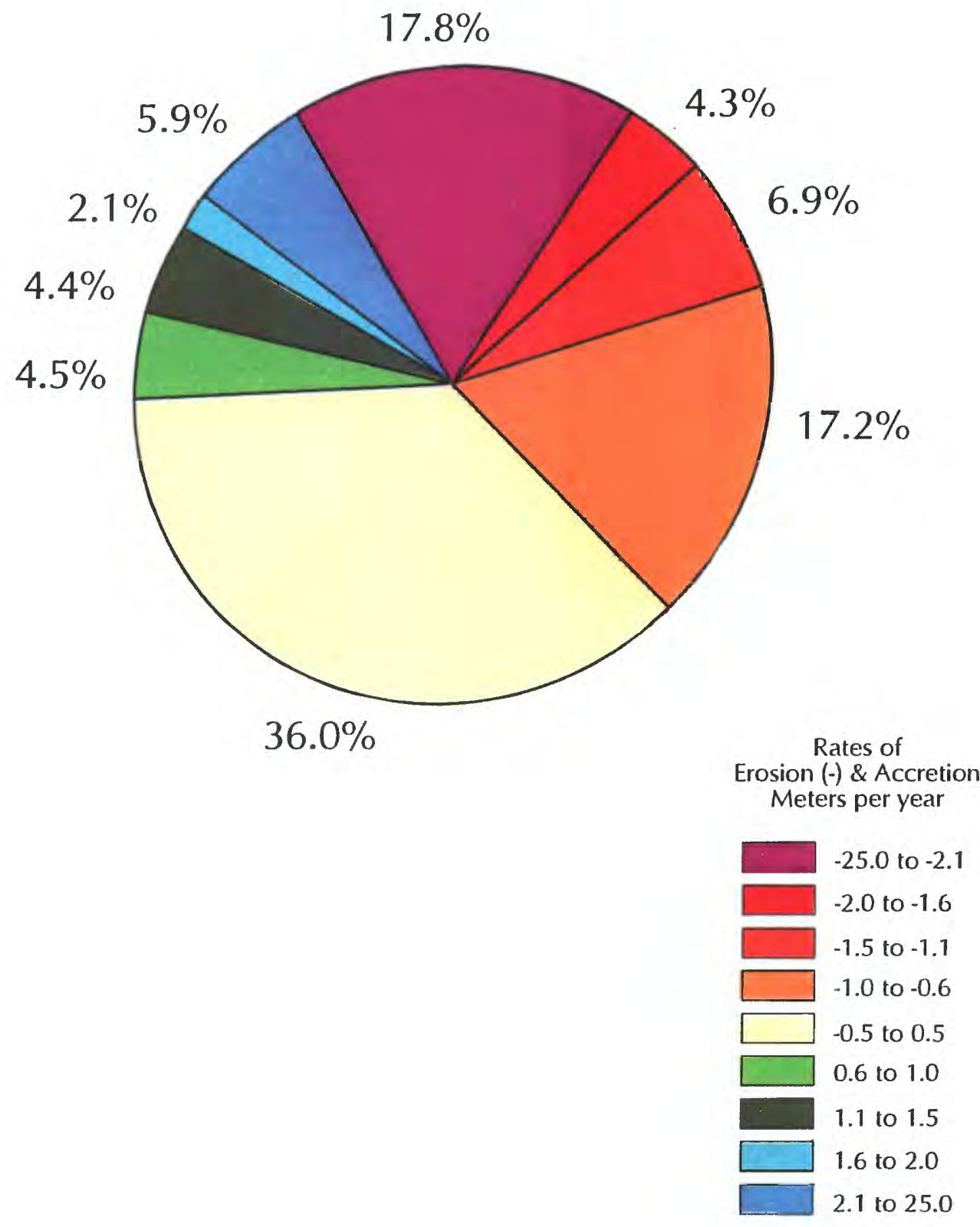

\title{
DATA FOR MIDDLE ATLANTIC COAST WEIGHTED BY LENGTH OF COAST
}

\author{
FIGURE 1
}




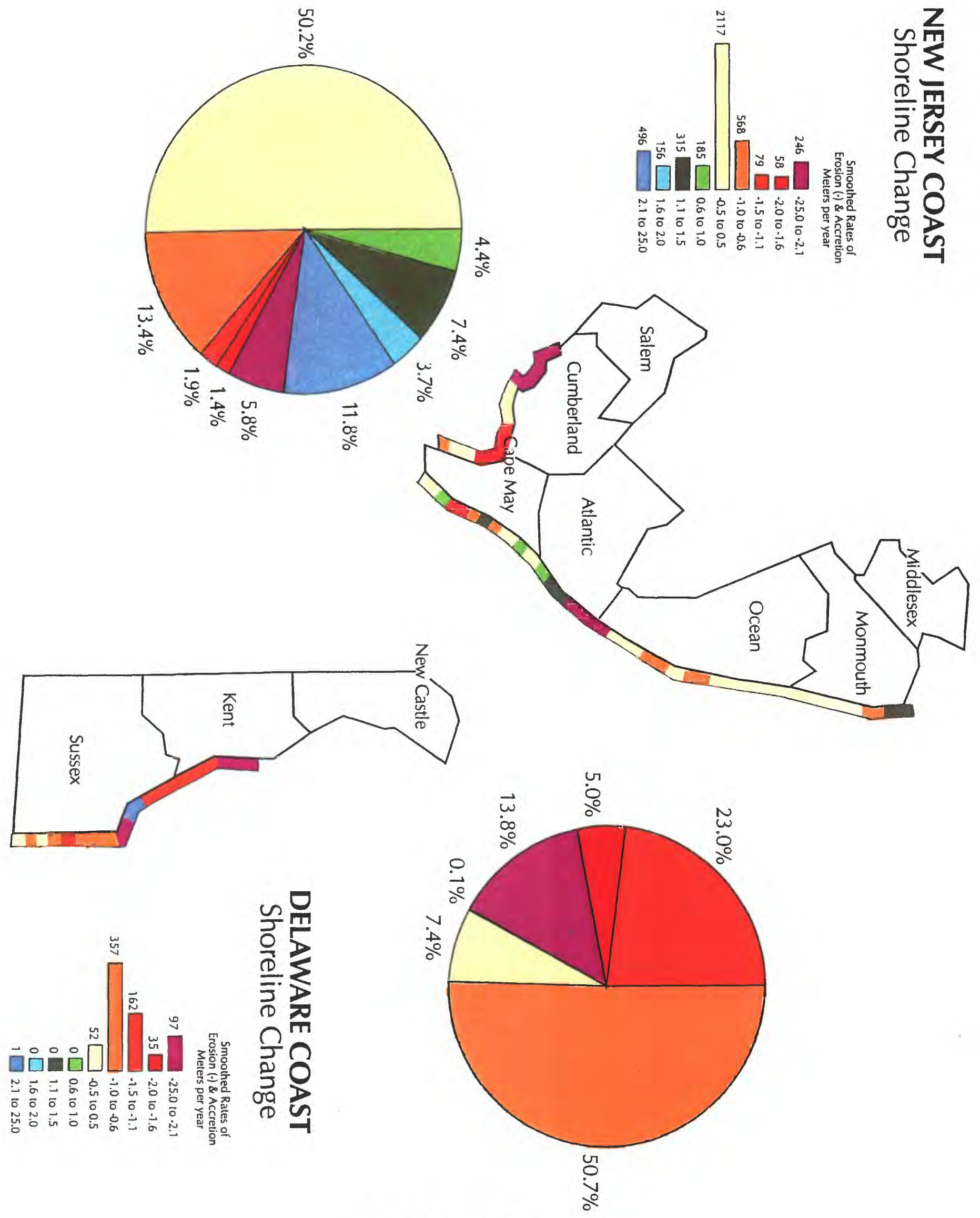

FIGURE 2A 


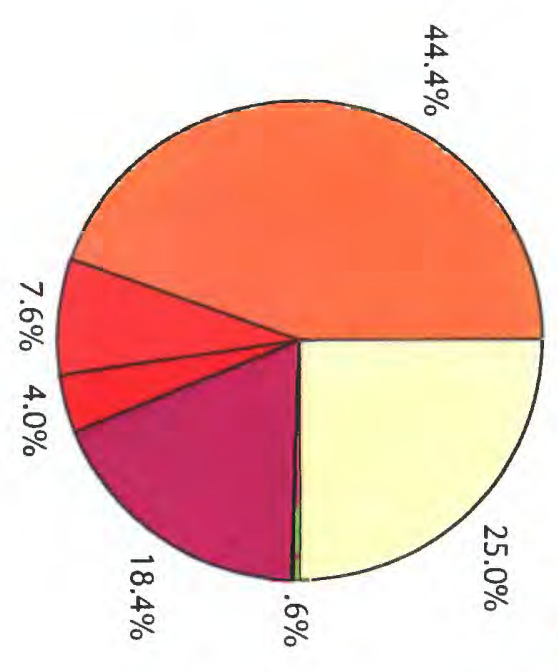

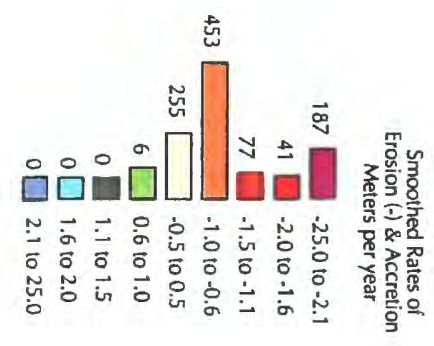
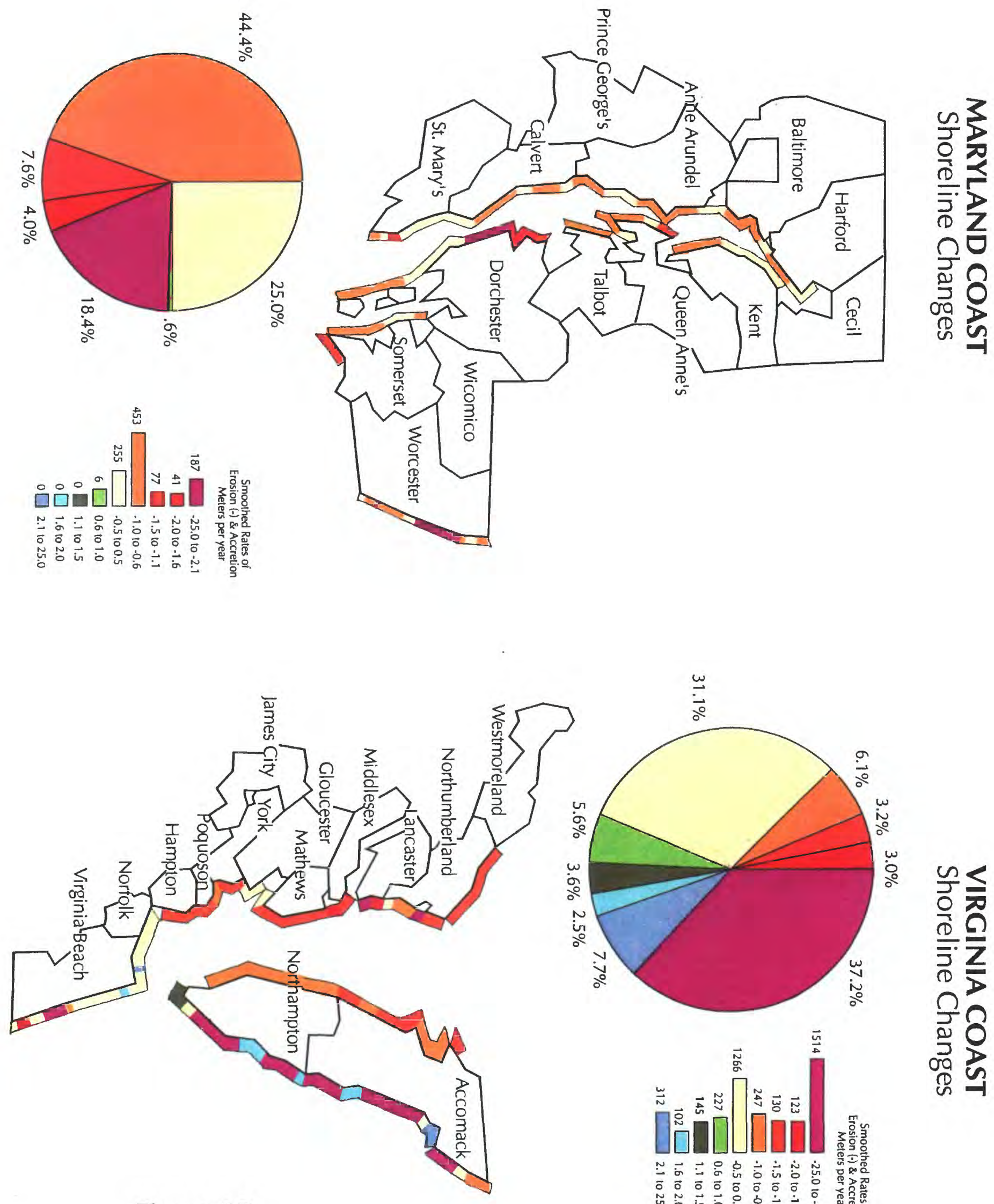

Figure $2 B$

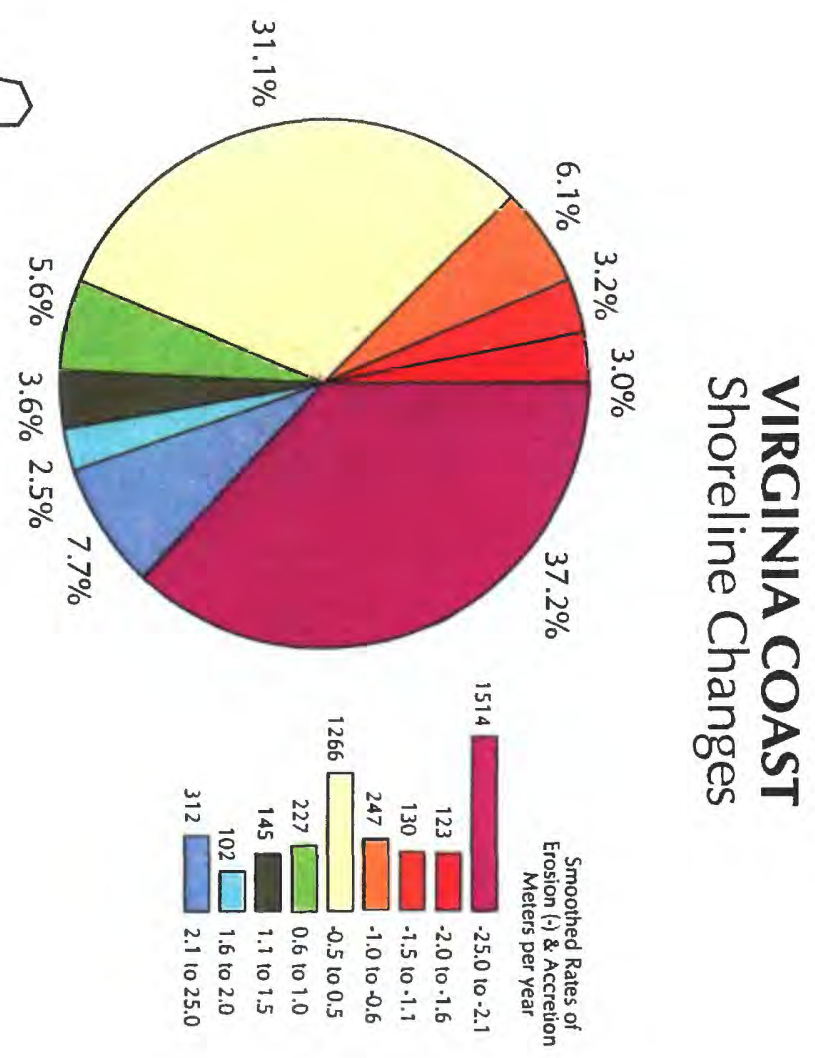




\section{NORTH CAROLINA COAST \\ Shoreline Changes}

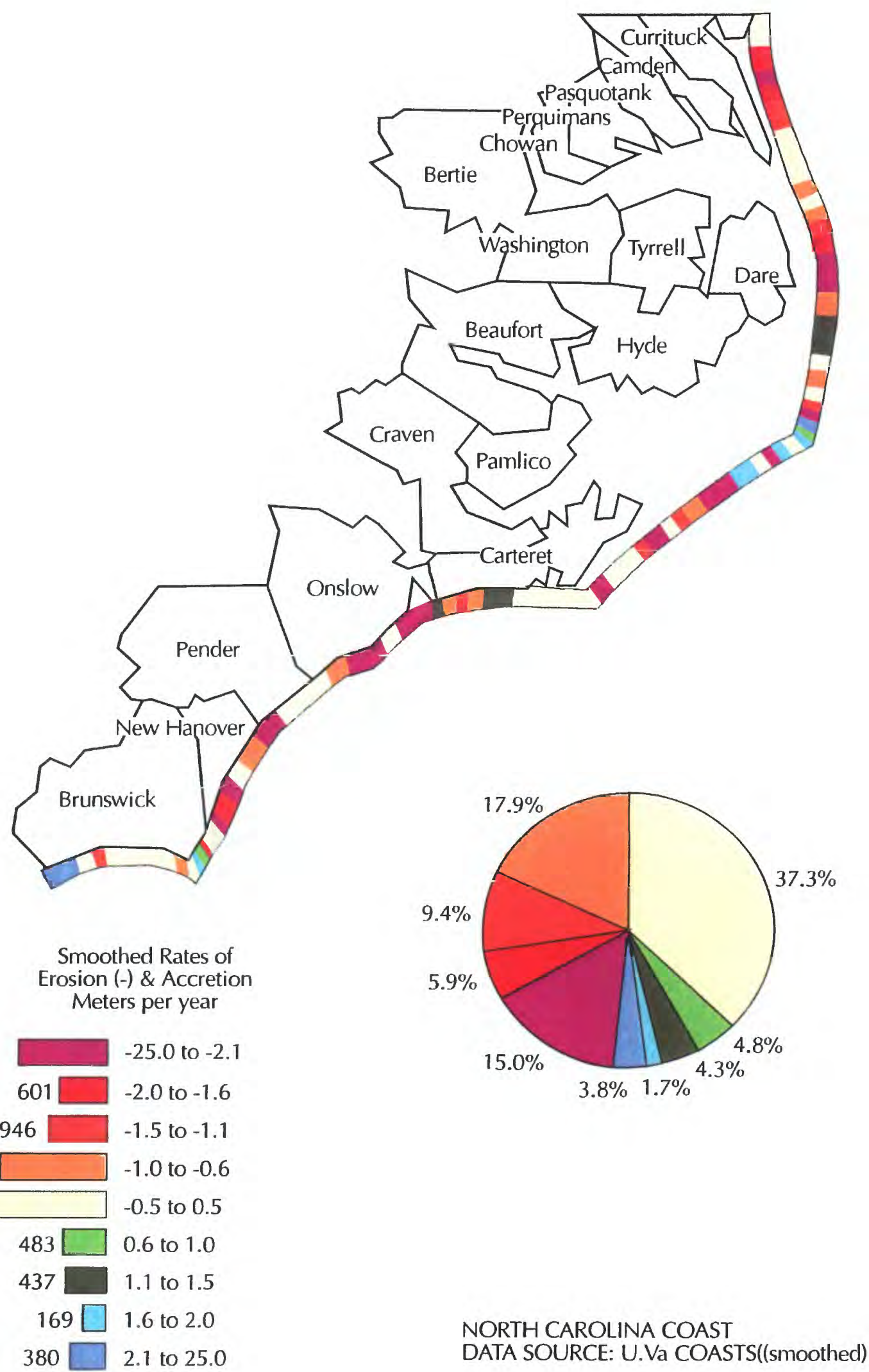




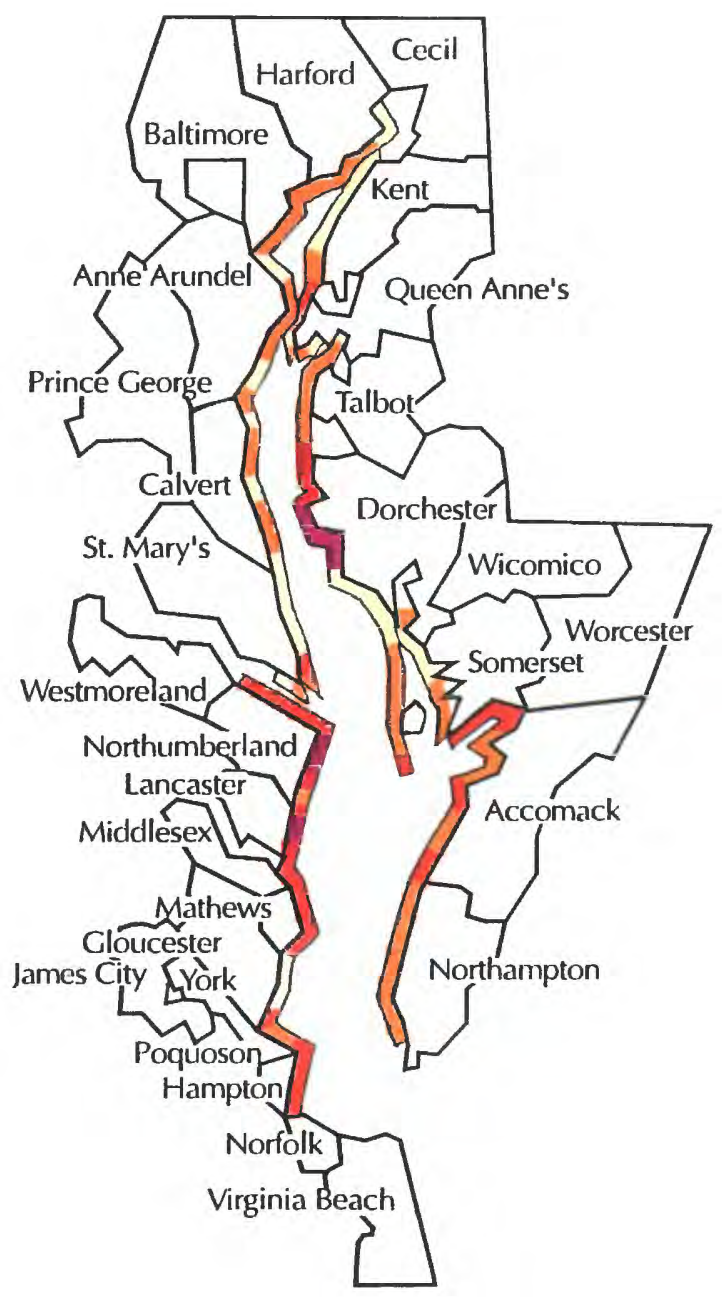

\section{CHESAPEAKE BAY AREA \\ Shoreline Change}

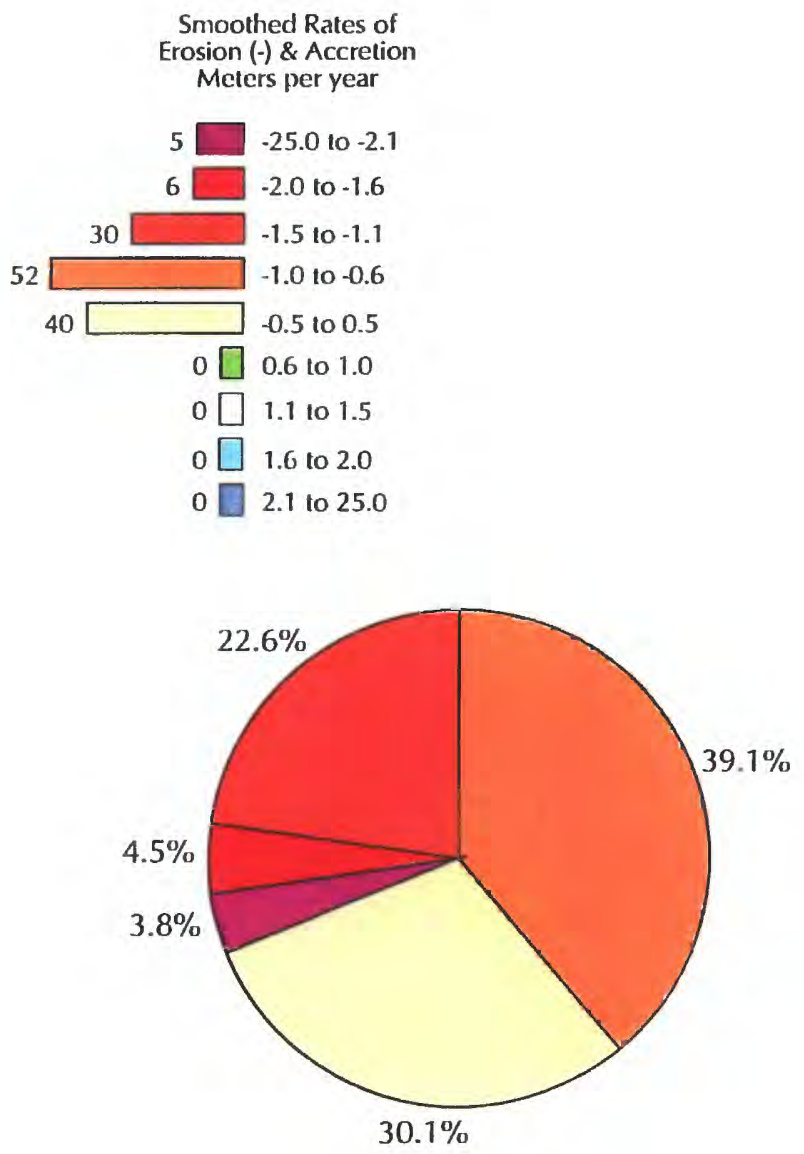

\section{DELAWARE \& NEW JERSEY BAY AREA Shoreline Change}

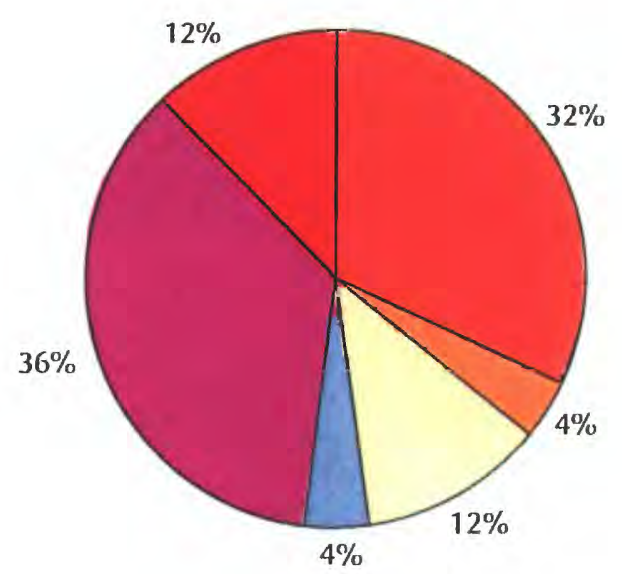

FIGURE 2D

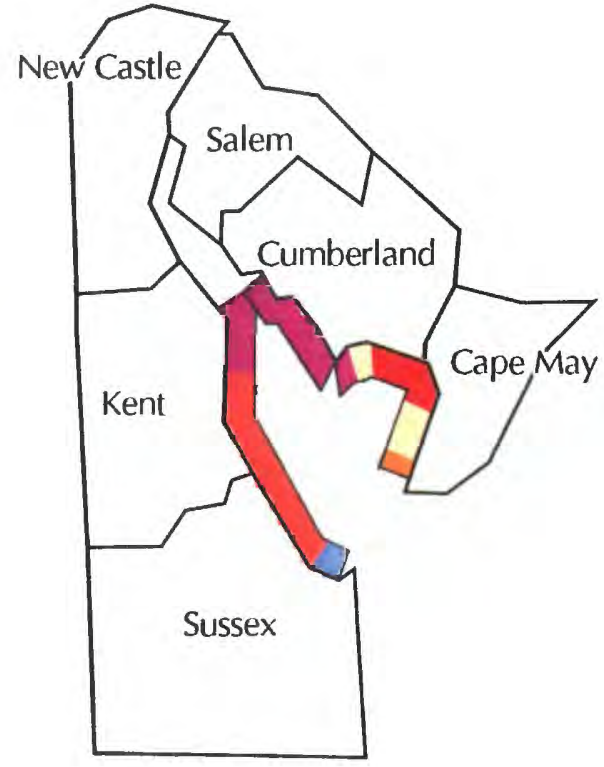




\section{CAPE HENRY, VA to CAPE HATTERAS \\ Shoreline Change}
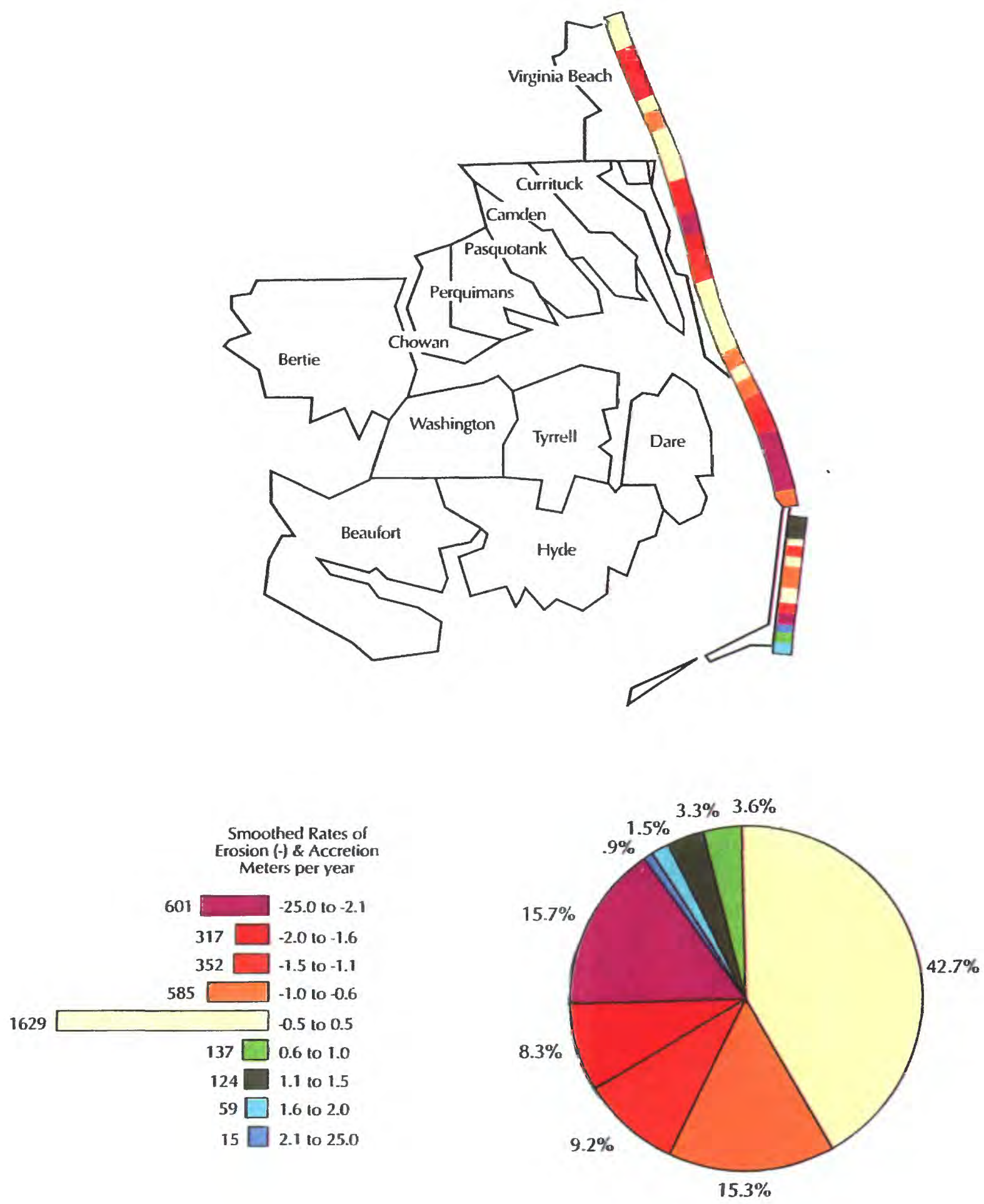

FIGURE 3A 


\section{CHINCOTEAGUE to CAPE CHARLES, VIRGINIA \\ Shoreline Change}

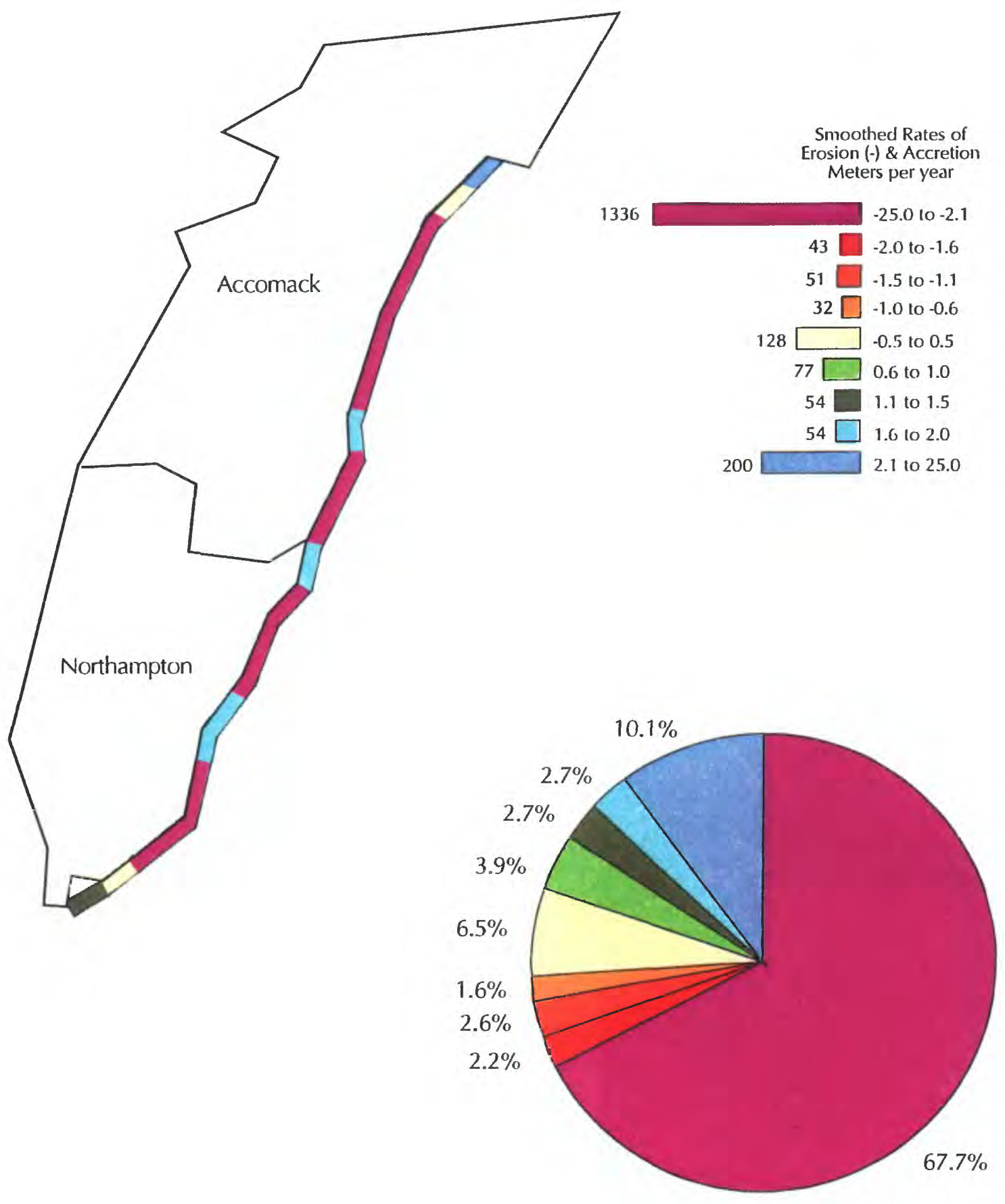




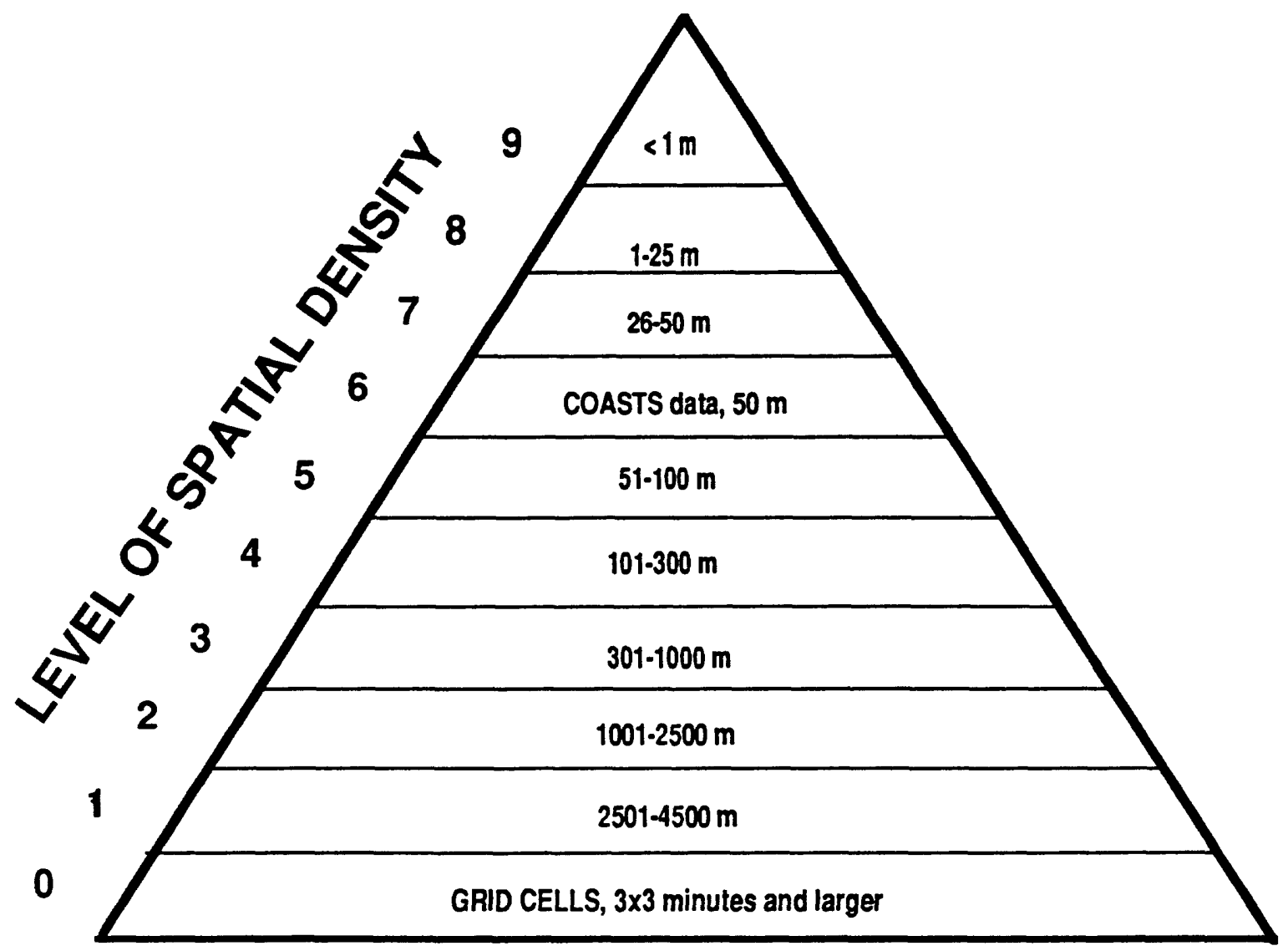

PYRAMIDAL DATA FILE STRUCTURE

FIGURE 4 
All transect locations were plotted by the computer as points on a boundary file along the shore side of each coastal county (see color strip maps shown as figure 2). A smoothed data value associated with each transect was imported and placed, as a colored symbol, in one of the nine rate categories. Each smoothed data rate was a transformed value, representing a running average of a 98-transect (3-mi) segment bracketing each point in succession. The point symbols were clustered into similar color categories which facilitated a visual distinction among the shoreline rates plotted on the computer-generated maps. For reaches of the coast where similar patterns of change were not easily discerned, a 5-km (3-mi) minimum mapping unit was used to group adjacent transects into a representative pattern.

The color strip for the middle Atlantic coast map created from the above method contains 102 segments of variable length, visually grouped into one of nine shoreline change categories. In total, these segments, which are positioned parallel to the shore, represent nearly 20,000 data points at $50-\mathrm{m}(150-\mathrm{ft})$ intervals along the coast from the northern boundary of New Jersey to the southern boundary of North Carolina. The main advantage of this method -- color coding and clustering individual transect values -- is that the results, displayed parallel to the coastline, are an easily understood depiction of shoreline change trends. (Note: The minor differences between the color patterns in the 1:2,000,000-scale map and the color-strip maps presented in this report are due to differences in methodologies.)

While visual displays of clustered categories are useful to a wide group of individuals, quantitative interpretation of categorized data is not acceptable. A pilot methodology to quantify the trends of shoreline change was developed for this project. Using latitude divisions (0.04 degrees) corresponding to the minimum mapping unit of the 1:2,000,000-scale map, a representative vertical shore profile was produced. The arithmetic mean for all data within each segment in the profile was calculated and displayed in the color category corresponding to the mean rate.

The principal advantage of the profile method is that each segment represents a numerically defined unit and the profile can be described using standard statistics. The main disadvantage, of course, is that the histogram does not parallel the coastline. For sections of the coastline oriented north/south, the two methods display similar trends in shoreline change. However, in reaches of coast where the actual shore orientation diverges from the north/south histogram abstraction, the three-mile segments are derived from a greater number of data points. For the southern third of the histogram, for example, below Cape Hatteras, a greater smoothing of information is represented by the three-mile segments. Individual extremes in erosion and accretion cancel each other out.

The data used for the middle Atlantic coast map are appended in hard copy to this report, and a high density floppy disk is included. The data are arranged in rows, from north to south, showing transect number, rate of change, and latitude and longitude (expressed with a negative sign for the western hemisphere). 


\section{ACKNOWLEDGMENTS}

Several individuals and groups have been involved in the production of the middle Atlantic coast map and data supplement. The authors acknowledge the contributions of Mark Crowell; Stephen Leatherman; Bruce Hayden; J.V. Byrne; G.L. Anderson; Espey, Houston \& Associates; J.T. Singewald, Jr.; T.H. Slaughter; and the U.S. Army Corps of Engineers. In addition, other individuals working on the map included Coastal Research Associate staff members, Jeannie Lewis Smith, Julia Bryce, and David Beaudry. Special appreciation is given to Robert Smith for the map design and cartographic work. Finally, we thank Max Ethridge, Richard Witmer, and Larry Amos, the National Mapping Division, U.S. Geological Survey, Reston, for their encouragement and support during the duration of the project. 


\section{REFERENCES CITED}

Anders, F.J., and Byrnes, M.R., 1991, Accuracy of shoreline change rates as determined from maps and aerial photographs: Shore and Beach, v. 59 , no. 1, p. 17-26.

Crowell, M., and Leatherman, S., 1989, Long-term erosion rate determinations from historical shoreline change data: Federal Insurance Agency Risk Studies Division, Federal Emergency Management Agency, Washington, D.C., 35 p.

Crowell, M., Leatherman, S., and Buckley, M., 1991, Historical shoreline change error analysis and mapping accuracy: Journal of Coastal Research, v. 7, no. 3, p. 839-852.

Dolan, R., Hayden, B., and Heywood, J., 1978, A new photogrammetric method for determining shoreline erosion: Coastal Engineering, v. 2, p. 21-39.

Dolan, R., Hayden, B., May, P., and May, S., 1980, The reliability of shoreline change measurements from aerial photographs: Shore and Beach, v. 48, p. 22-29.

Dolan, R., Fenster, M.S., and Holme, S.J., 1991, Temporal analysis of shoreline recession and accretion: Journal of Coastal Research, v. 7, no. 3, p. 723-744.

Dolan, R., Fenster, M.S., and Holme, S.J., 1992, Spatial analysis of shoreline recession and accretion: Journal of Coastal Research, v. 8 , no. 2, p. 263-285.

Federal Emergency Management Agency, 1988, Code of Federal regulations, chapter 1, part 63: Washington, D.C.

Foster, E.R., and Savage, R.J., 1989, Methods of historical shoreline analysis, in, Magoon, O.T., Converse, H., Miner, D., Tobin, L.T., and Clark, D. (eds.), Coastal Zone '89, v. 5: American Society of Civil Engineers, p. 4434-4448.

Leatherman, S., 1983, Shoreline mapping - a comparison of techniques: Shore and Beach, v. 51, p. 28-33.

May, S., Kimball, W., Grandy, N., and Dolan, R., 1982, The coastal erosion information system (CEIS): Shore and Beach, v. 50, no. 1, p. 19-25.

Morton, R.A., 1991, Accurate shoreline mapping: past, present, and future: Coastal Sediments '91, American Society of Civil Engineers, v. 1, p. 997-1010.

National Research Council, 1990, Managing coastal erosion: National Academy Press, Washington, D.C., 182 p. 
North Carolina Division of Coastal Management, 1986, Long term average annual erosion rates updated through 1986: North Carolina Division of Coastal Zone Management, Raleigh, 8 p.

Savage, R.J., and Foster, E.R., 1989, Historical shoreline change in southeast Florida, in, Magoon, O.T., Converse, H., Miner, D., Tobin, L.T., and Clark, D. (eds.), Coastal Zone '89, v. 5: American Society of Civil Engineers, p. 4406-4433.

Smith, G.L., and Zarillo, G.A., 1990, Calculating long-term shoreline recession rates using aerial photographic and beach profiling techniques: Journal of Coastal Research, v. 6, no. 1, p. 111-120.

White, W.A., and Morton, R.A., 1987, Historical shoreline changes in San Antonio, Espiritu Santu, and Mesquite Bays, Texas Gulf Coast: Bureau of Economic Geology, University of Texas, Austin, Geological Circular 87-1, 41 p. 


\section{THE DATA TABULATION}

1) All transect rates are in meters per year.

2) Longitude is expressed as a negative for transects in the western hemishere.

3) The tabulation of the data does not necessarily reflect a geographic continuum along the coast because the transects are sorted and printed by latitude and longitude. Adjacent rows in the data tabulation may refer to opposite sides of a barrier island, for example. Therefore, abrupt changes in rate from one transect to the next may be attributed to a complicated configuration of the shoreline from the ocean to the bays.

4) The majority of values reflect rates based on thirty or more years of data, but in some areas such as the Virginia Barrier Islands, the length of record is much shorter; therefore, those data points are of questionable reliability.

5) The symbol "NA" in the data tabulation indicates questionable data points. 


\begin{tabular}{|c|c|c|c|c|c|c|c|c|c|c|c|c|c|c|}
\hline TR\# & RATE & LONG & LAT & ST & TR\# & RATE & LONG & LAT & ST & TR\# & RATE & LONG & LAT & ST \\
\hline 1 & 2.81 & -74.00681 & 40.47515 & NJ & 73 & 2.49 & -73.98431 & 40.45031 & $\mathrm{NJ}$ & 145 & -1.55 & -73.98247 & 40.4188 & NJ \\
\hline 2 & 2.59 & -74.00632 & 40.47492 & $\mathrm{NJ}$ & 74 & 2.55 & -73.98407 & 40.4499 & NJ & 146 & -1.52 & -73.98242 & 40.41835 & NJ \\
\hline 3 & 2.53 & -74.00572 & 40.47479 & $\mathrm{NJ}$ & 75 & 2.53 & -73.98392 & 40.44946 & NJ & 147 & -1.38 & -73.98216 & 40.41792 & NJ \\
\hline 4 & 2.5 & -74.00513 & 40.47466 & NJ & 76 & 2.65 & -73.98367 & 40.44904 & NJ & 148 & -1.02 & -73.9816 & 40.41753 & $\mathrm{NJ}$ \\
\hline 5 & 2.3 & .74 .00459 & 40.47447 & NJ & 77 & 2.8 & -73.98346 & 40.44862 & NJ & 149 & 0.04 & -73.97997 & 40.41725 & $\mathrm{NJ}$ \\
\hline 6 & 2.02 & -74.0041 & 40.47423 & NJ & 78 & 2.89 & -73.98328 & 40.44819 & NJ & 150 & 0.22 & -73.97971 & 40.41682 & NJ \\
\hline 7 & 1.73 & -74.00359 & 40.474 & NJ & 79 & 2.94 & -73.98311 & 40.44775 & NJ & 151 & 0.18 & -73.97974 & 40.41636 & NJ \\
\hline 8 & 1.67 & -74.0031 & 40.47375 & NJ & 80 & 2.95 & -73.98302 & 40.44729 & NJ & 152 & 0.06 & -73.97987 & 40.41589 & $\mathrm{NJ}$ \\
\hline 9 & 3.29 & -73.9977 & 40.47358 & NJ & 81 & 3.01 & -73.98289 & 40.44683 & NJ & 153 & -0.08 & -73.97998 & 40.41543 & $\mathrm{NJ}$ \\
\hline 10 & 1.67 & -74.00259 & 40.4735 & NJ & 82 & 2.99 & -73.98283 & 40.44635 & NJ & 154 & -0.18 & -73.98007 & 40.41496 & $\mathrm{NJ}$ \\
\hline 11 & 1.52 & .74 .00214 & 40.47321 & NJ & 83 & 2.83 & -73.98277 & 40.44604 & NJ & 155 & -0.28 & -73.98009 & 40.41451 & $\mathrm{NJ}$ \\
\hline 12 & 3.15 & -73.99738 & 40.47312 & NJ & 84 & 2.94 & -73.98277 & 40.44588 & NJ & 156 & -0.35 & -73.97997 & 40.41406 & $\mathrm{NJ}$ \\
\hline 13 & 1.36 & -74.00166 & 540.47294 & NJ & 85 & 2.8 & -73.98276 & 540.44559 & NJ & 157 & 0.13 & -73.97935 & 40.41367 & NJ \\
\hline 14 & 1.28 & -74.00119 & 40.47268 & NJ & 86 & 2.82 & .73 .98277 & 740.44538 & NJ & 158 & -0.19 & -73.97935 & 40.41324 & $\mathrm{NJ}$ \\
\hline 15 & 2.98 & -73.99712 & 40.47256 & NJ & 87 & 2.75 & -73.98276 & 540.44513 & NJ & 159 & -0.2 & -73.97935 & 40.41322 & $\mathrm{NJ}$ \\
\hline 16 & 1.28 & -74.00072 & 40.47241 & $\mathrm{NJ}$ & 88 & 2.69 & -73.98271 & 40.44469 & NJ & 160 & -0.32 & -73.97942 & 40.41279 & $\mathrm{NJ}$ \\
\hline 17 & 1.15 & -74.00035 & 40.472 & $\mathrm{NJ}$ & 89 & 2.54 & -73.98267 & 70.44423 & NJ & 161 & -0.48 & -73.97946 & 40.41234 & $\mathrm{NJ}$ \\
\hline 18 & 2.78 & -73.99695 & 40.47192 & NJ & 90 & 2.41 & -73.98259 & 40.44379 & NJ & 162 & -0.58 & -73.97945 & 40.41189 & $\mathrm{NJ}$ \\
\hline 19 & 1.03 & -74.00002 & 40.47157 & $\mathrm{NJ}$ & 91 & 2.3 & -73.98251 & 40.44334 & NJ & 163 & -0.78 & -73.97939 & 40.41144 & $\mathrm{NJ}$ \\
\hline 20 & 2.52 & -73.99684 & 40.47121 & $\mathrm{NJ}$ & 92 & 2.21 & -73.98241 & 40.4429 & NJ & 164 & -0.89 & -73.97926 & 40.41099 & $\mathrm{NJ}$ \\
\hline 21 & 2.2 & -73.99673 & 40.47046 & NJ & 93 & 2.11 & -73.98228 & 340.44246 & $\mathrm{NJ}$ & 165 & -0.84 & -73.9789 & 40.41056 & $\mathrm{NJ}$ \\
\hline 22 & 1.77 & .73 .99672 & 40.46964 & NJ & 94 & 1.99 & -73.98215 & 540.44202 & $\mathrm{NJ}$ & 166 & -0.91 & -73.97893 & 40.41011 & $\mathrm{NJ}$ \\
\hline 23 & 1.46 & -73.99672 & 40.46924 & NJ & 95 & 1.88 & -73.98206 & 60.44157 & $\mathrm{NJ}$ & 167 & -0.89 & -73.97887 & 40.40966 & $\mathrm{NJ}$ \\
\hline 24 & 1.15 & -73.9968 & 40.46871 & NJ & 96 & 1.2 & -73.98201 & 40.44112 & NJ & 168 & -0.87 & .73 .9789 & 40.40921 & $\mathrm{NJ}$ \\
\hline 25 & 1.29 & -73.9968 & 40.46868 & NJ & 97 & 1.11 & -73.98196 & 40.44067 & NJ & 169 & -0.86 & -73.97871 & 40.40876 & $\mathrm{NJ}$ \\
\hline 26 & 1.37 & -73.99644 & 40.46828 & NJ & 98 & 1 & -73.98198 & 340.44022 & NJ & 170 & -0.88 & -73.97855 & 40.40832 & $\mathrm{NJ}$ \\
\hline 27 & 0.95 & -73.99672 & 40.46824 & $\mathrm{NJ}$ & 99 & 0.88 & -73.98203 & 40.43976 & $\mathrm{NJ}$ & 171 & -0.86 & -73.97836 & 40.40788 & $\mathrm{NJ}$ \\
\hline 28 & 0.78 & -73.99661 & 40.46778 & $\mathrm{NJ}$ & 100 & 0.82 & -73.98209 & 40.4393 & $\mathrm{NJ}$ & 172 & -0.63 & -73.97743 & 40.40747 & $\mathrm{NJ}$ \\
\hline 29 & 0.36 & .73 .99648 & 40.46737 & NJ & 101 & 0.73 & -73.98215 & 540.43883 & NJ & 173 & -0.81 & -73.97754 & 40.40701 & $\mathrm{NJ}$ \\
\hline 30 & 0.54 & -73.99646 & 40.46733 & NJ & 102 & 0.69 & -73.98222 & 40.43837 & $\mathrm{NJ}$ & 174 & -0.98 & -73.97763 & 40.40655 & $\mathrm{NJ}$ \\
\hline 31 & 0.23 & -73.99634 & 40.46688 & NJ & 103 & 0.61 & -73.98235 & 40.43791 & NJ & 175 & -1.19 & -73.97769 & 40.4061 & $\mathrm{NJ}$ \\
\hline 32 & 0.24 & -73.99615 & 40.46644 & NJ & 104 & 0.51 & -73.98245 & 50.43744 & NJ & 176 & -1.37 & -73.97772 & 40.40565 & $\mathrm{NJ}$ \\
\hline 33 & 0.01 & -73.99593 & 40.46603 & NJ & 105 & 0.43 & -73.98253 & 40.43698 & NJ & 177 & -1.38 & -73.97769 & 40.4052 & $\mathrm{NJ}$ \\
\hline 34 & 0.02 & -73.99577 & 40.46558 & $\mathrm{NJ}$ & 106 & 0.37 & -73.9826 & 40.43652 & $\mathrm{NJ}$ & 178 & -1.06 & -73.97726 & 40.40477 & $\mathrm{NJ}$ \\
\hline 35 & -0.05 & -73.99557 & 40.46516 & NJ & 107 & 0.37 & -73.98267 & 40.43605 & NJ & 179 & -1.16 & -73.97714 & 40.40432 & $\mathrm{NJ}$ \\
\hline 36 & -0.17 & -73.99533 & 40.46475 & NJ & 108 & 0.3 & -73.98276 & 40.43559 & NJ & 180 & -1.28 & -73.97731 & 40.40386 & $\mathrm{NJ}$ \\
\hline 37 & -0.29 & -73.99512 & 240.46433 & NJ & 109 & 0.26 & -73.9828 & 40.43513 & NJ & 181 & -1.42 & -73.97742 & 40.40341 & NJ \\
\hline 38 & -0.25 & -73.99489 & 940.46391 & NJ & 110 & 0.24 & -73.98285 & 540.43467 & NJ & 182 & -1.5 & -73.97751 & 40.40295 & $\mathrm{NJ}$ \\
\hline 39 & -0.2 & -73.99472 & 240.46347 & NJ & 111 & 0.2 & -73.98288 & 840.43421 & $\mathrm{NJ}$ & 183 & -1.51 & -73.97752 & 40.4025 & NJ \\
\hline 40 & -0.24 & -73.99455 & 540.46304 & $\mathrm{NJ}$ & 112 & 0.14 & -73.98293 & 340.43375 & $\mathrm{NJ}$ & 184 & -1.51 & -73.97746 & 40.40205 & $\mathrm{NJ}$ \\
\hline 41 & -0.18 & -73.99432 & 240.46262 & NJ & 113 & 0.11 & -73.98296 & 640.43329 & NJ & 185 & -1.28 & -73.97731 & 40.40161 & $\mathrm{NJ}$ \\
\hline 42 & -0.09 & -73.99411 & 40.4622 & NJ & 114 & 0.12 & -73.983 & 40.43284 & $\mathrm{NJ}$ & 186 & -0.5 & -73.97615 & 40.40121 & NJ \\
\hline 43 & 0.02 & .73 .9939 & 40.46178 & $\mathrm{NJ}$ & 115 & 0.03 & -73.98302 & 240.43238 & NJ & 187 & -0.75 & -73.97636 & 40.40075 & NJ \\
\hline 44 & 0.09 & -73.99368 & 840.46136 & NJ & 116 & -0.03 & -73.98305 & 540.43192 & NJ & 188 & -0.66 & -73.97633 & 40.4003 & $\mathrm{NJ}$ \\
\hline 45 & 0.06 & .73 .99348 & 3 40.46093 & NJ & 117 & -0.1 & -73.98312 & 240.43146 & NJ & 189 & -0.65 & -73.97629 & 40.39985 & NJ \\
\hline 46 & 0.15 & -73.99327 & 740.46051 & NJ & 118 & -0.09 & -73.98303 & 340.43101 & NJ & 190 & -0.69 & -73.97644 & 40.39939 & NJ \\
\hline 47 & 0.84 & -73.99281 & 140.46017 & $\mathrm{NJ}$ & 119 & -0.17 & -73.98308 & 840.43055 & NJ & 191 & -0.75 & -73.97655 & 40.39893 & $\mathrm{NJ}$ \\
\hline 48 & 0.97 & .73 .99243 & 40.45982 & $\mathrm{NJ}$ & 120 & -0.26 & -73.98312 & 240.43009 & NJ & 192 & -0.69 & -73.97658 & 40.39848 & NJ \\
\hline 49 & 1.05 & -73.99205 & 540.45946 & NJ & 121 & -0.32 & -73.98309 & 940.42964 & $\mathrm{NJ}$ & 193 & -0.64 & -73.9765 & 40.39803 & $\mathrm{NJ}$ \\
\hline 50 & 1.08 & .73 .9917 & 40.45909 & $\mathrm{NJ}$ & 122 & -0.41 & .73 .98306 & 640.42919 & NJ & 194 & -0.61 & -73.97612 & 40.3976 & $\mathrm{NJ}$ \\
\hline 51 & 1.14 & -73.99133 & 30.45872 & NJ & 123 & -0.52 & -73.98305 & 540.42874 & $\mathrm{NJ}$ & 195 & -0.75 & -73.9762 & 40.39715 & NJ \\
\hline 52 & 1.14 & -73.99098 & 340.45835 & NJ & 124 & -0.65 & -73.983 & 40.42829 & NJ & 196 & -0.75 & -73.97626 & 40.39669 & NJ \\
\hline 53 & 1.15 & -73.99062 & 240.45799 & NJ & 125 & -0.74 & -73.98294 & 440.42784 & NJ & 197 & -0.77 & -73.97623 & 40.39624 & $\mathrm{NJ}$ \\
\hline 54 & 1.2 & -73.99026 & 540.45762 & NJ & 126 & -0.8 & -73.98285 & 540.4274 & NJ & 198 & -0.79 & -73.97617 & 40.39579 & NJ \\
\hline 55 & 1.31 & -73.98981 & 40.45728 & $\mathrm{NJ}$ & 127 & -0.87 & -73.98282 & 240.42694 & NJ & 199 & -0.88 & -73.9761 & 40.39535 & NJ \\
\hline 56 & 1.37 & .73 .9894 & 40.45693 & $\mathrm{NJ}$ & 128 & -0.9 & -73.98276 & 640.4265 & $\mathrm{NJ}$ & 200 & -1 & -73.97604 & 40.3949 & $\mathrm{NJ}$ \\
\hline 57 & 1.42 & -73.98897 & 40.45659 & $\mathrm{NJ}$ & 129 & -0.88 & -73.98273 & 340.42605 & NJ & 201 & -0.97 & -73.97598 & 40.39445 & NJ \\
\hline 58 & 1.43 & -73.98862 & 240.45622 & $\mathrm{NJ}$ & 130 & -0.9 & -73.9827 & 40.42559 & $\mathrm{NJ}$ & 202 & -0.97 & -73.97594 & 40.394 & NJ \\
\hline 59 & 1.45 & -73.98824 & 40.45586 & $\mathrm{NJ}$ & 131 & -0.96 & -73.98271 & 40.42514 & $\mathrm{NJ}$ & 203 & -0.99 & -73.97588 & 40.39355 & NJ \\
\hline 60 & 1.43 & -73.9879 & 40.45549 & NJ & 132 & -1 & -73.9827 & 40.42469 & NJ & 204 & -0.93 & -73.97581 & 40.3931 & NJ \\
\hline 61 & 1.46 & -73.98756 & 640.45511 & NJ & 133 & -1.07 & -73.9827 & 40.42423 & NJ & 205 & -0.89 & -73.97575 & 40.39265 & $\mathrm{NJ}$ \\
\hline 62 & 1.53 & -73.98721 & 40.45474 & NJ & 134 & -1.11 & -73.98264 & 740.42378 & $\mathrm{NJ}$ & 206 & -0.77 & -73.97543 & 40.39222 & $\mathrm{NJ}$ \\
\hline 63 & 1.62 & -73.98683 & 40.45438 & NJ & 135 & -1.19 & -73.9826 & 40.42333 & $\mathrm{NJ}$ & 207 & -0.78 & -73.97552 & 40.39176 & NJ \\
\hline 64 & 1.72 & -73.98647 & 40.45401 & NJ & 136 & -1.3 & -73.9826 & 40.42288 & $\mathrm{NJ}$ & 208 & -0.77 & -73.97554 & 40.39131 & NJ \\
\hline 65 & 1.75 & -73.98621 & 40.45361 & NJ & 137 & -1.43 & -73.98264 & 440.42242 & NJ & 209 & -0.42 & -73.97511 & 40.39088 & NJ \\
\hline 66 & 1.8 & -73.98593 & 40.45321 & NJ & 138 & -1.54 & -73.98264 & 440.42196 & NJ & 210 & -0.68 & -73.97528 & 40.39042 & $\mathrm{NJ}$ \\
\hline 67 & 1.84 & -73.98569 & 40.4528 & NJ & 139 & -1.56 & -73.98268 & 840.4215 & NJ & 211 & -0.63 & -73.97536 & 40.38997 & NJ \\
\hline 68 & 1.93 & -73.98543 & 40.4524 & NJ & 140 & -1.52 & -73.98267 & 740.42105 & NJ & 212 & -0.65 & -73.97545 & 40.38951 & NJ \\
\hline 69 & 2.08 & -73.98509 & 40.45202 & NJ & 141 & -1.52 & -73.9827 & 40.42059 & NJ & 213 & -0.72 & .73 .97539 & 40.38906 & NJ \\
\hline 70 & 2.16 & -73.98489 & 40.45159 & NJ & 142 & -1.51 & -73.98264 & 440.42015 & NJ & 214 & -0.76 & -73.97536 & 40.38861 & $\mathrm{NJ}$ \\
\hline 71 & 2.3 & -73.9847 & 40.45117 & NJ & 143 & -1.52 & -73.98259 & 940.4197 & NJ & 215 & -0.78 & -73.97527 & 40.38817 & NJ \\
\hline 72 & 2.41 & -73.98451 & 40.45074 & NJ & 144 & -1.54 & -73.98251 & $\begin{array}{l}1 \quad 40.41925 \\
\end{array}$ & NJ & 216 & -0.84 & -73.9752 & 40.38772 & NJ \\
\hline
\end{tabular}




\begin{tabular}{|c|c|c|c|c|c|c|c|c|c|c|c|c|c|c|}
\hline TR\# & RATE & LONG & LAT & ST & TR\# & RATE & LONG & LAT & ST & TR\# & RATE & LONG & LAT & ST \\
\hline 217 & -0.88 & -73.97514 & 40.38727 & NJ & 289 & -0.49 & -73.97282 & 40.3549 & $\mathrm{NJ}$ & 361 & -0.61 & -73.9761 & 40.32372 & $\mathrm{NJ}$ \\
\hline 218 & -0.91 & -73.97508 & 40.38682 & $\mathrm{NJ}$ & 290 & -0.34 & -73.97246 & 40.35446 & $\mathrm{NJ}$ & 362 & -0.66 & -73.97624 & 40.32328 & $\mathrm{NJ}$ \\
\hline 219 & -0.93 & -73.97507 & 40.38637 & NJ & 291 & -0.42 & -73.9726 & 40.35401 & $\mathrm{NJ}$ & 363 & -0.74 & -73.97638 & 40.32284 & $\mathrm{NJ}$ \\
\hline 220 & -0.91 & -73.97501 & 40.38592 & $\mathrm{NJ}$ & 292 & -0.38 & -73.97272 & 40.35355 & $\mathrm{NJ}$ & 364 & -0.84 & -73.97656 & 40.32241 & $\mathrm{NJ}$ \\
\hline 221 & -0.88 & -73.97496 & 40.38547 & $\mathrm{NJ}$ & 293 & -0.03 & -73.97238 & 40.35311 & $\mathrm{NJ}$ & 365 & -0.37 & -73.97594 & 40.32189 & $\mathrm{NJ}$ \\
\hline 222 & -0.85 & -73.97493 & 40.38502 & NJ & 294 & -0.45 & -73.97284 & 40.35264 & $\mathrm{NJ}$ & 366 & -0.41 & -73.97588 & 40.32142 & $\mathrm{NJ}$ \\
\hline 223 & -0.84 & -73.9749 & 40.38457 & NJ & 295 & -0.41 & -73.97296 & 40.35219 & $\mathrm{NJ}$ & 367 & -0.54 & -73.97615 & 40.321 & $\mathrm{NJ}$ \\
\hline 224 & -0.79 & -73.97485 & 40.38412 & NJ & 296 & -0.4 & -73.97298 & 40.35173 & NJ & 368 & -0.56 & -73.97629 & 40.32056 & $\mathrm{NJ}$ \\
\hline 225 & -0.78 & -73.97482 & 40.38367 & $\mathrm{NJ}$ & 297 & -0.39 & -73.97299 & 40.35128 & $\mathrm{NJ}$ & 369 & -0.51 & -73.97633 & 40.32011 & $\mathrm{NJ}$ \\
\hline 226 & -0.77 & -73.97478 & 40.38323 & $\mathrm{NJ}$ & 298 & -0.41 & -73.97301 & 40.35083 & $\mathrm{NJ}$ & 370 & -0.47 & -73.97626 & 40.31965 & $\mathrm{NJ}$ \\
\hline 227 & -0.74 & -73.97472 & 40.38278 & $\mathrm{NJ}$ & 299 & -0.41 & -73.97302 & 40.35038 & $\mathrm{NJ}$ & 371 & -0.57 & -73.97646 & 40.31921 & $\mathrm{NJ}$ \\
\hline 228 & -0.77 & -73.9747 & 40.38233 & $\mathrm{NJ}$ & 300 & -0.41 & -73.97304 & 40.34993 & $\mathrm{NJ}$ & 372 & -0.62 & -73.97664 & 40.31878 & $\mathrm{NJ}$ \\
\hline 229 & -0.78 & -73.97469 & 40.38188 & NJ & 301 & -0.08 & -73.97256 & 40.3495 & $\mathrm{NJ}$ & 373 & -0.55 & -73.97659 & 40.31832 & $\mathrm{NJ}$ \\
\hline 230 & -0.79 & -73.97466 & 40.38143 & NJ & 302 & -0.48 & -73.97308 & 40.34902 & $\mathrm{NJ}$ & 374 & -0.64 & -73.97672 & 40.31788 & $\mathrm{NJ}$ \\
\hline 231 & -0.81 & -73.97464 & 40.38098 & $\mathrm{NJ}$ & 303 & -0.58 & -73.97333 & 40.34856 & $\mathrm{NJ}$ & 375 & -0.6 & -73.97668 & 40.31742 & $\mathrm{NJ}$ \\
\hline 232 & -0.44 & -73.97404 & 40.38055 & NJ & 304 & -0.63 & -73.97343 & 40.34811 & $N J$ & 376 & -0.63 & -73.97659 & 40.31696 & $\mathrm{NJ}$ \\
\hline 233 & -0.62 & -73.97424 & 40.38009 & NJ & 305 & -0.68 & -73.97354 & 40.34765 & $\mathrm{NJ}$ & 377 & -0.7 & -73.97682 & 40.31652 & $\mathrm{NJ}$ \\
\hline 234 & -0.67 & -73.97437 & 40.37964 & $\mathrm{NJ}$ & 306 & -0.73 & -73.97366 & 40.34719 & $\mathrm{NJ}$ & 378 & -0.75 & -73.97701 & 40.31609 & $\mathrm{NJ}$ \\
\hline 235 & -0.7 & -73.97441 & 40.37918 & NJ & 307 & -0.79 & -73.97375 & 40.34674 & $\mathrm{NJ}$ & 379 & -0.77 & -73.97717 & 40.31565 & $\mathrm{NJ}$ \\
\hline 236 & -0.74 & -73.97444 & 40.37873 & $\mathrm{NJ}$ & 308 & -0.84 & -73.97388 & 40.34628 & $\mathrm{NJ}$ & 380 & -0.75 & -73.97726 & 40.31521 & $\mathrm{NJ}$ \\
\hline 237 & -0.71 & -73.9745 & 40.37828 & $\mathrm{NJ}$ & 309 & -0.91 & -73.97395 & 40.34583 & $\mathrm{NJ}$ & 381 & -0.74 & .73 .97733 & 40.31476 & $\mathrm{NJ}$ \\
\hline 238 & -0.71 & -73.97447 & 40.37783 & $\mathrm{NJ}$ & 310 & -0.99 & -73.97401 & 40.34537 & $\mathrm{NJ}$ & 382 & -0.78 & -73.9774 & 40.31431 & $\mathrm{NJ}$ \\
\hline 239 & -0.67 & -73.97446 & 40.37738 & $\mathrm{NJ}$ & 311 & -1.05 & -73.97406 & 40.34492 & $\mathrm{NJ}$ & 383 & 0.3 & -73.97595 & 40.3137 & $\mathrm{NJ}$ \\
\hline 240 & -0.65 & -73.97443 & 40.37693 & NJ & 312 & -0.89 & -73.9738 & 40.34448 & $\mathrm{NJ}$ & 384 & -0.62 & -73.97714 & 40.31337 & $\mathrm{NJ}$ \\
\hline 241 & -0.65 & -73.9744 & 40.37648 & $\mathrm{NJ}$ & 313 & -0.71 & -73.97343 & 40.34405 & $\mathrm{NJ}$ & 385 & -0.72 & -73.97737 & 40.31295 & $\mathrm{NJ}$ \\
\hline 242 & -0.66 & -73.9744 & 40.37603 & $\mathrm{NJ}$ & 314 & -1.06 & -73.97385 & 40.34358 & $\mathrm{NJ}$ & 386 & -0.73 & -73.97758 & 40.31251 & $\mathrm{NJ}$ \\
\hline 243 & 0.07 & -73.97437 & 40.37558 & $\mathrm{NJ}$ & 315 & -1.05 & -73.974 & 40.34312 & $\mathrm{NJ}$ & 387 & -0.72 & -73.97768 & 40.31207 & $\mathrm{NJ}$ \\
\hline 244 & -1.02 & -73.97435 & 40.37513 & $\mathrm{NJ}$ & 316 & -1.1 & -73.97411 & 40.34267 & $\mathrm{NJ}$ & 388 & -0.59 & -73.97758 & 40.31161 & $\mathrm{NJ}$ \\
\hline 245 & -1.02 & -73.97433 & 40.37468 & $\mathrm{NJ}$ & 317 & -1.12 & -73.97415 & 40.34221 & $\mathrm{NJ}$ & 389 & 0.34 & -73.97636 & 40.31102 & $\mathrm{NJ}$ \\
\hline 246 & -1.05 & -73.97432 & 40.37423 & NJ & 318 & -1.11 & -73.97417 & 40.34176 & $\mathrm{NJ}$ & 390 & -0.28 & -73.97728 & 40.31066 & $\mathrm{NJ}$ \\
\hline 247 & -1.08 & -73.9743 & 40.37378 & NJ & 319 & -1.11 & -73.9742 & 40.34131 & $\mathrm{NJ}$ & 391 & -0.3 & -73.97739 & 40.31022 & $\mathrm{NJ}$ \\
\hline 248 & -1.13 & -73.97429 & 40.37332 & $\mathrm{NJ}$ & 320 & -1.06 & -73.9742 & 40.34086 & $\mathrm{NJ}$ & 392 & -0.31 & -73.97748 & 40.30977 & $\mathrm{NJ}$ \\
\hline 249 & -1.18 & -73.97427 & 40.37287 & $\mathrm{NJ}$ & 321 & -0.93 & -73.9742 & 40.34041 & NJ & 393 & -0.29 & -73.97745 & 40.30932 & $\mathrm{NJ}$ \\
\hline 250 & -1.25 & -73.97417 & 40.37243 & $\mathrm{NJ}$ & 322 & -0.78 & -73.97421 & 40.33995 & $\mathrm{NJ}$ & 394 & -0.3 & -73.97765 & 40.30888 & $\mathrm{NJ}$ \\
\hline 251 & -0.66 & -73.97316 & 40.37202 & $\mathrm{NJ}$ & 323 & -0.77 & -73.97421 & 40.3395 & $\mathrm{NJ}$ & 395 & -0.31 & -73.97774 & 40.30844 & $\mathrm{NJ}$ \\
\hline 252 & -0.77 & -73.97342 & 40.37156 & NJ & 324 & -0.71 & -73.97426 & 40.33905 & $\mathrm{NJ}$ & 396 & -0.24 & -73.97768 & 40.30798 & $\mathrm{NJ}$ \\
\hline 253 & -0.84 & -73.97351 & 40.3711 & $\mathrm{NJ}$ & 325 & -0.75 & -73.97429 & 40.3386 & $\mathrm{NJ}$ & 397 & -0.31 & -73.97778 & 40.30753 & $\mathrm{NJ}$ \\
\hline 254 & -0.89 & -73.97356 & 40.37065 & $\mathrm{NJ}$ & 326 & -0.78 & -73.97435 & 40.33814 & $\mathrm{NJ}$ & 398 & -0.37 & -73.97794 & 40.30709 & $\mathrm{NJ}$ \\
\hline 255 & -0.98 & -73.97368 & 40.37019 & $\mathrm{NJ}$ & 327 & -0.81 & -73.97437 & 40.33769 & $\mathrm{NJ}$ & 399 & -0.27 & -73.97797 & 40.30664 & $\mathrm{NJ}$ \\
\hline 256 & -1.04 & -73.97375 & 40.36974 & $\mathrm{NJ}$ & 328 & -0.83 & -73.9744 & 40.33724 & $\mathrm{NJ}$ & 400 & -0.22 & -73.978 & 40.30619 & $\mathrm{NJ}$ \\
\hline 257 & -1.12 & .73 .97379 & 40.36929 & $\mathrm{NJ}$ & 329 & -0.84 & -73.97443 & 40.33679 & $\mathrm{NJ}$ & 401 & -0.31 & -73.97816 & 40.30576 & $\mathrm{NJ}$ \\
\hline 258 & -1.1 & -73.97372 & 40.36884 & $\mathrm{NJ}$ & 330 & -0.76 & -73.97427 & 40.33634 & $\mathrm{NJ}$ & 402 & -0.28 & -73.97815 & 40.3053 & $\mathrm{NJ}$ \\
\hline 259 & -1.05 & .73 .97353 & 40.3684 & $\mathrm{NJ}$ & 331 & -0.77 & -73.97446 & 40.33588 & $\mathrm{NJ}$ & 403 & -0.22 & -73.97801 & 40.30483 & $\mathrm{NJ}$ \\
\hline 260 & -1.09 & -73.97353 & 40.36795 & NJ & 332 & -0.72 & -73.97453 & 40.33543 & $\mathrm{NJ}$ & 404 & -0.3 & -73.97821 & 40.3044 & $\mathrm{NJ}$ \\
\hline 261 & -0.83 & -73.97324 & 40.36751 & $\mathrm{NJ}$ & 333 & -0.79 & -73.97459 & 40.33498 & $\mathrm{NJ}$ & 405 & -0.24 & -73.97818 & 40.30394 & $\mathrm{NJ}$ \\
\hline 262 & -1.21 & -73.97359 & 40.36704 & $\mathrm{NJ}$ & 334 & -0.72 & -73.97456 & 40.33453 & $\mathrm{NJ}$ & 406 & 0.78 & -73.9769 & 40.30335 & $\mathrm{NJ}$ \\
\hline 263 & -0.79 & -73.97307 & 40.36661 & $\mathrm{NJ}$ & 335 & -0.35 & -73.97408 & 40.3341 & NJ & 407 & 1.47 & -73.97598 & 40.30279 & $\mathrm{NJ}$ \\
\hline 264 & -0.64 & -73.97269 & 40.36618 & $\mathrm{NJ}$ & 336 & 0.01 & -73.97357 & 40.33367 & $\mathrm{NJ}$ & 408 & -0.01 & -73.97803 & 40.30256 & $\mathrm{NJ}$ \\
\hline 265 & -0.78 & -73.97284 & 40.36572 & NJ & 337 & 0.38 & -73.97314 & 40.33324 & $\mathrm{NJ}$ & 409 & -0.05 & -73.97826 & 40.30213 & $\mathrm{NJ}$ \\
\hline 266 & -0.84 & -73.97292 & 40.36527 & $\mathrm{NJ}$ & 338 & 0.16 & -73.97305 & 40.33279 & $\mathrm{NJ}$ & 410 & -0.08 & .73 .97836 & 40.30169 & $\mathrm{NJ}$ \\
\hline 267 & -0.89 & -73.97295 & 40.36481 & $\mathrm{NJ}$ & 339 & 0.02 & -73.97353 & 40.33232 & $\mathrm{NJ}$ & 411 & -0.1 & .73 .97849 & 40.30124 & $\mathrm{NJ}$ \\
\hline 268 & -0.88 & .73 .97295 & 40.36436 & $\mathrm{NJ}$ & 340 & -0.09 & -73.97383 & 40.33185 & $\mathrm{NJ}$ & 412 & -0.06 & -73.97855 & 40.30079 & NJ \\
\hline 269 & -0.66 & -73.97273 & 40.36392 & $\mathrm{NJ}$ & 341 & -0.14 & -73.97412 & 40.33139 & $\mathrm{NJ}$ & 413 & 0.05 & -73.9785 & 40.30033 & $\mathrm{NJ}$ \\
\hline 270 & -0.77 & -73.97276 & 40.36347 & $\mathrm{NJ}$ & 342 & -0.14 & -73.97417 & 40.33124 & $\mathrm{NJ}$ & 414 & -0.01 & -73.97868 & 40.2999 & $\mathrm{NJ}$ \\
\hline 271 & -0.76 & -73.97278 & 40.36302 & NJ & 343 & -0.13 & -73.9742 & 40.33093 & $\mathrm{NJ}$ & 415 & -0.06 & -73.97887 & 40.29947 & $\mathrm{NJ}$ \\
\hline 272 & -0.75 & -73.97276 & 40.36257 & $\mathrm{NJ}$ & 344 & -0.12 & -73.97421 & 40.3308 & $\mathrm{NJ}$ & 416 & -0.14 & -73.97906 & 40.29903 & $\mathrm{NJ}$ \\
\hline 273 & -0.51 & -73.97237 & 40.36213 & $\mathrm{NJ}$ & 345 & -0.1 & -73.97427 & 40.33048 & $\mathrm{NJ}$ & 417 & -0.16 & -73.97913 & 40.29858 & $\mathrm{NJ}$ \\
\hline 274 & -0.67 & -73.9725 & 40.36168 & $\mathrm{NJ}$ & 346 & -0.09 & -73.97427 & 40.33035 & $\mathrm{NJ}$ & 418 & -0.21 & -73.97929 & 40.29815 & $\mathrm{NJ}$ \\
\hline 275 & -0.74 & .73 .97263 & 40.36122 & $\mathrm{NJ}$ & 347 & -0.12 & -73.9744 & 40.3299 & $\mathrm{NJ}$ & 419 & -0.19 & -73.97932 & 40.2977 & $\mathrm{NJ}$ \\
\hline 276 & -0.74 & -73.97272 & 40.36076 & $\mathrm{NJ}$ & 348 & -0.11 & -73.97449 & 40.32946 & $\mathrm{NJ}$ & 420 & -0.06 & -73.97923 & 40.29723 & $\mathrm{NJ}$ \\
\hline 277 & -0.75 & -73.97279 & 40.36031 & $\mathrm{NJ}$ & 349 & -0.13 & -73.97456 & 40.32901 & $\mathrm{NJ}$ & 421 & -0.02 & -73.97925 & 40.29678 & $\mathrm{NJ}$ \\
\hline 278 & -0.72 & .73 .97272 & 40.35986 & $\mathrm{NJ}$ & 350 & -0.08 & -73.97462 & 40.32857 & $\mathrm{NJ}$ & 422 & -0.09 & -73.97945 & 40.29634 & $\mathrm{NJ}$ \\
\hline 279 & -0.41 & -73.97247 & 40.35942 & $\mathrm{NJ}$ & 351 & -0.06 & -73.97475 & 40.32812 & $\mathrm{NJ}$ & 423 & 0.27 & -73.97903 & 40.29585 & $\mathrm{NJ}$ \\
\hline 280 & -0.49 & -73.97266 & 40.35896 & $\mathrm{NJ}$ & 352 & -0.1 & -73.97485 & 40.32768 & $\mathrm{NJ}$ & 424 & -0.12 & -73.97968 & 40.29546 & $\mathrm{NJ}$ \\
\hline 281 & -0.49 & -73.97273 & 40.35851 & $\mathrm{NJ}$ & 353 & -0.15 & -73.97499 & 40.32724 & $\mathrm{NJ}$ & 425 & -0.07 & -73.97974 & 40.29501 & $\mathrm{NJ}$ \\
\hline 282 & -0.52 & -73.97279 & 40.35806 & $\mathrm{NJ}$ & 354 & 0.45 & .73 .97423 & 40.3267 & $\mathrm{NJ}$ & 426 & 0.12 & -73.97952 & 40.29454 & $\mathrm{NJ}$ \\
\hline 283 & -0.55 & -73.97281 & 40.3576 & $\mathrm{NJ}$ & 355 & -0.44 & .73 .9754 & 40.32637 & $\mathrm{NJ}$ & 427 & -0.02 & -73.97984 & 40.29411 & $\mathrm{NJ}$ \\
\hline 284 & -0.55 & -73.97282 & 40.35715 & $\mathrm{NJ}$ & 356 & -0.51 & -73.97562 & 40.32594 & $\mathrm{NJ}$ & 428 & 0.41 & -73.97945 & 40.29362 & $\mathrm{NJ}$ \\
\hline 285 & -0.55 & -73.97282 & 40.3567 & $\mathrm{NJ}$ & 357 & -0.61 & -73.97578 & 40.32551 & $\mathrm{NJ}$ & 429 & -0.05 & -73.98018 & 40.29324 & $\mathrm{NJ}$ \\
\hline 286 & -0.53 & -73.97285 & 40.35625 & $\mathrm{NJ}$ & 358 & -0.43 & -73.97571 & 40.32504 & $\mathrm{NJ}$ & 430 & -0.04 & -73.98029 & 40.2928 & $\mathrm{NJ}$ \\
\hline 287 & -0.49 & -73.97287 & 40.3558 & NJ & 359 & -0.49 & -73.97585 & 40.3246 & $\mathrm{NJ}$ & 431 & -0.03 & -73.98036 & 40.29235 & $\mathrm{NJ}$ \\
\hline 288 & -0.5 & -73.97289 & 40.35535 & $\mathrm{NJ}$ & 360 & -0.56 & -73.97598 & 40.32416 & $\mathrm{NJ}$ & 432 & -0.05 & -73.9805 & 40.29191 & $\mathrm{NJ}$ \\
\hline
\end{tabular}




\begin{tabular}{|c|c|c|c|c|c|c|c|c|c|c|c|c|c|c|}
\hline TR\# & RATE & LONG & LAT & ST & TR\# & RATE & LONG & LAT & ST & TR\# & RATE & LONG & LAT & ST \\
\hline 433 & 0.28 & -73.98016 & 540.29142 & NJ & 505 & -0.1 & -73.98849 & 40.25958 & $\mathrm{NJ}$ & 577 & 0.14 & -73.99698 & 40.22818 & $\mathrm{NJ}$ \\
\hline 434 & -0.05 & -73.98074 & 40.29103 & NJ & 506 & -0.09 & -73.98856 & 40.25913 & NJ & 578 & 0.22 & -73.99699 & 40.22772 & NJ \\
\hline 435 & -0.02 & -73.98082 & 40.29058 & NJ & 507 & -0.08 & -73.98863 & 40.25868 & $\mathrm{NJ}$ & 579 & 0.11 & .73 .99731 & 40.22731 & $\mathrm{NJ}$ \\
\hline 436 & 0.07 & -73.9807 & 40.29011 & NJ & 508 & -0.06 & -73.98869 & 40.25823 & NJ & 580 & 0.07 & -73.99757 & 40.2269 & $\mathrm{NJ}$ \\
\hline 437 & -0.02 & -73.98106 & 40.2897 & NJ & 509 & 1 & -73.98727 & 40.25763 & $\mathrm{NJ}$ & 581 & 0.03 & -73.99776 & 40.22647 & NJ \\
\hline 438 & -0.05 & -73.98123 & 40.28926 & $\mathrm{NJ}$ & 510 & -0.08 & -73.98869 & 40.25733 & NJ & 582 & 0.13 & -73.99782 & 40.22602 & $\mathrm{NJ}$ \\
\hline 439 & -0.09 & -73.98135 & 40.28882 & NJ & 511 & 0.36 & -73.98833 & 40.25683 & $\mathrm{NJ}$ & 583 & 0.34 & -73.99771 & 40.22553 & $\mathrm{NJ}$ \\
\hline 440 & 0 & -73.98129 & 40.28836 & $\mathrm{NJ}$ & 512 & -0.16 & -73.98904 & 40.25645 & NJ & 584 & 0.17 & .73 .99812 & 40.22515 & $\mathrm{NJ}$ \\
\hline 441 & 0 & -73.98135 & 40.28791 & NJ & 513 & -0.43 & -73.98933 & 40.25603 & NJ & 585 & 0.12 & -73.99838 & 40.22474 & $\mathrm{NJ}$ \\
\hline 442 & -0.01 & -73.98148 & 40.28747 & $\mathrm{NJ}$ & 514 & -0.42 & -73.98959 & 40.2556 & NJ & 586 & 0.18 & .73 .99841 & 40.22428 & NJ \\
\hline 443 & 0.46 & -73.98087 & 40.28695 & $\mathrm{NJ}$ & 515 & -0.45 & -73.98982 & 40.25517 & NJ & 587 & 0.95 & -73.99756 & 40.22364 & $\mathrm{NJ}$ \\
\hline 444 & -0.08 & -73.98157 & 40.28657 & NJ & 516 & -0.44 & -73.99002 & 40.25474 & NJ & 588 & 0.95 & -73.99773 & 40.22321 & NJ \\
\hline 445 & 0.05 & -73.9816 & 40.28612 & NJ & 517 & -0.34 & -73.99013 & 40.2543 & NJ & 589 & 0.28 & -73.9986 & 40.22292 & $\mathrm{NJ}$ \\
\hline 446 & 0.09 & -73.98151 & 40.28565 & $\mathrm{NJ}$ & 518 & -0.3 & -73.99019 & 40.25385 & NJ & 590 & 0.21 & -73.99879 & 40.22249 & NJ \\
\hline 447 & -0.03 & -73.98181 & 40.28523 & NJ & 519 & -0.22 & -73.99019 & 40.25339 & NJ & 591 & 0.36 & -73.99884 & 40.22204 & NJ \\
\hline 448 & -0.12 & -73.98196 & 40.28479 & NJ & 520 & -0.14 & -73.98991 & 40.25291 & NJ & 592 & 0.32 & -73.99886 & 40.22157 & NJ \\
\hline 449 & -0.18 & -73.98215 & 40.28436 & NJ & 521 & -0.27 & -73.99023 & 40.25249 & $\mathrm{NJ}$ & 593 & 0.25 & -73.99922 & 40.22118 & $\mathrm{NJ}$ \\
\hline 450 & -0.19 & -73.98228 & 40.28392 & NJ & 522 & -0.32 & -73.99049 & 40.25206 & $\mathrm{NJ}$ & 594 & 0.21 & -73.99951 & 40.22077 & $\mathrm{NJ}$ \\
\hline 451 & -0.13 & -73.98232 & 40.28347 & NJ & 523 & -0.27 & -73.99068 & 40.25163 & $\mathrm{NJ}$ & 595 & 0.22 & -73.99973 & 40.22035 & $\mathrm{NJ}$ \\
\hline 452 & -0.15 & -73.98239 & 40.28302 & $\mathrm{NJ}$ & 524 & -0.18 & -73.99075 & 40.25118 & $\mathrm{NJ}$ & 596 & 0.2 & -73.99985 & 40.21991 & $\mathrm{NJ}$ \\
\hline 453 & -0.2 & -73.98251 & 40.28258 & $\mathrm{NJ}$ & 525 & 0.75 & -73.98959 & 40.2506 & $\mathrm{NJ}$ & 597 & 0.22 & -73.99992 & 40.21946 & $\mathrm{NJ}$ \\
\hline 454 & 0.34 & -73.98193 & 40.28206 & NJ & 526 & -0.34 & -73.99098 & 40.2503 & $\mathrm{NJ}$ & 598 & 0.31 & .73 .99994 & 40.219 & $\mathrm{NJ}$ \\
\hline 455 & -0.09 & -73.98259 & 40.28168 & NJ & 527 & -0.41 & -73.99132 & 40.24988 & $\mathrm{NJ}$ & 599 & 0.41 & -73.99982 & 40.21851 & $\mathrm{NJ}$ \\
\hline 456 & -0.08 & -73.98265 & 40.28123 & NJ & 528 & -0.46 & -73.99159 & 40.24945 & $\mathrm{NJ}$ & 600 & 0.33 & -74.00014 & 40.2181 & $\mathrm{NJ}$ \\
\hline 457 & -0.06 & -73.9826 & 40.28077 & NJ & 529 & -0.46 & -73.99159 & 40.24943 & $\mathrm{NJ}$ & 601 & 0.3 & -74.00038 & 40.21769 & $\mathrm{NJ}$ \\
\hline 458 & -0.12 & -73.98285 & 40.28034 & NJ & 530 & -0.51 & -73.99181 & 40.24902 & $\mathrm{NJ}$ & 602 & 0.32 & -74.00047 & 40.21724 & $\mathrm{NJ}$ \\
\hline 459 & -0.08 & -73.98294 & 40.2799 & $\mathrm{NJ}$ & 531 & -0.51 & -73.99181 & 40.24901 & NJ & 603 & 0.38 & -74.00052 & 40.21679 & $\mathrm{NJ}$ \\
\hline 460 & 0.03 & -73.98283 & 40.27943 & NJ & 532 & -0.46 & -73.99197 & 40.24858 & $\mathrm{NJ}$ & 604 & 0.31 & -74.00041 & 40.2163 & $\mathrm{NJ}$ \\
\hline 461 & -0.04 & -73.98305 & 40.279 & NJ & 533 & -0.46 & -73.99208 & 40.24813 & $\mathrm{NJ}$ & 605 & 0.21 & -74.00078 & 40.21591 & $\mathrm{NJ}$ \\
\hline 462 & -0.05 & -73.98318 & 40.27856 & NJ & 534 & -0.55 & .73 .99237 & 40.24773 & $\mathrm{NJ}$ & 606 & 0.09 & .74 .00107 & 40.2155 & $\mathrm{NJ}$ \\
\hline 463 & 0.23 & -73.98286 & 40.27807 & NJ & 535 & -0.6 & -73.99258 & 40.2473 & NJ & 607 & 0.1 & -74.00143 & 40.21511 & $\mathrm{NJ}$ \\
\hline 464 & -0.14 & -73.98328 & 40.27766 & NJ & 536 & -0.59 & -73.99274 & 40.24687 & NJ & 608 & 0.18 & -74.00153 & 40.21466 & $\mathrm{NJ}$ \\
\hline 465 & -0.21 & -73.98352 & 40.27723 & NJ & 537 & -0.33 & -73.99249 & 40.24635 & NJ & 609 & 0.2 & -74.00165 & 40.21422 & $\mathrm{NJ}$ \\
\hline 466 & -0.23 & -73.98373 & 40.2768 & NJ & 538 & -0.36 & .73 .99268 & 40.24593 & NJ & 610 & 0.1 & -74.00192 & 40.21381 & NJ \\
\hline 467 & -0.19 & -73.9838 & 40.27635 & NJ & 539 & 0.72 & -73.99133 & 40.2452 & $\mathrm{NJ}$ & 611 & 0.05 & -74.00217 & 40.21339 & $\mathrm{NJ}$ \\
\hline 468 & -0.03 & -73.98361 & 40.27588 & NJ & 540 & -0.44 & -73.99284 & 40.24503 & $\mathrm{NJ}$ & 612 & 0.1 & -74.00227 & 40.21295 & NJ \\
\hline 469 & -0.3 & -73.98395 & 40.27546 & NJ & 541 & -0.5 & -73.99318 & 40.24463 & NJ & 613 & 0.15 & -74.00233 & 40.2125 & $\mathrm{NJ}$ \\
\hline 470 & -0.22 & -73.98412 & 40.27502 & $\mathrm{NJ}$ & 542 & -0.45 & -73.99344 & 40.24422 & NJ & 614 & 0.11 & -74.00256 & 40.21207 & $\mathrm{NJ}$ \\
\hline 471 & -0.16 & -73.98422 & 40.27458 & NJ & 543 & -0.45 & -73.99358 & 40.24378 & NJ & 615 & 0.06 & -74.00279 & 40.21165 & NJ \\
\hline 472 & -0.14 & -73.98433 & 40.27413 & $\mathrm{NJ}$ & 544 & -0.39 & -73.99342 & 40.24328 & NJ & 616 & -0.01 & -74.00296 & 40.21122 & $\mathrm{NJ}$ \\
\hline 473 & -0.06 & -73.98433 & 40.27368 & $\mathrm{NJ}$ & 545 & -0.37 & -73.9935 & 40.24283 & NJ & 617 & 0.01 & -74.00308 & 40.21078 & NJ \\
\hline 474 & -0.06 & -73.98453 & 40.27325 & $\mathrm{NJ}$ & 546 & -0.23 & .73 .99347 & 40.24236 & NJ & 618 & 0.71 & -74.00227 & 40.21016 & $\mathrm{NJ}$ \\
\hline 475 & -0.06 & -73.98466 & 40.27281 & NJ & 547 & -0.17 & -73.99365 & 40.24193 & NJ & 619 & -0.14 & -74.00352 & 40.20994 & $\mathrm{NJ}$ \\
\hline 476 & -0.01 & -73.98479 & 40.27236 & $\mathrm{NJ}$ & 548 & -0.31 & -73.99397 & 40.24153 & $\mathrm{NJ}$ & 620 & -0.23 & -74.00375 & 40.20952 & $\mathrm{NJ}$ \\
\hline 477 & -0.02 & -73.98486 & 40.27192 & NJ & 549 & -0.35 & -73.99416 & 40.2411 & NJ & 621 & -0.1 & -74.00401 & 40.20911 & $\mathrm{NJ}$ \\
\hline 478 & -0.01 & -73.98495 & 40.27147 & NJ & 550 & -0.34 & -73.99429 & 40.24067 & NJ & 622 & -0.12 & -74.0042 & 40.20868 & $\mathrm{NJ}$ \\
\hline 479 & NA & -73.98509 & 40.27103 & NJ & 551 & -0.3 & -73.99438 & 40.24022 & $\mathrm{NJ}$ & 623 & -0.39 & .74 .00439 & 40.20825 & $\mathrm{NJ}$ \\
\hline 480 & -0.09 & -73.98528 & 40.2706 & NJ & 552 & -0.23 & -73.99443 & 40.23976 & $\mathrm{NJ}$ & 624 & -0.47 & -74.00462 & 40.20783 & NJ \\
\hline 481 & -0.09 & -73.98535 & 40.27015 & NJ & 553 & -0.31 & -73.99471 & 40.23935 & $\mathrm{NJ}$ & 625 & -0.51 & .74 .00485 & 40.20741 & NJ \\
\hline 482 & 0.51 & -73.98468 & 40.26962 & NJ & 554 & -0.33 & -73.99484 & 40.23892 & $\mathrm{NJ}$ & 626 & .0 .5 & -74.00499 & 40.20697 & NJ \\
\hline 483 & 0.51 & -73.98477 & 40.26918 & NJ & 555 & -0.32 & -73.995 & 40.23848 & NJ & 627 & -0.51 & .74 .00514 & 40.20654 & $\mathrm{NJ}$ \\
\hline 484 & -0.09 & -73.98572 & 40.26883 & NJ & 556 & -0.29 & -73.99509 & 40.23803 & NJ & 628 & -0.54 & -74.00533 & 40.20611 & $\mathrm{NJ}$ \\
\hline 485 & -0.1 & -73.98586 & 40.26839 & NJ & 557 & 0.05 & -73.99483 & 40.23752 & $\mathrm{NJ}$ & 629 & -0.51 & -74.00543 & 40.20567 & $\mathrm{NJ}$ \\
\hline 486 & -0.09 & -73.98596 & 40.26794 & NJ & 558 & 0.48 & -73.99451 & 40.23652 & NJ & 630 & -0.46 & -74.00552 & 40.20522 & $\mathrm{NJ}$ \\
\hline 487 & 0.46 & -73.98535 & 40.26742 & NJ & 559 & 0.29 & -73.9949 & 40.23614 & $\mathrm{NJ}$ & 631 & -0.34 & -74.00555 & 40.20476 & $\mathrm{NJ}$ \\
\hline 488 & -0.09 & -73.98627 & 40.26707 & NJ & 560 & 0.16 & .73 .99518 & 40.23573 & $\mathrm{NJ}$ & 632 & -0.45 & -74.00584 & 40.20435 & $\mathrm{NJ}$ \\
\hline 489 & -0.1 & -73.98639 & 40.26663 & NJ & 561 & 0.13 & -73.99538 & 40.2353 & $\mathrm{NJ}$ & 633 & -0.5 & -74.00606 & 40.20393 & $\mathrm{NJ}$ \\
\hline 490 & 0.48 & -73.9857 & 40.2661 & NJ & 562 & 0.13 & -73.99554 & 40.23487 & $\mathrm{NJ}$ & 634 & -0.55 & -74.00633 & 40.20352 & $\mathrm{NJ}$ \\
\hline 491 & -0.09 & -73.98656 & 40.26574 & NJ & 563 & 0.03 & -73.99582 & 40.23446 & $\mathrm{NJ}$ & 635 & -0.53 & -74.00644 & 40.20308 & $\mathrm{NJ}$ \\
\hline 492 & 0.57 & -73.98589 & 40.26521 & NJ & 564 & -0.07 & -73.99609 & 40.23405 & $\mathrm{NJ}$ & 636 & -0.51 & -74.00659 & 40.20264 & $\mathrm{NJ}$ \\
\hline 493 & 0.55 & -73.98605 & 40.26477 & NJ & 565 & -0.16 & -73.99632 & 40.23363 & $\mathrm{NJ}$ & 637 & -0.53 & -74.00677 & 40.20221 & $\mathrm{NJ}$ \\
\hline 494 & -0.17 & -73.98698 & 40.26442 & NJ & 566 & -0.2 & -73.99651 & 40.2332 & $\mathrm{NJ}$ & 638 & -0.44 & -74.00676 & 40.20174 & $\mathrm{NJ}$ \\
\hline 495 & 0.51 & -73.98637 & 40.2639 & NJ & 567 & -0.28 & -73.99673 & 40.23278 & $\mathrm{NJ}$ & 639 & -0.35 & -74.00677 & 40.20128 & $\mathrm{NJ}$ \\
\hline 496 & 0.49 & .73 .98651 & 40.26346 & NJ & 568 & -0.34 & .73 .99687 & 40.23234 & NJ & 640 & -0.43 & .74 .00703 & 40.20087 & $\mathrm{NJ}$ \\
\hline 497 & -0.27 & -73.98763 & 40.26312 & NJ & 569 & -0.39 & .73 .99696 & 40.2319 & $\mathrm{NJ}$ & 641 & -0.56 & -74.00737 & 40.20047 & $\mathrm{NJ}$ \\
\hline 498 & -0.27 & -73.98778 & 40.26268 & NJ & 570 & -0.41 & -73.99707 & 40.23145 & NJ & 642 & -0.6 & -74.00755 & 40.20004 & NJ \\
\hline 499 & -0.27 & -73.98767 & 40.26222 & $\mathrm{NJ}$ & 571 & -0.38 & -73.99712 & 40.23099 & $\mathrm{NJ}$ & 643 & -0.59 & -74.00769 & 40.1996 & $\mathrm{NJ}$ \\
\hline 500 & -0.28 & -73.98801 & 40.2618 & $\mathrm{NJ}$ & 572 & 0.02 & -73.99702 & 40.23051 & $\mathrm{NJ}$ & 644 & -0.57 & -74.00783 & 40.19916 & NJ \\
\hline 501 & -0.29 & -73.98817 & 40.26136 & NJ & 573 & 0.25 & -73.99622 & 40.22988 & NJ & 645 & -0.54 & -74.00795 & 40.19872 & NJ \\
\hline 502 & -0.23 & -73.98817 & 40.26091 & NJ & 574 & 0.05 & -73.99651 & 40.22948 & NJ & 646 & -0.46 & -74.00795 & 40.19826 & NJ \\
\hline 503 & -0.21 & -73.98834 & 40.26047 & NJ & 575 & 0.05 & -73.99677 & 40.22906 & NJ & 647 & -0.47 & -74.00804 & 40.19781 & NJ \\
\hline 504 & -0.13 & -73.9883 & 40.26001 & NJ & 576 & 0.08 & -73.99693 & 40.22863 & $\mathrm{NJ}$ & 648 & 0.59 & -74.00677 & 40.1971 & NJ \\
\hline
\end{tabular}




\begin{tabular}{|c|c|c|c|c|c|c|c|c|c|c|c|c|c|c|}
\hline TR\# & RATE & LONG & LAT & ST & TR\# & RATE & LONG & LAT & ST & TR\# & RATE & LONG & LAT & ST \\
\hline 649 & -0.42 & -74.00829 & 940.19693 & NJ & 721 & -0.04 & -74.01787 & 40.16442 & $\mathrm{NJ}$ & 793 & -0.16 & -74.02798 & 340.13269 & $\mathrm{NJ}$ \\
\hline 650 & -0.52 & .74 .00853 & 40.19651 & NJ & 722 & -0.06 & -74.01807 & 40.16399 & NJ & 794 & -0.24 & -74.02821 & 40.13227 & $\mathrm{NJ}$ \\
\hline 651 & -0.55 & .74 .00867 & 40.19608 & NJ & 723 & $\mathrm{NA}$ & -74.01814 & 40.16354 & NJ & 795 & -0.24 & -74.02843 & 40.13184 & NJ \\
\hline 652 & -0.56 & -74.00877 & 40.19563 & $\mathrm{NJ}$ & 724 & 1.06 & -74.01691 & 40.16283 & $\mathrm{NJ}$ & 796 & -0.21 & -74.02853 & 40.1314 & $\mathrm{NJ}$ \\
\hline 653 & -0.47 & -74.00876 & 540.19516 & NJ & 725 & -0.09 & -74.0183 & 40.16264 & NJ & 797 & -0.23 & -74.02869 & 40.13096 & $\mathrm{NJ}$ \\
\hline 654 & 1.02 & -74.00685 & 540.19432 & NJ & 726 & -0.14 & -74.01857 & 40.16223 & $\mathrm{NJ}$ & 798 & -0.2 & -74.02884 & 40.13053 & NJ \\
\hline 655 & -0.07 & .74 .00845 & 540.19417 & NJ & 727 & -0.11 & -74.01881 & 40.16181 & $\mathrm{NJ}$ & 799 & -0.09 & -74.02881 & 40.13006 & NJ \\
\hline 656 & -0.17 & -74.00873 & 340.19376 & NJ & 728 & -0.04 & -74.01901 & 40.16139 & $\mathrm{NJ}$ & 800 & -0.1 & -74.02866 & 540.12958 & $\mathrm{NJ}$ \\
\hline 657 & -0.17 & -74.0089 & 40.19333 & NJ & 729 & -0.02 & -74.01913 & 40.16095 & $\mathrm{NJ}$ & 801 & -0.19 & -74.02905 & 540.12918 & $\mathrm{NJ}$ \\
\hline 658 & -0.12 & -74.00896 & 540.19288 & NJ & 730 & 0.1 & -74.01915 & 40.16048 & $\mathrm{NJ}$ & 802 & -0.26 & -74.0293 & 40.12876 & NJ \\
\hline 659 & -0.06 & -74.00897 & 740.19241 & NJ & 731 & 0.21 & -74.01881 & 40.15995 & $\mathrm{NJ}$ & 803 & -0.26 & -74.02953 & 40.12833 & $\mathrm{NJ}$ \\
\hline 660 & 0.09 & -74.00883 & 340.19192 & NJ & 732 & 0.14 & -74.0192 & 40.15956 & $\mathrm{NJ}$ & 804 & -0.26 & -74.02979 & 40.12791 & NJ \\
\hline 661 & -0.16 & -74.0092 & 40.19153 & NJ & 733 & 0.12 & -74.01949 & 40.15915 & NJ & 805 & -0.24 & -74.02998 & 40.12748 & NJ \\
\hline 662 & -0.25 & -74.00938 & 8 40.1911 & NJ & 734 & 0.12 & -74.01968 & 40.15873 & $\mathrm{NJ}$ & 806 & -0.28 & .74 .03003 & 40.12703 & NJ \\
\hline 663 & -0.21 & -74.0094 & 40.19064 & NJ & 735 & 0.14 & -74.01981 & 40.15829 & $\mathrm{NJ}$ & 807 & 0.14 & -74.02968 & 40.12652 & NJ \\
\hline 664 & -0.16 & -74.00935 & 540.19017 & NJ & 736 & 0.19 & -74.01976 & 40.15781 & $\mathrm{NJ}$ & 808 & -0.34 & -74.03004 & 40.12612 & $\mathrm{NJ}$ \\
\hline 665 & -0.21 & -74.00945 & 540.18972 & NJ & 737 & 0.05 & -74.01988 & 40.15737 & NJ & 809 & -0.34 & -74.0303 & 40.1257 & NJ \\
\hline 666 & -0.12 & -74.00964 & 40.18929 & NJ & 738 & -0.09 & -74.0202 & 40.15697 & $\mathrm{NJ}$ & 810 & -0.34 & .74 .03049 & 40.12526 & NJ \\
\hline 667 & -0.43 & -74.00978 & 40.18886 & NJ & 739 & -0.1 & -74.02046 & 40.15656 & NJ & 811 & 0.28 & .74 .02998 & 40.12474 & NJ \\
\hline 668 & -0.27 & .74 .0098 & 40.18839 & $\mathrm{NJ}$ & 740 & -0.11 & -74.02063 & 40.15612 & $\mathrm{NJ}$ & 812 & -0.13 & -74.03041 & 40.12434 & $\mathrm{NJ}$ \\
\hline 669 & 0.77 & -74.00858 & 340.18769 & $\mathrm{NJ}$ & 741 & -0.07 & -74.02071 & 40.15567 & $\mathrm{NJ}$ & 813 & -0.05 & -74.03056 & 540.1239 & $\mathrm{NJ}$ \\
\hline 670 & 1.12 & .74 .00793 & 340.18617 & $\mathrm{NJ}$ & 742 & 0.06 & -74.02074 & 40.15522 & $\mathrm{NJ}$ & 814 & 0.61 & -74.02989 & 40.12335 & $\mathrm{NJ}$ \\
\hline 671 & 0.93 & -74.00838 & 40.18579 & NJ & 743 & 0.13 & -74.02065 & 40.15474 & NJ & 815 & 0.13 & -74.03061 & 40.12299 & $\mathrm{NJ}$ \\
\hline 672 & 0.92 & -74.00874 & 40.1854 & NJ & 744 & -0.06 & .74 .02101 & 40.15434 & NJ & 816 & -0.21 & -74.03098 & 40.12258 & NJ \\
\hline 673 & 0.89 & -74.00906 & 540.18499 & NJ & 745 & -0.14 & -74.02127 & 40.15393 & NJ & 817 & -0.14 & -74.03094 & 40.12212 & $\mathrm{NJ}$ \\
\hline 674 & 0.83 & -74.00935 & 540.18459 & NJ & 746 & -0.15 & -74.02148 & 40.1535 & NJ & 818 & -0.11 & -74.03099 & 40.12167 & $\mathrm{NJ}$ \\
\hline 675 & 0.78 & -74.00963 & 40.18418 & NJ & 747 & -0.11 & .74 .02159 & 40.15306 & NJ & 819 & -0.21 & .74 .03127 & 40.12125 & NJ \\
\hline 676 & 0.75 & -74.0099 & 40.18377 & $\mathrm{NJ}$ & 748 & 0 & -74.02136 & 40.15255 & $\mathrm{NJ}$ & 820 & -0.09 & -74.0314 & 40.12081 & NJ \\
\hline 677 & 0.71 & -74.01015 & 540.18335 & NJ & 749 & -0.01 & -74.02116 & 40.15205 & $\mathrm{NJ}$ & 821 & -0.03 & .74 .03111 & 40.12032 & $\mathrm{NJ}$ \\
\hline 678 & 0.69 & -74.01041 & 40.18294 & NJ & 750 & -0.11 & -74.02156 & 40.15166 & $\mathrm{NJ}$ & 822 & -0.08 & -74.03149 & 40.11991 & NJ \\
\hline 679 & 0.69 & -74.01065 & 540.18252 & NJ & 751 & -0.15 & -74.02191 & 40.15126 & NJ & 823 & -0.03 & -74.03168 & 40.11948 & NJ \\
\hline 680 & 0.69 & -74.01085 & 540.18209 & NJ & 752 & -0.19 & -74.02216 & 40.15085 & NJ & 824 & 0.5 & .74 .03108 & 40.11894 & NJ \\
\hline 681 & 0.63 & -74.01105 & 540.18167 & NJ & 753 & -0.19 & -74.02235 & 40.15042 & NJ & 825 & -0.11 & -74.03172 & 40.11857 & NJ \\
\hline 682 & 0.57 & -74.01126 & 540.18124 & NJ & 754 & -0.18 & -74.02248 & 40.14998 & $\mathrm{NJ}$ & 826 & -0.14 & -74.03197 & 40.11815 & $\mathrm{NJ}$ \\
\hline 683 & 0.52 & .74 .01146 & 540.18082 & NJ & 755 & -0.09 & -74.02209 & 40.14944 & NJ & 827 & -0.21 & -74.03217 & 40.11772 & $\mathrm{NJ}$ \\
\hline 684 & 0.48 & -74.01163 & 340.18038 & $\mathrm{NJ}$ & 756 & -0.14 & -74.02245 & 40.14904 & $\mathrm{NJ}$ & 828 & 0.16 & -74.03188 & 40.11722 & $\mathrm{NJ}$ \\
\hline 685 & 0.45 & -74.01178 & 40.17995 & $\mathrm{NJ}$ & 757 & -0.13 & -74.02274 & 40.14864 & $\mathrm{NJ}$ & 829 & 0.64 & -74.03133 & 40.11668 & $\mathrm{NJ}$ \\
\hline 686 & 0.46 & -74.0119 & 40.17951 & NJ & 758 & -0.11 & -74.02293 & 40.14821 & NJ & 830 & $=0.02$ & .74 .03195 & 40.11631 & $\mathrm{NJ}$ \\
\hline 687 & 0.44 & -74.01207 & 740.17908 & $\mathrm{NJ}$ & 759 & -0.1 & -74.02307 & 40.14777 & $\mathrm{NJ}$ & 831 & 0.03 & -74.03217 & 40.11589 & $\mathrm{NJ}$ \\
\hline 688 & 0.48 & -74.01216 & 540.17863 & $\mathrm{NJ}$ & 760 & -0.05 & -74.02295 & 40.14728 & NJ & 832 & 0.07 & -74.03221 & 40.11544 & NJ \\
\hline 689 & 0.55 & -74.01224 & 40.17818 & NJ & 761 & -0.08 & -74.0228 & 40.14679 & $\mathrm{NJ}$ & 833 & 0.12 & -74.03214 & 40.11497 & $\mathrm{NJ}$ \\
\hline 690 & 0.44 & -74.01201 & 40.17767 & NJ & 762 & -0.17 & -74.02316 & 40.1464 & NJ & 834 & 0.08 & -74.03236 & 40.11454 & NJ \\
\hline 691 & 0.35 & -74.01244 & 40.17729 & NJ & 763 & -0.18 & -74.02341 & 40.14598 & NJ & 835 & 0.09 & -74.03258 & 40.11411 & $\mathrm{NJ}$ \\
\hline 692 & 0.31 & -74.01277 & 40.17689 & NJ & 764 & -0.19 & -74.02359 & 40.14555 & NJ & 836 & 0.15 & -74.03265 & 40.11367 & NJ \\
\hline 693 & 0.29 & -74.01308 & 340.17649 & NJ & 765 & -0.11 & -74.0237 & 40.14511 & NJ & 837 & 0.12 & -74.03252 & 40.11319 & $\mathrm{NJ}$ \\
\hline 694 & 0.22 & -74.01331 & 40.17607 & NJ & 766 & 0.05 & -74.02368 & 40.14464 & NJ & 838 & 0.89 & -74.03188 & 40.11264 & NJ \\
\hline 695 & 0.25 & -74.01352 & 40.17564 & NJ & 767 & 0.02 & -74.02373 & 40.14418 & NJ & 839 & 0.21 & -74.0322 & 40.11223 & NJ \\
\hline 696 & 0.29 & -74.01369 & 40.17521 & $\mathrm{NJ}$ & 768 & -0.03 & -74.02403 & 40.14378 & NJ & 840 & 0.18 & -74.03252 & 40.11182 & NJ \\
\hline 697 & 0.34 & -74.01376 & 640.17476 & NJ & 769 & 0.05 & -74.02428 & 40.14336 & NJ & 841 & 0.04 & -74.03278 & 40.11139 & $\mathrm{NJ}$ \\
\hline 698 & 1.19 & -74.01294 & 40.17414 & NJ & 770 & 0.05 & .74 .02448 & 40.14294 & NJ & 842 & 0.08 & -74.03297 & 40.11096 & NJ \\
\hline 699 & 0.34 & -74.01401 & 40.17388 & $\mathrm{NJ}$ & 771 & 0.11 & -74.02464 & 40.14251 & NJ & 843 & 0.08 & -74.033 & 40.11051 & NJ \\
\hline 700 & 0.29 & -74.01428 & 40.17347 & NJ & 772 & 0.19 & -74.02467 & 40.14204 & NJ & 844 & 0.11 & .74 .03265 & 40.11001 & NJ \\
\hline 701 & 0.33 & -74.01451 & 40.17305 & NJ & 773 & 0.19 & -74.0247 & 40.14159 & NJ & 845 & 0.5 & -74.0329 & 40.10958 & NJ \\
\hline 702 & 0.37 & -74.01465 & 40.17261 & $\mathrm{NJ}$ & 774 & 0.02 & -74.02502 & 40.14119 & NJ & 846 & 0.56 & -74.03313 & 40.10915 & NJ \\
\hline 703 & 0.46 & -74.01463 & 40.17215 & $\mathrm{NJ}$ & 775 & 0 & -74.02539 & 40.14057 & NJ & 847 & 0.16 & -74.03336 & 40.10873 & $\mathrm{NJ}$ \\
\hline 704 & 0.33 & -74.01485 & 40.17172 & $\mathrm{NJ}$ & 776 & -0.02 & -74.02554 & 40.14013 & NJ & 848 & 0.09 & -74.03354 & 40.1083 & NJ \\
\hline 705 & 0.2 & .74 .01518 & 40.17132 & $\mathrm{NJ}$ & 777 & 0.07 & -74.02554 & 40.13968 & NJ & 849 & -0.67 & -74.03371 & 40.10786 & NJ \\
\hline 706 & 0.13 & -74.01546 & 40.17091 & NJ & 778 & 0.06 & -74.02513 & 40.13916 & NJ & 850 & -0.68 & -74.03387 & 40.10743 & NJ \\
\hline 707 & 0.13 & -74.01569 & 40.17049 & NJ & 779 & -0.12 & .74 .02554 & 40.13876 & NJ & 851 & -0.67 & -74.03387 & 40.10697 & NJ \\
\hline 708 & 0.12 & -74.01588 & 340.17007 & $\mathrm{NJ}$ & 780 & -0.19 & .74 .02583 & 40.13834 & NJ & 852 & -0.72 & -74.03401 & 40.10653 & $\mathrm{NJ}$ \\
\hline 709 & 0.16 & -74.01605 & 40.16963 & NJ & 781 & -0.26 & -74.02605 & 40.13792 & NJ & 853 & -0.75 & -74.03389 & 40.10606 & NJ \\
\hline 710 & 0.23 & -74.01607 & 40.16917 & NJ & 782 & -0.3 & -74.02628 & 40.13749 & NJ & 854 & -0.73 & -74.03407 & 40.10563 & NJ \\
\hline 711 & 0.18 & -74.01622 & 40.16874 & NJ & 783 & -0.23 & -74.0264 & 40.13705 & NJ & 855 & -0.75 & -74.0341 & 40.10517 & $\mathrm{NJ}$ \\
\hline 712 & 0.02 & -74.01659 & 40.16834 & $\mathrm{NJ}$ & 784 & 0.48 & -74.02583 & 40.13651 & NJ & 856 & -0.66 & -74.03424 & 40.10473 & NJ \\
\hline 713 & -0.11 & -74.01685 & 40.16793 & $\mathrm{NJ}$ & 785 & -0.1 & .74 .02657 & 40.13616 & NJ & 857 & -0.68 & -74.03409 & 40.10425 & NJ \\
\hline 714 & -0.12 & -74.01706 & 40.16751 & NJ & 786 & -0.11 & .74 .02689 & 40.13574 & NJ & 858 & -0.62 & -74.03427 & 40.10382 & NJ \\
\hline 715 & -0.13 & -74.01726 & 40.16708 & NJ & 787 & -0.1 & .74 .02713 & 40.13532 & NJ & 859 & -0.47 & -74.03439 & 40.10338 & NJ \\
\hline 716 & -0.1 & -74.01736 & 50.16664 & NJ & 788 & -0.12 & -74.0273 & 40.13489 & NJ & 860 & -0.11 & -74.03418 & 40.10289 & NJ \\
\hline 717 & -0.05 & -74.01746 & 40.16619 & $\mathrm{NJ}$ & 789 & -0.11 & -74.0275 & 40.13446 & NJ & 861 & 0.87 & -74.03299 & 40.10227 & NJ \\
\hline 718 & 0.12 & -74.01743 & 40.16572 & $\mathrm{NJ}$ & 790 & -0.11 & -74.02757 & 40.13401 & NJ & 862 & 1.99 & -74.03154 & 40.10161 & NJ \\
\hline 719 & 0.14 & .74 .01735 & 40.16524 & NJ & 791 & 0.04 & -74.02754 & 40.13355 & NJ & 863 & -0.07 & -74.03444 & 40.10156 & NJ \\
\hline 720 & 0.03 & .74 .01764 & 40.16484 & NJ & 792 & -0.02 & -74.02776 & 40.13312 & NJ & 864 & 1.05 & -74.033 & 40.1009 & NJ \\
\hline
\end{tabular}




\begin{tabular}{|c|c|c|c|c|c|c|c|c|c|c|c|c|c|c|}
\hline TR\# & RATE & LONG & LAT & ST & TR\# & RATE & LONG & LAT & ST & TR\# & RATE & LONG & LAT & ST \\
\hline 865 & 1.98 & -74.03192 & 40.10029 & NJ & 937 & -0.03 & -74.04208 & 40.06876 & $\mathrm{NJ}$ & 1009 & -0.08 & -74.04962 & 40.03686 & $\mathrm{NJ}$ \\
\hline 866 & 0.94 & -74.03226 & 40.09988 & NJ & 938 & -0.02 & -74.04205 & 40.06829 & NJ & 1010 & 0 & -74.04976 & 40.03642 & NJ \\
\hline 867 & 0.87 & -74.03264 & 40.09947 & NJ & 939 & 0.04 & -74.04219 & 40.06786 & NJ & 1011 & -0.03 & -74.04984 & 40.03597 & $\mathrm{NJ}$ \\
\hline 868 & 0.82 & -74.03293 & 40.09906 & NJ & 940 & 0.03 & -74.0423 & 40.06741 & NJ & 1012 & -0.06 & -74.04991 & 40.03552 & NJ \\
\hline 869 & 0.77 & -74.03313 & 40.09863 & NJ & 941 & -0.01 & -74.04234 & 40.06696 & NJ & 1013 & -0.08 & -74.05 & 40.03508 & NJ \\
\hline 870 & 0.77 & -74.03337 & 40.0982 & $\mathrm{NJ}$ & 942 & 0.02 & -74.0425 & 40.06653 & NJ & 1014 & -0.1 & -74.05013 & 40.03464 & NJ \\
\hline 871 & 0.81 & -74.03358 & 40.09778 & NJ & 943 & -0.02 & -74.04257 & 40.06608 & NJ & 1015 & -0.05 & -74.05025 & 40.0342 & $\mathrm{NJ}$ \\
\hline 872 & 0.8 & -74.0338 & 40.09735 & $\mathrm{NJ}$ & 944 & -0.03 & -74.04259 & 40.06562 & $\mathrm{NJ}$ & 1016 & -0.05 & -74.0504 & 40.03376 & $\mathrm{NJ}$ \\
\hline 873 & 0.8 & -74.03403 & 40.09692 & NJ & 945 & -0.05 & -74.04276 & 40.06519 & NJ & 1017 & -0.06 & -74.05049 & 40.03332 & $\mathrm{NJ}$ \\
\hline 874 & 0.73 & -74.03423 & 40.09649 & NJ & 946 & -0.03 & -74.04292 & 40.06476 & NJ & 1018 & -0.1 & -74.0506 & 40.03287 & $\mathrm{NJ}$ \\
\hline 875 & 0.71 & -74.03445 & 40.09607 & NJ & 947 & -0.06 & -74.04308 & 40.06432 & NJ & 1019 & -0.09 & -74.0507 & 40.03243 & $\mathrm{NJ}$ \\
\hline 876 & 0.64 & -74.03465 & 40.09564 & NJ & 948 & -0.1 & -74.04318 & 40.06388 & NJ & 1020 & -0.06 & -74.05084 & 40.03199 & $\mathrm{NJ}$ \\
\hline 877 & 0.61 & -74.03485 & 540.09521 & NJ & 949 & -0.12 & -74.04333 & 40.06344 & NJ & 1021 & -0.07 & -74.05095 & 40.03155 & $\mathrm{NJ}$ \\
\hline 878 & 0.6 & -74.03503 & 40.09477 & NJ & 950 & -0.24 & -74.04347 & 40.063 & NJ & 1022 & -0.07 & -74.05107 & 40.03111 & $\mathrm{NJ}$ \\
\hline 879 & 0.58 & -74.03522 & 40.09434 & NJ & 951 & -0.35 & -74.04356 & 40.06256 & $\mathrm{NJ}$ & 1023 & -0.06 & -74.05119 & 40.03067 & $\mathrm{NJ}$ \\
\hline 880 & 0.57 & -74.03542 & 40.09391 & NJ & 952 & -0.36 & -74.04367 & 40.06211 & NJ & 1024 & -0.05 & -74.05132 & 40.03023 & NJ \\
\hline 881 & 0.57 & -74.03561 & 40.09348 & NJ & 953 & -0.34 & -74.04379 & 40.06167 & $\mathrm{NJ}$ & 1025 & -0.05 & -74.05144 & 40.02978 & NJ \\
\hline 882 & 0.51 & -74.03581 & 40.09305 & NJ & 954 & -0.34 & -74.04385 & 40.06122 & NJ & 1026 & -0.09 & -74.05154 & 40.02934 & $\mathrm{NJ}$ \\
\hline 883 & 0.49 & -74.036 & 40.09262 & NJ & 955 & -0.31 & -74.04395 & 40.06078 & NJ & 1027 & -0.12 & -74.05167 & 40.0289 & $\mathrm{NJ}$ \\
\hline 884 & 0.47 & -74.03616 & 540.09219 & NJ & 956 & -0.32 & -74.04404 & 40.06033 & NJ & 1028 & -0.12 & -74.05177 & 40.02846 & $\mathrm{NJ}$ \\
\hline 885 & 0.46 & -74.03635 & 40.09175 & NJ & 957 & -0.3 & -74.04413 & 40.05989 & NJ & 1029 & -0.1 & -74.0519 & 40.02802 & $\mathrm{NJ}$ \\
\hline 886 & 0.46 & -74.03647 & 40.09131 & NJ & 958 & -0.35 & -74.04422 & 40.05944 & NJ & 1030 & -0.08 & -74.05202 & 40.02758 & NJ \\
\hline 887 & 0.45 & -74.03661 & 40.09088 & $\mathrm{NJ}$ & 959 & -0.38 & -74.0443 & 40.059 & NJ & 1031 & -0.1 & -74.05212 & 40.02714 & NJ \\
\hline 888 & 0.44 & -74.03677 & 40.09044 & NJ & 960 & -0.47 & -74.04436 & 40.05855 & NJ & 1032 & -0.1 & -74.05223 & 40.02669 & $\mathrm{NJ}$ \\
\hline 889 & 0.4 & -74.03697 & 40.09001 & NJ & 961 & -0.45 & -74.04449 & 40.05811 & NJ & 1033 & -0.12 & -74.05232 & 40.02625 & NJ \\
\hline 890 & 0.37 & -74.03708 & 40.08957 & $\mathrm{NJ}$ & 962 & -0.41 & -74.04462 & 40.05767 & NJ & 1034 & -0.14 & -74.05244 & 40.02581 & $\mathrm{NJ}$ \\
\hline 891 & 0.36 & -74.03722 & 40.08913 & $\mathrm{NJ}$ & 963 & -0.39 & -74.04469 & 40.05722 & NJ & 1035 & -0.14 & -74.05257 & 40.02537 & $\mathrm{NJ}$ \\
\hline 892 & 0.31 & -74.03737 & 40.08869 & $\mathrm{NJ}$ & 964 & -0.38 & -74.04475 & 40.05677 & NJ & 1036 & -0.14 & -74.05266 & 40.02492 & $\mathrm{NJ}$ \\
\hline 893 & 0.31 & -74.03749 & 40.08825 & NJ & 965 & -0.36 & -74.04486 & 40.05633 & NJ & 1037 & -0.15 & -74.05276 & 40.02448 & NJ \\
\hline 894 & 0.27 & -74.03767 & 40.08782 & $\mathrm{NJ}$ & 966 & -0.37 & -74.04495 & 40.05588 & NJ & 1038 & -0.19 & -74.05286 & 40.02403 & $\mathrm{NJ}$ \\
\hline 895 & 0.27 & -74.03781 & 40.08738 & NJ & 967 & -0.38 & -74.04504 & 40.05544 & NJ & 1039 & -0.21 & -74.05295 & 40.02359 & $\mathrm{NJ}$ \\
\hline 896 & 0.24 & -74.03806 & 40.08696 & NJ & 968 & -0.37 & -74.04517 & 40.055 & NJ & 1040 & -0.19 & -74.05307 & 40.02315 & $\mathrm{NJ}$ \\
\hline 897 & 0.12 & -74.03818 & 40.08652 & NJ & 969 & -0.35 & -74.04527 & 40.05456 & NJ & 1041 & -0.12 & -74.05318 & 40.0227 & $\mathrm{NJ}$ \\
\hline 898 & 0.12 & -74.0383 & 40.08608 & $\mathrm{NJ}$ & 970 & -0.34 & -74.04536 & 40.05411 & NJ & 1042 & -0.08 & -74.05328 & 40.02226 & $\mathrm{NJ}$ \\
\hline 899 & 0.1 & -74.03841 & 40.08564 & $\mathrm{NJ}$ & 971 & -0.28 & -74.04547 & 40.05367 & NJ & 1043 & -0.08 & -74.05341 & 40.02182 & NJ \\
\hline 900 & 0.03 & -74.03853 & 40.08519 & $\mathrm{NJ}$ & 972 & -0.24 & .74 .04558 & 40.05322 & NJ & 1044 & -0.08 & -74.05351 & 40.02138 & $\mathrm{NJ}$ \\
\hline 901 & 0 & -74.03864 & 40.08475 & $\mathrm{NJ}$ & 973 & -0.17 & -74.04568 & 40.05278 & NJ & 1045 & -0.1 & -74.05362 & 40.02094 & $\mathrm{NJ}$ \\
\hline 902 & -0.02 & -74.03876 & 640.08431 & $\mathrm{NJ}$ & 974 & -0.2 & -74.04582 & 40.05234 & NJ & 1046 & -0.13 & -74.05367 & 40.02073 & $\mathrm{NJ}$ \\
\hline 903 & -0.04 & -74.03886 & 640.08387 & $\mathrm{NJ}$ & 975 & -0.15 & -74.04594 & 40.0519 & NJ & 1047 & -0.1 & -74.05377 & 40.02029 & NJ \\
\hline 904 & -0.02 & -74.03899 & 940.08343 & $\mathrm{NJ}$ & 976 & -0.08 & -74.04607 & 40.05146 & NJ & 1048 & -0.09 & -74.05386 & 40.01984 & $\mathrm{NJ}$ \\
\hline 905 & -0.01 & -74.03912 & 240.08299 & $\mathrm{NJ}$ & 977 & -0.1 & -74.04617 & 40.05102 & NJ & 1049 & -0.08 & -74.05397 & 40.0194 & $\mathrm{NJ}$ \\
\hline 906 & 0.01 & -74.03922 & 240.08254 & $\mathrm{NJ}$ & 978 & -0.11 & -74.0463 & 40.05058 & NJ & 1050 & -0.08 & -74.05409 & 40.01896 & $\mathrm{NJ}$ \\
\hline 907 & 0.01 & -74.03934 & 40.0821 & NJ & 979 & -0.17 & .74 .04642 & 40.05014 & NJ & 1051 & -0.08 & -74.05418 & 40.01851 & $\mathrm{NJ}$ \\
\hline 908 & 0 & -74.03946 & 640.08166 & NJ & 980 & -0.16 & .74 .04652 & 40.0497 & NJ & 1052 & -0.01 & .74 .05429 & 40.01807 & $\mathrm{NJ}$ \\
\hline 909 & NA & -74.0396 & 40.08122 & NJ & 981 & -0.21 & -74.04665 & 40.04925 & NJ & 1053 & 0.03 & -74.0544 & 40.01763 & $\mathrm{NJ}$ \\
\hline 910 & -0.05 & -74.0397 & 40.08078 & NJ & 982 & -0.18 & -74.04674 & 40.04881 & NJ & 1054 & -0.04 & -74.05453 & 40.01719 & $\mathrm{NJ}$ \\
\hline 911 & -0.06 & -74.03983 & 3 40.08034 & NJ & 983 & -0.12 & -74.04684 & 40.04837 & $\mathrm{NJ}$ & 1055 & -0.05 & -74.05463 & 40.01674 & $\mathrm{NJ}$ \\
\hline 912 & -0.1 & -74.03992 & 240.07989 & NJ & 984 & -0.14 & -74.04697 & 40.04793 & $\mathrm{NJ}$ & 1056 & -0.04 & .74 .05473 & 40.0163 & $\mathrm{NJ}$ \\
\hline 913 & -0.11 & -74.03998 & 8 40.07945 & NJ & 985 & -0.13 & -74.04707 & 40.04748 & NJ & 1057 & -0.07 & -74.05486 & 40.01586 & NJ \\
\hline 914 & -0.12 & -74.04008 & 840.079 & NJ & 986 & -0.13 & -74.04718 & 40.04704 & NJ & 1058 & -0.08 & -74.05498 & 40.01542 & $\mathrm{NJ}$ \\
\hline 915 & -0.18 & -74.04016 & 640.07856 & NJ & 987 & -0.09 & -74.04729 & 40.0466 & NJ & 1059 & -0.04 & -74.05508 & 40.01498 & NJ \\
\hline 916 & -0.18 & -74.04024 & 40.07811 & NJ & 988 & -0.12 & -74.04738 & 40.04615 & NJ & 1060 & -0.06 & -74.05521 & 40.01454 & $\mathrm{NJ}$ \\
\hline 917 & -0.2 & -74.04033 & 40.07767 & NJ & 989 & -0.11 & -74.04747 & 40.04571 & $\mathrm{NJ}$ & 1061 & -0.12 & -74.05531 & 40.01409 & $\mathrm{NJ}$ \\
\hline 918 & -0.19 & -74.04047 & 40.07722 & NJ & 990 & -0.14 & -74.04758 & 40.04527 & NJ & 1062 & -0.17 & -74.05544 & 40.01365 & NJ \\
\hline 919 & -0.12 & -74.04054 & 40.07678 & NJ & 991 & -0.12 & -74.04768 & 40.04482 & $\mathrm{NJ}$ & 1063 & -0.13 & -74.05556 & 40.01321 & $\mathrm{NJ}$ \\
\hline 920 & -0.14 & -74.04063 & 40.07633 & NJ & 992 & -0.21 & -74.04779 & 40.04438 & NJ & 1064 & -0.14 & -74.05566 & 40.01277 & NJ \\
\hline 921 & -0.14 & -74.0407 & 40.07589 & NJ & 993 & -0.2 & -74.04788 & 40.04394 & NJ & 1065 & -0.19 & -74.05579 & 40.01233 & $\mathrm{NJ}$ \\
\hline 922 & -0.11 & -74.04076 & 540.07544 & $\mathrm{NJ}$ & 994 & -0.16 & -74.04797 & 40.04349 & NJ & 1066 & -0.01 & -74.05589 & 40.01188 & $\mathrm{NJ}$ \\
\hline 923 & -0.1 & -74.04082 & 40.07499 & NJ & 995 & -0.15 & -74.0481 & 40.04305 & NJ & 1067 & -0.05 & -74.05602 & 40.01144 & NJ \\
\hline 924 & -0.09 & -74.04092 & 40.07454 & NJ & 996 & -0.13 & -74.0482 & 40.04261 & $\mathrm{NJ}$ & 1068 & -0.09 & -74.05615 & 40.011 & NJ \\
\hline 925 & -0.08 & -74.04105 & 40.0741 & NJ & 997 & -0.11 & -74.04832 & 40.04216 & NJ & 1069 & -0.04 & -74.05626 & 40.01056 & NJ \\
\hline 926 & -0.1 & -74.04117 & 740.07366 & NJ & 998 & -0.17 & -74.04842 & 40.04172 & NJ & 1070 & -0.03 & -74.0564 & 40.01012 & NJ \\
\hline 927 & -0.12 & -74.04129 & 940.07322 & $\mathrm{NJ}$ & 999 & -0.16 & -74.04854 & 40.04128 & $\mathrm{NJ}$ & 1071 & -0.04 & -74.0565 & 40.00968 & NJ \\
\hline 928 & -0.12 & -74.04138 & 840.07278 & $\mathrm{NJ}$ & 1000 & -0.13 & -74.04865 & 40.04084 & $\mathrm{NJ}$ & 1072 & -0.07 & -74.05663 & 40.00924 & NJ \\
\hline 929 & -0.12 & -74.0414 & 40.07232 & NJ & 1001 & -0.18 & -74.04875 & 40.04039 & NJ & 1073 & -0.09 & -74.05673 & 40.0088 & NJ \\
\hline 930 & -0.13 & -74.04146 & 640.07187 & NJ & 1002 & -0.19 & -74.04887 & 40.03995 & NJ & 1074 & -0.13 & -74.05685 & 40.00835 & $\mathrm{NJ}$ \\
\hline 931 & -0.11 & -74.04161 & 140.07144 & $\mathrm{NJ}$ & 1003 & -0.17 & -74.04898 & 40.03951 & NJ & 1075 & -0.12 & -74.05698 & 40.00791 & NJ \\
\hline 932 & -0.08 & -74.0417 & 40.07099 & $\mathrm{NJ}$ & 1004 & -0.12 & -74.0491 & 40.03907 & NJ & 1076 & -0.07 & -74.0571 & 40.00747 & NJ \\
\hline 933 & -0.06 & -74.04172 & 240.07053 & $\mathrm{NJ}$ & 1005 & -0.08 & -74.04921 & 40.03863 & NJ & 1077 & -0.06 & -74.05722 & 40.00703 & NJ \\
\hline 934 & -0.06 & -74.04173 & 3 40.07008 & $\mathrm{NJ}$ & 1006 & -0.14 & -74.04933 & 40.03819 & NJ & 1078 & $\mathrm{NA}$ & -74.05734 & 40.00659 & NJ \\
\hline 935 & -0.07 & -74.0419 & 40.06965 & NJ & 1007 & -0.12 & -74.04942 & 40.03774 & NJ & 1079 & -0.01 & -74.05746 & 40.00615 & NJ \\
\hline 936 & -0.05 & -74.04205 & 540.06921 & NJ & 1008 & -0.09 & -74.04951 & 40.0373 & NJ & 1080 & -0.02 & -74.05759 & 40.00571 & NJ \\
\hline
\end{tabular}




\begin{tabular}{|c|c|c|c|c|c|c|c|c|c|c|c|c|c|c|}
\hline TR\# & RATE & LONG & LAT & ST & TR\# & RATE & LONG & LAT & ST & TR\# & RATE & LONG & LAT & ST \\
\hline 1081 & 0 & -74.05768 & 840.00526 & $\mathrm{NJ}$ & 1153 & 0.1 & -74.06516 & 539.97335 & $\mathrm{NJ}$ & 1225 & 0.55 & .74 .06982 & 39.94108 & $\mathrm{NJ}$ \\
\hline 1082 & 0.04 & -74.05779 & 40.00482 & $\mathrm{NJ}$ & 1154 & 0.43 & -74.0649 & 39.97286 & NJ & 1226 & 0.5 & -74.07002 & 39.94065 & $\mathrm{NJ}$ \\
\hline 1083 & 0.09 & -74.05791 & 40.00438 & NJ & 1155 & 0.21 & -74.06526 & 539.97245 & $\mathrm{NJ}$ & 1227 & 0.47 & -74.07016 & 39.94021 & $\mathrm{NJ}$ \\
\hline 1084 & 0.12 & -74.05811 & 40.00395 & $\mathrm{NJ}$ & 1156 & 0.26 & -74.06535 & 539.97201 & NJ & 1228 & 0.49 & -74.07031 & 39.93978 & $\mathrm{NJ}$ \\
\hline 1085 & 0.18 & -74.0582 & 40.0035 & NJ & 1157 & 0.28 & .74 .06544 & 439.97156 & $\mathrm{NJ}$ & 1229 & 0.47 & -74.07043 & 39.93934 & $\mathrm{NJ}$ \\
\hline 1086 & 0.25 & -74.05832 & 40.00306 & NJ & 1158 & 0.24 & -74.06554 & 439.97112 & NJ & 1230 & 0.49 & -74.07056 & 39.93889 & $\mathrm{NJ}$ \\
\hline 1087 & 0.25 & -74.05846 & 540.00262 & NJ & 1159 & 0.57 & -74.06522 & 239.97062 & NJ & 1231 & 0.48 & -74.07066 & 39.93845 & NJ \\
\hline 1088 & 0.26 & -74.05855 & 540.00218 & $\mathrm{NJ}$ & 1160 & 0.23 & -74.06564 & 439.97022 & NJ & 1232 & 0.48 & -74.07079 & 39.93801 & $\mathrm{NJ}$ \\
\hline 1089 & 0.21 & -74.05862 & 40.00173 & NJ & 1161 & 0.23 & -74.06577 & 739.96978 & NJ & 1233 & 0.44 & -74.07092 & 39.93757 & NJ \\
\hline 1090 & 0.16 & -74.05872 & 40.00129 & NJ & 1162 & 0.23 & .74 .06586 & 539.96933 & $\mathrm{NJ}$ & 1234 & 0.8 & -74.07076 & 39.93709 & $\mathrm{NJ}$ \\
\hline 1091 & 0.15 & -74.05878 & 40.00084 & NJ & 1163 & 0.2 & -74.0659 & 39.96888 & $\mathrm{NJ}$ & 1235 & 0.91 & -74.07072 & 39.93663 & NJ \\
\hline 1092 & 0.14 & -74.05885 & 540.00039 & NJ & 1164 & 0.13 & -74.06599 & 39.96844 & $\mathrm{NJ}$ & 1236 & 1.06 & -74.07065 & 39.93617 & NJ \\
\hline 1093 & 0.11 & -74.05894 & 439.99995 & NJ & 1165 & 0.11 & -74.06607 & 739.96799 & NJ & 1237 & 1.08 & -74.07076 & 39.93573 & $\mathrm{NJ}$ \\
\hline 1094 & 0.11 & -74.05902 & 239.9995 & NJ & 1166 & 0.11 & -74.06618 & 839.96755 & $\mathrm{NJ}$ & 1238 & 1.06 & -74.07085 & 39.93528 & NJ \\
\hline 1095 & 0.09 & -74.05913 & 39.99906 & NJ & 1167 & 0.09 & -74.06625 & 539.9671 & $\mathrm{NJ}$ & 1239 & 0.81 & -74.07129 & 39.93488 & NJ \\
\hline 1096 & 0.1 & -74.05925 & 539.99862 & NJ & 1168 & 0.09 & -74.06631 & 39.96665 & $\mathrm{NJ}$ & 1240 & 0.83 & -74.07141 & 39.93444 & NJ \\
\hline 1097 & 0.1 & -74.05939 & 39.99818 & NJ & 1169 & 0.45 & -74.0659 & 39.96615 & $\mathrm{NJ}$ & 1241 & 0.91 & -74.07146 & 39.93399 & NJ \\
\hline 1098 & 0.13 & -74.05949 & 39.99773 & NJ & 1170 & 0.03 & -74.06644 & 439.96575 & NJ & 1242 & 0.91 & -74.07159 & 39.93355 & NJ \\
\hline 1099 & 0.14 & .74 .05959 & 39.99729 & NJ & 1171 & 0.03 & -74.06648 & 39.9653 & NJ & 1243 & 0.89 & .74 .07173 & 39.93311 & $\mathrm{NJ}$ \\
\hline 1100 & 0.12 & -74.05968 & 39.99685 & NJ & 1172 & 0 & -74.06651 & 39.96485 & $\mathrm{NJ}$ & 1244 & 0.89 & -74.07179 & 39.93266 & $\mathrm{NJ}$ \\
\hline 1101 & 0.11 & -74.05975 & 39.9964 & NJ & 1173 & -0.04 & -74.06654 & 49.9644 & $\mathrm{NJ}$ & 1245 & 0.87 & -74.07191 & 39.93222 & $\mathrm{NJ}$ \\
\hline 1102 & 0.09 & -74.05988 & 39.99596 & $\mathrm{NJ}$ & 1174 & -0.06 & -74.06656 & 539.96395 & $\mathrm{NJ}$ & 1246 & 0.86 & -74.07204 & 39.93178 & $\mathrm{NJ}$ \\
\hline 1103 & 0.12 & -74.05998 & 39.99552 & NJ & 1175 & -0.11 & -74.06662 & 39.9635 & $\mathrm{NJ}$ & 1247 & 0.78 & -74.07219 & 39.93134 & $\mathrm{NJ}$ \\
\hline 1104 & 0.12 & -74.06009 & 39.99507 & NJ & 1176 & -0.13 & -74.0667 & 39.96305 & NJ & 1248 & 0.72 & -74.07233 & 39.93091 & NJ \\
\hline 1105 & 0.15 & -74.06023 & 39.99463 & $\mathrm{NJ}$ & 1177 & -0.17 & .74 .06676 & 39.9626 & NJ & 1249 & 0.68 & -74.07248 & 39.93047 & $\mathrm{NJ}$ \\
\hline 1106 & 0.18 & -74.06033 & 39.99419 & NJ & 1178 & -0.25 & -74.06683 & 39.96215 & NJ & 1250 & 0.75 & -74.07249 & 39.93001 & $\mathrm{NJ}$ \\
\hline 1107 & 0.19 & -74.06044 & 39.99375 & $\mathrm{NJ}$ & 1179 & 0.02 & -74.0667 & 39.96168 & NJ & 1251 & 0.83 & -74.07249 & 39.92956 & $\mathrm{NJ}$ \\
\hline 1108 & 0.22 & -74.06055 & 39.99331 & $\mathrm{NJ}$ & 1180 & -0.31 & -74.06694 & 39.96126 & NJ & 1252 & 0.83 & -74.07262 & 39.92912 & $\mathrm{NJ}$ \\
\hline 1109 & 0.2 & -74.06065 & 39.99286 & NJ & 1181 & -0.3 & .74 .06702 & 39.96081 & $\mathrm{NJ}$ & 1253 & 0.8 & -74.07274 & 39.92867 & $\mathrm{NJ}$ \\
\hline 1110 & 0.17 & -74.06075 & 39.99242 & NJ & 1182 & -0.28 & -74.06708 & 39.96036 & NJ & 1254 & 0.8 & -74.07288 & 39.92824 & $\mathrm{NJ}$ \\
\hline 1111 & 0.12 & -74.06087 & 39.99198 & $\mathrm{NJ}$ & 1183 & -0.26 & -74.06714 & 39.95991 & NJ & 1255 & 0.84 & -74.07294 & 39.92779 & $\mathrm{NJ}$ \\
\hline 1112 & 0.09 & -74.06096 & 539.99153 & $\mathrm{NJ}$ & 1184 & -0.23 & -74.06721 & 39.95946 & $\mathrm{NJ}$ & 1256 & 0.87 & -74.07304 & 39.92734 & $\mathrm{NJ}$ \\
\hline 1113 & 0.11 & -74.0611 & 39.99109 & NJ & 1185 & -0.33 & -74.06723 & 39.95901 & NJ & 1257 & 0.87 & -74.07317 & 39.9269 & $\mathrm{NJ}$ \\
\hline 1114 & 0.08 & -74.0612 & 39.99065 & NJ & 1186 & -0.24 & -74.06729 & 39.95856 & NJ & 1258 & 0.91 & -74.07323 & 39.92645 & $\mathrm{NJ}$ \\
\hline 1115 & 0.11 & -74.06131 & 39.99021 & $\mathrm{NJ}$ & 1187 & -0.18 & -74.06734 & 39.95811 & $\mathrm{NJ}$ & 1259 & 0.91 & .74 .0733 & 39.92601 & $\mathrm{NJ}$ \\
\hline 1116 & 0.2 & -74.06142 & 39.98976 & NJ & 1188 & -0.17 & -74.06738 & 39.95766 & NJ & 1260 & 0.92 & -74.07339 & 39.92556 & $\mathrm{NJ}$ \\
\hline 1117 & 0.19 & -74.06154 & 39.98932 & NJ & 1189 & -0.1 & -74.06743 & 39.95721 & NJ & 1261 & 0.82 & -74.07349 & 39.92512 & NJ \\
\hline 1118 & 0.23 & -74.06165 & 39.98888 & NJ & 1190 & -0.05 & -74.06744 & 39.95675 & $\mathrm{NJ}$ & 1262 & 0.67 & -74.07361 & 39.92468 & $\mathrm{NJ}$ \\
\hline 1119 & 0.22 & -74.06175 & 39.98844 & NJ & 1191 & -0.07 & -74.06749 & 39.9563 & NJ & 1263 & 0.65 & -74.07372 & 39.92423 & $\mathrm{NJ}$ \\
\hline 1120 & 0.23 & -74.06184 & 39.98799 & NJ & 1192 & -0.14 & -74.06757 & 39.95586 & NJ & 1264 & 0.77 & -74.07376 & 39.92378 & $\mathrm{NJ}$ \\
\hline 1121 & 0.13 & -74.06197 & 39.98755 & NJ & 1193 & -0.09 & -74.06766 & 539.95541 & $\mathrm{NJ}$ & 1265 & 0.8 & -74.07387 & 39.92334 & $\mathrm{NJ}$ \\
\hline 1122 & 0.11 & -74.06207 & 39.98711 & NJ & 1194 & -0.13 & -74.06772 & 39.95497 & $\mathrm{NJ}$ & 1266 & 0.73 & -74.07411 & 39.92292 & NJ \\
\hline 1123 & 0.11 & -74.06216 & 39.98666 & NJ & 1195 & -0.17 & -74.06776 & 39.95451 & NJ & 1267 & 0.77 & -74.07414 & 39.92246 & $\mathrm{NJ}$ \\
\hline 1124 & 0.11 & -74.06229 & 39.98622 & NJ & 1196 & -0.17 & -74.06784 & 39.95407 & NJ & 1268 & 0.51 & -74.07457 & 39.92206 & NJ \\
\hline 1125 & 0.1 & -74.06238 & 39.98578 & NJ & 1197 & -0.11 & -74.06792 & 39.95362 & $\mathrm{NJ}$ & 1269 & 0.49 & -74.07472 & 39.92162 & $\mathrm{NJ}$ \\
\hline 1126 & 0.07 & -74.06253 & 39.98534 & NJ & 1198 & -0.1 & -74.06802 & 39.95318 & $\mathrm{NJ}$ & 1270 & 0.48 & -74.07481 & 39.92118 & $\mathrm{NJ}$ \\
\hline 1127 & -0.03 & -74.06264 & 39.9849 & NJ & 1199 & -0.1 & .74 .06808 & 39.95273 & NJ & 1271 & 0.5 & -74.07494 & 39.92074 & $\mathrm{NJ}$ \\
\hline 1128 & 0.06 & -74.06271 & 39.98445 & $\mathrm{NJ}$ & 1200 & -0.1 & -74.06816 & 39.95228 & NJ & 1272 & 0.48 & -74.07501 & 39.92029 & $\mathrm{NJ}$ \\
\hline 1129 & 0.12 & -74.06282 & 39.98401 & NJ & 1201 & -0.09 & -74.06825 & 39.95184 & NJ & 1273 & 0.37 & -74.07518 & 39.91986 & $\mathrm{NJ}$ \\
\hline 1130 & 0.1 & -74.06294 & 39.98357 & NJ & 1202 & -0.03 & -74.06833 & 39.95139 & $\mathrm{NJ}$ & 1274 & 0.37 & -74.07532 & 39.91942 & $\mathrm{NJ}$ \\
\hline 1131 & 0.05 & -74.06305 & 39.98312 & NJ & 1203 & -0.03 & .74 .06842 & 39.95095 & $\mathrm{NJ}$ & 1275 & 0.41 & -74.07539 & 39.91897 & $\mathrm{NJ}$ \\
\hline 1132 & 0.02 & -74.06316 & 39.98268 & $\mathrm{NJ}$ & 1204 & 0.03 & -74.06847 & 39.9505 & $\mathrm{NJ}$ & 1276 & 0.44 & -74.0755 & 39.91853 & NJ \\
\hline 1133 & 0.03 & -74.06326 & 39.98224 & NJ & 1205 & 0.02 & -74.06854 & 39.95005 & $\mathrm{NJ}$ & 1277 & 0.47 & -74.07558 & 39.91808 & $\mathrm{NJ}$ \\
\hline 1134 & 0.05 & -74.06335 & 39.98179 & NJ & 1206 & 0.07 & .74 .0686 & 39.9496 & $\mathrm{NJ}$ & 1278 & 0.44 & -74.07571 & 39.91764 & $\mathrm{NJ}$ \\
\hline 1135 & 0.07 & -74.06345 & 39.98135 & NJ & 1207 & 0.12 & -74.06868 & 39.94915 & NJ & 1279 & 0.44 & -74.07579 & 39.9172 & NJ \\
\hline 1136 & 0.03 & -74.06355 & 39.98091 & NJ & 1208 & 0.18 & -74.06876 & 39.94871 & $\mathrm{NJ}$ & 1280 & 0.43 & -74.07587 & 39.91675 & NJ \\
\hline 1137 & 0.02 & -74.06367 & 39.98046 & NJ & 1209 & 0.26 & -74.06882 & 39.94826 & NJ & 1281 & 0.4 & -74.07594 & 39.9163 & NJ \\
\hline 1138 & 0.01 & -74.0638 & 39.98003 & NJ & 1210 & 0.29 & -74.06891 & 39.94781 & $\mathrm{NJ}$ & 1282 & 0.34 & -74.0761 & 39.91586 & $\mathrm{NJ}$ \\
\hline 1139 & 0.01 & -74.06396 & 39.97959 & NJ & 1211 & 0.28 & -74.06902 & 39.94737 & NJ & 1283 & 0.32 & -74.07619 & 39.91542 & NJ \\
\hline 1140 & 0.01 & -74.06403 & 39.97914 & $\mathrm{NJ}$ & 1212 & 0.32 & .74 .06908 & 39.94692 & NJ & 1284 & 0.27 & -74.07629 & 39.91498 & NJ \\
\hline 1141 & -0.01 & -74.06407 & 39.97869 & $\mathrm{NJ}$ & 1213 & 0.28 & .74 .06914 & 39.94647 & $\mathrm{NJ}$ & 1285 & 0.22 & -74.0764 & 39.91453 & NJ \\
\hline 1142 & 0.02 & -74.06413 & 39.97824 & NJ & 1214 & 0.36 & -74.06918 & 39.94602 & NJ & 1286 & 0.22 & -74.07648 & 39.91409 & NJ \\
\hline 1143 & 0.03 & -74.06415 & 39.97779 & NJ & 1215 & 0.41 & .74 .06924 & 39.94558 & NJ & 1287 & 0.18 & -74.07658 & 39.91365 & NJ \\
\hline 1144 & 0.34 & -74.06396 & 39.97731 & NJ & 1216 & 0.4 & -74.06935 & 39.94513 & NJ & 1288 & 0.19 & -74.07669 & 39.9132 & NJ \\
\hline 1145 & 0.02 & -74.06429 & 39.97689 & NJ & 1217 & 0.41 & -74.06943 & 39.94468 & NJ & 1289 & 0.23 & -74.0768 & 39.91276 & NJ \\
\hline 1146 & 0.08 & -74.06442 & 39.97645 & NJ & 1218 & 0.37 & .74 .06953 & 39.94424 & NJ & 1290 & 0.16 & -74.07703 & 39.91233 & NJ \\
\hline 1147 & 0.08 & -74.06453 & 39.97601 & NJ & 1219 & 0.44 & -74.06956 & 39.94379 & NJ & 1291 & 0.19 & -74.0771 & 39.91189 & NJ \\
\hline 1148 & 0.1 & -74.06467 & 39.97557 & $\mathrm{NJ}$ & 1220 & 0.51 & -74.06963 & 39.94334 & NJ & 1292 & 0.24 & -74.07718 & 39.91144 & NJ \\
\hline 1149 & 0.12 & -74.06474 & 39.97512 & NJ & 1221 & 0.6 & -74.06947 & 39.94287 & $\mathrm{NJ}$ & 1293 & 0.17 & -74.0773 & 39.911 & NJ \\
\hline 1150 & 0.09 & -74.06485 & 39.97468 & NJ & 1222 & 1.66 & -74.06842 & 39.94228 & NJ & 1294 & 0.1 & -74.07745 & 39.91056 & NJ \\
\hline 1151 & 0.14 & -74.06496 & 39.97424 & $\mathrm{NJ}$ & 1223 & 0.72 & -74.06946 & 39.94195 & NJ & 1295 & 0.08 & -74.07756 & 39.91012 & NJ \\
\hline 1152 & 0.09 & -74.06508 & 39.9738 & $\mathrm{NJ}$ & 1224 & 0.61 & -74.06966 & 39.94152 & NJ & 1296 & 0.07 & -74.07761 & 39.90967 & NJ \\
\hline
\end{tabular}




\begin{tabular}{|c|c|c|c|c|c|c|c|c|c|c|c|c|c|c|}
\hline TR\# & RATE & LONG & LAT & ST & IR\# & RATE & LONG & LAT & ST & TR\# & RATE & LONG & LAT & ST \\
\hline 1297 & 0.12 & -74.07768 & 339.90922 & NJ & 1369 & -0.13 & .74 .0822 & 39.87693 & NJ & 1441 & -0.17 & -74.08617 & 39.84463 & $\mathrm{NJ}$ \\
\hline 1298 & 0.14 & -74.07776 & 39.90877 & $\mathrm{NJ}$ & 1370 & -0.08 & -74.08221 & 39.87646 & NJ & 1442 & -0.18 & .74 .08623 & 39.84418 & $\mathrm{NJ}$ \\
\hline 1299 & 0.17 & -74.07784 & 39.90833 & NJ & 1371 & -0.08 & -74.08224 & 39.87601 & NJ & 1443 & -0.13 & -74.0863 & 39.84374 & $\mathrm{NJ}$ \\
\hline 1300 & 0.2 & -74.07776 & 39.90786 & NJ & 1372 & -0.07 & -74.08241 & 39.87557 & NJ & 1444 & -0.13 & .74 .0864 & 39.84329 & $\mathrm{NJ}$ \\
\hline 1301 & 0.16 & -74.0779 & 39.90742 & NJ & 1373 & -0.17 & -74.08258 & 39.87513 & NJ & 1445 & -0.15 & -74.08646 & 39.84284 & NJ \\
\hline 1302 & 0.12 & -74.07809 & 39.90699 & $\mathrm{NJ}$ & 1374 & -0.15 & -74.0827 & 39.87469 & $\mathrm{NJ}$ & 1446 & -0.14 & -74.08652 & 39.8424 & NJ \\
\hline 1303 & 0.05 & -74.07819 & 39.90655 & NJ & 1375 & -0.15 & -74.08282 & 39.87424 & NJ & 1447 & -0.12 & -74.08662 & 39.84195 & $\mathrm{NJ}$ \\
\hline 1304 & 0.07 & -74.07814 & 39.90608 & NJ & 1376 & -0.07 & -74.08286 & 39.87379 & NJ & 1448 & -0.12 & -74.08669 & 39.8415 & $\mathrm{NJ}$ \\
\hline 1305 & 0.07 & -74.07817 & 39.90563 & NJ & 1377 & -0.1 & -74.08289 & 39.87334 & NJ & 1449 & -0.02 & -74.08675 & 39.84105 & $\mathrm{NJ}$ \\
\hline 1306 & 0.12 & -74.07826 & 39.90519 & NJ & 1378 & -0.07 & -74.08287 & 39.87289 & NJ & 1450 & -0.02 & -74.08681 & 39.84061 & $\mathrm{NJ}$ \\
\hline 1307 & 0.12 & -74.07832 & 39.90474 & NJ & 1379 & -0.07 & -74.08282 & 39.87243 & NJ & 1451 & -0.04 & -74.0869 & 39.84016 & $\mathrm{NJ}$ \\
\hline 1308 & 0.07 & -74.07835 & 39.90429 & NJ & 1380 & -0.03 & -74.08276 & 39.87198 & NJ & 1452 & -0.02 & -74.08698 & 39.83971 & $\mathrm{NJ}$ \\
\hline 1309 & 0.04 & -74.07841 & 39.90384 & NJ & 1381 & -0.02 & -74.08276 & 39.87153 & NJ & 1453 & -0.05 & -74.08704 & 39.83927 & $\mathrm{NJ}$ \\
\hline 1310 & 0.03 & -74.07846 & 639.90339 & NJ & 1382 & -0.01 & -74.08286 & 39.87108 & NJ & 1454 & -0.14 & -74.0871 & 39.83882 & $\mathrm{NJ}$ \\
\hline 1311 & 0.05 & -74.07851 & 39.90294 & NJ & 1383 & 0.02 & -74.08299 & 39.87064 & NJ & 1455 & -0.2 & -74.08716 & 39.83837 & $\mathrm{NJ}$ \\
\hline 1312 & 0.03 & -74.07864 & 39.9025 & NJ & 1384 & 0.07 & -74.08313 & 39.87019 & NJ & 1456 & -0.28 & -74.08719 & 39.83792 & NJ \\
\hline 1313 & -0.02 & -74.07874 & 39.90205 & NJ & 1385 & 0.07 & -74.08325 & 39.86975 & $\mathrm{NJ}$ & 1457 & -0.27 & -74.0872 & 39.83747 & $\mathrm{NJ}$ \\
\hline 1314 & -0.03 & -74.07884 & 39.90161 & NJ & 1386 & 0.11 & -74.08331 & 39.8693 & NJ & 1458 & -0.26 & .74 .08723 & 39.83702 & $\mathrm{NJ}$ \\
\hline 1315 & 0.01 & -74.07896 & 39.90117 & NJ & 1387 & 0.09 & -74.08342 & 39.86886 & NJ & 1459 & -0.25 & -74.08728 & 39.83657 & $\mathrm{NJ}$ \\
\hline 1316 & 0.05 & -74.07909 & 39.90073 & $\mathrm{NJ}$ & 1388 & 0.13 & -74.08351 & 39.86841 & NJ & 1460 & -0.28 & -74.08736 & 39.83612 & NJ \\
\hline 1317 & -0.04 & -74.07916 & 39.90028 & $\mathrm{NJ}$ & 1389 & 0.15 & -74.08359 & 39.86796 & NJ & 1461 & -0.27 & -74.08743 & 39.83568 & NJ \\
\hline 1318 & -0.04 & -74.07922 & 39.89983 & $\mathrm{NJ}$ & 1390 & 0.16 & -74.08365 & 39.86752 & NJ & 1462 & -0.17 & -74.08746 & 39.83523 & NJ \\
\hline 1319 & -0.07 & -74.07928 & 39.89938 & NJ & 1391 & 0.16 & -74.08369 & 39.86707 & NJ & 1463 & -0.16 & -74.08751 & 39.83478 & NJ \\
\hline 1320 & -0.1 & -74.07938 & 39.89894 & NJ & 1392 & 0.14 & -74.08377 & 39.86662 & NJ & 1464 & -0.21 & -74.08757 & 39.83433 & NJ \\
\hline 1321 & -0.08 & -74.07944 & 39.89849 & $\mathrm{NJ}$ & 1393 & 0.01 & -74.08388 & 39.86618 & NJ & 1465 & -0.28 & -74.08763 & 39.83388 & NJ \\
\hline 1322 & -0.14 & -74.07948 & 39.89804 & NJ & 1394 & 0.05 & -74.08389 & 39.86573 & NJ & 1466 & -0.34 & -74.08771 & 39.83343 & NJ \\
\hline 1323 & -0.14 & -74.07956 & 39.89759 & NJ & 1395 & 0.09 & -74.08398 & 39.86528 & NJ & 1467 & -0.31 & -74.08777 & 39.83299 & NJ \\
\hline 1324 & -0.11 & -74.07961 & 39.89714 & $\mathrm{NJ}$ & 1396 & 0.09 & -74.08408 & 39.86483 & NJ & 1468 & -0.29 & -74.08786 & 39.83254 & NJ \\
\hline 1325 & -0.08 & -74.07967 & 39.89669 & $\mathrm{NJ}$ & 1397 & 0.1 & .74 .08411 & 39.86438 & NJ & 1469 & -0.29 & -74.08792 & 39.83209 & NJ \\
\hline 1326 & -0.13 & -74.07976 & 39.89625 & NJ & 1398 & 0.18 & -74.08408 & 39.86393 & NJ & 1470 & -0.3 & -74.088 & 39.83165 & NJ \\
\hline 1327 & -0.16 & -74.07983 & 39.8958 & $\mathrm{NJ}$ & 1399 & 0.23 & -74.08414 & 39.86348 & NJ & 1471 & -0.27 & -74.088 & 39.83119 & $\mathrm{NJ}$ \\
\hline 1328 & -0.24 & -74.07994 & 39.89536 & NJ & 1400 & 0.21 & -74.08421 & 39.86303 & NJ & 1472 & -0.26 & -74.08797 & 39.83074 & NJ \\
\hline 1329 & -0.32 & -74.08 & 39.89491 & $\mathrm{NJ}$ & 1401 & 0.22 & -74.08427 & 39.86259 & NJ & 1473 & -0.29 & -74.088 & 39.83029 & NJ \\
\hline 1330 & -0.32 & -74.08003 & 39.89446 & NJ & 1402 & 0.25 & -74.08432 & 39.86214 & NJ & 1474 & -0.33 & -74.08804 & 39.82984 & NJ \\
\hline 1331 & -0.41 & -74.08009 & 39.89401 & NJ & 1403 & 0.27 & -74.08435 & 39.86169 & NJ & 1475 & -0.33 & -74.08809 & 39.82939 & NJ \\
\hline 1332 & -0.48 & -74.08015 & 39.89356 & $\mathrm{NJ}$ & 1404 & 0.28 & -74.0844 & 39.86124 & NJ & 1476 & -0.34 & -74.08813 & 39.82894 & NJ \\
\hline 1333 & -0.53 & -74.08025 & 39.89312 & $\mathrm{NJ}$ & 1405 & 0.2 & -74.08446 & 39.86079 & NJ & 1477 & -0.27 & -74.08818 & 39.82849 & NJ \\
\hline 1334 & -0.5 & -74.08037 & 39.89268 & NJ & 1406 & 0.16 & -74.0845 & 39.86034 & NJ & 1478 & -0.3 & -74.08824 & 39.82805 & NJ \\
\hline 1335 & -0.48 & -74.08051 & 39.89224 & NJ & 1407 & 0.14 & -74.0845 & 39.85989 & NJ & 1479 & -0.31 & -74.08827 & 39.8276 & NJ \\
\hline 1336 & -0.49 & -74.08058 & 39.89179 & $\mathrm{NJ}$ & 1408 & 0.09 & -74.08455 & 39.85944 & NJ & 1480 & -0.21 & -74.08833 & 39.82715 & NJ \\
\hline 1337 & -0.49 & -74.08066 & 39.89134 & $\mathrm{NJ}$ & 1409 & 0.08 & -74.08463 & 39.85899 & NJ & 1481 & -0.25 & -74.08839 & 39.8267 & NJ \\
\hline 1338 & -0.51 & -74.0807 & 39.89089 & $\mathrm{NJ}$ & 1410 & 0.07 & -74.08466 & 39.85854 & NJ & 1482 & -0.17 & -74.08842 & 39.82625 & NJ \\
\hline 1339 & -0.46 & -74.08073 & 39.89044 & $\mathrm{NJ}$ & 1411 & 0.14 & -74.08464 & 39.85809 & NJ & 1483 & -0.1 & -74.08846 & 39.8258 & NJ \\
\hline 1340 & -0.41 & -74.08076 & 39.88999 & $\mathrm{NJ}$ & 1412 & 0.25 & .74 .08463 & 39.85764 & NJ & 1484 & -0.09 & -74.08855 & 39.82535 & NJ \\
\hline 1341 & -0.4 & .74 .08083 & 39.88954 & NJ & 1413 & 0.28 & -74.08463 & 39.85719 & NJ & 1485 & -0.01 & -74.08858 & 39.8249 & NJ \\
\hline 1342 & -0.36 & -74.08089 & 39.88909 & NJ & 1414 & 0.24 & -74.08476 & 39.85674 & NJ & 1486 & -0.02 & -74.08862 & 39.82446 & NJ \\
\hline 1343 & -0.37 & -74.08096 & 39.88864 & NJ & 1415 & 0.23 & -74.08482 & 39.85629 & NJ & 1487 & -0.05 & .74 .08865 & 39.82401 & NJ \\
\hline 1344 & -0.44 & -74.08095 & 39.88819 & NJ & 1416 & 0.22 & -74.08488 & 39.85585 & NJ & 1488 & -0.11 & -74.08868 & 39.82356 & NJ \\
\hline 1345 & -0.52 & -74.08096 & 39.88773 & $\mathrm{NJ}$ & 1417 & 0.21 & .74 .08495 & 39.8554 & NJ & 1489 & -0.08 & -74.08871 & 39.82311 & NJ \\
\hline 1346 & -0.56 & -74.08109 & 39.88729 & NJ & 1418 & 0.1 & -74.08508 & 39.85496 & NJ & 1490 & -0.07 & -74.08878 & 39.82266 & NJ \\
\hline 1347 & -0.55 & .74 .08118 & 39.88685 & NJ & 1419 & 0.1 & -74.08511 & 39.85451 & NJ & 1491 & -0.1 & -74.08887 & 39.82221 & NJ \\
\hline 1348 & -0.54 & -74.08127 & 39.8864 & NJ & 1420 & 0.13 & -74.08516 & 39.85406 & NJ & 1492 & -0.11 & -74.08888 & 39.82176 & NJ \\
\hline 1349 & -0.5 & -74.08125 & 39.88594 & NJ & 1421 & 0.11 & -74.08521 & 39.85361 & NJ & 1493 & -0.09 & -74.08894 & 39.82131 & NJ \\
\hline 1350 & -0.52 & -74.08134 & 39.8855 & $\mathrm{NJ}$ & 1422 & 0.05 & -74.08524 & 39.85316 & NJ & 1494 & -0.12 & -74.08899 & 39.82086 & NJ \\
\hline 1351 & -0.49 & -74.08141 & 39.88505 & $\mathrm{NJ}$ & 1423 & -0.03 & -74.08533 & 39.85271 & $\mathrm{NJ}$ & 1495 & -0.09 & -74.08904 & 39.82041 & $\mathrm{NJ}$ \\
\hline 1352 & -0.47 & -74.08145 & 39.8846 & $\mathrm{NJ}$ & 1424 & -0.08 & -74.08542 & 39.85227 & NJ & 1496 & -0.06 & -74.0891 & 39.81997 & NJ \\
\hline 1353 & -0.45 & -74.08148 & 39.88415 & NJ & 1425 & -0.09 & -74.08553 & 39.85182 & NJ & 1497 & -0.14 & -74.08911 & 39.81952 & NJ \\
\hline 1354 & -0.42 & -74.08159 & 39.8837 & NJ & 1426 & -0.08 & -74.08557 & 39.85137 & NJ & 1498 & -0.17 & -74.08916 & 39.81907 & NJ \\
\hline 1355 & -0.4 & -74.08167 & 39.88326 & $\mathrm{NJ}$ & 1427 & -0.09 & -74.08565 & 39.85093 & NJ & 1499 & -0.24 & -74.0892 & 39.81862 & NJ \\
\hline 1356 & -0.38 & -74.08171 & 39.88281 & $\mathrm{NJ}$ & 1428 & -0.04 & -74.0854 & 39.85046 & NJ & 1500 & -0.23 & -74.08928 & 39.81817 & NJ \\
\hline 1357 & -0.37 & -74.08174 & 39.88235 & NJ & 1429 & -0.02 & -74.08546 & 39.85001 & NJ & 1501 & -0.25 & -74.08939 & 39.81773 & $\mathrm{NJ}$ \\
\hline 1358 & -0.32 & -74.08174 & 39.8819 & $\mathrm{NJ}$ & 1430 & -0.05 & -74.08551 & 39.84956 & NJ & 1502 & -0.25 & -74.08949 & 39.81728 & NJ \\
\hline 1359 & -0.27 & -74.08177 & 39.88144 & NJ & 1431 & -0.05 & -74.08557 & 39.84911 & NJ & 1503 & -0.28 & -74.08952 & 39.81683 & NJ \\
\hline 1360 & -0.27 & .74 .08185 & 39.881 & NJ & 1432 & -0.05 & -74.08565 & 39.84867 & NJ & 1504 & -0.25 & -74.08955 & 39.81638 & NJ \\
\hline 1361 & -0.27 & -74.08191 & 39.88055 & $\mathrm{NJ}$ & 1433 & -0.15 & -74.086 & 39.84824 & NJ & 1505 & -0.2 & -74.0896 & 39.81593 & NJ \\
\hline 1362 & -0.24 & -74.08195 & 39.8801 & $\mathrm{NJ}$ & 1434 & -0.16 & -74.08575 & 39.84777 & $\mathrm{NJ}$ & 1506 & -0.14 & -74.08963 & 39.81548 & NJ \\
\hline 1363 & -0.22 & -74.08202 & 39.87965 & NJ & 1435 & -0.14 & -74.08583 & 39.84732 & NJ & 1507 & -0.14 & -74.08968 & 39.81503 & $\mathrm{NJ}$ \\
\hline 1364 & -0.16 & -74.08202 & 39.87919 & NJ & 1436 & -0.12 & -74.08589 & 39.84687 & NJ & 1508 & -0.17 & -74.08971 & 39.81458 & NJ \\
\hline 1365 & -0.13 & -74.08203 & 39.87874 & $\mathrm{NJ}$ & 1437 & -0.1 & -74.08594 & 39.84642 & NJ & 1509 & -0.14 & -74.08978 & 39.81414 & NJ \\
\hline 1366 & -0.15 & -74.08202 & 39.87828 & NJ & 1438 & -0.14 & -74.086 & 39.84598 & NJ & 1510 & -0.09 & .74 .08984 & 39.81369 & NJ \\
\hline 1367 & -0.2 & -74.08206 & 39.87783 & NJ & 1439 & -0.22 & -74.08607 & 39.84553 & NJ & 1511 & -0.08 & -74.08995 & 39.81324 & NJ \\
\hline 1368 & -0.18 & -74.08214 & 39.87738 & NJ & 1440 & -0.22 & -74.08611 & 39.84508 & NJ & 1512 & -0.08 & -74.09003 & 39.8128 & NJ \\
\hline
\end{tabular}




\begin{tabular}{|c|c|c|c|c|c|c|c|c|c|c|c|c|c|c|}
\hline TR\# & RATE & LONG & LAT & ST & I TR\# & RATE & LONG & LAT & ST & TR\# & RATE & LONG & LAT & ST \\
\hline 1513 & -0.06 & -74.09009 & 39.81235 & $\mathrm{NJ}$ & 1585 & -1.92 & -74.09464 & 39.78127 & $\mathrm{NJ}$ & 1657 & -0.07 & -74.11205 & 539.74362 & $\mathrm{NJ}$ \\
\hline 1514 & -0.03 & -74.09015 & 59.8119 & $\mathrm{NJ}$ & 1586 & -1.88 & .74 .09473 & 39.78083 & $\mathrm{NJ}$ & 1658 & -0.15 & -74.1124 & 39.74324 & $\mathrm{NJ}$ \\
\hline 1515 & -0.07 & -74.09019 & 39.81145 & $\mathrm{NJ}$ & 1587 & -1.81 & -74.0948 & 39.78038 & $\mathrm{NJ}$ & 1659 & -0.18 & -74.1127 & 39.74285 & $\mathrm{NJ}$ \\
\hline 1516 & -0.13 & -74.09023 & 39.811 & NJ & 1588 & -1.78 & -74.09486 & 39.77993 & $\mathrm{NJ}$ & 1660 & -0.25 & -74.11298 & 839.74245 & $\mathrm{NJ}$ \\
\hline 1517 & -0.12 & -74.09024 & 39.81055 & $\mathrm{NJ}$ & 1589 & -1.7 & -74.09499 & 39.77949 & $\mathrm{NJ}$ & 1661 & -0.35 & -74.11324 & 439.74205 & $\mathrm{NJ}$ \\
\hline 1518 & -0.44 & -74.09058 & 39.80858 & NJ & 1590 & -1.59 & -74.09508 & 39.77904 & $\mathrm{NJ}$ & 1662 & -0.38 & -74.11356 & 539.74166 & $\mathrm{NJ}$ \\
\hline 1519 & -0.55 & -74.09065 & 39.8081 & NJ & 1591 & -1.36 & -74.09511 & 39.77859 & NJ & 1663 & -0.37 & -74.11385 & 539.74127 & $\mathrm{NJ}$ \\
\hline 1520 & -0.58 & -74.0907 & 39.80763 & $\mathrm{NJ}$ & 1592 & -1.02 & -74.09514 & 39.77814 & $\mathrm{NJ}$ & 1664 & -0.33 & -74.11411 & 39.74086 & $\mathrm{NJ}$ \\
\hline 1521 & -0.62 & -74.09081 & 39.80715 & $\mathrm{NJ}$ & 1593 & -0.72 & -74.09517 & 39.77768 & $\mathrm{NJ}$ & 1665 & -0.33 & -74.11433 & 39.74045 & $\mathrm{NJ}$ \\
\hline 1522 & -0.59 & .74 .0909 & 39.80668 & NJ & 1594 & -0.49 & -74.09525 & 39.77724 & $\mathrm{NJ}$ & 1666 & -0.35 & -74.1145 & 39.74002 & $\mathrm{NJ}$ \\
\hline 1523 & -0.57 & -74.09097 & 39.8062 & NJ & 1595 & -0.22 & -74.09534 & 39.77679 & $\mathrm{NJ}$ & 1667 & -0.42 & -74.11475 & 539.73961 & $\mathrm{NJ}$ \\
\hline 1524 & -0.56 & -74.09103 & 39.80573 & $\mathrm{NJ}$ & 1596 & 0.11 & -74.09543 & 39.77635 & $\mathrm{NJ}$ & 1668 & -0.43 & -74.11504 & 39.73922 & $\mathrm{NJ}$ \\
\hline 1525 & -0.57 & .74 .09109 & 39.80525 & $\mathrm{NJ}$ & 1597 & 0.56 & -74.09547 & 39.7759 & NJ & 1669 & -0.47 & -74.11531 & 39.73881 & $\mathrm{NJ}$ \\
\hline 1526 & -0.6 & -74.09117 & 39.80478 & $\mathrm{NJ}$ & 1598 & 0.98 & -74.09555 & 39.77545 & NJ & 1670 & -0.48 & -74.11554 & 39.7384 & $\mathrm{NJ}$ \\
\hline 1527 & -0.58 & -74.09122 & 39.80431 & NJ & 1599 & 1.78 & -74.09557 & 39.77499 & $\mathrm{NJ}$ & 1671 & -0.55 & -74.11571 & 39.73797 & $\mathrm{NJ}$ \\
\hline 1528 & -0.57 & -74.09129 & 39.80383 & NJ & 1600 & -0.18 & -74.0956 & 39.77454 & $\mathrm{NJ}$ & 1672 & -0.6 & -74.11601 & 39.73758 & $\mathrm{NJ}$ \\
\hline 1529 & -0.61 & -74.09132 & 39.80336 & $\mathrm{NJ}$ & 1601 & 0.12 & -74.09566 & 39.77409 & NJ & 1673 & -0.65 & -74.11629 & 39.73717 & $\mathrm{NJ}$ \\
\hline 1530 & -0.6 & -74.09142 & 39.80288 & $\mathrm{NJ}$ & 1602 & 0.74 & -74.0957 & 39.77363 & NJ & 1674 & -0.66 & -74.11655 & 539.73677 & $\mathrm{NJ}$ \\
\hline 1531 & -0.58 & -74.09142 & 39.80281 & $\mathrm{NJ}$ & 1603 & 2.93 & -74.09706 & 39.76512 & NJ & 1675 & -0.67 & -74.11678 & 39.73636 & NJ \\
\hline 1532 & -0.59 & -74.09148 & 39.80241 & NJ & 1604 & 2.83 & -74.09702 & 39.76465 & NJ & 1676 & -0.71 & -74.11694 & 39.73593 & NJ \\
\hline 1533 & -0.57 & -74.09149 & 39.80236 & $\mathrm{NJ}$ & 1605 & -1.76 & -74.10233 & 39.76454 & $\mathrm{NJ}$ & 1677 & -0.77 & -74.11714 & 49.7355 & $\mathrm{NJ}$ \\
\hline 1534 & -0.65 & -74.09154 & 39.80194 & $\mathrm{NJ}$ & 1606 & -1.4 & -74.10168 & 39.76419 & $\mathrm{NJ}$ & 1678 & -0.74 & -74.11742 & 39.7351 & NJ \\
\hline 1535 & -0.61 & -74.09154 & 39.80191 & $\mathrm{NJ}$ & 1607 & -1.12 & -74.10144 & 39.76394 & NJ & 1679 & -0.74 & -74.11768 & 39.7347 & $\mathrm{NJ}$ \\
\hline 1536 & -0.62 & -74.09158 & 39.80147 & $\mathrm{NJ}$ & 1608 & -1.17 & -74.10139 & 39.76361 & $\mathrm{NJ}$ & 1680 & -0.76 & -74.11792 & 39.73429 & $\mathrm{NJ}$ \\
\hline 1537 & -0.62 & -74.09158 & 39.80146 & $\mathrm{NJ}$ & 1609 & -1.09 & -74.10139 & 39.76348 & $\mathrm{NJ}$ & 1681 & -0.74 & -74.1181 & 39.73386 & NJ \\
\hline 1538 & -0.73 & -74.09164 & 39.80101 & $\mathrm{NJ}$ & 1610 & -1.27 & -74.10146 & 39.76314 & $\mathrm{NJ}$ & 1682 & -0.77 & -74.11833 & 39.73344 & $\mathrm{NJ}$ \\
\hline 1539 & -0.74 & -74.09164 & 39.801 & $\mathrm{NJ}$ & 1611 & -1.48 & .74 .10159 & 39.76269 & $\mathrm{NJ}$ & 1683 & -0.7 & -74.11861 & 39.73304 & $\mathrm{NJ}$ \\
\hline 1540 & -0.85 & -74.09167 & 39.80056 & $\mathrm{NJ}$ & 1612 & -1.76 & -74.10178 & 39.76227 & $\mathrm{NJ}$ & 1684 & -0.66 & -74.11887 & 39.73264 & $\mathrm{NJ}$ \\
\hline 1541 & -0.84 & -74.09167 & 39.80053 & $\mathrm{NJ}$ & 1613 & -2.06 & -74.10197 & 39.76184 & $\mathrm{NJ}$ & 1685 & -0.62 & -74.11913 & 39.73224 & $\mathrm{NJ}$ \\
\hline 1542 & -0.91 & -74.09174 & 39.80011 & $\mathrm{NJ}$ & 1614 & -2.25 & -74.10217 & 39.76142 & $\mathrm{NJ}$ & 1686 & -0.68 & -74.11925 & 39.73179 & $\mathrm{NJ}$ \\
\hline 1543 & -0.91 & -74.09174 & 39.80005 & NJ & 1615 & -2.27 & -74.10229 & 39.76096 & $\mathrm{NJ}$ & 1687 & -0.79 & -74.11945 & 39.73137 & $\mathrm{NJ}$ \\
\hline 1544 & -0.95 & -74.09178 & 39.79965 & $\mathrm{NJ}$ & 1616 & -2.32 & -74.1024 & 39.76051 & $\mathrm{NJ}$ & 1688 & -0.83 & .74 .11974 & 39.73097 & $\mathrm{NJ}$ \\
\hline 1545 & -0.91 & -74.09186 & 39.79921 & $\mathrm{NJ}$ & 1617 & -2.38 & -74.10265 & 39.7601 & $\mathrm{NJ}$ & 1689 & -0.87 & -74.12001 & 39.73057 & $\mathrm{NJ}$ \\
\hline 1546 & -0.84 & .74 .09193 & 39.79876 & $\mathrm{NJ}$ & 1618 & -2.25 & -74.1028 & 39.75966 & $\mathrm{NJ}$ & 1690 & -0.86 & -74.12024 & 39.73016 & $\mathrm{NJ}$ \\
\hline 1547 & -0.83 & -74.09204 & 39.79832 & $\mathrm{NJ}$ & 1619 & -2.09 & -74.10295 & 39.75922 & $\mathrm{NJ}$ & 1691 & -0.79 & -74.12039 & 39.72972 & $\mathrm{NJ}$ \\
\hline 1548 & -0.83 & -74.09215 & 39.79787 & $\mathrm{NJ}$ & 1620 & -1.96 & -74.1031 & 39.75878 & $\mathrm{NJ}$ & 1692 & -0.8 & -74.12061 & 39.7293 & $\mathrm{NJ}$ \\
\hline 1549 & -0.81 & -74.09222 & 39.79743 & $\mathrm{NJ}$ & 1621 & -1.79 & -74.10323 & 39.75833 & $\mathrm{NJ}$ & 1693 & -0.77 & -74.12088 & 39.7289 & $\mathrm{NJ}$ \\
\hline 1550 & -0.79 & -74.09222 & 39.79697 & NJ & 1622 & -1.59 & .74 .10332 & 39.75788 & $\mathrm{NJ}$ & 1694 & -0.65 & -74.12112 & 39.72849 & $\mathrm{NJ}$ \\
\hline 1551 & -0.78 & -74.09233 & 39.79653 & $\mathrm{NJ}$ & 1623 & -1.26 & -74.10329 & 39.75738 & $\mathrm{NJ}$ & 1695 & -0.61 & -74.12131 & 39.72806 & NJ \\
\hline 1552 & -0.82 & -74.09239 & 39.79608 & $\mathrm{NJ}$ & 1624 & 0.81 & -74.10114 & 39.75618 & $\mathrm{NJ}$ & 1696 & -0.26 & -74.12131 & 39.72757 & $\mathrm{NJ}$ \\
\hline 1553 & -0.86 & -74.0925 & 39.79563 & $\mathrm{NJ}$ & 1625 & 0.18 & -74.10217 & 39.75603 & $\mathrm{NJ}$ & 1697 & -0.58 & -74.12172 & 39.72722 & $\mathrm{NJ}$ \\
\hline 1554 & -0.86 & -74.09258 & 39.79519 & $\mathrm{NJ}$ & 1626 & -0.38 & -74.10251 & 39.75565 & $\mathrm{NJ}$ & 1698 & -0.63 & -74.12202 & 39.72683 & $\mathrm{NJ}$ \\
\hline 1555 & -0.87 & -74.09264 & 39.79474 & $\mathrm{NJ}$ & 1627 & -0.24 & -74.10268 & 39.75521 & $\mathrm{NJ}$ & 1699 & -0.71 & -74.12228 & 39.72642 & $\mathrm{NJ}$ \\
\hline 1556 & -0.89 & -74.09271 & 39.79429 & $\mathrm{NJ}$ & 1628 & 0.12 & -74.10277 & 39.75476 & $\mathrm{NJ}$ & 1700 & -0.78 & -74.12248 & 39.726 & $\mathrm{NJ}$ \\
\hline 1557 & -0.9 & -74.0928 & 39.79384 & $\mathrm{NJ}$ & 1629 & 0.43 & -74.10281 & 39.75428 & $\mathrm{NJ}$ & 1701 & -0.74 & -74.12267 & 39.72557 & $\mathrm{NJ}$ \\
\hline 1558 & -0.94 & -74.09288 & 39.7934 & $\mathrm{NJ}$ & 1630 & 2.37 & -74.10133 & 39.7533 & NJ & 1702 & -0.76 & .74 .12291 & 39.72516 & $\mathrm{NJ}$ \\
\hline 1559 & -1.01 & -74.09294 & 39.79295 & $\mathrm{NJ}$ & 1631 & 2.58 & -74.10141 & 39.75284 & $\mathrm{NJ}$ & 1703 & -0.75 & .74 .12318 & 39.72476 & $\mathrm{NJ}$ \\
\hline 1560 & -1.1 & -74.09299 & 39.7925 & $\mathrm{NJ}$ & 1632 & 2.71 & -74.10156 & 39.75239 & $\mathrm{NJ}$ & 1704 & -0.7 & -74.12341 & 39.72435 & NJ \\
\hline 1561 & -1.17 & -74.09308 & 39.79205 & $\mathrm{NJ}$ & 1633 & 2.65 & -74.10188 & 39.75201 & $\mathrm{NJ}$ & 1705 & -0.64 & -74.12361 & 39.72392 & $\mathrm{NJ}$ \\
\hline 1562 & -1.2 & -74.09312 & 39.7916 & $\mathrm{NJ}$ & 1634 & 2.58 & .74 .10226 & 39.75165 & $\mathrm{NJ}$ & 1706 & -0.57 & -74.12376 & 39.72348 & $\mathrm{NJ}$ \\
\hline 1563 & -1.26 & -74.09325 & 39.79116 & $\mathrm{NJ}$ & 1635 & 2.4 & .74 .1028 & 39.75134 & $\mathrm{NJ}$ & 1707 & -0.59 & -74.12395 & 39.72306 & $\mathrm{NJ}$ \\
\hline 1564 & -1.3 & -74.09335 & 39.79072 & $\mathrm{NJ}$ & 1636 & 2.23 & -74.1033 & 39.75101 & $\mathrm{NJ}$ & 1708 & -0.54 & -74.12421 & 39.72265 & $\mathrm{NJ}$ \\
\hline 1565 & -1.34 & .74 .09344 & 39.79027 & $\mathrm{NJ}$ & 1637 & 2.05 & -74.10384 & 39.7507 & NJ & 1709 & -0.48 & -74.12447 & 39.72225 & $\mathrm{NJ}$ \\
\hline 1566 & -1.37 & -74.09354 & 39.78982 & $\mathrm{NJ}$ & 1638 & 1.92 & -74.10429 & 39.75036 & $\mathrm{NJ}$ & 1710 & -0.52 & -74.12468 & 39.72183 & $\mathrm{NJ}$ \\
\hline 1567 & -1.44 & -74.09361 & 39.78938 & $\mathrm{NJ}$ & 1639 & 1.8 & .74 .10475 & 39.75002 & NJ & 1711 & -0.54 & -74.12483 & 39.72139 & $\mathrm{NJ}$ \\
\hline 1568 & -1.44 & -74.09367 & 39.78893 & $\mathrm{NJ}$ & 1640 & 1.69 & -74.10518 & 39.74967 & $\mathrm{NJ}$ & 1712 & -0.55 & -74.12497 & 39.72094 & $\mathrm{NJ}$ \\
\hline 1569 & -1.48 & -74.09377 & 39.78848 & $\mathrm{NJ}$ & 1641 & 1.62 & .74 .10558 & 39.74931 & $\mathrm{NJ}$ & 1713 & -0.89 & -74.1252 & 39.72053 & $\mathrm{NJ}$ \\
\hline 1570 & -1.47 & -74.09375 & 39.78802 & $\mathrm{NJ}$ & 1642 & 1.54 & .74 .10596 & 39.74895 & $\mathrm{NJ}$ & 1714 & -0.97 & -74.12544 & 39.72012 & $\mathrm{NJ}$ \\
\hline 1571 & -1.45 & -74.09373 & 39.78756 & $\mathrm{NJ}$ & 1643 & 1.44 & -74.10638 & 39.7486 & $\mathrm{NJ}$ & 1715 & -1.01 & -74.12572 & 39.71972 & $\mathrm{NJ}$ \\
\hline 1572 & -1.46 & -74.09377 & 39.78711 & $\mathrm{NJ}$ & 1644 & 1.16 & -74.10686 & 39.74827 & $\mathrm{NJ}$ & 1716 & -0.98 & -74.12592 & 39.71929 & $\mathrm{NJ}$ \\
\hline 1573 & -1.47 & -74.0938 & 39.78665 & $\mathrm{NJ}$ & 1645 & 0.99 & -74.10736 & 39.74794 & $\mathrm{NJ}$ & 1717 & -0.98 & -74.1261 & 39.71887 & $\mathrm{NJ}$ \\
\hline 1574 & -1.52 & -74.09383 & 39.7862 & NJ & 1646 & 0.81 & -74.10786 & 39.74762 & $\mathrm{NJ}$ & 1718 & -0.98 & -74.12631 & 39.71845 & $\mathrm{NJ}$ \\
\hline 1575 & -1.55 & -74.09392 & 39.78575 & $\mathrm{NJ}$ & 1647 & 0.67 & -74.10832 & 39.74728 & $\mathrm{NJ}$ & 1719 & -0.95 & -74.12656 & 39.71804 & $\mathrm{NJ}$ \\
\hline 1576 & -1.58 & -74.09399 & 39.78531 & $\mathrm{NJ}$ & 1648 & 0.56 & .74 .1087 & 39.74692 & $\mathrm{NJ}$ & 1720 & -0.92 & -74.12679 & 39.71763 & $\mathrm{NJ}$ \\
\hline 1577 & -1.66 & -74.09407 & 39.78486 & $\mathrm{NJ}$ & 1649 & 0.45 & .74 .10913 & 39.74657 & $\mathrm{NJ}$ & 1721 & -0.9 & -74.12701 & 39.71721 & $\mathrm{NJ}$ \\
\hline 1578 & -1.74 & -74.09413 & 39.78441 & $\mathrm{NJ}$ & 1650 & 0.35 & .74 .10954 & 39.74622 & NJ & 1722 & -0.86 & -74.12724 & 39.7168 & $\mathrm{NJ}$ \\
\hline 1579 & -1.77 & -74.09419 & 39.78396 & $\mathrm{NJ}$ & 1651 & 0.32 & -74.10986 & 39.74583 & $\mathrm{NJ}$ & 1723 & -0.81 & -74.12749 & 39.71639 & $\mathrm{NJ}$ \\
\hline 1580 & -1.78 & -74.09419 & 39.7835 & $\mathrm{NJ}$ & 1652 & 0.23 & .74 .11024 & 39.74547 & NJ & 1724 & -0.8 & -74.12775 & 39.71598 & $\mathrm{NJ}$ \\
\hline 1581 & -1.82 & -74.09425 & 39.78305 & $N J$ & 1653 & 0.22 & -74.11057 & 39.74509 & NJ & 1725 & -0.82 & -74.12796 & 39.71556 & $\mathrm{NJ}$ \\
\hline 1582 & -1.84 & -74.09433 & 39.7826 & $\mathrm{NJ}$ & 1654 & 0.12 & -74.11096 & 39.74472 & $\mathrm{NJ}$ & 1726 & -0.82 & -74.12816 & 39.71514 & $\mathrm{NJ}$ \\
\hline 1583 & -1.87 & .74 .09444 & 39.78216 & $\mathrm{NJ}$ & 1655 & 0.04 & -74.11131 & 39.74435 & $\mathrm{NJ}$ & 1727 & -0.88 & -74.12839 & 39.71473 & $\mathrm{NJ}$ \\
\hline 1584 & -1.87 & -74.0945 & 39.78171 & NJ & 1656 & -0.04 & -74.11172 & 39.744 & $\mathrm{NJ}$ & 1728 & -0.59 & -74.12813 & 39.71415 & $\mathrm{NJ}$ \\
\hline
\end{tabular}




\begin{tabular}{|c|c|c|c|c|c|c|c|c|c|c|c|c|c|c|}
\hline TR\# & RATE & LONG & LAT & ST & TR\# & RATE & LONG & LAT & ST & TR\# & RATE & LONG & LAT & ST \\
\hline 1729 & -1.05 & -74.12874 & 39.71386 & NJ & 1801 & -1.13 & -74.14566 & 39.68505 & NJ & 1873 & -0.28 & .74 .16698 & 39.65712 & $\mathrm{NJ}$ \\
\hline 1730 & -0.99 & -74.12897 & 39.71345 & NJ & 1802 & -1.14 & -74.1459 & 39.68464 & NJ & 1874 & -0.25 & -74.1673 & 39.65674 & $\mathrm{NJ}$ \\
\hline 1731 & -0.98 & -74.1292 & 39.71304 & NJ & 1803 & -1.14 & -74.14619 & 39.68425 & NJ & 1875 & -0.23 & .74 .16756 & 39.65634 & $\mathrm{NJ}$ \\
\hline 1732 & -0.98 & -74.12939 & 39.71261 & NJ & 1804 & -1.15 & -74.14647 & 39.68385 & NJ & 1876 & -0.16 & -74.16783 & 39.65594 & $\mathrm{NJ}$ \\
\hline 1733 & -0.85 & -74.12956 & 39.71218 & NJ & 1805 & -1.1 & -74.14676 & 39.68346 & NJ & 1877 & -0.03 & -74.16815 & 39.65556 & $\mathrm{NJ}$ \\
\hline 1734 & -0.78 & -74.12978 & 39.71176 & NJ & 1806 & -1.16 & -74.14706 & 39.68308 & NJ & 1878 & 0.01 & -74.16833 & 39.65512 & $\mathrm{NJ}$ \\
\hline 1735 & -0.75 & -74.13002 & 39.71134 & NJ & 1807 & -1.16 & -74.14732 & 39.68268 & $\mathrm{NJ}$ & 1879 & -0.09 & -74.16869 & 39.65476 & $\mathrm{NJ}$ \\
\hline 1736 & -0.71 & -74.1302 & 39.71091 & NJ & 1808 & -1.07 & .74 .14757 & 39.68226 & NJ & 1880 & -0.02 & .74 .16904 & 39.6544 & $\mathrm{NJ}$ \\
\hline 1737 & -0.64 & -74.13031 & 39.71046 & NJ & 1809 & -1.11 & -74.14784 & 39.68186 & NJ & 1881 & -0.04 & .74 .16937 & 39.65403 & $\mathrm{NJ}$ \\
\hline 1738 & -0.69 & -74.13045 & 39.71001 & NJ & 1810 & -1.11 & -74.14812 & 39.68147 & NJ & 1882 & -0.08 & -74.16971 & 39.65366 & $\mathrm{NJ}$ \\
\hline 1739 & -0.8 & -74.13065 & 39.70959 & NJ & 1811 & -1.13 & -74.14842 & 39.68108 & NJ & 1883 & -0.05 & -74.17006 & 39.6533 & $\mathrm{NJ}$ \\
\hline 1740 & -0.88 & -74.13091 & 39.70919 & NJ & 1812 & -1.16 & -74.14873 & 39.6807 & NJ & 1884 & -0.07 & -74.1703 & 39.65289 & $\mathrm{NJ}$ \\
\hline 1741 & -0.94 & -74.13113 & 39.70878 & NJ & 1813 & -1.14 & -74.149 & 39.6803 & NJ & 1885 & -0.16 & -74.17072 & 39.65255 & $\mathrm{NJ}$ \\
\hline 1742 & -1.01 & -74.1313 & 39.70834 & NJ & 1814 & -1.21 & -74.14931 & 39.67992 & $\mathrm{NJ}$ & 1886 & -0.12 & -74.17104 & 39.65217 & $\mathrm{NJ}$ \\
\hline 1743 & -0.54 & -74.13104 & 39.70777 & NJ & 1815 & -1.19 & -74.14961 & 39.67954 & $\mathrm{NJ}$ & 1887 & -0.08 & -74.1713 & 39.65177 & NJ \\
\hline 1744 & -1.02 & -74.13165 & 39.70748 & NJ & 1816 & -1.17 & -74.14989 & 39.67913 & NJ & 1888 & -0.11 & -74.1716 & 39.65138 & $\mathrm{NJ}$ \\
\hline 1745 & -1.05 & -74.13194 & 39.70708 & NJ & 1817 & -1.16 & -74.15012 & 39.67872 & NJ & 1889 & -0.11 & .74 .17191 & 39.651 & $\mathrm{NJ}$ \\
\hline 1746 & -1.05 & -74.13226 & 39.7067 & NJ & 1818 & -1.13 & -74.15039 & 39.67832 & $\mathrm{NJ}$ & 1890 & -0.06 & -74.17216 & 39.6506 & $\mathrm{NJ}$ \\
\hline 1747 & -1 & -74.13252 & 39.70629 & NJ & 1819 & -1.11 & -74.1507 & 39.67793 & NJ & 1891 & -0.05 & -74.17236 & 39.65017 & $\mathrm{NJ}$ \\
\hline 1748 & -1.01 & -74.13254 & 39.70624 & NJ & 1820 & -1.1 & -74.15099 & 39.67755 & $\mathrm{NJ}$ & 1892 & -0.06 & -74.17271 & 39.6498 & $\mathrm{NJ}$ \\
\hline 1749 & -0.98 & -74.13269 & 39.70586 & NJ & 1821 & -1.07 & -74.15128 & 39.67716 & NJ & 1893 & -0.09 & -74.17307 & 39.64944 & $\mathrm{NJ}$ \\
\hline 1750 & -0.99 & -74.13271 & 39.7058 & NJ & 1822 & -0.97 & -74.15157 & 39.67676 & NJ & 1894 & -0.12 & -74.17343 & 39.64908 & $\mathrm{NJ}$ \\
\hline 1751 & -1.05 & -74.13284 & 39.70533 & $\mathrm{NJ}$ & 1823 & -0.97 & .74 .15186 & 39.67638 & NJ & 1895 & -0.16 & -74.17381 & 39.64874 & $\mathrm{NJ}$ \\
\hline 1752 & -1.1 & -74.13303 & 39.70489 & $\mathrm{NJ}$ & 1824 & -0.89 & -74.15215 & 39.67599 & $\mathrm{NJ}$ & 1896 & -0.1 & -74.17419 & 39.64838 & $\mathrm{NJ}$ \\
\hline 1753 & -1.09 & -74.1333 & 39.7045 & NJ & 1825 & -0.88 & -74.15248 & 39.67562 & NJ & 1897 & -0.1 & -74.1743 & 39.64791 & $\mathrm{NJ}$ \\
\hline 1754 & -1.1 & -74.13358 & 39.7041 & $\mathrm{NJ}$ & 1826 & -0.9 & -74.15282 & 39.67524 & NJ & 1898 & -0.13 & -74.17462 & 39.64753 & $\mathrm{NJ}$ \\
\hline 1755 & -1.08 & -74.13383 & 39.7037 & NJ & 1827 & -0.94 & -74.15312 & 39.67486 & NJ & 1899 & -0.14 & -74.1749 & 39.64714 & $\mathrm{NJ}$ \\
\hline 1756 & -1.04 & -74.13405 & 39.70328 & NJ & 1828 & -0.94 & -74.15343 & 39.67448 & NJ & 1900 & -0.11 & -74.17514 & 39.64673 & $\mathrm{NJ}$ \\
\hline 1757 & -1.07 & -74.13419 & 39.70281 & NJ & 1829 & -0.97 & -74.15376 & 39.67411 & NJ & 1901 & -0.08 & -74.17548 & 39.64635 & $\mathrm{NJ}$ \\
\hline 1758 & -1.08 & -74.13437 & 39.70238 & NJ & 1830 & -1.01 & -74.15414 & 39.67375 & NJ & 1902 & -0.03 & -74.1758 & 39.64598 & $\mathrm{NJ}$ \\
\hline 1759 & -1.15 & -74.13466 & 39.70199 & NJ & 1831 & -1.01 & -74.15446 & 39.67338 & $\mathrm{NJ}$ & 1903 & 0 & -74.1761 & 39.64559 & $\mathrm{NJ}$ \\
\hline 1760 & -1.2 & -74.13495 & 39.7016 & NJ & 1832 & -0.99 & -74.15475 & 39.67299 & NJ & 1904 & -0.06 & -74.17641 & 39.64521 & $\mathrm{NJ}$ \\
\hline 1761 & -1.23 & -74.13519 & 39.70119 & NJ & 1833 & -0.99 & -74.15503 & 39.67259 & NJ & 1905 & -0.07 & -74.17671 & 39.64483 & $\mathrm{NJ}$ \\
\hline 1762 & -1.16 & -74.13535 & 39.70073 & NJ & 1834 & -0.93 & -74.15535 & 39.67221 & NJ & 1906 & -0.09 & -74.17703 & 39.64445 & NJ \\
\hline 1763 & -1.06 & -74.13547 & 39.70027 & $\mathrm{NJ}$ & 1835 & -0.91 & -74.15565 & 39.67183 & NJ & 1907 & -0.12 & -74.17732 & 39.64406 & $\mathrm{NJ}$ \\
\hline 1764 & -1.16 & -74.13579 & 39.69989 & NJ & 1836 & -0.85 & -74.15585 & 39.6714 & NJ & 1908 & -0.07 & -74.17764 & 39.64368 & $\mathrm{NJ}$ \\
\hline 1765 & -1.19 & -74.13612 & 39.69952 & NJ & 1837 & -0.8 & -74.1561 & 39.67098 & $\mathrm{NJ}$ & 1909 & -0.02 & -74.17799 & 39.64332 & $\mathrm{NJ}$ \\
\hline 1766 & -1.22 & -74.13641 & 39.69913 & NJ & 1838 & -0.83 & -74.15637 & 39.67059 & $\mathrm{NJ}$ & 1910 & 0.02 & -74.17825 & 39.64291 & NJ \\
\hline 1767 & -1.19 & -74.13663 & 39.6987 & NJ & 1839 & -0.82 & -74.15666 & 39.6702 & $\mathrm{NJ}$ & 1911 & 0.07 & -74.1786 & 39.64255 & $\mathrm{NJ}$ \\
\hline 1768 & -1.08 & -74.13681 & 39.69826 & NJ & 1840 & -0.77 & -74.15698 & 39.66982 & NJ & 1912 & 0.12 & -74.17892 & 39.64217 & $\mathrm{NJ}$ \\
\hline 1769 & -1.11 & -74.13705 & 39.69786 & NJ & 1841 & -0.73 & -74.15729 & 39.66944 & NJ & 1913 & -0.46 & -74.17912 & 39.64193 & NJ \\
\hline 1770 & -1.3 & -74.13737 & 39.69747 & NJ & 1842 & -0.72 & -74.15756 & 39.66904 & $\mathrm{NJ}$ & 1914 & -0.49 & -74.17943 & 39.64154 & $\mathrm{NJ}$ \\
\hline 1771 & -1.28 & -74.13766 & 39.69709 & NJ & 1843 & -0.73 & -74.15778 & 39.66862 & NJ & 1915 & -0.52 & -74.17973 & 39.64116 & NJ \\
\hline 1772 & -1.28 & -74.13788 & 39.69666 & NJ & 1844 & -0.62 & -74.158 & 39.6682 & NJ & 1916 & -0.48 & -74.17999 & 39.64076 & $\mathrm{NJ}$ \\
\hline 1773 & -1.25 & -74.13806 & 39.69622 & NJ & 1845 & -0.59 & -74.15826 & 39.66779 & $\mathrm{NJ}$ & 1917 & -0.49 & -74.18015 & 39.64031 & NJ \\
\hline 1774 & -1.16 & -74.13834 & 39.69582 & NJ & 1846 & -0.54 & -74.15857 & 39.66741 & NJ & 1918 & -0.5 & -74.1804 & 39.6399 & $\mathrm{NJ}$ \\
\hline 1775 & -1.13 & -74.13864 & 39.69544 & NJ & 1847 & -0.52 & -74.15892 & 39.66704 & $\mathrm{NJ}$ & 1919 & -0.47 & -74.18069 & 39.63951 & NJ \\
\hline 1776 & -1.15 & -74.13895 & 39.69506 & NJ & 1848 & -0.51 & -74.15926 & 39.66667 & NJ & 1920 & -0.45 & -74.18102 & 39.63913 & NJ \\
\hline 1777 & -1.14 & -74.13921 & 39.69465 & NJ & 1849 & -0.5 & -74.15958 & 39.6663 & NJ & 1921 & -0.45 & -74.18134 & 39.63875 & NJ \\
\hline 1778 & -1.17 & -74.1394 & 39.69422 & NJ & 1850 & -0.53 & -74.15984 & 39.66589 & NJ & 1922 & -0.47 & -74.1817 & 39.6384 & $\mathrm{NJ}$ \\
\hline 1779 & -1.19 & -74.1396 & 39.69379 & NJ & 1851 & -0.53 & .74 .16003 & 39.66546 & NJ & 1923 & -0.52 & -74.18195 & 39.63799 & $\mathrm{NJ}$ \\
\hline 1780 & -1.14 & -74.13986 & 39.69338 & NJ & 1852 & -0.52 & -74.16034 & 39.66508 & NJ & 1924 & -0.51 & -74.18228 & 39.63762 & NJ \\
\hline 1781 & -1.12 & -74.14018 & 39.69301 & $\mathrm{NJ}$ & 1853 & -0.43 & -74.16069 & 39.66472 & NJ & 1925 & -0.51 & -74.18262 & 39.63725 & $\mathrm{NJ}$ \\
\hline 1782 & -1.17 & -74.14047 & 39.69261 & NJ & 1854 & -0.36 & -74.16104 & 39.66435 & NJ & 1926 & -0.57 & -74.18303 & 39.63691 & NJ \\
\hline 1783 & -1.1 & -74.14072 & 39.69221 & NJ & 1855 & -0.34 & -74.16138 & 39.66398 & NJ & 1927 & -0.59 & -74.18336 & 39.63654 & NJ \\
\hline 1784 & -1.05 & -74.14091 & 39.69178 & NJ & 1856 & -0.34 & -74.16173 & 39.66362 & NJ & 1928 & -0.55 & -74.18362 & 39.63614 & $\mathrm{NJ}$ \\
\hline 1785 & -1.01 & -74.14114 & 39.69136 & NJ & 1857 & -0.3 & -74.16202 & 39.66323 & NJ & 1929 & -0.52 & -74.18362 & 39.63562 & $\mathrm{NJ}$ \\
\hline 1786 & -1.09 & -74.14149 & 39.69099 & NJ & 1858 & -0.23 & -74.16212 & 39.66276 & NJ & 1930 & -0.51 & -74.18407 & 39.6353 & NJ \\
\hline 1787 & -1.13 & -74.14178 & 39.69061 & NJ & 1859 & -0.32 & -74.16248 & 39.66239 & $\mathrm{NJ}$ & 1931 & -0.47 & -74.1844 & 39.63492 & $\mathrm{NJ}$ \\
\hline 1788 & -1.1 & -74.14206 & 39.69021 & $\mathrm{NJ}$ & 1860 & -0.32 & -74.16281 & 39.66202 & $\mathrm{NJ}$ & 1932 & -0.45 & -74.18471 & 39.63454 & $\mathrm{NJ}$ \\
\hline 1789 & -1.08 & -74.14232 & 39.6898 & $\mathrm{NJ}$ & 1861 & -0.29 & -74.16316 & 39.66166 & $\mathrm{NJ}$ & 1933 & -0.51 & -74.185 & 39.63415 & $\mathrm{NJ}$ \\
\hline 1790 & -1.05 & -74.14253 & 39.68937 & NJ & 1862 & -0.27 & -74.16348 & 39.66129 & NJ & 1934 & -0.56 & -74.18524 & 39.63374 & $\mathrm{NJ}$ \\
\hline 1791 & -1.09 & -74.14279 & 39.68897 & $\mathrm{NJ}$ & 1863 & -0.28 & -74.16383 & 39.66092 & NJ & 1935 & -0.59 & -74.18549 & 39.63333 & $\mathrm{NJ}$ \\
\hline 1792 & -1.13 & -74.1431 & 39.68859 & $\mathrm{NJ}$ & 1864 & -0.33 & -74.16409 & 39.66051 & $\mathrm{NJ}$ & 1936 & -0.52 & -74.18578 & 39.63294 & $\mathrm{NJ}$ \\
\hline 1793 & -1.16 & -74.14342 & 39.68821 & NJ & 1865 & -0.32 & -74.16431 & 39.66009 & NJ & 1937 & -0.52 & -74.18604 & 39.63253 & $\mathrm{NJ}$ \\
\hline 1794 & -1.11 & -74.14372 & 39.68783 & NJ & 1866 & -0.27 & -74.16466 & 39.65972 & NJ & 1938 & -0.48 & -74.1862 & 39.63209 & $\mathrm{NJ}$ \\
\hline 1795 & -1.01 & -74.14401 & 39.68744 & NJ & 1867 & -0.26 & -74.16498 & 39.65935 & NJ & 1939 & -0.39 & -74.18636 & 39.63164 & $\mathrm{NJ}$ \\
\hline 1796 & -0.99 & -74.14426 & 39.68702 & NJ & 1868 & -0.24 & -74.1653 & 39.65897 & NJ & 1940 & -0.42 & -74.18663 & 39.63124 & $\mathrm{NJ}$ \\
\hline 1797 & -1.02 & -74.14449 & 39.6866 & NJ & 1869 & -0.25 & -74.16573 & 39.65865 & NJ & 1941 & -0.41 & -74.18695 & 39.63086 & $\mathrm{NJ}$ \\
\hline 1798 & -1.05 & -74.14478 & 39.68622 & NJ & 1870 & -0.23 & -74.16603 & 39.65826 & NJ & 1942 & -0.49 & -74.18727 & 39.63048 & $\mathrm{NJ}$ \\
\hline 1799 & -1.12 & -74.14508 & 39.68583 & NJ & 1871 & -0.24 & -74.16618 & 39.65781 & NJ & 1943 & -0.49 & -74.18758 & 39.6301 & $\mathrm{NJ}$ \\
\hline 1800 & -1.17 & -74.14539 & 39.68545 & $\mathrm{NJ}$ & 1872 & -0.32 & -74.1666 & 39.65747 & NJ & 1944 & -0.46 & -74.18788 & 39.62972 & NJ \\
\hline
\end{tabular}




\begin{tabular}{|c|c|c|c|c|c|c|c|c|c|c|c|c|c|c|}
\hline TR\# & RATE & LONG & LAT & ST & TR\# & RATE & LONG & LAT & ST & TR\# & RATE & LONG & LAT & ST \\
\hline 1945 & -0.57 & -74.18822 & 39.62935 & $\mathrm{NJ}$ & 2017 & -0.04 & .74 .20818 & 39.60082 & $\mathrm{NJ}$ & 2089 & -0.32 & .74 .22856 & 39.57218 & NJ \\
\hline 1946 & -0.6 & -74.18889 & 39.62913 & $\mathrm{NJ}$ & 2018 & -0.05 & -74.20848 & 39.60043 & NJ & 2090 & -0.32 & -74.22881 & 39.57178 & $\mathrm{NJ}$ \\
\hline 1947 & -0.58 & -74.18884 & 39.62859 & NJ & 2019 & -0.05 & -74.20863 & 39.59998 & $\mathrm{NJ}$ & 2091 & -0.28 & -74.229 & 39.57134 & $\mathrm{NJ}$ \\
\hline 1948 & -0.58 & -74.18913 & 39.6282 & $\mathrm{NJ}$ & 2020 & -0.07 & -74.20892 & 39.5996 & NJ & 2092 & -0.29 & -74.22919 & 39.57091 & $\mathrm{NJ}$ \\
\hline 1949 & -0.59 & -74.18941 & 39.6278 & NJ & 2021 & -0.09 & -74.20921 & 39.5992 & $\mathrm{NJ}$ & 2093 & -0.24 & -74.22948 & 39.57052 & $\mathrm{NJ}$ \\
\hline 1950 & -0.61 & -74.18971 & 39.62742 & NJ & 2022 & -0.05 & -74.20947 & 39.5988 & NJ & 2094 & -0.25 & -74.22978 & 39.57014 & NJ \\
\hline 1951 & -0.6 & -74.19006 & 39.62705 & $\mathrm{NJ}$ & 2023 & -0.06 & -74.20979 & 39.59842 & NJ & 2095 & -0.21 & -74.2301 & 39.56976 & $\mathrm{NJ}$ \\
\hline 1952 & -0.64 & -74.19035 & 39.62666 & NJ & 2024 & -0.1 & -74.21008 & 39.59803 & $\mathrm{NJ}$ & 2096 & -0.19 & -74.23042 & 39.56938 & NJ \\
\hline 1953 & -0.66 & -74.19063 & 39.62627 & NJ & 2025 & -0.14 & -74.21046 & 39.59768 & NJ & 2097 & -0.11 & -74.23071 & 39.56899 & $\mathrm{NJ}$ \\
\hline 1954 & -0.61 & -74.1909 & 39.62587 & $\mathrm{NJ}$ & 2026 & -0.15 & -74.21085 & 39.59733 & $\mathrm{NJ}$ & 2098 & -0.12 & -74.23093 & 39.56857 & NJ \\
\hline 1955 & -0.7 & -74.19122 & 39.62549 & NJ & 2027 & -0.12 & -74.2112 & 39.59696 & $\mathrm{NJ}$ & 2099 & -0.02 & -74.23111 & 39.56813 & $\mathrm{NJ}$ \\
\hline 1956 & -0.72 & -74.19153 & 39.62511 & NJ & 2028 & -0.11 & -74.21147 & 39.59657 & $\mathrm{NJ}$ & 2100 & -0.02 & -74.23132 & 39.56771 & NJ \\
\hline 1957 & -0.71 & -74.19182 & 39.62472 & NJ & 2029 & -0.01 & -74.21156 & 39.59609 & NJ & 2101 & -0.04 & -74.23164 & 39.56733 & NJ \\
\hline 1958 & -0.69 & -74.19209 & 39.62431 & $\mathrm{NJ}$ & 2030 & -0.15 & -74.21198 & 39.59575 & $\mathrm{NJ}$ & 2102 & -0.07 & .74 .23196 & 39.56696 & $\mathrm{NJ}$ \\
\hline 1959 & -0.65 & -74.19235 & 39.62391 & $\mathrm{NJ}$ & 2031 & -0.26 & -74.2123 & 39.59538 & NJ & 2103 & -0.08 & -74.2323 & 39.56658 & $\mathrm{NJ}$ \\
\hline 1960 & -0.71 & -74.19264 & 39.62352 & NJ & 2032 & .0 .26 & -74.21269 & 39.59503 & NJ & 2104 & -0.07 & -74.23257 & 39.56618 & NJ \\
\hline 1961 & -0.72 & -74.19295 & 39.62313 & $\mathrm{NJ}$ & 2033 & -0.26 & .74 .21304 & 39.59467 & NJ & 2105 & -0.04 & -74.23273 & 39.56574 & NJ \\
\hline 1962 & -0.68 & -74.19322 & 39.62274 & NJ & 2034 & -0.24 & -74.21333 & 39.59428 & $\mathrm{NJ}$ & 2106 & -0.02 & -74.23296 & 39.56532 & NJ \\
\hline 1963 & -0.62 & -74.19366 & 39.62242 & $\mathrm{NJ}$ & 2035 & -0.27 & -74.21359 & 39.59388 & $\mathrm{NJ}$ & 2107 & -0.05 & -74.23328 & 39.56495 & NJ \\
\hline 1964 & -0.59 & -74.19374 & 39.62193 & NJ & 2036 & -0.27 & -74.21362 & 39.59338 & NJ & 2108 & -0.03 & -74.23366 & 39.56459 & $\mathrm{NJ}$ \\
\hline 1965 & -0.51 & -74.19402 & 39.62154 & NJ & 2037 & -0.27 & -74.21387 & 39.59296 & NJ & 2109 & 0.01 & -74.23399 & 39.56422 & $\mathrm{NJ}$ \\
\hline 1966 & -0.5 & -74.19427 & 39.62113 & NJ & 2038 & -0.31 & -74.21417 & 39.59258 & NJ & 2110 & -0.03 & -74.23427 & 39.56382 & $\mathrm{NJ}$ \\
\hline 1967 & -0.41 & -74.19453 & 39.62073 & NJ & 2039 & -0.29 & -74.21455 & 39.59222 & NJ & 2111 & -0.05 & -74.23445 & 39.56339 & $\mathrm{NJ}$ \\
\hline 1968 & -0.38 & -74.19478 & 39.62032 & NJ & 2040 & -0.28 & -74.21497 & 39.59189 & NJ & 2112 & -0.18 & -74.23454 & 39.56292 & $\mathrm{NJ}$ \\
\hline 1969 & -0.36 & -74.19508 & 39.61994 & NJ & 2041 & -0.3 & -74.21521 & 39.59148 & NJ & 2113 & -0.27 & -74.23492 & 39.56256 & $\mathrm{NJ}$ \\
\hline 1970 & -0.4 & -74.19534 & 39.61953 & NJ & 2042 & -0.37 & -74.21556 & 39.59112 & NJ & 2114 & -0.3 & -74.23529 & 39.5622 & $\mathrm{NJ}$ \\
\hline 1971 & -0.37 & -74.19556 & 39.6191 & NJ & 2043 & -0.4 & -74.21584 & 39.59071 & NJ & 2115 & -0.29 & -74.23566 & 39.56184 & NJ \\
\hline 1972 & -0.35 & -74.19579 & 39.61868 & NJ & 2044 & -0.37 & -74.21609 & 39.59032 & NJ & 2116 & -0.21 & -74.23602 & 39.56147 & $\mathrm{NJ}$ \\
\hline 1973 & -0.33 & -74.19603 & 39.61827 & NJ & 2045 & -0.34 & -74.21635 & 39.58991 & NJ & 2117 & -0.19 & -74.23636 & 39.5611 & $\mathrm{NJ}$ \\
\hline 1974 & -0.39 & -74.1963 & 39.61788 & NJ & 2046 & -0.27 & .74 .21664 & 39.58952 & NJ & 2118 & -0.15 & -74.23665 & 39.56071 & $\mathrm{NJ}$ \\
\hline 1975 & -0.36 & -74.19659 & 39.61748 & $\mathrm{NJ}$ & 2047 & -0.25 & -74.21684 & 39.58909 & NJ & 2119 & -0.1 & -74.23685 & 39.56028 & $\mathrm{NJ}$ \\
\hline 1976 & -0.31 & -74.19688 & 39.61709 & NJ & 2048 & -0.27 & -74.21713 & 39.5887 & NJ & 2120 & -0.08 & -74.237 & 39.55984 & $\mathrm{NJ}$ \\
\hline 1977 & -0.27 & -74.19711 & 39.61668 & NJ & 2049 & -0.26 & -74.21738 & 39.58829 & NJ & 2121 & -0.09 & -74.23738 & 39.55948 & NJ \\
\hline 1978 & -0.29 & -74.1974 & 39.61629 & NJ & 2050 & -0.29 & -74.21765 & 39.58789 & NJ & 2122 & -0.12 & -74.23776 & 39.55913 & $N J$ \\
\hline 1979 & -0.3 & -74.19769 & 39.6159 & NJ & 2051 & -0.33 & -74.21791 & 39.58749 & NJ & 2123 & -0.16 & -74.23813 & 39.55877 & $\mathrm{NJ}$ \\
\hline 1980 & -0.32 & -74.19794 & 39.61549 & NJ & 2052 & -0.32 & -74.21819 & 39.58709 & NJ & 2124 & -0.17 & -74.23848 & 39.5584 & $\mathrm{NJ}$ \\
\hline 1981 & -0.31 & -74.1982 & 39.61508 & NJ & 2053 & -0.33 & -74.21848 & 39.5867 & NJ & 2125 & -0.2 & -74.23878 & 39.55801 & $\mathrm{NJ}$ \\
\hline 1982 & -0.38 & -74.1985 & 39.6147 & NJ & 2054 & -0.33 & -74.21883 & 39.58633 & NJ & 2126 & -0.14 & -74.23895 & 39.55757 & $\mathrm{NJ}$ \\
\hline 1983 & -0.4 & -74.19875 & 39.61429 & NJ & 2055 & -0.31 & -74.21915 & 39.58596 & NJ & 2127 & -0.48 & -74.23924 & 39.55719 & $\mathrm{NJ}$ \\
\hline 1984 & -0.39 & -74.19901 & 39.61388 & NJ & 2056 & -0.29 & -74.21948 & 39.58559 & NJ & 2128 & -0.6 & -74.23967 & 39.55684 & NJ \\
\hline 1985 & -0.3 & -74.19923 & 39.61347 & NJ & 2057 & -0.27 & -74.21974 & 39.58518 & NJ & 2129 & -0.69 & -74.24005 & 39.55649 & NJ \\
\hline 1986 & -0.27 & -74.19949 & 39.61306 & $\mathrm{NJ}$ & 2058 & -0.28 & -74.21999 & 39.58477 & NJ & 2130 & -0.74 & -74.24043 & 39.55614 & NJ \\
\hline 1987 & -0.29 & -74.19975 & 39.61266 & NJ & 2059 & -0.32 & -74.22023 & 39.58436 & NJ & 2131 & -0.76 & -74.24078 & 39.55577 & NJ \\
\hline 1988 & -0.26 & -74.20004 & 39.61227 & NJ & 2060 & -0.32 & -74.22047 & 39.58395 & NJ & 2132 & -0.86 & -74.241 & 39.55535 & $\mathrm{NJ}$ \\
\hline 1989 & -0.2 & -74.20033 & 39.61187 & $\mathrm{NJ}$ & 2061 & -0.27 & -74.22075 & 39.58355 & NJ & 2133 & -1.07 & -74.24126 & 39.55494 & $\mathrm{NJ}$ \\
\hline 1990 & -0.16 & -74.20058 & 39.61147 & NJ & 2062 & -0.24 & -74.22101 & 39.58315 & NJ & 2134 & -1.18 & -74.2417 & 39.55461 & NJ \\
\hline 1991 & -0.11 & -74.20081 & 39.61105 & NJ & 2063 & -0.27 & -74.22118 & 39.5827 & $\mathrm{NJ}$ & 2135 & -1.29 & -74.24213 & 39.55427 & NJ \\
\hline 1992 & -0.15 & -74.201 & 39.61062 & NJ & 2064 & -0.24 & -74.22147 & 39.58231 & $\mathrm{NJ}$ & 2136 & -1.41 & -74.24251 & 39.55392 & NJ \\
\hline 1993 & -0.17 & -74.20128 & 39.61022 & NJ & 2065 & -0.2 & -74.22176 & 39.58193 & NJ & 2137 & -1.47 & -74.2429 & 39.55356 & NJ \\
\hline 1994 & -0.17 & -74.20152 & 39.60981 & NJ & 2066 & -0.19 & -74.22205 & 39.58154 & NJ & 2138 & -1.52 & -74.24327 & 39.55321 & NJ \\
\hline 1995 & -0.22 & -74.20178 & 39.6094 & $\mathrm{NJ}$ & 2067 & -0.16 & -74.22231 & 39.58113 & $\mathrm{NJ}$ & 2139 & -1.54 & -74.24361 & 39.55283 & NJ \\
\hline 1996 & -0.25 & -74.20204 & 39.609 & NJ & 2068 & -0.16 & -74.22258 & 39.58073 & NJ & 2140 & -1.64 & -74.2438 & 39.5524 & NJ \\
\hline 1997 & -0.19 & -74.20232 & 39.6086 & $\mathrm{NJ}$ & 2069 & -0.17 & -74.22285 & 39.58034 & NJ & 2141 & -1.86 & -74.24442 & 39.55213 & NJ \\
\hline 1998 & -0.12 & -74.20258 & 39.6082 & $\mathrm{NJ}$ & 2070 & -0.18 & -74.22318 & 39.57996 & $\mathrm{NJ}$ & 2142 & -2.02 & -74.24483 & 39.55179 & NJ \\
\hline 1999 & -0.08 & -74.20287 & 39.60781 & NJ & 2071 & -0.18 & -74.22346 & 39.57957 & $\mathrm{NJ}$ & 2143 & -2.22 & -74.24527 & 39.55146 & NJ \\
\hline 2000 & -0.05 & -74.20314 & 39.60741 & $\mathrm{NJ}$ & 2072 & -0.13 & -74.22375 & 39.57918 & $\mathrm{NJ}$ & 2144 & -2.4 & -74.2457 & 39.55112 & NJ \\
\hline 2001 & 0.01 & -74.20341 & 39.60701 & $\mathrm{NJ}$ & 2073 & -0.16 & -74.22398 & 39.57876 & $\mathrm{NJ}$ & 2145 & -2.53 & -74.24608 & 39.55077 & NJ \\
\hline 2002 & -0.05 & -74.20369 & 39.60661 & $\mathrm{NJ}$ & 2074 & -0.2 & -74.22424 & 39.57835 & $\mathrm{NJ}$ & 2146 & -2.75 & -74.24654 & 39.55044 & NJ \\
\hline 2003 & -0.04 & -74.20403 & 39.60624 & $\mathrm{NJ}$ & 2075 & -0.2 & -74.22452 & 39.57796 & $\mathrm{NJ}$ & 2147 & -2.87 & -74.24689 & 39.55008 & NJ \\
\hline 2004 & -0.02 & -74.20432 & 39.60585 & $\mathrm{NJ}$ & 2076 & -0.11 & -74.22482 & 39.57757 & $\mathrm{NJ}$ & 2148 & -2.97 & -74.24724 & 39.54971 & NJ \\
\hline 2005 & -0.01 & -74.20462 & 39.60547 & $\mathrm{NJ}$ & 2077 & -0.12 & -74.22511 & 39.57718 & NJ & 2149 & -3.28 & -74.2476 & 39.54935 & NJ \\
\hline 2006 & -0.02 & -74.20488 & 39.60507 & $\mathrm{NJ}$ & 2078 & -0.07 & -74.22539 & 39.57678 & $\mathrm{NJ}$ & 2150 & -3.4 & -74.24802 & 39.549 & NJ \\
\hline 2007 & -0.03 & -74.20509 & 39.60464 & $\mathrm{NJ}$ & 2079 & -0.09 & -74.22565 & 39.57638 & $\mathrm{NJ}$ & 2151 & -3.55 & -74.24849 & 39.54868 & NJ \\
\hline 2008 & 0.05 & -74.20563 & 39.60436 & $\mathrm{NJ}$ & 2080 & -0.1 & -74.22604 & 39.57574 & $\mathrm{NJ}$ & 2152 & -3.68 & -74.24886 & 39.54831 & NJ \\
\hline 2009 & 0.05 & -74.2059 & 39.60396 & NJ & 2081 & -0.11 & -74.22633 & 39.57535 & $\mathrm{NJ}$ & 2153 & -3.83 & -74.24919 & 39.54794 & NJ \\
\hline 2010 & 0.05 & -74.20618 & 39.60356 & NJ & 2082 & -0.1 & -74.22667 & 39.57498 & $\mathrm{NJ}$ & 2154 & -3.9 & -74.24953 & 39.54757 & NJ \\
\hline 2011 & -0.03 & -74.20651 & 39.6032 & NJ & 2083 & -0.12 & -74.22696 & 39.57458 & $\mathrm{NJ}$ & 2155 & -3.94 & -74.24985 & 39.54719 & NJ \\
\hline 2012 & 0.02 & -74.20676 & 39.60278 & NJ & 2084 & -0.09 & -74.22723 & 39.57419 & $\mathrm{NJ}$ & 2156 & -4.08 & -74.25015 & 39.54681 & NJ \\
\hline 2013 & 0 & -74.20702 & 39.60238 & NJ & 2085 & -0.16 & -74.22746 & 39.57377 & NJ & 2157 & -4.18 & -74.25055 & 39.54646 & NJ \\
\hline 2014 & 0.07 & -74.20724 & 39.60196 & NJ & 2086 & -0.17 & -74.22768 & 39.57335 & $\mathrm{NJ}$ & 2158 & -4.28 & -74.25092 & 39.54609 & NJ \\
\hline 2015 & 0.04 & -74.20758 & 39.60159 & NJ & 2087 & -0.26 & -74.22797 & 39.57296 & $\mathrm{NJ}$ & 2159 & -4.44 & -74.25127 & 39.54573 & $\mathrm{NJ}$ \\
\hline 2016 & 0 & -74.20776 & 39.60115 & NJ & 2088 & -0.32 & -74.22829 & 39.57258 & $\mathrm{NJ}$ & 2160 & -4.58 & -74.25157 & 39.54534 & NJ \\
\hline
\end{tabular}




\begin{tabular}{|c|c|c|c|c|c|c|c|c|c|c|c|c|c|c|}
\hline TR\# & RATE & LONG & LAT & ST & TR\# & RATE & LONG & LAT & ST & TR\# & RATE & LONG & LAT & ST \\
\hline 2161 & -4.63 & -74.25189 & 39.54496 & $\mathrm{NJ}$ & 2233 & -9.42 & -74.27356 & 39.51962 & NJ & 2305 & 4 & -74.31207 & 39.46739 & NJ \\
\hline 2162 & -4.73 & -74.25224 & 39.5446 & NJ & 2234 & -9.42 & -74.2737 & 39.51935 & $\mathrm{NJ}$ & 2306 & 3.93 & -74.31238 & 39.467 & $\mathrm{NJ}$ \\
\hline 2163 & -4.78 & -74.25259 & 39.54423 & $\mathrm{NJ}$ & 2235 & -9.35 & -74.2738 & 39.51921 & NJ & 2307 & 3.86 & -74.31271 & 39.46663 & NJ \\
\hline 2164 & -4.78 & -74.25288 & 39.54384 & $\mathrm{NJ}$ & 2236 & -9.31 & -74.27399 & 39.51894 & NJ & 2308 & 3.76 & -74.31305 & 39.46627 & NJ \\
\hline 2165 & -4.82 & -74.2532 & 39.54346 & $\mathrm{NJ}$ & 2237 & -9.3 & -74.27409 & 39.51882 & $\mathrm{NJ}$ & 2309 & 3.61 & .74 .31339 & 39.4659 & $\mathrm{NJ}$ \\
\hline 2166 & -4.74 & -74.25352 & 39.54308 & NJ & 2238 & -9.25 & -74.27432 & 39.51856 & $\mathrm{NJ}$ & 2310 & 3.43 & -74.31374 & 39.46553 & $\mathrm{NJ}$ \\
\hline 2167 & -4.78 & -74.25381 & 39.54269 & $\mathrm{NJ}$ & 2239 & -9.3 & .74 .27441 & 39.51844 & $\mathrm{NJ}$ & 2311 & 3.22 & -74.31409 & 39.46518 & $\mathrm{NJ}$ \\
\hline 2168 & -4.84 & -74.2541 & 39.5423 & NJ & 2240 & -9.25 & -74.27466 & 39.51818 & $\mathrm{NJ}$ & 2312 & 3.12 & -74.31442 & 39.46482 & NJ \\
\hline 2169 & -4.8 & -74.25443 & 39.54191 & NJ & 2241 & -9.33 & -74.27507 & 39.51787 & NJ & 2313 & 3 & -74.31477 & 39.46446 & $\mathrm{NJ}$ \\
\hline 2170 & -4.78 & -74.25476 & 39.54154 & $\mathrm{NJ}$ & 2242 & -9.43 & -74.2755 & 39.51756 & $\mathrm{NJ}$ & 2314 & 2.9 & -74.31511 & 39.46409 & $\mathrm{NJ}$ \\
\hline 2171 & -4.81 & -74.2551 & 39.54117 & $\mathrm{NJ}$ & 2243 & -9.5 & -74.27589 & 39.51723 & NJ & 2315 & 2.71 & -74.31549 & 39.46374 & NJ \\
\hline 2172 & -4.9 & -74.25542 & 39.54079 & NJ & 2244 & -9.52 & -74.27625 & 39.51686 & NJ & 2316 & 2.48 & -74.31587 & 39.4634 & $\mathrm{NJ}$ \\
\hline 2173 & -5.01 & -74.25586 & 39.54046 & $\mathrm{NJ}$ & 2245 & -9.45 & -74.27657 & 39.51647 & NJ & 2317 & 2.34 & -74.31619 & 39.46303 & NJ \\
\hline 2174 & -5.48 & -74.25607 & 39.54004 & $\mathrm{NJ}$ & 2246 & -9.42 & -74.27692 & 39.51611 & NJ & 2318 & 2.24 & -74.31653 & 39.46266 & $\mathrm{NJ}$ \\
\hline 2175 & -5.53 & -74.25639 & 39.53965 & $\mathrm{NJ}$ & 2247 & -9.43 & -74.27728 & 39.51577 & $\mathrm{NJ}$ & 2319 & 2.15 & -74.31686 & 39.4623 & $\mathrm{NJ}$ \\
\hline 2176 & -5.51 & -74.25667 & 39.53926 & NJ & 2248 & -9.41 & -74.27766 & 39.51542 & NJ & 2320 & 2.02 & -74.3172 & 39.46192 & NJ \\
\hline 2177 & -5.59 & -74.25703 & 39.5389 & NJ & 2249 & -9.41 & -74.27802 & 39.51506 & $\mathrm{NJ}$ & 2321 & 1.88 & -74.3175 & 39.46154 & $\mathrm{NJ}$ \\
\hline 2178 & -5.65 & -74.25732 & 39.5385 & $\mathrm{NJ}$ & 2250 & -9.4 & -74.27838 & 39.5147 & NJ & 2322 & 1.64 & -74.3179 & 39.4612 & $\mathrm{NJ}$ \\
\hline 2179 & -5.68 & -74.25757 & 39.5381 & NJ & 2251 & -9.4 & -74.27875 & 39.51435 & NJ & 2323 & 1.51 & -74.31819 & 39.46081 & $\mathrm{NJ}$ \\
\hline 2180 & -5.71 & -74.25786 & 39.5377 & NJ & 2252 & -9.42 & -74.27911 & 39.514 & NJ & 2324 & 1.45 & -74.31844 & 39.46039 & $\mathrm{NJ}$ \\
\hline 2181 & -5.78 & -74.25816 & 39.53732 & $\mathrm{NJ}$ & 2253 & -9.45 & -74.27951 & 39.51367 & $\mathrm{NJ}$ & 2325 & 1.27 & -74.31883 & 39.46006 & NJ \\
\hline 2182 & -5.53 & -74.25845 & 39.53693 & NJ & 2254 & -9.5 & -74.27992 & 39.51335 & $\mathrm{NJ}$ & 2326 & 1.14 & -74.31917 & 39.45969 & NJ \\
\hline 2183 & -5.47 & -74.25873 & 39.53653 & $\mathrm{NJ}$ & 2255 & -9.56 & -74.28033 & 39.51304 & $\mathrm{NJ}$ & 2327 & 1.01 & -74.31949 & 39.45932 & NJ \\
\hline 2184 & .5 .49 & -74.25896 & 39.53611 & $\mathrm{NJ}$ & 2256 & -9.63 & -74.28076 & 39.51273 & $\mathrm{NJ}$ & 2328 & 0.89 & -74.31982 & 39.45894 & NJ \\
\hline 2185 & -5.55 & -74.2592 & 39.53571 & $\mathrm{NJ}$ & 2257 & -9.69 & -74.28117 & 39.51241 & $\mathrm{NJ}$ & 2329 & 0.76 & -74.32013 & 39.45856 & NJ \\
\hline 2186 & -5.64 & -74.25948 & 39.53531 & NJ & 2258 & -9.77 & -74.2816 & 39.5121 & NJ & 2330 & 0.68 & -74.32043 & 39.45818 & NJ \\
\hline 2187 & -5.67 & -74.25972 & 39.5349 & $\mathrm{NJ}$ & 2259 & -9.85 & -74.28203 & 39.5118 & $\mathrm{NJ}$ & 2331 & 0.61 & -74.32076 & 39.45781 & NJ \\
\hline 2188 & -5.57 & -74.25993 & 39.53448 & $\mathrm{NJ}$ & 2260 & -9.87 & -74.28242 & 39.51147 & NJ & 2332 & 0.55 & -74.32109 & 39.45743 & NJ \\
\hline 2189 & -5.61 & -74.26004 & 39.53402 & $\mathrm{NJ}$ & 2261 & -9.82 & -74.28281 & 39.51113 & NJ & 2333 & 0.52 & -74.3214 & 39.45705 & NJ \\
\hline 2190 & -5.66 & -74.26041 & 39.53366 & $\mathrm{NJ}$ & 2262 & -9.73 & -74.28317 & 39.51077 & NJ & 2334 & 0.52 & -74.32169 & 39.45665 & NJ \\
\hline 2191 & -5.64 & -74.26067 & 39.53325 & NJ & 2263 & -9.69 & -74.28355 & 39.51044 & NJ & 2335 & 0.48 & -74.32199 & 39.45627 & $\mathrm{NJ}$ \\
\hline 2192 & -5.63 & -74.26091 & 39.53284 & NJ & 2264 & -9.63 & -74.28394 & 39.5101 & $\mathrm{NJ}$ & 2336 & 0.49 & -74.32224 & 39.45585 & NJ \\
\hline 2193 & -5.63 & -74.26099 & 39.53237 & $\mathrm{NJ}$ & 2265 & -9.55 & -74.28432 & 39.50975 & $\mathrm{NJ}$ & 2337 & 0.45 & -74.32256 & 39.45547 & NJ \\
\hline 2194 & -5.85 & -74.26152 & 39.53208 & NJ & 2266 & -9.49 & -74.28468 & 39.5094 & NJ & 2338 & 0.45 & -74.32283 & 39.45507 & $\mathrm{NJ}$ \\
\hline 2195 & -5.98 & -74.26193 & 39.53173 & $\mathrm{NJ}$ & 2267 & -9.44 & -74.28506 & 39.50906 & NJ & 2339 & 0.47 & -74.3231 & 39.45467 & NJ \\
\hline 2196 & -6.18 & -74.26233 & 39.53138 & $\mathrm{NJ}$ & 2268 & -9.45 & -74.28552 & 39.50877 & NJ & 2340 & 0.55 & -74.32336 & 39.45425 & $\mathrm{NJ}$ \\
\hline 2197 & -6.29 & -74.26265 & 39.531 & $\mathrm{NJ}$ & 2269 & -9.48 & -74.286 & 39.5085 & $\mathrm{NJ}$ & 2341 & 0.56 & -74.32365 & 39.45386 & $\mathrm{NJ}$ \\
\hline 2198 & -6.71 & -74.26367 & 39.53089 & NJ & 2270 & -9.53 & -74.28648 & 39.50824 & NJ & 2342 & 0.62 & -74.32391 & 39.45345 & $\mathrm{NJ}$ \\
\hline 2199 & -7.56 & -74.26419 & 39.53059 & $\mathrm{NJ}$ & 2271 & -9.56 & -74.28696 & 39.50797 & NJ & 2343 & 0.67 & -74.32417 & 39.45303 & $\mathrm{NJ}$ \\
\hline 2200 & -7.78 & -74.26463 & 39.53026 & $\mathrm{NJ}$ & 2272 & -9.5 & -74.28746 & 39.50773 & NJ & 2344 & 0.7 & -74.32443 & 39.45263 & NJ \\
\hline 2201 & -7.99 & -74.26517 & 39.52996 & NJ & 2273 & -9.11 & -74.28792 & 39.50744 & NJ & 2345 & 0.78 & -74.32466 & 39.4522 & NJ \\
\hline 2202 & -8.17 & -74.26559 & 39.52962 & $\mathrm{NJ}$ & 2274 & -11.55 & -74.2944 & 39.50732 & $\mathrm{NJ}$ & 2346 & 0.81 & -74.32495 & 39.4518 & $\mathrm{NJ}$ \\
\hline 2203 & -8.36 & -74.26598 & 39.52927 & $\mathrm{NJ}$ & 2275 & -9.03 & -74.28841 & 39.50718 & $\mathrm{NJ}$ & 2347 & 0.85 & -74.32529 & 39.45143 & $\mathrm{NJ}$ \\
\hline 2204 & -8.46 & -74.26634 & 39.52891 & $\mathrm{NJ}$ & 2276 & -9.03 & -74.28889 & 39.50692 & $\mathrm{NJ}$ & 2348 & 0.89 & -74.32562 & 39.45107 & NJ \\
\hline 2205 & -8.59 & -74.26671 & 39.52854 & NJ & 2277 & -9.11 & -74.28941 & 39.50668 & $\mathrm{NJ}$ & 2349 & 0.85 & -74.32599 & 39.45072 & $\mathrm{NJ}$ \\
\hline 2206 & -8.71 & -74.26707 & 39.52818 & NJ & 2278 & -11.84 & -74.30002 & 39.50653 & NJ & 2350 & 0.8 & -74.32629 & 39.45033 & NJ \\
\hline 2207 & -8.77 & -74.26743 & 39.52782 & $\mathrm{NJ}$ & 2279 & -9.12 & -74.28995 & 39.50646 & $\mathrm{NJ}$ & 2351 & 0.77 & -74.32655 & 39.44992 & $\mathrm{NJ}$ \\
\hline 2208 & -8.84 & -74.26781 & 39.52746 & $\mathrm{NJ}$ & 2280 & -9.75 & -74.29228 & 39.50634 & NJ & 2352 & -0.33 & -74.32825 & 39.44978 & NJ \\
\hline 2209 & -8.88 & -74.26816 & 39.52709 & NJ & 2281 & -9.11 & -74.29054 & 39.50629 & $\mathrm{NJ}$ & 2353 & 0.78 & -74.32681 & 39.44951 & $\mathrm{NJ}$ \\
\hline 2210 & -8.87 & -74.26852 & 39.52673 & NJ & 2282 & -9.32 & -74.29132 & 39.50624 & $\mathrm{NJ}$ & 2354 & -0.37 & -74.32861 & 39.44943 & $\mathrm{NJ}$ \\
\hline 2211 & -8.86 & -74.26884 & 39.52635 & $\mathrm{NJ}$ & 2283 & -10.7 & -74.29997 & 39.50587 & NJ & 2355 & -0.27 & -74.32883 & 39.44898 & $\mathrm{NJ}$ \\
\hline 2212 & -8.89 & -74.26917 & 39.52597 & $\mathrm{NJ}$ & 2284 & -9.7 & -74.3 & 39.50527 & $\mathrm{NJ}$ & 2356 & -0.84 & -74.32968 & 39.44891 & $\mathrm{NJ}$ \\
\hline 2213 & -8.89 & -74.26947 & 39.52559 & $\mathrm{NJ}$ & 2285 & -17.41 & -74.30023 & 39.5048 & NJ & 2357 & 1.26 & -74.33076 & 39.44449 & NJ \\
\hline 2214 & -8.92 & -74.26976 & 39.52519 & NJ & 2286 & -17.13 & -74.30042 & 39.50431 & $\mathrm{NJ}$ & 2358 & 2.06 & -74.33055 & 39.44381 & $\mathrm{NJ}$ \\
\hline 2215 & -8.96 & -74.27005 & 39.5248 & NJ & 2287 & -16.88 & -74.30061 & 39.50384 & $\mathrm{NJ}$ & 2359 & 3.98 & -74.33028 & 39.4431 & $\mathrm{NJ}$ \\
\hline 2216 & -9.03 & -74.27036 & 39.52441 & NJ & 2288 & -16.66 & -74.30087 & 39.5034 & $\mathrm{NJ}$ & 2360 & 7.21 & -74.32739 & 39.44088 & $\mathrm{NJ}$ \\
\hline 2217 & -9.6 & -74.27065 & 39.52403 & NJ & 2289 & -16.45 & -74.3011 & 39.50294 & NJ & 2361 & 7.68 & -74.32719 & 39.4402 & $\mathrm{NJ}$ \\
\hline 2218 & -9.64 & -74.27097 & 39.52365 & $\mathrm{NJ}$ & 2290 & -16.26 & -74.30133 & 39.50249 & $\mathrm{NJ}$ & 2362 & 7.97 & -74.32735 & 39.43973 & $\mathrm{NJ}$ \\
\hline 2219 & -9.63 & -74.27124 & 39.52325 & NJ & 2291 & -16.07 & -74.30157 & 39.50205 & $\mathrm{NJ}$ & 2363 & 8.43 & -74.32755 & 39.43929 & $\mathrm{NJ}$ \\
\hline 2220 & -9.63 & -74.27151 & 39.52285 & NJ & 2292 & -15.9 & -74.30185 & 39.50162 & $\mathrm{NJ}$ & 2364 & 9.31 & -74.3275 & 39.43869 & $\mathrm{NJ}$ \\
\hline 2221 & -9.64 & -74.27182 & 39.52247 & $\mathrm{NJ}$ & 2293 & 7.29 & -74.30832 & 39.47196 & NJ & 2365 & 5.54 & -74.32768 & 39.43824 & NJ \\
\hline 2222 & -9.66 & .74 .27209 & 39.52207 & NJ & 2294 & 6.03 & -74.30862 & 39.47157 & $\mathrm{NJ}$ & 2366 & 4.45 & -74.32806 & 39.43789 & NJ \\
\hline 2223 & -9.67 & -74.27238 & 39.52168 & $\mathrm{NJ}$ & 2295 & 5.69 & -74.30885 & 39.47115 & $\mathrm{NJ}$ & 2367 & 3.07 & -74.32837 & 39.43751 & $\mathrm{NJ}$ \\
\hline 2224 & -9.89 & -74.27245 & 39.52155 & $\mathrm{NJ}$ & 2296 & 5.45 & -74.30916 & 39.47076 & $\mathrm{NJ}$ & 2368 & 2.13 & -74.32867 & 39.43712 & NJ \\
\hline 2225 & -9.64 & -74.27263 & 39.52127 & $\mathrm{NJ}$ & 2297 & 5.25 & -74.30945 & 39.47037 & $\mathrm{NJ}$ & 2369 & 1.85 & -74.32899 & 39.43675 & $\mathrm{NJ}$ \\
\hline 2226 & -9.81 & -74.27272 & 39.52112 & NJ & 2298 & 5.18 & -74.30974 & 39.46997 & NJ & 2370 & 1.7 & -74.32924 & 39.43633 & $\mathrm{NJ}$ \\
\hline 2227 & -9.57 & -74.27286 & 39.52086 & $\mathrm{NJ}$ & 2299 & 5.07 & -74.31004 & 39.46959 & $\mathrm{NJ}$ & 2371 & 1.53 & -74.32957 & 39.43597 & NJ \\
\hline 2228 & -9.76 & -74.27296 & 39.52068 & $\mathrm{NJ}$ & 2300 & 4.91 & -74.31036 & 39.46921 & NJ & 2372 & 1.6 & -74.32988 & 39.43558 & $\mathrm{NJ}$ \\
\hline 2229 & -9.53 & -74.2731 & 39.52045 & NJ & 2301 & 4.7 & -74.3107 & 39.46884 & $\mathrm{NJ}$ & 2373 & 1.65 & -74.33022 & 39.43521 & NJ \\
\hline 2230 & -9.69 & -74.27321 & 39.52024 & NJ & 2302 & 4.52 & -74.31104 & 39.46847 & $\mathrm{NJ}$ & 2374 & 1.4 & -74.33061 & 39.43487 & NJ \\
\hline 2231 & -9.48 & -74.27333 & 39.52003 & NJ & 2303 & 4.35 & -74.31139 & 39.46811 & $\mathrm{NJ}$ & 2375 & 1.28 & -74.33096 & 39.43451 & NJ \\
\hline 2232 & -9.59 & -74.27347 & 39.5198 & $\mathrm{NJ}$ & 2304 & 4.16 & -74.31174 & 39.46776 & $\mathrm{NJ}$ & 2376 & 1.14 & .74 .33133 & 39.43417 & NJ \\
\hline
\end{tabular}




\begin{tabular}{|c|c|c|c|c|c|c|c|c|c|c|c|c|c|c|}
\hline TR\# & RATE & LONG & LAT & ST & _TR\# & RATE & LONG & LAT & ST & TR\# & RATE & LONG & LAT & ST \\
\hline 2377 & 1.02 & -.74 .33173 & 339.43383 & NJ & 2449 & -2.52 & -74.3595 & 39.40943 & $\mathrm{NJ}$ & 2521 & 2.12 & -74.3904 & 39.38516 & $5 \mathrm{NJ}$ \\
\hline 2378 & -1.18 & -74.33211 & 139.43349 & $\mathrm{NJ}$ & 2450 & -2.32 & -74.35983 & 39.40907 & $\mathrm{NJ}$ & 2522 & 2.1 & -74.39079 & 39.38483 & $\mathrm{NJ}$ \\
\hline 2379 & -1.17 & -74.33247 & 739.43315 & NJ & 2451 & -2.12 & -74.36021 & 139.40873 & $\mathrm{NJ}$ & 2523 & 2.11 & -74.39116 & 539.38448 & $\mathrm{NJ}$ \\
\hline 2380 & -1.18 & -74.33286 & 639.43281 & $\mathrm{NJ}$ & 2452 & -1.98 & -74.3606 & 39.40838 & $\mathrm{NJ}$ & 2524 & 2.09 & -74.39156 & 639.38414 & $\mathrm{NJ}$ \\
\hline 2381 & -1.2 & -74.33324 & 439.43246 & $\mathrm{NJ}$ & 2453 & -1.8 & -74.36096 & 639.40803 & NJ & 2525 & 2.07 & -74.39194 & 39.3838 & $\mathrm{NJ}$ \\
\hline 2382 & -1.23 & -74.33366 & 639.43214 & $\mathrm{NJ}$ & 2454 & -1.62 & -74.36131 & 39.40768 & $\mathrm{NJ}$ & 2526 & 1.98 & -74.39243 & 39.38352 & $\mathrm{NJ}$ \\
\hline 2383 & -1.28 & -74.33411 & 139.43184 & $\mathrm{NJ}$ & 2455 & -1.42 & -74.3616 & 39.40729 & $\mathrm{NJ}$ & 2527 & 2.02 & -74.39276 & 539.38315 & $\mathrm{NJ}$ \\
\hline 2384 & -1.31 & -74.3345 & 39.43151 & $\mathrm{NJ}$ & 2456 & -1.27 & -74.36195 & 539.40693 & $\mathrm{NJ}$ & 2528 & 1.98 & .74 .39314 & 39.38281 & $\mathrm{NJ}$ \\
\hline 2385 & -1.31 & -74.33492 & 239.43118 & $\mathrm{NJ}$ & 2457 & -1.11 & -74.36237 & 739.4066 & $\mathrm{NJ}$ & 2529 & 1.94 & .74 .39352 & 239.38248 & $\mathrm{NJ}$ \\
\hline 2386 & -1.35 & -74.33533 & 339.43086 & $\mathrm{NJ}$ & 2458 & -0.93 & -74.3627 & 39.40623 & NJ & 2530 & 1.92 & .74 .39391 & 39.38213 & $\mathrm{NJ}$ \\
\hline 2387 & -1.38 & -74.33572 & 239.43053 & $\mathrm{NJ}$ & 2459 & -0.84 & -74.36311 & 39.40591 & NJ & 2531 & 1.88 & .74 .39433 & 39.38181 & $\mathrm{NJ}$ \\
\hline 2388 & -1.4 & -74.33615 & 539.43021 & $\mathrm{NJ}$ & 2460 & -0.78 & -74.36351 & 139.40558 & $\mathrm{NJ}$ & 2532 & 1.83 & -74.39474 & 49.38149 & $\mathrm{NJ}$ \\
\hline 2389 & -1.49 & -74.33656 & 639.42989 & NJ & 2461 & -0.71 & -74.36389 & 39.40523 & $\mathrm{NJ}$ & 2533 & 1.75 & -74.3952 & 39.3812 & $\mathrm{NJ}$ \\
\hline 2390 & -1.51 & -74.33696 & 639.42956 & $\mathrm{NJ}$ & 2462 & -0.65 & -74.36426 & 639.40489 & $\mathrm{NJ}$ & 2534 & 1.83 & -74.39551 & 39.38081 & $\mathrm{NJ}$ \\
\hline 2391 & -1.56 & -74.33736 & 639.42922 & $\mathrm{NJ}$ & 2463 & -0.52 & -74.36462 & 39.40454 & NJ & 2535 & 1.73 & -74.39597 & 39.38052 & $\mathrm{NJ}$ \\
\hline 2392 & -1.55 & -74.33771 & 139.42887 & $\mathrm{NJ}$ & 2464 & -0.36 & -74.36497 & 79.40418 & $\mathrm{NJ}$ & 2536 & 1.77 & .74 .39635 & 39.38017 & $\mathrm{NJ}$ \\
\hline 2393 & -1.56 & -74.33807 & 739.42851 & $\mathrm{NJ}$ & 2465 & -0.19 & -74.36531 & 39.40381 & $\mathrm{NJ}$ & 2537 & 1.76 & .74 .39679 & 39.37986 & $\mathrm{NJ}$ \\
\hline 2394 & -1.55 & -74.33849 & 939.42819 & $\mathrm{NJ}$ & 2466 & -0.06 & -74.3656 & 39.40342 & $\mathrm{NJ}$ & 2538 & 1.63 & -74.39729 & 39.37959 & $\mathrm{NJ}$ \\
\hline 2395 & -1.58 & -74.33893 & 339.42789 & NJ & 2467 & 0.08 & -74.36601 & 39.4031 & NJ & 2539 & 1.63 & -74.39771 & 39.37926 & $\mathrm{NJ}$ \\
\hline 2396 & -1.65 & -74.33934 & 439.42756 & NJ & 2468 & 0.21 & -74.36647 & 739.40279 & NJ & 2540 & 1.64 & -74.39816 & 39.37897 & $\mathrm{NJ}$ \\
\hline 2397 & -1.7 & -74.33978 & 839.42725 & $\mathrm{NJ}$ & 2469 & 0.19 & -74.36688 & 39.40247 & $\mathrm{NJ}$ & 2541 & 1.69 & -74.39859 & 39.37866 & $\mathrm{NJ}$ \\
\hline 2398 & -1.71 & -74.34016 & 639.42691 & $\mathrm{NJ}$ & 2470 & 0.23 & -74.36726 & 539.40213 & $\mathrm{NJ}$ & 2542 & 1.72 & -74.39903 & 39.37835 & $\mathrm{NJ}$ \\
\hline 2399 & -1.77 & -74.34056 & 639.42658 & $\mathrm{NJ}$ & 2471 & 0.28 & -74.36768 & 39.4018 & $\mathrm{NJ}$ & 2543 & 1.76 & -74.39949 & 39.37805 & $\mathrm{NJ}$ \\
\hline 2400 & -1.85 & -74.34094 & 439.42625 & $\mathrm{NJ}$ & 2472 & 0.26 & -74.36806 & 39.40146 & NJ & 2544 & 1.74 & -74.39998 & 39.37778 & $\mathrm{NJ}$ \\
\hline 2401 & -1.84 & -74.34135 & 539.42591 & $\mathrm{NJ}$ & 2473 & 0.35 & -74.36845 & 599.40113 & $\mathrm{NJ}$ & 2545 & 1.77 & -74.40041 & 39.37746 & $\mathrm{NJ}$ \\
\hline 2402 & -1.93 & -74.34174 & 439.42558 & $\mathrm{NJ}$ & 2474 & 0.48 & -74.36884 & 39.40079 & $\mathrm{NJ}$ & 2546 & 1.79 & -74.40088 & 39.37717 & $\mathrm{NJ}$ \\
\hline 2403 & -2.01 & -74.34215 & 539.42525 & $\mathrm{NJ}$ & 2475 & 0.64 & .74 .36917 & 39.40043 & NJ & 2547 & 1.82 & -74.40135 & 39.37688 & $\mathrm{NJ}$ \\
\hline 2404 & -2.08 & -74.34254 & 499.42491 & $\mathrm{NJ}$ & 2476 & 0.77 & -74.36955 & 39.40009 & NJ & 2548 & 1.89 & -74.40179 & 39.37658 & $\mathrm{NJ}$ \\
\hline 2405 & -2.15 & -74.34294 & 439.42459 & NJ & 2477 & 0.98 & -74.37 & 39.39978 & $\mathrm{NJ}$ & 2549 & 1.97 & -74.40228 & 39.37629 & $\mathrm{NJ}$ \\
\hline 2406 & -2.21 & -74.34332 & 239.42425 & $\mathrm{NJ}$ & 2478 & 1.05 & -74.37042 & 239.39946 & $\mathrm{NJ}$ & 2550 & 2.06 & -74.40274 & 39.376 & $\mathrm{NJ}$ \\
\hline 2407 & -2.24 & -74.34373 & 339.42392 & $\mathrm{NJ}$ & 2479 & 1.03 & -74.37083 & 39.39914 & $\mathrm{NJ}$ & 2551 & 2.22 & -74.40318 & 39.37569 & $\mathrm{NJ}$ \\
\hline 2408 & -2.21 & -74.34415 & 539.4236 & $\mathrm{NJ}$ & 2480 & 0.97 & -74.37128 & 399.39883 & $\mathrm{NJ}$ & 2552 & 2.4 & -74.40364 & 39.37539 & $\mathrm{NJ}$ \\
\hline 2409 & -2.24 & -74.34454 & 439.42326 & NJ & 2481 & 1.18 & -74.37172 & 39.39852 & NJ & 2553 & 2.66 & -74.40405 & 39.37507 & $\mathrm{NJ}$ \\
\hline 2410 & -2.27 & -74.34491 & 139.42292 & $\mathrm{NJ}$ & 2482 & 1.28 & -74.37216 & 539.39822 & NJ & 2554 & 3.03 & .74 .40446 & 39.37475 & NJ \\
\hline 2411 & -2.33 & -74.34528 & 839.42257 & $\mathrm{NJ}$ & 2483 & 1.43 & -74.37254 & 39.39788 & $\mathrm{NJ}$ & 2555 & -0.71 & -74.41312 & 39.37181 & NJ \\
\hline 2412 & -2.4 & -74.34566 & 539.42222 & $\mathrm{NJ}$ & 2484 & 1.47 & -74.373 & 39.39758 & NJ & 2556 & 1.19 & -74.41144 & 39.3696 & $\mathrm{NJ}$ \\
\hline 2413 & -2.46 & -74.34602 & 239.42187 & $\mathrm{NJ}$ & 2485 & 1.51 & -74.37334 & 49.39722 & $\mathrm{NJ}$ & 2557 & 1.75 & -74.41095 & 39.36846 & NJ \\
\hline 2414 & -2.49 & -74.34641 & 39.42153 & NJ & 2486 & 1.59 & -74.37378 & 39.39691 & $\mathrm{NJ}$ & 2558 & 2.41 & -74.41042 & 39.36728 & $\mathrm{NJ}$ \\
\hline 2415 & -2.54 & -74.34674 & 439.42117 & $\mathrm{NJ}$ & 2487 & 1.66 & -74.37428 & 39.39664 & $\mathrm{NJ}$ & 2559 & 4.17 & -74.40895 & 39.36525 & NJ \\
\hline 2416 & -2.6 & .74 .34709 & 39.4208 & $\mathrm{NJ}$ & 2488 & 1.71 & -74.37468 & 39.3963 & $\mathrm{NJ}$ & 2560 & 4.63 & -74.40871 & 39.36433 & $\mathrm{NJ}$ \\
\hline 2417 & -2.66 & -74.34744 & 49.42045 & $\mathrm{NJ}$ & 2489 & 1.74 & -74.37511 & 39.39599 & NJ & 2561 & 4.21 & -74.40923 & 39.36411 & NJ \\
\hline 2418 & -2.66 & -74.34779 & 39.42009 & $\mathrm{NJ}$ & 2490 & 1.76 & -74.37553 & 39.39568 & NJ & 2562 & 3.9 & .74 .40965 & 39.3638 & NJ \\
\hline 2419 & -2.72 & .74 .34822 & 239.41977 & $\mathrm{NJ}$ & 2491 & 1.8 & -74.37598 & 39.39537 & $\mathrm{NJ}$ & 2563 & 3.65 & -74.41006 & 39.36348 & $\mathrm{NJ}$ \\
\hline 2420 & -2.75 & -74.34857 & 39.41941 & NJ & 2492 & 1.8 & -74.37646 & 39.39508 & $\mathrm{NJ}$ & 2564 & 3.53 & -74.41042 & 39.3631 & NJ \\
\hline 2421 & -2.76 & -74.34894 & 39.41907 & $\mathrm{NJ}$ & 2493 & 1.83 & -74.37691 & | 39.39478 & $\mathrm{NJ}$ & 2565 & 3.39 & -74.41081 & 39.36277 & NJ \\
\hline 2422 & -2.82 & -74.3493 & 39.41872 & $\mathrm{NJ}$ & 2494 & 1.92 & .74 .3773 & 39.39445 & $\mathrm{NJ}$ & 2566 & 3.54 & -74.41104 & 39.36229 & NJ \\
\hline 2423 & -2.88 & -74.34969 & 39.41837 & $\mathrm{NJ}$ & 2495 & 1.94 & -74.37779 & 39.39417 & NJ & 2567 & 3.42 & .74 .41158 & 39.36208 & NJ \\
\hline 2424 & -2.97 & -74.35008 & 339.41804 & $\mathrm{NJ}$ & 2496 & 2 & -74.37825 & 39.39387 & NJ & 2568 & 3.36 & -74.41203 & 39.3618 & NJ \\
\hline 2425 & -3.06 & -74.35048 & 39.41771 & $\mathrm{NJ}$ & 2497 & 2.18 & -74.37878 & 39.39362 & $\mathrm{NJ}$ & 2569 & 3.33 & -74.41245 & 39.36148 & NJ \\
\hline 2426 & -3.11 & -74.35088 & 39.41737 & NJ & 2498 & 2.37 & -74.37926 & 59.39332 & NJ & 2570 & 3.32 & -74.41284 & 39.36115 & NJ \\
\hline 2427 & -3.18 & -74.35129 & 39.41705 & NJ & 2499 & 2.63 & -74.3797 & 39.39302 & NJ & 2571 & 3.29 & -74.41324 & 39.36082 & $\mathrm{NJ}$ \\
\hline 2428 & -3.22 & -74.35167 & 39.41671 & NJ & 2500 & 5.04 & -74.38148 & 39.39181 & NJ & 2572 & 2.97 & -74.41386 & 39.36069 & $\mathrm{NJ}$ \\
\hline 2429 & -3.32 & .74 .35204 & 39.41636 & $\mathrm{NJ}$ & 2501 & 4.07 & -74.38199 & 39.39154 & $\mathrm{NJ}$ & 2573 & 2.77 & -74.41438 & 39.36047 & NJ \\
\hline 2430 & -3.38 & -74.35239 & 39.416 & NJ & 2502 & 3.47 & -74.38242 & 39.39121 & NJ & 2574 & 2.55 & -74.41484 & 39.3602 & NJ \\
\hline 2431 & -3.42 & -74.35275 & 39.41565 & NJ & 2503 & 3.12 & -74.3829 & 39.39095 & NJ & 2575 & 2.83 & -74.41479 & 39.35946 & NJ \\
\hline 2432 & .3 .46 & -74.3531 & 39.41529 & $\mathrm{NJ}$ & 2504 & 2.78 & -74.38341 & 39.39067 & $\mathrm{NJ}$ & 2576 & 1.34 & .74 .41631 & 39.35945 & NJ \\
\hline 2433 & -3.47 & -74.35345 & 59.41494 & $\mathrm{NJ}$ & 2505 & 2.34 & -74.38393 & 39.39041 & $\mathrm{NJ}$ & 2577 & 0.85 & -74.41679 & 39.35919 & NJ \\
\hline 2434 & -3.47 & -74.35378 & 39.41455 & NJ & 2506 & 1.91 & -74.38438 & 39.39011 & $\mathrm{NJ}$ & 2578 & 2.6 & .74 .41512 & 39.35906 & $\mathrm{NJ}$ \\
\hline 2435 & -3.39 & -74.35406 & 39.41416 & $\mathrm{NJ}$ & 2507 & 1.73 & -74.38483 & 39.3898 & $\mathrm{NJ}$ & 2579 & 0.34 & .74 .41737 & 39.35903 & $\mathrm{NJ}$ \\
\hline 2436 & -3.48 & -74.35469 & 39.41396 & $\mathrm{NJ}$ & 2508 & 1.83 & -74.38515 & 39.38943 & $\mathrm{NJ}$ & 2580 & -0.04 & .74 .41788 & 39.35879 & NJ \\
\hline 2437 & -3.53 & -74.3548 & 39.41347 & NJ & 2509 & 1.83 & -74.3857 & 39.38918 & $\mathrm{NJ}$ & 2581 & 0.34 & -74.41835 & 39.35853 & $\mathrm{NJ}$ \\
\hline 2438 & -3.49 & -74.35519 & 39.41313 & $\mathrm{NJ}$ & 2510 & 1.89 & -74.38608 & 39.38884 & $\mathrm{NJ}$ & 2582 & 0.66 & .74 .41881 & 39.35826 & $\mathrm{NJ}$ \\
\hline 2439 & -3.51 & -74.35561 & 39.41281 & NJ & 2511 & 1.9 & -74.38652 & 39.38853 & $\mathrm{NJ}$ & 2583 & 0.87 & -74.41924 & 39.35795 & NJ \\
\hline 2440 & -3.53 & -74.35602 & 39.41248 & NJ & 2512 & 2.01 & -74.38683 & 39.38815 & $\mathrm{NJ}$ & 2584 & 1.93 & -74.44043 & 39.35186 & $\mathrm{NJ}$ \\
\hline 2441 & -3.58 & -74.35641 & 39.41216 & NJ & 2513 & 1.94 & -74.38731 & 39.38787 & $\mathrm{NJ}$ & 2585 & 1.97 & .74 .44096 & 39.35169 & $\mathrm{NJ}$ \\
\hline 2442 & -3.61 & -74.35681 & 39.41182 & NJ & 2514 & 1.97 & -74.3877 & 39.38753 & $\mathrm{NJ}$ & 2586 & 2.04 & -74.4415 & 39.35149 & $\mathrm{NJ}$ \\
\hline 2443 & -3.54 & -74.35719 & 39.41148 & $\mathrm{NJ}$ & 2515 & 2.06 & -74.38805 & 39.38717 & $\mathrm{NJ}$ & 2587 & 2.1 & -74.44202 & 39.3513 & $\mathrm{NJ}$ \\
\hline 2444 & -3.38 & -74.3576 & 39.41116 & NJ & 2516 & 2.1 & -74.38843 & 39.38683 & $\mathrm{NJ}$ & 2588 & 2.14 & -74.44257 & 39.35111 & $\mathrm{NJ}$ \\
\hline 2445 & -3.22 & -74.35799 & 39.41081 & NJ & 2517 & 2.1 & -74.38881 & 39.38649 & $\mathrm{NJ}$ & 2589 & 2.18 & .74 .44308 & 39.35093 & $\mathrm{NJ}$ \\
\hline 2446 & -3.07 & -74.35838 & 39.41048 & $\mathrm{NJ}$ & 2518 & 2.14 & -74.38918 & 39.38614 & $\mathrm{NJ}$ & 2590 & 2.2 & .74 .44362 & 39.35074 & $\mathrm{NJ}$ \\
\hline 2447 & -2.89 & -74.35875 & 39.41013 & $\mathrm{NJ}$ & 2519 & 2.15 & -74.38959 & 39.38581 & $\mathrm{NJ}$ & 2591 & 2.22 & .74 .44415 & 39.35055 & $\mathrm{NJ}$ \\
\hline 2448 & -2.72 & -74.35913 & 39.40979 & NJ & 2520 & 2.19 & -74.38995 & 39.38546 & $\mathrm{NJ}$ & 2592 & 2.23 & .74 .44469 & 39.35035 & NJ \\
\hline
\end{tabular}




\begin{tabular}{|c|c|c|c|c|c|c|c|c|c|c|c|c|c|c|}
\hline TR\# & RATE & LONG & LAT & ST & TR\# & RATE & LONG & LAT & ST & TR\# & RATE & LONG & LAT & ST \\
\hline 2593 & 2.25 & -74.44521 & 39.35015 & $\mathrm{NJ}$ & 2665 & 1.02 & $\begin{array}{l}-74.4818 \\
\end{array}$ & 39.33446 & NJ & 2737 & -0.12 & -74.51674 & 39.31669 & $\overline{\mathrm{NJ}}$ \\
\hline 2594 & 2.27 & -74.44572 & 39.34994 & $\mathrm{NJ}$ & 2666 & 0.98 & -74.4823 & 39.33424 & NJ & 2738 & -0.23 & -74.51724 & 39.31646 & NJ \\
\hline 2595 & 2.31 & -74.44623 & 39.34973 & NJ & 2667 & 0.99 & -74.48282 & 39.33403 & NJ & 2739 & -0.32 & -74.51772 & 39.31619 & NJ \\
\hline 2596 & 2.33 & -74.44675 & 539.34952 & $\mathrm{NJ}$ & 2668 & 0.95 & -74.48331 & 39.33379 & NJ & 2740 & -0.36 & -74.51817 & 39.31592 & $\mathrm{NJ}$ \\
\hline 2597 & 2.34 & -74.44727 & 39.34933 & NJ & 2669 & 0.97 & -74.48381 & 39.33357 & NJ & 2741 & -0.37 & -74.51865 & 39.31565 & $\mathrm{NJ}$ \\
\hline 2598 & 2.37 & -74.44778 & 8 39.34912 & $\mathrm{NJ}$ & 2670 & 0.91 & -74.48431 & 39.33334 & NJ & 2742 & -0.35 & -74.5191 & 39.31537 & $\mathrm{NJ}$ \\
\hline 2599 & 2.37 & -74.4483 & 39.34892 & $\mathrm{NJ}$ & 2671 & 0.85 & -74.48482 & 39.33312 & NJ & 2743 & -0.32 & -74.51958 & 39.3151 & NJ \\
\hline 2600 & 2.37 & -74.44884 & 39.34872 & $\mathrm{NJ}$ & 2672 & 0.8 & -74.48532 & 39.33289 & $\mathrm{NJ}$ & 2744 & -0.24 & -74.52003 & 39.31482 & NJ \\
\hline 2601 & 2.38 & -74.44934 & 39.3485 & NJ & 2673 & 0.8 & -74.48582 & 39.33268 & $\mathrm{NJ}$ & 2745 & -0.22 & -74.52048 & 39.31453 & $\mathrm{NJ}$ \\
\hline 2602 & 2.37 & -74.44986 & 539.3483 & $\mathrm{NJ}$ & 2674 & 0.8 & .74 .48634 & 39.33246 & NJ & 2746 & -0.3 & -74.52094 & 39.31425 & NJ \\
\hline 2603 & 2.37 & -74.45038 & 39.34809 & $\mathrm{NJ}$ & 2675 & 0.78 & .74 .48685 & 39.33223 & NJ & 2747 & -0.34 & -74.52138 & 39.31395 & $\mathrm{NJ}$ \\
\hline 2604 & 2.39 & -74.45088 & 39.34787 & $\mathrm{NJ}$ & 2676 & 0.76 & .74 .48734 & 39.332 & NJ & 2748 & -0.32 & -74.52182 & 39.31365 & $\mathrm{NJ}$ \\
\hline 2605 & 2.41 & -74.45139 & 39.34765 & NJ & 2677 & 0.77 & -74.48784 & 39.33177 & $\mathrm{NJ}$ & 2749 & -0.32 & -74.52226 & 39.31335 & $\mathrm{NJ}$ \\
\hline 2606 & 2.44 & -74.45189 & 39.34741 & NJ & 2678 & 0.74 & .74 .48834 & 39.33154 & $\mathrm{NJ}$ & 2750 & -0.29 & -74.52271 & 39.31304 & $\mathrm{NJ}$ \\
\hline 2607 & 2.45 & -74.45239 & 39.3472 & $\mathrm{NJ}$ & 2679 & 0.72 & .74 .48885 & 39.33132 & NJ & 2751 & -0.29 & .74 .52313 & 39.31272 & $\mathrm{NJ}$ \\
\hline 2608 & 2.45 & -74.4529 & 39.34697 & NJ & 2680 & 0.68 & -74.48933 & 39.33108 & NJ & 2752 & -0.33 & -74.52357 & 39.31243 & $\mathrm{NJ}$ \\
\hline 2609 & 2.44 & -74.4534 & 39.34674 & NJ & 2681 & 0.65 & .74 .48982 & 39.33083 & NJ & 2753 & -0.27 & .74 .52402 & 39.31212 & $\mathrm{NJ}$ \\
\hline 2610 & 2.44 & .74 .45389 & 39.34651 & $\mathrm{NJ}$ & 2682 & 0.62 & .74 .49033 & 39.3306 & $\mathrm{NJ}$ & 2754 & -0.3 & -74.52448 & 39.31183 & $\mathrm{NJ}$ \\
\hline 2611 & 2.4 & -74.45441 & 39.34629 & $\mathrm{NJ}$ & 2683 & 0.64 & .74 .4908 & 39.33035 & NJ & 2755 & -0.23 & -74.5249 & 39.31153 & NJ \\
\hline 2612 & 2.4 & -74.4549 & 39.34605 & NJ & 2684 & 0.61 & -74.49129 & 39.33012 & NJ & 2756 & -0.27 & -74.52534 & 39.31123 & $\mathrm{NJ}$ \\
\hline 2613 & 2.33 & -74.4554 & 39.34583 & NJ & 2685 & 0.58 & -74.49178 & 39.32987 & NJ & 2757 & -0.29 & .74 .52579 & 39.31091 & NJ \\
\hline 2614 & 2.28 & -74.45592 & 39.34561 & $\mathrm{NJ}$ & 2686 & 0.56 & .74 .49226 & 39.32962 & $\mathrm{NJ}$ & 2758 & -0.22 & -74.5262 & 39.31058 & $\mathrm{NJ}$ \\
\hline 2615 & 2.27 & -74.45641 & 39.34539 & NJ & 2687 & 0.61 & -74.49275 & 39.32938 & NJ & 2759 & -0.22 & -74.5266 & 39.31023 & NJ \\
\hline 2616 & 2.25 & -74.45692 & 39.34517 & NJ & 2688 & 0.61 & .74 .49324 & 39.32914 & NJ & 2760 & -0.27 & .74 .52702 & 39.30991 & $\mathrm{NJ}$ \\
\hline 2617 & 2.25 & -74.45741 & 39.34495 & NJ & 2689 & 0.54 & -74.49371 & 39.32889 & NJ & 2761 & -0.24 & -74.52747 & 39.3096 & NJ \\
\hline 2618 & 2.23 & .74 .45795 & 39.34475 & $\mathrm{NJ}$ & 2690 & 0.59 & -74.4942 & 39.32862 & NJ & 2762 & -0.35 & -74.52789 & 39.30928 & NJ \\
\hline 2619 & 2.26 & -74.45844 & 39.34451 & $\mathrm{NJ}$ & 2691 & 0.55 & -74.49467 & 39.32838 & NJ & 2763 & -0.3 & .74 .52831 & 39.30895 & NJ \\
\hline 2620 & 2.26 & -74.45895 & 39.3443 & NJ & 2692 & 0.59 & .74 .49516 & 39.32813 & $\mathrm{NJ}$ & 2764 & -0.25 & -74.52872 & 39.3086 & NJ \\
\hline 2621 & 1.69 & -74.45947 & 39.3441 & $\mathrm{NJ}$ & 2693 & 0.64 & .74 .49564 & 39.32787 & NJ & 2765 & -0.16 & -74.52911 & 39.30826 & NJ \\
\hline 2622 & 1.66 & -74.46001 & 39.34391 & $\mathrm{NJ}$ & 2694 & 0.57 & -74.49661 & 39.32738 & $\mathrm{NJ}$ & 2766 & -0.27 & .74 .52954 & 39.30792 & $\mathrm{NJ}$ \\
\hline 2623 & 1.67 & -74.46051 & 39.34369 & NJ & 2695 & 0.93 & -74.4959 & 39.32734 & NJ & 2767 & -0.3 & -74.52995 & 39.3076 & NJ \\
\hline 2624 & 1.69 & -74.46103 & 39.34348 & $\mathrm{NJ}$ & 2696 & 0.49 & -74.49712 & 39.32716 & $\mathrm{NJ}$ & 2768 & -0.26 & .74 .53036 & 39.30726 & $\mathrm{NJ}$ \\
\hline 2625 & 1.65 & -74.46153 & 39.34326 & $\mathrm{NJ}$ & 2697 & 0.35 & .74 .49762 & 39.32693 & $\mathrm{NJ}$ & 2769 & -0.29 & -74.53076 & 39.30689 & NJ \\
\hline 2626 & 1.64 & -74.46204 & +39.34303 & $\mathrm{NJ}$ & 2698 & 0.3 & -74.49809 & 39.32667 & NJ & 2770 & -0.29 & .74 .53116 & 39.30654 & $\mathrm{NJ}$ \\
\hline 2627 & 1.59 & -74.46254 & 39.34281 & $\mathrm{NJ}$ & 2699 & 0.28 & -74.4986 & 39.32643 & NJ & 2771 & -0.31 & -74.53159 & 39.3062 & NJ \\
\hline 2628 & 1.64 & -74.46304 & 39.34259 & $\mathrm{NJ}$ & 2700 & 0.33 & -74.49905 & 39.32617 & NJ & 2772 & -0.29 & -74.53198 & 39.30586 & NJ \\
\hline 2629 & 1.57 & -74.46355 & 39.34236 & $\mathrm{NJ}$ & 2701 & 0.37 & -74.49956 & 39.32592 & NJ & 2773 & -0.27 & -74.53241 & 39.30553 & NJ \\
\hline 2630 & 1.6 & .74 .46407 & 39.34215 & $\mathrm{NJ}$ & 2702 & 0.44 & -74.50003 & 39.32567 & NJ & 2774 & -0.29 & -74.53284 & 39.3052 & NJ \\
\hline 2631 & 1.6 & -74.46457 & 39.34193 & $\mathrm{NJ}$ & 2703 & 0.46 & -74.50047 & 39.32539 & NJ & 2775 & -0.3 & .74 .53325 & 39.30487 & NJ \\
\hline 2632 & 1.53 & -74.46507 & 39.34172 & $\mathrm{NJ}$ & 2704 & 0.44 & .74 .50093 & 39.32509 & NJ & 2776 & -0.27 & -74.53368 & 39.30456 & NJ \\
\hline 2633 & 1.56 & -74.46561 & 39.34153 & $\mathrm{NJ}$ & 2705 & 0.43 & -74.50142 & 39.32485 & NJ & 2777 & -0.26 & .74 .5341 & 39.30425 & $\mathrm{NJ}$ \\
\hline 2634 & 1.53 & -74.46613 & 39.34131 & $\mathrm{NJ}$ & 2706 & 0.35 & -74.50194 & 39.32465 & NJ & 2778 & -0.22 & -74.53448 & 39.30385 & NJ \\
\hline 2635 & 1.55 & .74 .46663 & 39.3411 & $\mathrm{NJ}$ & 2707 & 0.32 & -74.50243 & 39.3244 & NJ & 2779 & -2.25 & .74 .53833 & 39.30356 & NJ \\
\hline 2636 & 1.55 & -74.46713 & 39.34088 & NJ & 2708 & 0.3 & -74.50291 & 39.32415 & NJ & 2780 & -0.97 & -74.53625 & 39.30353 & $\mathrm{NJ}$ \\
\hline 2637 & 1.52 & -74.46765 & 39.34067 & $\mathrm{NJ}$ & 2709 & 0.22 & -74.50339 & 39.32389 & NJ & 2781 & -1.41 & -74.53693 & 39.30349 & $\mathrm{NJ}$ \\
\hline 2638 & 1.51 & -74.46815 & 39.34045 & $\mathrm{NJ}$ & 2710 & 0.14 & -74.50388 & 39.32365 & NJ & 2782 & -1.74 & -74.53757 & 39.30344 & $\mathrm{NJ}$ \\
\hline 2639 & 1.49 & -74.46866 & 39.34022 & $\mathrm{NJ}$ & 2711 & 0.2 & -74.50433 & 39.32338 & NJ & 2783 & -0.1 & -74.53484 & 39.30344 & NJ \\
\hline 2640 & 1.45 & -74.46918 & 39.34001 & $\mathrm{NJ}$ & 2712 & 0.13 & .74 .50482 & 39.32312 & NJ & 2784 & -2.49 & -74.53891 & 39.30342 & $\mathrm{NJ}$ \\
\hline 2641 & 1.42 & -74.46968 & 39.33979 & $\mathrm{NJ}$ & 2713 & 0.14 & -74.50529 & 39.32286 & NJ & 2785 & 0.05 & .74 .53516 & 39.30298 & $\mathrm{NJ}$ \\
\hline 2642 & 1.41 & -74.47018 & 39.33956 & NJ & 2714 & 0.13 & -74.50574 & 39.32257 & NJ & 2786 & 4.86 & -74.55128 & 39.29271 & NJ \\
\hline 2643 & 1.37 & -74.47067 & 39.33933 & NJ & 2715 & 0.24 & -74.50613 & 39.32221 & NJ & 2787 & 4.22 & -74.55177 & 39.29239 & $\mathrm{NJ}$ \\
\hline 2644 & 1.34 & -74.47119 & 39.33911 & $\mathrm{NJ}$ & 2716 & 0.27 & .74 .50659 & 39.32192 & NJ & 2788 & 3.93 & -74.55202 & 39.29198 & $\mathrm{NJ}$ \\
\hline 2645 & 1.36 & -74.47168 & 39.33888 & NJ & 2717 & 0.23 & -74.50706 & 39.32166 & NJ & 2789 & 3.8 & -74.55219 & 39.29154 & NJ \\
\hline 2646 & 1.35 & -74.47218 & 39.33866 & $\mathrm{NJ}$ & 2718 & 0.22 & -74.50752 & 39.32138 & NJ & 2790 & 3.78 & -74.55234 & 39.2911 & $\mathrm{NJ}$ \\
\hline 2647 & 1.31 & -74.47269 & 39.33843 & NJ & 2719 & 0.2 & -74.508 & 39.32112 & $\mathrm{NJ}$ & 2791 & 4.74 & -74.55185 & 39.29043 & $\mathrm{NJ}$ \\
\hline 2648 & 1.35 & -74.47321 & 39.33822 & $\mathrm{NJ}$ & 2720 & 0.14 & -74.50847 & 39.32086 & $\mathrm{NJ}$ & 2792 & 4.04 & -74.55241 & 39.29013 & $\mathrm{NJ}$ \\
\hline 2649 & 1.34 & .74 .47369 & 39.33798 & NJ & 2721 & 0.12 & -74.50896 & 39.32061 & NJ & 2793 & 3.88 & -74.55263 & 39.28971 & $\mathrm{NJ}$ \\
\hline 2650 & 1.35 & -74.4742 & 39.33775 & $\mathrm{NJ}$ & 2722 & 0.45 & -74.50999 & 39.3202 & NJ & 2794 & 3.77 & -74.55281 & 39.28928 & $\mathrm{NJ}$ \\
\hline 2651 & 1.37 & -74.4747 & 39.33753 & NJ & 2723 & 0.28 & -74.51051 & 39.32001 & NJ & 2795 & 3.83 & -74.55292 & 39.28882 & $\mathrm{NJ}$ \\
\hline 2652 & 1.33 & .74 .4752 & 39.33731 & NJ & 2724 & 0.16 & -74.51102 & 39.31977 & NJ & 2796 & 4.17 & -74.55269 & 39.28824 & NJ \\
\hline 2653 & 1.31 & -74.47569 & 39.33708 & NJ & 2725 & 1.32 & -74.50891 & 39.31969 & NJ & 2797 & 4.35 & -74.55316 & 39.28792 & $\mathrm{NJ}$ \\
\hline 2654 & 1.28 & -74.47621 & 39.33686 & NJ & 2726 & 0.14 & -74.51151 & 39.31953 & NJ & 2798 & 3.29 & -74.55353 & 39.28755 & NJ \\
\hline 2655 & 1.3 & .74 .47672 & 39.33663 & NJ & 2727 & 0.15 & .74 .51199 & 39.31928 & $\mathrm{NJ}$ & 2799 & 2.98 & -74.55373 & 39.28713 & $\mathrm{NJ}$ \\
\hline 2656 & 1.32 & -74.4772 & 39.33639 & $\mathrm{NJ}$ & 2728 & 0.14 & -74.51247 & 39.31903 & NJ & 2800 & 3.44 & -74.55313 & 39.28641 & NJ \\
\hline 2657 & 1.28 & -74.47769 & 39.33615 & $\mathrm{NJ}$ & 2729 & 0.07 & -74.51294 & 39.31877 & NJ & 2801 & 2.61 & -74.55411 & 39.28627 & NJ \\
\hline 2658 & 1.31 & -74.4782 & 39.33593 & $\mathrm{NJ}$ & 2730 & -0.04 & -74.51341 & 39.31852 & NJ & 2802 & 2.33 & -74.55461 & 39.28596 & $\mathrm{NJ}$ \\
\hline 2659 & 1.3 & -74.47871 & 39.33573 & NJ & 2731 & -0.05 & .74 .51389 & 39.31824 & NJ & 2803 & 2.18 & -74.55502 & 39.28561 & NJ \\
\hline 2660 & 1.23 & -74.47922 & 39.33551 & NJ & 2732 & -0.06 & -74.51434 & 39.31797 & NJ & 2804 & 2.07 & -74.5553 & 39.28522 & $\mathrm{NJ}$ \\
\hline 2661 & 1.19 & -74.47975 & 39.33532 & $\mathrm{NJ}$ & 2733 & -0.06 & -74.51482 & 39.3177 & NJ & 2805 & 2.2 & -74.55544 & 39.28476 & NJ \\
\hline 2662 & 1.12 & -74.48026 & 39.3351 & NJ & 2734 & -0.03 & -74.51529 & 39.31744 & NJ & 2806 & 2.07 & -74.55586 & 39.28442 & NJ \\
\hline 2663 & 1.08 & -74.48077 & 39.33488 & NJ & 2735 & 0 & .74 .51575 & 39.31714 & NJ & 2807 & 1.94 & .74 .55646 & 39.28414 & NJ \\
\hline 2664 & 1.04 & -74.48128 & 39.33467 & NJ & 2736 & -0.05 & -74.51624 & 39.31691 & NJ & 2808 & 2.02 & -74.55698 & 39.28383 & NJ \\
\hline
\end{tabular}




\begin{tabular}{|c|c|c|c|c|c|c|c|c|c|c|c|c|c|c|}
\hline TR\# & RATE & LONG & LAT & ST & t TR\# & RATE & LONG & LAT & ST & TR\# & RATE & LONG & LAT & ST \\
\hline 2809 & 2.26 & -74.55727 & 39.28345 & NJ & 2881 & 0.27 & -74.59012 & 39.26524 & $\mathrm{NJ}$ & 2953 & 0.45 & -74.61044 & 39.24838 & $\mathrm{NJ}$ \\
\hline 2810 & 2.23 & -74.55774 & 39.28312 & NJ & 2882 & 0.34 & -74.59055 & 39.26493 & $\mathrm{NJ}$ & 2954 & 0.27 & -74.61092 & 39.24827 & $\mathrm{NJ}$ \\
\hline 2811 & 2.37 & -74.55815 & 39.28277 & $\mathrm{NJ}$ & 2883 & 0.34 & -74.59097 & 39.26463 & NJ & 2955 & 0.41 & -74.61086 & 39.24805 & NJ \\
\hline 2812 & 2.53 & -74.55846 & 39.28239 & $\mathrm{NJ}$ & 2884 & 0.36 & -74.5914 & 39.26432 & NJ & 2956 & 0.3 & -74.61124 & 39.24789 & $\mathrm{NJ}$ \\
\hline 2813 & 3.36 & -74.55795 & 39.28171 & NJ & 2885 & 0.37 & -74.59183 & 39.26401 & NJ & 2957 & 0.34 & -74.61124 & 39.24771 & NJ \\
\hline 2814 & 2.79 & -74.55841 & 39.28151 & NJ & 2886 & 0.42 & -74.59225 & 39.2637 & NJ & 2958 & 0.3 & .74 .61159 & 39.24753 & $\mathrm{NJ}$ \\
\hline 2815 & 3.13 & -74.55858 & 39.28144 & NJ & 2887 & 0.45 & -74.59267 & 39.26338 & NJ & 2959 & 0.42 & -74.6116 & 39.24736 & $\mathrm{NJ}$ \\
\hline 2816 & 3.45 & -74.55753 & 39.28134 & NJ & 2888 & 0.48 & -74.59306 & 39.26306 & NJ & 2960 & 0.27 & -74.61198 & 39.2472 & $\mathrm{NJ}$ \\
\hline 2817 & 2.81 & -74.55891 & 39.28127 & NJ & 2889 & 0.52 & -74.59348 & 39.26273 & NJ & 2961 & 0.38 & -74.61197 & 39.24701 & NJ \\
\hline 2818 & 3.13 & -74.55913 & 39.28114 & NJ & 2890 & 0.53 & -74.59389 & 39.26241 & NJ & 2962 & 0.24 & -74.61234 & 39.24685 & NJ \\
\hline 2819 & 2.79 & -74.55937 & 39.281 & NJ & 2891 & 0.57 & -74.59428 & 39.26209 & $\mathrm{NJ}$ & 2963 & 0.34 & -74.61235 & 39.24666 & $\mathrm{NJ}$ \\
\hline 2820 & 3.14 & -74.55963 & 39.28083 & NJ & 2892 & 0.58 & -74.5947 & 39.26177 & $\mathrm{NJ}$ & 2964 & 0.18 & -74.61276 & 39.24654 & $\mathrm{NJ}$ \\
\hline 2821 & 2.78 & -74.5598 & 39.2807 & NJ & 2893 & 0.59 & -74.59508 & 39.26144 & $\mathrm{NJ}$ & 2965 & 0.24 & -74.61275 & 39.24633 & $\mathrm{NJ}$ \\
\hline 2822 & 3.16 & -74.56007 & 39.28049 & NJ & 2894 & 0.62 & -74.59549 & 39.26111 & NJ & 2966 & 0.19 & -74.61311 & 39.24618 & $\mathrm{NJ}$ \\
\hline 2823 & 2.84 & -74.56021 & 39.28037 & $\mathrm{NJ}$ & 2895 & 0.63 & -74.5959 & 39.2608 & $\mathrm{NJ}$ & 2967 & 0.24 & -74.61311 & 39.24598 & $\mathrm{NJ}$ \\
\hline 2824 & 2.93 & -74.56058 & 39.28 & $\mathrm{NJ}$ & 2896 & 0.6 & -74.59631 & 39.26048 & NJ & 2968 & 0.15 & -74.61351 & 39.24585 & NJ \\
\hline 2825 & 3.18 & -74.56073 & 39.27939 & NJ & 2897 & 0.59 & -74.59673 & 39.26016 & NJ & 2969 & 0.21 & -74.61351 & 39.24564 & NJ \\
\hline 2826 & 3.01 & -74.56126 & 39.2792 & NJ & 2898 & 0.56 & -74.59715 & 39.25985 & NJ & 2970 & 0.15 & .74 .61388 & 39.2455 & $\mathrm{NJ}$ \\
\hline 2827 & 3.01 & -74.56177 & 39.27897 & NJ & 2899 & 0.58 & -74.59753 & 39.25952 & NJ & 2971 & 0.18 & -74.61386 & 39.24528 & NJ \\
\hline 2828 & 2.87 & -74.56226 & 39.27871 & NJ & 2900 & 0.59 & -74.59795 & 39.25919 & NJ & 2972 & 0.12 & -74.61424 & 39.24515 & $\mathrm{NJ}$ \\
\hline 2829 & 2.92 & -74.56271 & 39.27844 & NJ & 2901 & 0.59 & -74.59834 & 39.25887 & $\mathrm{NJ}$ & 2973 & 0.19 & .74 .61424 & 39.24494 & $\mathrm{NJ}$ \\
\hline 2830 & 3.06 & -74.56316 & 39.27815 & $\mathrm{NJ}$ & 2902 & 0.58 & -74.59872 & 39.25853 & NJ & 2974 & 0.06 & -74.61461 & 39.2448 & $\mathrm{NJ}$ \\
\hline 2831 & 3.15 & -74.56354 & 39.27779 & NJ & 2903 & 0.56 & .74 .59914 & 39.25821 & NJ & 2975 & 0.13 & -74.61462 & 39.2446 & NJ \\
\hline 2832 & 3.65 & -74.56384 & 39.27735 & NJ & 2904 & 0.51 & -74.59958 & 39.25792 & NJ & 2976 & 0.03 & -74.61501 & 39.24447 & NJ \\
\hline 2833 & 3.56 & -74.56441 & 39.27718 & NJ & 2905 & 0.48 & -74.60001 & 39.2576 & NJ & 2977 & 0.19 & .74 .61497 & 39.24424 & $\mathrm{NJ}$ \\
\hline 2834 & 3.27 & .74 .56563 & 39.27698 & $\mathrm{NJ}$ & 2906 & 0.46 & -74.60039 & 39.25727 & NJ & 2978 & 0.07 & -74.61533 & 39.24408 & $\mathrm{NJ}$ \\
\hline 2835 & 3.56 & -74.5649 & 39.27696 & NJ & 2907 & 0.46 & -74.60078 & 39.25694 & NJ & 2979 & 0.19 & -74.61534 & 39.24388 & $\mathrm{NJ}$ \\
\hline 2836 & 3.19 & -74.5661 & 39.27671 & NJ & 2908 & 0.49 & -74.6012 & 39.25662 & NJ & 2980 & 0.07 & -74.61569 & 39.24374 & NJ \\
\hline 2837 & 3.1 & -74.56654 & 39.27643 & NJ & 2909 & 0.43 & .74 .60158 & 39.25628 & NJ & 2981 & 0.14 & -74.61565 & 39.2435 & NJ \\
\hline 2838 & 3.03 & -74.56699 & 39.27613 & NJ & 2910 & 0.42 & .74 .60205 & 39.25601 & NJ & 2982 & 0.09 & -74.61601 & 39.24336 & $\mathrm{NJ}$ \\
\hline 2839 & 2.89 & -74.56808 & 39.27576 & $\mathrm{NJ}$ & 2911 & 0.4 & .74 .60248 & 39.2557 & $\mathrm{NJ}$ & 2983 & 0.18 & -74.61601 & 39.24315 & $\mathrm{NJ}$ \\
\hline 2840 & 3.59 & -74.56725 & 39.27564 & $\mathrm{NJ}$ & 2912 & 0.45 & -74.60281 & 39.25533 & NJ & 2984 & 0.09 & -74.61636 & 39.243 & $\mathrm{NJ}$ \\
\hline 2841 & 2.84 & -74.56862 & 39.27557 & $\mathrm{NJ}$ & 2913 & 0.85 & -74.60281 & 39.25524 & NJ & 2985 & 0.13 & -74.61639 & 39.24281 & $\mathrm{NJ}$ \\
\hline 2842 & 2.81 & -74.56914 & 39.27536 & NJ & 2914 & 0.37 & -74.60329 & 39.25505 & NJ & 2986 & 0.09 & .74 .61671 & 39.24265 & NJ \\
\hline 2843 & 2.86 & -74.56961 & 39.2751 & NJ & 2915 & 0.76 & -74.60321 & 39.25491 & NJ & 2987 & 0.2 & -74.61673 & 39.24244 & NJ \\
\hline 2844 & 3.12 & -74.5701 & 39.27486 & NJ & 2916 & 0.39 & .74 .60362 & 39.25468 & NJ & 2988 & 0.13 & -74.61703 & 39.24226 & NJ \\
\hline 2845 & 2.85 & -74.57054 & 39.27456 & $\mathrm{NJ}$ & 2917 & 0.77 & -74.60361 & 39.25457 & NJ & 2989 & 0.16 & -74.61707 & 39.24207 & $\mathrm{NJ}$ \\
\hline 2846 & 2.79 & -74.57185 & 39.27444 & $\mathrm{NJ}$ & 2918 & 0.33 & .74 .60403 & 39.25437 & NJ & 2990 & 0.11 & -74.61742 & 39.24192 & $\mathrm{NJ}$ \\
\hline 2847 & 2.44 & -74.57251 & 39.27437 & NJ & 2919 & 0.74 & -74.60403 & 39.25425 & NJ & 2991 & 0.17 & -74.61742 & 39.24171 & NJ \\
\hline 2848 & 2.38 & -74.57298 & 39.27411 & $\mathrm{NJ}$ & 2920 & 0.33 & .74 .60443 & 39.25403 & NJ & 2992 & 0.11 & -74.61777 & 39.24156 & $\mathrm{NJ}$ \\
\hline 2849 & 3.52 & -74.57076 & 39.27403 & NJ & 2921 & 0.7 & .74 .60442 & 39.2539 & NJ & 2993 & 0.16 & -74.61778 & 39.24136 & NJ \\
\hline 2850 & 2.37 & -74.57346 & 39.27385 & NJ & 2922 & 0.36 & -74.60478 & 39.25367 & NJ & 2994 & 0.1 & -74.61812 & 39.2412 & NJ \\
\hline 2851 & 2.36 & -74.57393 & 39.2736 & NJ & 2923 & 0.7 & -74.60474 & 39.25353 & NJ & 2995 & 0.11 & -74.61816 & 39.24103 & NJ \\
\hline 2852 & 2.23 & -74.57439 & 39.27331 & NJ & 2924 & 0.3 & .74 .60519 & 39.25336 & NJ & 2996 & 0.07 & -74.6185 & 39.24086 & NJ \\
\hline 2853 & 2.19 & -74.57481 & 39.27301 & NJ & 2925 & 0.64 & .74 .60506 & 39.25315 & NJ & 2997 & 0.12 & -74.61855 & 39.24068 & NJ \\
\hline 2854 & 1.91 & -74.57608 & 39.27283 & $\mathrm{NJ}$ & 2926 & 0.26 & -74.60561 & 39.25303 & NJ & 2998 & 0.05 & -74.61887 & 39.24052 & $\mathrm{NJ}$ \\
\hline 2855 & 2.17 & -74.57527 & 39.27273 & $\mathrm{NJ}$ & 2927 & 0.6 & -74.60545 & 39.25282 & $\mathrm{NJ}$ & 2999 & 0.13 & -74.61893 & 39.24034 & $\mathrm{NJ}$ \\
\hline 2856 & 1.8 & -74.57664 & 39.27266 & NJ & 2928 & 0.2 & -74.60602 & 39.25272 & NJ & 3000 & 0.05 & -74.61925 & 39.24017 & NJ \\
\hline 2857 & 1.75 & -74.57716 & 39.27246 & NJ & 2929 & 0.6 & -74.60587 & 39.2525 & NJ & 3001 & 0.03 & -74.61958 & 39.2398 & NJ \\
\hline 2858 & 1.73 & -74.57765 & 39.27221 & NJ & 2930 & 0.16 & -74.60641 & 39.25239 & NJ & 3002 & -0.05 & -74.61996 & 39.23947 & NJ \\
\hline 2859 & 1.69 & -74.57814 & 39.27197 & NJ & 2931 & 0.58 & -74.60626 & 39.25216 & NJ & 3003 & 0.04 & .74 .62022 & 39.23904 & NJ \\
\hline 2860 & 1.67 & -74.57863 & 39.27173 & NJ & 2932 & 0.22 & -74.60674 & 39.25202 & NJ & 3004 & -0.01 & -74.62062 & 39.23871 & NJ \\
\hline 2861 & 1.45 & -74.5806 & 39.27073 & NJ & 2933 & 0.59 & -74.60667 & 39.25183 & NJ & 3005 & -0.01 & .74 .62097 & 39.23835 & $\mathrm{NJ}$ \\
\hline 2862 & 1.45 & -74.58107 & 39.27045 & $\mathrm{NJ}$ & 2934 & 0.27 & -74.6071 & 39.25166 & NJ & 3006 & -0.03 & -74.62131 & 39.23799 & NJ \\
\hline 2863 & 1.53 & -74.58153 & 39.27017 & NJ & 2935 & 0.59 & -74.60707 & 39.2515 & NJ & 3007 & -0.02 & -74.62161 & 39.2376 & NJ \\
\hline 2864 & 1.51 & -74.58202 & 39.26989 & $\mathrm{NJ}$ & 2936 & 0.29 & .74 .60748 & 39.25132 & NJ & 3008 & -0.02 & -74.62195 & 39.23723 & NJ \\
\hline 2865 & 1.08 & -74.58315 & 39.26947 & NJ & 2937 & 0.58 & -74.60751 & 39.25118 & NJ & 3009 & -0.08 & .74 .62234 & 39.23689 & NJ \\
\hline 2866 & 0.97 & -74.58363 & 39.26921 & NJ & 2938 & 0.27 & .74 .60789 & 39.251 & NJ & 3010 & -0.19 & -74.62277 & 39.23659 & NJ \\
\hline 2867 & 2.39 & -74.58171 & 39.26909 & NJ & 2939 & 0.55 & -74.60789 & 39.25084 & NJ & 3011 & -0.17 & -74.62309 & 39.23621 & NJ \\
\hline 2868 & 0.91 & .74 .58418 & 39.26898 & NJ & 2940 & 0.25 & -74.60828 & 39.25067 & $\mathrm{NJ}$ & 3012 & -0.13 & .74 .62341 & 39.23582 & NJ \\
\hline 2869 . & 0.85 & .74 .58466 & 39.2687 & NJ & 2941 & 0.49 & -74.60828 & 39.2505 & NJ & 3013 & -0.14 & .74 .62376 & 39.23546 & NJ \\
\hline 2870 & 0.86 & -74.58511 & 39.26842 & NJ & 2942 & 0.25 & .74 .60866 & 39.25033 & NJ & 3014 & -0.1 & -74.62405 & 39.23506 & NJ \\
\hline 2871 & 0.85 & -74.58559 & 39.26814 & NJ & 2943 & 0.46 & .74 .60864 & 39.25015 & NJ & 3015 & -0.12 & -74.62442 & 39.23472 & NJ \\
\hline 2872 & 0.72 & -74.58606 & 39.26787 & NJ & 2944 & 0.25 & -74.60902 & 39.24998 & NJ & 3016 & -0.16 & -74.6248 & 39.23437 & NJ \\
\hline 2873 & 0.62 & -74.5865 & 39.26757 & NJ & 2945 & 0.41 & -74.60901 & 39.2498 & NJ & 3017 & -0.12 & .74 .62509 & 39.23398 & NJ \\
\hline 2874 & 0.56 & -74.58696 & 39.26728 & NJ & 2946 & 0.23 & -74.60942 & 39.24965 & NJ & 3018 & -0.19 & -74.62547 & 39.23364 & NJ \\
\hline 2875 & 0.55 & -74.5874 & 39.26698 & NJ & 2947 & 0.36 & -74.60938 & 39.24945 & NJ & 3019 & -0.22 & -74.62584 & 39.23329 & NJ \\
\hline 2876 & 0.56 & -74.58784 & 39.26668 & NJ & 2948 & 0.23 & -74.60979 & 39.24931 & NJ & 3020 & -0.27 & -74.62621 & 39.23294 & $\mathrm{NJ}$ \\
\hline 2877 & 0.53 & -74.5883 & 39.2664 & NJ & 2949 & 0.41 & -74.60977 & 39.24911 & NJ & 3021 & -0.27 & -74.62651 & 39.23255 & NJ \\
\hline 2878 & 0.45 & -74.58875 & 39.2661 & NJ & 2950 & 0.29 & -74.61012 & 39.24894 & $\mathrm{NJ}$ & 3022 & -0.27 & -74.62685 & 39.23217 & NJ \\
\hline 2879 & 0.37 & -74.58928 & 39.26587 & NJ & 2951 & 0.43 & -74.61009 & 39.24874 & NJ & 3023 & -0.3 & -74.6272 & 39.23181 & NJ \\
\hline 2880 & 0.3 & -74.58971 & 39.26555 & NJ & 2952 & 0.32 & -74.61047 & 39.24857 & NJ & 3024 & -0.21 & -74.62744 & 39.23138 & NJ \\
\hline
\end{tabular}




\begin{tabular}{|c|c|c|c|c|c|c|c|c|c|c|c|c|c|c|}
\hline TR\# & RATE & LONG & LAT & ST & I TR\# & RATE & LONG & LAT & ST & TR\# & RATE & LONG & LAT & ST \\
\hline 3025 & -0.21 & -74.62776 & 39.231 & $\mathrm{NJ}$ & 3097 & 2.12 & -74.64149 & 39.21361 & $\mathrm{NJ}$ & 3169 & -1.14 & -74.67004 & 39.18133 & $\overline{\mathrm{NJ}}$ \\
\hline 3026 & -0.23 & -74.62811 & 39.23064 & NJ & 3098 & 1.8 & -74.64165 & 539.21313 & NJ & 3170 & -1.17 & -74.67036 & 39.18095 & NJ \\
\hline 3027 & -0.27 & -74.62846 & 39.23028 & NJ & 3099 & 1.6 & -74.64182 & 39.21269 & NJ & 3171 & -1.19 & -74.6707 & 39.18058 & NJ \\
\hline 3028 & -0.28 & -74.62878 & 39.22991 & NJ & 3100 & 1.44 & -74.642 & 39.21224 & $\mathrm{NJ}$ & 3172 & -1.23 & -74.67104 & 39.18022 & $\mathrm{NJ}$ \\
\hline 3029 & -0.29 & -74.629112 & 39.22953 & $\mathrm{NJ}$ & 3101 & 1.14 & -74.6422 & 39.21179 & NJ & 3173 & -1.24 & -74.67139 & 39.17986 & NJ \\
\hline 3030 & -0.28 & -74.62943 & 39.22914 & NJ & 3102 & 0.85 & -74.64236 & 539.21135 & NJ & 3174 & -1.22 & -74.67168 & 39.17946 & $\mathrm{NJ}$ \\
\hline 3031 & 0.13 & -74.62944 & 39.22901 & NJ & 3103 & 0.49 & -74.64262 & 239.21093 & NJ & 3175 & -1.24 & -74.67215 & 39.17917 & NJ \\
\hline 3032 & -0.31 & -74.62978 & 39.22878 & $\mathrm{NJ}$ & 3104 & 0.01 & -74.6429 & 39.21054 & NJ & 3176 & -1.25 & -74.67245 & 39.17878 & $\mathrm{NJ}$ \\
\hline 3033 & 0.06 & -74.62978 & 39.22865 & NJ & 3105 & -0.55 & -74.64317 & 39.21014 & NJ & 3177 & -1.28 & -74.67276 & 39.1784 & $\mathrm{NJ}$ \\
\hline 3034 & -0.37 & -74.63013 & 39.22843 & $\mathrm{NJ}$ & 3106 & -0.61 & -74.64348 & 39.20975 & $\mathrm{NJ}$ & 3178 & -1.3 & -74.67307 & 39.17802 & NJ \\
\hline 3035 & 0.04 & -74.63011 & 39.22828 & NJ & 3107 & -0.29 & -74.64386 & 59.20941 & NJ & 3179 & -1.28 & -74.67337 & 39.17763 & $\mathrm{NJ}$ \\
\hline 3036 & -0.4 & -74.63046 & 39.22806 & NJ & 3108 & -0.02 & .74 .64427 & 39.20908 & NJ & 3180 & -1.28 & -74.67365 & 39.17724 & $\mathrm{NJ}$ \\
\hline 3037 & 0.04 & -74.63045 & 39.22791 & NJ & 3109 & 0.28 & -74.64471 & 39.20877 & NJ & 3181 & -1.27 & -74.67392 & 39.17684 & $\mathrm{NJ}$ \\
\hline 3038 & -0.4 & .74 .63078 & 39.22767 & $\mathrm{NJ}$ & 3110 & -1.04 & -74.64946 & 539.20257 & $\mathrm{NJ}$ & 3182 & -1.27 & -74.67424 & 39.17647 & $\mathrm{NJ}$ \\
\hline 3039 & 0.03 & -74.63075 & 39.22752 & NJ & 3111 & -0.82 & -74.64954 & 39.20206 & NJ & 3183 & -1.26 & -74.67456 & 39.17609 & $\mathrm{NJ}$ \\
\hline 3040 & -0.43 & -74.63112 & 39.2273 & NJ & 3112 & -0.57 & -74.64964 & 39.20158 & NJ & 3184 & -1.24 & -74.6749 & 39.17572 & NJ \\
\hline 3041 & 0.02 & -74.63104 & 39.22714 & NJ & 3113 & -0.4 & -74.64983 & 39.20113 & NJ & 3185 & -1.24 & -74.6752 & 39.17534 & NJ \\
\hline 3042 & -0.42 & -74.63142 & 39.22691 & NJ & 3114 & -0.36 & -74.65009 & 39.20072 & NJ & 3186 & -1.26 & -74.67551 & 39.17496 & $\mathrm{NJ}$ \\
\hline 3043 & -0.05 & -74.63139 & 39.22678 & NJ & 3115 & -0.43 & -74.65039 & 39.20034 & NJ & 3187 & -1.16 & -74.6758 & 39.17456 & $\mathrm{NJ}$ \\
\hline 3044 & -0.39 & -74.63165 & 39.22647 & NJ & 3116 & -0.54 & -74.65074 & 39.19998 & NJ & 3188 & -1.13 & -74.67612 & 39.17418 & $\mathrm{NJ}$ \\
\hline 3045 & -0.04 & -74.63171 & 39.22641 & NJ & 3117 & -0.69 & -74.65112 & 39.19964 & NJ & 3189 & -1.12 & -74.67638 & 39.17379 & $\mathrm{NJ}$ \\
\hline 3046 & -0.35 & .74 .63199 & 39.2261 & NJ & 3118 & -0.75 & -74.65154 & 39.19931 & NJ & 3190 & -1.08 & -74.6767 & 39.17341 & $\mathrm{NJ}$ \\
\hline 3047 & -0.02 & .74 .63203 & 39.22603 & NJ & 3119 & -0.71 & -74.65186 & 539.19893 & NJ & 3191 & -1.06 & -74.67699 & 39.17302 & $\mathrm{NJ}$ \\
\hline 3048 & -0.32 & -74.63229 & 39.22571 & NJ & 3120 & -0.67 & -74.65218 & 39.19856 & NJ & 3192 & -1.07 & .74 .67729 & 39.17263 & $\mathrm{NJ}$ \\
\hline 3049 & 0.01 & -74.63235 & 39.22565 & NJ & 3121 & -0.52 & .74 .65248 & 39.19818 & NJ & 3193 & -1.09 & .74 .67758 & 39.17224 & $\mathrm{NJ}$ \\
\hline 3050 & -0.27 & -74.63261 & 39.22533 & NJ & 3122 & -0.52 & -74.65289 & 39.19785 & NJ & 3194 & -1.02 & -74.67787 & 39.17185 & $\mathrm{NJ}$ \\
\hline 3051 & 0.05 & -74.63266 & 39.22527 & NJ & 3123 & -0.53 & -74.65327 & 39.19751 & NJ & 3195 & -1.03 & -74.67815 & 39.17145 & $\mathrm{NJ}$ \\
\hline 3052 & -0.27 & -74.63292 & 39.22493 & NJ & 3124 & -0.56 & -74.6537 & 39.19719 & NJ & 3196 & -1.01 & .74 .67845 & 39.17107 & NJ \\
\hline 3053 & NA & -74.63297 & 39.22488 & NJ & 3125 & -0.57 & -74.6541 & 39.19685 & NJ & 3197 & -0.98 & -74.67873 & 39.17067 & $\mathrm{NJ}$ \\
\hline 3054 & -0.23 & -74.63324 & 39.22455 & NJ & 3126 & -0.58 & -74.6545 & 39.19652 & NJ & 3198 & -0.97 & -74.67903 & 39.17028 & $\mathrm{NJ}$ \\
\hline 3055 & -0.47 & .74 .63329 & 39.2245 & NJ & 3127 & -0.56 & -74.65488 & 39.19618 & NJ & 3199 & -0.89 & .74 .67932 & 39.16989 & NJ \\
\hline 3056 & -0.21 & -74.63354 & 39.22417 & NJ & 3128 & -0.58 & -74.65527 & 39.19584 & $\mathrm{NJ}$ & 3200 & -0.89 & -74.67961 & 39.1695 & NJ \\
\hline 3057 & -0.43 & -74.63358 & 39.22412 & NJ & 3129 & -0.59 & -74.65562 & 39.19549 & NJ & 3201 & -0.85 & -74.67992 & 39.16912 & NJ \\
\hline 3058 & -0.23 & .74 .63387 & 39.22378 & NJ & 3130 & -0.57 & .74 .65598 & 39.19513 & NJ & 3202 & -0.83 & -74.68019 & 39.16872 & $\mathrm{NJ}$ \\
\hline 3059 & -0.46 & -74.6339 & 39.22374 & NJ & 3131 & -0.62 & -74.6564 & 39.1948 & NJ & 3203 & -0.82 & -74.68047 & 39.16832 & $\mathrm{NJ}$ \\
\hline 3060 & -0.28 & -74.63417 & 39.22339 & NJ & 3132 & -0.75 & -74.65688 & 39.1945 & NJ & 3204 & -0.82 & -74.68076 & 39.16793 & NJ \\
\hline 3061 & -0.43 & -74.63416 & 39.22333 & NJ & 3133 & -0.83 & -74.65729 & 39.19418 & NJ & 3205 & -0.83 & -74.68102 & 39.16752 & NJ \\
\hline 3062 & -0.43 & -74.63451 & 39.22302 & NJ & 3134 & -0.96 & -74.65771 & 139.19387 & NJ & 3206 & -0.8 & -74.6813 & 39.16713 & NJ \\
\hline 3063 & -0.43 & -74.63446 & 39.22295 & NJ & 3135 & -0.96 & -74.6581 & 39.19352 & NJ & 3207 & -0.8 & -74.68159 & 39.16674 & NJ \\
\hline 3064 & -0.45 & -74.6348 & 39.22263 & NJ & 3136 & -1.03 & -74.65855 & 539.19322 & NJ & 3208 & -0.77 & -74.68184 & 39.16633 & $\mathrm{NJ}$ \\
\hline 3065 & -0.45 & -74.63477 & 39.22257 & NJ & 3137 & -0.97 & -74.65887 & 739.19284 & $\mathrm{NJ}$ & 3209 & -0.8 & -74.68214 & 39.16595 & NJ \\
\hline 3066 & -0.46 & -74.63512 & 39.22224 & $\mathrm{NJ}$ & 3138 & -1.09 & -74.65932 & 239.19253 & NJ & 3210 & -0.78 & -74.68242 & 39.16555 & NJ \\
\hline 3067 & -0.45 & -74.63506 & 39.22217 & NJ & 3139 & -1.08 & -74.6597 & 39.19219 & NJ & 3211 & -0.76 & -74.68269 & 39.16514 & NJ \\
\hline 3068 & -0.53 & -74.63544 & 39.22186 & NJ & 3140 & -1.04 & -74.66005 & 539.19183 & NJ & 3212 & -0.71 & -74.68295 & 39.16474 & $\mathrm{NJ}$ \\
\hline 3069 & -0.53 & -74.63535 & 39.22178 & $\mathrm{NJ}$ & 3141 & -1.04 & -74.66042 & 239.19148 & $\mathrm{NJ}$ & 3213 & -0.68 & -74.68323 & 39.16434 & NJ \\
\hline 3070 & -0.57 & -74.63574 & 39.22147 & NJ & 3142 & -1.11 & -74.66081 & 139.19115 & NJ & 3214 & -0.64 & -74.68349 & 39.16393 & $\mathrm{NJ}$ \\
\hline 3071 & -0.57 & -74.63564 & 39.2214 & NJ & 3143 & -1.12 & -74.66116 & 539.19078 & NJ & 3215 & -0.61 & -74.68372 & 39.1635 & NJ \\
\hline 3072 & -0.51 & -74.63605 & 39.22108 & NJ & 3144 & -1.13 & -74.66153 & 339.19043 & $\mathrm{NJ}$ & 3216 & -0.54 & -74.68394 & 39.16308 & $\mathrm{NJ}$ \\
\hline 3073 & -0.51 & -74.63594 & 39.22101 & NJ & 3145 & -1.16 & -74.66191 & 139.19009 & NJ & 3217 & -0.42 & -74.68416 & 39.16265 & NJ \\
\hline 3074 & -0.56 & -74.63634 & 39.22067 & NJ & 3146 & -1.11 & -74.66222 & 39.1897 & NJ & 3218 & -0.39 & -74.68439 & 39.16223 & $\mathrm{NJ}$ \\
\hline 3075 & -0.55 & -74.63626 & 39.22064 & NJ & 3147 & -0.56 & -74.662 & 39.18905 & NJ & 3219 & -0.27 & -74.6846 & 39.1618 & NJ \\
\hline 3076 & -0.52 & -74.63664 & 39.22029 & NJ & 3148 & -1.12 & -74.66289 & 39.18897 & NJ & 3220 & -0.17 & -74.68477 & 39.16135 & NJ \\
\hline 3077 & -0.52 & -74.6366 & 39.22026 & NJ & 3149 & -1.18 & -74.66328 & 339.18864 & NJ & 3221 & -0.04 & -74.68478 & 39.16081 & NJ \\
\hline 3078 & -0.44 & -74.6369 & 39.21989 & NJ & 3150 & -1.2 & -74.66367 & 39.18829 & NJ & 3222 & -0.01 & .74 .6851 & 39.16043 & NJ \\
\hline 3079 & -0.44 & -74.63695 & 39.21989 & NJ & 3151 & -1.22 & .74 .664 & 39.18793 & NJ & 3223 & -0.14 & -74.68542 & 39.16006 & NJ \\
\hline 3080 & -0.42 & -74.63721 & 39.2195 & $\mathrm{NJ}$ & 3152 & -1.24 & -74.66435 & 539.18757 & $\mathrm{NJ}$ & 3224 & -0.17 & -74.68568 & 39.15966 & $\mathrm{NJ}$ \\
\hline 3081 & -0.42 & -74.63724 & 39.2195 & NJ & 3153 & -1.25 & .74 .66476 & 539.18724 & NJ & 3225 & -0.15 & -74.686 & 39.15928 & NJ \\
\hline 3082 & -0.25 & -74.63747 & 39.21909 & NJ & 3154 & -1.27 & -74.66512 & 39.18689 & $\mathrm{NJ}$ & 3226 & -0.16 & -74.68633 & 39.15891 & NJ \\
\hline 3083 & -0.25 & -74.6375 & 39.21907 & NJ & 3155 & -1.24 & -74.66547 & 39.18652 & NJ & 3227 & -0.16 & -74.68661 & 39.15851 & NJ \\
\hline 3084 & -0.33 & -74.63776 & 39.2187 & NJ & 3156 & -1.25 & -74.66579 & 39.18615 & NJ & 3228 & -0.13 & .74 .68684 & 39.15809 & NJ \\
\hline 3085 & -0.4 & -74.63808 & 39.21832 & NJ & 3157 & -1.16 & -74.66608 & 39.18576 & $\mathrm{NJ}$ & 3229 & -0.13 & -74.68713 & 39.1577 & NJ \\
\hline 3086 & -0.44 & .74 .6384 & 39.21795 & NJ & 3158 & -1.08 & -74.66637 & 39.18537 & NJ & 3230 & -0.23 & -74.68748 & 39.15734 & $\mathrm{NJ}$ \\
\hline 3087 & -0.52 & -74.6387 & 39.21757 & NJ & 3159 & -1.1 & -74.66673 & 39.18502 & NJ & 3231 & -0.36 & -74.68784 & 39.15698 & NJ \\
\hline 3088 & -0.56 & -74.63899 & 39.21718 & NJ & 3160 & -1.16 & -74.66716 & 39.1847 & NJ & 3232 & -0.41 & -74.68816 & 39.15661 & NJ \\
\hline 3089 & -0.54 & -74.63933 & 39.21681 & NJ & 3161 & -1.22 & .74 .66756 & 39.18436 & NJ & 3233 & -0.4 & -74.68846 & 39.15622 & NJ \\
\hline 3090 & -0.38 & -74.63963 & 39.21643 & NJ & 3162 & -1.21 & -74.66788 & 39.18398 & NJ & 3234 & -0.43 & -74.68874 & 39.15582 & NJ \\
\hline 3091 & -0.05 & -74.63989 & 39.21602 & NJ & 3163 & -1.1 & -74.66818 & 39.18361 & NJ & 3235 & -0.35 & -74.68901 & 39.15542 & NJ \\
\hline 3092 & -0.37 & -74.64018 & 39.21563 & NJ & 3164 & -1 & -74.66853 & 39.18324 & NJ & 3236 & -0.27 & -74.68925 & 39.15501 & NJ \\
\hline 3093 & -0.34 & -74.64047 & 39.21524 & NJ & 3165 & -0.95 & .74 .66885 & 39.18288 & NJ & 3237 & -0.23 & -74.68948 & 39.15459 & NJ \\
\hline 3094 & -0.36 & .74 .64079 & 39.21486 & NJ & 3166 & -0.94 & -74.6692 & 39.18251 & NJ & 3238 & -0.26 & -74.68982 & 39.15422 & NJ \\
\hline 3095 & -0.36 & -74.64107 & 39.21446 & NJ & 3167 & -0.99 & -74.66943 & 39.18209 & NJ & 3239 & -0.29 & -74.69016 & 39.15385 & NJ \\
\hline 3096 & -0.31 & .74 .6413 & 39.21404 & NJ & 3168 & -1.09 & -74.66975 & 39.18172 & NJ & 3240 & -0.3 & -74.69044 & 39.15346 & NJ \\
\hline
\end{tabular}




\begin{tabular}{|c|c|c|c|c|c|c|c|c|c|c|c|c|c|c|}
\hline TR\# & RATE & LONG & LAT & ST & TR\# & RATE & LONG & LAT & ST & TR\# & RATE & LONG & LAT & ST \\
\hline 3241 & -0.35 & -74.69075 & 539.15308 & NJ & 3313 & 1.62 & -74.70528 & 39.10455 & NJ & 3385 & 1.59 & -74.71704 & 39.09366 & $\mathrm{NJ}$ \\
\hline 3242 & -0.33 & -74.69102 & 239.15268 & NJ & 3314 & 1.23 & -74.7054 & 39.10435 & NJ & 3386 & 1.5 & -74.71745 & 539.09334 & NJ \\
\hline 3243 & -0.22 & -74.6913 & 39.15228 & NJ & 3315 & 1.23 & -74.7054 & 39.10435 & NJ & 3387 & 1.5 & -74.71745 & 539.09334 & $\mathrm{NJ}$ \\
\hline 3244 & -0.17 & -74.69168 & 8 39.15193 & NJ & 3316 & 1.6 & -74.70558 & 39.10408 & NJ & 3388 & 1.56 & -74.71785 & 539.09301 & $\mathrm{NJ}$ \\
\hline 3245 & -0.2 & -74.69203 & 339.15158 & NJ & 3317 & 1.6 & -74.70558 & 39.10408 & NJ & 3389 & 1.56 & -74.71785 & 539.09301 & $\mathrm{NJ}$ \\
\hline 3246 & -0.23 & -74.69237 & 739.15121 & NJ & 3318 & 1.39 & -74.70567 & 39.10394 & $\mathrm{NJ}$ & 3390 & 1.57 & -74.71825 & 539.09267 & $\mathrm{NJ}$ \\
\hline 3247 & -0.23 & -74.69266 & 639.15082 & NJ & 3319 & 1.39 & -74.70567 & 39.10394 & NJ & 3391 & 1.57 & -74.71825 & 539.09267 & $\mathrm{NJ}$ \\
\hline 3248 & -0.23 & -74.69336 & 639.1501 & NJ & 3320 & 1.63 & -74.7059 & 39.10362 & NJ & 3392 & 1.56 & -74.71861 & 39.09233 & $\mathrm{NJ}$ \\
\hline 3249 & 1.05 & .74 .692 & 39.14993 & NJ & 3321 & 1.63 & -74.7059 & 39.10362 & NJ & 3393 & 1.56 & -74.71861 & 39.09233 & NJ \\
\hline 3250 & -0.34 & -74.69373 & 339.14975 & NJ & 3322 & 1.3 & -74.70596 & 539.10355 & $\mathrm{NJ}$ & 3394 & 1.48 & -74.71906 & 539.09201 & $\mathrm{NJ}$ \\
\hline 3251 & -0.4 & -74.69408 & 839.14939 & NJ & 3323 & 1.3 & -74.70596 & 539.10355 & $\mathrm{NJ}$ & 3395 & 1.48 & -74.71906 & 539.09201 & $\mathrm{NJ}$ \\
\hline 3252 & -0.34 & -74.69441 & 139.14902 & NJ & 3324 & 1.28 & -74.70625 & 39.10316 & $\mathrm{NJ}$ & 3396 & 1.51 & -74.71935 & 539.09163 & $\mathrm{NJ}$ \\
\hline 3253 & -0.36 & -74.69472 & 239.14864 & NJ & 3325 & 1.28 & -74.70625 & 39.10316 & NJ & 3397 & 1.51 & -74.71935 & 59.09163 & $\mathrm{NJ}$ \\
\hline 3254 & -0.33 & -74.69502 & 239.14825 & NJ & 3326 & 1.77 & -74.70625 & 39.10315 & NJ & 3398 & 1.51 & -74.71973 & 39.09128 & $\mathrm{NJ}$ \\
\hline 3255 & -0.3 & -74.69528 & 839.14785 & NJ & 3327 & 1.77 & -74.70625 & 39.10315 & NJ & 3399 & 1.51 & -74.71973 & 39.09128 & $\mathrm{NJ}$ \\
\hline 3256 & -0.29 & -74.69553 & 339.14744 & NJ & 3328 & 1.36 & -74.70654 & 39.10277 & NJ & 3400 & 1.43 & -74.72012 & 39.09096 & NJ \\
\hline 3257 & -0.17 & -74.69582 & 239.14705 & NJ & 3329 & 1.36 & -74.70654 & 39.10277 & NJ & 3401 & 1.43 & -74.72012 & 29.09096 & $\mathrm{NJ}$ \\
\hline 3258 & -0.18 & -74.69614 & 39.14667 & NJ & 3330 & 1.95 & -74.70659 & 39.10269 & $\mathrm{NJ}$ & 3402 & 1.36 & -74.72058 & 39.09065 & $\mathrm{NJ}$ \\
\hline 3259 & -0.21 & -74.69644 & 439.14628 & NJ & 3331 & 1.95 & -74.70659 & 39.10269 & $\mathrm{NJ}$ & 3403 & 1.36 & -74.72058 & 39.09065 & $\mathrm{VJ}$ \\
\hline 3260 & -0.28 & -74.69678 & 839.14592 & NJ & 3332 & 1.4 & -74.70683 & 39.10238 & $\mathrm{NJ}$ & 3404 & 1.24 & -74.72105 & 39.09036 & NJ \\
\hline 3261 & -0.3 & -74.69708 & 8 39.14554 & NJ & 3333 & 1.4 & -74.70683 & 39.10238 & $\mathrm{NJ}$ & 3405 & 1.24 & -74.72105 & 59.09036 & $\mathrm{NJ}$ \\
\hline 3262 & -0.24 & -74.69734 & 439.14513 & NJ & 3334 & 1.43 & -74.70712 & 39.10198 & NJ & 3406 & 1.1 & -74.72147 & 39.09004 & NJ \\
\hline 3263 & -0.17 & -74.69754 & 439.14469 & NJ & 3335 & 1.43 & -74.70712 & 39.10198 & $\mathrm{NJ}$ & 3407 & 1.1 & -74.72147 & 39.09004 & $\mathrm{NJ}$ \\
\hline 3264 & -0.12 & -74.69778 & 8 39.14428 & NJ & 3336 & 1.44 & -74.70744 & 39.10161 & $\mathrm{NJ}$ & 3408 & 0.94 & -74.72185 & 39.0897 & $\mathrm{NJ}$ \\
\hline 3265 & 0.58 & -74.69743 & 39.14355 & $\mathrm{NJ}$ & 3337 & 1.44 & -74.70744 & 39.10161 & $\mathrm{NJ}$ & 3409 & 0.94 & -74.72185 & 39.0897 & NJ \\
\hline 3266 & 0.06 & -74.69826 & 639.14344 & NJ & 3338 & 1.27 & -74.70779 & 39.10125 & $\mathrm{NJ}$ & 3410 & 0.85 & -74.72224 & 39.08937 & $\mathrm{NJ}$ \\
\hline 3267 & 0.14 & -74.6985 & 39.14302 & NJ & 3339 & 1.27 & -74.70779 & 39.10125 & $\mathrm{NJ}$ & 3411 & 0.85 & -74.72224 & 39.08937 & $\mathrm{NJ}$ \\
\hline 3268 & 0.06 & -74.69882 & 239.14265 & NJ & 3340 & 1.3 & -74.70816 & 39.1009 & NJ & 3412 & 0.84 & -74.72266 & 39.08903 & $\mathrm{NJ}$ \\
\hline 3269 & -0.08 & -74.69917 & 739.14229 & NJ & 3341 & 1.3 & -74.70816 & 39.1009 & $\mathrm{NJ}$ & 3413 & 0.84 & -74.72266 & 39.08903 & $\mathrm{NJ}$ \\
\hline 3270 & -0.16 & -74.69949 & 39.14191 & $\mathrm{NJ}$ & 3342 & 1.28 & -74.7085 & 39.10054 & $\mathrm{NJ}$ & 3414 & 0.82 & -74.72304 & 39.0887 & $\mathrm{NJ}$ \\
\hline 3271 & -0.13 & -74.69972 & 239.14149 & NJ & 3343 & 1.28 & -74.7085 & 39.10054 & $\mathrm{NJ}$ & 3415 & 0.82 & -74.72304 & 39.0887 & $\mathrm{NJ}$ \\
\hline 3272 & -5.46 & -74.7103 & 39.11122 & NJ & 3344 & 1.16 & -74.70885 & 39.10018 & $\mathrm{NJ}$ & 3416 & 0.79 & -74.72342 & 39.08835 & $\mathrm{NJ}$ \\
\hline 3273 & $\mathrm{NA}$ & -74.7103 & 39.11122 & NJ & 3345 & 1.16 & -74.70885 & 39.10018 & $\mathrm{NJ}$ & 3417 & 0.79 & -74.72342 & 39.08835 & NJ \\
\hline 3274 & -4.44 & -74.70975 & 539.11085 & NJ & 3346 & 1.05 & -74.70924 & 39.09984 & $\mathrm{NJ}$ & 3418 & 0.7 & -74.72382 & 39.08802 & NJ \\
\hline 3275 & -4.44 & -74.70975 & 539.11085 & NJ & 3347 & 1.05 & -74.70924 & 39.09984 & $\mathrm{NJ}$ & 3419 & 0.7 & -74.72382 & 39.08802 & $\mathrm{NJ}$ \\
\hline 3276 & -3.82 & -74.70924 & 39.11048 & NJ & 3348 & 1.17 & -74.70959 & 39.09949 & $\mathrm{NJ}$ & 3420 & 0.6 & -74.7242 & 39.08768 & $\mathrm{NJ}$ \\
\hline 3277 & -3.82 & -74.70924 & 439.11048 & $\mathrm{NJ}$ & 3349 & 1.17 & -74.70959 & 39.09949 & $\mathrm{NJ}$ & 3421 & 0.6 & -74.7242 & 39.08768 & NJ \\
\hline 3278 & -3.29 & -74.70892 & 239.11007 & NJ & 3350 & 1.26 & -74.71001 & 39.09916 & $\mathrm{NJ}$ & 3422 & 0.59 & -74.72459 & 39.08735 & NJ \\
\hline 3279 & -3.29 & -74.70892 & 239.11007 & NJ & 3351 & 1.26 & -74.71001 & 39.09916 & NJ & 3423 & 0.59 & -74.72459 & 39.08735 & $\mathrm{NJ}$ \\
\hline 3280 & -2.58 & -74.70828 & 8 39.10972 & NJ & 3352 & 1.3 & -74.71042 & 39.09883 & $\mathrm{NJ}$ & 3424 & 0.5 & -74.72496 & 39.087 & $\mathrm{NJ}$ \\
\hline 3281 & -2.58 & -74.70828 & 8 39.10972 & NJ & 3353 & 1.3 & -74.71042 & 39.09883 & $\mathrm{NJ}$ & 3425 & 0.5 & -74.72496 & 59.087 & $\mathrm{NJ}$ \\
\hline 3282 & -2.3 & -74.70798 & 8 39.10932 & NJ & 3354 & 1.35 & -74.71085 & 39.09852 & NJ & 3426 & 0.45 & -74.72536 & 39.08666 & NJ \\
\hline 3283 & -2.3 & -74.70798 & 8 39.10932 & NJ & 3355 & 1.35 & -74.71085 & 39.09852 & $\mathrm{NJ}$ & 3427 & 0.45 & -74.72536 & 39.08666 & NJ \\
\hline 3284 & -1.8 & -74.70757 & 39.10893 & NJ & 3356 & 1.37 & -74.71123 & 39.09818 & $\mathrm{NJ}$ & 3428 & 0.45 & -74.72572 & 39.08632 & $\mathrm{NJ}$ \\
\hline 3285 & -1.8 & -74.70757 & 39.10893 & NJ & 3357 & 1.37 & -74.71123 & 39.09818 & $\mathrm{NJ}$ & 3429 & 0.45 & -74.72572 & 39.08632 & $\mathrm{NJ}$ \\
\hline 3286 & -1.33 & -74.70708 & 3 39.10856 & $\mathrm{NJ}$ & 3358 & 1.39 & -74.71165 & 39.09786 & NJ & 3430 & 0.4 & -74.7261 & 39.08598 & NJ \\
\hline 3287 & -1.33 & -74.70708 & 8 39.10856 & $\mathrm{NJ}$ & 3359 & 1.39 & -74.71165 & 39.09786 & $\mathrm{NJ}$ & 3431 & 0.4 & -74.7261 & 39.08598 & NJ \\
\hline 3288 & -0.96 & -74.70657 & 39.10818 & NJ & 3360 & 1.41 & -74.71205 & 39.09753 & NJ & 3432 & 0.32 & -74.72649 & 39.08564 & NJ \\
\hline 3289 & -0.96 & -74.70657 & 39.10818 & NJ & 3361 & 1.41 & -74.71205 & 39.09753 & NJ & 3433 & 0.32 & -74.72649 & 39.08564 & NJ \\
\hline 3290 & 1 & -74.70564 & 439.10788 & NJ & 3362 & 1.4 & -74.71246 & 39.09721 & NJ & 3434 & 0.26 & -74.72688 & 39.0853 & $\mathrm{NJ}$ \\
\hline 3291 & 1 & -74.70564 & 49.10788 & $\mathrm{NJ}$ & 3363 & 1.4 & -74.71246 & 39.09721 & $\mathrm{NJ}$ & 3435 & 0.26 & -74.72688 & 39.0853 & $\mathrm{NJ}$ \\
\hline 3292 & 2.07 & -74.70486 & 539.10756 & $\mathrm{NJ}$ & 3364 & 1.47 & -74.71288 & 39.09688 & $\mathrm{NJ}$ & 3436 & 0.19 & -74.72726 & 39.08496 & $\mathrm{NJ}$ \\
\hline 3293 & 2.07 & -74.70486 & 539.10756 & $\mathrm{NJ}$ & 3365 & 1.47 & -74.71288 & 39.09688 & $\mathrm{NJ}$ & 3437 & 0.19 & -74.72726 & 39.08496 & NJ \\
\hline 3294 & 0.37 & -74.70531 & 139.10725 & NJ & 3366 & 1.53 & -74.71329 & 39.09655 & $\mathrm{NJ}$ & 3438 & 0.04 & -74.72765 & 39.08462 & $\mathrm{NJ}$ \\
\hline 3295 & 0.37 & -74.70531 & 139.10725 & $\mathrm{NJ}$ & 3367 & 1.53 & -74.71329 & 39.09655 & NJ & 3439 & 0.04 & -74.72765 & 39.08462 & $\mathrm{NJ}$ \\
\hline 3296 & 0.55 & -74.70518 & 839.10704 & $\mathrm{NJ}$ & 3368 & 1.52 & -74.7137 & 39.09623 & $\mathrm{NJ}$ & 3440 & -0.03 & -74.72801 & 39.08427 & $\mathrm{NJ}$ \\
\hline 3297 & 0.55 & -74.70518 & 3 39.10704 & NJ & 3369 & 1.52 & -74.7137 & 39.09623 & $\mathrm{NJ}$ & 3441 & -0.03 & -74.72801 & 39.08427 & NJ \\
\hline 3298 & 0.47 & -74.70509 & 39.10681 & $\mathrm{NJ}$ & 3370 & 1.49 & -74.71411 & 39.09591 & $\mathrm{NJ}$ & 3442 & -0.08 & -74.72836 & 39.08391 & $\mathrm{NJ}$ \\
\hline 3299 & 0.47 & -74.70509 & 39.10681 & $\mathrm{NJ}$ & 3371 & 1.49 & -74.71411 & 39.09591 & $\mathrm{NJ}$ & 3443 & -0.08 & -74.72836 & 39.08391 & NJ \\
\hline 3300 & 0.93 & -74.70494 & 439.10636 & $\mathrm{NJ}$ & 3372 & 1.45 & -74.71452 & 39.09558 & $\mathrm{NJ}$ & 3444 & -0.11 & -74.72873 & 39.08356 & NJ \\
\hline 3301 & 0.93 & -74.70494 & 439.10636 & $\mathrm{NJ}$ & 3373 & 1.45 & -74.71452 & 39.09558 & $\mathrm{NJ}$ & 3445 & -0.11 & -74.72873 & 39.08356 & NJ \\
\hline 3302 & 1.25 & -74.70493 & 39.10591 & $\mathrm{NJ}$ & 3374 & 1.47 & -74.71494 & 39.09526 & $\mathrm{NJ}$ & 3446 & -0.19 & -74.7291 & 39.08321 & NJ \\
\hline 3303 & 1.25 & .74 .70493 & 39.10591 & NJ & 3375 & 1.47 & -74.71494 & 39.09526 & NJ & 3447 & -0.19 & -74.7291 & 39.08321 & NJ \\
\hline 3304 & 1.41 & -74.70502 & 239.10546 & $\mathrm{NJ}$ & 3376 & 1.48 & -74.71536 & 39.09495 & NJ & 3448 & -0.25 & -74.72945 & 39.08285 & $\mathrm{NJ}$ \\
\hline 3305 & 1.41 & -74.70502 & 39.10546 & $\mathrm{NJ}$ & 3377 & 1.48 & -74.71536 & 39.09495 & $\mathrm{NJ}$ & 3449 & -0.25 & -74.72945 & 39.08285 & $\mathrm{NJ}$ \\
\hline 3306 & 0.83 & -74.70505 & 539.10525 & NJ & 3378 & 1.57 & -74.71577 & 39.09462 & $\mathrm{NJ}$ & 3450 & -0.31 & -74.72981 & 39.08251 & NJ \\
\hline 3307 & 0.83 & -74.70505 & 539.10525 & NJ & 3379 & 1.57 & -74.71577 & 39.09462 & $\mathrm{NJ}$ & 3451 & -0.31 & -74.72981 & 39.08251 & NJ \\
\hline 3308 & 1.6 & -74.70508 & 39.10501 & NJ & 3380 & 1.52 & -74.71622 & 39.09431 & $\mathrm{NJ}$ & 3452 & -0.32 & -74.73019 & 39.08216 & $\mathrm{NJ}$ \\
\hline 3309 & 1.6 & -74.70508 & 39.10501 & $\mathrm{NJ}$ & 3381 & 1.52 & -74.71622 & 39.09431 & $\mathrm{NJ}$ & 3453 & -0.32 & -74.73019 & 39.08216 & $\mathrm{NJ}$ \\
\hline 3310 & 1.04 & -74.70514 & 39.10476 & NJ & 3382 & 1.58 & -74.71663 & 39.09399 & $\mathrm{NJ}$ & 3454 & -0.39 & -74.73058 & 39.08182 & NJ \\
\hline 3311 & 1.04 & -74.70514 & 439.10476 & $\mathrm{NJ}$ & 3383 & 1.58 & -74.71663 & 39.09399 & $\mathrm{NJ}$ & 3455 & -0.39 & -74.73058 & 39.08182 & $\mathrm{NJ}$ \\
\hline 3312 & 1.62 & -74.70528 & 39.10455 & NJ & 3384 & 1.59 & .74 .71704 & 39.09366 & $\mathrm{NJ}$ & 3456 & -0.43 & -74.73093 & 39.08147 & NJ \\
\hline
\end{tabular}




\begin{tabular}{|c|c|c|c|c|c|c|c|c|c|c|c|c|c|c|}
\hline TR\# & RATE & LONG & LAT & ST & TR\# & RATE & LONG & LAT & ST & TR\# & RATE & LONG & LAT & ST \\
\hline 3457 & -0.43 & .74 .73093 & 39.08147 & NJ & 3529 & -1.17 & -74.74327 & 39.06841 & $\mathrm{NJ}$ & 3601 & 0.49 & -74.75363 & 39.05426 & $\mathrm{NJ}$ \\
\hline 3458 & -0.5 & -74.73134 & 39.08114 & NJ & 3530 & -0.78 & -74.74355 & 39.06801 & NJ & 3602 & 0.59 & -74.75398 & 39.0539 & NJ \\
\hline 3459 & -0.5 & -74.73134 & 39.08114 & $\mathrm{NJ}$ & 3531 & -1.03 & .74 .74355 & 39.06801 & $\mathrm{NJ}$ & 3603 & 0.44 & -74.75398 & 39.0539 & NJ \\
\hline 3460 & -0.52 & -74.73169 & 39.08078 & NJ & 3532 & -0.69 & -74.74382 & 39.06761 & $\mathrm{NJ}$ & 3604 & 0.61 & -74.75433 & 39.05354 & NJ \\
\hline 3461 & -0.52 & -74.73169 & 39.08078 & NJ & 3533 & -0.91 & -74.74382 & 39.06761 & NJ & 3605 & 0.37 & -74.75433 & 39.05354 & NJ \\
\hline 3462 & -0.5 & -74.73203 & 39.08042 & NJ & 3534 & -0.67 & -74.74409 & 39.06721 & NJ & 3606 & 0.61 & -74.75467 & 39.05318 & NJ \\
\hline 3463 & -0.5 & -74.73203 & 39.08042 & $\mathrm{NJ}$ & 3535 & -0.85 & -74.74409 & 39.06721 & NJ & 3607 & 0.37 & -74.75467 & 39.05318 & NJ \\
\hline 3464 & -0.55 & -74.73238 & 39.08006 & $\mathrm{NJ}$ & 3536 & -0.66 & -74.74442 & 39.06684 & NJ & 3608 & 0.56 & -74.75499 & 39.0528 & NJ \\
\hline 3465 & -0.55 & -74.73238 & 39.08006 & NJ & 3537 & -0.86 & -74.74442 & 39.06684 & NJ & 3609 & 0.32 & -74.75499 & 39.0528 & NJ \\
\hline 3466 & -0.6 & -74.73273 & 39.0797 & $\mathrm{NJ}$ & 3538 & -0.65 & -74.74474 & 39.06646 & NJ & 3610 & 0.51 & -74.75528 & 39.05241 & NJ \\
\hline 3467 & -0.6 & -74.73273 & 39.0797 & $\mathrm{NJ}$ & 3539 & -0.86 & -74.74474 & 39.06646 & NJ & 3611 & 0.27 & -74.75528 & 39.05241 & NJ \\
\hline 3468 & -0.72 & -74.73311 & 39.07936 & $\mathrm{NJ}$ & 3540 & -0.57 & -74.745 & 39.06605 & NJ & 3612 & 0.48 & -74.7556 & 39.05204 & NJ \\
\hline 3469 & -0.72 & -74.73311 & 39.07936 & $\mathrm{NJ}$ & 3541 & -0.78 & -74.745 & 39.06605 & $\mathrm{NJ}$ & 3613 & 0.29 & -74.7556 & 39.05204 & $\mathrm{NJ}$ \\
\hline 3470 & -0.79 & -74.73347 & 39.07901 & $\mathrm{NJ}$ & 3542 & -0.5 & .74 .74527 & 39.06565 & NJ & 3614 & 0.49 & -74.75594 & 39.05167 & NJ \\
\hline 3471 & -0.79 & -74.73347 & 39.07901 & $\mathrm{NJ}$ & 3543 & -0.68 & .74 .74527 & 39.06565 & NJ & 3615 & 0.38 & -74.75594 & 39.05167 & $\mathrm{NJ}$ \\
\hline 3472 & -0.84 & -74.73383 & 39.07865 & $\mathrm{NJ}$ & 3544 & -0.38 & -74.74551 & 39.06523 & $\mathrm{NJ}$ & 3616 & 0.5 & .74 .75626 & 39.05129 & NJ \\
\hline 3473 & -0.84 & .74 .73383 & 39.07865 & $\mathrm{NJ}$ & 3545 & -0.55 & .74 .74551 & 39.06523 & NJ & 3617 & 0.35 & -74.75626 & 39.05129 & NJ \\
\hline 3474 & -0.9 & -74.73418 & 39.07829 & $\mathrm{NJ}$ & 3546 & -0.29 & .74 .74579 & 39.06483 & $\mathrm{NJ}$ & 3618 & 0.55 & -74.75656 & 39.05091 & NJ \\
\hline 3475 & -0.9 & -74.73418 & 39.07829 & $\mathrm{NJ}$ & 3547 & -0.45 & -74.74579 & 39.06483 & NJ & 3619 & 0.34 & -74.75656 & 539.05091 & $\mathrm{NJ}$ \\
\hline 3476 & -0.89 & -74.73454 & 39.07795 & $\mathrm{NJ}$ & 3548 & -0.24 & -74.74606 & 39.06444 & NJ & 3620 & 0.49 & -74.75684 & 39.05051 & NJ \\
\hline 3477 & -0.89 & -74.73454 & 39.07795 & NJ & 3549 & -0.41 & -74.74606 & 39.06444 & NJ & 3621 & 0.28 & -74.75684 & 39.05051 & NJ \\
\hline 3478 & -1.03 & -74.73492 & 39.0776 & $\mathrm{NJ}$ & 3550 & -0.16 & -74.74635 & 39.06404 & NJ & 3622 & 0.42 & -74.75703 & 39.05007 & NJ \\
\hline 3479 & -1.03 & -74.73492 & 39.0776 & $\mathrm{NJ}$ & 3551 & -0.38 & -74.74635 & 39.06404 & NJ & 3623 & 0.35 & -74.75703 & 39.05007 & NJ \\
\hline 3480 & -1.04 & -74.73528 & 39.07724 & $\mathrm{NJ}$ & 3552 & -0.08 & -74.74661 & 39.06363 & NJ & 3624 & 0.28 & -74.7574 & 39.04971 & NJ \\
\hline 3481 & -1.04 & -74.73528 & 39.07724 & $\mathrm{NJ}$ & 3553 & -0.31 & -74.74661 & 39.06363 & NJ & 3625 & 0.21 & -74.7574 & 39.04971 & NJ \\
\hline 3482 & -1.03 & -74.73563 & 39.07688 & $\mathrm{NJ}$ & 3554 & -0.04 & -74.74687 & 39.06322 & NJ & 3626 & 0.3 & -74.75775 & 39.04936 & NJ \\
\hline 3483 & -1.03 & -74.73563 & 39.07688 & NJ & 3555 & -0.24 & -74.74687 & 39.06322 & NJ & 3627 & 0.12 & -74.75775 & 39.04936 & NJ \\
\hline 3484 & -1.03 & -74.73598 & 39.07653 & NJ & 3556 & 0.02 & -74.74713 & 39.06281 & NJ & 3628 & 0.36 & -74.7581 & 39.049 & NJ \\
\hline 3485 & -1.03 & -74.73598 & 39.07653 & NJ & 3557 & -0.17 & -74.74713 & 39.06281 & NJ & 3629 & 0.09 & -74.7581 & 39.049 & NJ \\
\hline 3486 & -0.94 & -74.73631 & 39.07617 & $\mathrm{NJ}$ & 3558 & 0.03 & -74.74744 & 39.06243 & NJ & 3630 & 0.33 & -74.75842 & 39.04863 & NJ \\
\hline 3487 & -1.05 & -74.73631 & 39.07617 & NJ & 3559 & -0.18 & -74.74744 & 39.06243 & $\mathrm{NJ}$ & 3631 & -0.04 & -74.75842 & 39.04863 & NJ \\
\hline 3488 & -0.95 & -74.73663 & 39.07579 & NJ & 3560 & 0.03 & -74.74774 & 39.06205 & NJ & 3632 & 0.21 & .74 .75877 & 39.04826 & NJ \\
\hline 3489 & -1.12 & -74.73663 & 39.07579 & NJ & 3561 & -0.17 & -74.74774 & 39.06205 & $\mathrm{NJ}$ & 3633 & -0.2 & -74.75877 & 39.04826 & NJ \\
\hline 3490 & -1 & -74.73698 & 39.07543 & NJ & 3562 & 0.08 & -74.74805 & 39.06166 & NJ & 3634 & 0.12 & -74.75909 & 39.0479 & $\mathrm{NJ}$ \\
\hline 3491 & -1.15 & -74.73698 & 39.07543 & NJ & 3563 & -0.17 & -74.74805 & 39.06166 & NJ & 3635 & -0.3 & -74.75909 & 39.0479 & NJ \\
\hline 3492 & -1.11 & -74.73738 & 39.0751 & NJ & 3564 & 0.15 & -74.74829 & 39.06125 & NJ & 3636 & 0.03 & -74.75943 & 39.04753 & NJ \\
\hline 3493 & -1.24 & -74.73738 & 39.0751 & NJ & 3565 & -0.1 & -74.74829 & 39.06125 & $\mathrm{NJ}$ & 3637 & -0.43 & -74.75943 & 39.04753 & NJ \\
\hline 3494 & -1.13 & -74.73772 & 39.07472 & NJ & 3566 & 0.25 & -74.74854 & 39.06083 & NJ & 3638 & -0.02 & -74.75972 & 39.04713 & NJ \\
\hline 3495 & -1.25 & -74.73772 & 39.07472 & NJ & 3567 & NA & -74.74854 & 39.06083 & NJ & 3639 & -0.44 & -74.75972 & 39.04713 & $\mathrm{NJ}$ \\
\hline 3496 & -1.15 & -74.73807 & 39.07437 & NJ & 3568 & 0.35 & -74.74879 & 39.06042 & NJ & 3640 & -0.02 & -74.76003 & 39.04675 & NJ \\
\hline 3497 & -1.31 & -74.73807 & 39.07437 & $\mathrm{NJ}$ & 3569 & 0.11 & -74.74879 & 39.06042 & NJ & 3641 & -0.41 & .74 .76003 & 39.04675 & $\mathrm{NJ}$ \\
\hline 3498 & -1.16 & -74.7384 & 39.074 & NJ & 3570 & 0.46 & -74.74905 & 39.06001 & NJ & 3642 & -0.08 & -74.76031 & 39.04636 & $\mathrm{NJ}$ \\
\hline 3499 & -1.34 & -74.7384 & 39.074 & NJ & 3571 & 0.22 & -74.74905 & 39.06001 & NJ & 3643 & -0.41 & -74.76031 & 39.04636 & $\mathrm{NJ}$ \\
\hline 3500 & -1.14 & -74.73872 & 39.07363 & NJ & 3572 & 0.55 & -74.7493 & 39.0596 & NJ & 3644 & -0.13 & -74.76062 & 39.04597 & $\mathrm{NJ}$ \\
\hline 3501 & -1.19 & -74.73872 & 39.07363 & $\mathrm{NJ}$ & 3573 & 0.35 & -74.7493 & 39.0596 & NJ & 3645 & -0.44 & -74.76062 & 39.04597 & $\mathrm{NJ}$ \\
\hline 3502 & -1.12 & -74.73904 & 39.07325 & $\mathrm{NJ}$ & 3574 & 0.6 & -74.74957 & 39.05919 & NJ & 3646 & -0.17 & -74.76086 & 39.04556 & $\mathrm{NJ}$ \\
\hline 3503 & -1.07 & -74.73904 & 39.07325 & NJ & 3575 & 0.46 & -74.74957 & 39.05919 & NJ & 3647 & -0.41 & -74.76086 & 39.04556 & $\mathrm{NJ}$ \\
\hline 3504 & -1.16 & -74.7394 & 39.07289 & NJ & 3576 & 0.59 & -74.74986 & 39.0588 & NJ & 3648 & -0.15 & -74.76105 & 39.04511 & NJ \\
\hline 3505 & -1.14 & -74.7394 & 39.07289 & $\mathrm{NJ}$ & 3577 & 0.55 & -74.74986 & 39.0588 & NJ & 3649 & -0.36 & -74.76105 & 39.04511 & NJ \\
\hline 3506 & -1.17 & -74.73975 & 39.07254 & NJ & 3578 & 0.56 & -74.7502 & 39.05843 & NJ & 3650 & -0.27 & -74.76141 & 39.04475 & NJ \\
\hline 3507 & -1.22 & -74.73975 & 39.07254 & NJ & 3579 & 0.6 & -74.7502 & 39.05843 & NJ & 3651 & -0.45 & -74.76141 & 39.04475 & NJ \\
\hline 3508 & -1.15 & -74.74007 & 39.07216 & NJ & 3580 & 0.57 & -74.7505 & 39.05806 & NJ & 3652 & -0.3 & -74.76175 & 39.04439 & NJ \\
\hline 3509 & -1.25 & -74.74007 & 39.07216 & NJ & 3581 & 0.62 & -74.7505 & 39.05806 & NJ & 3653 & -0.48 & -74.76175 & 39.04439 & NJ \\
\hline 3510 & -1.15 & -74.7404 & 39.07179 & NJ & 3582 & 0.51 & -74.7509 & 39.05772 & NJ & 3654 & -0.36 & -74.76208 & 39.04402 & $\mathrm{NJ}$ \\
\hline 3511 & -1.28 & -74.7404 & 39.07179 & NJ & 3583 & 0.48 & -74.7509 & 39.05772 & NJ & 3655 & -0.51 & -74.76208 & 39.04402 & NJ \\
\hline 3512 & -1.09 & -74.74071 & 39.07141 & NJ & 3584 & 0.49 & -74.75124 & 39.05735 & NJ & 3656 & -0.48 & -74.76244 & 39.04366 & NJ \\
\hline 3513 & -1.26 & -74.74071 & 39.07141 & NJ & 3585 & 0.38 & -74.75124 & 39.05735 & NJ & 3657 & -0.56 & -74.76244 & 39.04366 & $\mathrm{NJ}$ \\
\hline 3514 & -1.02 & -74.74101 & 39.07103 & NJ & 3586 & 0.46 & -74.75157 & 39.05698 & NJ & 3658 & -0.57 & -74.76274 & 39.04328 & NJ \\
\hline 3515 & -1.2 & -74.74101 & 39.07103 & NJ & 3587 & 0.33 & -74.75157 & 39.05698 & NJ & 3659 & -0.6 & -74.76274 & 39.04328 & NJ \\
\hline 3516 & -1.05 & -74.74133 & 39.07065 & NJ & 3588 & 0.41 & -74.75192 & 39.05663 & NJ & 3660 & -0.69 & -74.76308 & 39.04291 & NJ \\
\hline 3517 & -1.17 & -74.74133 & 39.07065 & NJ & 3589 & 0.3 & .74 .75192 & 39.05663 & NJ & 3661 & -0.73 & -74.76308 & 39.04291 & NJ \\
\hline 3518 & -1.01 & -74.74162 & 39.07026 & NJ & 3590 & 0.4 & -74.75224 & 39.05625 & NJ & 3662 & -0.73 & -74.76337 & 39.04253 & NJ \\
\hline 3519 & -1.16 & -74.74162 & 39.07026 & $\mathrm{NJ}$ & 3591 & 0.29 & -74.75224 & 39.05625 & NJ & 3663 & -0.75 & -74.76337 & 39.04253 & $\mathrm{NJ}$ \\
\hline 3520 & -0.99 & -74.74197 & 39.0699 & $\mathrm{NJ}$ & 3592 & 0.41 & .74 .75256 & 39.05588 & NJ & 3664 & -0.75 & -74.76363 & 39.04211 & $\mathrm{NJ}$ \\
\hline 3521 & -1.19 & -74.74197 & 39.0699 & $\mathrm{NJ}$ & 3593 & 0.29 & -74.75256 & 39.05588 & NJ & 3665 & -0.71 & -74.76363 & 39.04211 & NJ \\
\hline 3522 & -1.02 & -74.74234 & 39.06955 & $\mathrm{NJ}$ & 3594 & 0.43 & -74.75282 & 39.05547 & NJ & 3666 & -0.79 & -74.76385 & 39.04169 & NJ \\
\hline 3523 & -1.2 & -74.74234 & 39.06955 & $\mathrm{NJ}$ & 3595 & 0.35 & -74.75282 & 39.05547 & NJ & 3667 & -0.73 & -74.76385 & 39.04169 & NJ \\
\hline 3524 & -0.95 & -74.74266 & 39.06917 & $\mathrm{NJ}$ & 3596 & 0.46 & -74.75311 & 39.05508 & NJ & 3668 & -0.93 & -74.76421 & 39.04133 & NJ \\
\hline 3525 & -1.16 & -74.74266 & 39.06917 & NJ & 3597 & 0.42 & -74.75311 & 39.05508 & NJ & 3669 & -0.77 & -74.76421 & 39.04133 & NJ \\
\hline 3526 & -0.93 & -74.74295 & 39.06879 & NJ & 3598 & 0.49 & -74.75339 & 39.05468 & NJ & 3670 & -1.02 & -74.76454 & 39.04096 & NJ \\
\hline 3527 & -1.19 & -74.74295 & 39.06879 & $\mathrm{NJ}$ & 3599 & 0.44 & -74.75339 & 39.05468 & NJ & 3671 & -0.75 & -74.76454 & 39.04096 & NJ \\
\hline 3528 & -0.86 & -74.74327 & 39.06841 & $\mathrm{NJ}$ & 3600 & 0.59 & -74.75363 & 39.05426 & NJ & 3672 & -1.07 & -74.76485 & 39.04058 & NJ \\
\hline
\end{tabular}




\begin{tabular}{|c|c|c|c|c|c|c|c|c|c|c|c|c|c|c|}
\hline TR\# & RATE & LONG & LAT & ST & I TR\# & RATE & LONG & LAT & ST & TR\# & RATE & LONG & LAT & ST \\
\hline 3673 & -0.75 & -74.76485 & 539.04058 & $\mathrm{NJ}$ & 3745 & -6.52 & -74.77779 & 39.02786 & $\mathrm{NJ}$ & 3817 & 7.29 & .74 .78856 & 38.99445 & $\overline{\mathrm{NJ}}$ \\
\hline 3674 & -1.18 & .74 .76508 & 839.04016 & NJ & 3746 & -6.48 & -74.77815 & 39.02752 & $\mathrm{NJ}$ & 3818 & 7.32 & -74.78893 & 38.99411 & NJ \\
\hline 3675 & -0.8 & .74 .76508 & 839.04016 & $\mathrm{NJ}$ & 3747 & -6.48 & -74.77815 & 39.02752 & $\mathrm{NJ}$ & 3819 & 7.31 & -74.78894 & 38.99411 & NJ \\
\hline 3676 & -1.56 & .74 .76558 & 839.03989 & NJ & 3748 & -6.82 & .74 .77852 & 39.02716 & $\mathrm{NJ}$ & 3820 & 7.27 & -74.78932 & 38.99378 & NJ \\
\hline 3677 & -1.15 & .74 .76558 & 839.03989 & NJ & 3749 & -6.82 & -74.77852 & 39.02716 & NJ & 3821 & 7.25 & -74.78932 & 38.99377 & NJ \\
\hline 3678 & -1.73 & -74.76598 & 839.03955 & $\mathrm{NJ}$ & 3750 & -3.3 & -74.7789 & 39.02682 & $\mathrm{NJ}$ & 3822 & 7.23 & -74.78972 & 38.99345 & NJ \\
\hline 3679 & -1.26 & -74.76598 & 839.03955 & $\mathrm{NJ}$ & 3751 & -3.15 & -74.77927 & 39.02647 & NJ & 3823 & 7.2 & -74.78973 & 38.99345 & NJ \\
\hline 3680 & -1.89 & -74.76634 & 439.0392 & $\mathrm{NJ}$ & 3752 & -2.8 & -74.77962 & 39.02611 & $\mathrm{NJ}$ & 3824 & 7.19 & .74 .79016 & 38.99316 & $\mathrm{NJ}$ \\
\hline 3681 & -1.38 & -74.76634 & 439.0392 & NJ & 3753 & -0.47 & -74.79152 & 39.00997 & NJ & 3825 & 7.17 & -74.79018 & 38.99316 & NJ \\
\hline 3682 & -2.05 & -74.76668 & 839.03884 & NJ & 3754 & -0.46 & -74.79131 & 39.00955 & NJ & 3826 & 7.14 & -74.79063 & 38.99289 & NJ \\
\hline 3683 & -1.5 & -74.76668 & 839.03884 & NJ & 3755 & -0.58 & -74.79112 & 39.00912 & NJ & 3827 & 7.13 & -74.79063 & 38.99289 & NJ \\
\hline 3684 & -2.25 & -74.76704 & 439.03848 & $\mathrm{NJ}$ & 3756 & -0.89 & -74.79111 & 39.00862 & NJ & 3828 & 7.07 & -74.79114 & 38.99266 & NJ \\
\hline 3685 & -1.53 & -74.76704 & 439.03848 & NJ & 3757 & -0.91 & -74.79089 & 39.00821 & NJ & 3829 & 7.06 & -74.79115 & 38.99265 & NJ \\
\hline 3686 & -2.46 & -74.76738 & 839.03812 & $\mathrm{NJ}$ & 3758 & -1.46 & -74.79079 & 39.00774 & NJ & 3830 & 6.97 & -74.79161 & 38.99239 & NJ \\
\hline 3687 & -1.67 & -74.76738 & 839.03812 & NJ & 3759 & -1.46 & -74.79073 & 39.00727 & NJ & 3831 & 6.95 & -74.79163 & 38.99238 & NJ \\
\hline 3688 & -2.62 & -74.7677 & 39.03774 & $\mathrm{NJ}$ & 3760 & -1.83 & .74 .7907 & 39.00712 & $\mathrm{NJ}$ & 3832 & 6.85 & -74.79205 & 38.99211 & $\mathrm{NJ}$ \\
\hline 3689 & -1.73 & -74.7677 & 39.03774 & $\mathrm{NJ}$ & 3761 & -2.12 & .74 .7906 & 39.00682 & NJ & 3833 & 6.82 & -74.79207 & 38.9921 & NJ \\
\hline 3690 & -2.72 & .74 .76804 & 439.03737 & NJ & 3762 & -2.6 & -74.79057 & 39.00666 & NJ & 3834 & 6.74 & -74.79259 & 38.99189 & NJ \\
\hline 3691 & -1.74 & -74.76804 & 439.03737 & NJ & 3763 & -2.12 & -74.7905 & 39.00636 & NJ & 3835 & 6.72 & -74.7926 & 38.99188 & NJ \\
\hline 3692 & -2.8 & .74 .76836 & 639.037 & NJ & 3764 & -2.51 & .74 .79042 & 39.00619 & NJ & 3836 & 6.64 & -74.79306 & 38.99162 & NJ \\
\hline 3693 & -1.76 & -74.76836 & 639.037 & $\mathrm{NJ}$ & 3765 & -2.05 & -74.79034 & 39.00591 & $\mathrm{NJ}$ & 3837 & 6.63 & -74.79308 & 38.99161 & NJ \\
\hline 3694 & -2.87 & -74.76865 & 539.03661 & NJ & 3766 & -2.02 & -74.79031 & 39.00573 & NJ & 3838 & 6.56 & -74.79353 & 38.99136 & NJ \\
\hline 3695 & -1.71 & -74.76865 & 539.03661 & $\mathrm{NJ}$ & 3767 & -2 & -74.79021 & 39.00547 & NJ & 3839 & 6.55 & -74.79355 & 38.99135 & NJ \\
\hline 3696 & -3.01 & .74 .76894 & 439.03622 & NJ & 3768 & -1.79 & -74.79013 & 39.00527 & NJ & 3840 & 6.47 & -74.79401 & 38.99109 & NJ \\
\hline 3697 & -1.81 & -74.76894 & 439.03622 & NJ & 3769 & -1.7 & -74.79002 & 39.00504 & NJ & 3841 & 6.45 & -74.79402 & 38.99108 & $\mathrm{NJ}$ \\
\hline 3698 & -3.1 & .74 .76924 & 439.03583 & NJ & 3770 & -0.98 & .74 .78992 & 39.0048 & NJ & 3842 & 6.4 & -74.79443 & 38.9908 & NJ \\
\hline 3699 & -1.79 & -74.76924 & 439.03583 & $\mathrm{NJ}$ & 3771 & -1.3 & -74.78972 & 39.00465 & NJ & 3843 & 6.38 & .74 .79446 & 38.99079 & $\mathrm{NJ}$ \\
\hline 3700 & -3.13 & -74.7695 & 39.03543 & $\mathrm{NJ}$ & 3772 & -0.08 & -74.7892 & 39.00435 & $\mathrm{NJ}$ & 3844 & 6.31 & -74.79491 & 38.99052 & NJ \\
\hline 3701 & -1.64 & -74.7695 & 39.03543 & $\mathrm{NJ}$ & 3773 & 0.02 & -74.78899 & 39.00429 & NJ & 3845 & 6.28 & -74.79492 & 38.99051 & NJ \\
\hline 3702 & -2.45 & .74 .7692 & 39.0347 & NJ & 3774 & 1.12 & -74.78888 & 39.00397 & NJ & 3846 & 6.19 & -74.79539 & 38.99028 & NJ \\
\hline 3703 & -0.68 & -74.7692 & 39.0347 & NJ & 3775 & 0.62 & -74.78883 & 39.00383 & NJ & 3847 & 6.19 & -74.79543 & 38.99026 & NJ \\
\hline 3704 & -3.29 & -74.76997 & 739.03458 & NJ & 3776 & 2.31 & -74.7887 & 39.00354 & NJ & 3848 & 6.05 & -74.7959 & 38.99004 & NJ \\
\hline 3705 & -1.62 & -74.76997 & 739.03458 & NJ & 3777 & 1.19 & -74.7886 & 39.00336 & NJ & 3849 & 6.03 & -74.79593 & 38.99002 & NJ \\
\hline 3706 & -3.43 & -74.77029 & 939.03421 & $\mathrm{NJ}$ & 3778 & 3.35 & -74.78841 & 39.00314 & $\mathrm{NJ}$ & 3850 & 5.9 & -74.79642 & 38.98981 & $\mathrm{NJ}$ \\
\hline 3707 & -1.59 & -74.77029 & 939.03421 & NJ & 3779 & 1.57 & .74 .78786 & 39.00287 & NJ & 3851 & 5.9 & -74.79645 & 38.98979 & NJ \\
\hline 3708 & -3.55 & -74.77063 & 339.03384 & $\mathrm{NJ}$ & 3780 & 2.1 & -74.78784 & 39.00286 & NJ & 3852 & 5.8 & -74.79691 & 38.98957 & NJ \\
\hline 3709 & -1.65 & -74.77063 & 339.03384 & NJ & 3781 & 2.79 & -74.7876 & 39.00239 & NJ & 3853 & 5.78 & -74.79695 & 38.98955 & $\mathrm{NJ}$ \\
\hline 3710 & -3.66 & -74.77094 & 439.03346 & NJ & 3782 & 3.54 & -74.78735 & 39.00192 & NJ & 3854 & 5.67 & .74 .79739 & 38.98931 & NJ \\
\hline 3711 & -1.75 & -74.77094 & 439.03346 & NJ & 3783 & 4.29 & -74.78677 & 39.00143 & NJ & 3855 & 5.64 & -74.79744 & 38.98929 & NJ \\
\hline 3712 & -3.87 & -74.77122 & 239.03307 & $\mathrm{NJ}$ & 3784 & 3.36 & -74.78677 & 39.00138 & $\mathrm{NJ}$ & 3856 & 5.53 & -74.79791 & 38.98908 & NJ \\
\hline 3713 & -1.85 & -74.77122 & 239.03307 & NJ & 3785 & 4.72 & -74.78693 & 39.00099 & NJ & 3857 & 5.51 & -74.79796 & 38.98907 & NJ \\
\hline 3714 & -3.98 & -74.77151 & 139.03268 & $\mathrm{NJ}$ & 3786 & 3.8 & -74.78697 & 39.00089 & NJ & 3858 & 5.42 & -74.7984 & 38.98883 & NJ \\
\hline 3715 & -1.83 & -74.77151 & 139.03268 & NJ & 3787 & 5.16 & -74.78694 & 39.00054 & NJ & 3859 & 5.4 & -74.79845 & 38.9888 & NJ \\
\hline 3716 & -6.09 & -74.77432 & 239.03258 & NJ & 3788 & 4.36 & -74.78687 & 39.00017 & NJ & 3860 & 5.28 & -74.7989 & 38.98859 & NJ \\
\hline 3717 & -4.06 & -74.77432 & 239.03258 & NJ & 3789 & 5.62 & -74.78687 & 39.00008 & NJ & 3861 & 5.25 & -74.79895 & 38.98857 & NJ \\
\hline 3718 & -4.08 & -74.7718 & 39.03228 & $\mathrm{NJ}$ & 3790 & 6.12 & -74.7867 & 38.99962 & NJ & 3862 & 5.18 & -74.79938 & 38.98833 & NJ \\
\hline 3719 & -1.86 & -74.7718 & 39.03228 & NJ & 3791 & 5.01 & -74.78664 & 38.99947 & NJ & 3863 & 5.18 & -74.79942 & 38.9883 & NJ \\
\hline 3720 & -4.09 & -74.77203 & 339.03185 & NJ & 3792 & 5.22 & -74.78654 & 38.99928 & NJ & 3864 & 5.09 & -74.79985 & 38.98807 & NJ \\
\hline 3721 & -1.77 & -74.77203 & 339.03185 & NJ & 3793 & 6.72 & -74.7865 & 38.99915 & NJ & 3865 & 5.07 & -74.79991 & 38.98804 & NJ \\
\hline 3722 & -6.46 & -74.77367 & 739.03166 & NJ & 3794 & 7.25 & -74.78635 & 38.99869 & NJ & 3866 & 4.97 & -74.80035 & 38.98782 & NJ \\
\hline 3723 & -4.4 & -74.77367 & 739.03166 & NJ & 3795 & 5.94 & -74.78629 & 38.99852 & NJ & 3867 & 4.95 & -74.80042 & 38.9878 & NJ \\
\hline 3724 & -6.8 & -74.77411 & 139.03135 & $\mathrm{NJ}$ & 3796 & 5.99 & -74.78629 & 38.99843 & NJ & 3868 & 4.84 & .74 .80086 & 38.98759 & NJ \\
\hline 3725 & -4.71 & -74.77411 & 139.03135 & NJ & 3797 & 7.7 & -74.78629 & 38.99823 & NJ & 3869 & 4.81 & -74.80093 & 38.98757 & NJ \\
\hline 3726 & -7.2 & -74.77444 & 439.03098 & NJ & 3798 & 8.17 & -74.78613 & 38.99777 & NJ & 3870 & 4.68 & -74.80138 & 38.98737 & NJ \\
\hline 3727 & -5.01 & -74.77444 & 439.03098 & NJ & 3799 & 6.62 & -74.78612 & 38.9977 & NJ & 3871 & 4.67 & -74.80145 & 38.98733 & NJ \\
\hline 3728 & -5.33 & -74.77477 & 739.03061 & $\mathrm{NJ}$ & 3800 & 6.67 & -74.78609 & 38.99763 & NJ & 3872 & 4.59 & -74.80185 & 38.9871 & NJ \\
\hline 3729 & -5.33 & -74.77477 & 739.03061 & $\mathrm{NJ}$ & 3801 & 8.65 & -74.78601 & 38.99731 & NJ & 3873 & 4.56 & -74.80193 & 38.98707 & NJ \\
\hline 3730 & -5.69 & .74 .77515 & 539.03027 & NJ & 3802 & 7.14 & -74.78607 & 38.997 & NJ & 3874 & 4.46 & -74.80235 & 38.98686 & NJ \\
\hline 3731 & -5.69 & -74.77515 & 539.03027 & NJ & 3803 & 7.13 & -74.78607 & 38.99697 & NJ & 3875 & 4.43 & .74 .80244 & 38.98683 & $\mathrm{NJ}$ \\
\hline 3732 & -6.12 & -74.77551 & 139.02992 & $\mathrm{NJ}$ & 3804 & 7.19 & -74.78641 & 38.99662 & NJ & 3876 & 4.33 & -74.80287 & 38.98663 & NJ \\
\hline 3733 & -6.12 & -74.77551 & 139.02992 & NJ & 3805 & 7.19 & -74.78642 & 38.99661 & NJ & 3877 & 4.3 & .74 .80295 & 38.98659 & $\mathrm{NJ}$ \\
\hline 3734 & -6.41 & -74.77592 & 239.02959 & NJ & 3806 & 7.13 & -74.78685 & 38.99634 & $\mathrm{NJ}$ & 3878 & 4.18 & -74.80338 & 38.9864 & $\mathrm{NJ}$ \\
\hline 3735 & -6.41 & -74.77592 & 239.02959 & $\mathrm{NJ}$ & 3807 & 7.11 & -74.78687 & 38.99632 & $\mathrm{NJ}$ & 3879 & 4.14 & -74.80347 & 38.98636 & $\mathrm{NJ}$ \\
\hline 3736 & -6.48 & -74.77631 & 139.02926 & $\mathrm{NJ}$ & 3808 & 7.06 & -74.78731 & 38.99606 & NJ & 3880 & 4.02 & -74.80389 & 38.98617 & NJ \\
\hline 3737 & -6.48 & -74.77631 & 139.02926 & NJ & 3809 & 7.04 & -74.78732 & 38.99604 & NJ & 3881 & 3.99 & -74.80399 & 38.98613 & NJ \\
\hline 3738 & -6.59 & -74.77669 & 939.02891 & NJ & 3810 & 7.03 & -74.78749 & 38.99555 & $\mathrm{NJ}$ & 3882 & 3.9 & .74 .8044 & 38.98594 & $\mathrm{NJ}$ \\
\hline 3739 & .6 .59 & -74.77669 & 939.02891 & $\mathrm{NJ}$ & 3811 & 7.01 & -74.78751 & 38.99554 & NJ & 3883 & 3.85 & -74.8045 & 38.9859 & NJ \\
\hline 3740 & -6.73 & -74.77707 & 739.02857 & $\mathrm{NJ}$ & 3812 & 7.09 & -74.78784 & 38.99518 & NJ & 3884 & 3.72 & -74.80493 & 38.98572 & NJ \\
\hline 3741 & -6.73 & -74.77707 & 739.02857 & $\mathrm{NJ}$ & 3813 & 7.07 & -74.78784 & 38.99517 & $\mathrm{NJ}$ & 3885 & 3.68 & -74.80502 & 38.98568 & NJ \\
\hline 3742 & -6.7 & -74.77744 & 439.02822 & NJ & 3814 & 7.16 & -74.78818 & 38.99481 & NJ & 3886 & 3.54 & -74.80547 & 38.98551 & $\mathrm{NJ}$ \\
\hline 3743 & -6.7 & -74.77744 & 439.02822 & NJ & 3815 & 7.16 & -74.78819 & 38.9948 & NJ & 3887 & 3.5 & -74.80557 & 38.98547 & $\mathrm{NJ}$ \\
\hline 3744 & -6.52 & -74.77779 & 939.02786 & $\mathrm{NJ}$ & 3816 & 7.31 & -74.78854 & 38.99446 & NJ & 3888 & 3.4 & -74.80597 & 38.98527 & NJ \\
\hline
\end{tabular}




\begin{tabular}{|c|c|c|c|c|c|c|c|c|c|c|c|c|c|c|}
\hline TR\# & RATE & LONG & LAT & ST & TR\# & RATE & LONG & LAT & ST & TR\# & RATE & LONG & LAT & ST \\
\hline 3889 & 3.35 & -74.80608 & 338.98522 & NJ & 3961 & 1.13 & -74.8232 & 38.97568 & $\mathrm{NJ}$ & 4033 & 2.35 & -74.83823 & 338.96448 & $\mathrm{NJ}$ \\
\hline 3890 & 3.27 & -74.80646 & 538.98502 & NJ & 3962 & 1.13 & -74.82349 & 38.97549 & $\mathrm{NJ}$ & 4034 & 2.42 & -74.83849 & 98.96428 & $\mathrm{NJ}$ \\
\hline 3891 & 3.26 & -74.80655 & 38.98496 & NJ & 3963 & 1.15 & -74.82362 & 238.97537 & NJ & 4035 & 2.59 & -74.83862 & 238.96415 & $\mathrm{NJ}$ \\
\hline 3892 & 3.16 & -74.80696 & 538.98478 & NJ & 3964 & 1.17 & -74.82387 & 738.97516 & $\mathrm{NJ}$ & 4036 & 2.71 & -74.83888 & 838.96396 & $\mathrm{NJ}$ \\
\hline 3893 & 3.12 & -74.80708 & 38.98473 & NJ & 3965 & 1.16 & -74.82404 & 38.97506 & $\mathrm{NJ}$ & 4037 & 2.86 & -74.83905 & 538.96385 & $\mathrm{NJ}$ \\
\hline 3894 & 3.04 & -74.80746 & 538.98453 & NJ & 3966 & 1.16 & -74.8243 & 38.97486 & $\mathrm{NJ}$ & 4038 & 2.99 & -74.83931 & 138.96365 & $\mathrm{NJ}$ \\
\hline 3895 & 3.02 & -74.80756 & 538.98447 & NJ & 3967 & 1.17 & -74.82446 & 538.97476 & $\mathrm{NJ}$ & 4039 & 3.13 & -74.83946 & 638.96354 & $\mathrm{NJ}$ \\
\hline 3896 & 2.91 & .74 .80797 & 38.9843 & $\mathrm{NJ}$ & 3968 & 1.12 & -74.82477 & 738.97459 & $\mathrm{NJ}$ & 4040 & 3.22 & -74.83974 & 438.96334 & $\mathrm{NJ}$ \\
\hline 3897 & 2.88 & -74.80809 & 38.98425 & NJ & 3969 & 1.12 & -74.82494 & 438.97448 & $\mathrm{NJ}$ & 4041 & 3.38 & -74.83987 & 738.96322 & $\mathrm{NJ}$ \\
\hline 3898 & 2.8 & -74.80849 & 38.98406 & NJ & 3970 & 1.15 & -74.82516 & 638.97426 & $\mathrm{NJ}$ & 4042 & 3.54 & -74.8401 & 38.96299 & $\mathrm{NJ}$ \\
\hline 3899 & 2.76 & -74.80859 & 38.98401 & NJ & 3971 & 1.14 & -74.82532 & 238.97415 & $\mathrm{NJ}$ & 4043 & 3.71 & -74.84024 & 438.96287 & $\mathrm{NJ}$ \\
\hline 3900 & 2.68 & -74.80898 & 38.98382 & NJ & 3972 & 1.12 & -74.82561 & 38.97397 & $\mathrm{NJ}$ & 4044 & 3.86 & -74.84048 & 838.96265 & $\mathrm{NJ}$ \\
\hline 3901 & 2.67 & -74.8091 & 38.98376 & NJ & 3973 & 1.11 & -74.82578 & 38.97386 & $\mathrm{NJ}$ & 4045 & 4.06 & -74.84062 & 238.96253 & $\mathrm{NJ}$ \\
\hline 3902 & 2.65 & -74.80943 & 38.98354 & $\mathrm{NJ}$ & 3974 & 1.08 & -74.82607 & 738.9737 & $\mathrm{NJ}$ & 4046 & 4.18 & -74.8409 & 38.96234 & $\mathrm{NJ}$ \\
\hline 3903 & 2.64 & -74.80956 & 538.98348 & NJ & 3975 & 1.08 & -74.82623 & 38.97359 & $\mathrm{NJ}$ & 4047 & 4.52 & -74.84105 & 538.96222 & $\mathrm{NJ}$ \\
\hline 3904 & 2.58 & -74.80991 & 38.98328 & NJ & 3976 & 1.06 & -74.82652 & 238.97342 & $\mathrm{NJ}$ & 4048 & 4.66 & -74.84131 & 38.96202 & $\mathrm{NJ}$ \\
\hline 3905 & 2.56 & -74.81003 & 38.98322 & NJ & 3977 & 1.08 & -74.82668 & 38.97329 & $\mathrm{NJ}$ & 4049 & 5.01 & -74.84148 & 838.96192 & NJ \\
\hline 3906 & 2.49 & -74.81039 & 38.98303 & $\mathrm{NJ}$ & 3978 & 1.05 & -74.82697 & 738.97313 & $\mathrm{NJ}$ & 4050 & 5.21 & -74.84174 & 48.96172 & NJ \\
\hline 3907 & 2.48 & -74.8105 & 38.98295 & $\mathrm{NJ}$ & 3979 & 1.04 & -74.82713 & 38.97302 & $\mathrm{NJ}$ & 4051 & 5.84 & -74.84187 & 38.9616 & $\mathrm{NJ}$ \\
\hline 3908 & 2.4 & -74.81088 & 38.98277 & $\mathrm{NJ}$ & 3980 & 1.04 & -74.82739 & 38.97283 & $\mathrm{NJ}$ & 4052 & 7.67 & -74.84213 & 38.96139 & $\mathrm{NJ}$ \\
\hline 3909 & 2.39 & -74.811 & 38.98271 & $\mathrm{NJ}$ & 3981 & 1.03 & -74.82755 & 38.97271 & $\mathrm{NJ}$ & 4053 & 7.37 & -74.84225 & 538.96126 & $\mathrm{NJ}$ \\
\hline 3910 & 2.37 & -74.81136 & 538.98251 & $\mathrm{NJ}$ & 3982 & 1.02 & -74.82782 & 238.97252 & $\mathrm{NJ}$ & 4054 & 7.35 & -74.8425 & 38.96104 & NJ \\
\hline 3911 & 2.34 & -74.81148 & 38.98244 & $\mathrm{NJ}$ & 3983 & 1.03 & -74.82799 & 38.97242 & $\mathrm{NJ}$ & 4055 & 7.17 & -74.84267 & 738.96094 & NJ \\
\hline 3912 & 2.33 & -74.81178 & 38.98221 & $\mathrm{NJ}$ & 3984 & 1.07 & -74.82825 & 58.97221 & $\mathrm{NJ}$ & 4056 & 7.14 & -74.84296 & 538.96076 & $\mathrm{NJ}$ \\
\hline 3913 & 2.31 & -74.8119 & 38.98214 & $\mathrm{NJ}$ & 3985 & 1.08 & -74.8284 & 38.9721 & $\mathrm{NJ}$ & 4057 & 7.04 & -74.84309 & 38.96064 & $\mathrm{NJ}$ \\
\hline 3914 & 2.27 & -74.81226 & 538.98194 & $\mathrm{NJ}$ & 3986 & 1.07 & -74.82867 & 38.97192 & $\mathrm{NJ}$ & 4058 & 7.05 & -74.84334 & 438.96042 & $\mathrm{NJ}$ \\
\hline 3915 & 2.25 & -74.81239 & 38.98188 & $\mathrm{NJ}$ & 3987 & 1.1 & -74.82883 & 38.97179 & $\mathrm{NJ}$ & 4059 & 6.68 & -74.84346 & 538.96029 & $\mathrm{NJ}$ \\
\hline 3916 & 2.19 & -74.81276 & 538.9817 & $\mathrm{NJ}$ & 3988 & 1.09 & -74.8291 & 38.97162 & $\mathrm{NJ}$ & 4060 & 6.73 & -74.84369 & 38.96005 & $\mathrm{NJ}$ \\
\hline 3917 & 2.19 & -74.81288 & 38.98163 & $\mathrm{NJ}$ & 3989 & 1.09 & -74.82927 & 738.9715 & $\mathrm{NJ}$ & 4061 & 6.47 & -74.84386 & 538.95995 & NJ \\
\hline 3918 & 2.16 & -74.81322 & 38.98143 & $\mathrm{NJ}$ & 3990 & 1.11 & -74.82951 & 38.97131 & $\mathrm{NJ}$ & 4062 & 6.32 & -74.84411 & 38.95975 & $\mathrm{NJ}$ \\
\hline 3919 & 2.15 & -74.81335 & 58.98136 & $\mathrm{NJ}$ & 3991 & 1.12 & -74.82968 & 38.97119 & $\mathrm{NJ}$ & 4063 & 6.14 & .74 .84425 & 538.95963 & $\mathrm{NJ}$ \\
\hline 3920 & 2.1 & -74.81371 & 38.98117 & NJ & 3992 & 1.15 & .74 .82993 & 38.97099 & $\mathrm{NJ}$ & 4064 & 5.93 & -74.84451 & 38.95943 & $\mathrm{NJ}$ \\
\hline 3921 & 2.08 & -74.81384 & 38.9811 & $\mathrm{NJ}$ & 3993 & 1.13 & -74.83011 & 38.97089 & $\mathrm{NJ}$ & 4065 & 5.57 & -74.84467 & 38.95931 & $\mathrm{NJ}$ \\
\hline 3922 & 2.01 & -74.81421 & 38.98094 & $\mathrm{NJ}$ & 3994 & 1.16 & -74.83035 & 58.97068 & $\mathrm{NJ}$ & 4066 & 5.43 & -74.84492 & 38.9591 & $\mathrm{NJ}$ \\
\hline 3923 & 1.99 & -74.81436 & 538.98087 & $\mathrm{NJ}$ & 3995 & 1.15 & -74.83052 & 38.97057 & $\mathrm{NJ}$ & 4067 & 5.07 & -74.84506 & 538.95899 & NJ \\
\hline 3924 & 1.94 & -74.8147 & 38.98068 & $\mathrm{NJ}$ & 3996 & 1.19 & -74.83078 & 38.97038 & $\mathrm{NJ}$ & 4068 & 4.96 & -74.84532 & 38.95877 & NJ \\
\hline 3925 & 1.92 & -74.81483 & 38.98061 & $\mathrm{NJ}$ & 3997 & 1.21 & -74.83093 & 38.97026 & $\mathrm{NJ}$ & 4069 & 4.74 & -74.84544 & 38.95866 & $\mathrm{NJ}$ \\
\hline 3926 & 1.88 & -74.81516 & 38.98041 & $\mathrm{NJ}$ & 3998 & 1.23 & -74.83119 & 38.97006 & $\mathrm{NJ}$ & 4070 & 4.67 & -74.8457 & 38.95843 & $\mathrm{NJ}$ \\
\hline 3927 & 1.87 & -74.81529 & 38.98033 & $\mathrm{NJ}$ & 3999 & 1.26 & -74.83134 & 438.96993 & $\mathrm{NJ}$ & 4071 & 4.5 & -74.84584 & 38.95832 & $\mathrm{NJ}$ \\
\hline 3928 & 1.86 & -74.8156 & 38.98012 & $\mathrm{NJ}$ & 4000 & 1.27 & -74.83159 & 38.96973 & $\mathrm{NJ}$ & 4072 & 4.41 & -74.84608 & 38.9581 & $\mathrm{NJ}$ \\
\hline 3929 & 1.84 & -74.81573 & 38.98004 & $\mathrm{NJ}$ & 4001 & 1.27 & -74.83176 & 538.96962 & $\mathrm{NJ}$ & 4073 & 4.23 & -74.84624 & 38.95799 & $\mathrm{NJ}$ \\
\hline 3930 & 1.8 & -74.81606 & 538.97985 & $\mathrm{NJ}$ & 4002 & 1.31 & -74.832 & 38.96942 & $\mathrm{NJ}$ & 4074 & 4.14 & -74.8465 & 38.95777 & NJ \\
\hline 3931 & 1.77 & -74.81622 & 38.97978 & $\mathrm{NJ}$ & 4003 & 1.36 & -74.83215 & 38.96929 & $\mathrm{NJ}$ & 4075 & 4.01 & -74.8466 & 38.95764 & $\mathrm{NJ}$ \\
\hline 3932 & 1.74 & -74.81656 & 38.9796 & $\mathrm{NJ}$ & 4004 & 1.37 & -74.83241 & 38.9691 & $\mathrm{NJ}$ & 4076 & 3.88 & -74.84689 & 38.95746 & $\mathrm{NJ}$ \\
\hline 3933 & 1.75 & -74.81668 & 38.9795 & $\mathrm{NJ}$ & 4005 & 1.39 & -74.83255 & 38.96897 & $\mathrm{NJ}$ & 4077 & 3.76 & -74.84703 & 38.95734 & $\mathrm{NJ}$ \\
\hline 3934 & 1.71 & -74.81702 & 38.97932 & NJ & 4006 & 1.4 & -74.83281 & 38.96877 & $\mathrm{NJ}$ & 4078 & 3.72 & -74.84729 & 38.95712 & $\mathrm{NJ}$ \\
\hline 3935 & 1.68 & -74.81717 & 38.97924 & $\mathrm{NJ}$ & 4007 & 1.39 & -74.83298 & 38.96867 & $\mathrm{NJ}$ & 4079 & 3.63 & -74.84741 & 38.957 & $\mathrm{NJ}$ \\
\hline 3936 & 1.67 & -74.81747 & 38.97905 & $\mathrm{NJ}$ & 4008 & 1.4 & -74.83324 & 38.96847 & $\mathrm{NJ}$ & 4080 & 3.55 & -74.84767 & 38.95678 & $\mathrm{NJ}$ \\
\hline 3937 & 1.67 & -74.81761 & 38.97895 & $\mathrm{NJ}$ & 4009 & 1.42 & -74.83339 & 38.96835 & $\mathrm{NJ}$ & 4081 & 3.45 & -74.84779 & 38.95667 & $\mathrm{NJ}$ \\
\hline 3938 & 1.67 & -74.81792 & 38.97875 & $\mathrm{NJ}$ & 4010 & 1.45 & -74.83365 & 38.96815 & $\mathrm{NJ}$ & 4082 & 3.42 & -74.84804 & 38.95643 & $\mathrm{NJ}$ \\
\hline 3939 & 1.69 & -74.81802 & 38.97864 & $\mathrm{NJ}$ & 4011 & 1.46 & -74.83382 & 38.96804 & $\mathrm{NJ}$ & 4083 & 3.34 & -74.84814 & 38.95631 & $\mathrm{NJ}$ \\
\hline 3940 & 1.63 & -74.81836 & 38.97847 & $\mathrm{NJ}$ & 4012 & 1.46 & -74.83408 & 38.96785 & $\mathrm{NJ}$ & 4084 & 3.24 & -74.84843 & 38.9561 & $\mathrm{NJ}$ \\
\hline 3941 & 1.61 & -74.81851 & 38.97838 & $\mathrm{NJ}$ & 4013 & 1.47 & -74.83421 & 38.96773 & $\mathrm{NJ}$ & 4085 & 3.16 & -74.84857 & 38.956 & $\mathrm{NJ}$ \\
\hline 3942 & 1.55 & -74.81885 & 38.97822 & $\mathrm{NJ}$ & 4014 & 1.48 & -74.83447 & 38.96752 & $\mathrm{NJ}$ & 4086 & 3.13 & -74.8488 & 38.95574 & $\mathrm{NJ}$ \\
\hline 3943 & 1.53 & -74.81902 & 38.97814 & $\mathrm{NJ}$ & 4015 & 1.48 & -74.83463 & 38.96741 & $\mathrm{NJ}$ & 4087 & 3.05 & -74.84892 & 38.95563 & $\mathrm{NJ}$ \\
\hline 3944 & 1.5 & .74 .81932 & 38.97795 & $\mathrm{NJ}$ & 4016 & 1.5 & -74.83488 & 38.96721 & NJ & 4088 & 2.95 & -74.84921 & 38.95543 & $\mathrm{NJ}$ \\
\hline 3945 & 1.5 & -74.81946 & 38.97785 & $\mathrm{NJ}$ & 4017 & 1.54 & -74.83502 & 38.96708 & $\mathrm{NJ}$ & 4089 & 2.87 & -74.84932 & 38.9553 & $\mathrm{NJ}$ \\
\hline 3946 & 1.49 & -74.81976 & 38.97766 & $\mathrm{NJ}$ & 4018 & 1.58 & -74.83528 & 38.96688 & $\mathrm{NJ}$ & 4090 & 2.77 & -74.84959 & 38.95508 & $\mathrm{NJ}$ \\
\hline 3947 & 1.49 & -74.81992 & 38.97757 & $\mathrm{NJ}$ & 4019 & 1.58 & -74.83543 & 38.96677 & NJ & 4091 & 2.66 & .74 .84972 & 38.95498 & $\mathrm{NJ}$ \\
\hline 3948 & 1.48 & -74.82021 & 38.97737 & $\mathrm{NJ}$ & 4020 & 1.62 & -74.83569 & 38.96657 & $\mathrm{NJ}$ & 4092 & 2.55 & -74.84998 & 38.95475 & $\mathrm{NJ}$ \\
\hline 3949 & 1.46 & -74.82036 & 38.97728 & $\mathrm{NJ}$ & 4021 & 1.67 & -74.83583 & 38.96643 & NJ & 4093 & 2.39 & -74.85011 & 38.95466 & $\mathrm{NJ}$ \\
\hline 3950 & 1.39 & -74.82069 & 38.97713 & $\mathrm{NJ}$ & 4022 & 1.69 & -74.83609 & 38.96624 & NJ & 4094 & 2.21 & -74.8504 & 38.95444 & $\mathrm{NJ}$ \\
\hline 3951 & 1.37 & -74.82086 & 38.97704 & $\mathrm{NJ}$ & 4023 & 1.73 & -74.83624 & 38.96612 & NJ & 4095 & 2.01 & -74.85052 & 38.95434 & $\mathrm{NJ}$ \\
\hline 3952 & 1.34 & -74.82117 & 38.97686 & $\mathrm{NJ}$ & 4024 & 1.79 & -74.8365 & 38.96592 & NJ & 4096 & 1.72 & .74 .85083 & 38.95414 & $\mathrm{NJ}$ \\
\hline 3953 & 1.32 & -74.82133 & 38.97677 & $\mathrm{NJ}$ & 4025 & 1.85 & -74.83664 & 38.96579 & NJ & 4097 & 1.49 & -74.85097 & 38.95405 & $\mathrm{NJ}$ \\
\hline 3954 & 1.3 & .74 .82162 & 38.97658 & $\mathrm{NJ}$ & 4026 & 1.9 & -74.83688 & 38.96558 & NJ & 4098 & 1.23 & .74 .85123 & 38.95381 & $\mathrm{NJ}$ \\
\hline 3955 & 1.29 & -74.82179 & 38.97649 & NJ & 4027 & 1.94 & -74.83704 & 38.96546 & $\mathrm{NJ}$ & 4099 & 1.09 & -74.85135 & 38.95371 & $\mathrm{NJ}$ \\
\hline 3956 & 1.24 & -74.82211 & 38.97632 & $\mathrm{NJ}$ & 4028 & 2.01 & -74.83728 & 38.96525 & NJ & 4100 & 0.94 & -74.85161 & 38.95347 & $\mathrm{NJ}$ \\
\hline 3957 & 1.21 & -74.82228 & 38.97623 & $\mathrm{NJ}$ & 4029 & 2.04 & -74.83743 & 38.96514 & NJ & 4101 & 0.86 & -74.85175 & 38.95337 & $\mathrm{NJ}$ \\
\hline 3958 & 1.18 & -74.82257 & 38.97605 & NJ & 4030 & 2.09 & -74.83769 & 38.96494 & $\mathrm{NJ}$ & 4102 & 0.78 & -74.85199 & 38.95313 & $\mathrm{NJ}$ \\
\hline 3959 & 1.17 & -74.82272 & 38.97595 & $\mathrm{NJ}$ & 4031 & 2.2 & -74.83783 & 38.96481 & $\mathrm{NJ}$ & 4103 & 0.7 & -74.85213 & 38.95304 & $\mathrm{NJ}$ \\
\hline 3960 & 1.15 & -74.82301 & 38.97577 & $\mathrm{NJ}$ & 4032 & 2.28 & -74.83809 & 38.96461 & $\mathrm{NJ}$ & 4104 & 0.58 & -74.85243 & 38.95284 & $\mathrm{NJ}$ \\
\hline
\end{tabular}




\begin{tabular}{|c|c|c|c|c|c|c|c|c|c|c|c|c|c|c|}
\hline TR\# & RATE & LONG & LAT & ST & TR\# & RATE & LONG & LAT & ST & TR\# & RATE & LONG & LAT & ST \\
\hline 4105 & 0.53 & -74.85255 & 38.95273 & NJ & 4177 & 3.93 & -74.86743 & 38.94291 & NJ & 4249 & -2.86 & -74.88924 & 38.93968 & NJ \\
\hline 4106 & 0.46 & -74.85281 & 38.9525 & NJ & 4178 & -1.76 & -74.8754 & 38.94289 & NJ & 4250 & 7.86 & -74.86469 & 38.93962 & NJ \\
\hline 4107 & 0.41 & -74.85294 & 38.9524 & NJ & 4179 & -1.96 & -74.87546 & 38.94287 & NJ & 4251 & -2.91 & -74.88951 & 38.93955 & NJ \\
\hline 4108 & 0.36 & -74.8532 & 38.95216 & NJ & 4180 & -2.33 & -74.87778 & 38.94284 & NJ & 4252 & 8.44 & -74.86458 & 38.93949 & NJ \\
\hline 4109 & 0.34 & -74.85332 & 38.95205 & NJ & 4181 & -2.9 & -74.87836 & 38.94282 & NJ & 4253 & -2.67 & -74.88974 & 38.93943 & NJ \\
\hline 4110 & 0.33 & -74.85356 & 38.9518 & NJ & 4182 & -3.01 & -74.87842 & 38.94281 & $\mathrm{NJ}$ & 4254 & 7.14 & -74.86809 & 38.93938 & $\mathrm{NJ}$ \\
\hline 4111 & 0.33 & -74.85367 & 38.95169 & NJ & 4183 & 5.36 & -74.8631 & 38.94281 & NJ & 4255 & -2.7 & -74.89006 & 38.93928 & NJ \\
\hline 4112 & 0.37 & -74.85391 & 38.95145 & NJ & 4184 & -2.22 & -74.87656 & 38.94274 & NJ & 4256 & -2.53 & -74.89023 & 38.93918 & NJ \\
\hline 4113 & 0.4 & -74.85403 & 38.95134 & NJ & 4185 & -3.29 & -74.8795 & 38.94274 & $\mathrm{NJ}$ & 4257 & 7.71 & -74.86801 & 38.93914 & NJ \\
\hline 4114 & 0.48 & -74.85431 & 38.95111 & NJ & 4186 & -3.13 & -74.87892 & 38.94274 & NJ & 4258 & -2.52 & -74.89059 & 38.939 & NJ \\
\hline 4115 & 0.54 & -74.85442 & 38.951 & NJ & 4187 & -2.25 & -74.8766 & 38.94273 & $\mathrm{NJ}$ & 4259 & -2.38 & -74.89075 & 38.93895 & NJ \\
\hline 4116 & 0.64 & -74.85468 & 38.95076 & NJ & 4188 & -3.13 & -74.87898 & 38.94271 & NJ & 4260 & -2.37 & -74.89116 & 38.9388 & $\mathrm{NJ}$ \\
\hline 4117 & 0.71 & -74.8548 & 38.95065 & NJ & 4189 & -1.95 & -74.87592 & 38.94271 & $\mathrm{NJ}$ & 4261 & -2.27 & -74.89127 & 38.93876 & $\mathrm{NJ}$ \\
\hline 4118 & 0.85 & -74.85506 & 38.95041 & NJ & 4190 & 5.71 & -74.86319 & 38.9427 & NJ & 4262 & 9.52 & -74.86389 & 38.93867 & NJ \\
\hline 4119 & 0.9 & -74.85516 & 38.95031 & NJ & 4191 & -3.46 & -74.88007 & 38.94267 & NJ & 4263 & -2.23 & -74.89171 & 38.93861 & NJ \\
\hline 4120 & 0.96 & -74.85544 & 38.95007 & NJ & 4192 & -3.48 & -74.8808 & 38.94266 & NJ & 4264 & -2.12 & -74.89178 & 38.93857 & $\mathrm{NJ}$ \\
\hline 4121 & 1.02 & -74.85556 & 38.94998 & NJ & 4193 & -3.6 & -74.88065 & 38.94266 & NJ & 4265 & -2.06 & -74.89226 & 38.93837 & $\mathrm{NJ}$ \\
\hline 4122 & 1.13 & -74.85585 & 38.94976 & NJ & 4194 & -2.19 & -74.87712 & 38.94265 & NJ & 4266 & -1.95 & -74.89229 & 38.93833 & NJ \\
\hline 4123 & 1.21 & -74.85596 & 38.94966 & NJ & 4195 & -2.23 & -74.87717 & 38.94264 & $\mathrm{NJ}$ & 4267 & 8.72 & -74.86732 & 38.93831 & $\mathrm{NJ}$ \\
\hline 4124 & 1.3 & -74.85623 & 38.94941 & NJ & 4196 & -3.32 & .74 .87956 & 38.94263 & NJ & 4268 & -1.94 & .74 .89281 & 38.93815 & NJ \\
\hline 4125 & 1.35 & -74.85634 & 38.94932 & NJ & 4197 & 5.89 & -74.86325 & 38.94261 & NJ & 4269 & -1.86 & -74.89279 & 38.93807 & $\mathrm{NJ}$ \\
\hline 4126 & 1.43 & -74.85663 & 38.94909 & NJ & 4198 & -3.75 & -74.88123 & 38.9426 & NJ & 4270 & 9.88 & -74.86333 & 38.93798 & NJ \\
\hline 4127 & 1.5 & -74.85674 & 38.94899 & NJ & 4199 & -3.63 & -74.88138 & 38.94258 & $\mathrm{NJ}$ & 4271 & -1.79 & -74.89336 & 38.93787 & NJ \\
\hline 4128 & 1.58 & -74.85701 & 38.94875 & NJ & 4200 & -3.86 & -74.88177 & 38.94249 & NJ & 4272 & 10.59 & -74.8632 & 38.93784 & NJ \\
\hline 4129 & 1.66 & -74.8571 & 38.94864 & NJ & 4201 & 4.72 & -74.87077 & 38.94249 & NJ & 4273 & 10.96 & -74.86693 & 38.93782 & NJ \\
\hline 4130 & 1.8 & -74.85735 & 38.94837 & NJ & 4202 & -3.82 & -74.88193 & 38.94244 & $\mathrm{NJ}$ & 4274 & -1.72 & -74.89328 & 38.9378 & $\mathrm{NJ}$ \\
\hline 4131 & 1.86 & -74.85745 & 38.94828 & NJ & 4203 & -3.38 & -74.8801 & 38.94243 & $\mathrm{NJ}$ & 4275 & -1.59 & -74.89377 & 38.93753 & NJ \\
\hline 4132 & 1.97 & -74.85776 & 38.94806 & NJ & 4204 & -2.28 & -74.87762 & 38.94239 & NJ & 4276 & -1.6 & -74.89389 & 38.93751 & NJ \\
\hline 4133 & 2.08 & -74.85786 & 38.94796 & NJ & 4205 & -3.95 & -74.88234 & 38.94235 & NJ & 4277 & 9.74 & -74.86664 & 38.93747 & NJ \\
\hline 4134 & 2.26 & -74.85812 & 38.94769 & NJ & 4206 & -3.81 & -74.88249 & 38.94233 & NJ & 4278 & -1.4 & -74.89426 & 38.93725 & NJ \\
\hline 4135 & 2.36 & -74.85822 & 38.9476 & NJ & 4207 & 5.66 & -74.86349 & 38.94233 & $\mathrm{NJ}$ & 4279 & -1.39 & -74.89442 & 38.93715 & NJ \\
\hline 4136 & 2.5 & -74.85847 & 38.94734 & $\mathrm{NJ}$ & 4208 & 6.1 & -74.86351 & 38.9423 & NJ & 4280 & 11.62 & -74.86252 & 38.93702 & $\mathrm{NJ}$ \\
\hline 4137 & 2.6 & -74.85857 & 38.94723 & NJ & 4209 & -3.93 & -74.8829 & 38.94224 & NJ & 4281 & -1.18 & -74.8947 & 38.9369 & NJ \\
\hline 4138 & 2.72 & -74.85881 & 38.94695 & NJ & 4210 & 6.26 & -74.86357 & 38.94222 & NJ & 4282 & 10.81 & -74.86595 & 38.93663 & NJ \\
\hline 4139 & 2.78 & -74.85889 & 38.94685 & NJ & 4211 & -3.77 & -74.88306 & 38.94221 & $\mathrm{NJ}$ & 4283 & 14.21 & -74.86198 & 38.93648 & $\mathrm{NJ}$ \\
\hline 4140 & 2.92 & -74.85916 & 38.94658 & NJ & 4212 & -3.86 & -74.88347 & 38.9421 & NJ & 4284 & 11.74 & -74.86201 & 38.93647 & NJ \\
\hline 4141 & 3.02 & -74.85925 & 38.9465 & NJ & 4213 & -3.78 & -74.88359 & 38.94206 & $\mathrm{NJ}$ & 4285 & -0.76 & -74.8949 & 38.93635 & $\mathrm{NJ}$ \\
\hline 4142 & 3.24 & -74.85954 & 38.94625 & $\mathrm{NJ}$ & 4214 & -3.88 & -74.88403 & 38.94194 & NJ & 4286 & -0.75 & -74.89503 & 38.93629 & $\mathrm{NJ}$ \\
\hline 4143 & 3.36 & -74.85965 & 38.94617 & NJ & 4215 & -3.74 & -74.88412 & 38.9419 & NJ & 4287 & -0.85 & -74.89546 & 38.93623 & $\mathrm{NJ}$ \\
\hline 4144 & 3.49 & -74.85992 & 38.94591 & NJ & 4216 & 6.46 & -74.86382 & 38.94189 & $\mathrm{NJ}$ & 4288 & -0.82 & -74.89558 & 38.93617 & NJ \\
\hline 4145 & 3.61 & -74.86005 & 38.94583 & NJ & 4217 & 5.98 & -74.86388 & 38.94182 & $\mathrm{NJ}$ & 4289 & 11.34 & -74.86554 & 38.93613 & $\mathrm{NJ}$ \\
\hline 4146 & 3.73 & -74.86034 & 38.94558 & NJ & 4218 & 6.64 & -74.86388 & 38.94181 & NJ & 4290 & -0.85 & -74.89603 & 38.93611 & $\mathrm{NJ}$ \\
\hline 4147 & 3.85 & -74.86043 & 38.9455 & NJ & 4219 & -3.81 & -74.88458 & 38.94176 & NJ & 4291 & -0.84 & -74.89613 & 38.93604 & $\mathrm{NJ}$ \\
\hline 4148 & 4 & -74.8607 & 38.94524 & NJ & 4220 & -3.66 & -74.88467 & 38.94173 & NJ & 4292 & -0.87 & -74.89659 & 38.93599 & $\mathrm{NJ}$ \\
\hline 4149 & 4.13 & -74.86079 & 38.94515 & NJ & 4221 & 5.35 & -74.87009 & 38.94165 & NJ & 4293 & -0.85 & -74.8967 & 38.93593 & $\mathrm{NJ}$ \\
\hline 4150 & 4.26 & -74.86108 & 38.94489 & $\mathrm{NJ}$ & 4222 & -3.77 & -74.88515 & 38.94157 & NJ & 4294 & -0.9 & -74.89716 & 38.93587 & $\mathrm{NJ}$ \\
\hline 4151 & 4.34 & -74.86118 & 38.94481 & NJ & 4223 & -3.64 & -74.88519 & 38.94155 & NJ & 4295 & -0.88 & -74.89726 & 38.93581 & NJ \\
\hline 4152 & 4.45 & -74.86147 & 38.94456 & $\mathrm{NJ}$ & 4224 & 6.84 & -74.86414 & 38.94151 & NJ & 4296 & -0.92 & -74.89771 & 38.93572 & NJ \\
\hline 4153 & 1.96 & -74.8688 & 38.94455 & NJ & 4225 & 7.89 & -74.86421 & 38.94144 & NJ & 4297 & -0.89 & -74.89781 & 38.93567 & NJ \\
\hline 4154 & 4.56 & -74.86156 & 38.94447 & NJ & 4226 & -3.72 & -74.8857 & 38.94136 & $\mathrm{NJ}$ & 4298 & -0.94 & -74.89827 & 38.93558 & NJ \\
\hline 4155 & 4.66 & -74.86185 & 38.94421 & NJ & 4227 & -3.6 & -74.88573 & 38.94135 & NJ & 4299 & -0.91 & -74.89836 & 38.93553 & $\mathrm{NJ}$ \\
\hline 4156 & 4.71 & -74.86194 & 38.94413 & NJ & 4228 & 5.91 & -74.86606 & 38.94127 & NJ & 4300 & -1.01 & -74.89867 & 38.93544 & $\mathrm{NJ}$ \\
\hline 4157 & 4.85 & -74.86218 & 38.94384 & $\mathrm{NJ}$ & 4229 & 5.27 & -74.86958 & 38.9412 & NJ & 4301 & -0.95 & -74.89925 & 38.93526 & $\mathrm{NJ}$ \\
\hline 4158 & 4.96 & -74.86227 & 38.94376 & NJ & 4230 & -3.58 & -74.88623 & 38.94114 & $\mathrm{NJ}$ & 4302 & -0.91 & -74.89938 & 38.93522 & NJ \\
\hline 4159 & 4.72 & -74.86229 & 38.94372 & NJ & 4231 & 6.25 & -74.86595 & 38.94114 & NJ & 4303 & -0.89 & -74.89943 & 38.9352 & $\mathrm{NJ}$ \\
\hline 4160 & 2.13 & -74.8726 & 38.94347 & NJ & 4232 & -3.67 & -74.88625 & 38.94113 & NJ & 4304 & -0.93 & -74.89983 & 38.93507 & $\mathrm{NJ}$ \\
\hline 4161 & 5.12 & -74.86253 & 38.94346 & NJ & 4233 & 8.26 & -74.86447 & 38.94112 & $\mathrm{NJ}$ & 4305 & -0.86 & -74.89996 & 38.93502 & NJ \\
\hline 4162 & 5.25 & -74.86261 & 38.94338 & $\mathrm{NJ}$ & 4234 & 8.71 & -74.86453 & 38.94105 & NJ & 4306 & -0.89 & -74.89993 & 38.93502 & NJ \\
\hline 4163 & 1.82 & -74.87315 & 38.94337 & NJ & 4235 & -3.5 & -74.88673 & 38.94092 & NJ & 4307 & -0.48 & -74.89877 & 38.93495 & NJ \\
\hline 4164 & 1.94 & -74.8732 & 38.94336 & NJ & 4236 & -3.62 & -74.8868 & 38.9409 & $\mathrm{NJ}$ & 4308 & -0.4 & -74.8987 & 38.93492 & NJ \\
\hline 4165 & 1.12 & -74.87373 & 38.94327 & NJ & 4237 & 5.78 & -74.86938 & 38.94082 & NJ & 4309 & -0.9 & -74.90041 & 38.93487 & NJ \\
\hline 4166 & 5.05 & -74.8627 & 38.94327 & NJ & 4238 & 9.34 & -74.86481 & 38.94074 & $\mathrm{NJ}$ & 4310 & -0.84 & .74 .9005 & 38.93483 & NJ \\
\hline 4167 & 1.76 & -74.87376 & 38.94326 & NJ & 4239 & -3.34 & -74.88725 & 38.94071 & NJ & 4311 & -0.87 & -74.9005 & 38.93483 & NJ \\
\hline 4168 & 5.24 & -74.86275 & 38.94322 & NJ & 4240 & -3.48 & -74.88734 & 38.94066 & NJ & 4312 & -0.88 & -74.90097 & 38.93467 & NJ \\
\hline 4169 & -0.18 & .74 .8743 & 38.94317 & NJ & 4241 & -3.25 & -74.88777 & 38.94047 & NJ & 4313 & -0.82 & -74.90102 & 38.93465 & NJ \\
\hline 4170 & -0.26 & -74.87433 & 38.94316 & NJ & 4242 & -3.35 & -74.88789 & 38.9404 & NJ & 4314 & -0.85 & -74.90105 & 38.93464 & NJ \\
\hline 4171 & 5.4 & -74.86287 & 38.94309 & NJ & 4243 & 7.31 & -74.86526 & 38.94031 & NJ & 4315 & -0.85 & -74.90155 & 38.93447 & NJ \\
\hline 4172 & -1.05 & -74.87485 & 38.94304 & NJ & 4244 & -3.12 & -74.88824 & 38.94018 & NJ & 4316 & -0.8 & -74.90155 & 38.93447 & $\mathrm{NJ}$ \\
\hline 4173 & -1.68 & -74.87489 & 38.94302 & NJ & 4245 & -3.18 & -74.88843 & 38.94008 & $\mathrm{NJ}$ & 4317 & -0.82 & -74.9016 & 38.93445 & NJ \\
\hline 4174 & 3.32 & -74.87106 & 38.94302 & NJ & 4246 & 6.69 & -74.8687 & 38.93998 & NJ & 4318 & -0.76 & -74.90207 & 38.93429 & NJ \\
\hline 4175 & -2.08 & -74.87605 & 38.94301 & NJ & 4247 & -2.97 & -74.88873 & 38.93993 & NJ & 4319 & -0.81 & -74.90213 & 38.93427 & NJ \\
\hline 4176 & 5.54 & .74 .86295 & 38.943 & NJ & 4248 & -3.01 & -74.88898 & 38.93982 & NJ & 4320 & -0.78 & -74.90216 & 38.93426 & NJ \\
\hline
\end{tabular}




\begin{tabular}{|c|c|c|c|c|c|c|c|c|c|c|c|c|c|c|}
\hline TR\# & RATE & LONG & LAT & ST & TR\# & RATE & LONG & LAT & ST & TR\# & RATE & LONG & LAT & ST \\
\hline 4321 & -0.73 & -74.9026 & 38.9341 & $\mathrm{NJ}$ & 4393 & -1.1 & -74.93558 & 38.93056 & NJ & 23 & -2.39 & -75.08717 & 38.78808 & $\mathrm{DE}$ \\
\hline 4322 & -0.76 & -74.90269 & 38.93407 & $\mathrm{NJ}$ & 4394 & -0.38 & .74 .91595 & 38.93053 & NJ & 24 & -2.36 & -75.0871 & 38.78763 & DE \\
\hline 4323 & -0.74 & -74.90271 & 38.93407 & NJ & 4395 & -0.35 & -74.91653 & 38.93043 & $\mathrm{NJ}$ & 25 & -2.39 & -75.08698 & 38.78719 & $\mathrm{DE}$ \\
\hline 4324 & -0.68 & -74.90314 & 38.93392 & NJ & 4396 & 0.3 & -74.91309 & 38.93026 & $\mathrm{NJ}$ & 26 & -2.55 & -75.08685 & 38.78674 & $\mathrm{DE}$ \\
\hline 4325 & -0.69 & -74.90385 & 38.93368 & $\mathrm{NJ}$ & 4397 & -0.12 & -74.91711 & 38.93013 & NJ & 27 & -2.65 & -75.08675 & 38.7863 & DE \\
\hline 4326 & -0.49 & -74.90327 & 38.93366 & $\mathrm{NJ}$ & 4398 & -0.2 & -74.91942 & 38.93008 & $\mathrm{NJ}$ & 28 & -2.77 & -75.08672 & 38.78585 & DE \\
\hline 4327 & -0.67 & -74.90443 & 38.93349 & $\mathrm{NJ}$ & 4399 & -0.22 & -74.91885 & 38.93008 & $\mathrm{NJ}$ & 29 & -2.87 & -75.08664 & 38.7854 & $\mathrm{DE}$ \\
\hline 4328 & -0.64 & .74 .90501 & 38.9333 & NJ & 4400 & -0.11 & -74.92 & 38.92997 & $\mathrm{NJ}$ & 30 & -2.93 & -75.08658 & 38.78495 & $\mathrm{DE}$ \\
\hline 4329 & -0.59 & -74.90559 & 38.93311 & $\mathrm{NJ}$ & 4401 & 0.04 & -74.91827 & 38.92992 & $\mathrm{NJ}$ & 31 & -2.96 & -75.0865 & 38.78451 & $\mathrm{DE}$ \\
\hline 4330 & -0.55 & -74.90616 & 38.93291 & $\mathrm{NJ}$ & 4402 & 0 & -74.92058 & 38.92983 & NJ & 32 & -3.09 & -75.08647 & 38.78406 & DE \\
\hline 4331 & -0.45 & -74.90674 & 38.93265 & $\mathrm{NJ}$ & 4403 & 0.23 & -74.91769 & 38.92976 & $\mathrm{NJ}$ & 33 & -3.09 & .75 .0864 & 38.78361 & $\mathrm{DE}$ \\
\hline 4332 & -4.24 & -74.94479 & 38.93222 & $\mathrm{NJ}$ & 4404 & 0.15 & -74.92114 & 38.92968 & $\mathrm{NJ}$ & 34 & -3.06 & -75.08633 & 38.78316 & $\mathrm{DE}$ \\
\hline 4333 & -0.35 & -74.90788 & 38.93221 & $\mathrm{NJ}$ & 4405 & 0.21 & -74.92288 & 38.92956 & NJ & 35 & -3.08 & -75.08624 & 38.78272 & $\mathrm{DE}$ \\
\hline 4334 & -4.24 & -74.94537 & 38.93219 & $\mathrm{NJ}$ & 4406 & 0.23 & -74.92346 & 38.92954 & $\mathrm{NJ}$ & 36 & -3.12 & -75.08614 & 38.78227 & $\mathrm{DE}$ \\
\hline 4335 & -4.26 & -74.94595 & 38.93218 & $\mathrm{NJ}$ & 4407 & 0.25 & -74.92807 & 38.92953 & NJ & 37 & -3.14 & -75.08606 & 38.78182 & $\mathrm{DE}$ \\
\hline 4336 & -4.26 & -74.94421 & 38.93218 & NJ & 4408 & 0.26 & -74.92865 & 38.92952 & $\mathrm{NJ}$ & 38 & -3.21 & -75.08595 & 38.78138 & $\mathrm{DE}$ \\
\hline 4337 & -0.46 & .74 .90846 & 38.93214 & NJ & 4409 & 0.23 & .74 .92923 & 38.92951 & $\mathrm{NJ}$ & 39 & -3.19 & -75.08582 & 38.78094 & $\mathrm{DE}$ \\
\hline 4338 & -4.22 & -74.94653 & 38.93213 & NJ & 4410 & 0.27 & -74.92751 & 38.92951 & NJ & 40 & -3.26 & -75.08569 & 38.78049 & $\mathrm{DE}$ \\
\hline 4339 & -4.15 & -74.9471 & 38.93209 & $\mathrm{NJ}$ & 4411 & 0.24 & -74.92232 & 38.92948 & NJ & 41 & -3.29 & -75.08557 & 38.78005 & $\mathrm{DE}$ \\
\hline 4340 & -4.21 & -74.94363 & 38.93209 & $\mathrm{NJ}$ & 4412 & 0.31 & -74.92172 & 38.92948 & $\mathrm{NJ}$ & 42 & -3.31 & -75.08543 & 38.7796 & $\mathrm{DE}$ \\
\hline 4341 & -4.08 & -74.94768 & 38.93208 & $\mathrm{NJ}$ & 4413 & 0.32 & -74.92979 & 38.92947 & NJ & 43 & -3.33 & -75.08528 & 38.77916 & $\mathrm{DE}$ \\
\hline 4342 & -4.16 & -74.94307 & 38.93206 & NJ & 4414 & 0.32 & -74.92404 & 38.92946 & NJ & 44 & -3.29 & -75.08511 & 38.77872 & $\mathrm{DE}$ \\
\hline 4343 & -4.02 & -74.94826 & 38.93204 & $\mathrm{NJ}$ & 4415 & 0.47 & -74.92693 & 38.92945 & NJ & 45 & -3.3 & -75.08496 & 38.77828 & $\mathrm{DE}$ \\
\hline 4344 & -4.11 & -74.94249 & 38.93202 & NJ & 4416 & 0.43 & -74.93037 & 38.92935 & NJ & 46 & -3.3 & -75.08479 & 38.77784 & $\mathrm{DE}$ \\
\hline 4345 & -3.93 & -74.94884 & 38.93198 & NJ & 4417 & 0.43 & -74.92461 & 38.92935 & NJ & 47 & -3.19 & -75.08463 & 38.7774 & $\mathrm{DE}$ \\
\hline 4346 & -4.04 & -74.94191 & 38.93198 & NJ & 4418 & 0.51 & -74.92635 & 38.92932 & NJ & 48 & -3.09 & -75.08443 & 38.77696 & $\mathrm{DE}$ \\
\hline 4347 & -3.94 & -74.94135 & 38.93195 & $\mathrm{NJ}$ & 4419 & 0.59 & -74.92519 & 38.92926 & $\mathrm{NJ}$ & 49 & -3.03 & -75.08426 & 38.77652 & $\mathrm{DE}$ \\
\hline 4348 & -0.44 & -74.90906 & 38.93194 & $\mathrm{NJ}$ & 4420 & 0.52 & -74.93153 & 38.92922 & NJ & 50 & -3 & -75.08408 & 38.77608 & $\mathrm{DE}$ \\
\hline 4349 & -3.84 & -74.94942 & 38.93192 & $\mathrm{NJ}$ & 4421 & 0.52 & -74.93095 & 38.92921 & NJ & 51 & -2.94 & -75.08391 & 38.77564 & $\mathrm{DE}$ \\
\hline 4350 & -3.85 & -74.94077 & 38.9319 & $\mathrm{NJ}$ & 4422 & 0.78 & -74.92577 & 38.92917 & NJ & 52 & -2.85 & -75.08369 & 38.7752 & $\mathrm{DE}$ \\
\hline 4351 & -3.7 & -74.95 & 38.93188 & $\mathrm{NJ}$ & 4423 & 0.58 & -74.93211 & 38.92914 & NJ & 53 & -2.78 & -75.08354 & 38.77476 & $\mathrm{DE}$ \\
\hline 4352 & -3.57 & -74.95056 & 38.93184 & $\mathrm{NJ}$ & 4424 & 0.67 & -74.93269 & 38.92903 & NJ & 54 & -2.7 & -75.08339 & 38.77431 & $\mathrm{DE}$ \\
\hline 4353 & -3.8 & .74 .94019 & 38.93183 & $\mathrm{NJ}$ & 4425 & 0.74 & -74.93326 & 38.92893 & NJ & 55 & -2.63 & -75.08324 & 38.77387 & $\mathrm{DE}$ \\
\hline 4354 & -3.55 & -74.95114 & 38.93181 & $\mathrm{NJ}$ & 4426 & 0.83 & -74.93384 & 38.92883 & $\mathrm{NJ}$ & 56 & -2.54 & -75.08302 & 38.77343 & $\mathrm{DE}$ \\
\hline 4355 & -3.71 & -74.93961 & 38.93176 & NJ & 4427 & 0.89 & -74.93442 & 38.92875 & NJ & 57 & -2.51 & -75.08289 & 38.77299 & $\mathrm{DE}$ \\
\hline 4356 & -3.51 & -74.95172 & 38.93175 & $\mathrm{NJ}$ & 4428 & 1.36 & -74.935 & 38.92823 & NJ & 58 & -2.54 & -75.08284 & 38.77254 & $\mathrm{DE}$ \\
\hline 4357 & -0.43 & -74.90962 & 38.93175 & $\mathrm{NJ}$ & 4429 & -0.7 & -74.98333 & 39.01667 & NJ & 59 & -2.49 & -75.08276 & 38.7721 & $\mathrm{DE}$ \\
\hline 4358 & -3.61 & -74.93903 & 38.93171 & NJ & 4430 & 0.3 & -74.96667 & 39.06667 & $\mathrm{NJ}$ & 60 & -2.38 & -75.08261 & 38.77165 & $\mathrm{DE}$ \\
\hline 4359 & -3.41 & -74.95229 & 38.9317 & NJ & 4431 & -0.1 & -74.93333 & 39.11666 & NJ & 61 & -2.29 & -75.08244 & 38.77121 & $\mathrm{DE}$ \\
\hline 4360 & -3.47 & -74.93845 & 38.93168 & NJ & 4432 & -2 & -74.91666 & 39.16667 & $\mathrm{NJ}$ & 62 & -2.25 & -75.08241 & 38.77076 & $\mathrm{DE}$ \\
\hline 4361 & -3.31 & -74.95287 & 38.93161 & $\mathrm{NJ}$ & 4433 & -2 & -74.95 & 39.21667 & NJ & 63 & -2.27 & -75.08247 & 38.77031 & $\mathrm{DE}$ \\
\hline 4362 & -3.28 & -74.93788 & 38.9316 & $\mathrm{NJ}$ & 4434 & -2 & .75 & 39.21667 & NJ & 64 & -2.3 & -75.08247 & 38.76985 & $\mathrm{DE}$ \\
\hline 4363 & -3.18 & -74.95345 & 38.93156 & $\mathrm{NJ}$ & 4435 & -0.1 & -75.05 & 39.21667 & NJ & 65 & -2.28 & -75.08247 & 38.7694 & $\mathrm{DE}$ \\
\hline 4364 & -0.37 & -74.9102 & 38.93155 & $\mathrm{NJ}$ & 4436 & -3 & -75.1 & 39.21667 & NJ & 66 & -2.31 & -75.0825 & 38.76895 & $\mathrm{DE}$ \\
\hline 4365 & 0.48 & -74.90732 & 38.93155 & $\mathrm{NJ}$ & 4437 & -3 & -75.15 & 39.21667 & $\mathrm{NJ}$ & 67 & -2.26 & -75.08247 & 38.7685 & $\mathrm{DE}$ \\
\hline 4366 & -3.08 & -74.95403 & 38.9315 & $\mathrm{NJ}$ & 4438 & -3 & -75.2 & 39.21667 & $\mathrm{NJ}$ & 68 & -2.23 & -75.08237 & 38.76805 & $\mathrm{DE}$ \\
\hline 4367 & -2.63 & -74.95747 & 38.93145 & $\mathrm{NJ}$ & 4439 & -3 & -75.2 & 39.26667 & NJ & 69 & -2.16 & -75.08223 & 38.76761 & $\mathrm{DE}$ \\
\hline 4368 & -2.91 & -74.95461 & 38.93145 & $\mathrm{NJ}$ & 4440 & -3 & -75.25 & 39.3 & $\mathrm{NJ}$ & 70 & -1.98 & -75.08194 & 38.76718 & $\mathrm{DE}$ \\
\hline 4369 & -3.06 & -74.9373 & 38.93145 & $\mathrm{NJ}$ & 4441 & -3 & -75.3 & 39.31667 & $\mathrm{NJ}$ & 71 & -1.95 & -75.08191 & 38.76673 & $\mathrm{DE}$ \\
\hline 4370 & -2.49 & .74 .95805 & 38.93144 & NJ & 4442 & -3 & -75.43333 & 39.25 & NJ & 72 & -1.9 & -75.08188 & 38.76628 & $\mathrm{DE}$ \\
\hline 4371 & -2.72 & -74.95691 & 38.93142 & NJ & 1 & -3 & -75.43333 & 39.2 & $\mathrm{DE}$ & 73 & -1.82 & -75.0818 & 38.76583 & $\mathrm{DE}$ \\
\hline 4372 & -2.33 & -74.95863 & 38.93141 & NJ & 2 & -3 & -75.43333 & 39.15 & $\mathrm{DE}$ & 74 & -1.83 & -75.0818 & 38.76538 & $\mathrm{DE}$ \\
\hline 4373 & -0.36 & -74.91077 & 38.93136 & $\mathrm{NJ}$ & 3 & -1.5 & -75.43333 & 39.1 & $\mathrm{DE}$ & 75 & -1.92 & -75.08182 & 38.76493 & $\mathrm{DE}$ \\
\hline 4374 & -2.8 & -74.95518 & 38.93134 & $\mathrm{NJ}$ & 4 & -1.5 & -75.43333 & 39.05 & $\mathrm{DE}$ & 76 & -1.97 & -75.08182 & 38.76447 & $\mathrm{DE}$ \\
\hline 4375 & -2.14 & -74.95921 & 38.93133 & $\mathrm{NJ}$ & 5 & -1.5 & -75.35 & 39.05 & $\mathrm{DE}$ & 77 & -1.95 & -75.08174 & 38.76403 & $\mathrm{DE}$ \\
\hline 4376 & -1.92 & -74.95979 & 38.93124 & $\mathrm{NJ}$ & 6 & -1.5 & -75.33334 & 39 & $\mathrm{DE}$ & 78 & -2.07 & -75.08182 & 38.76357 & $\mathrm{DE}$ \\
\hline 4377 & -2.64 & -74.93672 & 38.93124 & $\mathrm{NJ}$ & 7 & -1.5 & -75.33334 & 38.95 & $\mathrm{DE}$ & 79 & -2.15 & -75.08186 & 38.76311 & $\mathrm{DE}$ \\
\hline 4378 & -2.73 & -74.95575 & 38.93123 & $\mathrm{NJ}$ & 8 & -1.5 & -75.3 & 38.9 & $\mathrm{DE}$ & 80 & -2.25 & -75.08192 & 38.76266 & $\mathrm{DE}$ \\
\hline 4379 & -0.34 & -74.91135 & 38.93122 & $\mathrm{NJ}$ & 9 & -1.5 & -75.25 & 38.86666 & $\mathrm{DE}$ & 81 & -2.31 & -75.08195 & 38.7622 & $\mathrm{DE}$ \\
\hline 4380 & -2.72 & -74.95633 & 38.93121 & $\mathrm{NJ}$ & 10 & -1.5 & -75.2 & 38.83333 & $\mathrm{DE}$ & 82 & -2.35 & -75.082 & 38.76175 & $\mathrm{DE}$ \\
\hline 4381 & -1.65 & .74 .96037 & 38.93111 & $\mathrm{NJ}$ & 11 & NA & -75.15 & 38.81667 & $\mathrm{DE}$ & 83 & -2.35 & -75.082 & 38.7613 & $\mathrm{DE}$ \\
\hline 4382 & -0.34 & -74.91193 & 38.9311 & $\mathrm{NJ}$ & 12 & -0.96 & -75.08836 & 38.79297 & $\mathrm{DE}$ & 84 & -2.42 & -75.08203 & 38.76084 & $\mathrm{DE}$ \\
\hline 4383 & -2.19 & -74.93616 & 38.93097 & $\mathrm{NJ}$ & 13 & -1.37 & -75.08823 & 38.79253 & $\mathrm{DE}$ & 85 & -2.5 & -75.08208 & 38.76039 & $\mathrm{DE}$ \\
\hline 4384 & -1.34 & -74.96094 & 38.93095 & $\mathrm{NJ}$ & 14 & -1.57 & -75.0881 & 38.79208 & $\mathrm{DE}$ & 86 & -2.47 & -75.08208 & 38.75994 & $\mathrm{DE}$ \\
\hline 4385 & -0.24 & -74.91251 & 38.93092 & $\mathrm{NJ}$ & 15 & -1.77 & -75.088 & 38.79164 & $\mathrm{DE}$ & 87 & -2.49 & -75.08205 & 38.75949 & $\mathrm{DE}$ \\
\hline 4386 & -0.7 & -74.9621 & 38.93091 & NJ & 16 & -1.93 & -75.08788 & 38.7912 & $\mathrm{DE}$ & 88 & -2.53 & -75.08206 & 38.75903 & $\mathrm{DE}$ \\
\hline 4387 & -0.41 & -74.91367 & 38.93087 & $\mathrm{NJ}$ & 17 & -2.01 & -75.08775 & 38.79075 & $\mathrm{DE}$ & 89 & -2.54 & -75.08203 & 38.75858 & $\mathrm{DE}$ \\
\hline 4388 & -0.95 & -74.96152 & 38.93086 & $\mathrm{NJ}$ & 18 & -2.08 & -75.08765 & 38.79031 & $\mathrm{DE}$ & 90 & -2.54 & -75.08195 & 38.75814 & $\mathrm{DE}$ \\
\hline 4389 & -0.37 & -74.96268 & 38.93085 & $\mathrm{NJ}$ & 19 & -2.11 & -75.08755 & 38.78986 & $\mathrm{DE}$ & 91 & -2.54 & -75.08191 & 38.75769 & $\mathrm{DE}$ \\
\hline 4390 & -0.42 & -74.91423 & 38.93079 & $\mathrm{NJ}$ & 20 & -2.18 & -75.08743 & 38.78942 & $\mathrm{DE}$ & 92 & -2.48 & .75 .08179 & 38.75724 & $\mathrm{DE}$ \\
\hline 4391 & -0.43 & -74.91481 & 38.9307 & NJ & 21 & -2.24 & -75.08736 & 38.78897 & $\mathrm{DE}$ & 93 & -2.45 & -75.08173 & 38.7568 & $\mathrm{DE}$ \\
\hline 4392 & -0.42 & .74 .91539 & 38.93061 & $\mathrm{NJ}$ & 22 & -2.28 & .75 .08727 & 38.78852 & $\mathrm{DE}$ & 94 & -2.41 & -75.08162 & 38.75635 & $\mathrm{DE}$ \\
\hline
\end{tabular}




\begin{tabular}{|c|c|c|c|c|c|c|c|c|c|c|c|c|c|c|}
\hline TR\# & RATE & LONG & LAT & ST & TR\# & RATE & LONG & LAT & ST & TR\# & RATE & LONG & LAT & ST \\
\hline 95 & -2.32 & -75.08147 & 38.75591 & $\overline{D E}$ & 167 & -0.94 & -75.07701 & 38.72366 & $\mathrm{DE}$ & 239 & -0.71 & -75.07169 & 38.69133 & $\mathrm{DE}$ \\
\hline 96 & -2.19 & -75.08127 & 38.75547 & $\mathrm{DE}$ & 168 & -0.92 & -75.07693 & 38.72321 & $\mathrm{DE}$ & 240 & -0.73 & -75.07156 & 38.69089 & $\mathrm{DE}$ \\
\hline 97 & -2.1 & -75.08118 & 38.75502 & $\mathrm{DE}$ & 169 & -0.85 & -75.07681 & 38.72277 & $\mathrm{DE}$ & 241 & -0.72 & -75.07146 & 38.69045 & $\mathrm{DE}$ \\
\hline 98 & -2.08 & -75.08115 & 38.75457 & $\mathrm{DE}$ & 170 & -0.87 & -75.07672 & 38.72232 & $\mathrm{DE}$ & 242 & -0.69 & -75.07137 & 38.69 & $\mathrm{DE}$ \\
\hline 99 & -2.1 & -75.08113 & 38.75412 & DE & 171 & -0.87 & -75.07666 & 38.72187 & DE & 243 & -0.69 & -75.07123 & 38.68956 & $\mathrm{DE}$ \\
\hline 100 & -2.25 & -75.08119 & 38.75367 & DE & 172 & -0.86 & -75.0766 & 38.72143 & $\mathrm{DE}$ & 244 & -0.69 & -75.07112 & 38.68911 & DE \\
\hline 101 & -2.16 & -75.08113 & 38.75322 & $\mathrm{DE}$ & 173 & -0.84 & -75.07652 & 38.72098 & $\mathrm{DE}$ & 245 & -0.61 & -75.07098 & 38.68867 & $\mathrm{DE}$ \\
\hline 102 & -2.19 & -75.08112 & 38.75277 & $\mathrm{DE}$ & 174 & -0.83 & -75.07639 & 38.72054 & $\mathrm{DE}$ & 246 & -0.63 & -75.07088 & 38.68823 & $\mathrm{DE}$ \\
\hline 103 & -2.18 & -75.08107 & 38.75232 & $\mathrm{DE}$ & 175 & -0.85 & -75.07637 & 38.72008 & $\mathrm{DE}$ & 247 & -0.64 & -75.07074 & 38.68779 & $\mathrm{DE}$ \\
\hline 104 & -2.16 & -75.08101 & 38.75187 & $\mathrm{DE}$ & 176 & -0.82 & -75.07629 & 38.71964 & $\mathrm{DE}$ & 248 & -0.66 & -75.07062 & 38.68734 & $\mathrm{DE}$ \\
\hline 105 & -2.16 & -75.08101 & 38.75142 & DE & 177 & -0.82 & -75.07623 & 38.71919 & $\mathrm{DE}$ & 249 & -0.71 & -75.07053 & 38.6869 & $\mathrm{DE}$ \\
\hline 106 & -2.19 & -75.08096 & 38.75097 & DE & 178 & -0.76 & -75.07611 & 38.71875 & $\mathrm{DE}$ & 250 & -0.71 & -75.07039 & 38.68645 & $\mathrm{DE}$ \\
\hline 107 & -2.21 & -75.08093 & 38.75052 & DE & 179 & -0.76 & -75.07602 & 38.7183 & $\mathrm{DE}$ & 251 & -0.71 & -75.07027 & 38.68601 & $\mathrm{DE}$ \\
\hline 108 & -2.21 & -75.0809 & 38.75007 & DE & 180 & -0.75 & -75.07596 & 38.71785 & DE & 252 & -0.71 & -75.07014 & 38.68557 & $\mathrm{DE}$ \\
\hline 109 & -2.2 & -75.08086 & 38.74962 & $\mathrm{DE}$ & 181 & -0.82 & -75.07591 & 38.7174 & $\mathrm{DE}$ & 253 & -0.77 & -75.07005 & 38.68512 & $\mathrm{DE}$ \\
\hline 110 & -2.22 & -75.08086 & 38.74917 & $\mathrm{DE}$ & 182 & -0.84 & -75.07585 & 38.71696 & $\mathrm{DE}$ & 254 & -0.79 & -75.06996 & 38.68468 & $\mathrm{DE}$ \\
\hline 111 & -2.2 & -75.08089 & 38.74872 & DE & 183 & -0.79 & -75.07571 & 38.71651 & $\mathrm{DE}$ & 255 & -0.72 & -75.06982 & 38.68423 & $\mathrm{DE}$ \\
\hline 112 & -2.26 & -75.08086 & 38.74827 & $\mathrm{DE}$ & 184 & -0.78 & -75.07567 & 38.71606 & $\mathrm{DE}$ & 256 & -0.67 & -75.06976 & 38.68379 & $\mathrm{DE}$ \\
\hline 113 & -2.23 & -75.08083 & 38.74782 & $D E$ & 185 & -0.75 & -75.07559 & 38.71562 & $D E$ & 257 & -0.66 & -75.06966 & 38.68334 & $\mathrm{DE}$ \\
\hline 114 & -2.21 & -75.0808 & 38.74737 & $\mathrm{DE}$ & 186 & -0.71 & -75.0755 & 38.71517 & $\mathrm{DE}$ & 258 & -0.66 & -75.0696 & 38.68289 & $\mathrm{DE}$ \\
\hline 115 & -2.25 & -75.08078 & 38.74691 & $\mathrm{DE}$ & 187 & -0.64 & -75.07542 & 38.71472 & $\mathrm{DE}$ & 259 & -0.69 & -75.06953 & 38.68245 & $D E$ \\
\hline 116 & -2.21 & -75.08075 & 38.74646 & $\mathrm{DE}$ & 188 & -0.69 & .75 .07542 & 38.71427 & $\mathrm{DE}$ & 260 & -0.67 & -75.06947 & 38.682 & $\mathrm{DE}$ \\
\hline 117 & -2.18 & -75.08072 & 38.74601 & $\mathrm{DE}$ & 189 & -0.66 & .75 .07536 & 38.71382 & $\mathrm{DE}$ & 261 & -0.66 & .75 .0694 & 38.68155 & $\mathrm{DE}$ \\
\hline 118 & -2.15 & -75.0807 & 38.74556 & $\mathrm{DE}$ & 190 & -0.62 & -75.07527 & 38.71338 & $\mathrm{DE}$ & 262 & -0.7 & -75.06934 & 38.6811 & $\mathrm{DE}$ \\
\hline 119 & -2.17 & -75.08064 & 38.74512 & $\mathrm{DE}$ & 191 & -0.64 & -75.07524 & 38.71293 & $D E$ & 263 & -0.71 & -75.06927 & 38.68066 & $\mathrm{DE}$ \\
\hline 120 & -2.08 & -75.08058 & 38.74467 & $\mathrm{DE}$ & 192 & -0.64 & -75.07516 & 38.71234 & $\mathrm{DE}$ & 264 & -0.68 & -75.0692 & 38.68021 & $\mathrm{DE}$ \\
\hline 121 & -2.06 & -75.08054 & 38.74422 & $\mathrm{DE}$ & 193 & -0.59 & -75.07507 & 38.71189 & $\mathrm{DE}$ & 265 & -0.66 & -75.06912 & 38.67976 & $\mathrm{DE}$ \\
\hline 122 & -2.08 & -75.08051 & 38.74377 & $\mathrm{DE}$ & 194 & -0.57 & -75.075 & 38.71145 & $\mathrm{DE}$ & 266 & -0.7 & -75.06903 & 38.67932 & $\mathrm{DE}$ \\
\hline 123 & -2.07 & .75 .08043 & 38.74332 & $\mathrm{DE}$ & 195 & -0.56 & -75.07494 & 38.711 & $\mathrm{DE}$ & 267 & -0.69 & -75.06894 & 38.67887 & $\mathrm{DE}$ \\
\hline 124 & -2.04 & .75 .08037 & 38.74287 & $\mathrm{DE}$ & 196 & -0.58 & -75.07489 & 38.71055 & $\mathrm{DE}$ & 268 & -0.64 & -75.06883 & 38.67843 & $\mathrm{DE}$ \\
\hline 125 & -2.01 & -75.08032 & 38.74242 & $\mathrm{DE}$ & 197 & -0.56 & -75.07481 & 38.7101 & $\mathrm{DE}$ & 269 & -0.65 & -75.06876 & 38.67798 & $\mathrm{DE}$ \\
\hline 126 & -2.01 & -75.08026 & 38.74198 & $D E$ & 198 & -0.58 & -75.07478 & 38.70965 & $\mathrm{DE}$ & 270 & -0.61 & -75.06865 & 38.67754 & $\mathrm{DE}$ \\
\hline 127 & -1.99 & .75 .08022 & 38.74153 & $\mathrm{DE}$ & 199 & -0.57 & -75.07475 & 38.7092 & $\mathrm{DE}$ & 271 & -0.65 & -75.06857 & 38.67709 & $\mathrm{DE}$ \\
\hline 128 & -1.96 & -75.08017 & 38.74108 & $\mathrm{DE}$ & 200 & -0.58 & -75.07471 & 38.70875 & $\mathrm{DE}$ & 272 & -0.63 & -75.06848 & 38.67664 & $\mathrm{DE}$ \\
\hline 129 & -1.96 & -75.08012 & 38.74063 & $\mathrm{DE}$ & 201 & -0.59 & -75.07468 & 38.7083 & $D E$ & 273 & -0.62 & -75.06839 & 38.6762 & $\mathrm{DE}$ \\
\hline 130 & -1.96 & -75.08006 & 38.74018 & $\mathrm{DE}$ & 202 & -0.61 & -75.07465 & 38.70785 & $\mathrm{DE}$ & 274 & -0.64 & -75.06831 & 38.67575 & $\mathrm{DE}$ \\
\hline 131 & -1.93 & -75.08002 & 38.73973 & $\mathrm{DE}$ & 203 & -0.61 & -75.0746 & 38.7074 & $\mathrm{DE}$ & 275 & -0.62 & -75.06824 & 38.6753 & $\mathrm{DE}$ \\
\hline 132 & -1.93 & -75.07997 & 38.73928 & $\mathrm{DE}$ & 204 & -0.61 & -75.07455 & 38.70695 & $\mathrm{DE}$ & 276 & -0.62 & -75.06815 & 38.67486 & $\mathrm{DE}$ \\
\hline 133 & -1.88 & -75.07986 & 38.73884 & $\mathrm{DE}$ & 205 & -0.61 & .75 .07451 & 38.70651 & $\mathrm{DE}$ & 277 & -0.66 & -75.0681 & 38.67441 & $\mathrm{DE}$ \\
\hline 134 & -1.87 & -75.07977 & 38.73839 & $\mathrm{DE}$ & 206 & -0.64 & -75.07446 & 38.70606 & $\mathrm{DE}$ & 278 & -0.67 & .75 .06799 & 38.67396 & $D E$ \\
\hline 135 & -1.67 & -75.07944 & 38.73796 & $\mathrm{DE}$ & 207 & -0.64 & .75 .0744 & 38.70561 & $\mathrm{DE}$ & 279 & -0.64 & -75.06795 & 38.67352 & $\mathrm{DE}$ \\
\hline 136 & -1.71 & -75.07948 & 38.73751 & $\mathrm{DE}$ & 208 & -0.65 & -75.07434 & 38.70516 & $\mathrm{DE}$ & 280 & -0.62 & -75.06786 & 38.67307 & $\mathrm{DE}$ \\
\hline 137 & -1.73 & -75.07947 & 38.73706 & $\mathrm{DE}$ & 209 & -0.62 & -75.0743 & 38.70471 & $\mathrm{DE}$ & 281 & -0.65 & -75.06778 & 38.67262 & $\mathrm{DE}$ \\
\hline 138 & -1.71 & -75.07944 & 38.73661 & $\mathrm{DE}$ & 210 & -0.63 & .75 .07425 & 38.70426 & $\mathrm{DE}$ & 282 & -0.64 & .75 .06767 & 38.67218 & $\mathrm{DE}$ \\
\hline 139 & -1.71 & -75.07939 & 38.73616 & $\mathrm{DE}$ & 211 & -0.63 & -75.07417 & 38.70382 & $\mathrm{DE}$ & 283 & -0.59 & -75.06761 & 38.67173 & $\mathrm{DE}$ \\
\hline 140 & -1.68 & -75.07938 & 38.73571 & $\mathrm{DE}$ & 212 & -0.62 & -75.0741 & 38.70337 & $\mathrm{DE}$ & 284 & -0.59 & -75.06752 & 38.67129 & $\mathrm{DE}$ \\
\hline 141 & -1.64 & -75.07935 & 38.73526 & $D E$ & 213 & -0.64 & -75.07408 & 38.70292 & $\mathrm{DE}$ & 285 & -0.65 & -75.06746 & 38.67084 & $\mathrm{DE}$ \\
\hline 142 & -1.6 & -75.07928 & 38.73481 & DE & 214 & -0.63 & -75.07394 & 38.70248 & DE & 286 & -0.69 & -75.0674 & 38.67039 & $\mathrm{DE}$ \\
\hline 143 & -1.63 & -75.07925 & 38.73436 & $\mathrm{DE}$ & 215 & -0.59 & .75 .07384 & 38.70203 & $\mathrm{DE}$ & 287 & -0.68 & -75.06735 & 38.66994 & $\mathrm{DE}$ \\
\hline 144 & -1.58 & -75.07916 & 38.73391 & $D E$ & 216 & -0.59 & -75.07378 & 38.70158 & $\mathrm{DE}$ & 288 & -0.66 & -75.06728 & 38.66949 & $\mathrm{DE}$ \\
\hline 145 & -1.52 & -75.07912 & 38.73346 & $\mathrm{DE}$ & 217 & -0.56 & -75.07368 & 38.70114 & $\mathrm{DE}$ & 289 & -0.7 & -75.06725 & 38.66904 & $\mathrm{DE}$ \\
\hline 146 & -1.46 & -75.07903 & 38.73302 & $\mathrm{DE}$ & 218 & -0.6 & -75.07362 & 38.70069 & $\mathrm{DE}$ & 290 & -0.69 & -75.06718 & 38.6686 & DE \\
\hline 147 & -1.39 & -75.07889 & 38.73257 & $\mathrm{DE}$ & 219 & -0.61 & -75.07355 & 38.70024 & DE & 291 & -0.71 & -75.06715 & 38.66815 & $D E$ \\
\hline 148 & -1.34 & -75.07872 & 38.73213 & $\mathrm{DE}$ & 220 & -0.62 & .75 .07344 & 38.6998 & DE & 292 & -0.69 & -75.06709 & 38.6677 & DE \\
\hline 149 & -1.22 & .75 .07851 & 38.7317 & $\mathrm{DE}$ & 221 & -0.62 & .75 .07336 & 38.69935 & $D E$ & 293 & -0.71 & -75.06706 & 38.66725 & $\mathrm{DE}$ \\
\hline 150 & -1.15 & -75.07837 & 38.73125 & $\mathrm{DE}$ & 222 & -0.62 & -75.07329 & 38.69891 & $\mathrm{DE}$ & 294 & -0.69 & -75.06699 & 38.6668 & $\mathrm{DE}$ \\
\hline 151 & -1.17 & -75.07831 & 38.7308 & DE & 223 & -0.57 & -75.07317 & 38.69846 & $\mathrm{DE}$ & 295 & -0.66 & -75.06696 & 38.66635 & DE \\
\hline 152 & -1.17 & -75.07828 & 38.73036 & $\mathrm{DE}$ & 224 & -0.59 & -75.07307 & 38.69802 & DE & 296 & -0.67 & -75.06691 & 38.6659 & $\mathrm{DE}$ \\
\hline 153 & -1.13 & -75.0782 & 38.72991 & $\mathrm{DE}$ & 225 & -0.58 & -75.07297 & 38.69757 & DE & 297 & -0.66 & -75.0668 & 38.66546 & DE \\
\hline 154 & -1.14 & -75.07816 & 38.72946 & $\mathrm{DE}$ & 226 & -0.6 & -75.07292 & 38.69712 & $\mathrm{DE}$ & 298 & -0.61 & -75.06676 & 38.66501 & $D E$ \\
\hline 155 & -1.13 & -75.07809 & 38.72901 & $\mathrm{DE}$ & 227 & -0.57 & -75.07281 & 38.69668 & DE & 299 & -0.59 & -75.06673 & 38.66456 & DE \\
\hline 156 & -1.1 & -75.07799 & 38.72857 & $\mathrm{DE}$ & 228 & -0.6 & -75.07274 & 38.69623 & DE & 300 & -0.55 & -75.06664 & 38.66411 & $\mathrm{DE}$ \\
\hline 157 & -1.02 & .75 .07785 & 38.72812 & DE & 229 & -0.59 & -75.07263 & 38.69579 & $\mathrm{DE}$ & 301 & -0.51 & -75.06654 & 38.66367 & $D E$ \\
\hline 158 & -1.05 & -75.07774 & 38.72768 & DE & 230 & -0.59 & -75.07257 & 38.69534 & DE & 302 & -0.53 & -75.06651 & 38.66322 & DE \\
\hline 159 & -1.09 & -75.0777 & 38.72723 & $D E$ & 231 & -0.59 & -75.07248 & 38.69489 & DE & 303 & -0.49 & -75.06641 & 38.66277 & $\mathrm{DE}$ \\
\hline 160 & -1.05 & -75.07762 & 38.72678 & $\mathrm{DE}$ & 232 & -0.58 & -75.07239 & 38.69445 & DE & 304 & -0.5 & -75.06638 & 38.66232 & $\mathrm{DE}$ \\
\hline 161 & -1.03 & -75.0775 & 38.72634 & $\mathrm{DE}$ & 233 & -0.56 & -75.0723 & 38.694 & DE & 305 & -0.52 & -75.06636 & 38.66187 & DE \\
\hline 162 & -0.99 & -75.07739 & 38.72589 & $\mathrm{DE}$ & 234 & -0.62 & -75.07224 & 38.69356 & $\mathrm{DE}$ & 306 & -0.5 & -75.06633 & 38.66142 & DE \\
\hline 163 & -1.02 & -75.07735 & 38.72544 & $\mathrm{DE}$ & 235 & -0.62 & -75.0721 & 38.69311 & $\mathrm{DE}$ & 307 & -0.49 & -75.06631 & 38.66097 & DE \\
\hline 164 & -0.99 & -75.07729 & 38.725 & $\mathrm{DE}$ & 236 & -0.65 & -75.07202 & 38.69267 & $\mathrm{DE}$ & 308 & -0.51 & -75.06625 & 38.66052 & $\mathrm{DE}$ \\
\hline 165 & -1 & .75 .07721 & 38.72455 & $\mathrm{DE}$ & 237 & -0.65 & -75.07188 & 38.69222 & $\mathrm{DE}$ & 309 & -0.48 & -75.06619 & 38.66007 & DE \\
\hline 166 & -0.93 & -75.07706 & 38.72411 & $\mathrm{DE}$ & 238 & -0.67 & -75.07179 & 38.69178 & DE & 310 & -0.49 & -75.06615 & 38.65962 & DE \\
\hline
\end{tabular}




\begin{tabular}{|c|c|c|c|c|c|c|c|c|c|c|c|c|c|c|}
\hline TR\# & RATE & LONG & LAT & ST & TR\# & RATE & LONG & LAT & ST & TR\# & RATE & LONG & LAT & ST \\
\hline 311 & -0.47 & -75.06606 & 538.65918 & $\mathrm{DE}$ & 383 & -1.83 & -75.06497 & 38.62668 & $\mathrm{DE}$ & 455 & -0.69 & -75.05911 & 38.59053 & $\mathrm{DE}$ \\
\hline 312 & -0.43 & -75.06604 & 38.65873 & $\mathrm{DE}$ & 384 & -1.84 & -75.0649 & 38.62623 & $\mathrm{DE}$ & 456 & -0.7 & -75.05907 & 38.59008 & DE \\
\hline 313 & -0.43 & -75.06598 & 38.65828 & $\mathrm{DE}$ & 385 & -1.87 & -75.06488 & 38.62578 & $\mathrm{DE}$ & 457 & -0.69 & -75.05901 & 38.58963 & $\mathrm{DE}$ \\
\hline 314 & -0.42 & -75.06598 & 38.65783 & $\mathrm{DE}$ & 386 & -1.83 & -75.06479 & 38.62534 & $\mathrm{DE}$ & 458 & -0.62 & -75.05894 & 38.58918 & DE \\
\hline 315 & -0.42 & -75.06595 & 38.65738 & $\mathrm{DE}$ & 387 & -1.87 & -75.06474 & 38.62489 & $\mathrm{DE}$ & 459 & -0.63 & -75.05888 & 38.58873 & $\mathrm{DE}$ \\
\hline 316 & -0.45 & -75.06593 & 38.65693 & $\mathrm{DE}$ & 388 & -1.9 & -75.06471 & 38.62444 & $\mathrm{DE}$ & 460 & -0.67 & -75.05885 & 38.58828 & DE \\
\hline 317 & -0.48 & -75.06593 & 38.65647 & $\mathrm{DE}$ & 389 &,-1.92 & -75.06464 & 38.62399 & $\mathrm{DE}$ & 461 & -0.64 & -75.05879 & 38.58784 & $\mathrm{DE}$ \\
\hline 318 & -0.52 & -75.06593 & 38.65602 & $\mathrm{DE}$ & 390 & -1.93 & .75 .06458 & 38.62354 & $\mathrm{DE}$ & 462 & -0.62 & -75.05873 & 38.58739 & DE \\
\hline 319 & -0.55 & -75.0659 & 38.65557 & $\mathrm{DE}$ & 391 & -1.87 & -75.06451 & 38.6231 & $\mathrm{DE}$ & 463 & -0.64 & .75 .05869 & 38.58694 & DE \\
\hline 320 & -0.58 & -75.06589 & 38.65512 & $\mathrm{DE}$ & 392 & -1.95 & -75.06447 & 38.62265 & $\mathrm{DE}$ & 464 & -0.66 & .75 .05869 & 38.58649 & $\mathrm{DE}$ \\
\hline 321 & -0.64 & -75.06592 & 38.65466 & $\mathrm{DE}$ & 393 & -1.94 & .75 .06439 & 38.6222 & $\mathrm{DE}$ & 465 & -0.67 & -75.05865 & 38.58604 & $\mathrm{DE}$ \\
\hline 322 & -0.71 & -75.06596 & 38.65421 & $\mathrm{DE}$ & 394 & -1.92 & -75.06435 & 38.62175 & $\mathrm{DE}$ & 466 & -0.63 & -75.05856 & 38.58559 & $\mathrm{DE}$ \\
\hline 323 & -0.73 & -75.06593 & 38.65376 & $\mathrm{DE}$ & 395 & -1.96 & -75.06429 & 38.6213 & $\mathrm{DE}$ & 467 & -0.64 & -75.05852 & 38.58514 & $\mathrm{DE}$ \\
\hline 324 & -0.77 & -75.06595 & 38.6533 & $\mathrm{DE}$ & 396 & -2.01 & -75.06422 & 38.62085 & $\mathrm{DE}$ & 468 & -0.66 & -75.05849 & 38.58469 & $\mathrm{DE}$ \\
\hline 325 & -0.81 & .75 .06593 & 38.65285 & $\mathrm{DE}$ & 397 & -2.02 & -75.06415 & 38.62041 & $\mathrm{DE}$ & 469 & -0.7 & -75.05846 & 38.58424 & $\mathrm{DE}$ \\
\hline 326 & -0.8 & -75.06592 & 38.6524 & $\mathrm{DE}$ & 398 & -2.09 & -75.06413 & 38.61996 & $\mathrm{DE}$ & 470 & -0.71 & -75.05838 & 38.58379 & $\mathrm{DE}$ \\
\hline 327 & -0.81 & .75 .0659 & 38.65195 & $\mathrm{DE}$ & 399 & -2.17 & -75.0641 & 38.61951 & $\mathrm{DE}$ & 471 & -0.7 & -75.05833 & 38.58335 & DE \\
\hline 328 & -0.84 & -75.06592 & 38.6515 & $\mathrm{DE}$ & 400 & -2.24 & -75.06409 & 38.61905 & $\mathrm{DE}$ & 472 & -0.65 & -75.05829 & 38.5829 & $\mathrm{DE}$ \\
\hline 329 & -0.8 & -75.06589 & 38.65105 & $\mathrm{DE}$ & 401 & -2.31 & -75.06404 & 38.61861 & $\mathrm{DE}$ & 473 & -0.61 & -75.05824 & 38.58245 & $\mathrm{DE}$ \\
\hline 330 & -0.79 & -75.06586 & 38.6506 & $\mathrm{DE}$ & 402 & -2.43 & -75.06403 & 38.61815 & $\mathrm{DE}$ & 474 & -0.62 & -75.05818 & 38.582 & $\mathrm{DE}$ \\
\hline 331 & -0.79 & -75.06586 & 38.65014 & $\mathrm{DE}$ & 403 & -2.45 & -75.06398 & 38.61771 & $\mathrm{DE}$ & 475 & -0.59 & .75 .05814 & 38.58155 & $\mathrm{DE}$ \\
\hline 332 & -0.74 & -75.06584 & 38.64969 & $\mathrm{DE}$ & 404 & -2.56 & -75.06396 & 38.61725 & $\mathrm{DE}$ & 476 & -0.59 & -75.05807 & 38.5811 & $\mathrm{DE}$ \\
\hline 333 & -0.73 & -75.06583 & 38.64924 & $\mathrm{DE}$ & 405 & -2.6 & .75 .06392 & 38.6168 & $\mathrm{DE}$ & 477 & -0.58 & .75 .05798 & 38.58065 & $\mathrm{DE}$ \\
\hline 334 & -0.71 & -75.0658 & 38.64879 & $\mathrm{DE}$ & 406 & -2.68 & -75.06387 & 38.61635 & $\mathrm{DE}$ & 478 & -0.53 & .75 .05792 & 38.58021 & $\mathrm{DE}$ \\
\hline 335 & -0.67 & -75.06578 & 38.64834 & $\mathrm{DE}$ & 407 & -2.7 & -75.06383 & 38.61591 & $\mathrm{DE}$ & 479 & -0.47 & -75.05785 & 38.57976 & $\mathrm{DE}$ \\
\hline 336 & -0.67 & -75.06577 & 38.64789 & $\mathrm{DE}$ & 408 & -2.76 & -75.0638 & 38.61546 & $\mathrm{DE}$ & 480 & -0.46 & -75.05775 & 38.57931 & $\mathrm{DE}$ \\
\hline 337 & -0.67 & -75.0657 & 38.64744 & $\mathrm{DE}$ & 409 & -2.8 & -75.06378 & 38.61501 & $\mathrm{DE}$ & 481 & -0.51 & -75.05777 & 38.57886 & $\mathrm{DE}$ \\
\hline 338 & -0.67 & -75.06564 & 38.64699 & $\mathrm{DE}$ & 410 & -2.37 & -75.06342 & 38.61154 & $\mathrm{DE}$ & 482 & -0.44 & -75.05769 & 38.57841 & $\mathrm{DE}$ \\
\hline 339 & -0.66 & -75.06563 & 38.64654 & $\mathrm{DE}$ & 411 & -2.35 & -75.06326 & 38.61109 & $\mathrm{DE}$ & 483 & -0.4 & .75 .05766 & 538.57796 & $\mathrm{DE}$ \\
\hline 340 & -0.6 & -75.06554 & 38.6461 & $\mathrm{DE}$ & 412 & -2.29 & -75.06313 & 38.61065 & $\mathrm{DE}$ & 484 & -0.4 & -75.05763 & 38.57751 & $\mathrm{DE}$ \\
\hline 341 & -0.62 & -75.06552 & 38.64564 & $\mathrm{DE}$ & 413 & -2.19 & -75.06294 & 38.61021 & $\mathrm{DE}$ & 485 & -0.44 & -75.0576 & 38.57706 & $\mathrm{DE}$ \\
\hline 342 & -0.6 & -75.06549 & 38.64519 & $\mathrm{DE}$ & 414 & -2.09 & -75.06271 & 38.60977 & $\mathrm{DE}$ & 486 & -0.48 & -75.05757 & 38.57661 & $\mathrm{DE}$ \\
\hline 343 & -0.58 & -75.06544 & 38.64474 & $\mathrm{DE}$ & 415 & -2.03 & -75.06245 & 38.60933 & $\mathrm{DE}$ & 487 & -0.48 & -75.05754 & 38.57616 & $\mathrm{DE}$ \\
\hline 344 & -0.52 & -75.06538 & 38.6443 & $\mathrm{DE}$ & 416 & -0.73 & -75.06042 & 38.60897 & $\mathrm{DE}$ & 488 & -0.46 & -75.0575 & 38.57571 & $\mathrm{DE}$ \\
\hline 345 & -0.53 & -75.0654 & 38.64384 & $\mathrm{DE}$ & 417 & -0.02 & -75.05913 & 38.60767 & DE & 489 & -0.39 & -75.05743 & 38.57526 & DE \\
\hline 346 & -0.51 & -75.06535 & 38.64339 & $\mathrm{DE}$ & 418 & -0.8 & -75.06021 & 38.60717 & $\mathrm{DE}$ & 490 & -0.33 & -75.05737 & 38.57482 & $\mathrm{DE}$ \\
\hline 347 & -0.52 & -75.06534 & 38.64294 & $\mathrm{DE}$ & 419 & -0.95 & -75.06026 & 38.60672 & $\mathrm{DE}$ & 491 & -0.33 & -75.05734 & 38.57437 & $\mathrm{DE}$ \\
\hline 348 & -0.48 & -75.06532 & 238.64249 & $\mathrm{DE}$ & 420 & -0.99 & -75.06029 & 38.60627 & $\mathrm{DE}$ & 492 & -0.3 & -75.05734 & 38.57392 & $\mathrm{DE}$ \\
\hline 349 & -0.46 & -75.06532 & 238.64204 & $\mathrm{DE}$ & 421 & -0.98 & -75.0603 & 38.60581 & $\mathrm{DE}$ & 493 & -0.3 & -75.05734 & 38.57346 & $\mathrm{DE}$ \\
\hline 350 & -0.46 & .75 .06532 & 38.64159 & $\mathrm{DE}$ & 422 & -0.94 & -75.06026 & 38.60537 & $\mathrm{DE}$ & 494 & -0.32 & -75.05734 & 38.57301 & $\mathrm{DE}$ \\
\hline 351 & -0.46 & -75.06532 & 238.64113 & $\mathrm{DE}$ & 423 & -0.91 & -75.06017 & 38.60492 & $\mathrm{DE}$ & 495 & -0.29 & -75.05734 & 38.57256 & $\mathrm{DE}$ \\
\hline 352 & -0.48 & -75.06532 & 38.64068 & $\mathrm{DE}$ & 424 & -0.77 & -75.06007 & 38.60447 & $\mathrm{DE}$ & 496 & -0.26 & -75.05733 & 38.57211 & $\mathrm{DE}$ \\
\hline 353 & -0.53 & -75.06535 & 538.64023 & $\mathrm{DE}$ & 425 & -0.72 & .75 .05997 & 38.60403 & $\mathrm{DE}$ & 497 & -0.29 & -75.05734 & 38.57166 & $\mathrm{DE}$ \\
\hline 354 & -0.51 & -75.06538 & 38.63977 & $\mathrm{DE}$ & 426 & -0.6 & -75.05981 & 38.60358 & $\mathrm{DE}$ & 498 & -0.3 & -75.05733 & 38.57121 & $\mathrm{DE}$ \\
\hline 355 & -0.57 & -75.06541 & 38.63932 & $\mathrm{DE}$ & 427 & -0.51 & -75.05968 & 38.60314 & $\mathrm{DE}$ & 499 & -0.27 & -75.0573 & 38.57076 & $\mathrm{DE}$ \\
\hline 356 & -0.59 & -75.06541 & 38.63886 & $\mathrm{DE}$ & 428 & -0.47 & -75.05957 & 38.60269 & $\mathrm{DE}$ & 500 & -0.21 & -75.05725 & 38.57031 & $\mathrm{DE}$ \\
\hline 357 & -0.65 & -75.06544 & 38.63841 & $\mathrm{DE}$ & 429 & -0.48 & -75.05956 & 38.60224 & $\mathrm{DE}$ & 501 & -0.23 & -75.05727 & 38.56986 & $\mathrm{DE}$ \\
\hline 358 & -0.7 & -75.06546 & 38.63796 & $\mathrm{DE}$ & 430 & -0.46 & -75.05959 & 38.60179 & $\mathrm{DE}$ & 502 & -0.2 & -75.05717 & 38.56941 & $\mathrm{DE}$ \\
\hline 359 & -0.77 & -75.06548 & 38.6375 & $\mathrm{DE}$ & 431 & -0.49 & -75.05962 & 38.60133 & $\mathrm{DE}$ & 503 & -0.17 & -75.05716 & 38.56896 & $\mathrm{DE}$ \\
\hline 360 & -0.8 & -75.06549 & 38.63705 & $\mathrm{DE}$ & 432 & -0.58 & -75.05966 & 38.60088 & $\mathrm{DE}$ & 504 & -0.17 & -75.05716 & 38.56851 & $\mathrm{DE}$ \\
\hline 361 & -0.83 & -75.06549 & 38.6366 & $\mathrm{DE}$ & 433 & -0.62 & -75.05969 & 38.60043 & $\mathrm{DE}$ & 505 & -0.17 & -75.05711 & 38.56806 & $\overline{D E}$ \\
\hline 362 & -0.88 & -75.06549 & 38.63614 & $\mathrm{DE}$ & 434 & -0.64 & -75.05972 & 38.59998 & $\mathrm{DE}$ & 506 & -0.23 & -75.05713 & 38.56761 & $\mathrm{DE}$ \\
\hline 363 & -0.94 & -75.06551 & 38.63569 & $\mathrm{DE}$ & 435 & -0.67 & -75.05975 & 38.59952 & $\mathrm{DE}$ & 507 & -0.29 & -75.05713 & 38.56716 & $\mathrm{DE}$ \\
\hline 364 & -1.02 & -75.06552 & 38.63524 & $\mathrm{DE}$ & 436 & -0.71 & -75.05975 & 38.59907 & $\mathrm{DE}$ & 508 & -0.3 & -75.05707 & 38.56671 & $\mathrm{DE}$ \\
\hline 365 & -1.05 & -75.06557 & 38.63478 & $\mathrm{DE}$ & 437 & -0.69 & -75.05974 & 38.59862 & $\mathrm{DE}$ & 509 & -0.37 & -75.05707 & 38.56626 & $\mathrm{DE}$ \\
\hline 366 & -1.11 & -75.0656 & 38.63433 & $\mathrm{DE}$ & 438 & -0.7 & -75.05972 & 38.59817 & $\mathrm{DE}$ & 510 & -0.34 & -75.05701 & 38.56581 & $\mathrm{DE}$ \\
\hline 367 & -1.16 & -75.0656 & 38.63387 & $\mathrm{DE}$ & 439 & -0.69 & -75.05971 & 38.59772 & $\mathrm{DE}$ & 511 & -0.3 & -75.05695 & 38.56536 & $\mathrm{DE}$ \\
\hline 368 & -1.19 & -75.06561 & 38.63342 & $\mathrm{DE}$ & 440 & -0.66 & -75.05966 & 538.59727 & $\mathrm{DE}$ & 512 & -0.23 & -75.05692 & 38.56491 & $\mathrm{DE}$ \\
\hline 369 & -1.24 & -75.0656 & 38.63297 & $\mathrm{DE}$ & 441 & -0.65 & -75.05966 & 538.59682 & $\mathrm{DE}$ & 513 & -0.23 & -75.05688 & 38.56446 & $\mathrm{DE}$ \\
\hline 370 & -1.31 & -75.06557 & 38.63252 & $\mathrm{DE}$ & 442 & -0.66 & -75.05963 & 38.59637 & $\mathrm{DE}$ & 514 & -0.19 & -75.05688 & 38.56401 & $\mathrm{DE}$ \\
\hline 371 & -1.33 & -75.06554 & 38.63207 & $\mathrm{DE}$ & 443 & -0.63 & -75.05959 & 38.59592 & $\mathrm{DE}$ & 515 & -0.18 & -75.0569 & 38.56356 & $\mathrm{DE}$ \\
\hline 372 & -1.4 & -75.06555 & 38.63161 & $\mathrm{DE}$ & 444 & -0.59 & -75.05951 & 38.59547 & $\mathrm{DE}$ & 516 & -0.18 & -75.05693 & 38.56311 & $\mathrm{DE}$ \\
\hline 373 & -1.48 & -75.06554 & 38.63116 & $\mathrm{DE}$ & 445 & -0.58 & -75.05945 & 38.59503 & $\mathrm{DE}$ & 517 & -0.21 & -75.05693 & 38.56265 & $\mathrm{DE}$ \\
\hline 374 & -1.52 & -75.06549 & 38.63071 & $\mathrm{DE}$ & 446 & -0.55 & -75.05939 & 38.59458 & $\mathrm{DE}$ & 518 & -0.2 & .75 .05695 & 38.5622 & $\mathrm{DE}$ \\
\hline 375 & -1.55 & -75.06541 & 38.63027 & $\mathrm{DE}$ & 447 & -0.52 & -75.05933 & 38.59413 & $\mathrm{DE}$ & 519 & -0.21 & -75.05701 & 38.56175 & $\mathrm{DE}$ \\
\hline 376 & -1.6 & -75.06535 & 38.62982 & $\mathrm{DE}$ & 448 & -0.51 & -75.0593 & 38.59368 & $\mathrm{DE}$ & 520 & -0.23 & .75 .05698 & 38.5613 & $\mathrm{DE}$ \\
\hline 377 & -1.64 & -75.06531 & 38.62937 & $\mathrm{DE}$ & 449 & -0.51 & -75.05928 & 38.59323 & $\mathrm{DE}$ & 521 & -0.26 & -75.05702 & 38.56085 & $\mathrm{DE}$ \\
\hline 378 & -1.63 & -75.06522 & 38.62892 & $\mathrm{DE}$ & 450 & -0.55 & -75.05927 & 38.59278 & $\mathrm{DE}$ & 522 & -0.28 & -75.05704 & 38.56039 & $\mathrm{DE}$ \\
\hline 379 & -1.67 & -75.06522 & 38.62847 & $\mathrm{DE}$ & 451 & -0.59 & -75.0592 & 38.59233 & $\mathrm{DE}$ & 523 & -0.28 & -75.05702 & 38.55994 & $\mathrm{DE}$ \\
\hline 380 & -1.71 & -75.06514 & 38.62803 & $\mathrm{DE}$ & 452 & -0.62 & -75.05917 & 38.59188 & $\mathrm{DE}$ & 524 & -0.3 & -75.05702 & 38.55949 & $\mathrm{DE}$ \\
\hline 381 & -1.75 & -75.06509 & 38.62758 & $\mathrm{DE}$ & 453 & -0.69 & -75.05917 & 38.59143 & $\mathrm{DE}$ & 525 & -0.35 & -75.05701 & 38.55904 & $\mathrm{DE}$ \\
\hline 382 & -1.77 & .75 .06503 & 38.62713 & $\mathrm{DE}$ & 454 & -0.67 & .75 .05911 & 38.59098 & $\mathrm{DE}$ & 526 & -0.44 & -75.05702 & 38.55859 & $\mathrm{DE}$ \\
\hline
\end{tabular}




\begin{tabular}{|c|c|c|c|c|c|c|c|c|c|c|c|c|c|c|}
\hline TR\# & RATE & LONG & LAT & ST & TR\# & RATE & LONG & LAT & ST & TR\# & RATE & LONG & LAT & ST \\
\hline 527 & -0.48 & -75.05701 & 138.55814 & $\mathrm{DE}$ & 599 & -0.81 & -75.0533 & 38.52492 & $\overline{\mathrm{DE}}$ & 671 & -0.95 & .75 .05093 & 38.49254 & $\mathrm{DE}$ \\
\hline 528 & -0.78 & -75.05693 & 38.55679 & $\mathrm{DE}$ & 600 & -0.84 & -75.05327 & 38.52447 & $\mathrm{DE}$ & 672 & -0.97 & -75.0509 & 38.49209 & $\mathrm{DE}$ \\
\hline 529 & -0.87 & -75.05695 & 538.55634 & $\mathrm{DE}$ & 601 & -0.88 & -75.05325 & 38.52402 & $\mathrm{DE}$ & 673 & -1 & -75.05089 & 38.49164 & $\mathrm{DE}$ \\
\hline 530 & -0.89 & -75.05693 & 38.55589 & $\mathrm{DE}$ & 602 & -0.89 & -75.05322 & 38.52357 & $\mathrm{DE}$ & 674 & -0.98 & -75.05087 & 38.49119 & $\mathrm{DE}$ \\
\hline 531 & -0.95 & -75.05688 & 838.55544 & $\mathrm{DE}$ & 603 & -0.94 & -75.05316 & 38.52312 & $\mathrm{DE}$ & 675 & -1.05 & -75.05086 & 38.49074 & $\mathrm{DE}$ \\
\hline 532 & -0.93 & -75.05682 & 238.55499 & $\mathrm{DE}$ & 604 & -0.97 & -75.05312 & 38.52267 & $\mathrm{DE}$ & 676 & -1.04 & -75.05084 & 38.49029 & $\mathrm{DE}$ \\
\hline 533 & -0.97 & -75.05681 & 38.55454 & $\mathrm{DE}$ & 605 & -0.92 & .75 .05312 & 38.52222 & $\mathrm{DE}$ & 677 & -1.06 & -75.05084 & 38.48984 & $\mathrm{DE}$ \\
\hline 534 & -0.96 & -75.05673 & 338.55409 & $\mathrm{DE}$ & 606 & -0.95 & -75.05309 & 38.52177 & $\mathrm{DE}$ & 678 & -1.1 & -75.0508 & 38.48939 & $\mathrm{DE}$ \\
\hline 535 & -0.97 & -75.05669 & 938.55364 & $\mathrm{DE}$ & 607 & -0.99 & -75.05305 & 38.52132 & $\mathrm{DE}$ & 679 & -1.16 & -75.05077 & 38.48894 & $\mathrm{DE}$ \\
\hline 536 & -0.95 & -75.05666 & 638.55319 & $\mathrm{DE}$ & 608 & -1.01 & -75.05305 & 38.52087 & $\mathrm{DE}$ & 680 & -1.19 & -75.05077 & 38.48849 & $\mathrm{DE}$ \\
\hline 537 & -1.01 & -75.05664 & 438.55274 & $\mathrm{DE}$ & 609 & -1.06 & -75.05302 & 38.52042 & $\mathrm{DE}$ & 681 & -1.22 & -75.05078 & 38.48804 & $\mathrm{DE}$ \\
\hline 538 & -1 & -75.05663 & 338.55229 & DE & 610 & -1 & -75.05299 & 38.51997 & $\mathrm{DE}$ & 682 & -1.19 & -75.0507 & 38.48759 & $\mathrm{DE}$ \\
\hline 539 & -1.02 & -75.05659 & 938.55184 & $\mathrm{DE}$ & 611 & -0.97 & -75.05296 & 38.51952 & $\mathrm{DE}$ & 683 & -1.17 & -75.05064 & 38.48714 & $\mathrm{DE}$ \\
\hline 540 & -1.08 & -75.05656 & 638.55139 & $\mathrm{DE}$ & 612 & -0.91 & -75.05295 & 38.51907 & $\mathrm{DE}$ & 684 & -1.17 & .75 .0506 & 38.48669 & $\mathrm{DE}$ \\
\hline 541 & -1.05 & -75.05653 & 338.55094 & $\mathrm{DE}$ & 613 & -0.88 & -75.05293 & 38.51862 & $\mathrm{DE}$ & 685 & -1.11 & -75.05051 & 38.48625 & $\mathrm{DE}$ \\
\hline 542 & -1.03 & -75.05647 & 738.55049 & $\mathrm{DE}$ & 614 & -0.84 & -75.05292 & 38.51817 & $\mathrm{DE}$ & 686 & -1.15 & .75 .05051 & 38.48579 & $\mathrm{DE}$ \\
\hline 543 & -0.96 & -75.05637 & 738.55005 & $\mathrm{DE}$ & 615 & -0.83 & -75.05289 & 38.51772 & $\mathrm{DE}$ & 687 & -1.16 & -75.05049 & 38.48534 & DE \\
\hline 544 & -0.98 & -75.05627 & 738.5496 & DE & 616 & -0.91 & -75.05286 & 38.51727 & $\mathrm{DE}$ & 688 & -1.2 & -75.05048 & 38.48489 & DE \\
\hline 545 & -0.87 & -75.05617 & 738.54915 & $D E$ & 617 & -0.77 & .75 .05286 & 38.51682 & $\mathrm{DE}$ & 689 & -1.16 & -75.05046 & 38.48444 & $\mathrm{DE}$ \\
\hline 546 & -0.81 & .75 .05602 & 238.54871 & $\mathrm{DE}$ & 618 & -0.9 & .75 .05286 & 38.51637 & $\mathrm{DE}$ & 690 & -1.13 & .75 .05042 & 38.48399 & $\mathrm{DE}$ \\
\hline 547 & -0.84 & -75.05589 & 38.54826 & $\mathrm{DE}$ & 619 & -0.81 & -75.05284 & 38.51592 & $\mathrm{DE}$ & 691 & -1.19 & -75.05042 & 38.48354 & $\mathrm{DE}$ \\
\hline 548 & -0.8 & -75.05582 & 238.54782 & $\mathrm{DE}$ & 620 & -0.86 & -75.05283 & 38.51547 & $\mathrm{DE}$ & 692 & -1.16 & -75.05037 & 38.48309 & $\mathrm{DE}$ \\
\hline 549 & -0.78 & -75.05568 & 838.54737 & $\mathrm{DE}$ & 621 & -0.85 & -75.0528 & 38.51502 & $\mathrm{DE}$ & 693 & -1.19 & -75.05035 & 38.48264 & $\mathrm{DE}$ \\
\hline 550 & -0.69 & -75.05553 & 38.54693 & $\mathrm{DE}$ & 622 & -0.94 & -75.05289 & 38.51456 & $\mathrm{DE}$ & 694 & -1.17 & -75.05034 & 38.48219 & $\mathrm{DE}$ \\
\hline 551 & -0.65 & -75.0554 & 38.54648 & $\mathrm{DE}$ & 623 & -0.97 & -75.05289 & 38.51411 & $\mathrm{DE}$ & 695 & -1.2 & -75.05031 & 38.48174 & $\mathrm{DE}$ \\
\hline 552 & -0.7 & -75.05531 & 138.54603 & $\mathrm{DE}$ & 624 & -1.01 & -75.05286 & 38.51366 & $\mathrm{DE}$ & 696 & -1.15 & -75.05023 & 38.48129 & $\mathrm{DE}$ \\
\hline 553 & -0.58 & .75 .05518 & 838.54559 & $\mathrm{DE}$ & 625 & -0.97 & -75.05283 & 38.51321 & $\mathrm{DE}$ & 697 & -1.16 & .75 .05016 & 38.48085 & $\mathrm{DE}$ \\
\hline 554 & -0.58 & -75.05507 & 78.54514 & $\mathrm{DE}$ & 626 & -0.97 & -75.05278 & 38.51276 & $\mathrm{DE}$ & 698 & -1.14 & -75.05016 & 38.4804 & $\mathrm{DE}$ \\
\hline 555 & -0.63 & -75.05498 & 838.5447 & $\mathrm{DE}$ & 627 & -0.97 & -75.05273 & 38.51231 & $\mathrm{DE}$ & 699 & -1.13 & -75.05009 & 38.47995 & $\mathrm{DE}$ \\
\hline 556 & -0.6 & -75.05482 & 238.54425 & DE & 628 & -0.96 & -75.05275 & 38.51186 & $\mathrm{DE}$ & 700 & -1.15 & -75.05005 & 38.4795 & $\mathrm{DE}$ \\
\hline 557 & -0.63 & -75.0547 & 38.54381 & $\mathrm{DE}$ & 629 & -1.01 & -75.05273 & 38.51141 & $D E$ & 701 & -1.16 & -75.05 & 38.47905 & $\mathrm{DE}$ \\
\hline 558 & -0.69 & -75.05463 & 38.54336 & $\mathrm{DE}$ & 630 & -1.06 & -75.05272 & 38.51096 & $\mathrm{DE}$ & 702 & -1.11 & -75.04996 & 38.4786 & $\mathrm{DE}$ \\
\hline 559 & -0.72 & -75.0545 & 38.54291 & $\mathrm{DE}$ & 631 & -1.05 & -75.05264 & 38.51051 & $\mathrm{DE}$ & 703 & -1.11 & -75.04997 & 38.47815 & $\mathrm{DE}$ \\
\hline 560 & -0.8 & -75.05446 & 638.54246 & $\mathrm{DE}$ & 632 & -1.08 & -75.05264 & 38.51006 & $\mathrm{DE}$ & 704 & -1.07 & -75.04994 & 38.4777 & $\mathrm{DE}$ \\
\hline 561 & -0.84 & -75.05441 & 38.54201 & $D E$ & 633 & -1.12 & .75 .05264 & 38.50961 & $D E$ & 705 & -1.08 & -75.04991 & 38.47725 & $D E$ \\
\hline 562 & -0.91 & -75.05429 & 98.54157 & $\mathrm{DE}$ & 634 & -1.15 & -75.05263 & 38.50916 & $D E$ & 706 & -1.08 & -75.0499 & 38.4768 & $\mathrm{DE}$ \\
\hline 563 & -0.95 & -75.05428 & 338.54112 & DE & 635 & -1.13 & -75.05263 & 38.50871 & $D E$ & 707 & -1.12 & -75.04988 & 38.47635 & $\mathrm{DE}$ \\
\hline 564 & -0.92 & -75.05423 & 38.54067 & DE & 636 & -1.18 & -75.05264 & 38.50826 & $D E$ & 708 & -1.1 & -75.04984 & 38.4759 & $\mathrm{DE}$ \\
\hline 565 & -0.99 & -75.05421 & 38.54022 & $\mathrm{DE}$ & 637 & -1.12 & -75.05261 & 38.50781 & $D E$ & 709 & -1.07 & -75.04984 & 38.47545 & $\mathrm{DE}$ \\
\hline 566 & -1.03 & -75.05412 & 38.53977 & $\mathrm{DE}$ & 638 & -1.15 & -75.05254 & 38.50736 & $D E$ & 710 & -1.09 & -75.0498 & 38.475 & $\mathrm{DE}$ \\
\hline 567 & -0.98 & -75.05402 & 38.53932 & $\mathrm{DE}$ & 639 & -1.16 & -75.05252 & 38.50691 & $\mathrm{DE}$ & 711 & -1.03 & -75.04976 & 38.47455 & $\mathrm{DE}$ \\
\hline 568 & -1.06 & -75.05402 & 38.53887 & $\mathrm{DE}$ & 640 & -1.15 & -75.05246 & 38.50646 & $D E$ & 712 & -1.06 & -75.04973 & 38.4741 & $\mathrm{DE}$ \\
\hline 569 & -1.02 & -75.05399 & 38.53842 & DE & 641 & -1.2 & -75.05243 & 38.50601 & DE & 713 & -1.04 & -75.04971 & 38.47365 & $\mathrm{DE}$ \\
\hline 570 & -1.1 & -75.05397 & 38.53797 & DE & 642 & -1.16 & -75.05235 & 38.50556 & $D E$ & 714 & -1.02 & -75.04971 & 38.4732 & $\mathrm{DE}$ \\
\hline 571 & -1.01 & -75.05396 & 38.53752 & DE & 643 & -1.19 & -75.05229 & 38.50511 & $\mathrm{DE}$ & 715 & -1.06 & -75.04968 & 38.47275 & $\mathrm{DE}$ \\
\hline 572 & -1.08 & -75.05396 & 38.53707 & DE & 644 & -1.14 & -75.05223 & 38.50467 & $\mathrm{DE}$ & 716 & -1.09 & -75.04968 & 38.4723 & $\mathrm{DE}$ \\
\hline 573 & -1.05 & -75.05394 & 38.53662 & $\mathrm{DE}$ & 645 & -1.01 & .75 .05217 & 38.50422 & $\mathrm{DE}$ & 717 & -1.06 & -75.04968 & 38.47184 & $\mathrm{DE}$ \\
\hline 574 & -1.05 & -75.05396 & 38.53617 & $\mathrm{DE}$ & 646 & -1.04 & -75.05212 & 38.50377 & $\mathrm{DE}$ & 718 & -1.05 & -75.04967 & 38.47139 & $\mathrm{DE}$ \\
\hline 575 & -1.06 & .75 .05392 & 38.53572 & $\mathrm{DE}$ & 647 & -1.08 & -75.05209 & 38.50332 & $\mathrm{DE}$ & 719 & -1.07 & -75.04965 & 38.47094 & $\mathrm{DE}$ \\
\hline 576 & -1.05 & -75.05388 & 38.53527 & $\mathrm{DE}$ & 648 & -1.12 & -75.05206 & 38.50287 & $\mathrm{DE}$ & 720 & -1.07 & -75.04964 & 38.47049 & $\mathrm{DE}$ \\
\hline 577 & -1.04 & -75.05392 & 38.53482 & $\mathrm{DE}$ & 649 & -0.97 & -75.05186 & 38.50243 & $\mathrm{DE}$ & 721 & -1.07 & -75.04962 & 38.47004 & $\mathrm{DE}$ \\
\hline 578 & -1.12 & -75.05391 & 38.53437 & $D E$ & 650 & -0.95 & -75.05183 & 38.50198 & $\mathrm{DE}$ & 722 & -1.04 & -75.04961 & 38.46959 & $\mathrm{DE}$ \\
\hline 579 & -1.16 & -75.05391 & 38.53392 & DE & 651 & -0.93 & -75.05176 & 38.50153 & $D E$ & 723 & -1.05 & -75.04958 & 38.46914 & $\mathrm{DE}$ \\
\hline 580 & -1.17 & -75.05392 & 38.53346 & DE & 652 & -0.94 & -75.05173 & 38.50108 & DE & 724 & -1.06 & -75.04956 & 38.46869 & $\mathrm{DE}$ \\
\hline 581 & -1.21 & -75.05391 & 38.53301 & DE & 653 & -0.93 & -75.0517 & 38.50063 & $D E$ & 725 & -1.12 & -75.04955 & 38.46824 & $\mathrm{DE}$ \\
\hline 582 & -1.23 & -75.05391 & 38.53256 & $D E$ & 654 & -0.97 & -75.05168 & 38.50018 & $D E$ & 726 & -1.14 & -75.04955 & 38.46779 & $\mathrm{DE}$ \\
\hline 583 & -1.18 & -75.05389 & 38.53211 & DE & 655 & -0.95 & -75.05164 & 38.49973 & DE & 727 & -1.15 & -75.04956 & 38.46734 & $\mathrm{DE}$ \\
\hline 584 & -1.18 & -75.05389 & 38.53166 & $\mathrm{DE}$ & 656 & -0.88 & -75.05161 & 38.49928 & $\mathrm{DE}$ & 728 & -1.14 & -75.04959 & 38.46689 & $\mathrm{DE}$ \\
\hline 585 & -1.18 & .75 .05386 & 38.53121 & $\mathrm{DE}$ & 657 & -0.92 & -75.05153 & 38.49883 & $\mathrm{DE}$ & 729 & -1.15 & -75.04959 & 38.46643 & $\mathrm{DE}$ \\
\hline 586 & -1.16 & .75 .05386 & 38.53076 & $\mathrm{DE}$ & 658 & -0.93 & -75.0515 & 38.49838 & DE & 730 & -1.28 & -75.04958 & 38.46598 & $\mathrm{DE}$ \\
\hline 587 & -1.13 & -75.05383 & 38.53031 & DE & 659 & -0.98 & -75.05147 & 38.49793 & DE & 731 & -1.33 & -75.04958 & 38.46553 & $\mathrm{DE}$ \\
\hline 588 & -1.11 & -75.0538 & 38.52986 & $D E$ & 660 & -0.94 & -75.05141 & 38.49748 & $\mathrm{DE}$ & 732 & -1.24 & -75.04958 & 38.46508 & $\mathrm{DE}$ \\
\hline 589 & -1.09 & -75.05377 & 38.52941 & $D E$ & 661 & -0.88 & -75.05138 & 38.49703 & DE & 733 & -1.18 & -75.04953 & 38.46463 & $\mathrm{DE}$ \\
\hline 590 & -1.03 & .75 .05371 & 38.52896 & $\mathrm{DE}$ & 662 & -0.91 & -75.05135 & 38.49658 & $\mathrm{DE}$ & 734 & -1.12 & -75.04948 & 38.46418 & $\mathrm{DE}$ \\
\hline 591 & -1.02 & .75 .05363 & 38.52851 & $\mathrm{DE}$ & 663 & -0.98 & -75.05133 & 38.49613 & DE & 735 & -1.23 & -75.04951 & 38.46373 & $\mathrm{DE}$ \\
\hline 592 & -0.94 & -75.05354 & 38.52807 & $\mathrm{DE}$ & 664 & -0.92 & -75.05124 & 38.49569 & $\mathrm{DE}$ & 736 & -1.34 & -75.04951 & 38.46328 & $\mathrm{DE}$ \\
\hline 593 & -0.99 & -75.05351 & 38.52762 & DE & 665 & -0.9 & -75.05119 & 38.49524 & DE & 737 & -1.38 & -75.04955 & 38.46283 & $D E$ \\
\hline 594 & -0.9 & -75.05347 & 38.52717 & $\mathrm{DE}$ & 666 & -0.9 & -75.05112 & 38.49479 & $\mathrm{DE}$ & 738 & -1.38 & -75.04951 & 38.46238 & DE \\
\hline 595 & -0.88 & -75.05342 & 38.52672 & $D E$ & 667 & -0.89 & -75.05107 & 38.49434 & DE & 739 & -1.31 & -75.04955 & 38.46192 & $D E$ \\
\hline 596 & -0.92 & .75 .05341 & 38.52627 & $\mathrm{DE}$ & 668 & -0.89 & -75.05103 & 38.49389 & $\mathrm{DE}$ & 740 & -1.18 & -75.04953 & 38.46147 & $\mathrm{DE}$ \\
\hline 597 & -0.85 & -75.05334 & 38.52582 & $\mathrm{DE}$ & 669 & -0.87 & -75.05098 & 38.49344 & $\mathrm{DE}$ & 741 & -1.26 & -75.04955 & 38.46102 & $\mathrm{DE}$ \\
\hline 598 & -0.81 & -75.05328 & 38.52537 & $\mathrm{DE}$ & 670 & -0.97 & -75.05095 & 38.49299 & $\mathrm{DE}$ & 742 & -1.23 & -75.04951 & 38.46057 & $\mathrm{DE}$ \\
\hline
\end{tabular}




\begin{tabular}{|c|c|c|c|c|c|c|c|c|c|c|c|c|c|c|}
\hline TR\# & RATE & LONG & LAT & ST & I TR\# & RATE & LONG & LAT & ST & TR\# & RATE & LONG & LAT & ST \\
\hline 743 & -1.12 & -75.04955 & 538.46012 & $\mathrm{DE}$ & 52 & -0.6 & -75.0485 & 38.4282 & MD & 124 & -1.6 & -75.0563 & 38.3959 & MD \\
\hline 744 & -1.12 & -75.04955 & 538.45967 & $\mathrm{DE}$ & 53 & -0.4 & -75.0486 & 38.4277 & MD & 125 & -1.5 & -75.0564 & 38.3955 & MD \\
\hline 745 & -1.13 & -75.04958 & 838.45922 & $\mathrm{DE}$ & 54 & -0.1 & -75.0487 & 38.4273 & MD & 126 & -1.3 & -75.0566 & 38.395 & MD \\
\hline 746 & -1.18 & -75.04961 & 138.45876 & $\mathrm{DE}$ & 55 & -0.3 & -75.0487 & 38.4268 & $M D$ & 127 & -1.4 & -75.0567 & 38.3946 & MD \\
\hline 747 & -1.13 & .75 .04959 & 938.45831 & $\mathrm{DE}$ & 56 & -0.5 & -75.0488 & 38.4264 & MD & 128 & -1.5 & .75 .0568 & 38.3941 & MD \\
\hline 748 & -1.11 & -75.04958 & 838.45786 & $D E$ & 57 & -0.5 & -75.0489 & 38.4259 & MD & 129 & -1.4 & -75.057 & 38.3936 & MD \\
\hline 749 & -1.09 & .75 .04958 & 838.45741 & $\mathrm{DE}$ & 58 & -0.5 & -75.049 & 38.4254 & $\mathrm{MD}$ & 130 & -1.3 & -75.0571 & 38.3932 & MD \\
\hline 750 & -1.07 & -75.04961 & 138.45696 & $\mathrm{DE}$ & 59 & -0.7 & -75.0491 & 38.425 & MD & 131 & -1.3 & -75.0572 & 38.3927 & MD \\
\hline 751 & -1.1 & -75.04964 & 438.45651 & $\mathrm{DE}$ & 60 & -0.8 & -75.0492 & 38.4245 & MD & 132 & -1.3 & -75.0574 & 38.3923 & MD \\
\hline 752 & -1.05 & -75.04961 & 138.45606 & $\mathrm{DE}$ & 61 & -0.8 & -75.0493 & 38.4241 & $\mathrm{MD}$ & 133 & -1.3 & -75.0575 & 38.3918 & MD \\
\hline 753 & -1.05 & -75.04961 & 138.45561 & $\mathrm{DE}$ & 62 & -0.9 & -75.0494 & 38.4236 & MD & 134 & -1.3 & -75.0576 & 38.3914 & MD \\
\hline 754 & -1.1 & -75.04962 & 238.45515 & $\mathrm{DE}$ & 63 & -0.9 & -75.0494 & 38.4231 & MD & 135 & -1.4 & -75.0578 & 38.3909 & MD \\
\hline 755 & -1.16 & -75.0497 & 38.4547 & $\mathrm{DE}$ & 64 & -0.9 & -75.0495 & 38.4227 & MD & 136 & -1.5 & -75.0579 & 38.3905 & MD \\
\hline 756 & -1.17 & -75.04971 & 138.45425 & $\mathrm{DE}$ & 65 & -0.9 & -75.0496 & 38.4222 & $\mathrm{MD}$ & 137 & -1.5 & -75.058 & 38.39 & MD \\
\hline 757 & -1.17 & -75.04973 & 338.4538 & $\mathrm{DE}$ & 66 & -0.9 & -75.0497 & 38.4218 & MD & 138 & -1.4 & -75.0582 & 38.3895 & MD \\
\hline 758 & -1.2 & -75.04974 & 438.45334 & $\mathrm{DE}$ & 67 & -0.7 & -75.0498 & 38.4213 & MD & 139 & -1.4 & -75.0583 & 38.3891 & MD \\
\hline 759 & -1.15 & -75.04974 & 438.45289 & $\mathrm{DE}$ & 68 & -0.5 & -75.0499 & 38.4208 & MD & 140 & -1.3 & -75.0584 & 38.3886 & MD \\
\hline 760 & -1.16 & -75.0498 & 38.45244 & $\mathrm{DE}$ & 69 & -0.6 & -75.05 & 38.4204 & MD & 141 & -1.1 & .75 .0586 & 38.3882 & MD \\
\hline 761 & -1.2 & -75.04985 & 538.45199 & $\mathrm{DE}$ & 70 & -0.7 & -75.0501 & 38.4199 & MD & 142 & -0.9 & -75.0587 & 38.3877 & MD \\
\hline 762 & -1.23 & -75.0499 & 38.45153 & $\mathrm{DE}$ & 71 & -0.6 & -75.0501 & 38.4195 & MD & 143 & -0.7 & -75.0588 & 38.3873 & MD \\
\hline 763 & -1.19 & -75.04993 & 38.45108 & $\mathrm{DE}$ & 72 & -0.5 & .75 .0502 & 38.419 & MD & 144 & -0.6 & -75.059 & 38.3868 & MD \\
\hline 1 & -0.6 & -75.0454 & 38.4511 & MD & 73 & -0.5 & -75.0503 & 38.4185 & MD & 145 & -0.6 & -75.0591 & 38.3863 & MD \\
\hline 2 & -0.7 & -75.0454 & 38.4507 & MD & 74 & -0.6 & .75 .0504 & 38.4181 & MD & 146 & -0.5 & -75.0592 & 38.3859 & MD \\
\hline 3 & -0.6 & .75 .0454 & 38.4502 & MD & 75 & -0.6 & -75.0505 & 38.4176 & MD & 147 & -0.4 & .75 .0594 & 38.3854 & MD \\
\hline 4 & -0.5 & .75 .0454 & 38.4497 & MD & 76 & -0.6 & .75 .0506 & 38.4171 & MD & 148 & -0.2 & -75.0595 & 38.385 & $\mathrm{MD}$ \\
\hline 5 & -0.6 & .75 .0454 & 38.4493 & MD & 77 & -0.6 & .75 .0507 & 38.4167 & MD & 149 & -0.2 & -75.0596 & 38.3845 & MD \\
\hline 6 & -0.7 & .75 .0454 & 38.4488 & $M D$ & 78 & -0.6 & -75.0508 & 38.4162 & MD & 150 & -0.1 & -75.0598 & 38.3841 & MD \\
\hline 7 & -0.5 & -75.0454 & 38.4483 & MD & 79 & -0.5 & -75.0508 & 38.4158 & MD & 151 & -0.1 & -75.0599 & 38.3836 & MD \\
\hline 8 & -0.4 & -75.0454 & 38.4479 & MD & 80 & -0.5 & -75.0509 & 38.4153 & MD & 152 & -0.1 & -75.06 & 38.3832 & MD \\
\hline 9 & -0.5 & -75.0455 & 38.4474 & MD & 81 & .0 .6 & .75 .051 & 38.4148 & MD & 153 & 0.2 & -75.0602 & 38.3827 & MD \\
\hline 10 & -0.5 & -75.0455 & 38.4469 & MD & 82 & -0.7 & -75.0511 & 38.4144 & MD & 154 & 0.4 & -75.0603 & 38.3822 & MD \\
\hline 11 & -0.5 & -75.0455 & 38.4465 & $\mathrm{MD}$ & 83 & -0.8 & -75.0512 & 38.4139 & MD & 155 & 0.5 & -75.0604 & 38.3818 & MD \\
\hline 12 & -0.4 & -75.0455 & 38.446 & MD & 84 & -0.9 & -75.0513 & 38.4135 & MD & 156 & 0.5 & -75.0606 & 38.3813 & MD \\
\hline 13 & -0.4 & -75.0455 & 38.4456 & MD & 85 & -1 & -75.0514 & 38.413 & MD & 157 & 0.5 & -75.0607 & 38.3809 & $M D$ \\
\hline 14 & -0.5 & -75.0455 & 38.4451 & MD & 86 & -1.2 & -75.0515 & 38.4125 & MD & 158 & 0.5 & -75.0608 & 38.3804 & MD \\
\hline 15 & -0.4 & -75.0455 & 38.4446 & MD & 87 & -1.1 & .75 .0515 & 38.4121 & MD & 159 & 0.5 & -75.061 & 38.38 & MD \\
\hline 16 & -0.4 & .75 .0455 & 38.4442 & MD & 88 & -1.1 & -75.0516 & 38.4116 & MD & 160 & 0.2 & -75.061 & 38.38 & MD \\
\hline 17 & -0.4 & -75.0456 & 38.4437 & $\mathrm{MD}$ & 89 & -1.2 & -75.0517 & 38.4112 & MD & 161 & 0.4 & -75.0611 & 38.3795 & $\mathrm{MD}$ \\
\hline 18 & -0.3 & -75.0456 & 38.4432 & $\mathrm{MD}$ & 90 & -1.1 & .75 .0519 & 38.411 & $\mathrm{MD}$ & 162 & 0 & -75.0611 & 38.3795 & $\mathrm{MD}$ \\
\hline 19 & -0.2 & .75 .0456 & 38.4428 & MD & 91 & -1.3 & -75.0518 & 38.4107 & $\mathrm{MD}$ & 163 & 0 & -75.0613 & 38.3791 & MD \\
\hline 20 & -0.3 & -75.0458 & 38.4425 & $\mathrm{MD}$ & 92 & -1.2 & -75.052 & 38.4105 & MD & 164 & 0.1 & -75.0614 & 38.3786 & MD \\
\hline 21 & 0 & -75.0456 & 38.4423 & MD & 93 & -1.3 & -75.0521 & 38.4101 & MD & 165 & 0 & -75.0616 & 38.3782 & MD \\
\hline 22 & -0.4 & -75.0459 & 38.442 & MD & 94 & -1.3 & -75.0523 & 38.4096 & MD & 166 & -0.2 & -75.0617 & 38.3777 & MD \\
\hline 23 & -0.5 & -75.0459 & 38.4416 & MD & 95 & -1.2 & -75.0524 & 38.4092 & MD & 167 & -0.1 & -75.0618 & 38.3773 & MD \\
\hline 24 & -0.5 & -75.046 & 38.4411 & MD & 96 & -1 & -75.0525 & 38.4087 & $\mathrm{MD}$ & 168 & -0.1 & -75.062 & 38.3768 & MD \\
\hline 25 & -0.5 & -75.0461 & 38.4406 & MD & 97 & -0.9 & -75.0527 & 38.4082 & MD & 169 & -0.1 & -75.0621 & 38.3764 & MD \\
\hline 26 & -0.5 & -75.0462 & 38.4402 & MD & 98 & -0.7 & -75.0528 & 38.4078 & MD & 170 & -0.1 & -75.0623 & 38.3759 & $M D$ \\
\hline 27 & -0.5 & -75.0463 & 38.4397 & MD & 99 & -0.6 & -75.0529 & 38.4073 & MD & 171 & -0.2 & -75.0624 & 38.3755 & MD \\
\hline 28 & -0.3 & -75.0464 & 38.4393 & MD & 100 & -0.6 & -75.0531 & 38.4069 & MD & 172 & -0.2 & -75.0626 & 38.375 & MD \\
\hline 29 & -0.5 & -75.0465 & 38.4388 & MD & 101 & -0.6 & -75.0532 & 38.4064 & MD & 173 & 0 & .75 .0627 & 38.3746 & MD \\
\hline 30 & -0.7 & -75.0466 & 38.4383 & MD & 102 & -0.5 & -75.0533 & 38.406 & MD & 174 & 0.1 & -75.0629 & 38.3741 & $\mathrm{MD}$ \\
\hline 31 & -0.8 & -75.0466 & 38.4379 & MD & 103 & -0.6 & -75.0535 & 38.4055 & MD & 175 & 0.1 & .75 .063 & 38.3737 & $\mathrm{MD}$ \\
\hline 32 & -0.8 & -75.0467 & 38.4374 & MD & 104 & -0.7 & -75.0536 & 38.4051 & MD & 176 & 0.1 & -75.0631 & 38.3732 & $\mathrm{MD}$ \\
\hline 33 & -0.6 & -75.0468 & 38.437 & MD & 105 & -0.9 & -75.0537 & 38.4046 & MD & 177 & 0 & -75.0633 & 38.3728 & $\mathrm{MD}$ \\
\hline 34 & -0.4 & -75.0469 & 38.4365 & MD & 106 & -1 & .75 .0539 & 38.4041 & MD & 178 & -0.1 & -75.0634 & 38.3723 & MD \\
\hline 35 & -0.6 & .75 .047 & 38.436 & MD & 107 & -1 & .75 .054 & 38.4037 & MD & 179 & -0.1 & -75.0636 & 38.3719 & MD \\
\hline 36 & -0.7 & -75.0471 & 38.4356 & MD & 108 & -1.1 & -75.0541 & 38.4032 & MD & 180 & -0.1 & -75.0637 & 38.3714 & MD \\
\hline 37 & -0.8 & -75.0472 & 38.4351 & MD & 109 & -1.2 & -75.0543 & 38.4028 & MD & 181 & -0.2 & .75 .0639 & 38.371 & MD \\
\hline 38 & -0.8 & -75.0473 & 38.4346 & $\mathrm{MD}$ & 110 & -1.3 & -75.0544 & 38.4023 & MD & 182 & -0.3 & -75.064 & 38.3705 & MD \\
\hline 39 & -0.7 & -75.0473 & 38.4342 & MD & 111 & -1.3 & -75.0545 & 38.4019 & $\mathrm{MD}$ & 183 & -0.2 & -75.0641 & 38.37 & MD \\
\hline 40 & -0.6 & -75.0474 & 38.4337 & MD & 112 & -1.2 & -75.0547 & 38.4014 & MD & 184 & -0.2 & -75.0643 & 38.3696 & MD \\
\hline 41 & -0.7 & -75.0475 & 38.4333 & MD & 113 & -1.3 & .75 .0548 & 38.4009 & MD & 185 & -0.2 & -75.0644 & 38.3691 & MD \\
\hline 42 & -0.8 & -75.0476 & 38.4328 & MD & 114 & -1.4 & -75.0549 & 38.4005 & MD & 186 & -0.2 & -75.0646 & 38.3687 & MD \\
\hline 43 & -0.8 & -75.0477 & 38.4323 & MD & 115 & -1.3 & -75.0551 & 38.4 & MD & 187 & -0.3 & -75.0647 & 38.3682 & MD \\
\hline 44 & -0.9 & -75.0478 & 38.4319 & MD & 116 & -1.3 & -75.0552 & 38.3996 & MD & 188 & -0.4 & -75.0649 & 38.3678 & MD \\
\hline 45 & -0.8 & -75.0479 & 38.4314 & MD & 117 & -1.5 & .75 .0553 & 38.3991 & MD & 189 & -0.3 & -75.065 & 38.3673 & MD \\
\hline 46 & -0.7 & -75.048 & 38.431 & MD & 118 & -1.6 & .75 .0555 & 38.3987 & $\mathrm{MD}$ & 190 & -0.1 & -75.0652 & 38.3669 & MD \\
\hline 47 & -0.7 & -75.048 & 38.4305 & MD & 119 & -1.7 & -75.0556 & 38.3982 & MD & 191 & -0.2 & -75.0653 & 38.3664 & MD \\
\hline 48 & -0.7 & -75.0481 & 38.43 & MD & 120 & -1.7 & -75.0557 & 38.3978 & MD & 192 & -0.2 & -75.0654 & 38.366 & MD \\
\hline 49 & -0.6 & -75.0482 & 38.4296 & MD & 121 & -1.6 & -75.0559 & 38.3973 & MD & 193 & -0.3 & .75 .0656 & 38.3655 & $\mathrm{MD}$ \\
\hline 50 & -0.5 & .75 .0483 & 38.4291 & MD & 122 & -1.6 & .75 .056 & 38.3968 & MD & 194 & -0.3 & -75.0657 & 38.3651 & MD \\
\hline 51 & -0.5 & -75.0484 & 38.4287 & MD & 123 & -1.6 & .75 .0561 & 38.3964 & MD & 195 & -0.3 & -75.0659 & 38.3646 & MD \\
\hline
\end{tabular}




\begin{tabular}{|c|c|c|c|c|c|c|c|c|c|c|c|c|c|c|}
\hline TR\# & RATE & LONG & LAT & ST & I TR\# & RATE & LONG & LAT & ST & TR\# & RATE & LONG & LAT & ST \\
\hline 196 & -0.3 & -75.066 & 38.3642 & MD & 268 & 1.2 & -75.0791 & 38.3327 & MD & 340 & -8.8 & -75.101 & 38.3021 & MD \\
\hline 197 & -0.3 & -75.0662 & 38.3637 & MD & 269 & 1.4 & -75.0793 & 38.3323 & MD & 341 & -9.3 & -75.0999 & 38.3017 & MD \\
\hline 198 & -0.2 & -75.0663 & 38.3633 & MD & 270 & 1.6 & -75.0795 & 38.3319 & MD & 342 & -8.7 & -75.1012 & 38.3017 & MD \\
\hline 199 & -0.2 & -75.0664 & 38.3628 & MD & 271 & 1.9 & -75.0797 & 38.3314 & MD & 343 & -9.4 & -75.1002 & 38.3013 & $M D$ \\
\hline 200 & -0.2 & .75 .0666 & 38.3624 & MD & 272 & 2.1 & -75.0799 & 38.331 & MD & 344 & -8.8 & -75.1013 & 38.3013 & MD \\
\hline 201 & -0.1 & -75.0667 & 38.3619 & MD & 273 & 2.2 & -75.0801 & 38.3306 & MD & 345 & -8.9 & -75.1015 & 38.3008 & MD \\
\hline 202 & 0 & -75.0669 & 38.3615 & MD & 274 & 2.3 & -75.0804 & 38.3301 & $\mathrm{MD}$ & 346 & -8.7 & -75.1017 & 38.3004 & MD \\
\hline 203 & 0 & -75.067 & 38.361 & MD & 275 & 2.4 & -75.0806 & 38.3297 & $\mathrm{MD}$ & 347 & -8.5 & -75.1018 & 38.2999 & MD \\
\hline 204 & 0.1 & -75.0672 & 38.3606 & MD & 276 & 2.5 & -75.0808 & 38.3292 & $\mathrm{MD}$ & 348 & -8.4 & -75.102 & 38.2995 & $\mathrm{MD}$ \\
\hline 205 & 0.1 & -75.0673 & 38.3601 & MD & 277 & 2.7 & -75.081 & 38.3288 & MD & 349 & -8.3 & -75.1022 & 38.299 & MD \\
\hline 206 & 0 & -75.0675 & 38.3597 & $M D$ & 278 & 3 & -75.0812 & 38.3284 & MD & 350 & -8.3 & -75.1023 & 38.2986 & MD \\
\hline 207 & 0 & -75.0676 & 38.3592 & MD & 279 & 3.3 & -75.0814 & 38.3279 & MD & 351 & -8.2 & -75.1025 & 38.2981 & MD \\
\hline 208 & 0 & -75.0677 & 38.3587 & MD & 280 & 3.5 & -75.0817 & 38.3275 & MD & 352 & -8.1 & -75.1027 & 38.2977 & MD \\
\hline 209 & 0 & -75.0679 & 38.3583 & MD & 281 & 3.7 & -75.0819 & 38.3271 & MD & 353 & -8.1 & -75.1028 & 38.2973 & MD \\
\hline 210 & -0.1 & -75.068 & 38.3578 & MD & 282 & 3.8 & -75.0821 & 38.3266 & MD & 354 & -8 & .75 .103 & 38.2968 & MD \\
\hline 211 & 0 & -75.0682 & 38.3574 & MD & 283 & 4 & -75.0823 & 38.3262 & MD & 355 & -7.9 & -75.1032 & 38.2964 & MD \\
\hline 212 & 0.1 & -75.0683 & 38.3569 & MD & 284 & 4.2 & -75.0825 & 38.3258 & MD & 356 & -7.8 & .75 .1033 & 38.2959 & MD \\
\hline 213 & 0.1 & -75.0685 & 38.3565 & MD & 285 & 4.2 & .75 .0827 & 38.3253 & MD & 357 & -7.8 & -75.1035 & 38.2955 & MD \\
\hline 214 & 0.2 & -75.0686 & 38.356 & MD & 286 & 4.1 & -75.083 & 38.3249 & MD & 358 & -7.9 & -75.1037 & 38.295 & $\mathrm{MD}$ \\
\hline 215 & 0.2 & -75.0687 & 38.3556 & MD & 287 & 4.3 & -75.0832 & 38.3245 & MD & 359 & -7.9 & .75 .1038 & 38.2946 & $\mathrm{MD}$ \\
\hline 216 & 0.2 & -75.0689 & 38.3551 & MD & 288 & 4.4 & .75 .0834 & 38.324 & MD & 360 & -7.8 & -75.104 & 38.2942 & $\mathrm{MD}$ \\
\hline 217 & 0 & -75.069 & 38.3547 & MD & 289 & 4.4 & -75.0836 & 38.3236 & MD & 361 & -7.6 & -75.1042 & 38.2937 & $M D$ \\
\hline 218 & -0.2 & -75.0692 & 38.3542 & MD & 290 & 4.3 & -75.0838 & 38.3232 & MD & 362 & -7.4 & -75.1043 & 38.2933 & MD \\
\hline 219 & -0.2 & -75.0693 & 38.3538 & MD & 291 & -2 & -75.0861 & 38.3207 & MD & 363 & -7.3 & -75.1045 & 38.2928 & MD \\
\hline 220 & -0.3 & -75.0695 & 38.3533 & MD & 292 & -6.9 & -75.0864 & 38.3203 & MD & 364 & -7.2 & .75 .1047 & 38.2924 & MD \\
\hline 221 & -0.2 & -75.0696 & 38.3529 & MD & 293 & -9.8 & -75.0867 & 38.3199 & MD & 365 & -7.1 & -75.1048 & 38.2919 & MD \\
\hline 222 & -0.1 & -75.0698 & 38.3524 & MD & 294 & -7.4 & -75.087 & 38.3195 & MD & 366 & -6.9 & -75.105 & 38.2915 & MD \\
\hline 223 & 0.1 & -75.0699 & 38.352 & MD & 295 & -5 & -75.0873 & 38.3191 & MD & 367 & -6.8 & -75.1052 & 38.291 & MD \\
\hline 224 & 0.3 & -75.07 & 38.3515 & MD & 296 & -5.4 & -75.0876 & 38.3187 & $M D$ & 368 & -6.7 & -75.1053 & 38.2906 & MD \\
\hline 225 & 0.2 & -75.0702 & 38.3511 & MD & 297 & -5.9 & -75.0878 & 38.3183 & MD & 369 & -6.6 & -75.1055 & 38.2902 & MD \\
\hline 226 & 0.2 & -75.0703 & 38.3506 & MD & 298 & -6.2 & -75.0881 & 38.3179 & $\mathrm{MD}$ & 370 & -6.6 & -75.1057 & 38.2897 & MD \\
\hline 227 & 0 & -75.0705 & 38.3502 & MD & 299 & -6.6 & -75.0884 & 38.3175 & MD & 371 & -6.7 & -75.1058 & 38.2893 & MD \\
\hline 228 & -0.1 & -75.0706 & 38.3497 & MD & 300 & -6.7 & -75.0887 & 38.3171 & MD & 372 & -6.6 & -75.106 & 38.2888 & MD \\
\hline 229 & -0.1 & -75.0708 & 38.3493 & MD & 301 & -6.9 & -75.089 & 38.3167 & $\mathrm{MD}$ & 373 & -6.5 & -75.1061 & 38.2884 & MD \\
\hline 230 & -0.1 & -75.0709 & 38.3488 & MD & 302 & -7.1 & -75.0893 & 38.3163 & MD & 374 & -6.4 & -75.1063 & 38.2879 & MD \\
\hline 231 & -0.2 & -75.071 & 38.3488 & MD & 303 & -7.3 & -75.0896 & 38.3159 & MD & 375 & -6.3 & -75.1065 & 38.2875 & MD \\
\hline 232 & -0.1 & -75.0713 & 38.3484 & MD & 304 & -7.2 & -75.0899 & 38.3155 & MD & 376 & -6.3 & -75.1066 & 38.2871 & MD \\
\hline 233 & -0.2 & -75.0715 & 38.348 & MD & 305 & -7.2 & -75.0902 & 38.3151 & MD & 377 & -6.2 & -75.1068 & 38.2866 & MD \\
\hline 234 & -0.2 & -75.0717 & 38.3475 & MD & 306 & -7.4 & -75.0905 & 38.3146 & $\mathrm{MD}$ & 378 & -5.9 & -75.107 & 38.2862 & $\mathrm{MD}$ \\
\hline 235 & -0.2 & -75.0719 & 38.3471 & MD & 307 & -7.7 & -75.0908 & 38.3142 & MD & 379 & -5.6 & -75.1071 & 38.2857 & MD \\
\hline 236 & -0.3 & -75.0721 & 38.3467 & MD & 308 & -7.7 & -75.0911 & 38.3138 & $M D$ & 380 & -5.5 & -75.1073 & 38.2853 & MD \\
\hline 237 & -0.3 & -75.0723 & 38.3462 & MD & 309 & -7.6 & -75.0914 & 38.3134 & MD & 381 & -5.4 & -75.1075 & 38.2848 & MD \\
\hline 238 & -0.2 & -75.0726 & 38.3458 & MD & 310 & -7.6 & -75.0917 & 38.313 & $\mathrm{MD}$ & 382 & -5.4 & .75 .1076 & 38.2844 & MD \\
\hline 239 & -0.2 & -75.0728 & 38.3453 & MD & 311 & -7.5 & -75.092 & 38.3126 & MD & 383 & -5.4 & -75.1078 & 38.2839 & MD \\
\hline 240 & -0.1 & -75.073 & 38.3449 & MD & 312 & -8 & -75.0923 & 38.3122 & MD & 384 & .5 .3 & -75.108 & 38.2835 & MD \\
\hline 241 & -0.1 & -75.0732 & 38.3445 & MD & 313 & -8.4 & .75 .0926 & 38.3118 & $\mathrm{MD}$ & 385 & -5.2 & -75.1081 & 38.2831 & $\mathrm{MD}$ \\
\hline 242 & 0 & -75.0734 & 38.344 & MD & 314 & -8.6 & -75.0929 & 38.3114 & $\mathrm{MD}$ & 386 & -5.1 & -75.1083 & 38.2826 & MD \\
\hline 243 & 0 & -75.0736 & 38.3436 & MD & 315 & -8.8 & -75.0931 & 38.311 & $\mathrm{MD}$ & 387 & -5.1 & -75.1085 & 38.2822 & MD \\
\hline 244 & 0.1 & -75.0739 & 38.3432 & MD & 316 & -8.9 & .75 .0934 & 38.3106 & $\mathrm{MD}$ & 388 & -5 & -75.1086 & 38.2817 & MD \\
\hline 245 & 0 & -75.0741 & 38.3427 & MD & 317 & -9 & -75.0937 & 38.3102 & MD & 389 & -4.9 & -75.1088 & 38.2813 & MD \\
\hline 246 & -0.2 & -75.0743 & 38.3423 & MD & 318 & -9.1 & .75 .094 & 38.3098 & MD & 390 & -4.7 & .75 .109 & 38.2808 & $\mathrm{MD}$ \\
\hline 247 & -0.3 & -75.0745 & 38.3419 & MD & 319 & -9.1 & -75.0943 & 38.3094 & MD & 391 & -4.5 & -75.1091 & 38.2804 & MD \\
\hline 248 & -0.4 & -75.0747 & 38.3414 & MD & 320 & -9.2 & -75.0946 & 38.309 & MD & 392 & -4.6 & -75.1093 & 38.28 & MD \\
\hline 249 & -0.4 & -75.0749 & 38.341 & MD & 321 & -9.3 & -75.0949 & 38.3086 & MD & 393 & -4.6 & -75.1095 & 38.2795 & MD \\
\hline 250 & -0.4 & -75.0752 & 38.3406 & MD & 322 & -9.3 & .75 .0952 & 38.3082 & MD & 394 & -4.6 & -75.1096 & 38.2791 & $\mathrm{MD}$ \\
\hline 251 & -0.4 & -75.0754 & 38.3401 & MD & 323 & -9.4 & -75.0955 & 38.3078 & MD & 395 & -4.5 & -75.1098 & 38.2786 & MD \\
\hline 252 & -0.4 & -75.0756 & 38.3397 & MD & 324 & -9.4 & -75.0958 & 38.3074 & MD & 396 & -4.5 & -75.11 & 38.2782 & MD \\
\hline 253 & -0.6 & -75.0758 & 38.3393 & MD & 325 & -9.3 & -75.0961 & 38.307 & MD & 397 & -4.5 & -75.1101 & 38.2777 & MD \\
\hline 254 & -0.8 & .75 .076 & 38.3388 & MD & 326 & -9.4 & -75.0964 & 38.3066 & $\mathrm{MD}$ & 398 & -4.4 & -75.1103 & 38.2773 & MD \\
\hline 255 & -0.5 & -75.0762 & 38.3384 & MD & 327 & -9.5 & -75.0967 & 38.3062 & MD & 399 & -4.3 & -75.1105 & 38.2768 & MD \\
\hline 256 & -0.3 & -75.0765 & 38.3379 & MD & 328 & -9.5 & -75.097 & 38.3058 & MD & 400 & -4.1 & -75.1106 & 38.2764 & MD \\
\hline 257 & -0.3 & -75.0767 & 38.3375 & MD & 329 & -9.5 & -75.0973 & 38.3054 & $M D$ & 401 & -4 & -75.1108 & 38.276 & MD \\
\hline 258 & -0.3 & -75.0769 & 38.3371 & MD & 330 & -9.3 & -75.0976 & 38.3049 & MD & 402 & -3.9 & -75.111 & 38.2755 & MD \\
\hline 259 & 0 & -75.0771 & 38.3366 & MD & 331 & -9.2 & -75.0979 & 38.3045 & MD & 403 & -5 & -75.1139 & 38.2752 & MD \\
\hline 260 & 0.4 & -75.0773 & 38.3362 & MD & 332 & -9.2 & -75.0982 & 38.3041 & MD & 404 & -3.8 & -75.1111 & 38.2751 & MD \\
\hline 261 & 0.5 & -75.0775 & 38.3358 & MD & 333 & -9.2 & -75.0984 & 38.3037 & MD & 405 & -4.8 & -75.1141 & 38.2748 & MD \\
\hline 262 & 0.5 & -75.0778 & 38.3353 & MD & 334 & -9.3 & -75.0987 & 38.3033 & MD & 406 & -3.6 & -75.1113 & 38.2746 & MD \\
\hline 263 & 0.7 & -75.078 & 38.3349 & MD & 335 & -8.8 & -75.1007 & 38.303 & MD & 407 & -4.5 & -75.1143 & 38.2743 & MD \\
\hline 264 & 0.8 & -75.0782 & 38.3345 & MD & 336 & -9.4 & -75.099 & 38.3029 & MD & 408 & -3.4 & -75.1115 & 38.2742 & MD \\
\hline 265 & 1 & -75.0784 & 38.334 & MD & 337 & -8.8 & -75.1008 & 38.3026 & MD & 409 & -4.2 & -75.1145 & 38.2739 & MD \\
\hline 266 & 1.1 & -75.0786 & 38.3336 & MD & 338 & -9.3 & -75.0993 & 38.3025 & MD & 410 & -4 & -75.1148 & 38.2735 & MD \\
\hline 267 & 1.2 & -75.0788 & 38.3332 & MD & 339 & -9.2 & -75.0996 & 38.3021 & MD & 411 & -3.9 & -75.115 & 38.273 & MD \\
\hline
\end{tabular}




\begin{tabular}{|c|c|c|c|c|c|c|c|c|c|c|c|c|c|c|}
\hline TR\# & RATE & LONG & LAT & ST & TR\# & RATE & LONG & LAT & ST & TR\# & RATE & LONG & LAT & ST \\
\hline 412 & -3.7 & -75.1152 & 38.2726 & $\mathrm{MD}$ & 484 & -0.5 & -75.1299 & 38.2425 & $\mathrm{MD}$ & 556 & -1.1 & -75.1439 & 38.2124 & $\mathrm{MD}$ \\
\hline 413 & -3.5 & -75.1154 & 38.2722 & MD & 485 & -0.5 & -75.1301 & 38.2421 & MD & 557 & -1.1 & -75.1441 & 38.212 & MD \\
\hline 414 & -3.3 & -75.1156 & 38.2717 & MD & 486 & -0.2 & -75.1304 & 38.2417 & MD & 558 & -1 & -75.1443 & 38.2115 & MD \\
\hline 415 & -3.2 & -75.1158 & 38.2713 & $\mathrm{MD}$ & 487 & 0.1 & -75.1306 & 38.2412 & MD & 559 & -1 & -75.1445 & 38.2111 & MD \\
\hline 416 & -3 & -75.1161 & 38.2708 & MD & 488 & 0.1 & -75.1308 & 38.2408 & $\mathrm{MD}$ & 560 & -1 & -75.1447 & 38.2106 & $\mathrm{MD}$ \\
\hline 417 & -2.9 & -75.1163 & 38.2704 & MD & 489 & 0.2 & -75.131 & 38.2404 & MD & 561 & -1 & -75.1449 & 38.2102 & MD \\
\hline 418 & -2.7 & -75.1165 & 38.27 & MD & 490 & 0.2 & -75.1312 & 38.2399 & MD & 562 & -0.8 & -75.145 & 38.2097 & MD \\
\hline 419 & -2.5 & -75.1167 & 38.2695 & MD & 491 & 0.2 & -75.1314 & 38.2395 & MD & 563 & -0.6 & .75 .1452 & 38.2093 & MD \\
\hline 420 & -2.3 & -75.1169 & 38.2691 & MD & 492 & 0.3 & .75 .1316 & 38.2391 & MD & 564 & -0.6 & -75.1454 & 38.2089 & MD \\
\hline 421 & -2.2 & -75.1171 & 38.2687 & MD & 493 & 0.3 & -75.1319 & 38.2386 & MD & 565 & -0.7 & -75.1456 & 38.2084 & MD \\
\hline 422 & -2.1 & -75.1173 & 38.2682 & MD & 494 & 0.2 & -75.1321 & 38.2382 & MD & 566 & -0.6 & -75.1458 & 38.208 & MD \\
\hline 423 & -2 & -75.1176 & 38.2678 & MD & 495 & 0 & -75.1323 & 38.2378 & MD & 567 & -0.6 & -75.146 & 38.2075 & MD \\
\hline 424 & -2 & -75.1178 & 38.2674 & MD & 496 & -0.1 & -75.1325 & 38.2373 & MD & 568 & -0.6 & -75.1462 & 38.2071 & MD \\
\hline 425 & -1.9 & -75.118 & 38.2669 & MD & 497 & -0.3 & -75.1327 & 38.2369 & MD & 569 & -0.6 & -75.1464 & 38.2067 & MD \\
\hline 426 & -1.8 & -75.1182 & 38.2665 & MD & 498 & -0.3 & -75.1329 & 38.2365 & MD & 570 & -0.4 & -75.1466 & 38.2062 & MD \\
\hline 427 & -1.7 & -75.1184 & 38.2661 & MD & 499 & -0.3 & -75.1332 & 38.236 & MD & 571 & -0.3 & -75.1468 & 38.2058 & MD \\
\hline 428 & -1.7 & -75.1186 & 38.2656 & MD & 500 & -0.6 & -75.1334 & 38.2356 & MD & 572 & -0.2 & -75.1469 & 38.2053 & MD \\
\hline 429 & -1.7 & -75.1189 & 38.2652 & MD & 501 & -0.9 & -75.1336 & 38.2352 & MD & 573 & -0.1 & -75.1471 & 38.2049 & MD \\
\hline 430 & -1.9 & -75.1191 & 38.2648 & MD & 502 & -0.8 & -75.1338 & 38.2347 & MD & 574 & -1 & -75.1473 & 38.2045 & MD \\
\hline 431 & -2 & -75.1193 & 38.2643 & MD & 503 & -0.7 & -75.134 & 38.2343 & MD & 575 & -1.1 & -75.1475 & 38.204 & MD \\
\hline 432 & -3.8 & -75.1195 & 38.2639 & MD & 504 & -0.7 & -75.1342 & 38.2339 & MD & 576 & -1.1 & -75.1477 & 38.2036 & MD \\
\hline 433 & -3.9 & -75.1197 & 38.2634 & MD & 505 & -0.7 & -75.1344 & 38.2334 & MD & 577 & -1.1 & -75.1479 & 38.2031 & MD \\
\hline 434 & -3.7 & -75.1199 & 38.263 & $\mathrm{MD}$ & 506 & -0.7 & -75.1347 & 38.233 & MD & 578 & -1.1 & -75.1481 & 38.2027 & MD \\
\hline 435 & -3.5 & -75.1201 & 38.2626 & MD & 507 & -0.7 & -75.1349 & 38.2326 & MD & 579 & -1.1 & -75.1483 & 38.2023 & MD \\
\hline 436 & -3.5 & -75.1204 & 38.2621 & MD & 508 & -0.8 & -75.1351 & 38.2321 & $M D$ & 580 & -1.1 & -75.1485 & 38.2018 & MD \\
\hline 437 & -3.5 & -75.1206 & 38.2617 & MD & 509 & -1 & -75.1353 & 38.2317 & MD & 581 & -1.1 & -75.1487 & 38.2014 & MD \\
\hline 438 & -3.6 & -75.1208 & 38.2613 & MD & 510 & -1 & -75.1355 & 38.2313 & MD & 582 & -1.1 & -75.1488 & 38.2009 & MD \\
\hline 439 & -3.8 & -75.121 & 38.2608 & MD & 511 & -1 & -75.1357 & 38.2308 & MD & 583 & -1.1 & -75.149 & 38.2005 & MD \\
\hline 440 & -3.7 & -75.1212 & 38.2604 & MD & 512 & -0.9 & -75.136 & 38.2304 & MD & 584 & -1 & -75.1492 & 38.2 & MD \\
\hline 441 & -3.7 & -75.1214 & 38.26 & MD & 513 & -0.9 & -75.1362 & 38.2299 & MD & 585 & -1 & -75.1494 & 38.1996 & $\mathrm{MD}$ \\
\hline 442 & -3.7 & -75.1217 & 38.2595 & MD & 514 & -0.9 & -75.1364 & 38.2295 & MD & 586 & -0.8 & -75.1496 & 38.1992 & MD \\
\hline 443 & -3.8 & -75.1219 & 38.2591 & MD & 515 & -1 & -75.1366 & 38.2291 & MD & 587 & -0.7 & -75.1498 & 38.1987 & MD \\
\hline 444 & -3.7 & -75.1221 & 38.2587 & MD & 516 & -1.1 & -75.1368 & 38.2286 & MD & 588 & -0.4 & -75.15 & 38.1983 & MD \\
\hline 445 & -3.6 & -75.1223 & 38.2582 & MD & 517 & -1.3 & -75.137 & 38.2282 & MD & 589 & 0 & -75.1502 & 38.1978 & MD \\
\hline 446 & -3.5 & -75.1225 & 38.2578 & MD & 518 & -1.3 & -75.1373 & 38.2278 & MD & 590 & 0.1 & -75.1504 & 38.1974 & MD \\
\hline 447 & -3.5 & -75.1227 & 38.2574 & MD & 519 & -1.3 & -75.1375 & 38.2273 & MD & 591 & 0.2 & -75.1506 & 38.197 & MD \\
\hline 448 & -3.2 & -75.123 & 38.2569 & MD & 520 & -1.3 & -75.1377 & 38.2269 & MD & 592 & 0 & -75.1507 & 38.1965 & MD \\
\hline 449 & -2.9 & -75.1232 & 38.2565 & MD & 521 & -1.3 & -75.1379 & 38.2265 & MD & 593 & -0.1 & -75.1509 & 38.1961 & MD \\
\hline 450 & -3 & -75.1234 & 38.2561 & MD & 522 & -1.3 & -75.1381 & 38.226 & MD & 594 & -0.2 & -75.1511 & 38.1956 & $\mathrm{MD}$ \\
\hline 451 & -3.2 & -75.1236 & 38.2556 & MD & 523 & -1.2 & -75.1383 & 38.2256 & MD & 595 & -0.2 & -75.1513 & 38.1952 & MD \\
\hline 452 & -3.1 & -75.1238 & 38.2552 & MD & 524 & -1.3 & -75.1385 & 38.2252 & MD & 596 & -0.3 & -75.1515 & 38.1948 & MD \\
\hline 453 & -2.9 & -75.124 & 38.2547 & MD & 525 & -1.3 & -75.1388 & 38.2247 & MD & 597 & -0.4 & -75.1517 & 38.1943 & MD \\
\hline 454 & -2.7 & -75.1242 & 38.2543 & MD & 526 & -1.5 & -75.139 & 38.2243 & MD & 598 & -0.4 & -75.1519 & 38.1939 & MD \\
\hline 455 & -2.4 & -75.1245 & 38.2539 & MD & 527 & -1.7 & -75.1392 & 38.2239 & MD & 599 & 0.7 & -75.1521 & 38.1934 & $\mathrm{MD}$ \\
\hline 456 & -2.3 & -75.1247 & 38.2534 & MD & 528 & -1.8 & -75.1394 & 38.2234 & MD & 600 & 0.7 & -75.1523 & 38.193 & MD \\
\hline 457 & -2.2 & -75.1249 & 38.253 & MD & 529 & -1.8 & -75.1396 & 38.223 & MD & 601 & 0.7 & -75.1525 & 38.1926 & MD \\
\hline 458 & -2.3 & -75.1251 & 38.2526 & MD & 530 & -1.8 & -75.1398 & 38.2226 & MD & 602 & 0.7 & -75.1526 & 38.1921 & MD \\
\hline 459 & -2.4 & -75.1253 & 38.2521 & MD & 531 & -1.8 & -75.1401 & 38.2221 & MD & 603 & 0.7 & -75.1528 & 38.1917 & MD \\
\hline 460 & -2.3 & -75.1255 & 38.2517 & MD & 532 & -1.9 & -75.1403 & 38.2217 & MD & 604 & 0.7 & -75.153 & 38.1912 & MD \\
\hline 461 & -2.2 & -75.1258 & 38.2513 & MD & 533 & -2 & -75.1405 & 38.2213 & MD & 605 & 0.6 & -75.1532 & 38.1908 & MD \\
\hline 462 & -2.1 & -75.126 & 38.2508 & MD & 534 & -2 & -75.1407 & 38.2208 & MD & 606 & 0.7 & -75.1534 & 38.1903 & MD \\
\hline 463 & -2.1 & -75.1262 & 38.2504 & MD & 535 & -2 & -75.1409 & 38.2204 & MD & 607 & 0.7 & -75.1536 & 38.1899 & MD \\
\hline 464 & -2 & -75.1264 & 38.25 & MD & 536 & -2 & -75.1411 & 38.22 & MD & 608 & 0.7 & -75.1538 & 38.1895 & $\mathrm{MD}$ \\
\hline 465 & -1.9 & -75.1266 & 38.2495 & MD & 537 & -2 & -75.1413 & 38.2195 & MD & 609 & 0.8 & -75.154 & 38.189 & MD \\
\hline 466 & -1.7 & -75.1268 & 38.2491 & MD & 538 & -1.7 & -75.1416 & 38.2191 & MD & 610 & 0.9 & -75.1542 & 38.1886 & MD \\
\hline 467 & -1.6 & -75.127 & 38.2487 & MD & 539 & -1.5 & -75.1418 & 38.2187 & MD & 611 & 0.9 & -75.1544 & 38.1881 & MD \\
\hline 468 & -1.6 & -75.1273 & 38.2482 & MD & 540 & -1.3 & -75.142 & 38.2182 & MD & 612 & 0.9 & -75.1545 & 38.1877 & MD \\
\hline 469 & -1.7 & -75.1275 & 38.2478 & MD & 541 & -1.1 & -75.1422 & 38.2178 & MD & 613 & 0.9 & -75.1547 & 38.1873 & MD \\
\hline 470 & -1.4 & -75.1277 & 38.2473 & MD & 542 & -1.2 & -75.1424 & 38.2174 & MD & 614 & -0.4 & -75.1542 & 38.187 & MD \\
\hline 471 & -1.2 & -75.1279 & 38.2469 & MD & 543 & -1.3 & -75.1426 & 38.2169 & MD & 615 & 1 & -75.1549 & 38.1868 & MD \\
\hline 472 & -3.1 & -75.1281 & 38.2465 & MD & 544 & -1.6 & -75.142 & 38.2168 & MD & 616 & -0.5 & -75.1544 & 38.1866 & MD \\
\hline 473 & -3.2 & -75.1283 & 38.246 & MD & 545 & -1.2 & -75.1429 & 38.2165 & MD & 617 & 1 & -75.1551 & 38.1864 & MD \\
\hline 474 & -2.6 & -75.1282 & 38.246 & MD & 546 & -1.5 & -75.1422 & 38.2164 & MD & 618 & -0.6 & -75.1545 & 38.1861 & MD \\
\hline 475 & -3.4 & -75.1286 & 38.2456 & MD & 547 & -1.2 & -75.1431 & 38.2161 & MD & 619 & -0.6 & -75.1547 & 38.1857 & MD \\
\hline 476 & -2.6 & -75.1284 & 38.2456 & MD & 548 & -1.4 & -75.1424 & 38.2159 & MD & 620 & -0.6 & -75.1549 & 38.1852 & MD \\
\hline 477 & -3.7 & -75.1288 & 38.2452 & MD & 549 & -1.3 & -75.1426 & 38.2155 & MD & 621 & -0.6 & -75.155 & 38.1848 & MD \\
\hline 478 & -2.7 & -75.1286 & 38.2451 & MD & 550 & -1.4 & -75.1428 & 38.215 & MD & 622 & -0.7 & -75.1552 & 38.1843 & MD \\
\hline 479 & -2.7 & -75.1288 & 38.2447 & MD & 551 & -1.6 & -75.143 & 38.2146 & MD & 623 & -0.8 & -75.1554 & 38.1839 & MD \\
\hline 480 & -2.5 & -75.1291 & 38.2443 & MD & 552 & -1.5 & -75.1431 & 38.2142 & MD & 624 & -0.6 & -75.1555 & 38.1834 & MD \\
\hline 481 & -2.3 & .75 .1293 & 38.2438 & MD & 553 & -1.3 & -75.1433 & 38.2137 & MD & 625 & -0.4 & -75.1557 & 38.183 & MD \\
\hline 482 & -2.2 & -75.1295 & 38.2434 & MD & 554 & -1.2 & -75.1435 & 38.2133 & MD & 626 & -0.6 & -75.1559 & 38.1825 & MD \\
\hline 483 & -0.6 & -75.1297 & 38.243 & MD & 555 & -1.1 & -75.1437 & 38.2128 & MD & 627 & -0.8 & -75.156 & 38.1821 & MD \\
\hline
\end{tabular}




\begin{tabular}{|c|c|c|c|c|c|c|c|c|c|c|c|c|c|c|}
\hline TR\# & RATE & LONG & LAT & ST & TR\# & RATE & LONG & LAT & ST & TR\# & RATE & LONG & LAT & ST \\
\hline 628 & -0.8 & -75.1562 & 38.1816 & MD & 700 & -1 & -75.1677 & 38.1507 & $\mathrm{MD}$ & 772 & -0.8 & -75.1796 & 38.1193 & $\overline{\mathrm{MD}}$ \\
\hline 629 & -0.8 & -75.1564 & 38.1812 & $\mathrm{MD}$ & 701 & -1 & -75.1678 & 38.1503 & MD & 773 & -0.8 & -75.1798 & 38.1188 & MD \\
\hline 630 & -1 & -75.1565 & 38.1807 & $\mathrm{MD}$ & 702 & -1.1 & -75.168 & 38.1498 & MD & 774 & -0.8 & -75.18 & 38.1184 & MD \\
\hline 631 & -1.1 & -75.1567 & 38.1803 & MD & 703 & -1.2 & -75.1682 & 38.1494 & MD & 775 & -0.8 & -75.1802 & 38.118 & MD \\
\hline 632 & -1.2 & -75.1569 & 38.1798 & MD & 704 & -1 & -75.1683 & 38.1489 & MD & 776 & -0.6 & -75.1804 & 38.1176 & MD \\
\hline 633 & -1.3 & -75.157 & 38.1794 & MD & 705 & -0.8 & -75.1685 & 38.1485 & MD & 777 & -0.5 & -75.1806 & 38.1171 & MD \\
\hline 634 & -1 & -75.1572 & 38.1789 & MD & 706 & -0.9 & -75.1686 & 38.148 & MD & 778 & -0.5 & -75.1808 & 38.1167 & MD \\
\hline 635 & -0.8 & -75.1574 & 38.1785 & MD & 707 & -1 & -75.1688 & 38.1476 & MD & 779 & -0.5 & -75.1811 & 38.1163 & MD \\
\hline 636 & -0.7 & -75.1576 & 38.178 & MD & 708 & -0.9 & -75.1689 & 38.1471 & MD & 780 & -0.4 & -75.1813 & 38.1158 & MD \\
\hline 637 & -0.6 & -75.1577 & 38.1776 & $\mathrm{MD}$ & 709 & -0.8 & -75.1691 & 38.1467 & MD & 781 & -0.2 & -75.1815 & 38.1154 & MD \\
\hline 638 & -0.4 & -75.1579 & 38.1771 & MD & 710 & -0.7 & -75.1693 & 38.1462 & MD & 782 & -0.4 & -75.1817 & 38.115 & MD \\
\hline 639 & -0.3 & -75.1581 & 38.1767 & MD & 711 & -0.5 & -75.1694 & 38.1458 & MD & 783 & -0.7 & -75.1819 & 38.1145 & MD \\
\hline 640 & -0.3 & -75.1582 & 38.1763 & MD & 712 & -0.3 & -75.1696 & 38.1453 & MD & 784 & -0.8 & -75.1821 & 38.1141 & MD \\
\hline 641 & -0.3 & -75.1584 & 38.1758 & MD & 713 & -0.1 & .75 .1697 & 38.1449 & MD & 785 & -0.8 & -75.1823 & 38.1137 & MD \\
\hline 642 & -0.4 & -75.1586 & 38.1754 & $\mathrm{MD}$ & 714 & -0.2 & -75.1699 & 38.1444 & MD & 786 & -1 & -75.1825 & 38.1132 & MD \\
\hline 643 & -0.5 & -75.1587 & 38.1749 & MD & 715 & -0.4 & -75.17 & 38.144 & MD & 787 & -1.2 & -75.1827 & 38.1128 & MD \\
\hline 644 & -0.6 & -75.1589 & 38.1745 & MD & 716 & -0.4 & -75.1702 & 38.1435 & MD & 788 & -1.4 & -75.183 & 38.1124 & MD \\
\hline 645 & -0.7 & -75.1591 & 38.174 & MD & 717 & -0.3 & -75.1703 & 38.1431 & MD & 789 & -1.6 & -75.1832 & 38.1119 & MD \\
\hline 646 & -0.7 & -75.1592 & 38.1736 & MD & 718 & -0.3 & -75.1705 & 38.1426 & MD & 790 & -1.2 & -75.1834 & 38.1115 & MD \\
\hline 647 & -0.6 & -75.1594 & 38.1731 & MD & 719 & -0.3 & -75.1707 & 38.1422 & MD & 791 & -0.8 & -75.1836 & 38.1111 & MD \\
\hline 648 & -0.6 & -75.1596 & 38.1727 & MD & 720 & -0.3 & -75.1708 & 38.1417 & MD & 792 & -0.8 & -75.1838 & 38.1106 & MD \\
\hline 649 & -0.6 & -75.1597 & 38.1722 & MD & 721 & -0.3 & -75.171 & 38.1413 & MD & 793 & -0.8 & -75.184 & 38.1102 & MD \\
\hline 650 & -0.4 & -75.1599 & 38.1718 & MD & 722 & -0.5 & -75.1711 & 38.1408 & MD & 794 & -0.8 & -75.1842 & 38.1098 & MD \\
\hline 651 & -0.2 & -75.1601 & 38.1713 & MD & 723 & -0.7 & -75.1713 & 38.1404 & MD & 795 & -0.8 & -75.1844 & 38.1093 & MD \\
\hline 652 & -0.1 & -75.1602 & 38.1709 & $\mathrm{MD}$ & 724 & -0.7 & -75.1714 & 38.1399 & MD & 796 & -0.8 & -75.1847 & 38.1089 & MD \\
\hline 653 & 0 & -75.1604 & 38.1704 & MD & 725 & -0.6 & -75.1716 & 38.1395 & MD & 797 & -0.9 & -75.1849 & 38.1085 & MD \\
\hline 654 & -0.3 & -75.1606 & 38.17 & MD & 726 & -0.5 & -75.1718 & 38.139 & MD & 798 & -0.8 & -75.1851 & 38.108 & MD \\
\hline 655 & -0.6 & -75.1607 & 38.1695 & $\mathrm{MD}$ & 727 & -0.4 & -75.1719 & 38.1386 & MD & 799 & -0.8 & -75.1853 & 38.1076 & MD \\
\hline 656 & -0.6 & -75.1609 & 38.1691 & MD & 728 & -0.6 & -75.1721 & 38.1381 & MD & 800 & -0.7 & -75.1855 & 38.1072 & MD \\
\hline 657 & -0.6 & -75.1611 & 38.1686 & MD & 729 & -0.7 & -75.1722 & 38.1377 & MD & 801 & -0.7 & -75.1857 & 38.1067 & MD \\
\hline 658 & -0.5 & -75.1612 & 38.1682 & MD & 730 & -0.6 & -75.1724 & 38.1372 & MD & 802 & -0.6 & -75.1859 & 38.1063 & MD \\
\hline 659 & -0.4 & -75.1614 & 38.1677 & MD & 731 & -0.4 & -75.1725 & 38.1368 & MD & 803 & -0.5 & -75.1861 & 38.1059 & $M D$ \\
\hline 660 & -0.4 & -75.1616 & 38.1673 & MD & 732 & -0.7 & -75.1727 & 38.1363 & MD & 804 & -0.6 & -75.1863 & 38.1054 & MD \\
\hline 661 & -0.4 & -75.1617 & 38.1668 & $\mathrm{MD}$ & 733 & -1 & -75.1728 & 38.1359 & MD & 805 & -0.8 & -75.1866 & 38.105 & MD \\
\hline 662 & -0.6 & -75.1619 & 38.1664 & MD & 734 & -1.1 & -75.173 & 38.1354 & MD & 806 & -1 & -75.1868 & 38.1046 & MD \\
\hline 663 & -0.9 & -75.1621 & 38.1659 & MD & 735 & -1.2 & -75.1732 & 38.135 & MD & 807 & -1.2 & -75.187 & 38.1041 & MD \\
\hline 664 & -0.8 & -75.1622 & 38.1655 & MD & 736 & -1.3 & -75.1733 & 38.1345 & MD & 808 & -1 & -75.1872 & 38.1037 & MD \\
\hline 665 & -0.8 & -75.1624 & 38.1651 & MD & 737 & -1.5 & -75.1735 & 38.1341 & MD & 809 & -0.9 & -75.1874 & 38.1033 & MD \\
\hline 666 & -0.8 & -75.1626 & 38.1646 & MD & 738 & -1.3 & -75.1736 & 38.1336 & MD & 810 & -0.9 & -75.1876 & 38.1029 & MD \\
\hline 667 & -0.8 & -75.1627 & 38.1642 & MD & 739 & -1.2 & -75.1738 & 38.1332 & MD & 811 & -0.9 & -75.1878 & 38.1024 & $M D$ \\
\hline 668 & -0.8 & -75.1629 & 38.1637 & MD & 740 & -1.1 & -75.1739 & 38.1327 & MD & 812 & -0.7 & -75.188 & 38.102 & MD \\
\hline 669 & -0.8 & -75.1631 & 38.1633 & MD & 741 & -1.1 & -75.1741 & 38.1323 & MD & 813 & -0.5 & -75.1882 & 38.1016 & MD \\
\hline 670 & -0.8 & -75.1633 & 38.1628 & MD & 742 & -1 & -75.1743 & 38.1318 & MD & 814 & -0.4 & -75.1885 & 38.1011 & MD \\
\hline 671 & -0.8 & -75.1634 & 38.1624 & MD & 743 & -1 & -75.1744 & 38.1314 & MD & 815 & -0.4 & -75.1887 & 38.1007 & MD \\
\hline 672 & -0.7 & -75.1636 & 38.1619 & MD & 744 & -0.8 & -75.1746 & 38.1309 & MD & 816 & -0.4 & -75.1889 & 38.1003 & MD \\
\hline 673 & -0.6 & -75.1638 & 38.1615 & MD & 745 & -0.6 & -75.1747 & 38.1305 & MD & 817 & -0.3 & -75.1891 & 38.0998 & MD \\
\hline 674 & -0.7 & -75.1639 & 38.161 & MD & 746 & -0.6 & -75.1749 & 38.13 & MD & 818 & -0.4 & -75.1893 & 38.0994 & MD \\
\hline 675 & -0.8 & -75.1641 & 38.1606 & MD & 747 & -0.6 & -75.175 & 38.1296 & MD & 819 & -0.6 & -75.1895 & 38.099 & MD \\
\hline 676 & -0.6 & -75.1643 & 38.1601 & MD & 748 & -0.6 & -75.1752 & 38.1291 & MD & 820 & -0.4 & -75.1897 & 38.0985 & MD \\
\hline 677 & -0.4 & -75.1644 & 38.1597 & MD & 749 & -0.6 & -75.1753 & 38.1287 & MD & 821 & -0.3 & -75.1899 & 38.0981 & MD \\
\hline 678 & -0.6 & -75.1646 & 38.1592 & MD & 750 & -0.5 & -75.1755 & 38.1282 & MD & 822 & -0.5 & -75.1901 & 38.0977 & MD \\
\hline 679 & -0.8 & -75.1648 & 38.1588 & MD & 751 & -0.4 & -75.1757 & 38.1278 & MD & 823 & -1.9 & -75.1901 & 38.0974 & MD \\
\hline 680 & -0.8 & -75.1649 & 38.1583 & MD & 752 & -0.4 & -75.1758 & 38.1273 & MD & 824 & -0.7 & -75.1904 & 38.0972 & MD \\
\hline 681 & -0.9 & -75.1651 & 38.1579 & MD & 753 & -0.5 & -75.176 & 38.1269 & MD & 825 & -1.6 & -75.1904 & 38.097 & MD \\
\hline 682 & -0.8 & -75.1653 & 38.1574 & MD & 754 & -0.3 & -75.1761 & 38.1264 & MD & 826 & -0.9 & -75.1906 & 38.0968 & MD \\
\hline 683 & -0.8 & -75.1654 & 38.157 & MD & 755 & -0.7 & -75.1762 & 38.1262 & MD & 827 & -1.3 & -75.1906 & 38.0966 & MD \\
\hline 684 & -1.1 & -75.1655 & 38.157 & MD & 756 & -0.1 & -75.1763 & 38.126 & MD & 828 & -1.1 & -75.1908 & 38.0964 & MD \\
\hline 685 & -1 & -75.1657 & 38.1566 & MD & 757 & -0.7 & -75.1764 & 38.1258 & MD & 829 & -1 & -75.1909 & 38.0962 & MD \\
\hline 686 & -0.9 & -75.1656 & 38.1565 & MD & 758 & -0.6 & -75.1766 & 38.1253 & MD & 830 & -1.2 & -75.1911 & 38.0957 & MD \\
\hline 687 & -1 & -75.1658 & 38.1561 & MD & 759 & -0.6 & -75.1768 & 38.1249 & MD & 831 & -1.4 & -75.1914 & 38.0953 & MD \\
\hline 688 & -1 & -75.1658 & 38.1561 & MD & 760 & -0.8 & .75 .177 & 38.1245 & MD & 832 & -1.2 & -75.1917 & 38.0949 & MD \\
\hline 689 & -0.9 & -75.166 & 38.1557 & MD & 761 & -1 & -75.1773 & 38.124 & MD & 833 & -1.2 & -75.1919 & 38.0945 & MD \\
\hline 690 & -1 & -75.1661 & 38.1552 & MD & 762 & -1.3 & -75.1775 & 38.1236 & MD & 834 & -1.3 & -75.1922 & 38.0941 & $\mathrm{MD}$ \\
\hline 691 & -1 & -75.1663 & 38.1548 & MD & 763 & -1.6 & -75.1777 & 38.1232 & MD & 835 & -1.5 & -75.1925 & 38.0937 & MD \\
\hline 692 & -1.1 & -75.1664 & 38.1543 & MD & 764 & -1.7 & -75.1779 & 38.1227 & MD & 836 & -1.2 & -75.1927 & 38.0933 & MD \\
\hline 693 & -1.2 & -75.1666 & 38.1539 & MD & 765 & -1.7 & -75.1781 & 38.1223 & MD & 837 & -1 & -75.193 & 38.0928 & MD \\
\hline 694 & -1.2 & -75.1668 & 38.1534 & MD & 766 & -1.8 & -75.1783 & 38.1219 & MD & 838 & -1.1 & -75.1932 & 38.0924 & MD \\
\hline 695 & -1.2 & -75.1669 & 38.153 & MD & 767 & -1.8 & -75.1785 & 38.1214 & MD & 839 & -1.2 & -75.1935 & 38.092 & MD \\
\hline 696 & -1.3 & -75.1671 & 38.1525 & MD & 768 & -1.6 & -75.1787 & 38.121 & MD & 840 & -1.4 & -75.1938 & 38.0916 & MD \\
\hline 697 & -1.5 & -75.1672 & 38.1521 & MD & 769 & -1.4 & -75.1789 & 38.1206 & MD & 841 & -1.6 & -75.194 & 38.0912 & MD \\
\hline 698 & -1.3 & -75.1674 & 38.1516 & MD & 770 & -1.1 & -75.1792 & 38.1201 & MD & 842 & -1.4 & -75.1943 & 38.0908 & MD \\
\hline 699 & -1.1 & -75.1675 & 38.1512 & MD & 771 & -0.9 & -75.1794 & 38.1197 & MD & 843 & -1.2 & -75.1946 & 38.0904 & MD \\
\hline
\end{tabular}




\begin{tabular}{|c|c|c|c|c|c|c|c|c|c|c|c|c|c|c|}
\hline TR\# & RATE & LONG & LAT & ST & TR\# & RATE & LONG & LAT & ST & TR\# & RATE & LONG & LAT & ST \\
\hline 844 & -1 & -75.1948 & 38.0899 & MD & 916 & -0.8 & -75.2138 & 38.0613 & MD & 988 & -0.2 & -75.2344 & 38.0332 & MD \\
\hline 845 & -0.8 & -75.1951 & 38.0895 & MD & 917 & -1 & -75.2141 & 38.0609 & MD & 989 & 0.2 & -75.2347 & 38.0328 & MD \\
\hline 846 & -0.8 & -75.1953 & 38.0891 & MD & 918 & -1 & -75.2144 & 38.0605 & MD & 990 & 0 & -75.235 & 38.0324 & MD \\
\hline 847 & -0.8 & -75.1956 & 38.0887 & MD & 919 & -1 & -75.2147 & 38.0601 & MD & 991 & -0.3 & -75.2353 & 38.032 & MD \\
\hline 848 & -0.7 & -75.1959 & 38.0883 & MD & 920 & -1 & -75.215 & 38.0597 & MD & 992 & -0.3 & -75.2357 & 38.0316 & MD \\
\hline 849 & -0.5 & -75.1961 & 38.0879 & MD & 921 & -1 & -75.2153 & 38.0593 & MD & 993 & -0.4 & -75.236 & 38.0313 & MD \\
\hline 850 & -0.7 & -75.1964 & 38.0875 & MD & 922 & -0.8 & -75.2156 & 38.0589 & MD & 994 & -0.2 & -75.2363 & 38.0309 & MD \\
\hline 851 & -1 & -75.1966 & 38.087 & MD & 923 & -0.6 & -75.2158 & 38.0585 & MD & 995 & 0 & -75.2366 & 38.0305 & MD \\
\hline 852 & -1 & -75.1969 & 38.0866 & MD & 924 & -0.4 & -75.2161 & 38.0581 & MD & 996 & -0.2 & -75.2369 & 38.0301 & MD \\
\hline 853 & -1.1 & -75.1972 & 38.0862 & MD & 925 & -0.2 & -75.2164 & 38.0577 & MD & 997 & -0.3 & -75.2372 & 38.0297 & MD \\
\hline 854 & -1.1 & -75.1974 & 38.0858 & MD & 926 & -0.3 & -75.2167 & 38.0573 & MD & 998 & -0.2 & .75 .2375 & 38.0293 & MD \\
\hline 855 & -1.2 & -75.1977 & 38.0854 & MD & 927 & -0.4 & -75.217 & 38.0569 & MD & 999 & -0.2 & -75.2378 & 38.0289 & MD \\
\hline 856 & -1.1 & -75.198 & 38.085 & MD & 928 & -0.4 & -75.2173 & 38.0565 & MD & 1000 & -0.1 & -75.2381 & 38.0285 & MD \\
\hline 857 & -1 & -75.1982 & 38.0846 & MD & 929 & -0.3 & -75.2176 & 38.0561 & MD & 1001 & -0.1 & -75.2384 & 38.0281 & MD \\
\hline 858 & -0.9 & -75.1985 & 38.0841 & MD & 930 & -0.3 & -75.2179 & 38.0557 & MD & 1002 & 0 & -75.2388 & 38.0277 & MD \\
\hline 859 & -0.9 & -75.1987 & 38.0837 & MD & 931 & -0.2 & -75.2182 & 38.0553 & MD & 1003 & 0 & -75.2391 & 38.0273 & MD \\
\hline 860 & -0.8 & -75.199 & 38.0833 & MD & 932 & -0.3 & -75.2185 & 38.0549 & MD & 1004 & 0.1 & -75.2394 & 38.0269 & MD \\
\hline 861 & -0.8 & -75.1993 & 38.0829 & MD & 933 & -0.4 & -75.2188 & 38.0545 & MD & 1005 & 0.1 & -75.2397 & 38.0265 & MD \\
\hline 862 & -1.1 & -75.1995 & 38.0825 & MD & 934 & -0.3 & -75.2191 & 38.0541 & MD & 1006 & -1.4 & -75.8 & 37.98333 & MD \\
\hline 863 & -1.4 & -75.1998 & 38.0821 & MD & 935 & -0.2 & -75.2194 & 38.0537 & MD & 1007 & -1.4 & -75.85 & 37.96667 & MD \\
\hline 864 & -1.3 & -75.2001 & 38.0817 & MD & 936 & -0.4 & -75.2197 & 38.0533 & MD & 1008 & -1.4 & -75.9 & 37.93333 & MD \\
\hline 865 & -1.2 & -75.2003 & 38.0813 & MD & 937 & -0.6 & -75.22 & 38.0529 & MD & 1009 & -0.6 & -75.91666 & 37.98333 & MD \\
\hline 866 & -1 & -75.2006 & 38.0808 & MD & 938 & -0.6 & -75.2203 & 38.0525 & MD & 1010 & -0.6 & -75.91666 & 38.03333 & MD \\
\hline 867 & -1 & -75.2008 & 38.0804 & MD & 939 & -0.6 & -75.2206 & 38.0521 & MD & 1011 & -0.6 & -75.91666 & 38.08333 & MD \\
\hline 868 & -0.9 & -75.2011 & 38.08 & MD & 940 & -0.7 & -75.2209 & 38.0517 & MD & 1012 & -0.2 & -75.91666 & 38.13334 & MD \\
\hline 869 & -0.9 & -75.2014 & 38.0796 & MD & 941 & -0.8 & -75.2211 & 38.0513 & MD & 1013 & -0.2 & -75.96667 & 38.13334 & MD \\
\hline 870 & -1 & -75.2016 & 38.0792 & MD & 942 & -0.8 & -75.2214 & 38.0509 & MD & 1014 & -0.2 & -75.96667 & 38.18333 & MD \\
\hline 871 & -1.1 & -75.2019 & 38.0788 & MD & 943 & -0.8 & -75.2217 & 38.0505 & MD & 1015 & -0.6 & -75.96667 & 38.23333 & MD \\
\hline 872 & -1.1 & -75.2022 & 38.0784 & MD & 944 & -0.8 & -75.222 & 38.0501 & MD & 1016 & -0.6 & -76.06667 & 38 & MD \\
\hline 873 & -1.1 & -75.2024 & 38.0779 & MD & 945 & -0.9 & -75.2223 & 38.0497 & MD & 1017 & -0.6 & -76.06667 & 38.05 & MD \\
\hline 874 & -1 & -75.2027 & 38.0775 & MD & 946 & -0.9 & -75.2226 & 38.0493 & MD & 1018 & -0.6 & -76.06667 & 38.1 & MD \\
\hline 875 & -1 & -75.2029 & 38.0771 & MD & 947 & -0.9 & -75.2229 & 38.0489 & MD & 1019 & -0.6 & -76.1 & 38.15 & MD \\
\hline 876 & -1 & -75.2032 & 38.0767 & MD & 948 & -0.6 & -75.2232 & 38.0485 & MD & 1020 & -0.4 & -76.1 & 38.2 & MD \\
\hline 877 & -1 & -75.2035 & 38.0763 & MD & 949 & -0.4 & -75.2235 & 38.0481 & MD & 1021 & -0.3 & -76.15 & 38.25 & MD \\
\hline 878 & -1.2 & -75.2037 & 38.0759 & MD & 950 & -0.4 & -75.2238 & 38.0477 & MD & 1022 & -0.3 & -76.2 & 38.25 & MD \\
\hline 879 & -1.4 & -75.204 & 38.0755 & MD & 951 & -0.4 & -75.2241 & 38.0473 & MD & 1023 & -0.3 & -76.21667 & 38.3 & MD \\
\hline 880 & -1.2 & -75.2042 & 38.075 & MD & 952 & -0.4 & -75.2244 & 38.0469 & MD & 1024 & -0.3 & -76.25 & 38.35 & MD \\
\hline 881 & -1.1 & -75.2045 & 38.0746 & MD & 953 & -0.4 & -75.2247 & 38.0465 & MD & 1025 & -0.3 & -76.3 & 38.35 & MD \\
\hline 882 & -1.1 & -75.2048 & 38.0742 & MD & 954 & -0.3 & -75.225 & 38.0461 & MD & 1026 & -3.8 & -76.25 & 38.4 & MD \\
\hline 883 & -1.1 & -75.205 & 38.0738 & MD & 955 & -0.3 & -75.2253 & 38.0457 & MD & 1027 & -3.8 & -76.3 & 38.4 & MD \\
\hline 884 & -1.2 & -75.2053 & 38.0734 & MD & 956 & -0.3 & -75.2256 & 38.0453 & MD & 1028 & -3.8 & -76.31667 & 38.45 & MD \\
\hline 885 & -1.3 & -75.2056 & 38.073 & MD & 957 & -0.3 & -75.2259 & 38.0449 & MD & 1029 & -3.8 & -76.35 & 38.5 & MD \\
\hline 886 & -1.1 & -75.2058 & 38.0726 & MD & 958 & -0.2 & -75.2262 & 38.0445 & MD & 1030 & -1.6 & -76.36667 & 38.55 & MD \\
\hline 887 & -0.9 & -75.2061 & 38.0721 & MD & 959 & -0.1 & -75.2264 & 38.0441 & MD & 1031 & -1.6 & -76.33334 & 38.6 & MD \\
\hline 888 & -1.2 & -75.2063 & 38.0717 & MD & 960 & -0.1 & -75.2267 & 38.0437 & MD & 1032 & -1.2 & .76 .3 & 38.65 & MD \\
\hline 889 & -1.4 & -75.2066 & 38.0713 & MD & 961 & -0.1 & -75.227 & 38.0433 & MD & 1033 & -0.7 & -76.36667 & 38.7 & MD \\
\hline 890 & -1.4 & -75.2069 & 38.0709 & MD & 962 & -0.2 & -75.2273 & 38.0429 & MD & 1034 & -0.7 & -76.36667 & 38.75 & MD \\
\hline 891 & -1.5 & -75.2071 & 38.0705 & MD & 963 & -0.2 & -75.2276 & 38.0425 & MD & 1035 & -0.7 & .76 .35 & 38.8 & MD \\
\hline 892 & -1.6 & -75.2074 & 38.0701 & MD & 964 & -0.2 & -75.2279 & 38.0421 & MD & 1036 & -0.7 & -76.41666 & 38.78333 & MD \\
\hline 893 & -1.7 & -75.2077 & 38.0697 & MD & 965 & -0.2 & -75.2276 & 38.0419 & MD & 1037 & -0.7 & -76.31667 & 38.85 & MD \\
\hline 894 & -1 & -75.2079 & 38.0693 & MD & 966 & -0.1 & -75.2282 & 38.0417 & MD & 1038 & -0.5 & -76.28333 & 38.9 & MD \\
\hline 895 & -1.8 & -75.2079 & 38.0692 & MD & 967 & -0.2 & -75.2279 & 38.0415 & MD & 1039 & -1 & .76 .38333 & 38.86666 & MD \\
\hline 896 & -1.2 & -75.2082 & 38.0689 & MD & 968 & -0.2 & -75.2282 & 38.0411 & MD & 1040 & -1 & -76.38333 & 38.91667 & MD \\
\hline 897 & -1.9 & -75.2082 & 38.0688 & MD & 969 & -0.2 & -75.2285 & 38.0407 & MD & 1041 & -0.5 & -76.38333 & 38.96667 & MD \\
\hline 898 & -1.3 & -75.2085 & 38.0685 & MD & 970 & -0.2 & -75.2288 & 38.0403 & MD & 1042 & -1.1 & -76.36667 & 39.01667 & MD \\
\hline 899 & -1.5 & -75.2088 & 38.0681 & MD & 971 & -0.1 & -75.2291 & 38.0399 & MD & 1043 & -1.1 & -76.33334 & 39.06667 & MD \\
\hline 900 & -1.4 & -75.2091 & 38.0677 & MD & 972 & -0.2 & -75.2295 & 38.0395 & MD & 1044 & -0.7 & -76.25 & 39.1 & MD \\
\hline 901 & -1.2 & -75.2094 & 38.0673 & MD & 973 & -0.4 & -75.2298 & 38.0391 & MD & 1045 & -0.7 & -76.28333 & 39.15 & MD \\
\hline 902 & -1.3 & -75.2097 & 38.0669 & MD & 974 & -0.4 & -75.2301 & 38.0387 & MD & 1046 & -0.7 & -76.28333 & 39.2 & MD \\
\hline 903 & -1.4 & -75.21 & 38.0665 & MD & 975 & -0.4 & -75.2304 & 38.0384 & MD & 1047 & -0.3 & -76.23333 & 39.25 & MD \\
\hline 904 & -1.4 & -75.2103 & 38.0661 & MD & 976 & -0.3 & -75.2307 & 38.038 & MD & 1048 & -0.3 & -76.25 & 39.3 & MD \\
\hline 905 & -1.4 & -75.2105 & 38.0657 & MD & 977 & -0.2 & -75.231 & 38.0376 & MD & 1049 & -0.3 & -76.2 & 39.33333 & MD \\
\hline 906 & -1.4 & -75.2108 & 38.0653 & MD & 978 & -0.4 & -75.2313 & 38.0372 & MD & 1050 & -0.3 & -76.16666 & 39.38334 & MD \\
\hline 907 & -1.5 & -75.2111 & 38.0649 & MD & 979 & -0.5 & -75.2316 & 38.0368 & MD & 1051 & -0.3 & -76.11667 & 39.38334 & MD \\
\hline 908 & -1.4 & -75.2114 & 38.0645 & MD & 980 & -0.4 & -75.2319 & 38.0364 & $\mathrm{MD}$ & 1052 & -0.2 & -76.1 & 39.48333 & MD \\
\hline 909 & -1.3 & -75.2117 & 38.0641 & MD & 981 & -0.4 & -75.2322 & 38.036 & MD & 1053 & -0.4 & -76.15 & 39.45 & MD \\
\hline 910 & -1.1 & -75.212 & 38.0637 & MD & 982 & -0.2 & -75.2326 & 38.0356 & MD & 1054 & -0.7 & .76 .2 & 39.41667 & MD \\
\hline 911 & -0.9 & -75.2123 & 38.0633 & MD & 983 & -0.1 & -75.2329 & 38.0352 & MD & 1055 & -0.7 & -76.25 & 39.38334 & MD \\
\hline 912 & -1 & -75.2126 & 38.0629 & MD & 984 & -0.3 & -75.2332 & 38.0348 & $\mathrm{MD}$ & 1056 & -0.4 & -76.3 & 39.35 & MD \\
\hline 913 & -1.1 & -75.2129 & 38.0625 & MD & 985 & -0.6 & -75.2335 & 38.0344 & MD & 1057 & -0.4 & -76.3 & 39.3 & MD \\
\hline 914 & -0.9 & -75.2132 & 38.0621 & MD & 986 & -0.6 & -75.2338 & 38.034 & MD & 1058 & -0.6 & -76.35 & 39.33333 & MD \\
\hline 915 & -0.7 & -75.2135 & 38.0617 & MD & 987 & -0.5 & -75.2341 & 38.0336 & MD & 1059 & -0.6 & -76.4 & 39.31667 & MD \\
\hline
\end{tabular}

MID-ATLANTIC MAP DATA/USGS/NJ.DE.MD.VA 


\begin{tabular}{|c|c|c|c|c|c|c|c|c|c|c|c|c|c|c|}
\hline TR\# & RATE & LONG & LAT & ST & TR\# & RATE & LONG & LAT & ST & TR\# & RATE & LONG & LAT & ST \\
\hline 1060 & -0.7 & -76.41666 & 539.26667 & MD & 45 & -2.2 & -75.2524 & 38.0099 & $\overline{\mathrm{VA}}$ & 117 & 0.5 & -75.2734 & 37.9826 & $\overline{V A}$ \\
\hline 1061 & -0.7 & -76.46667 & 739.23333 & MD & 46 & -2.1 & -75.2527 & 38.0095 & VA & 118 & 0.3 & -75.2737 & 37.9822 & VA \\
\hline 1062 & -0.3 & -76.46667 & 39.18333 & MD & 47 & -1.9 & -75.253 & 38.0091 & VA & 119 & 0.4 & -75.274 & 37.9818 & VA \\
\hline 1063 & -0.5 & .76 .46667 & 39.13334 & MD & 48 & -1.8 & -75.2533 & 38.0087 & VA & 120 & 0.5 & -75.2743 & 37.9814 & VA \\
\hline 1064 & -0.8 & -76.45 & 39.08333 & MD & 49 & -1.6 & -75.2537 & 38.0084 & VA & 121 & 0.6 & -75.2747 & 37.9811 & VA \\
\hline 1065 & -0.8 & -76.43333 & 39.03333 & MD & 50 & -1.4 & -75.254 & 38.008 & VA & 122 & 0.8 & -75.275 & 37.9807 & VA \\
\hline 1066 & -0.8 & -76.46667 & 38.98333 & MD & 51 & -1.4 & -75.2543 & 38.0076 & VA & 123 & 0.6 & .75 .2753 & 37.9803 & VA \\
\hline 1067 & -0.2 & -76.46667 & 38.93333 & MD & 52 & -1.4 & -75.2546 & 38.0072 & VA & 124 & 0.5 & -75.2756 & 37.9799 & VA \\
\hline 1068 & -0.2 & -76.51667 & 38.9 & MD & 53 & -1.5 & -75.2549 & 38.0068 & vA & 125 & 0.6 & -75.2759 & 37.9795 & VA \\
\hline 1069 & -0.2 & -76.51667 & 738.85 & MD & 54 & -1.6 & -75.2552 & 38.0064 & $\mathrm{VA}$ & 126 & 0.7 & -75.2763 & 37.9791 & VA \\
\hline 1070 & -0.2 & -76.36667 & 38.81667 & MD & 55 & -1.5 & -75.2555 & 38.006 & VA & 127 & 0.6 & -75.2766 & 37.9787 & VA \\
\hline 1071 & -0.9 & -76.6 & 38.78333 & MD & 56 & -1.5 & .75 .2559 & 38.0056 & $\mathrm{VA}$ & 128 & 0.6 & -75.2769 & 37.9783 & VA \\
\hline 1072 & -0.9 & -76.56667 & 38.73333 & MD & 57 & -1.6 & -75.2562 & 38.0052 & VA & 129 & 0.3 & -75.2772 & 37.978 & VA \\
\hline 1073 & -0.4 & -76.55 & 38.68333 & MD & 58 & -1.7 & -75.2565 & 38.0048 & VA & 130 & 0 & -75.2776 & 37.9776 & VA \\
\hline 1074 & -0.6 & -76.55 & 38.63334 & MD & 59 & -1.5 & -75.2568 & 38.0044 & $\mathrm{VA}$ & 131 & 0.2 & -75.2779 & 37.9772 & VA \\
\hline 1075 & -0.6 & -76.55 & 38.58333 & MD & 60 & -1.4 & -75.2571 & 38.004 & VA & 132 & 0.3 & -75.2782 & 37.9768 & VA \\
\hline 1076 & -0.2 & -76.53333 & 38.53333 & MD & 61 & -1.4 & -75.2574 & 38.0037 & $V_{A}$ & 133 & 0.5 & -75.2785 & 37.9764 & VA \\
\hline 1077 & -0.8 & -76.5 & 38.48333 & MD & 62 & -1.4 & -75.2577 & 38.0033 & $\mathrm{VA}$ & 134 & 0.7 & -75.2788 & 37.976 & VA \\
\hline 1078 & -0.8 & .76 .45 & 38.45 & MD & 63 & -1.4 & -75.2581 & 38.0029 & VA & 135 & 0.7 & -75.2792 & 37.9756 & VA \\
\hline 1079 & -0.1 & -76.41666 & 638.4 & MD & 64 & -1.5 & -75.2584 & 38.0025 & VA & 136 & 0.7 & -75.2795 & 37.9752 & VA \\
\hline 1080 & -0.1 & -76.41666 & 538.35 & MD & 65 & -1.5 & -75.2587 & 38.0021 & VA & 137 & 1 & -75.2798 & 37.9749 & VA \\
\hline 1081 & -0.5 & -76.41666 & 538.3 & MD & 66 & -1.6 & -75.259 & 38.0017 & VA & 138 & 1.3 & -75.2801 & 37.9745 & VA \\
\hline 1082 & -0.3 & -76.4 & 38.25 & MD & 67 & -1.5 & -75.2593 & 38.0013 & VA & 139 & 1.5 & -75.2805 & 37.9741 & VA \\
\hline 1083 & -0.3 & -76.38333 & 38.2 & MD & 68 & -1.5 & -75.2596 & 38.0009 & $\mathrm{VA}_{\mathrm{A}}$ & 140 & 1.7 & -75.2808 & 37.9737 & VA \\
\hline 1084 & -0.3 & .76 .33334 & 38.18333 & MD & 69 & -1.5 & .75 .2599 & 38.0005 & VA & 141 & 1.7 & -75.2811 & 37.9733 & VA \\
\hline 1085 & -1.2 & -76.36667 & 38.13334 & MD & 70 & -1.5 & -75.2602 & 38.0001 & VA & 142 & 1.7 & -75.2814 & 37.9729 & VA \\
\hline 1086 & -0.7 & -76.35 & 38.08333 & MD & 71 & -1.6 & -75.2606 & 37.9997 & $\mathrm{VA}$ & 143 & 1.8 & -75.2817 & 37.9725 & VA \\
\hline 1087 & -0.5 & -76.4 & 38.11666 & MD & 72 & -1.7 & -75.2609 & 37.9993 & VA & 144 & 2 & -75.2821 & 37.9721 & VA \\
\hline 1 & 0.2 & -75.24 & 38.0261 & VA & 73 & -1.5 & -75.2612 & 37.999 & VA & 145 & 1.8 & .75 .2824 & 37.9718 & VA \\
\hline 2 & 0.2 & -75.2403 & 38.0257 & VA & 74 & -1.3 & -75.2615 & 37.9986 & VA & 146 & 1.7 & -75.2827 & 37.9714 & VA \\
\hline 3 & 0.2 & -75.2406 & 38.0253 & VA & 75 & -1.2 & -75.2618 & 37.9982 & VA & 147 & 1.7 & -75.283 & 37.971 & VA \\
\hline 4 & 0.2 & -75.2409 & 38.0249 & $\mathrm{VA}$ & 76 & -1.2 & -75.2621 & 37.9978 & VA & 148 & 1.6 & -75.2834 & 37.9706 & VA \\
\hline 5 & 0.1 & -75.2412 & 38.0245 & VA & 77 & -1 & -75.2624 & 37.9974 & VA & 149 & 1.8 & -75.2837 & 37.9702 & VA \\
\hline 6 & 0 & -75.2415 & 38.0242 & VA & 78 & -0.8 & -75.2628 & 37.997 & VA & 150 & 1.9 & -75.284 & 37.9698 & VA \\
\hline 7 & 0.1 & -75.2419 & 38.0238 & VA & 79 & -0.7 & -75.2631 & 37.9966 & VA & 151 & 1.9 & -75.2843 & 37.9694 & VA \\
\hline 8 & 0.2 & -75.2422 & 38.0234 & VA & 80 & -0.7 & -75.2634 & 37.9962 & VA & 152 & 1.9 & -75.2846 & 37.969 & VA \\
\hline 9 & 0.2 & -75.2425 & 38.023 & VA & 81 & -0.7 & .75 .2637 & 37.9958 & $\mathrm{VA}$ & 153 & 1.6 & -75.285 & 37.9687 & VA \\
\hline 10 & 0.1 & -75.2428 & 38.0226 & VA & 82 & -0.8 & -75.264 & 37.9954 & VA & 154 & 1.3 & -75.2853 & 37.9683 & VA \\
\hline 11 & 0 & -75.2431 & 38.0222 & VA & 83 & -0.8 & -75.2643 & 37.995 & VA & 155 & 1.5 & -75.2856 & 37.9679 & VA \\
\hline 12 & -0.2 & -75.2434 & 38.0218 & VA & 84 & -0.9 & -75.2646 & 37.9946 & VA & 156 & 1.7 & -75.2859 & 37.9675 & VA \\
\hline 13 & -0.2 & -75.2437 & 38.0214 & $\mathrm{VA}$ & 85 & -0.8 & -75.265 & 37.9943 & VA & 157 & 1.6 & -75.2863 & 37.9671 & VA \\
\hline 14 & -0.4 & -75.244 & 38.021 & VA & 86 & -0.8 & -75.2653 & 37.9939 & VA & 158 & 1.6 & -75.2866 & 37.9667 & VA \\
\hline 15 & -0.2 & -75.2443 & 38.0206 & VA & 87 & -0.7 & -75.2656 & 37.9935 & VA & 159 & 1.6 & -75.2869 & 37.9663 & VA \\
\hline 16 & -0.2 & -75.2446 & 38.0202 & VA & 88 & -0.6 & -75.2659 & 37.9931 & VA & 160 & 1.5 & -75.2872 & 37.9659 & VA \\
\hline 17 & -0.3 & -75.245 & 38.0198 & $\mathrm{VA}$ & 89 & -0.4 & -75.2662 & 37.9927 & VA & 161 & 1.5 & -75.2876 & 37.9656 & VA \\
\hline 18 & -0.4 & -75.2453 & 38.0194 & $\mathrm{VA}$ & 90 & -0.2 & -75.2665 & 37.9923 & $\mathrm{VA}$ & 162 & 1.5 & -75.2879 & 37.9652 & VA \\
\hline 19 & -0.6 & -75.2456 & 38.019 & $\mathrm{VA}$ & 91 & -0.2 & -75.2668 & 37.9919 & $\mathrm{VA}$ & 163 & 1.5 & -75.2882 & 37.9648 & VA \\
\hline 20 & -0.8 & -75.2459 & 38.0186 & VA & 92 & -0.3 & -75.2672 & 37.9915 & VA & 164 & 1.4 & -75.2885 & 37.9644 & VA \\
\hline 21 & -0.8 & -75.2462 & 38.0182 & VA & 93 & -0.2 & -75.2675 & 37.9911 & VA & 165 & 1.2 & -75.2888 & 37.964 & VA \\
\hline 22 & -0.9 & -75.2465 & 38.0178 & VA & 94 & -0.1 & -75.2678 & 37.9907 & VA & 166 & 0.9 & -75.2892 & 37.9636 & VA \\
\hline 23 & -0.8 & -75.2468 & 38.0174 & VA & 95 & 0.1 & .75 .2681 & 37.9903 & VA & 167 & 1 & -75.2895 & 37.9632 & VA \\
\hline 24 & -0.8 & -75.2471 & 38.0171 & VA & 96 & 0.2 & -75.2684 & 37.9899 & VA & 168 & 0.6 & -75.2897 & 37.9628 & VA \\
\hline 25 & -0.9 & -75.2474 & 38.0167 & VA & 97 & 0.1 & -75.2687 & 37.9896 & VA & 169 & 1.1 & -75.2898 & 37.9628 & VA \\
\hline 26 & -0.9 & -75.2477 & 38.0163 & VA & 98 & 0 & -75.269 & 37.9892 & VA & 170 & 1.1 & -75.2901 & 37.9625 & VA \\
\hline 27 & -1 & -75.2481 & 38.0159 & VA & 99 & 0.2 & -75.2682 & 37.9888 & VA & 171 & 0.8 & -75.29 & 37.9624 & VA \\
\hline 28 & -1.1 & -75.2484 & 38.0155 & VA & 100 & 0 & -75.2694 & 37.9888 & VA & 172 & 1.1 & -75.2905 & 37.9621 & VA \\
\hline 29 & -1 & -75.248 & 38.0154 & VA & 101 & 0.2 & -75.2685 & 37.9884 & VA & 173 & 1 & -75.2902 & 37.962 & VA \\
\hline 30 & -1.1 & -75.2487 & 38.0151 & VA & 102 & -0.1 & -75.2697 & 37.9884 & VA & 174 & 1.2 & -75.2905 & 37.9616 & VA \\
\hline 31 & -1.2 & -75.2483 & 38.015 & $\mathrm{VA}$ & 103 & 0.2 & -75.2688 & 37.988 & VA & 175 & 1 & -75.2908 & 37.9611 & VA \\
\hline 32 & -1.1 & -75.249 & 38.0147 & $\mathrm{VA}$ & 104 & 0.1 & -75.2692 & 37.9876 & VA & 176 & 0.8 & -75.2911 & 37.9607 & VA \\
\hline 33 & -1.5 & -75.2486 & 38.0146 & VA & 105 & 0.2 & -75.2695 & 37.9873 & VA & 177 & 1.2 & -75.2913 & 37.9603 & VA \\
\hline 34 & -1.7 & -75.2489 & 38.0142 & VA & 106 & 0.3 & -75.2698 & 37.9869 & VA & 178 & 1.5 & -75.2916 & 37.9599 & VA \\
\hline 35 & -1.9 & -75.2493 & 38.0138 & VA & 107 & 0.1 & -75.2701 & 37.9865 & VA & 179 & 1.3 & -75.2919 & 37.9595 & VA \\
\hline 36 & -2.2 & -75.2496 & 38.0134 & VA & 108 & 0 & .75 .2705 & 37.9861 & VA & 180 & 1.1 & -75.2922 & 37.9591 & VA \\
\hline 37 & -2 & -75.2499 & 38.0131 & VA & 109 & -0.1 & -75.2708 & 37.9857 & VA & 181 & 1 & -75.2924 & 37.9587 & VA \\
\hline 38 & -1.8 & -75.2502 & 38.0127 & VA & 110 & -0.3 & -75.2711 & 37.9853 & VA & 182 & 0.8 & -75.2927 & 37.9583 & VA \\
\hline 39 & -1.9 & -75.2505 & 38.0123 & VA & 111 & -0.2 & .75 .2714 & 37.9849 & VA & 183 & 0.7 & -75.293 & 37.9578 & VA \\
\hline 40 & -2 & -75.2508 & 38.0119 & $\mathrm{VA}$ & 112 & -0.2 & -75.2717 & 37.9845 & VA & 184 & 0.7 & -75.2933 & 37.9574 & VA \\
\hline 41 & -1.9 & -75.2511 & 38.0115 & VA & 113 & -0.1 & -75.2721 & 37.9842 & VA & 185 & 0.7 & -75.2935 & 37.957 & VA \\
\hline 42 & -1.8 & -75.2515 & 38.0111 & VA & 114 & 0 & -75.2724 & 37.9838 & $\mathrm{VA}$ & 186 & 0.8 & -75.2938 & 37.9566 & VA \\
\hline 43 & -2 & -75.2518 & 38.0107 & VA & 115 & 0.3 & -75.2727 & 37.9834 & VA & 187 & 0.6 & -75.2941 & 37.9562 & VA \\
\hline 44 & -2.3 & -75.2521 & 38.0103 & VA & 116 & 0.6 & -75.273 & 37.983 & VA & 188 & 0.5 & -75.2944 & 37.9558 & VA \\
\hline
\end{tabular}




\begin{tabular}{|c|c|c|c|c|c|c|c|c|c|c|c|c|c|c|}
\hline TR\# & RATE & LONG & LAT & ST & I TR\# & RATE & LONG & LAT & ST & TR\# & RATE & LONG & LAT & ST \\
\hline 189 & 0.4 & -75.2946 & 37.9554 & $\overline{V A}$ & 261 & -0.7 & -75.3126 & 37.9272 & $\overline{V A}$ & 333 & -4 & -75.33 & 37.8979 & VA \\
\hline 190 & 0.2 & -75.2949 & 37.955 & VA & 262 & -0.8 & -75.3129 & 37.9268 & VA & 334 & -4 & -75.3303 & 37.8974 & VA \\
\hline 191 & 0.3 & -75.2952 & 37.9545 & VA & 263 & -1 & -75.3131 & 37.9263 & VA & 335 & -3.9 & -75.3305 & 37.897 & VA \\
\hline 192 & 0.3 & -75.2955 & 37.9541 & VA & 264 & -1.3 & -75.3134 & 37.9259 & VA & 336 & -3.7 & -75.3308 & 37.8966 & VA \\
\hline 193 & 0.2 & -75.2957 & 37.9537 & VA & 265 & -1.1 & -75.3136 & 37.9255 & VA & 337 & -3.8 & -75.3311 & 37.8962 & VA \\
\hline 194 & 0.1 & -75.296 & 37.9533 & VA & 266 & -1 & -75.3139 & 37.9251 & VA & 338 & -3.8 & -75.3313 & 37.8958 & VA \\
\hline 195 & 0 & -75.2963 & 37.9529 & VA & 267 & -1 & -75.3141 & 37.9246 & VA & 339 & -3.8 & -75.3316 & 37.8954 & VA \\
\hline 196 & 0 & -75.2966 & 37.9525 & VA & 268 & -1.1 & -75.3143 & 37.9242 & VA & 340 & -3.8 & -75.3319 & 37.895 & VA \\
\hline 197 & 0 & -75.2968 & 37.9521 & VA & 269 & -1 & -75.3146 & 37.9238 & VA & 341 & -3.8 & -75.3321 & 37.8945 & VA \\
\hline 198 & 0 & -75.2971 & 37.9517 & $V_{A}$ & 270 & -1 & .75 .3148 & 37.9234 & $\mathrm{VA}_{\mathrm{A}}$ & 342 & -3.7 & -75.3324 & 37.8941 & VA \\
\hline 199 & 0 & -75.2974 & 37.9512 & VA & 271 & -1 & -75.3151 & 37.9229 & VA & 343 & -3.8 & -75.3327 & 37.8937 & VA \\
\hline 200 & -0.1 & .75 .2977 & 37.9508 & VA & 272 & -1 & -75.3153 & 37.9225 & $\mathrm{VA}$ & 344 & -4 & -75.3329 & 37.8933 & VA \\
\hline 201 & 0 & -75.2979 & 37.9504 & VA & 273 & -1.3 & -75.3156 & 37.9221 & $\mathrm{VA}_{\mathrm{A}}$ & 345 & -4 & -75.3332 & 37.8929 & VA \\
\hline 202 & 0 & -75.2982 & 37.95 & VA & 274 & -1.6 & -75.3158 & 37.9217 & VA & 346 & -4.1 & -75.3335 & 37.8925 & VA \\
\hline 203 & -0.3 & -75.2985 & 37.9496 & VA & 275 & -1.7 & -75.316 & 37.9212 & VA & 347 & -4.3 & -75.3337 & 37.892 & VA \\
\hline 204 & -0.6 & -75.2988 & 37.9492 & VA & 276 & -1.8 & -75.3163 & 37.9208 & VA & 348 & -4.4 & -75.334 & 37.8916 & VA \\
\hline 205 & -0.5 & .75 .299 & 37.9488 & VA & 277 & -1.8 & -75.3165 & 37.9204 & VA & 349 & -4.4 & -75.3343 & 37.8912 & VA \\
\hline 206 & -0.4 & -75.2993 & 37.9484 & $\mathrm{VA}$ & 278 & -1.9 & -75.3168 & 37.92 & VA & 350 & -4.5 & -75.3345 & 37.8908 & VA \\
\hline 207 & -0.4 & -75.2996 & 37.9479 & VA & 279 & -2.1 & -75.317 & 37.9195 & $\mathrm{VA}$ & 351 & -4.5 & -75.3348 & 37.8904 & $\mathrm{VA}$ \\
\hline 208 & -0.4 & -75.2999 & 37.9475 & VA & 280 & -2.3 & -75.3172 & 37.9191 & VA & 352 & -4.6 & .75 .3351 & 37.89 & VA \\
\hline 209 & -0.2 & -75.3001 & 37.9471 & VA & 281 & -2.3 & -75.3175 & 37.9187 & VA & 353 & -4.8 & -75.3353 & 37.8895 & VA \\
\hline 210 & 0.1 & .75 .3004 & 37.9467 & VA & 282 & -2.4 & -75.3177 & 37.9183 & VA & 354 & -5.1 & -75.3356 & 37.8891 & VA \\
\hline 211 & -0.2 & -75.3007 & 37.9463 & VA & 283 & -2.4 & .75 .318 & 37.9178 & VA & 355 & -5.1 & -75.3359 & 37.8887 & VA \\
\hline 212 & -0.5 & -75.301 & 37.9459 & $\mathrm{VA}$ & 284 & -2.5 & -75.3182 & 37.9174 & $\mathrm{VA}$ & 356 & -5.1 & -75.3361 & 37.8883 & VA \\
\hline 213 & -0.3 & -75.3012 & 37.9455 & $\mathrm{VA}$ & 285 & -2.5 & -75.3185 & 37.917 & $\mathrm{VA}_{\mathrm{A}}$ & 357 & -5 & -75.3364 & 37.8879 & VA \\
\hline 214 & -0.1 & -75.3015 & 37.9451 & VA & 286 & -2.5 & -75.3187 & 37.9166 & VA & 358 & -5 & -75.3367 & 37.8875 & VA \\
\hline 215 & -0.1 & -75.3018 & 37.9446 & VA & 287 & -2.6 & -75.3189 & 37.9161 & VA & 359 & -5.1 & -75.3369 & 37.8871 & VA \\
\hline 216 & -0.2 & -75.3021 & 37.9442 & VA & 288 & -2.8 & -75.3192 & 37.9157 & VA & 360 & -5.1 & -75.3372 & 37.8866 & VA \\
\hline 217 & -0.3 & -75.3023 & 37.9438 & VA & 289 & -2.8 & -75.3194 & 37.9153 & VA & 361 & -4.9 & .75 .3375 & 37.8862 & VA \\
\hline 218 & -0.4 & -75.3026 & 37.9434 & VA & 290 & -2.9 & -75.3197 & 37.9149 & VA & 362 & -4.8 & -75.3377 & 37.8858 & VA \\
\hline 219 & -0.3 & -75.3029 & 37.943 & VA & 291 & -2.9 & -75.3199 & 37.9144 & VA & 363 & -4.7 & -75.338 & 37.8854 & VA \\
\hline 220 & -0.2 & -75.3032 & 37.9426 & VA & 292 & -2.9 & -75.3202 & 37.914 & VA & 364 & -4.7 & -75.3383 & 37.885 & VA \\
\hline 221 & -0.2 & -75.3034 & 37.9422 & VA & 293 & -2.9 & -75.3204 & 37.9136 & VA & 365 & -4.7 & -75.3386 & 37.8846 & VA \\
\hline 222 & -0.2 & -75.3037 & 37.9418 & VA & 294 & -2.9 & -75.3206 & 37.9132 & VA & 366 & -4.8 & -75.3388 & 37.8841 & VA \\
\hline 223 & -0.1 & -75.304 & 37.9413 & VA & 295 & -3 & -75.3209 & 37.9127 & VA & 367 & -4.8 & -75.3391 & 37.8837 & VA \\
\hline 224 & -0.1 & -75.3043 & 37.9409 & $\mathrm{VA}$ & 296 & -3.1 & -75.3211 & 37.9123 & VA & 368 & -4.9 & -75.3394 & 37.8833 & VA \\
\hline 225 & -0.2 & .75 .3045 & 37.9405 & VA & 297 & -3.3 & -75.3214 & 37.9119 & VA & 369 & -4.9 & -75.3396 & 37.8829 & $\mathrm{VA}$ \\
\hline 226 & -0.4 & -75.3048 & 37.9401 & VA & 298 & -3.5 & -75.3216 & 37.9115 & VA & 370 & -4.9 & -75.3399 & 37.8825 & VA \\
\hline 227 & -0.4 & -75.3051 & 37.9397 & VA & 299 & -3.4 & -75.3219 & 37.911 & VA & 371 & -5 & -75.3402 & 37.8821 & VA \\
\hline 228 & -0.5 & -75.3054 & 37.9393 & VA & 300 & -3.3 & -75.3221 & 37.9106 & VA & 372 & -5.1 & -75.3404 & 37.8817 & VA \\
\hline 229 & -0.6 & -75.3056 & 37.9389 & VA & 301 & -3.3 & -75.3223 & 37.9102 & VA & 373 & -5.1 & -75.3407 & 37.8812 & VA \\
\hline 230 & -0.8 & -75.3059 & 37.9385 & $\mathrm{VA}_{\mathrm{A}}$ & 302 & -3.2 & -75.3226 & 37.9098 & vA & 374 & -5.2 & -75.341 & 37.8808 & VA \\
\hline 231 & -0.7 & -75.3062 & 37.938 & VA & 303 & -3.4 & -75.3228 & 37.9093 & VA & 375 & -5.4 & -75.3412 & 37.8804 & VA \\
\hline 232 & -0.6 & -75.3065 & 37.9376 & $\mathrm{VA}$ & 304 & -3.6 & -75.3231 & 37.9089 & $\mathrm{VA}$ & 376 & -5.6 & -75.3415 & 37.88 & VA \\
\hline 233 & -0.4 & -75.3067 & 37.9372 & VA & 305 & -3.8 & -75.3233 & 37.9085 & $\mathrm{VA}$ & 377 & -5.5 & -75.3418 & 37.8796 & VA \\
\hline 234 & -0.3 & -75.307 & 37.9368 & $\mathrm{VA}$ & 306 & -4.1 & -75.3235 & 37.9081 & VA & 378 & -5.5 & -75.342 & 37.8792 & VA \\
\hline 235 & -0.2 & -75.3073 & 37.9364 & VA & 307 & -4.2 & .75 .3238 & 37.9076 & VA & 379 & NA & -75.4114 & 37.879 & VA \\
\hline 236 & -0.2 & -75.3076 & 37.936 & VA & 308 & -4.3 & -75.324 & 37.9072 & VA & 380 & -5.9 & -75.3422 & 37.879 & VA \\
\hline 237 & -0.3 & -75.3078 & 37.9357 & $V_{A}$ & 309 & -5.3 & -75.3241 & 37.907 & VA & 381 & $\mathrm{NA}$ & -75.4119 & 37.8788 & VA \\
\hline 238 & -0.3 & -75.3078 & 37.9356 & VA & 310 & -4.6 & -75.3243 & 37.9068 & vA & 382 & -5.8 & -75.3423 & 37.8787 & VA \\
\hline 239 & -0.3 & -75.308 & 37.9353 & VA & 311 & -5.2 & -75.3244 & 37.9066 & VA & 383 & -6.1 & -75.3425 & 37.8786 & VA \\
\hline 240 & -0.4 & -75.3081 & 37.9352 & $\mathrm{VA}$ & 312 & -4.9 & -75.3245 & 37.9064 & $\mathrm{VA}$ & 384 & -19.3 & -75.4125 & 37.8785 & VA \\
\hline 241 & -0.3 & -75.3083 & 37.9348 & VA & 313 & -5.1 & -75.3246 & 37.9062 & VA & 385 & -14.5 & -75.413 & 37.8783 & VA \\
\hline 242 & -0.3 & -75.3084 & 37.9347 & VA & 314 & -5 & -75.3249 & 37.9058 & VA & 386 & -6.1 & -75.3426 & 37.8783 & VA \\
\hline 243 & -0.4 & -75.3085 & 37.9344 & VA & 315 & -4.8 & -75.3252 & 37.9053 & VA & 387 & -6.3 & -75.3429 & 37.8782 & VA \\
\hline 244 & -0.3 & -75.3087 & 37.9343 & VA & 316 & -4.7 & -75.3254 & 37.9049 & VA & 388 & -9.7 & -75.4135 & 37.8781 & VA \\
\hline 245 & -0.5 & -75.3088 & 37.934 & VA & 317 & -4.8 & -75.3257 & 37.9045 & $V_{A}$ & 389 & -6.3 & -75.414 & 37.8778 & VA \\
\hline 246 & -0.7 & -75.309 & 37.9336 & $\mathrm{VA}$ & 318 & -4.8 & -75.326 & 37.9041 & $V_{A}$ & 390 & -6.5 & -75.3432 & 37.8778 & VA \\
\hline 247 & -0.6 & -75.3093 & 37.9331 & VA & 319 & -4.9 & -75.3262 & 37.9037 & VA & 391 & -2.9 & -75.4145 & 37.8776 & VA \\
\hline 248 & -0.6 & -75.3095 & 37.9327 & VA & 320 & -5 & -75.3265 & 37.9033 & VA & 392 & -1.6 & -75.415 & 37.8774 & VA \\
\hline 249 & -0.6 & -75.3097 & 37.9323 & VA & 321 & -4.8 & -75.3268 & 37.9028 & VA & 393 & -6.1 & -75.3435 & 37.8774 & VA \\
\hline 250 & -0.6 & -75.31 & 37.9319 & VA & 322 & -4.6 & -75.327 & 37.9024 & $\mathrm{VA}$ & 394 & -0.3 & -75.4155 & 37.8771 & VA \\
\hline 251 & -0.6 & -75.3102 & 37.9314 & $v_{A}$ & 323 & -4.6 & .75 .3273 & 37.902 & VA & 395 & -5.8 & -75.3438 & 37.8771 & VA \\
\hline 252 & -0.5 & -75.3105 & 37.931 & VA & 324 & -4.7 & -75.3276 & 37.9016 & VA & 396 & -2 & .75 .416 & 37.8769 & VA \\
\hline 253 & -0.5 & -75.3107 & 37.9306 & $\mathrm{VA}$ & 325 & -4.9 & -75.3278 & 37.9012 & VA & 397 & -3.7 & -75.4165 & 37.8767 & VA \\
\hline 254 & -0.5 & -75.3109 & 37.9302 & $\mathrm{VA}$ & 326 & -5 & -75.3281 & 37.9008 & $\mathrm{VA}_{\mathrm{A}}$ & 398 & -5.7 & -75.3442 & 37.8767 & VA \\
\hline 255 & -0.7 & -75.3112 & 37.9297 & $\mathrm{VA}$ & 327 & -4.9 & -75.3284 & 37.9004 & $\mathrm{VA}$ & 399 & -2.8 & -75.417 & 37.8764 & VA \\
\hline 256 & -0.8 & .75 .3114 & 37.9293 & VA & 328 & -4.7 & .75 .3286 & 37.8999 & VA & 400 & -5.6 & -75.3445 & 37.8763 & VA \\
\hline 257 & -0.8 & -75.3117 & 37.9289 & VA & 329 & -4.5 & -75.3289 & 37.8995 & VA & 401 & 9 & -75.4175 & 37.8762 & VA \\
\hline 258 & -0.8 & -75.3119 & 37.9285 & VA & 330 & -4.2 & -75.3292 & 37.8991 & $\mathrm{VA}$ & 402 & 8.3 & -75.418 & 37.876 & VA \\
\hline 259 & -0.8 & -75.3122 & 37.928 & $\mathrm{VA}$ & 331 & -4.1 & -75.3295 & 37.8987 & VA & 403 & -5.7 & -75.3448 & 37.8759 & VA \\
\hline 260 & -0.7 & -75.3124 & 37.9276 & $\mathrm{VA}$ & 332 & -4 & -75.3297 & 37.8983 & $\mathrm{VA}$ & 404 & 7.6 & -75.4185 & 37.8757 & VA \\
\hline
\end{tabular}




\begin{tabular}{|c|c|c|c|c|c|c|c|c|c|c|c|c|c|c|}
\hline TR\# & RATE & LONG & LAT & ST & TR\# & RATE & LONG & LAT & ST & TR\# & RATE & LONG & LAT & ST \\
\hline 405 & 7.3 & -75.419 & 37.8755 & $\mathrm{VA}$ & 4777 & 5.6 & -75.3979 & 37.8684 & $\mathrm{VA}$ & 549 & -3.8 & -75.3573 & 37.8611 & $\overline{V A}$ \\
\hline 406 & -5.9 & -75.3452 & 37.8755 & VA & 478 & 5.7 & -75.4337 & 37.8683 & VA & 550 & -1.1 & -75.4444 & 37.8609 & VA \\
\hline 407 & 6.9 & -75.4195 & 37.8753 & VA & 479 & 5.6 & -75.3974 & 37.8682 & VA & 551 & 10.7 & -75.3834 & 37.8608 & VA \\
\hline 408 & -5.8 & -75.3455 & 37.8751 & VA & 480 & -6 & -75.3514 & 37.8681 & VA & 552 & -4.1 & -75.3576 & 37.8607 & VA \\
\hline 409 & 6.9 & -75.42 & 37.875 & $\mathrm{VA}$ & 481 & 5.9 & -75.397 & 37.8679 & $\mathrm{VA}$ & 553 & -1.3 & -75.4448 & 37.8606 & VA \\
\hline 410 & 6.9 & -75.4205 & 37.8748 & VA & 482 & 5.3 & -75.4342 & 37.8679 & VA & 554 & 11.1 & -75.3829 & 37.8605 & VA \\
\hline 411 & -5.7 & -75.3458 & 37.8747 & VA & 483 & 6.2 & -75.3965 & 37.8677 & VA & 555 & 11.1 & -75.3824 & 37.8603 & VA \\
\hline 412 & 6.8 & -75.421 & 37.8746 & VA & 484 & -5.8 & -75.3517 & 37.8677 & VA & 556 & -1.4 & -75.4453 & 37.8603 & VA \\
\hline 413 & 6.6 & -75.4216 & 37.8743 & VA & 485 & 5 & -75.4346 & 37.8676 & VA & 557 & -3.9 & -75.358 & 37.8603 & VA \\
\hline 414 & -5.5 & -75.3461 & 37.8743 & VA & 486 & 6.9 & -75.396 & 37.8674 & VA & 558 & 11.1 & -75.3819 & 37.86 & VA \\
\hline 415 & 6.4 & -75.4221 & 37.8741 & VA & 487 & 4.6 & -75.4351 & 37.8673 & VA & 559 & -1.5 & -75.4457 & 37.86 & VA \\
\hline 416 & -5.3 & -75.3465 & 37.8739 & VA & 488 & -5.9 & -75.352 & 37.8673 & VA & 560 & -3.8 & -75.3583 & 37.86 & VA \\
\hline 417 & 6.2 & -75.4226 & 37.8738 & VA & 489 & 7.6 & .75 .3955 & 37.8672 & VA & 561 & 11.4 & .75 .3815 & 37.8598 & VA \\
\hline 418 & 6.1 & -75.4231 & 37.8736 & VA & 490 & 4.3 & -75.4355 & 37.867 & VA & 562 & -1.7 & -75.4462 & 37.8597 & VA \\
\hline 419 & -5.5 & -75.3468 & 37.8736 & VA & 491 & 8.1 & -75.395 & 37.8669 & VA & 563 & -3.5 & -75.3586 & 37.8596 & VA \\
\hline 420 & 6 & -75.4236 & 37.8734 & VA & 492 & -6 & -75.3524 & 37.8669 & VA & 564 & 11.8 & -75.381 & 37.8595 & VA \\
\hline 421 & $\mathrm{NA}$ & -75.4071 & 37.8733 & VA & 493 & 8.7 & -75.3945 & 37.8667 & VA & 565 & -1.8 & -75.4466 & 37.8594 & VA \\
\hline 422 & -5.7 & -75.3471 & 37.8732 & VA & 494 & 3.9 & -75.436 & 37.8667 & VA & 566 & 11.5 & .75 .3805 & 37.8593 & VA \\
\hline 423 & 5.8 & -75.4241 & 37.8731 & VA & 495 & -5.8 & -75.3527 & 37.8666 & VA & 567 & -3.2 & -75.3589 & 37.8592 & VA \\
\hline 424 & $\mathrm{NA}$ & -75.4066 & 37.873 & VA & 496 & 9.3 & -75.394 & 37.8664 & VA & 568 & -1.9 & -75.4471 & 37.8591 & VA \\
\hline 425 & 13.2 & -75.4246 & 37.8729 & VA & 497 & 3.5 & -75.4364 & 37.8664 & VA & 569 & 11.3 & -75.38 & 37.859 & VA \\
\hline 426 & NA & -75.4062 & 37.8728 & VA & 498 & 10 & -75.3936 & 37.8662 & VA & 570 & 11.5 & -75.3795 & 37.8588 & VA \\
\hline 427 & -5.6 & -75.3475 & 37.8728 & VA & 499 & -5.6 & .75 .353 & 37.8662 & VA & 571 & -1.9 & -75.4475 & 37.8588 & VA \\
\hline 428 & 11.5 & -75.4251 & 37.8727 & VA & 500 & 3 & -75.4369 & 37.8661 & $\mathrm{VA}$ & 572 & -2.7 & -75.3593 & 37.8588 & VA \\
\hline 429 & NA & -75.4057 & 37.8725 & VA & 501 & 9.6 & -75.3931 & 37.8659 & VA & 573 & -1.9 & -75.4479 & 37.8585 & VA \\
\hline 430 & 9.7 & -75.4256 & 37.8724 & VA & 502 & 3 & -75.4373 & 37.8658 & VA & 574 & 11.6 & .75 .379 & 37.8585 & VA \\
\hline 431 & -5.5 & -75.3478 & 37.8724 & VA & 503 & -5.5 & -75.3534 & 37.8658 & VA & 575 & -2.2 & -75.3596 & 37.8584 & VA \\
\hline 432 & 9.6 & -75.4052 & 37.8723 & VA & 504 & 9.2 & -75.3926 & 37.8656 & VA & 576 & -1.9 & -75.4484 & 37.8582 & VA \\
\hline 433 & 9.3 & -75.4261 & 37.8722 & $V_{A}$ & 505 & 3.1 & -75.4377 & 37.8655 & VA & 577 & 11.5 & -75.3785 & 37.8582 & VA \\
\hline 434 & 9.6 & -75.4047 & 37.872 & VA & 506 & 9.1 & -75.3921 & 37.8654 & VA & 578 & 11.3 & -75.3781 & 37.858 & VA \\
\hline 435 & 8.9 & -75.4266 & 37.872 & VA & 507 & -5.4 & -75.3537 & 37.8654 & VA & 579 & -1.6 & -75.3599 & 37.858 & VA \\
\hline 436 & -5.6 & -75.3481 & 37.872 & VA & 508 & 2.5 & -75.4382 & 37.8652 & VA & 580 & -1.7 & -75.4488 & 37.8579 & VA \\
\hline 437 & 9.6 & -75.4042 & 37.8718 & VA & 509 & 8.9 & -75.3916 & 37.8651 & $\mathrm{VA}$ & 581 & 11 & -75.3776 & 37.8577 & VA \\
\hline 438 & 8.5 & -75.4271 & 37.8717 & VA & 510 & -5 & -75.354 & 37.865 & VA & 582 & -1.5 & -75.4493 & 37.8576 & VA \\
\hline 439 & -5.7 & -75.3484 & 37.8716 & VA & 511 & 8.8 & -75.3911 & 37.8649 & VA & 583 & -0.9 & -75.3602 & 37.8576 & VA \\
\hline 440 & 8 & -75.4276 & 37.8715 & VA & 512 & 2 & -75.4386 & 37.8649 & VA & 584 & 10.6 & -75.3771 & 37.8575 & VA \\
\hline 441 & 9.1 & -75.4037 & 37.8715 & VA & 513 & 8.6 & -75.3907 & 37.8646 & VA & 585 & -1.6 & -75.4497 & 37.8573 & VA \\
\hline 442 & 8.5 & -75.4033 & 37.8713 & VA & 514 & 1.7 & -75.4391 & 37.8646 & $\mathrm{VA}$ & 586 & 10 & -75.3766 & 37.8572 & VA \\
\hline 443 & 7.9 & -75.4281 & 37.8713 & VA & 515 & -4.6 & -75.3543 & 37.8646 & $\mathrm{VA}$ & 587 & 0.4 & -75.3606 & 37.8572 & VA \\
\hline 444 & -5.8 & -75.3488 & 37.8712 & VA & 516 & 8.6 & -75.3902 & 37.8644 & VA & 588 & 9.4 & -75.3761 & 37.857 & VA \\
\hline 445 & 7.9 & -75.4028 & 37.871 & VA & 517 & 1.4 & -75.4395 & 37.8643 & $\mathrm{VA}$ & 589 & -1.8 & -75.4502 & 37.8569 & VA \\
\hline 446 & 7.8 & -75.4286 & 37.871 & $\mathrm{VA}$ & 518 & -4.5 & -75.3547 & 37.8642 & VA & 590 & 1.7 & -75.3609 & 37.8568 & VA \\
\hline 447 & 7.7 & -75.4291 & 37.8708 & VA & 519 & 8.6 & -75.3897 & 37.8641 & $\mathrm{VA}$ & 591 & 9.5 & -75.3756 & 37.8567 & VA \\
\hline 448 & -5.8 & -75.3491 & 37.8708 & VA & 520 & 1.1 & -75.44 & 37.864 & $\mathrm{VA}$ & 592 & -2 & -75.4506 & 37.8566 & VA \\
\hline 449 & 7.3 & -75.4023 & 37.8707 & VA & 521 & 8.3 & -75.3892 & 37.8639 & VA & 593 & 9.7 & -75.3752 & 37.8565 & VA \\
\hline 450 & 7.6 & -75.4296 & 37.8706 & VA & 522 & -4.4 & -75.355 & 37.8638 & VA & 594 & 3.5 & -75.3612 & 37.8565 & VA \\
\hline 451 & 7.1 & -75.4018 & 37.8705 & VA & 523 & 0.8 & .75 .4404 & 37.8637 & VA & 595 & -2.3 & .75 .4511 & 37.8563 & VA \\
\hline 452 & -5.7 & -75.3494 & 37.8704 & VA & 524 & 8 & -75.3887 & 37.8636 & VA & 596 & 8.9 & -75.3747 & 37.8562 & VA \\
\hline 453 & 7.6 & -75.4301 & 37.8703 & VA & 525 & -4.1 & -75.3553 & 37.8635 & VA & 597 & 5.3 & -75.3616 & 37.8561 & VA \\
\hline 454 & 7 & -75.4013 & 37.8702 & VA & 526 & 0.5 & -75.4408 & 37.8634 & VA & 598 & 8.2 & -75.3742 & 37.856 & VA \\
\hline 455 & 7.6 & -75.4307 & 37.8701 & VA & 527 & 8.6 & -75.3882 & 37.8633 & $\mathrm{VA}$ & 599 & -2.3 & -75.4515 & 37.856 & VA \\
\hline 456 & -5.7 & -75.3497 & 37.8701 & VA & 528 & 9 & -75.3878 & 37.8631 & VA & 600 & 7.4 & -75.3737 & 37.8557 & VA \\
\hline 457 & 6.7 & -75.4008 & 37.87 & $\mathrm{VA}$ & 529 & 0.2 & -75.4413 & 37.8631 & $\mathrm{VA}$ & 601 & -2.2 & -75.4519 & 37.8557 & VA \\
\hline 458 & 7.6 & -75.4312 & 37.8698 & VA & 530 & -4 & -75.3557 & 37.8631 & VA & 602 & 12.5 & -75.3619 & 37.8557 & VA \\
\hline 459 & 6.3 & -75.4003 & 37.8697 & VA & 531 & -0.1 & -75.4417 & 37.8628 & VA & 603 & -2 & -75.4524 & 37.8554 & VA \\
\hline 460 & -5.8 & -75.3501 & 37.8697 & VA & 532 & 9.5 & -75.3873 & 37.8628 & VA & 604 & 16 & -75.3622 & 37.8553 & VA \\
\hline 461 & 7.6 & -75.4317 & 37.8696 & VA & 533 & -3.9 & -75.356 & 37.8627 & VA & 605 & -1.8 & .75 .4528 & 37.8551 & VA \\
\hline 462 & 5.9 & -75.3999 & 37.8695 & VA & 534 & 9.9 & -75.3868 & 37.8626 & VA & 606 & 3.4 & -75.3625 & 37.8549 & VA \\
\hline 463 & 6.8 & -75.432 & 37.8695 & VA & 535 & -0.3 & -75.4422 & 37.8624 & VA & 607 & -1.7 & -75.4533 & 37.8548 & VA \\
\hline 464 & 7.4 & -75.4322 & 37.8694 & VA & 536 & 10 & -75.3863 & 37.8623 & VA & 608 & -1.6 & -75.4537 & 37.8545 & VA \\
\hline 465 & -6 & -75.3504 & 37.8693 & VA & 537 & -3.8 & -75.3563 & 37.8623 & VA & 609 & 5.1 & -75.3629 & 37.8545 & VA \\
\hline 466 & 7.2 & -75.3994 & 37.8692 & VA & 538 & 10 & -75.3858 & 37.8621 & VA & 610 & -1.5 & -75.4542 & 37.8542 & VA \\
\hline 467 & 6.8 & -75.4324 & 37.8692 & VA & 539 & -0.5 & -75.4426 & 37.8621 & VA & 611 & 0.3 & -75.3632 & 37.8541 & VA \\
\hline 468 & 7.2 & -75.4327 & 37.8691 & VA & 540 & -3.7 & -75.3566 & 37.8619 & VA & 612 & -1.5 & -75.4546 & 37.8539 & VA \\
\hline 469 & 6.4 & -75.3989 & 37.869 & VA & 541 & 9.7 & -75.3853 & 37.8618 & VA & 613 & 0.9 & -75.3635 & 37.8537 & VA \\
\hline 470 & 7.1 & -75.4332 & 37.8689 & $\mathrm{VA}$ & 542 & -0.8 & -75.4431 & 37.8618 & VA & 614 & -1.5 & -75.455 & 37.8536 & VA \\
\hline 471 & 6.5 & -75.4329 & 37.8689 & VA & 543 & 9.3 & -75.3848 & 37.8616 & VA & 615 & -1.5 & -75.4555 & 37.8533 & VA \\
\hline 472 & -6 & -75.3507 & 37.8689 & VA & 544 & -0.8 & -75.4435 & 37.8615 & $\mathrm{VA}$ & 616 & 9.2 & -75.3639 & 37.8533 & VA \\
\hline 473 & 6.9 & -75.4337 & 37.8687 & VA & 545 & -3.6 & -75.357 & 37.8615 & $\mathrm{VA}$ & 617 & -1.6 & -75.4559 & 37.853 & VA \\
\hline 474 & 5.5 & -75.3984 & 37.8687 & VA & 546 & 9.8 & -75.3844 & 37.8613 & $\mathrm{VA}$ & 618 & 17.3 & -75.3642 & 37.853 & VA \\
\hline 475 & 6.1 & -75.4333 & 37.8686 & VA & 547 & -0.9 & .75 .444 & 37.8612 & VA & 619 & -1.7 & -75.4564 & 37.8527 & VA \\
\hline 476 & -6.1 & -75.3511 & 37.8685 & VA & 548 & 10.3 & -75.3839 & 37.8611 & VA & 620 & -1.7 & -75.4568 & 37.8524 & VA \\
\hline
\end{tabular}




\begin{tabular}{|c|c|c|c|c|c|c|c|c|c|c|c|c|c|c|}
\hline TR\# & RATE & LONG & LAT & ST & TR\# & RATE & LONG & LAT & ST & TR\# & RATE & LONG & LAT & ST \\
\hline 621 & -1.8 & -75.4573 & 37.8521 & VA & 693 & -1.8 & -75.4834 & 37.8273 & VA & 765 & -9.6 & -75.5065 & 37.8011 & VA \\
\hline 622 & -1.8 & -75.4577 & 37.8518 & VA & 694 & -1.8 & -75.4838 & 37.827 & VA & 766 & -7.5 & -75.5061 & 37.801 & VA \\
\hline 623 & -1.9 & -75.4582 & 37.8514 & VA & 695 & -0.1 & -75.4837 & 37.8266 & VA & 767 & -9.8 & -75.5067 & 37.8007 & VA \\
\hline 624 & -1.9 & -75.4586 & 37.8511 & VA & 696 & -1.7 & -75.4842 & 37.8266 & VA & 768 & -10 & .75 .507 & 37.8003 & VA \\
\hline 625 & -1.9 & -75.459 & 37.8508 & VA & 697 & -0.1 & -75.484 & 37.8263 & $\mathrm{VA}$ & 769 & -10.2 & -75.5073 & 37.7999 & VA \\
\hline 626 & -1.9 & -75.4595 & 37.8505 & VA & 698 & -1.8 & -75.4845 & 37.8263 & VA & 770 & -10.3 & -75.5075 & 37.7995 & VA \\
\hline 627 & -1.9 & -75.4599 & 37.8502 & VA & 699 & -0.3 & -75.4843 & 37.8259 & $\mathrm{VA}$ & 771 & -10.4 & -75.5078 & 37.7991 & VA \\
\hline 628 & -0.9 & -75.4601 & 37.8501 & $\mathrm{VA}$ & 700 & -1.9 & -75.4849 & 37.8259 & VA & 772 & -10.4 & -75.5081 & 37.7987 & VA \\
\hline 629 & -1.6 & -75.4604 & 37.8499 & VA & 701 & -0.4 & -75.4847 & 37.8255 & $\mathrm{VA}$ & 773 & -10.4 & -75.5083 & 37.7982 & VA \\
\hline 630 & -1 & -75.4605 & 37.8497 & VA & 702 & -0.5 & -75.485 & 37.8251 & $\mathrm{VA}$ & 774 & -10.4 & -75.5086 & 37.7978 & VA \\
\hline 631 & -1.3 & -75.4608 & 37.8496 & VA & 703 & -0.6 & -75.4853 & 37.8247 & VA & 775 & -10.4 & -75.5089 & 37.7974 & VA \\
\hline 632 & -1 & -75.4608 & 37.8493 & VA & 704 & -0.7 & -75.4857 & 37.8243 & VA & 776 & -10.5 & -75.5091 & 37.797 & VA \\
\hline 633 & -1.1 & .75 .4612 & 37.849 & VA & 705 & -0.8 & .75 .486 & 37.824 & VA & 777 & -10.7 & -75.5094 & 37.7966 & VA \\
\hline 634 & -1.1 & -75.4616 & 37.8486 & VA & 706 & -1.1 & -75.4864 & 37.8236 & $\mathrm{VA}$ & 778 & -10.9 & -75.5097 & 37.7962 & VA \\
\hline 635 & -1.1 & -75.4619 & 37.8483 & VA & 707 & -1.3 & -75.4867 & 37.8232 & $\mathrm{VA}$ & 779 & -11.1 & -75.5099 & 37.7958 & VA \\
\hline 636 & -1.1 & -75.4623 & 37.8479 & VA & 708 & -1.8 & -75.487 & 37.8228 & VA & 780 & -11.2 & -75.5102 & 37.7953 & VA \\
\hline 637 & -1.1 & -75.4627 & 37.8475 & VA & 709 & -2.2 & -75.4874 & 37.8224 & $\mathrm{VA}$ & 781 & -11.4 & -75.5105 & 37.7949 & VA \\
\hline 638 & -1.1 & -75.463 & 37.8472 & VA & 710 & -2.8 & -75.4877 & 37.822 & $\mathrm{VA}$ & 782 & -11.4 & -75.5107 & 37.7945 & VA \\
\hline 639 & -1.2 & -75.4634 & 37.8468 & VA & 711 & -3.4 & -75.488 & 37.8217 & $\mathrm{VA}_{\mathrm{A}}$ & 783 & -11.4 & .75 .511 & 37.7941 & VA \\
\hline 640 & -1.2 & -75.4638 & 37.8465 & VA & 712 & -3.6 & -75.4884 & 37.8213 & $\mathrm{VA}$ & 784 & -11.4 & -75.5113 & 37.7937 & VA \\
\hline 641 & -1.1 & -75.4642 & 37.8461 & $\mathrm{VA}$ & 713 & -3.9 & -75.4887 & 37.8209 & $\mathrm{VA}$ & 785 & -11.4 & -75.5115 & 37.7933 & VA \\
\hline 642 & -1 & -75.4645 & 37.8457 & VA & 714 & -4.1 & -75.489 & 37.8205 & VA & 786 & -11.3 & -75.5118 & 37.7929 & VA \\
\hline 643 & -1 & -75.4649 & 37.8454 & VA & 715 & -4.3 & -75.4894 & 37.8201 & VA & 787 & -11.2 & -75.5121 & 37.7924 & VA \\
\hline 644 & -0.8 & -75.4653 & 37.845 & VA & 716 & -4.3 & -75.4897 & 37.8197 & $V A$ & 788 & -11.3 & -75.5123 & 37.792 & VA \\
\hline 645 & -0.7 & .75 .4656 & 37.8446 & VA & 717 & -4.3 & -75.49 & 37.8194 & VA & 789 & -11.4 & -75.5126 & 37.7916 & VA \\
\hline 646 & -0.7 & -75.466 & 37.8443 & $\mathrm{VA}$ & 718 & -4.3 & -75.4904 & 37.819 & VA & 790 & -11.1 & -75.5129 & 37.7912 & $\mathrm{VA}$ \\
\hline 647 & -0.7 & -75.4664 & 37.8439 & VA & 719 & -4.3 & -75.4907 & 37.8186 & VA & 791 & -10.9 & -75.5131 & 37.7908 & VA \\
\hline 648 & -0.6 & -75.4667 & 37.8436 & $V_{A}$ & 720 & -4.5 & -75.491 & 37.8182 & VA & 792 & -11.1 & -75.5134 & 37.7904 & VA \\
\hline 649 & -0.6 & -75.4671 & 37.8432 & VA & 721 & -4.7 & -75.4914 & 37.8178 & VA & 793 & -11.2 & -75.5137 & 37.79 & VA \\
\hline 650 & -0.5 & -75.4675 & 37.8428 & VA & 722 & -4 & -75.4917 & 37.8174 & $\mathrm{VA}$ & 794 & -11.2 & -75.5139 & 37.7895 & VA \\
\hline 651 & -0.5 & -75.4679 & 37.8425 & VA & 723 & -3.2 & -75.4921 & 37.8171 & $\mathrm{VA}$ & 795 & -11.2 & -75.5142 & 37.7891 & VA \\
\hline 652 & -0.2 & .75 .4682 & 37.8421 & VA & 724 & -0.8 & -75.4924 & 37.8167 & $\mathrm{VA}$ & 796 & -11.2 & -75.5145 & 37.7887 & VA \\
\hline 653 & 0 & -75.4686 & 37.8418 & $\mathrm{VA}$ & 725 & 1.6 & -75.4927 & 37.8163 & $\mathrm{VA}$ & 797 & -11.1 & -75.5147 & 37.7883 & VA \\
\hline 654 & 0 & -75.469 & 37.8414 & VA & 726 & -3.1 & -75.4931 & 37.8159 & $\mathrm{VA}$ & 798 & -11 & -75.515 & 37.7879 & VA \\
\hline 655 & -0.1 & -75.4693 & 37.841 & VA & 727 & -1.7 & -75.4934 & 37.8155 & $\mathrm{VA}$ & 799 & -10.9 & -75.5152 & 37.7875 & VA \\
\hline 656 & -0.1 & -75.4697 & 37.8407 & VA & 728 & -0.6 & -75.4937 & 37.8151 & VA & 800 & -10.9 & -75.5155 & 37.7871 & VA \\
\hline 657 & -0.2 & -75.4701 & 37.8403 & VA & 729 & 0.7 & -75.4941 & 37.8148 & VA & 801 & -10.9 & -75.5158 & 37.7867 & VA \\
\hline 658 & -0.1 & -75.4705 & 37.84 & VA & 730 & 2.1 & -75.4944 & 37.8144 & $\mathrm{VA}$ & 802 & -10.7 & -75.516 & 37.7862 & VA \\
\hline 659 & -0.1 & -75.4708 & 37.8396 & VA & 731 & -4.3 & -75.4947 & 37.814 & $\mathrm{VA}$ & 803 & -10.5 & -75.5163 & 37.7858 & VA \\
\hline 660 & 0 & -75.4712 & 37.8392 & VA & 732 & -6.1 & -75.4951 & 37.8136 & VA & 804 & -10.4 & -75.5166 & 37.7854 & VA \\
\hline 661 & 0.1 & -75.4716 & 37.8389 & $V_{A}$ & 733 & -7.9 & -75.4954 & 37.8132 & VA & 805 & -10.2 & -75.5168 & 37.785 & VA \\
\hline 662 & 0.1 & -75.4719 & 37.8385 & VA & 734 & -6.5 & -75.4957 & 37.8128 & VA & 806 & -10.2 & -75.5171 & 37.7846 & VA \\
\hline 663 & 0.1 & -75.4723 & 37.8382 & VA & 735 & -5.2 & -75.4961 & 37.8125 & VA & 807 & -10.2 & -75.5174 & 37.7842 & VA \\
\hline 664 & 0.1 & .75 .4727 & 37.8378 & $\mathrm{VA}$ & 736 & -5.1 & -75.4964 & 37.8121 & VA & 808 & -10.2 & -75.5176 & 37.7838 & VA \\
\hline 665 & 0.1 & -75.473 & 37.8374 & VA & 737 & -5.1 & -75.4967 & 37.8117 & VA & 809 & -10.2 & -75.5179 & 37.7833 & VA \\
\hline 666 & 0.1 & -75.4734 & 37.8371 & VA & 738 & -5.3 & -75.4971 & 37.8113 & VA & 810 & -10 & -75.5182 & 37.7829 & VA \\
\hline 667 & 0.2 & -75.4738 & 37.8367 & VA & 739 & -5.3 & -75.4974 & 37.8109 & $\mathrm{VA}$ & 811 & -9.8 & .75 .5184 & 37.7825 & VA \\
\hline 668 & 0.2 & -75.4742 & 37.8364 & VA & 740 & -5.5 & -75.4977 & 37.8105 & VA & 812 & -9.6 & -75.5187 & 37.7821 & VA \\
\hline 669 & 0.1 & -75.4745 & 37.836 & VA & 741 & -5.7 & -75.4981 & 37.8102 & VA & 813 & -9.5 & -75.519 & 37.7817 & VA \\
\hline 670 & 0.2 & -75.4749 & 37.8356 & $\mathrm{VA}_{\mathrm{A}}$ & 742 & -5.8 & -75.4984 & 37.8098 & VA & 814 & -9.3 & -75.5192 & 37.7813 & VA \\
\hline 671 & 0.2 & -75.4753 & 37.8353 & VA & 743 & -6 & -75.4988 & 37.8094 & VA & 815 & -9.1 & -75.5195 & 37.7809 & VA \\
\hline 672 & 0.1 & -75.4756 & 37.8349 & VA & 744 & -6 & -75.4991 & 37.809 & VA & 816 & -8.8 & -75.5198 & 37.7804 & VA \\
\hline 673 & 0.1 & -75.476 & 37.8346 & VA & 745 & -6 & -75.4994 & 37.8086 & VA & 817 & -8.6 & -75.52 & 37.78 & VA \\
\hline 674 & 0.1 & -75.4764 & 37.8342 & $\mathrm{VA}$ & 746 & -6.1 & -75.4998 & 37.8082 & $\mathrm{VA}$ & 818 & -8.3 & -75.5203 & 37.7796 & VA \\
\hline 675 & 0 & -75.4768 & 37.8338 & VA & 747 & -6.1 & -75.5001 & 37.8079 & VA & 819 & -8 & -75.5206 & 37.7792 & VA \\
\hline 676 & 0.1 & -75.4771 & 37.8335 & VA & 748 & -6.2 & -75.5004 & 37.8075 & VA & 820 & -7.8 & -75.5208 & 37.7788 & VA \\
\hline 677 & 0.2 & -75.4775 & 37.8331 & VA & 749 & -6.2 & -75.5008 & 37.8071 & VA & 821 & -7.5 & -75.5211 & 37.7784 & VA \\
\hline 678 & 0.1 & -75.4779 & 37.8328 & VA & 750 & -6.2 & -75.5011 & 37.8067 & VA & 822 & -7.3 & -75.5214 & 37.778 & VA \\
\hline 679 & -0.1 & -75.4782 & 37.8324 & VA & 751 & -6.3 & -75.5014 & 37.8063 & $\mathrm{VA}$ & 823 & -7.1 & -75.5216 & 37.7775 & VA \\
\hline 680 & -0.1 & -75.4786 & 37.832 & $\mathrm{VA}$ & 752 & -6.4 & -75.5018 & 37.8059 & $\mathrm{VA}$ & 824 & -6.9 & -75.5219 & 37.7771 & VA \\
\hline 681 & -0.2 & -75.479 & 37.8317 & $\mathrm{VA}$ & 753 & -6.4 & -75.5021 & 37.8056 & $\mathrm{VA}$ & 825 & -6.6 & -75.5222 & 37.7767 & VA \\
\hline 682 & -0.3 & -75.4793 & 37.8313 & VA & 754 & -6.5 & -75.5024 & 37.8052 & VA & 826 & -6.4 & -75.5224 & 37.7763 & VA \\
\hline 683 & -0.5 & -75.4797 & 37.8309 & $\mathrm{VA}$ & 755 & -6.7 & -75.5028 & 37.8048 & VA & 827 & -6.2 & .75 .5227 & 37.7759 & VA \\
\hline 684 & -0.6 & -75.4801 & 37.8306 & VA & 756 & -6.5 & -75.5031 & 37.8044 & VA & 828 & -6.7 & -75.523 & 37.7755 & VA \\
\hline 685 & -0.7 & -75.4805 & 37.8302 & VA & 757 & -6.4 & -75.5034 & 37.804 & VA & 829 & -7.1 & -75.5232 & 37.7751 & VA \\
\hline 686 & -0.8 & -75.4808 & 37.8299 & $\mathrm{VA}$ & 758 & -6.3 & -75.5038 & 37.8036 & VA & 830 & -8.5 & -75.5235 & 37.7746 & VA \\
\hline 687 & -1 & -75.4812 & 37.8295 & VA & 759 & -6.1 & -75.5041 & 37.8033 & $\mathrm{VA}$ & 831 & -7.9 & -75.5235 & 37.7742 & VA \\
\hline 688 & -1.1 & -75.4816 & 37.8291 & $\mathrm{VA}$ & 760 & -6.3 & -75.5045 & 37.8029 & $\mathrm{VA}$ & 832 & -9.8 & -75.5238 & 37.7742 & VA \\
\hline 689 & -1.3 & .75 .4819 & 37.8288 & VA & 761 & -6.5 & .75 .5048 & 37.8025 & VA & 833 & -5.4 & -75.5237 & 37.7738 & VA \\
\hline 690 & -1.5 & -75.4823 & 37.8284 & VA & 762 & -6.7 & -75.5051 & 37.8021 & VA & 834 & -5.7 & -75.524 & 37.7738 & VA \\
\hline 691 & -1.6 & -75.4827 & 37.8281 & VA & 763 & -6.9 & -75.5055 & 37.8017 & VA & 835 & -0.2 & .75 .524 & 37.7734 & VA \\
\hline 692 & -1.7 & -75.483 & 37.8277 & VA & 764 & -7.2 & -75.5058 & 37.8013 & VA & 836 & -7.5 & -75.5243 & 37.7734 & VA \\
\hline
\end{tabular}




\begin{tabular}{|c|c|c|c|c|c|c|c|c|c|c|c|c|c|c|}
\hline TR\# & RATE & LONG & LAT & ST & TR\# & RATE & LONG & LAT & ST & TR\# & RATE & LONG & LAT & ST \\
\hline 837 & 5 & -75.5242 & 37.7729 & VA & 909 & -6.8 & -75.5421 & 37.7438 & $\mathrm{VA}$ & 981 & -28.1 & -75.558 & 37.7152 & VA \\
\hline 838 & 5.4 & -75.5245 & 37.7725 & VA & 910 & -6.6 & -75.5423 & 37.7434 & VA & 982 & -27.8 & -75.5582 & 37.7147 & VA \\
\hline 839 & 5.7 & -75.5247 & 37.7721 & VA & 911 & -6.4 & -75.5426 & 37.743 & VA & 983 & -27.6 & -75.5583 & 37.7143 & VA \\
\hline 840 & 5.4 & -75.525 & 37.7717 & VA & 912 & -6.2 & -75.5428 & 37.7426 & VA & 984 & -27.3 & -75.5585 & 37.7139 & VA \\
\hline 841 & 5.2 & -75.5252 & 37.7713 & VA & 913 & -6.5 & -75.543 & 37.7421 & VA & 985 & -28.6 & -75.5587 & 37.7134 & VA \\
\hline 842 & 5.1 & -75.5255 & 37.7708 & VA & 914 & -6.8 & -75.5433 & 37.7417 & VA & 986 & -29.8 & -75.5589 & 37.713 & VA \\
\hline 843 & 5 & -75.5257 & 37.7704 & VA & 915 & -7 & -75.5435 & 37.7413 & VA & 987 & -16 & -75.5591 & 37.7125 & VA \\
\hline 844 & 5.5 & -75.526 & 37.77 & VA & 916 & -7.1 & -75.5438 & 37.7409 & VA & 988 & -16.2 & -75.5593 & 37.7121 & VA \\
\hline 845 & 5.9 & -75.5262 & 37.7696 & VA & 917 & -7.2 & -75.544 & 37.7405 & VA & 989 & -16.5 & -75.5595 & 37.7116 & VA \\
\hline 846 & 6.9 & -75.5265 & 37.7691 & VA & 918 & -7.3 & -75.5443 & 37.74 & VA & 990 & -30.5 & -75.5597 & 37.7112 & VA \\
\hline 847 & 7.9 & -75.5267 & 37.7687 & VA & 919 & -7.4 & .75 .5445 & 37.7396 & VA & 991 & -26.8 & -75.5598 & 37.7108 & VA \\
\hline 848 & 4.1 & -75.527 & 37.7683 & VA & 920 & -7.5 & -75.5448 & 37.7392 & VA & 992 & -23 & -75.56 & 37.7103 & VA \\
\hline 849 & 0.4 & -75.5272 & 37.7679 & VA & 921 & -7.7 & -75.545 & 37.7388 & VA & 993 & -22.2 & -75.5602 & 37.7099 & VA \\
\hline 850 & -2.1 & -75.5275 & 37.7674 & VA & 922 & -8 & -75.5452 & 37.7384 & VA & 994 & -21.4 & -75.5604 & 37.7094 & VA \\
\hline 851 & -4.6 & -75.5277 & 37.767 & VA & 923 & -8.3 & -75.5455 & 37.7379 & VA & 995 & -21.1 & -75.5606 & 37.709 & VA \\
\hline 852 & -6.4 & -75.528 & 37.7666 & VA & 924 & -8.6 & -75.5457 & 37.7375 & VA & 996 & -20.7 & -75.5608 & 37.7085 & VA \\
\hline 853 & -8 & -75.5282 & 37.7662 & VA & 925 & -8.7 & -75.546 & 37.7371 & VA & 997 & -20.7 & -75.561 & 37.7081 & VA \\
\hline 854 & -8.2 & -75.5285 & 37.7658 & VA & 926 & -8.7 & -75.5462 & 37.7367 & VA & 998 & -20.7 & -75.5612 & 37.7077 & VA \\
\hline 855 & -8.4 & -75.5287 & 37.7653 & VA & 927 & -8.7 & -75.5465 & 37.7363 & VA & 999 & -20.6 & -75.5613 & 37.7072 & VA \\
\hline 856 & -8.4 & -75.529 & 37.7649 & VA & 928 & -8.6 & -75.5467 & 37.7358 & VA & 1000 & -20.5 & -75.5615 & 37.7068 & VA \\
\hline 857 & -8.4 & -75.5292 & 37.7645 & VA & 929 & -8.9 & -75.547 & 37.7354 & VA & 1001 & -20.3 & -75.5617 & 37.7063 & VA \\
\hline 858 & -8.5 & -75.5295 & 37.7641 & VA & 930 & -9.3 & -75.5472 & 37.735 & VA & 1002 & -20.2 & -75.5619 & 37.7059 & VA \\
\hline 859 & -8.6 & -75.5297 & 37.7636 & VA & 931 & -9.5 & -75.5475 & 37.7346 & VA & 1003 & -19.5 & -75.5621 & 37.7054 & VA \\
\hline 860 & -8.5 & -75.53 & 37.7632 & VA & 932 & -9.8 & -75.5477 & 37.7341 & VA & 1004 & -18.9 & -75.5623 & 37.705 & VA \\
\hline 861 & -8.4 & -75.5302 & 37.7628 & VA & 933 & -10.5 & -75.5479 & 37.7337 & VA & 1005 & -18.9 & -75.5625 & 37.7046 & VA \\
\hline 862 & -8.6 & -75.5305 & 37.7624 & VA & 934 & -11.2 & -75.5482 & 37.7333 & VA & 1006 & -18.7 & -75.5627 & 37.7041 & VA \\
\hline 863 & -8.7 & -75.5307 & 37.762 & VA & 935 & -12.5 & -75.5484 & 37.7329 & VA & 1007 & -18.5 & -75.5628 & 37.7037 & VA \\
\hline 864 & -8.9 & -75.531 & 37.7615 & VA & 936 & -13.7 & -75.5487 & 37.7325 & VA & 1008 & -18.2 & -75.563 & 37.7032 & VA \\
\hline 865 & -9.1 & -75.5312 & 37.7611 & VA & 937 & -15.4 & -75.5489 & 37.732 & VA & 1009 & -17.9 & -75.5632 & 37.7028 & VA \\
\hline 866 & -9.1 & -75.5315 & 37.7607 & VA & 938 & -17.1 & -75.5492 & 37.7316 & VA & 1010 & -17.7 & -75.5634 & 37.7024 & VA \\
\hline 867 & -9.1 & -75.5317 & 37.7603 & VA & 939 & -15.6 & -75.5494 & 37.7312 & VA & 1011 & -17.7 & -75.5636 & 37.7019 & VA \\
\hline 868 & -9.2 & -75.532 & 37.7598 & VA & 940 & -17 & -75.5497 & 37.7308 & VA & 1012 & -17.7 & -75.5638 & 37.7015 & VA \\
\hline 869 & -9.3 & -75.5322 & 37.7594 & VA & 941 & -18.3 & -75.5499 & 37.7304 & VA & 1013 & -17.6 & -75.564 & 37.701 & VA \\
\hline 870 & -9.1 & -75.5325 & 37.759 & VA & 942 & -22.8 & -75.5501 & 37.7299 & VA & 1014 & -17.5 & -75.5642 & 37.7006 & VA \\
\hline 871 & -8.9 & -75.5327 & 37.7586 & VA & 943 & -22.7 & -75.5504 & 37.7295 & VA & 1015 & -16.6 & -75.5613 & 37.6997 & VA \\
\hline 872 & -8.3 & -75.533 & 37.7582 & VA & 944 & -22.5 & -75.5506 & 37.7291 & VA & 1016 & -16.4 & -75.5615 & 37.6992 & VA \\
\hline 873 & -7.7 & -75.5332 & 37.7577 & VA & 945 & -22.8 & -75.5509 & 37.7287 & VA & 1017 & -16.3 & -75.5617 & 37.6988 & VA \\
\hline 874 & -7.1 & -75.5335 & 37.7573 & VA & 946 & -23 & -75.5511 & 37.7282 & VA & 1018 & -16.2 & -75.5619 & 37.6983 & VA \\
\hline 875 & -6.6 & -75.5337 & 37.7569 & VA & 947 & -23.1 & -75.5514 & 37.7278 & VA & 1019 & -16.2 & -75.5621 & 37.6979 & VA \\
\hline 876 & -6.4 & -75.534 & 37.7565 & VA & 948 & -23.2 & -75.5516 & 37.7274 & VA & 1020 & -16.1 & -75.5623 & 37.6975 & VA \\
\hline 877 & -6.2 & -75.5342 & 37.756 & VA & 949 & -23.4 & -75.5519 & 37.727 & VA & 1021 & -16.2 & -75.5625 & 37.697 & VA \\
\hline 878 & -6 & -75.5345 & 37.7556 & VA & 950 & -23.6 & -75.5521 & 37.7266 & VA & 1022 & -16.4 & -75.5627 & 37.6966 & VA \\
\hline 879 & -5.7 & -75.5347 & 37.7552 & VA & 951 & -23.8 & -75.5524 & 37.7261 & VA & 1023 & -16.2 & -75.5629 & 37.6961 & VA \\
\hline 880 & -5.2 & -75.535 & 37.7548 & VA & 952 & -24.1 & -75.5526 & 37.7257 & VA & 1024 & -16.1 & -75.5631 & 37.6957 & VA \\
\hline 881 & -4.6 & -75.5352 & 37.7544 & VA & 953 & -24.3 & -75.5528 & 37.7253 & VA & 1025 & $\cdot 16.1$ & -75.5632 & 37.6952 & VA \\
\hline 882 & -4.3 & -75.5355 & 37.7539 & VA & 954 & -24.5 & -75.5531 & 37.7249 & VA & 1026 & -16.1 & -75.5634 & 37.6948 & VA \\
\hline 883 & -3.9 & -75.5357 & 37.7535 & VA & 955 & -24.8 & -75.5533 & 37.7245 & VA & 1027 & -15.9 & -75.5636 & 37.6944 & VA \\
\hline 884 & -3.9 & -75.536 & 37.7531 & VA & 956 & -25.2 & -75.5536 & 37.724 & VA & 1028 & -15.7 & -75.5638 & 37.6939 & VA \\
\hline 885 & -3.9 & -75.5362 & 37.7527 & VA & 957 & -25.6 & -75.5538 & 37.7236 & VA & 1029 & -15.9 & -75.564 & 37.6935 & VA \\
\hline 886 & -4.1 & -75.5365 & 37.7522 & VA & 958 & -26.1 & -75.5541 & 37.7232 & VA & 1030 & -16.1 & -75.5642 & 37.693 & VA \\
\hline 887 & -4.3 & -75.5367 & 37.7518 & VA & 959 & -26.4 & -75.5543 & 37.7228 & VA & 1031 & -16.4 & -75.5644 & 37.6926 & VA \\
\hline 888 & -4.5 & -75.537 & 37.7514 & VA & 960 & -26.8 & -75.5546 & 37.7224 & VA & 1032 & -16.8 & -75.5646 & 37.6922 & VA \\
\hline 889 & -4.6 & -75.5372 & 37.751 & VA & 961 & -27 & -75.5548 & 37.7219 & VA & 1033 & -17.4 & -75.5648 & 37.6917 & VA \\
\hline 890 & -5 & -75.5375 & 37.7505 & VA & 962 & -27.1 & -75.555 & 37.7215 & VA & 1034 & -18 & .75 .565 & 37.6913 & VA \\
\hline 891 & -5.4 & -75.5377 & 37.7501 & VA & 963 & -27.6 & -75.5553 & 37.7211 & VA & 1035 & -23.2 & -75.5651 & 37.6908 & VA \\
\hline 892 & -5.4 & -75.538 & 37.7497 & VA & 964 & -28 & -75.5555 & 37.7207 & VA & 1036 & -28.4 & -75.5653 & 37.6904 & VA \\
\hline 893 & -5.5 & .75 .5382 & 37.7493 & VA & 965 & -28.2 & .75 .5558 & 37.7202 & $\mathrm{VA}$ & 1037 & -27.7 & -75.5655 & 37.69 & VA \\
\hline 894 & -5.6 & -75.5385 & 37.7489 & VA & 966 & -28.4 & -75.556 & 37.7198 & VA & 1038 & -27.1 & -75.5657 & 37.6895 & VA \\
\hline 895 & -5.7 & -75.5387 & 37.7484 & VA & 967 & -28.6 & -75.5563 & 37.7194 & VA & 1039 & -26.2 & -75.5659 & 37.6891 & VA \\
\hline 896 & -5.8 & .75 .539 & 37.748 & VA & 968 & -28.7 & -75.5565 & 37.719 & VA & 1040 & -25.3 & -75.5661 & 37.6886 & VA \\
\hline 897 & -5.9 & -75.5392 & 37.7476 & VA & 969 & -28.2 & -75.5565 & 37.7187 & VA & 1041 & -16.7 & -75.5663 & 37.6882 & VA \\
\hline 898 & -6.1 & -75.5395 & 37.7472 & VA & 970 & -28.7 & -75.5568 & 37.7186 & VA & 1042 & -18 & -75.5665 & 37.6878 & VA \\
\hline 899 & -6.4 & -75.5403 & 37.7468 & VA & 971 & -28.2 & -75.5567 & 37.7183 & VA & 1043 & -11.7 & -75.5667 & 37.6873 & VA \\
\hline 900 & -6.2 & -75.5397 & 37.7467 & VA & 972 & -28.7 & -75.557 & 37.7181 & VA & 1044 & -11.9 & -75.5669 & 37.6869 & VA \\
\hline 901 & -6.4 & -75.5406 & 37.7464 & VA & 973 & -28.4 & -75.5568 & 37.7178 & VA & 1045 & -11.7 & -75.567 & 37.6864 & VA \\
\hline 902 & -6.3 & -75.54 & 37.7463 & VA & 974 & -28.7 & -75.5573 & 37.7177 & $\mathrm{VA}$ & 1046 & -11.6 & -75.5672 & 37.686 & VA \\
\hline 903 & -6.4 & -75.5408 & 37.7459 & VA & 975 & -28.6 & -75.557 & 37.7174 & VA & 1047 & -11.9 & -75.5674 & 37.6855 & VA \\
\hline 904 & -6.4 & -75.5402 & 37.7459 & VA & 976 & -28.7 & -75.5575 & 37.7173 & VA & 1048 & -12.1 & -75.5676 & 37.6851 & VA \\
\hline 905 & -6.7 & -75.5411 & 37.7455 & VA & 977 & -28.6 & -75.5572 & 37.7169 & VA & 1049 & -12.6 & -75.5678 & 37.6847 & VA \\
\hline 906 & -7 & -75.5413 & 37.7451 & VA & 978 & -28.6 & -75.5574 & 37.7165 & VA & 1050 & -13 & -75.568 & 37.6842 & VA \\
\hline 907 & -7 & -75.5416 & 37.7447 & VA & 979 & -28.5 & -75.5576 & 37.7161 & VA & 1051 & -13.2 & -75.5682 & 37.6838 & VA \\
\hline 908 & -7 & -75.5418 & 37.7443 & VA & 980 & -28.4 & -75.5578 & 37.7156 & VA & 1052 & -13.3 & -75.5684 & 37.6833 & VA \\
\hline
\end{tabular}

MID-ATLANTIC MAP DATA/USGS/NJ.DE.MD.VA 


\begin{tabular}{|c|c|c|c|c|c|c|c|c|c|c|c|c|c|c|}
\hline TR\# & RATE & LONG & LAT & ST & TR\# & RATE & LONG & LAT & ST & TR\# & RATE & LONG & LAT & ST \\
\hline 1053 & -13.9 & -75.5686 & 37.6829 & $\overline{V A}$ & 1125 & -4.2 & -75.5838 & 37.6477 & VA & 1197 & -6.7 & -75.5971 & 37.6168 & VA \\
\hline 1054 & -14.5 & -75.5688 & 37.6825 & $\mathrm{VA}$ & 1126 & -4.5 & .75 .584 & 37.6472 & VA & 1198 & .7 & -75.5973 & 37.6163 & VA \\
\hline 1055 & -14.6 & -75.5689 & 37.682 & VA & 1127 & -4.4 & .75 .5842 & 37.6468 & $\mathrm{VA}$ & 1199 & -7.2 & -75.5975 & 37.6159 & VA \\
\hline 1056 & -14.7 & -75.5691 & 37.6816 & VA & 1128 & -4.3 & -75.5844 & 37.6463 & VA & 1200 & -7.3 & -75.5977 & 37.6155 & VA \\
\hline 1057 & -15.9 & -75.5693 & 37.6811 & VA & 1129 & -4.5 & -75.5846 & 37.6459 & VA & 1201 & -7.4 & -75.5979 & 37.615 & VA \\
\hline 1058 & -17.1 & -75.5695 & 37.6807 & VA & 1130 & -4.6 & -75.5848 & 37.6455 & VA & 1202 & -7.5 & -75.5981 & 37.6146 & VA \\
\hline 1059 & -14.6 & -75.5697 & 37.6803 & VA & 1131 & -4.6 & -75.5849 & 37.645 & VA & 1203 & -7.4 & -75.5983 & 37.6141 & VA \\
\hline 1060 & -16.2 & .75 .5699 & 37.6798 & VA & 1132 & -4.7 & -75.5851 & 37.6446 & VA & 1204 & -7.3 & -75.5985 & 37.6137 & VA \\
\hline 1061 & -6.9 & -75.5701 & 37.6794 & VA & 1133 & -4.6 & .75 .5853 & 37.6441 & VA & 1205 & -7.3 & -75.5986 & 37.6132 & VA \\
\hline 1062 & -6.6 & -75.5703 & 37.6789 & VA & 1134 & -4.6 & -75.5855 & 37.6437 & VA & 1206 & -7.2 & -75.5988 & 37.6128 & VA \\
\hline 1063 & -6 & -75.5705 & 37.6785 & VA & 1135 & -4.7 & -75.5857 & 37.6433 & VA & 1207 & -7.2 & -75.599 & 37.6124 & VA \\
\hline 1064 & -5.6 & -75.5707 & 37.6781 & VA & 1136 & -4.7 & -75.5859 & 37.6428 & VA & 1208 & -7.2 & .75 .5992 & 37.6119 & VA \\
\hline 1065 & NA & -75.572 & 37.6748 & VA & 1137 & -4.6 & -75.5861 & 37.6424 & VA & 1209 & -7.3 & -75.5994 & 37.6115 & VA \\
\hline 1066 & NA & -75.5728 & 37.6731 & VA & 1138 & -4.5 & -75.5863 & 37.6419 & VA & 1210 & -7.4 & -75.5996 & 37.611 & VA \\
\hline 1067 & NA & -75.573 & 37.6726 & $\mathrm{VA}$ & 1139 & -4.6 & -75.5865 & 37.6415 & $\mathrm{VA}$ & 1211 & -7.5 & -75.5998 & 37.6106 & VA \\
\hline 1068 & NA & -75.5732 & 37.6722 & VA & 1140 & -4.7 & -75.5866 & 37.6411 & VA & 1212 & -7.6 & -75.6 & 37.6101 & VA \\
\hline 1069 & -13.2 & -75.5734 & 37.6717 & VA & 1141 & -4.6 & -75.5868 & 37.6406 & $\mathrm{VA}$ & 1213 & -7.7 & .75 .6001 & 37.6097 & VA \\
\hline 1070 & -10 & -75.5736 & 37.6713 & VA & 1142 & -4.5 & -75.587 & 37.6402 & VA & 1214 & -7.9 & -75.6003 & 37.6093 & VA \\
\hline 1071 & -11.9 & -75.5738 & 37.6709 & VA & 1143 & -4.4 & -75.5872 & 37.6397 & VA & 1215 & -7.9 & -75.6005 & 37.6088 & VA \\
\hline 1072 & -13.8 & -75.574 & 37.6704 & $\mathrm{VA}$ & 1144 & -4.3 & -75.5874 & 37.6393 & $\mathrm{VA}$ & 1216 & -7.9 & -75.6007 & 37.6084 & VA \\
\hline 1073 & -14.1 & -75.5741 & 37.67 & VA & 1145 & -4.5 & -75.5876 & 37.6389 & $\mathrm{VA}$ & 1217 & -7.7 & -75.6009 & 37.6079 & VA \\
\hline 1074 & -10.1 & -75.5743 & 37.6696 & $\mathrm{VA}$ & 1146 & -4.6 & -75.5878 & 37.6384 & $\mathrm{VA}$ & 1218 & -7.5 & -75.6011 & 37.6075 & VA \\
\hline 1075 & -12.3 & -75.5745 & 37.6691 & VA & 1147 & -4.6 & -75.588 & 37.638 & VA & 1219 & -7.5 & -75.6013 & 37.607 & VA \\
\hline 1076 & -10.9 & -75.5747 & 37.6687 & VA & 1148 & -4.6 & -75.5882 & 37.6376 & VA & 1220 & -7.5 & -75.6015 & 37.6066 & VA \\
\hline 1077 & -11.4 & -75.5749 & 37.6682 & VA & 1149 & -4.5 & -75.5883 & 37.6371 & VA & 1221 & -1.3 & -75.5981 & 37.6063 & VA \\
\hline 1078 & -11.9 & -75.5751 & 37.6678 & VA & 1150 & -4.4 & -75.5885 & 37.6367 & VA & 1222 & -7.2 & -75.6016 & 37.6062 & VA \\
\hline 1079 & -12.1 & -75.5753 & 37.6674 & VA & 1151 & -4.3 & -75.5887 & 37.6362 & VA & 1223 & -0.9 & -75.5981 & 37.6058 & VA \\
\hline 1080 & -12.2 & -75.5755 & 37.6669 & VA & 1152 & -4.3 & -75.5889 & 37.6358 & $\mathrm{VA}$ & 1224 & -6.9 & -75.6018 & 37.6057 & VA \\
\hline 1081 & -12.3 & -75.5757 & 37.6665 & $\mathrm{VA}$ & 1153 & -4.4 & -75.5891 & 37.6354 & VA & 1225 & -0.7 & -75.598 & 37.6053 & VA \\
\hline 1082 & -12.4 & -75.5759 & 37.666 & VA & 1154 & -4.4 & -75.5893 & 37.6349 & VA & 1226 & -6.4 & -75.602 & 37.6053 & VA \\
\hline 1083 & -12.2 & -75.5761 & 37.6656 & VA & 1155 & -4.5 & -75.5895 & 37.6345 & VA & 1227 & -0.5 & -75.598 & 37.6049 & VA \\
\hline 1084 & -12 & -75.5763 & 37.6652 & VA & 1156 & -4.6 & -75.5897 & 37.634 & VA & 1228 & -5.9 & -75.6022 & 37.6048 & VA \\
\hline 1085 & -11.6 & -75.5764 & 37.6647 & $\mathrm{VA}$ & 1157 & -4.4 & -75.5899 & 37.6336 & $\mathrm{VA}$ & 1229 & -0.4 & -75.5979 & 37.6044 & VA \\
\hline 1086 & -11.2 & -75.5766 & 37.6643 & $\mathrm{VA}$ & 1158 & -4.3 & -75.59 & 37.6332 & VA & 1230 & -5.3 & .75 .6024 & 37.6044 & VA \\
\hline 1087 & -10.6 & -75.5768 & 37.6638 & VA & 1159 & -4.3 & -75.5902 & 37.6327 & $\mathrm{VA}$ & 1231 & -4.8 & -75.6026 & 37.604 & VA \\
\hline 1088 & -10 & -75.577 & 37.6634 & VA & 1160 & -4.2 & -75.5904 & 37.6323 & VA & 1232 & -0.2 & -75.5979 & 37.6039 & VA \\
\hline 1089 & -9.7 & -75.5772 & 37.663 & $\mathrm{VA}$ & 1161 & -4.3 & -75.5908 & 37.6318 & VA & 1233 & -0.2 & -75.5978 & 37.6035 & VA \\
\hline 1090 & -9.4 & -75.5774 & 37.6625 & VA & 1162 & -4.3 & -75.5906 & 37.6318 & VA & 1234 & -4.4 & -75.6028 & 37.6035 & VA \\
\hline 1091 & -9.3 & -75.5776 & 37.6621 & VA & 1163 & -4.6 & -75.591 & 37.6314 & VA & 1235 & -3.9 & -75.603 & 37.6031 & VA \\
\hline 1092 & -9.2 & -75.5778 & 37.6616 & VA & 1164 & -4.3 & -75.5908 & 37.6314 & $v_{A}$ & 1236 & -0.2 & -75.5978 & 37.603 & VA \\
\hline 1093 & -9.1 & -75.578 & 37.6612 & VA & 1165 & -4.7 & -75.5911 & 37.6309 & $\mathrm{VA}_{\mathrm{A}}$ & 1237 & -3.4 & -75.6031 & 37.6026 & VA \\
\hline 1094 & -9 & -75.5782 & 37.6608 & VA & 1166 & -4.9 & -75.5913 & 37.6305 & VA & 1238 & 0 & -75.5977 & 37.6025 & VA \\
\hline 1095 & -8.9 & -75.5784 & 37.6603 & VA & 1167 & -4.9 & -75.5915 & 37.63 & VA & 1239 & -2.9 & -75.6033 & 37.6022 & VA \\
\hline 1096 & -8.7 & -75.5786 & 37.6599 & VA & 1168 & -4.8 & -75.5917 & 37.6296 & VA & 1240 & 0.2 & -75.5977 & 37.6021 & VA \\
\hline 1097 & -8.4 & -75.5787 & 37.6594 & VA & 1169 & -4.8 & -75.5919 & 37.6292 & VA & 1241 & -2.8 & -75.6035 & 37.6017 & VA \\
\hline 1098 & -8 & -75.5789 & 37.659 & $\mathrm{VA}_{\mathrm{A}}$ & 1170 & -4.9 & -75.5921 & 37.6287 & $\mathrm{VA}$ & 1242 & 0.6 & .75 .5976 & 37.6016 & VA \\
\hline 1099 & -7.5 & -75.5791 & 37.6586 & VA & 1171 & -4.9 & -75.5923 & 37.6283 & VA & 1243 & -2.7 & -75.6037 & 37.6013 & VA \\
\hline 1100 & -7 & -75.5793 & 37.6581 & VA & 1172 & -5 & -75.5925 & 37.6278 & $V_{A}$ & 1244 & 0.9 & -75.5976 & 37.6011 & VA \\
\hline 1101 & -6.6 & -75.5795 & 37.6577 & $\mathrm{VA}$ & 1173 & -5 & -75.5926 & 37.6274 & $\mathrm{VA}$ & 1245 & 1.2 & -75.5975 & 37.6007 & VA \\
\hline 1102 & -4.3 & .75 .5797 & 37.6573 & $\mathrm{VA}$ & 1174 & -5.1 & -75.5928 & 37.627 & VA & 1246 & 1.4 & -75.5975 & 37.6002 & VA \\
\hline 1103 & -6.1 & -75.5797 & 37.6572 & VA & 1175 & -5.1 & -75.593 & 37.6265 & VA & 1247 & 1.7 & -75.5974 & 37.5997 & VA \\
\hline 1104 & -4 & -75.5799 & 37.6569 & VA & 1176 & -5.1 & -75.5932 & 37.6261 & VA & 1248 & 1.9 & -75.5974 & 37.5993 & VA \\
\hline 1105 & -3.9 & -75.58 & 37.6564 & VA & 1177 & -5.2 & -75.5934 & 37.6256 & VA & 1249 & 2.1 & -75.5973 & 37.5988 & VA \\
\hline 1106 & -3.9 & -75.5802 & 37.656 & VA & 1178 & -5.2 & -75.5936 & 37.6252 & VA & 1250 & 2.2 & -75.5973 & 37.5983 & VA \\
\hline 1107 & -3.7 & -75.5804 & 37.6556 & VA & 1179 & -5.4 & -75.5938 & 37.6247 & VA & 1251 & 2.5 & .75 .5972 & 37.5979 & VA \\
\hline 1108 & -3.5 & -75.5806 & 37.6551 & VA & 1180 & -5.5 & -75.594 & 37.6243 & $\mathrm{VA}$ & 1252 & 2.7 & -75.5972 & 37.5974 & VA \\
\hline 1109 & -3.5 & -75.5808 & 37.6547 & VA & 1181 & -5.6 & -75.5941 & 37.6239 & VA & 1253 & 3 & -75.5971 & 37.5969 & VA \\
\hline 1110 & -3.5 & -75.581 & 37.6543 & VA & 1182 & -5.7 & -75.5943 & 37.6234 & VA & 1254 & 3.4 & -75.5971 & 37.5965 & VA \\
\hline 1111 & -3.4 & -75.5812 & 37.6538 & VA & 1183 & -5.6 & -75.5945 & 37.623 & VA & 1255 & 3.8 & -75.597 & 37.596 & VA \\
\hline 1112 & -3.4 & -75.5814 & 37.6534 & VA & 1184 & -5.6 & -75.5947 & 37.6225 & VA & 1256 & 4.2 & -75.597 & 37.5955 & VA \\
\hline 1113 & -3.5 & -75.5816 & 37.6529 & $\mathrm{VA}_{\mathrm{A}}$ & 1185 & -5.6 & -75.5949 & 37.6221 & VA & 1257 & 4.5 & -75.5969 & 37.5951 & VA \\
\hline 1114 & -3.6 & -75.5817 & 37.6525 & VA & 1186 & -5.6 & -75.5951 & 37.6216 & vA & 1258 & 4.8 & -75.5969 & 37.5946 & VA \\
\hline 1115 & -3.5 & -75.5819 & 37.6521 & VA & 1187 & -5.7 & -75.5953 & 37.6212 & VA & 1259 & 4.9 & -75.5968 & 37.5941 & VA \\
\hline 1116 & -3.5 & -75.5821 & 37.6516 & VA & 1188 & -5.8 & -75.5955 & 37.6208 & VA & 1260 & 5.1 & -75.5968 & 37.5937 & VA \\
\hline 1117 & -3.6 & -75.5823 & 37.6512 & VA & 1189 & -6.1 & -75.5956 & 37.6203 & VA & 1261 & 5.2 & -75.5967 & 37.5932 & VA \\
\hline 1118 & -3.7 & -75.5825 & 37.6507 & VA & 1190 & -6.3 & -75.5958 & 37.6199 & VA & 1262 & 5.3 & -75.5967 & 37.5927 & VA \\
\hline 1119 & -3.6 & -75.5827 & 37.6503 & VA & 1191 & -6.3 & -75.596 & 37.6194 & $\mathrm{VA}$ & 1263 & 5.7 & & 37.5923 & VA \\
\hline 1120 & -3.5 & -75.5829 & 37.6499 & VA & 1192 & -6.2 & -75.5962 & 37.619 & $\mathrm{VA}$ & 1264 & 6.2 & -75.5966 & 37.5918 & VA \\
\hline 1121 & -3.6 & -75.5831 & 37.6494 & VA & 1193 & -6.1 & -75.5964 & 37.6185 & $\mathrm{VA}$ & 1265 & 6.7 & -75.5965 & 37.5913 & VA \\
\hline 1122 & -3.8 & -75.5833 & 37.649 & VA & 1194 & -6 & -75.5966 & 37.6181 & $\mathrm{VA}$ & 1266 & 7.2 & -75.5965 & 37.5909 & VA \\
\hline 1123 & -3.8 & .75 .5834 & 37.6485 & VA & 1195 & -6.2 & -75.5968 & 37.6177 & VA & 1267 & 0.6 & -75.5964 & 37.5904 & VA \\
\hline 1124 & -3.9 & -75.5836 & 37.6481 & VA & 1196 & -6.4 & -75.597 & 37.6172 & VA & 1268 & 0.3 & -75.5964 & 37.59 & VA \\
\hline
\end{tabular}




\begin{tabular}{|c|c|c|c|c|c|c|c|c|c|c|c|c|c|c|}
\hline TR\# & RATE & LONG & LAT & ST & TR\# & RATE & LONG & LAT & ST & TR\# & RATE & LONG & LAT & ST \\
\hline 1269 & 0.8 & -75.5963 & 37.5895 & VA & 1341 & -6.5 & -75.6032 & 37.5541 & VA & 1413 & -5 & -75.6241 & 37.5259 & VA \\
\hline 1270 & 1.3 & -75.5963 & 37.589 & VA & 1342 & -6.6 & -75.6035 & 37.5537 & VA & 1414 & -5 & -75.6244 & 37.5255 & VA \\
\hline 1271 & 2.3 & -75.5962 & 37.5886 & VA & 1343 & -6.4 & -75.6038 & 37.5533 & VA & 1415 & -5.1 & -75.6247 & 37.5251 & VA \\
\hline 1272 & 3.2 & -75.5962 & 37.5881 & VA & 1344 & -6.2 & -75.6041 & 37.5529 & VA & 1416 & -5.2 & -75.625 & 37.5247 & VA \\
\hline 1273 & 4.8 & -75.5961 & 37.5876 & VA & 1345 & -6.2 & -75.6044 & 37.5525 & VA & 1417 & -5.3 & -75.6253 & 37.5243 & VA \\
\hline 1274 & 6.3 & -75.5961 & 37.5872 & VA & 1346 & -6.2 & -75.6047 & 37.5521 & VA & 1418 & -5.4 & -75.6256 & 37.5239 & VA \\
\hline 1275 & 7.4 & -75.596 & 37.5867 & VA & 1347 & -6.1 & -75.605 & 37.5517 & VA & 1419 & -5.4 & -75.6259 & 37.5235 & VA \\
\hline 1276 & 8.5 & -75.596 & 37.5862 & VA & 1348 & -6.1 & -75.6053 & 37.5513 & VA & 1420 & -5.4 & -75.6262 & 37.5231 & VA \\
\hline 1277 & 8.1 & -75.5959 & 37.5858 & VA & 1349 & -5.8 & -75.6056 & 37.5509 & VA & 1421 & -5.4 & -75.6265 & 37.5227 & VA \\
\hline 1278 & 8.8 & -75.5959 & 37.5853 & VA & 1350 & -5.5 & -75.6059 & 37.5505 & VA & 1422 & -5.4 & -75.6268 & 37.5223 & VA \\
\hline 1279 & 5.9 & -75.5958 & 37.5848 & VA & 1351 & -5.7 & .75 .6062 & 37.5501 & VA & 1423 & -8.2 & -75.6255 & 37.5221 & VA \\
\hline 1280 & 5.8 & -75.5958 & 37.5844 & VA & 1352 & -5.9 & -75.6065 & 37.5497 & VA & 1424 & -5.5 & .75 .6271 & 37.5219 & VA \\
\hline 1281 & 22.2 & .75 .5957 & 37.5839 & VA & 1353 & -6 & -75.6068 & 37.5493 & VA & 1425 & -10 & -75.6258 & 37.5216 & VA \\
\hline 1282 & 22.9 & -75.5957 & 37.5834 & VA & 1354 & -6.8 & -75.6069 & 37.5492 & VA & 1426 & -5.7 & -75.6274 & 37.5215 & VA \\
\hline 1283 & 23.4 & -75.5956 & 37.583 & VA & 1355 & -6.1 & .75 .6071 & 37.5489 & VA & 1427 & -8.3 & .75 .626 & 37.5212 & VA \\
\hline 1284 & 24.1 & -75.5956 & 37.5825 & VA & 1356 & -6.8 & -75.6072 & 37.5488 & VA & 1428 & -6.6 & -75.6263 & 37.5208 & VA \\
\hline 1285 & -4.1 & -75.5951 & 37.5778 & VA & 1357 & -6.9 & -75.6075 & 37.5484 & VA & 1429 & -6.4 & -75.6265 & 37.5204 & VA \\
\hline 1286 & -2.8 & -75.595 & 37.5774 & VA & 1358 & -7 & -75.6078 & 37.548 & VA & 1430 & -6.2 & -75.6268 & 37.52 & VA \\
\hline 1287 & -1.4 & -75.595 & 37.5769 & VA & 1359 & -6.9 & -75.6081 & 37.5476 & VA & 1431 & -6.4 & -75.627 & 37.5195 & VA \\
\hline 1288 & -0.5 & -75.5949 & 37.5764 & VA & 1360 & -6.8 & -75.6084 & 37.5472 & VA & 1432 & -6.6 & -75.6273 & 37.5191 & VA \\
\hline 1289 & 0.4 & -75.5949 & 37.576 & VA & 1361 & -6.7 & -75.6087 & 37.5468 & VA & 1433 & -6.5 & -75.6275 & 37.5187 & VA \\
\hline 1290 & 1.3 & -75.5948 & 37.5755 & VA & 1362 & -6.6 & -75.609 & 37.5464 & VA & 1434 & -6.4 & -75.6278 & 37.5183 & VA \\
\hline 1291 & 2.1 & -75.5948 & 37.575 & VA & 1363 & -6.6 & -75.6093 & 37.546 & VA & 1435 & -6.7 & -75.628 & 37.5179 & VA \\
\hline 1292 & 2.9 & -75.5947 & 37.5746 & VA & 1364 & -6.6 & -75.6096 & 37.5456 & VA & 1436 & -7 & .75 .6283 & 37.5174 & VA \\
\hline 1293 & 3.8 & -75.5947 & 37.5741 & VA & 1365 & -6.8 & -75.6099 & 37.5452 & VA & 1437 & -7.1 & -75.6285 & 37.517 & VA \\
\hline 1294 & 11.8 & -75.5891 & 37.5729 & VA & 1366 & -7 & -75.6102 & 37.5448 & VA & 1438 & -7.3 & -75.6288 & 37.5166 & VA \\
\hline 1295 & 10.5 & -75.5894 & 37.5725 & VA & 1367 & -6.9 & -75.6105 & 37.5444 & VA & 1439 & -7.2 & -75.629 & 37.5162 & VA \\
\hline 1296 & -0.2 & -75.5897 & 37.5721 & VA & 1368 & -6.8 & -75.6108 & 37.544 & VA & 1440 & -7.1 & -75.6293 & 37.5158 & VA \\
\hline 1297 & 2.6 & -75.59 & 37.5717 & VA & 1369 & -7 & -75.6111 & 37.5436 & VA & 1441 & -7.3 & -75.6295 & 37.5153 & VA \\
\hline 1298 & 5.4 & -75.5903 & 37.5713 & VA & 1370 & -7.1 & -75.6114 & 37.5432 & VA & 1442 & -7.5 & -75.6298 & 37.5149 & VA \\
\hline 1299 & 5.9 & -75.5906 & 37.5709 & VA & 1371 & -7.1 & -75.6116 & 37.5428 & VA & 1443 & -7.6 & .75 .63 & 37.5145 & VA \\
\hline 1300 & 6.4 & -75.5909 & 37.5705 & VA & 1372 & -7.1 & -75.6119 & 37.5424 & $V_{A}$ & 1444 & -7.7 & -75.6303 & 37.5141 & VA \\
\hline 1301 & 5.6 & -75.5912 & 37.5701 & VA & 1373 & -7.1 & -75.6122 & 37.542 & VA & 1445 & -7.7 & -75.6305 & 37.5137 & VA \\
\hline 1302 & 4.8 & -75.5915 & 37.5697 & VA & 1374 & -7.1 & -75.6125 & 37.5416 & VA & 1446 & -7.9 & -75.6308 & 37.5132 & VA \\
\hline 1303 & 6.1 & -75.5918 & 37.5693 & VA & 1375 & -7 & -75.6128 & 37.5412 & VA & 1447 & -8 & -75.6311 & 37.5128 & VA \\
\hline 1304 & 6.2 & -75.5921 & 37.5689 & VA & 1376 & -6.8 & -75.6131 & 37.5408 & VA & 1448 & -8.2 & -75.6313 & 37.5124 & VA \\
\hline 1305 & 0 & -75.5924 & 37.5685 & VA & 1377 & -6.7 & -75.6134 & 37.5404 & VA & 1449 & -8.6 & -75.6316 & 37.512 & VA \\
\hline 1306 & -6.2 & -75.5927 & 37.5681 & VA & 1378 & -6.6 & -75.6137 & 37.54 & VA & 1450 & -8.9 & -75.6318 & 37.5116 & VA \\
\hline 1307 & -7 & -75.593 & 37.5677 & VA & 1379 & -6.5 & -75.614 & 37.5396 & VA & 1451 & -8.8 & -75.6321 & 37.5111 & VA \\
\hline 1308 & -7.8 & -75.5933 & 37.5673 & VA & 1380 & -6.4 & -75.6143 & 37.5392 & VA & 1452 & -8.7 & -75.6323 & 37.5107 & VA \\
\hline 1309 & -6.8 & -75.5936 & 37.5669 & VA & 1381 & -6.1 & -75.6146 & 37.5388 & VA & 1453 & -9 & -75.6326 & 37.5103 & VA \\
\hline 1310 & -5.9 & -75.5939 & 37.5665 & VA & 1382 & -5.9 & -75.6149 & 37.5384 & VA & 1454 & -9.3 & -75.6328 & 37.5099 & VA \\
\hline 1311 & -5.5 & -75.5942 & 37.5661 & VA & 1383 & -5.9 & -75.6152 & 37.538 & VA & 1455 & -9.2 & -75.6331 & 37.5095 & VA \\
\hline 1312 & .2 & -75.5945 & 37.5657 & VA & 1384 & -5.9 & -75.6155 & 37.5376 & VA & 1456 & -9.1 & -75.6333 & 37.509 & VA \\
\hline 1313 & -1.8 & -75.5948 & 37.5653 & VA & 1385 & -6 & -75.6158 & 37.5372 & VA & 1457 & -9.1 & -75.6336 & 37.5086 & VA \\
\hline 1314 & -1.5 & -75.5951 & 37.5649 & VA & 1386 & -6.1 & -75.6161 & 37.5368 & VA & 1458 & -9.1 & -75.6338 & 37.5082 & VA \\
\hline 1315 & -1.7 & -75.5954 & 37.5645 & VA & 1387 & -6 & -75.6164 & 37.5364 & VA & 1459 & -9.2 & -75.6341 & 37.5078 & VA \\
\hline 1316 & -1.8 & -75.5957 & 37.5641 & VA & 1388 & -5.9 & -75.6167 & 37.536 & VA & 1460 & -9.3 & -75.6343 & 37.5074 & VA \\
\hline 1317 & -1.9 & -75.596 & 37.5637 & VA & 1389 & -5.7 & -75.617 & 37.5355 & VA & 1461 & -9.6 & -75.6346 & 37.507 & VA \\
\hline 1318 & -2 & -75.5963 & 37.5633 & VA & 1390 & -5.5 & -75.6173 & 37.5351 & $V_{A}$ & 1462 & -9.8 & -75.6348 & 37.5065 & VA \\
\hline 1319 & -2.1 & -75.5966 & 37.5629 & VA & 1391 & -5.5 & -75.6176 & 37.5347 & VA & 1463 & -9.9 & -75.6351 & 37.5061 & VA \\
\hline 1320 & -2.2 & -75.5969 & 37.5625 & VA & 1392 & -5.4 & -75.6179 & 37.5343 & VA & 1464 & -10 & -75.6353 & 37.5057 & VA \\
\hline 1321 & -2.4 & -75.5972 & 37.5621 & VA & 1393 & -5.5 & -75.6182 & 37.5339 & VA & 1465 & -10.1 & -75.6356 & 37.5053 & VA \\
\hline 1322 & -2.6 & .75 .5975 & 37.5617 & VA & 1394 & -5.7 & -75.6185 & 37.5335 & VA & 1466 & -10.2 & -75.6358 & 37.5049 & VA \\
\hline 1323 & -2.9 & -75.5978 & 37.5613 & VA & 1395 & -5.6 & -75.6188 & 37.5331 & VA & 1467 & -10.2 & -75.6361 & 37.5044 & VA \\
\hline 1324 & -3.1 & -75.5981 & 37.5609 & VA & 1396 & -5.5 & .75 .6191 & 37.5327 & VA & 1468 & -10.4 & -75.6363 & 37.504 & VA \\
\hline 1325 & -3.4 & -75.5984 & 37.5605 & VA & 1397 & -5.5 & -75.6194 & 37.5323 & VA & 1469 & -10.5 & -75.6366 & 37.5036 & VA \\
\hline 1326 & -3.7 & -75.5987 & 37.5601 & VA & 1398 & -5.4 & -75.6197 & 37.5319 & VA & 1470 & -10.5 & .75 .6368 & 37.5032 & VA \\
\hline 1327 & .4 & -75.599 & 37.5597 & VA & 1399 & -5.5 & -75.62 & 37.5315 & VA & 1471 & -10.6 & -75.6371 & 37.5028 & VA \\
\hline 1328 & -4.2 & -75.5993 & 37.5593 & VA & 1400 & -5.5 & -75.6203 & 37.5311 & VA & 1472 & -10.7 & -75.6374 & 37.5023 & VA \\
\hline 1329 & -4.3 & -75.5996 & 37.5589 & VA & 1401 & -5.5 & -75.6206 & 37.5307 & VA & 1473 & -11 & -75.6376 & 37.5019 & VA \\
\hline 1330 & -5.2 & -75.5999 & 37.5585 & VA & 1402 & -5.5 & -75.6209 & 37.5303 & VA & 1474 & -11.2 & -75.6379 & 37.5015 & VA \\
\hline 1331 & -5.4 & -75.6002 & 37.5581 & VA & 1403 & -5.4 & -75.6212 & 37.5299 & $\mathrm{VA}$ & 1475 & -11.4 & -75.6381 & 37.5011 & VA \\
\hline 1332 & -5.5 & -75.6005 & 37.5577 & VA & 1404 & -5.4 & -75.6215 & 37.5295 & VA & 1476 & -11.6 & -75.6384 & 37.5007 & VA \\
\hline 1333 & -5.7 & -75.6008 & 37.5573 & VA & 1405 & -5.4 & -75.6218 & 37.5291 & VA & 1477 & -11.7 & -75.6386 & 37.5002 & VA \\
\hline 1334 & -5.9 & -75.6011 & 37.5569 & VA & 1406 & -5.4 & -75.6221 & 37.5287 & $V_{A}$ & 1478 & -11.8 & -75.6389 & 37.4998 & VA \\
\hline 1335 & -6 & -75.6014 & 37.5565 & VA & 1407 & -5.2 & -75.6223 & 37.5283 & VA & 1479 & -11.8 & -75.6391 & 37.4994 & VA \\
\hline 1336 & -6.1 & -75.6017 & 37.5561 & VA & 1408 & -5 & -75.6226 & 37.5279 & VA & 1480 & -11.8 & -75.6394 & 37.499 & VA \\
\hline 1337 & -6.2 & -75.602 & 37.5557 & VA & 1409 & -5 & .75 .6229 & 37.5275 & VA & 1481 & -11.8 & -75.6396 & 37.49 .86 & VA \\
\hline 1338 & -6.2 & -75.6023 & 37.5553 & VA & 1410 & -5 & -75.6232 & 37.5271 & VA & 1482 & -11.8 & -75.6399 & 37.4981 & VA \\
\hline 1339 & -6.3 & -75.6026 & 37.5549 & VA & 1411 & -5 & -75.6235 & 37.5267 & $V_{A}$ & 1483 & -12 & -75.6401 & 37.4977 & VA \\
\hline 1340 & -6.4 & -75.6029 & 37.5545 & VA & 1412 & .5 & -75.6238 & 37.5263 & $\mathrm{VA}$ & 1484 & -12.1 & -75.6404 & 37.4973 & VA \\
\hline
\end{tabular}




\begin{tabular}{|c|c|c|c|c|c|c|c|c|c|c|c|c|c|c|}
\hline TR\# & RATE & LONG & LAT & ST & TR\# & RATE & LONG & LAT & ST & TR\# & RATE & LONG & LAT & ST \\
\hline 1485 & -14.1 & -75.6406 & 37.4969 & $\mathrm{VA}$ & 1557 & -5 & -75.6553 & 37.4662 & $\mathrm{VA}$ & 1629 & 3 & -75.6618 & 37.43 & $\overline{\mathrm{VA}}$ \\
\hline 1486 & -16.1 & -75.6409 & 37.4965 & VA & 1558 & 15.4 & -75.6555 & 37.4657 & $\mathrm{VA}$ & 1630 & 3.7 & -75.6622 & 37.4296 & VA \\
\hline 1487 & -14.2 & -75.6411 & 37.496 & VA & 1559 & 3 & -75.651 & 37.4605 & $\mathrm{VA}$ & 1631 & 4.3 & -75.6626 & 37.4293 & VA \\
\hline 1488 & -12.3 & -75.6414 & 37.4956 & VA & 1560 & 0.6 & -75.6511 & 37.4601 & $\mathrm{VA}$ & 1632 & 4.6 & -75.663 & 37.429 & VA \\
\hline 1489 & -12.4 & -75.6416 & 37.4952 & VA & 1561 & -9 & -75.6512 & 37.4596 & $V_{A}$ & 1633 & 4.9 & .75 .6634 & 37.4286 & VA \\
\hline 1490 & -12.5 & -75.6419 & 37.4948 & VA & 1562 & -6.2 & .75 .6512 & 37.4591 & VA & 1634 & 4.9 & -75.6638 & 37.4283 & VA \\
\hline 1491 & -12.4 & -75.6421 & 37.4944 & VA & 1563 & -2.6 & -75.6513 & 37.4587 & $\mathrm{VA}$ & 1635 & 4.9 & -75.6642 & 37.4279 & VA \\
\hline 1492 & -12.3 & -75.6424 & 37.4939 & VA & 1564 & -2.4 & -75.6514 & 37.4582 & VA & 1636 & 4.2 & -75.6646 & 37.4276 & VA \\
\hline 1493 & -12.5 & -75.6426 & 37.4935 & VA & 1565 & -2.1 & -75.6515 & 37.4578 & $v_{A}$ & 1637 & 3.7 & -75.665 & 37.4272 & VA \\
\hline 1494 & -12.7 & -75.6429 & 37.4931 & VA & 1566 & -1.9 & -75.6516 & 37.4573 & VA & 1638 & 4.1 & -75.6654 & 37.4269 & VA \\
\hline 1495 & -12.6 & -75.6401 & 37.4924 & VA & 1567 & -1.7 & .75 .6517 & 37.4568 & VA & 1639 & 4.5 & -75.6658 & 37.4265 & VA \\
\hline 1496 & -12.9 & -75.6403 & 37.4919 & VA & 1568 & -1.2 & -75.6518 & 37.4564 & VA & 1640 & 4.3 & -75.6662 & 37.4262 & VA \\
\hline 1497 & -13 & -75.6406 & 37.4915 & VA & 1569 & -0.7 & -75.6519 & 37.4559 & VA & 1641 & 4.2 & -75.6666 & 37.4259 & VA \\
\hline 1498 & -13.2 & -75.6408 & 37.4911 & VA & 1570 & -0.3 & -75.652 & 37.4555 & VA & 1642 & 3.9 & -75.667 & 37.4255 & VA \\
\hline 1499 & -13.4 & -75.6411 & 37.4907 & VA & 1571 & 0.2 & -75.6521 & 37.455 & VA & 1643 & 3.7 & -75.6674 & 37.4252 & VA \\
\hline 1500 & -13.6 & -75.6413 & 37.4902 & VA & 1572 & 0.7 & -75.6522 & 37.4545 & $V_{A}$ & 1644 & 3.7 & -75.6677 & 37.4248 & VA \\
\hline 1501 & -13.6 & -75.6416 & 37.4898 & VA & 1573 & 1.2 & -75.6523 & 37.4541 & $\mathrm{VA}$ & 1645 & 3.8 & -75.6681 & 37.4245 & VA \\
\hline 1502 & -13.7 & -75.6418 & 37.4894 & VA & 1574 & 1.4 & -75.6523 & 37.4536 & $V_{A}$ & 1646 & 4.1 & .75 .6685 & 37.4241 & VA \\
\hline 1503 & -13.7 & -75.6421 & 37.489 & VA & 1575 & 1.6 & .75 .6524 & 37.4532 & $V_{A}$ & 1647 & 4.3 & -75.6689 & 37.4238 & VA \\
\hline 1504 & -13.7 & -75.6423 & 37.4886 & VA & 1576 & 1.8 & -75.6525 & 37.4527 & VA & 1648 & 4.9 & -75.6693 & 37.4235 & VA \\
\hline 1505 & -13.9 & -75.6425 & 37.4881 & VA & 1577 & 2.1 & -75.6526 & 37.4522 & $V_{A}$ & 1649 & 5.4 & -75.6697 & 37.4231 & VA \\
\hline 1506 & -14.1 & -75.6428 & 37.4877 & VA & 1578 & 2.5 & -75.6527 & 37.4518 & $v_{A}$ & 1650 & 5.6 & -75.6701 & 37.4228 & VA \\
\hline 1507 & -14.1 & -75.643 & 37.4873 & VA & 1579 & 3 & -75.6528 & 37.4513 & VA & 1651 & 5.7 & -75.6705 & 37.4224 & VA \\
\hline 1508 & -14.1 & -75.6433 & 37.4869 & VA & 1580 & 3.8 & .75 .6529 & 37.4509 & $\mathrm{VA}_{\mathrm{A}}$ & 1652 & 5.9 & -75.6709 & 37.4221 & VA \\
\hline 1509 & -14.3 & -75.6435 & 37.4864 & VA & 1581 & 4.5 & .75 .653 & 37.4504 & $V_{A}$ & 1653 & 6.1 & -75.6713 & 37.4217 & VA \\
\hline 1510 & -14.5 & -75.6438 & 37.486 & VA & 1582 & 5.1 & -75.6531 & 37.45 & VA & 1654 & 6.1 & -75.6717 & 37.4214 & VA \\
\hline 1511 & -14.5 & -75.644 & 37.4856 & VA & 1583 & 5.7 & -75.6532 & 37.4495 & VA & 1655 & 6.1 & -75.6721 & 37.421 & VA \\
\hline 1512 & -14.5 & -75.6443 & 37.4852 & VA & 1584 & 6.8 & -75.6533 & 37.449 & VA & 1656 & 6 & -75.6725 & 37.4207 & VA \\
\hline 1513 & -14.5 & -75.6445 & 37.4847 & VA & 1585 & 7.8 & -75.6534 & 37.4486 & VA & 1657 & 5.9 & -75.6729 & 37.4204 & VA \\
\hline 1514 & -14.5 & -75.6447 & 37.4843 & VA & 1586 & 8.5 & -75.6534 & 37.4481 & VA & 1658 & 5.9 & .75 .6733 & 37.42 & VA \\
\hline 1515 & -14.5 & -75.645 & 37.4839 & VA & 1587 & 9.2 & -75.6535 & 37.4477 & VA & 1659 & 5.9 & -75.6737 & 37.4197 & VA \\
\hline 1516 & -14.5 & -75.6452 & 37.4835 & $\mathrm{VA}$ & 1588 & 10 & .75 .6536 & 37.4472 & VA & 1660 & 5.8 & -75.6741 & 37.4193 & VA \\
\hline 1517 & -14.5 & -75.6455 & 37.4831 & VA & 1589 & 10.8 & -75.6537 & 37.4467 & VA & 1661 & 5.7 & -75.6745 & 37.419 & VA \\
\hline 1518 & -14.6 & -75.6457 & 37.4826 & $\mathrm{VA}$ & 1590 & 11.8 & -75.6538 & 37.4463 & $\mathrm{VA}$ & 1662 & 5.8 & -75.6749 & 37.4186 & $\mathrm{VA}$ \\
\hline 1519 & -14.6 & -75.646 & 37.4822 & $\mathrm{VA}$ & 1591 & 12.9 & -75.6539 & 37.4458 & $\mathrm{VA}$ & 1663 & 5.9 & -75.6753 & 37.4183 & VA \\
\hline 1520 & -14.5 & -75.6462 & 37.4818 & VA & 1592 & 13.7 & -75.654 & 37.4454 & VA & 1664 & 5.9 & -75.6757 & 37.418 & VA \\
\hline 1521 & -14.4 & .75 .6465 & 37.4814 & $\mathrm{VA}$ & 1593 & 14.6 & .75 .6541 & 37.4449 & VA & 1665 & 5.9 & -75.6761 & 37.4176 & VA \\
\hline 1522 & -14.3 & -75.6467 & 37.4809 & VA & 1594 & 15 & -75.6542 & 37.4444 & VA & 1666 & 5.9 & .75 .6765 & 37.4173 & VA \\
\hline 1523 & -14.3 & -75.647 & 37.4805 & VA & 1595 & 15.3 & -75.6543 & 37.444 & $V_{A}$ & 1667 & 5.9 & -75.6769 & 37.4169 & VA \\
\hline 1524 & -14.3 & -75.6472 & 37.4801 & $\mathrm{VA}$ & 1596 & 14.9 & -75.6544 & 37.4435 & $V_{A}$ & 1668 & 5.9 & -75.6772 & 37.4166 & VA \\
\hline 1525 & -14.1 & -75.6474 & 37.4797 & VA & 1597 & 14.4 & -75.6545 & 37.4431 & VA & 1669 & 5.9 & -75.6776 & 37.4162 & VA \\
\hline 1526 & -13.9 & -75.6477 & 37.4793 & VA & 1598 & 13.9 & -75.6545 & 37.4426 & VA & 1670 & 5.9 & -75.678 & 37.4159 & VA \\
\hline 1527 & -13.6 & -75.6479 & 37.4788 & $\mathrm{VA}$ & 1599 & 13.4 & -75.6546 & 37.4421 & $\mathrm{VA}$ & 1671 & 5.9 & -75.6784 & 37.4155 & VA \\
\hline 1528 & -13.4 & -75.6482 & 37.4784 & $\mathrm{VA}$ & 1600 & 12.9 & -75.6547 & 37.4417 & VA & 1672 & 5.9 & -75.6788 & 37.4152 & VA \\
\hline 1529 & -13.4 & -75.6484 & 37.478 & VA & 1601 & 12.3 & -75.6548 & 37.4412 & $\mathrm{VA}_{\mathrm{A}}$ & 1673 & 5.9 & -75.6792 & 37.4149 & VA \\
\hline 1530 & -13.4 & -75.6487 & 37.4776 & VA & 1602 & 12.2 & -75.6549 & 37.4408 & VA & 1674 & 4.2 & -75.6795 & 37.4148 & VA \\
\hline 1531 & -13.1 & -75.6489 & 37.4771 & VA & 1603 & 12 & -75.655 & 37.4403 & $V_{A}$ & 1675 & 5.8 & -75.6796 & 37.4145 & VA \\
\hline 1532 & -12.9 & -75.6492 & 37.4767 & VA & 1604 & 12 & -75.6551 & 37.4399 & VA & 1676 & 4 & -75.6798 & 37.4144 & VA \\
\hline 1533 & -12.7 & -75.6494 & 37.4763 & VA & 1605 & 12 & -75.6552 & 37.4394 & VA & 1677 & 5.7 & -75.68 & 37.4142 & VA \\
\hline 1534 & -12.5 & -75.6496 & 37.4759 & VA & 1606 & 11.6 & -75.6553 & 37.4389 & VA & 1678 & 3.9 & -75.6801 & 37.414 & VA \\
\hline 1535 & -12.5 & -75.6499 & 37.4755 & VA & 1607 & 11.1 & -75.6554 & 37.4385 & VA & 1679 & 3.8 & .75 .6804 & 37.4136 & VA \\
\hline 1536 & -12.5 & -75.6501 & 37.475 & VA & 1608 & 10.1 & -75.6555 & 37.438 & $v_{A}$ & 1680 & 3.8 & -75.6807 & 37.4132 & VA \\
\hline 1537 & -12.5 & -75.6504 & 37.4746 & VA & 1609 & 9 & -75.6556 & 37.4376 & $V_{A}$ & 1681 & 3.7 & -75.681 & 37.4128 & VA \\
\hline 1538 & -12.5 & -75.6506 & 37.4742 & $V_{A}$ & 1610 & 8.2 & -75.6556 & 37.4371 & VA & 1682 & 3.7 & -75.6813 & 37.4124 & VA \\
\hline 1539 & -12.7 & -75.6509 & 37.4738 & VA & 1611 & 7.5 & -75.6557 & 37.4366 & $\mathrm{VA}_{\mathrm{A}}$ & 1683 & 3.7 & -75.6816 & 37.412 & VA \\
\hline 1540 & -13 & -75.6511 & 37.4733 & VA & 1612 & 13.8 & -75.6551 & 37.4358 & VA & 1684 & 3.7 & -75.6819 & 37.4116 & VA \\
\hline 1541 & -13.5 & -75.6514 & 37.4729 & VA & 1613 & 13.4 & -75.6555 & 37.4355 & VA & 1685 & 3.7 & -75.6822 & 37.4112 & VA \\
\hline 1542 & -13.9 & -75.6516 & 37.4725 & $\mathrm{VA}$ & 1614 & 12.8 & .75 .6559 & 37.4351 & $\mathrm{VA}$ & 1686 & 3.5 & -75.6825 & 37.4108 & VA \\
\hline 1543 & -14.4 & -75.6519 & 37.4721 & VA & 1615 & 12.2 & -75.6563 & 37.4348 & VA & 1687 & 3.3 & -75.6828 & 37.4104 & VA \\
\hline 1544 & -14.8 & -75.6521 & 37.4717 & VA & 1616 & 11.3 & -75.6567 & 37.4345 & VA & 1688 & 3 & -75.6832 & 37.4101 & VA \\
\hline 1545 & -15.4 & -75.6523 & 37.4712 & VA & 1617 & 10.4 & -75.6571 & 37.4341 & $v_{A}$ & 1689 & 2.6 & -75.6835 & 37.4097 & VA \\
\hline 1546 & -15.9 & -75.6526 & 37.4708 & $\mathrm{VA}$ & 1618 & 9.5 & -75.6575 & 37.4338 & $\mathrm{VA}_{\mathrm{A}}$ & 1690 & 2.3 & -75.6838 & 37.4093 & VA \\
\hline 1547 & -17.1 & -75.6528 & 37.4704 & $\mathrm{VA}_{\mathrm{A}}$ & 1619 & 8.5 & -75.6579 & 37.4334 & VA & 1691 & 1.9 & -75.6841 & 37.4089 & VA \\
\hline 1548 & -18.4 & -75.6531 & 37.47 & VA & 1620 & 7.6 & -75.6582 & 37.4331 & $\mathrm{VA}$ & 1692 & 1.6 & -75.6844 & 37.4085 & VA \\
\hline 1549 & -19.6 & -75.6533 & 37.4695 & $V_{A}$ & 1621 & 6.8 & -75.6586 & 37.4327 & VA & 1693 & 1.2 & -75.6847 & 37.4081 & VA \\
\hline 1550 & -20.9 & -75.6536 & 37.4691 & VA & 1622 & 5.8 & -75.659 & 37.4324 & $\mathrm{VA}$ & 1694 & 0.8 & -75.685 & 37.4077 & VA \\
\hline 1551 & -7.7 & -75.6538 & 37.4687 & VA & 1623 & 4.9 & -75.6594 & 37.432 & VA & 1695 & 0.3 & -75.6853 & 37.4073 & VA \\
\hline 1552 & -8.3 & -75.6541 & 37.4683 & VA & 1624 & 3.9 & -75.6598 & 37.4317 & $\mathrm{VA}_{\mathrm{A}}$ & 1696 & 0 & -75.6856 & 37.4069 & VA \\
\hline 1553 & -8.5 & -75.6543 & 37.4678 & VA & 1625 & 3 & -75.6602 & 37.4314 & $\mathrm{VA}_{\mathrm{A}}$ & 1697 & -0.3 & -75.6859 & 37.4065 & VA \\
\hline 1554 & -8.7 & -75.6545 & 37.4674 & $\mathrm{VA}$ & 1626 & 2 & -75.6606 & 37.431 & $\mathrm{VA}$ & 1698 & -0.7 & -75.6862 & 37.4061 & VA \\
\hline 1555 & -12.8 & -75.6548 & 37.467 & VA & 1627 & 1 & -75.661 & 37.4307 & $\mathrm{VA}$ & 1699 & -1 & -75.6865 & 37.4057 & VA \\
\hline 1556 & -17 & -75.655 & 37.4666 & $\mathrm{VA}$ & 1628 & 2 & .75 .6614 & 37.4303 & VA & 1700 & -1.4 & -75.6868 & 37.4053 & VA \\
\hline
\end{tabular}




\begin{tabular}{|c|c|c|c|c|c|c|c|c|c|c|c|c|c|c|}
\hline TR\# & RATE & LONG & LAT & ST & TR\# & RATE & LONG & LAT & ST & TR\# & RATE & LONG & LAT & ST \\
\hline 1701 & -1.7 & -75.6871 & 37.4049 & VA & 1773 & 0.1 & -75.7036 & 37.3794 & $\overline{\mathrm{VA}}$ & 1845 & 7.1 & -75.7184 & 37.3436 & $\mathrm{VA}$ \\
\hline 1702 & -2.2 & -75.6874 & 37.4045 & VA & 1774 & 1 & -75.7038 & 37.3789 & VA & 1846 & 5.7 & -75.7186 & 37.3431 & VA \\
\hline 1703 & -2.6 & -75.6877 & 37.4041 & VA & 1775 & 1.9 & -75.704 & 37.3785 & VA & 1847 & 4.3 & -75.7188 & 37.3427 & VA \\
\hline 1704 & -3 & -75.688 & 37.4037 & VA & 1776 & 2.8 & -75.7042 & 37.3781 & VA & 1848 & 4 & -75.719 & 37.3423 & VA \\
\hline 1705 & -3.5 & -75.6883 & 37.4033 & VA & 1777 & 3.2 & -75.7045 & 37.3776 & VA & 1849 & 3.7 & -75.7192 & 37.3418 & VA \\
\hline 1706 & -3.9 & -75.6886 & 37.4029 & VA & 1778 & 3.7 & -75.7047 & 37.3772 & VA & 1850 & 3.2 & -75.7194 & 37.3414 & VA \\
\hline 1707 & -4.3 & -75.6889 & 37.4025 & VA & 1779 & 4.2 & -75.7049 & 37.3768 & VA & 1851 & 2.8 & -75.7196 & 37.341 & VA \\
\hline 1708 & -4.7 & -75.6892 & 37.4021 & VA & 1780 & 4.7 & -75.7051 & 37.3763 & VA & 1852 & 2.4 & -75.7199 & 37.3405 & VA \\
\hline 1709 & -5 & -75.6895 & 37.4017 & VA & 1781 & 5.5 & -75.7053 & 37.3759 & VA & 1853 & 2.1 & -75.7201 & 37.3401 & VA \\
\hline 1710 & -5.2 & -75.6898 & 37.4013 & VA & 1782 & 6.3 & -75.7055 & 37.3755 & $\mathrm{VA}$ & 1854 & 1.7 & .75 .7203 & 37.3397 & VA \\
\hline 1711 & -5.4 & -75.6901 & 37.4009 & VA & 1783 & 6.8 & -75.7058 & 37.375 & VA & 1855 & 1.2 & -75.7205 & 37.3392 & VA \\
\hline 1712 & -5.4 & -75.6904 & 37.4005 & VA & 1784 & 7.3 & -75.706 & 37.3746 & VA & 1856 & -1.6 & -75.7207 & 37.3388 & VA \\
\hline 1713 & -5.4 & -75.6907 & 37.4001 & VA & 1785 & 7.9 & -75.7062 & 37.3742 & VA & 1857 & -2.3 & -75.7209 & 37.3384 & VA \\
\hline 1714 & -5.4 & -75.691 & 37.3997 & VA & 1786 & 8.5 & -75.7064 & 37.3737 & VA & 1858 & -2.7 & -75.7211 & 37.3379 & VA \\
\hline 1715 & -5.4 & -75.6913 & 37.3993 & VA & 1787 & 9.4 & -75.7066 & 37.3733 & VA & 1859 & 9.7 & -75.7213 & 37.3375 & VA \\
\hline 1716 & -5.7 & -75.6916 & 37.3989 & VA & 1788 & 10.3 & -75.7068 & 37.3728 & VA & 1860 & 8.7 & -75.7216 & 37.3371 & VA \\
\hline 1717 & -6.1 & -75.6919 & 37.3985 & VA & 1789 & 3.4 & -75.707 & 37.3724 & VA & 1861 & 7.6 & -75.7218 & 37.3366 & VA \\
\hline 1718 & -6.4 & -75.6922 & 37.3981 & VA & 1790 & 4.6 & -75.7073 & 37.372 & VA & 1862 & 6.8 & -75.722 & 37.3362 & VA \\
\hline 1719 & -6.6 & -75.6925 & 37.3977 & VA & 1791 & 6 & -75.7075 & 37.3715 & VA & 1863 & 5.9 & -75.7222 & 37.3358 & VA \\
\hline 1720 & -6.7 & -75.6928 & 37.3973 & VA & 1792 & 7.5 & -75.7077 & 37.3711 & VA & 1864 & 5.1 & -75.7224 & 37.3353 & VA \\
\hline 1721 & -6.8 & -75.6931 & 37.3969 & VA & 1793 & 10.5 & -75.7079 & 37.3707 & VA & 1865 & 4.3 & -75.7226 & 37.3349 & VA \\
\hline 1722 & -7 & -75.6934 & 37.3965 & VA & 1794 & 13.5 & -75.7081 & 37.3702 & VA & 1866 & 3.5 & -75.7228 & 37.3345 & VA \\
\hline 1723 & -7.3 & -75.6937 & 37.3961 & $\mathrm{VA}$ & 1795 & 11.7 & -75.7083 & 37.3698 & VA & 1867 & 2.8 & -75.723 & 37.334 & $\mathrm{VA}$ \\
\hline 1724 & -7.6 & -75.6941 & 37.3958 & $\mathrm{VA}$ & 1796 & 14.6 & -75.7086 & 37.3694 & VA & 1868 & 2.1 & -75.7233 & 37.3336 & VA \\
\hline 1725 & -8 & .75 .6944 & 37.3954 & VA & 1797 & 5.9 & -75.7088 & 37.3689 & VA & 1869 & -1.4 & -75.7257 & 37.3333 & VA \\
\hline 1726 & -8.1 & -75.6947 & 37.395 & VA & 1798 & 6.1 & -75.709 & 37.3685 & VA & 1870 & 1.4 & -75.7235 & 37.3332 & VA \\
\hline 1727 & -8.2 & -75.695 & 37.3946 & VA & 1799 & 7.2 & -75.7092 & 37.3681 & VA & 1871 & -2.3 & -75.7261 & 37.333 & VA \\
\hline 1728 & -8.3 & -75.6953 & 37.3942 & VA & 1800 & 8.4 & -75.7094 & 37.3676 & VA & 1872 & -2.7 & -75.7265 & 37.3326 & VA \\
\hline 1729 & -8.5 & -75.6956 & 37.3938 & $\mathrm{VA}$ & 1801 & 10.7 & -75.7096 & 37.3672 & VA & 1873 & 2.3 & -75.7269 & 37.3323 & VA \\
\hline 1730 & -8.7 & -75.6959 & 37.3934 & VA & 1802 & 13.1 & -75.7098 & 37.3668 & VA & 1874 & 1.8 & -75.7273 & 37.332 & VA \\
\hline 1731 & -8.9 & -75.6962 & 37.393 & VA & 1803 & 29.9 & -75.7101 & 37.3663 & VA & 1875 & 1.4 & -75.7277 & 37.3316 & VA \\
\hline 1732 & -9.7 & -75.6969 & 37.3929 & VA & 1804 & NA & -75.7103 & 37.3659 & VA & 1876 & 1 & -75.7281 & 37.3313 & VA \\
\hline 1733 & -9 & -75.6965 & 37.3926 & VA & 1805 & $\mathrm{NA}$ & -75.7105 & 37.3654 & VA & 1877 & 0.7 & -75.7285 & 37.3309 & VA \\
\hline 1734 & -9.6 & -75.6971 & 37.3924 & VA & 1806 & NA & -75.7107 & 37.365 & VA & 1878 & 0.3 & -75.7289 & 37.3306 & VA \\
\hline 1735 & -9.2 & -75.6968 & 37.3922 & VA & 1807 & NA & -75.7109 & 37.3646 & VA & 1879 & -0.2 & -75.7293 & 37.3303 & VA \\
\hline 1736 & -9.6 & -75.6973 & 37.392 & VA & 1808 & NA & -75.7111 & 37.3641 & VA & 1880 & -0.5 & -75.7297 & 37.3299 & VA \\
\hline 1737 & -9.5 & -75.6971 & 37.3918 & VA & 1809 & NA & -75.7114 & 37.3637 & VA & 1881 & -0.9 & -75.7301 & 37.3296 & VA \\
\hline 1738 & -9.5 & -75.6976 & 37.3916 & VA & 1810 & $\mathrm{NA}$ & -75.7116 & 37.3633 & VA & 1882 & -1.1 & .75 .7305 & 37.3292 & VA \\
\hline 1739 & -9.7 & -75.6974 & 37.3914 & VA & 1811 & $\mathrm{NA}$ & -75.7118 & 37.3628 & VA & 1883 & -1.4 & -75.7309 & 37.3289 & $V A$ \\
\hline 1740 & -9.4 & -75.6978 & 37.3911 & VA & 1812 & NA & -75.712 & 37.3624 & VA & 1884 & -1.6 & -75.7313 & 37.3286 & VA \\
\hline 1741 & -9.8 & -75.6977 & 37.391 & VA & 1813 & NA & -75.7116 & 37.3575 & VA & 1885 & -1.9 & -75.7317 & 37.3282 & VA \\
\hline 1742 & -9.4 & -75.698 & 37.3907 & VA & 1814 & NA & -75.7118 & 37.357 & VA & 1886 & -2.1 & -75.7321 & 37.3279 & $\mathrm{VA}$ \\
\hline 1743 & -9.9 & -75.698 & 37.3906 & VA & 1815 & $\mathrm{NA}$ & -75.712 & 37.3566 & VA & 1887 & -2.3 & -75.7325 & 37.3276 & VA \\
\hline 1744 & -9.4 & -75.6982 & 37.3903 & VA & 1816 & NA & -75.7122 & 37.3562 & VA & 1888 & -2.5 & -75.7329 & 37.3272 & VA \\
\hline 1745 & -10 & -75.6983 & 37.3902 & VA & 1817 & $\mathrm{NA}$ & -75.7124 & 37.3557 & VA & 1889 & -2.8 & -75.7333 & 37.3269 & VA \\
\hline 1746 & -9.3 & -75.6984 & 37.3898 & VA & 1818 & NA & -75.7126 & 37.3553 & VA & 1890 & -3.1 & -75.7337 & 37.3265 & VA \\
\hline 1747 & -10.1 & -75.6986 & 37.3898 & VA & 1819 & $\mathrm{NA}$ & -75.7128 & 37.3549 & VA & 1891 & -3.3 & -75.7341 & 37.3262 & VA \\
\hline 1748 & -9.2 & -75.6986 & 37.3894 & VA & 1820 & -37.7 & -75.7131 & 37.3544 & VA & 1892 & -3.5 & -75.7345 & 37.3259 & VA \\
\hline 1749 & -10.2 & -75.6989 & 37.3894 & VA & 1821 & -34.9 & -75.7133 & 37.354 & VA & 1893 & -3.7 & -75.7349 & 37.3255 & VA \\
\hline 1750 & -10.3 & -75.6992 & 37.389 & VA & 1822 & -33.5 & -75.7135 & 37.3536 & VA & 1894 & -3.8 & -75.7353 & 37.3252 & VA \\
\hline 1751 & -8.9 & -75.6989 & 37.3889 & VA & 1823 & -32.2 & -75.7137 & 37.3531 & $\mathrm{VA}$ & 1895 & -4 & -75.7357 & 37.3248 & VA \\
\hline 1752 & -8.7 & -75.6991 & 37.3885 & VA & 1824 & .30 .8 & -75.7139 & 37.3527 & VA & 1896 & -4.1 & -75.7361 & 37.3245 & VA \\
\hline 1753 & -8.5 & -75.6993 & 37.3881 & VA & 1825 & -29.4 & -75.7141 & 37.3523 & VA & 1897 & -4.2 & -75.7365 & 37.3242 & VA \\
\hline 1754 & -8.3 & -75.6995 & 37.3876 & VA & 1826 & -27.8 & .75 .7143 & 37.3518 & VA & 1898 & -4.1 & -75.7369 & 37.3238 & VA \\
\hline 1755 & -8.1 & -75.6997 & 37.3872 & VA & 1827 & .26 .3 & .75 .7145 & 37.3514 & VA & 1899 & .4 & -75.7373 & 37.3235 & VA \\
\hline 1756 & -7.8 & -75.6999 & 37.3868 & VA & 1828 & -25.2 & -75.7148 & 37.351 & VA & 1900 & -4.2 & -75.7377 & 37.3231 & VA \\
\hline 1757 & -7.5 & -75.7001 & 37.3863 & VA & 1829 & -24.1 & -75.715 & 37.3505 & VA & 1901 & -4.3 & -75.7381 & 37.3228 & VA \\
\hline 1758 & -7.1 & -75.7004 & 37.3859 & VA & 1830 & -23.3 & -75.7152 & 37.3501 & VA & 1902 & -4.5 & -75.7385 & 37.3225 & VA \\
\hline 1759 & -6.8 & -75.7006 & 37.3855 & VA & 1831 & -18.1 & -75.7154 & 37.3497 & VA & 1903 & -4.7 & -75.7389 & 37.3221 & VA \\
\hline 1760 & -6.4 & -75.7008 & 37.385 & VA & 1832 & -15.6 & -75.7156 & 37.3492 & VA & 1904 & -4.9 & -75.7393 & 37.3218 & VA \\
\hline 1761 & -6 & -75.701 & 37.3846 & VA & 1833 & -13.2 & -75.7158 & 37.3488 & VA & 1905 & -5 & -75.7397 & 37.3214 & VA \\
\hline 1762 & -5.6 & -75.7012 & 37.3842 & VA & 1834 & -11.6 & -75.716 & 37.3484 & VA & 1906 & -5.3 & -75.7401 & 37.3211 & VA \\
\hline 1763 & -5.1 & -75.7014 & 37.3837 & VA & 1835 & -9.9 & .75 .7162 & 37.3479 & VA & 1907 & -5.6 & -75.7405 & 37.3208 & VA \\
\hline 1764 & -4.7 & -75.7017 & 37.3833 & VA & 1836 & -7.5 & -75.7165 & 37.3475 & VA & 1908 & -5.6 & -75.7409 & 37.3204 & VA \\
\hline 1765 & -4.3 & -75.7019 & 37.3829 & VA & 1837 & -5 & -75.7167 & 37.347 & VA & 1909 & -5.6 & -75.7413 & 37.3201 & VA \\
\hline 1766 & -4 & -75.7021 & 37.3824 & VA & 1838 & -1.6 & -75.7169 & 37.3466 & VA & 1910 & -5.5 & -75.7417 & 37.3197 & VA \\
\hline 1767 & -3.7 & -75.7023 & 37.382 & VA & 1839 & 1.9 & -75.7171 & 37.3462 & VA & 1911 & -5.4 & -75.7421 & 37.3194 & VA \\
\hline 1768 & -3.3 & -75.7025 & 37.3815 & VA & 1840 & 3.1 & -75.7173 & 37.3457 & VA & 1912 & -5.5 & -75.7425 & 37.3191 & VA \\
\hline 1769 & -2.8 & -75.7027 & 37.3811 & VA & 1841 & 4.3 & -75.7175 & 37.3453 & VA & 1913 & -5.6 & -75.7429 & 37.3187 & VA \\
\hline 1770 & -2.3 & -75.7029 & 37.3807 & VA & 1842 & 4.9 & -75.7177 & 37.3449 & VA & 1914 & -5.7 & -75.7433 & 37.3184 & VA \\
\hline 1771 & -1.6 & -75.7032 & 37.3802 & VA & 1843 & 5.4 & -75.7179 & 37.3444 & VA & 1915 & -5.9 & -75.7437 & 37.318 & VA \\
\hline 1772 & -0.9 & -75.7034 & 37.3798 & VA & 1844 & 6.3 & -75.7182 & 37.344 & VA & 1916 & -5.9 & -75.7441 & 37.3177 & VA \\
\hline
\end{tabular}

MID-ATLANTIC MAP DATA/USGS/NJ.DE.MD.VA 


\begin{tabular}{|c|c|c|c|c|c|c|c|c|c|c|c|c|c|c|}
\hline TR\# & RATE & LONG & LAT & ST & TR\# & RATE & LONG & LAT & ST & TR\# & RATE & LONG & LAT & ST \\
\hline 1917 & -5.9 & -75.7445 & 37.3174 & VA & 1989 & 54.3 & -75.7796 & 37.2944 & $\overline{\mathrm{VA}}$ & 2061 & -2 & -75.7826 & 37.2686 & $\overline{\mathrm{VA}}$ \\
\hline 1918 & -6 & -75.7449 & 37.317 & VA & 1990 & -6.8 & -75.772 & 37.2942 & VA & 2062 & -1.6 & -75.7827 & 37.2681 & VA \\
\hline 1919 & -6.1 & -75.7453 & 37.3167 & VA & 1991 & 52.2 & -75.7796 & 37.294 & VA & 2063 & -1.1 & -75.7829 & 37.2677 & VA \\
\hline 1920 & -6.2 & -75.7457 & 37.3163 & VA & 1992 & -7.5 & -75.7724 & 37.2939 & VA & 2064 & -0.7 & -75.783 & 37.2672 & VA \\
\hline 1921 & -6.3 & -75.7461 & 37.316 & VA & 1993 & -8.2 & -75.7728 & 37.2936 & VA & 2065 & -0.6 & -75.7831 & 37.2668 & VA \\
\hline 1922 & -6.4 & -75.7465 & 37.3157 & VA & 1994 & 50.1 & -75.7797 & 37.2935 & VA & 2066 & -0.5 & -75.7833 & 37.2663 & VA \\
\hline 1923 & -6.4 & -75.7469 & 37.3153 & VA & 1995 & -18.3 & -75.7732 & 37.2932 & VA & 2067 & -0.6 & -75.7834 & 37.2659 & VA \\
\hline 1924 & -6.3 & -75.7473 & 37.315 & VA & 1996 & 49.1 & -75.7797 & 37.2931 & VA & 2068 & -0.7 & -75.7835 & 37.2654 & VA \\
\hline 1925 & -6.1 & -75.7477 & 37.3147 & VA & 1997 & -28.3 & -75.7736 & 37.2929 & VA & 2069 & -0.9 & -75.7836 & 37.265 & VA \\
\hline 1926 & -6 & -75.7481 & 37.3143 & VA & 1998 & 48.1 & -75.7798 & 37.2926 & VA & 2070 & -1.2 & -75.7838 & 37.2645 & VA \\
\hline 1927 & -5.9 & -75.7485 & 37.314 & VA & 1999 & -28.3 & -75.774 & 37.2925 & VA & 2071 & -1.7 & -75.7839 & 37.2641 & VA \\
\hline 1928 & -5.9 & -75.7489 & 37.3136 & VA & 2000 & -28.3 & -75.7744 & 37.2922 & VA & 2072 & -2.1 & -75.784 & 37.2636 & VA \\
\hline 1929 & -5.9 & -75.7493 & 37.3133 & VA & 2001 & 47.4 & -75.7798 & 37.2921 & VA & 2073 & -2.3 & -75.7842 & 37.2631 & VA \\
\hline 1930 & -6 & -75.7497 & 37.313 & VA & 2002 & -15.8 & -75.7748 & 37.2919 & VA & 2074 & -2.4 & -75.7843 & 37.2627 & VA \\
\hline 1931 & -6.1 & -75.7501 & 37.3126 & VA & 2003 & 43.5 & -75.7799 & 37.2917 & VA & 2075 & -2.9 & -75.7844 & 37.2622 & VA \\
\hline 1932 & -6.1 & -75.7505 & 37.3123 & VA & 2004 & -3.2 & -75.7752 & 37.2915 & VA & 2076 & -3.3 & -75.7845 & 37.2618 & VA \\
\hline 1933 & -6.1 & -75.7509 & 37.3119 & VA & 2005 & 40.8 & -75.7799 & 37.2912 & VA & 2077 & -3.8 & -75.7847 & 37.2613 & VA \\
\hline 1934 & -6.2 & -75.7513 & 37.3116 & VA & 2006 & -2.7 & -75.7756 & 37.2912 & VA & 2078 & -4.3 & -75.7848 & 37.2609 & VA \\
\hline 1935 & -6.3 & -75.7517 & 37.3113 & VA & 2007 & -2.2 & -75.776 & 37.2909 & VA & 2079 & -6.1 & -75.7849 & 37.2604 & VA \\
\hline 1936 & -6.1 & -75.7521 & 37.3109 & VA & 2008 & 38.1 & -75.78 & 37.2907 & VA & 2080 & -7.8 & -75.785 & 37.26 & VA \\
\hline 1937 & -5.9 & -75.7525 & 37.3106 & VA & 2009 & -2.3 & -75.7764 & 37.2905 & VA & 2081 & -6.6 & -75.7852 & 37.2595 & VA \\
\hline 1938 & -5.9 & -75.7529 & 37.3102 & VA & 2010 & 18 & -75.78 & 37.2903 & VA & 2082 & -5.4 & -75.7853 & 37.2591 & VA \\
\hline 1939 & -10.1 & -75.7531 & 37.3101 & VA & 2011 & -2.5 & -75.7768 & 37.2902 & VA & 2083 & -5.8 & -75.7854 & 37.2586 & VA \\
\hline 1940 & -5.9 & -75.7533 & 37.3099 & VA & 2012 & -2.1 & -75.7801 & 37.2898 & VA & 2084 & -6.3 & -75.7856 & 37.2582 & VA \\
\hline 1941 & -10 & -75.7535 & 37.3098 & VA & 2013 & -2.5 & -75.7772 & 37.2898 & VA & 2085 & -7.1 & -75.7857 & 37.2577 & VA \\
\hline 1942 & -9.7 & .75 .7539 & 37.3094 & VA & 2014 & -2.5 & -75.7776 & 37.2895 & VA & 2086 & -7.8 & -75.7858 & 37.2572 & VA \\
\hline 1943 & -9.5 & -75.7543 & 37.3091 & VA & 2015 & -2.7 & -75.7801 & 37.2894 & VA & 2087 & -8.4 & -75.7859 & 37.2568 & VA \\
\hline 1944 & -9.2 & -75.7547 & 37.3088 & VA & 2016 & -3.3 & -75.7802 & 37.2889 & VA & 2088 & -9 & -75.7861 & 37.2563 & VA \\
\hline 1945 & -9 & -75.7551 & 37.3084 & VA & 2017 & -3.4 & -75.7802 & 37.2884 & VA & 2089 & -9 & -75.7862 & 37.2559 & VA \\
\hline 1946 & -8.7 & -75.7555 & 37.3081 & VA & 2018 & -3.5 & -75.7802 & 37.288 & VA & 2090 & -9 & -75.7863 & 37.2554 & VA \\
\hline 1947 & -8.4 & -75.7559 & 37.3078 & VA & 2019 & -3.5 & -75.7803 & 37.2875 & VA & 2091 & -8.9 & -75.7865 & 37.255 & VA \\
\hline 1948 & -8.2 & -75.7563 & 37.3074 & VA & 2020 & -3.5 & -75.7803 & 37.287 & VA & 2092 & -8.9 & -75.7866 & 37.2545 & VA \\
\hline 1949 & -7.9 & -75.7567 & 37.3071 & VA & 2021 & -4 & -75.7804 & 37.2866 & VA & 2093 & -8.8 & -75.7867 & 37.2541 & VA \\
\hline 1950 & -7.6 & -75.7571 & 37.3067 & VA & 2022 & -4.5 & -75.7804 & 37.2861 & VA & 2094 & -8.7 & -75.7868 & 37.2536 & VA \\
\hline 1951 & -7.3 & -75.7575 & 37.3064 & VA & 2023 & -4.6 & -75.7805 & 37.2856 & VA & 2095 & -8.7 & -75.787 & 37.2532 & VA \\
\hline 1952 & -7.7 & -75.7579 & 37.3061 & VA & 2024 & -4.7 & -75.7805 & 37.2852 & VA & 2096 & -8.7 & -75.7871 & 37.2527 & VA \\
\hline 1953 & -8.1 & -75.7583 & 37.3057 & VA & 2025 & -4.8 & -75.7806 & 37.2847 & VA & 2097 & -9.1 & .75 .7872 & 37.2523 & VA \\
\hline 1954 & -8.1 & -75.7587 & 37.3054 & VA & 2026 & -4.9 & -75.7806 & 37.2843 & VA & 2098 & -9.6 & -75.7874 & 37.2518 & VA \\
\hline 1955 & -8.1 & -75.7591 & 37.3051 & VA & 2027 & -4.5 & -75.7807 & 37.2838 & $V_{A}$ & 2099 & -9.9 & -75.7875 & 37.2514 & VA \\
\hline 1956 & -8 & -75.7595 & 37.3047 & VA & 2028 & -4.2 & -75.7807 & 37.2833 & VA & 2100 & -10.3 & -75.7876 & 37.2509 & VA \\
\hline 1957 & -7.9 & -75.7599 & 37.3044 & VA & 2029 & -3.9 & -75.7808 & 37.2829 & VA & 2101 & -10.3 & -75.7877 & 37.2504 & VA \\
\hline 1958 & -7.7 & -75.7603 & 37.304 & VA & 2030 & -3.7 & -75.7808 & 37.2824 & VA & 2102 & -10.3 & -75.7879 & 37.25 & VA \\
\hline 1959 & -7.5 & -75.7607 & 37.3037 & VA & 2031 & -3.6 & -75.7809 & 37.2819 & VA & 2103 & -10.8 & -75.788 & 37.2495 & VA \\
\hline 1960 & -7.7 & -75.7611 & 37.3034 & VA & 2032 & -3.5 & -75.7809 & 37.2815 & VA & 2104 & -11.3 & -75.7881 & 37.2491 & VA \\
\hline 1961 & -7.9 & -75.7615 & 37.303 & VA & 2033 & -3.4 & $\begin{array}{l}-75.7809 \\
\end{array}$ & 37.281 & VA & 2105 & -12.9 & -75.7883 & 37.2486 & VA \\
\hline 1962 & -7.8 & -75.7619 & 37.3027 & VA & 2034 & -3.3 & -75.781 & 37.2805 & VA & 2106 & -14.4 & -75.7884 & 37.2482 & VA \\
\hline 1963 & -7.7 & -75.7623 & 37.3023 & VA & 2035 & -3.2 & -75.781 & 37.2801 & VA & 2107 & -15.8 & -75.7885 & 37.2477 & $\mathrm{VA}$ \\
\hline 1964 & -7.1 & -75.7627 & 37.302 & VA & 2036 & -3.1 & .75 .7811 & 37.2796 & VA & 2108 & -17.2 & -75.7886 & 37.2473 & VA \\
\hline 1965 & -6.5 & -75.7631 & 37.3017 & VA & 2037 & -2.8 & -75.7811 & 37.2792 & VA & 2109 & -17.4 & -75.7888 & 37.2468 & VA \\
\hline 1966 & -6 & -75.7635 & 37.3013 & VA & 2038 & -2.4 & -75.7812 & 37.2787 & VA & 2110 & -17.6 & -75.7889 & 37.2464 & VA \\
\hline 1967 & -5.6 & -75.7639 & 37.301 & VA & 2039 & -2.3 & -75.7812 & 37.2782 & VA & 2111 & -18.4 & -75.7841 & 37.2327 & VA \\
\hline 1968 & -5.2 & -75.7643 & 37.3007 & VA & 2040 & -2.1 & -75.7813 & 37.2778 & VA & 2112 & -19 & -75.7843 & 37.2323 & $\mathrm{VA}$ \\
\hline 1969 & -4.8 & -75.7647 & 37.3003 & VA & 2041 & -2.3 & -75.7813 & 37.2773 & VA & 2113 & -47.8 & -75.7845 & 37.2319 & VA \\
\hline 1970 & -4.7 & -75.7651 & 37.3 & VA & 2042 & -2.4 & -75.7814 & 37.2768 & VA & 2114 & -46.8 & -75.7846 & 37.2314 & VA \\
\hline 1971 & -4.7 & -75.7655 & 37.2996 & VA & 2043 & -2.4 & -75.7814 & 37.2764 & VA & 2115 & -45.7 & -75.7848 & 37.231 & VA \\
\hline 1972 & -4.6 & -75.7659 & 37.2993 & VA & 2044 & -2.3 & -75.7815 & 37.2759 & VA & 2116 & -44.7 & -75.785 & 37.2305 & VA \\
\hline 1973 & -4.5 & -75.7663 & 37.299 & VA & 2045 & -2.5 & -75.7815 & 37.2755 & VA & 2117 & -43.6 & -75.7852 & 37.2301 & VA \\
\hline 1974 & -4.4 & -75.7668 & 37.2986 & VA & 2046 & -2.8 & -75.7815 & 37.275 & VA & 2118 & -42.2 & -75.7854 & 37.2296 & VA \\
\hline 1975 & -4.3 & -75.7672 & 37.2983 & VA & 2047 & -3 & -75.7816 & 37.2745 & VA & 2119 & -40.7 & -75.7856 & 37.2292 & VA \\
\hline 1976 & -3.9 & -75.7676 & 37.298 & VA & 2048 & -3.1 & -75.7816 & 37.2741 & VA & 2120 & -40.2 & -75.7858 & 37.2288 & VA \\
\hline 1977 & -3.6 & -75.768 & 37.2976 & VA & 2049 & -3 & -75.7817 & 37.2736 & VA & 2121 & -39.8 & -75.786 & 37.2283 & VA \\
\hline 1978 & -3.4 & -75.7684 & 37.2973 & VA & 2050 & -2.8 & -75.7817 & 37.2731 & VA & 2122 & -38.9 & -75.7861 & 37.2279 & VA \\
\hline 1979 & -3.2 & -75.7688 & 37.2969 & VA & 2051 & -2.9 & -75.7818 & 37.2727 & VA & 2123 & -37.9 & -75.7863 & 37.2274 & VA \\
\hline 1980 & -3.3 & -75.7692 & 37.2966 & VA & 2052 & -3 & -75.7818 & 37.2722 & VA & 2124 & -37.6 & -75.7865 & 37.227 & VA \\
\hline 1981 & -3.4 & -75.7696 & 37.2963 & VA & 2053 & -2.5 & -75.7819 & 37.2717 & VA & 2125 & -37.2 & -75.7867 & 37.2266 & VA \\
\hline 1982 & -3.9 & -75.77 & 37.2959 & VA & 2054 & -2.1 & -75.7819 & 37.2713 & VA & 2126 & -36.9 & -75.7869 & 37.2261 & VA \\
\hline 1983 & -4.5 & -75.7704 & 37.2956 & VA & 2055 & -1.7 & -75.782 & 37.2708 & VA & 2127 & -36.7 & -75.7871 & 37.2257 & VA \\
\hline 1984 & NA & -75.7795 & 37.2954 & VA & 2056 & -1.4 & -75.782 & 37.2704 & VA & 2128 & -36.2 & -75.7873 & 37.2252 & VA \\
\hline 1985 & -5 & -75.7708 & 37.2953 & VA & 2057 & -1 & -75.7821 & 37.2699 & VA & 2129 & -35.6 & -75.7875 & 37.2248 & VA \\
\hline 1986 & $\mathrm{NA}$ & -75.7796 & 37.2949 & VA & 2058 & -2.7 & -75.7824 & 37.2695 & VA & 2130 & -34.9 & -75.7876 & 37.2244 & VA \\
\hline 1987 & -5.6 & -75.7712 & 37.2949 & VA & 2059 & -0.7 & -75.7821 & 37.2694 & VA & 2131 & -34.2 & -75.7878 & 37.2239 & VA \\
\hline 1988 & -6.2 & -75.7716 & 37.2946 & VA & 2060 & -2.4 & -75.7825 & 37.269 & VA & 2132 & -33.5 & -75.788 & 37.2235 & VA \\
\hline
\end{tabular}

MID-ATLANTIC MAP DATA/USGS/NJ.DE.MD.VA 


\begin{tabular}{|c|c|c|c|c|c|c|c|c|c|c|c|c|c|c|}
\hline TR\# & RATE & LONG & LAT & ST & TR\# & RATE & LONG & LAT & ST & TR\# & RATE & LONG & LAT & ST \\
\hline 2133 & -32.7 & -75.7882 & 37.223 & VA & 2205 & -5.2 & -75.8011 & 37.1911 & VA & 2277 & -8.2 & .75 .8317 & 37.1655 & VA \\
\hline 2134 & -32 & -75.7884 & 37.2226 & $\mathrm{VA}$ & 2206 & -5.2 & -75.8014 & 37.1906 & $\mathrm{VA}$ & 2278 & -8.2 & -75.8321 & 37.1652 & VA \\
\hline 2135 & -31.3 & -75.7886 & 37.2222 & VA & 2207 & -5.2 & -75.8017 & 37.1902 & VA & 2279 & -8.3 & -75.8325 & 37.1649 & VA \\
\hline 2136 & -30.8 & -75.7888 & 37.2217 & $\mathrm{VA}$ & 2208 & -5.3 & -75.8019 & 37.1898 & $\mathrm{VA}$ & 2280 & -8.3 & -75.8329 & 37.1646 & $\mathrm{VA}$ \\
\hline 2137 & -30.3 & -75.789 & 37.2213 & $\mathrm{VA}$ & 2209 & -5.4 & -75.8022 & 37.1894 & $\mathrm{VA}$ & 2281 & -8.3 & -75.8334 & 37.1643 & VA \\
\hline 2138 & -30.3 & -75.7891 & 37.2208 & VA & 2210 & -5.4 & -75.8025 & 37.189 & VA & 2282 & -8.5 & -75.8338 & 37.164 & VA \\
\hline 2139 & -30.3 & -75.7893 & 37.2204 & VA & 2211 & -5.4 & -75.8027 & 37.1886 & VA & 2283 & -8.7 & -75.8342 & 37.1636 & VA \\
\hline 2140 & -26.4 & -75.7895 & 37.2199 & VA & 2212 & -5.6 & -75.803 & 37.1882 & VA & 2284 & .8 .9 & .75 .8347 & 37.1633 & VA \\
\hline 2141 & -22.4 & -75.7897 & 37.2195 & VA & 2213 & -5.7 & -75.8033 & 37.1878 & VA & 2285 & -9 & -75.8351 & 37.163 & VA \\
\hline 2142 & -21.1 & -75.7899 & 37.2191 & $\mathrm{VA}$ & 2214 & -5.8 & -75.8036 & 37.1873 & $\mathrm{VA}$ & 2286 & -9 & -75.8355 & 37.1627 & VA \\
\hline 2143 & -19.8 & -75.7901 & 37.2186 & $\mathrm{VA}$ & 2215 & -5.9 & -75.8038 & 37.1869 & $\mathrm{VA}$ & 2287 & -9 & -75.8359 & 37.1624 & VA \\
\hline 2144 & -18.8 & -75.7903 & 37.2182 & $\mathrm{VA}$ & 2216 & -6.4 & -75.8041 & 37.1865 & $\mathrm{VA}$ & 2288 & -8.9 & .75 .8364 & 37.1621 & VA \\
\hline 2145 & -17.7 & -75.7905 & 37.2177 & VA & 2217 & -6.8 & -75.8044 & 37.1861 & VA & 2289 & -8.7 & .75 .8368 & 37.1618 & VA \\
\hline 2146 & -16.7 & -75.7906 & 37.2173 & VA & 2218 & -6.8 & -75.8046 & 37.1857 & VA & 2290 & -9 & -75.8372 & 37.1614 & VA \\
\hline 2147 & -15.6 & -75.7908 & 37.2169 & VA & 2219 & -6.8 & -75.8049 & 37.1853 & VA & 2291 & -9.4 & -75.8376 & 37.1611 & VA \\
\hline 2148 & -14.8 & -75.791 & 37.2164 & VA & 2220 & -7 & -75.8052 & 37.1849 & VA & 2292 & -9.7 & -75.8381 & 37.1608 & VA \\
\hline 2149 & -13.9 & -75.7912 & 37.216 & $\mathrm{VA}$ & 2221 & -7.1 & -75.8055 & 37.1845 & VA & 2293 & -10.1 & -75.8385 & 37.1605 & VA \\
\hline 2150 & -13.2 & -75.7914 & 37.2155 & $\mathrm{VA}$ & 2222 & -7.1 & -75.8057 & 37.184 & $\mathrm{VA}$ & 2294 & -10.5 & .75 .8389 & 37.1602 & VA \\
\hline 2151 & -12.5 & -75.7916 & 37.2151 & VA & 2223 & -7 & -75.806 & 37.1836 & VA & 2295 & -11 & -75.8394 & 37.1599 & VA \\
\hline 2152 & -12.1 & -75.7918 & 37.2147 & VA & 2224 & -6.8 & -75.8063 & 37.1832 & VA & 2296 & -11.6 & -75.8398 & 37.1596 & VA \\
\hline 2153 & -11.6 & .75 .792 & 37.2142 & VA & 2225 & -6.6 & -75.8066 & 37.1828 & VA & 2297 & -12.2 & -75.8402 & 37.1592 & VA \\
\hline 2154 & -11.3 & -75.7921 & 37.2138 & VA & 2226 & -6.5 & -75.8068 & 37.1824 & VA & $\therefore 98$ & -12.3 & -75.8406 & 37.1589 & VA \\
\hline 2155 & -11 & -75.7923 & 37.2133 & VA & 2227 & -6.4 & -75.8071 & 37.182 & VA & 2299 & -12.5 & -75.8411 & 37.1586 & $\mathrm{VA}$ \\
\hline 2156 & -10.3 & -75.7925 & 37.2129 & VA & 2228 & -6.3 & -75.8074 & 37.1816 & VA & 2300 & -12.6 & -75.8415 & 37.1583 & VA \\
\hline 2157 & -9.7 & -75.7927 & 37.2125 & VA & 2229 & -6.1 & -75.8076 & 37.1812 & VA & 2301 & -12.7 & -75.8419 & 37.158 & VA \\
\hline 2158 & -9 & -75.7929 & 37.212 & VA & 2230 & -5.7 & -75.8079 & 37.1807 & VA & 2302 & -12.6 & -75.8424 & 37.1577 & VA \\
\hline 2159 & -8.2 & -75.7931 & 37.2116 & VA & 2231 & -5.4 & -75.8082 & 37.1803 & VA & 2303 & -11.8 & .75 .8427 & 37.1575 & VA \\
\hline 2160 & -7.2 & -75.7933 & 37.2111 & VA & 2232 & -5.3 & -75.8085 & 37.1799 & VA & 2304 & -12.5 & -75.8428 & 37.1574 & VA \\
\hline 2161 & -6.3 & -75.7935 & 37.2107 & VA & 2233 & -5.2 & -75.8087 & 37.1795 & VA & 2305 & -12 & -75.8431 & 37.1572 & VA \\
\hline 2162 & 0.4 & -75.7936 & 37.2102 & VA & 2234 & -5.5 & -75.809 & 37.1791 & VA & 2306 & -12 & -75.8435 & 37.1569 & VA \\
\hline 2163 & 7.1 & -75.7938 & 37.2098 & $\mathrm{VA}$ & 2235 & -5.7 & -75.8093 & 37.1787 & VA & 2307 & -12 & -75.844 & 37.1566 & VA \\
\hline 2164 & 3.7 & -75.794 & 37.2094 & $\mathrm{VA}$ & 2236 & -5.8 & .75 .8095 & 37.1783 & VA & 2308 & -12.3 & .75 .8444 & 37.1563 & VA \\
\hline 2165 & 0.2 & -75.7942 & 37.2089 & $\mathrm{VA}$ & 2237 & -5.9 & .75 .8098 & 37.1779 & VA & 2309 & -12.7 & -75.8448 & 37.1559 & VA \\
\hline 2166 & -0.6 & -75.7944 & 37.2085 & VA & 2238 & -5.5 & -75.8101 & 37.1774 & VA & 2310 & -13 & -75.8452 & 37.1556 & VA \\
\hline 2167 & -1.7 & -75.7946 & 37.208 & VA & 2239 & 2.4 & -75.8158 & 37.1771 & VA & 2311 & -13.2 & -75.8457 & 37.1553 & VA \\
\hline 2168 & -1.8 & -75.7948 & 37.2076 & $\mathrm{VA}$ & 2240 & -5 & -75.8104 & 37.177 & VA & 2312 & -13.3 & .75 .8461 & 37.155 & VA \\
\hline 2169 & -1.9 & -75.795 & 37.2072 & $\mathrm{VA}$ & 2241 & 2.8 & -75.8162 & 37.1768 & VA & 2313 & -13.4 & -75.8465 & 37.1547 & VA \\
\hline 2170 & -1.8 & -75.7951 & 37.2067 & VA & 2242 & 2.7 & -75.8167 & 37.1765 & VA & 2314 & -13.6 & -75.847 & 37.1544 & VA \\
\hline 2171 & -1.7 & -75.7953 & 37.2063 & VA & 2243 & 2.3 & -75.8171 & 37.1762 & VA & 2315 & -13.7 & -75.8474 & 37.1541 & VA \\
\hline 2172 & -2.2 & -75.7955 & 37.2058 & VA & 2244 & -16.4 & -75.8175 & 37.1759 & VA & 2316 & -13.9 & -75.8478 & 37.1537 & VA \\
\hline 2173 & -2.6 & -75.7957 & 37.2054 & VA & 2245 & -32.4 & -75.818 & 37.1756 & VA & 2317 & -14.1 & -75.8482 & 37.1534 & VA \\
\hline 2174 & 8.1 & -75.7927 & 37.2038 & VA & 2246 & -49.6 & -75.8184 & 37.1753 & $\mathrm{VA}$ & 2318 & -14.7 & -75.8487 & 37.1531 & VA \\
\hline 2175 & 15.3 & -75.793 & 37.2034 & VA & 2247 & -45.1 & -75.8188 & 37.1749 & VA & 2319 & -15.3 & .75 .8491 & 37.1528 & $\mathrm{VA}$ \\
\hline 2176 & 16.2 & -75.7932 & 37.203 & VA & 2248 & -27 & -75.8192 & 37.1746 & VA & 2320 & -15.8 & -75.8495 & 37.1525 & VA \\
\hline 2177 & 12.2 & -75.7935 & 37.2026 & VA & 2249 & -5.6 & -75.8197 & 37.1743 & VA & 2321 & -16.3 & -75.8499 & 37.1522 & VA \\
\hline 2178 & 11.5 & -75.7938 & 37.2022 & VA & 2250 & -4.9 & .75 .8201 & 37.174 & VA & 2322 & -16.5 & .75 .8504 & 37.1519 & VA \\
\hline 2179 & 10.8 & -75.794 & 37.2018 & VA & 2251 & -4.3 & -75.8205 & 37.1737 & VA & 2323 & -16.7 & -75.8508 & 37.1515 & VA \\
\hline 2180 & 10.3 & -75.7943 & 37.2014 & VA & 2252 & -5.6 & -75.821 & 37.1734 & VA & 2324 & -16.5 & .75 .8512 & 37.1512 & VA \\
\hline 2181 & 9.7 & -75.7946 & 37.201 & VA & 2253 & -6.8 & -75.8214 & 37.1731 & VA & 2325 & -16.3 & .75 .8517 & 37.1509 & VA \\
\hline 2182 & 9.3 & -75.7949 & 37.2005 & VA & 2254 & -7.8 & -75.8218 & 37.1727 & VA & 2326 & -16.2 & -75.8521 & 37.1506 & VA \\
\hline 2183 & 8.9 & -75.7951 & 37.2001 & $\mathrm{VA}$ & 2255 & -8.7 & .75 .8222 & 37.1724 & $\mathrm{VA}$ & 2327 & -16.2 & .75 .8525 & 37.1503 & VA \\
\hline 2184 & 7.7 & -75.7954 & 37.1997 & $\mathrm{VA}$ & 2256 & -8.6 & -75.8227 & 37.1721 & VA & 2328 & -15.8 & .75 .8529 & 37.15 & VA \\
\hline 2185 & 6.6 & -75.7957 & 37.1993 & $\mathrm{VA}$ & 2257 & -8.5 & -75.8231 & 37.1718 & $\mathrm{VA}$ & 2329 & -15.5 & .75 .8534 & 37.1497 & VA \\
\hline 2186 & 5 & -75.7959 & 37.1989 & VA & 2258 & -8.3 & -75.8235 & 37.1715 & VA & 2330 & -14.6 & -75.8538 & 37.1493 & VA \\
\hline 2187 & 3.5 & -75.7962 & 37.1985 & VA & 2259 & -8.2 & -75.8239 & 37.1712 & VA & 2331 & -13.7 & -75.8542 & 37.149 & VA \\
\hline 2188 & 2.4 & -75.7965 & 37.1981 & VA & 2260 & -8.3 & -75.8244 & 37.1709 & VA & 2332 & -13.6 & -75.8547 & 37.1487 & VA \\
\hline 2189 & 1.4 & -75.7968 & 37.1977 & VA & 2261 & -8.5 & -75.8248 & 37.1705 & VA & 2333 & -13.4 & -75.8551 & 37.1484 & VA \\
\hline 2190 & 0.2 & -75.797 & 37.1972 & $\mathrm{VA}$ & 2262 & -8.4 & -75.8252 & 37.1702 & VA & 2334 & -12.7 & -75.8555 & 37.1481 & VA \\
\hline 2191 & -1 & -75.7973 & 37.1968 & VA & 2263 & -8.3 & -75.8257 & 37.1699 & VA & 2335 & -12 & -75.8559 & 37.1478 & VA \\
\hline 2192 & -1.1 & -75.7976 & 37.1964 & VA & 2264 & -8.2 & -75.8261 & 37.1696 & VA & 2336 & -11.5 & -75.8564 & 37.1475 & VA \\
\hline 2193 & -1.2 & -75.7979 & 37.196 & VA & 2265 & -8 & -75.8265 & 37.1693 & VA & 2337 & -11 & -75.8568 & 37.1472 & VA \\
\hline 2194 & -1.7 & -75.7981 & 37.1956 & VA & 2266 & -7.6 & -75.8269 & 37.169 & VA & 2338 & -8.9 & -75.8572 & 37.1468 & VA \\
\hline 2195 & -2.3 & -75.7984 & 37.1952 & VA & 2267 & -7.3 & -75.8274 & 37.1687 & VA & 2339 & -7 & -75.8577 & 37.1465 & VA \\
\hline 2196 & -2.1 & -75.7987 & 37.1948 & VA & 2268 & .7 .3 & -75.8278 & 37.1683 & VA & 2340 & -12.2 & -75.8581 & 37.1462 & VA \\
\hline 2197 & -1.9 & .75 .7989 & 37.1944 & VA & 2269 & -7.3 & -75.8282 & 37.168 & VA & 2341 & -11.9 & -75.8585 & 37.1459 & VA \\
\hline 2198 & -2.2 & -75.7992 & 37.1939 & VA & 2270 & -8 & -75.8287 & 37.1677 & VA & 2342 & -11.8 & .75 .8589 & 37.1456 & VA \\
\hline 2199 & -2.4 & -75.7995 & 37.1935 & $\mathrm{VA}$ & 2271 & -8.7 & -75.8291 & 37.1674 & VA & 2343 & -0.9 & -75.8594 & 37.1453 & VA \\
\hline 2200 & -3.6 & -75.7998 & 37.1931 & VA & 2272 & -8.5 & -75.8295 & 37.1671 & VA & 2344 & -4.6 & -75.8598 & 37.145 & VA \\
\hline 2201 & -4.7 & -75.8 & 37.1927 & $\mathrm{VA}$ & 2273 & -8.3 & -75.8299 & 37.1668 & VA & 2345 & -8.3 & -75.8602 & 37.1446 & VA \\
\hline 2202 & -4.9 & -75.8003 & 37.1923 & $\mathrm{VA}$ & 2274 & -8.2 & .75 .8304 & 37.1665 & VA & 2346 & -9 & -75.8607 & 37.1443 & VA \\
\hline 2203 & .5 & -75.8006 & 37.1919 & $\mathrm{VA}$ & 2275 & -8 & -75.8308 & 37.1662 & VA & 2347 & -9.7 & -75.8611 & 37.144 & VA \\
\hline 2204 & -5.1 & -75.8008 & 37.1915 & $\mathrm{VA}$ & 2276 & -8.1 & -75.8312 & 37.1658 & VA & 2348 & -9.6 & -75.8615 & 37.1437 & VA \\
\hline
\end{tabular}




\begin{tabular}{|c|c|c|c|c|c|c|c|c|c|c|c|c|c|c|}
\hline TR\# & RATE & LONG & LAT & ST & TR\# & RATE & LONG & LAT & ST & TR\# & RATE & LONG & LAT & ST \\
\hline 2349 & -9.4 & -75.8619 & 37.1434 & $\overline{\mathrm{VA}}$ & 2421 & -1.9 & -75.89 & 37.1199 & VA & 2493 & -3.6 & -75.9376 & 37.0879 & VA \\
\hline 2350 & -9.2 & -75.8624 & 37.1431 & VA & 2422 & -1.7 & -75.8904 & 37.1195 & VA & 2494 & -2.1 & -75.938 & 37.0876 & VA \\
\hline 2351 & -9 & -75.8628 & 37.1428 & VA & 2423 & -1.6 & -75.8908 & 37.1192 & VA & 2495 & -1.8 & -75.9385 & 37.0873 & VA \\
\hline 2352 & -8.6 & -75.8632 & 37.1424 & VA & 2424 & -0.7 & -75.8912 & 37.1188 & VA & 2496 & -1.6 & -75.9389 & 37.0871 & VA \\
\hline 2353 & -8.2 & -75.8636 & 37.1421 & VA & 2425 & 0.2 & -75.8916 & 37.1185 & $\mathrm{VA}$ & 2497 & -2.3 & -75.9394 & 37.0868 & VA \\
\hline 2354 & -7.6 & -75.8641 & 37.1418 & $\mathrm{VA}$ & 2426 & 0.7 & -75.892 & 37.1181 & VA & 2498 & -3 & -75.9399 & 37.0865 & VA \\
\hline 2355 & -7.1 & -75.8645 & 37.1415 & VA & 2427 & 1.2 & -75.8924 & 37.1178 & VA & 2499 & -3.9 & -75.9403 & 37.0862 & VA \\
\hline 2356 & -6.6 & -75.8649 & 37.1412 & VA & 2428 & 1.5 & -75.8928 & 37.1174 & VA & 2500 & 4.2 & -75.9856 & 37.0859 & VA \\
\hline 2357 & -6.1 & -75.8654 & 37.1409 & VA & 2429 & 1.7 & -75.8931 & 37.1171 & VA & 2501 & -4.7 & -75.9408 & 37.0859 & VA \\
\hline 2358 & -5.8 & -75.8658 & 37.1406 & VA & 2430 & 2.4 & -75.8935 & 37.1167 & VA & 2502 & 6.3 & -75.985 & 37.0858 & VA \\
\hline 2359 & -5.6 & -75.8662 & 37.1402 & VA & 2431 & 3.1 & -75.8939 & 37.1164 & VA & 2503 & 1.8 & .75 .9845 & 37.0857 & VA \\
\hline 2360 & -5.9 & -75.8666 & 37.1399 & VA & 2432 & 2.4 & -75.8938 & 37.1155 & VA & 2504 & -5 & -75.9412 & 37.0857 & VA \\
\hline 2361 & -6.3 & -75.8671 & 37.1396 & VA & 2433 & 3 & -75.8944 & 37.1154 & VA & 2505 & 3.6 & -75.9839 & 37.0856 & VA \\
\hline 2362 & -5.5 & -75.8675 & 37.1393 & VA & 2434 & 3 & -75.8949 & 37.1152 & VA & 2506 & 5.4 & -75.9833 & 37.0855 & VA \\
\hline 2363 & -4.7 & -75.8679 & 37.139 & VA & 2435 & 3.1 & -75.8954 & 37.115 & VA & 2507 & 6.7 & -75.9827 & 37.0854 & VA \\
\hline 2364 & -7.4 & -75.8687 & 37.1389 & VA & 2436 & 3 & -75.896 & 37.1148 & VA & 2508 & -5.3 & -75.9417 & 37.0854 & VA \\
\hline 2365 & -4.2 & -75.8684 & 37.1387 & VA & 2437 & 2.8 & -75.8965 & 37.1146 & VA & 2509 & 7.9 & -75.9822 & 37.0853 & VA \\
\hline 2366 & -7.3 & -75.869 & 37.1386 & VA & 2438 & 2.5 & -75.897 & 37.1144 & VA & 2510 & 9.1 & -75.9816 & 37.0852 & VA \\
\hline 2367 & -3.8 & -75.8688 & 37.1384 & VA & 2439 & 2.3 & -75.8976 & 37.1142 & VA & 2511 & 10.4 & -75.981 & 37.0851 & VA \\
\hline 2368 & -6.9 & -75.8694 & 37.1382 & VA & 2440 & 2.1 & -75.8981 & 37.114 & VA & 2512 & -5.7 & -75.9422 & 37.0851 & VA \\
\hline 2369 & -6.4 & -75.8698 & 37.1379 & VA & 2441 & 1.9 & -75.8986 & 37.1138 & VA & 2513 & 11.3 & -75.9804 & 37.085 & VA \\
\hline 2370 & -6.1 & -75.8702 & 37.1375 & VA & 2442 & 2 & -75.8992 & 37.1137 & VA & 2514 & 12.3 & -75.9799 & 37.0849 & VA \\
\hline 2371 & -5.7 & -75.8706 & 37.1372 & VA & 2443 & 2.1 & -75.8997 & 37.1135 & VA & 2515 & 14.6 & -75.9787 & 37.0848 & VA \\
\hline 2372 & -5.4 & -75.871 & 37.1368 & VA & 2444 & 2.1 & -75.9002 & 37.1133 & VA & 2516 & 13.4 & -75.9793 & 37.0848 & VA \\
\hline 2373 & -5 & -75.8714 & 37.1365 & VA & 2445 & 2.1 & -75.9008 & 37.1131 & VA & 2517 & -6.1 & -75.9426 & 37.0848 & VA \\
\hline 2374 & -4.8 & -75.8718 & 37.1361 & VA & 2446 & 2.2 & -75.9013 & 37.1129 & VA & 2518 & 15.6 & -75.9781 & 37.0847 & VA \\
\hline 2375 & -4.5 & -75.8722 & 37.1358 & VA & 2447 & 2.3 & -75.9018 & 37.1127 & VA & 2519 & 16.7 & -75.9776 & 37.0846 & VA \\
\hline 2376 & -4.2 & -75.8725 & 37.1354 & VA & 2448 & 2.3 & -75.9023 & 37.1125 & VA & 2520 & 16.9 & -75.977 & 37.0845 & VA \\
\hline 2377 & -4 & -75.8729 & 37.1351 & VA & 2449 & 2.4 & -75.9029 & 37.1123 & VA & 2521 & -6.4 & .75 .9431 & 37.0845 & VA \\
\hline 2378 & -3.7 & -75.8733 & 37.1348 & VA & 2450 & 2.4 & -75.9034 & 37.1121 & $\mathrm{VA}$ & 2522 & 17.2 & -75.9764 & 37.0844 & VA \\
\hline 2379 & -3.5 & -75.8737 & 37.1344 & VA & 2451 & 2.4 & -75.9039 & 37.112 & VA & 2523 & 16 & -75.9759 & 37.0843 & VA \\
\hline 2380 & -3 & -75.8741 & 37.1341 & VA & 2452 & 2.2 & -75.9045 & 37.1118 & VA & 2524 & 14.7 & -75.9753 & 37.0842 & VA \\
\hline 2381 & -2.6 & -75.8745 & 37.1337 & VA & 2453 & 1.9 & -75.905 & 37.1116 & VA & 2525 & -6.7 & -75.9436 & 37.0842 & VA \\
\hline 2382 & -2.2 & -75.8749 & 37.1334 & VA & 2454 & 1.6 & -75.9055 & 37.1114 & VA & 2526 & 10.8 & -75.9747 & 37.0841 & VA \\
\hline 2383 & -1.7 & -75.8753 & 37.133 & VA & 2455 & 1.2 & -75.9061 & 37.1112 & VA & 2527 & 6.8 & -75.9741 & 37.084 & VA \\
\hline 2384 & -1.1 & -75.8757 & 37.1327 & VA & 2456 & 0.9 & -75.9066 & 37.111 & VA & 2528 & -6.8 & -75.944 & 37.084 & VA \\
\hline 2385 & -0.5 & -75.876 & 37.1323 & VA & 2457 & 0.7 & -75.9071 & 37.1108 & VA & 2529 & 2.7 & -75.9736 & 37.0839 & VA \\
\hline 2386 & 0.1 & -75.8764 & 37.132 & VA & 2458 & 0.6 & -75.9077 & 37.1106 & VA & 2530 & -1.4 & -75.973 & 37.0838 & VA \\
\hline 2387 & 0.7 & -75.8768 & 37.1316 & VA & 2459 & 0.5 & -75.9082 & 37.1104 & VA & 2531 & -3.3 & -75.9724 & 37.0837 & VA \\
\hline 2388 & 1.6 & -75.8772 & 37.1313 & VA & 2460 & 0.5 & -75.9087 & 37.1103 & VA & 2532 & -6.8 & -75.9445 & 37.0837 & VA \\
\hline 2389 & 2.4 & -75.8776 & 37.1309 & VA & 2461 & 0.5 & -75.9093 & 37.1101 & VA & 2533 & -5.3 & -75.9718 & 37.0836 & VA \\
\hline 2390 & 3.8 & -75.878 & 37.1306 & VA & 2462 & 0.3 & -75.9098 & 37.1099 & VA & 2534 & -4.4 & -75.9713 & 37.0835 & VA \\
\hline 2391 & 5.2 & -75.8784 & 37.1303 & VA & 2463 & 0.2 & -75.9103 & 37.1097 & VA & 2535 & -3.5 & -75.9707 & 37.0834 & VA \\
\hline 2392 & 7.1 & -75.8788 & 37.1299 & VA & 2464 & 0.2 & -75.9109 & 37.1095 & VA & 2536 & -6.9 & -75.9449 & 37.0834 & VA \\
\hline 2393 & 8.9 & -75.8792 & 37.1296 & VA & 2465 & 0.2 & -75.9114 & 37.1093 & VA & 2537 & -3.2 & -75.9701 & 37.0833 & VA \\
\hline 2394 & -7 & -75.8795 & 37.1292 & VA & 2466 & 0.2 & -75.9119 & 37.1091 & VA & 2538 & -3 & -75.9695 & 37.0832 & VA \\
\hline 2395 & -6.8 & -75.8799 & 37.1289 & VA & 2467 & 0.2 & -75.9125 & 37.1089 & VA & 2539 & -2.8 & -75.969 & 37.0831 & VA \\
\hline 2396 & -6 & -75.8803 & 37.1285 & VA & 2468 & -0.1 & -75.913 & 37.1088 & VA & 2540 & -7 & -75.9454 & 37.0831 & VA \\
\hline 2397 & -5.1 & -75.8807 & 37.1282 & VA & 2469 & -0.3 & -75.9135 & 37.1086 & VA & 2541 & -2.6 & -75.9684 & 37.083 & VA \\
\hline 2398 & -4.7 & -75.8811 & 37.1278 & VA & 2470 & -0.6 & -75.9141 & 37.1084 & VA & 2542 & -2.2 & -75.9678 & 37.0829 & VA \\
\hline 2399 & -4.4 & -75.8815 & 37.1275 & VA & 2471 & -0.9 & -75.9146 & 37.1082 & VA & 2543 & -1.8 & -75.9673 & 37.0828 & VA \\
\hline 2400 & -4 & -75.8819 & 37.1271 & VA & 2472 & -1.1 & -75.9151 & 37.108 & VA & 2544 & -7.3 & -75.9459 & 37.0828 & VA \\
\hline 2401 & -3.5 & -75.8823 & 37.1268 & VA & 2473 & -1.4 & -75.9157 & 37.1078 & VA & 2545 & -0.9 & -75.9667 & 37.0827 & VA \\
\hline 2402 & -2.4 & -75.8826 & 37.1264 & VA & 2474 & -1.6 & -75.9162 & 37.1076 & VA & 2546 & -0.2 & -75.9661 & 37.0826 & VA \\
\hline 2403 & 11.3 & -75.883 & 37.1261 & VA & 2475 & -1.9 & -75.9167 & 37.1074 & VA & 2547 & -7.5 & -75.9463 & 37.0826 & VA \\
\hline 2404 & 7.4 & -75.8834 & 37.1257 & VA & 2476 & -2.4 & -75.9173 & 37.1072 & VA & 2548 & -0.9 & -75.965 & 37.0825 & VA \\
\hline 2405 & 3.5 & -75.8838 & 37.1254 & VA & 2477 & -2.8 & -75.9178 & 37.1071 & VA & 2549 & -0.5 & -75.9655 & 37.0825 & VA \\
\hline 2406 & 4.2 & -75.8842 & 37.1251 & VA & 2478 & -4.4 & -75.9183 & 37.1069 & VA & 2550 & -1 & -75.9644 & 37.0824 & VA \\
\hline 2407 & 4.9 & -75.8846 & 37.1247 & VA & 2479 & -6.1 & -75.9189 & 37.1067 & VA & 2551 & -1.1 & -75.9638 & 37.0823 & VA \\
\hline 2408 & 4.1 & -75.885 & 37.1244 & VA & 2480 & -9 & -75.9194 & 37.1065 & VA & 2552 & -7.8 & -75.9468 & 37.0823 & VA \\
\hline 2409 & 3.3 & -75.8854 & 37.124 & VA & 2481 & -12 & -75.9199 & 37.1063 & VA & 2553 & -0.9 & -75.9632 & 37.0822 & VA \\
\hline 2410 & 2.7 & -75.8858 & 37.1237 & VA & 2482 & NA & -75.9325 & 37.091 & VA & 2554 & -0.7 & -75.9627 & 37.0821 & VA \\
\hline 2411 & 2.1 & -75.8861 & 37.1233 & VA & 2483 & 10 & -75.933 & 37.0907 & VA & 2555 & -0.6 & -75.9621 & 37.082 & VA \\
\hline 2412 & 1.6 & -75.8865 & 37.123 & VA & 2484 & -10.4 & -75.9334 & 37.0904 & VA & 2556 & -8.1 & -75.9472 & 37.082 & VA \\
\hline 2413 & 1 & -75.8869 & 37.1226 & VA & 2485 & -10.9 & -75.9339 & 37.0902 & VA & 2557 & -0.5 & -75.9615 & 37.0819 & VA \\
\hline 2414 & 0.3 & -75.8873 & 37.1223 & VA & 2486 & -11.6 & -75.9343 & 37.0899 & VA & 2558 & -0.4 & -75.961 & 37.0818 & VA \\
\hline 2415 & -0.3 & -75.8877 & 37.1219 & VA & 2487 & -11.2 & -75.9348 & 37.0896 & VA & 2559 & -0.2 & -75.9604 & 37.0817 & VA \\
\hline 2416 & -0.9 & -75.8881 & 37.1216 & VA & 2488 & -10.9 & -75.9353 & 37.0893 & VA & 2560 & -7.9 & -75.9477 & 37.0817 & VA \\
\hline 2417 & -1.4 & .75 .8885 & 37.1212 & VA & 2489 & -9.5 & -75.9357 & 37.089 & VA & 2561 & 1.8 & -75.9598 & 37.0816 & VA \\
\hline 2418 & -1.6 & -75.8889 & 37.1209 & VA & 2490 & -8.1 & -75.9362 & 37.0888 & VA & 2562 & 3.7 & -75.9592 & 37.0815 & VA \\
\hline 2419 & -1.9 & -75.8893 & 37.1206 & VA & 2491 & -6.6 & -75.9366 & 37.0885 & VA & 2563 & 3.8 & -75.9587 & 37.0814 & VA \\
\hline 2420 & -1.9 & -75.8896 & 37.1202 & VA & 2492 & -5.1 & -75.9371 & 37.0882 & VA & 2564 & -7.7 & -75.9482 & 37.0814 & VA \\
\hline
\end{tabular}




\begin{tabular}{|c|c|c|c|c|c|c|c|c|c|c|c|c|c|c|}
\hline TR\# & RATE & LONG & LAT & ST & TR\# & RATE & LONG & LAT & ST & TR\# & RATE & LONG & LAT & ST \\
\hline 2565 & 3.9 & -75.9581 & 37.0813 & $\mathrm{VA}$ & 2637 & -0.4 & .76 .2914 & 36.9735 & VA & 2709 & 0.1 & -76.2529 & 36.9602 & VA \\
\hline 2566 & 0.4 & -75.9575 & 37.0812 & VA & 2638 & -0.6 & -76.2909 & 36.9734 & VA & 2710 & 0.2 & .76 .2525 & 36.9599 & VA \\
\hline 2567 & -3.2 & -75.9569 & 37.0811 & $\mathrm{VA}_{\mathrm{A}}$ & 2639 & -1.1 & -76.2903 & 36.9733 & VA & 2711 & 0.3 & -76.252 & 36.9596 & VA \\
\hline 2568 & -3.8 & -75.9564 & 37.081 & VA & 2640 & -1.4 & -76.2898 & 36.9732 & VA & 2712 & 0.4 & -76.2516 & 36.9593 & VA \\
\hline 2569 & -4.4 & -75.9558 & 37.0809 & VA & 2641 & -1.3 & -76.2892 & 36.973 & VA & 2713 & 0.5 & -76.2511 & 36.959 & VA \\
\hline 2570 & -5 & -75.9552 & 37.0808 & VA & 2642 & -1.1 & -76.2886 & 36.9729 & VA & 2714 & 0.5 & -76.2506 & 36.9588 & VA \\
\hline 2571 & -5.6 & -75.9546 & 37.0807 & VA & 2643 & 0.1 & .76 .2881 & 36.9728 & VA & 2715 & 0.4 & .76 .2502 & 36.9585 & VA \\
\hline 2572 & -6 & -75.9541 & 37.0806 & VA & 2644 & 1.3 & -76.2875 & 36.9727 & $\mathrm{VA}_{\mathrm{A}}$ & 2716 & 0.4 & -76.2497 & 36.9582 & VA \\
\hline 2573 & -6.5 & -75.9535 & 37.0805 & VA & 2645 & 0.5 & -76.287 & 36.9726 & VA & 2717 & 0 & -76.2493 & 36.9579 & VA \\
\hline 2574 & -6.8 & -75.9529 & 37.0804 & VA & 2646 & -0.3 & -76.2864 & 36.9724 & VA & 2718 & -0.3 & -76.2488 & 36.9577 & VA \\
\hline 2575 & -7.2 & -75.9524 & 37.0803 & VA & 2647 & -0.6 & -76.2859 & 36.9723 & VA & 2719 & -0.3 & -76.2484 & 36.9574 & VA \\
\hline 2576 & -7.2 & -75.9512 & 37.0802 & VA & 2648 & -1 & -76.2853 & 36.9722 & VA & 2720 & -0.3 & -76.2479 & 36.9571 & VA \\
\hline 2577 & -7.2 & -75.9518 & 37.0802 & VA & 2649 & -0.8 & -76.2848 & 36.9721 & VA & 2721 & -0.4 & -76.2474 & 36.9568 & VA \\
\hline 2578 & -7.3 & -75.9506 & 37.0801 & VA & 2650 & -0.7 & -76.2842 & 36.972 & VA & 2722 & -0.4 & -76.247 & 36.9565 & VA \\
\hline 2579 & -0.6 & .76 & 37.16667 & VA & 2651 & -0.1 & -76.2837 & 36.9719 & VA & 2723 & -0.3 & .76 .2465 & 36.9563 & VA \\
\hline 2580 & -0.6 & -76.03333 & 37.21667 & VA & 2652 & 0.4 & -76.2831 & 36.9717 & VA & 2724 & -0.3 & -76.2461 & 36.956 & VA \\
\hline 2581 & -0.6 & -76.05 & 37.26667 & VA & 2653 & 0.7 & -76.2825 & 36.9716 & VA & 2725 & -0.4 & -76.2456 & 36.9557 & VA \\
\hline 2582 & -0.6 & -76.05 & 37.31667 & VA & 2654 & 0.9 & -76.282 & 36.9715 & VA & 2726 & -0.5 & -76.2451 & 36.9554 & VA \\
\hline 2583 & -0.7 & -76.03333 & 37.36666 & VA & 2655 & 1.4 & -76.2814 & 36.9714 & VA & 2727 & -0.3 & -76.2447 & 36.9551 & VA \\
\hline 2584 & -0.8 & -76 & 37.41667 & VA & 2656 & 2 & -76.2809 & 36.9713 & VA & 2728 & -0.2 & -76.2442 & 36.9549 & VA \\
\hline 2585 & -0.8 & -76 & 37.46667 & VA & 2657 & 2.3 & -76.2803 & 36.9711 & VA & 2729 & -0.2 & -76.2438 & 36.9546 & VA \\
\hline 2586 & -0.8 & -76 & 37.51667 & VA & 2658 & 2.6 & -76.2798 & 36.971 & VA & 2730 & -0.3 & -76.2433 & 36.9543 & VA \\
\hline 2587 & -1.4 & -75.96667 & 37.56667 & VA & 2659 & 1.2 & -76.2792 & 36.9709 & VA & 2731 & -0.3 & -76.2429 & 36.954 & VA \\
\hline 2588 & -1.5 & -75.95 & 37.61666 & VA & 2660 & -0.1 & -76.2787 & 36.9708 & VA & 2732 & -0.3 & -76.2424 & 36.9538 & VA \\
\hline 2589 & -1 & -75.91666 & 37.66667 & VA & 2661 & -1.3 & -76.2781 & 36.9707 & VA & 2733 & -0.2 & -76.2419 & 36.9535 & VA \\
\hline 2590 & -1.5 & -75.88333 & 37.71667 & VA & 2662 & -2.4 & -76.2775 & 36.9705 & VA & 2734 & -0.1 & -76.2415 & 36.9532 & VA \\
\hline 2591 & -1.5 & -75.85 & 37.76667 & VA & 2663 & -1.6 & -76.277 & 36.9704 & VA & 2735 & -0.2 & -76.241 & 36.9529 & VA \\
\hline 2592 & -1.5 & -75.83334 & 37.81667 & VA & 2664 & -0.7 & -76.2764 & 36.9703 & VA & 2736 & -0.3 & -76.2406 & 36.9526 & VA \\
\hline 2593 & -0.8 & -75.78333 & 37.83333 & VA & 2665 & -0.7 & -76.2759 & 36.9702 & VA & 2737 & -0.1 & -76.2401 & 36.9524 & VA \\
\hline 2594 & -0.8 & -75.73333 & 37.86666 & VA & 2666 & -0.6 & -76.2753 & 36.9701 & $\mathrm{VA}_{\mathrm{A}}$ & 2738 & 0.1 & -76.2397 & 36.9521 & VA \\
\hline 2595 & -0.8 & -75.73333 & 37.91667 & VA & 2667 & -0.6 & -76.2748 & 36.97 & VA & 2739 & 0 & -76.2392 & 36.9518 & VA \\
\hline 2596 & -0.8 & -75.78333 & 37.91667 & VA & 2668 & -0.6 & -76.2742 & 36.9698 & VA & 2740 & -0.2 & -76.2387 & 36.9515 & VA \\
\hline 2597 & -1.2 & -75.75 & 37.96667 & VA & 2669 & -0.6 & -76.2737 & 36.9697 & VA & 2741 & -0.2 & -76.2383 & 36.9512 & VA \\
\hline 2598 & -1.2 & -76.01667 & 37.85 & VA & 2670 & -0.7 & -76.2731 & 36.9696 & VA & 2742 & -0.2 & -76.2378 & 36.951 & VA \\
\hline 2599 & -0.6 & -76.03333 & 37.9 & VA & 2671 & -0.5 & -76.2726 & 36.9695 & VA & 2743 & -0.1 & -76.2374 & 36.9507 & VA \\
\hline 2600 & -0.6 & -76.06667 & 37.95 & VA & 2672 & -0.4 & -76.272 & 36.9694 & VA & 2744 & -0.1 & -76.2369 & 36.9504 & VA \\
\hline 2601 & -1.5 & -76.5 & 38.03333 & VA & 2673 & -0.7 & -76.2714 & 36.9692 & VA & 2745 & -0.1 & -76.2365 & 36.9501 & VA \\
\hline 2602 & -1.5 & -76.46667 & 37.98333 & VA & 2674 & -1.1 & -76.2709 & 36.9691 & VA & 2746 & -0.1 & -76.236 & 36.9498 & VA \\
\hline 2603 & -1.5 & -76.41666 & 37.98333 & VA & 2675 & -1 & -76.2703 & 36.969 & VA & 2747 & -0.2 & -76.2355 & 36.9496 & VA \\
\hline 2604 & -1.5 & .76 .36667 & 37.96667 & VA & 2676 & -0.9 & -76.2698 & 36.9689 & VA & 2748 & -0.3 & -76.2351 & 36.9493 & VA \\
\hline 2605 & -1.5 & -76.31667 & 37.95 & VA & 2677 & -0.9 & -76.2692 & 36.9688 & $\mathrm{VA}_{\mathrm{A}}$ & 2749 & -0.3 & -76.2346 & 36.949 & VA \\
\hline 2606 & -1.5 & -76.26667 & 37.93333 & VA & 2678 & -0.8 & -76.2687 & 36.9687 & VA & 2750 & -0.3 & -76.2342 & 36.9487 & VA \\
\hline 2607 & -1.8 & -76.28333 & 37.88334 & VA & 2679 & 0.1 & -76.2681 & 36.9685 & VA & 2751 & -0.3 & -76.2337 & 36.9485 & VA \\
\hline 2608 & -1.6 & -76.3 & 37.83333 & VA & 2680 & 1 & -76.2676 & 36.9684 & $\mathrm{VA}_{\mathrm{A}}$ & 2752 & -0.4 & -76.2332 & 36.9482 & VA \\
\hline 2609 & -0.8 & -76.33334 & 37.78333 & VA & 2681 & 0.2 & -76.267 & 36.9683 & VA & 2753 & 0 & .76 .234 & 36.948 & VA \\
\hline 2610 & -0.4 & -76.33334 & 37.73333 & VA & 2682 & -0.6 & -76.2664 & 36.9682 & VA & 2754 & -0.2 & -76.2328 & 36.9479 & VA \\
\hline 2611 & -1.9 & -76.35 & 37.68333 & VA & 2683 & -0.6 & -76.2659 & 36.9681 & VA & 2755 & 0.1 & -76.2335 & 36.9478 & VA \\
\hline 2612 & -2.2 & -76.31667 & 37.63334 & VA & 2684 & -0.6 & .76 .2653 & 36.9679 & VA & 2756 & 0 & -76.2323 & 36.9476 & VA \\
\hline 2613 & -1.2 & -76.33334 & 37.58333 & VA & 2685 & -0.5 & -76.2648 & 36.9678 & VA & 2757 & 0.1 & -76.233 & 36.9476 & VA \\
\hline 2614 & -1.8 & -76.31667 & 37.53333 & VA & 2686 & -0.3 & -76.2642 & 36.9677 & VA & 2758 & 0.1 & -76.2324 & 36.9474 & VA \\
\hline 2615 & -1.4 & -76.28333 & 37.48333 & VA & 2687 & -0.4 & -76.2637 & 36.9676 & VA & 2759 & 0 & -76.2319 & 36.9473 & VA \\
\hline 2616 & -1.4 & -76.28333 & 37.43333 & VA & 2688 & -0.4 & -76.2631 & 36.9675 & VA & 2760 & 0.1 & -76.2319 & 36.9473 & VA \\
\hline 2617 & -1.3 & -76.28333 & 37.38334 & VA & 2689 & -0.4 & .76 .2626 & 36.9674 & VA & 2761 & 0 & -76.2314 & 36.9471 & VA \\
\hline 2618 & -1.3 & -76.28333 & 37.33333 & VA & 2690 & -0.4 & -76.262 & 36.9672 & VA & 2762 & 0.1 & -76.2314 & 36.9471 & VA \\
\hline 2619 & -0.2 & -76.33334 & 37.36666 & VA & 2691 & 0 & -76.2612 & 36.9652 & VA & 2763 & -0.1 & -76.2308 & 36.9469 & VA \\
\hline 2620 & -0.5 & -76.4 & 37.31667 & VA & 2692 & -0.2 & -76.2607 & 36.9649 & VA & 2764 & 0 & -76.231 & 36.9468 & VA \\
\hline 2621 & -0.4 & -76.4 & 37.26667 & VA & 2693 & 0 & -76.2603 & 36.9646 & VA & 2765 & -0.3 & -76.2303 & 36.9467 & VA \\
\hline 2622 & -0.4 & -76.45 & 37.28333 & VA & 2694 & 0.1 & -76.2598 & 36.9643 & VA & 2766 & -0.3 & -76.2298 & 36.9466 & VA \\
\hline 2623 & -1.1 & -76.46667 & 37.23333 & VA & 2695 & -0.1 & -76.2593 & 36.9641 & VA & 2767 & -0.1 & -76.2305 & 36.9465 & VA \\
\hline 2624 & -1.2 & -76.41666 & 37.23333 & VA & 2696 & -0.4 & -76.2589 & 36.9638 & VA & 2768 & -0.2 & -76.2292 & 36.9464 & VA \\
\hline 2625 & -0.8 & .76 .4 & 37.18333 & VA & 2697 & -0.6 & -76.2584 & 36.9635 & VA & 2769 & -0.3 & -76.2287 & 36.9462 & VA \\
\hline 2626 & -0.8 & -76.35 & 37.18333 & VA & 2698 & -0.7 & -76.258 & 36.9632 & VA & 2770 & -0.4 & -76.2282 & 36.946 & VA \\
\hline 2627 & -1.4 & -76.31667 & 37.13334 & VA & 2699 & -0.5 & .76 .2575 & 36.963 & VA & 2771 & -0.2 & -76.2276 & 36.9459 & VA \\
\hline 2628 & -1.4 & -76.31667 & 37.08333 & VA & 2700 & -0.3 & -76.257 & 36.9627 & VA & 2772 & 0 & -76.2271 & 36.9457 & VA \\
\hline 2629 & -1.4 & -76.33334 & 37.03333 & VA & 2701 & -0.5 & -76.2566 & 36.9624 & VA & 2773 & 0 & -76.2266 & 36.9455 & VA \\
\hline 2630 & 0.3 & -76.2953 & 36.9743 & VA & 2702 & -0.6 & -76.2561 & 36.9621 & VA & 2774 & 0 & -76.226 & 36.9453 & VA \\
\hline 2631 & 0.4 & -76.2948 & 36.9742 & VA & 2703 & -0.3 & -76.2557 & 36.9618 & VA & 2775 & 0 & -76.2255 & 36.9451 & VA \\
\hline 2632 & 0.5 & -76.2942 & 36.9741 & $\mathrm{VA}_{\mathrm{A}}$ & 2704 & 0 & -76.2552 & 36.9616 & $\mathrm{VA}_{\mathrm{A}}$ & 2776 & -0.1 & -76.225 & 36.945 & VA \\
\hline 2633 & 0.1 & -76.2936 & 36.974 & VA & 2705 & 0 & -76.2548 & 36.9613 & VA & 2777 & -0.2 & -76.2244 & 36.9448 & VA \\
\hline 2634 & -0.2 & -76.2931 & 36.9739 & VA & 2706 & 0 & -76.2543 & 36.961 & VA & 2778 & -0.3 & -76.2239 & 36.9446 & VA \\
\hline 2635 & -0.2 & -76.2925 & 36.9737 & VA & 2707 & 0 & -76.2538 & 36.9607 & VA & 2779 & -0.3 & -76.2234 & 36.9444 & VA \\
\hline 2636 & -0.3 & -76.292 & 36.9736 & VA & 2708 & 0 & -76.2534 & 36.9604 & VA & 2780 & -0.3 & -76.2228 & 36.9443 & VA \\
\hline
\end{tabular}




\begin{tabular}{|c|c|c|c|c|c|c|c|c|c|c|c|c|c|c|}
\hline TR\# & RATE & LONG & LAT & ST & TR\# & RATE & LONG & LAT & ST & TR\# & RATE & LONG & LAT & ST \\
\hline 2781 & -0.3 & -76.2223 & 36.9441 & $\mathrm{VA}$ & 2853 & -0.5 & -76.0269 & 36.9349 & VA & 2925 & 2.8 & -76.0432 & 36.9323 & $\overline{\mathrm{VA}}$ \\
\hline 2782 & -0.3 & -76.2218 & 36.9439 & VA & 2854 & 2.2 & -76.0378 & 36.9349 & VA & 2926 & 3 & -76.0437 & 36.932 & VA \\
\hline 2783 & -0.3 & -76.2212 & 36.9437 & VA & 2855 & 0.2 & -76.1912 & 36.9348 & VA & 2927 & 3.2 & -76.0442 & 36.9318 & VA \\
\hline 2784 & -0.3 & -76.2207 & 36.9435 & VA & 2856 & -0.3 & -76.0263 & 36.9348 & VA & 2928 & 0 & -76.1743 & 36.9317 & VA \\
\hline 2785 & -0.3 & -76.2202 & 36.9434 & VA & 2857 & -0.5 & -76.0257 & 36.9348 & VA & 2929 & -0.3 & -76.1738 & 36.9316 & VA \\
\hline 2786 & -0.3 & -76.2196 & 36.9432 & VA & 2858 & 0.2 & -76.1907 & 36.9347 & VA & 2930 & -0.6 & -76.1732 & 36.9315 & VA \\
\hline 2787 & -0.3 & -76.2191 & 36.943 & VA & 2859 & 2.4 & -76.0382 & 36.9347 & VA & 2931 & 3.2 & -76.0447 & 36.9315 & VA \\
\hline 2788 & -0.1 & -76.2185 & 36.9428 & VA & 2860 & -0.7 & -76.0252 & 36.9347 & VA & 2932 & -0.8 & -76.004 & 36.9315 & VA \\
\hline 2789 & -0.1 & -76.218 & 36.9427 & VA & 2861 & -0.3 & -76.0246 & 36.9347 & VA & 2933 & -0.8 & -76.1727 & 36.9314 & VA \\
\hline 2790 & 0 & -76.2175 & 36.9425 & VA & 2862 & 0.2 & -76.1901 & 36.9346 & VA & 2934 & -0.9 & -76.1721 & 36.9313 & VA \\
\hline 2791 & -0.1 & -76.2169 & 36.9423 & VA & 2863 & 0 & -76.024 & 36.9346 & VA & 2935 & 3.2 & -76.0452 & 36.9313 & VA \\
\hline 2792 & -0.2 & -76.2164 & 36.9421 & VA & 2864 & 0.3 & -76.1896 & 36.9345 & VA & 2936 & -1.2 & -76.1715 & 36.9312 & VA \\
\hline 2793 & 0 & -76.2159 & 36.9419 & $\mathrm{VA}_{\mathrm{A}}$ & 2865 & 2.4 & -76.0387 & 36.9345 & VA & 2937 & -1.2 & -76.0036 & 36.9312 & VA \\
\hline 2794 & 0.3 & -76.2153 & 36.9418 & VA & 2866 & 0.1 & -76.0234 & 36.9345 & VA & 2938 & -1.6 & -76.171 & 36.9311 & VA \\
\hline 2795 & 0.2 & -76.2148 & 36.9416 & VA & 2867 & 0.3 & -76.0229 & 36.9345 & VA & 2939 & 3 & -76.0457 & 36.9311 & VA \\
\hline 2796 & 0 & -76.2143 & 36.9414 & VA & 2868 & 0.4 & -76.189 & 36.9344 & VA & 2940 & -1.4 & -76.1704 & 36.9309 & VA \\
\hline 2797 & 0 & -76.2137 & 36.9412 & VA & 2869 & 0.3 & .76 .0223 & 36.9344 & $\mathrm{VA}$ & 2941 & -1.5 & .76 .0031 & 36.9309 & VA \\
\hline 2798 & 0 & -76.2132 & 36.9411 & $V_{A}$ & 2870 & 0.5 & -76.1884 & 36.9343 & VA & 2942 & -1.1 & .76 .1698 & 36.9308 & VA \\
\hline 2799 & -0.1 & -76.2127 & 36.9409 & VA & 2871 & -0.2 & -76.0212 & 36.9343 & VA & 2943 & 2.8 & -76.0462 & 36.9308 & VA \\
\hline 2800 & -0.3 & -76.2121 & 36.9407 & VA & 2872 & 0.2 & -76.0217 & 36.9343 & VA & 2944 & -1.2 & -76.1693 & 36.9307 & VA \\
\hline 2801 & -0.4 & -76.2116 & 36.9405 & VA & 2873 & 0.5 & -76.1879 & 36.9342 & VA & 2945 & -0.4 & -76.1644 & 36.9307 & VA \\
\hline 2802 & -0.6 & -76.2111 & 36.9403 & VA & 2874 & -0.5 & -76.0206 & 36.9342 & VA & 2946 & -1.3 & -76.1687 & 36.9306 & VA \\
\hline 2803 & -0.4 & -76.2105 & 36.9402 & VA & 2875 & 2.5 & -76.0392 & 36.9342 & VA & 2947 & 2.9 & -76.0467 & 36.9306 & VA \\
\hline 2804 & -0.3 & -76.21 & 36.94 & VA & 2876 & 0.7 & -76.1873 & 36.9341 & VA & 2948 & -1.7 & -76.0027 & 36.9306 & VA \\
\hline 2805 & -0.3 & -76.2095 & 36.9398 & VA & 2877 & -0.8 & -76.02 & 36.9341 & VA & 2949 & -0.6 & .76 .1639 & 36.9306 & VA \\
\hline 2806 & -0.3 & -76.2089 & 36.9396 & VA & 2878 & -1 & -76.0194 & 36.9341 & VA & 2950 & -1.4 & -76.1681 & 36.9305 & VA \\
\hline 2807 & -0.4 & -76.2084 & 36.9395 & VA & 2879 & 0.9 & -76.1867 & 36.934 & VA & 2951 & -1.6 & -76.1676 & 36.9304 & VA \\
\hline 2808 & -0.6 & -76.2079 & 36.9393 & VA & 2880 & -1.2 & -76.0189 & 36.934 & VA & 2952 & -0.5 & -76.1633 & 36.9304 & VA \\
\hline 2809 & -0.7 & -76.2073 & 36.9391 & VA & 2881 & -1.2 & -76.0183 & 36.934 & VA & 2953 & -1.7 & -76.167 & 36.9303 & VA \\
\hline 2810 & -0.9 & -76.2068 & 36.9389 & VA & 2882 & 2.7 & -76.0397 & 36.934 & VA & 2954 & -1.6 & -76.0023 & 36.9303 & VA \\
\hline 2811 & -0.9 & -76.2063 & 36.9388 & VA & 2883 & 0.9 & -76.1862 & 36.9339 & VA & 2955 & 3 & -76.0472 & 36.9303 & VA \\
\hline 2812 & -0.9 & -76.2057 & 36.9386 & VA & 2884 & -1.2 & -76.0177 & 36.9339 & VA & 2956 & -0.4 & -76.1628 & 36.9303 & VA \\
\hline 2813 & -0.8 & -76.2052 & 36.9384 & VA & 2885 & 0.9 & -76.1856 & 36.9338 & VA & 2957 & -2 & -76.1665 & 36.9302 & VA \\
\hline 2814 & -0.6 & -76.2047 & 36.9382 & VA & 2886 & -1.2 & -76.0171 & 36.9338 & VA & 2958 & -0.7 & -76.1622 & 36.9302 & VA \\
\hline 2815 & -0.6 & -76.2041 & 36.938 & VA & 2887 & -1.2 & -76.0166 & 36.9338 & VA & 2959 & -1.8 & -76.1659 & 36.9301 & VA \\
\hline 2816 & -0.6 & -76.2036 & 36.9379 & VA & 2888 & 0.9 & -76.185 & 36.9337 & VA & 2960 & 2.9 & -76.0477 & 36.9301 & VA \\
\hline 2817 & -0.6 & -76.2031 & 36.9377 & VA & 2889 & 2.9 & -76.0402 & 36.9337 & $\mathrm{VA}$ & 2961 & -1.6 & -76.1653 & 36.93 & VA \\
\hline 2818 & -0.6 & -76.2025 & 36.9375 & VA & 2890 & -1.2 & .76 .016 & 36.9337 & VA & 2962 & -0.9 & -76.1617 & 36.93 & VA \\
\hline 2819 & -0.8 & -76.202 & 36.9373 & VA & 2891 & 0.9 & -76.1845 & 36.9336 & VA & 2963 & -1.4 & -76.0018 & 36.93 & VA \\
\hline 2820 & -0.9 & -76.2015 & 36.9372 & VA & 2892 & -1.4 & -76.0149 & 36.9336 & VA & 2964 & -1.6 & -76.1648 & 36.9299 & VA \\
\hline 2821 & 0.1 & -76.2019 & 36.9368 & VA & 2893 & -1.3 & -76.0154 & 36.9336 & VA & 2965 & -0.9 & -76.1611 & 36.9299 & VA \\
\hline 2822 & 0.1 & -76.2014 & 36.9367 & VA & 2894 & 3 & -76.0407 & 36.9335 & VA & 2966 & -1.6 & -76.1642 & 36.9298 & VA \\
\hline 2823 & 0.2 & -76.2008 & 36.9366 & VA & 2895 & -1.3 & -76.0143 & 36.9335 & VA & 2967 & 2.9 & -76.0482 & 36.9298 & VA \\
\hline 2824 & 0.3 & -76.2003 & 36.9365 & VA & 2896 & 1.1 & -76.1839 & 36.9334 & VA & 2968 & -0.9 & -76.1606 & 36.9297 & VA \\
\hline 2825 & 0.3 & -76.1997 & 36.9364 & $\mathrm{VA}$ & 2897 & -1 & -76.0131 & 36.9334 & VA & 2969 & -1.5 & -76.0014 & 36.9297 & VA \\
\hline 2826 & 0.2 & -76.1991 & 36.9363 & VA & 2898 & -1.2 & -76.0137 & 36.9334 & VA & 2970 & -0.9 & -76.16 & 36.9296 & VA \\
\hline 2827 & 0.2 & -76.1986 & 36.9362 & VA & 2899 & 1.4 & -76.1834 & 36.9333 & VA & 2971 & 2.6 & -76.0487 & 36.9296 & VA \\
\hline 2828 & 0.2 & -76.198 & 36.9361 & VA & 2900 & -0.9 & -76.0126 & 36.9333 & VA & 2972 & -0.9 & -76.1595 & 36.9295 & VA \\
\hline 2829 & 0.2 & -76.1974 & 36.9359 & VA & 2901 & -0.9 & -76.012 & 36.9333 & VA & 2973 & -1.6 & -76.0009 & 36.9294 & VA \\
\hline 2830 & 0.1 & -76.1969 & 36.9358 & VA & 2902 & 1 & -76.1828 & 36.9332 & VA & 2974 & 2.4 & -76.0492 & 36.9294 & VA \\
\hline 2831 & 0 & -76.1963 & 36.9357 & VA & 2903 & 3.2 & -76.0412 & 36.9332 & VA & 2975 & -0.9 & -76.1589 & 36.9293 & VA \\
\hline 2832 & 2 & -76.0363 & 36.9357 & VA & 2904 & -0.9 & -76.0114 & 36.9332 & VA & 2976 & -1 & .76 .1584 & 36.9292 & VA \\
\hline 2833 & -0.1 & -76.1957 & 36.9356 & VA & 2905 & 0.6 & -76.1822 & 36.9331 & VA & 2977 & 2.1 & -76.0497 & 36.9291 & VA \\
\hline 2834 & 0 & -76.1952 & 36.9355 & VA & 2906 & -0.9 & -76.0103 & 36.9331 & VA & 2978 & -1.8 & -76.0005 & 36.9291 & VA \\
\hline 2835 & 1 & -76.0326 & 36.9355 & VA & 2907 & -0.9 & -76.0108 & 36.9331 & VA & 2979 & -1 & -76.1578 & 36.929 & VA \\
\hline 2836 & 0.8 & -76.032 & 36.9355 & VA & 2908 & 1 & -76.1817 & 36.933 & VA & 2980 & -1 & -76.1573 & 36.9289 & VA \\
\hline 2837 & 0 & -76.1946 & 36.9354 & VA & 2909 & -0.8 & -76.0097 & 36.933 & VA & 2981 & 1.8 & -76.0501 & 36.9289 & VA \\
\hline 2838 & 2 & -76.0368 & 36.9354 & VA & 2910 & 3 & -76.0417 & 36.933 & VA & 2982 & -1.3 & -76.1567 & 36.9288 & VA \\
\hline 2839 & 0.8 & -76.0315 & 36.9354 & VA & 2911 & 1.4 & -76.1811 & 36.9329 & VA & 2983 & -2.1 & -76.0001 & 36.9288 & VA \\
\hline 2840 & 0 & -76.1941 & 36.9353 & VA & 2912 & -0.7 & -76.0091 & 36.9329 & VA & 2984 & -1.7 & -76.1562 & 36.9286 & VA \\
\hline 2841 & 0.6 & -76.0303 & 36.9353 & VA & 2913 & -0.8 & .76 .0085 & 36.9329 & VA & 2985 & 1.8 & -76.0506 & 36.9286 & VA \\
\hline 2842 & 0.8 & -76.0309 & 36.9353 & VA & 2914 & 1.3 & -76.1805 & 36.9328 & VA & 2986 & -1.5 & -76.1556 & 36.9285 & VA \\
\hline 2843 & 0 & -76.1935 & 36.9352 & VA & 2915 & -1 & -76.0074 & 36.9328 & VA & 2987 & -1.4 & -76.1551 & 36.9284 & VA \\
\hline 2844 & 0.6 & -76.0292 & 36.9352 & VA & 2916 & 2.9 & -76.0422 & 36.9328 & VA & 2988 & -1.9 & -75.9996 & 36.9284 & VA \\
\hline 2845 & 2 & -76.0373 & 36.9352 & $\mathrm{VA}$ & 2917 & -0.9 & -76.008 & 36.9328 & $\mathrm{VA}$ & 2989 & 1.7 & -76.0511 & 36.9284 & VA \\
\hline 2846 & 0.4 & -76.0298 & 36.9352 & VA & 2918 & 1.3 & -76.18 & 36.9327 & VA & 2990 & -1.4 & -76.1545 & 36.9282 & VA \\
\hline 2847 & 0 & -76.1929 & 36.9351 & VA & 2919 & -1 & -76.0068 & 36.9327 & VA & 2991 & 2.2 & -76.0516 & 36.9282 & VA \\
\hline 2848 & 0.8 & -76.0286 & 36.9351 & VA & 2920 & 1.3 & -76.1794 & 36.9326 & VA & 2992 & -1.4 & -76.154 & 36.9281 & VA \\
\hline 2849 & 0 & -76.1924 & 36.935 & VA & 2921 & 1.4 & -76.1788 & 36.9325 & VA & 2993 & -1.7 & -75.9992 & 36.9281 & VA \\
\hline 2850 & -0.7 & -76.0275 & 36.935 & VA & 2922 & 2.8 & -76.0427 & 36.9325 & VA & 2994 & -1.6 & -76.1534 & 36.9279 & VA \\
\hline 2851 & 0.8 & -76.028 & 36.935 & VA & 2923 & 2 & -76.1783 & 36.9324 & VA & 2995 & 2.8 & -76.0521 & 36.9279 & VA \\
\hline 2852 & 0.1 & -76.1918 & 36.9349 & VA & 2924 & 2.8 & -76.1777 & 36.9323 & $\mathrm{VA}$ & 2996 & -1.8 & -76.1529 & 36.9278 & VA \\
\hline
\end{tabular}




\begin{tabular}{|c|c|c|c|c|c|c|c|c|c|c|c|c|c|c|}
\hline TR\# & RATE & LONG & LAT & ST & TR\# & RATE & LONG & LAT & ST & TR\# & RATE & LONG & LAT & ST \\
\hline 2997 & -1.4 & -75.9988 & 36.9278 & $\overline{V A}$ & 3069 & -0.4 & -76.1331 & 36.9228 & $\overline{\mathrm{VA}}$ & 3141 & 0 & -76.1165 & 36.9181 & $\overline{\mathrm{VA}}$ \\
\hline 2998 & -1.8 & -76.1523 & 36.9277 & VA & 3070 & 1.5 & -76.0625 & 36.9228 & VA & 3142 & 0.4 & -76.0766 & 36.9181 & VA \\
\hline 2999 & 2.5 & -76.0526 & 36.9277 & VA & 3071 & -0.3 & -76.1325 & 36.9226 & VA & 3143 & 0.1 & -76.116 & 36.918 & VA \\
\hline 3000 & -1.9 & -76.1518 & 36.9275 & VA & 3072 & 1.3 & -76.063 & 36.9226 & VA & 3144 & 0.7 & -76.0772 & 36.918 & VA \\
\hline 3001 & -1 & -75.9983 & 36.9275 & VA & 3073 & -0.3 & -75.9914 & 36.9226 & VA & 3145 & 0.8 & .76 .0777 & 36.9179 & VA \\
\hline 3002 & -1.6 & -76.1512 & 36.9274 & VA & 3074 & -0.1 & -76.132 & 36.9225 & VA & 3146 & 0.1 & -76.1155 & 36.9178 & VA \\
\hline 3003 & 2.2 & -76.0531 & 36.9274 & VA & 3075 & -0.6 & -76.1314 & 36.9224 & VA & 3147 & 2.2 & -75.9878 & 36.9178 & VA \\
\hline 3004 & -1.4 & -76.1507 & 36.9272 & VA & 3076 & 1.4 & -76.0635 & 36.9223 & VA & 3148 & 0.9 & -76.0783 & 36.9177 & VA \\
\hline 3005 & 2.2 & -76.0536 & 36.9272 & $\mathrm{VA}_{\mathrm{A}}$ & 3077 & -1.2 & -76.1309 & 36.9222 & VA & 3149 & 0.2 & -76.1149 & 36.9176 & VA \\
\hline 3006 & -1 & -75.9979 & 36.9272 & VA & 3078 & -1.1 & -76.1303 & 36.9221 & VA & 3150 & 1 & -76.0789 & 36.9176 & VA \\
\hline 3007 & -1.4 & -76.1501 & 36.9271 & VA & 3079 & 1.5 & -76.064 & 36.9221 & VA & 3151 & 0.5 & -76.1144 & 36.9175 & VA \\
\hline 3008 & -1.4 & -76.1496 & 36.927 & VA & 3080 & -1 & -76.1298 & 36.9219 & VA & 3152 & 1.1 & -76.0794 & 36.9175 & VA \\
\hline 3009 & -0.9 & -75.9975 & 36.9269 & $\mathrm{vA}_{\mathrm{A}}$ & 3081 & 1.3 & -76.0645 & 36.9219 & VA & 3153 & 2.1 & .75 .9877 & 36.9174 & VA \\
\hline 3010 & 2.2 & -76.0541 & 36.9269 & VA & 3082 & -0.6 & -76.1292 & 36.9218 & VA & 3154 & 0.9 & -76.08 & 36.9174 & VA \\
\hline 3011 & -1.3 & -76.149 & 36.9268 & VA & 3083 & -0.2 & -76.1287 & 36.9217 & VA & 3155 & 0.8 & -76.1138 & 36.9173 & VA \\
\hline 3012 & -1.2 & -76.1485 & 36.9267 & VA & 3084 & 1.1 & .76 .065 & 36.9216 & VA & 3156 & 0.7 & -76.0806 & 36.9173 & VA \\
\hline 3013 & 2.6 & -76.0546 & 36.9267 & VA & 3085 & 0 & .76 .1281 & 36.9215 & VA & 3157 & 0.5 & -76.0811 & 36.9172 & VA \\
\hline 3014 & -0.9 & -75.997 & 36.9266 & $V_{A}$ & 3086 & -0.1 & -76.1273 & 36.9215 & VA & 3158 & 0.9 & -76.1133 & 36.9171 & VA \\
\hline 3015 & -1.4 & -76.1479 & 36.9265 & VA & 3087 & 0.3 & -76.1276 & 36.9214 & VA & 3159 & 0.4 & -76.0817 & 36.9171 & VA \\
\hline 3016 & 3 & -76.0551 & 36.9265 & VA & 3088 & 1.1 & -76.0655 & 36.9214 & VA & 3160 & 1.1 & -76.1128 & 36.917 & VA \\
\hline 3017 & -1.5 & -76.1474 & 36.9264 & VA & 3089 & -0.3 & -76.1268 & 36.9213 & VA & 3161 & 0.6 & -76.0822 & 36.917 & VA \\
\hline 3018 & -1.4 & -76.1468 & 36.9263 & VA & 3090 & 0 & -76.127 & 36.9212 & VA & 3162 & 1.9 & -75.9876 & 36.9169 & VA \\
\hline 3019 & -1 & -75.9966 & 36.9263 & $\mathrm{VA}$ & 3091 & -0.3 & -76.1265 & 36.9211 & $\mathrm{VA}$ & 3163 & 0.8 & .76 .0828 & 36.9169 & VA \\
\hline 3020 & 2.7 & -76.0556 & 36.9262 & VA & 3092 & -0.4 & -76.1262 & 36.9211 & $\mathrm{VA}$ & 3164 & 0.8 & -76.1122 & 36.9168 & VA \\
\hline 3021 & -1.2 & -76.1463 & 36.9261 & VA & 3093 & -0.6 & -76.1257 & 36.921 & VA & 3165 & 0.9 & -76.0834 & 36.9168 & VA \\
\hline 3022 & -1.4 & -76.1457 & 36.926 & VA & 3094 & -0.6 & -76.1252 & 36.9208 & VA & 3166 & 1.1 & -76.0839 & 36.9167 & VA \\
\hline 3023 & 2.4 & -76.0561 & 36.926 & VA & 3095 & 0.4 & -76.0625 & 36.9208 & VA & 3167 & 0.4 & -76.1117 & 36.9166 & VA \\
\hline 3024 & -0.9 & -75.9962 & 36.926 & VA & 3096 & 0.2 & -76.0631 & 36.9207 & VA & 3168 & 1.2 & -76.0845 & 36.9166 & VA \\
\hline 3025 & -1.5 & -76.1452 & 36.9258 & VA & 3097 & -0.6 & -76.1246 & 36.9206 & $\mathrm{VA}$ & 3169 & 0.3 & -76.1111 & 36.9165 & VA \\
\hline 3026 & -1.4 & -76.1446 & 36.9257 & VA & 3098 & -1.2 & -75.9885 & 36.9206 & VA & 3170 & 1.7 & -75.9875 & 36.9165 & VA \\
\hline 3027 & -0.9 & -75.9957 & 36.9257 & VA & 3099 & 0 & -76.0637 & 36.9206 & VA & 3171 & 1.3 & -76.0851 & 36.9164 & VA \\
\hline 3028 & 2.3 & -76.0566 & 36.9257 & $v_{A}$ & 3100 & -0.5 & -76.1241 & 36.9205 & VA & 3172 & 0.2 & -76.1106 & 36.9163 & VA \\
\hline 3029 & -1.4 & -76.1441 & 36.9256 & $V_{A}$ & 3101 & 0 & -76.0642 & 36.9205 & VA & 3173 & 1.8 & -76.0856 & 36.9163 & VA \\
\hline 3030 & 2.2 & -76.0571 & 36.9255 & $\mathrm{VA}$ & 3102 & -0.4 & -76.1235 & 36.9203 & VA & 3174 & 2.4 & -76.0862 & 36.9162 & VA \\
\hline 3031 & -1.6 & -76.1435 & 36.9254 & VA & 3103 & 0 & -76.0648 & 36.9203 & VA & 3175 & -2 & -76.1101 & 36.9161 & VA \\
\hline 3032 & -0.9 & -75.9953 & 36.9254 & VA & 3104 & 0 & -76.0653 & 36.9202 & VA & 3176 & 2.4 & -76.0868 & 36.9161 & VA \\
\hline 3033 & -1.8 & -76.143 & 36.9253 & VA & 3105 & -0.2 & -76.123 & 36.9201 & VA & 3177 & -1.9 & -76.1095 & 36.916 & VA \\
\hline 3034 & 2.1 & -76.0576 & 36.9252 & VA & 3106 & 0 & -76.0659 & 36.9201 & VA & 3178 & 1.7 & -75.9873 & 36.916 & VA \\
\hline 3035 & -2.1 & -76.1424 & 36.9251 & VA & 3107 & -0.7 & -75.9884 & 36.9201 & VA & 3179 & 2.4 & -76.0873 & 36.916 & VA \\
\hline 3036 & -1 & -75.9949 & 36.9251 & VA & 3108 & 0 & -76.1225 & 36.92 & VA & 3180 & 2.5 & -76.0879 & 36.9159 & VA \\
\hline 3037 & -2.3 & -76.1419 & 36.925 & VA & 3109 & -0.1 & -76.0665 & 36.92 & VA & 3181 & -1.8 & -76.109 & 36.9158 & VA \\
\hline 3038 & 2 & -76.0581 & 36.925 & VA & 3110 & -0.1 & -76.067 & 36.9199 & VA & 3182 & 2.6 & -76.0884 & 36.9158 & VA \\
\hline 3039 & -2.3 & -76.1413 & 36.9249 & VA & 3111 & 0 & -76.1219 & 36.9198 & VA & 3183 & -1.7 & -76.1084 & 36.9157 & VA \\
\hline 3040 & 1.9 & -76.0586 & 36.9248 & $\mathrm{VA}_{\mathrm{A}}$ & 3112 & 0.1 & -76.0676 & 36.9198 & VA & 3184 & 3 & -76.089 & 36.9157 & VA \\
\hline 3041 & -2.2 & -76.1408 & 36.9247 & VA & 3113 & 0.3 & -76.0682 & 36.9197 & $\mathrm{vA}_{\mathrm{A}}$ & 3185 & 1.6 & -75.9872 & 36.9156 & VA \\
\hline 3042 & -1.1 & -75.9944 & 36.9247 & VA & 3114 & 0.3 & -75.9883 & 36.9197 & VA & 3186 & 3.3 & -76.0896 & 36.9156 & VA \\
\hline 3043 & -2.2 & -76.1402 & 36.9246 & VA & 3115 & 0 & -76.1214 & 36.9196 & VA & 3187 & -1.1 & -76.1079 & 36.9155 & VA \\
\hline 3044 & 1.8 & -76.0591 & 36.9245 & $v_{A}$ & 3116 & 0.1 & -76.0687 & 36.9196 & VA & 3188 & 3.5 & -76.0901 & 36.9155 & VA \\
\hline 3045 & -2.1 & -76.1397 & 36.9244 & VA & 3117 & 0.1 & -76.1208 & 36.9195 & VA & 3189 & 3.7 & -76.0907 & 36.9154 & VA \\
\hline 3046 & -1.2 & -75.994 & 36.9244 & VA & 3118 & -0.1 & -76.0693 & 36.9195 & VA & 3190 & -0.7 & -76.1074 & 36.9153 & VA \\
\hline 3047 & -2.5 & -76.1391 & 36.9243 & VA & 3119 & -0.3 & -76.0699 & 36.9194 & VA & 3191 & 2.9 & -76.0913 & 36.9153 & VA \\
\hline 3048 & 1.7 & -76.0596 & 36.9243 & VA & 3120 & 0.2 & -76.1203 & 36.9193 & VA & 3192 & -0.2 & -76.1068 & 36.9152 & VA \\
\hline 3049 & -2.9 & -76.1386 & 36.9242 & $\mathrm{VA}_{\mathrm{A}}$ & 3121 & -0.4 & -76.0704 & 36.9193 & VA & 3193 & 2.1 & -76.0918 & 36.9151 & VA \\
\hline 3050 & -1.3 & -75.9936 & 36.9241 & VA & 3122 & -0.3 & -76.071 & 36.9192 & VA & 3194 & 1.5 & -75.9871 & 36.9151 & VA \\
\hline 3051 & -2.9 & -76.138 & 36.924 & VA & 3123 & 1.4 & -75.9882 & 36.9192 & VA & 3195 & 0.2 & -76.1063 & 36.915 & VA \\
\hline 3052 & 1.6 & -76.0601 & 36.924 & VA & 3124 & 0 & -76.1198 & 36.9191 & VA & 3196 & 0.8 & -76.0924 & 36.915 & VA \\
\hline 3053 & -2.9 & -76.1375 & 36.9239 & VA & 3125 & -0.2 & -76.1192 & 36.919 & VA & 3197 & -0.4 & -76.0929 & 36.9149 & VA \\
\hline 3054 & 1.4 & -76.0606 & 36.9238 & VA & 3126 & -0.3 & -76.0715 & 36.919 & VA & 3198 & -0.3 & -76.1057 & 36.9148 & VA \\
\hline 3055 & -1.4 & -75.9931 & 36.9238 & VA & 3127 & -0.3 & -76.0721 & 36.9189 & VA & 3199 & -0.8 & -76.1052 & 36.9147 & VA \\
\hline 3056 & -2.7 & -76.1369 & 36.9237 & VA & 3128 & -0.2 & -76.1187 & 36.9188 & VA & 3200 & 1.4 & -75.987 & 36.9147 & VA \\
\hline 3057 & -2.4 & -76.1364 & 36.9236 & VA & 3129 & -0.3 & -76.0727 & 36.9188 & VA & 3201 & -1.3 & -76.1047 & 36.9145 & VA \\
\hline 3058 & -1.7 & -76.1358 & 36.9235 & VA & 3130 & 1.8 & -75.9881 & 36.9188 & VA & 3202 & -1.9 & -76.1041 & 36.9143 & VA \\
\hline 3059 & 1.3 & -76.0611 & 36.9235 & VA & 3131 & -0.2 & -76.0732 & 36.9187 & VA & 3203 & 0.4 & -76.0963 & 36.9143 & VA \\
\hline 3060 & -1.2 & -75.9927 & 36.9235 & VA & 3132 & -0.1 & -76.1182 & 36.9186 & VA & 3204 & -1.4 & -76.1036 & 36.9142 & VA \\
\hline 3061 & -0.9 & -76.1353 & 36.9233 & VA & 3133 & -0.1 & -76.0738 & 36.9186 & VA & 3205 & 1.4 & -75.9869 & 36.9142 & VA \\
\hline 3062 & 1.4 & -76.0616 & 36.9233 & VA & 3134 & -0.1 & -76.1176 & 36.9185 & VA & 3206 & 2.1 & -76.0969 & 36.9142 & VA \\
\hline 3063 & -0.5 & -76.1347 & 36.9232 & $\mathrm{VA}$ & 3135 & -0.3 & -76.0744 & 36.9185 & VA & 3207 & 3.8 & -76.0975 & 36.9141 & VA \\
\hline 3064 & -1 & -75.9922 & 36.9232 & $\mathrm{VA}$ & 3136 & -0.4 & -76.0749 & 36.9184 & VA & 3208 & -0.9 & -76.103 & 36.914 & VA \\
\hline 3065 & -0.1 & -76.1342 & 36.9231 & VA & 3137 & 0 & -76.1171 & 36.9183 & VA & 3209 & 2.4 & -76.098 & 36.914 & VA \\
\hline 3066 & 1.6 & -76.062 & 36.9231 & VA & 3138 & -0.1 & -76.0755 & 36.9183 & VA & 3210 & 1.1 & -76.1025 & 36.9138 & VA \\
\hline 3067 & -0.3 & -76.1336 & 36.9229 & VA & 3139 & 2.3 & -75.9879 & 36.9183 & VA & 3211 & 1.1 & -76.0986 & 36.9138 & VA \\
\hline 3068 & -0.7 & -75.9918 & 36.9229 & $\mathrm{VA}$ & 3140 & 0.1 & -76.076 & 36.9182 & VA & 3212 & -1.2 & -76.102 & 36.9137 & VA \\
\hline
\end{tabular}




\begin{tabular}{|c|c|c|c|c|c|c|c|c|c|c|c|c|c|c|}
\hline TR\# & RATE & LONG & LAT & ST & TR\# & RATE & LONG & LAT & ST & TR\# & RATE & LONG & LAT & ST \\
\hline 3213 & 0.9 & -76.0991 & 36.9137 & $\mathrm{VA}$ & 3285 & -0.4 & -75.9827 & 36.8945 & VA & 3357 & -0.3 & -75.9724 & 36.8628 & $\overline{V A}$ \\
\hline 3214 & 1.4 & -75.9867 & 36.9137 & VA & 3286 & -0.5 & -75.9826 & 36.894 & VA & 3358 & -0.3 & -75.9723 & 36.8624 & VA \\
\hline 3215 & 0.8 & -76.0997 & 36.9136 & VA & 3287 & -0.5 & -75.9825 & 36.8936 & VA & 3359 & -0.3 & -75.9722 & 36.8619 & VA \\
\hline 3216 & -1.5 & -76.1014 & 36.9135 & VA & 3288 & -0.5 & -75.9824 & 36.8931 & VA & 3360 & -0.4 & -75.9721 & 36.8615 & VA \\
\hline 3217 & 0.9 & -76.1003 & 36.9135 & VA & 3289 & -0.5 & -75.9822 & 36.8927 & VA & 3361 & -0.3 & -75.9719 & 36.861 & VA \\
\hline 3218 & 0.9 & -76.1008 & 36.9134 & VA & 3290 & -0.5 & -75.9821 & 36.8922 & VA & 3362 & -0.2 & -75.9718 & 36.8606 & VA \\
\hline 3219 & -1.9 & -76.1009 & 36.9133 & VA & 3291 & -0.4 & -75.982 & 36.8917 & VA & 3363 & -0.3 & -75.9717 & 36.8601 & VA \\
\hline 3220 & 1 & -76.1014 & 36.9133 & VA & 3292 & -0.2 & -75.9819 & 36.8913 & VA & 3364 & -0.3 & -75.9716 & 36.8597 & VA \\
\hline 3221 & 1.3 & -75.9866 & 36.9133 & VA & 3293 & -0.2 & -75.9817 & 36.8908 & VA & 3365 & -0.3 & -75.9714 & 36.8592 & VA \\
\hline 3222 & -1.7 & -76.1003 & 36.9132 & VA & 3294 & -0.2 & -75.9816 & 36.8904 & VA & 3366 & -0.3 & -75.9713 & 36.8587 & VA \\
\hline 3223 & -1.4 & -76.0998 & 36.913 & VA & 3295 & -0.2 & -75.9815 & 36.8899 & VA & 3367 & -0.3 & -75.9712 & 36.8583 & VA \\
\hline 3224 & -1.5 & -76.0993 & 36.9128 & VA & 3296 & -0.2 & -75.9814 & 36.8895 & VA & 3368 & -0.3 & -75.9711 & 36.8578 & VA \\
\hline 3225 & 1.2 & -75.9865 & 36.9128 & VA & 3297 & -0.2 & -75.9812 & 36.889 & VA & 3369 & -0.1 & -75.971 & 36.8574 & VA \\
\hline 3226 & -1.8 & -76.0987 & 36.9127 & VA & 3298 & -0.2 & -75.9811 & 36.8886 & VA & 3370 & 0 & -75.9708 & 36.8569 & VA \\
\hline 3227 & -1.7 & -76.0982 & 36.9125 & VA & 3299 & -0.2 & -75.981 & 36.8881 & VA & 3371 & 0 & -75.9707 & 36.8565 & VA \\
\hline 3228 & 1.3 & -75.9864 & 36.9124 & $\mathrm{VA}$ & 3300 & -0.1 & -75.9809 & 36.8877 & VA & 3372 & 0 & -75.9706 & 36.856 & VA \\
\hline 3229 & -1.4 & -76.0977 & 36.9123 & VA & 3301 & -0.2 & -75.9807 & 36.8872 & VA & 3373 & 0 & -75.9705 & 36.8556 & VA \\
\hline 3230 & -1.3 & -76.0971 & 36.9122 & VA & 3302 & -0.2 & -75.9806 & 36.8868 & VA & 3374 & 0 & -75.9703 & 36.8551 & VA \\
\hline 3231 & -1.3 & -76.0966 & 36.912 & VA & 3303 & -0.3 & -75.9805 & 36.8863 & VA & 3375 & 0 & -75.9702 & 36.8547 & VA \\
\hline 3232 & 1.4 & -75.9863 & 36.9119 & VA & 3304 & -0.4 & -75.9804 & 36.8858 & VA & 3376 & 0.1 & -75.9701 & 36.8542 & VA \\
\hline 3233 & -0.9 & -76.096 & 36.9118 & VA & 3305 & -0.2 & -75.9802 & 36.8854 & VA & 3377 & 0 & -75.97 & 36.8537 & VA \\
\hline 3234 & -0.3 & -76.0955 & 36.9117 & VA & 3306 & 0 & -75.9801 & 36.8849 & VA & 3378 & 0 & -75.9699 & 36.8533 & VA \\
\hline 3235 & -1 & -76.095 & 36.9115 & VA & 3307 & 0.1 & -75.98 & 36.8845 & VA & 3379 & -0.1 & -75.9697 & 36.8528 & VA \\
\hline 3236 & 1.2 & -75.9862 & 36.9115 & VA & 3308 & 0.1 & -75.9799 & 36.884 & VA & 3380 & -0.2 & -75.9696 & 36.8524 & VA \\
\hline 3237 & -1.8 & -76.0944 & 36.9113 & VA & 3309 & 0.1 & .75 .9797 & 36.8836 & VA & 3381 & -0.1 & -75.9695 & 36.8519 & VA \\
\hline 3238 & -0.8 & -76.0939 & 36.9112 & VA & 3310 & 0 & -75.9796 & 36.8831 & VA & 3382 & -0.1 & -75.9694 & 36.8515 & VA \\
\hline 3239 & 0.2 & -76.0933 & 36.911 & VA & 3311 & 0.1 & -75.9795 & 36.8827 & VA & 3383 & -0.1 & -75.9692 & 36.851 & VA \\
\hline 3240 & 1 & -75.986 & 36.911 & VA & 3312 & 0.2 & -75.9794 & 36.8822 & VA & 3384 & -0.1 & -75.9691 & 36.8506 & VA \\
\hline 3241 & -1.1 & -76.0928 & 36.9108 & VA & 3313 & 0.2 & -75.9792 & 36.8818 & VA & 3385 & -0.2 & -75.969 & 36.8501 & VA \\
\hline 3242 & -2.5 & -76.0923 & 36.9107 & VA & 3314 & 0.1 & -75.9791 & 36.8813 & VA & 3386 & -0.2 & -75.9689 & 36.8496 & VA \\
\hline 3243 & 25 & -76.0917 & 36.9105 & VA & 3315 & 0.2 & -75.979 & 36.8809 & VA & 3387 & -0.2 & -75.9688 & 36.8492 & VA \\
\hline 3244 & 1.1 & -75.9859 & 36.9105 & VA & 3316 & 0.2 & -75.9789 & 36.8804 & VA & 3388 & -0.2 & -75.9686 & 36.8487 & VA \\
\hline 3245 & 54.9 & -76.0912 & 36.9103 & VA & 3317 & 0.1 & -75.9787 & 36.88 & VA & 3389 & -0.2 & -75.9685 & 36.8483 & VA \\
\hline 3246 & 27.8 & -76.0906 & 36.9102 & VA & 3318 & -0.1 & -75.9786 & 36.8795 & VA & 3390 & -0.2 & -75.9684 & 36.8478 & VA \\
\hline 3247 & 1.2 & -75.9858 & 36.9101 & VA & 3319 & -0.1 & -75.9785 & 36.879 & VA & 3391 & -0.3 & -75.9683 & 36.8474 & VA \\
\hline 3248 & 0.6 & -76.0901 & 36.91 & VA & 3320 & -0.1 & -75.9784 & 36.8786 & VA & 3392 & -0.3 & -75.9681 & 36.8469 & VA \\
\hline 3249 & 1.2 & -75.9857 & 36.9096 & VA & 3321 & -0.1 & -75.9782 & 36.8781 & VA & 3393 & -0.3 & -75.968 & 36.8465 & VA \\
\hline 3250 & 1 & -75.9856 & 36.9092 & VA & 3322 & 0 & -75.9781 & 36.8777 & VA & 3394 & -0.3 & -75.9679 & 36.846 & VA \\
\hline 3251 & 1 & -75.9854 & 36.9087 & VA & 3323 & -0.2 & -75.978 & 36.8772 & VA & 3395 & -0.2 & -75.9678 & 36.8456 & VA \\
\hline 3252 & 1 & -75.9853 & 36.9083 & VA & 3324 & -0.4 & -75.9779 & 36.8768 & VA & 3396 & -0.2 & -75.9677 & 36.8451 & VA \\
\hline 3253 & 1 & -75.9852 & 36.9078 & VA & 3325 & -0.5 & -75.9777 & 36.8763 & VA & 3397 & 0 & -75.9675 & 36.8446 & VA \\
\hline 3254 & 1 & -75.9851 & 36.9074 & VA & 3326 & -0.6 & -75.9776 & 36.8759 & VA & 3398 & 0.3 & -75.9674 & 36.8442 & VA \\
\hline 3255 & 1 & -75.985 & 36.9069 & VA & 3327 & -0.7 & -75.9775 & 36.8754 & VA & 3399 & 0.4 & -75.9673 & 36.8437 & VA \\
\hline 3256 & 1 & -75.9848 & 36.9064 & VA & 3328 & -0.9 & -75.9774 & 36.875 & $V_{A}$ & 3400 & 0.6 & -75.9672 & 36.8433 & VA \\
\hline 3257 & 1 & -75.9847 & 36.906 & $\mathrm{VA}$ & 3329 & -0.8 & -75.9772 & 36.8745 & VA & 3401 & 0.5 & -75.967 & 36.8428 & VA \\
\hline 3258 & 1 & -75.9846 & 36.9055 & VA & 3330 & -0.7 & -75.9771 & 36.8741 & $\mathrm{VA}_{\mathrm{A}}$ & 3402 & 0.5 & -75.9669 & 36.8424 & VA \\
\hline 3259 & 0.9 & -75.9845 & 36.9051 & VA & 3331 & -0.7 & -75.977 & 36.8736 & VA & 3403 & 0.5 & -75.9668 & 36.8419 & VA \\
\hline 3260 & 0.9 & -75.9844 & 36.9046 & VA & 3332 & -0.7 & -75.9769 & 36.8731 & VA & 3404 & 0.4 & -75.9667 & 36.8415 & VA \\
\hline 3261 & 0.8 & -75.9842 & 36.9042 & VA & 3333 & -0.7 & -75.9767 & 36.8727 & VA & 3405 & 0.4 & -75.9665 & 36.841 & VA \\
\hline 3262 & 0.7 & -75.9841 & 36.9037 & VA & 3334 & -0.6 & -75.9766 & 36.8722 & VA & 3406 & 0.3 & -75.9664 & 36.8405 & VA \\
\hline 3263 & 0.9 & -75.984 & 36.9032 & $\mathrm{VA}$ & 3335 & -0.7 & -75.9765 & 36.8718 & VA & 3407 & 0.3 & -75.9663 & 36.8401 & VA \\
\hline 3264 & 1 & -75.9839 & 36.9028 & VA & 3336 & -0.9 & -75.9764 & 36.8713 & $\mathrm{VA}$ & 3408 & 0.3 & -75.9662 & 36.8396 & VA \\
\hline 3265 & 1 & -75.9838 & 36.9023 & VA & 3337 & -0.9 & -75.9762 & 36.8709 & VA & 3409 & 0.3 & -75.9661 & 36.8392 & VA \\
\hline 3266 & 1 & -75.9836 & 36.9019 & $\mathrm{VA}$ & 3338 & -1 & -75.9761 & 36.8704 & VA & 3410 & 0.3 & -75.9659 & 36.8387 & VA \\
\hline 3267 & 1.2 & -75.9835 & 36.9014 & VA & 3339 & -1.1 & .75 .976 & 36.87 & VA & 3411 & 0.3 & -75.9658 & 36.8383 & VA \\
\hline 3268 & 1.4 & -75.9834 & 36.901 & VA & 3340 & -1.2 & -75.9759 & 36.8695 & VA & 3412 & 0.3 & -75.9657 & 36.8378 & VA \\
\hline 3269 & 1.4 & -75.9833 & 36.9005 & VA & 3341 & -1.2 & -75.9757 & 36.8691 & VA & 3413 & 0.2 & -75.9656 & 36.8374 & VA \\
\hline 3270 & 1.4 & -75.9832 & 36.9001 & VA & 3342 & -1.1 & -75.9756 & 36.8686 & $\mathrm{VA}$ & 3414 & 0.2 & -75.9654 & 36.8369 & VA \\
\hline 3271 & 1.4 & -75.983 & 36.8996 & VA & 3343 & -1.1 & -75.9755 & 36.8682 & VA & 3415 & 0.2 & -75.9653 & 36.8365 & VA \\
\hline 3272 & 1.4 & -75.9829 & 36.8991 & VA & 3344 & -1.1 & -75.9754 & 36.8677 & VA & 3416 & 0.3 & -75.9652 & 36.836 & VA \\
\hline 3273 & 1.5 & -75.9828 & 36.8987 & VA & 3345 & -0.4 & -75.9737 & 36.8674 & VA & 3417 & 0.1 & -75.966 & 36.836 & VA \\
\hline 3274 & 1.6 & -75.9827 & 36.8982 & VA & 3346 & -1.2 & -75.9752 & 36.8673 & VA & 3418 & 0.1 & -75.9659 & 36.8355 & VA \\
\hline 3275 & 0 & -75.9837 & 36.8981 & VA & 3347 & -0.5 & -75.9735 & 36.8669 & VA & 3419 & 0.2 & -75.9658 & 36.8351 & VA \\
\hline 3276 & 1.7 & -75.9826 & 36.8978 & VA & 3348 & -1.2 & -75.9751 & 36.8668 & VA & 3420 & 0.2 & -75.9657 & 36.8346 & VA \\
\hline 3277 & 0 & -75.9836 & 36.8976 & $\mathrm{VA}$ & 3349 & -0.4 & -75.9734 & 36.8665 & VA & 3421 & 0.2 & -75.9656 & 36.8342 & VA \\
\hline 3278 & 1.9 & -75.9824 & 36.8973 & $\mathrm{VA}$ & 3350 & -0.4 & -75.9733 & 36.866 & VA & 3422 & 0.2 & -75.9655 & 36.8337 & VA \\
\hline 3279 & -0.1 & .75 .9835 & 36.8972 & $V_{A}$ & 3351 & -0.4 & -75.9732 & 36.8656 & VA & 3423 & 0.2 & -75.9653 & 36.8332 & VA \\
\hline 3280 & -0.2 & -75.9834 & 36.8967 & VA & 3352 & -0.5 & -75.973 & 36.8651 & VA & 3424 & 0.3 & -75.9652 & 36.8328 & VA \\
\hline 3281 & -0.3 & -75.9832 & 36.8963 & VA & 3353 & -0.4 & -75.9729 & 36.8647 & VA & 3425 & 0.3 & -75.9651 & 36.8323 & VA \\
\hline 3282 & -0.4 & -75.9831 & 36.8958 & VA & 3354 & -0.4 & -75.9728 & 36.8642 & VA & 3426 & 0.3 & -75.965 & 36.8319 & VA \\
\hline 3283 & -0.4 & -75.983 & 36.8954 & VA & 3355 & -0.4 & -75.9727 & 36.8638 & VA & 3427 & 0.5 & -75.9649 & 36.8314 & VA \\
\hline 3284 & -0.4 & -75.9829 & 36.8949 & VA & 3356 & -0.4 & -75.9726 & 36.8633 & VA & 3428 & 0.7 & -75.9648 & 36.8309 & VA \\
\hline
\end{tabular}




\begin{tabular}{|c|c|c|c|c|c|c|c|c|c|c|c|c|c|c|}
\hline TR\# & RATE & LONG & LAT & ST & IR\# & RATE & LONG & LAT & ST & TR\# & RATE & LONG & LAT & ST \\
\hline 3429 & 0.3 & -75.9647 & 36.8305 & $\overline{V A}$ & 3501 & 0.2 & -75.9575 & 36.7986 & $\overline{\mathrm{VA}}$ & 3573 & 0.3 & -75.9466 & 36.7677 & $\overline{V A}$ \\
\hline 3430 & -0.2 & -75.9646 & 36.83 & VA & 3502 & 0.2 & -75.9574 & 36.7981 & VA & 3574 & 0.3 & -75.9464 & 36.7673 & VA \\
\hline 3431 & 0.2 & -75.9645 & 36.8296 & VA & 3503 & 0.1 & -75.9573 & 36.7977 & VA & 3575 & 0.1 & -75.9463 & 36.7668 & VA \\
\hline 3432 & 0.6 & -75.9644 & 36.8291 & VA & 3504 & -0.1 & -75.9571 & 36.7972 & VA & 3576 & 0 & -75.9461 & 36.7664 & VA \\
\hline 3433 & 0.6 & -75.9643 & 36.8287 & VA & 3505 & -0.1 & -75.957 & 36.7968 & VA & 3577 & 0 & -75.9459 & 36.7659 & VA \\
\hline 3434 & 0.6 & -75.9642 & 36.8282 & VA & 3506 & -0.1 & -75.9569 & 36.7963 & $\mathrm{VA}$ & 3578 & -0.1 & -75.9458 & 36.7655 & VA \\
\hline 3435 & 0.4 & -75.9641 & 36.8277 & VA & 3507 & -0.2 & -75.9567 & 36.7959 & VA & 3579 & -0.2 & -75.9456 & 36.7651 & VA \\
\hline 3436 & 0.3 & -75.964 & 36.8273 & VA & 3508 & -0.3 & -75.9566 & 36.7954 & VA & 3580 & -0.3 & -75.9454 & 36.7646 & VA \\
\hline 3437 & 0.3 & -75.9638 & 36.8268 & VA & 3509 & -0.3 & -75.9565 & 36.7949 & VA & 3581 & -0.3 & -75.9453 & 36.7642 & VA \\
\hline 3438 & 0.2 & -75.9637 & 36.8264 & VA & 3510 & -0.2 & -75.9563 & 36.7945 & VA & 3582 & -0.4 & -75.9451 & 36.7637 & VA \\
\hline 3439 & 0.1 & -75.9636 & 36.8259 & VA & 3511 & -0.2 & -75.9562 & 36.794 & VA & 3583 & -0.3 & -75.9449 & 36.7633 & VA \\
\hline 3440 & 0 & -75.9635 & 36.8255 & VA & 3512 & -0.1 & -75.956 & 36.7936 & VA & 3584 & -0.3 & -75.9448 & 36.7628 & VA \\
\hline 3441 & 0 & -75.9634 & 36.825 & VA & 3513 & -0.1 & -75.9559 & 36.7931 & VA & 3585 & -0.2 & -75.9446 & 36.7624 & VA \\
\hline 3442 & -0.1 & -75.9633 & 36.8245 & VA & 3514 & -0.1 & -75.9558 & 36.7927 & VA & 3586 & -0.2 & -75.9444 & 36.7619 & VA \\
\hline 3443 & -0.1 & -75.9632 & 36.8241 & VA & 3515 & -0.1 & -75.9556 & 36.7922 & VA & 3587 & 0 & -75.9443 & 36.7615 & VA \\
\hline 3444 & -0.2 & -75.9631 & 36.8236 & VA & 3516 & -0.1 & -75.9555 & 36.7918 & VA & 3588 & 0.1 & -75.9441 & 36.7611 & VA \\
\hline 3445 & -0.2 & -75.963 & 36.8232 & VA & 3517 & -0.1 & -75.9554 & 36.7913 & VA & 3589 & 0.3 & -75.9439 & 36.7606 & VA \\
\hline 3446 & -0.3 & -75.9629 & 36.8227 & VA & 3518 & -0.1 & -75.9552 & 36.7909 & VA & 3590 & 0.4 & -75.9438 & 36.7602 & VA \\
\hline 3447 & -0.3 & -75.9628 & 36.8223 & VA & 3519 & -0.1 & -75.9551 & 36.7904 & VA & 3591 & 0.3 & -75.9436 & 36.7597 & VA \\
\hline 3448 & -0.3 & -75.9627 & 36.8218 & VA & 3520 & -0.1 & -75.955 & 36.79 & VA & 3592 & 0.1 & -75.9435 & 36.7593 & VA \\
\hline 3449 & -0.4 & -75.9626 & 36.8213 & VA & 3521 & 0 & -75.9548 & 36.7895 & VA & 3593 & 0.1 & -75.9433 & 36.7588 & VA \\
\hline 3450 & -0.4 & -75.9625 & 36.8209 & VA & 3522 & 0 & -75.9547 & 36.7891 & VA & 3594 & 0 & -75.9431 & 36.7584 & VA \\
\hline 3451 & -0.4 & -75.9623 & 36.8204 & VA & 3523 & -0.1 & -75.9546 & 36.7886 & VA & 3595 & 0 & -75.943 & 36.758 & VA \\
\hline 3452 & -0.4 & -75.9622 & 36.82 & VA & 3524 & -0.2 & -75.9544 & 36.7882 & VA & 3596 & 0.1 & -75.9428 & 36.7575 & VA \\
\hline 3453 & -0.4 & -75.9621 & 36.8195 & VA & 3525 & -0.2 & -75.9543 & 36.7877 & VA & 3597 & 0 & -75.9426 & 36.7571 & VA \\
\hline 3454 & -0.5 & -75.962 & 36.819 & VA & 3526 & -0.3 & .75 .9542 & 36.7873 & VA & 3598 & -0.1 & .75 .9425 & 36.7566 & VA \\
\hline 3455 & -0.6 & -75.9619 & 36.8186 & VA & 3527 & -0.3 & -75.954 & 36.7868 & VA & 3599 & -0.1 & -75.9423 & 36.7562 & VA \\
\hline 3456 & -0.6 & -75.9618 & 36.8181 & VA & 3528 & -0.3 & -75.9539 & 36.7864 & VA & 3600 & -0.1 & -75.9421 & 36.7557 & VA \\
\hline 3457 & -0.6 & -75.9617 & 36.8177 & VA & 3529 & $-0.4^{-}$ & -75.9538 & 36.7859 & VA & 3601 & -0.2 & -75.942 & 36.7553 & VA \\
\hline 3458 & -0.6 & -75.9616 & 36.8172 & VA & 3530 & -0.4 & -75.9536 & 36.7855 & VA & 3602 & -0.2 & -75.9418 & 36.7548 & VA \\
\hline 3459 & -0.7 & -75.9615 & 36.8168 & VA & 3531 & -0.4 & -75.9535 & 36.785 & VA & 3603 & -0.3 & -75.9416 & 36.7544 & VA \\
\hline 3460 & -0.8 & -75.9614 & 36.8163 & VA & 3532 & -0.4 & -75.9533 & 36.7846 & VA & 3604 & -0.4 & -75.9415 & 36.754 & VA \\
\hline 3461 & -0.8 & -75.9613 & 36.8158 & VA & 3533 & -0.4 & -75.9532 & 36.7841 & VA & 3605 & -0.5 & -75.9413 & 36.7535 & VA \\
\hline 3462 & -0.8 & -75.9612 & 36.8154 & VA & 3534 & -0.3 & -75.9531 & 36.7836 & VA & 3606 & -0.5 & -75.9411 & 36.7531 & VA \\
\hline 3463 & -0.8 & -75.9611 & 36.8149 & VA & 3535 & -0.3 & -75.9529 & 36.7832 & VA & 3607 & -0.5 & -75.941 & 36.7526 & VA \\
\hline 3464 & -0.8 & -75.961 & 36.8145 & VA & 3536 & -0.3 & -75.9528 & 36.7827 & VA & 3608 & -0.6 & -75.9408 & 36.7522 & VA \\
\hline 3465 & -0.8 & -75.9608 & 36.814 & VA & 3537 & -0.2 & .75 .9527 & 36.7823 & VA & 3609 & -0.5 & -75.9406 & 36.7517 & VA \\
\hline 3466 & -0.8 & -75.9607 & 36.8136 & VA & 3538 & -0.1 & -75.9525 & 36.7818 & VA & 3610 & -0.4 & -75.9405 & 36.7513 & VA \\
\hline 3467 & -0.8 & -75.9606 & 36.8131 & VA & 3539 & -0.1 & -75.9524 & 36.7814 & VA & 3611 & -0.4 & -75.9403 & 36.7509 & VA \\
\hline 3468 & -0.8 & -75.9605 & 36.8126 & VA & 3540 & -0.1 & -75.9523 & 36.7809 & VA & 3612 & -0.4 & -75.9401 & 36.7504 & VA \\
\hline 3469 & -0.7 & -75.9604 & 36.8122 & VA & 3541 & -0.2 & -75.9521 & 36.7805 & VA & 3613 & -0.5 & -75.94 & 36.75 & VA \\
\hline 3470 & -0.6 & -75.9603 & 36.8117 & VA & 3542 & -0.2 & -75.952 & 36.78 & VA & 3614 & -0.6 & -75.9398 & 36.7495 & VA \\
\hline 3471 & -0.5 & -75.9602 & 36.8113 & VA & 3543 & -0.2 & -75.9519 & 36.7796 & VA & 3615 & -0.5 & -75.9396 & 36.7491 & VA \\
\hline 3472 & -0.4 & -75.9601 & 36.8108 & VA & 3544 & -0.2 & -75.9517 & 36.7791 & VA & 3616 & -0.5 & -75.9395 & 36.7486 & VA \\
\hline 3473 & -0.4 & -75.96 & 36.8104 & VA & 3545 & -0.1 & -75.9516 & 36.7787 & VA & 3617 & -0.7 & -75.9393 & 36.7482 & VA \\
\hline 3474 & -0.5 & -75.9599 & 36.8099 & VA & 3546 & -0.1 & -75.9515 & 36.7782 & VA & 3618 & -0.8 & -75.9391 & 36.7477 & VA \\
\hline 3475 & -0.4 & -75.9598 & 36.8094 & VA & 3547 & -0.1 & .75 .9513 & 36.7778 & VA & 3619 & -0.8 & -75.939 & 36.7473 & VA \\
\hline 3476 & -0.3 & -75.9597 & 36.809 & VA & 3548 & -0.2 & -75.9512 & 36.7773 & VA & 3620 & -0.9 & -75.9388 & 36.7469 & VA \\
\hline 3477 & -1.4 & -75.9596 & 36.8085 & VA & 3549 & -0.2 & -75.951 & 36.7769 & VA & 3621 & -0.9 & -75.9386 & 36.7464 & VA \\
\hline 3478 & -2.5 & -75.9595 & 36.8081 & VA & 3550 & -0.3 & -75.9509 & 36.7764 & VA & 3622 & -1 & -75.9385 & 36.746 & VA \\
\hline 3479 & -1.4 & -75.9593 & 36.8076 & VA & 3551 & -0.3 & -75.9508 & 36.776 & VA & 3623 & -0.9 & -75.9383 & 36.7455 & VA \\
\hline 3480 & -0.3 & -75.9592 & 36.8071 & VA & 3552 & -0.3 & -75.9506 & 36.7755 & VA & 3624 & -0.8 & -75.9381 & 36.7451 & VA \\
\hline 3481 & -0.3 & -75.9591 & 36.8067 & VA & 3553 & -0.3 & -75.9505 & 36.7751 & VA & 3625 & -0.8 & -75.938 & 36.7446 & VA \\
\hline 3482 & -0.4 & -75.959 & 36.8062 & VA & 3554 & 1.1 & -75.9493 & 36.7748 & VA & 3626 & -1.1 & -75.938 & 36.7446 & VA \\
\hline 3483 & -0.3 & -75.9589 & 36.8058 & VA & 3555 & -0.3 & -75.9504 & 36.7746 & VA & 3627 & -0.8 & -75.9378 & 36.7442 & VA \\
\hline 3484 & -0.3 & -75.9588 & 36.8053 & VA & 3556 & 1.1 & -75.9491 & 36.7744 & VA & 3628 & -1.1 & -75.9379 & 36.7441 & VA \\
\hline 3485 & -0.2 & -75.9587 & 36.8049 & VA & 3557 & -0.4 & -75.9502 & 36.7742 & VA & 3629 & -1 & -75.9377 & 36.7437 & VA \\
\hline 3486 & 0.5 & -75.9594 & 36.8049 & VA & 3558 & 1.1 & -75.9489 & 36.7739 & VA & 3630 & -1 & -75.9375 & 36.7432 & VA \\
\hline 3487 & -0.1 & .75 .9586 & 36.8044 & VA & 3559 & -0.4 & -75.9501 & 36.7737 & VA & 3631 & -0.9 & -75.9373 & 36.7428 & VA \\
\hline 3488 & 0.6 & .75 .9593 & 36.8044 & VA & 3560 & 1.1 & -75.9488 & 36.7735 & VA & 3632 & -0.8 & -75.9372 & 36.7423 & VA \\
\hline 3489 & 0.5 & -75.9592 & 36.804 & VA & 3561 & 0.9 & -75.9486 & 36.773 & VA & 3633 & -0.6 & -75.937 & 36.7419 & VA \\
\hline 3490 & 0.4 & -75.959 & 36.8035 & VA & 3562 & 0.8 & -75.9484 & 36.7726 & VA & 3634 & -0.6 & -75.9368 & 36.7414 & VA \\
\hline 3491 & 0.4 & -75.9589 & 36.8031 & VA & 3563 & 0.8 & -75.9483 & 36.7722 & VA & 3635 & -0.6 & -75.9367 & 36.741 & VA \\
\hline 3492 & 0.4 & -75.9588 & 36.8026 & VA & 3564 & 0.8 & -75.9481 & 36.7717 & VA & 3636 & -0.5 & -75.9365 & 36.7405 & VA \\
\hline 3493 & 0.4 & -75.9586 & 36.8022 & VA & 3565 & 0.7 & -75.9479 & 36.7713 & VA & 3637 & -0.4 & -75.9363 & 36.7401 & VA \\
\hline 3494 & 0.3 & -75.9585 & 36.8017 & VA & 3566 & 0.6 & -75.9478 & 36.7708 & VA & 3638 & -0.3 & -75.9361 & 36.7397 & VA \\
\hline 3495 & 0.2 & -75.9583 & 36.8013 & VA & 3567 & 0.6 & -75.9476 & 36.7704 & VA & 3639 & -0.3 & -75.936 & 36.7392 & VA \\
\hline 3496 & 0.1 & -75.9582 & 36.8008 & VA & 3568 & 0.5 & -75.9474 & 36.7699 & VA & 3640 & -0.2 & -75.9358 & 36.7388 & VA \\
\hline 3497 & 0.1 & -75.9581 & 36.8004 & VA & 3569 & 0.4 & -75.9473 & 36.7695 & VA & 3641 & -0.2 & -75.9356 & 36.7383 & VA \\
\hline 3498 & 0.1 & -75.9579 & 36.7999 & VA & 3570 & 0.3 & -75.9471 & 36.769 & VA & 3642 & -0.3 & -75.9355 & 36.7379 & VA \\
\hline 3499 & 0.1 & .75 .9578 & 36.7995 & VA & 3571 & 0.3 & -75.9469 & 36.7686 & VA & 3643 & -0.4 & -75.9353 & 36.7374 & VA \\
\hline 3500 & 0.2 & -75.9577 & 36.799 & VA & 3572 & 0.3 & -75.9468 & 36.7682 & VA & 3644 & -0.5 & -75.9351 & 36.737 & VA \\
\hline
\end{tabular}




\begin{tabular}{|c|c|c|c|c|c|c|c|c|c|c|c|c|c|c|}
\hline TR\# & RATE & LONG & LAT & ST & TR\# & RATE & LONG & LAT & ST & TR\# & RATE & LONG & LAT & ST \\
\hline 3645 & -0.5 & -75.9349 & 36.7365 & $\overline{V A}$ & 3717 & -2.5 & -75.9213 & 36.7063 & $\overline{\mathrm{VA}}$ & 3789 & -1.7 & -75.9076 & 36.6759 & $\overline{\mathrm{VA}}$ \\
\hline 3646 & -0.5 & -75.9348 & 36.7361 & VA & 3718 & -2.5 & -75.9211 & 36.7059 & VA & 3790 & -1.7 & -75.9074 & 36.6754 & VA \\
\hline 3647 & -0.4 & -75.9346 & 36.7357 & VA & 3719 & -2.5 & -75.9209 & 36.7054 & VA & 3791 & -1.6 & -75.9072 & 36.675 & VA \\
\hline 3648 & -0.4 & -75.9344 & 36.7352 & VA & 3720 & -2.5 & -75.9207 & 36.705 & VA & 3792 & -1.7 & -75.9069 & 36.6746 & VA \\
\hline 3649 & -0.4 & -75.9342 & 36.7348 & VA & 3721 & -2.4 & -75.9205 & 36.7045 & VA & 3793 & -1.7 & -75.9067 & 36.6741 & VA \\
\hline 3650 & -0.5 & -75.9341 & 36.7343 & VA & 3722 & -2.5 & -75.9203 & 36.7041 & VA & 3794 & -1.7 & -75.9065 & 36.6737 & VA \\
\hline 3651 & -0.5 & -75.9339 & 36.7339 & VA & 3723 & -2.5 & -75.9201 & 36.7037 & VA & 3795 & -1.7 & -75.9063 & 36.6733 & VA \\
\hline 3652 & -0.6 & -75.9337 & 36.7334 & VA & 3724 & -2.6 & -75.9199 & 36.7032 & VA & 3796 & -1.6 & -75.906 & 36.6729 & VA \\
\hline 3653 & -0.7 & -75.9336 & 36.733 & VA & 3725 & -2.6 & -75.9197 & 36.7028 & VA & 3797 & -1.5 & -75.9058 & 36.6724 & VA \\
\hline 3654 & -0.7 & -75.9334 & 36.7325 & VA & 3726 & -2.6 & -75.9195 & 36.7024 & VA & 3798 & -1.4 & -75.9056 & 36.672 & VA \\
\hline 3655 & -0.7 & -75.9332 & 36.7321 & VA & 3727 & -2.6 & -75.9193 & 36.7019 & VA & 3799 & -1.3 & -75.9054 & 36.6716 & VA \\
\hline 3656 & -0.7 & -75.933 & 36.7316 & VA & 3728 & -2.7 & .75 .9191 & 36.7015 & VA & 3800 & -1.4 & -75.9051 & 36.6711 & VA \\
\hline 3657 & -0.6 & -75.9329 & 36.7312 & VA & 3729 & -2.7 & -75.9189 & 36.701 & VA & 3801 & -1.4 & -75.9049 & 36.6707 & VA \\
\hline 3658 & -0.6 & -75.9327 & 36.7308 & VA & 3730 & -2.7 & -75.9187 & 36.7006 & $\mathrm{VA}_{\mathrm{A}}$ & 3802 & -1.4 & -75.9047 & 36.6703 & VA \\
\hline 3659 & -0.6 & -75.9325 & 36.7303 & VA & 3731 & -2.6 & -75.9185 & 36.7002 & VA & 3803 & -1.5 & -75.9045 & 36.6699 & VA \\
\hline 3660 & -0.7 & -75.9324 & 36.7299 & VA & 3732 & -2.6 & -75.9184 & 36.6997 & VA & 3804 & -1.5 & -75.9042 & 36.6694 & VA \\
\hline 3661 & -0.7 & -75.9322 & 36.7294 & VA & 3733 & -2.5 & -75.9182 & 36.6993 & VA & 3805 & -1.4 & -75.904 & 36.669 & VA \\
\hline 3662 & -0.9 & -75.932 & 36.729 & VA & 3734 & -2.4 & -75.918 & 36.6989 & VA & 3806 & -1.6 & -75.9038 & 36.6686 & VA \\
\hline 3663 & -1.1 & -75.9318 & 36.7285 & VA & 3735 & -2.3 & -75.9178 & 36.6984 & $\mathrm{VA}$ & 3807 & -1.7 & -75.9036 & 36.6681 & VA \\
\hline 3664 & -1.2 & -75.9317 & 36.7281 & VA & 3736 & -2.3 & -75.9176 & 36.698 & VA & 3808 & -1.7 & -75.9033 & 36.6677 & VA \\
\hline 3665 & -1.3 & -75.9315 & 36.7276 & VA & 3737 & -2.3 & -75.9174 & 36.6975 & VA & 3809 & -1.6 & -75.9031 & 36.6673 & VA \\
\hline 3666 & -1.4 & -75.9313 & 36.7272 & VA & 3738 & -2.3 & -75.9172 & 36.6971 & VA & 3810 & -1.5 & -75.9029 & 36.6669 & VA \\
\hline 3667 & -1.4 & -75.9312 & 36.7268 & VA & 3739 & -2.2 & -75.917 & 36.6967 & VA & 3811 & -1.4 & -75.9027 & 36.6664 & VA \\
\hline 3668 & -1.5 & -75.931 & 36.7263 & VA & 3740 & -2.1 & -75.9168 & 36.6962 & VA & 3812 & -1.4 & -75.9024 & 36.666 & VA \\
\hline 3669 & -1.6 & -75.9308 & 36.7259 & VA & 3741 & -2.1 & -75.9166 & 36.6958 & VA & 3813 & -1.4 & -75.9022 & 36.6656 & VA \\
\hline 3670 & -1.6 & -75.9306 & 36.7254 & VA & 3742 & -2 & -75.9164 & 36.6954 & VA & 3814 & -1.4 & -75.902 & 36.6651 & VA \\
\hline 3671 & -1.6 & -75.9305 & 36.725 & VA & 3743 & -1.9 & -75.9162 & 36.6949 & VA & 3815 & -1.5 & -75.9018 & 36.6647 & VA \\
\hline 3672 & -1.7 & -75.9303 & 36.7245 & VA & 3744 & -1.8 & -75.916 & 36.6945 & VA & 3816 & -1.5 & -75.9015 & 36.6643 & VA \\
\hline 3673 & -1.8 & -75.9301 & 36.7241 & VA & 3745 & -1.8 & -75.9158 & 36.694 & VA & 3817 & -1.6 & -75.9013 & 36.6639 & VA \\
\hline 3674 & -1.9 & -75.93 & 36.7236 & VA & 3746 & -1.8 & -75.9156 & 36.6936 & VA & 3818 & -1.7 & -75.9011 & 36.6634 & VA \\
\hline 3675 & -1.9 & -75.9298 & 36.7232 & VA & 3747 & -1.9 & -75.9154 & 36.6932 & VA & 3819 & -1.9 & -75.9009 & 36.663 & VA \\
\hline 3676 & -2 & -75.9296 & 36.7227 & VA & 3748 & -1.8 & -75.9152 & 36.6927 & VA & 3820 & -2 & -75.9006 & 36.6626 & VA \\
\hline 3677 & -2 & -75.9294 & 36.7223 & $\mathrm{VA}$ & 3749 & -1.7 & -75.915 & 36.6923 & $\mathrm{VA}$ & 3821 & -2.1 & -75.9004 & 36.6622 & VA \\
\hline 3678 & -1.9 & -75.9293 & 36.7219 & VA & 3750 & -1.9 & -75.9148 & 36.6918 & VA & 3822 & -2.1 & -75.9002 & 36.6617 & VA \\
\hline 3679 & -1.9 & -75.9291 & 36.7214 & VA & 3751 & -2 & -75.9146 & 36.6914 & VA & 3823 & -2.1 & -75.9 & 36.6613 & VA \\
\hline 3680 & -2 & -75.9289 & 36.721 & VA & 3752 & -2 & -75.9144 & 36.691 & VA & 3824 & -1.9 & -75.8997 & 36.6609 & VA \\
\hline 3681 & -2.1 & -75.9287 & 36.7205 & VA & 3753 & -2.1 & -75.9142 & 36.6905 & VA & 3825 & -1.8 & -75.8995 & 36.6604 & VA \\
\hline 3682 & -2.1 & -75.9286 & 36.7201 & VA & 3754 & -2.1 & -75.914 & 36.6901 & VA & 3826 & -1.9 & -75.8993 & 36.66 & VA \\
\hline 3683 & -2 & -75.9284 & 36.7196 & VA & 3755 & -2.1 & -75.9138 & 36.6897 & VA & 3827 & -2 & -75.8991 & 36.6596 & VA \\
\hline 3684 & -1.9 & -75.9282 & 36.7192 & VA & 3756 & -2.1 & -75.9136 & 36.6892 & VA & 3828 & -2 & -75.8988 & 36.6592 & VA \\
\hline 3685 & -1.9 & -75.9281 & 36.7187 & VA & 3757 & -2.1 & -75.9134 & 36.6888 & VA & 3829 & -2.1 & -75.8986 & 36.6587 & VA \\
\hline 3686 & -1.9 & -75.9279 & 36.7183 & VA & 3758 & -2.1 & -75.9132 & 36.6883 & VA & 3830 & -2.1 & -75.8984 & 36.6583 & VA \\
\hline 3687 & -2 & -75.9277 & 36.7179 & VA & 3759 & -2.1 & -75.913 & 36.6879 & VA & 3831 & -2.1 & -75.8982 & 36.6579 & VA \\
\hline 3688 & -2 & -75.9275 & 36.7174 & VA & 3760 & -2.1 & -75.9128 & 36.6875 & VA & 3832 & -2 & -75.8979 & 36.6574 & VA \\
\hline 3689 & -2 & -75.9274 & 36.717 & VA & 3761 & -2.1 & -75.9126 & 36.687 & VA & 3833 & -1.9 & -75.8977 & 36.657 & VA \\
\hline 3690 & -2 & -75.9272 & 36.7165 & VA & 3762 & -2.1 & -75.9124 & 36.6866 & VA & 3834 & -1.9 & -75.8975 & 36.6566 & VA \\
\hline 3691 & -2 & -75.927 & 36.7161 & $\mathrm{VA}$ & 3763 & -2.1 & -75.9122 & 36.6862 & VA & 3835 & -1.8 & -75.8973 & 36.6562 & VA \\
\hline 3692 & -2.1 & -75.9269 & 36.7156 & VA & 3764 & -2.1 & -75.912 & 36.6857 & VA & 3836 & -1.9 & -75.897 & 36.6557 & VA \\
\hline 3693 & -2.3 & -75.9267 & 36.7152 & VA & 3765 & -2.1 & -75.9118 & 36.6853 & VA & 3837 & 1.1 & -75.8977 & 36.6555 & VA \\
\hline 3694 & -2.3 & -75.9265 & 36.7147 & VA & 3766 & -2.1 & -75.9116 & 36.6848 & VA & 3838 & -1.9 & -75.8968 & 36.6553 & VA \\
\hline 3695 & -2.7 & -75.9251 & 36.7146 & VA & 3767 & -2.4 & -75.9123 & 36.6848 & VA & 3839 & 1.1 & -75.8975 & 36.6551 & VA \\
\hline 3696 & -2.3 & -75.9263 & 36.7143 & VA & 3768 & -2.1 & -75.9114 & 36.6844 & VA & 3840 & 1.3 & -75.8973 & 36.6547 & VA \\
\hline 3697 & -2.7 & -75.9249 & 36.7142 & VA & 3769 & -2.4 & -75.9121 & 36.6844 & VA & 3841 & 1.4 & -75.8971 & 36.6542 & VA \\
\hline 3698 & -2.3 & -75.9262 & 36.7138 & VA & 3770 & -2.4 & -75.9119 & 36.684 & VA & 3842 & 1.3 & -75.8969 & 36.6538 & VA \\
\hline 3699 & -2.8 & -75.9247 & 36.7137 & VA & 3771 & -2.3 & -75.9117 & 36.6836 & $V_{A}$ & 3843 & 1.2 & -75.8967 & 36.6534 & VA \\
\hline 3700 & -2.4 & -75.926 & 36.7134 & VA & 3772 & -2.3 & -75.9114 & 36.6831 & VA & 3844 & 1.1 & .75 .8965 & 36.6529 & VA \\
\hline 3701 & -2.8 & -75.9245 & 36.7133 & VA & 3773 & -2.3 & -75.9112 & 36.6827 & VA & 3845 & 1.1 & -75.8962 & 36.6525 & VA \\
\hline 3702 & -2.7 & -75.9243 & 36.7129 & VA & 3774 & -2.3 & -75.911 & 36.6823 & VA & 3846 & 1.1 & -75.896 & 36.6521 & VA \\
\hline 3703 & -2.7 & -75.9241 & 36.7124 & VA & 3775 & -2.2 & -75.9108 & 36.6818 & VA & 3847 & 1.2 & -75.8958 & 36.6516 & VA \\
\hline 3704 & -2.8 & -75.9239 & 36.712 & VA & 3776 & -2.2 & -75.9105 & 36.6814 & VA & 3848 & 1.2 & -75.8956 & 36.6512 & VA \\
\hline 3705 & -2.9 & -75.9237 & 36.7116 & VA & 3777 & -2.1 & -75.9103 & 36.681 & VA & 3849 & 1.3 & -75.8954 & 36.6508 & VA \\
\hline 3706 & -2.9 & -75.9235 & 36.7111 & VA & 3778 & -2 & -75.9101 & 36.6806 & VA & 3850 & 1.3 & -75.8952 & 36.6503 & VA \\
\hline 3707 & -2.8 & -75.9233 & 36.7107 & VA & 3779 & -1.9 & -75.9099 & 36.6801 & VA & 3851 & 1.3 & -75.895 & 36.6499 & VA \\
\hline 3708 & -2.9 & -75.9231 & 36.7102 & VA & 3780 & -1.9 & -75.9096 & 36.6797 & VA & 3852 & 1.1 & -75.8948 & 36.6495 & VA \\
\hline 3709 & -3 & -75.9229 & 36.7098 & VA & 3781 & -1.8 & -75.9094 & 36.6793 & VA & 3853 & 1 & -75.8946 & 36.649 & VA \\
\hline 3710 & -3 & -75.9227 & 36.7094 & VA & 3782 & -1.8 & -75.9092 & 36.6788 & VA & 3854 & 0.9 & -75.8944 & 36.6486 & VA \\
\hline 3711 & -3 & -75.9225 & 36.7089 & VA & 3783 & -1.7 & -75.909 & 36.6784 & VA & 3855 & 0.9 & -75.8942 & 36.6482 & VA \\
\hline 3712 & -3 & -75.9223 & 36.7085 & VA & 3784 & -1.7 & -75.9087 & 36.678 & VA & 3856 & 0.9 & -75.894 & 36.6477 & VA \\
\hline 3713 & -3 & -75.9221 & 36.7081 & VA & 3785 & -1.7 & -75.9085 & 36.6776 & VA & 3857 & 1 & -75.8938 & 36.6473 & VA \\
\hline 3714 & -2.9 & -75.9219 & 36.7076 & VA & 3786 & -1.7 & -75.9083 & 36.6771 & VA & 3858 & 1 & -75.8936 & 36.6469 & VA \\
\hline 3715 & -2.8 & -75.9217 & 36.7072 & VA & 3787 & -1.7 & -75.9081 & 36.6767 & VA & 3859 & 1 & -75.8934 & 36.6464 & VA \\
\hline 3716 & -2.7 & -75.9215 & 36.7067 & VA & 3788 & -1.7 & -75.9078 & 36.6763 & VA & 3860 & 0.8 & -75.8932 & 36.646 & VA \\
\hline
\end{tabular}




\begin{tabular}{|c|c|c|c|c|c|c|c|c|c|c|c|c|c|c|}
\hline TR\# & RATE & LONG & LAT & ST & TR\# & RATE & LONG & LAT & ST & TR\# & RATE & LONG & LAT & ST \\
\hline 3861 & 0.7 & -75.893 & 36.6456 & VA & 3933 & 0.2 & -75.8797 & 36.6141 & $\overline{\mathrm{VA}}$ & 4005 & -0.7 & -75.8691 & 36.5829 & VA \\
\hline 3862 & 0.7 & -75.8928 & 36.6451 & VA & 3934 & 0.2 & -75.8795 & 36.6137 & VA & 4006 & -0.6 & -75.869 & 36.5825 & VA \\
\hline 3863 & 0.7 & -75.8925 & 36.6447 & VA & 3935 & 0.1 & -75.8793 & 36.6132 & VA & 4007 & -0.5 & -75.8689 & 36.582 & VA \\
\hline 3864 & 0.8 & -75.8923 & 36.6443 & VA & 3936 & 0 & -75.8792 & 36.6128 & $\mathrm{VA}$ & 4008 & -0.4 & -75.8688 & 36.5816 & VA \\
\hline 3865 & 0.8 & -75.8921 & 36.6438 & VA & 3937 & 0 & -75.879 & 36.6123 & VA & 4009 & -0.2 & -75.8687 & 36.5811 & VA \\
\hline 3866 & 0.9 & -75.8919 & 36.6434 & VA & 3938 & -0.1 & -75.8789 & 36.6119 & $\mathrm{VA}$ & 4010 & -0.2 & -75.8686 & 36.5806 & VA \\
\hline 3867 & 1 & -75.8917 & 36.643 & VA & 3939 & -0.2 & -75.8787 & 36.6114 & VA & 4011 & -0.2 & -75.8685 & 36.5802 & VA \\
\hline 3868 & 1.1 & -75.8915 & 36.6425 & VA & 3940 & -0.3 & -75.8785 & 36.611 & VA & 4012 & -0.2 & -75.8683 & 36.5797 & VA \\
\hline 3869 & 1.3 & -75.8913 & 36.6421 & VA & 3941 & -0.4 & -75.8784 & 36.6105 & VA & 4013 & -0.1 & -75.8682 & 36.5793 & VA \\
\hline 3870 & 1.3 & -75.8911 & 36.6417 & $\mathrm{VA}$ & 3942 & -0.4 & -75.8782 & 36.6101 & VA & 4014 & -0.1 & -75.8681 & 36.5788 & VA \\
\hline 3871 & 1.4 & .75 .8909 & 36.6412 & VA & 3943 & -0.5 & -75.8781 & 36.6096 & VA & 4015 & -0.1 & -75.868 & 36.5783 & VA \\
\hline 3872 & 1.5 & -75.8907 & 36.6408 & VA & 3944 & -0.6 & -75.8779 & 36.6092 & VA & 4016 & -0.1 & -75.8679 & 36.5779 & VA \\
\hline 3873 & 1.6 & -75.8905 & 36.6403 & $\mathrm{VA}$ & 3945 & -0.7 & -75.8777 & 36.6087 & $\mathrm{VA}$ & 4017 & 0 & -75.8678 & 36.5774 & VA \\
\hline 3874 & 1.7 & -75.8903 & 36.6399 & VA & 3946 & -0.7 & -75.8776 & 36.6083 & VA & 4018 & 0.1 & -75.8677 & 36.577 & VA \\
\hline 3875 & 1.7 & -75.8901 & 36.6395 & VA & 3947 & -0.7 & -75.8774 & 36.6078 & VA & 4019 & 0.2 & -75.8676 & 36.5765 & VA \\
\hline 3876 & 1.8 & -75.8899 & 36.639 & VA & 3948 & -1 & -75.8773 & 36.6074 & VA & 4020 & 0.2 & -75.8675 & 36.5761 & VA \\
\hline 3877 & 1.8 & -75.8897 & 36.6386 & VA & 3949 & -1.2 & -75.8771 & 36.6069 & $\mathrm{VA}$ & 4021 & 0.2 & -75.8674 & 36.5756 & VA \\
\hline 3878 & 1.8 & -75.8895 & 36.6382 & VA & 3950 & -1.2 & -75.8769 & 36.6065 & VA & 4022 & 0.2 & -75.8673 & 36.5751 & VA \\
\hline 3879 & 1.8 & -75.8893 & 36.6377 & VA & 3951 & -1.2 & -75.8768 & 36.606 & $\mathrm{VA}$ & 4023 & 0.2 & -75.8672 & 36.5747 & VA \\
\hline 3880 & 1.8 & -75.8891 & 36.6373 & VA & 3952 & -1.1 & -75.8766 & 36.6056 & $\mathrm{VA}$ & 4024 & 0.2 & -75.8671 & 36.5742 & VA \\
\hline 3881 & 1.8 & -75.8888 & 36.6369 & VA & 3953 & -1 & -75.8765 & 36.6051 & $\mathrm{VA}$ & 4025 & 0.1 & -75.867 & 36.5738 & VA \\
\hline 3882 & 1.8 & -75.8886 & 36.6364 & VA & 3954 & -0.9 & -75.8763 & 36.6047 & VA & 4026 & 0.1 & .75 .8669 & 36.5733 & VA \\
\hline 3883 & 1.8 & -75.8884 & 36.636 & VA & 3955 & -0.7 & -75.8761 & 36.6043 & VA & 4027 & 0.1 & -75.8668 & 36.5729 & VA \\
\hline 3884 & 1.8 & -75.8882 & 36.6356 & VA & 3956 & -0.8 & -75.876 & 36.6038 & VA & 4028 & 0 & -75.8667 & 36.5724 & VA \\
\hline 3885 & 1.8 & -75.888 & 36.6351 & VA & 3957 & -0.9 & -75.8758 & 36.6034 & $\mathrm{VA}$ & 4029 & -0.1 & .75 .8666 & 36.5719 & VA \\
\hline 3886 & 1.9 & -75.8878 & 36.6347 & VA & 3958 & -0.9 & -75.8757 & 36.6029 & VA & 4030 & -0.1 & -75.8665 & 36.5715 & VA \\
\hline 3887 & 2 & -75.8876 & 36.6343 & VA & 3959 & -0.9 & -75.8755 & 36.6025 & $\mathrm{VA}$ & 4031 & -0.1 & -75.8664 & 36.571 & VA \\
\hline 3888 & 2 & -75.8874 & 36.6338 & VA & 3960 & -1 & -75.8753 & 36.602 & VA & 4032 & -0.1 & -75.8663 & 36.5706 & VA \\
\hline 3889 & 2 & -75.8872 & 36.6334 & VA & 3961 & -1.1 & -75.8752 & 36.6016 & VA & 4033 & -0.1 & -75.8662 & 36.5701 & VA \\
\hline 3890 & 2.1 & -75.887 & 36.633 & VA & 3962 & -1 & -75.875 & 36.6011 & VA & 4034 & 0 & -75.8661 & 36.5697 & VA \\
\hline 3891 & 2.2 & -75.8868 & 36.6325 & VA & 3963 & -1 & -75.8748 & 36.6007 & VA & 4035 & 0.1 & -75.866 & 36.5692 & VA \\
\hline 3892 & 2.2 & -75.8866 & 36.6321 & VA & 3964 & -1 & -75.8747 & 36.6002 & VA & 4036 & 0.1 & -75.8658 & 36.5687 & VA \\
\hline 3893 & 2.2 & -75.8864 & 36.6317 & VA & 3965 & -1 & -75.8745 & 36.5998 & VA & 4037 & 0 & -75.8657 & 36.5683 & VA \\
\hline 3894 & 2.2 & -75.8862 & 36.6312 & VA & 3966 & -1 & -75.8744 & 36.5993 & VA & 4038 & -0.2 & -75.8656 & 36.5678 & VA \\
\hline 3895 & 2.2 & -75.886 & 36.6308 & VA & 3967 & -1.1 & -75.8742 & 36.5989 & VA & 4039 & -0.4 & -75.8655 & 36.5674 & VA \\
\hline 3896 & 2.1 & .75 .8858 & 36.6304 & VA & 3968 & -1.1 & -75.874 & 36.5984 & VA & 4040 & -0.4 & -75.8654 & 36.5669 & VA \\
\hline 3897 & 2 & -75.8856 & 36.6299 & VA & 3969 & -1.1 & -75.8739 & 36.598 & VA & 4041 & -0.5 & -75.8653 & 36.5664 & VA \\
\hline 3898 & 1.8 & -75.8854 & 36.6295 & VA & 3970 & -1.1 & -75.8737 & 36.5975 & VA & 4042 & -0.5 & -75.8652 & 36.566 & VA \\
\hline 3899 & 1.7 & -75.8851 & 36.6291 & VA & 3971 & -1.1 & -75.8736 & 36.5971 & VA & 4043 & -0.5 & -75.8651 & 36.5655 & VA \\
\hline 3900 & 1.6 & -75.8849 & 36.6286 & VA & 3972 & -1.2 & -75.8734 & 36.5966 & VA & 4044 & -0.5 & -75.865 & 36.5651 & VA \\
\hline 3901 & 1.6 & -75.8847 & 36.6282 & VA & 3973 & -1.2 & -75.8732 & 36.5962 & VA & 4045 & -0.6 & -75.8649 & 36.5646 & VA \\
\hline 3902 & 1.3 & -75.8845 & 36.6278 & VA & 3974 & -1.4 & -75.8731 & 36.5957 & VA & 4046 & -0.6 & -75.8648 & 36.5642 & VA \\
\hline 3903 & 1.1 & -75.8843 & 36.6273 & VA & 3975 & -1.5 & -75.8729 & 36.5953 & VA & 4047 & -0.3 & -75.8656 & 36.5642 & VA \\
\hline 3904 & 1 & -75.8841 & 36.6269 & VA & 3976 & -1.7 & -75.8719 & 36.5953 & VA & 4048 & -0.6 & -75.8647 & 36.5637 & VA \\
\hline 3905 & 0.9 & -75.8839 & 36.6265 & $\mathrm{VA}$ & 3977 & -1.4 & -75.8728 & 36.5948 & VA & 4049 & -0.1 & -75.8655 & 36.5637 & VA \\
\hline 3906 & 0.9 & -75.8837 & 36.626 & VA & 3978 & -1.5 & -75.8718 & 36.5948 & VA & 4050 & -0.1 & -75.8654 & 36.5633 & VA \\
\hline 3907 & 0.9 & -75.8835 & 36.6256 & VA & 3979 & -1.4 & -75.8726 & 36.5944 & VA & 4051 & -0.2 & -75.8653 & 36.5628 & VA \\
\hline 3908 & 2.2 & -75.8837 & 36.6253 & VA & 3980 & -1.4 & -75.8717 & 36.5944 & VA & 4052 & -0.2 & -75.8653 & 36.5623 & VA \\
\hline 3909 & 2.1 & .75 .8835 & 36.6249 & VA & 3981 & -1.2 & -75.8716 & 36.5939 & VA & 4053 & -0.1 & -75.8652 & 36.5619 & VA \\
\hline 3910 & 2.2 & -75.8834 & 36.6244 & VA & 3982 & -1.2 & -75.8715 & 36.5935 & VA & 4054 & -0.2 & -75.8651 & 36.5614 & VA \\
\hline 3911 & 2.2 & -75.8832 & 36.624 & VA & 3983 & -1.1 & -75.8714 & 36.593 & VA & 4055 & -0.2 & -75.865 & 36.5609 & VA \\
\hline 3912 & 2 & -75.883 & 36.6235 & VA & 3984 & -1.2 & -75.8713 & 36.5925 & VA & 4056 & -0.1 & -75.8649 & 36.5605 & VA \\
\hline 3913 & 1.9 & -75.8829 & 36.6231 & VA & 3985 & -1.4 & -75.8712 & 36.5921 & VA & 4057 & -0.1 & -75.8648 & 36.56 & VA \\
\hline 3914 & 1.9 & -75.8827 & 36.6226 & $V A$ & 3986 & -1.4 & -75.8711 & 36.5916 & $\mathrm{VA}$ & 4058 & -0.1 & -75.8647 & 36.5596 & VA \\
\hline 3915 & 1.9 & -75.8826 & 36.6222 & VA & 3987 & -1.4 & -75.871 & 36.5912 & $\mathrm{VA}$ & 4059 & -0.1 & -75.8646 & 36.5591 & VA \\
\hline 3916 & 1.7 & -75.8824 & 36.6217 & VA & 3988 & -1.3 & -75.8708 & 36.5907 & $\mathrm{VA}$ & 4060 & -0.1 & -75.8645 & 36.5586 & VA \\
\hline 3917 & 1.6 & -75.8822 & 36.6213 & VA & 3989 & -1.2 & -75.8707 & 36.5902 & VA & 4061 & -0.1 & -75.8644 & 36.5582 & VA \\
\hline 3918 & 1.4 & .75 .8821 & 36.6208 & VA & 3990 & -1.2 & -75.8706 & 36.5898 & VA & 4062 & 0 & -75.8644 & 36.5577 & VA \\
\hline 3919 & 1.1 & .75 .8819 & 36.6204 & VA & 3991 & -1.1 & -75.8705 & 36.5893 & VA & 4063 & -0.1 & -75.8643 & 36.5572 & VA \\
\hline 3920 & 1 & -75.8818 & 36.6199 & $\mathrm{VA}$ & 3992 & -1.2 & -75.8704 & 36.5889 & VA & 4064 & -0.1 & -75.8642 & 36.5568 & VA \\
\hline 3921 & 0.9 & -75.8816 & 36.6195 & VA & 3993 & -1.2 & -75.8703 & 36.5884 & VA & 4065 & -0.2 & .75 .8641 & 36.5563 & VA \\
\hline 3922 & 0.9 & -75.8814 & 36.619 & VA & 3994 & -1.2 & -75.8702 & 36.588 & VA & 4066 & 0 & -75.864 & 36.5559 & VA \\
\hline 3923 & 0.9 & -75.8813 & 36.6186 & VA & 3995 & -1.1 & -75.8701 & 36.5875 & VA & 4067 & 0.1 & -75.8639 & 36.5554 & VA \\
\hline 3924 & 0.8 & -75.8811 & 36.6181 & VA & 3996 & -1.1 & -75.87 & 36.587 & VA & 4068 & 0.1 & -75.8638 & 36.5549 & VA \\
\hline 3925 & 0.7 & .75 .8809 & 36.6177 & VA & 3997 & -1.1 & -75.8699 & 36.5866 & VA & 4069 & 0.2 & -75.8637 & 36.5545 & VA \\
\hline 3926 & 0.5 & -75.8808 & 36.6172 & VA & 3998 & -1.1 & -75.8698 & 36.5861 & $\mathrm{VA}$ & 4070 & 0.2 & -75.8636 & 36.554 & VA \\
\hline 3927 & 0.4 & -75.8806 & 36.6168 & VA & 3999 & -1 & -75.8697 & 36.5857 & $\mathrm{VA}$ & 4071 & 0.2 & -75.8635 & 36.5536 & VA \\
\hline 3928 & 0.4 & -75.8805 & 36.6163 & VA & 4000 & -1 & -75.8696 & 36.5852 & VA & 4072 & 0.3 & -75.8635 & 36.5531 & VA \\
\hline 3929 & 0.4 & -75.8803 & 36.6159 & VA & 4001 & -1 & -75.8695 & 36.5848 & VA & 4073 & 0.4 & -75.8634 & 36.5526 & VA \\
\hline 3930 & 0.4 & .75 .8801 & 36.6155 & VA & 4002 & -0.9 & -75.8694 & 36.5843 & VA & 4074 & 0.5 & -75.8633 & 36.5522 & VA \\
\hline 3931 & 0.4 & -75.88 & 36.615 & VA & 4003 & -0.9 & -75.8693 & 36.5838 & VA & 4075 & 0.7 & -75.8632 & 36.5517 & VA \\
\hline \multirow{3}{*}{3932} & 0.3 & -75.8798 & 36.6146 & VA & 4004 & -0.8 & -75.8692 & 36.5834 & VA & 4076 & 0.8 & -75.8631 & 36.5512 & VA \\
\hline & & & & & & & & & & 4077 & 0.9 & -75.863 & 36.5508 & VA \\
\hline & & & & & & & & & & 4078 & 1.1 & .75 .8629 & 36.5503 & VA \\
\hline
\end{tabular}




\begin{tabular}{|c|c|c|c|c|c|c|c|c|c|c|c|c|c|c|}
\hline TR\# & RATE & LONG & LAT & ST & TR\# & RATE & LONG & LAT & ST & TR\# & RATE & LONG & LAT & ST \\
\hline TR\# & RATE & LON & LAT & ST & 72 & 0.3 & -75.8568 & 36.5177 & $\mathrm{NC}$ & 144 & 0.5 & -75.8488 & 36.4862 & NC \\
\hline 1 & 1.2 & -75.8628 & 36.5499 & NC & 73 & 0.1 & -75.8567 & 36.5173 & NC & 145 & 0.5 & -75.8487 & 36.4857 & NC \\
\hline 2 & 1.3 & -75.8627 & 36.5494 & $\mathrm{NC}$ & 74 & 0.1 & -75.8566 & 36.5168 & $\mathrm{NC}$ & 146 & 0.4 & -75.8486 & 36.4853 & NC \\
\hline 3 & 1.3 & -75.8626 & 36.5489 & $\mathrm{NC}$ & 75 & 0.1 & -75.8565 & 36.5163 & $\mathrm{NC}$ & 147 & 0.4 & -75.8485 & 36.4848 & NC \\
\hline 4 & 1.2 & -75.8626 & 36.5485 & NC & 76 & 0.1 & -75.8564 & 36.5159 & NC & 148 & 0.5 & -75.8483 & 36.4844 & NC \\
\hline 5 & 1.2 & -75.8625 & 36.548 & $\mathrm{NC}$ & 77 & 0.2 & -75.8563 & 36.5154 & NC & 149 & 0.6 & -75.8482 & 36.4839 & NC \\
\hline 6 & 1.2 & -75.8624 & 36.5475 & NC & 78 & 0 & -75.8562 & 36.515 & NC & 150 & 0.6 & -75.8481 & 36.4835 & NC \\
\hline 7 & 1.3 & -75.8623 & 36.5471 & NC & 79 & -0.1 & -75.8561 & 36.5145 & NC & 151 & 0.6 & -75.848 & 36.483 & NC \\
\hline 8 & 1.3 & -75.8622 & 36.5466 & NC & 80 & 0 & -75.856 & 36.5141 & NC & 152 & 0.4 & -75.8479 & 36.4826 & NC \\
\hline 9 & 1.3 & -75.8621 & 36.5462 & NC & 81 & 0.2 & -75.8559 & 36.5136 & NC & 153 & 0.4 & -75.8477 & 36.4821 & NC \\
\hline 10 & 1.3 & -75.862 & 36.5457 & $\mathrm{NC}$ & 82 & 0.1 & -75.8558 & 36.5131 & NC & 154 & 0.2 & -75.8476 & 36.4817 & NC \\
\hline 11 & 1.3 & -75.8619 & 36.5452 & NC & 83 & -0.1 & -75.8557 & 36.5127 & NC & 155 & 0.3 & -75.8475 & 36.4812 & NC \\
\hline 12 & 1.3 & -75.8618 & 36.5448 & NC & 84 & -0.2 & -75.8556 & 36.5122 & $\mathrm{NC}$ & 156 & 0.3 & -75.8474 & 36.4808 & NC \\
\hline 13 & 1.2 & -75.8617 & 36.5443 & NC & 85 & -0.1 & -75.8555 & 36.5118 & NC & 157 & 0.4 & -75.8473 & 36.4803 & NC \\
\hline 14 & 1.1 & -75.8617 & 36.5438 & NC & 86 & -0.2 & -75.8554 & 36.5113 & NC & 158 & 0.4 & -75.8471 & 36.4799 & NC \\
\hline 15 & 1.1 & -75.8616 & 36.5434 & NC & 87 & -0.3 & -75.8553 & 36.5109 & NC & 159 & -2.3 & -75.8486 & 36.4794 & NC \\
\hline 16 & 1 & -75.8615 & 36.5429 & NC & 88 & -0.3 & -75.8552 & 36.5104 & NC & 160 & 0.3 & -75.847 & 36.4794 & NC \\
\hline 17 & 0.9 & -75.8614 & 36.5425 & NC & 89 & -0.3 & -75.8551 & 36.5099 & NC & 161 & -2.3 & -75.8484 & 36.4789 & NC \\
\hline 18 & 0.8 & -75.8613 & 36.542 & NC & 90 & -0.1 & -75.855 & 36.5095 & NC & 162 & -2.1 & -75.8483 & 36.4785 & NC \\
\hline 19 & 0.7 & -75.8612 & 36.5415 & NC & 91 & 0.2 & -75.8549 & 36.509 & NC & 163 & -2 & -75.8482 & 36.478 & NC \\
\hline 20 & 0.6 & -75.8611 & 36.5411 & NC & 92 & 0.1 & -75.8548 & 36.5086 & NC & 164 & -2.1 & -75.848 & 36.4776 & NC \\
\hline 21 & 0.5 & -75.861 & 36.5406 & NC & 93 & 0.1 & -75.8547 & 36.5081 & NC & 165 & -2.2 & -75.8479 & 36.4771 & NC \\
\hline 22 & 0.5 & -75.8609 & 36.5402 & NC & 94 & 0.2 & -75.8546 & 36.5077 & NC & 166 & -2.2 & -75.8478 & 36.4767 & NC \\
\hline 23 & 0.4 & -75.8608 & 36.5397 & NC & 95 & 0.2 & -75.8545 & 36.5072 & NC & 167 & -2.1 & -75.8476 & 36.4762 & NC \\
\hline 24 & 0.4 & -75.8608 & 36.5392 & NC & 96 & 0.1 & -75.8544 & 36.5067 & NC & 168 & -2.2 & -75.8475 & 36.4758 & NC \\
\hline 25 & 0.5 & -75.8607 & 36.5388 & NC & 97 & -0.1 & -75.8543 & 36.5063 & NC & 169 & -2 & -75.8474 & 36.4753 & NC \\
\hline 26 & 0.4 & -75.8606 & 36.5383 & NC & 98 & 0 & -75.8542 & 36.5058 & NC & 170 & -2.2 & -75.8472 & 36.4749 & NC \\
\hline 27 & 0.3 & -75.8605 & 36.5378 & NC & 99 & 0 & -75.8541 & 36.5054 & NC & 171 & -2.2 & -75.8471 & 36.4744 & NC \\
\hline 28 & 0.3 & -75.8604 & 36.5374 & NC & 100 & 0 & -75.8538 & 36.5052 & NC & 172 & -2.1 & -75.847 & 36.474 & $\mathrm{NC}$ \\
\hline 29 & 0.3 & -75.8603 & 36.5369 & NC & 101 & -0.1 & -75.854 & 36.5049 & NC & 173 & -2.1 & -75.8468 & 36.4735 & NC \\
\hline 30 & 0.3 & -75.8602 & 36.5365 & NC & 102 & 0 & -75.8537 & 36.5047 & $\mathrm{NC}$ & 174 & -2.2 & -75.8467 & 36.4731 & NC \\
\hline 31 & 0.3 & -75.8601 & 36.536 & NC & 103 & -0.2 & .75 .8539 & 36.5044 & NC & 175 & -2.2 & -75.8466 & 36.4726 & NC \\
\hline 32 & 0.3 & -75.86 & 36.5355 & NC & 104 & -0.1 & -75.8536 & 36.5043 & NC & 176 & -1.9 & -75.8465 & 36.4722 & NC \\
\hline 33 & 0.3 & -75.8599 & 36.5351 & NC & 105 & -0.2 & -75.8535 & 36.5038 & NC & 177 & -1.8 & -75.8463 & 36.4717 & NC \\
\hline 34 & 0.3 & -75.8599 & 36.5346 & NC & 106 & -0.2 & -75.8534 & 36.5034 & NC & 178 & -1.8 & -75.8462 & 36.4713 & NC \\
\hline 35 & 0.3 & -75.8598 & 36.5341 & NC & 107 & -0.1 & -75.8532 & 36.5029 & NC & 179 & -1.8 & -75.8461 & 36.4708 & NC \\
\hline 36 & 0.4 & -75.8597 & 36.5337 & NC & 108 & -0.1 & -75.8531 & 36.5025 & NC & 180 & -1.7 & -75.8459 & 36.4704 & NC \\
\hline 37 & -0.8 & -75.8603 & 36.5333 & $\mathrm{NC}$ & 109 & 0 & -75.853 & 36.502 & NC & 181 & -1.6 & -75.8458 & 36.4699 & NC \\
\hline 38 & 0.4 & -75.8596 & 36.5332 & NC & 110 & 0 & -75.8529 & 36.5016 & NC & 182 & -1.6 & -75.8457 & 36.4695 & NC \\
\hline 39 & -0.9 & -75.8602 & 36.5328 & NC & 111 & 0.1 & -75.8528 & 36.5011 & NC & 183 & -1.5 & -75.8455 & 36.469 & NC \\
\hline 40 & -1 & -75.8601 & 36.5324 & NC & 112 & 0 & -75.8526 & 36.5007 & NC & 184 & -1.5 & -75.8454 & 36.4686 & NC \\
\hline 41 & -0.9 & -75.86 & 36.5319 & NC & 113 & -0.1 & -75.8525 & 36.5002 & NC & 185 & -1.5 & -75.8453 & 36.4681 & NC \\
\hline 42 & -1.1 & -75.8599 & 36.5315 & NC & 114 & -0.2 & -75.8524 & 36.4998 & NC & 186 & -1.7 & -75.8451 & 36.4677 & NC \\
\hline 43 & -1 & -75.8598 & 36.531 & NC & 115 & -0.3 & -75.8523 & 36.4993 & NC & 187 & -1.7 & -75.845 & 36.4672 & NC \\
\hline 44 & -0.9 & -75.8597 & 36.5305 & $\mathrm{NC}$ & 116 & -0.2 & -75.8522 & 36.4989 & NC & 188 & -1.7 & -75.8449 & 36.4668 & NC \\
\hline 45 & -0.8 & -75.8596 & 36.5301 & NC & 117 & -0.1 & -75.852 & 36.4984 & NC & 189 & -1.6 & -75.8448 & 36.4663 & NC \\
\hline 46 & -0.7 & -75.8595 & 36.5296 & NC & 118 & -0.2 & -75.8519 & 36.4979 & NC & 190 & -1.8 & -75.8446 & 36.4659 & NC \\
\hline 47 & -0.7 & .75 .8594 & 36.5292 & $\mathrm{NC}$ & 119 & -0.2 & -75.8518 & 36.4975 & NC & 191 & -2 & -75.8445 & 36.4654 & NC \\
\hline 48 & -0.5 & -75.8593 & 36.5287 & NC & 120 & -0.2 & -75.8517 & 36.497 & NC & 192 & -1.9 & -75.8444 & 36.465 & NC \\
\hline 49 & -0.4 & -75.8592 & 36.5282 & $\mathrm{NC}$ & 121 & -0.2 & -75.8516 & 36.4966 & NC & 193 & -1.9 & -75.8442 & 36.4645 & NC \\
\hline 50 & -0.4 & -75.8591 & 36.5278 & NC & 122 & -0.2 & -75.8514 & 36.4961 & NC & 194 & -2 & -75.8441 & 36.4641 & NC \\
\hline 51 & -0.4 & -75.859 & 36.5273 & NC & 123 & -0.1 & -75.8513 & 36.4957 & NC & 195 & -1.9 & -75.844 & 36.4636 & NC \\
\hline 52 & -0.3 & -75.8589 & 36.5269 & NC & 124 & 0 & -75.8512 & 36.4952 & $\mathrm{NC}$ & 196 & -2.1 & -75.8438 & 36.4632 & $\mathrm{NC}$ \\
\hline 53 & 0 & -75.8588 & 36.5264 & NC & 125 & 0.2 & -75.8511 & 36.4948 & NC & 197 & -2.2 & -75.8437 & 36.4627 & NC \\
\hline 54 & 0.1 & -75.8587 & 36.526 & NC & 126 & 0.2 & -75.851 & 36.4943 & NC & 198 & -2.2 & -75.8436 & 36.4623 & $\mathrm{NC}$ \\
\hline 55 & 0.2 & -75.8586 & 36.5255 & NC & 127 & 0.3 & -75.8508 & 36.4939 & NC & 199 & -2.3 & -75.8434 & 36.4618 & $\mathrm{NC}$ \\
\hline 56 & 0.3 & -75.8585 & 36.525 & NC & 128 & 0.1 & -75.8507 & 36.4934 & NC & 200 & -2.4 & -75.8433 & 36.4614 & $\mathrm{NC}$ \\
\hline 57 & 0.5 & -75.8584 & 36.5246 & NC & 129 & 0.1 & -75.8506 & 36.493 & NC & 201 & -2.4 & -75.8432 & 36.4609 & $\mathrm{NC}$ \\
\hline 58 & 0.5 & -75.8583 & 36.5241 & NC & 130 & 0.1 & -75.8505 & 36.4925 & NC & 202 & -2.4 & -75.8431 & 36.4605 & NC \\
\hline 59 & 0.4 & -75.8582 & 36.5237 & NC & 131 & 0 & .75 .8504 & 36.4921 & NC & 203 & -2.2 & -75.8429 & 36.46 & NC \\
\hline 60 & 0.4 & -75.8581 & 36.5232 & $\mathrm{NC}$ & 132 & 0 & -75.8503 & 36.4916 & $\mathrm{NC}$ & 204 & -2.3 & -75.8428 & 36.4596 & $\mathrm{NC}$ \\
\hline 61 & 0.3 & -75.858 & 36.5228 & NC & 133 & 0.1 & -75.8501 & 36.4912 & NC & 205 & -2.2 & -75.8427 & 36.4591 & NC \\
\hline 62 & 0.5 & -75.8579 & 36.5223 & NC & 134 & 0.2 & -75.85 & 36.4907 & NC & 206 & -2.1 & -75.8425 & 36.4587 & $\mathrm{NC}$ \\
\hline 63 & 0.6 & -75.8578 & 36.5218 & NC & 135 & 0.5 & -75.8499 & 36.4903 & $\mathrm{NC}$ & 207 & -2 & -75.8424 & 36.4582 & NC \\
\hline 64 & 0.6 & -75.8577 & 36.5214 & NC & 136 & 0.6 & -75.8498 & 36.4898 & $\mathrm{NC}$ & 208 & -2 & -75.8423 & 36.4578 & NC \\
\hline 65 & 0.7 & -75.8576 & 36.5209 & NC & 137 & 0.6 & -75.8497 & 36.4894 & NC & 209 & -2 & -75.8421 & 36.4573 & $\mathrm{NC}$ \\
\hline 66 & 0.5 & -75.8575 & 36.5205 & NC & 138 & 0.4 & -75.8495 & 36.4889 & $\mathrm{NC}$ & 210 & -2.1 & -75.842 & 36.4569 & NC \\
\hline 67 & 0.2 & -75.8574 & 36.52 & NC & 139 & 0.3 & -75.8494 & 36.4885 & $\mathrm{NC}$ & 211 & -2 & -75.8419 & 36.4564 & NC \\
\hline 68 & 0.3 & -75.8573 & 36.5196 & NC & 140 & 0.4 & .75 .8493 & 36.488 & NC & 212 & -2.1 & -75.8417 & 36.456 & NC \\
\hline 69 & 0.3 & -75.8572 & 36.5191 & NC & 141 & 0.6 & -75.8492 & 36.4876 & NC & 213 & -2.1 & -75.8416 & 36.4555 & NC \\
\hline 70 & 0.3 & -75.8571 & 36.5186 & NC & 142 & 0.6 & -75.8491 & 36.4871 & NC & 214 & -2 & -75.8415 & 36.4551 & NC \\
\hline 71 & 0 & -75.857 & 36.5182 & NC & 143 & 0.5 & -75.8489 & 36.4866 & $\mathrm{NC}$ & 215 & -2.1 & -75.8413 & 36.4546 & NC \\
\hline
\end{tabular}




\begin{tabular}{|c|c|c|c|c|c|c|c|c|c|c|c|c|c|c|}
\hline TR\# & RATE & LONG & LAT & ST & TR\# & RATE & LONG & LAT & ST & TR\# & RATE & LONG & LAT & ST \\
\hline 216 & -2.2 & -75.8412 & 36.4542 & NC & 288 & -3.5 & -75.8308 & 36.4231 & NC & 360 & -2.6 & -75.8238 & 36.391 & NC \\
\hline 217 & -2.3 & -75.8411 & 36.4537 & NC & 289 & -3.3 & -75.8307 & 36.4226 & NC & 361 & -1 & -75.8226 & 36.3909 & NC \\
\hline 218 & -2.2 & -75.841 & 36.4533 & NC & 290 & -0.1 & -75.8306 & 36.4222 & NC & 362 & -2.6 & -75.8237 & 36.3905 & NC \\
\hline 219 & -2 & -75.8408 & 36.4528 & NC & 291 & -3.3 & -75.8305 & 36.4217 & NC & 363 & -1 & -75.8224 & 36.3904 & NC \\
\hline 220 & -2.1 & -75.8407 & 36.4524 & NC & 292 & -3.4 & -75.8303 & 36.4213 & NC & 364 & -1.1 & -75.8223 & 36.39 & NC \\
\hline 221 & -2.2 & -75.839 & 36.452 & NC & 293 & 0.2 & -75.8312 & 36.4208 & NC & 365 & -1.1 & -75.8222 & 36.3895 & NC \\
\hline 222 & -2.2 & -75.8406 & 36.4519 & NC & 294 & -3.5 & -75.8302 & 36.4208 & NC & 366 & -1.1 & -75.8221 & 36.3891 & NC \\
\hline 223 & -2.3 & -75.8389 & 36.4515 & NC & 295 & 0.2 & -75.8311 & 36.4203 & NC & 367 & -1 & -75.822 & 36.3886 & NC \\
\hline 224 & -2.2 & -75.8404 & 36.4515 & NC & 296 & 0.1 & -75.831 & 36.4199 & NC & 368 & -1 & -75.8219 & 36.3882 & NC \\
\hline 225 & -2.3 & -75.8388 & 36.4511 & NC & 297 & 0 & -75.8308 & 36.4194 & NC & 369 & -1 & -75.8217 & 36.3877 & NC \\
\hline 226 & -2.1 & -75.8403 & 36.451 & NC & 298 & -0.2 & -75.8307 & 36.419 & NC & 370 & -1 & -75.8216 & 36.3873 & NC \\
\hline 227 & -2.3 & -75.8387 & 36.4506 & NC & 299 & -0.3 & -75.8306 & 36.4185 & NC & 371 & -1 & -75.8215 & 36.3868 & NC \\
\hline 228 & -2.3 & -75.8385 & 36.4502 & NC & 300 & -0.4 & -75.8305 & 36.4181 & NC & 372 & -1.2 & -75.8214 & 36.3864 & NC \\
\hline 229 & -2.3 & -75.8384 & 36.4497 & NC & 301 & -0.4 & -75.8304 & 36.4176 & NC & 373 & -1.3 & -75.8213 & 36.3859 & NC \\
\hline 230 & -2.3 & -75.8383 & 36.4493 & NC & 302 & -0.4 & -75.8303 & 36.4172 & NC & 374 & -1.4 & -75.8211 & 36.3855 & $\mathrm{NC}$ \\
\hline 231 & -2.3 & -75.8381 & 36.4488 & NC & 303 & -0.2 & -75.8302 & 36.4167 & NC & 375 & -1.3 & -75.821 & 36.385 & $\mathrm{NC}$ \\
\hline 232 & -2.1 & -75.838 & 36.4484 & NC & 304 & -0.2 & -75.8301 & 36.4163 & NC & 376 & -1.4 & -75.8209 & 36.3845 & NC \\
\hline 233 & -1.7 & -75.8379 & 36.4479 & NC & 305 & -0.1 & -75.83 & 36.4158 & NC & 377 & -1.5 & -75.8208 & 36.3841 & $\mathrm{NC}$ \\
\hline 234 & -1.7 & -75.8378 & 36.4475 & NC & 306 & -0.1 & -75.8298 & 36.4154 & $\mathrm{NC}$ & 378 & -1.6 & -75.8207 & 36.3836 & NC \\
\hline 235 & -1.8 & -75.8376 & 36.447 & NC & 307 & -0.1 & -75.8297 & 36.4149 & NC & 379 & -1.6 & -75.8206 & 36.3832 & NC \\
\hline 236 & -1.8 & -75.8375 & 36.4466 & NC & 308 & -0.3 & -75.8296 & 36.4145 & NC & 380 & -1.6 & -75.8204 & 36.3827 & NC \\
\hline 237 & -1.8 & -75.8374 & 36.4461 & NC & 309 & -0.4 & -75.8295 & 36.414 & NC & 381 & -1.6 & -75.8203 & 36.3823 & NC \\
\hline 238 & -1.8 & -75.8372 & 36.4457 & NC & 310 & -0.4 & -75.8294 & 36.4136 & NC & 382 & -1.8 & -75.8202 & 36.3818 & NC \\
\hline 239 & -1.9 & -75.8371 & 36.4452 & $\mathrm{NC}$ & 311 & -0.3 & -75.8293 & 36.4131 & NC & 383 & -1.8 & -75.8201 & 36.3814 & NC \\
\hline 240 & -1.8 & -75.837 & 36.4448 & $\mathrm{NC}$ & 312 & -0.3 & -75.8292 & 36.4127 & NC & 384 & -1.9 & -75.82 & 36.3809 & NC \\
\hline 241 & -1.9 & -75.8369 & 36.4443 & NC & 313 & -0.1 & -75.8291 & 36.4122 & NC & 385 & -1.9 & -75.8198 & 36.3805 & NC \\
\hline 242 & -2 & -75.8367 & 36.4439 & NC & 314 & -0.1 & -75.829 & 36.4118 & NC & 386 & -2 & -75.8197 & 36.38 & NC \\
\hline 243 & -2 & -75.8366 & 36.4434 & NC & 315 & -0.3 & -75.8288 & 36.4113 & NC & 387 & -2.1 & -75.8196 & 36.3796 & $\mathrm{NC}$ \\
\hline 244 & -2.2 & -75.8365 & 36.443 & NC & 316 & -0.5 & -75.8287 & 36.4108 & NC & 388 & -2.1 & -75.8195 & 36.3791 & $\mathrm{NC}$ \\
\hline 245 & -2.2 & -75.8364 & 36.4425 & NC & 317 & -0.5 & -75.8286 & 36.4104 & NC & 389 & -2.1 & -75.8194 & 36.3786 & $\mathrm{NC}$ \\
\hline 246 & -2.3 & -75.8362 & 36.442 & $\mathrm{NC}$ & 318 & -0.4 & -75.8285 & 36.4099 & NC & 390 & -2.1 & -75.8193 & 36.3782 & $\mathrm{NC}$ \\
\hline 247 & -2.4 & -75.8361 & 36.4416 & NC & 319 & -0.6 & -75.8284 & 36.4095 & NC & 391 & -2.1 & -75.8191 & 36.3777 & $\mathrm{NC}$ \\
\hline 248 & -2.5 & -75.836 & 36.4411 & NC & 320 & -0.8 & -75.8283 & 36.409 & NC & 392 & -2.2 & -75.819 & 36.3773 & $\mathrm{NC}$ \\
\hline 249 & -2.5 & -75.8358 & 36.4407 & NC & 321 & -0.8 & -75.8282 & 36.4086 & $\mathrm{NC}$ & 393 & -2.3 & -75.8189 & 36.3768 & $\mathrm{NC}$ \\
\hline 250 & -2.4 & -75.8357 & 36.4402 & NC & 322 & -0.8 & -75.8281 & 36.4081 & NC & 394 & -2.3 & -75.8188 & 36.3764 & NC \\
\hline 251 & -2.4 & -75.8356 & 36.4398 & NC & 323 & -0.8 & -75.828 & 36.4077 & NC & 395 & -2.3 & -75.8187 & 36.3759 & NC \\
\hline 252 & -2.4 & -75.8355 & 36.4393 & NC & 324 & -0.9 & -75.8278 & 36.4072 & NC & 396 & -2.4 & -75.8185 & 36.3755 & NC \\
\hline 253 & -2.4 & -75.8353 & 36.4389 & NC & 325 & -1.1 & -75.8277 & 36.4068 & NC & 397 & -2.5 & -75.8184 & 36.375 & NC \\
\hline 254 & -2.4 & -75.8352 & 36.4384 & NC & 326 & -1.3 & -75.8276 & 36.4063 & NC & 398 & -2.3 & -75.8183 & 36.3746 & NC \\
\hline 255 & -2.4 & -75.8351 & 36.438 & NC & 327 & -1.4 & -75.8275 & 36.4059 & NC & 399 & -2.1 & -75.8182 & 36.3741 & NC \\
\hline 256 & -2.5 & -75.8349 & 36.4375 & NC & 328 & -1.4 & -75.8274 & 36.4054 & NC & 400 & -2.1 & -75.8181 & 36.3737 & NC \\
\hline 257 & -2.4 & -75.8348 & 36.4371 & NC & 329 & -1.2 & -75.8273 & 36.405 & NC & 401 & -2 & -75.8179 & 36.3732 & NC \\
\hline 258 & -2.5 & -75.8347 & 36.4366 & NC & 330 & -1.3 & -75.8272 & 36.4045 & NC & 402 & -2 & -75.8178 & 36.3728 & $\mathrm{NC}$ \\
\hline 259 & -2.6 & -75.8346 & 36.4362 & NC & 331 & -1.5 & -75.8271 & 36.4041 & NC & 403 & -2 & -75.8177 & 36.3723 & NC \\
\hline 260 & -2.6 & -75.8344 & 36.4357 & NC & 332 & -1.3 & -75.8269 & 36.4036 & NC & 404 & -2.2 & -75.8176 & 36.3718 & NC \\
\hline 261 & -2.7 & -75.8343 & 36.4353 & NC & 333 & -1.3 & -75.8268 & 36.4032 & NC & 405 & -2.2 & -75.8175 & 36.3714 & NC \\
\hline 262 & -3 & -75.8342 & 36.4348 & NC & 334 & -1.2 & -75.8267 & 36.4027 & NC & 406 & -2.3 & -75.8174 & 36.3709 & NC \\
\hline 263 & -3.1 & -75.834 & 36.4344 & NC & 335 & -1.3 & -75.8266 & 36.4023 & NC & 407 & -2.4 & -75.8172 & 36.3705 & NC \\
\hline 264 & -3.1 & -75.8339 & 36.4339 & NC & 336 & -1.6 & -75.8265 & 36.4018 & NC & 408 & -2.6 & -75.8171 & 36.37 & NC \\
\hline 265 & -3.1 & -75.8338 & 36.4335 & NC & 337 & -1.6 & -75.8264 & 36.4014 & NC & 409 & -2.7 & -75.817 & 36.3696 & NC \\
\hline 266 & -3.1 & -75.8337 & 36.433 & NC & 338 & -1.6 & -75.8263 & 36.4009 & NC & 410 & -2.8 & -75.8169 & 36.3691 & NC \\
\hline 267 & -3.1 & -75.8335 & 36.4326 & NC & 339 & -1.7 & -75.8262 & 36.4005 & $\mathrm{NC}$ & 411 & -2.8 & -75.8168 & 36.3687 & NC \\
\hline 268 & -3.2 & -75.8334 & 36.4321 & NC & 340 & -1.8 & -75.8261 & 36.4 & NC & 412 & -2.9 & -75.8166 & 36.3682 & NC \\
\hline 269 & -3.3 & -75.8333 & 36.4317 & NC & 341 & -1.9 & -75.8259 & 36.3995 & NC & 413 & -3 & -75.8165 & 36.3678 & NC \\
\hline 270 & -3.4 & -75.8331 & 36.4312 & NC & 342 & -2 & -75.8258 & 36.3991 & NC & 414 & -2.8 & -75.8164 & 36.3673 & NC \\
\hline 271 & -3.4 & -75.833 & 36.4307 & NC & 343 & -2 & -75.8257 & 36.3986 & NC & 415 & -2.7 & -75.8163 & 36.3669 & NC \\
\hline 272 & -3.6 & -75.8329 & 36.4303 & NC & 344 & -2 & -75.8256 & 36.3982 & NC & 416 & -2.6 & -75.8162 & 36.3664 & NC \\
\hline 273 & -3.7 & -75.8328 & 36.4298 & NC & 345 & -1.9 & -75.8255 & 36.3977 & NC & 417 & -2.5 & -75.8161 & 36.3659 & NC \\
\hline 274 & -3.7 & -75.8326 & 36.4294 & NC & 346 & -2 & -75.8254 & 36.3973 & NC & 418 & -2.4 & -75.8159 & 36.3655 & NC \\
\hline 275 & -3.5 & -75.8325 & 36.4289 & NC & 347 & -2.1 & -75.8253 & 36.3968 & NC & 419 & -2.4 & -75.8158 & 36.365 & NC \\
\hline 276 & -3.6 & -75.8324 & 36.4285 & NC & 348 & -2.2 & -75.8252 & 36.3964 & NC & 420 & -2.4 & -75.8157 & 36.3646 & NC \\
\hline 277 & -3.7 & -75.8323 & 36.428 & NC & 349 & -2.3 & -75.8251 & 36.3959 & NC & 421 & -2.5 & -75.8156 & 36.3641 & NC \\
\hline 278 & -3.6 & -75.8321 & 36.4276 & NC & 350 & -2.2 & -75.8249 & 36.3955 & NC & 422 & -2.5 & -75.8155 & 36.3637 & NC \\
\hline 279 & -3.5 & -75.832 & 36.4271 & NC & 351 & -2.2 & -75.8248 & 36.395 & NC & 423 & -2.5 & -75.8153 & 36.3632 & NC \\
\hline 280 & -3.5 & -75.8319 & 36.4267 & NC & 352 & -2.1 & -75.8247 & 36.3946 & NC & 424 & -2.6 & -75.8152 & 36.3628 & NC \\
\hline 281 & -3.5 & -75.8317 & 36.4262 & NC & 353 & -2.3 & -75.8246 & 36.3941 & NC & 425 & -2.7 & -75.8151 & 36.3623 & NC \\
\hline 282 & -3.3 & -75.8316 & 36.4258 & NC & 354 & -2.4 & -75.8245 & 36.3937 & NC & 426 & -2.7 & -75.815 & 36.3619 & NC \\
\hline 283 & -3.1 & -75.8315 & 36.4253 & NC & 355 & -2.5 & .75 .8244 & 36.3932 & NC & 427 & -2.7 & -75.8149 & 36.3614 & NC \\
\hline 284 & -3.3 & -75.8314 & 36.4249 & NC & 356 & -2.6 & -75.8243 & 36.3928 & NC & 428 & -2.6 & -75.8148 & 36.361 & NC \\
\hline 285 & -3.3 & -75.8312 & 36.4244 & $\mathrm{NC}$ & 357 & -2.7 & -75.8242 & 36.3923 & NC & 429 & 0 & -75.8132 & 36.3609 & NC \\
\hline 286 & -3.4 & -75.8311 & 36.424 & NC & 358 & -2.7 & -75.8241 & 36.3919 & $\mathrm{NC}$ & 430 & -2.5 & -75.8146 & 36.3605 & NC \\
\hline 287 & -3.5 & -75.831 & 36.4235 & NC & 359 & -2.7 & -75.8239 & 36.3914 & NC & 431 & -0.1 & -75.8131 & 36.3604 & NC \\
\hline
\end{tabular}




\begin{tabular}{|c|c|c|c|c|c|c|c|c|c|c|c|c|c|c|}
\hline TR\# & RATE & LONG & LAT & ST & TR\# & RATE & LONG & LAT & ST & TR\# & RATE & LONG & LAT & ST \\
\hline 432 & -2.5 & -75.8145 & 36.3601 & NC & 504 & -2 & -75.8059 & 36.3299 & NC & 576 & -0.2 & -75.7945 & 36.299 & NC \\
\hline 433 & -0.1 & -75.813 & 36.36 & NC & 505 & -1.8 & -75.804 & 36.3297 & NC & 577 & -0.3 & -75.7944 & 36.2985 & NC \\
\hline 434 & -2.5 & -75.8144 & 36.3596 & NC & 506 & -2.1 & -75.8058 & 36.3295 & NC & 578 & -0.3 & -75.7942 & 36.2981 & NC \\
\hline 435 & -0.1 & -75.8128 & 36.3595 & NC & 507 & -2 & -75.8056 & 36.329 & NC & 579 & -0.4 & -75.794 & 36.2976 & $\mathrm{NC}$ \\
\hline 436 & -0.1 & -75.8127 & 36.3591 & NC & 508 & -2 & -75.8055 & 36.3286 & NC & 580 & -0.4 & -75.7939 & 36.2972 & NC \\
\hline 437 & -0.2 & -75.8126 & 36.3586 & NC & 509 & -1.8 & -75.8053 & 36.3281 & NC & 581 & -0.4 & -75.7937 & 36.2968 & NC \\
\hline 438 & -0.2 & -75.8124 & 36.3582 & NC & 510 & -1.6 & -75.8052 & 36.3277 & NC & 582 & -0.2 & -75.7935 & 36.2963 & NC \\
\hline 439 & -0.1 & -75.8123 & 36.3577 & NC & 511 & -1.7 & -75.805 & 36.3272 & NC & 583 & -0.3 & -75.7934 & 36.2959 & NC \\
\hline 440 & -0.2 & -75.8122 & 36.3573 & NC & 512 & -1.7 & -75.8049 & 36.3268 & NC & 584 & -0.2 & -75.7932 & 36.2954 & NC \\
\hline 441 & -0.1 & -75.812 & 36.3568 & NC & 513 & -1.7 & -75.8047 & 36.3263 & NC & 585 & -0.3 & -75.793 & 36.295 & NC \\
\hline 442 & -0.2 & -75.8119 & 36.3564 & NC & 514 & -1.7 & -75.8046 & 36.3259 & NC & 586 & -0.2 & -75.7929 & 36.2945 & NC \\
\hline 443 & -0.1 & -75.8118 & 36.3559 & NC & 515 & -1.8 & -75.8044 & 36.3254 & NC & 587 & -0.4 & -75.7927 & 36.2941 & NC \\
\hline 444 & -0.2 & -75.8116 & 36.3555 & NC & 516 & -1.8 & -75.8043 & 36.325 & NC & 588 & -0.4 & -75.7925 & 36.2936 & NC \\
\hline 445 & -0.4 & -75.8115 & 36.355 & NC & 517 & -1.7 & -75.8041 & 36.3245 & NC & 589 & -0.5 & -75.7924 & 36.2932 & NC \\
\hline 446 & -0.4 & -75.8114 & 36.3546 & NC & 518 & -1.6 & -75.804 & 36.3241 & NC & 590 & -0.5 & -75.7922 & 36.2928 & NC \\
\hline 447 & -0.5 & -75.8112 & 36.3541 & NC & 519 & -1.7 & -75.8038 & 36.3236 & NC & 591 & -0.6 & .75 .792 & 36.2923 & NC \\
\hline 448 & -0.6 & -75.8111 & 36.3537 & NC & 520 & -1.7 & -75.8037 & 36.3232 & NC & 592 & -0.5 & -75.7919 & 36.2919 & NC \\
\hline 449 & -0.5 & -75.811 & 36.3532 & NC & 521 & -1.7 & -75.8035 & 36.3227 & NC & 593 & -0.5 & -75.7917 & 36.2914 & NC \\
\hline 450 & -0.6 & -75.8108 & 36.3528 & NC & 522 & -1.8 & -75.8034 & 36.3223 & NC & 594 & -0.6 & -75.7915 & 36.291 & NC \\
\hline 451 & -0.5 & -75.8107 & 36.3523 & NC & 523 & -1.7 & -75.8032 & 36.3218 & NC & 595 & -0.8 & -75.7914 & 36.2905 & NC \\
\hline 452 & -0.5 & -75.8106 & 36.3519 & NC & 524 & -1.9 & -75.8031 & 36.3214 & NC & 596 & -0.8 & -75.7912 & 36.2901 & NC \\
\hline 453 & -0.5 & -75.8104 & 36.3514 & NC & 525 & -1.7 & -75.8029 & 36.321 & NC & 597 & -0.8 & -75.791 & 36.2896 & NC \\
\hline 454 & -0.5 & -75.8103 & 36.3509 & NC & 526 & -1.6 & -75.8028 & 36.3205 & NC & 598 & -0.8 & -75.7909 & 36.2892 & NC \\
\hline 455 & -0.3 & -75.8102 & 36.3505 & NC & 527 & -1.5 & -75.8026 & 36.3201 & NC & 599 & -0.8 & -75.7907 & 36.2887 & NC \\
\hline 456 & -0.4 & -75.81 & 36.35 & NC & 528 & -1.4 & -75.8025 & 36.3196 & NC & 600 & -0.7 & -75.7905 & 36.2883 & NC \\
\hline 457 & -0.4 & -75.8099 & 36.3496 & NC & 529 & -1.2 & -75.8023 & 36.3192 & NC & 601 & -0.8 & -75.7904 & 36.2879 & NC \\
\hline 458 & -0.4 & -75.8098 & 36.3491 & NC & 530 & -1.2 & -75.8022 & 36.3187 & NC & 602 & -0.7 & -75.7902 & 36.2874 & NC \\
\hline 459 & -0.3 & -75.8096 & 36.3487 & NC & 531 & -1.2 & -75.802 & 36.3183 & NC & 603 & -0.8 & -75.79 & 36.287 & NC \\
\hline 460 & -0.5 & -75.8095 & 36.3482 & $\mathrm{NC}$ & 532 & -1.1 & -75.8019 & 36.3178 & NC & 604 & -0.9 & -75.7899 & 36.2865 & NC \\
\hline 461 & -0.5 & -75.8094 & 36.3478 & NC & 533 & -1.2 & -75.8017 & 36.3174 & NC & 605 & -0.8 & -75.7897 & 36.2861 & NC \\
\hline 462 & -0.4 & -75.8092 & 36.3473 & NC & 534 & -1.2 & -75.8016 & 36.3169 & NC & 606 & -0.8 & -75.7896 & 36.2856 & NC \\
\hline 463 & -0.4 & -75.8091 & 36.3469 & NC & 535 & -1.1 & -75.8014 & 36.3165 & NC & 607 & -0.9 & -75.7894 & 36.2852 & NC \\
\hline 464 & -0.5 & -75.809 & 36.3464 & NC & 536 & -1 & -75.8013 & 36.316 & NC & 608 & -0.8 & -75.7892 & 36.2847 & NC \\
\hline 465 & -0.6 & -75.8088 & 36.346 & NC & 537 & -1 & -75.8011 & 36.3156 & NC & 609 & -0.8 & .75 .7891 & 36.2843 & NC \\
\hline 466 & -0.6 & -75.8087 & 36.3455 & NC & 538 & -1 & -75.801 & 36.3151 & NC & 610 & -0.8 & -75.7889 & 36.2839 & NC \\
\hline 467 & -0.7 & -75.8085 & 36.3451 & NC & 539 & -0.8 & -75.8008 & 36.3147 & NC & 611 & -0.6 & -75.7887 & 36.2834 & NC \\
\hline 468 & -0.8 & -75.8084 & 36.3446 & NC & 540 & -0.7 & -75.8007 & 36.3142 & NC & 612 & -0.5 & -75.7886 & 36.283 & NC \\
\hline 469 & -0.9 & -75.8083 & 36.3442 & NC & 541 & -0.7 & -75.8005 & 36.3138 & NC & 613 & -0.6 & -75.7884 & 36.2825 & NC \\
\hline 470 & -0.9 & -75.8081 & 36.3437 & NC & 542 & -0.7 & -75.8004 & 36.3133 & NC & 614 & -0.7 & -75.7882 & 36.2821 & NC \\
\hline 471 & -0.9 & -75.808 & 36.3433 & NC & 543 & -0.7 & -75.8002 & 36.3129 & NC & 615 & -0.5 & -75.7881 & 36.2816 & NC \\
\hline 472 & -0.8 & -75.8079 & 36.3428 & NC & 544 & -0.6 & -75.8001 & 36.3124 & NC & 616 & -0.5 & -75.7879 & 36.2812 & NC \\
\hline 473 & -0.8 & -75.8077 & 36.3424 & NC & 545 & -0.6 & -75.7999 & 36.312 & NC & 617 & -0.6 & .75 .7877 & 36.2807 & NC \\
\hline 474 & -0.7 & -75.8076 & 36.3419 & NC & 546 & -0.5 & -75.7998 & 36.3115 & NC & 618 & -0.6 & -75.7876 & 36.2803 & NC \\
\hline 475 & -0.7 & -75.8075 & 36.3415 & NC & 547 & -0.6 & -75.7996 & 36.3111 & NC & 619 & -0.5 & -75.7874 & 36.2798 & NC \\
\hline 476 & -0.8 & -75.8073 & 36.341 & NC & 548 & -0.6 & -75.7995 & 36.3106 & NC & 620 & -0.5 & -75.7872 & 36.2794 & NC \\
\hline 477 & -1 & -75.8072 & 36.3406 & NC & 549 & -0.6 & -75.7993 & 36.3102 & NC & 621 & -0.5 & -75.7871 & 36.279 & NC \\
\hline 478 & -0.9 & -75.8071 & 36.3401 & NC & 550 & -0.7 & -75.7992 & 36.3098 & NC & 622 & -0.5 & -75.7869 & 36.2785 & $\mathrm{NC}$ \\
\hline 479 & -1 & -75.8069 & 36.3396 & $\mathrm{NC}$ & 551 & -0.8 & -75.799 & 36.3093 & NC & 623 & -0.5 & -75.7867 & 36.2781 & NC \\
\hline 480 & -1.2 & -75.8068 & 36.3392 & NC & 552 & -0.8 & -75.7989 & 36.3089 & NC & 624 & -0.5 & -75.7866 & 36.2776 & $\mathrm{NC}$ \\
\hline 481 & -1.3 & -75.8067 & 36.3387 & NC & 553 & -0.9 & -75.7987 & 36.3084 & NC & 625 & -0.4 & -75.7864 & 36.2772 & NC \\
\hline 482 & -1.4 & -75.8065 & 36.3383 & NC & 554 & -0.9 & -75.7986 & 36.308 & NC & 626 & -0.5 & -75.7862 & 36.2767 & NC \\
\hline 483 & -1.6 & -75.8064 & 36.3378 & NC & 555 & -0.9 & -75.7984 & 36.3075 & NC & 627 & -0.5 & -75.7861 & 36.2763 & NC \\
\hline 484 & -1.6 & -75.8063 & 36.3374 & NC & 556 & -0.9 & -75.7983 & 36.3071 & NC & 628 & -0.5 & -75.7859 & 36.2758 & NC \\
\hline 485 & -1.6 & -75.8061 & 36.3369 & NC & 557 & -0.9 & -75.7981 & 36.3066 & NC & 629 & -0.5 & -75.7857 & 36.2754 & NC \\
\hline 486 & -1.6 & -75.806 & 36.3365 & NC & 558 & -1 & -75.798 & 36.3062 & NC & 630 & -0.5 & -75.7856 & 36.275 & NC \\
\hline 487 & -1.7 & -75.8059 & 36.336 & NC & 559 & -1 & -75.7978 & 36.3057 & NC & 631 & -0.5 & -75.7854 & 36.2745 & NC \\
\hline 488 & -1.8 & -75.8057 & 36.3356 & NC & 560 & -1 & -75.7977 & 36.3053 & NC & 632 & -0.5 & -75.7852 & 36.2741 & NC \\
\hline 489 & -1.8 & -75.8056 & 36.3351 & NC & 561 & -0.9 & -75.7975 & 36.3048 & NC & 633 & -0.5 & -75.7851 & 36.2736 & NC \\
\hline 490 & -1.8 & -75.8055 & 36.3347 & NC & 562 & -0.8 & -75.7974 & 36.3044 & NC & 634 & -0.5 & -75.7849 & 36.2732 & NC \\
\hline 491 & -1.9 & -75.8053 & 36.3342 & NC & 563 & -0.7 & -75.7972 & 36.3039 & NC & 635 & -0.5 & .75 .7847 & 36.2727 & NC \\
\hline 492 & -2 & .75 .8052 & 36.3338 & NC & 564 & -0.7 & -75.7971 & 36.3035 & NC & 636 & -0.4 & -75.7846 & 36.2723 & NC \\
\hline 493 & -2.1 & -75.8051 & 36.3333 & NC & 565 & -0.6 & -75.7969 & 36.303 & NC & 637 & -0.5 & -75.7844 & 36.2718 & NC \\
\hline 494 & -2.1 & -75.8049 & 36.3329 & NC & 566 & -0.5 & -75.7968 & 36.3026 & NC & 638 & -0.6 & -75.7842 & 36.2714 & NC \\
\hline 495 & -2 & -75.8048 & 36.3324 & NC & 567 & -0.6 & -75.7966 & 36.3021 & NC & 639 & -0.7 & -75.7841 & 36.2709 & $\mathrm{NC}$ \\
\hline 496 & -2 & -75.8047 & 36.332 & NC & 568 & -0.5 & -75.7965 & 36.3017 & NC & 640 & 0.4 & -75.7856 & 36.2708 & NC \\
\hline 497 & -1.9 & -75.8045 & 36.3315 & NC & 569 & -0.3 & -75.7954 & 36.3012 & NC & 641 & -0.6 & -75.7839 & 36.2705 & NC \\
\hline 498 & -1.9 & -75.8064 & 36.3313 & NC & 570 & -0.6 & -75.7963 & 36.3012 & NC & 642 & 0.3 & -75.7855 & 36.2704 & NC \\
\hline 499 & -1.9 & -75.8044 & 36.3311 & NC & 571 & -0.3 & -75.7952 & 36.3008 & NC & 643 & 0.2 & -75.7853 & 36.2699 & NC \\
\hline 500 & -2 & -75.8062 & 36.3308 & NC & 572 & -0.6 & -75.7962 & 36.3008 & NC & 644 & 0.2 & -75.7851 & 36.2695 & NC \\
\hline 501 & -1.8 & -75.8043 & 36.3306 & NC & 573 & -0.2 & -75.795 & 36.3003 & NC & 645 & 0.4 & -75.7849 & 36.2691 & NC \\
\hline 502 & -2 & -75.8061 & 36.3304 & NC & 574 & 0 & .75 .7949 & 36.2999 & NC & 646 & 0.5 & -75.7847 & 36.2686 & NC \\
\hline 503 & -1.8 & -75.8041 & 36.3302 & NC & 575 & -0.1 & -75.7947 & 36.2994 & NC & 647 & 0.5 & -75.7846 & 36.2682 & NC \\
\hline
\end{tabular}




\begin{tabular}{|c|c|c|c|c|c|c|c|c|c|c|c|c|c|c|}
\hline TR\# & RATE & LONG & LAT & ST & TR\# & RATE & LONG & LAT & ST & TR\# & RATE & LONG & LAT & ST \\
\hline 864 & 0.1 & -75.7432 & 36.1767 & NC & 936 & 0.3 & -75.73 & 36.1453 & NC & 1008 & 0.1 & -75.7136 & 36.1157 & NC \\
\hline 865 & 0.2 & -75.743 & 36.1763 & NC & 937 & 0.2 & -75.7298 & 36.1449 & NC & 1009 & 0.1 & -75.7133 & 36.1153 & NC \\
\hline 866 & 0.2 & -75.7428 & 36.1759 & NC & 938 & 0.2 & -75.7296 & 36.1444 & NC & 1010 & 0.1 & -75.7131 & 36.1149 & NC \\
\hline 867 & 0.2 & -75.7426 & 36.1754 & NC & 939 & 0.2 & -75.7293 & 36.144 & NC & 1011 & 0.2 & -75.7128 & 36.1145 & $\mathrm{NC}$ \\
\hline 868 & 0.2 & -75.7424 & 36.175 & NC & 940 & 0.2 & -75.7291 & 36.1436 & NC & 1012 & 0.2 & -75.7126 & 36.114 & NC \\
\hline 869 & 0.3 & .75 .7422 & 36.1746 & NC & 941 & 0.2 & -75.7289 & 36.1432 & NC & 1013 & 0.2 & -75.7123 & 36.1136 & NC \\
\hline 870 & 0.3 & -75.742 & 36.1741 & NC & 942 & 0.2 & -75.7287 & 36.1427 & NC & 1014 & 0.3 & -75.7121 & 36.1132 & NC \\
\hline 871 & 0.2 & -75.7418 & 36.1737 & NC & 943 & 0.3 & -75.7285 & 36.1423 & NC & 1015 & 0.2 & -75.7119 & 36.1128 & NC \\
\hline 872 & 0.2 & -75.7416 & 36.1732 & NC & 944 & 0.4 & -75.7282 & 36.1419 & NC & 1016 & 0.2 & -75.7116 & 36.1124 & NC \\
\hline 873 & 0.1 & -75.7414 & 36.1728 & NC & 945 & 0.4 & -75.728 & 36.1414 & NC & 1017 & 0.2 & -75.7114 & 36.1119 & NC \\
\hline 874 & 0 & -75.7412 & 36.1724 & NC & 946 & 0.4 & -75.7278 & 36.141 & NC & 1018 & 0.2 & -75.7111 & 36.1115 & NC \\
\hline 875 & 0.1 & -75.741 & 36.1719 & NC & 947 & 0.4 & -75.7276 & 36.1406 & NC & 1019 & 0.2 & -75.7109 & 36.1111 & NC \\
\hline 876 & 0 & -75.7408 & 36.1715 & NC & 948 & 0.3 & -75.7274 & 36.1402 & NC & 1020 & 0.2 & -75.7107 & 36.1107 & NC \\
\hline 877 & 0.1 & -75.7406 & 36.1711 & NC & 949 & 0.3 & -75.7272 & 36.1397 & NC & 1021 & 0 & -75.7104 & 36.1103 & NC \\
\hline 878 & 0.2 & -75.7404 & 36.1706 & NC & 950 & 0.5 & -75.7269 & 36.1393 & NC & 1022 & -0.1 & -75.7102 & 36.1098 & NC \\
\hline 879 & 0.1 & -75.7402 & 36.1702 & NC & 951 & 0.2 & -75.7267 & 36.1389 & NC & 1023 & 0.1 & -75.7099 & 36.1094 & NC \\
\hline 880 & 0.1 & -75.74 & 36.1698 & NC & 952 & 0.2 & -75.7265 & 36.1384 & NC & 1024 & -0.1 & -75.7097 & 36.109 & NC \\
\hline 881 & 0.1 & -75.7398 & 36.1693 & NC & 953 & 0.4 & -75.7263 & 36.138 & NC & 1025 & -0.2 & -75.7094 & 36.1086 & NC \\
\hline 882 & 0.2 & -75.7396 & 36.1689 & NC & 954 & 0.5 & -75.7261 & 36.1376 & NC & 1026 & -0.2 & -75.7092 & 36.1082 & NC \\
\hline 883 & 0.2 & -75.7394 & 36.1685 & NC & 955 & 0.4 & -75.7258 & 36.1372 & NC & 1027 & -0.1 & -75.709 & 36.1078 & NC \\
\hline 884 & 0.1 & -75.7392 & 36.168 & $\mathrm{NC}$ & 956 & 0.2 & -75.7256 & 36.1367 & NC & 1028 & -0.1 & -75.7087 & 36.1073 & NC \\
\hline 885 & 0 & -75.739 & 36.1676 & NC & 957 & 0.2 & -75.7254 & 36.1363 & NC & 1029 & 0 & -75.7085 & 36.1069 & NC \\
\hline 886 & 0 & -75.7388 & 36.1672 & NC & 958 & 0.1 & -75.7252 & 36.1359 & NC & 1030 & 0.1 & -75.7082 & 36.1065 & NC \\
\hline 887 & 0.1 & -75.7386 & 36.1667 & NC & 959 & 0 & -75.725 & 36.1354 & NC & 1031 & 0.1 & -75.708 & 36.1061 & NC \\
\hline 888 & 0.2 & -75.7384 & 36.1663 & NC & 960 & 0 & -75.7247 & 36.135 & NC & 1032 & 0 & -75.7077 & 36.1057 & NC \\
\hline 889 & 0.1 & -75.7382 & 36.1659 & NC & 961 & 0.2 & -75.7245 & 36.1346 & NC & 1033 & 0 & -75.7075 & 36.1052 & NC \\
\hline 890 & 0 & -75.738 & 36.1654 & NC & 962 & 0.3 & -75.7243 & 36.1342 & NC & 1034 & 0.1 & -75.7073 & 36.1048 & NC \\
\hline 891 & 0.1 & -75.7378 & 36.165 & NC & 963 & 0.2 & -75.7241 & 36.1337 & NC & 1035 & 0.2 & -75.707 & 36.1044 & NC \\
\hline 892 & 0.2 & -75.7376 & 36.1645 & NC & 964 & 0.1 & -75.7239 & 36.1333 & NC & 1036 & 0.2 & -75.7068 & 36.104 & NC \\
\hline 893 & 0.3 & -75.7374 & 36.1641 & NC & 965 & 1.2 & -75.7236 & 36.1329 & NC & 1037 & 0 & -75.7065 & 36.1036 & NC \\
\hline 894 & 0.3 & -75.7372 & 36.1637 & NC & 966 & 0.2 & -75.7234 & 36.1325 & NC & 1038 & 0 & -75.7063 & 36.1032 & NC \\
\hline 895 & 0.3 & -75.737 & 36.1632 & NC & 967 & 0.1 & -75.7232 & 36.132 & NC & 1039 & 0.1 & -75.706 & 36.1027 & NC \\
\hline 896 & 0.2 & -75.7368 & 36.1628 & NC & 968 & 0.1 & -75.723 & 36.1316 & NC & 1040 & 0.3 & -75.7058 & 36.1023 & NC \\
\hline 897 & 0.1 & -75.7366 & 36.1624 & NC & 969 & 0.1 & -75.7228 & 36.1312 & NC & 1041 & 0.2 & -75.7056 & 36.1019 & NC \\
\hline 898 & 0.1 & -75.7364 & 36.1619 & NC & 970 & -0.1 & -75.7225 & 36.1307 & NC & 1042 & 0.2 & -75.7053 & 36.1015 & NC \\
\hline 899 & 0.2 & -75.7363 & 36.1615 & NC & 971 & -0.1 & -75.7223 & 36.1303 & NC & 1043 & 0.1 & -75.7051 & 36.1011 & NC \\
\hline 900 & 0.3 & -75.7361 & 36.1611 & NC & 972 & -0.1 & -75.7221 & 36.1299 & NC & 1044 & 0 & -75.7048 & 36.1006 & NC \\
\hline 901 & 0.2 & -75.7359 & 36.1606 & NC & 973 & -0.1 & -75.7219 & 36.1295 & NC & 1045 & 0 & -75.7046 & 36.1002 & $\mathrm{NC}$ \\
\hline 902 & 0.2 & -75.7357 & 36.1602 & NC & 974 & -0.1 & -75.7217 & 36.129 & NC & 1046 & 0 & -75.7044 & 36.0998 & NC \\
\hline 903 & 0.2 & -75.7355 & 36.1598 & NC & 975 & -0.1 & -75.7214 & 36.1286 & NC & 1047 & 0 & -75.7041 & 36.0994 & NC \\
\hline 904 & 0.1 & -75.7353 & 36.1593 & NC & 976 & -0.1 & -75.7212 & 36.1282 & NC & 1048 & 0 & -75.7039 & 36.099 & NC \\
\hline 905 & 0.1 & -75.7351 & 36.1589 & NC & 977 & -0.1 & -75.721 & 36.1277 & NC & 1049 & 0 & -75.7036 & 36.0986 & NC \\
\hline 906 & 0.1 & -75.7349 & 36.1585 & NC & 978 & -0.1 & -75.7208 & 36.1273 & NC & 1050 & 0 & -75.7034 & 36.0981 & NC \\
\hline 907 & 0 & -75.7347 & 36.158 & NC & 979 & -0.2 & -75.7206 & 36.1269 & $\mathrm{NC}$ & 1051 & 0 & -75.7031 & 36.0977 & NC \\
\hline 908 & 0.1 & -75.7345 & 36.1576 & NC & 980 & -0.4 & -75.7203 & 36.1265 & NC & 1052 & 0 & -75.7029 & 36.0973 & NC \\
\hline 909 & 0.1 & -75.7343 & 36.1571 & NC & 981 & -0.5 & -75.7196 & 36.1262 & $\mathrm{NC}$ & 1053 & -0.1 & -75.7038 & 36.0971 & NC \\
\hline 910 & 0.2 & -75.7341 & 36.1567 & NC & 982 & -0.3 & -75.7201 & 36.126 & NC & 1054 & -0.1 & -75.7035 & 36.0967 & NC \\
\hline 911 & 0.1 & -75.7339 & 36.1563 & NC & 983 & -0.5 & -75.7194 & 36.1257 & NC & 1055 & -0.1 & .75 .7032 & 36.0963 & NC \\
\hline 912 & 0.1 & -75.7337 & 36.1558 & NC & 984 & -0.2 & -75.7199 & 36.1256 & NC & 1056 & -0.2 & -75.703 & 36.0959 & NC \\
\hline 913 & 0.3 & -75.7335 & 36.1554 & NC & 985 & -0.4 & -75.7191 & 36.1253 & $\mathrm{NC}$ & 1057 & -0.2 & -75.7027 & 36.0955 & NC \\
\hline 914 & 0.3 & -75.7333 & 36.155 & NC & 986 & -0.2 & -75.7189 & 36.1249 & $\mathrm{NC}$ & 1058 & -0.3 & -75.7025 & 36.0951 & NC \\
\hline 915 & -0.3 & -75.7346 & 36.1543 & NC & 987 & -0.3 & -75.7186 & 36.1245 & $\mathrm{NC}$ & 1059 & -0.3 & -75.7022 & 36.0947 & NC \\
\hline 916 & -0.3 & -75.7344 & 36.1539 & NC & 988 & -0.3 & -75.7184 & 36.1241 & NC & 1060 & -0.2 & -75.7019 & 36.0942 & NC \\
\hline 917 & -0.5 & -75.7342 & 36.1534 & NC & 989 & -0.2 & -75.7182 & 36.1237 & NC & 1061 & -0.1 & -75.7017 & 36.0938 & NC \\
\hline 918 & -0.4 & -75.734 & 36.153 & NC & 990 & -0.1 & -75.7179 & 36.1232 & NC & 1062 & -0.1 & -75.7014 & 36.0934 & NC \\
\hline 919 & -0.2 & -75.7337 & 36.1526 & NC & 991 & -0.1 & -75.7177 & 36.1228 & NC & 1063 & 0 & -75.7012 & 36.093 & NC \\
\hline 920 & 0.1 & -75.7335 & 36.1521 & NC & 992 & -0.2 & -75.7174 & 36.1224 & NC & 1064 & -0.2 & -75.7009 & 36.0926 & NC \\
\hline 921 & -0.2 & -75.7333 & 36.1517 & NC & 993 & -0.2 & -75.7172 & 36.122 & $\mathrm{NC}$ & 1065 & -0.3 & -75.7006 & 36.0922 & NC \\
\hline 922 & -0.1 & -75.7331 & 36.1513 & NC & 994 & -0.1 & -75.717 & 36.1216 & $\mathrm{NC}$ & 1066 & -0.3 & -75.7004 & 36.0918 & NC \\
\hline 923 & 0 & -75.7329 & 36.1509 & NC & 995 & 0 & -75.7167 & 36.1211 & NC & 1067 & -0.3 & -75.7001 & 36.0913 & NC \\
\hline 924 & -0.1 & -75.7326 & 36.1504 & NC & 996 & 0.1 & -75.7165 & 36.1207 & NC & 1068 & -0.6 & -75.6998 & 36.0909 & NC \\
\hline 925 & -0.3 & -75.7324 & 36.15 & NC & 997 & 0 & .75 .7162 & 36.1203 & NC & 1069 & -0.6 & -75.6996 & 36.0905 & NC \\
\hline 926 & -0.2 & -75.7322 & 36.1496 & NC & 998 & 0.1 & -75.716 & 36.1199 & $\mathrm{NC}$ & 1070 & -0.5 & -75.6993 & 36.0901 & NC \\
\hline 927 & -0.2 & -75.732 & 36.1491 & NC & 999 & 0.2 & -75.7157 & 36.1195 & NC & 1071 & -0.5 & .75 .6991 & 36.0897 & NC \\
\hline 928 & -0.1 & -75.7318 & 36.1487 & NC & 1000 & 0.1 & -75.7155 & 36.1191 & NC & 1072 & -0.8 & -75.6988 & 36.0893 & NC \\
\hline 929 & -0.1 & -75.7315 & 36.1483 & NC & 1001 & 0.2 & -75.7153 & 36.1186 & $\mathrm{NC}$ & 1073 & -0.9 & -75.6985 & 36.0889 & $\mathrm{NC}$ \\
\hline 930 & 0 & -75.7313 & 36.1479 & NC & 1002 & 0.2 & -75.715 & 36.1182 & $\mathrm{NC}$ & 1074 & -0.9 & -75.6983 & 36.0884 & NC \\
\hline 931 & 0.1 & -75.7311 & 36.1474 & NC & 1003 & 0.2 & -75.7148 & 36.1178 & $\mathrm{NC}$ & 1075 & -0.7 & -75.698 & 36.088 & NC \\
\hline 932 & 0.2 & -75.7309 & 36.147 & NC & 1004 & 0.2 & -75.7145 & 36.1174 & NC & 1076 & -1 & -75.6978 & 36.0876 & $\mathrm{NC}$ \\
\hline 933 & 0.2 & -75.7307 & 36.1466 & NC & 1005 & 0.2 & -75.7143 & 36.117 & NC & 1077 & -1 & -75.6975 & 36.0872 & NC \\
\hline 934 & 0.2 & -75.7304 & 36.1462 & NC & 1006 & 0.3 & -75.714 & 36.1165 & NC & 1078 & -0.9 & .75 .6972 & 36.0868 & $\mathrm{NC}$ \\
\hline 935 & 0.3 & -75.7302 & 36.1457 & NC & 1007 & 0.2 & -75.7138 & 36.1161 & NC & 1079 & -0.8 & -75.697 & 36.0864 & $\mathrm{NC}$ \\
\hline
\end{tabular}




\begin{tabular}{|c|c|c|c|c|c|c|c|c|c|c|c|c|c|c|}
\hline TR\# & RATE & LONG & LAT & ST & TR\# & RATE & LONG & LAT & ST & TR\# & RATE & LONG & LAT & ST \\
\hline 1080 & -0.6 & -75.6967 & 36.086 & NC & 1152 & -0.1 & -75.6771 & 36.0579 & NC & 1224 & 0.5 & -75.6594 & 36.0284 & NC \\
\hline 1081 & -0.6 & -75.6965 & 36.0855 & NC & 1153 & 0.1 & -75.6769 & 36.0575 & NC & 1225 & 0.4 & -75.6592 & 36.028 & NC \\
\hline 1082 & -0.7 & -75.6962 & 36.0851 & NC & 1154 & 0.3 & -75.6766 & 36.0571 & NC & 1226 & 0.4 & -75.6589 & 36.0276 & NC \\
\hline 1083 & -0.8 & -75.6959 & 36.0847 & NC & 1155 & 0.2 & -75.6763 & 36.0566 & $\mathrm{NC}$ & 1227 & 0.5 & -75.6587 & 36.0272 & NC \\
\hline 1084 & -0.7 & -75.6957 & 36.0843 & NC & 1156 & 0 & -75.676 & 36.0562 & NC & 1228 & 0.6 & -75.6584 & 36.0267 & NC \\
\hline 1085 & -0.7 & -75.6954 & 36.0839 & NC & 1157 & 0 & -75.6758 & 36.0558 & NC & 1229 & 0.8 & -75.6582 & 36.0263 & NC \\
\hline 1086 & -1 & -75.6952 & 36.0835 & NC & 1158 & -0.1 & -75.6755 & 36.0554 & NC & 1230 & 0.9 & -75.6579 & 36.0259 & NC \\
\hline 1087 & -1.1 & -75.6949 & 36.0831 & NC & 1159 & -0.3 & -75.6752 & 36.055 & NC & 1231 & 0.9 & -75.6577 & 36.0255 & NC \\
\hline 1088 & -1.2 & -75.6946 & 36.0827 & $\mathrm{NC}$ & 1160 & -0.4 & -75.6749 & 36.0546 & NC. & 1232 & 0.8 & -75.6574 & 36.0251 & NC \\
\hline 1089 & -1 & -75.6944 & 36.0822 & NC & 1161 & -0.6 & -75.6747 & 36.0542 & $\mathrm{NC}$ & 1233 & 0.9 & -75.6571 & 36.0247 & NC \\
\hline 1090 & -1 & -75.6941 & 36.0818 & NC & 1162 & -0.7 & -75.6744 & 36.0538 & NC & 1234 & 1.2 & -75.6569 & 36.0242 & NC \\
\hline 1091 & -1.1 & -75.6939 & 36.0814 & NC & 1163 & -0.8 & -75.6741 & 36.0534 & NC & 1235 & 0.8 & -75.6566 & 36.0238 & NC \\
\hline 1092 & -1.1 & -75.6936 & 36.081 & NC & 1164 & -0.8 & -75.6738 & 36.053 & NC & 1236 & 0.6 & -75.6564 & 36.0234 & NC \\
\hline 1093 & -1.1 & -75.6933 & 36.0806 & NC & 1165 & -1 & -75.6736 & 36.0526 & NC & 1237 & 0.7 & -75.6561 & 36.023 & NC \\
\hline 1094 & -1 & -75.6931 & 36.0802 & NC & 1166 & -1.1 & -75.6733 & 36.0522 & NC & 1238 & 0.7 & -75.6559 & 36.0226 & NC \\
\hline 1095 & -1 & -75.6928 & 36.0798 & NC & 1167 & -1.2 & -75.673 & 36.0518 & $\mathrm{NC}$ & 1239 & 0.8 & -75.6556 & 36.0222 & NC \\
\hline 1096 & -1.2 & -75.6926 & 36.0793 & NC & 1168 & -1.2 & -75.6727 & 36.0514 & NC & 1240 & 1 & -75.6554 & 36.0217 & NC \\
\hline 1097 & -1.4 & -75.6923 & 36.0789 & NC & 1169 & -1.1 & -75.6725 & 36.0509 & NC & 1241 & 1.1 & -75.6551 & 36.0213 & NC \\
\hline 1098 & -1.5 & -75.692 & 36.0785 & NC & 1170 & -1 & -75.6722 & 36.0505 & NC & 1242 & 1.1 & -75.6549 & 36.0209 & $\mathrm{NC}$ \\
\hline 1099 & -1.4 & -75.6918 & 36.0781 & NC & 1171 & -1.1 & -75.6719 & 36.0501 & NC & 1243 & 0.9 & -75.6546 & 36.0205 & NC \\
\hline 1100 & -1.3 & -75.6915 & 36.0777 & NC & 1172 & -1.2 & -75.6716 & 36.0497 & NC & 1244 & 1 & -75.6544 & 36.0201 & $\mathrm{NC}$ \\
\hline 1101 & -1.2 & -75.6913 & 36.0773 & $\mathrm{NC}$ & 1173 & -1.3 & -75.6714 & 36.0493 & NC & 1245 & 0.9 & -75.6541 & 36.0197 & NC \\
\hline 1102 & -1 & -75.691 & 36.0769 & NC & 1174 & -1.5 & -75.6711 & 36.0489 & NC & 1246 & 0.8 & -75.6538 & 36.0192 & NC \\
\hline 1103 & -1 & -75.6907 & 36.0764 & NC & 1175 & -1.6 & -75.6708 & 36.0485 & NC & 1247 & 1 & -75.6536 & 36.0188 & NC \\
\hline 1104 & -1 & -75.6905 & 36.076 & NC & 1176 & -1.7 & -75.6705 & 36.0481 & NC & 1248 & 0.9 & -75.6533 & 36.0184 & NC \\
\hline 1105 & -1.2 & -75.6902 & 36.0756 & NC & 1177 & -1.7 & -75.6703 & 36.0477 & NC & 1249 & 1 & -75.6531 & 36.018 & NC \\
\hline 1106 & -1.3 & -75.6899 & 36.0752 & $\mathrm{NC}$ & 1178 & -1.7 & -75.67 & 36.0473 & NC & 1250 & 1 & -75.6528 & 36.0176 & NC \\
\hline 1107 & -1.3 & -75.6897 & 36.0748 & NC & 1179 & -2 & -75.6697 & 36.0469 & NC & 1251 & 1.3 & -75.6526 & 36.0172 & NC \\
\hline 1108 & -1.4 & -75.6894 & 36.0744 & NC & 1180 & -2.1 & -75.6694 & 36.0465 & NC & 1252 & 1.2 & -75.6523 & 36.0167 & NC \\
\hline 1109 & -1.3 & -75.6892 & 36.074 & NC & 1181 & -2.1 & -75.6692 & 36.0461 & NC & 1253 & 0.9 & -75.6521 & 36.0163 & NC \\
\hline 1110 & -1.5 & -75.6889 & 36.0735 & NC & 1182 & -2.1 & -75.6689 & 36.0457 & NC & 1254 & 0.9 & -75.6518 & 36.0159 & NC \\
\hline 1111 & -1.5 & -75.6886 & 36.0731 & NC & 1183 & -2.1 & -75.6686 & 36.0452 & NC & 1255 & 1 & -75.6516 & 36.0155 & NC \\
\hline 1112 & -1.5 & -75.6884 & 36.0727 & NC & 1184 & -2.3 & -75.6683 & 36.0448 & NC & 1256 & 1.1 & -75.6513 & 36.0151 & NC \\
\hline 1113 & -1.5 & -75.6881 & 36.0723 & NC & 1185 & -2.3 & -75.6681 & 36.0444 & NC & 1257 & 1 & -75.6511 & 36.0147 & NC \\
\hline 1114 & -1.6 & -75.6879 & 36.0719 & NC & 1186 & -2.3 & -75.6678 & 36.044 & NC & 1258 & 1 & -75.6492 & 36.0142 & NC \\
\hline 1115 & -1.3 & -75.6876 & 36.0715 & NC & 1187 & -2.5 & -75.6675 & 36.0436 & NC & 1259 & 0.9 & -75.6508 & 36.0142 & NC \\
\hline 1116 & -1.1 & -75.6873 & 36.0711 & NC & 1188 & -2.6 & -75.6672 & 36.0432 & NC & 1260 & 0.9 & -75.6506 & 36.0138 & NC \\
\hline 1117 & -1.4 & -75.6871 & 36.0706 & NC & 1189 & -2.8 & -75.667 & 36.0428 & $\mathrm{NC}$ & 1261 & 1 & -75.649 & 36.0137 & NC \\
\hline 1118 & 0.1 & -75.6857 & 36.0705 & NC & 1190 & -3 & -75.6667 & 36.0424 & NC & 1262 & 1 & -75.6503 & 36.0134 & NC \\
\hline 1119 & -1.6 & -75.6868 & 36.0702 & NC & 1191 & -1.3 & -75.6678 & 36.0422 & NC & 1263 & 0.9 & -75.6487 & 36.0133 & $\mathrm{NC}$ \\
\hline 1120 & 0.4 & -75.6854 & 36.0701 & NC & 1192 & -1.3 & -75.6675 & 36.0417 & NC & 1264 & 0.8 & -75.6485 & 36.0129 & NC \\
\hline 1121 & -1.5 & -75.6866 & 36.0698 & NC & 1193 & -1.3 & -75.6673 & 36.0413 & NC. & 1265 & 0.9 & -75.6482 & 36.0125 & NC \\
\hline 1122 & 0.6 & -75.6851 & 36.0697 & NC & 1194 & -1.3 & -75.667 & 36.0409 & NC & 1266 & 0.9 & -75.648 & 36.0121 & NC \\
\hline 1123 & -1.6 & -75.6863 & 36.0694 & NC & 1195 & -1.3 & -75.6668 & 36.0405 & NC & 1267 & 0.8 & -75.6478 & 36.0117 & NC \\
\hline 1124 & 0.6 & -75.6848 & 36.0693 & NC & 1196 & -1.2 & -75.6665 & 36.0401 & NC & 1268 & 0.8 & -75.6475 & 36.0112 & NC \\
\hline 1125 & 0.7 & -75.6846 & 36.0689 & NC & 1197 & -1.5 & .75 .6663 & 36.0397 & NC & 1269 & 0.7 & -75.6473 & 36.0108 & NC \\
\hline 1126 & 0.7 & -75.6843 & 36.0685 & NC & 1198 & -1.7 & -75.666 & 36.0392 & NC & 1270 & 0.7 & -75.647 & 36.0104 & NC \\
\hline 1127 & 0.5 & -75.684 & 36.068 & NC & 1199 & -1.6 & -75.6658 & 36.0388 & NC & 1271 & 0.8 & -75.6468 & 36.01 & NC \\
\hline 1128 & 0.3 & -75.6837 & 36.0676 & NC & 1200 & -1.5 & -75.6655 & 36.0384 & NC & 1272 & 0.8 & -75.6465 & 36.0096 & NC \\
\hline 1129 & 0.2 & -75.6835 & 36.0672 & NC & 1201 & -1.3 & -75.6653 & 36.038 & NC & 1273 & 0.8 & -75.6463 & 36.0091 & NC \\
\hline 1130 & 0.2 & -75.6832 & 36.0668 & NC & 1202 & -1.2 & .75 .665 & 36.0376 & NC & 1274 & 0.7 & -75.646 & 36.0087 & NC \\
\hline 1131 & 0.3 & -75.6829 & 36.0664 & NC & 1203 & -1.2 & -75.6648 & 36.0372 & NC & 1275 & 0.7 & -75.6458 & 36.0083 & NC \\
\hline 1132 & 0.8 & -75.6826 & 36.066 & NC & 1204 & -1.2 & -75.6645 & 36.0367 & NC & 1276 & 0.7 & .75 .6456 & 36.0079 & NC \\
\hline 1133 & 0.8 & -75.6824 & 36.0656 & NC & 1205 & -1.1 & -75.6642 & 36.0363 & NC & 1277 & 0.6 & -75.6453 & 36.0075 & NC \\
\hline 1134 & 0.9 & -75.6821 & 36.0652 & NC & 1206 & -1.1 & -75.664 & 36.0359 & NC & 1278 & 0.5 & -75.6451 & 36.0071 & NC \\
\hline 1135 & 1 & -75.6818 & 36.0648 & NC & 1207 & -1 & -75.6637 & 36.0355 & NC & 1279 & 0.3 & -75.6448 & 36.0066 & $\mathrm{NC}$ \\
\hline 1136 & 0.9 & -75.6815 & 36.0644 & NC & 1208 & -0.9 & -75.6635 & 36.0351 & NC & 1280 & 0.4 & -75.6446 & 36.0062 & NC \\
\hline 1137 & 0.8 & -75.6813 & 36.064 & NC & 1209 & -0.7 & -75.6632 & 36.0347 & NC & 1281 & 0.4 & -75.6443 & 36.0058 & NC \\
\hline 1138 & 0.5 & -75.681 & 36.0636 & NC & 1210 & -0.5 & -75.663 & 36.0342 & NC & 1282 & 0.4 & -75.6441 & 36.0054 & NC \\
\hline 1139 & 0.7 & -75.6807 & 36.0632 & NC & 1211 & -0.3 & .75 .6627 & 36.0338 & NC & 1283 & 0.4 & -75.6439 & 36.005 & NC \\
\hline 1140 & 0.8 & -75.6804 & 36.0628 & NC & 1212 & -0.1 & -75.6625 & 36.0334 & NC & 1284 & 0.4 & -75.6436 & 36.0045 & $\mathrm{NC}$ \\
\hline 1141 & 0.8 & -75.6802 & 36.0623 & NC & 1213 & -0.1 & -75.6622 & 36.033 & NC & 1285 & 0.5 & -75.6434 & 36.0041 & NC \\
\hline 1142 & 0.7 & -75.6799 & 36.0619 & NC & 1214 & 0 & -75.662 & 36.0326 & NC & 1286 & 0.6 & -75.6431 & 36.0037 & NC \\
\hline 1143 & 0.4 & -75.6796 & 36.0615 & NC & 1215 & 0.1 & -75.6617 & 36.0322 & $\mathrm{NC}$ & 1287 & 0.5 & -75.6429 & 36.0033 & NC \\
\hline 1144 & 0 & -75.6793 & 36.0611 & NC & 1216 & 0.3 & -75.6615 & 36.0317 & NC & 1288 & 0.5 & -75.6426 & 36.0029 & NC \\
\hline 1145 & 0.2 & -75.6791 & 36.0607 & NC & 1217 & 0.3 & -75.6612 & 36.0313 & NC & 1289 & 0.5 & -75.6424 & 36.0025 & NC \\
\hline 1146 & 0.2 & -75.6788 & 36.0603 & NC & 1218 & 0.2 & -75.6609 & 36.0309 & NC & 1290 & 0.5 & -75.6421 & 36.002 & NC \\
\hline 1147 & 0.2 & -75.6785 & 36.0599 & NC & 1219 & 0.2 & -75.6607 & 36.0305 & NC & 1291 & 0.3 & -75.6419 & 36.0016 & NC \\
\hline 1148 & 0.1 & -75.6782 & 36.0595 & NC & 1220 & 0.4 & -75.6604 & 36.0301 & NC & 1292 & 0.3 & -75.6417 & 36.0012 & $\mathrm{NC}$ \\
\hline 1149 & -0.1 & -75.678 & 36.0591 & NC & 1221 & 0.4 & -75.6602 & 36.0297 & $\mathrm{NC}$ & 1293 & 0.3 & -75.6414 & 36.0008 & NC \\
\hline 1150 & -0.2 & -75.6777 & 36.0587 & NC & 1222 & 0.4 & -75.6599 & 36.0292 & NC & 1294 & 0.2 & -75.6412 & 36.0004 & NC \\
\hline 1151 & -0.2 & -75.6774 & 36.0583 & NC & 1223 & 0.4 & -75.6597 & 36.0288 & NC & 1295 & 0.2 & -75.6409 & 35.9999 & $\mathrm{NC}$ \\
\hline
\end{tabular}




\begin{tabular}{|c|c|c|c|c|c|c|c|c|c|c|c|c|c|c|}
\hline TR\# & RATE & LONG & LAT & ST & TR\# & RATE & LONG & LAT & ST & TR\# & RATE & LONG & LAT & ST \\
\hline 1296 & 0.2 & -75.6407 & 35.9995 & NC & 1368 & -0.5 & -75.6228 & 35.9699 & NC & 1440 & -1 & -75.6073 & 35.9404 & NC \\
\hline 1297 & 0.3 & -75.6404 & 35.9991 & NC & 1369 & -0.3 & -75.6226 & 35.9695 & NC & 1441 & -1 & -75.6071 & 35.9399 & NC \\
\hline 1298 & 0.3 & -75.6402 & 35.9987 & NC & 1370 & -0.4 & -75.6224 & 35.9691 & NC & 1442 & -0.9 & -75.6069 & 35.9395 & NC \\
\hline 1299 & 0.2 & -75.64 & 35.9983 & NC & 1371 & -0.6 & -75.6221 & 35.9686 & NC & 1443 & -1 & -75.6066 & 35.9391 & NC \\
\hline 1300 & 0.4 & -75.6397 & 35.9978 & NC & 1372 & -0.3 & -75.6219 & 35.9682 & NC & 1444 & -0.9 & -75.6064 & 35.9386 & NC \\
\hline 1301 & 0.4 & -75.6395 & 35.9974 & NC & 1373 & -0.3 & -75.6217 & 35.9678 & NC & 1445 & -0.9 & -75.6062 & 35.9382 & NC \\
\hline 1302 & 0.3 & -75.6392 & 35.997 & NC & 1374 & -0.4 & -75.6214 & 35.9674 & NC & 1446 & -0.9 & -75.6059 & 35.9378 & NC \\
\hline 1303 & 0.3 & -75.639 & 35.9966 & NC & 1375 & -0.4 & .75 .6212 & 35.9669 & NC & 1447 & -1 & -75.6057 & 35.9374 & NC \\
\hline 1304 & 0.3 & -75.6387 & 35.9962 & NC & 1376 & -0.3 & -75.6209 & 35.9665 & NC & 1448 & -1 & -75.6054 & 35.9369 & NC \\
\hline 1305 & 0.2 & -75.6385 & 35.9958 & NC & 1377 & -0.4 & -75.6207 & 35.9661 & NC & 1449 & -0.9 & -75.6052 & 35.9365 & NC \\
\hline 1306 & 0.1 & -75.6382 & 35.9953 & NC & 1378 & -0.5 & -75.6205 & 35.9657 & NC & 1450 & -0.8 & -75.605 & 35.9361 & NC \\
\hline 1307 & 0.2 & -75.638 & 35.9949 & NC & 1379 & -0.6 & -75.6202 & 35.9652 & NC & 1451 & -0.8 & -75.6047 & 35.9357 & NC \\
\hline 1308 & 0.1 & -75.6378 & 35.9945 & NC & 1380 & -0.6 & -75.62 & 35.9648 & NC & 1452 & -0.8 & -75.6045 & 35.9352 & NC \\
\hline 1309 & 0.1 & -75.6375 & 35.9941 & NC & 1381 & -0.7 & -75.6198 & 35.9644 & NC & 1453 & -0.8 & -75.6043 & 35.9348 & NC \\
\hline 1310 & 0.2 & -75.6373 & 35.9937 & NC & 1382 & -0.7 & -75.6195 & 35.964 & NC & 1454 & -0.7 & -75.604 & 35.9344 & NC \\
\hline 1311 & 0.3 & -75.637 & 35.9932 & NC & 1383 & -0.7 & -75.6193 & 35.9635 & NC & 1455 & -0.8 & .75 .6038 & 35.934 & NC \\
\hline 1312 & 0.1 & .75 .6368 & 35.9928 & NC & 1384 & -0.7 & -75.6191 & 35.9631 & NC & 1456 & -0.8 & .75 .6036 & 35.9335 & NC \\
\hline 1313 & 0 & -75.6365 & 35.9924 & NC & 1385 & -0.8 & -75.6188 & 35.9627 & NC & 1457 & -0.8 & -75.6033 & 35.9331 & NC \\
\hline 1314 & -0.2 & -75.6363 & 35.992 & NC & 1386 & -0.8 & -75.6186 & 35.9623 & NC & 1458 & -0.8 & .75 .6031 & 35.9327 & NC \\
\hline 1315 & -0.2 & -75.6361 & 35.9916 & NC & 1387 & -0.7 & -75.6184 & 35.9618 & NC & 1459 & -0.8 & .75 .6029 & 35.9322 & NC \\
\hline 1316 & -0.1 & .75 .6358 & 35.9912 & NC & 1388 & -0.8 & -75.6181 & 35.9614 & NC & 1460 & -0.8 & -75.6026 & 35.9318 & NC \\
\hline 1317 & -0.2 & -75.6356 & 35.9907 & NC & 1389 & -0.8 & -75.6179 & 35.961 & NC & 1461 & -0.8 & -75.6024 & 35.9314 & NC \\
\hline 1318 & -0.4 & -75.6353 & 35.9903 & NC & 1390 & -0.8 & -75.6177 & 35.9606 & NC & 1462 & -0.7 & -75.6022 & 35.931 & NC \\
\hline 1319 & -0.1 & -75.6351 & 35.9899 & NC & 1391 & -0.9 & -75.6174 & 35.9601 & NC & 1463 & -0.6 & -75.6019 & 35.9305 & NC \\
\hline 1320 & 0.3 & -75.6348 & 35.9895 & NC & 1392 & -1 & -75.6172 & 35.9597 & NC & 1464 & -0.6 & -75.6017 & 35.9301 & NC \\
\hline 1321 & 0 & -75.6346 & 35.9891 & NC & 1393 & -1 & -75.6169 & 35.9593 & NC & 1465 & -0.5 & -75.6014 & 35.9297 & NC \\
\hline 1322 & -0.1 & -75.6343 & 35.9886 & NC & 1394 & -0.9 & -75.6167 & 35.9589 & NC & 1466 & -0.4 & -75.6012 & 35.9293 & NC \\
\hline 1323 & -0.2 & -75.6341 & 35.9882 & NC & 1395 & -0.9 & -75.6165 & 35.9584 & NC & 1467 & -0.4 & -75.601 & 35.9288 & NC \\
\hline 1324 & -0.3 & -75.6339 & 35.9878 & NC & 1396 & -0.8 & -75.6162 & 35.958 & NC & 1468 & -0.3 & -75.6007 & 35.9284 & NC \\
\hline 1325 & -0.3 & -75.6336 & 35.9874 & NC & 1397 & -0.9 & -75.616 & 35.9576 & NC & 1469 & -0.4 & -75.6005 & 35.928 & NC \\
\hline 1326 & -0.2 & -75.6334 & 35.987 & NC & 1398 & -1.1 & .75 .6158 & 35.9572 & NC & 1470 & 0.2 & -75.603 & 35.9278 & NC \\
\hline 1327 & -0.2 & -75.6331 & 35.9866 & NC & 1399 & -0.7 & -75.6165 & 35.957 & NC & 1471 & -0.4 & -75.6003 & 35.9276 & NC \\
\hline 1328 & -0.2 & -75.6329 & 35.9861 & NC & 1400 & -1.2 & -75.6155 & 35.9567 & NC & 1472 & 0.2 & -75.6028 & 35.9274 & NC \\
\hline 1329 & -0.2 & -75.6326 & 35.9857 & NC & 1401 & -0.6 & -75.6163 & 35.9566 & NC & 1473 & -0.4 & -75.6 & 35.9271 & NC \\
\hline 1330 & 0 & -75.6315 & 35.9856 & NC & 1402 & -1.3 & -75.6153 & 35.9563 & NC & 1474 & 0.2 & -75.6025 & 35.927 & NC \\
\hline 1331 & -0.3 & -75.6324 & 35.9853 & NC & 1403 & -0.6 & -75.616 & 35.9561 & NC & 1475 & -0.4 & -75.5998 & 35.9267 & NC \\
\hline 1332 & 0 & -75.6313 & 35.9852 & NC & 1404 & -0.6 & -75.6158 & 35.9557 & NC & 1476 & 0 & .75 .6023 & 35.9265 & NC \\
\hline 1333 & -0.2 & -75.6311 & 35.9848 & NC & 1405 & -0.7 & -75.6156 & 35.9553 & NC & 1477 & 0 & -75.6021 & 35.9261 & NC \\
\hline 1334 & -0.2 & -75.6308 & 35.9844 & NC & 1406 & -0.7 & -75.6153 & 35.9549 & NC & 1478 & 0.4 & -75.6018 & 35.9257 & NC \\
\hline 1335 & -0.2 & -75.6306 & 35.9839 & NC & 1407 & -0.8 & -75.6151 & 35.9544 & NC & 1479 & 0 & -75.6016 & 35.9253 & NC \\
\hline 1336 & -0.2 & -75.6304 & 35.9835 & NC & 1408 & -0.9 & -75.6149 & 35.954 & NC & 1480 & -0.2 & -75.6014 & 35.9248 & NC \\
\hline 1337 & -0.3 & -75.6301 & 35.9831 & NC & 1409 & -0.9 & -75.6146 & 35.9536 & NC & 1481 & 0.1 & -75.6012 & 35.9244 & NC \\
\hline 1338 & -0.5 & -75.6299 & 35.9827 & NC & 1410 & -0.9 & -75.6144 & 35.9532 & NC & 1482 & 0 & -75.6009 & 35.924 & NC \\
\hline 1339 & -0.5 & -75.6296 & 35.9822 & NC & 1411 & -0.9 & -75.6141 & 35.9527 & NC & 1483 & -0.2 & -75.6007 & 35.9236 & NC \\
\hline 1340 & -0.4 & -75.6294 & 35.9818 & NC & 1412 & -0.9 & -75.6139 & 35.9523 & NC & 1484 & -0.4 & -75.6005 & 35.9231 & NC \\
\hline 1341 & -0.3 & -75.6292 & 35.9814 & NC & 1413 & -0.9 & -75.6137 & 35.9519 & NC & 1485 & 0 & -75.6002 & 35.9227 & NC \\
\hline 1342 & -0.2 & -75.6289 & 35.981 & NC & 1414 & -0.8 & -75.6134 & 35.9515 & NC & 1486 & 0 & -75.6 & 35.9223 & NC \\
\hline 1343 & -0.1 & -75.6287 & 35.9805 & NC & 1415 & -0.9 & -75.6132 & 35.951 & NC & 1487 & 0.1 & -75.5998 & 35.9219 & NC \\
\hline 1344 & -0.1 & -75.6285 & 35.9801 & NC & 1416 & -0.9 & -75.613 & 35.9506 & NC & 1488 & 0.1 & -75.5995 & 35.9214 & NC \\
\hline 1345 & -0.1 & -75.6282 & 35.9797 & NC & 1417 & -1 & -75.6127 & 35.9502 & NC & 1489 & 0 & -75.5993 & 35.921 & NC \\
\hline 1346 & -0.1 & -75.628 & 35.9793 & NC & 1418 & -0.9 & -75.6125 & 35.9497 & NC & 1490 & 0 & -75.5991 & 35.9206 & NC \\
\hline 1347 & 0 & -75.6278 & 35.9788 & NC & 1419 & -0.7 & -75.6123 & 35.9493 & NC & 1491 & 0.2 & -75.5988 & 35.9202 & NC \\
\hline 1348 & -0.1 & -75.6275 & 35.9784 & NC & 1420 & -0.7 & -75.612 & 35.9489 & NC & 1492 & 0.2 & -75.5986 & 35.9197 & NC \\
\hline 1349 & -0.2 & -75.6273 & 35.978 & NC & 1421 & -0.9 & -75.6118 & 35.9485 & NC & 1493 & 0 & -75.5984 & 35.9193 & NC \\
\hline 1350 & -0.1 & -75.6271 & 35.9776 & NC & 1422 & -0.9 & -75.6116 & 35.948 & NC & 1494 & 0.2 & -75.5981 & 35.9189 & NC \\
\hline 1351 & -0.2 & -75.6268 & 35.9771 & NC & 1423 & -1 & -75.6113 & 35.9476 & NC & 1495 & 0.3 & -75.5979 & 35.9185 & NC \\
\hline 1352 & -0.3 & -75.6266 & 35.9767 & NC & 1424 & -0.8 & -75.6111 & 35.9472 & NC & 1496 & 0.3 & -75.5977 & 35.918 & NC \\
\hline 1353 & -0.3 & -75.6264 & 35.9763 & NC & 1425 & -0.8 & -75.6109 & 35.9468 & NC & 1497 & 0.6 & -75.5975 & 35.9176 & NC \\
\hline 1354 & -0.1 & -75.6261 & 35.9759 & NC & 1426 & -0.8 & -75.6106 & 35.9463 & NC & 1498 & 0.5 & -75.5972 & 35.9172 & NC \\
\hline 1355 & 0.1 & -75.6259 & 35.9754 & NC & 1427 & -0.8 & -75.6104 & 35.9459 & NC & 1499 & 0.7 & -75.597 & 35.9168 & NC \\
\hline 1356 & -0.1 & -75.6256 & 35.975 & NC & 1428 & -0.7 & -75.6101 & 35.9455 & NC & 1500 & 0.8 & -75.5968 & 35.9164 & NC \\
\hline 1357 & -0.1 & -75.6254 & 35.9746 & NC & 1429 & -0.8 & -75.6099 & 35.9451 & NC & 1501 & 0.5 & -75.5965 & 35.9159 & NC \\
\hline 1358 & -0.1 & -75.6252 & 35.9742 & NC & 1430 & -0.9 & -75.6097 & 35.9446 & NC & 1502 & 0.4 & -75.5963 & 35.9155 & NC \\
\hline 1359 & -0.3 & -75.6249 & 35.9737 & NC & 1431 & -0.9 & -75.6094 & 35.9442 & NC & 1503 & 0.4 & -75.5961 & 35.9151 & NC \\
\hline 1360 & -0.2 & -75.6247 & 35.9733 & NC & 1432 & -0.8 & -75.6092 & 35.9438 & NC & 1504 & -1 & -75.5958 & 35.9147 & NC \\
\hline 1361 & -0.3 & -75.6245 & 35.9729 & NC & 1433 & -0.8 & -75.609 & 35.9433 & NC & 1505 & -0.9 & -75.5956 & 35.9142 & NC \\
\hline 1362 & -0.3 & -75.6242 & 35.9725 & $\mathrm{NC}$ & 1434 & -0.8 & -75.6087 & 35.9429 & $\mathrm{NC}$ & 1506 & -0.8 & -75.5954 & 35.9138 & NC \\
\hline 1363 & -0.4 & -75.624 & 35.972 & NC & 1435 & -0.7 & -75.6085 & 35.9425 & $\mathrm{NC}$ & 1507 & -0.7 & -75.5951 & 35.9134 & NC \\
\hline 1364 & -0.3 & -75.6238 & 35.9716 & NC & 1436 & -0.7 & .75 .6083 & 35.9421 & NC & 1508 & -0.4 & -75.5949 & 35.913 & NC \\
\hline 1365 & -0.4 & -75.6235 & 35.9712 & NC & 1437 & -0.7 & -75.608 & 35.9416 & NC & 1509 & -0.4 & -75.5947 & 35.9125 & NC \\
\hline 1366 & -0.6 & -75.6233 & 35.9708 & NC & 1438 & -0.8 & -75.6078 & 35.9412 & NC & 1510 & -0.3 & -75.5945 & 35.9121 & NC \\
\hline 1367 & -0.6 & -75.6231 & 35.9703 & NC & 1439 & -0.9 & -75.6076 & 35.9408 & NC & 1511 & -0.3 & -75.5942 & 35.9117 & NC \\
\hline
\end{tabular}




\begin{tabular}{|c|c|c|c|c|c|c|c|c|c|c|c|c|c|c|}
\hline TR\# & RATE & LONG & LAT & ST & TR\# & RATE & LONG & LAT & ST & TR\# & RATE & LONG & LAT & ST \\
\hline 1512 & -0.4 & -75.594 & 35.9113 & $\mathrm{NC}$ & 1584 & -0.9 & -75.5779 & 35.8818 & $\mathrm{NC}$ & 1656 & -3 & -75.5621 & 35.8516 & $\mathrm{NC}$ \\
\hline 1513 & -0.4 & -75.5938 & 35.9108 & NC & 1585 & -0.9 & -75.5777 & 35.8814 & NC & 1657 & -3.2 & -75.5619 & 35.8512 & NC \\
\hline 1514 & -0.4 & -75.5935 & 35.9104 & NC & 1586 & -0.8 & -75.5774 & 35.881 & NC & 1658 & -3.5 & -75.5616 & 35.8508 & NC \\
\hline 1515 & -0.2 & -75.5933 & 35.91 & $\mathrm{NC}$ & 1587 & -0.8 & -75.5772 & 35.8806 & $\mathrm{NC}$ & 1659 & -3.3 & -75.5614 & 35.8503 & NC \\
\hline 1516 & -0.1 & -75.5931 & 35.9096 & $\mathrm{NC}$ & 1588 & -0.9 & -75.577 & 35.8801 & $\mathrm{NC}$ & 1660 & -3.4 & -75.5612 & 35.8499 & NC \\
\hline 1517 & -0.2 & -75.5928 & 35.9091 & NC & 1589 & -0.9 & -75.5767 & 35.8797 & NC & 1661 & -3.3 & -75.561 & 35.8495 & NC \\
\hline 1518 & -0.2 & -75.5926 & 35.9087 & NC & 1590 & -0.8 & -75.5765 & 35.8793 & NC & 1662 & -3.2 & -75.5608 & 35.849 & NC \\
\hline 1519 & -0.4 & -75.5924 & 35.9083 & NC & 1591 & -0.9 & -75.5763 & 35.8789 & NC & 1663 & -3.4 & -75.5606 & 35.8486 & NC \\
\hline 1520 & -0.5 & -75.5921 & 35.9079 & NC & 1592 & -0.9 & -75.576 & 35.8785 & NC & 1664 & -3.4 & -75.5603 & 35.8482 & NC \\
\hline 1521 & -0.7 & -75.5919 & 35.9075 & NC & 1593 & -1 & -75.5758 & 35.878 & NC & 1665 & -3.4 & -75.5601 & 35.8477 & NC \\
\hline 1522 & -0.7 & -75.5917 & 35.907 & NC & 1594 & -1.1 & -75.5756 & 35.8776 & NC & 1666 & -3.4 & -75.5599 & 35.8473 & NC \\
\hline 1523 & -0.6 & -75.5915 & 35.9066 & NC & 1595 & -1.3 & -75.5754 & 35.8772 & $\mathrm{NC}$ & 1667 & -3.3 & -75.5597 & 35.8469 & NC \\
\hline 1524 & -0.8 & -75.5912 & 35.9062 & NC & 1596 & -1.3 & -75.5751 & 35.8768 & NC & 1668 & -3.2 & -75.5595 & 35.8465 & NC \\
\hline 1525 & -0.8 & -75.591 & 35.9058 & NC & 1597 & -1.4 & -75.5749 & 35.8763 & NC & 1669 & -3.2 & -75.5593 & 35.846 & NC \\
\hline 1526 & -0.8 & -75.5908 & 35.9053 & $\mathrm{NC}$ & 1598 & -1.6 & -75.5747 & 35.8759 & NC & 1670 & -3.1 & -75.5591 & 35.8456 & NC \\
\hline 1527 & -0.7 & -75.5905 & 35.9049 & NC & 1599 & -1.6 & -75.5744 & 35.8755 & $\mathrm{NC}$ & 1671 & -3 & -75.5588 & 35.8452 & $\mathrm{NC}$ \\
\hline 1528 & -0.8 & -75.5903 & 35.9045 & NC & 1600 & -1.5 & -75.5742 & 35.8751 & NC & 1672 & -3 & -75.5586 & 35.8447 & NC \\
\hline 1529 & -0.9 & -75.5901 & 35.9041 & NC & 1601 & -1.6 & -75.574 & 35.8746 & NC & 1673 & -3 & -75.5584 & 35.8443 & NC \\
\hline 1530 & -1.1 & -75.5898 & 35.9036 & NC & 1602 & -2 & -75.5737 & 35.8742 & NC & 1674 & -3 & -75.5582 & 35.8439 & NC \\
\hline 1531 & -0.9 & -75.5896 & 35.9032 & NC & 1603 & -2 & -75.5735 & 35.8738 & NC & 1675 & -3.2 & -75.558 & 35.8434 & NC \\
\hline 1532 & -0.9 & -75.5894 & 35.9028 & NC & 1604 & -2.1 & -75.5733 & 35.8734 & $\mathrm{NC}$ & 1676 & -3 & -75.5578 & 35.843 & NC \\
\hline 1533 & -0.8 & -75.5891 & 35.9024 & NC & 1605 & -2.1 & -75.573 & 35.873 & NC & 1677 & -3 & -75.5583 & 35.8429 & NC \\
\hline 1534 & -0.9 & -75.5889 & 35.9019 & NC & 1606 & -2.3 & -75.5728 & 35.8725 & NC & 1678 & -3 & -75.5575 & 35.8426 & NC \\
\hline 1535 & -1 & -75.5887 & 35.9015 & NC & 1607 & -2.7 & -75.5726 & 35.8721 & NC & 1679 & -3 & -75.5581 & 35.8425 & NC \\
\hline 1536 & -1.1 & -75.5884 & 35.9011 & NC & 1608 & -1.6 & -75.5722 & 35.8718 & NC & 1680 & -3.2 & -75.5573 & 35.8422 & NC \\
\hline 1537 & -1.1 & -75.5882 & 35.9007 & NC & 1609 & -1.9 & -75.5723 & 35.8717 & NC & 1681 & -3.2 & -75.5579 & 35.842 & NC \\
\hline 1538 & -1.2 & -75.588 & 35.9002 & NC & 1610 & -1.5 & -75.572 & 35.8714 & NC & 1682 & -3.2 & -75.5577 & 35.8416 & NC \\
\hline 1539 & -1.3 & -75.5878 & 35.8998 & NC & 1611 & -1.7 & -75.5718 & 35.8709 & NC & 1683 & -3.2 & -75.5575 & 35.8412 & NC \\
\hline 1540 & -1.2 & -75.5875 & 35.8994 & NC & 1612 & -1.8 & -75.5716 & 35.8705 & NC & 1684 & -2.9 & -75.5573 & 35.8407 & NC \\
\hline 1541 & -1.5 & -75.5874 & 35.8992 & NC & 1613 & -1.7 & -75.5713 & 35.8701 & NC & 1685 & -2.8 & -75.5571 & 35.8403 & NC \\
\hline 1542 & -1.2 & -75.5873 & 35.899 & NC & 1614 & -1.6 & -75.5711 & 35.8697 & NC & 1686 & -2.8 & -75.5569 & 35.8398 & NC \\
\hline 1543 & -1.6 & -75.5872 & 35.8987 & $\mathrm{NC}$ & 1615 & -1.7 & -75.5709 & 35.8692 & NC & 1687 & -2.7 & -75.5567 & 35.8394 & NC \\
\hline 1544 & -1.2 & -75.5871 & 35.8985 & NC & 1616 & -1.6 & -75.5707 & 35.8688 & NC & 1688 & -2.5 & -75.5565 & 35.839 & $\mathrm{NC}$ \\
\hline 1545 & -1.7 & -75.587 & 35.8983 & NC & 1617 & -1.6 & -75.5705 & 35.8684 & NC & 1689 & -2.4 & -75.5563 & 35.8385 & NC \\
\hline 1546 & -1.4 & -75.5867 & 35.8979 & NC & 1618 & -1.5 & -75.5703 & 35.8679 & NC & 1690 & -2.4 & -75.5561 & 35.8381 & NC \\
\hline 1547 & -1.5 & -75.5865 & 35.8975 & $\mathrm{NC}$ & 1619 & -1.4 & -75.57 & 35.8675 & NC & 1691 & -2.3 & -75.556 & 35.8377 & $\mathrm{NC}$ \\
\hline 1548 & -1.4 & -75.5863 & 35.897 & NC & 1620 & -1.5 & -75.5698 & 35.8671 & NC & 1692 & -2.2 & -75.5558 & 35.8372 & NC \\
\hline 1549 & -1.4 & -75.586 & 35.8966 & NC & 1621 & -1.6 & -75.5696 & 35.8666 & NC & 1693 & -2.3 & -75.5556 & 35.8368 & NC \\
\hline 1550 & -1.5 & -75.5858 & 35.8962 & NC & 1622 & -1.6 & -75.5694 & 35.8662 & NC & 1694 & -2.4 & -75.5554 & 35.8364 & NC \\
\hline 1551 & -1.7 & -75.5856 & 35.8958 & NC & 1623 & -1.6 & -75.5692 & 35.8658 & NC & 1695 & -2.4 & -75.5552 & 35.8359 & NC \\
\hline 1552 & -1.7 & -75.5853 & 35.8954 & NC & 1624 & -1.7 & -75.569 & 35.8654 & NC & 1696 & -2.7 & -75.555 & 35.8355 & NC \\
\hline 1553 & -1.8 & -75.5851 & 35.8949 & NC & 1625 & -1.7 & -75.5688 & 35.8649 & NC & 1697 & -3 & -75.5548 & 35.835 & NC \\
\hline 1554 & -1.8 & -75.5849 & 35.8945 & NC & 1626 & -2.1 & -75.5685 & 35.8645 & NC & 1698 & -3.2 & -75.5546 & 35.8346 & NC \\
\hline 1555 & -1.7 & -75.5846 & 35.8941 & NC & 1627 & -2 & -75.5683 & 35.8641 & NC & 1699 & -3.1 & -75.5544 & 35.8342 & NC \\
\hline 1556 & -1.7 & -75.5844 & 35.8937 & NC & 1628 & -1.7 & -75.5681 & 35.8636 & NC & 1700 & -3 & -75.5542 & 35.8337 & NC \\
\hline 1557 & -1.7 & -75.5842 & 35.8932 & NC & 1629 & -1.8 & -75.5679 & 35.8632 & $\mathrm{NC}$ & 1701 & -2.7 & -75.554 & 35.8333 & NC \\
\hline 1558 & -1.6 & -75.5839 & 35.8928 & NC & 1630 & -2 & -75.5677 & 35.8628 & NC & 1702 & -2.9 & -75.5538 & 35.8329 & NC \\
\hline 1559 & -1.7 & -75.5837 & 35.8924 & $\mathrm{NC}$ & 1631 & -2.1 & -75.5675 & 35.8623 & NC & 1703 & -2.8 & -75.5536 & 35.8324 & NC \\
\hline 1560 & -1.7 & -75.5835 & 35.892 & $\mathrm{NC}$ & 1632 & -2 & -75.5672 & 35.8619 & NC & 1704 & -2.6 & -75.5534 & 35.832 & NC \\
\hline 1561 & -1.7 & -75.5833 & 35.8915 & NC & 1633 & -2.1 & -75.567 & 35.8615 & $\mathrm{NC}$ & 1705 & -2.5 & -75.5532 & 35.8315 & $\mathrm{NC}$ \\
\hline 1562 & -1.6 & -75.583 & 35.8911 & NC & 1634 & -2.1 & -75.5668 & 35.8611 & $\mathrm{NC}$ & 1706 & -2.3 & -75.553 & 35.8311 & $\mathrm{NC}$ \\
\hline 1563 & -1.5 & -75.5828 & 35.8907 & $\mathrm{NC}$ & 1635 & -2.1 & -75.5666 & 35.8606 & NC & 1707 & -2.3 & -75.5528 & 35.8307 & $\mathrm{NC}$ \\
\hline 1564 & -1.4 & -75.5826 & 35.8903 & NC & 1636 & -2.1 & -75.5664 & 35.8602 & NC & 1708 & -3.9 & -75.5526 & 35.8302 & $\mathrm{NC}$ \\
\hline 1565 & -1.3 & -75.5823 & 35.8899 & NC & 1637 & -2.3 & -75.5662 & 35.8598 & NC & 1709 & -3.8 & -75.5524 & 35.8298 & $\mathrm{NC}$ \\
\hline 1566 & -1.3 & -75.5821 & 35.8894 & NC & 1638 & -2.4 & -75.566 & 35.8593 & NC & 1710 & -3.7 & -75.5522 & 35.8294 & $\mathrm{NC}$ \\
\hline 1567 & -1.2 & -75.5819 & 35.889 & NC & 1639 & -2.6 & -75.5657 & 35.8589 & NC & 1711 & -3.9 & -75.552 & 35.8289 & NC \\
\hline 1568 & -1.1 & -75.5816 & 35.8886 & NC & 1640 & -2.6 & -75.5655 & 35.8585 & NC & 1712 & -3.8 & -75.5518 & 35.8285 & NC \\
\hline 1569 & -1 & -75.5814 & 35.8882 & NC & 1641 & -2.5 & -75.5653 & 35.8581 & NC & 1713 & -3.7 & -75.5516 & 35.8281 & NC \\
\hline 1570 & -1 & -75.5812 & 35.8877 & NC & 1642 & -2.5 & -75.5651 & 35.8576 & NC & 1714 & -4 & -75.5514 & 35.8276 & NC \\
\hline 1571 & -1 & -75.5809 & 35.8873 & NC & 1643 & -2.7 & -75.5649 & 35.8572 & NC & 1715 & -3.8 & -75.5513 & 35.8272 & NC \\
\hline 1572 & -1 & -75.5807 & 35.8869 & NC & 1644 & -2.6 & -75.5647 & 35.8568 & NC & 1716 & -3.8 & -75.5511 & 35.8267 & NC \\
\hline 1573 & -1.1 & -75.5805 & 35.8865 & NC & 1645 & -2.7 & -75.5644 & 35.8563 & NC & 1717 & -3.8 & -75.5509 & 35.8263 & NC \\
\hline 1574 & -0.9 & -75.5802 & 35.8861 & NC & 1646 & -2.6 & -75.5642 & 35.8559 & NC & 1718 & -4 & -75.5507 & 35.8259 & NC \\
\hline 1575 & -0.9 & -75.58 & 35.8856 & $\mathrm{NC}$ & 1647 & -2.7 & .75 .564 & 35.8555 & NC & 1719 & -4.3 & -75.5505 & 35.8254 & $\mathrm{NC}$ \\
\hline 1576 & -0.9 & -75.5798 & 35.8852 & NC & 1648 & -2.8 & -75.5638 & 35.855 & NC & 1720 & -4.4 & -75.5503 & 35.825 & NC \\
\hline 1577 & -0.8 & -75.5795 & 35.8848 & $\mathrm{NC}$ & 1649 & -3 & -75.5636 & 35.8546 & $\mathrm{NC}$ & 1721 & -4.5 & -75.5501 & 35.8246 & NC \\
\hline 1578 & -0.6 & -75.5793 & 35.8844 & $\mathrm{NC}$ & 1650 & -2.8 & -75.5634 & 35.8542 & NC & 1722 & -4.8 & -75.5499 & 35.8241 & NC \\
\hline 1579 & -0.8 & -75.5791 & 35.8839 & NC & 1651 & -2.7 & -75.5631 & 35.8538 & NC & 1723 & -4.8 & -75.5497 & 35.8237 & NC \\
\hline 1580 & -0.9 & -75.5788 & 35.8835 & NC & 1652 & -2.7 & -75.5629 & 35.8533 & NC & 1724 & -4.7 & -75.5495 & 35.8233 & $\mathrm{NC}$ \\
\hline 1581 & -0.7 & -75.5786 & 35.8831 & $\mathrm{NC}$ & 1653 & -2.7 & -75.5627 & 35.8529 & $\mathrm{NC}$ & 1725 & -4.5 & -75.5493 & 35.8228 & NC \\
\hline 1582 & -0.8 & -75.5784 & 35.8827 & NC & 1654 & -2.5 & -75.5625 & 35.8525 & NC & 1726 & -4.5 & -75.5491 & 35.8224 & NC \\
\hline 1583 & -0.9 & -75.5781 & 35.8823 & NC & 1655 & -2.6 & -75.5623 & 35.852 & NC & 1727 & -4.7 & -75.5489 & 35.8219 & $\mathrm{NC}$ \\
\hline
\end{tabular}




\begin{tabular}{|c|c|c|c|c|c|c|c|c|c|c|c|c|c|c|}
\hline TR\# & RATE & LONG & LAT & ST & TR\# & RATE & LONG & LAT & ST & TR\# & RATE & LONG & LAT & ST \\
\hline 1728 & -4.3 & -75.5487 & 35.8215 & NC & 1800 & 18.4 & -75.5352 & 35.7914 & NC & 1872 & -3.1 & -75.5121 & 35.7644 & NC \\
\hline 1729 & -4.2 & -75.5485 & 35.8211 & $\mathrm{NC}$ & 1801 & 20.5 & -75.535 & 35.791 & $\mathrm{NC}$ & 1873 & -3.1 & -75.5119 & 35.764 & NC \\
\hline 1730 & -4.3 & -75.5483 & 35.8206 & NC & 1802 & 17.2 & -75.5348 & 35.7906 & $\mathrm{NC}$ & 1874 & -3.2 & -75.5117 & 35.7635 & NC \\
\hline 1731 & -4.3 & -75.5481 & 35.8202 & NC & 1803 & 20.2 & -75.5346 & 35.7901 & $\mathrm{NC}$ & 1875 & -3.1 & -75.5114 & 35.7631 & $\mathrm{NC}$ \\
\hline 1732 & -4.4 & -75.5479 & 35.8198 & $\mathrm{NC}$ & 1804 & 18.8 & -75.5344 & 35.7897 & $\mathrm{NC}$ & 1876 & -3 & -75.5112 & 35.7627 & $\mathrm{NC}$ \\
\hline 1733 & -4.5 & -75.5477 & 35.8193 & NC & 1805 & 18.3 & -75.5342 & 35.7892 & $\mathrm{NC}$ & 1877 & -3 & -75.511 & 35.7622 & NC \\
\hline 1734 & -4.4 & -75.5475 & 35.8189 & NC & 1806 & 9.4 & -75.5246 & 35.7888 & $\mathrm{NC}$ & 1878 & -3.4 & -75.51 & 35.7618 & $\mathrm{NC}$ \\
\hline 1735 & -4.3 & -75.5473 & 35.8184 & $\mathrm{NC}$ & 1807 & 16.8 & .75 .534 & 35.7888 & $\mathrm{NC}$ & 1879 & -2.8 & -75.5108 & 35.7618 & NC \\
\hline 1736 & -4.4 & -75.5471 & 35.818 & NC & 1808 & 8.6 & -75.5244 & 35.7884 & $\mathrm{NC}$ & 1880 & -3.2 & -75.5098 & 35.7614 & $\mathrm{NC}$ \\
\hline 1737 & -4.2 & -75.5469 & 35.8176 & NC & 1809 & 15.5 & -75.5338 & 35.7884 & $\mathrm{NC}$ & 1881 & -2.8 & -75.5106 & 35.7614 & NC \\
\hline 1738 & -4.2 & -75.5467 & 35.8171 & NC & 1810 & 8.7 & -75.5242 & 35.7879 & $\mathrm{NC}$ & 1882 & -2.9 & .75 .5103 & 35.761 & NC \\
\hline 1739 & -4.4 & -75.5466 & 35.8167 & NC & 1811 & 14.6 & -75.5336 & 35.7879 & $\mathrm{NC}$ & 1883 & -3.3 & -75.5095 & 35.7609 & NC \\
\hline 1740 & -4.4 & -75.5464 & 35.8163 & NC & 1812 & 7.8 & -75.524 & 35.7875 & $\mathrm{NC}$ & 1884 & -3.3 & -75.5093 & 35.7605 & NC \\
\hline 1741 & -4.5 & -75.5462 & 35.8158 & $\mathrm{NC}$ & 1813 & 13.1 & -75.5335 & 35.7875 & $\mathrm{NC}$ & 1885 & -3 & -75.5101 & 35.7605 & NC \\
\hline 1742 & -4.7 & -75.546 & 35.8154 & $\mathrm{NC}$ & 1814 & 4.4 & -75.5237 & 35.7871 & $\mathrm{NC}$ & 1886 & -3.3 & -75.5091 & 35.7601 & NC \\
\hline 1743 & -4.7 & -75.5458 & 35.815 & $\mathrm{NC}$ & 1815 & 10.7 & -75.5333 & 35.7871 & $\mathrm{NC}$ & 1887 & -3.2 & -75.5099 & 35.7601 & NC \\
\hline 1744 & -4.6 & -75.5456 & 35.8145 & $\mathrm{NC}$ & 1816 & -0.3 & -75.5235 & 35.7866 & $\mathrm{NC}$ & 1888 & -3.4 & -75.5088 & 35.7597 & NC \\
\hline 1745 & -4.5 & -75.5454 & 35.8141 & NC & 1817 & 9.6 & -75.5331 & 35.7866 & $\mathrm{NC}$ & 1889 & -3.4 & -75.5086 & 35.7592 & $\mathrm{NC}$ \\
\hline 1746 & -5.4 & -75.5452 & 35.8137 & $\mathrm{NC}$ & 1818 & -0.6 & -75.5233 & 35.7862 & $\mathrm{NC}$ & 1890 & -3.3 & -75.5084 & 35.7588 & NC \\
\hline 1747 & -4.5 & -75.5452 & 35.8136 & $\mathrm{NC}$ & 1819 & 8.7 & -75.5329 & 35.7862 & $\mathrm{NC}$ & 1891 & -3.3 & -75.5081 & 35.7584 & NC \\
\hline 1748 & -5.1 & -75.545 & 35.8133 & $\mathrm{NC}$ & 1820 & -0.9 & -75.5231 & 35.7858 & $\mathrm{NC}$ & 1892 & -3.3 & -75.5079 & 35.758 & NC \\
\hline 1749 & -4.5 & -75.545 & 35.8132 & $\mathrm{NC}$ & 1821 & 6.8 & -75.5327 & 35.7858 & $\mathrm{NC}$ & 1893 & -3.4 & -75.5077 & 35.7575 & NC \\
\hline 1750 & -5 & -75.5448 & 35.8128 & NC & 1822 & -0.9 & -75.5229 & 35.7854 & $\mathrm{NC}$ & 1894 & -3.4 & -75.5074 & 35.7571 & $\mathrm{NC}$ \\
\hline 1751 & -4.4 & -75.5448 & 35.8128 & NC & 1823 & 5.3 & -75.5325 & 35.7853 & $\mathrm{NC}$ & 1895 & -3.8 & -75.5072 & 35.7567 & NC \\
\hline 1752 & -4.7 & -75.5446 & 35.8124 & $\mathrm{NC}$ & 1824 & -0.8 & -75.5226 & 35.7849 & $\mathrm{NC}$ & 1896 & -3.7 & -75.507 & 35.7563 & NC \\
\hline 1753 & -4.8 & -75.5444 & 35.812 & $\mathrm{NC}$ & 1825 & 1.3 & -75.5224 & 35.7845 & $\mathrm{NC}$ & 1897 & -3.6 & -75.5067 & 35.7558 & NC \\
\hline 1754 & -4.5 & -75.5442 & 35.8115 & NC & 1826 & 3.3 & -75.5222 & 35.7841 & $\mathrm{NC}$ & 1898 & -3.7 & -75.5065 & 35.7554 & NC \\
\hline 1755 & -4.3 & -75.544 & 35.8111 & NC & 1827 & 2.4 & -75.522 & 35.7836 & $\mathrm{NC}$ & 1899 & -4 & -75.5063 & 35.755 & $\mathrm{NC}$ \\
\hline 1756 & -4.3 & -75.5438 & 35.8106 & NC & 1828 & 1.7 & -75.5218 & 35.7832 & NC & 1900 & -4.1 & -75.506 & 35.7546 & NC \\
\hline 1757 & -4 & -75.5436 & 35.8102 & NC & 1829 & 1.4 & -75.5215 & 35.7828 & $\mathrm{NC}$ & 1901 & -4.2 & -75.5058 & 35.7541 & NC \\
\hline 1758 & -3.8 & .75 .5434 & 35.8098 & $\mathrm{NC}$ & 1830 & 1.2 & -75.5213 & 35.7824 & $\mathrm{NC}$ & 1902 & -4.3 & -75.5056 & 35.7537 & NC \\
\hline 1759 & -3.8 & -75.5432 & 35.8093 & $\mathrm{NC}$ & 1831 & 0.1 & -75.5211 & 35.7819 & $\mathrm{NC}$ & 1903 & -4.3 & -75.5053 & 35.7533 & NC \\
\hline 1760 & -3.9 & -75.543 & 35.8089 & $\mathrm{NC}$ & 1832 & -1.1 & -75.5209 & 35.7815 & NC & 1904 & -4.3 & -75.5051 & 35.7529 & NC \\
\hline 1761 & -3.7 & -75.5429 & 35.8085 & NC & 1833 & $\mathrm{NA}$ & -75.5207 & 35.7811 & $\mathrm{NC}$ & 1905 & -4.3 & -75.5049 & 35.7524 & NC \\
\hline 1762 & -3.4 & -75.5427 & 35.808 & NC & 1834 & NA & -75.5204 & 35.7807 & $\mathrm{NC}$ & 1906 & -4.3 & -75.5046 & 35.752 & $\mathrm{NC}$ \\
\hline 1763 & -3.1 & -75.5425 & 35.8076 & $\mathrm{NC}$ & 1835 & -19.7 & -75.5202 & 35.7802 & $\mathrm{NC}$ & 1907 & -4.2 & -75.5044 & 35.7516 & NC \\
\hline 1764 & -2.7 & -75.5423 & 35.8072 & NC & 1836 & -33.2 & -75.52 & 35.7798 & $\mathrm{NC}$ & 1908 & -4 & -75.5042 & 35.7512 & NC \\
\hline 1765 & -2.5 & -75.5421 & 35.8067 & NC & 1837 & -28 & -75.5198 & 35.7794 & $\mathrm{NC}$ & 1909 & -4.2 & -75.5039 & 35.7507 & NC \\
\hline 1766 & -2.2 & -75.5419 & 35.8063 & NC & 1838 & -22.7 & -75.5196 & 35.7789 & NC & 1910 & -4.3 & -75.5037 & 35.7503 & $\mathrm{NC}$ \\
\hline 1767 & -2.1 & -75.5417 & 35.8058 & $\mathrm{NC}$ & 1839 & -16.4 & -75.5193 & 35.7785 & $\mathrm{NC}$ & 1911 & -4 & -75.5035 & 35.7499 & $\mathrm{NC}$ \\
\hline 1768 & -2.2 & -75.5415 & 35.8054 & $\mathrm{NC}$ & 1840 & -18.4 & -75.5191 & 35.7781 & NC & 1912 & -4.2 & -75.5032 & 35.7495 & NC \\
\hline 1769 & -1.8 & -75.5413 & 35.805 & $\mathrm{NC}$ & 1841 & -15.2 & -75.5189 & 35.7777 & NC & 1913 & -4.3 & -75.503 & 35.749 & NC \\
\hline 1770 & -1.2 & -75.5411 & 35.8045 & $\mathrm{NC}$ & 1842 & -11.9 & -75.5187 & 35.7772 & NC & 1914 & -4.1 & -75.5028 & 35.7486 & NC \\
\hline 1771 & -0.9 & -75.5409 & 35.8041 & $\mathrm{NC}$ & 1843 & -10.2 & -75.5185 & 35.7768 & NC & 1915 & -4.1 & -75.5025 & 35.7482 & NC \\
\hline 1772 & -0.6 & -75.5407 & 35.8037 & $\mathrm{NC}$ & 1844 & -19.5 & -75.5182 & 35.7764 & NC & 1916 & -4.3 & -75.5023 & 35.7478 & $\mathrm{NC}$ \\
\hline 1773 & -0.1 & -75.5405 & 35.8032 & $\mathrm{NC}$ & 1845 & -17.9 & -75.518 & 35.7759 & NC & 1917 & -4.3 & -75.5021 & 35.7473 & NC \\
\hline 1774 & 0.2 & -75.5403 & 35.8028 & $\mathrm{NC}$ & 1846 & -14.4 & -75.5178 & 35.7755 & NC & 1918 & -4.4 & -75.5018 & 35.7469 & NC \\
\hline 1775 & 0.7 & -75.5401 & 35.8023 & $\mathrm{NC}$ & 1847 & -13.2 & -75.5176 & 35.7751 & NC & 1919 & -4.8 & -75.5016 & 35.7465 & NC \\
\hline 1776 & 0.9 & -75.5399 & 35.8019 & $\mathrm{NC}$ & 1848 & -11.3 & -75.5174 & 35.7747 & $\mathrm{NC}$ & 1920 & -5 & -75.5013 & 35.7461 & $\mathrm{NC}$ \\
\hline 1777 & 1.5 & -75.5397 & 35.8015 & NC & 1849 & -11.3 & -75.5172 & 35.7742 & $\mathrm{NC}$ & 1921 & -5.2 & -75.5011 & 35.7456 & NC \\
\hline 1778 & 2 & -75.5395 & 35.801 & NC & 1850 & -11.1 & -75.5169 & 35.7738 & $\mathrm{NC}$ & 1922 & -5.4 & .75 .5009 & 35.7452 & NC \\
\hline 1779 & 2.5 & -75.5393 & 35.8006 & NC & 1851 & -10.7 & -75.5167 & 35.7734 & $\mathrm{NC}$ & 1923 & -5.5 & -75.5006 & 35.7448 & NC \\
\hline 1780 & 3 & -75.5391 & 35.8002 & $\mathrm{NC}$ & 1852 & -9.8 & -75.5165 & 35.7729 & $\mathrm{NC}$ & 1924 & -5.6 & -75.5004 & 35.7444 & NC \\
\hline 1781 & 3.5 & -75.5389 & 35.7997 & $\mathrm{NC}$ & 1853 & -8 & -75.5163 & 35.7725 & $\mathrm{NC}$ & 1925 & -5.6 & -75.5002 & 35.7439 & NC \\
\hline 1782 & 4.1 & -75.5387 & 35.7993 & $\mathrm{NC}$ & 1854 & -7.4 & -75.5161 & 35.7721 & $\mathrm{NC}$ & 1926 & -5.4 & -75.4999 & 35.7435 & NC \\
\hline 1783 & 4.5 & -75.5385 & 35.7989 & $\mathrm{NC}$ & 1855 & -6.5 & -75.5158 & 35.7717 & $\mathrm{NC}$ & 1927 & -5.4 & -75.4997 & 35.7431 & $\mathrm{NC}$ \\
\hline 1784 & 5 & -75.5383 & 35.7984 & NC & 1856 & -5.8 & -75.5156 & 35.7712 & $\mathrm{NC}$ & 1928 & -5.2 & -75.4995 & 35.7427 & NC \\
\hline 1785 & 5.6 & -75.5382 & 35.798 & NC & 1857 & -5.5 & -75.5154 & 35.7708 & $\mathrm{NC}$ & 1929 & -5.2 & -75.4992 & 35.7422 & NC \\
\hline 1786 & 6.3 & -75.538 & 35.7975 & $\mathrm{NC}$ & 1858 & -3.9 & -75.5152 & 35.7704 & $\mathrm{NC}$ & 1930 & -5.1 & -75.499 & 35.7418 & NC \\
\hline 1787 & 2.9 & -75.5378 & 35.7971 & NC & 1859 & -3.8 & -75.515 & 35.7699 & NC & 1931 & -5 & -75.4988 & 35.7414 & NC \\
\hline 1788 & 3.2 & -75.5376 & 35.7967 & NC & 1860 & -3.7 & -75.5147 & 35.7695 & NC & 1932 & -5 & -75.4985 & 35.741 & $\mathrm{NC}$ \\
\hline 1789 & 4 & -75.5374 & 35.7962 & NC & 1861 & -3.7 & -75.5145 & 35.7691 & NC & 1933 & -5.1 & -75.4983 & 35.7405 & NC \\
\hline 1790 & 4.8 & -75.5372 & 35.7958 & NC & 1862 & -3.8 & -75.5143 & 35.7687 & $\mathrm{NC}$ & 1934 & -5.2 & -75.4981 & 35.7401 & NC \\
\hline 1791 & 6.1 & -75.537 & 35.7954 & $\mathrm{NC}$ & 1863 & -3.4 & -75.5141 & 35.7682 & $\mathrm{NC}$ & 1935 & -5 & -75.4978 & 35.7397 & $\mathrm{NC}$ \\
\hline 1792 & 7.4 & -75.5368 & 35.7949 & $\mathrm{NC}$ & 1864 & -3 & -75.5139 & 35.7678 & $\mathrm{NC}$ & 1936 & -5.1 & -75.4976 & 35.7393 & NC \\
\hline 1793 & 9.1 & -75.5366 & 35.7945 & $\mathrm{NC}$ & 1865 & -3 & -75.5136 & 35.7674 & $\mathrm{NC}$ & 1937 & -4.9 & .75 .4974 & 35.7388 & $\mathrm{NC}$ \\
\hline 1794 & 10.8 & -75.5364 & 35.7941 & $\mathrm{NC}$ & 1866 & -2.9 & -75.5134 & 35.767 & $\mathrm{NC}$ & 1938 & -4.9 & -75.4971 & 35.7384 & $\mathrm{NC}$ \\
\hline 1795 & 11.1 & -75.5362 & 35.7936 & $\mathrm{NC}$ & 1867 & -3 & -75.5132 & 35.7665 & $\mathrm{NC}$ & 1939 & -4.9 & -75.4969 & 35.738 & $\mathrm{NC}$ \\
\hline 1796 & 11.3 & -75.536 & 35.7932 & $\mathrm{NC}$ & 1868 & -3.1 & -75.513 & 35.7661 & $\mathrm{NC}$ & 1940 & -4.8 & .75 .4967 & 35.7376 & NC \\
\hline 1797 & 11.4 & -75.5358 & 35.7927 & $\mathrm{NC}$ & 1869 & -3.3 & -75.5128 & 35.7657 & $\mathrm{NC}$ & 1941 & -4.7 & -75.4964 & 35.7371 & $\mathrm{NC}$ \\
\hline 1798 & 11.6 & -75.5356 & 35.7923 & NC & 1870 & -3.3 & -75.5125 & 35.7652 & $\mathrm{NC}$ & 1942 & -4.6 & -75.4962 & 35.7367 & NC \\
\hline 1799 & 12.8 & -75.5354 & 35.7919 & NC & 1871 & -3.1 & -75.5123 & 35.7648 & $\mathrm{NC}$ & 1943 & -4.6 & .75 .496 & 35.7363 & NC \\
\hline
\end{tabular}




\begin{tabular}{|c|c|c|c|c|c|c|c|c|c|c|c|c|c|c|}
\hline TR\# & RATE & LONG & LAT & ST & TR\# & RATE & LONG & LAT & ST & TR\# & RATE & LONG & LAT & ST \\
\hline 1944 & -4.5 & -75.4957 & 35.7359 & NC & 2016 & -1.2 & -75.485 & 35.7047 & $\mathrm{NC}$ & \begin{tabular}{|l|}
2088 \\
\end{tabular} & -1.7 & -75.4751 & 35.6735 & NC \\
\hline 1945 & -4.4 & -75.4955 & 35.7354 & NC & 2017 & -1.3 & -75.4848 & 35.7042 & NC & 2089 & -0.8 & .75 .475 & 35.673 & NC \\
\hline 1946 & -4.4 & -75.4953 & 35.735 & NC & 2018 & -1.6 & -75.4847 & 35.7038 & NC & 2090 & -1.4 & -75.4749 & 35.673 & NC \\
\hline 1947 & -4.2 & -75.495 & 35.7346 & NC & 2019 & -2 & -75.4838 & 35.7033 & $\mathrm{NC}$ & 2091 & -0.7 & -75.4749 & 35.6726 & NC \\
\hline 1948 & -4.1 & -75.4948 & 35.7342 & NC & 2020 & -1.5 & -75.4845 & 35.7033 & NC & 2092 & -0.3 & -75.4749 & 35.6721 & NC \\
\hline 1949 & -4.1 & -75.4946 & 35.7337 & $\mathrm{NC}$ & 2021 & -1.8 & -75.4843 & 35.7029 & $\mathrm{NC}$ & 2093 & -0.1 & -75.4748 & 35.6717 & NC \\
\hline 1950 & -4.2 & -75.4943 & 35.7333 & NC & 2022 & -2.1 & -75.4837 & 35.7028 & $\mathrm{NC}$ & 2094 & -0.1 & -75.4747 & 35.6712 & NC \\
\hline 1951 & -4.1 & -75.4941 & 35.7329 & NC & 2023 & -2.2 & -75.4835 & 35.7024 & NC & 2095 & 0 & -75.4746 & 35.6708 & NC \\
\hline 1952 & -3.4 & -75.4952 & 35.7327 & NC & 2024 & -2.4 & -75.4834 & 35.7019 & $\mathrm{NC}$ & 2096 & -0.1 & -75.4745 & 35.6703 & $\mathrm{NC}$ \\
\hline 1953 & -4.2 & -75.4939 & 35.7325 & $\mathrm{NC}$ & 2025 & -2.5 & -75.4833 & 35.7015 & NC & 2097 & -0.3 & -75.4744 & 35.6699 & NC \\
\hline 1954 & -3.1 & -75.495 & 35.7323 & $\mathrm{NC}$ & 2026 & -2.7 & -75.4831 & 35.701 & NC & 2098 & -0.4 & -75.4744 & 35.6694 & NC \\
\hline 1955 & -3.2 & -75.4949 & 35.7318 & NC & 2027 & -2.6 & -75.483 & 35.7006 & NC & 2099 & -0.4 & -75.4743 & 35.669 & $\mathrm{NC}$ \\
\hline 1956 & -3.1 & -75.4947 & 35.7314 & NC & 2028 & -2.7 & -75.4829 & 35.7001 & NC & 2100 & -2 & -75.4742 & 35.6685 & NC \\
\hline 1957 & -3 & -75.4946 & 35.7309 & NC & 2029 & -3 & -75.4827 & 35.6997 & NC & 2101 & -1.9 & -75.4741 & 35.668 & NC \\
\hline 1958 & -5.1 & -75.4944 & 35.7305 & NC & 2030 & -3 & -75.4826 & 35.6992 & NC & 2102 & -1.9 & -75.474 & 35.6676 & NC \\
\hline 1959 & -5 & -75.4942 & 35.73 & $\mathrm{NC}$ & 2031 & -3.2 & -75.4825 & 35.6988 & NC & 2103 & -1.8 & -75.4739 & 35.6671 & NC \\
\hline 1960 & -4.6 & -75.4941 & 35.7296 & $\mathrm{NC}$ & 2032 & -3.4 & -75.4823 & 35.6983 & NC & 2104 & -1.7 & -75.4739 & 35.6667 & NC \\
\hline 1961 & -4.5 & -75.4939 & 35.7291 & NC & 2033 & -3.4 & -75.4822 & 35.6979 & NC & 2105 & -1.3 & -75.4738 & 35.6662 & NC \\
\hline 1962 & -4.5 & -75.4937 & 35.7287 & NC & 2034 & -3.3 & -75.4821 & 35.6974 & NC & 2106 & -1.3 & -75.4737 & 35.6658 & NC \\
\hline 1963 & -4.6 & -75.4936 & 35.7282 & NC & 2035 & -3.4 & -75.4819 & 35.697 & NC & 2107 & -1 & -75.4736 & 35.6653 & NC \\
\hline 1964 & -4.7 & -75.4934 & 35.7278 & NC & 2036 & -3.4 & -75.4818 & 35.6965 & NC & 2108 & -0.8 & -75.4735 & 35.6649 & NC \\
\hline 1965 & -4.4 & -75.4933 & 35.7274 & NC & 2037 & -3.4 & -75.4817 & 35.6961 & NC & 2109 & -0.7 & -75.4734 & 35.6644 & NC \\
\hline 1966 & -4.1 & -75.4931 & 35.7269 & NC & 2038 & -3.3 & -75.4815 & 35.6956 & NC & 2110 & -0.4 & -75.4734 & 35.6639 & NC \\
\hline 1967 & -3.9 & -75.4929 & 35.7265 & NC & 2039 & -3.2 & -75.4814 & 35.6952 & NC & 2111 & -0.4 & -75.4733 & 35.6635 & NC \\
\hline 1968 & -3.8 & -75.4928 & 35.726 & NC & 2040 & -3.2 & -75.4813 & 35.6947 & NC & 2112 & -0.4 & -75.4732 & 35.663 & NC \\
\hline 1969 & -3.5 & -75.4926 & 35.7256 & NC & 2041 & -3 & -75.4812 & 35.6943 & NC & 2113 & -0.4 & -75.4731 & 35.6626 & $\mathrm{NC}$ \\
\hline 1970 & -3.4 & -75.4924 & 35.7251 & NC & 2042 & -2.9 & -75.481 & 35.6938 & NC & 2114 & -0.5 & -75.473 & 35.6621 & NC \\
\hline 1971 & -3.6 & -75.4923 & 35.7247 & NC & 2043 & -2.7 & -75.4809 & 35.6934 & NC & 2115 & -0.6 & -75.4729 & 35.6617 & NC \\
\hline 1972 & -3.7 & -75.4921 & 35.7242 & NC & 2044 & -2.4 & -75.4808 & 35.6929 & NC & 2116 & -0.5 & -75.4729 & 35.6612 & NC \\
\hline 1973 & -3.3 & -75.492 & 35.7238 & NC & 2045 & -2.4 & -75.4806 & 35.6924 & NC & 2117 & -0.3 & -75.4728 & 35.6608 & NC \\
\hline 1974 & -3.3 & -75.4918 & 35.7234 & $\mathrm{NC}$ & 2046 & -2.2 & -75.4805 & 35.692 & NC & 2118 & 0.1 & -75.4727 & 35.6603 & NC \\
\hline 1975 & -3.3 & -75.4916 & 35.7229 & NC & 2047 & -2.4 & -75.4804 & 35.6915 & NC & 2119 & 0.3 & -75.4726 & 35.6599 & NC \\
\hline 1976 & -3.1 & -75.4915 & 35.7225 & $\mathrm{NC}$ & 2048 & -2.6 & -75.4802 & 35.6911 & NC & 2120 & 0.3 & -75.4725 & 35.6594 & NC \\
\hline 1977 & -3 & -75.4913 & 35.722 & $\mathrm{NC}$ & 2049 & -1.6 & -75.4801 & 35.6906 & NC & 2121 & 0.5 & -75.4724 & 35.6589 & $\mathrm{NC}$ \\
\hline 1978 & -3 & -75.4912 & 35.7216 & NC & 2050 & -1.7 & -75.48 & 35.6902 & NC & 2122 & 0.6 & -75.4724 & 35.6585 & $\mathrm{NC}$ \\
\hline 1979 & -3.3 & -75.491 & 35.7211 & NC & 2051 & -1.8 & -75.4798 & 35.6897 & NC & 2123 & 0.6 & -75.4723 & 35.658 & NC \\
\hline 1980 & -3.5 & -75.4908 & 35.7207 & NC & 2052 & -1.8 & -75.4797 & 35.6893 & NC & 2124 & 0.8 & -75.4722 & 35.6576 & NC \\
\hline 1981 & -3.5 & -75.4907 & 35.7202 & NC & 2053 & -1.6 & -75.4796 & 35.6888 & NC & 2125 & 0.9 & -75.4721 & 35.6571 & NC \\
\hline 1982 & -3.4 & -75.4905 & 35.7198 & NC & 2054 & -1.6 & -75.4794 & 35.6884 & NC & 2126 & 1 & -75.472 & 35.6567 & NC \\
\hline 1983 & -3.4 & -75.4903 & 35.7193 & NC & 2055 & -1.8 & -75.4793 & 35.6879 & NC & 2127 & 1.1 & -75.4719 & 35.6562 & NC \\
\hline 1984 & -3.4 & -75.4902 & 35.7189 & NC & 2056 & -1.7 & -75.4792 & 35.6875 & NC & 2128 & 1.2 & -75.4719 & 35.6558 & $\mathrm{NC}$ \\
\hline 1985 & -3.2 & -75.49 & 35.7185 & NC & 2057 & -2.1 & -75.479 & 35.687 & NC & 2129 & 1 & -75.4718 & 35.6553 & NC \\
\hline 1986 & -3.1 & -75.4899 & 35.718 & NC & 2058 & -2.2 & -75.4789 & 35.6866 & NC & 2130 & 0.7 & -75.4717 & 35.6548 & NC \\
\hline 1987 & -3 & -75.4897 & 35.7176 & NC & 2059 & -2.1 & -75.4788 & 35.6861 & NC & 2131 & 0.6 & -75.4716 & 35.6544 & NC \\
\hline 1988 & -2.7 & -75.4895 & 35.7171 & NC & 2060 & -2.1 & -75.4786 & 35.6857 & NC & 2132 & 0.5 & -75.4715 & 35.6539 & NC \\
\hline 1989 & -2.6 & -75.4894 & 35.7167 & NC & 2061 & -2.3 & -75.4785 & 35.6852 & NC & 2133 & 0.4 & -75.4714 & 35.6535 & NC \\
\hline 1990 & -2.6 & -75.4892 & 35.7162 & NC & 2062 & -2.4 & -75.4784 & 35.6848 & NC & 2134 & 0.3 & -75.4714 & 35.653 & NC \\
\hline 1991 & -2.7 & -75.489 & 35.7158 & NC & 2063 & -1.9 & -75.4782 & 35.6843 & NC & 2135 & 0 & -75.4713 & 35.6526 & $\mathrm{NC}$ \\
\hline 1992 & -2.5 & -75.4889 & 35.7153 & NC & 2064 & -1.6 & -75.4781 & 35.6839 & NC & 2136 & -0.1 & -75.4712 & 35.6521 & NC \\
\hline 1993 & -2.5 & -75.4887 & 35.7149 & NC & 2065 & -1.9 & -75.478 & 35.6834 & NC & 2137 & 0.1 & -75.4711 & 35.6517 & NC \\
\hline 1994 & -2.3 & -75.4886 & 35.7145 & $\mathrm{NC}$ & 2066 & .2 & -75.4778 & 35.683 & NC & 2138 & 0.2 & -75.471 & 35.6512 & NC \\
\hline 1995 & -2.4 & -75.4884 & 35.714 & NC & 2067 & -2.2 & .75 .4777 & 35.6825 & NC & 2139 & 0.4 & -75.4709 & 35.6508 & $\mathrm{NC}$ \\
\hline 1996 & -2.3 & -75.4882 & 35.7136 & NC & 2068 & -2.5 & -75.4776 & 35.6821 & NC & 2140 & 0.3 & -75.4709 & 35.6503 & NC \\
\hline 1997 & -2.3 & -75.4881 & 35.7131 & NC & 2069 & -2.2 & -75.4774 & 35.6816 & NC & 2141 & 0 & -75.4708 & 35.6498 & NC \\
\hline 1998 & -2.3 & -75.4879 & 35.7127 & $\mathrm{NC}$ & 2070 & -2.4 & -75.4773 & 35.6811 & NC & 2142 & -0.1 & -75.4707 & 35.6494 & NC \\
\hline 1999 & -2.4 & -75.4877 & 35.7122 & $\mathrm{NC}$ & 2071 & -2.4 & -75.4772 & 35.6807 & $\mathrm{NC}$ & 2143 & -0.1 & -75.4706 & 35.6489 & $\mathrm{NC}$ \\
\hline 2000 & -2.2 & -75.4876 & 35.7118 & $\mathrm{NC}$ & 2072 & -2.2 & -75.477 & 35.6802 & NC & 2144 & -0.2 & -75.4705 & 35.6485 & $\mathrm{NC}$ \\
\hline 2001 & -2.4 & -75.4874 & 35.7113 & NC & 2073 & -2.2 & -75.4769 & 35.6798 & NC & 2145 & -0.2 & -75.4704 & 35.648 & NC \\
\hline 2002 & -2.4 & -75.4873 & 35.7109 & NC & 2074 & -2.1 & -75.4768 & 35.6793 & NC & 2146 & 0 & -75.4704 & 35.6476 & NC \\
\hline 2003 & -2.4 & -75.4871 & 35.7104 & NC & 2075 & -1.9 & -75.4767 & 35.6789 & NC & 2147 & -0.3 & -75.4703 & 35.6471 & NC \\
\hline 2004 & -2.3 & .75 .4869 & 35.71 & $\mathrm{NC}$ & 2076 & -2 & -75.4765 & 35.6784 & NC & 2148 & -0.4 & -75.4702 & 35.6467 & $\mathrm{NC}$ \\
\hline 2005 & -2.2 & -75.4868 & 35.7096 & NC & 2077 & -1.8 & -75.4764 & 35.678 & NC & 2149 & -0.4 & -75.4701 & 35.6462 & NC \\
\hline 2006 & -2.1 & -75.4866 & 35.7091 & NC & 2078 & -1.6 & -75.4763 & 35.6775 & NC & 2150 & -0.3 & -75.47 & 35.6457 & NC \\
\hline 2007 & -2 & -75.4865 & 35.7087 & NC & 2079 & -1.6 & -75.4761 & 35.6771 & NC & 2151 & -0.4 & -75.4699 & 35.6453 & NC \\
\hline 2008 & -1.7 & -75.4863 & 35.7082 & NC & 2080 & -1.6 & -75.476 & 35.6766 & NC & 2152 & -0.4 & -75.4699 & 35.6448 & $\mathrm{NC}$ \\
\hline 2009 & -1.8 & -75.4861 & 35.7078 & NC & 2081 & -1.8 & -75.4759 & 35.6762 & NC & 2153 & -1 & -75.4679 & 35.6446 & $\mathrm{NC}$ \\
\hline 2010 & $\begin{array}{l}-1.0 \\
-1.8\end{array}$ & -75.486 & 35.7073 & NC & 2082 & -1.7 & .75 .4757 & 35.6757 & NC & 2154 & -0.6 & -75.4698 & 35.6444 & NC \\
\hline 2011 & -1.5 & -75.4858 & 35.7069 & NC & 2083 & -1.6 & -75.4756 & 35.6753 & NC & 2155 & -1 & -75.4678 & 35.6442 & NC \\
\hline 2012 & -1.4 & -75.4856 & 35.7064 & NC & 2084 & -1.5 & -75.4755 & 35.6748 & NC & 2156 & -0.8 & -75.4697 & 35.6439 & NC \\
\hline 2013 & -1.4 & -75.4855 & 35.706 & NC & 2085 & -1.8 & -75.4753 & 35.6744 & NC & 2157 & -1.1 & -75.4677 & 35.6437 & NC \\
\hline 2014 & -1.5 & -75.4853 & 35.7056 & NC & 2086 & -2.1 & -75.4752 & 35.6739 & NC & 2158 & -0.8 & -75.4696 & 35.6435 & NC \\
\hline 2015 & -1.3 & -75.4852 & 35.7051 & NC & 2087 & -1 & .75 .4751 & 35.6735 & NC & 2159 & -0.9 & -75.4675 & 35.6433 & NC \\
\hline
\end{tabular}




\begin{tabular}{|c|c|c|c|c|c|c|c|c|c|c|c|c|c|c|}
\hline TR\# & RATE & LONG & LAT & ST & TR\# & RATE & LONG & LAT & ST & TR\# & RATE & LONG & LAT & ST \\
\hline 2160 & -0.8 & -75.4695 & 35.643 & NC & 2232 & -4.3 & -75.4578 & 35.6113 & NC & | 2304 & -2.5 & -75.4561 & 35.5779 & NC \\
\hline 2161 & -0.6 & -75.4674 & 35.6428 & NC & 2233 & -4.3 & -75.4577 & 35.6108 & NC & 2305 & -2.7 & -75.4561 & 35.5775 & NC \\
\hline 2162 & -0.8 & -75.4673 & 35.6424 & NC & 2234 & -4.4 & -75.4577 & 35.6103 & NC & 2306 & -2.8 & -75.4562 & 35.577 & NC \\
\hline 2163 & -0.9 & -75.4672 & 35.6419 & NC & 2235 & -4.6 & -75.4576 & 35.6099 & NC & 2307 & -2.8 & -75.4562 & 35.5765 & NC \\
\hline 2164 & -1 & -75.4671 & 35.6415 & NC & 2236 & -4.7 & -75.4576 & 35.6094 & NC & 2308 & -2.9 & -75.4562 & 35.5761 & NC \\
\hline 2165 & -1.1 & -75.467 & 35.641 & NC & 2237 & -4.5 & -75.4575 & 35.6089 & NC & 2309 & -2.8 & -75.4563 & 35.5756 & NC \\
\hline 2166 & -1.1 & -75.4668 & 35.6406 & NC & 2238 & -4.6 & -75.4575 & 35.6085 & NC & 2310 & -2.5 & -75.4563 & 35.5751 & NC \\
\hline 2167 & -1.2 & -75.4667 & 35.6401 & NC & 2239 & -4.6 & -75.4575 & 35.608 & NC & 2311 & -2.5 & -75.4564 & 35.5747 & NC \\
\hline 2168 & -1.3 & -75.4666 & 35.6397 & NC & 2240 & -4.6 & -75.4574 & 35.6075 & NC & 2312 & -2.3 & -75.4564 & 35.5742 & NC \\
\hline 2169 & -1.6 & -75.4665 & 35.6392 & NC & 2241 & -4.6 & -75.4574 & 35.6071 & NC & 2313 & -2.6 & -75.4565 & 35.5738 & NC \\
\hline 2170 & -1.8 & -75.4664 & 35.6388 & NC & 2242 & -4.5 & -75.4573 & 35.6066 & NC & 2314 & -2.8 & -75.4565 & 35.5733 & NC \\
\hline 2171 & -1.5 & -75.4662 & 35.6383 & NC & 2243 & -4.9 & -75.4573 & 35.6061 & NC & 2315 & -2.9 & -75.4565 & 35.5728 & NC \\
\hline 2172 & -1.3 & -75.4661 & 35.6379 & NC & 2244 & -4.8 & -75.4572 & 35.6057 & NC & 2316 & -2.5 & -75.4566 & 35.5724 & NC \\
\hline 2173 & -1.4 & -75.466 & 35.6374 & NC & 2245 & -5 & -75.4572 & 35.6052 & $\mathrm{NC}$ & 2317 & -2.5 & -75.4566 & 35.5719 & NC \\
\hline 2174 & -1.5 & -75.4659 & 35.637 & NC & 2246 & -5.1 & -75.4571 & 35.6047 & NC & 2318 & -2.2 & -75.4567 & 35.5714 & NC \\
\hline 2175 & -1.3 & -75.4658 & 35.6365 & NC & 2247 & -5.4 & .75 .4571 & 35.6042 & NC & 2319 & -2.1 & -75.4567 & 35.571 & NC \\
\hline 2176 & -1.2 & -75.4657 & 35.6361 & NC & 2248 & -5.6 & -75.4571 & 35.6038 & NC & 2320 & -1.7 & -75.4568 & 35.5705 & NC \\
\hline 2177 & -1.2 & -75.4655 & 35.6356 & NC & 2249 & -5.5 & -75.457 & 35.6033 & NC & 2321 & -1.4 & -75.4568 & 35.5701 & NC \\
\hline 2178 & -1.3 & -75.4654 & 35.6352 & NC & 2250 & -5.5 & -75.457 & 35.6028 & NC & 2322 & -1.1 & -75.4568 & 35.5696 & NC \\
\hline 2179 & -1.3 & -75.4653 & 35.6347 & NC & 2251 & -5.2 & -75.4569 & 35.6024 & NC & 2323 & -0.9 & -75.4569 & 35.5691 & NC \\
\hline 2180 & -1.3 & -75.4652 & 35.6343 & NC & 2252 & -5 & -75.4569 & 35.6019 & NC & 2324 & -0.7 & -75.4569 & 35.5687 & NC \\
\hline 2181 & -1.1 & -75.4651 & 35.6338 & NC & 2253 & -4.8 & -75.4568 & 35.6014 & NC & 2325 & -0.7 & -75.457 & 35.5682 & NC \\
\hline 2182 & -1.3 & -75.4649 & 35.6334 & NC & 2254 & -4.5 & -75.4568 & 35.601 & NC & 2326 & -0.5 & -75.457 & 35.5677 & NC \\
\hline 2183 & -1.5 & -75.4648 & 35.6329 & NC & 2255 & -4.5 & -75.4567 & 35.6005 & NC & 2327 & -0.3 & -75.4571 & 35.5673 & NC \\
\hline 2184 & -1.6 & .75 .4647 & 35.6325 & $\mathrm{NC}$ & 2256 & -4.1 & -75.4567 & 35.6 & NC & 2328 & 0 & -75.4571 & 35.5668 & NC \\
\hline 2185 & -1.5 & -75.4646 & 35.632 & NC & 2257 & -4 & -75.4566 & 35.5996 & NC & 2329 & 0 & -75.4571 & 35.5663 & NC \\
\hline 2186 & -1.5 & -75.4645 & 35.6316 & NC & 2258 & -4.1 & -75.4566 & 35.5991 & NC & 2330 & 0.2 & -75.4572 & 35.5659 & NC \\
\hline 2187 & -1.5 & -75.4644 & 35.6311 & $\mathrm{NC}$ & 2259 & -4.2 & -75.4566 & 35.5986 & NC & 2331 & 0.3 & -75.4572 & 35.5654 & NC \\
\hline 2188 & -1.7 & -75.4642 & 35.6307 & NC & 2260 & -4.1 & -75.4565 & 35.5982 & NC & 2332 & 0.4 & -75.4573 & 35.565 & NC \\
\hline 2189 & -1.5 & -75.4641 & 35.6302 & NC & 2261 & -4.3 & -75.4565 & 35.5977 & NC & 2333 & 0.8 & -75.4573 & 35.5645 & NC \\
\hline 2190 & -1.3 & -75.464 & 35.6298 & NC & 2262 & -4.3 & -75.4564 & 35.5972 & NC & 2334 & 0.6 & -75.4573 & 35.564 & NC \\
\hline 2191 & -1.8 & -75.4639 & 35.6293 & NC & 2263 & -4.1 & -75.4564 & 35.5968 & NC & 2335 & 0.5 & -75.4574 & 35.5636 & NC \\
\hline 2192 & -2.2 & -75.4638 & 35.6289 & NC & 2264 & -3.3 & -75.4563 & 35.5963 & NC & 2336 & 0.7 & -75.4574 & 35.5631 & NC \\
\hline 2193 & -2.2 & -75.4636 & 35.6284 & NC & 2265 & -3.7 & -75.4563 & 35.5958 & NC & 2337 & 0.8 & -75.4575 & 35.5626 & NC \\
\hline 2194 & -2.3 & -75.4635 & 35.628 & NC & 2266 & -3.4 & -75.4562 & 35.5954 & NC & 2338 & 0.7 & -75.4575 & 35.5622 & NC \\
\hline 2195 & -2.1 & -75.4634 & 35.6275 & NC & 2267 & -3.6 & -75.4562 & 35.5949 & NC & 2339 & 0.6 & .75 .4576 & 35.5617 & NC \\
\hline 2196 & -2 & -75.4633 & 35.6271 & NC & 2268 & -3.3 & -75.4562 & 35.5944 & NC & 2340 & 0.8 & .75 .4576 & 35.5612 & NC \\
\hline 2197 & -2.1 & -75.4632 & 35.6266 & NC & 2269 & -3.1 & -75.4561 & 35.594 & NC & 2341 & 0.7 & -75.4576 & 35.5608 & NC \\
\hline 2198 & -2.1 & -75.463 & 35.6262 & NC & 2270 & -3 & -75.4561 & 35.5935 & NC & 2342 & 0.7 & -75.4577 & 35.5603 & NC \\
\hline 2199 & -1.9 & -75.4629 & 35.6257 & NC & 2271 & .3 & .75 .456 & 35.593 & NC & 2343 & 0.7 & .75 .4577 & 35.5599 & NC \\
\hline 2200 & -2 & -75.4628 & 35.6253 & NC & 2272 & -3.1 & .75 .456 & 35.5926 & NC & 2344 & 0.8 & -75.4578 & 35.5594 & NC \\
\hline 2201 & -1.9 & -75.4627 & 35.6248 & NC & 2273 & -3.1 & -75.4559 & 35.5921 & NC & 2345 & 0.5 & -75.4578 & 35.5589 & NC \\
\hline 2202 & -1.9 & -75.4626 & 35.6244 & NC & 2274 & -3 & -75.4559 & 35.5916 & NC & 2346 & 0.4 & -75.4579 & 35.5585 & NC \\
\hline 2203 & -1.9 & -75.4625 & 35.6239 & NC & 2275 & -3 & -75.4558 & 35.5912 & NC & 2347 & 0.6 & -75.4579 & 35.558 & NC \\
\hline 2204 & -1.9 & -75.4623 & 35.6235 & NC & 2276 & -2.8 & -75.4558 & 35.5907 & NC & 2348 & 0.6 & -75.4579 & 35.5575 & NC \\
\hline 2205 & -2.1 & -75.4622 & 35.623 & NC & 2277 & -2.9 & -75.4557 & 35.5902 & $\mathrm{NC}$ & 2349 & 0.6 & -75.458 & 35.5571 & NC \\
\hline 2206 & -2.3 & -75.4621 & 35.6226 & NC & 2278 & -2.9 & -75.4557 & 35.5898 & $\mathrm{NC}$ & 2350 & 0.7 & -75.458 & 35.5566 & NC \\
\hline 2207 & -2.4 & -75.462 & 35.6221 & NC & 2279 & -2.8 & -75.4557 & 35.5893 & NC & 2351 & 0.7 & -75.4581 & 35.5562 & NC \\
\hline 2208 & -2.7 & -75.4619 & 35.6217 & $\mathrm{NC}$ & 2280 & -2.5 & -75.4556 & 35.5888 & NC & 2352 & 0.6 & -75.4581 & 35.5557 & NC \\
\hline 2209 & -2.6 & -75.4617 & 35.6212 & NC & 2281 & -2.1 & .75 .4556 & 35.5883 & NC & 2353 & 0.5 & -75.4582 & 35.5552 & NC \\
\hline 2210 & -2.8 & .75 .4616 & 35.6208 & NC & 2282 & -1.8 & -75.4555 & 35.5879 & NC & 2354 & 0.7 & -75.4582 & 35.5548 & NC \\
\hline 2211 & -3 & -75.4615 & 35.6203 & NC & 2283 & -1.7 & .75 .4555 & 35.5874 & NC & 2355 & 0.7 & -75.4582 & 35.5543 & NC \\
\hline 2212 & -3.2 & -75.4614 & 35.6199 & $\mathrm{NC}$ & 2284 & -1.6 & -75.4554 & 35.5869 & $\mathrm{NC}$ & 2356 & 0.6 & -75.4583 & 35.5538 & NC \\
\hline 2213 & -3.4 & -75.4613 & 35.6194 & $\mathrm{NC}$ & 2285 & -1.6 & -75.4554 & 35.5865 & NC & 2357 & 0.4 & -75.4583 & 35.5534 & NC \\
\hline 2214 & -3.6 & -75.4612 & 35.619 & $\mathrm{NC}$ & 2286 & -1.5 & -75.4553 & 35.586 & NC & 2358 & 0.6 & -75.4584 & 35.5529 & NC \\
\hline 2215 & -4 & -75.461 & 35.6185 & NC & 2287 & -1.1 & -75.4553 & 35.5855 & NC & 2359 & 0.4 & -75.4584 & 35.5524 & NC \\
\hline 2216 & -4.3 & -75.4609 & 35.6181 & NC & 2288 & -0.4 & -75.4553 & 35.5851 & NC & 2360 & 0.4 & -75.4584 & 35.552 & NC \\
\hline 2217 & -4.7 & -75.4608 & 35.6176 & $\mathrm{NC}$ & 2289 & -0.4 & -75.4552 & 35.5846 & NC & 2361 & 0.4 & -75.4585 & 35.5515 & NC \\
\hline 2218 & -4.8 & -75.4607 & 35.6172 & $\mathrm{NC}$ & 2290 & -0.3 & -75.4552 & 35.5841 & NC & 2362 & 0.4 & -75.4585 & 35.5511 & NC \\
\hline 2219 & -4.9 & -75.4606 & 35.6167 & $\mathrm{NC}$ & 2291 & -0.1 & -75.4551 & 35.5837 & NC & 2363 & 0.4 & -75.4586 & 35.5506 & NC \\
\hline 2220 & -4.9 & -75.4604 & 35.6163 & NC & 2292 & 0 & -75.4551 & 35.5832 & NC & 2364 & 0.3 & -75.4586 & 35.5501 & NC \\
\hline 2221 & -4.7 & -75.4603 & 35.6158 & NC & 2293 & 0.1 & -75.455 & 35.5827 & $\mathrm{NC}$ & 2365 & 0.2 & -75.4587 & 35.5497 & NC \\
\hline 2222 & -4.7 & -75.4602 & 35.6154 & NC & 2294 & 0 & -75.455 & 35.5823 & NC & 2366 & 0.3 & -75.4587 & 35.5492 & NC \\
\hline 2223 & -4.5 & -75.4601 & 35.6149 & NC & 2295 & -0.8 & -75.4549 & 35.5818 & NC & 2367 & -0.1 & -75.4598 & 35.5487 & NC \\
\hline 2224 & -4.4 & -75.46 & 35.6145 & NC & 2296 & -1 & -75.4549 & 35.5813 & NC & 2368 & -0.1 & -75.4599 & 35.5482 & NC \\
\hline 2225 & -4.5 & -75.4599 & 35.614 & NC & 2297 & -1.3 & -75.4548 & 35.5809 & NC & 2369 & -0.2 & -75.46 & 35.5478 & NC \\
\hline 2226 & -4.8 & -75.458 & 35.6136 & NC & 2298 & -1.6 & -75.4548 & 35.5804 & NC & 2370 & -0.1 & -75.4601 & 35.5473 & NC \\
\hline 2227 & -4.5 & -75.4597 & 35.6136 & NC & 2299 & -1.4 & -75.4559 & 35.5802 & NC & 2371 & -0.1 & -75.4602 & 35.5468 & NC \\
\hline 2228 & -4.5 & -75.458 & 35.6131 & NC & 2300 & -1.5 & -75.4559 & 35.5798 & NC & 2372 & 0 & -75.4604 & 35.5464 & NC \\
\hline 2229 & -4.4 & -75.4579 & 35.6127 & NC & 2301 & -1.6 & -75.456 & 35.5793 & NC & 2373 & -0.1 & -75.4605 & 35.5459 & NC \\
\hline 2230 & -4.3 & -75.4579 & 35.6122 & NC & 2302 & -1.9 & -75.456 & 35.5789 & NC & 2374 & -0.1 & -75.4606 & 35.5455 & NC \\
\hline 2231 & -4.1 & -75.4578 & 35.6117 & NC & 2303 & -2.3 & -75.456 & 35.5784 & NC & 2375 & -0.1 & -75.4607 & 35.545 & NC \\
\hline
\end{tabular}




\begin{tabular}{|c|c|c|c|c|c|c|c|c|c|c|c|c|c|c|}
\hline TR\# & RATE & LONG & LAT & ST & TR\# & RATE & LONG & LAT & ST & TR\# & RATE & LONG & LAT & ST \\
\hline 2376 & -0.1 & -75.4608 & 35.5446 & NC & 2448 & 1.6 & -75.4687 & 35.5148 & NC & 2520 & 0.2 & -75.4741 & 35.4837 & $\mathrm{NC}$ \\
\hline 2377 & -0.1 & -75.4609 & 35.5441 & NC & 2449 & 1.5 & -75.4688 & 35.5144 & NC & 2521 & 0.2 & -75.4741 & 35.4832 & NC \\
\hline 2378 & 0 & -75.4611 & 35.5437 & NC & 2450 & 1.5 & .75 .4689 & 35.5139 & NC & 2522 & 0 & -75.4742 & 35.4828 & NC \\
\hline 2379 & 1 & -75.4612 & 35.5432 & NC & 2451 & 1.5 & -75.469 & 35.5135 & NC & 2523 & -0.1 & -75.4742 & 35.4823 & NC \\
\hline 2380 & 0.8 & -75.4613 & 35.5427 & NC & 2452 & 1.7 & -75.4691 & 35.513 & NC & 2524 & -0.1 & -75.4743 & 35.4818 & $\mathrm{NC}$ \\
\hline 2381 & 0.8 & -75.4614 & 35.5423 & NC & 2453 & 1.6 & -75.4692 & 35.5126 & NC & 2525 & 0 & -75.4743 & 35.4814 & NC \\
\hline 2382 & 0.8 & -75.4615 & 35.5418 & NC & 2454 & 1.7 & -75.4693 & 35.5121 & NC & 2526 & 0.1 & -75.4744 & 35.4809 & NC \\
\hline 2383 & 1 & -75.4616 & 35.5414 & NC & 2455 & 1.9 & -75.4694 & 35.5116 & NC & 2527 & -0.1 & -75.4745 & 35.4804 & NC \\
\hline 2384 & 1.2 & -75.4618 & 35.5409 & NC & 2456 & 2 & -75.4695 & 35.5112 & NC & 2528 & -0.3 & -75.4745 & 35.48 & NC \\
\hline 2385 & 1.1 & -75.4619 & 35.5405 & NC & 2457 & 1.5 & -75.4696 & 35.5107 & NC & 2529 & -0.4 & -75.4746 & 35.4795 & NC \\
\hline 2386 & 0.9 & -75.462 & 35.54 & NC & 2458 & 1 & -75.4697 & 35.5103 & NC & 2530 & -0.4 & -75.4746 & 35.4791 & NC \\
\hline 2387 & 0.7 & -75.4621 & 35.5395 & NC & 2459 & 0.9 & -75.4698 & 35.5098 & NC & 2531 & -0.1 & -75.4747 & 35.4786 & NC \\
\hline 2388 & 0.6 & -75.4622 & 35.5391 & NC & 2460 & 0.8 & -75.4699 & 35.5094 & NC & 2532 & 0 & -75.4747 & 35.4781 & NC \\
\hline 2389 & 0.9 & .75 .4623 & 35.5386 & NC & 2461 & 0.7 & -75.47 & 35.5089 & NC & 2533 & -0.1 & -75.4748 & 35.4777 & NC \\
\hline 2390 & 1 & -75.4625 & 35.5382 & NC & 2462 & 0.7 & -75.4701 & 35.5084 & NC & 2534 & -0.3 & -75.4748 & 35.4772 & NC \\
\hline 2391 & 1.1 & -75.4626 & 35.5377 & NC & 2463 & 0.5 & -75.4702 & 35.508 & NC & 2535 & -0.3 & -75.4749 & 35.4767 & NC \\
\hline 2392 & 1.2 & -75.4627 & 35.5373 & NC & 2464 & 0.3 & -75.4703 & 35.5075 & NC & 2536 & -0.2 & -75.475 & 35.4763 & NC \\
\hline 2393 & 1.1 & -75.4628 & 35.5368 & NC & 2465 & 0.3 & -75.4704 & 35.5071 & NC & 2537 & -0.3 & .75 .475 & 35.4758 & NC \\
\hline 2394 & 1.4 & -75.4629 & 35.5364 & NC & 2466 & 0.5 & -75.4705 & 35.5066 & NC & 2538 & -0.6 & -75.4751 & 35.4753 & NC \\
\hline 2395 & 1 & -75.463 & 35.5359 & NC & 2467 & 0.3 & -75.4706 & 35.5062 & NC & 2539 & -0.5 & -75.4751 & 35.4749 & NC \\
\hline 2396 & 0.7 & -75.4632 & 35.5354 & NC & 2468 & 0.5 & -75.4707 & 35.5057 & NC & 2540 & -0.4 & -75.4752 & 35.4744 & NC \\
\hline 2397 & 1.1 & -75.4633 & 35.535 & NC & 2469 & 0.4 & -75.4708 & 35.5052 & NC & 2541 & -0.3 & -75.4752 & 35.4739 & NC \\
\hline 2398 & 1.4 & -75.4634 & 35.5345 & NC & 2470 & 0.2 & -75.4709 & 35.5048 & NC & 2542 & -0.2 & -75.4753 & 35.4735 & NC \\
\hline 2399 & 1.4 & -75.4635 & 35.5341 & NC & 2471 & 0.1 & -75.4709 & 35.5043 & NC & 2543 & -0.5 & -75.4754 & 35.473 & NC \\
\hline 2400 & 1.5 & -75.4636 & 35.5336 & NC & 2472 & 0.1 & -75.471 & 35.5039 & NC & 2544 & -0.8 & -75.4754 & 35.4725 & NC \\
\hline 2401 & 2 & -75.4637 & 35.5332 & NC & 2473 & 0.2 & -75.4711 & 35.5034 & NC & 2545 & -0.7 & -75.4755 & 35.4721 & $\mathrm{NC}$ \\
\hline 2402 & 2.4 & -75.4639 & 35.5327 & NC & 2474 & 0.1 & -75.4712 & 35.5029 & NC & 2546 & -0.7 & -75.4755 & 35.4716 & $\mathrm{NC}$ \\
\hline 2403 & 2.4 & -75.464 & 35.5322 & NC & 2475 & 0.1 & -75.4713 & 35.5025 & NC & 2547 & -0.6 & -75.4756 & 35.4711 & NC \\
\hline 2404 & 2.2 & -75.4641 & 35.5318 & NC & 2476 & 0.2 & -75.4714 & 35.502 & NC & 2548 & -0.6 & -75.4756 & 35.4707 & NC \\
\hline 2405 & 2.3 & -75.4642 & 35.5313 & NC & 2477 & -0.1 & -75.4715 & 35.5016 & NC & 2549 & -0.7 & -75.4757 & 35.4702 & NC \\
\hline 2406 & 2.5 & -75.4643 & 35.5309 & NC & 2478 & -0.1 & -75.4716 & 35.5011 & NC & 2550 & -1 & -75.4757 & 35.4698 & NC \\
\hline 2407 & 2.6 & -75.4644 & 35.5304 & NC & 2479 & -0.3 & -75.4717 & 35.5007 & NC & 2551 & -0.9 & -75.4758 & 35.4693 & NC \\
\hline 2408 & 2.8 & -75.4646 & 35.53 & NC & 2480 & -0.5 & -75.4718 & 35.5002 & NC & 2552 & -0.8 & -75.4759 & 35.4688 & NC \\
\hline 2409 & 3 & -75.4647 & 35.5295 & NC & 2481 & -0.6 & -75.4719 & 35.4997 & NC & 2553 & -0.9 & -75.4759 & 35.4684 & NC \\
\hline 2410 & 3.3 & -75.4648 & 35.5291 & NC & 2482 & -0.7 & -75.472 & 35.4993 & NC & 2554 & -0.7 & -75.476 & 35.4679 & NC \\
\hline 2411 & 3.4 & -75.4649 & 35.5286 & NC & 2483 & -0.8 & -75.4721 & 35.4988 & NC & 2555 & -0.7 & -75.476 & 35.4674 & $\mathrm{NC}$ \\
\hline 2412 & 3.3 & -75.465 & 35.5281 & NC & 2484 & -1 & -75.4722 & 35.4984 & NC & 2556 & -0.5 & -75.4761 & 35.467 & NC \\
\hline 2413 & 3.2 & -75.4651 & 35.5277 & NC & 2485 & -0.9 & -75.4723 & 35.4979 & NC & 2557 & -0.7 & -75.4761 & 35.4665 & NC \\
\hline 2414 & 3.2 & -75.4653 & 35.5272 & NC & 2486 & -0.8 & -75.4724 & 35.4975 & NC & 2558 & -0.7 & -75.4762 & 35.466 & NC \\
\hline 2415 & 3.1 & -75.4654 & 35.5268 & NC & 2487 & -0.8 & -75.4725 & 35.497 & NC & 2559 & -0.7 & -75.4763 & 35.4656 & NC \\
\hline 2416 & 2.9 & -75.4655 & 35.5263 & NC & 2488 & -0.7 & -75.4726 & 35.4965 & NC & 2560 & -0.7 & -75.4763 & 35.4651 & NC \\
\hline 2417 & 2.8 & -75.4656 & 35.5259 & NC & 2489 & -0.6 & -75.4727 & 35.4961 & NC & 2561 & -0.7 & -75.4764 & 35.4646 & NC \\
\hline 2418 & 2.8 & -75.4657 & 35.5254 & NC & 2490 & -0.6 & -75.4728 & 35.4956 & NC & 2562 & -0.6 & -75.4764 & 35.4642 & NC \\
\hline 2419 & 3 & -75.4658 & 35.5249 & NC & 2491 & -0.4 & -75.4729 & 35.4952 & NC & 2563 & -0.6 & -75.4765 & 35.4637 & NC \\
\hline 2420 & 3.4 & -75.466 & 35.5245 & NC & 2492 & 0 & -75.473 & 35.4947 & NC & 2564 & -0.7 & -75.4765 & 35.4632 & NC \\
\hline 2421 & 3.5 & -75.4661 & 35.524 & NC & 2493 & -0.1 & -75.4731 & 35.4943 & NC & 2565 & -0.7 & -75.4766 & 35.4628 & NC \\
\hline 2422 & 3.3 & -75.4662 & 35.5236 & NC & 2494 & -0.2 & -75.4732 & 35.4938 & NC & 2566 & -0.5 & -75.4766 & 35.4623 & NC \\
\hline 2423 & 3.5 & -75.4663 & 35.5231 & NC & 2495 & -0.2 & -75.4733 & 35.4933 & NC & 2567 & -0.5 & -75.4764 & 35.4619 & NC \\
\hline 2424 & 2.1 & -75.4664 & 35.5227 & NC & 2496 & -0.3 & -75.4734 & 35.4929 & NC & 2568 & -0.6 & -75.4767 & 35.4619 & $\mathrm{NC}$ \\
\hline 2425 & 2.3 & -75.4665 & 35.5222 & NC & 2497 & 0 & -75.4735 & 35.4924 & $\mathrm{NC}$ & 2569 & -0.4 & -75.4764 & 35.4614 & NC \\
\hline 2426 & 2.4 & -75.4667 & 35.5218 & NC & 2498 & 0.2 & -75.4736 & 35.492 & NC & 2570 & -0.6 & -75.4768 & 35.4614 & $\mathrm{NC}$ \\
\hline 2427 & 2.6 & -75.4668 & 35.5213 & NC & 2499 & 0.4 & -75.4731 & 35.4916 & NC & 2571 & -0.5 & -75.4765 & 35.461 & NC \\
\hline 2428 & 3.4 & -75.4674 & 35.5208 & NC & 2500 & 0.1 & -75.4737 & 35.4915 & NC & 2572 & -0.6 & -75.4768 & 35.4609 & NC \\
\hline 2429 & 2.8 & -75.4669 & 35.5208 & NC & 2501 & 0.5 & -75.4732 & 35.4911 & NC & 2573 & -0.3 & -75.4765 & 35.4605 & NC \\
\hline 2430 & 2.7 & -75.467 & 35.5204 & NC & 2502 & 0.3 & -75.4738 & 35.491 & NC & 2574 & -0.5 & -75.4769 & 35.4605 & NC \\
\hline 2431 & 3.3 & -75.4675 & 35.5203 & NC & 2503 & 0.7 & -75.4732 & 35.4907 & NC & 2575 & -0.5 & -75.4766 & 35.46 & NC \\
\hline 2432 & 3.2 & -75.4676 & 35.5199 & NC & 2504 & 0.2 & -75.4739 & 35.4906 & NC & 2576 & -0.6 & -75.4766 & 35.4596 & NC \\
\hline 2433 & 2.6 & -75.4671 & 35.5199 & NC & 2505 & 0.7 & .75 .4733 & 35.4902 & NC & 2577 & -0.8 & -75.4766 & 35.4591 & NC \\
\hline 2434 & 2.7 & -75.4672 & 35.5195 & NC & 2506 & 0.4 & -75.474 & 35.4901 & NC & 2578 & -1.2 & .75 .4767 & 35.4586 & NC \\
\hline 2435 & 2.9 & -75.4677 & 35.5194 & NC & 2507 & 0.8 & -75.4733 & 35.4897 & NC & 2579 & -0.9 & -75.4767 & 35.4582 & NC \\
\hline 2436 & 2.6 & -75.4678 & 35.519 & NC & 2508 & 0.9 & -75.4734 & 35.4893 & NC & 2580 & -1 & -75.4768 & 35.4577 & NC \\
\hline 2437 & 2.5 & -75.4674 & 35.519 & NC & 2509 & 0.8 & -75.4734 & 35.4888 & NC & 2581 & -1 & -75.4768 & 35.4573 & NC \\
\hline 2438 & 2.3 & -75.4675 & 35.5186 & NC & 2510 & 0.7 & -75.4735 & 35.4883 & NC & 2582 & -0.9 & -75.4768 & 35.4568 & NC \\
\hline 2439 & 2.4 & -75.4679 & 35.5185 & NC & 2511 & 0.7 & -75.4736 & 35.4879 & NC & 2583 & -1.1 & -75.4769 & 35.4563 & NC \\
\hline 2440 & 2.1 & -75.468 & 35.5181 & NC & 2512 & 0.8 & .75 .4736 & 35.4874 & NC & 2584 & -1 & -75.4769 & 35.4559 & NC \\
\hline 2441 & 2 & -75.4676 & 35.5181 & NC & 2513 & 0.8 & -75.4737 & 35.487 & NC & 2585 & -1 & -75.477 & 35.4554 & NC \\
\hline 2442 & 1.8 & -75.4681 & 35.5176 & NC & 2514 & 0.8 & -75.4737 & 35.4865 & NC & 2586 & -0.9 & -75.477 & 35.4549 & NC \\
\hline 2443 & 1.8 & -75.4682 & 35.5171 & NC & 2515 & 0.7 & -75.4738 & 35.486 & NC & 2587 & -0.9 & -75.477 & 35.4545 & NC \\
\hline 2444 & 1.8 & -75.4683 & 35.5167 & NC & 2516 & 0.6 & -75.4738 & 35.4856 & NC & 2588 & -0.9 & -75.4771 & 35.454 & NC \\
\hline 2445 & 1.6 & -75.4684 & 35.5162 & NC & 2517 & 0.5 & -75.4739 & 35.4851 & NC & 2589 & -0.9 & -75.4771 & 35.4535 & NC \\
\hline 2446 & 1.5 & -75.4685 & 35.5158 & NC & 2518 & 0.3 & -75.4739 & 35.4846 & NC & 2590 & -1.1 & -75.4771 & 35.4531 & NC \\
\hline 2447 & 1.6 & -75.4686 & 35.5153 & NC & 2519 & 0.2 & -75.474 & 35.4842 & NC & 2591 & -1.1 & -75.47 .72 & 35.4526 & NC \\
\hline
\end{tabular}




\begin{tabular}{|c|c|c|c|c|c|c|c|c|c|c|c|c|c|c|}
\hline TR\# & RATE & LONG & LAT & ST & TR\# & RATE & LONG & LAT & ST & TR\# & RATE & LONG & LAT & ST \\
\hline 2592 & -1.2 & -75.4772 & 35.4521 & NC & 2664 & 0.5 & -75.4803 & 35.4205 & $\mathrm{NC}$ & 2736 & 2 & -75.4847 & 35.3868 & NC \\
\hline 2593 & -1.2 & -75.4773 & 35.4517 & NC & 2665 & 0.4 & -75.4803 & 35.4201 & $\mathrm{NC}$ & 2737 & 1.3 & -75.4849 & 35.3863 & NC \\
\hline 2594 & -1.2 & -75.4773 & 35.4512 & NC & 2666 & 0.2 & .75 .4803 & 35.4196 & NC & 2738 & 0.8 & -75.485 & 35.3859 & $\mathrm{NC}$ \\
\hline 2595 & -1.3 & -75.4773 & 35.4507 & $\mathrm{NC}$ & 2667 & 0.1 & -75.4803 & 35.4192 & NC & 2739 & 0.7 & -75.4852 & 35.3854 & NC \\
\hline 2596 & -1.1 & -75.4774 & 35.4503 & NC & 2668 & -0.1 & .75 .4804 & 35.4187 & $\mathrm{NC}$ & 2740 & 0.6 & -75.4853 & 35.385 & NC \\
\hline 2597 & -1.4 & -75.4774 & 35.4498 & NC & 2669 & -0.1 & -75.4804 & 35.4182 & $\mathrm{NC}$ & 2741 & 0.6 & -75.4854 & 35.3845 & NC \\
\hline 2598 & -1.4 & -75.4775 & 35.4494 & NC & 2670 & -0.1 & -75.4804 & 35.4178 & $\mathrm{NC}$ & 2742 & 0.6 & -75.4856 & 35.3841 & NC \\
\hline 2599 & -1.5 & -75.4775 & 35.4489 & NC & 2671 & -0.1 & -75.4804 & 35.4173 & $\mathrm{NC}$ & 2743 & 0.3 & -75.4857 & 35.3836 & $\mathrm{NC}$ \\
\hline 2600 & -1.4 & -75.4775 & 35.4484 & NC & 2672 & 0 & -75.4804 & 35.4168 & NC & 2744 & 0 & -75.4859 & 35.3832 & NC \\
\hline 2601 & -1.2 & -75.4776 & 35.448 & NC & 2673 & -0.2 & -75.4804 & 35.4164 & NC & 2745 & 0 & -75.486 & 35.3827 & NC \\
\hline 2602 & -1.1 & -75.4776 & 35.4475 & NC & 2674 & -0.4 & -75.4805 & 35.4159 & $\mathrm{NC}$ & 2746 & 0.1 & -75.4862 & 35.3823 & NC \\
\hline 2603 & -1.2 & -75.4777 & 35.447 & NC & 2675 & -0.7 & -75.4805 & 35.4154 & $\mathrm{NC}$ & 2747 & 0.1 & -75.4863 & 35.3818 & NC \\
\hline 2604 & -1.1 & -75.4777 & 35.4466 & NC & 2676 & -1.1 & -75.4805 & 35.415 & NC & 2748 & 0.1 & -75.4865 & 35.3814 & NC \\
\hline 2605 & -1.3 & -75.4777 & 35.4461 & NC & 2677 & -1.1 & -75.4805 & 35.4145 & $\mathrm{NC}$ & 2749 & 0.2 & -75.4866 & 35.3809 & $\mathrm{NC}$ \\
\hline 2606 & -1.5 & -75.4778 & 35.4456 & NC & 2678 & -1.2 & -75.4805 & 35.4141 & $\mathrm{NC}$ & 2750 & 0.5 & -75.4867 & 35.3805 & NC \\
\hline 2607 & -1.4 & -75.4778 & 35.4452 & NC & 2679 & -1.2 & -75.4805 & 35.4136 & NC & 2751 & 0.3 & -75.4869 & 35.38 & NC \\
\hline 2608 & -1.3 & -75.4779 & 35.4447 & NC & 2680 & -1.1 & -75.4806 & 35.4131 & $\mathrm{NC}$ & 2752 & 0.2 & -75.487 & 35.3796 & NC \\
\hline 2609 & -1.3 & -75.4779 & 35.4442 & $\mathrm{NC}$ & 2681 & -1.1 & -75.4806 & 35.4127 & $\mathrm{NC}$ & 2753 & 0.5 & .75 .4872 & 35.3791 & NC \\
\hline 2610 & -1.2 & -75.4779 & 35.4438 & NC & 2682 & -1.1 & -75.4806 & 35.4122 & $\mathrm{NC}$ & 2754 & 0.5 & -75.4873 & 35.3787 & NC \\
\hline 2611 & -1.3 & -75.478 & 35.4433 & NC & 2683 & -1.1 & -75.4806 & 35.4117 & $\mathrm{NC}$ & 2755 & 0.6 & -75.4875 & 35.3782 & NC \\
\hline 2612 & -1.2 & -75.478 & 35.4428 & NC & 2684 & -1 & -75.4806 & 35.4113 & $\mathrm{NC}$ & 2756 & 0.8 & -75.4876 & 35.3778 & NC \\
\hline 2613 & -1.3 & -75.4781 & 35.4424 & NC & 2685 & -1.2 & -75.4806 & 35.4108 & NC & 2757 & 1.3 & -75.4878 & 35.3773 & NC \\
\hline 2614 & -1.1 & -75.4781 & 35.4419 & NC & 2686 & -1.3 & -75.4807 & 35.4103 & $\mathrm{NC}$ & 2758 & 1.7 & -75.4879 & 35.3768 & NC \\
\hline 2615 & -1.1 & -75.4781 & 35.4414 & NC & 2687 & -1.3 & -75.4807 & 35.4099 & $\mathrm{NC}$ & 2759 & 1.6 & -75.4881 & 35.3764 & NC \\
\hline 2616 & -1 & -75.4782 & 35.441 & NC & 2688 & -1.4 & -75.4807 & 35.4094 & NC & 2760 & 1.3 & -75.4882 & 35.3759 & NC \\
\hline 2617 & -0.8 & -75.4782 & 35.4405 & $\mathrm{NC}$ & 2689 & -1.1 & -75.4807 & 35.409 & $\mathrm{NC}$ & 2761 & 1.3 & -75.4883 & 35.3755 & NC \\
\hline 2618 & -0.6 & -75.4783 & 35.4401 & NC & 2690 & -0.9 & -75.4807 & 35.4085 & NC & 2762 & 1.2 & -75.4885 & 35.375 & NC \\
\hline 2619 & -0.4 & .75 .4783 & 35.4396 & $\mathrm{NC}$ & 2691 & -0.9 & -75.4807 & 35.408 & NC & 2763 & 1.1 & -75.4886 & 35.3746 & NC \\
\hline 2620 & -0.3 & -75.4783 & 35.4391 & NC & 2692 & -0.9 & -75.4808 & 35.4076 & NC & 2764 & 0.8 & -75.4888 & 35.3741 & NC \\
\hline 2621 & -0.5 & -75.4784 & 35.4387 & NC & 2693 & -1 & -75.4808 & 35.4071 & $\mathrm{NC}$ & 2765 & 0.7 & -75.4889 & 35.3737 & NC \\
\hline 2622 & -0.7 & -75.4784 & 35.4382 & NC & 2694 & -1 & -75.4808 & 35.4066 & $\mathrm{NC}$ & 2766 & 0.5 & -75.4891 & 35.3732 & NC \\
\hline 2623 & -0.8 & -75.4785 & 35.4377 & NC & 2695 & -1.1 & -75.4808 & 35.4062 & NC & 2767 & 0.4 & -75.4892 & 35.3728 & NC \\
\hline 2624 & -0.7 & -75.4785 & 35.4373 & NC & 2696 & -1.3 & -75.4808 & 35.4057 & $\mathrm{NC}$ & 2768 & 0.1 & -75.4894 & 35.3723 & NC \\
\hline 2625 & -0.8 & -75.4785 & 35.4368 & NC & 2697 & -1.1 & -75.4808 & 35.4053 & $\mathrm{NC}$ & 2769 & 0.1 & -75.4895 & 35.3719 & NC \\
\hline 2626 & -0.9 & -75.4786 & 35.4363 & NC & 2698 & -0.8 & -75.4809 & 35.4048 & $\mathrm{NC}$ & 2770 & 0.1 & -75.4896 & 35.3714 & NC \\
\hline 2627 & -0.8 & -75.4786 & 35.4359 & NC & 2699 & -0.7 & -75.4809 & 35.4043 & $\mathrm{NC}$ & 2771 & 0 & -75.4898 & 35.371 & NC \\
\hline 2628 & -0.7 & -75.4786 & 35.4354 & $\mathrm{NC}$ & 2700 & -0.5 & -75.4809 & 35.4039 & $\mathrm{NC}$ & 2772 & 0.3 & -75.4899 & 35.3705 & NC \\
\hline 2629 & -0.5 & -75.4787 & 35.4349 & $\mathrm{NC}$ & 2701 & -0.3 & -75.4809 & 35.4034 & NC & 2773 & 0.3 & -75.4901 & 35.3701 & NC \\
\hline 2630 & -0.4 & .75 .4787 & 35.4345 & NC & 2702 & -0.3 & -75.4809 & 35.4029 & NC & 2774 & 0.3 & -75.4902 & 35.3696 & NC \\
\hline 2631 & -0.3 & .75 .4788 & 35.434 & NC & 2703 & -0.2 & -75.4809 & 35.4025 & $\mathrm{NC}$ & 2775 & 0.2 & -75.4904 & 35.3692 & NC \\
\hline 2632 & -0.2 & -75.4788 & 35.4335 & $\mathrm{NC}$ & 2704 & 0 & -75.481 & 35.402 & NC & 2776 & 0.4 & -75.4905 & 35.3687 & NC \\
\hline 2633 & -0.3 & -75.4788 & 35.4331 & $\mathrm{NC}$ & 2705 & -0.3 & -75.481 & 35.4015 & NC & 2777 & 0.5 & -75.4907 & 35.3683 & NC \\
\hline 2634 & -0.4 & -75.4789 & 35.4326 & $\mathrm{NC}$ & 2706 & -0.3 & -75.481 & 35.4011 & NC & 2778 & 0.8 & -75.4917 & 35.3678 & NC \\
\hline 2635 & -0.2 & -75.4789 & 35.4322 & $\mathrm{NC}$ & 2707 & -0.3 & -75.481 & 35.4006 & $\mathrm{NC}$ & 2779 & 0.5 & -75.4908 & 35.3678 & NC \\
\hline 2636 & -0.2 & -75.479 & 35.4317 & NC & 2708 & -0.4 & -75.481 & 35.4002 & NC & 2780 & 0.6 & -75.491 & 35.3674 & NC \\
\hline 2637 & 0.1 & .75 .4799 & 35.4312 & $\mathrm{NC}$ & 2709 & -0.2 & -75.481 & 35.3997 & NC & 2781 & 0.8 & -75.4918 & 35.3673 & NC \\
\hline 2638 & -0.2 & -75.479 & 35.4312 & $\mathrm{NC}$ & 2710 & 0 & -75.4811 & 35.3992 & NC & 2782 & 0.6 & -75.4919 & 35.3669 & NC \\
\hline 2639 & -0.3 & -75.479 & 35.4308 & $\mathrm{NC}$ & 2711 & 0.2 & -75.4811 & 35.3981 & $\mathrm{NC}$ & 2783 & 0.9 & -75.4911 & 35.3669 & $\mathrm{NC}$ \\
\hline 2640 & 0 & -75.4799 & 35.4307 & NC & 2712 & 0.6 & -75.4812 & 35.3976 & NC & 2784 & 0.5 & -75.492 & 35.3664 & NC \\
\hline 2641 & 0 & -75.4799 & 35.4303 & $\mathrm{NC}$ & 2713 & 0.6 & -75.4814 & 35.3972 & NC & 2785 & 0.5 & -75.4921 & 35.3659 & NC \\
\hline 2642 & -0.2 & -75.4791 & 35.4303 & NC & 2714 & 0.6 & -75.4815 & 35.3967 & $\mathrm{NC}$ & 2786 & 0.6 & -75.4922 & 35.3655 & NC \\
\hline 2643 & -0.1 & -75.48 & 35.4298 & NC & 2715 & 0.5 & -75.4817 & 35.3963 & $\mathrm{NC}$ & 2787 & 0.6 & -75.4923 & 35.365 & NC \\
\hline 2644 & -0.2 & -75.4791 & 35.4298 & $\mathrm{NC}$ & 2716 & 0.8 & -75.4818 & 35.3958 & $\mathrm{NC}$ & 2788 & 0.9 & -75.4924 & 35.3646 & NC \\
\hline 2645 & -0.3 & -75.48 & 35.4293 & $\mathrm{NC}$ & 2717 & 0.8 & -75.482 & 35.3954 & $\mathrm{NC}$ & 2789 & 0.7 & .75 .4926 & 35.3641 & NC \\
\hline 2646 & -0.5 & -75.48 & 35.4289 & $\mathrm{NC}$ & 2718 & 0.8 & -75.4821 & 35.3949 & $\mathrm{NC}$ & 2790 & 0.7 & -75.4927 & 35.3637 & NC \\
\hline 2647 & -0.3 & -75.48 & 35.4284 & NC & 2719 & 0.8 & -75.4823 & 35.3945 & $\mathrm{NC}$ & 2791 & 0.7 & -75.4928 & 35.3632 & NC \\
\hline 2648 & -0.1 & .75 .48 & 35.428 & NC & 2720 & 0.9 & .75 .4824 & 35.394 & NC & 2792 & 0.7 & .75 .4929 & 35.3628 & NC \\
\hline 2649 & 0 & -75.48 & 35.4275 & NC & 2721 & 0.9 & -75.4825 & 35.3936 & NC & 2793 & 0.8 & -75.493 & 35.3623 & NC \\
\hline 2650 & 0 & -75.4801 & 35.427 & NC & 2722 & 1.1 & -75.4827 & 35.3931 & NC & 2794 & 0.8 & -75.4931 & 35.3618 & NC \\
\hline 2651 & -0.2 & -75.4801 & 35.4266 & NC & 2723 & 1.1 & -75.4828 & 35.3927 & NC & 2795 & 0.9 & -75.4932 & 35.3614 & NC \\
\hline 2652 & -0.1 & -75.4801 & 35.4261 & NC & 2724 & 1.4 & -75.483 & 35.3922 & $\mathrm{NC}$ & 2796 & 0.9 & .75 .4933 & 35.3609 & NC \\
\hline 2653 & 0.1 & .75 .4801 & 35.4256 & $\mathrm{NC}$ & 2725 & 1.7 & -75.4831 & 35.3918 & NC & 2797 & 0.8 & -75.4934 & 35.3605 & $\mathrm{NC}$ \\
\hline 2654 & 0.2 & -75.4801 & 35.4252 & NC & 2726 & 1.9 & -75.4833 & 35.3913 & NC & 2798 & 0.5 & -75.4935 & 35.36 & NC \\
\hline 2655 & 0 & -75.4801 & 35.4247 & NC & 2727 & 2.2 & -75.4834 & 35.3909 & NC & 2799 & 0 & -75.4936 & 35.3596 & NC \\
\hline 2656 & 0.1 & -75.4802 & 35.4242 & NC & 2728 & 2.1 & -75.4836 & 35.3904 & $\mathrm{NC}$ & 2800 & 0 & -75.4937 & 35.3591 & NC \\
\hline 2657 & 0 & -75.4802 & 35.4238 & $\mathrm{NC}$ & 2729 & 2.3 & -75.4837 & 35.39 & NC & 2801 & -0.1 & -75.4938 & 35.3586 & NC \\
\hline 2658 & -0.2 & -75.4802 & 35.4233 & $\mathrm{NC}$ & 2730 & 2.6 & -75.4838 & 35.3895 & $\mathrm{NC}$ & 2802 & -0.1 & -75.4939 & 35.3582 & NC \\
\hline 2659 & -0.3 & -75.4802 & 35.4229 & NC & 2731 & 2.5 & -75.484 & 35.3891 & $\mathrm{NC}$ & 2803 & 0 & -75.494 & 35.3577 & NC \\
\hline 2660 & -0.3 & -75.4802 & 35.4224 & NC & 2732 & 2.5 & -75.4841 & 35.3886 & NC & 2804 & -0.1 & -75.4941 & 35.3573 & NC \\
\hline 2661 & -0.2 & -75.4802 & 35.4219 & NC & 2733 & 2.6 & -75.4843 & 35.3881 & NC & 2805 & -0.3 & -75.4942 & 35.3568 & NC \\
\hline 2662 & 0 & -75.4803 & 35.4215 & NC & 2734 & 2.7 & -75.4844 & 35.3877 & NC & 2806 & -0.4 & -75.4943 & 35.3564 & NC \\
\hline 2663 & 0.2 & -75.4803 & 35.421 & NC & 2735 & 2.4 & -75.4846 & 35.3872 & NC & 2807 & -0.3 & -75.4944 & 35.3559 & NC \\
\hline
\end{tabular}




\begin{tabular}{|c|c|c|c|c|c|c|c|c|c|c|c|c|c|c|}
\hline TR\# & RATE & LONG & LAT & ST & TR\# & RATE & LONG & LAT & ST & TR\# & RATE & LONG & LAT & ST \\
\hline 2808 & 0 & -75.4945 & 35.3555 & NC & 2880 & -1.3 & -75.501 & 35.3231 & $\mathrm{NC}$ & 2952 & -2.5 & -75.5071 & 35.2911 & NC \\
\hline 2809 & 0.1 & -75.4946 & 35.355 & NC & 2881 & -1.2 & -75.5012 & 35.3226 & NC & 2953 & -2.8 & -75.5072 & 35.2907 & NC \\
\hline 2810 & 0.4 & -75.4947 & 35.3545 & NC & 2882 & -1.2 & -75.5013 & 35.3222 & NC & 2954 & -2.8 & -75.5073 & 35.2902 & NC \\
\hline 2811 & 0.4 & -75.4948 & 35.3541 & NC & 2883 & -1 & -75.5014 & 35.3217 & $\mathrm{NC}$ & 2955 & -2.8 & -75.5074 & 35.2898 & NC \\
\hline 2812 & 0.6 & -75.4949 & 35.3536 & NC & 2884 & -0.7 & -75.5015 & 35.3213 & $\mathrm{NC}$ & 2956 & -2.9 & -75.5075 & 35.2893 & NC \\
\hline 2813 & 0.8 & -75.4951 & 35.3532 & NC & 2885 & -0.9 & -75.5016 & 35.3208 & $\mathrm{NC}$ & 2957 & -2.8 & -75.5075 & 35.2888 & NC \\
\hline 2814 & 0.9 & -75.4952 & 35.3527 & NC & 2886 & -1 & -75.5018 & 35.3203 & $\mathrm{NC}$ & 2958 & -2.9 & -75.5076 & 35.2884 & NC \\
\hline 2815 & 0.7 & -75.4953 & 35.3523 & $\mathrm{NC}$ & 2887 & -1.1 & -75.5019 & 35.3199 & NC & 2959 & -3 & -75.5077 & 35.2879 & NC \\
\hline 2816 & 0.6 & -75.4954 & 35.3518 & NC & 2888 & -1.1 & -75.502 & 35.3194 & $\mathrm{NC}$ & 2960 & -2.9 & -75.5078 & 35.2875 & NC \\
\hline 2817 & 0.5 & -75.4955 & 35.3513 & NC & 2889 & -0.9 & -75.5021 & 35.319 & NC & 2961 & -2.8 & -75.5079 & 35.287 & NC \\
\hline 2818 & 0.1 & -75.4956 & 35.3509 & NC & 2890 & -0.6 & -75.5022 & 35.3185 & $\mathrm{NC}$ & 2962 & -3.2 & -75.508 & 35.2865 & NC \\
\hline 2819 & 0 & -75.4957 & 35.3504 & NC & 2891 & -0.6 & -75.5023 & 35.3181 & NC & 2963 & -3.4 & -75.5081 & 35.2861 & NC \\
\hline 2820 & -0.1 & -75.4958 & 35.35 & NC & 2892 & -0.6 & -75.5025 & 35.3176 & $\mathrm{NC}$ & 2964 & -3.4 & -75.5082 & 35.2856 & NC \\
\hline 2821 & 0.1 & -75.4959 & 35.3495 & NC & 2893 & -0.8 & -75.5026 & 35.3172 & $\mathrm{NC}$ & 2965 & -3.2 & -75.5082 & 35.2852 & NC \\
\hline 2822 & 0.2 & -75.496 & 35.3491 & NC & 2894 & -0.7 & -75.5027 & 35.3167 & $\mathrm{NC}$ & 2966 & -3.1 & -75.5083 & 35.2847 & NC \\
\hline 2823 & -0.3 & -75.4961 & 35.3486 & NC & 2895 & -0.8 & -75.5028 & 35.3163 & $\mathrm{NC}$ & 2967 & -3.3 & -75.5084 & 35.2842 & NC \\
\hline 2824 & -0.6 & -75.4962 & 35.3482 & NC & 2896 & -0.7 & .75 .5029 & 35.3158 & NC & 2968 & -3.3 & -75.5085 & 35.2838 & NC \\
\hline 2825 & -0.7 & -75.4963 & 35.3477 & NC & 2897 & -0.7 & -75.5031 & 35.3153 & NC & 2969 & -3.2 & -75.5086 & 35.2833 & $\mathrm{NC}$ \\
\hline 2826 & -0.6 & -75.4964 & 35.3472 & $\mathrm{NC}$ & 2898 & -0.8 & -75.5032 & 35.3149 & NC & 2970 & -3.3 & -75.5087 & 35.2828 & $\mathrm{NC}$ \\
\hline 2827 & -0.7 & -75.4965 & 35.3468 & NC & 2899 & -0.7 & -75.5033 & 35.3144 & $\mathrm{NC}$ & 2971 & -3.3 & -75.5088 & 35.2824 & $\mathrm{NC}$ \\
\hline 2828 & -0.6 & -75.4966 & 35.3463 & NC & 2900 & -0.6 & -75.5034 & 35.314 & NC & 2972 & -3.3 & -75.5089 & 35.2819 & NC \\
\hline 2829 & -0.7 & -75.4967 & 35.3459 & NC & 2901 & -0.7 & -75.5035 & 35.3135 & NC & 2973 & -3.1 & -75.5089 & 35.2815 & NC \\
\hline 2830 & -0.9 & -75.4968 & 35.3454 & NC & 2902 & -0.7 & -75.5036 & 35.3131 & NC & 2974 & -3.2 & -75.509 & 35.281 & NC \\
\hline 2831 & -1 & -75.4969 & 35.345 & NC & 2903 & -0.7 & .75 .5038 & 35.3126 & $\mathrm{NC}$ & 2975 & -3 & -75.5091 & 35.2805 & NC \\
\hline 2832 & -1.1 & -75.497 & 35.3445 & NC & 2904 & -1 & -75.5039 & 35.3122 & NC & 2976 & -2.8 & -75.5092 & 35.2801 & $\mathrm{NC}$ \\
\hline 2833 & -0.9 & -75.4971 & 35.344 & NC & 2905 & -1.2 & -75.504 & 35.3117 & $\mathrm{NC}$ & 2977 & -2.8 & -75.5093 & 35.2796 & $\mathrm{NC}$ \\
\hline 2834 & -0.9 & -75.4972 & 35.3436 & NC & 2906 & -1.5 & -75.5041 & 35.3112 & NC & 2978 & -2.7 & -75.5094 & 35.2792 & $\mathrm{NC}$ \\
\hline 2835 & -0.9 & -75.4973 & 35.3431 & NC & 2907 & -1.2 & -75.5042 & 35.3108 & NC & 2979 & -2.6 & -75.5094 & 35.2789 & NC \\
\hline 2836 & -0.8 & -75.4974 & 35.3427 & NC & 2908 & -1 & -75.5044 & 35.3103 & NC & 2980 & -2.9 & -75.5095 & 35.2787 & $\mathrm{NC}$ \\
\hline 2837 & -0.7 & -75.4976 & 35.3422 & NC & 2909 & -1.1 & -75.5045 & 35.3099 & NC & 2981 & -2.7 & -75.5095 & 35.2785 & $\mathrm{NC}$ \\
\hline 2838 & -0.6 & -75.4977 & 35.3418 & NC & 2910 & -1.2 & -75.5046 & 35.3094 & NC & 2982 & -3 & -75.5096 & 35.2782 & NC \\
\hline 2839 & -0.6 & -75.4978 & 35.3413 & NC & 2911 & -1.1 & -75.5047 & 35.309 & NC & 2983 & -2.8 & -75.5096 & 35.278 & $\mathrm{NC}$ \\
\hline 2840 & -0.5 & -75.4979 & 35.3409 & NC & 2912 & -1.3 & -75.5048 & 35.3085 & $\mathrm{NC}$ & 2984 & -3 & -75.5096 & 35.2778 & $\mathrm{NC}$ \\
\hline 2841 & -0.9 & -75.498 & 35.3404 & NC & 2913 & -1.2 & -75.5049 & 35.3081 & NC & 2985 & -2.9 & -75.5097 & 35.2776 & $\mathrm{NC}$ \\
\hline 2842 & -1.3 & -75.4981 & 35.3399 & NC & 2914 & -1.5 & -75.5051 & 35.3076 & NC & 2986 & -3 & -75.5097 & 35.2773 & NC \\
\hline 2843 & -1.6 & -75.4982 & 35.3395 & NC & 2915 & -1.4 & -75.504 & 35.3073 & NC & 2987 & -2.8 & -75.5098 & 35.2771 & $\mathrm{NC}$ \\
\hline 2844 & -1.8 & -75.4969 & 35.339 & NC & 2916 & -1.3 & -75.5052 & 35.3072 & NC & 2988 & -3.1 & -75.5098 & 35.2769 & $\mathrm{NC}$ \\
\hline 2845 & -1.7 & -75.4983 & 35.339 & NC & 2917 & -1.6 & -75.5041 & 35.3068 & NC & 2989 & -2.9 & -75.5099 & 35.2767 & $\mathrm{NC}$ \\
\hline 2846 & -1.8 & -75.497 & 35.3385 & NC & 2918 & -1.4 & -75.5053 & 35.3067 & NC & 2990 & -3.2 & -75.5099 & 35.2764 & $\mathrm{NC}$ \\
\hline 2847 & -1.7 & -75.4971 & 35.3381 & NC & 2919 & -1.7 & -75.5042 & 35.3063 & NC & 2991 & -3.1 & -75.51 & 35.2762 & $\mathrm{NC}$ \\
\hline 2848 & -1.6 & -75.4973 & 35.3376 & NC & 2920 & -2 & -75.5043 & 35.3059 & NC & 2992 & -3.2 & -75.5101 & 35.2757 & NC \\
\hline 2849 & -1.8 & -75.4974 & 35.3372 & NC & 2921 & -2.1 & -75.5044 & 35.3054 & NC & 2993 & -3.3 & -75.5102 & 35.2753 & NC \\
\hline 2850 & -1.8 & -75.4975 & 35.3367 & NC & 2922 & -2.5 & -75.5045 & 35.305 & NC & 2994 & -3.2 & -75.5103 & 35.2748 & NC \\
\hline 2851 & -1.8 & -75.4976 & 35.3363 & NC & 2923 & -2.4 & -75.5046 & 35.3045 & NC & 2995 & -3.2 & -75.5104 & 35.2744 & $\mathrm{NC}$ \\
\hline 2852 & -1.6 & -75.4977 & 35.3358 & NC & 2924 & -2.1 & -75.5047 & 35.304 & NC & 2996 & -3 & -75.5105 & 35.2739 & NC \\
\hline 2853 & -1.4 & -75.4978 & 35.3354 & NC & 2925 & -2.3 & -75.5047 & 35.3036 & NC & 2997 & -3 & -75.5106 & 35.2734 & NC \\
\hline 2854 & -1.4 & -75.498 & 35.3349 & NC & 2926 & -2.3 & -75.5048 & 35.3031 & NC & 2998 & -2.9 & -75.5107 & 35.273 & NC \\
\hline 2855 & -1.4 & -75.4981 & 35.3345 & NC & 2927 & -2.4 & -75.5049 & 35.3027 & NC & 2999 & -2.8 & -75.5108 & 35.2725 & NC \\
\hline 2856 & -1.2 & -75.4982 & 35.334 & NC & 2928 & -2.3 & -75.505 & 35.3022 & NC & 3000 & -2.5 & -75.5109 & 35.2721 & $\mathrm{NC}$ \\
\hline 2857 & -1.2 & -75.4983 & 35.3335 & NC & 2929 & -2.3 & -75.5051 & 35.3017 & NC & 3001 & -2.4 & -75.511 & 35.2716 & $\mathrm{NC}$ \\
\hline 2858 & -1.3 & -75.4984 & 35.3331 & NC & 2930 & -2.4 & -75.5052 & 35.3013 & NC & 3002 & -2.4 & -75.5111 & 35.2712 & $\mathrm{NC}$ \\
\hline 2859 & -1.3 & -75.4986 & 35.3326 & NC & 2931 & -2.4 & -75.5053 & 35.3008 & NC & 3003 & -2.4 & -75.5112 & 35.2707 & NC \\
\hline 2860 & -1 & -75.4987 & 35.3322 & NC & 2932 & -2.6 & -75.5054 & 35.3003 & NC & 3004 & -2.5 & -75.5113 & 35.2702 & NC \\
\hline 2861 & -1.3 & -75.4988 & 35.3317 & NC & 2933 & -2.6 & -75.5054 & 35.2999 & NC & 3005 & -2.6 & -75.5114 & 35.2698 & $\mathrm{NC}$ \\
\hline 2862 & -1.6 & -75.4989 & 35.3313 & NC & 2934 & -2.6 & -75.5055 & 35.2994 & NC & 3006 & -2.4 & -75.5115 & 35.2693 & NC \\
\hline 2863 & -2.2 & -75.499 & 35.3308 & NC & 2935 & -2.4 & -75.5056 & 35.299 & NC & 3007 & -2.3 & -75.5116 & 35.2689 & NC \\
\hline 2864 & -2.3 & -75.4991 & 35.3304 & NC & 2936 & -2.1 & -75.5057 & 35.2985 & $\mathrm{NC}$ & 3008 & -2.5 & -75.5117 & 35.2684 & NC \\
\hline 2865 & -2.3 & -75.4993 & 35.3299 & NC & 2937 & -2.3 & -75.5058 & 35.298 & $\mathrm{NC}$ & 3009 & -2.5 & -75.5118 & 35.268 & NC \\
\hline 2866 & -2.2 & -75.4994 & 35.3294 & NC & 2938 & -2.2 & -75.5059 & 35.2976 & NC & 3010 & -2.4 & -75.5119 & 35.2675 & NC \\
\hline 2867 & -2 & -75.4995 & 35.329 & NC & 2939 & -2.2 & -75.506 & 35.2971 & NC & 3011 & -2.4 & -75.512 & 35.267 & NC \\
\hline 2868 & -1.8 & -75.4996 & 35.3285 & NC & 2940 & -2 & -75.5061 & 35.2967 & NC & 3012 & -2.7 & -75.5121 & 35.2666 & NC \\
\hline 2869 & -1.7 & -75.4997 & 35.3281 & NC & 2941 & -2 & -75.5061 & 35.2962 & NC & 3013 & -2.8 & -75.5122 & 35.2661 & NC \\
\hline 2870 & -1.6 & -75.4999 & 35.3276 & NC & 2942 & -2 & -75.5062 & 35.2957 & NC & 3014 & -2.5 & -75.5123 & 35.2657 & NC \\
\hline 2871 & -1.7 & -75.5 & 35.3272 & NC & 2943 & -2 & -75.5063 & 35.2953 & NC & 3015 & -2.6 & -75.5124 & 35.2652 & $\mathrm{NC}$ \\
\hline 2872 & -1.6 & -75.5001 & 35.3267 & NC & 2944 & -1.9 & -75.5064 & 35.2948 & NC & 3016 & -2.6 & -75.5125 & 35.2648 & NC \\
\hline 2873 & -1.5 & -75.5002 & 35.3263 & NC & 2945 & -2.1 & -75.5065 & 35.2944 & NC & 3017 & -2.5 & -75.5126 & 35.2643 & $\mathrm{NC}$ \\
\hline 2874 & -1.3 & -75.5003 & 35.3258 & NC & 2946 & -2.1 & -75.5066 & 35.2939 & NC & 3018 & -2.4 & -75.5127 & 35.2638 & NC \\
\hline 2875 & -1.4 & -75.5004 & 35.3254 & NC & 2947 & -2.3 & -75.5067 & 35.2934 & NC & 3019 & -2.3 & -75.5128 & 35.2634 & NC \\
\hline 2876 & -1.3 & -75.5006 & 35.3249 & NC & 2948 & -2.6 & -75.5068 & 35.293 & NC & 3020 & -2.1 & -75.5129 & 35.2629 & NC \\
\hline 2877 & -1.4 & -75.5007 & 35.3244 & NC & 2949 & -2.7 & -75.5068 & 35.2925 & NC & 3021 & -1.9 & -75.513 & 35.2625 & NC \\
\hline 2878 & -1.5 & -75.5008 & 35.324 & NC & 2950 & -2.8 & -75.5069 & 35.2921 & NC & 3022 & -1.8 & -75.5131 & 35.262 & NC \\
\hline 2879 & -1.5 & -75.5009 & 35.3235 & NC & 2951 & -2.6 & -75.507 & 35.2916 & NC & 3023 & -1.6 & -75.5132 & 35.2615 & NC \\
\hline
\end{tabular}




\begin{tabular}{|c|c|c|c|c|c|c|c|c|c|c|c|c|c|c|}
\hline TR\# & RATE & LONG & LAT & ST & TR\# & RATE & LONG & LAT & ST & TR\# & RATE & LONG & LAT & ST \\
\hline 3024 & -1.5 & -75.5133 & 35.2611 & NC & 3096 & -1.8 & -75.5214 & 35.2309 & NC & | 3168 & 0.8 & -75.5958 & 35.2291 & NC \\
\hline 3025 & -1.4 & -75.5134 & 35.2606 & NC & 3097 & 1.4 & -75.5613 & 35.2308 & NC & 3169 & 0.7 & -75.5953 & 35.2291 & NC \\
\hline 3026 & -1.4 & -75.5135 & 35.2602 & NC & 3098 & 1.4 & -75.5624 & 35.2308 & NC & 3170 & 1.4 & -75.5985 & 35.2291 & NC \\
\hline 3027 & -1.4 & -75.5136 & 35.2597 & NC & 3099 & 1.5 & .75 .5619 & 35.2308 & NC & 3171 & -0.9 & -75.522 & 35.2291 & NC \\
\hline 3028 & -1.2 & -75.5137 & 35.2593 & $\mathrm{NC}$ & 3100 & 1.4 & -75.5641 & 35.2307 & $\mathrm{NC}$ & 3172 & 1.2 & -75.5991 & 35.229 & NC \\
\hline 3029 & -1.1 & -75.5138 & 35.2588 & NC & 3101 & 1.6 & -75.5636 & 35.2307 & NC & 3173 & 1.2 & -75.5996 & 35.2289 & NC \\
\hline 3030 & -0.9 & -75.5139 & 35.2583 & NC & 3102 & 1.5 & -75.563 & 35.2307 & NC & 3174 & 1.5 & -75.6002 & 35.2287 & NC \\
\hline 3031 & -0.9 & -75.514 & 35.2579 & NC & 3103 & 1.5 & -75.5647 & 35.2307 & NC & 3175 & -0.9 & -75.5221 & 35.2287 & NC \\
\hline 3032 & -0.6 & -75.5141 & 35.2574 & NC & 3104 & 1.5 & -75.5653 & 35.2306 & NC & 3176 & 1.3 & -75.6007 & 35.2286 & NC \\
\hline 3033 & -0.7 & -75.5142 & 35.257 & NC & 3105 & 1.3 & .75 .5664 & 35.2306 & NC & 3177 & 1.1 & -75.6013 & 35.2285 & NC \\
\hline 3034 & -0.9 & -75.5143 & 35.2565 & NC & 3106 & 1.3 & -75.5658 & 35.2306 & NC & 3178 & 1 & -75.6018 & 35.2284 & NC \\
\hline 3035 & -0.9 & -75.5144 & 35.2561 & NC & 3107 & 1.5 & -75.5687 & 35.2305 & NC & 3179 & 0.8 & -75.6024 & 35.2283 & NC \\
\hline 3036 & -0.8 & -75.5145 & 35.2556 & $\mathrm{NC}$ & 3108 & 1.5 & -75.5681 & 35.2305 & NC & 3180 & 1.1 & -75.6029 & 35.2282 & NC \\
\hline 3037 & -0.7 & -75.5146 & 35.2551 & NC & 3109 & 1.5 & -75.5675 & 35.2305 & $\mathrm{NC}$ & 3181 & 0.9 & -75.6035 & 35.2281 & $\mathrm{NC}$ \\
\hline 3038 & -1 & -75.5147 & 35.2547 & NC & 3110 & 1.3 & -75.567 & 35.2305 & NC & 3182 & 0.7 & -75.604 & 35.228 & NC \\
\hline 3039 & -1.1 & -75.5148 & 35.2542 & NC & 3111 & -1.5 & -75.5216 & 35.2305 & NC & 3183 & 0.6 & -75.6046 & 35.2279 & $\mathrm{NC}$ \\
\hline 3040 & -1.1 & -75.5149 & 35.2538 & NC & 3112 & 1.4 & -75.5709 & 35.2304 & NC & 3184 & 0.6 & -75.6051 & 35.2278 & NC \\
\hline 3041 & -1 & -75.515 & 35.2533 & NC & 3113 & 1.5 & -75.5704 & 35.2304 & NC & 3185 & 0.6 & -75.6057 & 35.2277 & NC \\
\hline 3042 & -1.6 & -75.5151 & 35.2529 & NC & 3114 & 1.6 & -75.5698 & 35.2304 & NC & 3186 & 0.6 & -75.6062 & 35.2276 & $\mathrm{NC}$ \\
\hline 3043 & -1.9 & -75.5152 & 35.2524 & NC & 3115 & 1.5 & -75.5692 & 35.2304 & NC & 3187 & 0.3 & -75.6068 & 35.2275 & NC \\
\hline 3044 & -2.2 & -75.5153 & 35.2519 & NC & 3116 & 1.5 & -75.5721 & 35.2303 & NC & 3188 & 0.2 & -75.6073 & 35.2274 & NC \\
\hline 3045 & -2.6 & -75.5154 & 35.2515 & NC & 3117 & 1.6 & -75.5715 & 35.2303 & NC & 3189 & 0.1 & -75.6079 & 35.2273 & NC \\
\hline 3046 & -2.9 & -75.5155 & 35.251 & NC & 3118 & 1.4 & -75.5726 & 35.2303 & NC & 3190 & 0.2 & -75.6085 & 35.2272 & NC \\
\hline 3047 & -3.5 & -75.5156 & 35.2506 & NC & 3119 & 1.1 & -75.5749 & 35.2302 & NC & 3191 & 0.3 & -75.609 & 35.2271 & NC \\
\hline 3048 & -3.9 & -75.5157 & 35.2501 & NC & 3120 & 1.2 & -75.5743 & 35.2302 & NC & 3192 & 0.1 & .75 .6096 & 35.227 & NC \\
\hline 3049 & -4.2 & -75.5158 & 35.2496 & NC & 3121 & 1.3 & -75.5738 & 35.2302 & NC & 3193 & -0.1 & -75.6101 & 35.2268 & NC \\
\hline 3050 & -4.4 & -75.5159 & 35.2492 & NC & 3122 & 1.4 & -75.5732 & 35.2302 & NC & 3194 & -0.2 & -75.6107 & 35.2267 & NC \\
\hline 3051 & -4.5 & -75.516 & 35.2487 & NC & 3123 & 1.1 & -75.576 & 35.2301 & NC & 3195 & 0 & -75.6112 & 35.2266 & NC \\
\hline 3052 & -4.4 & -75.5161 & 35.2483 & NC & 3124 & 1.2 & -75.5755 & 35.2301 & NC & 3196 & 0.1 & -75.6118 & 35.2265 & NC \\
\hline 3053 & -4.5 & -75.5162 & 35.2478 & NC & 3125 & 1.2 & -75.5766 & 35.2301 & NC & 3197 & 0 & -75.6123 & 35.2264 & NC \\
\hline 3054 & -4.5 & -75.5163 & 35.2474 & NC & 3126 & 1.3 & -75.5772 & 35.23 & NC & 3198 & 0 & -75.6129 & 35.2263 & NC \\
\hline 3055 & -4.6 & -75.5164 & 35.2469 & NC & 3127 & 1.5 & -75.5788 & 35.23 & NC & 3199 & 0 & -75.6134 & 35.2262 & NC \\
\hline 3056 & -4.4 & -75.5165 & 35.2463 & NC & 3128 & 1.5 & -75.5783 & 35.23 & NC & 3200 & 0 & -75.614 & 35.2261 & NC \\
\hline 3057 & -4.4 & -75.5166 & 35.2458 & NC & 3129 & 1.5 & -75.5777 & 35.23 & NC & 3201 & 0 & -75.6145 & 35.226 & NC \\
\hline 3058 & -4.3 & -75.5168 & 35.2454 & NC & 3130 & -1.3 & -75.5217 & 35.23 & NC & 3202 & -0.1 & -75.6151 & 35.2259 & NC \\
\hline 3059 & -4.2 & -75.5169 & 35.2449 & NC & 3131 & 1.3 & -75.5805 & 35.2299 & NC & 3203 & 0 & -75.6156 & 35.2258 & NC \\
\hline 3060 & -4 & -75.5171 & 35.2445 & NC & 3132 & 1.4 & -75.58 & 35.2299 & NC & 3204 & 0 & -75.6162 & 35.2257 & NC \\
\hline 3061 & -3.6 & -75.5172 & 35.244 & NC & 3133 & 1.4 & -75.5794 & 35.2299 & NC & 3205 & -0.1 & -75.6167 & 35.2256 & NC \\
\hline 3062 & -3.6 & -75.5174 & 35.2436 & NC & 3134 & 1.1 & -75.5811 & 35.2298 & NC & 3206 & -0.2 & -75.6173 & 35.2255 & NC \\
\hline 3063 & -3.5 & -75.5175 & 35.2431 & NC & 3135 & 1.5 & -75.5828 & 35.2298 & NC & 3207 & -0.3 & -75.6178 & 35.2254 & NC \\
\hline 3064 & -3.5 & -75.5176 & 35.2427 & $\mathrm{NC}$ & 3136 & 1.4 & -75.5822 & 35.2298 & $\mathrm{NC}$ & 3208 & -0.4 & -75.6184 & 35.2253 & NC \\
\hline 3065 & -3.5 & -75.5178 & 35.2422 & NC & 3137 & 1.3 & -75.5817 & 35.2298 & NC & 3209 & -0.1 & -75.6189 & 35.2252 & NC \\
\hline 3066 & -3.4 & -75.5179 & 35.2418 & $\mathrm{NC}$ & 3138 & 1.4 & -75.5834 & 35.2297 & NC & 3210 & -0.4 & -75.6195 & 35.2251 & NC \\
\hline 3067 & -3.2 & -75.5181 & 35.2413 & NC & 3139 & 1.2 & -75.5952 & 35.2297 & $\mathrm{NC}$ & 3211 & -0.5 & -75.62 & 35.2249 & NC \\
\hline 3068 & -3.4 & -75.5182 & 35.2409 & NC & 3140 & 1.5 & -75.5845 & 35.2297 & NC & 3212 & -0.3 & -75.6206 & 35.2248 & NC \\
\hline 3069 & -3.2 & -75.5184 & 35.2404 & NC & 3141 & 1.4 & -75.5839 & 35.2297 & $\mathrm{NC}$ & 3213 & -0.1 & -75.6211 & 35.2247 & NC \\
\hline 3070 & -3.2 & -75.5185 & 35.24 & NC & 3142 & 1.6 & -75.5862 & 35.2296 & NC & 3214 & -0.1 & -75.6217 & 35.2246 & NC \\
\hline 3071 & -3.2 & -75.5187 & 35.2395 & NC & 3143 & 1.7 & -75.5856 & 35.2296 & $\mathrm{NC}$ & 3215 & -0.1 & -75.6223 & 35.2245 & NC \\
\hline 3072 & -3.1 & -75.5188 & 35.2391 & NC & 3144 & 1.6 & -75.5851 & 35.2296 & $\mathrm{NC}$ & 3216 & -0.5 & -75.6228 & 35.2244 & NC \\
\hline 3073 & -3.1 & .75 .5189 & 35.2386 & NC & 3145 & 1.1 & -75.5958 & 35.2296 & NC & 3217 & -0.6 & -75.6234 & 35.2243 & NC \\
\hline 3074 & -3.1 & -75.5191 & 35.2382 & NC & 3146 & 1.6 & -75.5868 & 35.2296 & NC & 3218 & -0.7 & -75.6239 & 35.2242 & NC \\
\hline 3075 & -3.1 & -75.5192 & 35.2377 & NC & 3147 & -0.8 & -75.5218 & 35.2296 & $\mathrm{NC}$ & 3219 & -0.8 & -75.6245 & 35.2241 & NC \\
\hline 3076 & -3.3 & -75.5194 & 35.2372 & NC & 3148 & 1.2 & -75.5885 & 35.2295 & $\mathrm{NC}$ & 3220 & -0.5 & -75.625 & 35.224 & $\mathrm{NC}$ \\
\hline 3077 & -3.4 & -75.5195 & 35.2368 & NC & 3149 & 1.3 & -75.5879 & 35.2295 & $\mathrm{NC}$ & 3221 & 3.4 & -75.562 & 35.2239 & NC \\
\hline 3078 & -3.5 & -75.5197 & 35.2363 & NC & 3150 & 1.6 & -75.5873 & 35.2295 & $\mathrm{NC}$ & 3222 & -0.3 & -75.6256 & 35.2239 & NC \\
\hline 3079 & -3.4 & -75.5198 & 35.2359 & NC & 3151 & 1.2 & -75.5963 & 35.2295 & $\mathrm{NC}$ & 3223 & -0.3 & -75.6261 & 35.2238 & NC \\
\hline 3080 & -3.4 & -75.52 & 35.2354 & NC & 3152 & 0.9 & -75.589 & 35.2295 & NC & 3224 & 3 & -75.5614 & 35.2238 & NC \\
\hline 3081 & -3.4 & -75.5201 & 35.235 & NC & 3153 & 0.9 & -75.5902 & 35.2294 & $\mathrm{NC}$ & 3225 & -0.3 & -75.6267 & 35.2237 & NC \\
\hline 3082 & -3.1 & -75.5203 & 35.2345 & NC & 3154 & 1 & -75.5896 & 35.2294 & $\mathrm{NC}$ & 3226 & 2.9 & -75.5609 & 35.2236 & NC \\
\hline 3083 & -2.7 & .75 .5204 & 35.2341 & NC & 3155 & 1.2 & -75.5969 & 35.2294 & NC & 3227 & -0.4 & -75.6272 & 35.2236 & NC \\
\hline 3084 & -2.5 & -75.5205 & 35.2336 & NC & 3156 & 0.9 & -75.5907 & 35.2294 & $\mathrm{NC}$ & 3228 & -0.5 & -75.6278 & 35.2235 & NC \\
\hline 3085 & -2 & -75.5207 & 35.2332 & NC & 3157 & 0.7 & -75.5924 & 35.2293 & $\mathrm{NC}$ & 3229 & 2.9 & -75.5603 & 35.2235 & NC \\
\hline 3086 & -1.8 & -75.5208 & 35.2327 & NC & 3158 & 0.7 & .75 .5919 & 35.2293 & $\mathrm{NC}$ & 3230 & 3.1 & -75.5598 & 35.2234 & NC \\
\hline 3087 & -1.6 & -75.521 & 35.2323 & NC & 3159 & 0.8 & -75.5913 & 35.2293 & NC & 3231 & -0.6 & -75.6283 & 35.2234 & NC \\
\hline 3088 & -1.8 & -75.5211 & 35.2318 & NC & 3160 & 1.4 & -75.5974 & 35.2293 & NC & 3232 & -0.7 & -75.6289 & 35.2233 & NC \\
\hline 3089 & -1.9 & -75.5213 & 35.2314 & NC & 3161 & 0.5 & -75.593 & 35.2293 & NC & 3233 & 2.8 & -75.5592 & 35.2233 & NC \\
\hline 3090 & 1.4 & -75.5585 & 35.231 & NC & 3162 & 0.7 & -75.5941 & 35.2292 & NC & 3234 & 2.7 & -75.5587 & 35.2232 & NC \\
\hline 3091 & 1.4 & .75 .5579 & 35.231 & NC & 3163 & 0.6 & -75.5936 & 35.2292 & NC & 3235 & -0.7 & -75.6294 & 35.2232 & NC \\
\hline 3092 & 1.2 & -75.5602 & 35.2309 & NC & 3164 & 1.5 & -75.598 & 35.2292 & $\mathrm{NC}$ & 3236 & -0.5 & -75.63 & 35.223 & NC \\
\hline 3093 & 1.3 & -75.5596 & 35.2309 & NC & 3165 & 0.8 & -75.5947 & 35.2292 & NC & 3237 & 2.7 & -75.5581 & 35.223 & NC \\
\hline 3094 & 1.2 & -75.559 & 35.2309 & NC & 3166 & 0.8 & -75.597 & 35.2291 & $\mathrm{NC}$ & 3238 & 2.7 & -75.5576 & 35.2229 & NC \\
\hline 3095 & 1.4 & -75.5607 & 35.2309 & NC & 3167 & 0.8 & -75.5964 & 35.2291 & NC & 3239 & -0.5 & -75.6305 & 35.2229 & $\mathrm{NC}$ \\
\hline
\end{tabular}




\begin{tabular}{|c|c|c|c|c|c|c|c|c|c|c|c|c|c|c|}
\hline TR\# & RATE & LONG & LAT & ST & TR\# & RATE & LONG & LAT & ST & TR\# & RATE & LONG & LAT & ST \\
\hline 3240 & 2.6 & -75.557 & 35.2228 & NC & 3312 & 8 & -75.536 & 35.2183 & NC & 3384 & -0.6 & -75.6774 & 35.2092 & NC \\
\hline 3241 & 0.1 & -75.6323 & 35.2228 & NC & 3313 & 9.1 & -75.5355 & 35.2181 & NC & 3385 & -0.7 & -75.6779 & 35.209 & $\mathrm{NC}$ \\
\hline 3242 & -0.3 & -75.6311 & 35.2228 & NC & 3314 & -0.6 & -75.6479 & 35.2181 & NC & 3386 & -0.5 & -75.6784 & 35.2089 & NC \\
\hline 3243 & 2.5 & -75.5565 & 35.2227 & NC & 3315 & -0.9 & -75.6484 & 35.218 & NC & 3387 & -0.4 & -75.6789 & 35.2087 & NC \\
\hline 3244 & -0.2 & -75.6316 & 35.2227 & NC & 3316 & 10.1 & -75.5349 & 35.218 & NC & 3388 & -0.3 & -75.6795 & 35.2085 & NC \\
\hline 3245 & 0.2 & -75.6328 & 35.2226 & NC & 3317 & 11.5 & -75.5344 & 35.2179 & NC & 3389 & -0.2 & -75.68 & 35.2083 & NC \\
\hline 3246 & -0.1 & -75.6322 & 35.2226 & NC & 3318 & -1.1 & -75.6489 & 35.2178 & NC & 3390 & -0.5 & -75.6805 & 35.2081 & NC \\
\hline 3247 & 2.3 & -75.5559 & 35.2226 & NC & 3319 & 12.8 & -75.5338 & 35.2178 & NC & 3391 & -0.4 & -75.681 & 35.2079 & NC \\
\hline 3248 & 0.2 & -75.6334 & 35.2225 & NC & 3320 & 14.4 & -75.5333 & 35.2177 & NC & 3392 & -0.5 & -75.6815 & 35.2077 & NC \\
\hline 3249 & -0.2 & -75.6327 & 35.2225 & NC & 3321 & -0.9 & -75.6495 & 35.2177 & NC & 3393 & -0.6 & -75.6821 & 35.2076 & NC \\
\hline 3250 & 2.3 & -75.5554 & 35.2224 & NC & 3322 & -0.7 & -75.65 & 35.2175 & NC & 3394 & -0.6 & -75.6826 & 35.2074 & NC \\
\hline 3251 & -0.2 & -75.6333 & 35.2224 & NC & 3323 & 16.3 & -75.5327 & 35.2175 & NC & 3395 & -0.5 & -75.6831 & 35.2072 & NC \\
\hline 3252 & 0 & -75.6339 & 35.2223 & NC & 3324 & 18.1 & -75.5322 & 35.2174 & NC & 3396 & -0.5 & -75.6836 & 35.207 & NC \\
\hline 3253 & 2.2 & -75.5548 & 35.2223 & NC & 3325 & -0.7 & -75.6505 & 35.2173 & NC & 3397 & -0.6 & -75.6842 & 35.2068 & NC \\
\hline 3254 & 2.1 & -75.5543 & 35.2222 & NC & 3326 & 19.7 & -75.5316 & 35.2173 & NC & 3398 & -0.5 & -75.6847 & 35.2066 & $\mathrm{NC}$ \\
\hline 3255 & -0.1 & -75.6344 & 35.2222 & NC & 3327 & 20.9 & -75.5311 & 35.2172 & NC & 3399 & -0.5 & -75.6852 & 35.2064 & $\mathrm{NC}$ \\
\hline 3256 & 2.1 & -75.5537 & 35.2221 & NC & 3328 & -0.7 & -75.6511 & 35.2172 & NC & 3400 & -0.3 & -75.6857 & 35.2063 & $\mathrm{NC}$ \\
\hline 3257 & 2.2 & -75.5532 & 35.222 & NC & 3329 & 47.3 & -75.5305 & 35.2171 & NC & 3401 & -0.2 & -75.6862 & 35.2061 & $\mathrm{NC}$ \\
\hline 3258 & -0.3 & -75.635 & 35.222 & NC & 3330 & -0.7 & -75.6516 & 35.217 & NC & 3402 & -0.1 & -75.6868 & 35.2059 & $\mathrm{NC}$ \\
\hline 3259 & -0.4 & -75.6355 & 35.2218 & NC & 3331 & 51.1 & -75.53 & 35.2169 & NC & 3403 & -0.2 & -75.6873 & 35.2057 & $\mathrm{NC}$ \\
\hline 3260 & 2.1 & -75.5526 & 35.2218 & NC & 3332 & -0.7 & -75.6522 & 35.2169 & NC & 3404 & -0.1 & -75.6878 & 35.2055 & NC \\
\hline 3261 & 2.2 & -75.552 & 35.2217 & NC & 3333 & 54.6 & -75.5294 & 35.2168 & NC & 3405 & -0.1 & -75.6883 & 35.2053 & $\mathrm{NC}$ \\
\hline 3262 & -0.6 & -75.6361 & 35.2217 & NC & 3334 & -0.9 & -75.6527 & 35.2167 & NC & 3406 & 0 & -75.6889 & 35.2051 & NC \\
\hline 3263 & 2.3 & -75.5515 & 35.2216 & NC & 3335 & -0.9 & -75.6532 & 35.2165 & NC & 3407 & 0.1 & -75.6894 & 35.2049 & NC \\
\hline 3264 & 2.4 & -75.5509 & 35.2215 & NC & 3336 & -0.9 & -75.6538 & 35.2164 & NC & 3408 & 0.1 & -75.6899 & 35.2048 & $\mathrm{NC}$ \\
\hline 3265 & -0.6 & -75.6366 & 35.2215 & NC & 3337 & -0.9 & -75.6543 & 35.2162 & NC & 3409 & 0.1 & -75.6904 & 35.2046 & NC \\
\hline 3266 & -0.7 & -75.6371 & 35.2214 & NC & 3338 & -1.2 & -75.6548 & 35.2161 & NC & 3410 & 0.4 & -75.6909 & 35.2044 & NC \\
\hline 3267 & 1.8 & -75.5504 & 35.2214 & NC & 3339 & -1.4 & -75.6554 & 35.2159 & NC & 3411 & 0.7 & -75.6915 & 35.2042 & NC \\
\hline 3268 & 1.4 & -75.5498 & 35.2212 & NC & 3340 & -1.2 & -75.6559 & 35.2157 & NC & 3412 & 0.7 & -75.692 & 35.204 & NC \\
\hline 3269 & -0.7 & -75.6377 & 35.2212 & NC & 3341 & -1.1 & -75.6564 & 35.2156 & NC & 3413 & 0.9 & -75.6925 & 35.2038 & NC \\
\hline 3270 & 1.1 & -75.5493 & 35.2211 & NC & 3342 & -1.1 & -75.657 & 35.2154 & NC & 3414 & 0.9 & -75.693 & 35.2036 & NC \\
\hline 3271 & 0.7 & -75.5487 & 35.221 & NC & 3343 & -1 & -75.6575 & 35.2153 & NC & 3415 & 0.8 & -75.6936 & 35.2035 & NC \\
\hline 3272 & -0.7 & -75.6382 & 35.221 & NC & 3344 & -1.1 & -75.6581 & 35.2151 & NC & 3416 & 0.8 & -75.6941 & 35.2033 & NC \\
\hline 3273 & -0.7 & -75.6387 & 35.2209 & NC & 3345 & -1 & -75.6586 & 35.2149 & NC & 3417 & 0.9 & -75.6946 & 35.2031 & NC \\
\hline 3274 & 0.6 & -75.5482 & 35.2209 & NC & 3346 & -1 & -75.6591 & 35.2148 & NC & 3418 & 0.9 & -75.6951 & 35.2029 & NC \\
\hline 3275 & 0.5 & -75.5476 & 35.2208 & NC & 3347 & -1.1 & -75.6597 & 35.2146 & NC & 3419 & 1 & -75.6956 & 35.2027 & NC \\
\hline 3276 & -0.5 & -75.6393 & 35.2207 & NC & 3348 & -1.4 & -75.6602 & 35.2145 & NC & 3420 & 1 & -75.6962 & 35.2025 & NC \\
\hline 3277 & -0.6 & -75.6398 & 35.2206 & NC & 3349 & -1.6 & -75.6607 & 35.2143 & NC & 3421 & 0.9 & -75.6967 & 35.2023 & NC \\
\hline 3278 & 0.4 & -75.5471 & 35.2206 & NC & 3350 & -1.7 & -75.6613 & 35.2141 & NC & 3422 & 0.9 & -75.6972 & 35.2022 & NC \\
\hline 3279 & 0.3 & -75.5465 & 35.2205 & NC & 3351 & -1.9 & -75.6618 & 35.214 & NC & 3423 & 1 & -75.6977 & 35.202 & NC \\
\hline 3280 & -0.7 & -75.6403 & 35.2204 & NC & 3352 & -1.8 & .75 .6624 & 35.2138 & NC & 3424 & 0.9 & -75.6983 & 35.2018 & NC \\
\hline 3281 & 0.5 & -75.546 & 35.2204 & NC & 3353 & -1.9 & -75.6629 & 35.2136 & NC & 3425 & 0.9 & -75.6988 & 35.2016 & NC \\
\hline 3282 & 0.4 & -75.5454 & 35.2203 & NC & 3354 & -1.8 & -75.6634 & 35.2135 & NC & 3426 & 1.1 & -75.6993 & 35.2014 & NC \\
\hline 3283 & -0.6 & -75.6409 & 35.2202 & NC & 3355 & -1.6 & -75.664 & 35.2133 & NC & 3427 & 1.5 & -75.6998 & 35.2012 & NC \\
\hline 3284 & 0.7 & -75.5449 & 35.2202 & NC & 3356 & -1.6 & -75.6645 & 35.2132 & NC & 3428 & 1.5 & .75 .7004 & 35.201 & NC \\
\hline 3285 & 0.9 & -75.5443 & 35.2201 & NC & 3357 & -1.7 & -75.665 & 35.213 & NC & 3429 & 1.5 & -75.7009 & 35.2009 & NC \\
\hline 3286 & -0.6 & -75.6414 & 35.2201 & NC & 3358 & -1.6 & -75.6656 & 35.2128 & $\mathrm{NC}$ & 3430 & 1.6 & -75.7014 & 35.2007 & NC \\
\hline 3287 & -0.7 & -75.642 & 35.2199 & NC & 3359 & -1.5 & -75.6661 & 35.2127 & NC & 3431 & 1.5 & -75.7019 & 35.2005 & NC \\
\hline 3288 & 1.1 & -75.5438 & 35.2199 & NC & 3360 & -1.5 & -75.6666 & 35.2125 & NC & 3432 & 1.3 & -75.7024 & 35.2003 & NC \\
\hline 3289 & 1.4 & -75.5432 & 35.2198 & NC & 3361 & -1.5 & -75.6672 & 35.2124 & NC & 3433 & 1.2 & -75.703 & 35.2001 & NC \\
\hline 3290 & -0.9 & -75.6425 & 35.2197 & NC & 3362 & -1.4 & -75.669 & 35.2122 & NC & 3434 & 1.2 & -75.7035 & 35.1999 & NC \\
\hline 3291 & 1.5 & -75.5427 & 35.2197 & NC & 3363 & -1.7 & -75.6677 & 35.2122 & NC & 3435 & 1.2 & -75.704 & 35.1997 & NC \\
\hline 3292 & 1.7 & -75.5421 & 35.2196 & NC & 3364 & -1.3 & -75.6695 & 35.212 & NC & 3436 & 1.2 & -75.7045 & 35.1996 & NC \\
\hline 3293 & -0.9 & -75.643 & 35.2196 & NC & 3365 & -1.7 & -75.6683 & 35.212 & NC & 3437 & 1.4 & -75.704 & 35.1994 & NC \\
\hline 3294 & 1.9 & -75.5416 & 35.2195 & NC & 3366 & -1.5 & -75.6688 & 35.2119 & NC & 3438 & 1.3 & -75.7051 & 35.1994 & NC \\
\hline 3295 & -0.9 & -75.6436 & 35.2194 & NC & 3367 & -1.2 & -75.67 & 35.2118 & NC & 3439 & 1.5 & -75.7045 & 35.1992 & NC \\
\hline 3296 & 2.3 & -75.541 & 35.2193 & NC & 3368 & -1.3 & -75.6693 & 35.2117 & NC & 3440 & 1.7 & -75.705 & 35.199 & NC \\
\hline 3297 & -0.8 & -75.6441 & 35.2193 & NC & 3369 & -1 & -75.6706 & 35.2116 & $\mathrm{NC}$ & 3441 & 1.8 & -75.7056 & 35.1988 & NC \\
\hline 3298 & 2.6 & -75.5405 & 35.2192 & NC & 3370 & -1.4 & -75.6699 & 35.2116 & NC & 3442 & 1.7 & -75.7061 & 35.1985 & NC \\
\hline 3299 & 3.1 & -75.5399 & 35.2191 & NC & 3371 & -1.1 & -75.6711 & 35.2115 & NC & 3443 & 1.6 & -75.7066 & 35.1983 & NC \\
\hline 3300 & -0.7 & -75.6446 & 35.2191 & NC & 3372 & -1.3 & -75.6704 & 35.2114 & NC & 3444 & 1.3 & -75.7071 & 35.1981 & NC \\
\hline 3301 & 3.8 & -75.5394 & 35.219 & NC & 3373 & -1.2 & -75.6716 & 35.2113 & NC & 3445 & 1.1 & -75.7076 & 35.1979 & NC \\
\hline 3302 & 4.3 & -75.5388 & 35.2189 & NC & 3374 & -1.2 & -75.6721 & 35.2111 & NC & 3446 & 1 & -75.7081 & 35.1977 & NC \\
\hline 3303 & -0.4 & -75.6452 & 35.2189 & NC & 3375 & -1.3 & -75.6727 & 35.2109 & NC & 3447 & 0.9 & -75.7087 & 35.1975 & NC \\
\hline 3304 & -0.5 & -75.6457 & 35.2188 & NC & 3376 & -1.2 & -75.6732 & 35.2107 & NC & 3448 & 0.8 & -75.7092 & 35.1973 & NC \\
\hline 3305 & 5 & -75.5382 & 35.2187 & NC & 3377 & -1 & -75.6737 & 35.2105 & NC & 3449 & 0.9 & -75.7097 & 35.1971 & NC \\
\hline 3306 & 5.8 & -75.5377 & 35.2186 & NC & 3378 & -1.1 & -75.6742 & 35.2103 & NC & 3450 & 0.8 & -75.7102 & 35.1968 & NC \\
\hline 3307 & -0.5 & -75.6463 & 35.2186 & NC & 3379 & -0.9 & -75.6747 & 35.2102 & NC & 3451 & 0.7 & -75.7107 & 35.1966 & NC \\
\hline 3308 & -0.4 & -75.6468 & 35.2185 & NC & 3380 & -0.9 & -75.6753 & 35.21 & NC & 3452 & 0.8 & -75.7112 & 35.1964 & NC \\
\hline 3309 & 6.5 & -75.5371 & 35.2185 & $\mathrm{NC}$ & 3381 & -0.9 & .75 .6758 & 35.2098 & NC & 3453 & 0.6 & -75.7118 & 35.1962 & NC \\
\hline 3310 & 7.2 & -75.5366 & 35.2184 & NC & 3382 & -0.7 & -75.6763 & 35.2096 & NC & 3454 & 0.3 & -75.7123 & 35.196 & NC \\
\hline 3311 & -0.3 & -75.6473 & 35.2183 & NC & 3383 & -0.8 & -75.6768 & 35.2094 & NC & 3455 & 0.1 & -75.7128 & 35.1958 & NC \\
\hline
\end{tabular}




\begin{tabular}{|c|c|c|c|c|c|c|c|c|c|c|c|c|c|c|}
\hline TR\# & RATE & LONG & LAT & ST & TR\# & RATE & LONG & LAT & ST & TR\# & RATE & LONG & LAT & ST \\
\hline 3456 & -0.5 & -75.7133 & 35.1956 & NC & 3528 & 7.3 & -75.7735 & 35.1788 & NC & 3600 & -2.6 & -75.8096 & 35.1659 & NC \\
\hline 3457 & -1.1 & -75.7138 & 35.1954 & NC & 3529 & 6.4 & -75.7741 & 35.1786 & NC & 3601 & -2.3 & -75.8101 & 35.1657 & NC \\
\hline 3458 & -1.3 & -75.7143 & 35.1951 & NC & 3530 & 5.6 & -75.7746 & 35.1784 & NC & 3602 & -1.9 & -75.8106 & 35.1655 & NC \\
\hline 3459 & -1.6 & -75.7149 & 35.1949 & NC & 3531 & 5.6 & -75.7751 & 35.1782 & NC & 3603 & -2.2 & -75.8111 & 35.1654 & NC \\
\hline 3460 & -1.5 & -75.7154 & 35.1947 & NC & 3532 & 5.5 & -75.7756 & 35.1781 & NC & 3604 & -2.2 & -75.8117 & 35.1652 & NC \\
\hline 3461 & -1.5 & -75.7159 & 35.1945 & NC & 3533 & 5.1 & -75.7761 & 35.1779 & NC & 3605 & -2.6 & -75.8122 & 35.165 & NC \\
\hline 3462 & -1.4 & -75.7164 & 35.1943 & NC & 3534 & 4.7 & -75.7767 & 35.1777 & NC & 3606 & -3.4 & -75.8127 & 35.1648 & NC \\
\hline 3463 & -1.4 & -75.7169 & 35.1941 & NC & 3535 & 3.9 & -75.7772 & 35.1775 & NC & 3607 & -3.1 & -75.8132 & 35.1646 & NC \\
\hline 3464 & -1.7 & -75.7174 & 35.1939 & NC & 3536 & 3.7 & -75.7777 & 35.1773 & NC & 3608 & -3.1 & -75.8137 & 35.1644 & NC \\
\hline 3465 & -2 & -75.718 & 35.1937 & NC & 3537 & 2.7 & -75.7782 & 35.1772 & NC & 3609 & -3 & -75.8142 & 35.1642 & NC \\
\hline 3466 & -2.7 & -75.7185 & 35.1934 & NC & 3538 & 2 & -75.7788 & 35.177 & NC & 3610 & -3 & -75.8148 & 35.164 & NC \\
\hline 3467 & -3 & -75.719 & 35.1932 & NC & 3539 & 1.5 & -75.7793 & 35.1768 & NC & 3611 & -3 & -75.8153 & 35.1638 & NC \\
\hline 3468 & -3.1 & -75.7195 & 35.193 & NC & 3540 & 1.7 & -75.7798 & 35.1766 & NC & 3612 & -2.9 & -75.8158 & 35.1636 & NC \\
\hline 3469 & -3.3 & -75.72 & 35.1928 & $\mathrm{NC}$ & 3541 & 1.7 & -75.7803 & 35.1764 & NC & 3613 & -2.9 & -75.8163 & 35.1635 & NC \\
\hline 3470 & -3.7 & -75.7205 & 35.1926 & NC & 3542 & 1.9 & -75.7808 & 35.1763 & NC & 3614 & -3 & -75.8168 & 35.1633 & NC \\
\hline 3471 & -4 & -75.7211 & 35.1924 & NC & 3543 & 1.8 & -75.7814 & 35.1761 & NC & 3615 & -2.9 & -75.8173 & 35.1631 & NC \\
\hline 3472 & -4.5 & -75.7216 & 35.1922 & NC & 3544 & 1.5 & -75.7819 & 35.1759 & NC & 3616 & -2.7 & -75.8179 & 35.1629 & NC \\
\hline 3473 & -5.3 & -75.7221 & 35.192 & NC & 3545 & 1 & -75.7824 & 35.1757 & NC & 3617 & -2.9 & -75.8184 & 35.1627 & NC \\
\hline 3474 & -5.8 & -75.7226 & 35.1917 & NC & 3546 & 0.5 & -75.7829 & 35.1755 & NC & 3618 & -2.9 & -75.8189 & 35.1625 & NC \\
\hline 3475 & -6.3 & -75.7231 & 35.1915 & NC & 3547 & 0.1 & -75.7835 & 35.1753 & NC & 3619 & -2.6 & -75.8194 & 35.1623 & NC \\
\hline 3476 & -6.3 & -75.7236 & 35.1913 & NC & 3548 & -0.5 & -75.784 & 35.1752 & NC & 3620 & -2.3 & -75.8199 & 35.1621 & NC \\
\hline 3477 & -6.3 & -75.7242 & 35.1911 & NC & 3549 & -1 & -75.7845 & 35.175 & NC & 3621 & -2.4 & -75.8204 & 35.1619 & NC \\
\hline 3478 & -5.3 & -75.7247 & 35.1909 & NC & 3550 & -1.4 & -75.785 & 35.1748 & NC & 3622 & -2.5 & -75.821 & 35.1617 & NC \\
\hline 3479 & -4.4 & -75.7252 & 35.1907 & NC & 3551 & -1.7 & -75.7855 & 35.1746 & NC & 3623 & -2.7 & .75 .8215 & 35.1616 & NC \\
\hline 3480 & -4.1 & -75.7257 & 35.1905 & NC & 3552 & -2 & .75 .7861 & 35.1744 & NC & 3624 & -2.8 & -75.822 & 35.1614 & NC \\
\hline 3481 & -3.8 & -75.7262 & 35.1903 & NC & 3553 & -2 & -75.7866 & 35.1743 & NC & 3625 & -3 & -75.8225 & 35.1612 & NC \\
\hline 3482 & -3.6 & -75.7267 & 35.19 & NC & 3554 & -2 & -75.7871 & 35.1741 & NC & 3626 & -3.2 & -75.823 & 35.161 & NC \\
\hline 3483 & -3.5 & -75.7273 & 35.1898 & NC & 3555 & -1.7 & -75.7876 & 35.1739 & NC & 3627 & -3.4 & -75.8235 & 35.1608 & NC \\
\hline 3484 & -3.6 & -75.7278 & 35.1896 & NC & 3556 & -1.3 & -75.7882 & 35.1737 & NC & 3628 & -3.5 & -75.8241 & 35.1606 & NC \\
\hline 3485 & -3.9 & -75.7283 & 35.1894 & NC & 3557 & -1.6 & -75.7887 & 35.1735 & NC & 3629 & -3.1 & -75.8246 & 35.1604 & NC \\
\hline 3486 & -4.1 & .75 .7288 & 35.1892 & NC & 3558 & -1.7 & -75.7892 & 35.1734 & NC & 3630 & -2.8 & -75.8251 & 35.1602 & NC \\
\hline 3487 & -4.2 & -75.7293 & 35.189 & NC & 3559 & -2.4 & -75.7897 & 35.1732 & NC & 3631 & -3.3 & -75.8256 & 35.16 & NC \\
\hline 3488 & -4.2 & -75.7298 & 35.1888 & NC & 3560 & -3.3 & -75.7902 & 35.173 & NC & 3632 & -3.2 & -75.8261 & 35.1598 & NC \\
\hline 3489 & -4.3 & -75.7304 & 35.1886 & NC & 3561 & -2.8 & -75.7908 & 35.1728 & $\mathrm{NC}$ & 3633 & -3.3 & -75.8266 & 35.1597 & NC \\
\hline 3490 & -4.5 & -75.7309 & 35.1883 & NC & 3562 & -2.5 & -75.7913 & 35.1726 & NC & 3634 & -3 & -75.8272 & 35.1595 & NC \\
\hline 3491 & -4.7 & -75.7314 & 35.1881 & NC & 3563 & -2.6 & -75.7918 & 35.1725 & NC & 3635 & -3 & -75.8277 & 35.1593 & NC \\
\hline 3492 & -4.4 & -75.7319 & 35.1879 & NC & 3564 & -2.8 & -75.7923 & 35.1723 & NC & 3636 & -3.1 & -75.8282 & 35.1591 & NC \\
\hline 3493 & -3.9 & -75.7324 & 35.1877 & NC & 3565 & -3.2 & -75.7929 & 35.1721 & NC & 3637 & -2.8 & -75.8287 & 35.1589 & NC \\
\hline 3494 & -3.1 & -75.7329 & 35.1875 & NC & 3566 & -3.6 & -75.7934 & 35.1719 & $\mathrm{NC}$ & 3638 & -2.4 & -75.8292 & 35.1587 & NC \\
\hline 3495 & -2.4 & -75.7335 & 35.1873 & NC & 3567 & -3.7 & -75.7939 & 35.1717 & $\mathrm{NC}$ & 3639 & -2 & -75.8297 & 35.1585 & NC \\
\hline 3496 & -1.5 & -75.734 & 35.1871 & NC & 3568 & -4 & -75.7944 & 35.1716 & NC & 3640 & -1.9 & -75.8303 & 35.1583 & NC \\
\hline 3497 & -0.5 & -75.7345 & 35.1869 & NC & 3569 & -4 & -75.795 & 35.1714 & NC & 3641 & -2.5 & -75.8308 & 35.1581 & NC \\
\hline 3498 & 1.2 & -75.735 & 35.1866 & NC & 3570 & -3.8 & -75.7955 & 35.1712 & NC & 3642 & -2.7 & -75.8313 & 35.1579 & NC \\
\hline 3499 & 2.8 & -75.7355 & 35.1864 & NC & 3571 & -4 & -75.796 & 35.171 & NC & 3643 & -2.4 & -75.8318 & 35.1578 & NC \\
\hline 3500 & 4.3 & -75.736 & 35.1862 & NC & 3572 & -4 & -75.7965 & 35.1708 & NC & 3644 & -2.4 & -75.8323 & 35.1576 & NC \\
\hline 3501 & 5.9 & -75.7366 & 35.186 & NC & 3573 & -4 & -75.797 & 35.1707 & NC & 3645 & -1.8 & -75.8324 & 35.1575 & NC \\
\hline 3502 & 7.5 & -75.7371 & 35.1858 & NC & 3574 & -3.9 & -75.7976 & 35.1705 & NC & 3646 & -2.3 & -75.8328 & 35.1574 & NC \\
\hline 3503 & 9 & -75.7376 & 35.1856 & NC & 3575 & -3.4 & -75.7977 & 35.1703 & NC & 3647 & -2.1 & -75.8329 & 35.1573 & NC \\
\hline 3504 & 10.7 & -75.7381 & 35.1854 & NC & 3576 & -3.7 & -75.7981 & 35.1703 & NC & 3648 & -2.3 & -75.8334 & 35.1572 & NC \\
\hline 3505 & 12.3 & -75.7386 & 35.1852 & NC & 3577 & -3.5 & -75.7982 & 35.1701 & NC & 3649 & -2.3 & -75.8334 & 35.1571 & NC \\
\hline 3506 & 10.5 & -75.7391 & 35.1849 & NC & 3578 & -3.7 & -75.7986 & 35.1701 & NC & 3650 & -2.3 & -75.8339 & 35.1569 & NC \\
\hline 3507 & 14.7 & -75.7397 & 35.1847 & NC & 3579 & -3.4 & -75.7987 & 35.1699 & NC & 3651 & -2.4 & -75.8345 & 35.1567 & NC \\
\hline 3508 & -0.2 & -75.7402 & 35.1845 & NC & 3580 & -3.2 & -75.7993 & 35.1697 & NC & 3652 & -2.3 & -75.835 & 35.1565 & NC \\
\hline 3509 & 3.3 & -75.7407 & 35.1843 & NC & 3581 & -3.7 & -75.7998 & 35.1695 & NC & 3653 & -2.2 & -75.8355 & 35.1563 & NC \\
\hline 3510 & NA & -75.7641 & 35.182 & NC & 3582 & -4 & -75.8003 & 35.1693 & NC & 3654 & -2.4 & -75.836 & 35.1561 & NC \\
\hline 3511 & 61.7 & -75.7646 & 35.1818 & NC & 3583 & -3.5 & -75.8008 & 35.1692 & NC & 3655 & -2 & -75.8365 & 35.156 & NC \\
\hline 3512 & 36.6 & -75.7652 & 35.1817 & NC & 3584 & -2.9 & -75.8013 & 35.169 & NC & 3656 & -1.9 & .75 .837 & 35.1558 & NC \\
\hline 3513 & 16.2 & -75.7657 & 35.1815 & NC & 3585 & -3.1 & -75.8018 & 35.1688 & NC & 3657 & -2.2 & -75.8375 & 35.1556 & NC \\
\hline 3514 & 15.4 & -75.7662 & 35.1813 & NC & 3586 & -3.3 & -75.8024 & 35.1686 & NC & 3658 & -2.2 & -75.838 & 35.1554 & NC \\
\hline 3515 & 14.6 & -75.7667 & 35.1811 & NC & 3587 & -3.8 & -75.8029 & 35.1684 & NC & 3659 & -2.3 & -75.8386 & 35.1552 & NC \\
\hline 3516 & 13.4 & -75.7673 & 35.1809 & NC & 3588 & -3.9 & -75.8034 & 35.1682 & NC & 3660 & -2.6 & .75 .8391 & 35.155 & NC \\
\hline 3517 & 12.9 & -75.7678 & 35.1808 & NC & 3589 & -4 & -75.8039 & 35.168 & NC & 3661 & -2.7 & -75.8396 & 35.1548 & NC \\
\hline 3518 & 12.3 & -75.7683 & 35.1806 & NC & 3590 & -4 & -75.8044 & 35.1678 & NC & 3662 & -2.9 & -75.8401 & 35.1546 & NC \\
\hline 3519 & 11.6 & -75.7688 & 35.1804 & NC & 3591 & -4.1 & -75.8049 & 35.1676 & NC & 3663 & -2.9 & -75.8406 & 35.1544 & $\mathrm{NC}$ \\
\hline 3520 & 11 & -75.7693 & 35.1802 & NC & 3592 & -4.1 & -75.8055 & 35.1674 & NC & 3664 & -3.2 & -75.8411 & 35.1542 & NC \\
\hline 3521 & 11.1 & -75.7699 & 35.18 & NC & 3593 & -4.1 & -75.806 & 35.1673 & NC & 3665 & -2.3 & -75.8416 & 35.154 & NC \\
\hline 3522 & 11.3 & -75.7704 & 35.1799 & NC & 3594 & -4 & -75.8065 & 35.1671 & NC & 3666 & -1.8 & -75.8421 & 35.1538 & NC \\
\hline 3523 & 11.8 & -75.7709 & 35.1797 & NC & 3595 & -3.9 & -75.807 & 35.1669 & $\mathrm{NC}$ & 3667 & -1.9 & -75.8427 & 35.1536 & $\mathrm{NC}$ \\
\hline 3524 & 12.1 & -75.7714 & 35.1795 & NC & 3596 & -4 & -75.8075 & 35.1667 & NC & 3668 & -2 & .75 .8432 & 35.1534 & NC \\
\hline 3525 & 10.4 & -75.772 & 35.1793 & NC & 3597 & -3.9 & -75.808 & 35.1665 & NC & 3669 & -1.9 & -75.8437 & 35.1533 & NC \\
\hline 3526 & 8.4 & -75.7725 & 35.1791 & NC & 3598 & -3.7 & -75.8086 & 35.1663 & NC & 3670 & -2 & -75.8442 & 35.1531 & NC \\
\hline 3527 & 7.9 & -75.773 & 35.179 & NC & 3599 & -3.2 & -75.8091 & 35.1661 & NC & 3671 & -2.1 & -75.8447 & 35.1529 & $\mathrm{NC}$ \\
\hline
\end{tabular}




\begin{tabular}{|c|c|c|c|c|c|c|c|c|c|c|c|c|c|c|}
\hline TR\# & RATE & LONG & LAT & ST & TR\# & RATE & LONG & LAT & ST & TR\# & RATE & LONG & LAT & ST \\
\hline 3672 & -2.4 & -75.8452 & 35.1527 & $\mathrm{NC}$ & 3744 & -0.2 & -75.8803 & 35.1388 & NC & 3816 & -0.2 & -75.9145 & 35.1231 & NC \\
\hline 3673 & -2.5 & -75.8457 & 35.1525 & $\mathrm{NC}$ & 3745 & -0.6 & -75.8808 & 35.1386 & NC & 3817 & -0.1 & -75.915 & 35.1229 & NC \\
\hline 3674 & -3 & -75.8462 & 35.1523 & $\mathrm{NC}$ & 3746 & -0.1 & -75.8813 & 35.1384 & NC & 3818 & -0.1 & -75.9155 & 35.1226 & NC \\
\hline 3675 & -2.9 & -75.8468 & 35.1521 & NC & 3747 & -0.3 & -75.8818 & 35.1382 & NC & 3819 & -0.3 & -75.9159 & 35.1224 & NC \\
\hline 3676 & -2.5 & -75.8473 & 35.1519 & NC & 3748 & -0.8 & -75.8823 & 35.138 & NC & 3820 & 0 & -75.9164 & 35.1221 & NC \\
\hline 3677 & -2.5 & -75.8478 & 35.1517 & NC & 3749 & -0.8 & -75.8828 & 35.1378 & NC & 3821 & -0.2 & -75.9169 & 35.1219 & $\mathrm{NC}$ \\
\hline 3678 & -2.4 & -75.8483 & 35.1515 & NC & 3750 & -0.9 & .75 .8833 & 35.1375 & NC & 3822 & -0.5 & -75.9174 & 35.1217 & NC \\
\hline 3679 & -2.4 & -75.8488 & 35.1513 & $\mathrm{NC}$ & 3751 & -1.1 & -75.8838 & 35.1373 & NC & 3823 & -0.5 & -75.9179 & 35.1214 & NC \\
\hline 3680 & -2.5 & -75.8493 & 35.1511 & $\mathrm{NC}$ & 3752 & -1.1 & -75.8843 & 35.1371 & NC & 3824 & -0.3 & -75.9184 & 35.1212 & NC \\
\hline 3681 & -2.3 & -75.8498 & 35.1509 & NC & 3753 & -0.9 & -75.8847 & 35.1369 & NC & 3825 & -0.2 & -75.9189 & 35.121 & NC \\
\hline 3682 & -2.2 & -75.8503 & 35.1507 & NC & 3754 & -0.9 & -75.8852 & 35.1367 & NC & 3826 & -0.2 & -75.9194 & 35.1207 & NC \\
\hline 3683 & -1.7 & -75.8509 & 35.1506 & NC & 3755 & -0.5 & .75 .8857 & 35.1365 & NC & 3827 & -0.4 & -75.9198 & 35.1205 & NC \\
\hline 3684 & -2.2 & -75.8514 & 35.1504 & NC & 3756 & -0.1 & -75.8862 & 35.1362 & NC & 3828 & -0.5 & -75.9203 & 35.1202 & NC \\
\hline 3685 & -1.9 & -75.8519 & 35.1502 & NC & 3757 & -0.4 & -75.8867 & 35.136 & NC & 3829 & -0.5 & -75.9208 & 35.12 & NC \\
\hline 3686 & -1.5 & -75.8524 & 35.15 & NC & 3758 & -0.8 & -75.8872 & 35.1358 & NC & 3830 & -0.1 & -75.9213 & 35.1198 & NC \\
\hline 3687 & -1.7 & -75.8529 & 35.1498 & NC & 3759 & -1.1 & -75.8877 & 35.1356 & NC & 3831 & 0.2 & -75.9218 & 35.1195 & NC \\
\hline 3688 & -1.6 & -75.8534 & 35.1496 & NC & 3760 & -1.1 & -75.8882 & 35.1354 & NC & 3832 & 0.6 & -75.9223 & 35.1193 & NC \\
\hline 3689 & -1.7 & -75.8539 & 35.1494 & NC & 3761 & -1.3 & -75.8887 & 35.1352 & $\mathrm{NC}$ & 3833 & 0.5 & -75.9228 & 35.1191 & NC \\
\hline 3690 & -1.5 & -75.8544 & 35.1492 & NC & 3762 & -1.3 & -75.8892 & 35.1349 & NC & 3834 & 0.5 & -75.9233 & 35.1188 & NC \\
\hline 3691 & -1.7 & -75.855 & 35.149 & NC & 3763 & -0.9 & -75.8897 & 35.1347 & NC & 3835 & 0.8 & -75.9238 & 35.1186 & NC \\
\hline 3692 & -1.8 & -75.8555 & 35.1488 & NC & 3764 & -0.4 & -75.8902 & 35.1345 & NC & 3836 & 0.8 & -75.9242 & 35.1183 & NC \\
\hline 3693 & -1.6 & -75.856 & 35.1486 & NC & 3765 & -0.1 & .75 .8907 & 35.1343 & NC & 3837 & 0.7 & -75.9247 & 35.1181 & NC \\
\hline 3694 & -1.5 & -75.8565 & 35.1484 & $\mathrm{NC}$ & 3766 & 0.2 & -75.8912 & 35.1341 & $\mathrm{NC}$ & 3838 & 0.8 & -75.9252 & 35.1179 & NC \\
\hline 3695 & -1.7 & -75.857 & 35.1482 & NC & 3767 & 0.1 & -75.8917 & 35.1339 & NC & 3839 & 0.5 & -75.9257 & 35.1176 & NC \\
\hline 3696 & -2.2 & -75.8575 & 35.148 & NC & 3768 & 0.1 & -75.8922 & 35.1336 & NC & 3840 & 0.5 & -75.9262 & 35.1174 & NC \\
\hline 3697 & -2 & -75.858 & 35.1479 & NC & 3769 & 0 & -75.8927 & 35.1334 & NC & 3841 & 0.4 & -75.9267 & 35.1171 & NC \\
\hline 3698 & -2 & -75.8585 & 35.1477 & NC & 3770 & -0.2 & -75.8932 & 35.1332 & NC & 3842 & 0.1 & -75.9272 & 35.1169 & NC \\
\hline 3699 & -2 & -75.8591 & 35.1475 & NC & 3771 & -0.4 & -75.8937 & 35.133 & NC & 3843 & 0.5 & -75.9277 & 35.1167 & NC \\
\hline 3700 & -1.9 & -75.8596 & 35.1473 & $\mathrm{NC}$ & 3772 & -0.8 & -75.8942 & 35.1328 & NC & 3844 & 1.2 & -75.9282 & 35.1164 & NC \\
\hline 3701 & -1.9 & -75.8601 & 35.1471 & NC & 3773 & -0.9 & -75.8947 & 35.1326 & NC & 3845 & 0.7 & -75.9286 & 35.1162 & NC \\
\hline 3702 & -1.5 & -75.8606 & 35.1469 & $\mathrm{NC}$ & 3774 & -1.1 & -75.8952 & 35.1323 & NC & 3846 & 0.5 & -75.9291 & 35.116 & NC \\
\hline 3703 & -1.4 & -75.8611 & 35.1467 & NC & 3775 & -1.2 & -75.8957 & 35.1321 & NC & 3847 & 0.6 & -75.9296 & 35.1157 & NC \\
\hline 3704 & -0.9 & -75.8616 & 35.1465 & NC & 3776 & -0.9 & -75.8962 & 35.1319 & NC & 3848 & 0.7 & -75.9301 & 35.1155 & NC \\
\hline 3705 & -0.9 & -75.8621 & 35.1463 & $\mathrm{NC}$ & 3777 & -0.9 & -75.8966 & 35.1317 & $\mathrm{NC}$ & 3849 & 0.8 & -75.9306 & 35.1152 & NC \\
\hline 3706 & -0.7 & -75.8626 & 35.1461 & NC & 3778 & -0.5 & -75.8971 & 35.1315 & $\mathrm{NC}$ & 3850 & 2 & -75.9336 & 35.1151 & NC \\
\hline 3707 & -0.9 & -75.8632 & 35.1459 & NC & 3779 & -1 & -75.8976 & 35.1313 & $\mathrm{NC}$ & 3851 & 1.1 & -75.9311 & 35.115 & NC \\
\hline 3708 & -1 & -75.8637 & 35.1457 & NC & 3780 & -1 & -75.8981 & 35.131 & NC & 3852 & 2.3 & -75.9341 & 35.1148 & NC \\
\hline 3709 & -0.6 & -75.8642 & 35.1455 & NC & 3781 & -0.9 & -75.8986 & 35.1308 & NC & 3853 & 1.1 & -75.9316 & 35.1148 & NC \\
\hline 3710 & -0.8 & -75.8647 & 35.1453 & $\mathrm{NC}$ & 3782 & -1.4 & -75.8991 & 35.1306 & $\mathrm{NC}$ & 3854 & 2.5 & -75.9345 & 35.1145 & NC \\
\hline 3711 & -0.8 & -75.8652 & 35.1452 & $\mathrm{NC}$ & 3783 & -1.2 & -75.8996 & 35.1304 & NC & 3855 & 1.3 & -75.9321 & 35.1145 & $\mathrm{NC}$ \\
\hline 3712 & -0.8 & -75.8657 & 35.145 & NC & 3784 & -0.7 & .75 .9001 & 35.1302 & $\mathrm{NC}$ & 3856 & 2.9 & -75.935 & 35.1143 & $\mathrm{NC}$ \\
\hline 3713 & -0.8 & -75.8662 & 35.1448 & NC & 3785 & 0.6 & -75.9003 & 35.13 & NC & 3857 & 1.5 & -75.9326 & 35.1143 & $\mathrm{NC}$ \\
\hline 3714 & -1.6 & -75.8669 & 35.1447 & NC & 3786 & -0.6 & -75.9006 & 35.13 & NC & 3858 & 1.8 & -75.933 & 35.1141 & NC \\
\hline 3715 & -1 & -75.8667 & 35.1446 & NC & 3787 & 0.5 & -75.9008 & 35.1298 & NC & 3859 & 2.3 & -75.9354 & 35.114 & NC \\
\hline 3716 & -1.3 & -75.8674 & 35.1445 & NC & 3788 & -0.1 & -75.9011 & 35.1297 & NC & 3860 & 1.8 & -75.9335 & 35.1138 & NC \\
\hline 3717 & -1.2 & -75.8673 & 35.1444 & NC & 3789 & 0.5 & -75.9013 & 35.1295 & $\mathrm{NC}$ & 3861 & 1.9 & -75.9359 & 35.1137 & $\mathrm{NC}$ \\
\hline 3718 & -0.9 & -75.8679 & 35.1443 & NC & 3790 & 0.3 & -75.9018 & 35.1293 & NC & 3862 & 1.8 & -75.934 & 35.1136 & NC \\
\hline 3719 & -1.5 & -75.8678 & 35.1442 & NC & 3791 & 0 & -75.9023 & 35.129 & NC & 3863 & 1.5 & -75.9363 & 35.1134 & $\mathrm{NC}$ \\
\hline 3720 & -0.2 & -75.8684 & 35.144 & NC & 3792 & -0.1 & -75.9027 & 35.1288 & NC & 3864 & 1.1 & -75.9368 & 35.1132 & NC \\
\hline 3721 & -0.1 & -75.8689 & 35.1438 & NC & 3793 & -0.1 & -75.9032 & 35.1286 & $\mathrm{NC}$ & 3865 & 1 & -75.9373 & 35.1129 & $\mathrm{NC}$ \\
\hline 3722 & 0.3 & -75.8694 & 35.1436 & NC & 3794 & -0.2 & -75.9037 & 35.1283 & $\mathrm{NC}$ & 3866 & 1 & -75.9377 & 35.1126 & $\mathrm{NC}$ \\
\hline 3723 & 0 & -75.8699 & 35.1434 & NC & 3795 & -0.3 & -75.9042 & 35.1281 & $\mathrm{NC}$ & 3867 & 1.3 & -75.9382 & 35.1123 & NC \\
\hline 3724 & -0.5 & -75.8704 & 35.1432 & NC & 3796 & -0.3 & -75.9047 & 35.1279 & NC & 3868 & 1.4 & -75.9386 & 35.112 & NC \\
\hline 3725 & -0.6 & -75.8709 & 35.143 & NC & 3797 & 0.3 & -75.9052 & 35.1276 & $\mathrm{NC}$ & 3869 & 1.8 & -75.9391 & 35.1118 & NC \\
\hline 3726 & -0.7 & -75.8714 & 35.1427 & NC & 3798 & 0.9 & -75.9057 & 35.1274 & $\mathrm{NC}$ & 3870 & 2.2 & -75.9395 & 35.1115 & NC \\
\hline 3727 & -0.5 & -75.8719 & 35.1425 & NC & 3799 & 0.8 & -75.9062 & 35.1271 & $\mathrm{NC}$ & 3871 & 2.2 & -75.94 & 35.1112 & NC \\
\hline 3728 & -0.8 & -75.8724 & 35.1423 & $\mathrm{NC}$ & 3800 & 0.7 & -75.9067 & 35.1269 & $\mathrm{NC}$ & 3872 & 2.1 & -75.9404 & 35.1109 & NC \\
\hline 3729 & -0.9 & -75.8728 & 35.1421 & NC & 3801 & 0.3 & -75.9071 & 35.1267 & NC & 3873 & 1.9 & -75.9409 & 35.1107 & NC \\
\hline 3730 & -0.6 & -75.8733 & 35.1419 & NC & 3802 & 0 & -75.9076 & 35.1264 & $\mathrm{NC}$ & 3874 & 2.2 & .75 .9414 & 35.1104 & NC \\
\hline 3731 & -0.6 & -75.8738 & 35.1417 & $\mathrm{NC}$ & 3803 & -0.2 & -75.9081 & 35.1262 & $\mathrm{NC}$ & 3875 & 2 & -75.9418 & 35.1101 & $\mathrm{NC}$ \\
\hline 3732 & -0.6 & -75.8743 & 35.1414 & NC & 3804 & -0.4 & -75.9086 & 35.126 & $\mathrm{NC}$ & 3876 & 2 & -75.9423 & 35.1098 & NC \\
\hline 3733 & -0.7 & -75.8748 & 35.1412 & $\mathrm{NC}$ & 3805 & -0.3 & -75.9091 & 35.1257 & $\mathrm{NC}$ & 3877 & 2 & -75.9427 & 35.1096 & NC \\
\hline 3734 & -0.9 & -75.8753 & 35.141 & NC & 3806 & -0.1 & -75.9096 & 35.1255 & $\mathrm{NC}$ & 3878 & 1.8 & .75 .9432 & 35.1093 & NC \\
\hline 3735 & -1 & -75.8758 & 35.1408 & NC & 3807 & -0.4 & -75.9101 & 35.1252 & $\mathrm{NC}$ & 3879 & 1.7 & -75.9436 & 35.109 & NC \\
\hline 3736 & -1 & -75.8763 & 35.1406 & NC & 3808 & -0.5 & -75.9106 & 35.125 & NC & 3880 & 1.6 & -75.9441 & 35.1087 & NC \\
\hline 3737 & -1.1 & -75.8768 & 35.1404 & $\mathrm{NC}$ & 3809 & -0.5 & -75.9111 & 35.1248 & $\mathrm{NC}$ & 3881 & 1.8 & -75.9446 & 35.1084 & NC \\
\hline 3738 & -0.7 & -75.8773 & 35.1401 & NC & 3810 & -0.4 & -75.9115 & 35.1245 & $\mathrm{NC}$ & 3882 & 2.1 & -75.945 & 35.1082 & NC \\
\hline 3739 & -0.3 & -75.8778 & 35.1399 & NC & 3811 & -0.3 & -75.912 & 35.1243 & $\mathrm{NC}$ & 3883 & 2.4 & -75.9455 & 35.1079 & NC \\
\hline 3740 & 0.2 & -75.8783 & 35.1397 & $\mathrm{NC}$ & 3812 & 0 & -75.9125 & 35.124 & NC & 3884 & 2.6 & .75 .9459 & 35.1076 & NC \\
\hline 3741 & -0.1 & -75.8788 & 35.1395 & NC & 3813 & -0.1 & -75.913 & 35.1238 & NC & 3885 & 3.3 & -75.9464 & 35.1073 & NC \\
\hline 3742 & -0.3 & -75.8793 & 35.1393 & NC & 3814 & -0.1 & -75.9135 & 35.1236 & NC & 3886 & 3.9 & -75.9468 & 35.1071 & NC \\
\hline 3743 & -0.8 & -75.8798 & 35.1391 & NC & 3815 & -0.2 & -75.914 & 35.1233 & NC & 3887 & 3.9 & -75.9473 & 35.1068 & NC \\
\hline
\end{tabular}


TR\# RATE LONG LAT ST TR\# RATE LONG LAT $S T$ TR\# $\quad$ RATE LONG LAT ST

\begin{tabular}{|c|c|c|c|c|c|c|c|c|c|c|c|c|c|c|}
\hline 3888 & 4.1 & -75.9477 & 35.1065 & NC & 3960 & 2.5 & -75.9771 & 35.0852 & NC & 4032 & 12.6 & -76.0064 & 35.0625 & NC \\
\hline 3889 & 3.7 & -75.9482 & 35.1062 & NC & 3961 & 2.7 & -75.9775 & 35.0849 & NC & 4033 & 11.1 & -76.0068 & 35.0622 & NC \\
\hline 3890 & 3.4 & -75.9487 & 35.1059 & NC & 3962 & 2.8 & -75.9779 & 35.0845 & NC & 4034 & 9.7 & -76.0073 & 35.0619 & NC \\
\hline 3891 & 3.6 & -75.9491 & 35.1057 & NC & 3963 & 2.5 & -75.9782 & 35.0842 & NC & 4035 & 9.5 & -76.0077 & 35.0616 & NC \\
\hline 3892 & 3.6 & -75.9496 & 35.1054 & NC & 3964 & 2 & -75.9786 & 35.0838 & NC & 4036 & 9.4 & -76.0082 & 35.0614 & NC \\
\hline 3893 & 3.7 & -75.95 & 35.1051 & NC & 3965 & 2 & -75.979 & 35.0835 & NC & 4037 & -31.9 & -76.0087 & 35.0611 & NC \\
\hline 3894 & 3.7 & -75.9505 & 35.1048 & NC & 3966 & 1.6 & -75.9794 & 35.0832 & NC & 4038 & -32.1 & -76.0091 & 35.0608 & NC \\
\hline 3895 & 3.4 & -75.9509 & 35.1046 & NC & 3967 & 1.1 & -75.9798 & 35.0828 & NC & 4039 & .31 .3 & -76.0096 & 35.0606 & NC \\
\hline 3896 & 3.4 & -75.9514 & 35.1043 & NC & 3968 & 0.5 & -75.9802 & 35.0825 & NC & 4040 & -30.7 & -76.01 & 35.0603 & NC \\
\hline 3897 & 3.1 & -75.9519 & 35.104 & NC & 3969 & 0.4 & -75.9806 & 35.0821 & NC & 4041 & -16 & -76.0317 & 35.0557 & NC \\
\hline 3898 & 3 & -75.9523 & 35.1037 & NC & 3970 & 0.4 & -75.9809 & 35.0818 & NC & 4042 & -13.6 & -76.0322 & 35.0554 & NC \\
\hline 3899 & 3.3 & -75.9528 & 35.1034 & NC & 3971 & -0.1 & -75.9813 & 35.0815 & NC & 4043 & -11.7 & -76.0327 & 35.0552 & NC \\
\hline 3900 & 3.5 & -75.9532 & 35.1032 & NC & 3972 & -0.7 & -75.9817 & 35.0811 & $\mathrm{NC}$ & 4044 & -10.7 & -76.0332 & 35.055 & NC \\
\hline 3901 & 3.4 & -75.9537 & 35.1029 & $\mathrm{NC}$ & 3973 & -0.6 & -75.9821 & 35.0808 & NC & 4045 & -25.4 & -76.0337 & 35.0548 & NC \\
\hline 3902 & 3.4 & -75.9541 & 35.1026 & NC & 3974 & -0.6 & -75.9825 & 35.0804 & NC & 4046 & -24.8 & -76.0342 & 35.0546 & NC \\
\hline 3903 & 3.5 & -75.9546 & 35.1023 & NC & 3975 & -1.4 & .75 .9829 & 35.0801 & NC & 4047 & -23.5 & -76.0347 & 35.0543 & NC \\
\hline 3904 & 3.5 & .75 .955 & 35.1021 & NC & 3976 & -1.6 & -75.9832 & 35.0798 & NC & 4048 & -22.6 & -76.0352 & 35.0541 & NC \\
\hline 3905 & 3.6 & -75.9555 & 35.1018 & NC & 3977 & -2.3 & -75.9836 & 35.0794 & NC & 4049 & -20.3 & -76.0357 & 35.0539 & NC \\
\hline 3906 & 3.4 & -75.956 & 35.1015 & NC & 3978 & -2.8 & -75.984 & 35.0791 & $\mathrm{NC}$ & 4050 & -19.1 & -76.0362 & 35.0537 & NC \\
\hline 3907 & 3.4 & .75 .9564 & 35.1012 & NC & 3979 & -2.7 & -75.9844 & 35.0787 & $\mathrm{NC}$ & 4051 & -18.2 & -76.0367 & 35.0535 & NC \\
\hline 3908 & 3.5 & -75.9569 & 35.1009 & NC & 3980 & -2.7 & -75.9848 & 35.0784 & NC & 4052 & -16.8 & -76.0372 & 35.0533 & NC \\
\hline 3909 & 3.2 & -75.9573 & 35.1007 & NC & 3981 & -3.3 & -75.9852 & 35.078 & NC & 4053 & -15.8 & -76.0377 & 35.053 & NC \\
\hline 3910 & 3.2 & -75.9578 & 35.1004 & NC & 3982 & -3.8 & -75.9856 & 35.0777 & NC & 4054 & -13.7 & -76.0382 & 35.0528 & NC \\
\hline 3911 & 3.2 & -75.9582 & 35.1001 & NC & 3983 & -4.6 & -75.9859 & 35.0774 & NC & 4055 & -12.8 & -76.0387 & 35.0526 & $\mathrm{NC}$ \\
\hline 3912 & 3.3 & -75.9587 & 35.0998 & NC & 3984 & -5.2 & -75.9863 & 35.077 & NC & 4056 & -11.9 & .76 .0392 & 35.0524 & $\mathrm{NC}$ \\
\hline 3913 & 3.2 & -75.9592 & 35.0996 & NC & 3985 & -5.7 & -75.9867 & 35.0767 & NC & 4057 & -11 & -76.0397 & 35.0522 & NC \\
\hline 3914 & 3.1 & -75.9596 & 35.0993 & NC & 3986 & -6.2 & -75.9871 & 35.0763 & NC & 4058 & -10.2 & -76.0401 & 35.0519 & NC \\
\hline 3915 & 3.5 & -75.9601 & 35.099 & NC & 3987 & -5.8 & -75.9875 & 35.076 & NC & 4059 & -9.7 & -76.0406 & 35.0517 & $\mathrm{NC}$ \\
\hline 3916 & 3.7 & -75.9605 & 35.0987 & NC & 3988 & -5.4 & -75.9879 & 35.0757 & NC & 4060 & -9.2 & -76.0411 & 35.0515 & NC \\
\hline 3917 & 3.7 & -75.961 & 35.0985 & NC & 3989 & -4.7 & -75.9882 & 35.0753 & NC & 4061 & -8.8 & -76.0416 & 35.0513 & NC \\
\hline 3918 & 3.7 & -75.9614 & 35.0982 & NC & 3990 & -4.4 & -75.9886 & 35.075 & NC & 4062 & -8.3 & -76.0421 & 35.0511 & NC \\
\hline 3919 & 3.7 & -75.9619 & 35.0979 & NC & 3991 & -4.2 & -75.9876 & 35.0736 & NC & 4063 & -8 & -76.0426 & 35.0508 & NC \\
\hline 3920 & 4.1 & -75.9629 & 35.0978 & NC & 3992 & -3.6 & -75.9881 & 35.0733 & NC & 4064 & -7.7 & -76.0431 & 35.0506 & NC \\
\hline 3921 & 3.7 & -75.9623 & 35.0976 & NC & 3993 & -3.3 & -75.9885 & 35.0731 & NC & 4065 & -7.8 & -76.0436 & 35.0504 & NC \\
\hline 3922 & 3.9 & -75.9633 & 35.0975 & NC & 3994 & -2.8 & -75.989 & 35.0728 & NC & 4066 & -7.8 & -76.0441 & 35.0502 & NC \\
\hline 3923 & 3.9 & -75.9628 & 35.0973 & NC & 3995 & -2.6 & -75.9894 & 35.0725 & NC & 4067 & -7.9 & -76.0446 & 35.05 & NC \\
\hline 3924 & 3.9 & -75.9636 & 35.0971 & NC & 3996 & -4.1 & -75.9899 & 35.0722 & NC & 4068 & -7.8 & -76.0451 & 35.0498 & NC \\
\hline 3925 & 3.9 & -75.9633 & 35.0971 & NC & 3997 & -4.7 & -75.9903 & 35.072 & NC & 4069 & -7.8 & -76.0456 & 35.0495 & NC \\
\hline 3926 & 3.7 & -75.964 & 35.0968 & NC & 3998 & -5.4 & -75.9908 & 35.0717 & NC & 4070 & -7.7 & -76.0461 & 35.0493 & NC \\
\hline 3927 & 4.1 & -75.9644 & 35.0965 & NC & 3999 & -4.8 & -75.9913 & 35.0714 & NC & 4071 & -7.8 & -76.0466 & 35.0491 & NC \\
\hline 3928 & 4.5 & -75.9648 & 35.0961 & NC & 4000 & -4.1 & -75.9917 & 35.0712 & NC & 4072 & -7.9 & -76.0471 & 35.0489 & NC \\
\hline 3929 & 3.7 & -75.9652 & 35.0958 & NC & 4001 & -5 & -75.9922 & 35.0709 & NC & 4073 & -7.9 & -76.0476 & 35.0487 & NC \\
\hline 3930 & 3.3 & -75.9656 & 35.0954 & NC & 4002 & -5.7 & -75.9926 & 35.0706 & NC & 4074 & -7.8 & -76.0481 & 35.0484 & NC \\
\hline 3931 & 3 & -75.9659 & 35.0951 & NC & 4003 & -6.4 & -75.9931 & 35.0703 & NC & 4075 & -7.5 & -76.0486 & 35.0482 & NC \\
\hline 3932 & 2.6 & -75.9663 & 35.0948 & NC & 4004 & -6.8 & -75.9936 & 35.0701 & NC & 4076 & -7.5 & -76.0491 & 35.048 & NC \\
\hline 3933 & 2.6 & -75.9667 & 35.0944 & NC & 4005 & -7.3 & -75.994 & 35.0698 & NC & 4077 & -7.6 & -76.0496 & 35.0478 & NC \\
\hline 3934 & 2.7 & -75.9671 & 35.0941 & NC & 4006 & -8 & -75.9945 & 35.0695 & NC & 4078 & -7.6 & -76.0501 & 35.0476 & NC \\
\hline 3935 & 3.2 & -75.9675 & 35.0937 & NC & 4007 & -7.9 & -75.9949 & 35.0693 & NC & 4079 & -7.7 & -76.0506 & 35.0474 & NC \\
\hline 3936 & 3.7 & -75.9679 & 35.0934 & NC & 4008 & -7.8 & -75.9954 & 35.069 & NC & 4080 & -7.6 & -76.0511 & 35.0471 & NC \\
\hline 3937 & 3.6 & -75.9683 & 35.093 & NC & 4009 & -8.1 & -75.9958 & 35.0687 & NC & 4081 & -7.6 & -76.0516 & 35.0469 & NC \\
\hline 3938 & 3.6 & -75.9686 & 35.0927 & NC & 4010 & -8.5 & -75.9963 & 35.0684 & NC & 4082 & -7.7 & -76.0521 & 35.0467 & NC \\
\hline 3939 & 3.2 & -75.969 & 35.0924 & NC & 4011 & -8.9 & -75.9968 & 35.0682 & NC & 4083 & -7.5 & -76.0526 & 35.0465 & NC \\
\hline 3940 & 3 & -75.9694 & 35.092 & NC & 4012 & -8.7 & -75.9972 & 35.0679 & NC & 4084 & -7.5 & -76.0531 & 35.0463 & NC \\
\hline 3941 & 3 & -75.9698 & 35.0917 & NC & 4013 & -9 & -75.9977 & 35.0676 & NC & 4085 & -7.5 & -76.0536 & 35.046 & NC \\
\hline 3942 & 2.9 & -75.9702 & 35.0913 & NC & 4014 & -10 & -75.9981 & 35.0673 & NC & 4086 & -7.4 & -76.0541 & 35.0458 & NC \\
\hline 3943 & 3.2 & -75.9706 & 35.091 & NC & 4015 & -10.2 & -75.9986 & 35.0671 & NC & 4087 & -7.5 & -76.0546 & 35.0456 & NC \\
\hline 3944 & 3.6 & -75.9709 & 35.0907 & NC & 4016 & -10.6 & -75.999 & 35.0668 & NC & 4088 & -7.4 & -76.0551 & 35.0454 & NC \\
\hline 3945 & 3 & -75.9713 & 35.0903 & NC & 4017 & -11.8 & -75.9995 & 35.0665 & NC & 4089 & -7.5 & -76.0556 & 35.0452 & NC \\
\hline 3946 & 2.6 & -75.9717 & 35.09 & NC & 4018 & -13 & -76 & 35.0663 & NC & 4090 & -7.5 & -76.0561 & 35.045 & NC \\
\hline 3947 & 3.5 & -75.9721 & 35.0896 & NC & 4019 & -12.4 & -76.0004 & 35.066 & NC & 4091 & -7.5 & -76.0566 & 35.0447 & NC \\
\hline 3948 & 4.4 & -75.9725 & 35.0893 & NC & 4020 & -12.8 & -76.0009 & 35.0657 & NC & 4092 & -8 & -76.0547 & 35.0439 & NC \\
\hline 3949 & 4.3 & -75.9729 & 35.089 & NC & 4021 & -15.7 & -76.0013 & 35.0654 & NC & 4093 & -7.7 & -76.0551 & 35.0436 & NC \\
\hline 3950 & 4.1 & -75.9733 & 35.0886 & NC & 4022 & -1.9 & -76.0018 & 35.0652 & NC & 4094 & -7.7 & -76.0556 & 35.0434 & NC \\
\hline 3951 & 3.6 & -75.9736 & 35.0883 & NC & 4023 & -2.2 & -76.0022 & 35.0649 & NC & 4095 & -7.9 & -76.056 & 35.0431 & NC \\
\hline 3952 & 2.9 & -75.974 & 35.0879 & NC & 4024 & -2.5 & -76.0027 & 35.0646 & NC & 4096 & -7.8 & -76.0564 & 35.0428 & $\mathrm{NC}$ \\
\hline 3953 & 2.8 & -75.9744 & 35.0876 & NC & 4025 & -3.1 & -76.0032 & 35.0644 & NC & 4097 & -7.9 & -76.0569 & 35.0425 & NC \\
\hline 3954 & 3 & -75.9748 & 35.0873 & NC & 4026 & -3.8 & -76.0036 & 35.0641 & NC & 4098 & -7.7 & -76.0573 & 35.0422 & NC \\
\hline 3955 & 2.7 & -75.9752 & 35.0869 & NC & 4027 & -4.4 & -76.0041 & 35.0638 & NC & 4099 & -7.6 & .76 .0578 & 35.0419 & NC \\
\hline 3956 & 2.3 & -75.9756 & 35.0866 & NC & 4028 & -5 & -76.0045 & 35.0635 & NC & 4100 & -7.5 & -76.0582 & 35.0416 & NC \\
\hline 3957 & 2.9 & -75.9759 & 35.0862 & NC & 4029 & 12.8 & -76.005 & 35.0633 & NC & 4101 & -7.4 & -76.0586 & 35.0413 & NC \\
\hline 3958 & 3.3 & -75.9763 & 35.0859 & NC & 4030 & 12.3 & -76.0055 & 35.063 & NC & 4102 & -7.4 & -76.0591 & 35.041 & NC \\
\hline 3959 & 2.9 & -75.9767 & 35.0855 & NC & 4031 & 12.4 & -76.0059 & 35.0627 & NC & 4103 & -7.2 & -76.0595 & 35.0407 & NC \\
\hline
\end{tabular}




\begin{tabular}{|c|c|c|c|c|c|c|c|c|c|c|c|c|c|c|}
\hline TR\# & RATE & LONG & LAT & ST & TR\# & RATE & LONG & LAT & ST & TR\# & RATE & LONG & LAT & ST \\
\hline 4320 & -1.6 & -76.1491 & 34.9776 & $\mathrm{NC}$ & 4392 & -0.9 & -76.1776 & 34.9564 & $\mathrm{NC}$ & | 4464 & -1.7 & -76.2052 & 34.9338 & NC \\
\hline 4321 & -1.2 & -76.1495 & 34.9773 & $\mathrm{NC}$ & 4393 & -0.9 & -76.178 & 34.9561 & $\mathrm{NC}$ & 4465 & -1.8 & -76.2056 & 34.9335 & NC \\
\hline 4322 & -1.2 & -76.1499 & 34.977 & NC & 4394 & -0.9 & -76.1784 & 34.9557 & NC & 4466 & -1.9 & -76.206 & 34.9332 & NC \\
\hline 4323 & -1 & -76.1504 & 34.9767 & $\mathrm{NC}$ & 4395 & -1 & -76.1788 & 34.9554 & NC & 4467 & -1.9 & -76.2064 & 34.9328 & NC \\
\hline 4324 & -0.9 & -76.1508 & 34.9764 & NC & 4396 & -0.9 & -76.1792 & 34.9551 & NC & 4468 & -1.9 & -76.2068 & 34.9325 & NC \\
\hline 4325 & -0.7 & -76.1512 & 34.9761 & $\mathrm{NC}$ & 4397 & -0.8 & -76.1796 & 34.9548 & NC & 4469 & -2.1 & -76.2072 & 34.9322 & NC \\
\hline 4326 & -0.4 & -76.1516 & 34.9758 & $\mathrm{NC}$ & 4398 & -0.9 & -76.18 & 34.9544 & $\mathrm{NC}$ & 4470 & -2.2 & -76.2076 & 34.9318 & NC \\
\hline 4327 & -0.1 & -76.152 & 34.9754 & $\mathrm{NC}$ & 4399 & -1 & -76.1804 & 34.9541 & NC & 4471 & -2.3 & -76.208 & 34.9315 & NC \\
\hline 4328 & -3.3 & -76.1524 & 34.9751 & NC & 4400 & -0.9 & -76.1808 & 34.9538 & NC & 4472 & -2.1 & -76.2084 & 34.9312 & NC \\
\hline 4329 & -3.4 & -76.1529 & 34.9748 & $\mathrm{NC}$ & 4401 & -1.1 & -76.1812 & 34.9534 & $\mathrm{NC}$ & 4473 & -1.5 & -76.2087 & 34.9308 & NC \\
\hline 4330 & -3.3 & -76.1533 & 34.9745 & NC & 4402 & -1.1 & -76.1816 & 34.9531 & NC & 4474 & -1.8 & -76.2091 & 34.9305 & NC \\
\hline 4331 & -3.3 & -76.1537 & 34.9742 & $\mathrm{NC}$ & 4403 & -1.1 & -76.182 & 34.9528 & NC & 4475 & -2 & -76.2095 & 34.9302 & NC \\
\hline 4332 & -3.2 & -76.1541 & 34.9739 & NC & 4404 & -1.1 & -76.1824 & 34.9525 & NC & 4476 & -2.3 & -76.2099 & 34.9298 & NC \\
\hline 4333 & -3 & -76.1545 & 34.9736 & $\mathrm{NC}$ & 4405 & -1.1 & -76.1828 & 34.9521 & NC & 4477 & -2.5 & -76.2103 & 34.9295 & NC \\
\hline 4334 & -3 & -76.1549 & 34.9732 & $\mathrm{NC}$ & 4406 & -0.9 & -76.1832 & 34.9518 & $\mathrm{NC}$ & 4478 & -2.4 & -76.2107 & 34.9292 & NC \\
\hline 4335 & 1.1 & -76.1554 & 34.9729 & NC & 4407 & -0.7 & -76.1836 & 34.9515 & NC & 4479 & -2.4 & -76.2111 & 34.9288 & NC \\
\hline 4336 & 0.6 & -76.1558 & 34.9726 & $\mathrm{NC}$ & 4408 & -0.9 & -76.184 & 34.9511 & NC & 4480 & -2.1 & -76.2115 & 34.9285 & NC \\
\hline 4337 & 0.1 & -76.1562 & 34.9723 & NC & 4409 & -1.1 & -76.1844 & 34.9508 & NC & 4481 & -1.8 & -76.2119 & 34.9282 & NC \\
\hline 4338 & 0.1 & -76.1566 & 34.972 & $\mathrm{NC}$ & 4410 & -1.1 & -76.1848 & 34.9505 & $\mathrm{NC}$ & 4482 & -1.8 & -76.2123 & 34.9278 & NC \\
\hline 4339 & 0.2 & -76.157 & 34.9717 & $\mathrm{NC}$ & 4411 & -1.1 & -76.1852 & 34.9502 & NC & 4483 & -1.9 & -76.2127 & 34.9275 & NC \\
\hline 4340 & 0.2 & -76.1574 & 34.9714 & $\mathrm{NC}$ & 4412 & -1 & -76.1856 & 34.9498 & $\mathrm{NC}$ & 4484 & -1.5 & -76.2131 & 34.9272 & NC \\
\hline 4341 & 0.1 & -76.1579 & 34.9711 & NC & 4413 & -1 & -76.186 & 34.9495 & $\mathrm{NC}$ & 4485 & -1.2 & -76.2135 & 34.9268 & NC \\
\hline 4342 & 0.1 & -76.1583 & 34.9707 & NC & 4414 & -1 & -76.1864 & 34.9492 & NC & 4486 & -1.3 & -76.2139 & 34.9265 & NC \\
\hline 4343 & -0.1 & -76.1587 & 34.9704 & NC & 4415 & -1 & -76.1868 & 34.9488 & NC & 4487 & -1.5 & -76.2143 & 34.9262 & NC \\
\hline 4344 & -0.2 & -76.1591 & 34.9701 & NC & 4416 & -1 & -76.1872 & 34.9485 & $\mathrm{NC}$ & 4488 & -1.6 & -76.2147 & 34.9258 & NC \\
\hline 4345 & -0.3 & -76.1595 & 34.9698 & $\mathrm{NC}$ & 4417 & -0.9 & -76.1876 & 34.9482 & NC & 4489 & -1.8 & -76.2151 & 34.9255 & NC \\
\hline 4346 & -0.4 & -76.1599 & 34.9695 & NC & 4418 & -1 & -76.188 & 34.9479 & NC & 4490 & -1.6 & -76.2155 & 34.9252 & NC \\
\hline 4347 & -0.4 & -76.1604 & 34.9692 & NC & 4419 & -0.9 & -76.1884 & 34.9475 & $\mathrm{NC}$ & 4491 & -1.3 & -76.2158 & 34.9248 & NC \\
\hline 4348 & -0.5 & -76.1608 & 34.9689 & $\mathrm{NC}$ & 4420 & -1.1 & -76.1888 & 34.9472 & NC & 4492 & -1.1 & -76.2162 & 34.9245 & NC \\
\hline 4349 & -0.5 & -76.1612 & 34.9685 & $\mathrm{NC}$ & 4421 & -1.1 & -76.1892 & 34.9469 & NC & 4493 & -0.7 & -76.2166 & 34.9242 & NC \\
\hline 4350 & -0.6 & -76.1616 & 34.9682 & NC & 4422 & -1.1 & -76.1896 & 34.9465 & NC & 4494 & -1.3 & -76.217 & 34.9238 & NC \\
\hline 4351 & -0.5 & -76.162 & 34.9679 & NC & 4423 & -1 & -76.19 & 34.9462 & $\mathrm{NC}$ & 4495 & -1.9 & -76.2174 & 34.9235 & $\mathrm{NC}$ \\
\hline 4352 & -0.5 & -76.1624 & 34.9676 & $\mathrm{NC}$ & 4424 & -1.2 & -76.1904 & 34.9459 & NC & 4496 & -1.9 & -76.2178 & 34.9232 & NC \\
\hline 4353 & -0.6 & -76.1629 & 34.9673 & $\mathrm{NC}$ & 4425 & -1.4 & -76.1908 & 34.9456 & NC & 4497 & -1.8 & -76.2182 & 34.9228 & NC \\
\hline 4354 & -0.3 & -76.1633 & 34.967 & $\mathrm{NC}$ & 4426 & -1.2 & -76.1912 & 34.9452 & $\mathrm{NC}$ & 4498 & -1 & -76.2186 & 34.9225 & NC \\
\hline 4355 & 0 & -76.1637 & 34.9667 & $\mathrm{NC}$ & 4427 & -1.2 & .76 .1916 & 34.9449 & $\mathrm{NC}$ & 4499 & -0.2 & -76.219 & 34.9222 & NC \\
\hline 4356 & -0.1 & -76.1641 & 34.9663 & $\mathrm{NC}$ & 4428 & -1.3 & -76.192 & 34.9446 & NC & 4500 & -0.9 & -76.2194 & 34.9219 & NC \\
\hline 4357 & -0.1 & -76.1645 & 34.966 & NC & 4429 & -1.4 & -76.1924 & 34.9443 & $\mathrm{NC}$ & 4501 & -1.4 & -76.2198 & 34.9215 & NC \\
\hline 4358 & -0.1 & -76.1649 & 34.9657 & $\mathrm{NC}$ & 4430 & -1.4 & -76.1928 & 34.9439 & $\mathrm{NC}$ & 4502 & -1.5 & -76.2202 & 34.9212 & NC \\
\hline 4359 & -0.1 & -76.1654 & 34.9654 & $\mathrm{NC}$ & 4431 & -1.2 & -76.1932 & 34.9436 & $\mathrm{NC}$ & 4503 & -1.8 & -76.2206 & 34.9209 & NC \\
\hline 4360 & -0.1 & -76.1658 & 34.9651 & $\mathrm{NC}$ & 4432 & -1.2 & -76.1936 & 34.9433 & $\mathrm{NC}$ & 4504 & -1.8 & -76.221 & 34.9205 & NC \\
\hline 4361 & -0.2 & -76.1662 & 34.9648 & NC & 4433 & -1.2 & -76.194 & 34.9429 & NC & 4505 & -1.6 & -76.2214 & 34.9202 & NC \\
\hline 4362 & -0.1 & -76.1666 & 34.9645 & $\mathrm{NC}$ & 4434 & -1.3 & -76.1944 & 34.9426 & $\mathrm{NC}$ & 4506 & -1.7 & -76.2218 & 34.9199 & NC \\
\hline 4363 & -0.1 & -76.167 & 34.9641 & $\mathrm{NC}$ & 4435 & -1.3 & -76.1948 & 34.9423 & NC & 4507 & -1.8 & -76.2222 & 34.9195 & NC \\
\hline 4364 & -0.1 & -76.1674 & 34.9638 & $\mathrm{NC}$ & 4436 & -1.3 & -76.1952 & 34.942 & NC & 4508 & -1.6 & -76.2226 & 34.9192 & NC \\
\hline 4365 & -0.1 & -76.1679 & 34.9635 & NC & 4437 & -1.2 & -76.1956 & 34.9416 & $\mathrm{NC}$ & 4509 & -1.4 & -76.2229 & 34.9189 & NC \\
\hline 4366 & -0.2 & -76.1683 & 34.9632 & $\mathrm{NC}$ & 4438 & -1.3 & -76.196 & 34.9413 & $\mathrm{NC}$ & 4510 & -1.5 & -76.2233 & 34.9185 & NC \\
\hline 4367 & -0.2 & -76.1687 & 34.9629 & $\mathrm{NC}$ & 4439 & -1.3 & -76.1964 & 34.941 & $\mathrm{NC}$ & 4511 & -1.4 & -76.2237 & 34.9182 & NC \\
\hline 4368 & -0.2 & -76.1691 & 34.9626 & NC & 4440 & -1.4 & -76.1968 & 34.9406 & $\mathrm{NC}$ & 4512 & -1.3 & -76.2241 & 34.9179 & NC \\
\hline 4369 & -0.3 & -76.1704 & 34.9623 & $\mathrm{NC}$ & 4441 & -1.5 & -76.1972 & 34.9403 & NC & 4513 & -1.1 & -76.2245 & 34.9175 & NC \\
\hline 4370 & -0.3 & -76.1695 & 34.9623 & $\mathrm{NC}$ & 4442 & -1.5 & -76.1976 & 34.94 & NC & 4514 & -1.5 & -76.2249 & 34.9172 & NC \\
\hline 4371 & -0.4 & -76.1708 & 34.962 & $\mathrm{NC}$ & 4443 & -0.9 & .76 .1981 & 34.9398 & NC & 4515 & -2 & -76.2253 & 34.9169 & NC \\
\hline 4372 & -0.3 & -76.1699 & 34.9619 & NC & 4444 & -1.4 & -76.198 & 34.9397 & NC & 4516 & -1.5 & -76.2257 & 34.9165 & NC \\
\hline 4373 & -0.5 & -76.1712 & 34.9616 & NC & 4445 & -1 & -76.1985 & 34.9395 & $\mathrm{NC}$ & 4517 & -1.8 & -76.2253 & 34.9164 & NC \\
\hline 4374 & -0.4 & -76.1704 & 34.9616 & $\mathrm{NC}$ & 4446 & -1.1 & -76.1984 & 34.9393 & NC & 4518 & -1.1 & -76.2261 & 34.9162 & NC \\
\hline 4375 & -0.6 & -76.1716 & 34.9613 & NC & 4447 & -1.1 & -76.1989 & 34.9391 & NC & 4519 & -1.9 & -76.2257 & 34.9161 & NC \\
\hline 4376 & -0.4 & -76.1708 & 34.9613 & NC & 4448 & -1 & -76.1988 & 34.939 & NC & 4520 & -2 & -76.2261 & 34.9158 & $\mathrm{NC}$ \\
\hline 4377 & -0.8 & -76.172 & 34.961 & NC & 4449 & -1.1 & -76.1993 & 34.9388 & NC & 4521 & -1.9 & -76.2266 & 34.9155 & NC \\
\hline 4378 & -0.4 & -76.1712 & 34.961 & NC & 4450 & -1.3 & -76.1997 & 34.9385 & NC & 4522 & -1.9 & -76.227 & 34.9152 & NC \\
\hline 4379 & -0.9 & -76.1724 & 34.9607 & $\mathrm{NC}$ & 4451 & -1.3 & -76.2001 & 34.9381 & $\mathrm{NC}$ & 4523 & -1.6 & -76.2274 & 34.9148 & $\mathrm{NC}$ \\
\hline 4380 & -0.9 & -76.1728 & 34.9603 & NC & 4452 & -1.4 & -76.2005 & 34.9378 & NC & 4524 & -1.6 & -76.2278 & 34.9145 & NC \\
\hline 4381 & -0.9 & -76.1732 & 34.96 & $\mathrm{NC}$ & 4453 & -1.3 & .76 .2009 & 34.9375 & NC & 4525 & -1.6 & -76.2282 & 34.9142 & NC \\
\hline 4382 & -0.9 & -76.1736 & 34.9597 & NC & 4454 & -1.4 & -76.2013 & 34.9371 & $\mathrm{NC}$ & 4526 & -1.7 & -76.2286 & 34.9139 & NC \\
\hline 4383 & -1 & .76 .174 & 34.9593 & $\mathrm{NC}$ & 4455 & -1.5 & -76.2016 & 34.9368 & NC & 4527 & -1.6 & -76.2291 & 34.9136 & NC \\
\hline 4384 & -0.9 & -76.1744 & 34.959 & $\mathrm{NC}$ & 4456 & -1.4 & -76.202 & 34.9365 & $\mathrm{NC}$ & 4528 & -1.5 & -76.2295 & 34.9133 & NC \\
\hline 4385 & -0.9 & -76.1748 & 34.9587 & $\mathrm{NC}$ & 4457 & -1.5 & -76.2024 & 34.9361 & NC & 4529 & -1.1 & -76.2299 & 34.913 & $\mathrm{NC}$ \\
\hline 4386 & -0.9 & -76.1752 & 34.9584 & $\mathrm{NC}$ & 4458 & -1.5 & -76.2028 & 34.9358 & $\mathrm{NC}$ & 4530 & -1 & -76.2303 & 34.9127 & $\mathrm{NC}$ \\
\hline 4387 & -0.8 & -76.1756 & 34.958 & $\mathrm{NC}$ & 4459 & -1.3 & -76.2032 & 34.9355 & NC & 4531 & -1 & -76.2307 & 34.9124 & NC \\
\hline 4388 & -0.9 & -76.176 & 34.9577 & $\mathrm{NC}$ & 4460 & -1.6 & -76.2036 & 34.9351 & NC & 4532 & -0.9 & -76.2311 & 34.912 & $\mathrm{NC}$ \\
\hline 4389 & -1 & -76.1764 & 34.9574 & $\mathrm{NC}$ & 4461 & -1.7 & -76.204 & 34.9348 & NC & 4533 & -0.9 & -76.2316 & 34.9117 & $\mathrm{NC}$ \\
\hline 4390 & -1 & -76.1768 & 34.957 & $\mathrm{NC}$ & 4462 & -1.7 & -76.2044 & 34.9345 & NC & 4534 & -1 & -76.232 & 34.9114 & $\mathrm{NC}$ \\
\hline 4391 & -0.9 & -76.1772 & 34.9567 & NC & 4463 & -1.7 & -76.2048 & 34.9341 & NC & 4535 & -1.1 & -76.2324 & 34.9111 & NC \\
\hline
\end{tabular}




\begin{tabular}{|c|c|c|c|c|c|c|c|c|c|c|c|c|c|c|}
\hline TR\# & RATE & LONG & LAT & ST & TR\# & RATE & LONG & LAT & ST & TR\# & RATE & LONG & LAT & ST \\
\hline 4536 & -1.3 & -76.2328 & 34.9108 & NC & 4608 & -0.6 & -76.2614 & 34.8898 & NC & 4680 & -1 & -76.2893 & 34.8681 & NC \\
\hline 4537 & -1.5 & -76.2332 & 34.9105 & NC & 4609 & -0.4 & -76.2618 & 34.8895 & NC & 4681 & -1 & -76.2897 & 34.8678 & NC \\
\hline 4538 & -1.4 & -76.2336 & 34.9102 & $\mathrm{NC}$ & 4610 & -0.3 & -76.2622 & 34.8891 & NC & 4682 & -1 & -76.2901 & 34.8674 & NC \\
\hline 4539 & -1.3 & -76.2341 & 34.9099 & NC & 4611 & -0.2 & -76.2626 & 34.8888 & NC & 4683 & -1 & -76.2905 & 34.8671 & $\mathrm{NC}$ \\
\hline 4540 & -1.2 & -76.2345 & 34.9096 & NC & 4612 & 0 & -76.263 & 34.8885 & NC & 4684 & -1.1 & -76.2909 & 34.8668 & NC \\
\hline 4541 & -1.1 & -76.2349 & 34.9092 & $\mathrm{NC}$ & 4613 & 0.2 & -76.2634 & 34.8882 & $\mathrm{NC}$ & 4685 & -1.1 & -76.2912 & 34.8664 & NC \\
\hline 4542 & -1.2 & -76.2353 & 34.9089 & NC & 4614 & 0.2 & -76.2638 & 34.8879 & NC & 4686 & -1.3 & -76.2916 & 34.8661 & NC \\
\hline 4543 & -1.4 & -76.2357 & 34.9086 & NC & 4615 & 0.2 & -76.2642 & 34.8875 & NC & 4687 & -1.2 & -76.292 & 34.8657 & NC \\
\hline 4544 & -1.3 & -76.2361 & 34.9083 & NC & 4616 & 0.2 & -76.2646 & 34.8872 & NC & 4688 & -1.4 & -76.2924 & 34.8654 & NC \\
\hline 4545 & -1.3 & -76.2366 & 34.908 & NC & 4617 & 0.2 & -76.265 & 34.8869 & NC & 4689 & -1.5 & -76.2928 & 34.8651 & NC \\
\hline 4546 & -1.4 & -76.237 & 34.9077 & NC & 4618 & 0.2 & -76.2654 & 34.8866 & NC & 4690 & -1.5 & -76.2932 & 34.8647 & $\mathrm{NC}$ \\
\hline 4547 & -1.4 & -76.2374 & 34.9074 & NC & 4619 & 0.2 & -76.2658 & 34.8863 & NC & 4691 & -1.5 & -76.2936 & 34.8644 & $\mathrm{NC}$ \\
\hline 4548 & -1.2 & -76.2378 & 34.9071 & NC & 4620 & 0.2 & -76.2663 & 34.886 & NC & 4692 & -1.7 & -76.294 & 34.8641 & NC \\
\hline 4549 & -0.9 & -76.2382 & 34.9068 & NC & 4621 & 0.2 & -76.2667 & 34.8856 & NC & 4693 & -1.9 & -76.2944 & 34.8637 & NC \\
\hline 4550 & -1.1 & -76.2386 & 34.9064 & NC & 4622 & 0.3 & -76.2671 & 34.8853 & NC & 4694 & -2.1 & -76.2947 & 34.8634 & NC \\
\hline 4551 & -1.3 & -76.2391 & 34.9061 & NC & 4623 & 0.3 & -76.2675 & 34.885 & NC & 4695 & -2.2 & -76.2951 & 34.863 & NC \\
\hline 4552 & -1.5 & -76.2395 & 34.9058 & NC & 4624 & 0.3 & -76.2679 & 34.8847 & NC & 4696 & -2.4 & -76.2955 & 34.8627 & $\mathrm{NC}$ \\
\hline 4553 & -1.6 & -76.2399 & 34.9055 & NC & 4625 & 0.3 & -76.2683 & 34.8844 & NC & 4697 & -2.6 & -76.2959 & 34.8624 & NC \\
\hline 4554 & -1.5 & -76.2403 & 34.9052 & NC & 4626 & 0.4 & -76.2687 & 34.884 & NC & 4698 & -2.6 & -76.2963 & 34.862 & NC \\
\hline 4555 & -1.5 & -76.2407 & 34.9049 & NC & 4627 & 0.4 & -76.2691 & 34.8837 & NC & 4699 & -2.5 & -76.2967 & 34.8617 & NC \\
\hline 4556 & -1.2 & -76.2411 & 34.9046 & NC & 4628 & 0.4 & -76.2695 & 34.8834 & NC & 4700 & -2.6 & -76.2971 & 34.8614 & NC \\
\hline 4557 & -0.8 & -76.2416 & 34.9043 & NC & 4629 & 0.5 & -76.2699 & 34.8831 & NC & 4701 & -2.7 & -76.2975 & 34.861 & NC \\
\hline 4558 & -0.8 & -76.242 & 34.9039 & NC & 4630 & 0.8 & -76.2703 & 34.8828 & NC & 4702 & -2.8 & -76.2978 & 34.8607 & NC \\
\hline 4559 & -0.7 & -76.2424 & 34.9036 & NC & 4631 & 1.1 & -76.2707 & 34.8824 & NC & 4703 & -2.8 & -76.2982 & 34.8603 & NC \\
\hline 4560 & -0.7 & -76.2428 & 34.9033 & NC & 4632 & 1.5 & -76.2712 & 34.8821 & $\mathrm{NC}$ & 4704 & -2.7 & -76.2986 & 34.86 & NC \\
\hline 4561 & -0.8 & -76.2432 & 34.903 & NC & 4633 & 1.9 & -76.2716 & 34.8818 & NC & 4705 & -2.7 & -76.299 & 34.8597 & NC \\
\hline 4562 & -1.1 & -76.2436 & 34.9027 & NC & 4634 & 2.5 & -76.272 & 34.8815 & NC & 4706 & -2.9 & -76.2994 & 34.8593 & NC \\
\hline 4563 & -1.3 & -76.2441 & 34.9024 & NC & 4635 & 3.1 & -76.2724 & 34.8812 & NC & 4707 & -2.9 & -76.2998 & 34.859 & NC \\
\hline 4564 & -1.5 & -76.2445 & 34.9021 & NC & 4636 & 1.8 & -76.2728 & 34.8809 & NC & 4708 & -2.9 & -76.3002 & 34.8587 & NC \\
\hline 4565 & -1.7 & -76.2449 & 34.9018 & $\mathrm{NC}$ & 4637 & 3.5 & -76.2732 & 34.8805 & NC & 4709 & -2.9 & -76.3006 & 34.8583 & NC \\
\hline 4566 & -1.8 & -76.2453 & 34.9015 & NC & 4638 & 6.6 & -76.2736 & 34.8802 & NC & 4710 & -3 & -76.301 & 34.858 & NC \\
\hline 4567 & -1.8 & -76.2457 & 34.9011 & NC & 4639 & 9.6 & -76.274 & 34.8799 & NC & 4711 & -3 & -76.3013 & 34.8576 & NC \\
\hline 4568 & -1.8 & -76.2461 & 34.9008 & NC & 4640 & 3.3 & -76.2744 & 34.8796 & NC & 4712 & -3 & -76.3017 & 34.8573 & NC \\
\hline 4569 & -1.7 & -76.2466 & 34.9005 & NC & 4641 & 1.8 & -76.2748 & 34.8793 & NC & 4713 & -3.2 & -76.3021 & 34.857 & NC \\
\hline 4570 & -1.7 & -76.247 & 34.9002 & $\mathrm{NC}$ & 4642 & -1 & -76.2752 & 34.8789 & NC & 4714 & -3.2 & -76.3025 & 34.8566 & NC \\
\hline 4571 & -1.7 & -76.2474 & 34.8999 & NC & 4643 & -3.6 & -76.2756 & 34.8786 & NC & 4715 & -2.8 & -76.3029 & 34.8563 & NC \\
\hline 4572 & -1.6 & -76.2478 & 34.8996 & NC & 4644 & -3.5 & -76.2761 & 34.8783 & NC & 4716 & -2.5 & -76.3033 & 34.8559 & $\mathrm{NC}$ \\
\hline 4573 & -1.6 & -76.2482 & 34.8993 & NC & 4645 & -3.2 & -76.2765 & 34.878 & NC & 4717 & -2.4 & -76.3037 & 34.8556 & NC \\
\hline 4574 & -1.5 & -76.2486 & 34.899 & NC & 4646 & -4.1 & -76.2769 & 34.8777 & NC & 4718 & -2.3 & -76.3041 & 34.8553 & NC \\
\hline 4575 & -1.4 & -76.2491 & 34.8987 & NC & 4647 & 9.2 & -76.2773 & 34.8774 & NC & 4719 & -2.2 & -76.3045 & 34.8549 & NC \\
\hline 4576 & -1.4 & -76.2495 & 34.8983 & NC & 4648 & 5.8 & -76.2777 & 34.877 & NC & 4720 & -1.9 & -76.3048 & 34.8546 & NC \\
\hline 4577 & -1.3 & -76.2499 & 34.898 & NC & 4649 & 2.2 & -76.2781 & 34.8767 & NC & 4721 & -1.9 & -76.3052 & 34.8543 & NC \\
\hline 4578 & -1.2 & -76.2503 & 34.8977 & NC & 4650 & 1.3 & -76.2785 & 34.8764 & NC & 4722 & -2.1 & -76.3056 & 34.8539 & NC \\
\hline 4579 & -1.3 & -76.2507 & 34.8974 & NC & 4651 & 0.4 & -76.2789 & 34.8761 & NC & 4723 & -2.4 & -76.306 & 34.8536 & NC \\
\hline 4580 & -1.3 & -76.2511 & 34.8971 & NC & 4652 & 0.2 & -76.2793 & 34.8758 & NC & 4724 & -2.8 & -76.3064 & 34.8532 & NC \\
\hline 4581 & -1 & -76.2516 & 34.8968 & NC & 4653 & 0 & -76.2797 & 34.8754 & NC & 4725 & -3.1 & -76.3068 & 34.8529 & NC \\
\hline 4582 & -1.2 & -76.252 & 34.8965 & NC & 4654 & 0 & -76.2801 & 34.8751 & NC & 4726 & -3.5 & -76.3072 & 34.8526 & $\mathrm{NC}$ \\
\hline 4583 & -1.3 & -76.2524 & 34.8962 & NC & 4655 & -0.1 & -76.2805 & 34.8748 & NC & 4727 & -3.9 & -76.3076 & 34.8522 & $\mathrm{NC}$ \\
\hline 4584 & -1.3 & -76.2528 & 34.8959 & NC & 4656 & -0.1 & -76.281 & 34.8745 & NC & 4728 & -4.6 & -76.308 & 34.8519 & NC \\
\hline 4585 & -0.8 & -76.254 & 34.8955 & NC & 4657 & -0.1 & -76.2823 & 34.8742 & NC & 4729 & -5.4 & -76.3083 & 34.8516 & NC \\
\hline 4586 & -1.4 & -76.2532 & 34.8955 & NC & 4658 & 0 & -76.2814 & 34.8742 & NC & 4730 & -7.2 & -76.3088 & 34.8513 & NC \\
\hline 4587 & -0.9 & -76.2544 & 34.8952 & $\mathrm{NC}$ & 4659 & 0 & -76.2827 & 34.8739 & NC & 4731 & -6 & -76.3087 & 34.8512 & NC \\
\hline 4588 & -1.5 & -76.2536 & 34.8952 & NC & 4660 & 0 & -76.2818 & 34.8739 & NC & 4732 & -8.4 & -76.3092 & 34.851 & NC \\
\hline 4589 & -0.9 & -76.2548 & 34.8949 & NC & 4661 & 0.1 & -76.2831 & 34.8735 & NC & 4733 & -6.9 & -76.3091 & 34.8509 & $\mathrm{NC}$ \\
\hline 4590 & -1.4 & -76.2541 & 34.8949 & NC & 4662 & 0 & -76.2822 & 34.8735 & NC & 4734 & -9.1 & -76.3096 & 34.8506 & NC \\
\hline 4591 & -1.4 & -76.2545 & 34.8946 & NC & 4663 & 0.1 & -76.2835 & 34.8732 & NC & 4735 & -8 & -76.3095 & 34.8505 & NC \\
\hline 4592 & -0.8 & -76.2552 & 34.8945 & NC & 4664 & 0 & .76 .2826 & 34.8732 & NC & 4736 & -9.6 & -76.31 & 34.8503 & NC \\
\hline 4593 & -1.5 & -76.2549 & 34.8943 & NC & 4665 & 0 & -76.283 & 34.8729 & NC & 4737 & -9 & -76.3099 & 34.8502 & NC \\
\hline 4594 & -1.1 & -76.2556 & 34.8942 & NC & 4666 & 0.1 & -76.2839 & 34.8728 & NC & 4738 & -10.2 & -76.3103 & 34.8499 & NC \\
\hline 4595 & -1.4 & -76.256 & 34.8939 & NC & 4667 & 0 & -76.2842 & 34.8725 & NC & 4739 & -11 & -76.3107 & 34.8496 & $\mathrm{NC}$ \\
\hline 4596 & -1.3 & -76.2565 & 34.8936 & NC & 4668 & -0.1 & -76.2846 & 34.8722 & NC & 4740 & -11.7 & -76.3111 & 34.8493 & NC \\
\hline 4597 & -1.2 & -76.2569 & 34.8933 & NC & 4669 & -0.1 & -76.285 & 34.8718 & NC & 4741 & -12.5 & -76.3115 & 34.8489 & NC \\
\hline 4598 & -1.2 & -76.2573 & 34.893 & NC & 4670 & -0.3 & -76.2854 & 34.8715 & NC & 4742 & -14.2 & -76.3119 & 34.8486 & $\mathrm{NC}$ \\
\hline 4599 & -1.2 & .76 .2577 & 34.8926 & NC & 4671 & -0.3 & -76.2858 & 34.8712 & NC & 4743 & -2.1 & -76.3123 & 34.8482 & NC \\
\hline 4600 & -1.1 & -76.2581 & 34.8923 & NC & 4672 & -0.4 & -76.2862 & 34.8708 & NC & 4744 & -2.3 & -76.3127 & 34.8479 & NC \\
\hline 4601 & -1.1 & -76.2585 & 34.892 & NC & 4673 & -0.4 & -76.2866 & 34.8705 & NC & 4745 & -2.5 & -76.3131 & 34.8476 & $\mathrm{NC}$ \\
\hline 4602 & -1.2 & .76 .2589 & 34.8917 & NC & 4674 & -0.6 & -76.287 & 34.8701 & NC & 4746 & -2.4 & -76.3134 & 34.8472 & $\mathrm{NC}$ \\
\hline 4603 & -1.3 & -76.2593 & 34.8914 & NC & 4675 & -0.7 & -76.2874 & 34.8698 & NC & 4747 & -18.5 & -76.3138 & 34.8469 & NC \\
\hline 4604 & -1.1 & -76.2597 & 34.891 & NC & 4676 & -0.8 & -76.2877 & 34.8695 & NC & 4748 & -16.1 & -76.3142 & 34.8465 & NC \\
\hline 4605 & -1 & -76.2601 & 34.8907 & NC & 4677 & -0.9 & -76.2881 & 34.8691 & NC & 4749 & -14.9 & -76.3146 & 34.8462 & NC \\
\hline 4606 & -0.8 & -76.2605 & 34.8904 & NC & 4678 & -0.8 & -76.2885 & 34.8688 & NC & 4750 & -13.9 & -76.315 & 34.8458 & $\mathrm{NC}$ \\
\hline 4607 & -0.6 & -76.2609 & 34.8901 & NC & 4679 & -0.7 & -76.2889 & 34.8685 & NC & 4751 & -12.7 & -76.3154 & 34.8455 & NC \\
\hline
\end{tabular}




\begin{tabular}{|c|c|c|c|c|c|c|c|c|c|c|c|c|c|c|}
\hline TR\# & RATE & LONG & LAT & ST & TR\# & RATE & LONG & LAT & ST & TR\# & RATE & LONG & LAT & ST \\
\hline 4752 & -11.8 & -76.3158 & 34.8452 & NC & 4824 & -2.7 & -76.3422 & 34.8216 & NC & 4896 & -0.5 & -76.368 & 34.7976 & $\mathrm{NC}$ \\
\hline 4753 & -11 & -76.3162 & 34.8448 & NC & 4825 & -2.6 & -76.3426 & 34.8213 & NC & 4897 & -0.5 & -76.3684 & 34.7973 & NC \\
\hline 4754 & -10.7 & -76.3165 & 34.8445 & NC & 4826 & -2.5 & -76.3429 & 34.8209 & NC & 4898 & -0.5 & -76.3687 & 34.7969 & NC \\
\hline 4755 & -10.4 & -76.3169 & 34.8441 & NC & 4827 & -2.4 & -76.3433 & 34.8206 & NC & 4899 & -0.4 & -76.3691 & 34.7966 & NC \\
\hline 4756 & -9.9 & -76.3173 & 34.8438 & NC & 4828 & -2.4 & -76.3437 & 34.8203 & NC & 4900 & -0.6 & -76.3695 & 34.7962 & NC \\
\hline 4757 & -9.2 & -76.3177 & 34.8435 & NC & 4829 & -2 & -76.3441 & 34.8199 & NC & 4901 & -0.6 & -76.3698 & 34.7959 & NC \\
\hline 4758 & -8.5 & -76.3181 & 34.8431 & NC & 4830 & -2.1 & -76.3444 & 34.8196 & NC & 4902 & -0.6 & -76.3702 & 34.7955 & NC \\
\hline 4759 & -7.7 & -76.3185 & 34.8428 & NC & 4831 & -2 & -76.3448 & 34.8192 & NC & 4903 & -0.5 & -76.3706 & 34.7952 & NC \\
\hline 4760 & -7 & -76.3189 & 34.8424 & $\mathrm{NC}$ & 4832 & -1.9 & -76.3452 & 34.8189 & NC & 4904 & -0.7 & -76.371 & 34.7948 & NC \\
\hline 4761 & -6.8 & -76.3193 & 34.8421 & NC & 4833 & -1.8 & -76.3456 & 34.8185 & NC & 4905 & -0.8 & -76.3713 & 34.7945 & NC \\
\hline 4762 & -6.5 & -76.3196 & 34.8418 & NC & 4834 & -1.9 & -76.3459 & 34.8182 & NC & 4906 & -0.8 & -76.3717 & 34.7941 & NC \\
\hline 4763 & -6.2 & -76.32 & 34.8414 & NC & 4835 & -1.8 & -76.3463 & 34.8178 & NC & 4907 & -0.8 & -76.3721 & 34.7938 & NC \\
\hline 4764 & -6 & -76.3204 & 34.8411 & $\mathrm{NC}$ & 4836 & -1.7 & -76.3467 & 34.8175 & NC & 4908 & -0.9 & -76.3724 & 34.7934 & NC \\
\hline 4765 & -5.7 & -76.3208 & 34.8407 & $\mathrm{NC}$ & 4837 & -1.5 & -76.3471 & 34.8172 & NC & 4909 & -0.7 & -76.3728 & 34.7931 & NC \\
\hline 4766 & -5.8 & -76.3212 & 34.8404 & NC & 4838 & -1.5 & -76.3474 & 34.8168 & NC & 4910 & -0.7 & -76.3732 & 34.7927 & NC \\
\hline 4767 & -5.8 & -76.3216 & 34.8401 & NC & 4839 & -1.4 & -76.3478 & 34.8165 & NC & 4911 & -0.6 & -76.3735 & 34.7924 & NC \\
\hline 4768 & -5.8 & -76.322 & 34.8397 & $\mathrm{NC}$ & 4840 & -1.4 & -76.3482 & 34.8161 & $\mathrm{NC}$ & 4912 & -0.8 & -76.3739 & 34.792 & NC \\
\hline 4769 & -5.8 & -76.3224 & 34.8394 & $\mathrm{NC}$ & 4841 & -1.5 & -76.3486 & 34.8158 & NC & 4913 & -0.9 & .76 .3743 & 34.7917 & NC \\
\hline 4770 & -5.8 & -76.3227 & 34.839 & NC & 4842 & -1.5 & -76.3489 & 34.8154 & NC & 4914 & -0.7 & -76.3747 & 34.7913 & NC \\
\hline 4771 & -5.8 & -76.3231 & 34.8387 & NC & 4843 & -1.6 & -76.3493 & 34.8151 & NC & 4915 & -0.5 & -76.375 & 34.791 & NC \\
\hline 4772 & -5.7 & -76.3235 & 34.8383 & NC & 4844 & -1.6 & -76.3497 & 34.8148 & NC & 4916 & -0.5 & -76.3754 & 34.7906 & NC \\
\hline 4773 & -5.5 & -76.3239 & 34.838 & NC & 4845 & -1.7 & -76.3501 & 34.8144 & NC & 4917 & -0.3 & -76.3758 & 34.7903 & NC \\
\hline 4774 & -5.6 & -76.3243 & 34.8377 & $\mathrm{NC}$ & 4846 & -1.6 & -76.3504 & 34.8141 & NC & 4918 & -0.5 & -76.3761 & 34.7899 & NC \\
\hline 4775 & -5.7 & -76.3247 & 34.8373 & NC & 4847 & -1.7 & -76.3508 & 34.8137 & NC & 4919 & -0.5 & -76.3765 & 34.7896 & NC \\
\hline 4776 & -5.7 & -76.3251 & 34.837 & NC & 4848 & -1.5 & -76.3512 & 34.8134 & NC & 4920 & -0.3 & -76.3769 & 34.7892 & NC \\
\hline 4777 & -5.7 & -76.3255 & 34.8366 & NC & 4849 & -1.3 & -76.3516 & 34.813 & NC & 4921 & -0.3 & -76.3773 & 34.7889 & NC \\
\hline 4778 & -5.7 & -76.3258 & 34.8363 & NC & 4850 & -1.2 & -76.3519 & 34.8127 & NC & 4922 & -0.4 & -76.3776 & 34.7885 & NC \\
\hline 4779 & -5.7 & -76.3262 & 34.836 & NC & 4851 & -1.1 & -76.3523 & 34.8123 & NC & 4923 & -0.3 & -76.378 & 34.7882 & NC \\
\hline 4780 & -5.6 & -76.3266 & 34.8356 & NC & 4852 & -1.2 & -76.3527 & 34.812 & NC & 4924 & -0.3 & -76.3784 & 34.7878 & NC \\
\hline 4781 & -5.3 & -76.327 & 34.8353 & $\mathrm{NC}$ & 4853 & -1.2 & -76.3531 & 34.8117 & NC & 4925 & -0.3 & -76.3787 & 34.7875 & NC \\
\hline 4782 & -5.1 & -76.3274 & 34.8349 & NC & 4854 & -0.6 & -76.3534 & 34.8113 & NC & 4926 & -0.5 & -76.3791 & 34.7871 & NC \\
\hline 4783 & -4.9 & -76.3278 & 34.8346 & NC & 4855 & -0.2 & -76.3538 & 34.811 & NC & 4927 & -0.3 & -76.3795 & 34.7868 & NC \\
\hline 4784 & -5.2 & -76.3282 & 34.8343 & NC & 4856 & -0.2 & -76.3542 & 34.8106 & NC & 4928 & -0.5 & -76.3798 & 34.7864 & NC \\
\hline 4785 & -5.3 & -76.3286 & 34.8339 & NC & 4857 & -0.3 & -76.3546 & 34.8103 & NC & 4929 & -0.3 & -76.3802 & 34.7861 & NC \\
\hline 4786 & -5.2 & -76.3289 & 34.8336 & NC & 4858 & -0.1 & -76.3549 & 34.8099 & NC & 4930 & -0.2 & -76.3806 & 34.7857 & NC \\
\hline 4787 & -5.1 & -76.3293 & 34.8332 & NC & 4859 & 0 & -76.3553 & 34.8096 & NC & 4931 & -0.1 & -76.381 & 34.7854 & NC \\
\hline 4788 & -5.2 & -76.3297 & 34.8329 & NC & 4860 & -0.2 & -76.3557 & 34.8093 & NC & 4932 & -0.3 & -76.3813 & 34.785 & NC \\
\hline 4789 & -5.1 & -76.3301 & 34.8326 & NC & 4861 & -0.4 & -76.3561 & 34.8089 & $\mathrm{NC}$ & 4933 & -0.3 & -76.3817 & 34.7847 & NC \\
\hline 4790 & -5.1 & -76.3305 & 34.8322 & NC & 4862 & -0.3 & -76.3564 & 34.8086 & $\mathrm{NC}$ & 4934 & -0.3 & -76.3821 & 34.7843 & NC \\
\hline 4791 & -5.1 & -76.3309 & 34.8319 & NC & 4863 & -0.1 & -76.3568 & 34.8082 & NC & 4935 & -0.4 & -76.3824 & 34.784 & NC \\
\hline 4792 & -5.2 & -76.3313 & 34.8315 & NC & 4864 & -0.1 & -76.3572 & 34.8079 & $\mathrm{NC}$ & 4936 & -0.4 & -76.3828 & 34.7836 & NC \\
\hline 4793 & -5.2 & -76.3317 & 34.8312 & NC & 4865 & -0.2 & -76.3576 & 34.8075 & NC & 4937 & -0.6 & -76.3832 & 34.7833 & NC \\
\hline 4794 & -5.2 & -76.332 & 34.8308 & NC & 4866 & -0.4 & -76.3579 & 34.8072 & $\mathrm{NC}$ & 4938 & -0.5 & -76.3835 & 34.7829 & NC \\
\hline 4795 & -5.2 & -76.3324 & 34.8305 & $\mathrm{NC}$ & 4867 & -0.6 & -76.3583 & 34.8068 & $\mathrm{NC}$ & 4939 & -0.4 & -76.3839 & 34.7826 & NC \\
\hline 4796 & -5 & -76.3328 & 34.8302 & NC & 4868 & -0.5 & -76.3587 & 34.8065 & $\mathrm{NC}$ & 4940 & -0.3 & -76.3839 & 34.7824 & NC \\
\hline 4797 & -4.8 & -76.3332 & 34.8298 & NC & 4869 & -0.5 & -76.3591 & 34.8062 & $\mathrm{NC}$ & 4941 & -0.5 & -76.3843 & 34.7822 & NC \\
\hline 4798 & -4.7 & -76.3336 & 34.8295 & NC & 4870 & -0.4 & -76.3594 & 34.8058 & NC & 4942 & -0.5 & -76.3842 & 34.7821 & NC \\
\hline 4799 & -4.6 & -76.334 & 34.8291 & $\mathrm{NC}$ & 4871 & -0.2 & -76.3598 & 34.8055 & $\mathrm{NC}$ & 4943 & -0.7 & -76.3847 & 34.7819 & NC \\
\hline 4800 & -4.5 & -76.3344 & 34.8288 & NC & 4872 & 0.1 & -76.3598 & 34.8053 & NC & 4944 & -0.6 & -76.3845 & 34.7817 & NC \\
\hline 4801 & -4.3 & -76.3348 & 34.8285 & NC & 4873 & 0 & -76.3602 & 34.8051 & $\mathrm{NC}$ & 4945 & -0.9 & -76.3849 & 34.7813 & NC \\
\hline 4802 & -4.3 & -76.3351 & 34.8281 & NC & 4874 & 0.3 & -76.3602 & 34.805 & NC & 4946 & -0.7 & -76.3852 & 34.7809 & NC \\
\hline 4803 & -3.4 & -76.3354 & 34.8278 & NC & 4875 & 0.3 & -76.3606 & 34.8048 & $\mathrm{NC}$ & 4947 & -0.4 & -76.3856 & 34.7806 & NC \\
\hline 4804 & -4.2 & -76.3355 & 34.8278 & $\mathrm{NC}$ & 4876 & 0.3 & -76.3606 & 34.8046 & NC & 4948 & -0.3 & -76.3859 & 34.7802 & NC \\
\hline 4805 & -3.5 & -76.3358 & 34.8275 & NC & 4877 & 0.3 & -76.361 & 34.8043 & NC & 4949 & -0.2 & -76.3862 & 34.7798 & NC \\
\hline 4806 & -4.1 & -76.3359 & 34.8274 & $\mathrm{NC}$ & 4878 & 0.2 & -76.3613 & 34.8039 & $\mathrm{NC}$ & 4950 & -0.1 & -76.3866 & 34.7795 & NC \\
\hline 4807 & -3.4 & -76.3362 & 34.8271 & $\mathrm{NC}$ & 4879 & -0.1 & -76.3617 & 34.8036 & NC & 4951 & 0 & -76.3869 & 34.7791 & NC \\
\hline 4808 & -4.1 & -76.3363 & 34.8271 & $\mathrm{NC}$ & 4880 & 0 & -76.3621 & 34.8032 & NC & 4952 & -0.3 & -76.3873 & 34.7787 & NC \\
\hline 4809 & -3.5 & -76.3366 & 34.8268 & $\mathrm{NC}$ & 4881 & 0 & -76.3624 & 34.8029 & NC & 4953 & -0.6 & -76.3876 & 34.7784 & NC \\
\hline 4810 & -3.6 & -76.3369 & 34.8264 & $\mathrm{NC}$ & 4882 & 0 & -76.3628 & 34.8025 & NC & 4954 & -0.5 & -76.3879 & 34.778 & NC \\
\hline 4811 & -3.6 & -76.3373 & 34.8261 & $\mathrm{NC}$ & 4883 & -0.2 & -76.3632 & 34.8022 & NC & 4955 & -0.1 & -76.3883 & 34.7776 & NC \\
\hline 4812 & -3.3 & -76.3377 & 34.8258 & $\mathrm{NC}$ & 4884 & -0.2 & -76.3635 & 34.8018 & NC & 4956 & -0.2 & -76.3886 & 34.7773 & NC \\
\hline 4813 & -3.1 & -76.3381 & 34.8254 & $\mathrm{NC}$ & 4885 & -0.2 & -76.3639 & 34.8015 & $\mathrm{NC}$ & 4957 & -0.2 & -76.3889 & 34.7769 & NC \\
\hline 4814 & -3 & -76.3384 & 34.8251 & NC & 4886 & -0.1 & -76.3643 & 34.8011 & NC & 4958 & -0.4 & -76.3893 & 34.7765 & NC \\
\hline 4815 & -3 & -76.3388 & 34.8247 & $\mathrm{NC}$ & 4887 & -0.1 & -76.3647 & 34.8008 & NC & 4959 & -0.6 & -76.3896 & 34.7762 & NC \\
\hline 4816 & -3 & -76.3392 & 34.8244 & $\mathrm{NC}$ & 4888 & -0.1 & -76.365 & 34.8004 & NC & 4960 & -0.8 & -76.39 & 34.7758 & NC \\
\hline 4817 & -3 & -76.3396 & 34.824 & NC & 4889 & 0 & -76.3654 & 34.8001 & NC & 4961 & -0.7 & -76.3903 & 34.7754 & NC \\
\hline 4818 & -2.8 & -76.3399 & 34.8237 & NC & 4890 & 0 & -76.3658 & 34.7997 & NC & 4962 & -0.7 & -76.3906 & 34.775 & NC \\
\hline 4819 & -2.6 & -76.3403 & 34.8233 & NC & 4891 & -0.1 & -76.3661 & 34.7994 & NC & 4963 & -0.7 & -76.391 & 34.7747 & NC \\
\hline 4820 & -2.5 & -76.3407 & 34.823 & $\mathrm{NC}$ & 4892 & -0.5 & -76.3665 & 34.799 & NC & 4964 & -0.7 & -76.3913 & 34.7743 & NC \\
\hline 4821 & -2.7 & -76.3411 & 34.8227 & NC & 4893 & -0.7 & -76.3669 & 34.7987 & NC & 4965 & -1 & -76.3917 & 34.7739 & NC \\
\hline 4822 & -2.6 & -76.3414 & 34.8223 & $\mathrm{NC}$ & 4894 & -0.6 & -76.3672 & 34.7983 & NC & 4966 & -1.1 & -76.392 & 34.7736 & NC \\
\hline 4823 & -2.6 & -76.3418 & 34.822 & $\mathrm{NC}$ & 4895 & -0.5 & -76.3676 & 34.798 & NC & 4967 & -1.1 & -76.3923 & 34.7732 & NC \\
\hline
\end{tabular}




\begin{tabular}{|c|c|c|c|c|c|c|c|c|c|c|c|c|c|c|}
\hline TR\# & RATE & LONG & LAT & ST & TR\# & RATE & LONG & LAT & ST & TR\# & RATE & LONG & LAT & ST \\
\hline 4968 & -1.1 & -76.3927 & 34.7728 & NC & 5040 & -0.3 & -76.4147 & 34.7487 & NC & 5112 & -1 & -76.4379 & 34.723 & NC \\
\hline 4969 & -1.2 & -76.393 & 34.7725 & NC & 5041 & -0.2 & -76.4151 & 34.7483 & NC & 5113 & -1 & -76.4383 & 34.7227 & NC \\
\hline 4970 & -1.1 & -76.3934 & 34.7721 & NC & 5042 & -0.2 & .76 .4154 & 34.7479 & NC & 5114 & -1.1 & -76.4386 & 34.7223 & $\mathrm{NC}$ \\
\hline 4971 & -1 & -76.3937 & 34.7717 & NC & 5043 & -0.2 & -76.4157 & 34.7476 & NC & 5115 & -1.2 & -76.4389 & 34.7219 & NC \\
\hline 4972 & -0.9 & -76.394 & 34.7714 & NC & 5044 & -0.3 & -76.4161 & 34.7472 & NC & 5116 & -1.2 & -76.4393 & 34.7216 & $\mathrm{NC}$ \\
\hline 4973 & -0.9 & -76.3944 & 34.771 & NC & 5045 & -0.4 & -76.4164 & 34.7468 & NC & 5117 & -1.3 & -76.4396 & 34.7212 & NC \\
\hline 4974 & -1 & -76.3947 & 34.7706 & NC & 5046 & -0.3 & -76.4168 & 34.7464 & NC & 5118 & -1.2 & -76.4399 & 34.7208 & $\mathrm{NC}$ \\
\hline 4975 & -0.9 & -76.3951 & 34.7702 & NC & 5047 & -0.4 & -76.4171 & 34.7461 & NC & 5119 & -1 & -76.4403 & 34.7204 & $\mathrm{NC}$ \\
\hline 4976 & -0.6 & -76.3954 & 34.7699 & NC & 5048 & -0.3 & -76.4174 & 34.7457 & NC & 5120 & -0.7 & -76.4406 & 34.7201 & NC \\
\hline 4977 & -0.7 & -76.3957 & 34.7695 & NC & 5049 & 0.1 & -76.4178 & 34.7453 & NC & 5121 & -0.8 & -76.4409 & 34.7197 & NC \\
\hline 4978 & -0.5 & -76.3961 & 34.7691 & NC & 5050 & 0.4 & -76.4181 & 34.745 & NC & 5122 & -0.8 & -76.4413 & 34.7193 & NC \\
\hline 4979 & -0.5 & -76.3964 & 34.7688 & NC & 5051 & 0.4 & -76.4184 & 34.7446 & NC & 5123 & -0.8 & -76.4416 & 34.719 & NC \\
\hline 4980 & -0.2 & -76.3968 & 34.7684 & NC & 5052 & 0.2 & -76.4188 & 34.7442 & NC & 5124 & -0.4 & -76.442 & 34.7186 & $\mathrm{NC}$ \\
\hline 4981 & -0.1 & -76.3971 & 34.768 & NC & 5053 & 0.2 & -76.4191 & 34.7439 & NC & 5125 & -0.7 & -76.4423 & 34.7182 & NC \\
\hline 4982 & 0 & -76.3974 & 34.7677 & NC & 5054 & 0 & -76.4194 & 34.7435 & NC & 5126 & -0.7 & -76.4426 & 34.7178 & NC \\
\hline 4983 & 0.4 & -76.3978 & 34.7673 & NC & 5055 & 0 & -76.4198 & 34.7431 & NC & 5127 & -0.6 & -76.443 & 34.7175 & NC \\
\hline 4984 & 0.5 & -76.3981 & 34.7669 & NC & 5056 & 0.1 & -76.4201 & 34.7428 & NC & 5128 & -0.5 & .76 .4433 & 34.7171 & NC \\
\hline 4985 & 0.6 & -76.3985 & 34.7666 & NC & 5057 & 0.2 & -76.4204 & 34.7424 & NC & 5129 & -0.8 & -76.4436 & 34.7167 & NC \\
\hline 4986 & 0.6 & -76.3988 & 34.7662 & NC & 5058 & 0.1 & -76.4208 & 34.742 & NC & 5130 & -0.5 & -76.444 & 34.7164 & NC \\
\hline 4987 & 0.7 & -76.3991 & 34.7658 & NC & 5059 & 0.1 & -76.4211 & 34.7416 & NC & 5131 & -0.4 & -76.4443 & 34.716 & NC \\
\hline 4988 & 0.6 & -76.3995 & 34.7654 & NC & 5060 & 0 & -76.4214 & 34.7413 & NC & 5132 & -0.4 & -76.4446 & 34.7156 & NC \\
\hline 4989 & 0.5 & -76.3998 & 34.7651 & NC & 5061 & -0.3 & -76.4218 & 34.7409 & NC & 5133 & -0.3 & .76 .445 & 34.7153 & NC \\
\hline 4990 & 0.4 & -76.4002 & 34.7647 & NC & 5062 & -0.5 & -76.4221 & 34.7405 & NC & 5134 & -0.2 & -76.4453 & 34.7149 & NC \\
\hline 4991 & 0.4 & -76.4005 & 34.7643 & NC & 5063 & -0.5 & -76.4224 & 34.7402 & NC & 5135 & -0.3 & -76.4456 & 34.7145 & $\mathrm{NC}$ \\
\hline 4992 & 0.1 & -76.4008 & 34.764 & NC & 5064 & -0.5 & -76.4228 & 34.7398 & NC & 5136 & -0.5 & -76.446 & 34.7141 & NC \\
\hline 4993 & 0.1 & -76.4012 & 34.7636 & NC & 5065 & -0.2 & -76.4231 & 34.7394 & NC & 5137 & -0.5 & -76.4463 & 34.7138 & NC \\
\hline 4994 & 0 & -76.4015 & 34.7632 & NC & 5066 & -0.1 & -76.4234 & 34.7391 & NC & 5138 & -0.3 & -76.4466 & 34.7134 & NC \\
\hline 4995 & 0 & -76.4018 & 34.7629 & NC & 5067 & 0.1 & -76.4238 & 34.7387 & NC & 5139 & 0.1 & -76.447 & 34.713 & NC \\
\hline 4996 & 0.1 & -76.4022 & 34.7625 & NC & 5068 & 0 & -76.4241 & 34.7383 & NC & 5140 & 0.3 & -76.4473 & 34.7127 & NC \\
\hline 4997 & 0 & -76.4025 & 34.7621 & NC & 5069 & 0 & -76.4244 & 34.738 & NC & 5141 & 0.1 & -76.4477 & 34.7123 & $\mathrm{NC}$ \\
\hline 4998 & 0 & -76.4029 & 34.7618 & NC & 5070 & 0 & -76.4248 & 34.7376 & NC & 5142 & 0.1 & -76.448 & 34.7119 & $\mathrm{NC}$ \\
\hline 4999 & -0.1 & -76.4032 & 34.7614 & NC & 5071 & -0.3 & -76.4251 & 34.7372 & NC & 5143 & 0.1 & -76.4483 & 34.7116 & NC \\
\hline 5000 & 0 & -76.4035 & 34.761 & NC & 5072 & -0.5 & -76.4254 & 34.7369 & NC & 5144 & 0.1 & -76.4487 & 34.7112 & NC \\
\hline 5001 & 0 & -76.4039 & 34.7607 & NC & 5073 & -0.5 & -76.4258 & 34.7365 & NC & 5145 & 0 & -76.4485 & 34.711 & NC \\
\hline 5002 & 0.2 & -76.4042 & 34.7603 & NC & 5074 & -0.7 & -76.4261 & 34.7361 & NC & 5146 & 0 & -76.449 & 34.7108 & NC \\
\hline 5003 & 0.3 & -76.4046 & 34.7599 & NC & 5075 & -0.5 & -76.4264 & 34.7357 & NC & 5147 & 0.2 & -76.4489 & 34.7107 & $\mathrm{NC}$ \\
\hline 5004 & -0.4 & -76.4047 & 34.7597 & NC & 5076 & -0.4 & -76.4268 & 34.7354 & NC & 5148 & 0 & -76.4493 & 34.7104 & NC \\
\hline 5005 & 0.4 & -76.4049 & 34.7595 & NC & 5077 & -0.3 & -76.4269 & 34.7353 & NC & 5149 & 0.1 & -76.4492 & 34.7103 & NC \\
\hline 5006 & -0.4 & -76.4051 & 34.7594 & NC & 5078 & -0.4 & -76.4271 & 34.735 & NC & 5150 & 0.1 & -76.4495 & 34.7099 & NC \\
\hline 5007 & 0.4 & -76.4052 & 34.7592 & NC & 5079 & -0.3 & -76.4272 & 34.7349 & NC & 5151 & 0.2 & -76.4498 & 34.7095 & NC \\
\hline 5008 & -0.3 & -76.4054 & 34.759 & NC & 5080 & -0.5 & -76.4274 & 34.7346 & NC & 5152 & 0.2 & -76.4501 & 34.7091 & NC \\
\hline 5009 & 0.3 & -76.4056 & 34.7588 & NC & 5081 & -0.4 & -76.4275 & 34.7345 & NC & 5153 & 0.2 & -76.4504 & 34.7087 & NC \\
\hline 5010 & -0.4 & -76.4057 & 34.7586 & NC & 5082 & -0.6 & -76.4279 & 34.7341 & NC & 5154 & 0.2 & -76.4508 & 34.7084 & NC \\
\hline 5011 & 0.4 & -76.4059 & 34.7584 & NC & 5083 & -0.3 & .76 .4282 & 34.7338 & NC & 5155 & 0 & -76.4511 & 34.708 & NC \\
\hline 5012 & -0.3 & -76.4061 & 34.7583 & NC & 5084 & -0.1 & -76.4285 & 34.7334 & NC & 5156 & -0.1 & -76.4514 & 34.7076 & NC \\
\hline 5013 & 0.5 & -76.4063 & 34.7581 & NC & 5085 & -0.3 & -76.4289 & 34.733 & NC & 5157 & -0.2 & -76.4517 & 34.7072 & NC \\
\hline 5014 & -0.2 & -76.4064 & 34.7579 & NC & 5086 & -0.5 & -76.4292 & 34.7327 & NC & 5158 & -0.4 & -76.452 & 34.7068 & NC \\
\hline 5015 & 0.7 & -76.4066 & 34.7577 & NC & 5087 & -0.6 & -76.4296 & 34.7323 & NC & 5159 & -0.3 & -76.4524 & 34.7065 & NC \\
\hline 5016 & -0.1 & -76.4067 & 34.7575 & NC & 5088 & -0.7 & -76.4299 & 34.7319 & NC & 5160 & -0.2 & -76.4527 & 34.7061 & NC \\
\hline 5017 & -0.3 & -76.4071 & 34.7571 & NC & 5089 & -0.9 & -76.4302 & 34.7316 & NC & 5161 & -0.3 & -76.453 & 34.7057 & NC \\
\hline 5018 & -0.7 & -76.4074 & 34.7568 & NC & 5090 & -0.7 & -76.4306 & 34.7312 & NC & 5162 & -0.4 & -76.4533 & 34.7053 & NC \\
\hline 5019 & -0.5 & -76.4077 & 34.7564 & NC & 5091 & -0.9 & -76.4309 & 34.7308 & NC & 5163 & -0.3 & -76.4536 & 34.7049 & NC \\
\hline 5020 & -0.3 & -76.4081 & 34.756 & NC & 5092 & -0.9 & -76.4312 & 34.7304 & NC & 5164 & -0.3 & -76.4539 & 34.7045 & NC \\
\hline 5021 & -0.3 & -76.4084 & 34.7557 & NC & 5093 & -0.9 & -76.4316 & 34.7301 & NC & 5165 & -0.4 & -76.4543 & 34.7042 & NC \\
\hline 5022 & -0.3 & -76.4087 & 34.7553 & NC & 5094 & -1 & -76.4319 & 34.7297 & NC & 5166 & -0.6 & -76.4546 & 34.7038 & NC \\
\hline 5023 & -0.5 & -76.4091 & 34.7549 & NC & 5095 & -1 & -76.4322 & 34.7293 & NC & 5167 & -0.5 & -76.4549 & 34.7034 & NC \\
\hline 5024 & -0.7 & -76.4094 & 34.7546 & NC & 5096 & -0.9 & -76.4326 & 34.729 & NC & 5168 & -0.5 & -76.4552 & 34.703 & NC \\
\hline 5025 & -0.4 & -76.4097 & 34.7542 & NC & 5097 & -0.9 & -76.4329 & 34.7286 & NC & 5169 & -0.2 & -76.4555 & 34.7026 & NC \\
\hline 5026 & -0.1 & -76.4101 & 34.7538 & NC & 5098 & -1 & -76.4332 & 34.7282 & NC & 5170 & 0 & -76.4559 & 34.7023 & NC \\
\hline 5027 & 0 & -76.4104 & 34.7535 & NC & 5099 & -1.1 & -76.4336 & 34.7279 & NC & 5171 & 0.2 & -76.4562 & 34.7019 & NC \\
\hline 5028 & 0 & -76.4107 & 34.7531 & NC & 5100 & -1.2 & -76.4339 & 34.7275 & NC & 5172 & 0.3 & -76.4565 & 34.7015 & NC \\
\hline 5029 & -0.2 & -76.4111 & 34.7527 & NC & 5101 & -1.1 & -76.4342 & 34.7271 & NC & 5173 & 0.2 & -76.4568 & 34.7011 & $\mathrm{NC}$ \\
\hline 5030 & -0.4 & -76.4114 & 34.7524 & NC & 5102 & -1 & -76.4346 & 34.7267 & NC & 5174 & 0.2 & -76.4571 & 34.7007 & NC \\
\hline 5031 & -0.4 & -76.4117 & 34.752 & NC & 5103 & -1 & .76 .4349 & 34.7264 & NC & 5175 & 0.2 & -76.4574 & 34.7003 & NC \\
\hline 5032 & -0.3 & -76.4121 & 34.7516 & NC & 5104 & -1.1 & -76.4353 & 34.726 & NC & 5176 & 0.1 & -76.4578 & 34.7 & NC \\
\hline 5033 & -0.3 & -76.4124 & 34.7512 & NC & 5105 & -1.1 & -76.4356 & 34.7256 & NC & 5177 & -0.1 & -76.4581 & 34.6996 & NC \\
\hline 5034 & -0.5 & -76.4127 & 34.7509 & NC & 5106 & -1.1 & -76.4359 & 34.7253 & NC & 5178 & 0 & -76.4584 & 34.6992 & NC \\
\hline 5035 & -0.3 & -76.4131 & 34.7505 & NC & 5107 & -1 & -76.4363 & 34.7249 & NC & 5179 & -0.7 & -76.5881 & 34.699 & NC \\
\hline 5036 & -0.1 & -76.4134 & 34.7501 & NC & 5108 & -1 & -76.4366 & 34.7245 & NC & 5180 & -0.1 & -76.4587 & 34.6988 & NC \\
\hline 5037 & -0.2 & -76.4137 & 34.7498 & NC & 5109 & -1 & -76.4369 & 34.7241 & NC & 5181 & -0.4 & -76.5875 & 34.6988 & NC \\
\hline 5038 & -0.4 & -76.4141 & 34.7494 & NC & 5110 & -1 & -76.4373 & 34.7238 & NC & 5182 & 0 & -76.587 & 34.6987 & NC \\
\hline 5039 & -0.4 & -76.4144 & 34.749 & NC & 5111 & -1 & -76.4376 & 34.7234 & NC & 5183 & -0.3 & -76.5865 & 34.6985 & $\mathrm{NC}$ \\
\hline
\end{tabular}




\begin{tabular}{|c|c|c|c|c|c|c|c|c|c|c|c|c|c|c|}
\hline TR\# & RATE & LONG & LAT & ST & TR\# & RATE & LONG & LAT & ST & TR\# & RATE & LONG & LAT & ST \\
\hline 5184 & -0.5 & -76.5859 & 34.6984 & NC & 5256 & -0.4 & -76.5586 & 34.691 & NC & 5328 & 1.5 & -76.7326 & 34.6892 & NC \\
\hline 5185 & -0.2 & -76.459 & 34.6984 & NC & 5257 & -0.2 & -76.5582 & 34.6909 & NC & 5329 & 1.5 & -76.7321 & 34.6892 & NC \\
\hline 5186 & -0.7 & -76.5854 & 34.6982 & $\mathrm{NC}$ & 5258 & -0.4 & -76.5581 & 34.6908 & $\mathrm{NC}$ & 5330 & 0 & -76.763 & 34.6892 & NC \\
\hline 5187 & -0.9 & .76 .5849 & 34.6981 & NC & 5259 & 0 & -76.5577 & 34.6908 & $\mathrm{NC}$ & 5331 & -0.1 & -76.7625 & 34.6892 & NC \\
\hline 5188 & -0.2 & -76.4594 & 34.6981 & NC & 5260 & 0 & -76.4654 & 34.6908 & $\mathrm{NC}$ & 5332 & 0 & -76.7619 & 34.6892 & NC \\
\hline 5189 & -0.9 & -76.5843 & 34.6979 & $\mathrm{NC}$ & 5261 & -0.2 & -76.5576 & 34.6907 & NC & 5333 & 0.1 & -76.7614 & 34.6892 & NC \\
\hline 5190 & -0.9 & -76.5838 & 34.6978 & $\mathrm{NC}$ & 5262 & -0.5 & -76.5571 & 34.6906 & NC & 5334 & 0.1 & -76.7608 & 34.6892 & NC \\
\hline 5191 & -0.2 & -76.4597 & 34.6977 & $\mathrm{NC}$ & 5263 & -0.9 & -76.5566 & 34.6904 & $\mathrm{NC}$ & 5335 & -0.6 & -76.7664 & 34.6891 & NC \\
\hline 5192 & -0.8 & -76.5833 & 34.6977 & NC & 5264 & 0.1 & -76.4657 & 34.6904 & NC & 5336 & -0.6 & -76.7658 & 34.6891 & NC \\
\hline 5193 & -0.7 & -76.5827 & 34.6975 & NC & 5265 & -0.7 & -76.5561 & 34.6902 & NC & 5337 & -0.4 & -76.7653 & 34.6891 & NC \\
\hline 5194 & -0.7 & -76.5822 & 34.6974 & NC & 5266 & -0.5 & -76.5556 & 34.6901 & NC & 5338 & -0.2 & -76.7647 & 34.6891 & NC \\
\hline 5195 & -0.1 & -76.46 & 34.6973 & NC & 5267 & 0.2 & -76.466 & 34.69 & NC & 5339 & 0 & -76.7642 & 34.6891 & NC \\
\hline 5196 & -0.5 & -76.5817 & 34.6972 & NC & 5268 & -0.5 & -76.555 & 34.6899 & NC & 5340 & 0 & -76.7636 & 34.6891 & NC \\
\hline 5197 & -0.5 & -76.5811 & 34.6971 & NC & 5269 & -0.4 & -76.5545 & 34.6897 & NC & 5341 & 1.3 & -76.7315 & 34.6891 & NC \\
\hline 5198 & -0.5 & -76.5806 & 34.6969 & NC & 5270 & -0.2 & -76.4664 & 34.6897 & $\mathrm{NC}$ & 5342 & 1.3 & -76.731 & 34.6891 & NC \\
\hline 5199 & -0.2 & -76.4603 & 34.6969 & NC & 5271 & -0.4 & -76.554 & 34.6895 & $\mathrm{NC}$ & 5343 & -0.8 & -76.7703 & 34.6891 & NC \\
\hline 5200 & -0.4 & -76.58 & 34.6968 & NC & 5272 & 2 & -76.7349 & 34.6894 & NC & 5344 & -0.7 & -76.7697 & 34.6891 & NC \\
\hline 5201 & -0.4 & -76.5795 & 34.6966 & NC & 5273 & -0.4 & -76.5535 & 34.6894 & $\mathrm{NC}$ & 5345 & -1.2 & -76.7709 & 34.6891 & NC \\
\hline 5202 & 0 & -76.4606 & 34.6965 & NC & 5274 & 1.8 & -76.7343 & 34.6894 & $\mathrm{NC}$ & 5346 & -1.1 & -76.7703 & 34.6891 & NC \\
\hline 5203 & -0.5 & -76.579 & 34.6965 & NC & 5275 & 1.5 & -76.7423 & 34.6893 & $\mathrm{NC}$ & 5347 & -1 & -76.7697 & 34.6891 & NC \\
\hline 5204 & -0.7 & -76.5784 & 34.6963 & $\mathrm{NC}$ & 5276 & 1.7 & -76.7418 & 34.6893 & NC & 5348 & -1 & -76.7692 & 34.6891 & NC \\
\hline 5205 & 0.1 & -76.461 & 34.6962 & $\mathrm{NC}$ & 5277 & 1.8 & -76.7412 & 34.6893 & NC & 5349 & -1 & -76.7686 & 34.6891 & NC \\
\hline 5206 & -0.4 & -76.5779 & 34.6962 & NC & 5278 & 2 & -76.7407 & 34.6893 & NC & 5350 & -0.8 & -76.7681 & 34.6891 & NC \\
\hline 5207 & -0.2 & -76.5774 & 34.6961 & NC & 5279 & 2.1 & -76.7401 & 34.6893 & $\mathrm{NC}$ & 5351 & -0.7 & -76.7675 & 34.6891 & NC \\
\hline 5208 & -0.2 & -76.5768 & 34.6959 & NC & 5280 & 2.3 & -76.7396 & 34.6893 & NC & 5352 & -0.7 & -76.7669 & 34.6891 & NC \\
\hline 5209 & -0.2 & -76.5763 & 34.6958 & NC & 5281 & 2.3 & -76.739 & 34.6893 & NC & 5353 & -0.8 & -76.7714 & 34.689 & NC \\
\hline 5210 & 0.2 & -76.4613 & 34.6958 & NC & 5282 & 2.2 & -76.7384 & 34.6893 & NC & 5354 & -0.9 & -76.7708 & 34.689 & NC \\
\hline 5211 & -0.2 & -76.5758 & 34.6956 & NC & 5283 & 2.2 & -76.7379 & 34.6893 & NC & 5355 & 1.2 & -76.7304 & 34.689 & NC \\
\hline 5212 & -0.2 & -76.5752 & 34.6955 & NC & 5284 & -0.5 & -76.4667 & 34.6893 & NC & 5356 & -0.5 & -76.5524 & 34.689 & NC \\
\hline 5213 & 0.4 & -76.4616 & 34.6954 & NC & 5285 & 2.2 & -76.7373 & 34.6893 & NC & 5357 & 1.2 & -76.7299 & 34.689 & NC \\
\hline 5214 & -0.2 & -76.5747 & 34.6953 & NC & 5286 & 2.2 & -76.7368 & 34.6893 & NC & 5358 & 1 & -76.7293 & 34.6889 & NC \\
\hline 5215 & -0.2 & -76.5742 & 34.6952 & NC & 5287 & 2.3 & -76.7362 & 34.6893 & NC & 5359 & 1.1 & -76.7288 & 34.6889 & NC \\
\hline 5216 & 0.4 & -76.4619 & 34.695 & NC & 5288 & 2.2 & -76.7356 & 34.6893 & NC & 5360 & -0.4 & -76.467 & 34.6889 & NC \\
\hline 5217 & -0.1 & -76.5736 & 34.695 & NC & 5289 & 2.2 & -76.7351 & 34.6893 & NC & 5361 & -0.8 & -76.7731 & 34.6889 & NC \\
\hline 5218 & 0 & -76.5731 & 34.6949 & NC & 5290 & 2.2 & -76.7345 & 34.6893 & NC & 5362 & -0.7 & -76.7725 & 34.6889 & NC \\
\hline 5219 & -0.3 & -76.5726 & 34.6948 & NC & 5291 & 2.3 & -76.734 & 34.6893 & $\mathrm{NC}$ & 5363 & -0.8 & -76.7719 & 34.6889 & NC \\
\hline 5220 & -0.5 & -76.572 & 34.6946 & NC & 5292 & 2.4 & -76.7334 & 34.6893 & NC & 5364 & -0.8 & -76.7736 & 34.6888 & NC \\
\hline 5221 & 0.3 & -76.4622 & 34.6946 & NC & 5293 & 1.7 & -76.7338 & 34.6893 & NC & 5365 & 1.3 & -76.7282 & 34.6888 & NC \\
\hline 5222 & -0.5 & -76.5715 & 34.6945 & NC & 5294 & 1.6 & -76.7332 & 34.6893 & NC & 5366 & 1.4 & -76.7276 & 34.6888 & $\mathrm{NC}$ \\
\hline 5223 & -0.5 & -76.571 & 34.6943 & NC & 5295 & 1.5 & -76.7429 & 34.6893 & NC & 5367 & -0.9 & -76.7747 & 34.6888 & NC \\
\hline 5224 & 0.2 & -76.4625 & 34.6942 & NC & 5296 & 0.2 & -76.7602 & 34.6892 & $\mathrm{NC}$ & 5368 & -0.5 & .76 .5519 & 34.6888 & NC \\
\hline 5225 & -0.5 & -76.5704 & 34.6942 & NC & 5297 & 0.2 & -76.7597 & 34.6892 & NC & 5369 & -0.8 & -76.7742 & 34.6888 & NC \\
\hline 5226 & -0.4 & -76.5699 & 34.694 & NC & 5298 & 0.2 & -76.7591 & 34.6892 & NC & 5370 & -0.5 & -76.5514 & 34.6887 & NC \\
\hline 5227 & -0.3 & -76.5693 & 34.6939 & NC & 5299 & 0.3 & -76.7586 & 34.6892 & NC & 5371 & 1.4 & -76.7265 & 34.6887 & NC \\
\hline 5228 & 0.2 & -76.4629 & 34.6939 & NC & 5300 & 0.3 & -76.758 & 34.6892 & NC & 5372 & -0.8 & -76.7759 & 34.6887 & NC \\
\hline 5229 & -0.2 & -76.5688 & 34.6937 & NC & 5301 & 0.2 & -76.7574 & 34.6892 & NC & 5373 & -0.9 & -76.7753 & 34.6887 & NC \\
\hline 5230 & -0.2 & -76.5683 & 34.6936 & NC & 5302 & 0.2 & -76.7569 & 34.6892 & NC & 5374 & 1.4 & -76.7271 & 34.6887 & NC \\
\hline 5231 & 0.2 & -76.4632 & 34.6935 & NC & 5303 & 0.3 & .76 .7563 & 34.6892 & NC & 5375 & -1 & -76.7764 & 34.6886 & NC \\
\hline 5232 & -0.2 & -76.5677 & 34.6934 & $\mathrm{NC}$ & 5304 & 0.3 & -76.7558 & 34.6892 & NC & 5376 & 1.3 & -76.726 & 34.6886 & NC \\
\hline 5233 & -0.2 & -76.5672 & 34.6933 & NC & 5305 & 0.2 & -76.7552 & 34.6892 & NC & 5377 & 1.4 & -76.7254 & 34.6886 & NC \\
\hline 5234 & -0.2 & -76.5667 & 34.6932 & NC & 5306 & 0.1 & -76.7546 & 34.6892 & NC & 5378 & -1 & -76.7775 & 34.6886 & NC \\
\hline 5235 & 0.3 & -76.4635 & 34.6931 & NC & 5307 & 0.1 & -76.7541 & 34.6892 & NC & 5379 & -1.2 & -76.777 & 34.6886 & NC \\
\hline 5236 & -0.3 & -76.5661 & 34.693 & NC & 5308 & 0.1 & -76.7535 & 34.6892 & $\mathrm{NC}$ & 5380 & 1.4 & -76.7249 & 34.6885 & NC \\
\hline 5237 & -0.4 & -76.5656 & 34.6929 & $\mathrm{NC}$ & 5309 & 0.1 & -76.753 & 34.6892 & $\mathrm{NC}$ & 5381 & 1.4 & -76.7243 & 34.6885 & NC \\
\hline 5238 & 0.3 & -76.4638 & 34.6927 & NC & 5310 & 0.1 & -76.7524 & 34.6892 & $\mathrm{NC}$ & 5382 & -0.9 & -76.7792 & 34.6885 & NC \\
\hline 5239 & -0.3 & -76.5651 & 34.6927 & NC & 5311 & 0 & -76.7519 & 34.6892 & $\mathrm{NC}$ & 5383 & -1 & -76.7786 & 34.6885 & NC \\
\hline 5240 & -0.2 & -76.5645 & 34.6926 & NC & 5312 & 0 & -76.7513 & 34.6892 & NC & 5384 & -1 & -76.7781 & 34.6885 & NC \\
\hline 5241 & -0.5 & -76.564 & 34.6924 & NC & 5313 & 0.1 & -76.7507 & 34.6892 & NC & 5385 & -0.3 & -76.4673 & 34.6885 & NC \\
\hline 5242 & -0.7 & -76.5635 & 34.6923 & $\mathrm{NC}$ & 5314 & 0.2 & -76.7502 & 34.6892 & NC & 5386 & -0.4 & -76.5509 & 34.6885 & NC \\
\hline 5243 & 0.3 & -76.4641 & 34.6923 & $\mathrm{NC}$ & 5315 & 0.3 & -76.7496 & 34.6892 & NC & 5387 & 1.4 & -76.7232 & 34.6884 & NC \\
\hline 5244 & -0.3 & -76.5629 & 34.6921 & $\mathrm{NC}$ & 5316 & 0.3 & -76.7491 & 34.6892 & NC & 5388 & -0.8 & -76.7803 & 34.6884 & NC \\
\hline 5245 & 0.2 & -76.5624 & 34.692 & NC & 5317 & 0.3 & -76.7485 & 34.6892 & NC & 5389 & -0.8 & -76.7798 & 34.6884 & NC \\
\hline 5246 & 0.1 & -76.4645 & 34.692 & NC & 5318 & 0.4 & -76.7479 & 34.6892 & $\mathrm{NC}$ & 5390 & 1.4 & -76.7237 & 34.6884 & NC \\
\hline 5247 & 0.3 & -76.5619 & 34.6919 & NC & 5319 & 0.5 & -76.7474 & 34.6892 & NC & 5391 & -0.4 & -76.5503 & 34.6883 & NC \\
\hline 5248 & 0.4 & -76.5613 & 34.6917 & $\mathrm{NC}$ & 5320 & 0.5 & -76.7468 & 34.6892 & NC & 5392 & -0.6 & -76.782 & 34.6883 & NC \\
\hline 5249 & -0.1 & -76.4648 & 34.6916 & $\mathrm{NC}$ & 5321 & -0.5 & -76.553 & 34.6892 & $\mathrm{NC}$ & 5393 & -0.5 & -76.7814 & 34.6883 & NC \\
\hline 5250 & 0 & -76.5608 & 34.6916 & $\mathrm{NC}$ & 5322 & 0.7 & -76.7463 & 34.6892 & NC & 5394 & -0.7 & -76.7809 & 34.6883 & NC \\
\hline 5251 & -0.4 & -76.5602 & 34.6914 & $\mathrm{NC}$ & 5323 & 0.8 & -76.7457 & 34.6892 & $\mathrm{NC}$ & 5395 & 1.5 & -76.7226 & 34.6883 & NC \\
\hline 5252 & -0.4 & -76.5597 & 34.6913 & NC & 5324 & 0.9 & -76.7451 & 34.6892 & $\mathrm{NC}$ & 5396 & 1.6 & -76.7221 & 34.6883 & NC \\
\hline 5253 & -0.1 & -76.4651 & 34.6912 & NC & 5325 & 1 & -76.7446 & 34.6892 & $\mathrm{NC}$ & 5397 & -0.5 & -76.7826 & 34.6882 & NC \\
\hline 5254 & -0.4 & -76.5592 & 34.6911 & $\mathrm{NC}$ & 5326 & 1.1 & -76.744 & 34.6892 & $\mathrm{NC}$ & 5398 & 1.6 & -76.7215 & 34.6882 & NC \\
\hline 5255 & -0.4 & .76 .5587 & 34.6911 & NC & 5327 & 1.3 & -76.7435 & 34.6892 & NC & 5399 & 1.7 & -76.721 & 34.6882 & NC \\
\hline
\end{tabular}




\begin{tabular}{|c|c|c|c|c|c|c|c|c|c|c|c|c|c|c|}
\hline TR\# & RATE & LONG & LAT & ST & TR\# & RATE & LONG & LAT & ST & TR\# & RATE & LONG & LAT & ST \\
\hline 5400 & -0.5 & -76.7831 & 34.6882 & $\mathrm{NC}$ & 5472 & 1.6 & -76.7065 & 34.6869 & NC & 5544 & -1.4 & -76.6873 & 34.6857 & NC \\
\hline 5401 & 1.7 & -76.7204 & 34.6881 & NC & 5473 & -0.4 & -76.8027 & 34.6869 & NC & 5545 & -1.5 & -76.6867 & 34.6857 & $\mathrm{NC}$ \\
\hline 5402 & -0.3 & -76.4676 & 34.6881 & NC & 5474 & 1.1 & -76.7054 & 34.6868 & NC & 5546 & 9 & -76.6684 & 34.6857 & NC \\
\hline 5403 & -0.4 & -76.5498 & 34.6881 & NC & 5475 & -0.4 & -76.8038 & 34.6868 & $\mathrm{NC}$ & 5547 & -0.7 & -76.5425 & 34.6857 & $\mathrm{NC}$ \\
\hline 5404 & 1.7 & -76.7199 & 34.6881 & NC & 5476 & -0.4 & -76.8032 & 34.6868 & $\mathrm{NC}$ & 5548 & -1.5 & -76.8152 & 34.6857 & NC \\
\hline 5405 & -0.5 & -76.7848 & 34.6881 & NC & 5477 & 7.9 & -76.6702 & 34.6868 & $\mathrm{NC}$ & 5549 & -1.5 & -76.8146 & 34.6857 & NC \\
\hline 5406 & -0.4 & -76.7842 & 34.6881 & $\mathrm{NC}$ & 5478 & 1.4 & -76.7059 & 34.6868 & $\mathrm{NC}$ & 5550 & -1.3 & -76.689 & 34.6857 & $\mathrm{NC}$ \\
\hline 5407 & -0.4 & -76.7837 & 34.6881 & NC & 5479 & 0.4 & -76.7043 & 34.6867 & $\mathrm{NC}$ & 5551 & -1.4 & -76.6884 & 34.6857 & NC \\
\hline 5408 & -0.6 & -76.7865 & 34.688 & NC & 5480 & -0.6 & -76.8055 & 34.6867 & NC & 5552 & -1.5 & -76.6878 & 34.6857 & NC \\
\hline 5409 & -0.5 & -76.7859 & 34.688 & $\mathrm{NC}$ & 5481 & -0.5 & -76.8049 & 34.6867 & $\mathrm{NC}$ & 5553 & -1.2 & -76.6901 & 34.6856 & NC \\
\hline 5410 & -0.4 & -76.5493 & 34.688 & NC & 5482 & -0.5 & -76.8044 & 34.6867 & $\mathrm{NC}$ & 5554 & -1.3 & -76.6895 & 34.6856 & $\mathrm{NC}$ \\
\hline 5411 & -0.4 & -76.7854 & 34.688 & NC & 5483 & -0.5 & -76.5456 & 34.6867 & NC & 5555 & -1.4 & -76.8157 & 34.6856 & NC \\
\hline 5412 & 1.8 & -76.7193 & 34.688 & NC & 5484 & 0.8 & -76.7048 & 34.6867 & NC & 5556 & -1.6 & -76.6917 & 34.6856 & $\mathrm{NC}$ \\
\hline 5413 & 1.8 & -76.7187 & 34.688 & NC & 5485 & -0.7 & -76.8066 & 34.6866 & NC & 5557 & -1.3 & -76.6912 & 34.6856 & $\mathrm{NC}$ \\
\hline 5414 & -0.6 & -76.7876 & 34.6879 & NC & 5486 & -0.7 & -76.806 & 34.6866 & NC & 5558 & -1.2 & -76.6906 & 34.6856 & NC \\
\hline 5415 & -0.6 & -76.787 & 34.6879 & NC & 5487 & 0 & -76.7037 & 34.6866 & NC & 5559 & -1.4 & -76.8168 & 34.6855 & NC \\
\hline 5416 & 1.8 & -76.7182 & 34.6879 & NC & 5488 & -0.2 & -76.7032 & 34.6866 & $\mathrm{NC}$ & 5560 & -1.5 & -76.8163 & 34.6855 & NC \\
\hline 5417 & 1.9 & -76.7176 & 34.6879 & NC & 5489 & -0.7 & -76.8072 & 34.6866 & $\mathrm{NC}$ & 5561 & -1.9 & -76.6945 & 34.6855 & NC \\
\hline 5418 & 2 & -76.7171 & 34.6878 & NC & 5490 & -0.3 & -76.4689 & 34.6866 & NC & 5562 & -1.9 & .76 .694 & 34.6855 & NC \\
\hline 5419 & 2.1 & -76.7165 & 34.6878 & NC & 5491 & -0.9 & -76.5451 & 34.6866 & $\mathrm{NC}$ & 5563 & -0.5 & -76.4699 & 34.6855 & NC \\
\hline 5420 & -0.4 & -76.7893 & 34.6878 & $\mathrm{NC}$ & 5492 & -0.8 & -76.8077 & 34.6865 & NC & 5564 & -1.9 & -76.6934 & 34.6855 & NC \\
\hline 5421 & -0.3 & -76.468 & 34.6878 & NC & 5493 & -0.5 & -76.7026 & 34.6865 & NC & 5565 & -1.8 & -76.6929 & 34.6855 & NC \\
\hline 5422 & -0.4 & -76.5488 & 34.6878 & $\mathrm{NC}$ & 5494 & -0.8 & -76.7021 & 34.6865 & NC & 5566 & 0.6 & -76.4699 & 34.6855 & NC \\
\hline 5423 & -0.5 & -76.7887 & 34.6878 & $\mathrm{NC}$ & 5495 & 12.2 & -76.6697 & 34.6865 & NC & 5567 & -0.7 & -76.542 & 34.6855 & NC \\
\hline 5424 & -0.6 & -76.7882 & 34.6878 & NC & 5496 & -1.4 & -76.8074 & 34.6865 & NC & 5568 & -1.8 & -76.6923 & 34.6855 & NC \\
\hline 5425 & -0.5 & -76.7909 & 34.6877 & $\mathrm{NC}$ & 5497 & -0.9 & -76.8083 & 34.6865 & $\mathrm{NC}$ & 5569 & -1.2 & -76.8174 & 34.6854 & NC \\
\hline 5426 & -0.5 & -76.7904 & 34.6877 & $\mathrm{NC}$ & 5498 & -1.4 & -76.808 & 34.6864 & $\mathrm{NC}$ & 5570 & -2 & -76.6967 & 34.6854 & $\mathrm{NC}$ \\
\hline 5427 & -0.4 & -76.7898 & 34.6877 & NC & 5499 & -0.9 & -76.5446 & 34.6864 & $\mathrm{NC}$ & 5571 & -2 & -76.6962 & 34.6854 & NC \\
\hline 5428 & 2.1 & -76.716 & 34.6877 & $\mathrm{NC}$ & 5500 & -1.1 & -76.7015 & 34.6864 & NC & 5572 & -1.9 & -76.6956 & 34.6854 & NC \\
\hline 5429 & 2.1 & -76.7154 & 34.6877 & NC & 5501 & -1.3 & -76.7009 & 34.6864 & NC & 5573 & -1.9 & -76.6951 & 34.6854 & $\mathrm{NC}$ \\
\hline 5430 & -0.4 & -76.7915 & 34.6876 & $\mathrm{NC}$ & 5502 & -1.6 & -76.8085 & 34.6864 & $\mathrm{NC}$ & 5574 & 7.3 & -76.668 & 34.6854 & $\mathrm{NC}$ \\
\hline 5431 & -0.5 & -76.5482 & 34.6876 & $\mathrm{NC}$ & 5503 & -1.8 & -76.6998 & 34.6863 & $\mathrm{NC}$ & 5575 & -1.2 & -76.8179 & 34.6853 & $\mathrm{NC}$ \\
\hline 5432 & 2.1 & -76.7148 & 34.6876 & NC & 5504 & -1.7 & -76.8096 & 34.6863 & $\mathrm{NC}$ & 5576 & -1.2 & -76.8185 & 34.6853 & NC \\
\hline 5433 & 2 & -76.7143 & 34.6876 & $\mathrm{NC}$ & 5505 & -1.7 & -76.8091 & 34.6863 & $\mathrm{NC}$ & 5577 & -0.5 & -76.5415 & 34.6853 & NC \\
\hline 5434 & -0.4 & -76.7921 & 34.6876 & $\mathrm{NC}$ & 5506 & -1.5 & -76.7004 & 34.6863 & NC & 5578 & -1.2 & -76.8196 & 34.6852 & $\mathrm{NC}$ \\
\hline 5435 & -0.4 & -76.7926 & 34.6875 & NC & 5507 & -1.9 & .76 .6993 & 34.6862 & $\mathrm{NC}$ & 5579 & -1.1 & -76.8191 & 34.6852 & $\mathrm{NC}$ \\
\hline 5436 & 2.1 & -76.7137 & 34.6875 & $\mathrm{NC}$ & 5508 & -1.9 & -76.6987 & 34.6862 & NC & 5580 & -0.4 & -76.5409 & 34.6852 & $\mathrm{NC}$ \\
\hline 5437 & 2.2 & -76.7132 & 34.6875 & NC & 5509 & -0.2 & -76.4692 & 34.6862 & NC & 5581 & -0.5 & -76.4703 & 34.6852 & $\mathrm{NC}$ \\
\hline 5438 & -0.3 & -76.7937 & 34.6875 & $\mathrm{NC}$ & 5510 & -0.9 & -76.5441 & 34.6862 & NC & 5582 & 0.7 & -76.4702 & 34.6851 & $\mathrm{NC}$ \\
\hline 5439 & -0.4 & -76.7932 & 34.6875 & NC & 5511 & 10.9 & -76.6693 & 34.6862 & $\mathrm{NC}$ & 5583 & -1 & -76.8202 & 34.6851 & $\mathrm{NC}$ \\
\hline 5440 & -0.3 & -76.7943 & 34.6874 & NC & 5512 & -1.6 & -76.8102 & 34.6862 & NC & 5584 & 6 & -76.6675 & 34.6851 & $\mathrm{NC}$ \\
\hline 5441 & 2.2 & -76.7126 & 34.6874 & NC & 5513 & 3.7 & .76 .6773 & 34.6861 & NC & 5585 & -0.9 & -76.8207 & 34.685 & $\mathrm{NC}$ \\
\hline 5442 & 2.3 & -76.7121 & 34.6874 & NC & 5514 & -0.1 & -76.6767 & 34.6861 & NC & 5586 & -0.6 & -76.5404 & 34.685 & $\mathrm{NC}$ \\
\hline 5443 & -0.2 & -76.4683 & 34.6874 & NC & 5515 & -1.5 & -76.8107 & 34.6861 & NC & 5587 & -0.7 & -76.8213 & 34.685 & NC \\
\hline 5444 & -0.7 & -76.5477 & 34.6874 & $\mathrm{NC}$ & 5516 & -1.9 & -76.6982 & 34.6861 & $\mathrm{NC}$ & 5588 & -0.7 & -76.8224 & 34.6849 & $\mathrm{NC}$ \\
\hline 5445 & -0.3 & -76.7954 & 34.6874 & $\mathrm{NC}$ & 5517 & -1.9 & -76.6976 & 34.6861 & NC & 5589 & -0.7 & -76.8218 & 34.6849 & $\mathrm{NC}$ \\
\hline 5446 & -0.3 & -76.7949 & 34.6874 & $\mathrm{NC}$ & 5518 & -1.6 & -76.8113 & 34.6861 & NC & 5590 & -0.9 & -76.5399 & 34.6848 & NC \\
\hline 5447 & 2.4 & -76.711 & 34.6873 & NC & 5519 & 1.9 & -76.6784 & 34.6861 & $\mathrm{NC}$ & 5591 & -0.7 & -76.8229 & 34.6848 & $\mathrm{NC}$ \\
\hline 5448 & -0.5 & -76.7965 & 34.6873 & NC & 5520 & 2.3 & -76.6778 & 34.6861 & $\mathrm{NC}$ & 5592 & -0.4 & -76.4706 & 34.6848 & NC \\
\hline 5449 & -0.4 & -76.796 & 34.6873 & $\mathrm{NC}$ & 5521 & -1.6 & -76.8118 & 34.686 & $\mathrm{NC}$ & 5593 & 5.5 & -76.6671 & 34.6848 & $\mathrm{NC}$ \\
\hline 5450 & -0.9 & -76.5472 & 34.6873 & NC & 5522 & -0.8 & -76.5435 & 34.686 & NC & 5594 & -0.7 & -76.8235 & 34.6847 & NC \\
\hline 5451 & 2.4 & -76.7115 & 34.6873 & NC & 5523 & -1.4 & -76.6812 & 34.686 & NC & 5595 & -0.6 & -76.824 & 34.6847 & NC \\
\hline 5452 & -0.5 & -76.7971 & 34.6872 & $\mathrm{NC}$ & 5524 & -0.9 & -76.6806 & 34.686 & NC & 5596 & 0.7 & -76.4705 & 34.6847 & NC \\
\hline 5453 & 2.3 & -76.7104 & 34.6872 & NC & 5525 & -0.4 & -76.6801 & 34.686 & NC & 5597 & -0.8 & -76.5394 & 34.6846 & $\mathrm{NC}$ \\
\hline 5454 & 2 & -76.7098 & 34.6872 & NC & 5526 & 0.5 & -76.6795 & 34.686 & NC & 5598 & -0.5 & -76.8246 & 34.6846 & NC \\
\hline 5455 & -0.4 & -76.7982 & 34.6872 & NC & 5527 & 1.2 & -76.6789 & 34.686 & NC & 5599 & -0.6 & -76.8252 & 34.6846 & NC \\
\hline 5456 & -0.5 & -76.7977 & 34.6872 & $\mathrm{NC}$ & 5528 & 10.6 & -76.6688 & 34.686 & NC & 5600 & 14.6 & -76.6666 & 34.6845 & $\mathrm{NC}$ \\
\hline 5457 & 1.7 & -76.7087 & 34.6871 & NC & 5529 & -1.6 & -76.8124 & 34.686 & NC & 5601 & -0.7 & -76.5388 & 34.6845 & NC \\
\hline 5458 & -0.5 & -76.5467 & 34.6871 & NC & 5530 & -2.1 & -76.6828 & 34.6859 & NC & 5602 & -0.6 & -76.8257 & 34.6845 & $\mathrm{NC}$ \\
\hline 5459 & -0.6 & -76.7993 & 34.6871 & NC & 5531 & -0.7 & -76.543 & 34.6859 & $\mathrm{NC}$ & 5603 & -0.6 & .76 .8268 & 34.6844 & $\mathrm{NC}$ \\
\hline 5460 & -0.5 & -76.7988 & 34.6871 & $\mathrm{NC}$ & 5532 & -2.1 & -76.6823 & 34.6859 & $\mathrm{NC}$ & 5604 & -0.4 & -76.4709 & 34.6844 & $\mathrm{NC}$ \\
\hline 5461 & 1.7 & -76.7093 & 34.6871 & NC & 5533 & -1.8 & -76.6817 & 34.6859 & NC & 5605 & -0.7 & -76.8263 & 34.6844 & $\mathrm{NC}$ \\
\hline 5462 & 1.8 & -76.7076 & 34.687 & NC & 5534 & -1.6 & -76.8129 & 34.6859 & NC & 5606 & 14.9 & -76.6662 & 34.6843 & NC \\
\hline 5463 & -0.2 & -76.4686 & 34.687 & $\mathrm{NC}$ & 5535 & -2 & -76.684 & 34.6859 & $\mathrm{NC}$ & 5607 & -0.5 & -76.8279 & 34.6843 & $\mathrm{NC}$ \\
\hline 5464 & -0.6 & -76.801 & 34.687 & $\mathrm{NC}$ & 5536 & -2 & -76.6834 & 34.6859 & NC & 5608 & -0.5 & -76.8274 & 34.6843 & NC \\
\hline 5465 & -0.6 & -76.8005 & 34.687 & NC & 5537 & -1.6 & -76.6862 & 34.6858 & NC & 5609 & -0.5 & -76.5383 & 34.6843 & $\mathrm{NC}$ \\
\hline 5466 & -0.6 & -76.7999 & 34.687 & NC & 5538 & 0.2 & -76.4695 & 34.6858 & NC & 5610 & -0.5 & -76.8285 & 34.6842 & $\mathrm{NC}$ \\
\hline 5467 & 1.9 & -76.7082 & 34.687 & NC & 5539 & -1.7 & -76.6856 & 34.6858 & NC & 5611 & -0.4 & -76.5378 & 34.6841 & $\mathrm{NC}$ \\
\hline 5468 & -0.5 & -76.8021 & 34.6869 & NC & 5540 & -1.7 & -76.6851 & 34.6858 & NC & 5612 & -0.4 & -76.8296 & 34.6841 & NC \\
\hline 5469 & -0.5 & -76.8016 & 34.6869 & NC & 5541 & -1.8 & -76.6845 & 34.6858 & NC & 5613 & -0.4 & -76.829 & 34.6841 & $\mathrm{NC}$ \\
\hline 5470 & -0.2 & -76.5462 & 34.6869 & NC & 5542 & -1.7 & -76.8135 & 34.6858 & $\mathrm{NC}$ & 5614 & -0.4 & -76.4712 & 34.684 & NC \\
\hline 5471 & 1.9 & -76.7071 & 34.6869 & NC & 5543 & -1.6 & -76.8141 & 34.6858 & NC & 5615 & -0.1 & -76.8307 & 34.684 & $\mathrm{NC}$ \\
\hline
\end{tabular}




\begin{tabular}{|c|c|c|c|c|c|c|c|c|c|c|c|c|c|c|}
\hline TR\# & RATE & LONG & LAT & ST & TR\# & RATE & LONG & LAT & ST & TR\# & RATE & LONG & LAT & ST \\
\hline 5616 & -0.3 & -76.8302 & 34.684 & $\mathrm{NC}$ & 5688 & -1.3 & -76.8522 & 34.6815 & NC & 5760 & 9.8 & -76.6568 & 34.6783 & NC \\
\hline 5617 & 14.8 & -76.6657 & 34.684 & $\mathrm{NC}$ & 5689 & 13.2 & -76.6617 & 34.6814 & NC & 5761 & -0.5 & -76.8794 & 34.6783 & NC \\
\hline 5618 & 0 & -76.8313 & 34.6839 & NC & 5690 & -1.2 & -76.8533 & 34.6814 & NC & 5762 & -0.3 & -76.476 & 34.6783 & NC \\
\hline 5619 & -0.2 & -76.5373 & 34.6839 & NC & 5691 & -2.5 & -76.5294 & 34.6813 & NC & 5763 & -0.5 & -76.88 & 34.6782 & NC \\
\hline 5620 & 0 & -76.8324 & 34.6838 & NC & 5692 & -1.2 & -76.8544 & 34.6813 & NC & 5764 & -0.4 & -76.8805 & 34.6782 & NC \\
\hline 5621 & 0.1 & -76.8318 & 34.6838 & NC & 5693 & -1.2 & -76.8539 & 34.6813 & NC & 5765 & -0.4 & -76.8811 & 34.6781 & NC \\
\hline 5622 & 0 & -76.5368 & 34.6838 & NC & 5694 & -0.4 & -76.4734 & 34.6813 & NC & 5766 & -0.3 & -76.8822 & 34.678 & NC \\
\hline 5623 & 14.3 & -76.6653 & 34.6837 & NC & 5695 & -1.2 & -76.855 & 34.6812 & NC & 5767 & -0.4 & -76.8816 & 34.678 & NC \\
\hline 5624 & 0 & -76.8335 & 34.6837 & NC & 5696 & -1.2 & -76.8561 & 34.6811 & NC & 5768 & 9.3 & -76.6564 & 34.678 & NC \\
\hline 5625 & 0 & -76.8329 & 34.6837 & NC & 5697 & -1.6 & -76.5289 & 34.6811 & NC & 5769 & -0.3 & -76.8827 & 34.6779 & NC \\
\hline 5626 & -0.1 & -76.5362 & 34.6836 & NC & 5698 & -1.2 & -76.8555 & 34.6811 & NC & 5770 & -0.2 & -76.8826 & 34.6779 & NC \\
\hline 5627 & 0.1 & -76.834 & 34.6836 & NC & 5699 & 12.1 & .76 .6613 & 34.6811 & NC & 5771 & -0.4 & -76.4763 & 34.6779 & NC \\
\hline 5628 & -0.4 & -76.4715 & 34.6836 & NC & 5700 & -0.5 & -76.5284 & 34.681 & NC & 5772 & -0.4 & -76.8833 & 34.6778 & NC \\
\hline 5629 & 0.1 & -76.8351 & 34.6835 & $\mathrm{NC}$ & 5701 & -1.1 & -76.8567 & 34.681 & NC & 5773 & -0.3 & -76.8832 & 34.6778 & NC \\
\hline 5630 & 0.1 & -76.8346 & 34.6835 & $\mathrm{NC}$ & 5702 & -0.5 & -76.4737 & 34.681 & NC & 5774 & -0.3 & -76.8837 & 34.6777 & NC \\
\hline 5631 & -0.2 & -76.5357 & 34.6834 & NC & 5703 & -1.1 & -76.8572 & 34.6809 & NC & 5775 & 8.7 & -76.6559 & 34.6777 & $\mathrm{NC}$ \\
\hline 5632 & 0.2 & -76.8363 & 34.6834 & $\mathrm{NC}$ & 5704 & 11.1 & -76.6608 & 34.6809 & NC & 5776 & -0.3 & -76.8843 & 34.6777 & NC \\
\hline 5633 & 0.1 & -76.8357 & 34.6834 & NC & 5705 & -1.1 & -76.8578 & 34.6809 & NC & 5777 & -0.3 & -76.8848 & 34.6776 & NC \\
\hline 5634 & 13.9 & -76.6648 & 34.6834 & NC & 5706 & -1.2 & -76.8583 & 34.6808 & NC & 5778 & -0.1 & -76.8854 & 34.6775 & NC \\
\hline 5635 & 0.1 & -76.8368 & 34.6833 & NC & 5707 & -0.1 & -76.5279 & 34.6808 & NC & 5779 & 8.4 & -76.6555 & 34.6775 & NC \\
\hline 5636 & -0.4 & -76.4718 & 34.6832 & NC & 5708 & -1.1 & -76.8589 & 34.6807 & NC & 5780 & -0.3 & -76.4766 & 34.6775 & NC \\
\hline 5637 & 0.1 & -76.5352 & 34.6832 & NC & 5709 & -1.1 & -76.8594 & 34.6807 & NC & 5781 & -0.2 & -76.8865 & 34.6774 & NC \\
\hline 5638 & 0 & -76.8379 & 34.6832 & NC & 5710 & 0 & -76.5273 & 34.6806 & NC & 5782 & -0.2 & -76.8859 & 34.6774 & NC \\
\hline 5639 & 0 & -76.8374 & 34.6832 & NC & 5711 & 19.9 & -76.6604 & 34.6806 & NC & 5783 & -0.3 & -76.887 & 34.6773 & NC \\
\hline 5640 & 0.4 & -76.5347 & 34.6831 & NC & 5712 & -1.1 & -76.86 & 34.6806 & $\mathrm{NC}$ & 5784 & -0.3 & -76.8876 & 34.6772 & NC \\
\hline 5641 & 12.1 & -76.6644 & 34.6831 & NC & 5713 & -0.6 & -76.4741 & 34.6806 & NC & 5785 & -0.3 & -76.8881 & 34.6771 & NC \\
\hline 5642 & 0 & -76.8385 & 34.6831 & NC & 5714 & -1 & -76.8611 & 34.6805 & NC & 5786 & -0.4 & -76.4769 & 34.6771 & NC \\
\hline 5643 & -0.1 & -76.8396 & 34.683 & NC & 5715 & -1.1 & -76.8605 & 34.6805 & $\mathrm{NC}$ & 5787 & -0.3 & -76.8892 & 34.677 & NC \\
\hline 5644 & -0.1 & -76.839 & 34.683 & NC & 5716 & -1.1 & -76.8616 & 34.6804 & NC & 5788 & -0.4 & -76.8887 & 34.677 & $\mathrm{NC}$ \\
\hline 5645 & -0.1 & -76.8407 & 34.6829 & NC & 5717 & -1.3 & -76.8628 & 34.6803 & NC & 5789 & 10.5 & .76 .6544 & 34.677 & NC \\
\hline 5646 & -0.1 & -76.8401 & 34.6829 & NC & 5718 & -1.2 & -76.8622 & 34.6803 & NC & 5790 & 9.7 & -76.6539 & 34.6769 & NC \\
\hline 5647 & -0.3 & -76.4722 & 34.6829 & $\mathrm{NC}$ & 5719 & 18.5 & -76.6599 & 34.6803 & $\mathrm{NC}$ & 5791 & -0.3 & -76.8898 & 34.6769 & NC \\
\hline 5648 & 0.2 & -76.5341 & 34.6829 & NC & 5720 & -0.4 & -76.4744 & 34.6802 & $\mathrm{NC}$ & 5792 & 8.8 & -76.6533 & 34.6768 & NC \\
\hline 5649 & 10.4 & -76.6639 & 34.6828 & NC & 5721 & -1.4 & -76.8633 & 34.6802 & $\mathrm{NC}$ & 5793 & -0.4 & -76.8903 & 34.6768 & NC \\
\hline 5650 & -0.1 & -76.8413 & 34.6828 & NC & 5722 & -1.3 & -76.8639 & 34.6801 & NC & 5794 & -0.4 & -76.4772 & 34.6768 & NC \\
\hline 5651 & -0.3 & -76.8418 & 34.6827 & NC & 5723 & -1.1 & -76.8644 & 34.6801 & $\mathrm{NC}$ & 5795 & 8.5 & -76.6528 & 34.6767 & NC \\
\hline 5652 & -0.3 & -76.8424 & 34.6827 & NC & 5724 & -1 & -76.8655 & 34.68 & $\mathrm{NC}$ & 5796 & -0.4 & -76.8915 & 34.6767 & NC \\
\hline 5653 & 0 & -76.5336 & 34.6827 & NC & 5725 & -1 & -76.865 & 34.68 & NC & 5797 & -0.5 & -76.8909 & 34.6767 & NC \\
\hline 5654 & -0.4 & -76.8429 & 34.6826 & NC & 5726 & 17.2 & -76.6595 & 34.68 & $\mathrm{NC}$ & 5798 & -0.4 & -76.892 & 34.6766 & NC \\
\hline 5655 & 9.3 & -76.6635 & 34.6826 & NC & 5727 & -0.9 & -76.8661 & 34.6799 & NC & 5799 & 8.2 & -76.6522 & 34.6765 & NC \\
\hline 5656 & -0.4 & -76.8435 & 34.6826 & NC & 5728 & -0.8 & -76.8666 & 34.6798 & NC & 5800 & -0.5 & -76.8926 & 34.6765 & NC \\
\hline 5657 & -0.1 & -76.5331 & 34.6825 & NC & 5729 & -0.8 & -76.8672 & 34.6798 & NC & 5801 & 7.7 & -76.6517 & 34.6764 & NC \\
\hline 5658 & -0.4 & -76.844 & 34.6825 & $\mathrm{NC}$ & 5730 & -0.2 & -76.4747 & 34.6798 & NC & 5802 & -0.4 & -76.8937 & 34.6764 & NC \\
\hline 5659 & -0.4 & -76.4725 & 34.6825 & NC & 5731 & -0.7 & -76.8678 & 34.6797 & NC & 5803 & -0.4 & -76.8931 & 34.6764 & NC \\
\hline 5660 & -0.4 & -76.8451 & 34.6824 & $\mathrm{NC}$ & 5732 & 15.1 & -76.6591 & 34.6797 & NC & 5804 & -0.5 & -76.4775 & 34.6764 & NC \\
\hline 5661 & -0.4 & -76.8446 & 34.6824 & $\mathrm{NC}$ & 5733 & -0.8 & -76.8689 & 34.6796 & NC & 5805 & -0.3 & -76.8942 & 34.6763 & NC \\
\hline 5662 & -0.2 & -76.5326 & 34.6824 & $\mathrm{NC}$ & 5734 & -0.7 & -76.8683 & 34.6796 & NC & 5806 & 7.1 & -76.6512 & 34.6763 & NC \\
\hline 5663 & -1.2 & -76.845 & 34.6824 & NC & 5735 & -0.7 & -76.8694 & 34.6795 & NC & 5807 & 6.6 & -76.6506 & 34.6762 & NC \\
\hline 5664 & -1.1 & -76.8456 & 34.6823 & NC & 5736 & 12.2 & -76.6586 & 34.6794 & NC & 5808 & -0.4 & -76.8948 & 34.6762 & NC \\
\hline 5665 & -0.3 & -76.8457 & 34.6823 & NC & 5737 & -0.6 & -76.8705 & 34.6794 & NC & 5809 & -0.4 & -76.8953 & 34.6761 & NC \\
\hline 5666 & 17.9 & -76.6631 & 34.6823 & NC & 5738 & -0.6 & -76.87 & 34.6794 & NC & 5810 & 6.1 & -76.6501 & 34.6761 & NC \\
\hline 5667 & -1 & -76.8461 & 34.6823 & NC & 5739 & -0.1 & -76.475 & 34.6794 & NC & 5811 & -0.3 & -76.8964 & 34.676 & NC \\
\hline 5668 & -1 & -76.8467 & 34.6822 & NC & 5740 & -0.6 & -76.8711 & 34.6793 & NC & 5812 & -0.4 & -76.8959 & 34.676 & NC \\
\hline 5669 & -0.2 & -76.5321 & 34.6822 & NC & 5741 & -0.7 & -76.8716 & 34.6792 & NC & 5813 & 5.8 & -76.6495 & 34.676 & NC \\
\hline 5670 & -1.1 & -76.8472 & 34.6821 & NC & 5742 & 11.7 & -76.6582 & 34.6792 & $\mathrm{NC}$ & 5814 & -0.6 & -76.4779 & 34.676 & NC \\
\hline 5671 & -1.2 & -76.8478 & 34.6821 & NC & 5743 & -0.6 & -76.8722 & 34.6792 & NC & 5815 & 5.4 & -76.649 & 34.6759 & NC \\
\hline 5672 & -0.7 & -76.4728 & 34.6821 & NC & 5744 & -0.7 & -76.8727 & 34.6791 & NC & 5816 & -0.3 & -76.897 & 34.6759 & NC \\
\hline 5673 & -0.2 & -76.5315 & 34.682 & NC & 5745 & -0.7 & -76.8739 & 34.679 & $\mathrm{NC}$ & 5817 & -0.3 & .76 .8975 & 34.6758 & NC \\
\hline 5674 & -1.2 & -76.8483 & 34.682 & NC & 5746 & -0.6 & -76.8733 & 34.679 & $\mathrm{NC}$ & 5818 & -0.4 & -76.8981 & 34.6757 & NC \\
\hline 5675 & 15.9 & -76.6626 & 34.682 & NC & 5747 & -0.1 & -76.4753 & 34.679 & NC & 5819 & 5 & -76.6485 & 34.6757 & NC \\
\hline 5676 & -1.3 & -76.8489 & 34.6819 & NC & 5748 & 11 & -76.6577 & 34.6789 & NC & 5820 & -0.3 & -76.8987 & 34.6757 & NC \\
\hline 5677 & -1.4 & -76.8494 & 34.6819 & NC & 5749 & -0.8 & -76.8744 & 34.6789 & NC & 5821 & -0.3 & -76.8992 & 34.6756 & NC \\
\hline 5678 & -1.4 & -76.85 & 34.6818 & NC & 5750 & -0.8 & -76.875 & 34.6788 & NC & 5822 & 4.8 & -76.6479 & 34.6756 & NC \\
\hline 5679 & -0.4 & -76.531 & 34.6818 & NC & 5751 & -0.8 & -76.8755 & 34.6788 & NC & 5823 & -0.6 & -76.4782 & 34.6756 & $\mathrm{NC}$ \\
\hline 5680 & -0.5 & -76.5305 & 34.6817 & NC & 5752 & -0.8 & -76.8761 & 34.6787 & NC & 5824 & 4.5 & -76.6474 & 34.6755 & NC \\
\hline 5681 & -1.3 & -76.8505 & 34.6817 & NC & 5753 & -0.2 & -76.4756 & 34.6787 & NC & 5825 & -0.3 & -76.8998 & 34.6755 & NC \\
\hline 5682 & 14 & -76.6622 & 34.6817 & NC & 5754 & -0.8 & -76.8772 & 34.6786 & $\mathrm{NC}$ & 5826 & -0.3 & -76.9003 & 34.6754 & NC \\
\hline 5683 & -1.3 & -76.8511 & 34.6817 & NC & 5755 & -0.8 & -76.8766 & 34.6786 & NC & 5827 & 4 & -76.6468 & 34.6754 & $\mathrm{NC}$ \\
\hline 5684 & -0.5 & -76.4731 & 34.6817 & NC & 5756 & 10.4 & -76.6573 & 34.6786 & NC & 5828 & 3.5 & -76.6463 & 34.6753 & NC \\
\hline 5685 & -1.3 & -76.8517 & 34.6816 & NC & 5757 & -0.7 & -76.8777 & 34.6785 & NC & 5829 & -0.3 & -76.9014 & 34.6753 & NC \\
\hline 5686 & -1.5 & -76.53 & 34.6815 & NC & 5758 & -0.7 & -76.8783 & 34.6784 & NC & 5830 & -0.1 & -76.9009 & 34.6753 & NC \\
\hline 5687 & -1.2 & -76.8528 & 34.6815 & NC & 5759 & -0.6 & -76.8789 & 34.6784 & NC & 5831 & 3.1 & -76.6457 & 34.6752 & $\mathrm{NC}$ \\
\hline
\end{tabular}




\begin{tabular}{|c|c|c|c|c|c|c|c|c|c|c|c|c|c|c|}
\hline TR\# & RATE & LONG & LAT & ST & TR\# & RATE & LONG & LAT & ST & TR\# & RATE & LONG & LAT & ST \\
\hline 5832 & -0.5 & .76 .902 & 34.6752 & NC & 5904 & -1 & -76.6317 & 34.6722 & NC & 5976 & -2 & -76.6188 & 34.6692 & NC \\
\hline 5833 & -0.7 & -76.4785 & 34.6752 & NC & 5905 & -0.1 & -76.9223 & 34.6722 & NC & 5977 & -0.7 & -76.9427 & 34.6692 & NC \\
\hline 5834 & -0.4 & -76.9025 & 34.6751 & NC & 5906 & 0 & -76.481 & 34.6722 & NC & 5978 & -0.7 & -76.9432 & 34.6691 & NC \\
\hline 5835 & 2.3 & -76.6452 & 34.6751 & NC & 5907 & 0 & -76.9229 & 34.6721 & NC & 5979 & 0.6 & -76.4836 & 34.6691 & NC \\
\hline 5836 & -0.5 & -76.9036 & 34.675 & NC & 5908 & -1 & -76.6311 & 34.6721 & NC & 5980 & -2 & -76.6183 & 34.669 & NC \\
\hline 5837 & -0.5 & -76.9031 & 34.675 & NC & 5909 & -1.1 & -76.6306 & 34.672 & NC & 5981 & -0.7 & -76.9438 & 34.669 & NC \\
\hline 5838 & 1.6 & -76.6447 & 34.6749 & NC & 5910 & 0 & -76.9234 & 34.672 & NC & 5982 & -0.6 & -76.9443 & 34.6689 & NC \\
\hline 5839 & -0.4 & -76.9042 & 34.6749 & NC & 5911 & 0 & -76.924 & 34.6719 & NC & 5983 & -2 & -76.6178 & 34.6689 & NC \\
\hline 5840 & 1.2 & -76.6441 & 34.6748 & NC & 5912 & -1.2 & -76.6301 & 34.6719 & NC & 5984 & -0.6 & -76.9449 & 34.6688 & NC \\
\hline 5841 & -0.3 & -76.9047 & 34.6748 & NC & 5913 & 0 & -76.9251 & 34.6718 & NC & 5985 & -2 & -76.6173 & 34.6687 & NC \\
\hline 5842 & -0.7 & -76.4788 & 34.6748 & NC & 5914 & 0 & -76.9245 & 34.6718 & NC & 5986 & -0.6 & -76.946 & 34.6687 & NC \\
\hline 5843 & -0.3 & -76.9053 & 34.6747 & NC & 5915 & -1.4 & -76.6295 & 34.6718 & NC & 5987 & -0.6 & -76.9454 & 34.6687 & NC \\
\hline 5844 & 0.8 & -76.6436 & 34.6747 & NC & 5916 & 0 & -76.4813 & 34.6718 & NC & 5988 & 0.6 & -76.4839 & 34.6687 & NC \\
\hline 5845 & -0.2 & -76.9064 & 34.6746 & NC & 5917 & 0.1 & -76.9256 & 34.6717 & $\mathrm{NC}$ & 5989 & -0.6 & -76.9465 & 34.6686 & NC \\
\hline 5846 & -0.3 & -76.9058 & 34.6746 & NC & 5918 & -1.6 & -76.629 & 34.6716 & NC & 5990 & -2 & -76.6167 & 34.6685 & NC \\
\hline 5847 & 0.4 & -76.643 & 34.6746 & NC & 5919 & 0.2 & -76.9262 & 34.6716 & $\mathrm{NC}$ & 5991 & -0.6 & -76.9471 & 34.6685 & NC \\
\hline 5848 & 0.1 & -76.6425 & 34.6745 & NC & 5920 & 0.3 & -76.9267 & 34.6715 & NC & 5992 & -0.5 & -76.9476 & 34.6684 & NC \\
\hline 5849 & -0.3 & -76.907 & 34.6745 & NC & 5921 & -1.8 & -76.6284 & 34.6715 & $\mathrm{NC}$ & 5993 & 0.6 & -76.4842 & 34.6684 & NC \\
\hline 5850 & -0.8 & -76.4791 & 34.6745 & NC & 5922 & -1.8 & -76.6279 & 34.6714 & NC & 5994 & -1.9 & -76.6162 & 34.6683 & NC \\
\hline 5851 & -0.3 & -76.9075 & 34.6744 & NC & 5923 & 0.3 & -76.9278 & 34.6714 & NC & 5995 & -0.5 & -76.9487 & 34.6683 & NC \\
\hline 5852 & 0.1 & -76.642 & 34.6744 & $\mathrm{NC}$ & 5924 & 0.4 & -76.9273 & 34.6714 & $\mathrm{NC}$ & 5996 & -0.4 & .76 .9482 & 34.6683 & NC \\
\hline 5853 & 0.1 & -76.6414 & 34.6743 & NC & 5925 & 0.1 & -76.4817 & 34.6714 & $\mathrm{NC}$ & 5997 & -0.4 & -76.9493 & 34.6682 & NC \\
\hline 5854 & -0.3 & -76.9086 & 34.6743 & NC & 5926 & -1.9 & -76.6274 & 34.6713 & NC & 5998 & -1.9 & -76.6157 & 34.6682 & NC \\
\hline 5855 & -0.3 & -76.9081 & 34.6743 & NC & 5927 & 0.3 & -76.9284 & 34.6713 & $\mathrm{NC}$ & 5999 & -0.4 & -76.9498 & 34.6681 & NC \\
\hline 5856 & -0.3 & -76.9092 & 34.6742 & NC & 5928 & 0.2 & -76.9289 & 34.6712 & $\mathrm{NC}$ & 6000 & -0.4 & -76.9504 & 34.668 & NC \\
\hline 5857 & 0.1 & -76.6409 & 34.6741 & NC & 5929 & -2 & -76.6268 & 34.6712 & NC & 6001 & -2 & -76.6152 & 34.668 & NC \\
\hline 5858 & -0.3 & -76.9097 & 34.6741 & NC & 5930 & -2.1 & -76.6263 & 34.6711 & NC & 6002 & 0.6 & -76.4845 & 34.668 & NC \\
\hline 5859 & -0.9 & -76.4794 & 34.6741 & $\mathrm{NC}$ & 5931 & 0.2 & -76.9295 & 34.6711 & NC & 6003 & -0.4 & -76.9509 & 34.6679 & NC \\
\hline 5860 & -0.4 & -76.9103 & 34.674 & NC & 5932 & -2.1 & -76.6257 & 34.671 & NC & 6004 & -0.5 & -76.9515 & 34.6678 & NC \\
\hline 5861 & 0 & -76.6403 & 34.674 & NC & 5933 & 0.2 & -76.93 & 34.671 & NC & 6005 & -1.9 & -76.6146 & 34.6678 & NC \\
\hline 5862 & -0.4 & -76.9108 & 34.6739 & NC & 5934 & 0.3 & -76.482 & 34.671 & NC & 6006 & -0.5 & -76.952 & 34.6678 & NC \\
\hline 5863 & -0.4 & -76.6398 & 34.6739 & NC & 5935 & 0.3 & -76.9306 & 34.6709 & NC & 6007 & -0.5 & -76.9526 & 34.6677 & NC \\
\hline 5864 & -0.3 & -76.9114 & 34.6739 & NC & 5936 & 0.1 & -76.9311 & 34.6709 & NC & 6008 & -1.9 & -76.6141 & 34.6677 & NC \\
\hline 5865 & -0.4 & -76.9119 & 34.6738 & NC & 5937 & 0 & -76.9317 & 34.6708 & NC & 6009 & -0.5 & -76.9531 & 34.6676 & NC \\
\hline 5866 & -0.6 & -76.6393 & 34.6738 & NC & 5938 & -2.1 & -76.6252 & 34.6708 & $\mathrm{NC}$ & 6010 & 0.5 & -76.4848 & 34.6676 & NC \\
\hline 5867 & -0.4 & -76.9125 & 34.6737 & NC & 5939 & 0.1 & -76.9322 & 34.6707 & NC & 6011 & -0.5 & -76.9537 & 34.6675 & NC \\
\hline 5868 & -0.6 & -76.6387 & 34.6737 & NC & 5940 & -2.1 & -76.6247 & 34.6707 & NC & 6012 & -1.8 & -76.6136 & 34.6675 & NC \\
\hline 5869 & -0.8 & -76.4798 & 34.6737 & NC & 5941 & 0.3 & -76.4823 & 34.6707 & NC & 6013 & -0.5 & -76.9548 & 34.6674 & NC \\
\hline 5870 & -0.5 & -76.9136 & 34.6736 & NC & 5942 & -2.1 & -76.6241 & 34.6706 & NC & 6014 & -0.5 & -76.9542 & 34.6674 & $\mathrm{NC}$ \\
\hline 5871 & -0.5 & -76.913 & 34.6736 & $\mathrm{NC}$ & 5943 & 0 & -76.9328 & 34.6706 & NC & 6015 & -1.9 & -76.6131 & 34.6673 & NC \\
\hline 5872 & -0.7 & -76.6382 & 34.6736 & NC & 5944 & -0.1 & -76.9333 & 34.6705 & NC & 6016 & -0.4 & -76.9553 & 34.6673 & NC \\
\hline 5873 & -0.6 & -76.9142 & 34.6735 & NC & 5945 & -2.1 & -76.6236 & 34.6705 & NC & 6017 & -1.8 & -76.6125 & 34.6672 & NC \\
\hline 5874 & -0.8 & -76.6376 & 34.6735 & NC & 5946 & -0.2 & -76.9339 & 34.6705 & NC & 6018 & -0.4 & -76.9559 & 34.6672 & NC \\
\hline 5875 & -0.5 & -76.9147 & 34.6734 & NC & 5947 & -2.2 & -76.623 & 34.6704 & NC & 6019 & 0.6 & -76.4851 & 34.6672 & NC \\
\hline 5876 & -0.5 & -76.9158 & 34.6733 & $\mathrm{NC}$ & 5948 & -0.2 & -76.9344 & 34.6704 & NC & 6020 & -0.3 & -76.9564 & 34.6671 & NC \\
\hline 5877 & -0.4 & -76.9153 & 34.6733 & NC & 5949 & -2.3 & -76.6225 & 34.6703 & NC & 6021 & 0.4 & -76.9573 & 34.667 & $\mathrm{NC}$ \\
\hline 5878 & -0.9 & -76.6371 & 34.6733 & NC & 5950 & -0.1 & -76.935 & 34.6703 & NC & 6022 & -0.3 & -76.957 & 34.667 & NC \\
\hline 5879 & -0.5 & -76.4801 & 34.6733 & NC & 5951 & 0.4 & -76.4826 & 34.6703 & NC & 6023 & -1.9 & -76.612 & 34.667 & NC \\
\hline 5880 & -0.9 & -76.6366 & 34.6732 & NC & 5952 & 0 & -76.9355 & 34.6702 & NC & 6024 & 0.6 & -76.9578 & 34.6669 & $\mathrm{NC}$ \\
\hline 5881 & -0.4 & -76.9164 & 34.6732 & NC & 5953 & -2.3 & -76.6219 & 34.6702 & NC & 6025 & -0.3 & -76.9581 & 34.6669 & $\mathrm{NC}$ \\
\hline 5882 & -0.5 & -76.9169 & 34.6731 & NC & 5954 & 0 & -76.9361 & 34.6701 & NC & 6026 & -0.2 & -76.9575 & 34.6669 & $\mathrm{NC}$ \\
\hline 5883 & -0.9 & -76.636 & 34.6731 & NC & 5955 & -2.4 & -76.6214 & 34.67 & NC & 6027 & 0.6 & -76.9584 & 34.6668 & NC \\
\hline 5884 & -0.9 & -76.6355 & 34.673 & $\mathrm{NC}$ & 5956 & -0.2 & -76.9372 & 34.67 & NC & 6028 & -2 & -76.6115 & 34.6668 & NC \\
\hline 5885 & -0.5 & -76.9175 & 34.673 & NC & 5957 & -0.1 & -76.9366 & 34.67 & NC & 6029 & 0.5 & -76.4855 & 34.6668 & NC \\
\hline 5886 & -0.5 & -76.918 & 34.6729 & NC & 5958 & -2.4 & -76.6209 & 34.6699 & NC & 6030 & -2.1 & -76.611 & 34.6667 & NC \\
\hline 5887 & -0.9 & -76.6349 & 34.6729 & NC & 5959 & -0.3 & -76.9377 & 34.6699 & NC & 6031 & 0.6 & -76.9589 & 34.6667 & NC \\
\hline 5888 & -0.5 & -76.9186 & 34.6729 & $\mathrm{NC}$ & 5960 & 0.5 & -76.4829 & 34.6699 & $\mathrm{NC}$ & 6032 & 0.8 & -76.9595 & 34.6666 & NC \\
\hline 5889 & -0.3 & -76.4804 & 34.6729 & NC & 5961 & -0.5 & -76.9383 & 34.6698 & NC & 6033 & -2.2 & -76.6104 & 34.6665 & NC \\
\hline 5890 & -0.4 & -76.9191 & 34.6728 & NC & 5962 & -2.4 & -76.6203 & 34.6698 & NC & 6034 & 0.7 & -76.96 & 34.6665 & NC \\
\hline 5891 & -0.9 & -76.6344 & 34.6728 & NC & 5963 & -2.4 & -76.6198 & 34.6697 & NC & 6035 & 0.4 & -76.4858 & 34.6665 & NC \\
\hline 5892 & -1 & -76.6338 & 34.6727 & NC & 5964 & -0.4 & -76.9388 & 34.6697 & NC & 6036 & 0.6 & -76.9606 & 34.6664 & NC \\
\hline 5893 & -0.4 & -76.9197 & 34.6727 & NC & 5965 & -0.5 & -76.9399 & 34.6696 & NC & 6037 & 0.4 & -76.9611 & 34.6663 & $\mathrm{NC}$ \\
\hline 5894 & -0.4 & -76.9208 & 34.6726 & NC & 5966 & -0.4 & -76.9394 & 34.6696 & $\mathrm{NC}$ & 6038 & -2.1 & -76.6099 & 34.6663 & NC \\
\hline 5895 & -0.4 & -76.9202 & 34.6726 & NC & 5967 & -2.5 & -76.6192 & 34.6696 & NC & 6039 & -2.2 & -76.6094 & 34.6662 & NC \\
\hline 5896 & -1 & -76.6333 & 34.6726 & NC & 5968 & -0.5 & -76.9405 & 34.6695 & NC & 6040 & 0.3 & -76.9617 & 34.6662 & NC \\
\hline 5897 & -0.1 & -76.4807 & 34.6726 & NC & 5969 & -2.5 & -76.6187 & 34.6695 & NC & 6041 & 0.3 & -76.9622 & 34.6661 & NC \\
\hline 5898 & 0 & -76.9201 & 34.6725 & NC & 5970 & 0.6 & -76.4832 & 34.6695 & $\mathrm{NC}$ & 6042 & 0.3 & -76.4861 & 34.6661 & $\mathrm{NC}$ \\
\hline 5899 & 0 & -76.9207 & 34.6724 & NC & 5971 & -2 & -76.6194 & 34.6694 & $\mathrm{NC}$ & 6043 & -2.4 & -76.6089 & 34.666 & NC \\
\hline 5900 & -1 & -76.6328 & 34.6724 & NC & 5972 & -0.6 & -76.941 & 34.6694 & $\mathrm{NC}$ & 6044 & 0.2 & -76.9628 & 34.666 & NC \\
\hline 5901 & -0.1 & -76.9212 & 34.6723 & NC & 5973 & -2.6 & -76.6182 & 34.6694 & NC & 6045 & 0.1 & -76.9633 & 34.6659 & NC \\
\hline 5902 & -1 & -76.6322 & 34.6723 & $\mathrm{NC}$ & 5974 & -0.7 & -76.9416 & 34.6693 & $\mathrm{NC}$ & 6046 & 0.1 & -76.9638 & 34.6658 & NC \\
\hline 5903 & -0.1 & -76.9218 & 34.6723 & NC & 5975 & -0.8 & -76.9421 & 34.6692 & NC & 6047 & -2.3 & -76.6083 & 34.6658 & NC \\
\hline
\end{tabular}




\begin{tabular}{|c|c|c|c|c|c|c|c|c|c|c|c|c|c|c|}
\hline TR\# & RATE & LONG & LAT & ST & TR\# & RATE & LONG & LAT & ST & TR\# & RATE & LONG & LAT & ST \\
\hline 6048 & 0.1 & -76.9644 & 34.6657 & NC & 6120 & -2.6 & -76.5963 & 34.6619 & $\mathrm{NC}$ & 6192 & -1 & -77.0036 & 34.6583 & $\mathrm{NC}$ \\
\hline 6049 & 0.3 & -76.4864 & 34.6657 & NC & 6121 & -0.3 & -76.4896 & 34.6619 & $\mathrm{NC}$ & 6193 & 0.3 & -76.4915 & 34.6583 & NC \\
\hline 6050 & 0.1 & -76.9649 & 34.6656 & NC & 6122 & -0.2 & -76.9867 & 34.6618 & NC & 6194 & -1 & -77.0042 & 34.6582 & NC \\
\hline 6051 & -2.2 & -76.6078 & 34.6656 & NC & 6123 & -0.1 & -76.9862 & 34.6618 & NC & 6195 & -1 & -77.0047 & 34.6581 & NC \\
\hline 6052 & -2.2 & -76.6073 & 34.6655 & NC & 6124 & -2.6 & -76.5958 & 34.6618 & NC & 6196 & -1 & -77.0052 & 34.658 & NC \\
\hline 6053 & 0 & -76.9655 & 34.6655 & NC & 6125 & -0.1 & -76.9873 & 34.6617 & NC & 6197 & -3.3 & -76.5842 & 34.658 & NC \\
\hline 6054 & 0.1 & -76.966 & 34.6654 & NC & 6126 & -0.1 & -76.9878 & 34.6616 & NC & 6198 & 0.4 & -76.4917 & 34.6579 & NC \\
\hline 6055 & 0.2 & -76.9671 & 34.6653 & NC & 6127 & -2.7 & -76.5953 & 34.6616 & NC & 6199 & -0.9 & -77.0058 & 34.6578 & NC \\
\hline 6056 & 0.2 & -76.9666 & 34.6653 & NC & 6128 & -0.2 & -76.9884 & 34.6615 & NC & 6200 & -3.4 & -76.5837 & 34.6578 & NC \\
\hline 6057 & -2.1 & -76.6068 & 34.6653 & NC & 6129 & -0.3 & -76.4899 & 34.6615 & NC & 6201 & -0.9 & -77.0063 & 34.6577 & NC \\
\hline 6058 & 0.3 & -76.4867 & 34.6653 & NC & 6130 & -0.2 & -76.9889 & 34.6614 & NC & 6202 & -3.4 & -76.5833 & 34.6576 & NC \\
\hline 6059 & 0.3 & -76.9677 & 34.6652 & NC & 6131 & -2.8 & -76.5947 & 34.6614 & NC & 6203 & -0.8 & -77.0069 & 34.6576 & NC \\
\hline 6060 & 0.3 & -76.9682 & 34.6651 & NC & 6132 & -0.1 & -76.9895 & 34.6613 & NC & 6204 & -0.9 & -77.0074 & 34.6575 & NC \\
\hline 6061 & -2.1 & -76.6063 & 34.6651 & NC & 6133 & -2.9 & -76.5942 & 34.6612 & NC & 6205 & 0.3 & -76.492 & 34.6575 & NC \\
\hline 6062 & -2 & -76.6057 & 34.665 & NC & 6134 & -0.4 & -76.99 & 34.6612 & NC & 6206 & -3.4 & -76.5828 & 34.6574 & NC \\
\hline 6063 & 0.3 & -76.9687 & 34.665 & NC & 6135 & -3 & -76.5937 & 34.6611 & NC & 6207 & -0.7 & -77.008 & 34.6574 & NC \\
\hline 6064 & 0.3 & -76.9693 & 34.6649 & NC & 6136 & -0.4 & -76.9905 & 34.6611 & NC & 6208 & -0.6 & -77.0085 & 34.6573 & NC \\
\hline 6065 & 0.4 & -76.487 & 34.6649 & NC & 6137 & -0.4 & -76.4902 & 34.6611 & NC & 6209 & -3.4 & -76.5823 & 34.6571 & NC \\
\hline 6066 & -2.1 & -76.6052 & 34.6648 & NC & 6138 & -0.4 & -76.9911 & 34.661 & NC & 6210 & -0.6 & -77.009 & 34.6571 & NC \\
\hline 6067 & 0.2 & -76.9698 & 34.6648 & NC & 6139 & -0.3 & -76.9916 & 34.6609 & NC & 6211 & 0.3 & -76.4923 & 34.6571 & NC \\
\hline 6068 & 0.2 & -76.9704 & 34.6647 & NC & 6140 & -3 & -76.5932 & 34.6609 & NC & 6212 & -0.6 & -77.0096 & 34.657 & NC \\
\hline 6069 & 0.2 & -76.9709 & 34.6646 & NC & 6141 & 0 & -76.4899 & 34.6608 & NC & 6213 & -0.5 & -77.0101 & 34.6569 & NC \\
\hline 6070 & -2.1 & -76.6047 & 34.6646 & NC & 6142 & -0.2 & -76.9922 & 34.6608 & NC & 6214 & -3.5 & -76.5818 & 34.6569 & NC \\
\hline 6071 & 0.2 & -76.9715 & 34.6645 & NC & 6143 & -3.2 & -76.5926 & 34.6607 & NC & 6215 & -0.6 & -77.0107 & 34.6568 & NC \\
\hline 6072 & -2.2 & -76.6042 & 34.6645 & NC & 6144 & -0.2 & -76.9927 & 34.6607 & NC & 6216 & -0.6 & -77.0112 & 34.6567 & NC \\
\hline 6073 & 0.5 & -76.4874 & 34.6645 & NC & 6145 & -0.4 & -76.4905 & 34.6607 & NC & 6217 & -3.7 & -76.5813 & 34.6567 & NC \\
\hline 6074 & 0.1 & -76.972 & 34.6644 & NC & 6146 & -0.3 & -76.9933 & 34.6606 & NC & 6218 & 0.4 & -76.4925 & 34.6567 & NC \\
\hline 6075 & -2.2 & -76.6036 & 34.6643 & NC & 6147 & -3.3 & -76.5921 & 34.6606 & NC & 6219 & -0.6 & -77.0118 & 34.6566 & NC \\
\hline 6076 & 0.1 & -76.9726 & 34.6643 & NC & 6148 & -0.4 & -76.9938 & 34.6605 & NC & 6220 & -3.8 & -76.5808 & 34.6565 & NC \\
\hline 6077 & 0.1 & -76.9731 & 34.6642 & NC & 6149 & 0.3 & -76.4902 & 34.6604 & NC & 6221 & -0.6 & -77.0123 & 34.6564 & NC \\
\hline 6078 & 0.3 & -76.4877 & 34.6642 & NC & 6150 & -0.6 & -76.9944 & 34.6604 & NC & 6222 & -0.7 & -77.0128 & 34.6563 & NC \\
\hline 6079 & -2.3 & -76.6031 & 34.6641 & NC & 6151 & -3.3 & -76.5916 & 34.6604 & NC & 6223 & 0.5 & -76.4928 & 34.6563 & NC \\
\hline 6080 & 0.1 & -76.9737 & 34.6641 & NC & 6152 & -0.6 & -76.9949 & 34.6603 & NC & 6224 & -3.9 & -76.5803 & 34.6562 & NC \\
\hline 6081 & 0 & -76.9742 & 34.664 & NC & 6153 & -0.8 & -76.9944 & 34.6603 & NC & 6225 & -0.7 & -77.0134 & 34.6562 & NC \\
\hline 6082 & -2.3 & -76.6026 & 34.664 & NC & 6154 & -0.2 & -76.4908 & 34.6603 & NC & 6226 & -0.6 & -77.0139 & 34.6561 & NC \\
\hline 6083 & 0 & -76.9747 & 34.6639 & NC & 6155 & -0.8 & -76.9949 & 34.6602 & NC & 6227 & -0.4 & -77.0145 & 34.656 & NC \\
\hline 6084 & 0.1 & -76.9753 & 34.6638 & NC & 6156 & -3.3 & -76.5911 & 34.6602 & NC & 6228 & -3.9 & -76.5798 & 34.656 & NC \\
\hline 6085 & -2.3 & -76.6021 & 34.6638 & NC & 6157 & -3.2 & -76.5905 & 34.6601 & NC & 6229 & -0.4 & -77.015 & 34.6559 & NC \\
\hline 6086 & 0.2 & -76.488 & 34.6638 & NC & 6158 & -0.9 & -76.9955 & 34.6601 & NC & 6230 & -3.8 & -76.5793 & 34.6558 & NC \\
\hline 6087 & 0.2 & -76.9758 & 34.6637 & NC & 6159 & 0.4 & -76.4904 & 34.66 & NC & 6231 & 0.5 & -76.493 & 34.6558 & NC \\
\hline 6088 & -2.3 & -76.6015 & 34.6636 & NC & 6160 & 0.2 & -76.4912 & 34.66 & NC & 6232 & -0.4 & -77.0155 & 34.6557 & NC \\
\hline 6089 & 0.2 & -76.9764 & 34.6636 & $\mathrm{NC}$ & 6161 & -0.9 & -76.996 & 34.6599 & NC & 6233 & -3.7 & -76.5788 & 34.6556 & NC \\
\hline 6090 & 0.1 & -76.9769 & 34.6635 & NC & 6162 & -3.2 & -76.59 & 34.6599 & NC & 6234 & -0.5 & -77.0161 & 34.6556 & NC \\
\hline 6091 & -2.4 & -76.601 & 34.6634 & NC & 6163 & -1 & -76.9966 & 34.6598 & $\mathrm{NC}$ & 6235 & -0.6 & .77 .0166 & 34.6555 & NC \\
\hline 6092 & 0.1 & -76.9775 & 34.6634 & NC & 6164 & -3.3 & -76.5895 & 34.6597 & NC & 6236 & -0.6 & -77.0172 & 34.6554 & NC \\
\hline 6093 & 0.1 & -76.4883 & 34.6634 & NC & 6165 & -1.1 & -76.9971 & 34.6597 & $\mathrm{NC}$ & 6237 & 0.5 & -76.4933 & 34.6554 & NC \\
\hline 6094 & 0 & -76.978 & 34.6633 & NC & 6166 & -1.1 & -76.9977 & 34.6596 & NC & 6238 & -3.6 & -76.5783 & 34.6553 & NC \\
\hline 6095 & -2.5 & -76.6005 & 34.6633 & NC & 6167 & -3.4 & -76.589 & 34.6596 & NC & 6239 & -0.5 & -77.0177 & 34.6553 & NC \\
\hline 6096 & 0 & -76.9786 & 34.6632 & NC & 6168 & -1.1 & -76.9982 & 34.6595 & NC & 6240 & -0.5 & -77.0183 & 34.6552 & NC \\
\hline 6097 & -2.6 & -76.6 & 34.6631 & NC & 6169 & 0.4 & -76.4907 & 34.6595 & NC & 6241 & -3.5 & -76.5778 & 34.6551 & NC \\
\hline 6098 & -0.2 & -76.9791 & 34.6631 & NC & 6170 & -1.1 & -76.9987 & 34.6594 & $\mathrm{NC}$ & 6242 & -0.4 & -77.0188 & 34.655 & NC \\
\hline 6099 & -0.2 & -76.9796 & 34.663 & NC & 6171 & -3.5 & -76.5884 & 34.6594 & $\mathrm{NC}$ & 6243 & 0.4 & -76.4935 & 34.655 & NC \\
\hline 6100 & 0 & -76.4886 & 34.663 & NC & 6172 & -3.6 & -76.5879 & 34.6592 & $\mathrm{NC}$ & 6244 & -0.4 & -77.0193 & 34.6549 & NC \\
\hline 6101 & -2.6 & -76.5994 & 34.6629 & NC & 6173 & -1.1 & -76.9993 & 34.6592 & $\mathrm{NC}$ & 6245 & -3.2 & -76.5773 & 34.6549 & NC \\
\hline 6102 & -0.2 & -76.9802 & 34.6629 & NC & 6174 & -3.5 & -76.5874 & 34.6591 & $\mathrm{NC}$ & 6246 & -0.5 & -77.0199 & 34.6548 & NC \\
\hline 6103 & -0.2 & -76.9807 & 34.6628 & NC & 6175 & -1 & -76.9998 & 34.6591 & $\mathrm{NC}$ & 6247 & -3.3 & -76.5768 & 34.6547 & NC \\
\hline 6104 & -2.6 & -76.5989 & 34.6628 & NC & 6176 & 0.5 & -76.491 & 34.6591 & NC & 6248 & -0.4 & -77.0204 & 34.6547 & NC \\
\hline 6105 & -0.2 & -76.9813 & 34.6627 & NC & 6177 & -0.9 & -77.0004 & 34.659 & $\mathrm{NC}$ & 6249 & -0.5 & -77.021 & 34.6546 & NC \\
\hline 6106 & -0.1 & -76.9818 & 34.6626 & NC & 6178 & -3.6 & -76.5862 & 34.6589 & $\mathrm{NC}$ & 6250 & 0.3 & -76.4938 & 34.6546 & NC \\
\hline 6107 & -2.6 & -76.5984 & 34.6626 & NC & 6179 & -3.5 & -76.5869 & 34.6589 & $\mathrm{NC}$ & 6251 & -0.4 & -77.0215 & 34.6545 & NC \\
\hline 6108 & -0.2 & -76.4889 & 34.6626 & NC & 6180 & -0.9 & -77.0009 & 34.6589 & $\mathrm{NC}$ & 6252 & -3.3 & -76.5764 & 34.6544 & NC \\
\hline 6109 & -0.1 & -76.9824 & 34.6625 & $\mathrm{NC}$ & 6181 & -0.9 & -77.0014 & 34.6588 & $\mathrm{NC}$ & 6253 & -0.3 & -77.0221 & 34.6543 & $\mathrm{NC}$ \\
\hline 6110 & -2.6 & -76.5979 & 34.6624 & NC & 6182 & -0.9 & -77.002 & 34.6587 & NC & 6254 & -3.5 & -76.5759 & 34.6542 & NC \\
\hline 6111 & -0.1 & -76.9829 & 34.6624 & NC & 6183 & -3.6 & -76.5863 & 34.6587 & NC & 6255 & -0.3 & -77.0226 & 34.6542 & NC \\
\hline 6112 & -2.6 & -76.5973 & 34.6623 & NC & 6184 & -3.6 & -76.5857 & 34.6587 & NC & 6256 & 0.3 & -76.4941 & 34.6542 & NC \\
\hline 6113 & -0.1 & -76.9835 & 34.6623 & $\mathrm{NC}$ & 6185 & 0.3 & -76.4912 & 34.6587 & NC & 6257 & -0.2 & -77.0231 & 34.6541 & NC \\
\hline 6114 & -0.2 & -76.4893 & 34.6623 & NC & 6186 & -3.6 & -76.5852 & 34.6585 & $\mathrm{NC}$ & 6258 & -0.2 & -77.0237 & 34.654 & NC \\
\hline 6115 & -0.2 & -76.984 & 34.6622 & NC & 6187 & -3.8 & -76.5858 & 34.6585 & $\mathrm{NC}$ & 6259 & -3.5 & -76.5754 & 34.654 & NC \\
\hline 6116 & -0.3 & -76.9846 & 34.6621 & NC & 6188 & -0.9 & -77.0025 & 34.6585 & $\mathrm{NC}$ & 6260 & -0.2 & -77.0242 & 34.6539 & NC \\
\hline 6117 & -2.6 & -76.5968 & 34.6621 & NC & 6189 & -3.8 & -76.5853 & 34.6584 & NC & 6261 & -0.2 & -77.0248 & 34.6538 & NC \\
\hline 6118 & -0.3 & -76.9851 & 34.662 & NC & 6190 & -0.9 & -77.0031 & 34.6584 & NC & 6262 & -3.5 & -76.5749 & 34.6538 & NC \\
\hline 6119 & -0.2 & -76.9856 & 34.6619 & NC & 6191 & -3.5 & -76.5847 & 34.6583 & NC & 6263 & 0.2 & -76.4943 & 34.6538 & $\mathrm{NC}$ \\
\hline
\end{tabular}




\begin{tabular}{|c|c|c|c|c|c|c|c|c|c|c|c|c|c|c|}
\hline TR\# & RATE & LONG & LAT & ST & TR\# & RATE & LONG & LAT & ST & TR\# & RATE & LONG & LAT & ST \\
\hline 6264 & -0.2 & -77.0253 & 34.6536 & NC & 6336 & 0.7 & -76.4974 & 34.6489 & NC & 6408 & 1.5 & -76.5005 & 34.6439 & NC \\
\hline 6265 & -3.6 & -76.5744 & 34.6535 & NC & 6337 & -0.1 & -77.0454 & 34.6488 & NC & 6409 & -1.9 & -76.5554 & 34.6438 & NC \\
\hline 6266 & -0.2 & -77.0259 & 34.6535 & NC & 6338 & -2.9 & -76.564 & 34.6488 & NC & 6410 & -0.3 & -77.0652 & 34.6438 & NC \\
\hline 6267 & -0.2 & -77.0264 & 34.6534 & NC & 6339 & -0.1 & -77.0459 & 34.6487 & NC & 6411 & -0.4 & -77.0657 & 34.6437 & NC \\
\hline 6268 & 0.3 & -76.4946 & 34.6534 & NC & 6340 & -2.8 & .76 .5635 & 34.6486 & NC & 6412 & -0.4 & -77.0662 & 34.6436 & NC \\
\hline 6269 & -3.6 & -76.5739 & 34.6533 & NC & 6341 & -0.1 & -77.0464 & 34.6486 & NC & 6413 & -1.9 & -76.555 & 34.6435 & NC \\
\hline 6270 & -0.2 & -77.0269 & 34.6533 & NC & 6342 & -2.7 & -76.563 & 34.6484 & NC & 6414 & 1.4 & -76.5008 & 34.6435 & NC \\
\hline 6271 & -0.2 & -77.0275 & 34.6532 & NC & 6343 & 0 & -77.047 & 34.6484 & NC & 6415 & -0.4 & -77.0668 & 34.6434 & NC \\
\hline 6272 & -0.3 & -77.028 & 34.6531 & NC & 6344 & 0.7 & -76.4977 & 34.6484 & NC & 6416 & -0.4 & -77.0673 & 34.6433 & NC \\
\hline 6273 & -3.6 & -76.5734 & 34.6531 & NC & 6345 & 0 & -77.0475 & 34.6483 & NC & 6417 & -2 & -76.5547 & 34.6432 & NC \\
\hline 6274 & 0.2 & .76 .4948 & 34.653 & NC & 6346 & 0.1 & -77.048 & 34.6482 & NC & 6418 & -1.3 & -77.067 & 34.6432 & NC \\
\hline 6275 & -0.2 & -77.0286 & 34.6529 & NC & 6347 & -2.6 & -76.5626 & 34.6481 & NC & 6419 & -0.4 & -77.0678 & 34.6432 & NC \\
\hline 6276 & -3.6 & -76.5729 & 34.6529 & NC & 6348 & 0.1 & -77.0486 & 34.648 & NC & 6420 & 1.4 & -76.5011 & 34.6431 & NC \\
\hline 6277 & -0.3 & -77.0291 & 34.6528 & NC & 6349 & 0.9 & -76.498 & 34.648 & NC & 6421 & -1.3 & -77.0675 & 34.643 & NC \\
\hline 6278 & -0.2 & -77.0296 & 34.6527 & NC & 6350 & 0 & -77.0491 & 34.6479 & NC & 6422 & -1.2 & -77.0681 & 34.6429 & NC \\
\hline 6279 & -0.2 & -77.0302 & 34.6526 & NC & 6351 & -2.6 & -76.5621 & 34.6479 & NC & 6423 & -1.9 & -76.5543 & 34.6428 & NC \\
\hline 6280 & -3.6 & -76.5724 & 34.6526 & NC & 6352 & 0 & .77 .0496 & 34.6478 & NC & 6424 & -1.2 & -77.0686 & 34.6427 & NC \\
\hline 6281 & 0.2 & -76.4951 & 34.6526 & NC & 6353 & -2.6 & -76.5616 & 34.6477 & NC & 6425 & 1.3 & -76.5013 & 34.6427 & NC \\
\hline 6282 & 0.1 & -77.0309 & 34.6525 & NC & 6354 & -0.1 & -77.0502 & 34.6476 & NC & 6426 & -1.1 & -77.0691 & 34.6426 & NC \\
\hline 6283 & -0.1 & -77.0307 & 34.6525 & NC & 6355 & 1.2 & -76.4982 & 34.6476 & NC & 6427 & -2 & -76.5539 & 34.6425 & NC \\
\hline 6284 & -3.5 & -76.5719 & 34.6524 & NC & 6356 & -2.3 & -76.5611 & 34.6475 & NC & 6428 & -1.1 & -77.0696 & 34.6424 & NC \\
\hline 6285 & 0.1 & -77.0314 & 34.6524 & NC & 6357 & 0 & -77.0507 & 34.6475 & NC & 6429 & -1.1 & -77.0702 & 34.6423 & NC \\
\hline 6286 & 0.1 & -77.0313 & 34.6524 & NC & 6358 & -0.1 & -77.0512 & 34.6474 & NC & 6430 & 1.4 & -76.5016 & 34.6423 & NC \\
\hline 6287 & 0.1 & -77.0318 & 34.6522 & NC & 6359 & -2.1 & -76.5606 & 34.6472 & NC & 6431 & -1 & -77.0707 & 34.6421 & NC \\
\hline 6288 & -3.4 & -76.5714 & 34.6522 & $\mathrm{NC}$ & 6360 & 0 & -77.0518 & 34.6472 & NC & 6432 & -1.9 & -76.5535 & 34.6421 & NC \\
\hline 6289 & 0.2 & -77.032 & 34.6522 & NC & 6361 & 1.3 & -76.4985 & 34.6472 & NC & 6433 & -1 & -77.0712 & 34.642 & NC \\
\hline 6290 & 0.2 & -77.0325 & 34.6521 & NC & 6362 & 0 & -77.0523 & 34.6471 & NC & 6434 & 1.2 & -76.5018 & 34.6419 & NC \\
\hline 6291 & 0.3 & -76.4954 & 34.6521 & NC & 6363 & 0.1 & -77.0528 & 34.647 & NC & 6435 & -1 & -77.0718 & 34.6418 & NC \\
\hline 6292 & 0.2 & -77.033 & 34.652 & NC & 6364 & -2.1 & -76.5601 & 34.647 & NC & 6436 & -1.9 & -76.5532 & 34.6418 & NC \\
\hline 6293 & -3.4 & -76.5709 & 34.652 & NC & 6365 & 0 & -77.0534 & 34.6468 & NC & 6437 & -1 & -77.0723 & 34.6417 & NC \\
\hline 6294 & 0.2 & -77.0336 & 34.6518 & NC & 6366 & -2 & -76.5596 & 34.6468 & NC & 6438 & -1.8 & -76.5528 & 34.6415 & NC \\
\hline 6295 & 0.1 & -77.0341 & 34.6517 & NC & 6367 & 1.2 & -76.4987 & 34.6468 & NC & 6439 & -1.1 & -77.0728 & 34.6415 & NC \\
\hline 6296 & -3.5 & -76.5704 & 34.6517 & NC & 6368 & 0 & -77.0539 & 34.6467 & NC & 6440 & -1.2 & -77.0733 & 34.6414 & NC \\
\hline 6297 & 0.3 & -76.4956 & 34.6517 & NC & 6369 & -2 & -76.5591 & 34.6466 & NC & 6441 & 1.2 & -76.5021 & 34.6414 & NC \\
\hline 6298 & 0.1 & -77.0346 & 34.6516 & NC & 6370 & 0.1 & -77.0544 & 34.6466 & NC & 6442 & -1.2 & -77.0739 & 34.6412 & NC \\
\hline 6299 & -3.4 & -76.5699 & 34.6515 & NC & 6371 & 0.3 & -77.055 & 34.6464 & NC & 6443 & -1.6 & -76.5524 & 34.6411 & NC \\
\hline 6300 & 0.1 & -77.0352 & 34.6514 & NC & 6372 & 1.3 & -76.499 & 34.6464 & NC & 6444 & -1.4 & -77.0744 & 34.6411 & NC \\
\hline 6301 & -3.3 & -76.5695 & 34.6513 & NC & 6373 & -2 & -76.5586 & 34.6463 & NC & 6445 & 1.1 & -76.5024 & 34.641 & NC \\
\hline 6302 & 0 & -77.0357 & 34.6513 & NC & 6374 & 0.3 & -77.0555 & 34.6463 & NC & 6446 & -1.5 & -77.0749 & 34.6409 & $\mathrm{NC}$ \\
\hline 6303 & 0.3 & -76.4959 & 34.6513 & NC & 6375 & 0.3 & -77.0561 & 34.6461 & NC & 6447 & -1.5 & -76.552 & 34.6408 & NC \\
\hline 6304 & -0.1 & -77.0363 & 34.6511 & NC & 6376 & -1.8 & -76.5581 & 34.6461 & NC & 6448 & -1.6 & -77.0755 & 34.6408 & $\mathrm{NC}$ \\
\hline 6305 & -3.3 & -76.569 & 34.6511 & NC & 6377 & 0.3 & -77.0566 & 34.646 & NC & 6449 & -1.7 & -77.076 & 34.6406 & NC \\
\hline 6306 & -0.3 & -77.0368 & 34.651 & NC & 6378 & 1.3 & .76 .4992 & 34.646 & NC & 6450 & 1.1 & -76.5026 & 34.6406 & $\mathrm{NC}$ \\
\hline 6307 & -0.3 & -77.0373 & 34.6509 & NC & 6379 & 0.4 & -77.0571 & 34.6459 & NC & 6451 & -1.6 & -77.0765 & 34.6405 & $\mathrm{NC}$ \\
\hline 6308 & 0.3 & -76.4961 & 34.6509 & NC & 6380 & -1.7 & -76.5576 & 34.6459 & NC & 6452 & -1.4 & -76.5516 & 34.6404 & $\mathrm{NC}$ \\
\hline 6309 & -3.2 & -76.5685 & 34.6508 & NC & 6381 & -1.6 & -76.5571 & 34.6457 & NC & 6453 & -1.5 & -77.077 & 34.6403 & NC \\
\hline 6310 & -0.3 & -77.0379 & 34.6507 & NC & 6382 & 0.2 & -77.0577 & 34.6457 & NC & 6454 & -1.6 & -77.0776 & 34.6402 & NC \\
\hline 6311 & -3.1 & -76.568 & 34.6506 & NC & 6383 & 0.2 & -77.0582 & 34.6456 & NC & 6455 & 1 & -76.5029 & 34.6402 & NC \\
\hline 6312 & -0.2 & -77.0384 & 34.6506 & NC & 6384 & 1.3 & -76.4995 & 34.6456 & NC & 6456 & -1.3 & -76.5513 & 34.6401 & NC \\
\hline 6313 & -0.1 & -77.0389 & 34.6505 & NC & 6385 & 0.1 & -77.0587 & 34.6455 & NC & 6457 & -1.7 & -77.0781 & 34.64 & NC \\
\hline 6314 & 0.5 & -76.4964 & 34.6505 & NC & 6386 & -1.7 & -76.5566 & 34.6454 & NC & 6458 & -1.7 & -77.0786 & 34.6399 & NC \\
\hline 6315 & -3.1 & -76.5675 & 34.6504 & NC & 6387 & 0 & -77.0593 & 34.6453 & NC & 6459 & 0.8 & -76.5031 & 34.6398 & NC \\
\hline 6316 & 0 & -77.0395 & 34.6503 & NC & 6388 & 0 & -77.0598 & 34.6452 & NC & 6460 & -1.1 & -76.5509 & 34.6397 & NC \\
\hline 6317 & 0 & -77.04 & 34.6502 & NC & 6389 & -1.7 & -76.5561 & 34.6452 & NC & 6461 & -1.8 & -77.0791 & 34.6397 & NC \\
\hline 6318 & -3 & -76.567 & 34.6502 & NC & 6390 & -0.1 & -77.0603 & 34.6451 & NC & 6462 & -1.7 & -77.0797 & 34.6395 & NC \\
\hline 6319 & 0 & -77.0405 & 34.6501 & NC & 6391 & 1.4 & -76.4998 & 34.6451 & NC & 6463 & -1 & -76.5505 & 34.6394 & NC \\
\hline 6320 & 0.5 & -76.4967 & 34.6501 & NC & 6392 & -1.7 & -76.5557 & 34.645 & NC & 6464 & -1.6 & -77.0802 & 34.6394 & NC \\
\hline 6321 & -0.1 & -77.0411 & 34.6499 & NC & 6393 & -0.1 & -77.0609 & 34.6449 & NC & 6465 & 0.8 & -76.5034 & 34.6394 & NC \\
\hline 6322 & -2.8 & -76.5665 & 34.6499 & NC & 6394 & -1.7 & -76.5552 & 34.6448 & NC & 6466 & -1.6 & -77.0807 & 34.6392 & NC \\
\hline 6323 & -0.1 & -77.0416 & 34.6498 & NC & 6395 & 0 & -77.0614 & 34.6448 & NC & 6467 & -1.7 & -77.0813 & 34.6391 & NC \\
\hline 6324 & -0.1 & -77.0421 & 34.6497 & NC & 6396 & -0.1 & -77.0619 & 34.6447 & NC & 6468 & -0.8 & -76.5501 & 34.6391 & NC \\
\hline 6325 & -2.8 & -76.566 & 34.6497 & NC & 6397 & 1.5 & -76.5 & 34.6447 & NC & 6469 & 0.9 & -76.5037 & 34.639 & NC \\
\hline 6326 & 0.6 & -76.4969 & 34.6497 & NC & 6398 & -1.6 & -76.5547 & 34.6445 & NC & 6470 & -1.8 & -77.0818 & 34.6389 & NC \\
\hline 6327 & -2.7 & -76.5655 & 34.6495 & NC & 6399 & -0.1 & -77.0625 & 34.6445 & NC & 6471 & -1.8 & -77.0823 & 34.6388 & NC \\
\hline 6328 & -0.1 & .77 .0427 & 34.6495 & NC & 6400 & .2 .2 & -76.5562 & 34.6445 & NC & 6472 & -0.6 & -76.5497 & 34.6387 & NC \\
\hline 6329 & -0.1 & -77.0432 & 34.6494 & NC & 6401 & -0.1 & -77.063 & 34.6444 & NC & 6473 & -1.8 & -77.0828 & 34.6386 & NC \\
\hline 6330 & -2.8 & -76.565 & 34.6493 & NC & 6402 & -0.2 & -77.0635 & 34.6443 & NC & 6474 & 0.9 & -76.5039 & 34.6386 & NC \\
\hline 6331 & -0.2 & -77.0437 & 34.6493 & NC & 6403 & -1.8 & -76.5542 & 34.6443 & NC & 6475 & -1.8 & -77.0834 & 34.6385 & NC \\
\hline 6332 & 0.5 & -76.4972 & 34.6493 & NC & 6404 & 1.5 & -76.5003 & 34.6443 & NC & 6476 & -0.4 & -76.5494 & 34.6384 & NC \\
\hline 6333 & -0.2 & -77.0443 & 34.6491 & NC & 6405 & -2.2 & -76.5558 & 34.6442 & $\mathrm{NC}$ & 6477 & -1.6 & -77.0839 & 34.6383 & NC \\
\hline 6334 & -0.1 & -77.0448 & 34.649 & NC & 6406 & -0.3 & -77.0641 & 34.6441 & NC & 6478 & -1.4 & -77.0844 & 34.6382 & NC \\
\hline 6335 & -2.9 & -76.5645 & 34.649 & NC & 6407 & -0.3 & -77.0646 & 34.644 & NC & 6479 & 1.3 & -76.5042 & 34.6382 & NC \\
\hline
\end{tabular}




\begin{tabular}{|c|c|c|c|c|c|c|c|c|c|c|c|c|c|c|}
\hline 6480 & -1.2 & -77.085 & 34.638 & $\mathrm{NC}$ & 6552 & 0.4 & -77.1133 & 34.6311 & NC & 6624 & 1.6 & -77.1365 & 34.6261 & NC \\
\hline 6481 & -0.2 & -76.549 & 34.638 & NC & 6553 & -12.3 & -77.1138 & 34.631 & NC & 6625 & -2.4 & -76.5357 & 34.626 & $\mathrm{NC}$ \\
\hline 6482 & -1 & -77.0855 & 34.6379 & $\mathrm{NC}$ & 6554 & -22.6 & -77.1143 & 34.6309 & $\mathrm{NC}$ & 6626 & 1.7 & -77.1371 & 34.626 & NC \\
\hline 6483 & -1 & -77.086 & 34.6377 & $\mathrm{NC}$ & 6555 & 1.2 & -76.5088 & 34.6309 & NC & 6627 & 1.5 & -76.5116 & 34.6259 & NC \\
\hline 6484 & 0.1 & -76.5486 & 34.6377 & $\mathrm{NC}$ & 6556 & 3.3 & -76.541 & 34.6308 & $\mathrm{NC}$ & 6628 & -8.7 & -76.5625 & 34.6258 & $\mathrm{NC}$ \\
\hline 6485 & 1.3 & -76.5044 & 34.6377 & $\mathrm{NC}$ & 6557 & -20.1 & -77.1149 & 34.6308 & $\mathrm{NC}$ & 6629 & -3.3 & -76.5353 & 34.6257 & $\mathrm{NC}$ \\
\hline 6486 & -0.9 & -77.0865 & 34.6376 & $\mathrm{NC}$ & 6558 & -15.2 & -77.1154 & 34.6307 & NC & 6630 & 1.5 & -76.5118 & 34.6255 & NC \\
\hline 6487 & -0.9 & -77.0871 & 34.6374 & $\mathrm{NC}$ & 6559 & -8.2 & -77.116 & 34.6306 & NC & 6631 & -4.9 & -76.535 & 34.6254 & $\mathrm{NC}$ \\
\hline 6488 & -0.8 & -77.0876 & 34.6373 & NC & 6560 & 3.5 & -76.5407 & 34.6305 & $\mathrm{NC}$ & 6632 & -8.1 & -76.5624 & 34.6254 & NC \\
\hline 6489 & 0.3 & -76.5482 & 34.6373 & $\mathrm{NC}$ & 6561 & 1.3 & -76.509 & 34.6305 & NC & 6633 & -6.2 & -76.5346 & 34.625 & $\mathrm{NC}$ \\
\hline 6490 & 1.2 & -76.5047 & 34.6373 & $\mathrm{NC}$ & 6562 & -0.7 & -77.1165 & 34.6304 & NC & 6634 & 1.7 & -77.1343 & 34.625 & $\mathrm{NC}$ \\
\hline 6491 & -0.8 & -77.0881 & 34.6371 & $\mathrm{NC}$ & 6563 & 4.9 & -77.117 & 34.6303 & $\mathrm{NC}$ & 6635 & 1.6 & -76.5121 & 34.625 & NC \\
\hline 6492 & 0.5 & -76.5478 & 34.637 & $\mathrm{NC}$ & 6564 & 10.5 & -77.1176 & 34.6302 & $\mathrm{NC}$ & 6636 & -4.2 & -76.5622 & 34.6249 & NC \\
\hline 6493 & -0.8 & -77.0887 & 34.637 & $\mathrm{NC}$ & 6565 & 3.9 & -76.5403 & 34.6302 & NC & 6637 & 1.7 & -77.1348 & 34.6248 & $\mathrm{NC}$ \\
\hline 6494 & 1.2 & -76.5049 & 34.6369 & $\mathrm{NC}$ & 6566 & 11.4 & .77 .1181 & 34.6301 & $\mathrm{NC}$ & 6638 & 1.8 & -77.1353 & 34.6246 & $\mathrm{NC}$ \\
\hline 6495 & -0.7 & -77.0892 & 34.6368 & $\mathrm{NC}$ & 6567 & 1.4 & -76.5092 & 34.6301 & $\mathrm{NC}$ & 6639 & 1.5 & -76.5123 & 34.6246 & $\mathrm{NC}$ \\
\hline 6496 & 0.7 & -76.5475 & 34.6367 & $\mathrm{NC}$ & 6568 & 11.7 & -77.1187 & 34.63 & $\mathrm{NC}$ & 6640 & -3.5 & -76.5621 & 34.6245 & NC \\
\hline 6497 & -0.5 & -77.0897 & 34.6367 & $\mathrm{NC}$ & 6569 & 12.3 & -77.1192 & 34.6299 & NC & 6641 & 1.7 & -77.1358 & 34.6244 & NC \\
\hline 6498 & -0.5 & -77.0902 & 34.6365 & $\mathrm{NC}$ & 6570 & 3.9 & -76.5399 & 34.6298 & NC & 6642 & 1.6 & -77.1363 & 34.6242 & $\mathrm{NC}$ \\
\hline 6499 & 1.1 & -76.5052 & 34.6365 & $\mathrm{NC}$ & 6571 & 13.4 & -77.1197 & 34.6297 & $\mathrm{NC}$ & 6643 & 1.5 & -76.5125 & 34.6242 & $\mathrm{NC}$ \\
\hline 6500 & -0.3 & -77.0908 & 34.6364 & $\mathrm{NC}$ & 6572 & 1.4 & -76.5095 & 34.6297 & NC & 6644 & 1.7 & -77.1368 & 34.624 & $\mathrm{NC}$ \\
\hline 6501 & 1 & -76.5471 & 34.6363 & $\mathrm{NC}$ & 6573 & 12.5 & -77.1203 & 34.6296 & $\mathrm{NC}$ & 6645 & -1 & -76.5619 & 34.624 & $\mathrm{NC}$ \\
\hline 6502 & -0.3 & -77.0913 & 34.6362 & NC & 6574 & 11.6 & -77.1208 & 34.6295 & NC & 6646 & 1.8 & -77.1374 & 34.6238 & $\mathrm{NC}$ \\
\hline 6503 & -0.2 & -77.0918 & 34.6361 & $\mathrm{NC}$ & 6575 & 3.7 & -76.5395 & 34.6295 & NC & 6647 & 1.3 & -76.5128 & 34.6238 & NC \\
\hline 6504 & 1.1 & -76.5055 & 34.6361 & $\mathrm{NC}$ & 6576 & 10.7 & -77.1214 & 34.6294 & $\mathrm{NC}$ & 6648 & 1.8 & -77.1379 & 34.6236 & $\mathrm{NC}$ \\
\hline 6505 & 1.2 & -76.5467 & 34.636 & $\mathrm{NC}$ & 6577 & 9.5 & -77.1219 & 34.6293 & NC & 6649 & 10.7 & -76.5617 & 34.6236 & NC \\
\hline 6506 & 0.1 & -77.0924 & 34.6359 & $\mathrm{NC}$ & 6578 & 8.1 & -77.1225 & 34.6292 & $\mathrm{NC}$ & 6650 & 1.7 & -77.1384 & 34.6234 & $\mathrm{NC}$ \\
\hline 6507 & 0.4 & -77.0929 & 34.6357 & NC & 6579 & 1.4 & -76.5097 & 34.6292 & $\mathrm{NC}$ & 6651 & 1 & -76.513 & 34.6233 & NC \\
\hline 6508 & 1.1 & -76.5057 & 34.6357 & $\mathrm{NC}$ & 6580 & 1.7 & -76.5391 & 34.6291 & $\mathrm{NC}$ & 6652 & 1.7 & -77.1389 & 34.6232 & $\mathrm{NC}$ \\
\hline 6509 & 1.5 & .76 .5463 & 34.6356 & $\mathrm{NC}$ & 6581 & 6.7 & -77.123 & 34.629 & $\mathrm{NC}$ & 6653 & 8.9 & -76.5616 & 34.6231 & $\mathrm{NC}$ \\
\hline 6510 & 0.6 & -77.0934 & 34.6356 & $\mathrm{NC}$ & 6582 & 5.7 & -77.1235 & 34.6289 & $\mathrm{NC}$ & 6654 & 1.6 & -77.1394 & 34.623 & $\mathrm{NC}$ \\
\hline 6511 & 1 & -77.0939 & 34.6354 & $\mathrm{NC}$ & 6583 & 4.6 & -77.1241 & 34.6288 & $\mathrm{NC}$ & 6655 & 1.2 & -76.5132 & 34.6229 & $\mathrm{NC}$ \\
\hline 6512 & 1.6 & -76.546 & 34.6353 & $\mathrm{NC}$ & 6584 & 0.7 & -76.5388 & 34.6288 & $\mathrm{NC}$ & 6656 & 1.6 & -77.1399 & 34.6228 & NC \\
\hline 6513 & 0.8 & -77.0945 & 34.6353 & $\mathrm{NC}$ & 6585 & 1.3 & -76.5099 & 34.6288 & $\mathrm{NC}$ & 6657 & 7 & -76.5614 & 34.6227 & NC \\
\hline 6514 & 1.2 & -76.506 & 34.6353 & $\mathrm{NC}$ & 6586 & 3.8 & -77.1246 & 34.6287 & $\mathrm{NC}$ & 6658 & 1.8 & -77.1404 & 34.6226 & $\mathrm{NC}$ \\
\hline 6515 & 0.6 & -77.095 & 34.6351 & $\mathrm{NC}$ & 6587 & 3 & -77.1252 & 34.6286 & $\mathrm{NC}$ & 6659 & 1.4 & -76.5135 & 34.6225 & NC \\
\hline 6516 & 0.3 & -77.0955 & 34.635 & $\mathrm{NC}$ & 6588 & 2.4 & -77.1257 & 34.6285 & $\mathrm{NC}$ & 6660 & 1.8 & -77.1409 & 34.6224 & $\mathrm{NC}$ \\
\hline 6517 & 1.7 & -76.5456 & 34.6349 & $\mathrm{NC}$ & 6589 & 0.4 & -76.5384 & 34.6284 & $\mathrm{NC}$ & 6661 & 6.2 & -76.5612 & 34.6223 & NC \\
\hline 6518 & 1.2 & -76.5062 & 34.6349 & $\mathrm{NC}$ & 6590 & 1.2 & -76.5102 & 34.6284 & $\mathrm{NC}$ & 6662 & 1.8 & -77.1414 & 34.6222 & $\mathrm{NC}$ \\
\hline 6519 & -0.1 & -77.096 & 34.6348 & NC & 6591 & 2 & -77.1262 & 34.6283 & $\mathrm{NC}$ & 6663 & 1.4 & -76.5137 & 34.6221 & NC \\
\hline 6520 & -0.5 & -77.0966 & 34.6347 & $\mathrm{NC}$ & 6592 & 1.5 & -77.1268 & 34.6282 & NC & 6664 & 1.8 & -77.1419 & 34.622 & $\mathrm{NC}$ \\
\hline 6521 & 2.1 & -76.5452 & 34.6346 & $\mathrm{NC}$ & 6593 & 0 & -76.538 & 34.6281 & $\mathrm{NC}$ & 6665 & 1.8 & -77.1424 & 34.6218 & $\mathrm{NC}$ \\
\hline 6522 & -2.5 & -77.0971 & 34.6345 & $\mathrm{NC}$ & 6594 & 1.1 & -77.1273 & 34.6281 & NC & 6666 & 3.1 & -76.5611 & 34.6218 & $\mathrm{NC}$ \\
\hline 6523 & 1.1 & -76.5065 & 34.6345 & NC & 6595 & 1 & -77.1279 & 34.628 & NC & 6667 & 1.3 & -76.5139 & 34.6217 & $\mathrm{NC}$ \\
\hline 6524 & -12.4 & -77.0976 & 34.6344 & NC & 6596 & 1.2 & -76.5104 & 34.628 & $\mathrm{NC}$ & 6668 & 1.8 & -77.1429 & 34.6216 & $\mathrm{NC}$ \\
\hline 6525 & 2.5 & -76.5448 & 34.6343 & NC & 6597 & 0.8 & -77.1284 & 34.6279 & $\mathrm{NC}$ & 6669 & 1.7 & -77.1435 & 34.6214 & $\mathrm{NC}$ \\
\hline 6526 & -14.9 & -77.0982 & 34.6342 & NC & 6598 & -0.6 & -76.5376 & 34.6278 & $\mathrm{NC}$ & 6670 & 1.9 & -76.5609 & 34.6214 & $\mathrm{NC}$ \\
\hline 6527 & -16.4 & -77.0987 & 34.6341 & NC & 6599 & 0.7 & -77.1289 & 34.6278 & NC & 6671 & 1.7 & -77.144 & 34.6212 & $\mathrm{NC}$ \\
\hline 6528 & 1.2 & -76.5068 & 34.634 & NC & 6600 & 0.6 & -77.1295 & 34.6276 & $\mathrm{NC}$ & 6672 & 1.2 & -76.5142 & 34.6212 & NC \\
\hline 6529 & 2.9 & -76.5444 & 34.6339 & $\mathrm{NC}$ & 6601 & 1.2 & -76.5106 & 34.6276 & $\mathrm{NC}$ & 6673 & 1.7 & -77.1445 & 34.621 & NC \\
\hline 6530 & -18.3 & -77.0992 & 34.6339 & NC & 6602 & 0.3 & -77.13 & 34.6275 & $\mathrm{NC}$ & 6674 & 0.2 & -76.5608 & 34.6209 & $\mathrm{NC}$ \\
\hline 6531 & 1.5 & -76.5071 & 34.6339 & NC & 6603 & -1.1 & -76.5372 & 34.6274 & NC & 6675 & 1.7 & -77.145 & 34.6208 & NC \\
\hline 6532 & -19.1 & -77.0997 & 34.6338 & NC & 6604 & 0 & -77.1306 & 34.6274 & NC & 6676 & 1.2 & -76.5144 & 34.6208 & $\mathrm{NC}$ \\
\hline 6533 & -19.4 & -77.1003 & 34.6336 & NC & 6605 & 0.2 & -77.1311 & 34.6273 & NC & 6677 & 1.7 & -77.1455 & 34.6206 & $\mathrm{NC}$ \\
\hline 6534 & 3 & -76.5441 & 34.6336 & NC & 6606 & 0.1 & -77.1316 & 34.6272 & NC & 6678 & -0.1 & -76.5606 & 34.6205 & $\mathrm{NC}$ \\
\hline 6535 & 1.3 & -76.5073 & 34.6335 & $\mathrm{NC}$ & 6607 & -1.5 & -76.5369 & 34.6271 & $\mathrm{NC}$ & 6679 & 1.7 & -77.146 & 34.6204 & NC \\
\hline 6536 & 3.2 & -76.5437 & 34.6332 & $\mathrm{NC}$ & 6608 & -13.9 & -76.563 & 34.6271 & $\mathrm{NC}$ & 6680 & 1 & -76.5146 & 34.6204 & $\mathrm{NC}$ \\
\hline 6537 & 1.2 & -76.5076 & 34.633 & NC & 6609 & 0.4 & -77.1322 & 34.6271 & $\mathrm{NC}$ & 6681 & 1.7 & -77.1465 & 34.6202 & $\mathrm{NC}$ \\
\hline 6538 & 3.5 & -76.5433 & 34.6329 & $\mathrm{NC}$ & 6610 & 1.3 & -76.5109 & 34.6271 & NC & 6682 & -0.1 & -76.5604 & 34.62 & $\mathrm{NC}$ \\
\hline 6539 & 3.7 & -76.5429 & 34.6326 & $\mathrm{NC}$ & 6611 & 0.7 & -77.1327 & 34.6269 & NC & 6683 & 1.8 & -77.147 & 34.62 & $\mathrm{NC}$ \\
\hline 6540 & 1 & -76.5078 & 34.6326 & $\mathrm{NC}$ & 6612 & 1 & -77.1333 & 34.6268 & NC & 6684 & 1.1 & -76.5149 & 34.62 & $\mathrm{NC}$ \\
\hline 6541 & 4 & -76.5425 & 34.6322 & $\mathrm{NC}$ & 6613 & -9.9 & -76.5629 & 34.6267 & $\mathrm{NC}$ & 6685 & 1.7 & -77.1475 & 34.6198 & $\mathrm{NC}$ \\
\hline 6542 & 1.1 & -76.5081 & 34.6322 & $\mathrm{NC}$ & 6614 & -1.8 & -76.5365 & 34.6267 & $\mathrm{NC}$ & 6686 & 1.1 & -76.5603 & 34.6196 & $\mathrm{NC}$ \\
\hline 6543 & 4.2 & -76.5422 & 34.6319 & NC & 6615 & 1.2 & -77.1338 & 34.6267 & $\mathrm{NC}$ & 6687 & 1.7 & -77.148 & 34.6196 & NC \\
\hline 6544 & 0.9 & -76.5083 & 34.6318 & NC & 6616 & 1.3 & -76.5111 & 34.6267 & $\mathrm{NC}$ & 6688 & 1.1 & -76.5151 & 34.6196 & $\mathrm{NC}$ \\
\hline 6545 & 9.8 & -77.1111 & 34.6316 & $\mathrm{NC}$ & 6617 & 1.3 & -77.1344 & 34.6266 & $\mathrm{NC}$ & 6689 & 1.7 & -77.1485 & 34.6194 & $\mathrm{NC}$ \\
\hline 6546 & 4.3 & -76.5418 & 34.6315 & $\mathrm{NC}$ & 6618 & 1.4 & -77.1349 & 34.6265 & $\mathrm{NC}$ & 6690 & 1.6 & -77.149 & 34.6192 & $\mathrm{NC}$ \\
\hline 6547 & 7.5 & -77.1116 & 34.6315 & NC & 6619 & 1.5 & -77.1354 & 34.6264 & $\mathrm{NC}$ & 6691 & 2.1 & -76.5601 & 34.6192 & $\mathrm{NC}$ \\
\hline 6548 & 6.5 & -77.1122 & 34.6314 & $\mathrm{NC}$ & 6620 & -2 & -76.5361 & 34.6264 & $\mathrm{NC}$ & 6692 & 1 & -76.5153 & 34.6191 & $\mathrm{NC}$ \\
\hline 6549 & 3 & -77.1127 & 34.6313 & NC & 6621 & -7.7 & -76.5627 & 34.6263 & NC & 6693 & 1.6 & -77.1496 & 34.619 & $\mathrm{NC}$ \\
\hline 6550 & 1 & -76.5085 & 34.6313 & $\mathrm{NC}$ & 6622 & 1.4 & -76.5113 & 34.6263 & $\mathrm{NC}$ & 6694 & 1.6 & -77.1501 & 34.6188 & $\mathrm{NC}$ \\
\hline 6551 & 4 & -76.5414 & 34.6312 & $\mathrm{NC}$ & 6623 & 1.6 & -77.136 & 34.6262 & $\mathrm{NC}$ & 6695 & 2.5 & -76.56 & 34.6187 & NC \\
\hline
\end{tabular}




\begin{tabular}{|c|c|c|c|c|c|c|c|c|c|c|c|c|c|c|}
\hline TR\# & RATE & LONG & LAT & ST & TR\# & RATE & LONG & LAT & ST & TR\# & RATE & LONG & LAT & ST \\
\hline 6696 & 0.9 & -76.5156 & 34.6187 & NC & 6768 & -0.8 & -76.52 & 34.6107 & $\mathrm{NC}$ & 6840 & -1.3 & -77.1912 & 34.6031 & NC \\
\hline 6697 & 1.5 & -77.1506 & 34.6186 & NC & 6769 & -0.7 & .76 .5569 & 34.6103 & NC & 6841 & -1.3 & -77.1917 & 34.6029 & NC \\
\hline 6698 & 1.5 & -77.1511 & 34.6184 & NC & 6770 & -0.8 & .76 .5203 & 34.6103 & NC & 6842 & -2 & -76.5217 & 34.6028 & NC \\
\hline 6699 & 3.3 & -76.5598 & 34.6183 & NC & 6771 & -0.8 & -76.5205 & 34.6099 & NC & 6843 & -3.7 & -76.5541 & 34.6027 & NC \\
\hline 6700 & 0.9 & -76.5158 & 34.6183 & $\mathrm{NC}$ & 6772 & -0.7 & -76.5567 & 34.6098 & NC & 6844 & -1.5 & -77.1922 & 34.6027 & NC \\
\hline 6701 & 1.5 & -77.1516 & 34.6182 & NC & 6773 & -0.8 & -77.1728 & 34.6097 & NC & 6845 & -1.5 & -77.1927 & 34.6025 & NC \\
\hline 6702 & 1.5 & -77.1521 & 34.618 & NC & 6774 & -0.3 & -77.1733 & 34.6096 & NC & 6846 & -1.6 & -77.1933 & 34.6024 & NC \\
\hline 6703 & 1.4 & -77.1526 & 34.6179 & NC & 6775 & 0.1 & -77.1738 & 34.6094 & NC & 6847 & -3.8 & -76.554 & 34.6023 & NC \\
\hline 6704 & 0.9 & -76.516 & 34.6179 & NC & 6776 & -0.9 & -76.5565 & 34.6094 & NC & 6848 & -2 & -76.5219 & 34.6023 & NC \\
\hline 6705 & 2.6 & -76.5596 & 34.6178 & NC & 6777 & -0.9 & -76.5208 & 34.6094 & NC & 6849 & -1.6 & -77.1938 & 34.6022 & NC \\
\hline 6706 & 1.5 & -77.1531 & 34.6177 & NC & 6778 & -0.7 & -77.1743 & 34.6092 & NC & 6850 & -1.7 & -77.1943 & 34.602 & NC \\
\hline 6707 & 1.3 & -77.1536 & 34.6175 & NC & 6779 & -1.7 & -77.1748 & 34.609 & NC & 6851 & -2.1 & .76 .522 & 34.6019 & NC \\
\hline 6708 & 1.3 & -76.5595 & 34.6174 & NC & 6780 & -0.9 & -76.521 & 34.609 & NC & 6852 & -4 & -76.5538 & 34.6018 & NC \\
\hline 6709 & 0.8 & -76.5163 & 34.6174 & $\mathrm{NC}$ & 6781 & -1.1 & -76.5564 & 34.6089 & NC & 6853 & -1.7 & -77.1948 & 34.6018 & NC \\
\hline 6710 & 1.3 & -77.1541 & 34.6173 & NC & 6782 & -1.8 & -77.1754 & 34.6088 & NC & 6854 & -1.6 & -77.1953 & 34.6016 & NC \\
\hline 6711 & 1.2 & -77.1546 & 34.6171 & NC & 6783 & -1.8 & -77.1759 & 34.6086 & NC & 6855 & -3.1 & -76.5552 & 34.6014 & NC \\
\hline 6712 & 0.7 & -76.5165 & 34.617 & NC & 6784 & -1.1 & -76.5212 & 34.6086 & $\mathrm{NC}$ & 6856 & -1.6 & -77.1958 & 34.6014 & NC \\
\hline 6713 & 0.7 & -76.5593 & 34.6169 & NC & 6785 & -1.1 & -76.5562 & 34.6085 & $\mathrm{NC}$ & 6857 & -2 & -76.5221 & 34.6014 & NC \\
\hline 6714 & 1.2 & -77.1551 & 34.6169 & NC & 6786 & -1.4 & .77 .1764 & 34.6084 & $\mathrm{NC}$ & 6858 & -1.6 & -77.1963 & 34.6013 & NC \\
\hline 6715 & 1 & -77.1557 & 34.6167 & NC & 6787 & -0.6 & -77.1769 & 34.6083 & NC & 6859 & -1.7 & -77.1968 & 34.6011 & NC \\
\hline 6716 & 0.5 & -76.5168 & 34.6166 & NC & 6788 & -1.3 & -76.5215 & 34.6082 & NC & 6860 & -3.4 & -76.555 & 34.601 & NC \\
\hline 6717 & 0.3 & -76.5591 & 34.6165 & NC & 6789 & -1.5 & -76.5561 & 34.6081 & NC & 6861 & -2.1 & -76.5222 & 34.601 & NC \\
\hline 6718 & 0.8 & -77.1562 & 34.6165 & NC & 6790 & -0.6 & -77.1774 & 34.6081 & NC & 6862 & -1.7 & -77.1973 & 34.6009 & NC \\
\hline 6719 & 0.9 & -77.1567 & 34.6163 & $\mathrm{NC}$ & 6791 & -1.9 & -76.5203 & 34.6081 & NC & 6863 & -1.8 & -77.1979 & 34.6007 & NC \\
\hline 6720 & 0.3 & -76.517 & 34.6162 & NC & 6792 & -0.7 & -77.1779 & 34.6079 & NC & 6864 & -3.6 & -76.5547 & 34.6006 & NC \\
\hline 6721 & 0.9 & -77.1572 & 34.6161 & NC & 6793 & -0.7 & -77.1784 & 34.6077 & NC & 6865 & -1.8 & -77.1984 & 34.6005 & NC \\
\hline 6722 & 0.2 & -76.559 & 34.616 & NC & 6794 & -2.1 & -76.5204 & 34.6077 & NC & 6866 & -1.9 & -76.5223 & 34.6005 & NC \\
\hline 6723 & 0.8 & -77.1577 & 34.6159 & NC & 6795 & -1.8 & -76.5559 & 34.6076 & $\mathrm{NC}$ & 6867 & -1.7 & -77.1989 & 34.6003 & NC \\
\hline 6724 & 0.1 & -76.5172 & 34.6158 & NC & 6796 & -0.9 & -77.1789 & 34.6075 & $\mathrm{NC}$ & 6868 & -3.8 & -76.5545 & 34.6002 & NC \\
\hline 6725 & 0.8 & -77.1582 & 34.6157 & $\mathrm{NC}$ & 6797 & -1 & -77.1794 & 34.6073 & NC & 6869 & -1.7 & -77.1994 & 34.6001 & NC \\
\hline 6726 & 0.3 & -76.5588 & 34.6156 & $\mathrm{NC}$ & 6798 & -1.9 & -76.5557 & 34.6072 & NC & 6870 & -1.9 & -76.5225 & 34.6001 & NC \\
\hline 6727 & 0.7 & -77.1587 & 34.6155 & NC & 6799 & -1.1 & -77.18 & 34.6072 & NC & 6871 & -1.7 & -77.1999 & 34.6 & NC \\
\hline 6728 & 0.6 & -77.1592 & 34.6153 & $\mathrm{NC}$ & 6800 & -2.2 & -76.5205 & 34.6072 & $\mathrm{NC}$ & 6872 & -1.7 & -77.2004 & 34.5998 & NC \\
\hline 6729 & 0 & -76.5175 & 34.6153 & $\mathrm{NC}$ & 6801 & -1.1 & -77.1805 & 34.607 & NC & 6873 & -3.9 & -76.5542 & 34.5998 & NC \\
\hline 6730 & 0.9 & -76.5587 & 34.6152 & NC & 6802 & -1 & -77.181 & 34.6068 & NC & 6874 & -1.8 & -77.2009 & 34.5996 & NC \\
\hline 6731 & 0.5 & -77.1597 & 34.6151 & NC & 6803 & -2.2 & -76.5206 & 34.6068 & NC & 6875 & -1.7 & -76.5226 & 34.5996 & NC \\
\hline 6732 & 0.5 & -77.1602 & 34.6149 & NC & 6804 & -1.9 & -76.5556 & 34.6067 & NC & 6876 & -1.9 & -77.2014 & 34.5994 & NC \\
\hline 6733 & -0.2 & -76.5177 & 34.6149 & NC & 6805 & -1.2 & -77.1815 & 34.6066 & NC & 6877 & -4.1 & -76.5539 & 34.5994 & NC \\
\hline 6734 & 4.7 & -76.5585 & 34.6147 & NC & 6806 & -1.2 & -77.182 & 34.6064 & NC & 6878 & -2 & -77.2019 & 34.5992 & NC \\
\hline 6735 & 0.4 & -77.1607 & 34.6147 & NC & 6807 & -2 & -76.5554 & 34.6063 & NC & 6879 & -2.1 & -76.5227 & 34.5992 & NC \\
\hline 6736 & 0.4 & -77.1612 & 34.6145 & NC & 6808 & -2.1 & -76.5208 & 34.6063 & NC & 6880 & -2 & -77.2025 & 34.599 & NC \\
\hline 6737 & -0.3 & .76 .5179 & 34.6145 & NC & 6809 & -1.2 & -77.1825 & 34.6062 & NC & 6881 & -4.1 & -76.5537 & 34.599 & NC \\
\hline 6738 & 3.6 & -76.5583 & 34.6143 & NC & 6810 & -1.2 & -77.183 & 34.606 & NC & 6882 & -2.1 & -76.5228 & 34.5987 & NC \\
\hline 6739 & 0.4 & -77.1618 & 34.6143 & NC & 6811 & -1.3 & -77.1835 & 34.6059 & NC & 6883 & -4 & -76.5534 & 34.5986 & NC \\
\hline 6740 & 0.5 & -77.1623 & 34.6141 & NC & 6812 & -1.8 & -76.5209 & 34.6059 & NC & 6884 & -2.2 & -76.5229 & 34.5983 & NC \\
\hline 6741 & -0.4 & -76.5182 & 34.6141 & NC & 6813 & -2.1 & -76.5553 & 34.6058 & NC & 6885 & -3.9 & -76.5531 & 34.5981 & NC \\
\hline 6742 & 0.5 & -77.1628 & 34.6139 & NC & 6814 & -1.3 & -77.184 & 34.6057 & NC & 6886 & -2.4 & -76.5231 & 34.5978 & NC \\
\hline 6743 & 2.2 & -76.5582 & 34.6138 & NC & 6815 & -1.3 & -77.1846 & 34.6055 & NC & 6887 & -4.1 & -76.5529 & 34.5977 & NC \\
\hline 6744 & 0.4 & -77.1633 & 34.6137 & NC & 6816 & -2.3 & -76.5551 & 34.6054 & NC & 6888 & -2.2 & -76.5232 & 34.5974 & NC \\
\hline 6745 & -0.5 & -76.5184 & 34.6137 & NC & 6817 & -1.9 & -76.521 & 34.6054 & NC & 6889 & -4.3 & -76.5526 & 34.5973 & NC \\
\hline 6746 & 0.8 & -77.1638 & 34.6135 & NC & 6818 & -1.3 & -77.1851 & 34.6053 & NC & 6890 & -2 & -76.5233 & 34.597 & NC \\
\hline 6747 & 1.6 & -76.558 & 34.6134 & NC & 6819 & -1.3 & -77.1856 & 34.6051 & NC & 6891 & -4.2 & -76.5523 & 34.5969 & NC \\
\hline 6748 & 1.2 & -77.1643 & 34.6133 & NC & 6820 & -2.5 & -76.5549 & 34.605 & NC & 6892 & -4.3 & -76.5521 & 34.5965 & NC \\
\hline 6749 & -0.6 & -76.5186 & 34.6132 & NC & 6821 & -1.9 & -76.5211 & 34.605 & NC & 6893 & -2.1 & -76.5234 & 34.5965 & NC \\
\hline 6750 & -1.8 & -77.1648 & 34.6131 & NC & 6822 & -1.3 & -77.1861 & 34.6049 & NC & 6894 & -4.4 & -76.5518 & 34.5961 & NC \\
\hline 6751 & 1 & -76.5578 & 34.6129 & NC & 6823 & -1.3 & -77.1866 & 34.6048 & NC & 6895 & -1.8 & -76.5236 & 34.5961 & NC \\
\hline 6752 & -1.7 & -77.1653 & 34.6129 & NC & 6824 & -1.3 & -77.1871 & 34.6046 & NC & 6896 & -4.5 & -76.5516 & 34.5957 & NC \\
\hline 6753 & -0.5 & -76.5189 & 34.6128 & NC & 6825 & -2.7 & -76.5548 & 34.6045 & NC & 6897 & -1.6 & -76.5237 & 34.5956 & NC \\
\hline 6754 & -0.6 & -77.1658 & 34.6127 & NC & 6826 & -1.9 & -76.5213 & 34.6045 & NC & 6898 & -4.6 & .76 .5513 & 34.5953 & NC \\
\hline 6755 & 0.2 & -76.5577 & 34.6125 & NC & 6827 & -1.3 & -77.1876 & 34.6044 & NC & 6899 & -1.5 & -76.5238 & 34.5952 & NC \\
\hline 6756 & 0.3 & -77.1663 & 34.6125 & NC & 6828 & -1.3 & -77.1881 & 34.6042 & NC & 6900 & -4.7 & .76 .551 & 34.5949 & NC \\
\hline 6757 & -0.5 & -76.5191 & 34.6124 & NC & 6829 & -3 & -76.5546 & 34.6041 & NC & 6901 & -1.4 & -76.5239 & 34.5947 & NC \\
\hline 6758 & 6.1 & -77.1668 & 34.6123 & NC & 6830 & -2.1 & -76.5214 & 34.6041 & $\mathrm{NC}$ & 6902 & -4.9 & -76.5508 & 34.5945 & NC \\
\hline 6759 & -0.6 & -76.5575 & 34.6121 & NC & 6831 & -1.3 & -77.1887 & 34.604 & NC & 6903 & -1.2 & -77.2096 & 34.5943 & $\mathrm{NC}$ \\
\hline 6760 & -5.8 & -77.1673 & 34.6121 & NC & 6832 & -1.3 & -77.1892 & 34.6038 & NC & 6904 & -1.3 & -76.524 & 34.5943 & NC \\
\hline 6761 & -0.5 & -76.5193 & 34.612 & NC & 6833 & -1.3 & -77.1897 & 34.6037 & NC & 6905 & -5 & -76.5505 & 34.5941 & NC \\
\hline 6762 & -15.4 & -77.1679 & 34.6119 & NC & 6834 & -3.3 & -76.5544 & 34.6036 & NC & 6906 & -1.2 & -77.2101 & 34.5941 & NC \\
\hline 6763 & -0.8 & -76.5574 & 34.6116 & NC & 6835 & -2 & -76.5215 & 34.6036 & NC & 6907 & -1.2 & -77.2106 & 34.5938 & NC \\
\hline 6764 & -0.6 & -76.5196 & 34.6116 & NC & 6836 & -1.2 & -77.1902 & 34.6035 & NC & 6908 & -1 & -76.5242 & 34.5938 & NC \\
\hline 6765 & -0.7 & -76.5572 & 34.6112 & NC & 6837 & -1.3 & -77.1907 & 34.6033 & NC & 6909 & -5.2 & -76.5502 & 34.5937 & NC \\
\hline 6766 & -0.7 & -76.5198 & 34.6111 & NC & 6838 & -3.5 & -76.5543 & 34.6032 & NC & 6910 & -1.2 & -77.211 & 34.5936 & NC \\
\hline 6767 & -0.7 & -76.557 & 34.6107 & NC & 6839 & -1.9 & -76.5216 & 34.6032 & NC & 6911 & -1 & -76.5243 & 34.5934 & NC \\
\hline
\end{tabular}




\begin{tabular}{|c|c|c|c|c|c|c|c|c|c|c|c|c|c|c|}
\hline TR\# & RATE & LONG & LAT & ST & TR\# & RATE & LONG & LAT & ST & TR\# & RATE & LONG & LAT & ST \\
\hline 6912 & -5.2 & -76.55 & 34.5933 & NC & 6984 & -4.3 & -77.2278 & 34.5844 & $\mathrm{NC}$ & 7056 & -0.2 & -77.2517 & 34.5704 & NC \\
\hline 6913 & -1.2 & -77.2115 & 34.5933 & NC & 6985 & -3 & -77.2283 & 34.5842 & $\mathrm{NC}$ & 7057 & -0.1 & -77.2521 & 34.5701 & NC \\
\hline 6914 & -1.3 & -77.212 & 34.5931 & NC & 6986 & -7.1 & -76.5439 & 34.584 & $\mathrm{NC}$ & 7058 & -0.1 & -77.2526 & 34.5699 & NC \\
\hline 6915 & -5.4 & -76.5497 & 34.5929 & NC & 6987 & -3.4 & -76.5268 & 34.584 & NC & 7059 & 0 & -77.253 & 34.5696 & NC \\
\hline 6916 & -1.2 & -76.5244 & 34.5929 & NC & 6988 & -2.1 & -77.2287 & 34.5839 & NC & 7060 & 0 & -77.2535 & 34.5693 & NC \\
\hline 6917 & -1.3 & -77.2124 & 34.5928 & NC & 6989 & -1.5 & -77.2292 & 34.5837 & NC & 7061 & -0.1 & -77.254 & 34.569 & NC \\
\hline 6918 & -1.2 & -77.2129 & 34.5926 & NC & 6990 & -8.1 & -76.5437 & 34.5836 & NC & 7062 & -0.1 & -77.2544 & 34.5688 & NC \\
\hline 6919 & -5.6 & -76.5495 & 34.5925 & NC & 6991 & -3.5 & -76.5269 & 34.5836 & NC & 7063 & -0.2 & -77.2549 & 34.5685 & NC \\
\hline 6920 & -1.3 & -76.5245 & 34.5925 & NC & 6992 & -1.2 & -77.2297 & 34.5834 & NC & 7064 & -0.2 & -77.2553 & 34.5682 & NC \\
\hline 6921 & -1.2 & -77.2134 & 34.5923 & NC & 6993 & -0.8 & -77.2301 & 34.5832 & NC & 7065 & -0.3 & -77.2558 & 34.5679 & NC \\
\hline 6922 & -5.7 & -76.5492 & 34.5921 & NC & 6994 & -0.6 & -76.5434 & 34.5832 & NC & 7066 & -0.4 & -77.2562 & 34.5677 & NC \\
\hline 6923 & -1.1 & -77.2138 & 34.592 & NC & 6995 & -3.4 & -76.5271 & 34.5831 & NC & 7067 & -0.4 & -77.2567 & 34.5674 & NC \\
\hline 6924 & -1.4 & -76.5246 & 34.592 & NC & 6996 & -0.4 & -77.2306 & 34.5829 & NC & 7068 & -0.2 & -77.2571 & 34.5671 & NC \\
\hline 6925 & -1.3 & -77.2143 & 34.5918 & NC & 6997 & -0.5 & -76.5431 & 34.5828 & NC & 7069 & -0.2 & -77.2576 & 34.5668 & NC \\
\hline 6926 & -5.8 & -76.5489 & 34.5917 & NC & 6998 & 0 & -77.2311 & 34.5827 & NC & 7070 & -0.3 & -77.258 & 34.5666 & NC \\
\hline 6927 & -1.5 & -76.5248 & 34.5916 & NC & 6999 & -3.3 & -76.5272 & 34.5827 & NC & 7071 & -0.2 & -77.2585 & 34.5663 & NC \\
\hline 6928 & -1.2 & -77.2148 & 34.5915 & NC & 7000 & 0.8 & -77.2318 & 34.5825 & NC & 7072 & -0.2 & -77.2589 & 34.566 & NC \\
\hline 6929 & -1.1 & -77.2152 & 34.5913 & NC & 7001 & 0.4 & -77.2315 & 34.5824 & NC & 7073 & -0.2 & -77.2594 & 34.5657 & $\mathrm{NC}$ \\
\hline 6930 & -6.1 & -76.5487 & 34.5913 & NC & 7002 & -0.5 & -76.5429 & 34.5824 & NC & 7074 & -0.4 & -77.2598 & 34.5655 & NC \\
\hline 6931 & -1.2 & -76.5249 & 34.5911 & NC & 7003 & 0.6 & -77.232 & 34.5822 & NC & 7075 & -0.4 & -77.2603 & 34.5652 & $\mathrm{NC}$ \\
\hline 6932 & -1 & -77.2157 & 34.591 & NC & 7004 & 0.6 & -77.2323 & 34.5822 & NC & 7076 & -0.5 & -77.2607 & 34.5649 & NC \\
\hline 6933 & -6.3 & -76.5484 & 34.5909 & NC & 7005 & -3.1 & -76.5273 & 34.5822 & NC & 7077 & -0.5 & -77.2612 & 34.5646 & NC \\
\hline 6934 & -1 & -77.2162 & 34.5908 & NC & 7006 & 0.5 & -77.2327 & 34.582 & NC & 7078 & -0.6 & -77.2616 & 34.5644 & NC \\
\hline 6935 & -1.1 & -76.525 & 34.5907 & NC & 7007 & -0.4 & -76.5426 & 34.582 & NC & 7079 & -0.6 & -77.2621 & 34.5641 & NC \\
\hline 6936 & -1 & -77.2166 & 34.5905 & NC & 7008 & 0.8 & -77.2325 & 34.5819 & NC & 7080 & 0 & -77.2619 & 34.564 & NC \\
\hline 6937 & -6.5 & -76.5481 & 34.5905 & NC & 7009 & -2.8 & -76.5274 & 34.5818 & NC & 7081 & -0.6 & -77.2625 & 34.5638 & NC \\
\hline 6938 & -0.7 & -77.2171 & 34.5903 & NC & 7010 & 0.6 & -77.2332 & 34.5817 & NC & 7082 & 0.1 & -77.2623 & 34.5637 & NC \\
\hline 6939 & -1.2 & -76.5251 & 34.5903 & NC & 7011 & 0.6 & -76.5423 & 34.5816 & NC & 7083 & -0.6 & -77.263 & 34.5635 & NC \\
\hline 6940 & -6.8 & -76.5479 & 34.5901 & NC & 7012 & 0.4 & -77.2336 & 34.5814 & NC & 7084 & 0 & -77.2628 & 34.5634 & NC \\
\hline 6941 & -0.6 & .77 .2175 & 34.59 & NC & 7013 & -2.7 & -76.5276 & 34.5813 & NC & 7085 & -0.1 & -77.2632 & 34.5631 & NC \\
\hline 6942 & -0.3 & -77.218 & 34.5898 & NC & 7014 & 1.6 & -76.5421 & 34.5812 & NC & 7086 & -0.1 & -77.2636 & 34.5628 & NC \\
\hline 6943 & -1.1 & -76.5253 & 34.5898 & NC & 7015 & 0.2 & -77.2341 & 34.5811 & NC & 7087 & -0.1 & -77.264 & 34.5625 & NC \\
\hline 6944 & -6.8 & -76.5476 & 34.5897 & NC & 7016 & 0.1 & -77.2345 & 34.5809 & NC & 7088 & 0 & -77.2645 & 34.5622 & NC \\
\hline 6945 & -0.1 & -77.2185 & 34.5895 & NC & 7017 & -2.6 & -76.5277 & 34.5809 & NC & 7089 & -0.1 & -77.2649 & 34.5619 & NC \\
\hline 6946 & -0.9 & -76.5254 & 34.5894 & NC & 7018 & 2.5 & -76.5418 & 34.5808 & NC & 7090 & 0 & -77.2653 & 34.5616 & NC \\
\hline 6947 & 0.1 & -77.2189 & 34.5893 & NC & 7019 & 0.2 & -77.235 & 34.5806 & NC & 7091 & -0.1 & -77.2658 & 34.5613 & NC \\
\hline 6948 & -6.9 & -76.5473 & 34.5893 & NC & 7020 & 0.1 & -77.2354 & 34.5803 & NC & 7092 & -0.1 & -77.2662 & 34.561 & NC \\
\hline 6949 & 0.4 & -77.2194 & 34.589 & NC & 7021 & 0 & -77.2359 & 34.58 & NC & 7093 & 0 & -77.2666 & 34.5607 & NC \\
\hline 6950 & -7.1 & -76.5471 & 34.5889 & NC & 7022 & 0.1 & -77.2363 & 34.5798 & NC & 7094 & 0 & -77.2671 & 34.5604 & NC \\
\hline 6951 & -0.5 & -76.5255 & 34.5889 & NC & 7023 & 0.1 & -77.2368 & 34.5795 & NC & 7095 & -0.1 & -77.2675 & 34.5601 & NC \\
\hline 6952 & 1 & -77.2199 & 34.5888 & NC & 7024 & 0.1 & -77.2372 & 34.5792 & NC & 7096 & -0.2 & -77.2679 & 34.5598 & NC \\
\hline 6953 & 1.5 & -77.2203 & 34.5885 & NC & 7025 & 0.1 & -77.2377 & 34.5789 & NC & 7097 & -0.2 & -77.2683 & 34.5595 & NC \\
\hline 6954 & 0.7 & -76.5256 & 34.5885 & NC & 7026 & 0.1 & -77.2381 & 34.5787 & NC & 7098 & -0.3 & -77.2688 & 34.5592 & NC \\
\hline 6955 & -7.3 & -76.5468 & 34.5884 & NC & 7027 & 0 & -77.2386 & 34.5784 & NC & 7099 & -0.4 & -77.2692 & 34.5589 & NC \\
\hline 6956 & 6.6 & -77.2208 & 34.5882 & NC & 7028 & -0.1 & -77.239 & 34.5781 & NC & 7100 & -0.3 & -77.2696 & 34.5586 & NC \\
\hline 6957 & 9.4 & -77.2213 & 34.588 & NC & 7029 & -0.2 & -77.2395 & 34.5778 & NC & 7101 & -0.3 & -77.2701 & 34.5583 & NC \\
\hline 6958 & -7.4 & -76.5466 & 34.588 & NC & 7030 & -0.2 & -77.2399 & 34.5776 & NC & 7102 & -0.5 & -77.2705 & 34.558 & NC \\
\hline 6959 & 1.1 & -76.5257 & 34.588 & NC & 7031 & -0.1 & -77.2404 & 34.5773 & NC & 7103 & -0.5 & -77.2709 & 34.5577 & NC \\
\hline 6960 & 12.3 & -77.2217 & 34.5877 & NC & 7032 & -0.2 & -77.2408 & 34.577 & NC & 7104 & -0.4 & -77.2714 & 34.5574 & NC \\
\hline 6961 & -7.6 & -76.5463 & 34.5876 & NC & 7033 & -0.3 & -77.2413 & 34.5767 & NC & 7105 & -0.3 & -77.2718 & 34.5571 & NC \\
\hline 6962 & 1.2 & -76.5259 & 34.5876 & NC & 7034 & -0.4 & -77.2417 & 34.5765 & NC & 7106 & -0.2 & -77.2722 & 34.5568 & NC \\
\hline 6963 & 6.8 & -77.2222 & 34.5875 & NC & 7035 & -0.5 & -77.2422 & 34.5762 & NC & 7107 & -0.2 & -77.2726 & 34.5565 & NC \\
\hline 6964 & .7 .9 & -76.546 & 34.5872 & NC & 7036 & -0.5 & -77.2427 & 34.5759 & NC & 7108 & -0.2 & -77.2731 & 34.5562 & NC \\
\hline 6965 & 4.8 & -77.2227 & 34.5872 & NC & 7037 & -0.4 & -77.2431 & 34.5756 & NC & 7109 & -0.2 & -77.2735 & 34.5559 & NC \\
\hline 6966 & 1.3 & -76.526 & 34.5871 & NC & 7038 & -0.5 & -77.2436 & 34.5754 & NC & 7110 & -0.3 & -77.2739 & 34.5556 & NC \\
\hline 6967 & 1.1 & -77.2231 & 34.587 & NC & 7039 & -0.6 & -77.244 & 34.5751 & NC & 7111 & -0.5 & -77.2744 & 34.5553 & NC \\
\hline 6968 & -8.2 & -76.5458 & 34.5868 & NC & 7040 & -0.6 & -77.2445 & 34.5748 & NC & 7112 & -0.4 & -77.2748 & 34.555 & NC \\
\hline 6969 & -2.5 & -77.2236 & 34.5867 & NC & 7041 & -0.6 & -77.2449 & 34.5745 & NC & 7113 & -0.5 & -77.2752 & 34.5547 & NC \\
\hline 6970 & -1.7 & -76.5261 & 34.5867 & NC & 7042 & -0.6 & -77.2454 & 34.5743 & NC & 7114 & -0.4 & -77.2756 & 34.5544 & NC \\
\hline 6971 & -8.6 & -76.5455 & 34.5864 & NC & 7043 & -0.6 & -77.2458 & 34.574 & NC & 7115 & -0.4 & -77.2761 & 34.5541 & NC \\
\hline 6972 & -1.6 & -76.5262 & 34.5862 & NC & 7044 & -0.5 & -77.2463 & 34.5737 & NC & 7116 & -0.4 & -77.2765 & 34.5538 & NC \\
\hline 6973 & -5.1 & -76.5452 & 34.586 & NC & 7045 & -0.6 & -77.2467 & 34.5734 & NC & 7117 & -0.4 & -77.2769 & 34.5535 & NC \\
\hline 6974 & -3 & -76.5263 & 34.5858 & NC & 7046 & -0.5 & -77.2472 & 34.5732 & NC & 7118 & -0.5 & -77.2774 & 34.5533 & NC \\
\hline 6975 & -5.9 & -76.545 & 34.5856 & NC & 7047 & -0.5 & -77.2476 & 34.5729 & NC & 7119 & -0.4 & -77.2778 & 34.553 & NC \\
\hline 6976 & -3.2 & -76.5265 & 34.5853 & NC & 7048 & -0.5 & -77.2481 & 34.5726 & NC & 7120 & -0.4 & -77.2782 & 34.5527 & NC \\
\hline 6977 & -6.6 & -76.5447 & 34.5852 & NC & 7049 & -0.3 & -77.2485 & 34.5723 & NC & 7121 & -0.5 & -77.2787 & 34.5524 & NC \\
\hline 6978 & -8.1 & -77.2269 & 34.5849 & NC & 7050 & -0.2 & -77.249 & 34.5721 & NC & 7122 & -0.5 & -77.2791 & 34.5521 & NC \\
\hline 6979 & -3.2 & -76.5266 & 34.5849 & NC & 7051 & -0.2 & -77.2494 & 34.5718 & NC & 7123 & -0.4 & -77.2795 & 34.5518 & NC \\
\hline 6980 & -6.9 & -76.5444 & 34.5848 & NC & 7052 & -0.2 & -77.2499 & 34.5715 & NC & 7124 & -0.4 & -77.2799 & 34.5515 & NC \\
\hline 6981 & -6 & -77.2273 & 34.5847 & NC & 7053 & -0.2 & -77.2503 & 34.5712 & NC & 7125 & -0.5 & -77.2804 & 34.5512 & NC \\
\hline 6982 & -3.3 & -76.5267 & 34.5845 & NC & 7054 & -0.2 & -77.2508 & 34.571 & NC & 7126 & -0.3 & -77.2808 & 34.5509 & NC \\
\hline 6983 & -7.3 & .76 .5442 & 34.5844 & NC & 7055 & -0.3 & -77.2512 & 34.5707 & NC & 7127 & -0.3 & -77.2812 & 34.5506 & NC \\
\hline
\end{tabular}




\begin{tabular}{|c|c|c|c|c|c|c|c|c|c|c|c|c|c|c|}
\hline TR\# & RATE & LONG & LAT & ST & TR\# & RATE & LONG & LAT & ST & TR\# & RATE & LONG & LAT & ST \\
\hline 7128 & -0.3 & -77.2817 & 34.5503 & NC & 7200 & -3.1 & -77.3135 & 34.5299 & NC & 7272 & -0.4 & -77.3511 & 34.5139 & NC \\
\hline 7129 & -0.4 & -77.2821 & 34.55 & NC & 7201 & -3.3 & -77.314 & 34.5297 & NC & 7273 & -0.5 & -77.3516 & 34.5137 & $\mathrm{NC}$ \\
\hline 7130 & -0.4 & -77.2825 & 34.5497 & NC & 7202 & -3.5 & -77.3144 & 34.5294 & NC & 7274 & -0.6 & -77.3521 & 34.5135 & NC \\
\hline 7131 & -0.4 & -77.2829 & 34.5494 & NC & 7203 & -3.5 & .77 .3149 & 34.5291 & NC & 7275 & -0.7 & -77.3526 & 34.5133 & NC \\
\hline 7132 & -0.3 & -77.2834 & 34.5491 & NC & 7204 & -3.5 & -77.3153 & 34.5289 & NC & 7276 & -0.8 & -77.3531 & 34.5131 & $\mathrm{NC}$ \\
\hline 7133 & -0.2 & -77.2838 & 34.5488 & NC & 7205 & -3.6 & -77.3158 & 34.5286 & NC & 7277 & -1 & -77.3536 & 34.5129 & NC \\
\hline 7134 & -0.2 & -77.2842 & 34.5485 & NC & 7206 & -3.8 & -77.3163 & 34.5284 & NC & 7278 & -1 & -77.3541 & 34.5127 & NC \\
\hline 7135 & -0.2 & -77.2847 & 34.5482 & NC & 7207 & -3.9 & -77.3167 & 34.5281 & NC & 7279 & -1 & -77.3546 & 34.5125 & NC \\
\hline 7136 & -0.2 & -77.2851 & 34.5479 & NC & 7208 & -4.1 & .77 .3172 & 34.5278 & NC & 7280 & -1.1 & -77.3551 & 34.5123 & $\mathrm{NC}$ \\
\hline 7137 & -0.2 & -77.2855 & 34.5476 & NC & 7209 & -4.2 & -77.3177 & 34.5276 & NC & 7281 & -1.2 & -77.3556 & 34.5121 & NC \\
\hline 7138 & -0.2 & -77.286 & 34.5473 & NC & 7210 & -4.2 & -77.3181 & 34.5273 & NC & 7282 & -1.3 & -77.3561 & 34.5119 & NC \\
\hline 7139 & -0.2 & -77.2864 & 34.547 & NC & 7211 & -4.2 & -77.3186 & 34.527 & NC & 7283 & -1.3 & -77.3566 & 34.5118 & NC \\
\hline 7140 & -0.3 & -77.2868 & 34.5467 & NC & 7212 & -4.3 & -77.319 & 34.5268 & NC & 7284 & -1.4 & -77.3572 & 34.5116 & NC \\
\hline 7141 & -0.4 & -77.2872 & 34.5464 & NC & 7213 & -4.4 & -77.3195 & 34.5265 & NC & 7285 & -1.3 & -77.3577 & 34.5114 & $\mathrm{NC}$ \\
\hline 7142 & -0.6 & -77.2877 & 34.5461 & NC & 7214 & -4.5 & -77.32 & 34.5263 & NC & 7286 & -1.4 & -77.3582 & 34.5112 & $\mathrm{NC}$ \\
\hline 7143 & -0.8 & -77.2881 & 34.5458 & NC & 7215 & -4.6 & -77.3204 & 34.526 & NC & 7287 & -1.5 & -77.3587 & 34.511 & NC \\
\hline 7144 & -1 & -77.2885 & 34.5455 & NC & 7216 & -4.6 & -77.3209 & 34.5257 & NC & 7288 & -1 & -77.3586 & 34.5109 & $\mathrm{NC}$ \\
\hline 7145 & -1.1 & -77.289 & 34.5452 & NC & 7217 & -4.7 & -77.3213 & 34.5255 & NC & 7289 & -1.6 & -77.3592 & 34.5108 & NC \\
\hline 7146 & -1.1 & -77.2894 & 34.5449 & NC & 7218 & -4.9 & -77.3218 & 34.5252 & NC & 7290 & -1.1 & -77.3591 & 34.5107 & NC \\
\hline 7147 & -1.2 & -77.2898 & 34.5446 & NC & 7219 & -5.1 & -77.3223 & 34.5249 & NC & 7291 & -1.7 & -77.3597 & 34.5106 & $\mathrm{NC}$ \\
\hline 7148 & -1.1 & -77.2903 & 34.5443 & NC & 7220 & -5.2 & -77.3227 & 34.5247 & NC & 7292 & -1.2 & -77.3596 & 34.5104 & NC \\
\hline 7149 & -1 & -77.2907 & 34.544 & NC & 7221 & -5.2 & -77.3232 & 34.5244 & NC & 7293 & -1.2 & -77.3601 & 34.5102 & NC \\
\hline 7150 & -0.9 & -77.2911 & 34.5437 & NC & 7222 & -3 & -77.3247 & 34.524 & NC & 7294 & -1.3 & -77.3606 & 34.51 & $\mathrm{NC}$ \\
\hline 7151 & -0.8 & -77.2915 & 34.5434 & NC & 7223 & -3.1 & -77.3252 & 34.5238 & NC & 7295 & -1.4 & -77.361 & 34.5098 & NC \\
\hline 7152 & -0.1 & -77.2914 & 34.5425 & NC & 7224 & -3.1 & -77.3257 & 34.5236 & $\mathrm{NC}$ & 7296 & -1.4 & -77.3615 & 34.5095 & NC \\
\hline 7153 & -0.2 & -77.2919 & 34.5422 & NC & 7225 & -3.2 & -77.3262 & 34.5234 & NC & 7297 & -1.3 & -77.362 & 34.5093 & NC \\
\hline 7154 & -0.4 & -77.2923 & 34.542 & NC & 7226 & -3.5 & -77.3267 & 34.5232 & NC & 7298 & -1.3 & -77.3625 & 34.5091 & $\mathrm{NC}$ \\
\hline 7155 & -0.6 & -77.2928 & 34.5417 & NC & 7227 & -3.5 & -77.3272 & 34.523 & $\mathrm{NC}$ & 7299 & -1.3 & -77.363 & 34.5089 & NC \\
\hline 7156 & -0.5 & -77.2932 & 34.5415 & NC & 7228 & -3.6 & -77.3277 & 34.5228 & NC & 7300 & -1.4 & -77.3635 & 34.5086 & $\mathrm{NC}$ \\
\hline 7157 & -0.5 & -77.2937 & 34.5412 & NC & 7229 & -3.7 & -77.3282 & 34.5226 & $\mathrm{NC}$ & 7301 & -1.6 & -77.364 & 34.5084 & NC \\
\hline 7158 & -0.6 & -77.2942 & 34.5409 & $\mathrm{NC}$ & 7230 & -3.8 & -77.3288 & 34.5224 & NC & 7302 & -1.5 & -77.3645 & 34.5082 & $\mathrm{NC}$ \\
\hline 7159 & -0.7 & -77.2946 & 34.5407 & NC & 7231 & -4 & -77.3293 & 34.5223 & NC & 7303 & -1.7 & -77.365 & 34.508 & NC \\
\hline 7160 & -0.7 & -77.2951 & 34.5404 & NC & 7232 & -4.2 & -77.3298 & 34.5221 & NC & 7304 & -1.7 & -77.3654 & 34.5077 & NC \\
\hline 7161 & -0.8 & -77.2955 & 34.5401 & NC & 7233 & -4.5 & -77.3303 & 34.5219 & NC & 7305 & -1.7 & -77.3659 & 34.5075 & NC \\
\hline 7162 & -0.8 & -77.296 & 34.5399 & NC & 7234 & -4.9 & -77.3308 & 34.5217 & $\mathrm{NC}$ & 7306 & -1.6 & -77.3664 & 34.5073 & NC \\
\hline 7163 & -0.9 & -77.2965 & 34.5396 & NC & 7235 & -5.4 & -77.3313 & 34.5215 & NC & 7307 & -1.5 & -77.3669 & 34.5071 & $\mathrm{NC}$ \\
\hline 7164 & -1.1 & -77.2969 & 34.5394 & NC & 7236 & -6.1 & -77.3318 & 34.5213 & NC & 7308 & -1.4 & -77.3674 & 34.5068 & $\mathrm{NC}$ \\
\hline 7165 & -1.1 & -77.2974 & 34.5391 & NC & 7237 & -6.8 & -77.3323 & 34.5211 & $\mathrm{NC}$ & 7309 & -1.4 & -77.3679 & 34.5066 & NC \\
\hline 7166 & -1 & -77.2978 & 34.5388 & NC & 7238 & -7.4 & -77.3328 & 34.5209 & $\mathrm{NC}$ & 7310 & -1.4 & -77.3684 & 34.5064 & $\mathrm{NC}$ \\
\hline 7167 & -1.1 & -77.2983 & 34.5386 & NC & 7239 & -8.2 & -77.3333 & 34.5207 & NC & 7311 & -1.4 & -77.3689 & 34.5062 & $\mathrm{NC}$ \\
\hline 7168 & -1.1 & -77.2988 & 34.5383 & NC & 7240 & -10.1 & -77.3338 & 34.5205 & NC & 7312 & -1.4 & -77.3694 & 34.5059 & NC \\
\hline 7169 & -1 & -77.2992 & 34.538 & NC & 7241 & -17.3 & -77.3353 & 34.5199 & NC & 7313 & -1.4 & -77.3698 & 34.5057 & NC \\
\hline 7170 & -1.1 & -77.2997 & 34.5378 & NC & 7242 & -16 & -77.3359 & 34.5197 & NC & 7314 & -1.4 & -77.3703 & 34.5055 & NC \\
\hline 7171 & -1.1 & -77.3002 & 34.5375 & NC & 7243 & -15 & -77.3364 & 34.5195 & NC & 7315 & -1.4 & -77.3708 & 34.5053 & $\mathrm{NC}$ \\
\hline 7172 & -1.2 & -77.3006 & 34.5373 & NC & 7244 & -12.7 & -77.3369 & 34.5193 & NC & 7316 & -1.4 & -77.3713 & 34.505 & NC \\
\hline 7173 & -1.2 & -77.3011 & 34.537 & NC & 7245 & -10.4 & -77.3374 & 34.5191 & NC & 7317 & -1.4 & -77.3718 & 34.5048 & $\mathrm{NC}$ \\
\hline 7174 & -1.2 & -77.3015 & 34.5367 & NC & 7246 & -13.7 & -77.3379 & 34.5189 & NC & 7318 & -1.3 & -77.3723 & 34.5046 & NC \\
\hline 7175 & -1.2 & -77.302 & 34.5365 & NC & 7247 & -12.3 & -77.3384 & 34.5188 & NC & 7319 & -1.3 & -77.3728 & 34.5044 & NC \\
\hline 7176 & -1.3 & -77.3025 & 34.5362 & NC & 7248 & -10.4 & -77.3389 & 34.5186 & NC & 7320 & -1.3 & -77.3733 & 34.5041 & NC \\
\hline 7177 & -1.4 & -77.3029 & 34.536 & NC & 7249 & -6.1 & -77.3394 & 34.5184 & NC & 7321 & -1.2 & -77.3738 & 34.5039 & NC \\
\hline 7178 & -1.5 & -77.3034 & 34.5357 & $\mathrm{NC}$ & 7250 & 1 & -77.3399 & 34.5182 & NC & 7322 & -1.3 & -77.3742 & 34.5037 & NC \\
\hline 7179 & -1.6 & -77.3038 & 34.5354 & NC & 7251 & 1.7 & -77.3404 & 34.518 & NC & 7323 & -1.2 & -77.3747 & 34.5035 & NC \\
\hline 7180 & -1.6 & -77.3043 & 34.5352 & NC & 7252 & 1.4 & -77.3409 & 34.5178 & NC & 7324 & -1.2 & -77.3752 & 34.5032 & $\mathrm{NC}$ \\
\hline 7181 & -1.6 & -77.3048 & 34.5349 & $\mathrm{NC}$ & 7253 & 1.1 & -77.3414 & 34.5176 & NC & 7325 & -1.2 & -77.3757 & 34.503 & $\mathrm{NC}$ \\
\hline 7182 & -1.6 & -77.3052 & 34.5346 & NC & 7254 & 0.7 & -77.3419 & 34.5174 & NC & 7326 & -1.2 & -77.3762 & 34.5028 & NC \\
\hline 7183 & -1.7 & -77.3057 & 34.5344 & NC & 7255 & 0.4 & -77.3424 & 34.5172 & NC & 7327 & -1.1 & -77.3767 & 34.5026 & NC \\
\hline 7184 & -1.7 & -77.3061 & 34.5341 & NC & 7256 & 0.2 & -77.343 & 34.517 & NC & 7328 & -1.1 & -77.3772 & 34.5023 & NC \\
\hline 7185 & -1.8 & -77.3066 & 34.5339 & NC & 7257 & 0 & -77.3435 & 34.5168 & NC & 7329 & -1.1 & -77.3777 & 34.5021 & NC \\
\hline 7186 & -1.9 & -77.3071 & 34.5336 & NC & 7258 & 0.1 & -77.344 & 34.5166 & NC & 7330 & -1 & -77.3781 & 34.5019 & $\mathrm{NC}$ \\
\hline 7187 & -2 & -77.3075 & 34.5333 & NC & 7259 & 0.3 & .77 .3445 & 34.5164 & NC & 7331 & -1 & -77.3786 & 34.5017 & NC \\
\hline 7188 & -2 & -77.308 & 34.5331 & NC & 7260 & 0.3 & -77.345 & 34.5162 & NC & 7332 & -1.1 & -77.3791 & 34.5014 & NC \\
\hline 7189 & -2 & -77.3084 & 34.5328 & NC & 7261 & 0.3 & -77.3455 & 34.516 & NC & 7333 & -1.1 & -77.3796 & 34.5012 & NC \\
\hline 7190 & -2.1 & -77.3089 & 34.5325 & $\mathrm{NC}$ & 7262 & 0.3 & -77.346 & 34.5158 & NC & 7334 & -1.1 & -77.3801 & 34.501 & NC \\
\hline 7191 & -2.1 & -77.3094 & 34.5323 & NC & 7263 & 0.4 & .77 .3465 & 34.5156 & NC & 7335 & -1 & -77.3806 & 34.5008 & NC \\
\hline 7192 & -2.2 & -77.3098 & 34.532 & NC & 7264 & 0.4 & .77 .347 & 34.5154 & NC & 7336 & -1 & -77.3811 & 34.5005 & NC \\
\hline 7193 & -2.3 & -77.3103 & 34.5318 & $\mathrm{NC}$ & 7265 & 0.4 & -77.3475 & 34.5153 & NC & 7337 & -1 & -77.3816 & 34.5003 & NC \\
\hline 7194 & -2.3 & -77.3107 & 34.5315 & NC & 7266 & 0.3 & -77.348 & 34.5151 & NC & 7338 & -1 & -77.3821 & 34.5001 & NC \\
\hline 7195 & -2.3 & -77.3112 & 34.5312 & NC & 7267 & 0 & -77.3485 & 34.5149 & $\mathrm{NC}$ & 7339 & -1.1 & -77.3825 & 34.4999 & NC \\
\hline 7196 & -2.4 & -77.3117 & 34.531 & NC & 7268 & 0 & -77.349 & 34.5147 & $\mathrm{NC}$ & 7340 & -1 & -77.383 & 34.4996 & NC \\
\hline 7197 & -2.5 & -77.3121 & 34.5307 & NC & 7269 & -0.1 & -77.3495 & 34.5145 & $\mathrm{NC}$ & 7341 & -0.9 & -77.3835 & 34.4994 & NC \\
\hline 7198 & -2.6 & -77.3126 & 34.5304 & NC & 7270 & -0.3 & -77.3501 & 34.5143 & $\mathrm{NC}$ & 7342 & -0.8 & -77.384 & 34.4992 & NC \\
\hline 7199 & -2.9 & -77.313 & 34.5302 & NC & 7271 & -0.4 & -77.3506 & 34.5141 & NC & 7343 & -0.8 & -77.3845 & 34.499 & NC \\
\hline
\end{tabular}




\begin{tabular}{|c|c|c|c|c|c|c|c|c|c|c|c|c|c|c|}
\hline TR\# & RATE & LONG & LAT & ST & TR\# & RATE & LONG & LAT & ST & TR\# & RATE & LONG & LAT & ST \\
\hline 7344 & -0.8 & -77.385 & 34.4987 & $\mathrm{NC}$ & 7416 & -0.7 & -77.4183 & 34.4837 & $\mathrm{NC}$ & 7488 & -0.5 & -77.4526 & 34.4676 & $\mathrm{NC}$ \\
\hline 7345 & -0.7 & -77.3855 & 34.4985 & $\mathrm{NC}$ & 7417 & -0.7 & -77.4188 & 34.4835 & $\mathrm{NC}$ & 7489 & -0.5 & -77.4531 & 34.4674 & NC \\
\hline 7346 & -0.7 & -77.386 & 34.4983 & NC & 7418 & -0.7 & -77.4193 & 34.4832 & $\mathrm{NC}$ & 7490 & -0.5 & -77.4535 & 34.4672 & NC \\
\hline 7347 & -0.7 & -77.3865 & 34.4981 & $\mathrm{NC}$ & 7419 & -0.8 & -77.4198 & 34.483 & $\mathrm{NC}$ & 7491 & -0.5 & -77.454 & 34.4669 & $\mathrm{NC}$ \\
\hline 7348 & -0.6 & -77.3869 & 34.4978 & NC & 7420 & -0.7 & -77.4203 & 34.4828 & $\mathrm{NC}$ & 7492 & -0.7 & -77.4545 & 34.4667 & NC \\
\hline 7349 & -0.6 & -77.3874 & 34.4976 & $\mathrm{NC}$ & 7421 & -0.6 & -77.4208 & 34.4825 & $\mathrm{NC}$ & 7493 & -0.8 & -77.455 & 34.4665 & NC \\
\hline 7350 & -0.6 & -77.3879 & 34.4974 & NC & 7422 & -0.6 & -77.4213 & 34.4823 & $\mathrm{NC}$ & 7494 & -0.8 & -77.4555 & 34.4663 & NC \\
\hline 7351 & -0.5 & -77.3884 & 34.4972 & $\mathrm{NC}$ & 7423 & -0.6 & -77.4218 & 34.4821 & NC & 7495 & -0.8 & -77.456 & 34.466 & NC \\
\hline 7352 & -0.6 & -77.3889 & 34.4969 & $\mathrm{NC}$ & 7424 & -0.6 & -77.4223 & 34.4819 & NC & 7496 & -0.8 & -77.4565 & 34.4658 & NC \\
\hline 7353 & -0.7 & -77.3894 & 34.4967 & NC & 7425 & -0.7 & -77.4228 & 34.4816 & $\mathrm{NC}$ & 7497 & -0.8 & -77.457 & 34.4656 & NC \\
\hline 7354 & -0.7 & -77.3899 & 34.4965 & $\mathrm{NC}$ & 7426 & -0.6 & -77.4233 & 34.4814 & $\mathrm{NC}$ & 7498 & -0.8 & -77.4575 & 34.4653 & NC \\
\hline 7355 & -0.7 & -77.3904 & 34.4963 & NC & 7427 & -0.5 & .77 .4238 & 34.4812 & NC & 7499 & -0.9 & -77.458 & 34.4651 & NC \\
\hline 7356 & -1.3 & -77.3918 & 34.496 & NC & 7428 & -0.5 & -77.4242 & 34.4809 & $\mathrm{NC}$ & 7500 & -0.8 & -77.4579 & 34.4651 & NC \\
\hline 7357 & -0.7 & -77.3909 & 34.496 & NC & 7429 & -0.5 & -77.4247 & 34.4807 & $\mathrm{NC}$ & 7501 & -0.8 & -77.4584 & 34.4649 & NC \\
\hline 7358 & -1.2 & -77.3923 & 34.4958 & NC & 7430 & -0.5 & -77.4252 & 34.4805 & NC & 7502 & -1 & -77.4585 & 34.4648 & NC \\
\hline 7359 & -0.7 & .77 .3913 & 34.4958 & NC & 7431 & -0.6 & -77.4252 & 34.4804 & NC & 7503 & -0.8 & -77.4589 & 34.4647 & NC \\
\hline 7360 & -0.6 & -77.3918 & 34.4956 & NC & 7432 & -0.5 & -77.4257 & 34.4803 & $\mathrm{NC}$ & 7504 & -1 & -77.4589 & 34.4646 & NC \\
\hline 7361 & -1.2 & -77.3928 & 34.4955 & NC & 7433 & -0.7 & -77.4257 & 34.4802 & $\mathrm{NC}$ & 7505 & -1.1 & -77.4594 & 34.4643 & NC \\
\hline 7362 & -0.6 & -77.3923 & 34.4954 & $\mathrm{NC}$ & 7434 & -0.7 & -77.4262 & 34.4799 & $\mathrm{NC}$ & 7506 & -1.1 & -77.4599 & 34.4641 & NC \\
\hline 7363 & -1.2 & -77.3933 & 34.4953 & NC & 7435 & -0.6 & -77.4267 & 34.4797 & $\mathrm{NC}$ & 7507 & -1 & -77.4603 & 34.4638 & NC \\
\hline 7364 & -1.2 & -77.3938 & 34.4951 & $\mathrm{NC}$ & 7436 & -0.6 & -77.4272 & 34.4795 & $\mathrm{NC}$ & 7508 & -1 & -77.4608 & 34.4636 & NC \\
\hline 7365 & -0.6 & -77.3928 & 34.4951 & NC & 7437 & -0.5 & -77.4276 & 34.4793 & $\mathrm{NC}$ & 7509 & -1 & -77.4613 & 34.4633 & NC \\
\hline 7366 & -1.3 & -77.3943 & 34.4949 & NC & 7438 & -0.5 & -77.4281 & 34.479 & NC & 7510 & -1 & -77.4618 & 34.4631 & NC \\
\hline 7367 & -0.6 & -77.3933 & 34.4949 & NC & 7439 & -0.5 & -77.4286 & 34.4788 & NC & 7511 & -0.9 & -77.4622 & 34.4628 & NC \\
\hline 7368 & -1.2 & -77.3947 & 34.4946 & NC & 7440 & -0.5 & -77.4291 & 34.4786 & $\mathrm{NC}$ & 7512 & -0.8 & -77.4627 & 34.4626 & NC \\
\hline 7369 & -1.1 & -77.3952 & 34.4944 & NC & 7441 & -0.5 & -77.4296 & 34.4783 & $\mathrm{NC}$ & 7513 & -0.8 & -77.4632 & 34.4623 & $\mathrm{NC}$ \\
\hline 7370 & -1 & -77.3957 & 34.4942 & NC & 7442 & -0.5 & -77.4301 & 34.4781 & $\mathrm{NC}$ & 7514 & -0.8 & -77.4636 & 34.462 & $\mathrm{NC}$ \\
\hline 7371 & -0.9 & -77.3962 & 34.4939 & $\mathrm{NC}$ & 7443 & -0.5 & -77.4306 & 34.4779 & $\mathrm{NC}$ & 7515 & -0.7 & -77.4641 & 34.4618 & NC \\
\hline 7372 & -0.9 & -77.3967 & 34.4937 & NC & 7444 & -0.6 & -77.4311 & 34.4777 & $\mathrm{NC}$ & 7516 & -0.7 & -77.4646 & 34.4615 & NC \\
\hline 7373 & -1 & -77.3972 & 34.4935 & NC & 7445 & -0.5 & -77.4316 & 34.4774 & $\mathrm{NC}$ & 7517 & -0.7 & -77.465 & 34.4613 & NC \\
\hline 7374 & -1 & -77.3977 & 34.4933 & NC & 7446 & -0.6 & -77.432 & 34.4772 & $\mathrm{NC}$ & 7518 & -0.7 & -77.4655 & 34.461 & NC \\
\hline 7375 & -1 & -77.3982 & 34.493 & NC & 7447 & -0.6 & -77.4325 & 34.477 & $\mathrm{NC}$ & 7519 & -0.7 & -77.466 & 34.4608 & NC \\
\hline 7376 & -1 & -77.3987 & 34.4928 & NC & 7448 & -0.6 & -77.433 & 34.4767 & NC & 7520 & -0.7 & -77.4664 & 34.4605 & NC \\
\hline 7377 & -1 & -77.3992 & 34.4926 & NC & 7449 & -0.6 & -77.4335 & 34.4765 & $\mathrm{NC}$ & 7521 & -0.7 & -77.4669 & 34.4603 & NC \\
\hline 7378 & -0.9 & -77.3997 & 34.4923 & NC & 7450 & -0.6 & -77.434 & 34.4763 & $\mathrm{NC}$ & 7522 & -0.7 & -77.4674 & 34.46 & NC \\
\hline 7379 & -1 & -77.4002 & 34.4921 & NC & 7451 & -0.5 & -77.4345 & 34.4761 & $\mathrm{NC}$ & 7523 & -0.8 & -77.4678 & 34.4597 & NC \\
\hline 7380 & -0.9 & -77.4006 & 34.4919 & $\mathrm{NC}$ & 7452 & -0.6 & -77.435 & 34.4758 & $\mathrm{NC}$ & 7524 & -0.8 & -77.4683 & 34.4595 & NC \\
\hline 7381 & -0.9 & -77.4011 & 34.4917 & $\mathrm{NC}$ & 7453 & -0.5 & -77.4355 & 34.4756 & $\mathrm{NC}$ & 7525 & -0.9 & -77.4688 & 34.4592 & NC \\
\hline 7382 & -1 & -77.4016 & 34.4914 & NC & 7454 & -0.5 & .77 .436 & 34.4754 & $\mathrm{NC}$ & 7526 & -0.9 & -77.4693 & 34.459 & NC \\
\hline 7383 & -1 & -77.4021 & 34.4912 & NC & 7455 & -0.5 & -77.4364 & 34.4752 & $\mathrm{NC}$ & 7527 & -1 & -77.4697 & 34.4587 & NC \\
\hline 7384 & -0.9 & -77.4026 & 34.491 & NC & 7456 & -0.4 & -77.4369 & 34.4749 & $\mathrm{NC}$ & 7528 & -1.1 & -77.4702 & 34.4585 & NC \\
\hline 7385 & -0.8 & -77.4031 & 34.4908 & NC & 7457 & -0.5 & -77.4374 & 34.4747 & $\mathrm{NC}$ & 7529 & -1.1 & -77.4707 & 34.4582 & $\mathrm{NC}$ \\
\hline 7386 & -0.8 & -77.4036 & 34.4905 & NC & 7458 & -0.4 & -77.4379 & 34.4745 & $\mathrm{NC}$ & 7530 & -1.2 & -77.4711 & 34.458 & NC \\
\hline 7387 & -0.8 & -77.4041 & 34.4903 & $\mathrm{NC}$ & 7459 & -0.4 & -77.4384 & 34.4742 & $\mathrm{NC}$ & 7531 & -1.2 & -77.4716 & 34.4577 & NC \\
\hline 7388 & -0.9 & -77.4046 & 34.4901 & NC & 7460 & -0.4 & -77.4389 & 34.474 & $\mathrm{NC}$ & 7532 & -1.2 & -77.4721 & 34.4575 & NC \\
\hline 7389 & -0.9 & -77.4051 & 34.4898 & NC & 7461 & -0.3 & -77.4394 & 34.4738 & $\mathrm{NC}$ & 7533 & -1.1 & -77.4725 & 34.4572 & NC \\
\hline 7390 & -0.9 & -77.4056 & 34.4896 & $\mathrm{NC}$ & 7462 & -0.3 & -77.4399 & 34.4736 & $\mathrm{NC}$ & 7534 & -1.1 & .77 .473 & 34.4569 & NC \\
\hline 7391 & -0.8 & -77.4061 & 34.4894 & NC & 7463 & -0.3 & -77.4404 & 34.4733 & $\mathrm{NC}$ & 7535 & -1.1 & -77.4735 & 34.4567 & NC \\
\hline 7392 & -0.8 & -77.4065 & 34.4892 & NC & 7464 & -0.3 & -77.4408 & 34.4731 & $\mathrm{NC}$ & 7536 & -0.9 & -77.4739 & 34.4564 & $\mathrm{NC}$ \\
\hline 7393 & -0.8 & -77.407 & 34.4889 & NC & 7465 & -0.3 & -77.4413 & 34.4729 & $\mathrm{NC}$ & 7537 & -0.8 & -77.4744 & 34.4562 & NC \\
\hline 7394 & -0.7 & -77.4075 & 34.4887 & NC & 7466 & -0.3 & -77.4418 & 34.4726 & $\mathrm{NC}$ & 7538 & -0.8 & -77.4749 & 34.4559 & $\mathrm{NC}$ \\
\hline 7395 & -0.7 & -77.408 & 34.4885 & NC & 7467 & -0.3 & -77.4423 & 34.4724 & $\mathrm{NC}$ & 7539 & -0.8 & -77.4754 & 34.4557 & NC \\
\hline 7396 & -0.7 & -77.4085 & 34.4882 & NC & 7468 & -0.4 & -77.4428 & 34.4722 & NC & 7540 & -0.8 & -77.4758 & 34.4554 & NC \\
\hline 7397 & -0.5 & -77.409 & 34.488 & NC & 7469 & -0.3 & -77.4433 & 34.472 & $\mathrm{NC}$ & 7541 & -0.8 & -77.4763 & 34.4552 & $\mathrm{NC}$ \\
\hline 7398 & -0.6 & -77.4095 & 34.4878 & NC & 7470 & -0.4 & -77.4438 & 34.4717 & $\mathrm{NC}$ & 7542 & -0.8 & -77.4768 & 34.4549 & NC \\
\hline 7399 & -0.7 & -77.41 & 34.4876 & $\mathrm{NC}$ & 7471 & -0.4 & -77.4443 & 34.4715 & NC & 7543 & -0.8 & -77.4772 & 34.4546 & NC \\
\hline 7400 & -0.7 & -77.4105 & 34.4873 & NC & 7472 & -0.4 & -77.4447 & 34.4713 & NC & 7544 & -0.7 & -77.4777 & 34.4544 & NC \\
\hline 7401 & -0.6 & -77.411 & 34.4871 & NC & 7473 & -0.5 & -77.4452 & 34.471 & $\mathrm{NC}$ & 7545 & -0.5 & -77.4782 & 34.4541 & $\mathrm{NC}$ \\
\hline 7402 & -0.6 & -77.4115 & 34.4869 & $\mathrm{NC}$ & 7474 & -0.4 & .77 .4457 & 34.4708 & $\mathrm{NC}$ & 7546 & -0.5 & -77.4786 & 34.4539 & NC \\
\hline 7403 & -0.5 & -77.412 & 34.4866 & $\mathrm{NC}$ & 7475 & -0.3 & -77.4462 & 34.4706 & $\mathrm{NC}$ & 7547 & -0.4 & -77.4791 & 34.4536 & NC \\
\hline 7404 & -0.7 & -77.4124 & 34.4864 & NC & 7476 & -0.3 & -77.4467 & 34.4704 & $\mathrm{NC}$ & 7548 & -0.4 & -77.4796 & 34.4534 & $\mathrm{NC}$ \\
\hline 7405 & -0.8 & -77.4129 & 34.4862 & $\mathrm{NC}$ & 7477 & -0.4 & -77.4472 & 34.4701 & $\mathrm{NC}$ & 7549 & -0.4 & -77.48 & 34.4531 & $\mathrm{NC}$ \\
\hline 7406 & -0.8 & -77.4134 & 34.486 & NC & 7478 & -0.4 & .77 .4477 & 34.4699 & $\mathrm{NC}$ & 7550 & -0.3 & -77.4805 & 34.4529 & $\mathrm{NC}$ \\
\hline 7407 & -0.9 & -77.4139 & 34.4857 & $\mathrm{NC}$ & 7479 & -0.4 & -77.4482 & 34.4697 & $\mathrm{NC}$ & 7551 & -0.2 & -77.481 & 34.4526 & $\mathrm{NC}$ \\
\hline 7408 & -0.8 & -77.4144 & 34.4855 & $\mathrm{NC}$ & 7480 & -0.5 & -77.4487 & 34.4694 & $\mathrm{NC}$ & 7552 & -0.2 & -77.4815 & 34.4524 & $\mathrm{NC}$ \\
\hline 7409 & -0.8 & .77 .4149 & 34.4853 & $\mathrm{NC}$ & 7481 & -0.5 & -77.4491 & 34.4692 & $\mathrm{NC}$ & 7553 & -0.2 & .77 .4819 & 34.4521 & $\mathrm{NC}$ \\
\hline 7410 & -0.7 & -77.4154 & 34.485 & $\mathrm{NC}$ & 7482 & -0.5 & -77.4496 & 34.469 & $\mathrm{NC}$ & 7554 & -0.2 & -77.4824 & 34.4518 & $\mathrm{NC}$ \\
\hline 7411 & -0.7 & -77.4159 & 34.4848 & $\mathrm{NC}$ & 7483 & -0.4 & -77.4501 & 34.4688 & $\mathrm{NC}$ & 7555 & -0.1 & -77.4829 & 34.4516 & $\mathrm{NC}$ \\
\hline 7412 & -0.7 & .77 .4164 & 34.4846 & $\mathrm{NC}$ & 7484 & -0.5 & -77.4506 & 34.4685 & $\mathrm{NC}$ & 7556 & -0.1 & -77.4833 & 34.4513 & $\mathrm{NC}$ \\
\hline 7413 & -0.7 & .77 .4169 & 34.4844 & $\mathrm{NC}$ & 7485 & -0.5 & -77.4511 & 34.4683 & $\mathrm{NC}$ & 7557 & 0 & -77.4838 & 34.4511 & $\mathrm{NC}$ \\
\hline 7414 & -0.7 & -77.4174 & 34.4841 & $\mathrm{NC}$ & 7486 & -0.5 & -77.4516 & 34.4681 & NC & 7558 & -0.1 & -77.4843 & 34.4508 & $\mathrm{NC}$ \\
\hline 7415 & -0.7 & -77.4179 & 34.4839 & NC & 7487 & -0.5 & -77.4521 & 34.4679 & $\mathrm{NC}$ & 7559 & -0.1 & -77.4847 & 34.4506 & $\mathrm{NC}$ \\
\hline
\end{tabular}




\begin{tabular}{|c|c|c|c|c|c|c|c|c|c|c|c|c|c|c|}
\hline TR\# & RATE & LONG & LAT & ST & TR\# & RATE & LONG & LAT & ST & TR\# & RATE & LONG & LAT & ST \\
\hline 7560 & -0.1 & -77.4852 & 34.4503 & NC & 7632 & -0.1 & -77.5171 & 34.4316 & NC & 7704 & -0.4 & -77.5489 & 34.4123 & NC \\
\hline 7561 & -0.1 & -77.4857 & 34.4501 & NC & 7633 & -0.1 & -77.5176 & 34.4313 & NC & 7705 & -0.4 & -77.5494 & 34.4121 & NC \\
\hline 7562 & -0.2 & -77.4861 & 34.4498 & NC & 7634 & -0.2 & -77.518 & 34.431 & NC & 7706 & -0.4 & -77.5498 & 34.4118 & NC \\
\hline 7563 & -0.2 & -77.4866 & 34.4495 & NC & 7635 & -0.2 & -77.5185 & 34.4308 & NC & 7707 & -0.4 & -77.5503 & 34.4115 & NC \\
\hline 7564 & -0.2 & -77.4871 & 34.4493 & NC & 7636 & -0.2 & -77.5189 & 34.4305 & NC & 7708 & -0.4 & -77.5518 & 34.4114 & NC \\
\hline 7565 & -0.1 & -77.4875 & 34.449 & NC & 7637 & -0.2 & -77.5194 & 34.4302 & NC & 7709 & -0.4 & -77.5507 & 34.4113 & $\mathrm{NC}$ \\
\hline 7566 & -0.2 & -77.488 & 34.4488 & $\mathrm{NC}$ & 7638 & -0.2 & -77.5198 & 34.4299 & $\mathrm{NC}$ & 7710 & -0.4 & -77.5522 & 34.4111 & NC \\
\hline 7567 & -0.1 & -77.4885 & 34.4485 & NC & 7639 & -0.1 & -77.5203 & 34.4297 & NC & 7711 & -0.4 & -77.5512 & 34.411 & NC \\
\hline 7568 & -0.1 & .77 .489 & 34.4483 & NC & 7640 & -0.1 & -77.5207 & 34.4294 & NC & 7712 & -0.4 & -77.5527 & 34.4108 & NC \\
\hline 7569 & -0.2 & -77.4894 & 34.448 & NC & 7641 & 0 & -77.5208 & 34.4292 & NC & 7713 & -0.2 & -77.5516 & 34.4107 & NC \\
\hline 7570 & -0.4 & -77.4899 & 34.4478 & NC & 7642 & -0.1 & -77.5212 & 34.4291 & NC & 7714 & -0.5 & -77.5531 & 34.4105 & $\mathrm{NC}$ \\
\hline 7571 & 0.1 & -77.4898 & 34.4477 & NC & 7643 & -0.1 & -77.5213 & 34.4289 & NC & 7715 & -0.2 & -77.5521 & 34.4104 & $\mathrm{NC}$ \\
\hline 7572 & -0.4 & -77.4904 & 34.4475 & NC & 7644 & -0.1 & -77.5217 & 34.4287 & NC & 7716 & -0.4 & -77.5535 & 34.4102 & NC \\
\hline 7573 & 0.3 & -77.4903 & 34.4474 & NC & 7645 & -0.1 & -77.5222 & 34.4284 & NC & 7717 & -0.4 & -77.554 & 34.4099 & NC \\
\hline 7574 & 0.4 & -77.4907 & 34.4472 & NC & 7646 & -0.2 & .77 .5226 & 34.4281 & NC & 7718 & -0.4 & -77.5544 & 34.4097 & NC \\
\hline 7575 & 0.4 & -77.4912 & 34.4469 & NC & 7647 & -0.2 & -77.5231 & 34.4278 & NC & 7719 & -0.4 & -77.5548 & 34.4094 & NC \\
\hline 7576 & 0.4 & -77.4916 & 34.4466 & NC & 7648 & -0.2 & -77.5235 & 34.4276 & NC & 7720 & -0.4 & -77.5553 & 34.4091 & NC \\
\hline 7577 & 0.5 & -77.4921 & 34.4464 & NC & 7649 & -0.2 & -77.524 & 34.4273 & NC & 7721 & -0.4 & -77.5557 & 34.4088 & NC \\
\hline 7578 & 0.5 & -77.4925 & 34.4461 & NC & 7650 & -0.2 & -77.5244 & 34.427 & NC & 7722 & -0.4 & -77.5562 & 34.4085 & NC \\
\hline 7579 & 0.5 & -77.493 & 34.4458 & NC & 7651 & -0.2 & -77.5249 & 34.4268 & NC & 7723 & -0.3 & -77.5566 & 34.4082 & NC \\
\hline 7580 & 0.5 & -77.4934 & 34.4455 & NC & 7652 & -0.1 & -77.5253 & 34.4265 & NC & 7724 & -0.3 & .77 .557 & 34.4079 & NC \\
\hline 7581 & 0.5 & -77.4939 & 34.4453 & NC & 7653 & 0 & -77.5258 & 34.4262 & NC & 7725 & -0.3 & -77.5575 & 34.4076 & NC \\
\hline 7582 & 0.4 & -77.4943 & 34.445 & NC & 7654 & 0 & -77.5262 & 34.4259 & NC & 7726 & -0.3 & -77.5579 & 34.4073 & NC \\
\hline 7583 & 0.4 & -77.4948 & 34.4447 & NC & 7655 & -0.1 & -77.5267 & 34.4257 & NC & 7727 & -0.3 & -77.5583 & 34.407 & NC \\
\hline 7584 & 0.4 & -77.4953 & 34.4445 & NC & 7656 & -0.2 & -77.5271 & 34.4254 & NC & 7728 & -0.3 & -77.5588 & 34.4068 & NC \\
\hline 7585 & 0.4 & -77.4957 & 34.4442 & NC & 7657 & -0.2 & -77.5276 & 34.4251 & NC & 7729 & -0.4 & -77.5592 & 34.4065 & NC \\
\hline 7586 & 0.4 & -77.4962 & 34.4439 & NC & 7658 & -0.2 & -77.5281 & 34.4249 & NC & 7730 & -0.4 & -77.5596 & 34.4062 & NC \\
\hline 7587 & 0.3 & -77.4966 & 34.4437 & $\mathrm{NC}$ & 7659 & -0.4 & -77.5285 & 34.4246 & NC & 7731 & -0.4 & -77.5601 & 34.4059 & NC \\
\hline 7588 & 0.4 & -77.4971 & 34.4434 & NC & 7660 & -0.4 & -77.529 & 34.4243 & NC & 7732 & -0.5 & -77.5605 & 34.4056 & NC \\
\hline 7589 & 0.5 & -77.4975 & 34.4431 & NC & 7661 & -0.4 & -77.5294 & 34.424 & NC & 7733 & -0.5 & -77.5609 & 34.4053 & NC \\
\hline 7590 & 0.5 & -77.498 & 34.4429 & NC & 7662 & -0.5 & -77.5299 & 34.4238 & NC & 7734 & -0.5 & -77.5614 & 34.405 & NC \\
\hline 7591 & 0.6 & -77.4984 & 34.4426 & NC & 7663 & -0.6 & -77.5303 & 34.4235 & $\mathrm{NC}$ & 7735 & -0.5 & -77.5618 & 34.4047 & NC \\
\hline 7592 & 0.6 & -77.4989 & 34.4423 & NC & 7664 & -0.6 & -77.5308 & 34.4232 & NC & 7736 & -0.5 & -77.5622 & 34.4044 & NC \\
\hline 7593 & 0.7 & -77.4994 & 34.4421 & NC & 7665 & -0.6 & -77.5312 & 34.4229 & NC & 7737 & -0.4 & -77.5627 & 34.4041 & NC \\
\hline 7594 & 0.7 & -77.4998 & 34.4418 & NC & 7666 & -0.6 & -77.5317 & 34.4227 & NC & 7738 & -0.4 & -77.5631 & 34.4039 & NC \\
\hline 7595 & 0.6 & -77.5003 & 34.4415 & NC & 7667 & -0.5 & -77.5321 & 34.4224 & NC & 7739 & -0.3 & -77.5636 & 34.4036 & NC \\
\hline 7596 & 0.6 & -77.5007 & 34.4412 & NC & 7668 & -0.5 & -77.5326 & 34.4221 & NC & 7740 & -0.2 & -77.564 & 34.4033 & NC \\
\hline 7597 & 0.7 & -77.5012 & 34.441 & NC & 7669 & -0.6 & -77.533 & 34.4219 & NC & 7741 & -0.3 & -77.5644 & 34.403 & NC \\
\hline 7598 & 0.6 & -77.5016 & 34.4407 & NC & 7670 & -0.5 & -77.5335 & 34.4216 & NC & 7742 & -0.4 & -77.5649 & 34.4027 & NC \\
\hline 7599 & 0.6 & -77.5021 & 34.4404 & NC & 7671 & -0.4 & -77.534 & 34.4213 & NC & 7743 & -0.4 & -77.5653 & 34.4024 & NC \\
\hline 7600 & 0.6 & -77.5025 & 34.4402 & NC & 7672 & -0.3 & -77.5344 & 34.421 & NC & 7744 & -0.5 & -77.5657 & 34.4021 & NC \\
\hline 7601 & 0.6 & -77.503 & 34.4399 & NC & 7673 & -0.2 & -77.5349 & 34.4208 & NC & 7745 & -0.4 & -77.5662 & 34.4018 & NC \\
\hline 7602 & 0.7 & -77.5034 & 34.4396 & NC & 7674 & -0.3 & -77.5353 & 34.4205 & NC & 7746 & -0.4 & -77.5666 & 34.4015 & NC \\
\hline 7603 & 0.7 & -77.5039 & 34.4394 & $\mathrm{NC}$ & 7675 & -0.4 & -77.5358 & 34.4202 & NC & 7747 & -0.4 & -77.567 & 34.4012 & NC \\
\hline 7604 & 0.8 & -77.5044 & 34.4391 & NC & 7676 & -0.3 & -77.5362 & 34.42 & $\mathrm{NC}$ & 7748 & -0.3 & -77.5675 & 34.401 & $\mathrm{NC}$ \\
\hline 7605 & 0.8 & -77.5048 & 34.4388 & NC & 7677 & -0.3 & -77.5367 & 34.4197 & NC & 7749 & -0.2 & -77.5679 & 34.4007 & $\mathrm{NC}$ \\
\hline 7606 & 0.7 & -77.5053 & 34.4386 & $\mathrm{NC}$ & 7678 & -0.2 & -77.5371 & 34.4194 & $\mathrm{NC}$ & 7750 & -0.2 & -77.5683 & 34.4004 & $\mathrm{NC}$ \\
\hline 7607 & 0.8 & -77.5057 & 34.4383 & NC & 7679 & -0.2 & -77.5376 & 34.4191 & NC & 7751 & -0.2 & -77.5688 & 34.4001 & NC \\
\hline 7608 & 0.7 & -77.5062 & 34.438 & NC & 7680 & -0.3 & -77.538 & 34.4189 & NC & 7752 & -0.1 & -77.5692 & 34.3998 & NC \\
\hline 7609 & 0.6 & .77 .5066 & 34.4377 & NC & 7681 & -0.2 & -77.5385 & 34.4186 & NC & 7753 & -0.1 & -77.5696 & 34.3995 & NC \\
\hline 7610 & 0.6 & -77.5071 & 34.4375 & NC & 7682 & -0.1 & -77.5389 & 34.4183 & NC & 7754 & -0.3 & -77.5701 & 34.3992 & NC \\
\hline 7611 & 0.6 & -77.5075 & 34.4372 & NC & 7683 & 0 & -77.5394 & 34.4181 & NC & 7755 & -0.4 & -77.5705 & 34.3989 & NC \\
\hline 7612 & 0.5 & -77.508 & 34.4369 & $\mathrm{NC}$ & 7684 & 0 & -77.5398 & 34.4178 & NC & 7756 & -0.4 & -77.5709 & 34.3986 & NC \\
\hline 7613 & 0.6 & -77.5085 & 34.4367 & NC & 7685 & 0.1 & -77.5403 & 34.4175 & NC & 7757 & -0.4 & -77.5714 & 34.3983 & NC \\
\hline 7614 & 0.5 & -77.5089 & 34.4364 & NC & 7686 & 0.1 & -77.5408 & 34.4172 & NC & 7758 & -0.4 & -77.5718 & 34.3981 & NC \\
\hline 7615 & 0.4 & -77.5094 & 34.4361 & NC & 7687 & 0 & -77.5412 & 34.417 & NC & 7759 & -0.4 & -77.5723 & 34.3978 & NC \\
\hline 7616 & 0.4 & -77.5098 & 34.4359 & NC & 7688 & 0 & -77.5417 & 34.4167 & NC & 7760 & -0.4 & -77.5727 & 34.3975 & NC \\
\hline 7617 & 0.3 & -77.5103 & 34.4356 & NC & 7689 & 0 & -77.5421 & 34.4164 & NC & 7761 & -0.4 & -77.5731 & 34.3972 & NC \\
\hline 7618 & 0.2 & -77.5107 & 34.4353 & NC & 7690 & 0.1 & -77.5426 & 34.4162 & NC & 7762 & -0.4 & -77.5736 & 34.3969 & NC \\
\hline 7619 & 0.3 & -77.5112 & 34.4351 & NC & 7691 & 0.1 & -77.543 & 34.4159 & NC & 7763 & -0.4 & -77.574 & 34.3966 & NC \\
\hline 7620 & 0.2 & -77.5116 & 34.4348 & NC & 7692 & 0 & -77.5435 & 34.4156 & NC & 7764 & -0.4 & -77.5744 & 34.3963 & NC \\
\hline 7621 & 0.2 & -77.5121 & 34.4345 & NC & 7693 & 0 & -77.5439 & 34.4153 & NC & 7765 & -0.2 & -77.5749 & 34.396 & NC \\
\hline 7622 & 0.2 & -77.5125 & 34.4342 & NC & 7694 & -0.1 & -77.5444 & 34.4151 & NC & 7766 & -0.4 & -77.5753 & 34.3957 & NC \\
\hline 7623 & 0.2 & -77.513 & 34.434 & NC & 7695 & -0.2 & -77.5448 & 34.4148 & NC & 7767 & -0.5 & -77.5757 & 34.3954 & NC \\
\hline 7624 & 0.2 & -77.5135 & 34.4337 & NC & 7696 & -0.2 & -77.5453 & 34.4145 & NC & 7768 & -0.5 & -77.5762 & 34.3952 & NC \\
\hline 7625 & 0.2 & -77.5139 & 34.4334 & NC & 7697 & -0.1 & -77.5457 & 34.4142 & NC & 7769 & -0.4 & -77.5766 & 34.3949 & NC \\
\hline 7626 & 0.2 & -77.5144 & 34.4332 & NC & 7698 & -0.1 & -77.5462 & 34.414 & NC & 7770 & -0.4 & -77.577 & 34.3946 & NC \\
\hline 7627 & 0.1 & -77.5148 & 34.4329 & NC & 7699 & -0.2 & -77.5467 & 34.4137 & NC & 7771 & -0.3 & -77.5775 & 34.3943 & NC \\
\hline 7628 & 0.1 & -77.5153 & 34.4326 & NC & 7700 & -0.3 & -77.5471 & 34.4134 & NC & 7772 & -0.3 & -77.5779 & 34.394 & NC \\
\hline 7629 & 0.1 & -77.5157 & 34.4324 & NC & 7701 & -0.3 & -77.5476 & 34.4132 & NC & 7773 & -0.2 & -77.5783 & 34.3937 & NC \\
\hline 7630 & 0 & -77.5162 & 34.4321 & NC & 7702 & -0.3 & -77.548 & 34.4129 & NC & 7774 & -0.2 & -77.5788 & 34.3934 & NC \\
\hline 7631 & -0.1 & -77.5166 & 34.4318 & NC & 7703 & -0.3 & -77.5485 & 34.4126 & NC & 7775 & -0.2 & -77.5792 & 34.3931 & NC \\
\hline
\end{tabular}




\begin{tabular}{|c|c|c|c|c|c|c|c|c|c|c|c|c|c|c|}
\hline TR\# & RATE & LONG & LAT & ST & TR\# & RATE & LONG & LAT & ST & TR\# & RATE & LONG & LAT & ST \\
\hline 7776 & -0.2 & -77.5797 & 34.3928 & NC & 7848 & 0 & -77.611 & 34.3723 & NC & 7920 & 0.3 & -77.637 & 34.3499 & NC \\
\hline 7777 & -0.3 & -77.5801 & 34.3925 & NC & 7849 & -0.5 & -77.6081 & 34.3721 & NC & 7921 & 1.1 & -77.6374 & 34.3496 & NC \\
\hline 7778 & -0.3 & -77.5805 & 34.3923 & NC & 7850 & 0.1 & -77.6114 & 34.3719 & NC & 7922 & 0.6 & -77.6374 & 34.3496 & NC \\
\hline 7779 & -0.6 & -77.5812 & 34.3922 & NC & 7851 & -0.5 & -77.6085 & 34.3717 & NC & 7923 & 1.2 & -77.6378 & 34.3493 & NC \\
\hline 7780 & -0.3 & -77.581 & 34.392 & NC & 7852 & 0.1 & -77.6118 & 34.3716 & NC & 7924 & 1.5 & -77.6382 & 34.349 & $\mathrm{NC}$ \\
\hline 7781 & -0.6 & -77.5816 & 34.3919 & $\mathrm{NC}$ & 7853 & -0.5 & -77.6089 & 34.3714 & NC & 7925 & 1.7 & -77.6386 & 34.3486 & $\mathrm{NC}$ \\
\hline 7782 & -0.2 & -77.5814 & 34.3917 & NC & 7854 & 0.1 & -77.6122 & 34.3713 & NC & 7926 & 2 & -77.639 & 34.3483 & $\mathrm{NC}$ \\
\hline 7783 & -0.6 & -77.582 & 34.3916 & NC & 7855 & -0.5 & -77.6094 & 34.3711 & NC & 7927 & 2.3 & .77 .6394 & 34.348 & NC \\
\hline 7784 & -0.3 & -77.5818 & 34.3914 & NC & 7856 & 0.1 & -77.6125 & 34.3709 & NC & 7928 & 2.8 & -77.6398 & 34.3477 & NC \\
\hline 7785 & -0.6 & -77.5824 & 34.3913 & NC & 7857 & -0.5 & -77.6098 & 34.3708 & NC & 7929 & 3.3 & -77.6402 & 34.3474 & NC \\
\hline 7786 & -0.6 & -77.5829 & 34.391 & NC & 7858 & 0.1 & -77.6129 & 34.3706 & NC & 7930 & -5 & -77.6406 & 34.3471 & $\mathrm{NC}$ \\
\hline 7787 & -0.6 & -77.5833 & 34.3907 & NC & 7859 & 0 & -77.6133 & 34.3703 & NC & 7931 & NA & -77.6411 & 34.3467 & $\mathrm{NC}$ \\
\hline 7788 & -0.6 & -77.5837 & 34.3903 & NC & 7860 & 0 & -77.6137 & 34.3699 & NC & 7932 & -1.2 & -77.6415 & 34.3464 & $\mathrm{NC}$ \\
\hline 7789 & -0.6 & -77.5841 & 34.39 & NC & 7861 & 0 & -77.6141 & 34.3696 & NC & 7933 & -0.1 & -77.6419 & 34.3461 & $\mathrm{NC}$ \\
\hline 7790 & -0.6 & -77.5845 & 34.3897 & NC & 7862 & 0 & -77.6145 & 34.3693 & NC & 7934 & 0.3 & -77.6423 & 34.3458 & $\mathrm{NC}$ \\
\hline 7791 & -0.5 & -77.5849 & 34.3894 & NC & 7863 & -0.1 & .77 .6149 & 34.3689 & NC & 7935 & 0.9 & -77.6427 & 34.3455 & $\mathrm{NC}$ \\
\hline 7792 & -0.5 & -77.5853 & 34.3891 & NC & 7864 & -0.1 & -77.6153 & 34.3686 & NC & 7936 & 1.6 & -77.6431 & 34.3452 & $\mathrm{NC}$ \\
\hline 7793 & -0.6 & -77.5858 & 34.3888 & NC & 7865 & -0.2 & -77.6157 & 34.3683 & $\mathrm{NC}$ & 7937 & 2.1 & -77.6435 & 34.3448 & NC \\
\hline 7794 & -0.6 & -77.5862 & 34.3885 & NC & 7866 & -0.2 & .77 .616 & 34.3679 & NC & 7938 & -1.8 & -77.6439 & 34.3445 & $\mathrm{NC}$ \\
\hline 7795 & -0.7 & -77.5866 & 34.3882 & NC & 7867 & -0.1 & -77.6164 & 34.3676 & NC & 7939 & 0.4 & -77.6443 & 34.3442 & NC \\
\hline 7796 & -0.6 & -77.587 & 34.3879 & NC & 7868 & -0.1 & -77.6168 & 34.3673 & NC & 7940 & 1.6 & -77.6447 & 34.3439 & $\mathrm{NC}$ \\
\hline 7797 & -0.6 & -77.5874 & 34.3876 & NC & 7869 & 0 & -77.6172 & 34.3669 & NC & 7941 & 1.4 & -77.6451 & 34.3436 & $\mathrm{NC}$ \\
\hline 7798 & -0.5 & -77.5878 & 34.3872 & NC & 7870 & 0 & -77.6176 & 34.3666 & NC & 7942 & 2.8 & -77.6455 & 34.3433 & $\mathrm{NC}$ \\
\hline 7799 & -0.6 & -77.5882 & 34.3869 & NC & 7871 & 0 & -77.618 & 34.3663 & NC & 7943 & 4.3 & -77.6459 & 34.3429 & NC \\
\hline 7800 & -0.6 & -77.5887 & 34.3866 & NC & 7872 & -0.1 & -77.6184 & 34.3659 & NC & 7944 & 3.2 & -77.6463 & 34.3426 & NC \\
\hline 7801 & -0.6 & -77.5891 & 34.3863 & NC & 7873 & -0.2 & -77.6188 & 34.3656 & NC & 7945 & 2 & -77.6467 & 34.3423 & $\mathrm{NC}$ \\
\hline 7802 & -0.5 & -77.5895 & 34.386 & NC & 7874 & -0.1 & -77.6192 & 34.3653 & NC & 7946 & 1.9 & -77.6471 & 34.342 & NC \\
\hline 7803 & -0.5 & -77.5899 & 34.3857 & $\mathrm{NC}$ & 7875 & -0.1 & -77.6195 & 34.3649 & NC & 7947 & 1.9 & -77.6475 & 34.3417 & NC \\
\hline 7804 & -0.5 & -77.5903 & 34.3854 & NC & 7876 & -0.2 & -77.6199 & 34.3646 & NC & 7948 & 1.9 & -77.6479 & 34.3414 & $\mathrm{NC}$ \\
\hline 7805 & -0.6 & -77.5907 & 34.3851 & NC & 7877 & -0.2 & -77.6203 & 34.3643 & NC & 7949 & 1.5 & -77.6484 & 34.341 & $\mathrm{NC}$ \\
\hline 7806 & -0.6 & -77.5911 & 34.3848 & NC & 7878 & -0.3 & -77.6207 & 34.3639 & NC & 7950 & 1.4 & -77.6488 & 34.3407 & NC \\
\hline 7807 & -0.7 & -77.5916 & 34.3845 & NC & 7879 & -0.3 & -77.6211 & 34.3636 & NC & 7951 & 1.1 & -77.6492 & 34.3404 & $\mathrm{NC}$ \\
\hline 7808 & -0.6 & -77.592 & 34.3841 & NC & 7880 & -0.2 & -77.6215 & 34.3633 & NC & 7952 & 0.8 & -77.6496 & 34.3401 & NC \\
\hline 7809 & -0.6 & -77.5924 & 34.3838 & NC & 7881 & -0.2 & -77.6219 & 34.3629 & NC & 7953 & 0.4 & -77.65 & 34.3398 & $\mathrm{NC}$ \\
\hline 7810 & -0.5 & -77.5928 & 34.3835 & NC & 7882 & -0.1 & -77.6223 & 34.3626 & NC & 7954 & -0.1 & -77.6504 & 34.3395 & $\mathrm{NC}$ \\
\hline 7811 & -0.5 & -77.5932 & 34.3832 & NC & 7883 & 0 & -77.6227 & 34.3623 & NC & 7955 & -0.6 & -77.6508 & 34.3391 & $\mathrm{NC}$ \\
\hline 7812 & -0.5 & -77.5936 & 34.3829 & $\mathrm{NC}$ & 7884 & 0.1 & -77.623 & 34.3619 & NC & 7956 & -1 & -77.6512 & 34.3388 & NC \\
\hline 7813 & -0.5 & -77.594 & 34.3826 & NC & 7885 & 0.2 & .77 .6234 & 34.3616 & NC & 7957 & -2.2 & -77.6516 & 34.3385 & NC \\
\hline 7814 & -0.5 & -77.5945 & 34.3823 & NC & 7886 & 0.2 & -77.6238 & 34.3613 & NC & 7958 & 0.5 & -77.652 & 34.3382 & NC \\
\hline 7815 & -0.5 & -77.5949 & 34.382 & NC & 7887 & 0.2 & -77.6242 & 34.3609 & NC & 7959 & 0.7 & -77.6524 & 34.3379 & NC \\
\hline 7816 & -0.5 & -77.5953 & 34.3817 & NC & 7888 & 0.2 & -77.6246 & 34.3606 & NC & 7960 & 0.9 & -77.6528 & 34.3376 & NC \\
\hline 7817 & -0.5 & -77.5957 & 34.3814 & NC & 7889 & 0.2 & -77.625 & 34.3602 & NC & 7961 & 1.1 & -77.6532 & 34.3372 & NC \\
\hline 7818 & -0.5 & -77.5961 & 34.381 & NC & 7890 & 0.2 & -77.6254 & 34.3599 & NC & 7962 & 0.8 & -77.6536 & 34.3369 & NC \\
\hline 7819 & -0.5 & -77.5965 & 34.3807 & NC & 7891 & 0.2 & -77.6258 & 34.3596 & NC & 7963 & 15 & -77.654 & 34.3366 & $\mathrm{NC}$ \\
\hline 7820 & -0.6 & -77.5969 & 34.3804 & NC & 7892 & -0.1 & -77.6261 & 34.3592 & NC & 7964 & 5.9 & -77.6544 & 34.3363 & NC \\
\hline 7821 & -0.5 & -77.5973 & 34.3801 & NC & 7893 & -0.3 & -77.6265 & 34.3589 & NC & 7965 & -3.3 & -77.6548 & 34.336 & NC \\
\hline 7822 & -0.5 & -77.5978 & 34.3798 & NC & 7894 & -0.2 & -77.6269 & 34.3586 & NC & 7966 & -11.8 & -77.6552 & 34.3357 & NC \\
\hline 7823 & -0.5 & -77.5982 & 34.3795 & NC & 7895 & -0.2 & -77.6273 & 34.3582 & $\mathrm{NC}$ & 7967 & -10.6 & -77.6557 & 34.3353 & $\mathrm{NC}$ \\
\hline 7824 & -0.5 & -77.5986 & 34.3792 & $\mathrm{NC}$ & 7896 & -0.3 & -77.6277 & 34.3579 & $\mathrm{NC}$ & 7968 & -9.5 & -77.6561 & 34.335 & NC \\
\hline 7825 & -0.5 & -77.599 & 34.3789 & NC & 7897 & -0.3 & -77.6281 & 34.3576 & $\mathrm{NC}$ & 7969 & -8.2 & -77.6565 & 34.3347 & NC \\
\hline 7826 & -0.5 & -77.5994 & 34.3786 & NC & 7898 & -0.4 & -77.6285 & 34.3572 & NC & 7970 & -6.9 & -77.6569 & 34.3344 & $\mathrm{NC}$ \\
\hline 7827 & -0.5 & -77.5998 & 34.3783 & NC & 7899 & -0.5 & -77.6289 & 34.3569 & NC & 7971 & -0.6 & -77.6573 & 34.3341 & $\mathrm{NC}$ \\
\hline 7828 & -0.5 & -77.6002 & 34.3779 & NC & 7900 & -0.5 & -77.6293 & 34.3566 & NC & 7972 & 0.2 & -77.6577 & 34.3338 & NC \\
\hline 7829 & -0.6 & -77.6007 & 34.3776 & NC & 7901 & -0.4 & -77.6296 & 34.3562 & NC & 7973 & 0.9 & -77.6581 & 34.3334 & NC \\
\hline 7830 & -0.6 & -77.6011 & 34.3773 & NC & 7902 & -0.5 & -77.63 & 34.3559 & NC & 7974 & 1.2 & -77.6585 & 34.3331 & NC \\
\hline 7831 & -0.7 & -77.6015 & 34.377 & NC & 7903 & -0.6 & -77.6304 & 34.3556 & NC & 7975 & 1.4 & -77.6589 & 34.3328 & NC \\
\hline 7832 & -0.7 & -77.6019 & 34.3767 & NC & 7904 & -0.5 & -77.6308 & 34.3552 & $\mathrm{NC}$ & 7976 & 1.3 & -77.6593 & 34.3325 & NC \\
\hline 7833 & -0.6 & -77.6023 & 34.3764 & NC & 7905 & -0.5 & -77.6312 & 34.3549 & NC & 7977 & 1.3 & -77.6597 & 34.3322 & $\mathrm{NC}$ \\
\hline 7834 & -0.5 & -77.6027 & 34.3761 & NC & 7906 & -0.5 & -77.6316 & 34.3546 & NC & 7978 & 1 & -77.6601 & 34.3319 & NC \\
\hline 7835 & -0.3 & -77.6031 & 34.3758 & NC & 7907 & -0.5 & -77.632 & 34.3542 & NC & 7979 & 0.7 & -77.6605 & 34.3315 & $\mathrm{NC}$ \\
\hline 7836 & -0.1 & -77.6036 & 34.3755 & NC & 7908 & -0.6 & -77.6324 & 34.3539 & NC & 7980 & 0.2 & -77.6609 & 34.3312 & $\mathrm{NC}$ \\
\hline 7837 & 0 & -77.604 & 34.3752 & NC & 7909 & -0.6 & -77.6328 & 34.3536 & NC & 7981 & 0 & -77.6613 & 34.3309 & NC \\
\hline 7838 & 0.1 & -77.6044 & 34.3748 & NC & 7910 & -0.6 & -77.6331 & 34.3532 & NC & 7982 & -0.2 & -77.6617 & 34.3306 & $\mathrm{NC}$ \\
\hline 7839 & 0.2 & -77.6048 & 34.3745 & NC & 7911 & -0.5 & -77.6335 & 34.3529 & NC & 7983 & -0.4 & -77.6621 & 34.3303 & $\mathrm{NC}$ \\
\hline 7840 & 0 & -77.6052 & 34.3742 & NC & 7912 & -0.4 & -77.6339 & 34.3526 & NC & 7984 & 0.6 & -77.6625 & 34.33 & $\mathrm{NC}$ \\
\hline 7841 & -0.1 & -77.6056 & 34.3739 & NC & 7913 & -0.3 & -77.6343 & 34.3522 & NC & 7985 & 1.5 & -77.663 & 34.3296 & $\mathrm{NC}$ \\
\hline 7842 & -0.4 & -77.606 & 34.3736 & $\mathrm{NC}$ & 7914 & -0.3 & -77.6347 & 34.3519 & NC & 7986 & 1.9 & -77.6634 & 34.3293 & $\mathrm{NC}$ \\
\hline 7843 & -0.5 & -77.6065 & 34.3733 & $\mathrm{NC}$ & 7915 & -0.3 & -77.6351 & 34.3516 & $\mathrm{NC}$ & 7987 & -26.4 & -77.6658 & 34.3291 & NC \\
\hline 7844 & -0.5 & -77.6069 & 34.373 & NC & 7916 & -0.3 & -77.6355 & 34.3512 & NC & 7988 & 2.4 & -77.6638 & 34.329 & NC \\
\hline 7845 & -0.5 & .77 .6073 & 34.3727 & NC & 7917 & -0.2 & -77.6359 & 34.3509 & NC & 7989 & -26.4 & -77.6662 & 34.3288 & NC \\
\hline 7846 & 0 & -77.6106 & 34.3726 & NC & 7918 & -0.2 & -77.6363 & 34.3506 & NC & 7990 & 2.6 & -77.6642 & 34.3287 & NC \\
\hline 7847 & -0.5 & -77.6077 & 34.3724 & $\mathrm{NC}$ & 7919 & 0 & -77.6366 & 34.3502 & NC & 7991 & -26.5 & -77.6666 & 34.3284 & NC \\
\hline
\end{tabular}




\begin{tabular}{|c|c|c|c|c|c|c|c|c|c|c|c|c|c|c|}
\hline TR\# & RATE & LONG & LAT & ST & TR\# & RATE & LONG & LAT & ST & TR\# & RATE & LONG & LAT & ST \\
\hline 7992 & 3 & -77.6646 & 34.3284 & NC & 8064 & -1.7 & -77.692 & 34.3059 & NC & 8136 & 0.1 & -77.7221 & 34.2783 & $\mathrm{NC}$ \\
\hline 7993 & -26.5 & -77.6669 & 34.3281 & NC & 8065 & -1.9 & -77.6927 & 34.3058 & NC & 8137 & 0.1 & -77.7225 & 34.2779 & $\mathrm{NC}$ \\
\hline 7994 & 0.4 & -77.665 & 34.3281 & NC & 8066 & -1.9 & -77.6931 & 34.3054 & NC & 8138 & 0 & -77.7229 & 34.2776 & $\mathrm{NC}$ \\
\hline 7995 & -26.6 & -77.6673 & 34.3278 & NC & 8067 & -1.7 & -77.6934 & 34.3051 & NC & 8139 & -0.1 & -77.7233 & 34.2773 & NC \\
\hline 7996 & 0.5 & -77.6654 & 34.3277 & NC & 8068 & -1.7 & -77.6937 & 34.3047 & NC & 8140 & -0.2 & -77.7236 & 34.2769 & NC \\
\hline 7997 & -26.7 & -77.6677 & 34.3274 & NC & 8069 & -1.8 & -77.6941 & 34.3043 & NC & 8141 & -0.3 & -77.724 & 34.2766 & NC \\
\hline 7998 & -26.9 & -77.6681 & 34.3271 & NC & 8070 & -1.8 & -77.6944 & 34.304 & NC & 8142 & -0.2 & -77.7244 & 34.2762 & NC \\
\hline 7999 & -27.1 & -77.6685 & 34.3267 & NC & 8071 & -1.8 & -77.6947 & 34.3036 & NC & 8143 & -0.3 & -77.7248 & 34.2759 & NC \\
\hline 8000 & .27 .6 & -77.6688 & 34.3264 & NC & 8072 & -1.9 & -77.6951 & 34.3032 & NC & 8144 & -0.3 & -77.7251 & 34.2756 & NC \\
\hline 8001 & -0.7 & -77.6692 & 34.3261 & $\mathrm{NC}$ & 8073 & -1.8 & -77.6954 & 34.3029 & NC & 8145 & -0.4 & -77.7255 & 34.2752 & NC \\
\hline 8002 & -1 & -77.6696 & 34.3257 & NC & 8074 & -1.8 & .77 .6958 & 34.3025 & NC & 8146 & -0.3 & -77.7259 & 34.2749 & NC \\
\hline 8003 & -1.4 & -77.67 & 34.3254 & $\mathrm{NC}$ & 8075 & -1.7 & -77.6961 & 34.3021 & NC & 8147 & -0.3 & -77.7263 & 34.2745 & NC \\
\hline 8004 & -1.6 & -77.6704 & 34.3251 & NC & 8076 & -1.6 & -77.6964 & 34.3018 & NC & 8148 & -0.4 & -77.7267 & 34.2742 & NC \\
\hline 8005 & -1.8 & -77.6707 & 34.3247 & NC & 8077 & -1.6 & -77.6968 & 34.3014 & NC & 8149 & -0.3 & -77.727 & 34.2739 & NC \\
\hline 8006 & -1.8 & -77.6711 & 34.3244 & NC & 8078 & -1.6 & -77.6971 & 34.301 & $\mathrm{NC}$ & 8150 & -0.3 & -77.7274 & 34.2735 & NC \\
\hline 8007 & -1.9 & -77.6715 & 34.3241 & NC & 8079 & -1.6 & -77.6974 & 34.3007 & NC & 8151 & -0.3 & -77.7278 & 34.2732 & $\mathrm{NC}$ \\
\hline 8008 & -2 & -77.6719 & 34.3237 & NC & 8080 & -1.6 & -77.6978 & 34.3003 & NC & 8152 & -0.4 & -77.7282 & 34.2728 & NC \\
\hline 8009 & -2.1 & -77.6723 & 34.3234 & NC & 8081 & -1.6 & -77.6981 & 34.2999 & NC & 8153 & -0.4 & -77.7285 & 34.2725 & $\mathrm{NC}$ \\
\hline 8010 & -2.5 & -77.6726 & 34.323 & $\mathrm{NC}$ & 8082 & -1.5 & -77.6985 & 34.2995 & NC & 8154 & -0.5 & -77.7289 & 34.2722 & $\mathrm{NC}$ \\
\hline 8011 & -3.1 & -77.673 & 34.3227 & NC & 8083 & -1.4 & -77.6988 & 34.2992 & NC & 8155 & -0.4 & -77.7293 & 34.2718 & NC \\
\hline 8012 & 2 & -77.6734 & 34.3224 & NC & 8084 & -1.3 & -77.6991 & 34.2988 & $\mathrm{NC}$ & 8156 & -0.4 & .77 .7297 & 34.2715 & NC \\
\hline 8013 & 1.3 & -77.6738 & 34.322 & NC & 8085 & -1.3 & -77.6995 & 34.2984 & NC & 8157 & -0.4 & -77.73 & 34.2711 & NC \\
\hline 8014 & 1 & -77.6742 & 34.3217 & NC & 8086 & -1.1 & -77.6998 & 34.2981 & NC & 8158 & -0.4 & -77.7304 & 34.2708 & NC \\
\hline 8015 & -3.3 & -77.6745 & 34.3214 & NC & 8087 & -1 & -77.7002 & 34.2977 & NC & 8159 & -0.4 & -77.7308 & 34.2704 & NC \\
\hline 8016 & -2.7 & -77.6749 & 34.321 & NC & 8088 & -0.9 & -77.7005 & 34.2973 & NC & 8160 & -0.4 & -77.7312 & 34.2701 & NC \\
\hline 8017 & -2 & -77.6753 & 34.3207 & NC & 8089 & -0.7 & -77.7008 & 34.297 & NC & 8161 & -0.5 & -77.7315 & 34.2698 & NC \\
\hline 8018 & -4.1 & -77.6757 & 34.3203 & NC & 8090 & -0.4 & -77.7012 & 34.2966 & NC & 8162 & -0.6 & .77 .7319 & 34.2694 & NC \\
\hline 8019 & -3 & -77.6761 & 34.32 & NC & 8091 & -0.1 & -77.7015 & 34.2962 & NC & 8163 & -0.5 & -77.7323 & 34.2691 & NC \\
\hline 8020 & -2.5 & -77.6764 & 34.3197 & NC & 8092 & 0.2 & -77.7018 & 34.2959 & NC & 8164 & -0.5 & -77.7327 & 34.2687 & NC \\
\hline 8021 & -2.3 & -77.6768 & 34.3193 & NC & 8093 & 0.4 & -77.7022 & 34.2955 & NC & 8165 & -0.5 & -77.733 & 34.2684 & NC \\
\hline 8022 & -2.2 & -77.6772 & 34.319 & $\mathrm{NC}$ & 8094 & 0.8 & -77.7025 & 34.2951 & NC & 8166 & -0.4 & -77.7334 & 34.2681 & NC \\
\hline 8023 & -2 & -77.6776 & 34.3187 & NC & 8095 & 1.4 & -77.7029 & 34.2948 & NC & 8167 & -0.5 & -77.7338 & 34.2677 & NC \\
\hline 8024 & -2 & -77.678 & 34.3183 & $\mathrm{NC}$ & 8096 & 7 & -77.7032 & 34.2944 & $\mathrm{NC}$ & 8168 & -0.5 & -77.7342 & 34.2674 & $\mathrm{NC}$ \\
\hline 8025 & -2 & -77.6783 & 34.318 & NC & 8097 & 7.3 & -77.7035 & 34.294 & $\mathrm{NC}$ & 8169 & -0.5 & -77.7346 & 34.267 & NC \\
\hline 8026 & -2 & -77.6787 & 34.3177 & NC & 8098 & .9 .1 & -77.7039 & 34.2937 & $\mathrm{NC}$ & 8170 & -0.5 & -77.7349 & 34.2667 & NC \\
\hline 8027 & -2.1 & -77.6791 & 34.3173 & NC & 8099 & -12.4 & -77.7042 & 34.2933 & NC & 8171 & -0.5 & -77.7353 & 34.2664 & NC \\
\hline 8028 & -2.1 & -77.6795 & 34.317 & $\mathrm{NC}$ & 8100 & -12.4 & -77.7045 & 34.2929 & NC & 8172 & -0.5 & -77.7357 & 34.266 & NC \\
\hline 8029 & -2.1 & -77.6799 & 34.3166 & NC & 8101 & -16.3 & -77.7049 & 34.2926 & NC & 8173 & -0.6 & -77.7361 & 34.2657 & NC \\
\hline 8030 & -2.1 & -77.6803 & 34.3163 & NC & 8102 & -41.5 & -77.7052 & 34.2922 & NC & 8174 & -0.7 & -77.7364 & 34.2653 & NC \\
\hline 8031 & -2 & -77.6806 & 34.316 & NC & 8103 & $\mathrm{NA}$ & -77.7056 & 34.2918 & NC & 8175 & -0.6 & -77.7368 & 34.265 & NC \\
\hline 8032 & -2.1 & -77.681 & 34.3156 & NC & 8104 & -15.5 & -77.7089 & 34.2882 & $\mathrm{NC}$ & 8176 & -0.6 & -77.7372 & 34.2647 & NC \\
\hline 8033 & -2.2 & -77.6814 & 34.3153 & NC & 8105 & -10.1 & -77.7093 & 34.2878 & $\mathrm{NC}$ & 8177 & -0.6 & .77 .7376 & 34.2643 & NC \\
\hline 8034 & -2 & -77.6818 & 34.315 & $\mathrm{NC}$ & 8106 & -4.6 & -77.7096 & 34.2874 & NC & 8178 & -0.6 & -77.7379 & 34.264 & NC \\
\hline 8035 & -1.9 & -77.6822 & 34.3146 & $\mathrm{NC}$ & 8107 & -3.7 & -77.71 & 34.287 & NC & 8179 & -0.6 & -77.7383 & 34.2636 & NC \\
\hline 8036 & -1.9 & -77.6825 & 34.3143 & $\mathrm{NC}$ & 8108 & -2.9 & -77.7103 & 34.2867 & NC & 8180 & -0.6 & -77.7387 & 34.2633 & NC \\
\hline 8037 & -1.9 & -77.6829 & 34.314 & $\mathrm{NC}$ & 8109 & -5.1 & -77.7106 & 34.2863 & NC & 8181 & -0.6 & -77.7391 & 34.2629 & NC \\
\hline 8038 & -1.8 & -77.6833 & 34.3136 & $\mathrm{NC}$ & 8110 & -4.2 & -77.711 & 34.2859 & NC & 8182 & -0.6 & -77.7394 & 34.2626 & NC \\
\hline 8039 & -1.8 & -77.6837 & 34.3133 & $\mathrm{NC}$ & 8111 & -3.5 & -77.7113 & 34.2856 & NC & 8183 & -0.5 & -77.7398 & 34.2623 & NC \\
\hline 8040 & -1.8 & -77.6841 & 34.3129 & $\mathrm{NC}$ & 8112 & -2.8 & -77.7116 & 34.2852 & $\mathrm{NC}$ & 8184 & -0.5 & -77.7402 & 34.2619 & NC \\
\hline 8041 & -1.8 & -77.6844 & 34.3126 & $\mathrm{NC}$ & 8113 & -2.3 & -77.712 & 34.2848 & $\mathrm{NC}$ & 8185 & -0.4 & -77.7406 & 34.2616 & NC \\
\hline 8042 & -1.9 & -77.6848 & 34.3123 & $\mathrm{NC}$ & 8114 & -1.8 & -77.7123 & 34.2845 & $\mathrm{NC}$ & 8186 & -0.4 & -77.7409 & 34.2612 & NC \\
\hline 8043 & -1.9 & -77.6852 & 34.3119 & NC & 8115 & -1.3 & -77.7127 & 34.2841 & NC & 8187 & -0.3 & -77.7413 & 34.2609 & $\mathrm{NC}$ \\
\hline 8044 & -1.9 & -77.6856 & 34.3116 & NC & 8116 & -0.8 & -77.713 & 34.2837 & NC & 8188 & 1.1 & -77.7417 & 34.2608 & NC \\
\hline 8045 & -1.9 & -77.686 & 34.3113 & NC & 8117 & -0.7 & -77.7133 & 34.2834 & NC & 8189 & -0.3 & -77.7417 & 34.2606 & NC \\
\hline 8046 & -1.8 & -77.6863 & 34.3109 & NC & 8118 & -0.6 & -77.7137 & 34.283 & NC & 8190 & 0.9 & -77.742 & 34.2604 & NC \\
\hline 8047 & -1.8 & -77.6867 & 34.3106 & NC & 8119 & -0.5 & -77.714 & 34.2826 & NC & 8191 & -0.3 & -77.7421 & 34.2602 & NC \\
\hline 8048 & -1.9 & -77.6871 & 34.3102 & NC & 8120 & -0.6 & -77.7143 & 34.2823 & NC & 8192 & 0.6 & -77.7424 & 34.26 & $\mathrm{NC}$ \\
\hline 8049 & -1.9 & -77.6875 & 34.3099 & NC & 8121 & 0.2 & -77.718 & 34.282 & NC & 8193 & -0.4 & -77.7424 & 34.2599 & NC \\
\hline 8050 & -1.9 & -77.6879 & 34.3096 & NC & 8122 & -0.5 & -77.7147 & 34.2819 & NC & 8194 & 0.5 & -77.7427 & 34.2597 & $\mathrm{NC}$ \\
\hline 8051 & -1.9 & .77 .6882 & 34.3092 & NC & 8123 & 0.3 & -77.7184 & 34.2817 & NC & 8195 & 0.5 & -77.743 & 34.2593 & $\mathrm{NC}$ \\
\hline 8052 & -1.8 & -77.6886 & 34.3089 & NC & 8124 & -0.6 & -77.715 & 34.2815 & NC & 8196 & 0.5 & -77.7433 & 34.2589 & $\mathrm{NC}$ \\
\hline 8053 & -1.8 & -77.689 & 34.3086 & NC & 8125 & 0.3 & -77.7188 & 34.2814 & NC & 8197 & 0.4 & -77.7437 & 34.2585 & $\mathrm{NC}$ \\
\hline 8054 & -1.8 & -77.6894 & 34.3082 & NC & 8126 & -0.6 & -77.7154 & 34.2812 & NC & 8198 & 0.3 & -77.744 & 34.2582 & $\mathrm{NC}$ \\
\hline 8055 & -1.7 & -77.6898 & 34.3079 & NC & 8127 & 0.4 & -77.7191 & 34.281 & NC & 8199 & 0.3 & -77.7443 & 34.2578 & NC \\
\hline 8056 & -1.6 & -77.6901 & 34.3076 & NC & 8128 & -0.6 & -77.7157 & 34.2808 & NC & 8200 & 0.2 & -77.7446 & 34.2574 & NC \\
\hline 8057 & -1.6 & -77.6905 & 34.3072 & NC & 8129 & 0.4 & -77.7195 & 34.2807 & NC & 8201 & 0.1 & -77.745 & 34.257 & NC \\
\hline 8058 & -1.6 & -77.6917 & 34.3069 & NC & 8130 & 0.3 & .77 .7199 & 34.2803 & NC & 8202 & 0 & -77.7453 & 34.2567 & $\mathrm{NC}$ \\
\hline 8059 & -1.6 & -77.6909 & 34.3069 & NC & 8131 & 0.4 & -77.7203 & 34.28 & NC & 8203 & 0 & -77.7456 & 34.2563 & NC \\
\hline 8060 & -1.6 & -77.692 & 34.3065 & NC & 8132 & 0.3 & -77.7206 & 34.2797 & NC & 8204 & 0 & -77.7459 & 34.2559 & NC \\
\hline 8061 & -1.6 & -77.6913 & 34.3065 & NC & 8133 & 0.4 & -77.721 & 34.2793 & NC & 8205 & -0.1 & -77.7463 & 34.2555 & NC \\
\hline 8062 & -1.8 & .77 .6924 & 34.3062 & NC & 8134 & 0.3 & -77.7214 & 34.279 & NC & 8206 & -0.2 & -77.7466 & 34.2552 & $\mathrm{NC}$ \\
\hline 8063 & -1.7 & -77.6917 & 34.3062 & NC & 8135 & 0.2 & -77.7218 & 34.2786 & NC & 8207 & -0.1 & -77.7469 & 34.2548 & NC \\
\hline
\end{tabular}




\begin{tabular}{|c|c|c|c|c|c|c|c|c|c|c|c|c|c|c|}
\hline TR\# & RATE & LONG & LAT & ST & TR\# & RATE & LONG & LAT & ST & TR\# & RATE & LONG & LAT & ST \\
\hline 8208 & -0.1 & -77.7473 & 34.2544 & NC & 8280 & -0.1 & -77.769 & 34.2284 & NC & 8352 & 1.1 & -77.7896 & 34.1991 & NC \\
\hline 8209 & 0 & -77.7476 & 34.254 & NC & 8281 & -0.2 & -77.7692 & 34.228 & NC & 8353 & 1 & -77.7899 & 34.1987 & NC \\
\hline 8210 & 0.1 & -77.7479 & 34.2537 & NC & 8282 & -0.3 & -77.7695 & 34.2276 & NC & 8354 & 1 & -77.7902 & 34.1983 & $\mathrm{NC}$ \\
\hline 8211 & 0.1 & -77.7482 & 34.2533 & NC & 8283 & -0.2 & -77.7698 & 34.2271 & NC & 8355 & 1 & -77.7905 & 34.1979 & NC \\
\hline 8212 & 0.2 & -77.7486 & 34.2529 & NC & 8284 & -0.1 & -77.77 & 34.2267 & $\mathrm{NC}$ & 8356 & 1 & -77.7907 & 34.1976 & NC \\
\hline 8213 & 0.1 & -77.7489 & 34.2525 & NC & 8285 & -0.1 & -77.7703 & 34.2263 & NC & 8357 & 1 & -77.791 & 34.1972 & $\mathrm{NC}$ \\
\hline 8214 & 0.1 & -77.7492 & 34.2522 & NC & 8286 & -0.1 & -77.7706 & 34.2259 & NC & 8358 & 1 & -77.7913 & 34.1968 & $\mathrm{NC}$ \\
\hline 8215 & -0.1 & -77.7495 & 34.2518 & NC & 8287 & -0.1 & -77.7708 & 34.2255 & NC & 8359 & 1 & -77.7916 & 34.1964 & NC \\
\hline 8216 & -0.2 & -77.7499 & 34.2514 & NC & 8288 & -0.2 & -77.7711 & 34.2251 & NC & 8360 & 1 & -77.7919 & 34.196 & NC \\
\hline 8217 & -0.1 & -77.7502 & 34.251 & NC & 8289 & -0.3 & -77.7714 & 34.2247 & NC & 8361 & 1 & -77.7922 & 34.1956 & NC \\
\hline 8218 & 0 & -77.7505 & 34.2506 & NC & 8290 & -0.4 & -77.7716 & 34.2243 & NC & 8362 & 1.1 & -77.7925 & 34.1952 & NC \\
\hline 8219 & -0.1 & -77.7508 & 34.2503 & NC & 8291 & -0.4 & -77.7719 & 34.2239 & NC & 8363 & 1.2 & -77.7928 & 34.1948 & NC \\
\hline 8220 & -0.2 & -77.7512 & 34.2499 & NC & 8292 & -0.5 & -77.7722 & 34.2235 & NC & 8364 & 1.3 & -77.7931 & 34.1944 & NC \\
\hline 8221 & -0.2 & -77.7515 & 34.2495 & NC & 8293 & -0.7 & -77.7724 & 34.2231 & NC & 8365 & 1.4 & -77.7934 & 34.194 & NC \\
\hline 8222 & -0.2 & -77.7518 & 34.2491 & NC & 8294 & -0.8 & -77.7727 & 34.2227 & NC & 8366 & 1.7 & -77.7937 & 34.1936 & NC \\
\hline 8223 & -0.1 & -77.7522 & 34.2488 & NC & 8295 & -0.8 & -77.773 & 34.2223 & NC & 8367 & 1.7 & -77.794 & 34.1932 & $\mathrm{NC}$ \\
\hline 8224 & 0 & -77.7525 & 34.2484 & NC & 8296 & -0.8 & -77.7732 & 34.2219 & NC & 8368 & 1.8 & -77.7943 & 34.1928 & $\mathrm{NC}$ \\
\hline 8225 & 0.3 & -77.7528 & 34.248 & NC & 8297 & -0.7 & -77.7735 & 34.2214 & NC & 8369 & 1.7 & -77.7946 & 34.1924 & NC \\
\hline 8226 & 0.7 & -77.7531 & 34.2476 & NC & 8298 & -0.7 & -77.7737 & 34.221 & NC & 8370 & 1.7 & -77.7949 & 34.192 & NC \\
\hline 8227 & -0.8 & -77.7535 & 34.2473 & NC & 8299 & -0.5 & -77.774 & 34.2206 & NC & 8371 & 1.6 & -77.7952 & 34.1916 & $\mathrm{NC}$ \\
\hline 8228 & -0.4 & -77.7538 & 34.2469 & NC & 8300 & -0.3 & -77.7743 & 34.2202 & NC & 8372 & 1.5 & -77.7955 & 34.1912 & $\mathrm{NC}$ \\
\hline 8229 & -0.4 & -77.7541 & 34.2465 & NC & 8301 & 0 & -77.7745 & 34.2198 & NC & 8373 & 1.5 & -77.7958 & 34.1908 & $\mathrm{NC}$ \\
\hline 8230 & -0.5 & -77.7544 & 34.2461 & NC & 8302 & 0.5 & -77.7748 & 34.2194 & NC & 8374 & 1.5 & -77.796 & 34.1905 & NC \\
\hline 8231 & -0.3 & -77.7548 & 34.2458 & NC & 8303 & -4 & -77.7751 & 34.219 & NC & 8375 & 1.5 & -77.7963 & 34.1901 & NC \\
\hline 8232 & 0.1 & -77.7551 & 34.2454 & NC & 8304 & -4 & -77.7753 & 34.2186 & NC & 8376 & 1.5 & -77.7966 & 34.1897 & NC \\
\hline 8233 & 0.3 & -77.7554 & 34.245 & NC & 8305 & -5.4 & -77.7756 & 34.2182 & NC & 8377 & 1.5 & -77.7969 & 34.1893 & $\mathrm{NC}$ \\
\hline 8234 & 0.3 & -77.7558 & 34.2446 & NC & 8306 & -4.2 & -77.7759 & 34.2178 & NC & 8378 & 1.6 & -77.7972 & 34.1889 & NC \\
\hline 8235 & 0 & -77.7561 & 34.2443 & NC & 8307 & -2.8 & -77.7761 & 34.2174 & NC & 8379 & 1.6 & -77.7975 & 34.1885 & NC \\
\hline 8236 & -0.3 & -77.7564 & 34.2439 & NC & 8308 & 2.1 & -77.7764 & 34.217 & NC & 8380 & 1.7 & -77.7978 & 34.1881 & $\mathrm{NC}$ \\
\hline 8237 & -0.5 & -77.7567 & 34.2435 & NC & 8309 & 1.2 & -77.7767 & 34.2166 & NC & 8381 & 1.7 & -77.7981 & 34.1877 & NC \\
\hline 8238 & -0.7 & -77.7571 & 34.2431 & NC & 8310 & 0.4 & .77 .7769 & 34.2162 & NC & 8382 & 1.8 & -77.7984 & 34.1873 & NC \\
\hline 8239 & -0.5 & -77.7574 & 34.2427 & NC & 8311 & 0.1 & -77.7772 & 34.2157 & NC & 8383 & 1.9 & -77.7987 & 34.1869 & NC \\
\hline 8240 & -0.5 & -77.7577 & 34.2424 & $\mathrm{NC}$ & 8312 & -0.1 & -77.7775 & 34.2153 & $\mathrm{NC}$ & 8384 & 2 & -77.799 & 34.1865 & NC \\
\hline 8241 & -0.4 & -77.758 & 34.242 & $\mathrm{NC}$ & 8313 & -0.2 & -77.7777 & 34.2149 & NC & 8385 & 1.9 & -77.7993 & 34.1861 & $\mathrm{NC}$ \\
\hline 8242 & -0.5 & -77.7584 & 34.2416 & NC & 8314 & -0.3 & -77.778 & 34.2145 & NC & 8386 & 1.8 & -77.7996 & 34.1857 & $\mathrm{NC}$ \\
\hline 8243 & -0.2 & -77.7587 & 34.2412 & $\mathrm{NC}$ & 8315 & -0.1 & -77.7783 & 34.2141 & NC & 8387 & 1.9 & -77.7999 & 34.1853 & $\mathrm{NC}$ \\
\hline 8244 & 0 & -77.759 & 34.2409 & NC & 8316 & 1.7 & -77.7785 & 34.2137 & NC & 8388 & 2 & -77.8002 & 34.1849 & $\mathrm{NC}$ \\
\hline 8245 & -0.1 & -77.7593 & 34.2405 & NC & 8317 & 1.4 & -77.7788 & 34.2133 & NC & 8389 & 2.1 & -77.8005 & 34.1845 & NC \\
\hline 8246 & 0 & -77.7597 & 34.2401 & $\mathrm{NC}$ & 8318 & 1 & -77.7791 & 34.2129 & NC & 8390 & 2.3 & -77.8008 & 34.1841 & NC \\
\hline 8247 & 0 & -77.76 & 34.2397 & $\mathrm{NC}$ & 8319 & 0.9 & -77.7793 & 34.2125 & NC & 8391 & 2.4 & -77.8011 & 34.1837 & NC \\
\hline 8248 & -0.1 & -77.7603 & 34.2394 & NC & 8320 & 0.9 & -77.7796 & 34.2121 & NC & 8392 & 2.5 & -77.8013 & 34.1834 & NC \\
\hline 8249 & -0.4 & -77.7607 & 34.239 & NC & 8321 & 1 & -77.7799 & 34.2117 & NC & 8393 & 2.6 & -77.8016 & 34.183 & NC \\
\hline 8250 & -2 & -77.761 & 34.2386 & NC & 8322 & 1 & -77.7801 & 34.2113 & NC & 8394 & 2.8 & -77.8019 & 34.1826 & NC \\
\hline 8251 & -5.5 & -77.7613 & 34.2382 & NC & 8323 & 1.1 & -77.7804 & 34.2109 & NC & 8395 & 2.7 & -77.8022 & 34.1822 & $\mathrm{NC}$ \\
\hline 8252 & -3.1 & -77.7616 & 34.2379 & NC & 8324 & 1.1 & -77.7807 & 34.2105 & NC & 8396 & 2.3 & -77.8025 & 34.1818 & $\mathrm{NC}$ \\
\hline 8253 & -1.6 & -77.762 & 34.2375 & NC & 8325 & 1.1 & -77.7809 & 34.21 & NC & 8397 & 6.2 & -77.8047 & 34.1775 & $\mathrm{NC}$ \\
\hline 8254 & -0.7 & -77.7623 & 34.2371 & NC & 8326 & 1.5 & -77.7812 & 34.2096 & NC & 8398 & 6.8 & -77.805 & 34.1772 & $\mathrm{NC}$ \\
\hline 8255 & NA & -77.7626 & 34.2367 & NC & 8327 & 1.4 & -77.7815 & 34.2092 & NC & 8399 & 6.9 & -77.8053 & 34.1768 & $\mathrm{NC}$ \\
\hline 8256 & NA & -77.7629 & 34.2364 & NC & 8328 & 1.5 & -77.7817 & 34.2088 & NC & 8400 & 3.4 & -77.8056 & 34.1764 & NC \\
\hline 8257 & -0.7 & -77.7639 & 34.2361 & NC & 8329 & 1.5 & -77.7828 & 34.2082 & NC & 8401 & -0.3 & -77.806 & 34.176 & NC \\
\hline 8258 & -7.7 & -77.7633 & 34.236 & NC & 8330 & 1.5 & -77.7831 & 34.2078 & NC & 8402 & -1.1 & -77.8063 & 34.1757 & NC \\
\hline 8259 & -0.3 & -77.7642 & 34.2357 & NC & 8331 & 1.5 & -77.7834 & 34.2074 & NC & 8403 & -1.8 & -77.8066 & 34.1753 & NC \\
\hline 8260 & -5.7 & -77.7636 & 34.2356 & NC & 8332 & 1.5 & -77.7837 & 34.207 & NC & 8404 & -2.2 & -77.807 & 34.1749 & $\mathrm{NC}$ \\
\hline 8261 & -0.1 & -77.7644 & 34.2353 & NC & 8333 & 1.5 & -77.784 & 34.2066 & NC & 8405 & -2.5 & -77.8073 & 34.1745 & NC \\
\hline 8262 & -4 & -77.7639 & 34.2352 & NC & 8334 & 1.5 & -77.7843 & 34.2062 & $\mathrm{NC}$ & 8406 & -2.9 & -77.8076 & 34.1742 & $\mathrm{NC}$ \\
\hline 8263 & 0 & -77.7647 & 34.2349 & NC & 8335 & 1.5 & -77.7846 & 34.2058 & NC & 8407 & -3.3 & -77.8079 & 34.1738 & $\mathrm{NC}$ \\
\hline 8264 & -1.1 & -77.7642 & 34.2349 & NC & 8336 & 1.5 & -77.7849 & 34.2054 & NC & 8408 & -3.6 & -77.8083 & 34.1734 & $\mathrm{NC}$ \\
\hline 8265 & 0.2 & -77.765 & 34.2345 & NC & 8337 & 1.6 & -77.7852 & 34.205 & NC & 8409 & -3.8 & -77.8086 & 34.173 & $\mathrm{NC}$ \\
\hline 8266 & 0.4 & -77.7652 & 34.2341 & $\mathrm{NC}$ & 8338 & 1.6 & -77.7854 & 34.2047 & NC & 8410 & -3.8 & -77.8089 & 34.1727 & $\mathrm{NC}$ \\
\hline 8267 & 0.3 & -77.7655 & 34.2337 & NC & 8339 & 1.6 & -77.7857 & 34.2043 & NC & 8411 & -3.8 & -77.8092 & 34.1723 & NC \\
\hline 8268 & 0.2 & -77.7658 & 34.2333 & NC & 8340 & 1.6 & -77.786 & 34.2039 & NC & 8412 & -3.7 & -77.8096 & 34.1719 & NC \\
\hline 8269 & 0.1 & -77.766 & 34.2328 & NC & 8341 & 1.6 & -77.7863 & 34.2035 & NC & 8413 & -3.7 & -77.8099 & 34.1715 & $\mathrm{NC}$ \\
\hline 8270 & -0.1 & -77.7663 & 34.2324 & NC & 8342 & 1.6 & -77.7866 & 34.2031 & NC & 8414 & -3.4 & -77.8102 & 34.1712 & NC \\
\hline 8271 & -0.2 & -77.7666 & 34.232 & NC & 8343 & 1.6 & -77.7869 & 34.2027 & NC & 8415 & -3.2 & -77.8106 & 34.1708 & NC \\
\hline 8272 & -0.2 & -77.7668 & 34.2316 & NC & 8344 & 1.7 & -77.7872 & 34.2023 & NC & 8416 & -3 & -77.8109 & 34.1704 & $\mathrm{NC}$ \\
\hline 8273 & -0.2 & -77.7671 & 34.2312 & NC & 8345 & 1.5 & -77.7875 & 34.2019 & NC & 8417 & -2.7 & -77.8112 & 34.17 & NC \\
\hline 8274 & -0.2 & -77.7674 & 34.2308 & NC & 8346 & 1.5 & -77.7878 & 34.2015 & NC & 8418 & -2.7 & -77.8115 & 34.1697 & NC \\
\hline 8275 & -0.1 & -77.7676 & 34.2304 & $\mathrm{NC}$ & 8347 & 1.1 & -77.7881 & 34.2011 & NC & 8419 & -2.4 & -77.8119 & 34.1693 & NC \\
\hline 8276 & 0 & .77 .7679 & 34.23 & NC & 8348 & 1 & -77.7884 & 34.2007 & NC & 8420 & -2.2 & .77 .8122 & 34.1689 & NC \\
\hline 8277 & -0.2 & -77.7682 & 34.2296 & NC & 8349 & 1 & -77.7887 & 34.2003 & NC & 8421 & -2 & -77.8125 & 34.1685 & NC \\
\hline 8278 & -0.1 & -77.7684 & 34.2292 & NC & 8350 & 0.9 & -77.789 & 34.1999 & NC & 8422 & -1.6 & -77.8129 & 34.1682 & NC \\
\hline 8279 & -0.2 & -77.7687 & 34.2288 & $\mathrm{NC}$ & 8351 & 1 & -77.7893 & 34.1995 & NC & 8423 & -1.5 & -77.8132 & 34.1678 & NC \\
\hline
\end{tabular}




\begin{tabular}{|c|c|c|c|c|c|c|c|c|c|c|c|c|c|c|}
\hline TR\# & RATE & LONG & LAT & ST & TR\# & RATE & LONG & LAT & ST & TR\# & RATE & LONG & LAT & ST \\
\hline 8424 & -1.3 & -77.8135 & 34.1674 & NC & 8496 & -2.1 & -77.831 & 34.1407 & $\mathrm{NC}$ & 8568 & -1.8 & -77.8464 & 34.1107 & NC \\
\hline 8425 & -1.2 & -77.8138 & 34.167 & NC & 8497 & -2.1 & -77.8312 & 34.1403 & $\mathrm{NC}$ & 8569 & -1.8 & -77.8466 & 34.1102 & NC \\
\hline 8426 & -1.3 & -77.8142 & 34.1667 & NC & 8498 & -2.1 & -77.8314 & 34.1398 & NC & 8570 & -1.8 & -77.8468 & 34.1098 & NC \\
\hline 8427 & -1.4 & -77.8145 & 34.1663 & NC & 8499 & -2.2 & -77.8317 & 34.1394 & NC & 8571 & -1.9 & -77.847 & 34.1094 & NC \\
\hline 8428 & -1.5 & -77.8148 & 34.1659 & $\mathrm{NC}$ & 8500 & -2.3 & -77.8319 & 34.139 & $\mathrm{NC}$ & 8572 & -1.8 & -77.8472 & 34.1089 & NC \\
\hline 8429 & -1.6 & -77.8152 & 34.1655 & NC & 8501 & -2.3 & -77.8321 & 34.1386 & NC & 8573 & -1.7 & -77.8475 & 34.1085 & NC \\
\hline 8430 & -1.7 & -77.8155 & 34.1652 & NC & 8502 & -2.3 & -77.8323 & 34.1381 & NC & 8574 & -1.7 & -77.8477 & 34.1081 & NC \\
\hline 8431 & -1.7 & -77.8158 & 34.1648 & NC & 8503 & -2.3 & -77.8326 & 34.1377 & NC & 8575 & -1.8 & -77.8479 & 34.1077 & $\mathrm{NC}$ \\
\hline 8432 & -1.7 & -77.8161 & 34.1644 & NC & 8504 & -2.2 & -77.8328 & 34.1373 & $\mathrm{NC}$ & 8576 & -1.8 & -77.8481 & 34.1072 & NC \\
\hline 8433 & -1.6 & -77.8165 & 34.164 & NC & 8505 & -2.2 & -77.833 & 34.1369 & $\mathrm{NC}$ & 8577 & -1.8 & -77.8483 & 34.1068 & NC \\
\hline 8434 & -1.7 & -77.8168 & 34.1637 & NC & 8506 & -2.1 & -77.8332 & 34.1364 & $\mathrm{NC}$ & 8578 & -1.8 & -77.8485 & 34.1064 & $\mathrm{NC}$ \\
\hline 8435 & -1.9 & -77.8171 & 34.1633 & NC & 8507 & -2.1 & -77.8334 & 34.136 & NC & 8579 & -1.8 & -77.8488 & 34.106 & NC \\
\hline 8436 & -1.9 & -77.8175 & 34.1629 & NC & 8508 & -2.1 & -77.8337 & 34.1356 & NC & 8580 & -1.8 & -77.849 & 34.1055 & NC \\
\hline 8437 & -1.7 & -77.8178 & 34.1625 & NC & 8509 & -2.1 & -77.8339 & 34.1352 & $\mathrm{NC}$ & 8581 & -1.7 & -77.8492 & 34.1051 & NC \\
\hline 8438 & -1.5 & -77.8181 & 34.1622 & NC & 8510 & -2.1 & -77.8341 & 34.1347 & NC & 8582 & -1.7 & -77.8494 & 34.1047 & NC \\
\hline 8439 & -1.3 & -77.8184 & 34.1618 & NC & 8511 & -2 & -77.8343 & 34.1343 & NC & 8583 & -1.6 & -77.8496 & 34.1042 & NC \\
\hline 8440 & -1.2 & -77.8188 & 34.1614 & NC & 8512 & -1.9 & -77.8346 & 34.1339 & NC & 8584 & -1.5 & -77.8498 & 34.1038 & NC \\
\hline 8441 & -1.2 & -77.8191 & 34.161 & NC & 8513 & -1.8 & -77.8348 & 34.1335 & NC & 8585 & -1.5 & -77.85 & 34.1034 & NC \\
\hline 8442 & -1.3 & -77.8194 & 34.1607 & NC & 8514 & -1.8 & -77.835 & 34.133 & NC & 8586 & -1.4 & -77.8503 & 34.103 & NC \\
\hline 8443 & -1.2 & -77.8197 & 34.1603 & NC & 8515 & -1.7 & -77.8352 & 34.1326 & $\mathrm{NC}$ & 8587 & -1.4 & -77.8505 & 34.1025 & NC \\
\hline 8444 & -1 & -77.8201 & 34.1599 & NC & 8516 & -1.7 & -77.8355 & 34.1322 & NC & 8588 & -1.4 & -77.8507 & 34.1021 & NC \\
\hline 8445 & -0.9 & -77.8204 & 34.1595 & NC & 8517 & -1.7 & -77.8357 & 34.1318 & NC & 8589 & -1.5 & -77.852 & 34.1017 & NC \\
\hline 8446 & -0.8 & -77.8207 & 34.1592 & NC & 8518 & -1.8 & -77.8359 & 34.1313 & NC & 8590 & -1.5 & -77.8509 & 34.1017 & NC \\
\hline 8447 & -0.9 & -77.8211 & 34.1588 & NC & 8519 & -1.9 & -77.8361 & 34.1309 & NC & 8591 & -1.5 & -77.8522 & 34.1013 & NC \\
\hline 8448 & -1 & -77.8214 & 34.1584 & NC & 8520 & -1.9 & -77.8363 & 34.1305 & NC & 8592 & -1.4 & -77.8511 & 34.1012 & NC \\
\hline 8449 & -1 & -77.8217 & 34.158 & NC & 8521 & -2 & -77.8366 & 34.1301 & NC & 8593 & -1.6 & -77.8524 & 34.1008 & NC \\
\hline 8450 & -1.8 & -77.8221 & 34.1577 & NC & 8522 & -2.1 & -77.8368 & 34.1296 & NC & 8594 & -1.3 & -77.8513 & 34.1008 & NC \\
\hline 8451 & -1 & -77.822 & 34.1577 & NC & 8523 & -2.2 & -77.8369 & 34.1295 & NC & 8595 & -1.6 & -77.8526 & 34.1004 & NC \\
\hline 8452 & -1.8 & -77.8223 & 34.1573 & NC & 8524 & -2.1 & -77.837 & 34.1292 & NC & 8596 & -1.5 & -77.8528 & 34.1 & NC \\
\hline 8453 & -0.8 & -77.8224 & 34.1573 & NC & 8525 & -2.1 & -77.8371 & 34.1291 & NC & 8597 & -1.5 & -77.853 & 34.0995 & NC \\
\hline 8454 & -0.8 & -77.8227 & 34.1569 & NC & 8526 & -1.9 & -77.8373 & 34.1286 & NC & 8598 & -1.6 & -77.8532 & 34.0991 & NC \\
\hline 8455 & -1.9 & -77.8225 & 34.1568 & NC & 8527 & -1.9 & -77.8375 & 34.1282 & NC & 8599 & -1.7 & -77.8534 & 34.0987 & NC \\
\hline 8456 & -0.9 & -77.823 & 34.1565 & NC & 8528 & -2 & -77.8378 & 34.1278 & NC & 8600 & -1.7 & -77.8536 & 34.0983 & NC \\
\hline 8457 & -2 & -77.8228 & 34.1564 & NC & 8529 & -2 & -77.838 & 34.1274 & NC & 8601 & -1.7 & -77.8538 & 34.0978 & NC \\
\hline 8458 & -1.4 & -77.8234 & 34.1562 & NC & 8530 & -2.1 & -77.8382 & 34.1269 & NC & 8602 & -1.6 & -77.854 & 34.0974 & NC \\
\hline 8459 & -2 & -77.823 & 34.156 & NC & 8531 & -2.1 & -77.8384 & 34.1265 & NC & 8603 & -1.5 & -77.8542 & 34.097 & NC \\
\hline 8460 & -1.7 & -77.8237 & 34.1558 & NC & 8532 & -2.1 & -77.8386 & 34.1261 & NC & 8604 & -1.7 & -77.8544 & 34.0965 & NC \\
\hline 8461 & -2 & -77.8232 & 34.1556 & NC & 8533 & -2.1 & -77.8388 & 34.1256 & NC & 8605 & -1.9 & -77.8546 & 34.0961 & NC \\
\hline 8462 & -2 & -77.8234 & 34.1551 & NC & 8534 & -1.9 & -77.8391 & 34.1252 & NC & 8606 & -2 & -77.8548 & 34.0957 & NC \\
\hline 8463 & -2 & -77.8237 & 34.1547 & NC & 8535 & -1.9 & -77.8393 & 34.1248 & NC & 8607 & -2 & -77.855 & 34.0952 & NC \\
\hline 8464 & -2.1 & -77.8239 & 34.1543 & NC & 8536 & -2 & -77.8395 & 34.1244 & NC & 8608 & -2.1 & -77.8552 & 34.0948 & NC \\
\hline 8465 & -2.1 & -77.8241 & 34.1539 & NC & 8537 & -2 & -77.8397 & 34.1239 & NC & 8609 & -2.1 & -77.8554 & 34.0944 & NC \\
\hline 8466 & -2.1 & -77.8243 & 34.1534 & NC & 8538 & -2.1 & -77.8399 & 34.1235 & NC & 8610 & -2.1 & -77.8556 & 34.0939 & NC \\
\hline 8467 & -2.1 & -77.8245 & 34.153 & NC & 8539 & -2.1 & -77.8401 & 34.1231 & NC & 8611 & -2.1 & -77.8558 & 34.0935 & NC \\
\hline 8468 & -2.1 & -77.8248 & 34.1526 & $\mathrm{NC}$ & 8540 & -2 & -77.8403 & 34.1226 & NC & 8612 & -2.1 & -77.856 & 34.0931 & NC \\
\hline 8469 & -2.1 & -77.825 & 34.1522 & NC & 8541 & -2 & -77.8406 & 34.1222 & NC & 8613 & -2.2 & -77.8562 & 34.0926 & NC \\
\hline 8470 & -2.1 & -77.8252 & 34.1517 & NC & 8542 & -2 & -77.8408 & 34.1218 & NC & 8614 & -2.2 & -77.8564 & 34.0922 & NC \\
\hline 8471 & -2 & -77.8254 & 34.1513 & NC & 8543 & -2 & -77.841 & 34.1214 & NC & 8615 & -2.1 & -77.8566 & 34.0918 & NC \\
\hline 8472 & -2.1 & -77.8257 & 34.1509 & $\mathrm{NC}$ & 8544 & -2 & -77.8412 & 34.1209 & NC & 8616 & -2.2 & -77.8568 & 34.0914 & NC \\
\hline 8473 & -2.2 & -77.8259 & 34.1505 & NC & 8545 & -1.9 & -77.8414 & 34.1205 & NC & 8617 & -2.2 & -77.857 & 34.0909 & NC \\
\hline 8474 & -2.3 & -77.8261 & 34.15 & NC & 8546 & -2 & -77.8416 & 34.1201 & NC & 8618 & -2.3 & -77.8572 & 34.0905 & NC \\
\hline 8475 & -2.3 & -77.8263 & 34.1496 & NC & 8547 & -2 & -77.8419 & 34.1197 & NC & 8619 & -2.3 & -77.8574 & 34.0901 & NC \\
\hline 8476 & -2.3 & -77.8266 & 34.1492 & NC & 8548 & -1.9 & -77.8421 & 34.1192 & NC & 8620 & -2.3 & -77.8576 & 34.0896 & $\mathrm{NC}$ \\
\hline 8477 & -2.3 & -77.8268 & 34.1488 & NC & 8549 & -1.9 & -77.8423 & 34.1188 & NC & 8621 & -2.2 & -77.8578 & 34.0892 & NC \\
\hline 8478 & -2.3 & -77.827 & 34.1483 & NC & 8550 & -1.8 & -77.8425 & 34.1184 & NC & 8622 & -2.3 & -77.858 & 34.0888 & NC \\
\hline 8479 & -2.4 & -77.8272 & 34.1479 & NC & 8551 & -1.8 & -77.8427 & 34.1179 & NC & 8623 & -2.4 & -77.8582 & 34.0883 & NC \\
\hline 8480 & -2.2 & -77.8274 & 34.1475 & NC & 8552 & -1.8 & -77.8429 & 34.1175 & $\mathrm{NC}$ & 8624 & -2.4 & -77.8584 & 34.0879 & $\mathrm{NC}$ \\
\hline 8481 & -2.2 & -77.8277 & 34.1471 & NC & 8553 & -1.8 & -77.8431 & 34.1171 & NC & 8625 & -2.4 & -77.8586 & 34.0875 & NC \\
\hline 8482 & -2 & -77.8279 & 34.1466 & NC & 8554 & -1.9 & -77.8434 & 34.1167 & NC & 8626 & -2.4 & -77.8588 & 34.087 & $\mathrm{NC}$ \\
\hline 8483 & -2 & -77.8281 & 34.1462 & NC & 8555 & -1.9 & -77.8436 & 34.1162 & NC & 8627 & -2.3 & -77.859 & 34.0866 & $\mathrm{NC}$ \\
\hline 8484 & -1.9 & -77.8283 & 34.1458 & NC & 8556 & -1.8 & -77.8438 & 34.1158 & NC & 8628 & -2.3 & -77.8592 & 34.0862 & NC \\
\hline 8485 & -1.8 & -77.8286 & 34.1454 & NC & 8557 & -1.7 & -77.844 & 34.1154 & NC & 8629 & -2.2 & -77.8594 & 34.0858 & NC \\
\hline 8486 & -1.8 & -77.8288 & 34.1449 & NC & 8558 & -1.8 & -77.8442 & 34.1149 & NC & 8630 & -2.4 & -77.8596 & 34.0853 & NC \\
\hline 8487 & -1.9 & -77.829 & 34.1445 & NC & 8559 & -1.7 & -77.8444 & 34.1145 & $\mathrm{NC}$ & 8631 & -2.2 & -77.8598 & 34.0849 & NC \\
\hline 8488 & -1.9 & -77.8292 & 34.1441 & $\mathrm{NC}$ & 8560 & -1.7 & -77.8447 & 34.1141 & NC & 8632 & -2.2 & -77.86 & 34.0845 & NC \\
\hline 8489 & -1.9 & -77.8294 & 34.1437 & NC & 8561 & -1.8 & -77.8449 & 34.1137 & NC & 8633 & -2.1 & -77.8602 & 34.084 & NC \\
\hline 8490 & -2 & -77.8297 & 34.1432 & NC & 8562 & -1.8 & -77.8451 & 34.1132 & $\mathrm{NC}$ & 8634 & -2.1 & -77.8604 & 34.0836 & NC \\
\hline 8491 & -2.1 & -77.8299 & 34.1428 & NC & 8563 & -1.8 & -77.8453 & 34.1128 & $\mathrm{NC}$ & 8635 & -2 & -77.8606 & 34.0832 & NC \\
\hline 8492 & -2.1 & -77.8301 & 34.1424 & $\mathrm{NC}$ & 8564 & -1.8 & -77.8455 & 34.1124 & $\mathrm{NC}$ & 8636 & -2.1 & -77.8608 & 34.0827 & NC \\
\hline 8493 & -2.1 & -77.8303 & 34.142 & NC & 8565 & -1.9 & -77.8457 & 34.1119 & $\mathrm{NC}$ & 8637 & -2 & -77.861 & 34.0823 & NC \\
\hline 8494 & -2.1 & -77.8306 & 34.1415 & NC & 8566 & -1.8 & -77.846 & 34.1115 & $\mathrm{NC}$ & 8638 & -2.1 & -77.8612 & 34.0819 & NC \\
\hline 8495 & -2.1 & -77.8308 & 34.1411 & $\mathrm{NC}$ & 8567 & -1.8 & -77.8462 & 34.1111 & $\mathrm{NC}$ & 8639 & -2.1 & -77.8614 & 34.0814 & NC \\
\hline
\end{tabular}




\begin{tabular}{|c|c|c|c|c|c|c|c|c|c|c|c|c|c|c|}
\hline 8640 & -2.2 & -77.8616 & 34.081 & NC & 8712 & 0.4 & -77.8767 & 34.0507 & NC & 8784 & 0.8 & -77.8892 & 34.0199 & NC \\
\hline 8641 & -2.1 & -77.8618 & 34.0806 & NC & 8713 & 0.5 & -77.8769 & 34.0502 & NC & 8785 & 0.8 & -77.8894 & 34.0195 & NC \\
\hline 8642 & -2.1 & -77.862 & 34.0802 & NC & 8714 & 0.6 & -77.8771 & 34.0498 & NC & 8786 & 0.8 & -77.8896 & 34.019 & NC \\
\hline 8643 & -2.3 & .77 .8622 & 34.0797 & NC & 8715 & 0.7 & -77.8772 & 34.0494 & NC & 8787 & 0.7 & -77.8897 & 34.0186 & NC \\
\hline 8644 & -2.5 & -77.8624 & 34.0793 & NC & 8716 & 0.8 & -77.8774 & 34.0489 & NC & 8788 & 0.6 & -77.8899 & 34.0182 & NC \\
\hline 8645 & -2.7 & -77.8626 & 34.0789 & NC & 8717 & 0.8 & -77.8776 & 34.0485 & NC & 8789 & 0.6 & -77.8901 & 34.0177 & NC \\
\hline 8646 & -3.2 & -77.8628 & 34.0784 & NC & 8718 & 0.8 & -77.8777 & 34.048 & NC & 8790 & 0.6 & -77.8903 & 34.0173 & $\mathrm{NC}$ \\
\hline 8647 & -3.7 & -77.863 & 34.078 & NC & 8719 & 0.9 & -77.8779 & 34.0476 & NC & 8791 & 0.6 & -77.8904 & 34.0169 & $\mathrm{NC}$ \\
\hline 8648 & -4 & -77.8632 & 34.0776 & NC & 8720 & 0.9 & -77.8781 & 34.0471 & NC & 8792 & 0.5 & -77.8906 & 34.0164 & $\mathrm{NC}$ \\
\hline 8649 & -4.4 & -77.8634 & 34.0771 & NC & 8721 & 1 & -77.8782 & 34.0467 & NC & 8793 & 0.5 & -77.8908 & 34.016 & NC \\
\hline 8650 & -4.8 & -77.8636 & 34.0767 & NC & 8722 & 1 & -77.8784 & 34.0463 & NC & 8794 & 0.5 & -77.891 & 34.0156 & NC \\
\hline 8651 & -13.3 & -77.8638 & 34.0763 & NC & 8723 & 1.1 & -77.8786 & 34.0458 & NC & 8795 & 0.4 & -77.8911 & 34.0151 & $\mathrm{NC}$ \\
\hline 8652 & -15.1 & -77.864 & 34.0758 & NC & 8724 & 1.2 & -77.8787 & 34.0454 & NC & 8796 & 0.3 & -77.8913 & 34.0147 & NC \\
\hline 8653 & -16.9 & -77.8642 & 34.0754 & NC & 8725 & 1.2 & -77.8789 & 34.0449 & NC & 8797 & 0.3 & -77.8915 & 34.0142 & $\mathrm{NC}$ \\
\hline 8654 & -19.8 & -77.8644 & 34.075 & NC & 8726 & 1.2 & -77.8791 & 34.0445 & NC & 8798 & -0.3 & -77.8922 & 34.0141 & NC \\
\hline 8655 & -22.7 & -77.8646 & 34.0745 & NC & 8727 & 1.2 & -77.8792 & 34.0441 & NC & 8799 & 0.3 & -77.8917 & 34.0138 & NC \\
\hline 8656 & -8.7 & -77.8648 & 34.0741 & $\mathrm{NC}$ & 8728 & 1.3 & -77.8794 & 34.0436 & NC & 8800 & -0.4 & -77.8924 & 34.0137 & NC \\
\hline 8657 & -7.7 & -77.865 & 34.0737 & NC & 8729 & 0.2 & -77.8797 & 34.0435 & NC & 8801 & 0.2 & -77.8918 & 34.0134 & NC \\
\hline 8658 & -3.8 & -77.868 & 34.0736 & $\mathrm{NC}$ & 8730 & 1.2 & -77.8796 & 34.0432 & $\mathrm{NC}$ & 8802 & -0.5 & -77.8925 & 34.0132 & $\mathrm{NC}$ \\
\hline 8659 & -6.6 & .77 .8652 & 34.0733 & NC & 8731 & 0.2 & -77.8799 & 34.0431 & NC & 8803 & -0.5 & -77.8927 & 34.0128 & NC \\
\hline 8660 & -3.7 & .77 .8682 & 34.0732 & NC & 8732 & 0.2 & -77.8801 & 34.0426 & NC & 8804 & -0.6 & -77.8928 & 34.0123 & NC \\
\hline 8661 & -5.8 & -77.8654 & 34.0728 & NC & 8733 & 0.3 & -77.8802 & 34.0422 & NC & 8805 & -0.6 & -77.893 & 34.0119 & $\mathrm{NC}$ \\
\hline 8662 & -3.6 & -77.8683 & 34.0727 & $\mathrm{NC}$ & 8734 & 0.3 & -77.8804 & 34.0418 & NC & 8806 & -0.6 & -77.8931 & 34.0114 & $\mathrm{NC}$ \\
\hline 8663 & -3.1 & -77.8685 & 34.0723 & NC & 8735 & 0.2 & -77.8806 & 34.0413 & NC & 8807 & -0.8 & -77.8933 & 34.011 & NC \\
\hline 8664 & -3 & -77.8687 & 34.0718 & $\mathrm{NC}$ & 8736 & 0.2 & -77.8808 & 34.0409 & NC & 8808 & -0.7 & -77.8935 & 34.0106 & $\mathrm{NC}$ \\
\hline 8665 & -3 & -77.8688 & 34.0714 & NC & 8737 & 0.3 & -77.8809 & 34.0404 & NC & 8809 & -0.7 & -77.8936 & 34.0101 & $\mathrm{NC}$ \\
\hline 8666 & -2.7 & -77.869 & 34.071 & NC & 8738 & 0.3 & -77.8811 & 34.04 & $\mathrm{NC}$ & 8810 & -0.8 & -77.8938 & 34.0097 & NC \\
\hline 8667 & -2.6 & -77.8692 & 34.0705 & NC & 8739 & 0.4 & -77.8813 & 34.0396 & NC & 8811 & -0.8 & -77.8939 & 34.0092 & NC \\
\hline 8668 & -2.6 & -77.8693 & 34.0701 & NC & 8740 & 0.4 & -77.8815 & 34.0391 & NC & 8812 & -0.8 & -77.8941 & 34.0088 & NC \\
\hline 8669 & -2.6 & -77.8695 & 34.0696 & NC & 8741 & 0.5 & -77.8816 & 34.0387 & NC & 8813 & -0.8 & -77.8943 & 34.0083 & NC \\
\hline 8670 & -2.6 & -77.8697 & 34.0692 & $\mathrm{NC}$ & 8742 & 0.5 & -77.8818 & 34.0383 & NC & 8814 & -0.8 & -77.8944 & 34.0079 & NC \\
\hline 8671 & -2.6 & -77.8698 & 34.0688 & NC & 8743 & 0.6 & -77.882 & 34.0378 & NC & 8815 & -0.9 & -77.8946 & 34.0074 & NC \\
\hline 8672 & -2.6 & -77.87 & 34.0683 & NC & 8744 & 0.7 & -77.8822 & 34.0374 & NC & 8816 & -0.8 & -77.8947 & 34.007 & NC \\
\hline 8673 & -2.7 & -77.8702 & 34.0679 & $\mathrm{NC}$ & 8745 & 0.7 & -77.8823 & 34.037 & NC & 8817 & -0.7 & -77.8949 & 34.0066 & NC \\
\hline 8674 & -2.8 & -77.8703 & 34.0674 & NC & 8746 & 0.7 & -77.8825 & 34.0365 & NC & 8818 & -0.7 & -77.895 & 34.0061 & NC \\
\hline 8675 & -2.9 & -77.8705 & 34.067 & NC & 8747 & 0.7 & -77.8827 & 34.0361 & NC & 8819 & -0.7 & -77.8952 & 34.0057 & NC \\
\hline 8676 & -2.9 & -77.8707 & 34.0665 & NC & 8748 & 0.8 & -77.8829 & 34.0356 & NC & 8820 & -0.6 & -77.8954 & 34.0052 & NC \\
\hline 8677 & -3 & -77.8708 & 34.0661 & NC & 8749 & 0.9 & -77.883 & 34.0352 & NC & 8821 & -0.6 & -77.8955 & 34.0048 & NC \\
\hline 8678 & -3.1 & -77.871 & 34.0657 & NC & 8750 & 0.9 & -77.8832 & 34.0348 & NC & 8822 & -0.4 & -77.8957 & 34.0043 & $\mathrm{NC}$ \\
\hline 8679 & -3.2 & -77.8712 & 34.0652 & NC & 8751 & 1 & -77.8834 & 34.0343 & NC & 8823 & -0.4 & -77.8958 & 34.0039 & NC \\
\hline 8680 & -3.1 & -77.8714 & 34.0648 & NC & 8752 & 1 & -77.8836 & 34.0339 & NC & 8824 & -0.5 & -77.896 & 34.0035 & NC \\
\hline 8681 & -3.1 & -77.8715 & 34.0643 & $\mathrm{NC}$ & 8753 & 1.1 & -77.8837 & 34.0335 & NC & 8825 & -0.6 & -77.8961 & 34.003 & $\mathrm{NC}$ \\
\hline 8682 & -3.1 & -77.8717 & 34.0639 & NC & 8754 & 1 & -77.8839 & 34.033 & NC & 8826 & -0.3 & -77.8963 & 34.0026 & $\mathrm{NC}$ \\
\hline 8683 & -3.2 & -77.8719 & 34.0635 & NC & 8755 & 1 & -77.8841 & 34.0326 & NC & 8827 & -0.2 & -77.8965 & 34.0021 & $\mathrm{NC}$ \\
\hline 8684 & -3.3 & -77.872 & 34.063 & NC & 8756 & 1 & -77.8843 & 34.0321 & NC & 8828 & -0.2 & -77.8966 & 34.0017 & $\mathrm{NC}$ \\
\hline 8685 & -3.2 & -77.8722 & 34.0626 & NC & 8757 & 1 & -77.8845 & 34.0317 & NC & 8829 & -0.2 & -77.8968 & 34.0012 & $\mathrm{NC}$ \\
\hline 8686 & -3.2 & -77.8724 & 34.0621 & NC & 8758 & 1 & -77.8846 & 34.0313 & NC & 8830 & -0.2 & -77.8969 & 34.0008 & NC \\
\hline 8687 & -3.1 & -77.8725 & 34.0617 & NC & 8759 & 1 & -77.8848 & 34.0308 & NC & 8831 & -0.3 & -77.8971 & 34.0003 & NC \\
\hline 8688 & -3.1 & -77.8727 & 34.0613 & NC & 8760 & 1.1 & -77.885 & 34.0304 & NC & 8832 & -0.3 & -77.8972 & 33.9999 & $\mathrm{NC}$ \\
\hline 8689 & -3.1 & -77.8729 & 34.0608 & NC & 8761 & 1.1 & -77.8852 & 34.03 & $\mathrm{NC}$ & 8833 & -0.3 & -77.8974 & 33.9995 & $\mathrm{NC}$ \\
\hline 8690 & -2.9 & -77.873 & 34.0604 & NC & 8762 & 1.2 & -77.8853 & 34.0295 & NC & 8834 & -0.3 & -77.8976 & 33.999 & NC \\
\hline 8691 & -2.8 & -77.8732 & 34.0599 & NC & 8763 & 1.3 & -77.8855 & 34.0291 & NC & 8835 & -0.2 & -77.8977 & 33.9986 & $\mathrm{NC}$ \\
\hline 8692 & -2.7 & -77.8734 & 34.0595 & NC & 8764 & 1.3 & -77.8857 & 34.0287 & NC & 8836 & -0.3 & -77.8979 & 33.9981 & NC \\
\hline 8693 & -2.7 & -77.8735 & 34.0591 & NC & 8765 & 1.4 & -77.8859 & 34.0282 & $\mathrm{NC}$ & 8837 & -0.4 & -77.898 & 33.9977 & NC \\
\hline 8694 & -2.5 & -77.8737 & 34.0586 & NC & 8766 & 1.4 & -77.886 & 34.0278 & NC & 8838 & -0.3 & -77.8982 & 33.9972 & $\mathrm{NC}$ \\
\hline 8695 & -2.5 & -77.8739 & 34.0582 & NC & 8767 & 1.5 & -77.8862 & 34.0273 & NC & 8839 & -0.3 & -77.8984 & 33.9968 & NC \\
\hline 8696 & -2.3 & -77.874 & 34.0577 & NC & 8768 & 1.4 & -77.8864 & 34.0269 & $\mathrm{NC}$ & 8840 & -0.3 & -77.8985 & 33.9964 & $\mathrm{NC}$ \\
\hline 8697 & -2.1 & -77.8742 & 34.0573 & $\mathrm{NC}$ & 8769 & 1.3 & -77.8866 & 34.0265 & NC & 8841 & -0.5 & -77.8987 & 33.9959 & NC \\
\hline 8698 & -2 & -77.8744 & 34.0568 & NC & 8770 & 1.3 & -77.8867 & 34.026 & NC & 8842 & -0.5 & -77.8988 & 33.9955 & NC \\
\hline 8699 & -1.9 & -77.8745 & 34.0564 & NC & 8771 & 1.3 & -77.8869 & 34.0256 & NC & 8843 & -0.5 & -77.899 & 33.995 & NC \\
\hline 8700 & -1.6 & -77.8747 & 34.056 & NC & 8772 & 1.2 & -77.8871 & 34.0252 & NC & 8844 & -0.5 & -77.8991 & 33.9946 & NC \\
\hline 8701 & -1.3 & -77.8749 & 34.0555 & NC & 8773 & 1.2 & -77.8873 & 34.0247 & NC & 8845 & -0.6 & -77.8993 & 33.9941 & NC \\
\hline 8702 & -1 & -77.875 & 34.0551 & $\mathrm{NC}$ & 8774 & 1.1 & -77.8874 & 34.0243 & NC & 8846 & -0.6 & -77.8995 & 33.9937 & NC \\
\hline 8703 & -0.7 & -77.8752 & 34.0546 & $\mathrm{NC}$ & 8775 & 1.1 & -77.8876 & 34.0239 & NC & 8847 & -0.6 & -77.8996 & 33.9932 & NC \\
\hline 8704 & -0.6 & -77.8754 & 34.0542 & NC & 8776 & 1.1 & -77.8878 & 34.0234 & $\mathrm{NC}$ & 8848 & -0.6 & -77.8998 & 33.9928 & NC \\
\hline 8705 & -0.6 & -77.8755 & 34.0538 & NC & 8777 & 1 & -77.888 & 34.023 & NC & 8849 & -0.6 & -77.8999 & 33.9924 & NC \\
\hline 8706 & -0.4 & -77.8757 & 34.0533 & $\mathrm{NC}$ & 8778 & 0.9 & -77.8882 & 34.0225 & NC & 8850 & -0.6 & -77.9001 & 33.9919 & NC \\
\hline 8707 & -0.3 & -77.8759 & 34.0529 & $\mathrm{NC}$ & 8779 & 0.8 & -77.8883 & 34.0221 & NC & 8851 & -0.6 & -77.9002 & 33.9915 & NC \\
\hline 8708 & -0.2 & -77.876 & 34.0524 & $\mathrm{NC}$ & 8780 & 0.7 & -77.8885 & 34.0217 & NC & 8852 & -0.6 & -77.9004 & 33.991 & NC \\
\hline 8709 & 0 & -77.8762 & 34.052 & $\mathrm{NC}$ & 8781 & 0.7 & -77.8887 & 34.0212 & NC & 8853 & -0.6 & -77.9006 & 33.9906 & $\mathrm{NC}$ \\
\hline 8710 & 0.2 & -77.8764 & 34.0516 & $\mathrm{NC}$ & 8782 & 0.7 & -77.8889 & 34.0208 & $\mathrm{NC}$ & 8854 & -0.6 & -77.9007 & 33.9901 & NC \\
\hline 8711 & 0.2 & -77.8765 & 34.0511 & NC & 8783 & 0.8 & -77.889 & 34.0204 & NC & 8855 & -0.6 & -77.9009 & 33.9897 & $\mathrm{NC}$ \\
\hline
\end{tabular}




\begin{tabular}{|c|c|c|c|c|c|c|c|c|c|c|c|c|c|c|}
\hline TR\# & RATE & LONG & LAT & $S T$ & TR\# & RATE & LONG & LAT & ST & TR\# & RATE & LONG & LAT & ST \\
\hline 8856 & -0.5 & -77.901 & 33.9893 & $\mathrm{NC}$ & 8928 & -3.3 & -77.9112 & 33.9588 & NC & 9000 & 3.1 & .77 .928 & 33.9279 & $\mathrm{NC}$ \\
\hline 8857 & -0.5 & -77.9012 & 33.9888 & NC & 8929 & -3.3 & .77 .9114 & 33.9584 & NC & 9001 & 3.3 & -77.9282 & 33.9275 & NC \\
\hline 8858 & -0.6 & -77.9013 & 33.9884 & NC & 8930 & -3.3 & -77.9115 & 33.9579 & $\mathrm{NC}$ & 9002 & 3.3 & -77.9285 & 33.9271 & NC \\
\hline 8859 & -0.7 & -77.9015 & 33.9879 & NC & 8931 & -3.3 & -77.9117 & 33.9575 & $\mathrm{NC}$ & 9003 & 3.4 & -77.9287 & 33.9266 & $\mathrm{NC}$ \\
\hline 8860 & -0.6 & -77.9017 & 33.9875 & NC & 8932 & -3.2 & -77.9118 & 33.957 & NC & 9004 & 3.6 & -77.9289 & 33.9262 & $\mathrm{NC}$ \\
\hline 8861 & -0.5 & -77.9018 & 33.987 & NC & 8933 & -3.2 & -77.9119 & 33.9566 & $\mathrm{NC}$ & 9005 & 3.7 & -77.9291 & 33.9258 & NC \\
\hline 8862 & -0.6 & -77.902 & 33.9866 & NC & 8934 & -3.2 & -77.9121 & 33.9561 & NC & 9006 & 3.8 & -77.9294 & 33.9254 & NC \\
\hline 8863 & -0.7 & -77.9021 & 33.9861 & NC & 8935 & -3.3 & -77.9122 & 33.9557 & NC & 9007 & 5.6 & -77.9315 & 33.9251 & $\mathrm{NC}$ \\
\hline 8864 & -0.7 & -77.9023 & 33.9857 & NC & 8936 & -3.3 & -77.9124 & 33.9552 & NC & 9008 & 4 & -77.9296 & 33.9249 & $\mathrm{NC}$ \\
\hline 8865 & -0.6 & -77.9025 & 33.9853 & NC & 8937 & -3.1 & -77.9125 & 33.9548 & $\mathrm{NC}$ & 9009 & 5.7 & -77.9317 & 33.9247 & NC \\
\hline 8866 & -0.6 & -77.9026 & 33.9848 & NC & 8938 & -2.9 & -77.9127 & 33.9543 & NC & 9010 & 4.2 & -77.9298 & 33.9245 & $\mathrm{NC}$ \\
\hline 8867 & -0.5 & .77 .9028 & 33.9844 & NC & 8939 & -2.7 & -77.9128 & 33.9539 & NC & 9011 & 5.6 & -77.9318 & 33.9242 & $\mathrm{NC}$ \\
\hline 8868 & -0.9 & -77.9031 & 33.9839 & NC & 8940 & -2.5 & -77.913 & 33.9534 & NC & 9012 & 4.3 & -77.93 & 33.9241 & NC \\
\hline 8869 & -0.4 & -77.9029 & 33.9839 & $\mathrm{NC}$ & 8941 & -2.4 & -77.9131 & 33.953 & NC & 9013 & 5.6 & -77.932 & 33.9238 & $\mathrm{NC}$ \\
\hline 8870 & -0.8 & -77.9032 & 33.9835 & NC & 8942 & -2.8 & -77.9152 & 33.9525 & NC & 9014 & 4.6 & -77.9302 & 33.9237 & $\mathrm{NC}$ \\
\hline 8871 & -0.4 & .77 .9031 & 33.9835 & $\mathrm{NC}$ & 8943 & -3 & -77.9154 & 33.9521 & NC & 9015 & 5.7 & -77.9321 & 33.9233 & $\mathrm{NC}$ \\
\hline 8872 & -1 & -77.9034 & 33.983 & $\mathrm{NC}$ & 8944 & -3.1 & -77.9156 & 33.9517 & $\mathrm{NC}$ & 9016 & 4.7 & -77.9305 & 33.9232 & $\mathrm{NC}$ \\
\hline 8873 & -0.5 & -77.9032 & 33.983 & NC & 8945 & -3.4 & -77.9159 & 33.9512 & NC & 9017 & 5.7 & -77.9323 & 33.9229 & NC \\
\hline 8874 & -1.1 & -77.9035 & 33.9826 & $\mathrm{NC}$ & 8946 & -3.4 & -77.9161 & 33.9508 & $\mathrm{NC}$ & 9018 & 5.6 & -77.9325 & 33.9224 & $\mathrm{NC}$ \\
\hline 8875 & -0.5 & -77.9034 & 33.9826 & $\mathrm{NC}$ & 8947 & -3.4 & -77.9163 & 33.9504 & NC & 9019 & 5.6 & -77.9326 & 33.922 & $\mathrm{NC}$ \\
\hline 8876 & -1.2 & -77.9037 & 33.9821 & NC & 8948 & -3.3 & -77.9165 & 33.95 & NC & 9020 & 5.6 & -77.9328 & 33.9216 & $\mathrm{NC}$ \\
\hline 8877 & -1.2 & -77.9038 & 33.9817 & NC & 8949 & -3.2 & -77.9167 & 33.9495 & NC & 9021 & 5.5 & -77.933 & 33.9211 & $\mathrm{NC}$ \\
\hline 8878 & -1.2 & -77.904 & 33.9812 & NC & 8950 & -3.1 & -77.917 & 33.9491 & NC & 9022 & 5.5 & -77.9331 & 33.9207 & $\mathrm{NC}$ \\
\hline 8879 & -1.1 & -77.9041 & 33.9808 & NC & 8951 & -2.9 & -77.9172 & 33.9487 & NC & 9023 & 5.4 & -77.9333 & 33.9202 & $\mathrm{NC}$ \\
\hline 8880 & -1.2 & -77.9043 & 33.9803 & NC & 8952 & -2.7 & -77.9174 & 33.9483 & NC & 9024 & 5.3 & -77.9334 & 33.9198 & $\mathrm{NC}$ \\
\hline 8881 & -1.2 & -77.9044 & 33.9799 & NC & 8953 & -2.5 & -77.9176 & 33.9478 & NC & 9025 & 5.2 & -77.9336 & 33.9194 & $\mathrm{NC}$ \\
\hline 8882 & -1.3 & -77.9046 & 33.9794 & NC & 8954 & -2.4 & -77.9179 & 33.9474 & NC & 9026 & 5.1 & -77.9338 & 33.9189 & $\mathrm{NC}$ \\
\hline 8883 & -1.3 & -77.9047 & 33.979 & NC & 8955 & -2.3 & -77.9181 & 33.947 & NC & 9027 & 5 & -77.9339 & 33.9185 & $\mathrm{NC}$ \\
\hline 8884 & -1.3 & -77.9048 & 33.9785 & NC & 8956 & -2.1 & -77.9183 & 33.9466 & NC & 9028 & 4.9 & -77.9341 & 33.918 & NC \\
\hline 8885 & -1.3 & -77.905 & 33.9781 & NC & 8957 & -1.9 & -77.9185 & 33.9461 & NC & 9029 & 4.9 & -77.9343 & 33.9176 & $\mathrm{NC}$ \\
\hline 8886 & -1.3 & -77.9051 & 33.9776 & $\mathrm{NC}$ & 8958 & -1.8 & -77.9187 & 33.9457 & NC & 9030 & 4.8 & -77.9344 & 33.9171 & $\mathrm{NC}$ \\
\hline 8887 & -1.3 & -77.9053 & 33.9772 & NC & 8959 & -1.7 & -77.919 & 33.9453 & NC & 9031 & 4.4 & -77.9346 & 33.9167 & NC \\
\hline 8888 & -1.3 & -77.9054 & 33.9767 & $\mathrm{NC}$ & 8960 & -1.6 & -77.9192 & 33.9449 & NC & 9032 & 3.5 & -77.9347 & 33.9163 & $\mathrm{NC}$ \\
\hline 8889 & -1.3 & -77.9056 & 33.9763 & $\mathrm{NC}$ & 8961 & -1.6 & -77.9194 & 33.9444 & NC & 9033 & 2.5 & -77.9349 & 33.9158 & $\mathrm{NC}$ \\
\hline 8890 & -1.3 & -77.9057 & 33.9758 & NC & 8962 & -1.4 & -77.9196 & 33.944 & NC & 9034 & 1.6 & -77.9351 & 33.9154 & $\mathrm{NC}$ \\
\hline 8891 & -1.3 & -77.9059 & 33.9754 & $\mathrm{NC}$ & 8963 & -1.4 & -77.9198 & 33.9436 & NC & 9035 & 0.8 & -77.9352 & 33.9149 & $\mathrm{NC}$ \\
\hline 8892 & -1.2 & -77.906 & 33.9749 & NC & 8964 & -1.2 & -77.9201 & 33.9432 & NC & 9036 & -1.7 & -77.9354 & 33.9145 & $\mathrm{NC}$ \\
\hline 8893 & -1.2 & -77.9061 & 33.9745 & NC & 8965 & -1 & -77.9203 & 33.9427 & NC & 9037 & -4.6 & -77.9359 & 33.9132 & $\mathrm{NC}$ \\
\hline 8894 & -1.4 & -77.9063 & 33.974 & NC & 8966 & -0.9 & -77.9205 & 33.9423 & NC & 9038 & -4.8 & -77.936 & 33.9127 & $\mathrm{NC}$ \\
\hline 8895 & -1.3 & -77.9064 & 33.9736 & NC & 8967 & -1 & -77.9207 & 33.9419 & NC & 9039 & -5.1 & -77.9362 & 33.9123 & $\mathrm{NC}$ \\
\hline 8896 & -1.3 & -77.9066 & 33.9 .732 & NC & 8968 & -1 & -77.9209 & 33.9415 & NC & 9040 & -5.8 & -77.9364 & 33.9118 & $\mathrm{NC}$ \\
\hline 8897 & -1.4 & -77.9067 & 33.9727 & NC & 8969 & -0.8 & -77.9212 & 33.9411 & NC & 9041 & -2.4 & -77.9365 & 33.9114 & $\mathrm{NC}$ \\
\hline 8898 & -1.9 & -77.9069 & 33.9723 & NC & 8970 & -0.7 & -77.9214 & 33.9406 & NC & 9042 & -2.4 & -77.9367 & 33.9109 & $\mathrm{NC}$ \\
\hline 8899 & -2.2 & -77.907 & 33.9718 & NC & 8971 & -0.7 & -77.9216 & 33.9402 & $\mathrm{NC}$ & 9043 & -1.7 & -77.9368 & 33.9105 & $\mathrm{NC}$ \\
\hline 8900 & -2.3 & -77.9072 & 33.9714 & NC & 8972 & -0.7 & -77.9218 & 33.9398 & $\mathrm{NC}$ & 9044 & -2.5 & -77.937 & 33.9101 & $\mathrm{NC}$ \\
\hline 8901 & -2.4 & -77.9073 & 33.9709 & NC & 8973 & -0.7 & -77.9221 & 33.9394 & NC & 9045 & -3.3 & -77.9372 & 33.9096 & NC \\
\hline 8902 & -2.4 & -77.9075 & 33.9705 & NC & 8974 & -0.6 & -77.9223 & 33.9389 & NC & 9046 & -3.5 & -77.9373 & 33.9092 & $\mathrm{NC}$ \\
\hline 8903 & -3 & -77.9076 & 33.97 & NC & 8975 & -0.5 & -77.9225 & 33.9385 & NC & 9047 & -3.5 & -77.9375 & 33.9087 & $\mathrm{NC}$ \\
\hline 8904 & -3.2 & -77.9077 & 33.9696 & $\mathrm{NC}$ & 8976 & -0.3 & -77.9227 & 33.9381 & NC & 9048 & -3.5 & -77.9377 & 33.9083 & $\mathrm{NC}$ \\
\hline 8905 & -3.1 & -77.9079 & 33.9691 & $\mathrm{NC}$ & 8977 & 0 & .77 .9229 & 33.9377 & NC & 9049 & -3.5 & -77.9378 & 33.9079 & $\mathrm{NC}$ \\
\hline 8906 & -2.7 & -77.908 & 33.9687 & NC & 8978 & 0.2 & -77.9232 & 33.9372 & NC & 9050 & -3.2 & -77.938 & 33.9074 & $\mathrm{NC}$ \\
\hline 8907 & -2.4 & -77.9082 & 33.9682 & $\mathrm{NC}$ & 8979 & 0.4 & -77.9234 & 33.9368 & NC & 9051 & -2.9 & -77.9381 & 33.907 & $\mathrm{NC}$ \\
\hline 8908 & -2.3 & -77.9083 & 33.9678 & $\mathrm{NC}$ & 8980 & 0.4 & -77.9236 & 33.9364 & $\mathrm{NC}$ & 9052 & -2.6 & -77.9383 & 33.9065 & $\mathrm{NC}$ \\
\hline 8909 & -1.8 & -77.9085 & 33.9673 & NC & 8981 & 0.5 & -77.9238 & 33.936 & NC & 9053 & -2.3 & -77.9385 & 33.9061 & NC \\
\hline 8910 & -1.7 & -77.9086 & 33.9669 & NC & 8982 & 0.7 & -77.924 & 33.9355 & NC & 9054 & -2 & -77.9386 & 33.9056 & $\mathrm{NC}$ \\
\hline 8911 & -1.8 & -77.9088 & 33.9664 & NC & 8983 & 0.9 & -77.9243 & 33.9351 & NC & 9055 & 0 & -78.1974 & 33.9055 & NC \\
\hline 8912 & -1.5 & -77.9089 & 33.966 & NC & 8984 & 1.2 & -77.9245 & 33.9347 & NC & 9056 & 0 & -78.1969 & 33.9055 & NC \\
\hline 8913 & -1.7 & -77.909 & 33.9655 & NC & 8985 & 1.4 & -77.9247 & 33.9343 & NC & 9057 & 0 & -78.1963 & 33.9055 & NC \\
\hline 8914 & -2.6 & -77.9092 & 33.9651 & NC & 8986 & 1.6 & -77.9249 & 33.9338 & NC & 9058 & 0 & -78.1958 & 33.9055 & NC \\
\hline 8915 & -2.7 & -77.9093 & 33.9646 & NC & 8987 & 1.8 & -77.9252 & 33.9334 & NC & 9059 & -0.1 & -78.1952 & 33.9055 & NC \\
\hline 8916 & -2.9 & -77.9095 & 33.9642 & NC & 8988 & 2.1 & -77.9254 & 33.933 & NC & 9060 & -0.1 & -78.1947 & 33.9055 & NC \\
\hline 8917 & -2.9 & -77.9096 & 33.9637 & NC & 8989 & 2.4 & .77 .9256 & 33.9326 & NC & 9061 & 0 & -78.1941 & 33.9055 & NC \\
\hline 8918 & -3 & -77.9098 & 33.9633 & $\mathrm{NC}$ & 8990 & 2.4 & -77.9258 & 33.9322 & NC & 9062 & 0 & -78.1936 & 33.9055 & NC \\
\hline 8919 & -3.1 & -77.9099 & 33.9628 & NC & 8991 & 2.5 & .77 .926 & 33.9317 & NC & 9063 & 0 & -78.193 & 33.9055 & NC \\
\hline 8920 & -3.2 & -77.9101 & 33.9624 & NC & 8992 & 2.6 & -77.9263 & 33.9313 & NC & 9064 & 0 & -78.1924 & 33.9055 & NC \\
\hline 8921 & -3.1 & -77.9102 & 33.962 & NC & 8993 & 2.8 & -77.9265 & 33.9309 & NC & 9065 & -0.1 & .78 .1919 & 33.9055 & NC \\
\hline 8922 & -3.1 & -77.9104 & 33.9615 & NC & 8994 & 2.8 & -77.9267 & 33.9305 & NC & 9066 & 0.2 & -78.2656 & 33.9055 & NC \\
\hline 8923 & -3.1 & -77.9105 & 33.9611 & NC & 8995 & 2.8 & -77.9269 & 33.93 & $\mathrm{NC}$ & 9067 & 0 & -78.198 & 33.9055 & NC \\
\hline 8924 & -3.1 & -77.9106 & 33.9606 & NC & 8996 & 2.8 & -77.9271 & 33.9296 & NC & 9068 & -0.3 & -78.183 & 33.9054 & $\mathrm{NC}$ \\
\hline 8925 & -3.2 & -77.9108 & 33.9602 & NC & 8997 & 2.8 & -77.9274 & 33.9292 & NC & 9069 & -0.4 & .78 .1825 & 33.9054 & NC \\
\hline 8926 & -3.2 & -77.9109 & 33.9597 & $\mathrm{NC}$ & 8998 & 2.9 & -77.9276 & 33.9288 & $\mathrm{NC}$ & 9070 & -0.3 & -78.1819 & 33.9054 & NC \\
\hline 8927 & -3.3 & -77.9111 & 33.9593 & NC & 8999 & 3 & -77.9278 & 33.9283 & NC & 9071 & -0.4 & -78.1814 & 33.9054 & NC \\
\hline
\end{tabular}




\begin{tabular}{|c|c|c|c|c|c|c|c|c|c|c|c|c|c|c|}
\hline TR\# & RATE & LONG & LAT & ST & TR\# & RATE & LONG & LAT & ST & TR\# & RATE & LONG & LAT & ST \\
\hline 9072 & -0.4 & -78.1808 & 33.9054 & NC & 9144 & -0.6 & -78.1617 & 33.9052 & NC & 9216 & -5.3 & -78.24 & 33.905 & NC \\
\hline 9073 & -0.4 & -78.1803 & 33.9054 & NC & 9145 & -1.6 & -77.9388 & 33.9052 & $\mathrm{NC}$ & 9217 & -5.8 & -78.2395 & 33.905 & NC \\
\hline 9074 & -0.4 & -78.1797 & 33.9054 & NC & 9146 & 0.1 & -78.2037 & 33.9051 & $\mathrm{NC}$ & 9218 & -3.3 & -78.2389 & 33.905 & NC \\
\hline 9075 & -0.3 & -78.1792 & 33.9054 & NC & 9147 & -0.6 & -78.1606 & 33.9051 & $\mathrm{NC}$ & 9219 & -1.3 & -78.2384 & 33.905 & NC \\
\hline 9076 & -0.3 & -78.1786 & 33.9054 & NC & 9148 & -0.6 & -78.1601 & 33.9051 & NC & 9220 & NA & -78.2362 & 33.905 & NC \\
\hline 9077 & -0.3 & -78.1781 & 33.9054 & NC & 9149 & 0.6 & -78.27 & 33.9051 & NC & 9221 & NA & -78.2356 & 33.905 & NC \\
\hline 9078 & -0.4 & -78.1775 & 33.9054 & NC & 9150 & 0.6 & -78.2695 & 33.9051 & NC & 9222 & -3.1 & -78.2351 & 33.905 & NC \\
\hline 9079 & -0.3 & -78.1769 & 33.9054 & NC & 9151 & 0 & -78.2065 & 33.9051 & NC & 9223 & -2.2 & -78.2345 & 33.905 & NC \\
\hline 9080 & -0.4 & -78.1764 & 33.9054 & NC & 9152 & -0.1 & -78.206 & 33.9051 & $\mathrm{NC}$ & 9224 & -2.1 & -78.234 & 33.905 & NC \\
\hline 9081 & -0.3 & -78.1758 & 33.9054 & NC & 9153 & -0.1 & .78 .2054 & 33.9051 & $\mathrm{NC}$ & 9225 & -1.8 & -78.2334 & 33.905 & NC \\
\hline 9082 & -0.5 & -78.1753 & 33.9054 & NC & 9154 & 0 & -78.2049 & 33.9051 & NC & 9226 & -1.7 & -78.2329 & 33.905 & NC \\
\hline 9083 & -0.3 & -78.1747 & 33.9054 & NC & 9155 & 0.1 & -78.2043 & 33.9051 & NC & 9227 & -1.7 & -78.2323 & 33.905 & NC \\
\hline 9084 & -0.3 & -78.1742 & 33.9054 & NC & 9156 & -1.1 & -78.2301 & 33.905 & NC & 9228 & -1.5 & -78.2318 & 33.905 & NC \\
\hline 9085 & -0.4 & -78.1736 & 33.9054 & NC & 9157 & -1 & -78.2296 & 33.905 & NC & 9229 & -1.2 & -78.2312 & 33.905 & NC \\
\hline 9086 & -0.3 & -78.1731 & 33.9054 & NC & 9158 & -0.9 & -78.229 & 33.905 & $\mathrm{NC}$ & 9230 & -1 & -78.2307 & 33.905 & NC \\
\hline 9087 & -0.3 & -78.1725 & 33.9054 & NC & 9159 & 0.6 & -78.2711 & 33.905 & NC & 9231 & -0.3 & -78.2126 & 33.9049 & NC \\
\hline 9088 & -0.3 & -78.172 & 33.9054 & NC & 9160 & 0.6 & -78.2706 & 33.905 & NC & 9232 & -0.2 & -78.2121 & 33.9049 & NC \\
\hline 9089 & 0.2 & -78.2662 & 33.9054 & NC & 9161 & -0.1 & -78.266 & 33.905 & NC & 9233 & -0.3 & -78.2115 & 33.9049 & NC \\
\hline 9090 & 0.2 & -78.2667 & 33.9054 & NC & 9162 & -0.2 & -78.2654 & 33.905 & $\mathrm{NC}$ & 9234 & -0.2 & -78.2109 & 33.9049 & NC \\
\hline 9091 & 0.2 & -78.1971 & 33.9054 & NC & 9163 & -0.2 & -78.2649 & 33.905 & NC & 9235 & -0.3 & -78.2104 & 33.9049 & NC \\
\hline 9092 & 0 & -78.1913 & 33.9054 & NC & 9164 & -0.3 & -78.2643 & 33.905 & NC & 9236 & -0.3 & -78.2098 & 33.9049 & NC \\
\hline 9093 & 0 & -78.1908 & 33.9054 & NC & 9165 & -0.4 & -78.2638 & 33.905 & NC & 9237 & -0.6 & .78 .1579 & 33.9049 & NC \\
\hline 9094 & 0 & -78.1902 & 33.9054 & NC & 9166 & -0.5 & -78.2632 & 33.905 & NC & 9238 & -0.5 & -78.1573 & 33.9049 & NC \\
\hline 9095 & 0 & -78.1897 & 33.9054 & NC & 9167 & -0.5 & -78.2627 & 33.905 & NC & 9239 & 0.7 & -78.2717 & 33.9049 & NC \\
\hline 9096 & -0.1 & -78.1891 & 33.9054 & NC & 9168 & -0.5 & -78.2621 & 33.905 & NC & 9240 & 0.7 & -78.2722 & 33.9049 & NC \\
\hline 9097 & -0.1 & -78.1886 & 33.9054 & NC & 9169 & -0.4 & -78.2616 & 33.905 & NC & 9241 & -0.3 & -78.2154 & 33.9048 & NC \\
\hline 9098 & 0 & -78.188 & 33.9054 & NC & 9170 & -0.5 & -78.261 & 33.905 & $\mathrm{NC}$ & 9242 & -0.3 & -78.2148 & 33.9048 & NC \\
\hline 9099 & -0.1 & -78.1875 & 33.9054 & NC & 9171 & -0.6 & -78.2605 & 33.905 & NC & 9243 & -0.3 & .78 .2143 & 33.9048 & NC \\
\hline 9100 & -0.2 & -78.1869 & 33.9054 & NC & 9172 & -0.7 & -78.2599 & 33.905 & NC & 9244 & -0.3 & -78.2137 & 33.9048 & NC \\
\hline 9101 & -0.2 & -78.1864 & 33.9054 & NC & 9173 & -0.8 & -78.2594 & 33.905 & $\mathrm{NC}$ & 9245 & -0.3 & -78.2132 & 33.9048 & NC \\
\hline 9102 & -0.2 & -78.1858 & 33.9054 & NC & 9174 & -0.9 & -78.2588 & 33.905 & NC & 9246 & -0.5 & -78.1568 & 33.9048 & NC \\
\hline 9103 & -0.2 & -78.1852 & 33.9054 & NC & 9175 & -0.9 & -78.2583 & 33.905 & NC & 9247 & -0.5 & -78.1562 & 33.9048 & NC \\
\hline 9104 & -0.2 & -78.1847 & 33.9054 & NC & 9176 & -0.9 & -78.2577 & 33.905 & NC & 9248 & 0.9 & -78.2733 & 33.9048 & NC \\
\hline 9105 & -0.2 & -78.1841 & 33.9054 & NC & 9177 & -0.9 & -78.2572 & 33.905 & NC & 9249 & 0.8 & -78.2728 & 33.9048 & NC \\
\hline 9106 & -0.2 & -78.1836 & 33.9054 & NC & 9178 & -0.9 & -78.2566 & 33.905 & $\mathrm{NC}$ & 9250 & -1.3 & -77.939 & 33.9048 & NC \\
\hline 9107 & -0.5 & -78.1631 & 33.9053 & NC & 9179 & -0.9 & -78.2561 & 33.905 & NC & 9251 & -0.2 & -78.2159 & 33.9047 & NC \\
\hline 9108 & -0.5 & -78.1626 & 33.9053 & NC & 9180 & -0.8 & -78.2555 & 33.905 & NC & 9252 & -0.5 & -78.1557 & 33.9047 & NC \\
\hline 9109 & -0.5 & .78 .162 & 33.9053 & NC & 9181 & -0.9 & -78.255 & 33.905 & NC & 9253 & -0.6 & -78.1551 & 33.9047 & NC \\
\hline 9110 & -0.6 & -78.1634 & 33.9053 & NC & 9182 & -0.9 & -78.2544 & 33.905 & NC & 9254 & -0.5 & -78.1546 & 33.9047 & NC \\
\hline 9111 & -0.6 & -78.1628 & 33.9053 & NC & 9183 & -0.8 & -78.2538 & 33.905 & NC & 9255 & 0.8 & -78.2739 & 33.9047 & NC \\
\hline 9112 & 0.3 & -78.2673 & 33.9053 & NC & 9184 & -0.9 & -78.2533 & 33.905 & NC & 9256 & 0.9 & -78.2744 & 33.9047 & NC \\
\hline 9113 & 0.4 & -78.2678 & 33.9053 & NC & 9185 & -0.9 & -78.2527 & 33.905 & NC & 9257 & -0.3 & -78.2181 & 33.9047 & NC \\
\hline 9114 & 0.2 & -78.2004 & 33.9053 & NC & 9186 & -0.8 & -78.2522 & 33.905 & NC & 9258 & -0.2 & -78.2176 & 33.9047 & NC \\
\hline 9115 & 0.3 & -78.1999 & 33.9053 & NC & 9187 & -0.9 & -78.2516 & 33.905 & $\mathrm{NC}$ & 9259 & -0.1 & -78.217 & 33.9047 & NC \\
\hline 9116 & 0.3 & -78.1993 & 33.9053 & NC & 9188 & -0.9 & -78.2511 & 33.905 & NC & 9260 & -0.1 & -78.2165 & 33.9047 & NC \\
\hline 9117 & 0.1 & -78.1988 & 33.9053 & NC & 9189 & -0.8 & -78.2505 & 33.905 & $\mathrm{NC}$ & 9261 & -0.4 & -78.2204 & 33.9046 & NC \\
\hline 9118 & 0.1 & -78.1982 & 33.9053 & NC & 9190 & -0.8 & -78.25 & 33.905 & NC & 9262 & -0.4 & -78.2198 & 33.9046 & NC \\
\hline 9119 & 0.2 & -78.1977 & 33.9053 & NC & 9191 & -0.9 & -78.2494 & 33.905 & $\mathrm{NC}$ & 9263 & -0.5 & -78.2192 & 33.9046 & NC \\
\hline 9120 & -0.4 & -78.1714 & 33.9053 & NC & 9192 & -0.9 & -78.2489 & 33.905 & $\mathrm{NC}$ & 9264 & -0.3 & -78.2187 & 33.9046 & NC \\
\hline 9121 & -0.3 & -78.1709 & 33.9053 & NC & 9193 & -0.9 & -78.2483 & 33.905 & $\mathrm{NC}$ & 9265 & -0.6 & -78.154 & 33.9046 & NC \\
\hline 9122 & -0.3 & -78.1703 & 33.9053 & NC & 9194 & -1 & -78.2478 & 33.905 & NC & 9266 & -0.5 & -78.1535 & 33.9046 & NC \\
\hline 9123 & -0.3 & -78.1697 & 33.9053 & NC & 9195 & -1 & -78.2472 & 33.905 & NC & 9267 & 0.9 & -78.275 & 33.9046 & NC \\
\hline 9124 & -0.2 & -78.1692 & 33.9053 & NC & 9196 & -1 & -78.2467 & 33.905 & NC & 9268 & 0.8 & -78.2755 & 33.9046 & NC \\
\hline 9125 & -0.3 & -78.1686 & 33.9053 & NC & 9197 & -1 & -78.2461 & 33.905 & NC & 9269 & -0.2 & -78.2215 & 33.9046 & NC \\
\hline 9126 & -0.3 & -78.1681 & 33.9053 & NC & 9198 & -0.2 & -78.2093 & 33.905 & NC & 9270 & -0.2 & -78.2209 & 33.9046 & NC \\
\hline 9127 & -0.3 & -78.1675 & 33.9053 & NC & 9199 & -0.2 & -78.2087 & 33.905 & NC & 9271 & 0.8 & -78.2761 & 33.9045 & NC \\
\hline 9128 & -0.2 & -78.167 & 33.9053 & NC & 9200 & -0.1 & -78.2082 & 33.905 & NC & 9272 & -0.2 & -78.2242 & 33.9045 & NC \\
\hline 9129 & -0.2 & -78.1664 & 33.9053 & NC & 9201 & -0.1 & -78.2076 & 33.905 & NC & 9273 & -0.2 & -78.2237 & 33.9045 & $\mathrm{NC}$ \\
\hline 9130 & -0.3 & -78.1659 & 33.9053 & NC & 9202 & 0 & -78.2071 & 33.905 & NC & 9274 & -0.2 & -78.2231 & 33.9045 & NC \\
\hline 9131 & -0.3 & -78.1653 & 33.9053 & NC & 9203 & -0.6 & -78.1595 & 33.905 & NC & 9275 & -0.2 & -78.2226 & 33.9045 & NC \\
\hline 9132 & -0.4 & -78.1648 & 33.9053 & NC & 9204 & -0.6 & .78 .159 & 33.905 & NC & 9276 & -0.2 & -78.222 & 33.9045 & NC \\
\hline 9133 & -0.5 & -78.1642 & 33.9053 & NC & 9205 & -0.6 & -78.1584 & 33.905 & NC & 9277 & -0.5 & -78.1529 & 33.9045 & NC \\
\hline 9134 & -0.5 & -78.1637 & 33.9053 & NC & 9206 & -1 & -78.2456 & 33.905 & NC & 9278 & -0.5 & -78.1524 & 33.9045 & NC \\
\hline 9135 & -0.6 & -78.1612 & 33.9052 & NC & 9207 & -1 & -78.245 & 33.905 & NC & 9279 & -0.5 & -78.1518 & 33.9045 & NC \\
\hline 9136 & 0.5 & -78.2689 & 33.9052 & NC & 9208 & -1 & -78.2445 & 33.905 & NC & 9280 & 0.9 & -78.2766 & 33.9045 & $\mathrm{NC}$ \\
\hline 9137 & 0.5 & -78.2684 & 33.9052 & NC & 9209 & -1.4 & -78.2439 & 33.905 & NC & 9281 & -0.5 & -78.1507 & 33.9044 & $\mathrm{NC}$ \\
\hline 9138 & 0.1 & -78.2032 & 33.9052 & NC & 9210 & -2.1 & -78.2434 & 33.905 & NC & 9282 & 0.8 & -78.2777 & 33.9044 & NC \\
\hline 9139 & 0.2 & -78.2026 & 33.9052 & NC & 9211 & -2.7 & -78.2428 & 33.905 & NC & 9283 & 0.9 & -78.2772 & 33.9044 & $\mathrm{NC}$ \\
\hline 9140 & 0.2 & -78.2021 & 33.9052 & NC & 9212 & -3 & -78.2423 & 33.905 & $\mathrm{NC}$ & 9284 & -0.4 & -78.2276 & 33.9044 & NC \\
\hline 9141 & 0.2 & -78.2015 & 33.9052 & $\mathrm{NC}$ & 9213 & -3.4 & -78.2417 & 33.905 & NC & 9285 & -0.2 & -78.227 & 33.9044 & NC \\
\hline 9142 & 0.2 & -78.201 & 33.9052 & NC & 9214 & -3.6 & -78.2412 & 33.905 & $\mathrm{NC}$ & 9286 & -0.5 & -78.2264 & 33.9044 & NC \\
\hline 9143 & -0.5 & -78.1623 & 33.9052 & $\mathrm{NC}$ & 9215 & -4.4 & -78.2406 & 33.905 & NC & 9287 & -0.5 & -78.2259 & 33.9044 & $\mathrm{NC}$ \\
\hline
\end{tabular}




\begin{tabular}{|c|c|c|c|c|c|c|c|c|c|c|c|c|c|c|}
\hline TR\# & RATE & LONG & LAT & ST & TR\# & RATE & LONG & LAT & ST & TR\# & RATE & LONG & LAT & ST \\
\hline 9288 & -0.4 & -78.2253 & 33.9044 & NC & 9360 & -0.6 & -78.1319 & 33.903 & NC & 9432 & -0.1 & -78.3141 & 33.9011 & $\mathrm{NC}$ \\
\hline 9289 & -0.4 & -78.2248 & 33.9044 & $\mathrm{NC}$ & 9361 & 0.6 & -78.2931 & 33.903 & NC & 9433 & -0.1 & -78.3135 & 33.9011 & NC \\
\hline 9290 & -0.5 & -78.1512 & 33.9044 & NC & 9362 & -0.2 & -77.9396 & 33.903 & NC & 9434 & 0 & -78.1187 & 33.9011 & NC \\
\hline 9291 & -0.9 & -78.2287 & 33.9043 & NC & 9363 & -0.6 & -78.1308 & 33.9029 & NC & 9435 & -0.1 & -78.1182 & 33.901 & NC \\
\hline 9292 & -0.5 & -78.2281 & 33.9043 & NC & 9364 & -0.6 & -78.1303 & 33.9029 & NC & 9436 & -0.1 & -78.3152 & 33.901 & NC \\
\hline 9293 & -0.5 & -78.1501 & 33.9043 & NC & 9365 & 0.5 & -78.2942 & 33.9029 & NC & 9437 & -0.1 & -78.3146 & 33.901 & NC \\
\hline 9294 & -0.4 & -78.1496 & 33.9043 & NC & 9366 & 0.5 & -78.2937 & 33.9029 & NC & 9438 & -0.1 & -78.3157 & 33.9009 & NC \\
\hline 9295 & -0.4 & -78.149 & 33.9043 & NC & 9367 & -0.6 & -78.1314 & 33.9029 & NC & 9439 & -0.1 & -78.1176 & 33.9009 & NC \\
\hline 9296 & 0.8 & -78.2783 & 33.9043 & NC & 9368 & 0.5 & -78.2953 & 33.9028 & NC & 9440 & -0.1 & -78.3163 & 33.9009 & NC \\
\hline 9297 & 0.8 & -78.2788 & 33.9043 & NC & 9369 & 0.4 & -78.2948 & 33.9028 & NC & 9441 & -0.1 & -78.1171 & 33.9008 & NC \\
\hline 9298 & -1.3 & -78.2303 & 33.9043 & $\mathrm{NC}$ & 9370 & -0.6 & -78.1297 & 33.9028 & NC & 9442 & -0.1 & -78.3174 & 33.9008 & NC \\
\hline 9299 & -1.1 & -78.2298 & 33.9043 & NC & 9371 & -0.7 & -78.1292 & 33.9028 & NC & 9443 & 0 & -78.3168 & 33.9008 & NC \\
\hline 9300 & -1 & -78.2292 & 33.9043 & NC & 9372 & -0.8 & -78.1281 & 33.9027 & NC & 9444 & 0.8 & -77.9404 & 33.9008 & NC \\
\hline 9301 & -0.9 & -77.9391 & 33.9043 & NC & 9373 & -0.7 & -78.1275 & 33.9027 & NC & 9445 & -0.1 & -78.3179 & 33.9007 & NC \\
\hline 9302 & -0.4 & -78.1485 & 33.9042 & NC & 9374 & 0.6 & -78.2964 & 33.9027 & NC & 9446 & -0.1 & -78.1165 & 33.9007 & NC \\
\hline 9303 & -0.3 & -78.1479 & 33.9042 & NC & 9375 & 0.6 & -78.2959 & 33.9027 & NC & 9447 & -0.1 & -78.3185 & 33.9007 & NC \\
\hline 9304 & 0.8 & -78.2799 & 33.9042 & NC & 9376 & -0.7 & -78.1286 & 33.9027 & NC & 9448 & -0.1 & -78.3196 & 33.9006 & NC \\
\hline 9305 & 0.8 & -78.2794 & 33.9042 & NC & 9377 & -0.7 & -78.1264 & 33.9026 & NC & 9449 & -0.1 & -78.319 & 33.9006 & NC \\
\hline 9306 & -1.2 & -78.2309 & 33.9042 & NC & 9378 & 0.5 & -78.2975 & 33.9026 & NC & 9450 & -0.2 & -78.116 & 33.9006 & NC \\
\hline 9307 & 0.8 & -78.281 & 33.9041 & NC & 9379 & 0.6 & -78.297 & 33.9026 & NC & 9451 & -0.2 & -78.1154 & 33.9005 & NC \\
\hline 9308 & 0.8 & -78.2805 & 33.9041 & NC & 9380 & -0.7 & -78.127 & 33.9026 & NC & 9452 & -0.2 & -78.3207 & 33.9005 & NC \\
\hline 9309 & -0.3 & -78.1474 & 33.9041 & NC & 9381 & 0.4 & -78.2986 & 33.9025 & NC & 9453 & -0.1 & -78.3201 & 33.9005 & NC \\
\hline 9310 & -0.3 & -78.1468 & 33.9041 & NC & 9382 & 0.5 & -78.2981 & 33.9025 & NC & 9454 & -0.2 & -78.3212 & 33.9004 & NC \\
\hline 9311 & -0.3 & -78.1463 & 33.9041 & NC & 9383 & -0.7 & -78.1259 & 33.9025 & NC & 9455 & -0.2 & -78.1149 & 33.9004 & NC \\
\hline 9312 & 0.8 & -78.2821 & 33.904 & NC & 9384 & -0.7 & -78.1253 & 33.9025 & NC & 9456 & -0.3 & -78.3218 & 33.9004 & NC \\
\hline 9313 & 0.8 & -78.2816 & 33.904 & NC & 9385 & 0.1 & -77.9398 & 33.9025 & NC & 9457 & -0.2 & -78.1143 & 33.9003 & NC \\
\hline 9314 & -0.4 & -78.1457 & 33.904 & NC & 9386 & 0.2 & -78.2997 & 33.9024 & NC & 9458 & -0.2 & -78.1138 & 33.9003 & NC \\
\hline 9315 & -0.5 & -78.1452 & 33.904 & $\mathrm{NC}$ & 9387 & 0.3 & -78.2992 & 33.9024 & NC & 9459 & -0.3 & -78.3229 & 33.9003 & NC \\
\hline 9316 & 0.8 & -78.2827 & 33.9039 & NC & 9388 & 0.2 & -78.1269 & 33.9024 & NC & 9460 & -0.3 & -78.3223 & 33.9003 & NC \\
\hline 9317 & -0.5 & -78.1446 & 33.9039 & NC & 9389 & 0.3 & -78.1264 & 33.9023 & $\mathrm{NC}$ & 9461 & 0.9 & -77.9406 & 33.9003 & NC \\
\hline 9318 & -0.5 & -78.1441 & 33.9039 & NC & 9390 & 0.2 & -78.3008 & 33.9023 & NC & 9462 & -0.4 & -78.324 & 33.9002 & NC \\
\hline 9319 & 0.8 & -78.2832 & 33.9039 & NC & 9391 & 0.2 & -78.3003 & 33.9023 & NC & 9463 & -0.4 & -78.3234 & 33.9002 & NC \\
\hline 9320 & -0.7 & -77.9393 & 33.9039 & NC & 9392 & 0.2 & -78.3014 & 33.9022 & NC & 9464 & -0.2 & -78.1132 & 33.9002 & NC \\
\hline 9321 & -0.5 & -78.1435 & 33.9038 & NC & 9393 & 0.4 & -78.1258 & 33.9022 & NC & 9465 & -0.1 & -78.1127 & 33.9001 & NC \\
\hline 9322 & -0.5 & -78.143 & 33.9038 & NC & 9394 & 0.1 & -78.3019 & 33.9022 & NC & 9466 & -0.4 & -78.3251 & 33.9001 & NC \\
\hline 9323 & -0.5 & -78.1424 & 33.9038 & NC & 9395 & 0.1 & -78.3025 & 33.9021 & NC & 9467 & -0.4 & -78.3245 & 33.9001 & NC \\
\hline 9324 & 0.8 & -78.2843 & 33.9038 & NC & 9396 & 0.3 & -78.1253 & 33.9021 & NC & 9468 & -0.4 & -78.3256 & 33.9 & NC \\
\hline 9325 & 0.8 & -78.2838 & 33.9038 & NC & 9397 & 0.3 & -78.1247 & 33.9021 & NC & 9469 & -0.1 & -78.1122 & 33.9 & NC \\
\hline 9326 & 0.7 & -78.2854 & 33.9037 & NC & 9398 & 0 & -78.3031 & 33.9021 & NC & 9470 & -0.4 & -78.3262 & 33.9 & NC \\
\hline 9327 & 0.7 & -78.2849 & 33.9037 & NC & 9399 & 0 & -78.303 & 33.9021 & NC & 9471 & -0.1 & -78.1116 & 33.8999 & NC \\
\hline 9328 & -0.5 & -78.1419 & 33.9037 & NC & 9400 & 0.3 & -77.9399 & 33.9021 & NC & 9472 & -0.4 & -78.3273 & 33.8999 & NC \\
\hline 9329 & -0.5 & -78.1413 & 33.9037 & NC & 9401 & 0.1 & -78.3036 & 33.902 & NC & 9473 & -0.3 & -78.3267 & 33.8999 & NC \\
\hline 9330 & -0.5 & -78.1408 & 33.9036 & NC & 9402 & 0.1 & -78.3036 & 33.902 & NC & 9474 & 1 & -77.9407 & 33.8999 & NC \\
\hline 9331 & -0.4 & -78.1402 & 33.9036 & NC & 9403 & 0.1 & -78.1242 & 33.902 & NC & 9475 & -0.3 & -78.3278 & 33.8998 & NC \\
\hline 9332 & -0.5 & -78.1397 & 33.9036 & NC & 9404 & 0.1 & -78.3042 & 33.902 & NC & 9476 & -0.2 & -78.1111 & 33.8998 & NC \\
\hline 9333 & 0.7 & -78.2865 & 33.9036 & NC & 9405 & 0.2 & -78.1236 & 33.9019 & NC & 9477 & -0.3 & -78.3284 & 33.8998 & NC \\
\hline 9334 & 0.7 & -78.286 & 33.9036 & NC & 9406 & 0.1 & -78.3053 & 33.9019 & NC & 9478 & -0.3 & -78.3295 & 33.8997 & NC \\
\hline 9335 & -0.6 & -78.1386 & 33.9035 & NC & 9407 & 0.1 & -78.3047 & 33.9019 & NC & 9479 & -0.3 & -78.3289 & 33.8997 & NC \\
\hline 9336 & 0.6 & -78.2876 & 33.9035 & NC & 9408 & 0 & -78.3058 & 33.9018 & NC & 9480 & -0.2 & -78.1105 & 33.8997 & NC \\
\hline 9337 & 0.6 & -78.2871 & 33.9035 & NC & 9409 & 0.2 & -78.1231 & 33.9018 & NC & 9481 & -0.3 & -78.11 & 33.8996 & NC \\
\hline 9338 & -0.6 & -78.1391 & 33.9035 & NC & 9410 & -0.1 & -78.3064 & 33.9018 & NC & 9482 & -0.4 & -78.3306 & 33.8996 & NC \\
\hline 9339 & 0.6 & -78.2882 & 33.9034 & NC & 9411 & 0.2 & -78.1225 & 33.9017 & NC & 9483 & -0.4 & -78.33 & 33.8996 & NC \\
\hline 9340 & -0.6 & -78.138 & 33.9034 & NC & 9412 & 0 & -78.3075 & 33.9017 & NC & 9484 & -0.5 & -78.3311 & 33.8995 & NC \\
\hline 9341 & -0.6 & -78.1374 & 33.9034 & NC & 9413 & 0 & -78.3069 & 33.9017 & NC & 9485 & -0.2 & -78.1094 & 33.8995 & NC \\
\hline 9342 & -0.6 & -78.1369 & 33.9034 & NC & 9414 & 0.5 & -77.9401 & 33.9017 & NC & 9486 & -0.5 & -78.3317 & 33.8995 & NC \\
\hline 9343 & 0.6 & -78.2887 & 33.9034 & NC & 9415 & 0.1 & -78.308 & 33.9016 & NC & 9487 & -0.2 & -78.1089 & 33.8994 & NC \\
\hline 9344 & -0.4 & -77.9394 & 33.9034 & NC & 9416 & 0 & -78.122 & 33.9016 & NC & 9488 & -0.3 & -78.1083 & 33.8994 & NC \\
\hline 9345 & 0.6 & -78.2893 & 33.9033 & NC & 9417 & 0 & -78.3086 & 33.9016 & NC & 9489 & -0.6 & -78.3328 & 33.8994 & NC \\
\hline 9346 & -0.6 & -78.1363 & 33.9033 & NC & 9418 & 0.1 & -78.3097 & 33.9015 & NC & 9490 & -0.6 & -78.3322 & 33.8994 & NC \\
\hline 9347 & -0.7 & -78.1358 & 33.9033 & NC & 9419 & 0.1 & -78.3091 & 33.9015 & NC & 9491 & 1 & -77.9409 & 33.8994 & NC \\
\hline 9348 & 0.5 & -78.2898 & 33.9033 & NC & 9420 & 0 & -78.1214 & 33.9015 & NC & 9492 & -0.6 & -78.3339 & 33.8993 & NC \\
\hline 9349 & 0.6 & -78.2904 & 33.9032 & NC & 9421 & -0.1 & -78.1209 & 33.9014 & NC & 9493 & -0.6 & -78.3333 & 33.8993 & NC \\
\hline 9350 & -0.6 & -78.1352 & 33.9032 & NC & 9422 & 0 & -78.3108 & 33.9014 & NC & 9494 & -0.3 & -78.1078 & 33.8993 & NC \\
\hline 9351 & -0.6 & -78.1347 & 33.9032 & NC & 9423 & 0.1 & -78.3102 & 33.9014 & NC & 9495 & -0.3 & -78.1072 & 33.8992 & NC \\
\hline 9352 & -0.7 & -78.1341 & 33.9032 & NC & 9424 & 0 & -78.3113 & 33.9013 & NC & 9496 & -0.6 & -78.335 & 33.8992 & NC \\
\hline 9353 & 0.5 & -78.2909 & 33.9032 & NC & 9425 & 0 & -78.1203 & 33.9013 & NC & 9497 & -0.5 & -78.3344 & 33.8992 & NC \\
\hline 9354 & 0.5 & -78.292 & 33.9031 & NC & 9426 & 0 & -78.3119 & 33.9013 & NC & 9498 & -0.5 & -78.3355 & 33.8991 & NC \\
\hline 9355 & 0.5 & -78.2915 & 33.9031 & NC & 9427 & 0 & -78.1198 & 33.9012 & NC & 9499 & -0.3 & -78.1067 & 33.8991 & NC \\
\hline 9356 & -0.6 & -78.1336 & 33.9031 & NC & 9428 & 0 & -78.1193 & 33.9012 & NC & 9500 & -0.5 & -78.3361 & 33.8991 & NC \\
\hline 9357 & -0.7 & -78.133 & 33.9031 & NC & 9429 & -0.1 & -78.313 & 33.9012 & NC & 9501 & -0.3 & -78.1061 & 33.899 & NC \\
\hline 9358 & 0.6 & -78.2926 & 33.903 & NC & 9430 & -0.1 & -78.3124 & 33.9012 & NC & 9502 & -0.7 & -78.3372 & 33.899 & NC \\
\hline 9359 & -0.6 & -78.1325 & 33.903 & NC & 9431 & 0.6 & -77.9402 & 33.9012 & NC & 9503 & -0.6 & -78.3366 & 33.899 & $\mathrm{NC}$ \\
\hline
\end{tabular}


TR\#

RATE LONG LAT ST TR\#

RATE

LONG LAT

\begin{tabular}{|c|c|c|c|c|c|c|c|c|c|c|c|c|c|c|}
\hline 9504 & 1.1 & -77.9411 & 33.899 & NC & 9576 & NA & -78.3768 & 33.8967 & $\mathrm{NC}$ & 9648 & -3.2 & -78.3847 & 33.8943 & NC \\
\hline 9505 & -0.6 & -78.3377 & 33.8989 & NC & 9577 & 0.4 & -78.3567 & 33.8967 & $\mathrm{NC}$ & 9649 & -1.6 & -77.9428 & 33.8943 & NC \\
\hline 9506 & -0.4 & -78.1056 & 33.8989 & NC & 9578 & 0.7 & -78.3578 & 33.8966 & $\mathrm{NC}$ & 9650 & -1.7 & -78.0818 & 33.8942 & NC \\
\hline 9507 & -0.7 & -78.3383 & 33.8989 & NC & 9579 & 0.6 & -78.3573 & 33.8966 & $\mathrm{NC}$ & 9651 & -2 & -78.3852 & 33.8941 & NC \\
\hline 9508 & -0.8 & -78.3394 & 33.8988 & NC & 9580 & -0.6 & -78.0914 & 33.8966 & $\mathrm{NC}$ & 9652 & -1.5 & -78.0812 & 33.8941 & NC \\
\hline 9509 & -0.7 & -78.3388 & 33.8988 & $\mathrm{NC}$ & 9581 & -0.6 & -78.0908 & 33.8965 & $\mathrm{NC}$ & 9653 & -1.4 & -78.0807 & 33.894 & NC \\
\hline 9510 & -0.4 & -78.105 & 33.8988 & $\mathrm{NC}$ & 9582 & 0.8 & -78.3583 & 33.8965 & $\mathrm{NC}$ & 9654 & -1.5 & -78.0802 & 33.8939 & $\mathrm{NC}$ \\
\hline 9511 & -0.4 & -78.1045 & 33.8987 & $\mathrm{NC}$ & 9583 & 0.8 & -78.3589 & 33.8964 & $\mathrm{NC}$ & 9655 & -1.2 & -78.3857 & 33.8939 & NC \\
\hline 9512 & -0.9 & -78.3405 & 33.8987 & $\mathrm{NC}$ & 9584 & -0.7 & -78.0903 & 33.8964 & $\mathrm{NC}$ & 9656 & -1.6 & -77.9429 & 33.8939 & NC \\
\hline 9513 & -0.8 & -78.3399 & 33.8987 & NC & 9585 & 0.9 & -78.3594 & 33.8964 & NC & 9657 & -0.5 & -78.3862 & 33.8938 & NC \\
\hline 9514 & -0.6 & -78.3404 & 33.8986 & NC & 9586 & 1.7 & -77.942 & 33.8964 & $\mathrm{NC}$ & 9658 & -1.4 & -78.0796 & 33.8937 & NC \\
\hline 9515 & -0.9 & -78.341 & 33.8986 & $\mathrm{NC}$ & 9587 & -0.8 & -78.0897 & 33.8963 & $\mathrm{NC}$ & 9659 & -1.5 & -78.0791 & 33.8936 & NC \\
\hline 9516 & -0.4 & -78.104 & 33.8986 & $\mathrm{NC}$ & 9588 & 1.4 & -78.3605 & 33.8963 & $\mathrm{NC}$ & 9660 & -0.3 & -78.3867 & 33.8936 & NC \\
\hline 9517 & 1.3 & -77.9412 & 33.8986 & $\mathrm{NC}$ & 9589 & 1.1 & -78.36 & 33.8963 & $\mathrm{NC}$ & 9661 & -0.3 & -78.3873 & 33.8935 & $\mathrm{NC}$ \\
\hline 9518 & -0.4 & -78.1034 & 33.8985 & $\mathrm{NC}$ & 9590 & -0.6 & -78.0903 & 33.8962 & $\mathrm{NC}$ & 9662 & -1.5 & -78.0786 & 33.8935 & NC \\
\hline 9519 & -0.3 & -78.1029 & 33.8985 & NC & 9591 & 1.6 & -78.3611 & 33.8962 & $\mathrm{NC}$ & 9663 & -1.6 & -78.078 & 33.8934 & NC \\
\hline 9520 & -0.6 & -78.3415 & 33.8985 & $\mathrm{NC}$ & 9592 & -0.9 & -78.0892 & 33.8962 & NC & 9664 & -1.5 & -77.943 & 33.8934 & NC \\
\hline 9521 & -0.6 & -78.3409 & 33.8985 & $\mathrm{NC}$ & 9593 & -0.7 & -78.0898 & 33.8961 & $\mathrm{NC}$ & 9665 & -0.2 & -78.3878 & 33.8933 & NC \\
\hline 9522 & -0.3 & -78.1023 & 33.8984 & $\mathrm{NC}$ & 9594 & 2.1 & -78.3621 & 33.8961 & $\mathrm{NC}$ & 9666 & -1.6 & -78.0775 & 33.8932 & NC \\
\hline 9523 & -0.5 & -78.342 & 33.8984 & $\mathrm{NC}$ & 9595 & 1.9 & -78.3616 & 33.8961 & $\mathrm{NC}$ & 9667 & -0.2 & -78.3883 & 33.8931 & NC \\
\hline 9524 & -0.4 & -78.1018 & 33.8983 & $\mathrm{NC}$ & 9596 & 2.1 & -78.3627 & 33.896 & $\mathrm{NC}$ & 9668 & -1.5 & -78.0769 & 33.8931 & $\mathrm{NC}$ \\
\hline 9525 & -0.5 & -78.3431 & 33.8983 & $\mathrm{NC}$ & 9597 & -0.7 & -78.0893 & 33.896 & $\mathrm{NC}$ & 9669 & -0.1 & -78.3888 & 33.893 & NC \\
\hline 9526 & -0.5 & -78.3426 & 33.8983 & $\mathrm{NC}$ & 9598 & -0.8 & -78.0887 & 33.8959 & $\mathrm{NC}$ & 9670 & -1.5 & -78.0764 & 33.893 & NC \\
\hline 9527 & -0.5 & -78.3437 & 33.8982 & $\mathrm{NC}$ & 9599 & 2.3 & -78.3638 & 33.8959 & NC & 9671 & -1.6 & -77.9431 & 33.893 & NC \\
\hline 9528 & -0.4 & -78.1012 & 33.8982 & $\mathrm{NC}$ & 9600 & 2.1 & -78.3632 & 33.8959 & $\mathrm{NC}$ & 9672 & -1.5 & -78.0759 & 33.8929 & NC \\
\hline 9529 & -0.4 & -78.3442 & 33.8982 & $\mathrm{NC}$ & 9601 & 1.6 & -77.9422 & 33.8959 & $\mathrm{NC}$ & 9673 & -0.1 & -78.3894 & 33.8928 & $\mathrm{NC}$ \\
\hline 9530 & -0.4 & -78.1007 & 33.8981 & $\mathrm{NC}$ & 9602 & 2.5 & -78.3643 & 33.8958 & $\mathrm{NC}$ & 9674 & -1.4 & -78.0753 & 33.8927 & NC \\
\hline 9531 & -0.5 & -78.3447 & 33.8981 & $\mathrm{NC}$ & 9603 & 2.9 & -78.3649 & 33.8957 & NC & 9675 & -1.5 & -78.0748 & 33.8926 & NC \\
\hline 9532 & 1.3 & -77.9414 & 33.8981 & $\mathrm{NC}$ & 9604 & -0.8 & -78.0882 & 33.8957 & NC & 9676 & -0.4 & -78.3899 & 33.8926 & NC \\
\hline 9533 & -0.4 & -78.3453 & 33.898 & $\mathrm{NC}$ & 9605 & 3.1 & -78.3654 & 33.8957 & $\mathrm{NC}$ & 9677 & -1.4 & -78.0743 & 33.8925 & NC \\
\hline 9534 & -0.4 & -78.1001 & 33.898 & $\mathrm{NC}$ & 9606 & 2.7 & -78.3665 & 33.8956 & $\mathrm{NC}$ & 9678 & -0.6 & -78.3904 & 33.8925 & NC \\
\hline 9535 & -0.4 & -78.3458 & 33.898 & $\mathrm{NC}$ & 9607 & 2.9 & -78.366 & 33.8956 & $\mathrm{NC}$ & 9679 & -1.8 & -77.9432 & 33.8925 & NC \\
\hline 9536 & -0.5 & -78.0996 & 33.8979 & NC & 9608 & -0.9 & -78.0877 & 33.8956 & $\mathrm{NC}$ & 9680 & -1.3 & -78.0737 & 33.8924 & NC \\
\hline 9537 & -0.4 & -78.3464 & 33.8979 & NC & 9609 & -0.8 & -78.0871 & 33.8955 & $\mathrm{NC}$ & 9681 & -0.6 & -78.3909 & 33.8923 & NC \\
\hline 9538 & -0.4 & -78.099 & 33.8978 & NC & 9610 & 2.3 & -78.367 & 33.8955 & $\mathrm{NC}$ & 9682 & -1.4 & -78.0732 & 33.8922 & NC \\
\hline 9539 & -0.3 & -78.3475 & 33.8978 & $\mathrm{NC}$ & 9611 & 1.6 & -77.9424 & 33.8955 & NC & 9683 & -0.9 & -78.3914 & 33.8922 & NC \\
\hline 9540 & -0.3 & -78.3469 & 33.8978 & $\mathrm{NC}$ & 9612 & 1.9 & -78.3676 & 33.8954 & $\mathrm{NC}$ & 9684 & -1.4 & .78 .0727 & 33.8921 & NC \\
\hline 9541 & -0.2 & -78.348 & 33.8977 & NC & 9613 & -1 & -78.0866 & 33.8954 & $\mathrm{NC}$ & 9685 & -1.1 & -78.392 & 33.892 & NC \\
\hline 9542 & -0.4 & -78.0985 & 33.8977 & NC & 9614 & 1.6 & -78.3681 & 33.8954 & $\mathrm{NC}$ & 9686 & -1.4 & -78.0721 & 33.892 & NC \\
\hline 9543 & 1.4 & -77.9415 & 33.8977 & NC & 9615 & 1.5 & -78.3687 & 33.8953 & $\mathrm{NC}$ & 9687 & -1.8 & -77.9433 & 33.892 & NC \\
\hline 9544 & -0.4 & -78.0979 & 33.8976 & $\mathrm{NC}$ & 9616 & 1.2 & -78.3698 & 33.8952 & NC & 9688 & -1.3 & -78.0716 & 33.8919 & NC \\
\hline 9545 & -0.4 & -78.0974 & 33.8976 & $\mathrm{NC}$ & 9617 & 1.4 & -78.3692 & 33.8952 & $\mathrm{NC}$ & 9689 & -1.3 & -78.3925 & 33.8918 & NC \\
\hline 9546 & 0 & -78.3491 & 33.8976 & NC & 9618 & -1.2 & -78.086 & 33.8952 & $\mathrm{NC}$ & 9690 & -1.3 & -78.393 & 33.8917 & NC \\
\hline 9547 & -0.1 & -78.3486 & 33.8976 & NC & 9619 & -5.2 & -78.3815 & 33.8952 & $\mathrm{NC}$ & 9691 & -1.4 & -78.0711 & 33.8917 & NC \\
\hline 9548 & 0.1 & -78.3502 & 33.8975 & NC & 9620 & -5.2 & -78.382 & 33.8951 & $\mathrm{NC}$ & 9692 & -1.4 & -78.0705 & 33.8916 & NC \\
\hline 9549 & 0.1 & -78.3496 & 33.8975 & NC & 9621 & 0.7 & -78.3708 & 33.8951 & $\mathrm{NC}$ & 9693 & -1.9 & .77 .9434 & 33.8916 & NC \\
\hline 9550 & -0.5 & -78.0969 & 33.8975 & NC & 9622 & 0.9 & -78.3703 & 33.8951 & $\mathrm{NC}$ & 96 & -1.3 & -78.07 & 33.8915 & NC \\
\hline 9551 & 0 & -78.3507 & 33.8974 & NC & 9623 & -1.2 & -78.0855 & 33.8951 & $\mathrm{NC}$ & 95 & -1.5 & -78.3935 & 33.8915 & NC \\
\hline 9552 & -0.5 & -78.0963 & 33.8974 & $\mathrm{NC}$ & 9624 & 0.2 & -78.3714 & 33.895 & $\mathrm{NC}$ & 9696 & -1.4 & -78.0695 & 33.8914 & NC \\
\hline 9553 & 0.1 & -78.3513 & 33.8973 & NC & & -1.1 & -78.085 & 33.895 & $\mathrm{NC}$ & & -1.4 & & 33.8913 & NC \\
\hline 9554 & -0.5 & -78.0958 & 33.8973 & NC & 9626 & 1.6 & -77.9425 & 33.895 & NC & 969 & -1.5 & -78.0689 & 33.8912 & NC \\
\hline 9555 & 0.1 & -78.3518 & 33.8973 & NC & 96 & -0.7 & -78.3725 & 33.8949 & $\mathrm{NC}$ & & -1.1 & & 33.8912 & NC \\
\hline 9556 & -0.6 & -78.0952 & 33.8972 & NC & 9628 & -0.2 & -78.3719 & 33.8949 & $\mathrm{NC}$ & 970 & -1.5 & -78.0684 & 33.8911 & NC \\
\hline 9557 & NA & -78.3752 & 33.8972 & NC & 9629 & -1.2 & -78.0844 & 33.8949 & $\mathrm{NC}$ & & -1.9 & -77.9435 & 33.8911 & NC \\
\hline 9558 & 0.1 & -78.3524 & 33.8972 & NC & 9630 & -5.4 & -78.3826 & 33.8949 & NC & & -0.8 & -78.3951 & 33.891 & NC \\
\hline 9559 & 1.4 & -77.9417 & 33.8972 & NC & & -1.7 & & 33.8 & $\mathrm{NC}$ & & -1.4 & -78.0679 & 33.891 & $\mathrm{NC}$ \\
\hline 9560 & 0.1 & -78.3529 & 33.8971 & $\mathrm{NC}$ & & -1.4 & -78.3 & 33.8948 & $\mathrm{NC}$ & & -1.4 & -78.0673 & 33.8909 & NC \\
\hline 9561 & -0.6 & -78.0947 & 33.8971 & NC & 9633 & -1.2 & -78.0839 & 33.8947 & NC & 970 & -0.4 & -78.3956 & 33.8909 & NC \\
\hline 9562 & 0.2 & -78.3534 & 33.8971 & NC & & -5.1 & & 33.8947 & $\mathrm{NC}$ & & -1.3 & & 33.8907 & NC \\
\hline 9563 & -0.6 & -78.0941 & 33.897 & $\mathrm{NC}$ & & -2.7 & -78.3741 & 33.8947 & $\mathrm{NC}$ & & -0.2 & & & NC \\
\hline 9564 & NA & & 33.8 & NC & & -1.7 & & 33.8 & NC & & -1.9 & & & NC \\
\hline 9565 & 0.3 & -78.3545 & 33.897 & $\mathrm{NC}$ & & -4.7 & -78.3836 & 33.8946 & $\mathrm{NC}$ & & -1.3 & & 33.8906 & NC \\
\hline 9566 & 0.3 & -78.354 & 33.897 & $\mathrm{NC}$ & 96 & -1.1 & -78.3747 & 33.8946 & $\mathrm{NC}$ & 97 & -1.3 & -78.0657 & 33.8905 & NC \\
\hline 9567 & -0.5 & -78.0936 & 33.8969 & $\mathrm{NC}$ & 9639 & -1.4 & -78.0834 & 33.8946 & $\mathrm{NC}$ & & 0 & -78.3967 & 33.8905 & NC \\
\hline 9568 & -27 & -78.3763 & 33.8969 & $\mathrm{NC}$ & 9640 & 1.7 & -77.9427 & 33.8946 & $\mathrm{NC}$ & 9712 & 0.2 & -78.3972 & 33.8904 & NC \\
\hline 9569 & 0.2 & -78.3551 & 33.8969 & $\mathrm{NC}$ & 964 & -1.7 & -78.0828 & 33.8945 & $\mathrm{NC}$ & 9713 & -1.2 & -78.0652 & 33.8904 & NC \\
\hline 9570 & 0.3 & -78.3562 & 33.8968 & $\mathrm{NC}$ & 964 & -0.2 & -78.3757 & 33.8945 & $\mathrm{NC}$ & 9714 & 0.4 & -78.3977 & & NC \\
\hline 9571 & 0.3 & -78.3556 & 33.8968 & NC & 9643 & -0.7 & -78.3752 & 33.8945 & $\mathrm{NC}$ & 9715 & -1.1 & -78.0646 & 33.8902 & NC \\
\hline 9572 & -0.6 & -78.093 & 33.8968 & $\mathrm{NC}$ & 9644 & -1.6 & -78.0823 & 33.8944 & $\mathrm{NC}$ & 9716 & -2 & -77.9438 & 33.8902 & NC \\
\hline 9573 & 1.6 & -77.9419 & 33.8968 & $\mathrm{NC}$ & & -3.9 & & 33.8944 & $\mathrm{NC}$ & 9717 & -1 & -78.0641 & 33.8901 & NC \\
\hline 9574 & -0.6 & -78.0925 & 33.8967 & $\mathrm{NC}$ & 9646 & 0.4 & -78.3768 & 33.8944 & $\mathrm{NC}$ & 9718 & 0.5 & -78.3982 & 33.89 & NC \\
\hline 9575 & -0.6 & -78.0919 & 33.8967 & NC & 9647 & 0.1 & -78.3763 & 33.8944 & $\mathrm{NC}$ & 9719 & -0.9 & -78.0636 & 33.89 & $\mathrm{NC}$ \\
\hline
\end{tabular}




\begin{tabular}{|c|c|c|c|c|c|c|c|c|c|c|c|c|c|c|}
\hline TR\# & RATE & LONG & LAT & ST & TR\# & RATE & LONG & LAT & ST & TR\# & RATE & LONG & LAT & ST \\
\hline 9720 & -0.8 & -78.063 & 33.8899 & NC & 9792 & 1.2 & -78.0459 & 33.8856 & NC & 9864 & 0.2 & -78.0282 & 33.8814 & $\mathrm{NC}$ \\
\hline 9721 & 0.6 & -78.3988 & 33.8899 & NC & 9793 & -0.7 & -78.4128 & 33.8856 & NC & 9865 & -0.5 & -78.4286 & 33.8814 & NC \\
\hline 9722 & -2 & -77.9439 & 33.8898 & NC & 9794 & -0.8 & -78.4133 & 33.8855 & NC & 9866 & -0.5 & -78.4292 & 33.8813 & NC \\
\hline 9723 & -0.7 & -78.0625 & 33.8897 & NC & 9795 & 1.3 & -78.0454 & 33.8854 & $\mathrm{NC}$ & 9867 & 0.4 & -78.0277 & 33.8813 & NC \\
\hline 9724 & 0.6 & -78.3993 & 33.8897 & NC & 9796 & 1.2 & -78.0448 & 33.8853 & NC & 9868 & 0.7 & -78.0272 & 33.8812 & NC \\
\hline 9725 & 0.6 & -78.3998 & 33.8896 & NC & 9797 & -0.9 & -78.4139 & 33.8853 & NC & 9869 & -0.5 & -78.4297 & 33.8812 & NC \\
\hline 9726 & -0.7 & -78.062 & 33.8896 & NC & 9798 & 1.1 & -78.0443 & 33.8852 & NC & 9870 & 1 & -78.0266 & 33.8811 & NC \\
\hline 9727 & -0.7 & -78.0614 & 33.8895 & NC & 9799 & -0.9 & -78.4144 & 33.8852 & NC & 9871 & -1.5 & -77.9458 & 33.8811 & NC \\
\hline 9728 & 0.5 & -78.4003 & 33.8894 & NC & 9800 & -1.9 & -77.9449 & 33.8852 & NC & 9872 & -0.4 & -78.4302 & 33.881 & NC \\
\hline 9729 & -0.7 & -78.0609 & 33.8894 & NC & 9801 & -1 & -78.4149 & 33.8851 & NC & 9873 & -0.4 & -78.4308 & 33.8809 & NC \\
\hline 9730 & -2.1 & -77.944 & 33.8893 & NC & 9802 & 1.1 & -78.0438 & 33.8851 & NC & 9874 & 1.3 & -78.0261 & 33.8809 & NC \\
\hline 9731 & 0.5 & -78.4008 & 33.8892 & NC & 9803 & 0.9 & .78 .0432 & 33.8849 & $\mathrm{NC}$ & 9875 & 1.6 & -78.0256 & 33.8808 & NC \\
\hline 9732 & -0.7 & -78.0604 & 33.8892 & NC & 9804 & -1 & -78.4154 & 33.8849 & $\mathrm{NC}$ & 9876 & -0.2 & -78.4313 & 33.8807 & NC \\
\hline 9733 & -0.6 & -78.0598 & 33.8891 & NC & 9805 & -0.9 & -78.416 & 33.8848 & NC & 9877 & 1.9 & -78.025 & 33.8807 & NC \\
\hline 9734 & 0.4 & -78.4014 & 33.8891 & NC & 9806 & 0.9 & -78.0427 & 33.8848 & NC & 9878 & -0.2 & -78.4318 & 33.8806 & NC \\
\hline 9735 & -0.7 & -78.0593 & 33.889 & NC & 9807 & 1 & -78.0422 & 33.8847 & NC & 9879 & 2.3 & -78.0245 & 33.8806 & NC \\
\hline 9736 & 0.3 & -78.4019 & 33.8889 & NC & 9808 & -1.8 & -77.945 & 33.8847 & NC & 9880 & -1.5 & -77.9459 & 33.8806 & $\mathrm{NC}$ \\
\hline 9737 & -0.6 & -78.0588 & 33.8889 & NC & 9809 & 0.9 & -78.0416 & 33.8846 & $\mathrm{NC}$ & 9881 & -0.1 & .78 .4323 & 33.8805 & $\mathrm{NC}$ \\
\hline 9738 & -2.1 & -77.9441 & 33.8889 & NC & 9810 & -1 & -78.4165 & 33.8846 & $\mathrm{NC}$ & 9882 & 2.7 & -78.024 & 33.8804 & NC \\
\hline 9739 & 0.3 & -78.4024 & 33.8888 & NC & 9811 & -1 & -78.417 & 33.8845 & NC & 9883 & 0 & -78.4329 & 33.8803 & NC \\
\hline 9740 & -0.5 & -78.0582 & 33.8887 & NC & 9812 & -1 & -78.4176 & 33.8844 & NC & 9884 & 3.3 & -78.0234 & 33.8803 & NC \\
\hline 9741 & 0.2 & -78.4029 & 33.8886 & NC & 9813 & 0.9 & -78.0411 & 33.8844 & NC & 9885 & 0.2 & -78.4334 & 33.8802 & NC \\
\hline 9742 & -0.4 & -78.0577 & 33.8886 & NC & 9814 & 0.8 & -78.0405 & 33.8843 & NC & 9886 & 3.7 & -78.0229 & 33.8802 & NC \\
\hline 9743 & -0.2 & -78.0571 & 33.8885 & NC & 9815 & -1.7 & -77.9451 & 33.8843 & NC & 9887 & -1.4 & -77.946 & 33.8802 & NC \\
\hline 9744 & -0.1 & -78.0566 & 33.8884 & NC & 9816 & 0.8 & -78.04 & 33.8842 & NC & 9888 & 4.3 & -78.0224 & 33.8801 & NC \\
\hline 9745 & 0.2 & -78.4035 & 33.8884 & NC & 9817 & -1 & -78.4181 & 33.8842 & NC & 9889 & 0.3 & -78.4339 & 33.88 & NC \\
\hline 9746 & -2.1 & -77.9442 & 33.8884 & NC & 9818 & -1 & -78.4186 & 33.8841 & NC & 9890 & 0.2 & -78.4345 & 33.8799 & NC \\
\hline 9747 & 0.1 & -78.404 & 33.8883 & NC & 9819 & 0.7 & -78.0395 & 33.8841 & NC & 9891 & 4.9 & -78.0218 & 33.8799 & NC \\
\hline 9748 & -0.1 & -78.0561 & 33.8882 & NC & 9820 & -1 & -78.4191 & 33.884 & NC & 9892 & 6.2 & -78.0213 & 33.8798 & NC \\
\hline 9749 & -0.3 & -78.0555 & 33.8881 & $\mathrm{NC}$ & 9821 & 0.7 & -78.0389 & 33.8839 & NC & 9893 & 0.1 & -78.435 & 33.8798 & NC \\
\hline 9750 & 0 & -78.4045 & 33.8881 & NC & 9822 & 0.6 & -78.0384 & 33.8838 & NC & 9894 & 7 & -78.0207 & 33.8797 & NC \\
\hline 9751 & -0.4 & -78.055 & 33.888 & NC & 9823 & -1 & .78 .4197 & 33.8838 & NC & 9895 & -1.5 & -77.9461 & 33.8797 & NC \\
\hline 9752 & 0 & -78.405 & 33.8879 & NC & 9824 & -1.7 & -77.9452 & 33.8838 & NC & 9896 & 0.1 & -78.4355 & 33.8796 & NC \\
\hline 9753 & -0.4 & -78.0545 & 33.8879 & NC & 9825 & -1 & -78.4202 & 33.8837 & NC & 9897 & 8.2 & -78.0202 & 33.8796 & NC \\
\hline 9754 & -2.2 & -77.9443 & 33.8879 & NC & 9826 & 0.6 & -78.0379 & 33.8837 & NC & 9898 & 0.1 & -78.436 & 33.8795 & NC \\
\hline 9755 & -0.1 & -78.4056 & 33.8878 & NC & 9827 & 0.4 & -78.0373 & 33.8836 & NC & 9899 & 9 & -78.0197 & 33.8794 & NC \\
\hline 9756 & -0.4 & -78.0539 & 33.8877 & NC & 9828 & -0.9 & -78.4207 & 33.8835 & NC & 9900 & 0.1 & -78.4366 & 33.8793 & NC \\
\hline 9757 & -0.1 & -78.4061 & 33.8876 & NC & 9829 & 0.5 & -78.0368 & 33.8834 & NC & 9901 & -1.5 & -77.9463 & 33.8793 & NC \\
\hline 9758 & -0.3 & -78.0534 & 33.8876 & NC & 9830 & -0.9 & -78.4212 & 33.8834 & NC & 9902 & 0.1 & -78.4371 & 33.8792 & NC \\
\hline 9759 & -0.3 & -78.4066 & 33.8875 & NC & 9831 & -1.6 & -77.9453 & 33.8834 & $\mathrm{NC}$ & 9903 & 0 & -78.4376 & 33.8791 & $\mathrm{NC}$ \\
\hline 9760 & -2.2 & -77.9444 & 33.8875 & NC & 9832 & -0.9 & -78.4218 & 33.8833 & $\mathrm{NC}$ & 9904 & 0 & -78.4381 & 33.8789 & $\mathrm{NC}$ \\
\hline 9761 & -0.7 & -78.0539 & 33.8874 & NC & 9833 & 0.4 & -78.0363 & 33.8833 & NC & 9905 & 0 & -78.4387 & 33.8788 & NC \\
\hline 9762 & -0.5 & -78.0534 & 33.8873 & NC & 9834 & 0.4 & -78.0357 & 33.8832 & $\mathrm{NC}$ & 9906 & -1.4 & -77.9464 & 33.8788 & NC \\
\hline 9763 & -0.3 & -78.4071 & 33.8873 & $\mathrm{NC}$ & 9835 & -0.7 & -78.4223 & 33.8831 & NC & 9907 & 0 & -78.4392 & 33.8787 & NC \\
\hline 9764 & -0.3 & -78.0529 & 33.8872 & NC & 9836 & 0.3 & -78.0352 & 33.8831 & NC & 9908 & 0.1 & -78.4397 & 33.8785 & NC \\
\hline 9765 & -0.2 & -78.0523 & 33.8871 & NC & 9837 & -0.8 & -78.4228 & 33.883 & NC & 9909 & 0.1 & -78.4403 & 33.8784 & $\mathrm{NC}$ \\
\hline 9766 & -0.4 & -78.4076 & 33.8871 & $\mathrm{NC}$ & 9838 & 0.4 & -78.0347 & 33.8829 & NC & 9910 & -1.4 & -77.9465 & 33.8784 & $\mathrm{NC}$ \\
\hline 9767 & -0.4 & -78.4082 & 33.887 & NC & 9839 & -1.6 & -77.9454 & 33.8829 & NC & 9911 & 0 & -78.4408 & 33.8782 & NC \\
\hline 9768 & -2.2 & -77.9445 & 33.887 & NC & 9840 & 0.3 & -78.0341 & 33.8828 & NC & 9912 & 0 & -78.4413 & 33.8781 & $\mathrm{NC}$ \\
\hline 9769 & -0.1 & -78.0518 & 33.8869 & NC & 9841 & -0.9 & -78.4234 & 33.8828 & NC & 9913 & -0.4 & -78.4417 & 33.8781 & $\mathrm{NC}$ \\
\hline 9770 & -0.5 & -78.4087 & 33.8868 & NC & 9842 & -0.9 & -78.4239 & 33.8827 & NC & 9914 & 0.1 & -78.4418 & 33.878 & NC \\
\hline 9771 & 0.1 & -78.0513 & 33.8868 & NC & 9843 & 0.2 & -78.0336 & 33.8827 & NC & 9915 & -0.4 & -78.4422 & 33.8779 & $\mathrm{NC}$ \\
\hline 9772 & 0.1 & -78.0507 & 33.8867 & NC & 9844 & 0.2 & -78.0331 & 33.8826 & NC & 9916 & -1.4 & -77.9466 & 33.8779 & NC \\
\hline 9773 & 0.5 & -78.0502 & 33.8866 & NC & 9845 & -0.9 & -78.4244 & 33.8826 & NC & 9917 & -0.4 & -78.4427 & 33.8778 & NC \\
\hline 9774 & -0.6 & -78.4092 & 33.8866 & NC & 9846 & -1.5 & -77.9455 & 33.8825 & NC & 9918 & 0.1 & -78.4424 & 33.8778 & NC \\
\hline 9775 & -2.1 & -77.9446 & 33.8866 & NC & 9847 & 0.1 & -78.0325 & 33.8824 & NC & 9919 & -0.3 & -78.4433 & 33.8776 & $\mathrm{NC}$ \\
\hline 9776 & -0.8 & -78.4097 & 33.8865 & NC & 9848 & -0.7 & -78.4249 & 33.8824 & NC & 9920 & -0.3 & -78.4438 & 33.8775 & $\mathrm{NC}$ \\
\hline 9777 & 0.7 & -78.0496 & 33.8864 & NC & 9849 & -0.7 & -78.4255 & 33.8823 & NC & 9921 & -1.5 & -77.9467 & 33.8774 & NC \\
\hline 9778 & -0.7 & -78.4102 & 33.8863 & NC & 9850 & 0 & -78.032 & 33.8823 & NC & 9922 & -0.3 & -78.4443 & 33.8773 & NC \\
\hline 9779 & -0.7 & .78 .4103 & 33.8863 & NC & 9851 & -0.2 & -78.0315 & 33.8822 & $\mathrm{NC}$ & 9923 & -0.3 & -78.4448 & 33.8772 & NC \\
\hline 9780 & 0.7 & -78.0491 & 33.8863 & NC & 9852 & -0.6 & -78.426 & 33.8821 & NC & 9924 & -0.2 & -78.4453 & 33.877 & NC \\
\hline 9781 & -0.7 & -78.4107 & 33.8862 & NC & 9853 & -0.2 & -78.0309 & 33.8821 & NC & 9925 & -1.6 & -77.9468 & 33.877 & NC \\
\hline 9782 & 0.7 & -78.0486 & 33.8862 & NC & 9854 & -0.6 & -78.4265 & 33.882 & NC & 9926 & -0.3 & -78.4459 & 33.8769 & $\mathrm{NC}$ \\
\hline 9783 & 0.7 & -78.048 & 33.8861 & NC & 9855 & -1.5 & -77.9456 & 33.882 & NC & 9927 & -0.3 & -78.4464 & 33.8767 & NC \\
\hline 9784 & -2 & -77.9447 & 33.8861 & NC & 9856 & -0.6 & -78.4271 & 33.8819 & NC & 9928 & -0.4 & -78.4469 & 33.8766 & NC \\
\hline 9785 & -0.8 & -78.4112 & 33.886 & NC & 9857 & -0.3 & -78.0304 & 33.8819 & NC & 9929 & -1.6 & -77.9469 & 33.8765 & NC \\
\hline 9786 & 0.6 & -78.0475 & 33.8859 & NC & 9858 & -0.2 & -78.0298 & 33.8818 & $\mathrm{NC}$ & 9930 & -0.4 & -78.4474 & 33.8764 & NC \\
\hline 9787 & -0.8 & -78.4117 & 33.8859 & NC & 9859 & -0.6 & -78.4276 & 33.8817 & NC & 9931 & -0.4 & -78.448 & 33.8763 & $\mathrm{NC}$ \\
\hline 9788 & -0.7 & -78.4123 & 33.8858 & NC & 9860 & -0.1 & -78.0293 & 33.8817 & NC & 9932 & -0.3 & -78.4485 & 33.8761 & NC \\
\hline 9789 & 0.8 & -78.047 & 33.8858 & $\mathrm{NC}$ & 9861 & -0.5 & -78.4281 & 33.8816 & NC & 9933 & -1.8 & -77.947 & 33.8761 & NC \\
\hline 9790 & 1.1 & -78.0464 & 33.8857 & NC & 9862 & 0 & -78.0288 & 33.8816 & NC & 9934 & -0.2 & -78.449 & 33.876 & NC \\
\hline 9791 & -1.9 & -77.9448 & 33.8857 & NC & 9863 & -1.5 & -77.9457 & 33.8816 & NC & 9935 & -0.2 & -78.4495 & 33.8758 & $\mathrm{NC}$ \\
\hline
\end{tabular}




\begin{tabular}{|c|c|c|c|c|c|c|c|c|c|c|c|c|c|c|}
\hline TR\# & RATE & LONG & LAT & ST & TR\# & RATE & LONG & LAT & ST & TR\# & RATE & LONG & LAT & ST \\
\hline 9936 & -0.2 & -78.45 & 33.8756 & $\mathrm{NC}$ & 10008 & -0.1 & -78.4756 & 33.8681 & $\mathrm{NC}$ & 10080 & 2.8 & -78.5057 & 33.8617 & $\mathrm{NC}$ \\
\hline 9937 & -2 & -77.9471 & 33.8756 & NC & 10009 & -3.9 & -78.4789 & 33.868 & $\mathrm{NC}$ & 10081 & 2.8 & -78.5062 & 33.8616 & $\mathrm{NC}$ \\
\hline 9938 & -0.2 & -78.4506 & 33.8755 & $\mathrm{NC}$ & 10010 & -4.2 & -78.4794 & 33.8679 & $\mathrm{NC}$ & 10082 & 3.7 & -78.5096 & 33.8615 & $\mathrm{NC}$ \\
\hline 9939 & -0.3 & -78.4511 & 33.8753 & NC & 10011 & -1.4 & -77.9489 & 33.8679 & NC & 10083 & 2.9 & -78.5067 & 33.8614 & $\mathrm{NC}$ \\
\hline 9940 & -0.3 & -78.4516 & 33.8752 & $\mathrm{NC}$ & 10012 & -3.9 & -78.48 & 33.8678 & $\mathrm{NC}$ & 10084 & 0.8 & -77.9497 & 33.8614 & $\mathrm{NC}$ \\
\hline 9941 & -2.2 & -77.9472 & 33.8752 & NC & 10013 & -3.6 & -78.4805 & 33.8677 & NC & 10085 & 2.9 & -78.5073 & 33.8613 & $\mathrm{NC}$ \\
\hline 9942 & -0.3 & -78.4521 & 33.875 & NC & 10014 & -3.6 & -78.4811 & 33.8675 & $\mathrm{NC}$ & 10086 & 3.7 & -78.51 & 33.8613 & NC \\
\hline 9943 & -0.3 & -78.4526 & 33.8749 & NC & 10015 & -3.5 & -78.4816 & 33.8674 & $\mathrm{NC}$ & 10087 & 2.9 & -78.5078 & 33.8612 & NC \\
\hline 9944 & -0.3 & -78.4532 & 33.8747 & NC & 10016 & -1 & -77.949 & 33.8674 & $\mathrm{NC}$ & 10088 & 3 & -78.5083 & 33.8611 & NC \\
\hline 9945 & -2 & -77.9473 & 33.8747 & NC & 10017 & 5.2 & -78.4821 & 33.8673 & $\mathrm{NC}$ & 10089 & 3.7 & -78.5105 & 33.8611 & NC \\
\hline 9946 & -0.3 & -78.4537 & 33.8746 & NC & 10018 & 10.4 & -78.4827 & 33.8672 & NC & 10090 & 3.7 & -78.511 & 33.8609 & $\mathrm{NC}$ \\
\hline 9947 & -0.3 & -78.4542 & 33.8744 & $\mathrm{NC}$ & 10019 & 11 & -78.4832 & 33.867 & NC & 10091 & 3.1 & -78.5089 & 33.8609 & NC \\
\hline 9948 & -0.3 & -78.4547 & 33.8743 & NC & 10020 & -0.7 & -77.9491 & 33.867 & $\mathrm{NC}$ & 10092 & 0.9 & -77.9497 & 33.8609 & $\mathrm{NC}$ \\
\hline 9949 & -2 & -77.9474 & 33.8743 & NC & 10021 & 11.2 & -78.4837 & 33.8669 & NC & 10093 & 3.1 & -78.5094 & 33.8608 & NC \\
\hline 9950 & -0.2 & -78.4552 & 33.8741 & NC & 10022 & 11.6 & -78.4843 & 33.8668 & $\mathrm{NC}$ & 10094 & 3.1 & -78.51 & 33.8607 & $\mathrm{NC}$ \\
\hline 9951 & -0.1 & -78.4558 & 33.874 & NC & 10023 & 12.1 & -78.4848 & 33.8666 & $\mathrm{NC}$ & 10095 & 3.6 & -78.5115 & 33.8606 & $\mathrm{NC}$ \\
\hline 9952 & -0.2 & -78.4563 & 33.8738 & NC & 10024 & 11.5 & -78.4853 & 33.8665 & NC & 10096 & 3.2 & -78.5105 & 33.8606 & $\mathrm{NC}$ \\
\hline 9953 & -1.9 & -77.9475 & 33.8738 & NC & 10025 & -0.4 & -77.9492 & 33.8665 & NC & 10097 & 0.9 & -77.9497 & 33.8605 & NC \\
\hline 9954 & -0.3 & -78.4568 & 33.8736 & NC & 10026 & 10.8 & -78.4859 & 33.8664 & $\mathrm{NC}$ & 10098 & 3.6 & -78.512 & 33.8604 & $\mathrm{NC}$ \\
\hline 9955 & -0.2 & -78.4573 & 33.8735 & $\mathrm{NC}$ & 10027 & 8 & -78.4864 & 33.8663 & NC & 10099 & 3.6 & -78.5125 & 33.8602 & $\mathrm{NC}$ \\
\hline 9956 & -0.1 & -78.4579 & 33.8733 & $\mathrm{NC}$ & 10028 & 5.3 & -78.4869 & 33.8661 & $\mathrm{NC}$ & 10100 & 0.9 & -77.9497 & 33.86 & $\mathrm{NC}$ \\
\hline 9957 & -1.8 & -77.9476 & 33.8733 & NC & 10029 & 4.1 & -78.4875 & 33.866 & NC & 10101 & 3.5 & -78.5129 & 33.8599 & NC \\
\hline 9958 & -0.1 & -78.4584 & 33.8732 & $\mathrm{NC}$ & 10030 & -0.1 & -77.9493 & 33.866 & NC & 10102 & 3.4 & -78.5134 & 33.8597 & $\mathrm{NC}$ \\
\hline 9959 & -0.1 & -78.4589 & 33.873 & $\mathrm{NC}$ & 10031 & 2.9 & -78.488 & 33.8659 & $\mathrm{NC}$ & 10103 & 3.3 & -78.5139 & 33.8595 & $\mathrm{NC}$ \\
\hline 9960 & -0.2 & -78.4594 & 33.8729 & $\mathrm{NC}$ & 10032 & 2.4 & -78.4885 & 33.8658 & NC & 10104 & 0.8 & -77.9498 & 33.8595 & $\mathrm{NC}$ \\
\hline 9961 & -1.8 & -77.9477 & 33.8729 & $\mathrm{NC}$ & 10033 & 1.9 & -78.4891 & 33.8656 & $\mathrm{NC}$ & 10105 & 3.3 & -78.5144 & 33.8592 & $\mathrm{NC}$ \\
\hline 9962 & -0.2 & -78.4599 & 33.8727 & $\mathrm{NC}$ & 10034 & -0.1 & -77.9494 & 33.8656 & $\mathrm{NC}$ & 10106 & 0.7 & -77.9498 & 33.8591 & $\mathrm{NC}$ \\
\hline 9963 & -0.2 & -78.4605 & 33.8726 & NC & 10035 & 1.7 & -78.4896 & 33.8655 & $\mathrm{NC}$ & 10107 & 3.1 & -78.5149 & 33.859 & $\mathrm{NC}$ \\
\hline 9964 & -0.1 & -78.461 & 33.8724 & $\mathrm{NC}$ & 10036 & 1.4 & -78.4902 & 33.8654 & NC & 10108 & 3.1 & -78.5153 & 33.8588 & $\mathrm{NC}$ \\
\hline 9965 & -1.7 & -77.9478 & 33.8724 & $\mathrm{NC}$ & 10037 & 0.9 & -78.4907 & 33.8653 & $\mathrm{NC}$ & 10109 & 3 & -78.5158 & 33.8586 & $\mathrm{NC}$ \\
\hline 9966 & 0 & -78.4615 & 33.8723 & NC & 10038 & 0.5 & -78.4912 & 33.8651 & NC & 10110 & 0.7 & .77 .9498 & 33.8586 & NC \\
\hline 9967 & 0 & -78.462 & 33.8721 & $\mathrm{NC}$ & 10039 & 0.4 & -77.9496 & 33.8651 & $\mathrm{NC}$ & 10111 & 2.9 & -78.5163 & 33.8583 & NC \\
\hline 9968 & -0.1 & -78.4625 & 33.872 & NC & 10040 & -0.2 & -77.9495 & 33.8651 & NC & 10112 & 2.8 & -78.5168 & 33.8581 & NC \\
\hline 9969 & -1.8 & -77.9479 & 33.872 & NC & 10041 & 0.2 & -78.4918 & 33.865 & NC & 10113 & 0.7 & -77.9498 & 33.8581 & NC \\
\hline 9970 & 0 & -78.4631 & 33.8718 & NC & 10042 & -0.1 & -78.4923 & 33.8649 & NC & 10114 & 2.9 & -78.5173 & 33.8579 & NC \\
\hline 9971 & 0 & -78.4636 & 33.8717 & $\mathrm{NC}$ & 10043 & -0.5 & -78.4928 & 33.8647 & NC & 10115 & 0.7 & -77.9498 & 33.8577 & NC \\
\hline 9972 & 0 & -78.4641 & 33.8715 & $\mathrm{NC}$ & 10044 & 0 & -77.9496 & 33.8647 & NC & 10116 & 2.9 & -78.5178 & 33.8576 & NC \\
\hline 9973 & -1.8 & -77.948 & 33.8715 & $\mathrm{NC}$ & 10045 & 0.5 & -77.9496 & 33.8646 & NC & 10117 & 2.9 & -78.5182 & 33.8574 & NC \\
\hline 9974 & 0.1 & -78.4646 & 33.8713 & $\mathrm{NC}$ & 10046 & -0.7 & -78.4934 & 33.8646 & NC & 10118 & 3 & -78.5187 & 33.8572 & $\mathrm{NC}$ \\
\hline 9975 & 0.1 & -78.4652 & 33.8712 & $\mathrm{NC}$ & 10047 & -1.1 & -78.4939 & 33.8645 & NC & 10119 & 0.8 & .77 .9498 & 33.8572 & NC \\
\hline 9976 & -1.7 & -77.9481 & 33.8711 & NC & 10048 & -1.4 & -78.4944 & 33.8644 & NC & 10120 & 3.5 & -78.5192 & 33.8569 & $\mathrm{NC}$ \\
\hline 9977 & 0 & -78.4657 & 33.871 & NC & 10049 & -1.4 & -78.495 & 33.8642 & NC & 10121 & 0.8 & -77.9498 & 33.8568 & $\mathrm{NC}$ \\
\hline 9978 & 0 & -78.4662 & 33.8709 & NC & 10050 & 0.6 & -77.9496 & 33.8642 & NC & 10122 & 4 & -78.5197 & 33.8567 & NC \\
\hline 9979 & 0 & .78 .4667 & 33.8707 & NC & 10051 & 0.2 & -77.9497 & 33.8642 & NC & 10123 & 4.6 & -78.5202 & 33.8565 & $\mathrm{NC}$ \\
\hline 9980 & -0.1 & -78.4672 & 33.8706 & $\mathrm{NC}$ & 10052 & -1.4 & -78.4955 & 33.8641 & $\mathrm{NC}$ & 10124 & 5 & -78.5206 & 33.8563 & $\mathrm{NC}$ \\
\hline 9981 & -1.5 & -77.9482 & 33.8706 & $\mathrm{NC}$ & 10053 & -1.2 & -78.496 & 33.864 & $\mathrm{NC}$ & 10125 & 0.9 & -77.9498 & 33.8563 & NC \\
\hline 9982 & -0.1 & -78.4678 & 33.8704 & NC & 10054 & -1 & -78.4966 & 33.8639 & $\mathrm{NC}$ & 10126 & 7 & -78.5211 & 33.856 & NC \\
\hline 9983 & 0 & -78.4683 & 33.8703 & $\mathrm{NC}$ & 10055 & 0.2 & -77.9498 & 33.8638 & $\mathrm{NC}$ & 10127 & 8.8 & -78.5216 & 33.8558 & $\mathrm{NC}$ \\
\hline 9984 & 0.1 & -78.4688 & 33.8701 & $\mathrm{NC}$ & 10056 & 0.7 & -77.9497 & 33.8637 & $\mathrm{NC}$ & 10128 & 0.9 & -77.9498 & 33.8558 & $\mathrm{NC}$ \\
\hline 9985 & -1.6 & -77.9483 & 33.8701 & $\mathrm{NC}$ & 10057 & -0.8 & -78.4971 & 33.8637 & $\mathrm{NC}$ & 10129 & 4.6 & -78.5221 & 33.8556 & $\mathrm{NC}$ \\
\hline 9986 & 0.3 & -78.4693 & 33.87 & $\mathrm{NC}$ & 10058 & -0.6 & -78.4976 & 33.8636 & $\mathrm{NC}$ & 10130 & 0.9 & -77.9499 & 33.8554 & $\mathrm{NC}$ \\
\hline 9987 & 0.7 & -78.4698 & 33.8698 & NC & 10059 & -0.3 & -78.4982 & 33.8635 & NC & 10131 & 10.6 & -78.5226 & 33.8553 & NC \\
\hline 9988 & 0.9 & -78.4704 & 33.8697 & $\mathrm{NC}$ & 10060 & 0 & -78.4987 & 33.8633 & NC & 10132 & 10.6 & -78.5231 & 33.8551 & $\mathrm{NC}$ \\
\hline 9989 & -1.8 & -77.9484 & 33.8697 & NC & 10061 & 0.3 & -77.9499 & 33.8633 & NC & 10133 & 9.4 & -78.5235 & 33.8549 & $\mathrm{NC}$ \\
\hline 9990 & 0.8 & -78.4709 & 33.8695 & $\mathrm{NC}$ & 10062 & 0.3 & -78.4992 & 33.8632 & $\mathrm{NC}$ & 10134 & 1.1 & -77.9499 & 33.8549 & $\mathrm{NC}$ \\
\hline 9991 & 0.8 & -78.4714 & 33.8693 & NC & 10063 & 0.7 & -77.9497 & 33.8632 & NC & 10135 & 7.7 & -78.524 & 33.8547 & NC \\
\hline 9992 & 0.8 & -78.4719 & 33.8692 & $\mathrm{NC}$ & 10064 & 0.6 & -78.4998 & 33.8631 & $\mathrm{NC}$ & 10136 & 1.2 & -77.9499 & 33.8545 & $\mathrm{NC}$ \\
\hline 9993 & -1.8 & -77.9485 & 33.8692 & $\mathrm{NC}$ & 10065 & 1 & -78.5003 & 33.863 & $\mathrm{NC}$ & 10137 & 5.1 & -78.5245 & 33.8544 & $\mathrm{NC}$ \\
\hline 9994 & 0.8 & -78.4724 & 33.869 & $\mathrm{NC}$ & 10066 & 1.3 & -78.5009 & 33.8628 & $\mathrm{NC}$ & 10138 & 18.9 & -78.022 & 33.8542 & $\mathrm{NC}$ \\
\hline 9995 & 0.8 & -78.473 & 33.8689 & $\mathrm{NC}$ & 10067 & 0.7 & -77.9497 & 33.8628 & NC & 10139 & 5.3 & -78.525 & 33.8542 & $\mathrm{NC}$ \\
\hline 9996 & -2 & -78.4757 & 33.8688 & $\mathrm{NC}$ & 10068 & 1.5 & -78.5014 & 33.8627 & NC & 10140 & 18.6 & -78.0215 & 33.854 & $\mathrm{NC}$ \\
\hline 9997 & -1.6 & -77.9486 & 33.8688 & $\mathrm{NC}$ & 10069 & 1.8 & -78.5019 & 33.8626 & $\mathrm{NC}$ & 10141 & 5 & -78.5255 & 33.854 & $\mathrm{NC}$ \\
\hline 9998 & -2.3 & -78.4762 & 33.8687 & $\mathrm{NC}$ & 10070 & 1.9 & -78.5025 & 33.8625 & NC & 10142 & 1.3 & -77.9499 & 33.854 & NC \\
\hline 9999 & 1 & -78.4735 & 33.8687 & $\mathrm{NC}$ & 10071 & 2 & -78.503 & 33.8623 & NC & 10143 & 18.3 & .78 .021 & 33.8538 & NC \\
\hline 10000 & 1 & -78.474 & 33.8686 & NC & 10072 & 0.7 & -77.9497 & 33.8623 & NC & 10144 & 23.9 & -78.0205 & 33.8537 & $\mathrm{NC}$ \\
\hline 10001 & -2.6 & -78.4768 & 33.8685 & NC & 10073 & 2.1 & -78.5035 & 33.8622 & NC & 10145 & 5 & -78.5259 & 33.8537 & $\mathrm{NC}$ \\
\hline 10002 & -3.7 & -78.4773 & 33.8684 & NC & 10074 & 2.3 & -78.5041 & 33.8621 & NC & 10146 & 29.5 & -78.0199 & 33.8535 & NC \\
\hline 10003 & 1 & -78.4745 & 33.8684 & NC & 10075 & 2.4 & -78.5046 & 33.862 & NC & 10147 & 4.9 & -78.5264 & 33.8535 & NC \\
\hline 10004 & 0.3 & -78.4751 & 33.8683 & $\mathrm{NC}$ & 10076 & 3.9 & -78.5086 & 33.862 & NC & 10148 & 1.4 & -77.9499 & 33.8535 & NC \\
\hline 10005 & -4.5 & -78.4778 & 33.8683 & NC & 10077 & 3.8 & -78.5091 & 33.8618 & NC & 10149 & 4.9 & -78.5269 & 33.8533 & $\mathrm{NC}$ \\
\hline 10006 & -1.5 & -77.9488 & 33.8683 & $\mathrm{NC}$ & 10078 & 2.7 & -78.5051 & 33.8618 & NC & 10150 & 32.6 & -78.0194 & 33.8533 & $\mathrm{NC}$ \\
\hline 10007 & -3.5 & -78.4784 & 33.8682 & $\mathrm{NC}$ & 10079 & 0.8 & -77.9497 & 33.8618 & NC & 10151 & 35.6 & -78.0189 & 33.8531 & NC \\
\hline
\end{tabular}




\begin{tabular}{|c|c|c|c|c|c|c|c|c|c|c|c|c|c|c|}
\hline TR\# & RATE & LONG & LAT & ST & TR\# & RATE & LONG & LAT & ST & TR\# & RATE & LONG & LAT & ST \\
\hline 10152 & 1.5 & -77.9499 & 33.8531 & NC & 10224 & -0.9 & -78.0015 & 33.8471 & NC & 10296 & -2.2 & -77.9785 & 33.8379 & $\mathrm{NC}$ \\
\hline 10153 & 22.9 & -78.0184 & 33.853 & NC & 10225 & 11.1 & -78.5399 & 33.8471 & $\mathrm{NC}$ & 10297 & -2.3 & -77.9781 & 33.8377 & $\mathrm{NC}$ \\
\hline 10154 & 5 & -78.5274 & 33.853 & NC & 10226 & 3.1 & -77.9501 & 33.8471 & NC & 10298 & -2.4 & -77.9776 & 33.8374 & NC \\
\hline 10155 & 4.9 & -78.5279 & 33.8528 & NC & 10227 & -0.9 & -78.001 & 33.8469 & NC & 10299 & -2.6 & -77.9771 & 33.8372 & NC \\
\hline 10156 & -7.3 & -78.0179 & 33.8528 & NC & 10228 & 12.3 & -78.5404 & 33.8468 & NC & 10300 & -2.7 & -77.9766 & 33.837 & NC \\
\hline 10157 & 4.8 & -78.5283 & 33.8526 & NC & 10229 & -1 & -78.0005 & 33.8467 & NC & 10301 & -2.9 & -77.9761 & 33.8367 & $\mathrm{NC}$ \\
\hline 10158 & -9.3 & -78.0174 & 33.8526 & NC & 10230 & -0.9 & -78 & 33.8466 & NC & 10302 & -3 & -77.9757 & 33.8365 & NC \\
\hline 10159 & 1.6 & -77.9499 & 33.8526 & NC & 10231 & 16 & -78.5409 & 33.8466 & NC & 10303 & -3.1 & -77.9752 & 33.8363 & NC \\
\hline 10160 & 1.5 & -78.0169 & 33.8524 & NC & 10232 & 3.3 & -77.9501 & 33.8466 & NC & 10304 & -3.1 & -77.9747 & 33.8361 & NC \\
\hline 10161 & 4.6 & -78.5288 & 33.8524 & NC & 10233 & -0.8 & -77.9994 & 33.8464 & NC & 10305 & -3.4 & -77.9742 & 33.8358 & NC \\
\hline 10162 & -0.8 & -78.0164 & 33.8522 & NC & 10234 & -0.7 & .77 .9989 & 33.8462 & NC & 10306 & -3.6 & -77.9737 & 33.8356 & $\mathrm{NC}$ \\
\hline 10163 & 4.5 & -78.5293 & 33.8521 & NC & 10235 & 3.3 & -77.9501 & 33.8461 & NC & 10307 & -3.9 & -77.9733 & 33.8354 & $\mathrm{NC}$ \\
\hline 10164 & -3.6 & -78.0158 & 33.8521 & NC & 10236 & -0.6 & -77.9984 & 33.846 & NC & 10308 & -4.1 & -77.9728 & 33.8351 & NC \\
\hline 10165 & 1.7 & -77.9499 & 33.8521 & NC & 10237 & -0.6 & -77.9979 & 33.8459 & NC & 10309 & -4.4 & -77.9723 & 33.8349 & NC \\
\hline 10166 & -3.9 & -78.0153 & 33.8519 & NC & 10238 & -0.5 & -77.9974 & 33.8457 & NC & 10310 & -4.8 & -77.9718 & 33.8347 & NC \\
\hline 10167 & 4.2 & -78.5298 & 33.8519 & NC & 10239 & 3.4 & -77.9501 & 33.8457 & NC & 10311 & -5.2 & -77.9713 & 33.8345 & NC \\
\hline 10168 & -4.3 & -78.0148 & 33.8517 & NC & 10240 & -0.7 & -77.9969 & 33.8455 & NC & 10312 & -5.6 & -77.9708 & 33.8342 & $\mathrm{NC}$ \\
\hline 10169 & 3.9 & -78.5303 & 33.8517 & NC & 10241 & -0.8 & -77.9964 & 33.8453 & NC & 10313 & -6 & -77.9704 & 33.834 & $\mathrm{NC}$ \\
\hline 10170 & 1.9 & -77.9499 & 33.8517 & NC & 10242 & 3.5 & -77.9501 & 33.8452 & NC & 10314 & -6.5 & -77.9699 & 33.8338 & NC \\
\hline 10171 & -4 & -78.0143 & 33.8515 & NC & 10243 & -0.9 & -77.9959 & 33.8451 & NC & 10315 & -7 & -77.9694 & 33.8335 & $\mathrm{NC}$ \\
\hline 10172 & -3.8 & -78.0138 & 33.8514 & NC & 10244 & -1 & -77.9953 & 33.845 & NC & 10316 & -7.5 & -77.9689 & 33.8333 & NC \\
\hline 10173 & 3.6 & -78.5308 & 33.8514 & NC & 10245 & -1 & -77.9948 & 33.8448 & NC & 10317 & -8 & -77.9684 & 33.8331 & NC \\
\hline 10174 & -3.5 & -78.0133 & 33.8512 & NC & 10246 & 3.6 & -77.9501 & 33.8447 & NC & 10318 & -8.5 & -77.968 & 33.8329 & NC \\
\hline 10175 & 3.4 & -78.5312 & 33.8512 & NC & 10247 & -1 & -77.9943 & 33.8446 & NC & 10319 & -9.2 & -77.9675 & 33.8326 & NC \\
\hline 10176 & 2 & -77.95 & 33.8512 & NC & 10248 & -1 & -77.9938 & 33.8444 & NC & 10320 & -9.9 & -77.967 & 33.8324 & NC \\
\hline 10177 & 3 & -78.5317 & 33.851 & NC & 10249 & -1 & -77.9933 & 33.8443 & NC & 10321 & 14 & -77.9665 & 33.8322 & NC \\
\hline 10178 & -3.5 & -78.0128 & 33.851 & NC & 10250 & 3.7 & -77.9501 & 33.8443 & NC & 10322 & 11.7 & -77.966 & 33.8319 & NC \\
\hline 10179 & 2.7 & -78.5322 & 33.8508 & NC & 10251 & -0.9 & -77.9928 & 33.8441 & NC & & & & & \\
\hline 10180 & -3.2 & -78.0123 & 33.8508 & NC & 10252 & -1 & -77.9923 & 33.8439 & NC & & & & & \\
\hline 10181 & 2.1 & -77.95 & 33.8508 & NC & 10253 & 4 & -77.9501 & 33.8438 & NC & & & & & \\
\hline 10182 & -2.7 & -78.0117 & 33.8507 & NC & 10254 & -0.9 & -77.9918 & 33.8437 & NC & & & & & \\
\hline 10183 & 2.7 & -78.5327 & 33.8505 & NC & 10255 & -0.8 & -77.9912 & 33.8436 & NC & & & & & \\
\hline 10184 & -2.5 & -78.0112 & 33.8505 & NC & 10256 & -0.8 & -77.9907 & 33.8434 & NC & & & & & \\
\hline 10185 & 2.8 & -78.5332 & 33.8503 & NC & 10257 & 4.4 & -77.9502 & 33.8434 & NC & & & & & \\
\hline 10186 & -2.4 & -78.0107 & 33.8503 & NC & 10258 & -0.8 & -77.9902 & 33.8432 & NC & & & & & \\
\hline 10187 & 2.2 & -77.95 & 33.8503 & NC & 10259 & -0.7 & -77.9897 & 33.843 & NC & & & & & \\
\hline 10188 & -2.4 & -78.0102 & 33.8501 & NC & 10260 & 4.6 & -77.9502 & 33.8429 & NC & & & & & \\
\hline 10189 & 2.6 & -78.5336 & 33.8501 & NC & 10261 & -0.7 & -77.9892 & 33.8428 & NC & & & & & \\
\hline 10190 & -2.3 & -78.0097 & 33.8499 & NC & 10262 & -0.6 & -77.9887 & 33.8427 & NC & & & & & \\
\hline 10191 & 2.6 & -78.5341 & 33.8498 & NC & 10263 & -0.6 & -77.9882 & 33.8425 & NC & & & & & \\
\hline 10192 & -2.3 & -78.0092 & 33.8498 & NC & 10264 & -1.8 & -77.9881 & 33.8424 & NC & & & & & \\
\hline 10193 & 2.4 & -77.95 & 33.8498 & NC & 10265 & 4.8 & -77.9502 & 33.8424 & NC & & & & & \\
\hline 10194 & -2.3 & -78.0087 & 33.8496 & NC & 10266 & -0.5 & -77.9877 & 33.8423 & NC & & & & & \\
\hline 10195 & 2.7 & -78.5346 & 33.8496 & NC & 10267 & -1.8 & -77.9877 & 33.8422 & NC & & & & & \\
\hline 10196 & -2 & -78.0082 & 33.8494 & NC & 10268 & -0.5 & -77.9871 & 33.8421 & NC & & & & & \\
\hline 10197 & 2.7 & -78.5351 & 33.8494 & NC & 10269 & -0.4 & -77.9866 & 33.842 & NC & & & & & \\
\hline 10198 & 2.6 & -77.95 & 33.8494 & NC & 10270 & -1.7 & -77.9872 & 33.842 & NC & & & & & \\
\hline 10199 & -1.8 & -78.0076 & 33.8492 & NC & 10271 & 5 & -77.9502 & 33.842 & NC & & & & & \\
\hline 10200 & -1.8 & -78.0071 & 33.8491 & NC & 10272 & -1.8 & -77.9867 & 33.8418 & NC & & & & & \\
\hline 10201 & 2.9 & -78.5356 & 33.8491 & NC & 10273 & -1.8 & -77.9862 & 33.8415 & NC & & & & & \\
\hline 10202 & -1.6 & -78.0066 & 33.8489 & NC & 10274 & 5.5 & .77 .9502 & 33.8415 & NC & & & & & \\
\hline 10203 & 3.1 & -78.5361 & 33.8489 & NC & 10275 & -2 & -77.9857 & 33.8413 & NC & & & & & \\
\hline 10204 & 2.7 & -77.95 & 33.8489 & NC & 10276 & -2 & -77.9853 & 33.8411 & NC & & & & & \\
\hline 10205 & 3.3 & -78.5365 & 33.8487 & NC & 10277 & 5.9 & -77.9502 & 33.8411 & NC & & & & & \\
\hline 10206 & -1.5 & -78.0061 & 33.8487 & NC & 10278 & -2 & -77.9848 & 33.8408 & NC & & & & & \\
\hline 10207 & -1.4 & -78.0056 & 33.8485 & NC & 10279 & -2.1 & -77.9843 & 33.8406 & NC & & & & & \\
\hline 10208 & 3.6 & -78.537 & 33.8485 & NC & 10280 & 6.4 & -77.9502 & 33.8406 & $\mathrm{NC}$ & & & & & \\
\hline 10209 & 2.8 & -77.95 & 33.8484 & NC & 10281 & -2.1 & -77.9838 & 33.8404 & NC & & & & & \\
\hline 10210 & -1.3 & -78.0051 & 33.8483 & NC & 10282 & -2.2 & -77.9833 & 33.8402 & $\mathrm{NC}$ & & & & & \\
\hline 10211 & 4.3 & -78.5375 & 33.8482 & NC & 10283 & 7.5 & -77.9502 & 33.8401 & NC & & & & & \\
\hline 10212 & -1.2 & -78.0046 & 33.8482 & NC & 10284 & -2.3 & -77.9829 & 33.8399 & NC & & & & & \\
\hline 10213 & 4.9 & -78.538 & 33.848 & NC & 10285 & -2.3 & -77.9824 & 33.8397 & NC & & & & & \\
\hline 10214 & -1.1 & -78.0041 & 33.848 & NC & 10286 & -4.7 & -77.9502 & 33.8397 & NC & & & & & \\
\hline 10215 & 2.8 & -77.95 & 33.848 & NC & 10287 & -2.3 & -77.9819 & 33.8395 & NC & & & & & \\
\hline 10216 & -1 & -78.0035 & 33.8478 & NC & 10288 & -2.3 & -77.9814 & 33.8392 & NC & & & & & \\
\hline 10217 & 6.4 & -78.5385 & 33.8478 & NC & 10289 & -4.4 & -77.9503 & 33.8392 & NC & & & & & \\
\hline 10218 & -1 & -78.003 & 33.8476 & NC & 10290 & -2.3 & -77.9809 & 33.839 & NC & & & & & \\
\hline 10219 & 8 & -78.5389 & 33.8475 & NC & 10291 & -2.3 & -77.9805 & 33.8388 & NC & & & & & \\
\hline 10220 & -1.1 & -78.0025 & 33.8475 & NC & 10292 & -4.2 & -77.9503 & 33.8387 & NC & & & & & \\
\hline 10221 & 2.9 & -77.9501 & 33.8475 & NC & 10293 & -2.3 & -77.98 & 33.8386 & NC & & & & & \\
\hline 10222 & -1 & -78.002 & 33.8473 & NC & 10294 & -2.2 & -77.9795 & 33.8383 & NC & & & & & \\
\hline 10223 & 9.6 & -78.5394 & 33.8473 & NC & 10295 & -2.2 & -77.979 & 33.8381 & NC & & & & & \\
\hline
\end{tabular}

